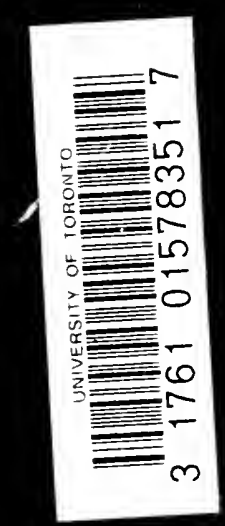




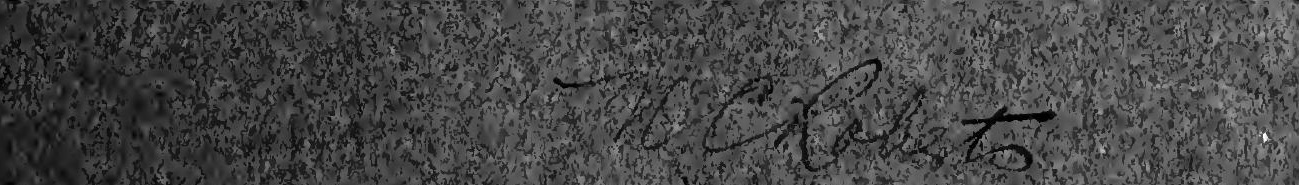

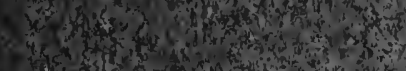

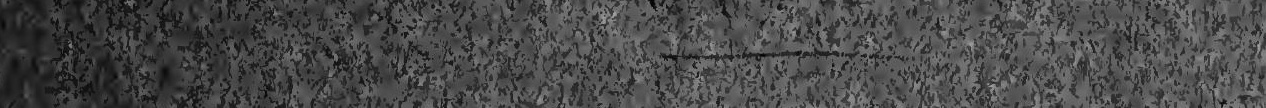

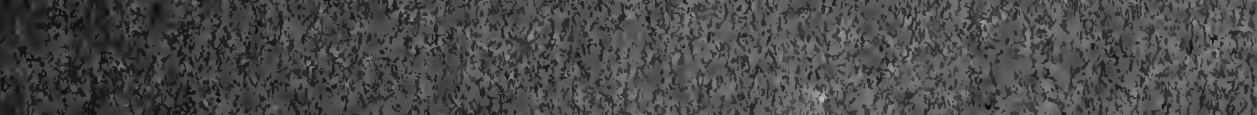
3.

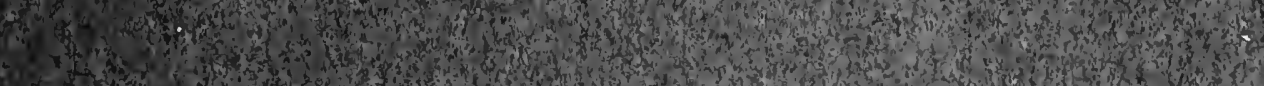

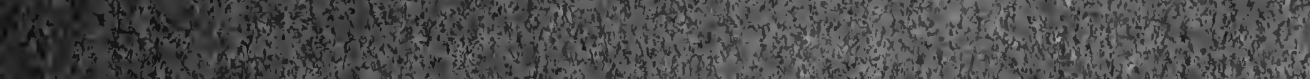

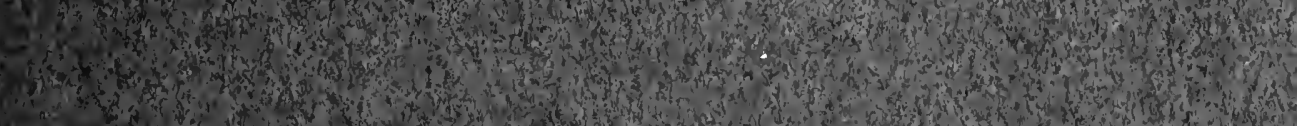

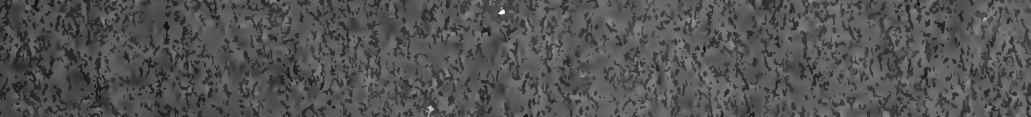

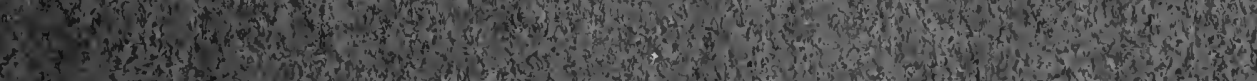

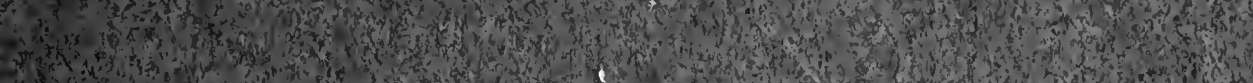

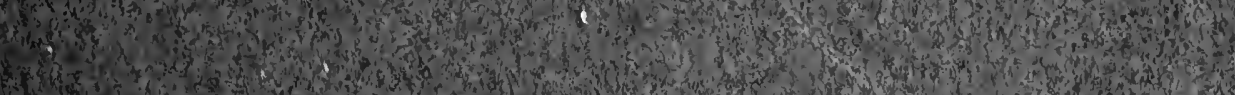
a

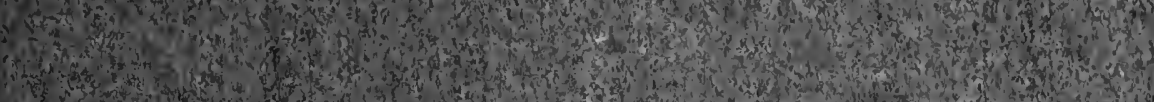
(2)

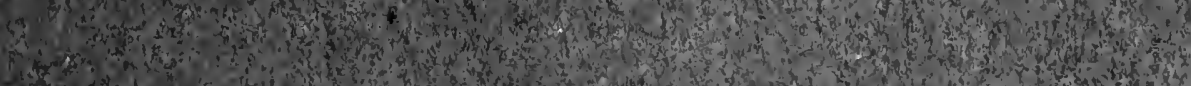

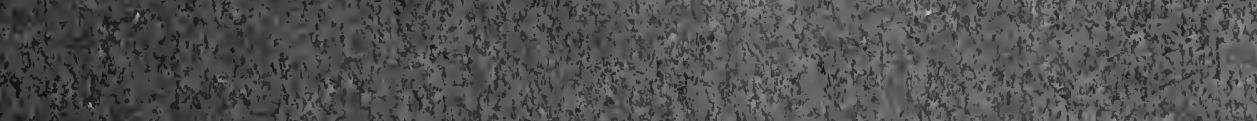
In

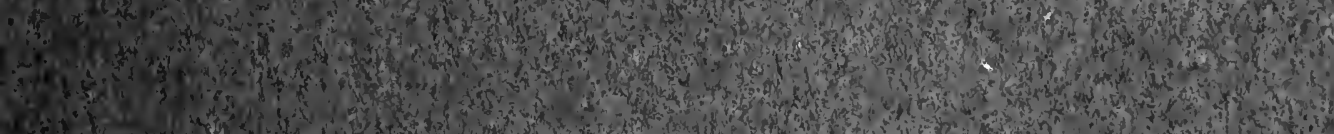

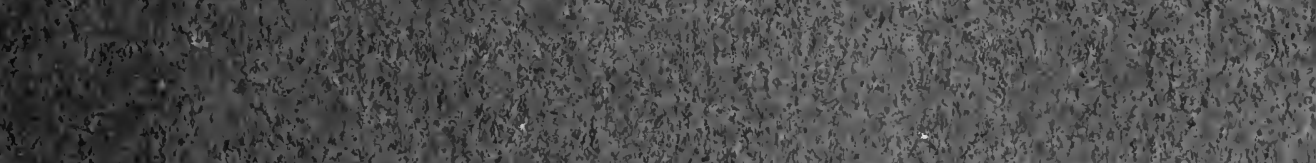

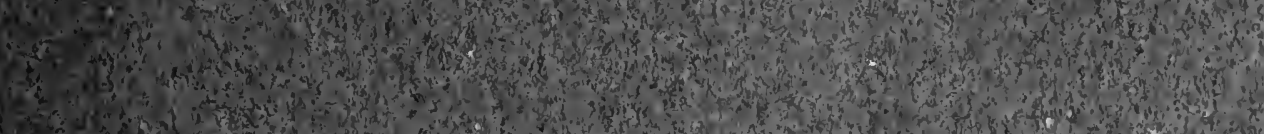

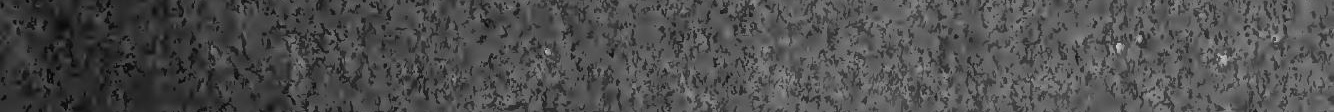

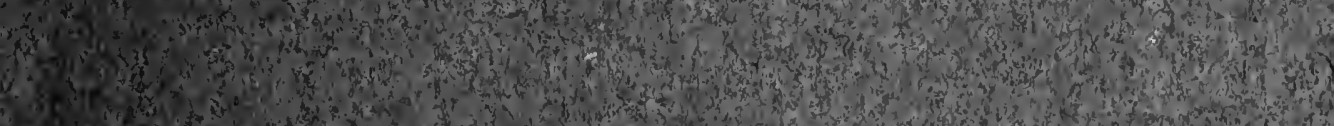
(3)

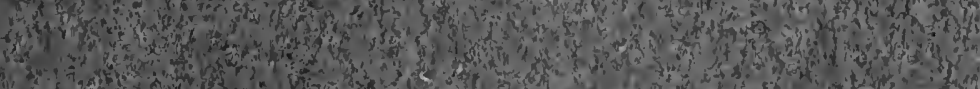

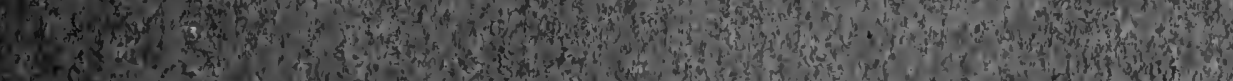
A to of 30 (f) 13. (1)

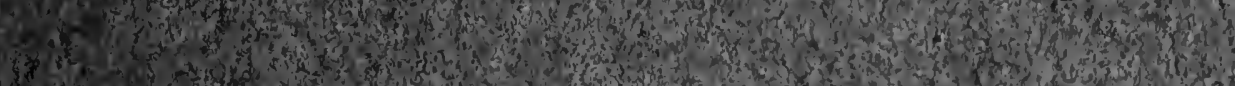
150. 19t (r) 
Digitized by the Internet'Archive in 2007 with funding from Microsoft Corporation 
M. 6. Poberts. 



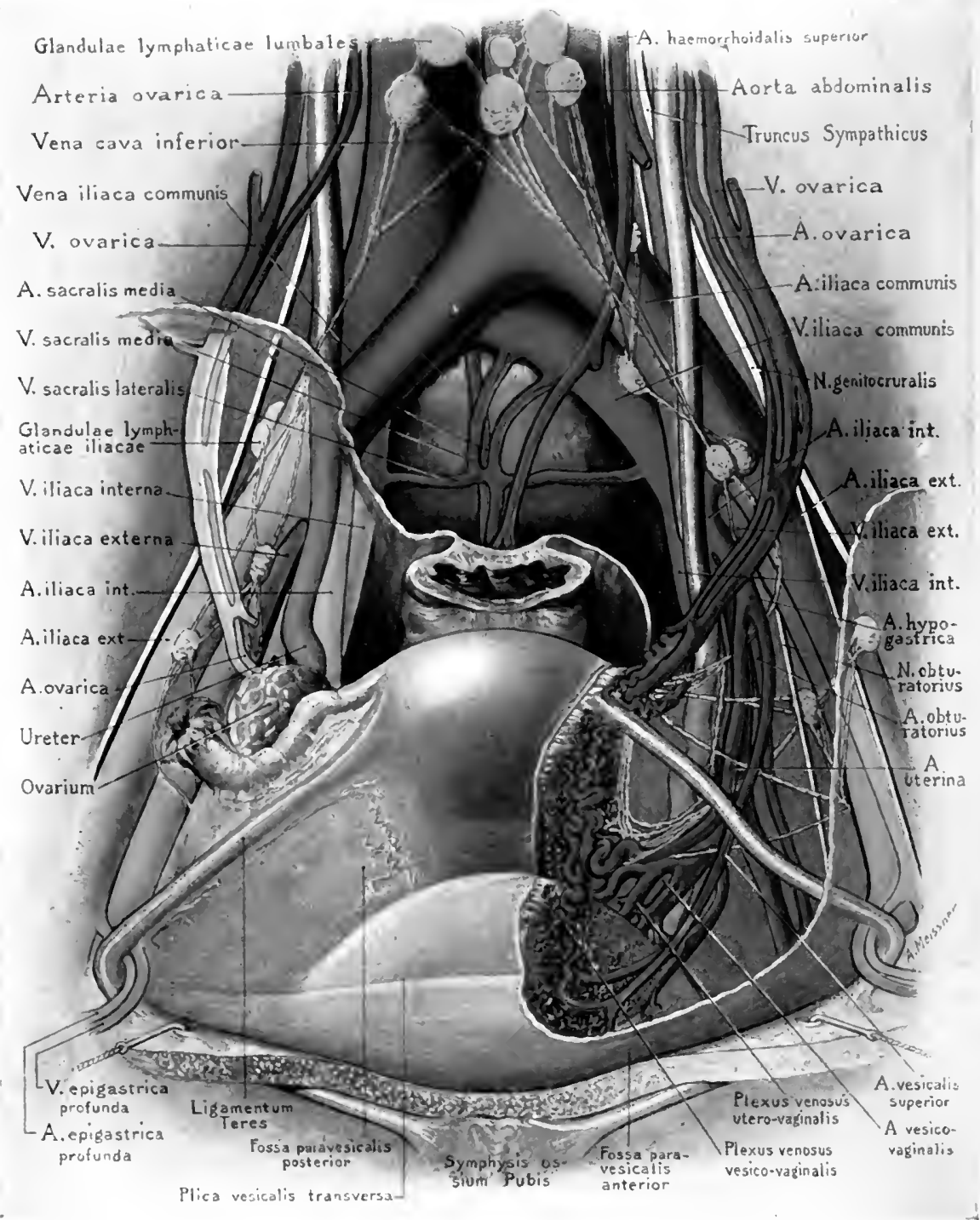

A STUDY FROM NUMEROUS DISSECTIONS AND PLATES. 
$D$

\title{
THE
}

\section{PRINCIPLES AND PRACTICE}

\section{OF \\ G Y N E C O L O G. \\ FOR}

\section{STUDENTS AND PRACTITIONERS.}

\author{
BY \\ E. C. InUDLEY, A. M., M. D.,
}

EX-PRESIDENT OF THE AMERICAN GYNECOLOGICAL SOCIETY; PROFESSOR OF GYNECOLOGY, NORTHWESTERN UNIVERSITY MIEDICAL SCIIOOL; GYNECOLOGIST TO ST. LUKE'S AND WESLEY hospitals, CHICAgo; EX-PRESIDENT OF THE CHICAgo GYNECOLOGICAL SOCIETY; ONE OF THE FOUNDERS OF CONGRES PÉRIODIQUE IXTERNATIONAL DE GYNÉCOLOGIE ET D'OBSTÉTRIQLE; FELLOW OF THE ROYAL SOCIETY OF NEDICINE, ENGLAND.

FIF'TH EDITION, REVISED AND ENLARGED.

WITH 431 ILLUSTRATIONS AND 20 FULL-PAGE PLATES IN COLORS AND MONOCHROME.
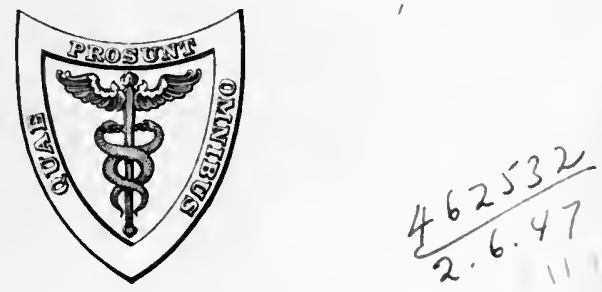

I. EA \& FEBIGER, PHILADELPHIA A ND NEW YORK. 
Entered according to Act of Congress, in the year 1908, by LEA \& FEBIGER,

In the Office of the Librarian of Congress. All rights reserved.

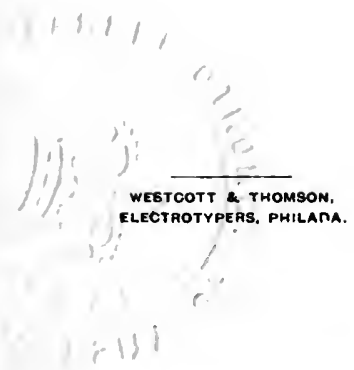


T O

THOMAS ADDIS EMMET

This Book is Gratefully

and Affectionately Dedicated. 
"THrs subject of man's body is of all other things in nature most susceptible of remedy; but then that remedy is most susceptible of error. For the same subtility of the subject doth cause large possibility and easy failing; and therefore the inquiry ought to be the more exact."

Francis Bacon, in the Second Book of the Proficience and Advancement of Learning. 


\section{PREFACE TO THE FIFTH EDITION.}

In accordance with the plan of the book as set forth in the prefaces to former editions I have divided the subjects, not in the usual manner of grouping in each part all the diverse diseases of some special organ, but so far as practicable have arranged them in pathological and etiological sequence. For example, infections and inflammations are brought together so that vulvovaginitis, metritis, salpingitis, ovaritis, peritonitis, and cellulitis may be studied in the combined forms whieh frequently they assune. In like manner, tumors are treated in another part, traumatisms in another, and displacements in another. Under this plan the student, it is thought, will have constantly before him the physiologieal and pathologieal unity of the reproductive system; on the other hand, if he considered all the diseases of each organ in a part by itself, he would find tunors, traumatisms, displacements, and other anomalies thrown in between the infections of that organ and causal or resultant infections in other parts of the pelvis, and thus might lose sight of the signifieance of morbid processes and the relations of those processes to one another.

I have endeavored to make a thoronghgoing revision of the text, which should include the recent advances in Gynecology, and in so doing have condensed, rewritten, and rearranged many parts, and in this way have found space for considerable new matter without materially enlarging the volume. The following chapters have been subjected to special changes and practical additions: Chapter XXIII., Treatment of Salpingitis, Ovaritis, and Pelvic Peritonitis; Chapter XXVII., Treatment of Myoma Uteri Chapter XXVIII., Treatment of Carcinoma Uteri ; Chapter XLV., Treatment of Descent of the Uterus; and Chapter XLVII., Treatment of Retroversion and Retroflexion. I have added two new chapters, an introductory chapter, and Chapter LV., on Ineontinence of Urine in Women.

All illustrations and plates have been reproduced from drawings especially made for the book. Forty now illustrations and full-page plates in color and monochrome have been added. Operative proeedures are set forth as they take place step by step in numerous series 
of drawings; for example, twenty-two drawings describe the steps of the different operations of Myomectomy and Hysteromyomectomy; thirty-two explain Perincal Lacerations and the steps of Perineorrhaphy. The surgical instrument catalogue element has been eliminated, all instruments, so far as practicable, being shown as they appear in actual work.

E. C. D.

100 State Street, Chicago.

1908. 


\section{CONTENTS.}

PART I.

GENERAL PRINCIPLES.

TATRODUCTION

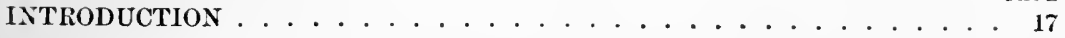

CHAPTER I.

THE PHYSIOLOGICAL PERIODS IN THE LIFE OF WOMAX . . . . . 21

CHAPTER II.

SEPTIC INFECTION AND ASEPTIC TECHNIQUE. . . . . . . . . . 32

CHAPTER III.

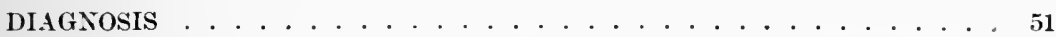

CHAPTER IV.

LOCAL TREATMENT . . . . . . . . . . . . . . . . 91

CHAPTER Y.

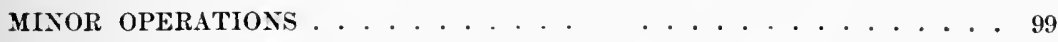

CHAPTER VI.

MAJOR OPERATIONS . . . . . . . . . . . . . . 120

CHAPTER VII.

DRAINAGE IN MAJOR OPERATIONS . . . . . . . . . . . 138

CHAPTER VIII.

AFTER-TREATMENT IN MAJOR OPERATIONS . . . . . . . 148

CHAPTER IX.

THE RELATIONS OF DRESS TO THE DISEASES OF WONEN . . . . 159 
INFECTIONS, INFLAMMATIONS, AND ALLIED DISORDERS.

CHAPTER $\mathbf{X}$.

GENERAL CONSIDERATIONS OF INFECTION AND INFLAMMATION OF

THE REPRODUCTIVE ORGANS . . . . . . . . . . . . . . . 168

CHAPTER XI.

VULVITIS, VULVOVAGINITIS, VAGINITIS 174

CHAPTER XII.

ECZEMA VULVA, HERPES VULYA, KRAUROSIS VULYA, PRURITUS

VULVE, HYPERESTHESIA VULVE, VAGINISMUS . . . . . . . 192

CHAPTER XIII.

METRITIS-INFLAMMATION OF THE UTERUS 202

CHAPTER XIV.

ACUTE METRITIS . . . . . . . . . . . . . . . 207

CHAPTER XV.

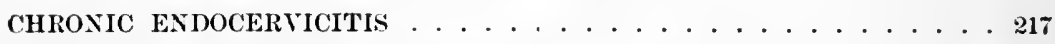

CHAPTER XVI.

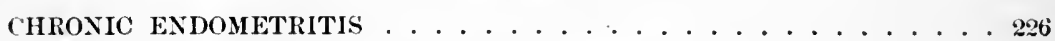

CHAPTER XVII.

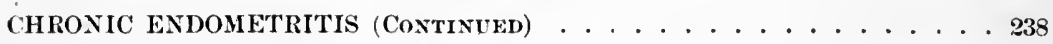

CHAPTER XVIII.

CHRONIC METRITIS 250

CHAPTER XIX.

PELIIC INFLAMMATION. . . . . . . . . . . . . . . 257

CHAPTER XX.

PELVIC CELLULITIS . . . . . . . . . . . . . . . 260

CHAPTER XXI.

INFLAMMATION OF THE UTERINE APPENDAGES-SALPINGITIS, OVA-

RITIS, PELVIC PERITONITIS . . . . . . . . . . . . 268 
CHAPTER XXII.

NON-SURGICAL TREATMENT OF PELVIC INFLAMMATION-SALPIN-

GITIS, OVARITIS, AND PELVIC PERITONITIS . . . . . . . . 28 i

CHAPTER XXIII.

SURGICAL TREATMENT OF SALPINGITIS, OVARITIS, AND PELIIC PERITONITIS . . . . . . . . . . . . . . . . . . 2991

CHAPTER XXIV.

URETHRITIS-URETHRITIS COMPLICATED BY PROLAPSE OF URETHRA -URETHRITIS COMPLICATED BY SUBURETHRAL ABSCESS-CYSTITIS-PYELITIS . . . . . . . . . . . . . . . 33

\section{P A R T I I .}

TUMORS, TUBAL PREGNANCY, MLLFORMLTIONS.

CHAPTER XXV.

TUMORS OF THE VULVA AND VAGINA . . . . . . . . . . . . 357

CHAPTER XXVI.

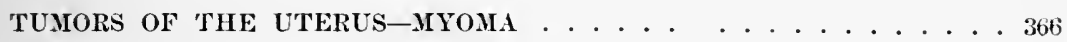

CHAPTER XXVII.

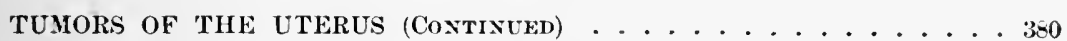

CHAPTER XXVIII.

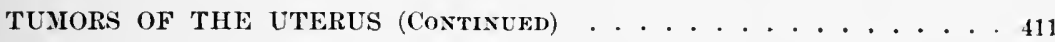

CHAPTER XXIX.

TUMORS OF THE UTERUS (CoNTINUED) . . . . . . . . . . . 431

CHAPTER XXX.

TUMORS OF 'THE UTERUS (Continued) ............. . 434

CHAPTER XXXI.

SOLID TUMORS OF THE OVARY .............. 4365

CHAPTER XXXII.

CLASSIFICATION, MODE OF DEVELOPMENT, AND PATHOLOGY OF OVARIAN AND PAROVARIAN CYSTS, AND OVARIAN HYDROCELE. 438 
SECONDARY CHANGES-SYMPTOMATOLOGY-DIAGNOSIS, PROGNOSIS, AND DIFFERENTIAL, DIAGNOSIS OF OVARIAN AND PAROVARIAN

CYSTS ........................ . . . . . . .

CHAPTER XXXIV.

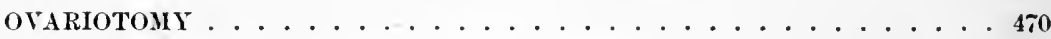

CHAPTER XXXV.

TUMORS OF THE FALLOPIAN TUBES, BROAD LIGAMENTS, ROUND

LIGAMENTS, AND LRINARY ORGANS. . . . . . . . . . 481

CHAPTER XXXVI.

TUBAL PREGNANCY ........................ 486

CHAPTER XXXVII.

EMBRYOLOGY OF THE GENITALIA AND CONGENITAL MALFORMATIONS 503

CHAPTER XXXVIII.

CONGENITAL GYNATRESIA WITH RETAINED MENSTRUAL FLUID. . 530

\section{P A R T I V.}

TRA U MA TISMS.

CHAPTER XXXIX.

NON-PUERPERAL, INJURIES OF THE VULYA, VAGINA, AND CERTIX

UTERI . . . . . . . . . . . . . . . . . . . 53 . . .

CHAPTER XL.

LACERATIONS OF THE PERINEUM AND PERINEAL REgION . . . . 538 CHAPTER XLI.

PERINEORRHAPHY . . . . . . . . . . . . . . . . . 549

CHAPTER XLII.

PUERPERAL LACERATION OF THE CERIIX UTERI . . . . . . . 565

CHAPTER XLIII.

GENITAL FISTULA ................. . . 593 
PART V.

DISPLACEMENTS OF THE UTERUS AND OTHER PELVIC ORGANS.

CHAPTER XLIV.

PAGE DISPLACEMENTS OF THE UTERUS. . . . . . . . . . . 627

CHAP'IER XLV.

MAL-LOCATIONS OF THE UTERUS . . . . . . . . . . . . 6355

CHAPTER XINI.

ETIOLOGY, SYMPTOMS, COURSE, DIAGNOSIS, AND PROGNOSIS OF RETRO-

VERSION AND RETROFLEXION . . . . . . . . . . . . . . 668

CHAPTER XLVII.

TREATMENT OF RETROVERSION AND RETROFLEXION . . . . . . 675

CHAPTER XLVIII.

ANTEVERSION AND ANTEFLEXION OF THE UTERUS: TORSION OF

THE UTERUS . . . . . . . . . . . . . . . . . . 710

CHAPTER XIIX.

INVERSION OF THE UTERUS. HERNIA OF THE UTERUS AND OVARY . 729

PART VI.

DISORDERS OF MENSTRUATION AND STERILITY AND INCONTINENCE OF URINE.

CHAPTER L.

PREMATURE MENSTRUATION AND PROTRACTED MENSTRUATION . . 743

CHAPTER LI.

AMENORRHEA AND SCANTY MENSTRUATION . . . . . . . . 745

CHAPTER LII.

UTERINE IEMORRHAGE-MENORRHAGIA AND METRORRHAGIA . . . 750

CHAPTER LIII.

DYSMENORRHGA AND PERIODIC INTERMENSTRUAL PAIN . . . . 756

CHAPTER LIV.

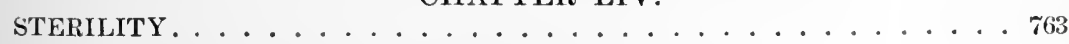

CHAPTER LV.

INCONTINENCE OF URINE IN WOMEN ................ . . . 



\section{PRINCIPLES AND PRACTICE OF GYNECOLOGY.}

\section{INTRODUCTION. ${ }^{1}$}

THE general law that progress in any direction is characterized by specialization, with its attendant classification and simplicity, has been exemplified in no great movement more strongly than in the development of scientific medicine during the last three decades. The late Samuel D. Gross, foremost general surgeon of his day, after a long period of active service as author, teacher, and practitioner, writing the preface to the sixth edition of his System of Surgery in October, 1882, thus early gives eredit to specialization for the unparalleled advances in modern surgery. Specialization, he says in substance, has penetrated with its methods and instruments of research the innermost recesses of the human body, and in a comparatively brief period has achieved triumphs which general surgery perlaps never would have accomplished.

In the earlier period, when the specialist confined himself to a particular organ, disregarding its relations to the general system, when frequently exclusive books appeared from this author, for example, on the stomach, or from that one on the brain, specialization was cumbersome, narrow, ineffective, and a hindrance to scientific medicine. Finally, the logical tendency to study each part, not by itself, but in its essential relations to the whole system, gave rise to such a welding together into a great unit of all the specialties that any organ, even though recognized in its individual importance and autonomy, at the same time was equally recognized as subject to general law. It then became apparent that physiological and pathological processes, such as circulation and infection, wcre substantially the same whatever the organ involved. Order then came out of chaos and specialization became a potent factor in the simplification and progress of medicine. From this time forward, laryngology, rhinology, orthopedics, ophthalmology, neurology, climatology, state medicine, obstetrics, and gynecology rapidly developed and became identified in all parts of the civilized world with remarkable groups of men who have strengthened scientific medicine by building up these departments to an extent unequaled in any other period of history.

In nearly all the medical teaching centers of America and Europe the most conspicuous specialty of modern medicine, gynecology, has enjoyed full recognition not only in the day of its early struggle,

\footnotetext{
1 From the author's presidential address, delivered at the annual meeting of the Ameriean Gynecological Society at Niagara Falls, 1905.
} 
but later in the period of its highest development, with the significant result that in dignity of position and in output of scientific produet it has in many respects outbalanced the department of general surgery itself.

The progress of gynecology has been marked by two pronounced periods: The first was an earlier period, characterized by great activity in the perfection of numerous plastic operations on the vaginal side of the pelvic floor. This development of minor plastic surgery calls to mind many familiar names at home and abroad, most conspicnous among them the names of two pioneers, Emmet and Marion-Sims. The second or later period was one of tremendous progress in the surgery of the obverse abdominal side of the pclvic floor. Now a third period is before us in which gynecology has taken to itself the whole field of abdominal surgery.

The early gynecologist was logically led by the anatomical, physiological, and pathological unity of the reproductive organs into the peritoneal surgery of the pelvic eavity and thence by anatomic continuity into that of the upper abdomen. In peritoneal surgery, his edueated touch, his special surgical judgment, and, above all, his training in the technic of plastic gynecology, placed him on a decided vantage ground over the general surgeon.

This widening of limitations to include the territory of abdominal surgery has given rise to an extraordinary and altogether interesting effort on the part of the general surgeon to promulgate the erroneous idea that the gynecologist has become a general surgeon and thereby has forced gynecology as a specialty into the background, where any one, even without special preparation, may practice it. Thus we hear of "The Merging of Gynecology into General Surgery," "The Passing of a Great Specialty," "The Expansion or Obliteration of a Specialty." We are told that gynecology is a finished subject, and that "he who runs mav read," that it is only a matter of a few operative procedures, that the technic of it is now perfected and readymade for the hand of any one, that soon in medical journals, in textbooks, in medical schools, in societies and hospitals, gynecology will be merged into general surgery and the name will be forgotten.

In considering this most recent attitude toward gynecology I do not refer to the practitioner who may be so situated that the most competent experts are not available; necessarily he may be compelled, to the best of his ability, not for himself alone, but in the interest of his patient, to undertake not only gynecology, but all the other specialties, nor do I deny that a general surgeon of sufficient versatility may carry on miscellaneous surgical work and at the same time, if he will undergo the necessary long and careful training, may acquire the special judgment, the special diagnostic and operative technic, essential to proficiency in the practice of a great specialty; but this admission does not weaken the indictment which I would offer against a type of general surgeon, whose number increases day by day, whose relation to this specialty is the outcome of a reasoning all his own, a reasoning from the plausible premise that "the gynecologist, having perfected and simplified his specialty, has found it too narrow and has 
expanded" to the specious conclusion that gynecology is an insignificant branch and that the gynecologist therefore has undertaken general surgery. This logic gives rise to a sophistry : if the gynecologist is a general surgeon, conversely the general surgeon is a gynecologist. As the times change and we change with them, this type of universal operator, quick to seize on and turn to his own account the intimation that this specialty has passed, with refinement neither of diagnostic nor operative technic, with no appreciation of his limitations, hypnotized by an apprenticeship of six weeks in some postgraduate school, or by no apprenticeship at all, emboldened by the fact that no one has called him to account, would make gynecology crude and common, would persuade the publie and the profession that it is a mere caudal appendix to surgery on which no one fears to tread. Let us for the moment dismiss the general discussion of the subject and imagine a private hospital conducted under certain practieal conditions of business management and promotion, with a year of active practice in capital operations, most of them belonging to this, forsooth, insignifieant branch of surgery and a mortality of 70 per cent., and then with this experience as a background, going on for an additional few weeks to eleven more consecutive abdominal operations and 100 per cent. of mortality. This is an extreme but nevertheless historical example, taken not from the dark ages of surgery, but from our own times. It would be painful to expose other instances.

Gynecology has not passed. We are not general surgeons. We are specialists in the diseases of women, and as our later transactions abundantly show, we are to a rapidly increasing extent specialists also in the wider field of abdominal surgery, a field in which the account on the ledger as it stands to-day will show general surgery indebted to us for a great part of its practical and scientific progress; the elaim is valid, for we were blazing the trail through this territory when it was an untrodden wilderness, and it is ours by right of discorery; we were giving laws to govern the conduet of the stranger in this field when it was unknown and uneonquered, and it is onrs by right of conquest; we received from the pioneers, our teachers, some of whom are with us now, the principles and precepts on which has been built up this most aggressive department of surgery, and it is ours by right of inheritanee.

Marion-Sims was not a general surgeon when he laid down the laws which to-day govern the surgery of the gall-bladder, when he foreshadowed the modern treatment of gunshot wounds of the abdomen and thereby set in motion a tide of general abdominal surgery of which the ebb flow, particularly in the upper zones of the abdomen, where we have joinerl hands with the general surgeon, is alrearly overdue. Do the traditions which properly belong to us count for nothing? Shall we retire into the background? Shall we organize a society of the Cincinnati, enter into our second childhood, and live on the memories of the past? Is our work done? Shall we say, "Troy has been, we have been Trojans"? If our work is done, why should we not go at once into voluntary liquidation? Why should a special society hold another meeting? But so long as in the diseases 
of women there are practical and scientific problems to be solred, our work is not done. Does not the increased strain of modern life, notwithstanding improved knowledge of sanitation and lygiene, bring about exaggerations of pathology which will demand not less but more of the gynecologist? If we do not respect our own specialty, who will? Let us consider, for example, the every-day subject of dysmenorrhea, about which as yet we know but little; the causes of eclampsia, of which we know less; the purpose of menstruation, of which we know nothing; the unknown eonditions, which in one case will supply defense against general septic peritonitis and in another apparently similar case will open the way to a rapidly fatal peritoneal infection. Let us reflect that we have not spoken the last word on the surgical treatment of descent, retroversion, and other deviations of the pelvic organs; let us consider whether in the next thirty years we or the general surgeons are going to make such improvements in practical gynecology that the hysteropexies, the hysterorrhaphies, the suspensions, the fixations, and a number of other procedures may look to our successors as crude and irrational as the elamp and routine use of the drainage-tube in ovariotomy look to us at the present time.

And now, supplementary to this discussion, may I offer a suggestion? In recent years, abdominal surgery has so far engrossed the mind of the gynecologist, not to mention that of the general surgeon, that by comparison the minor plastic work to some extent has been neglected or given into incompetent hands. It is perhaps not too much to say that our fathers in their day did better plastic surgery than we are doing in ours. Indeed, a revival of interest may be necessary in order to save this part of gynecology from becoming a lost art. There is now accumulating a very appreciable number of patients on whom plastic operations, some of them repeated on the same patient many times over, have been performed with indifferent or injurions results. Many such patients need to have the work undone or done over again. And this class of cases now, therefore, is making an increasing demand on the attention of the competent gynecologist. It is time, therefore, without losing sight of the claims of capital surgery, to bestow adequate attention on the homely every-day problems of minor gynecology. 


\section{PART I. \\ GENERAL PRINCIPLES.}

\section{CHA P TER I.}

\section{THE PHYSIOLOGICAL PERIODS IN THE LIFE OF WOMAN.}

Is embryonic life and early infancy the physiological lines that mark the distinction of sex nearly coincide, and anatomical differences have little more than potential significance; as childhood recedes these lines diverge; as maturity progresses they separate more and more; finally, in old age they draw together until in the second childhood, as in the first, they again nearly coincide. Development from infancy to maturity and decline from maturity to senility are common alike to man and to woman. In man the anatomical and physiological changes from the time of birth to the period of youth and virility and the cessation of sexual power in old age are gradual and even processes, relatively free from special outlay of energy, unmarked by specially critical periods, and unattended by pronounced nervous or mental disturbance. In woman these transition-periods are characterized by greater expenditure of energy, by more rapid sexual change, and by more distinct nervous and psychic phenomena; they are the critical turning-points in her life. At the first crisis-puberty -the reproductive organs, more complicated than those of the male and hitherto unproductive, suddenly become the centre of great and rapid development; from this period forward until the second and final crisis-the menopause-her vital forces are especially subject to the exactions of menstruation and maternity.

The life of woman may be divided into five periods, each corresponding to a special phase of her sexual existence; they are infancy, puberty, maturity, the menopanse, and senility.

\section{INFANCY.}

Infancy includes the first ten or twelve years of life, and, although a period of great pathological significance, is rather a subject of pædiatrics than of gynecology. During this period the reproductive organs are, for the most part, functionally dormant; they are undergoing a gradual development preparatory to the more rapid and radical changes of puberty. Infections and inflammations occasionally arise; neoplasms and traumatisms are rare; congenital malfornations, if present, usually are overlooked until the period of puberty or maturity, when, by reason of some defect in the function of men- 
struation, coitus, or parturition, they become evident ; displacenents of the infantile uterus, although possible, have little or no clinical significance.

\section{PUBERTY.}

Puberty is the critical transition-period in which the child becomes the woman. The relations and influences of this period are fundimental, both in the reproductive organs and in the entire woman, so that upon the normal course of it depends much of the after health, comfort, and usefulness of the individual.

The Anatomical Basis of puberty is the full physical development of the reproductive organs. The infantile uterus is small, soft, and plastic; it varies in size from that of early infancy (Figure 1) to that of the child-uterus just before puberty ; at the beginning of puberty the uterine canal would measure, perhaps, two inches; when fully developed at the end of puberty it should measure two and onehalf inches.

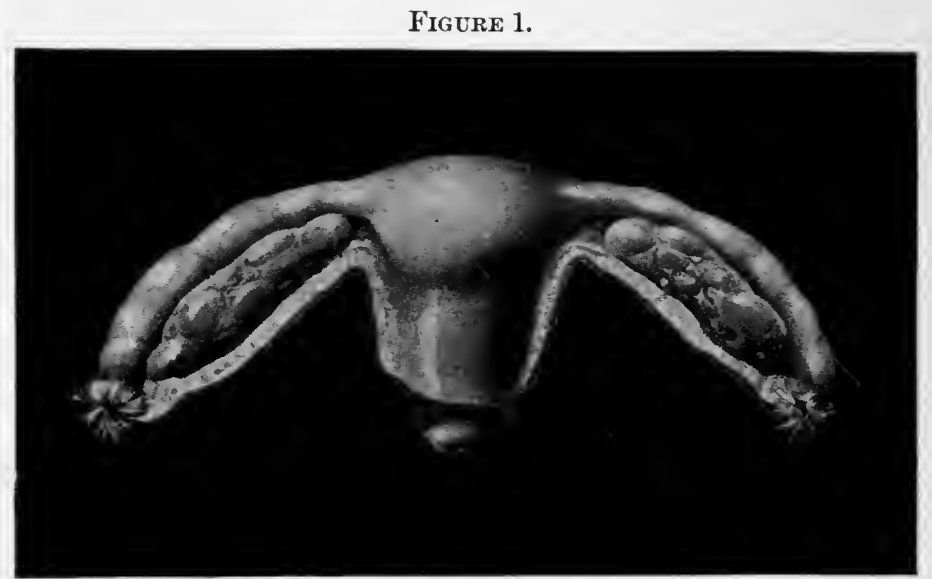

Uterus, Fallopian tubes, and ovaries of an infant one month old. Natural size.

The cervix of the infantile uterus is two-thirds, and the corpus one-third, as long as the entire organ. These proportions when the organ is fully developed at the end of puberty are reversed-that is, the corpus represents two-thirds and the cervix only one-third of the length of the mature uterus. At maturity the longitudinal axis extending from the os externum to the fundus measures three inches; the transverse axis of the corpus uteri measured laterally from horn to horn is two inches, and measured by the longest anteroposterior diameter is one inch. The fundus of the infantile uterus is flat; the fundus of the mature uterns is convex and dome-shaped. The mucosa of the infantile uterus presents an arbor vito arrangement throughout the corpus and cervix; at maturity this arrangement is confined to the cervix.

Developmental changes similar in extent to those above outlined occur in the ovaries and in the other genital organs. Puberty is marked also by enlargement of the pelvis and breasts, by the appear- 
ance of hair on the mons veneris, vulva, and armpits, and by general rounding out of the form with adipose tissue.

The Physiological Features of puberty are the onset of menstruation and ovulation and notable psychic changes, all of which indicate that the reproduetive organs and the sexual nervous organization are approaching maturity and that the girl is preparing for maternity.

\section{Menstruation.}

Menstruation is characterized by a bloody mucous discharge from the uterus; this discharge contains epithelial cells from the uterus and vagina; it begins with puberty, and, unless interrupted by uterogestation and lactation or by disease, normally recurs in regular periods until the time of the menopause. The phenomena of menstruation are both general and local.

I. The General Phenomena of Menstruation are as follows :

1. Slight elevation of pulse-rate and temperature at the onset.

2. Tendency to slight physical depression and inactivity.

3. Sensations of heat and cold.

4. Swelling of the breasts and thyroid gland.

5. Discomfort and throbbing in the head, weight in the pelvis and back, and irritability of the bladder.

These disturbances are subject to wide variations. In some cases they are absent; in others they are so slight as almost to eseape notice, or so severe as to render life miserable and useless. Painful menstruation-that is, dysmenorrhoa-is always proof of some pathological condition. See Chapter LIII.

II. The Local Phenomena of Menstruation are recognized in three stages :

1. Stage of invasion-discharge of mucus.

2. Stage of persistence-flow of blood.

3. Stage of decline-discharge of mucus.

Discharge of mucus before and after the flow of blood is an essential part of the menstrual flux; in lower animals the menstrual discharge, if present at all, is entirely of mucus. In the human race the lower the intelleetual scale, the greater the relative quantity of mueus; the higher the scale, the greater the relative quantity of blood.

Amenorrhœa.-Amenorrhœa is the absence of menstruation; it may be physiological or pathological.

Physiological Amenorrhœa.-Physiological absence of menstruation occurs :

1. Prior to puberty.

2. At irregular intermenstrual periods during the establishment of puberty.

3. During pregnancy and lactation.

4. At irregular intermenstrual periods during the climacteric.

5. After the menopause.

Pathological Amenorrhœa.-A discussion of the pathological causes of amenorrhcea may be found in Chapter LI.

Age of First Menstruation.-The age at which menstruation first 
appears varies widely with individuals. Climate and heredity, especially the former, are determining factors. In the United States it first appears on the average about the fourteenth or fifteenth year, sometimes as early as the ninth or tenth, or as late as the eightecnth. In the Areties the average age is sixteen years and in the tropics ten or eleven.

Precocious, Protracted, and Scanty Menstruation will be presented in Chapters L. and LI.

Frequency of Menstruation.-The human menstrual cycle covers a period of about twenty-cight days. Variations of a few days are common and usually harmless.

Quantity of Menstrual Discharge and Duration of Flow.-The average amount of menstrual fluid lost in a single period is from six to eight ounces; the minimum is two, and the maximum, ten ounces. A plethoric, well-nourished woman may menstruate freely for eight or ten days without ill effect, and may lose an amount of blood which would undermine seriously the strength of an anæmic, poorly nourished woman. What would be normal for one woman, therefore, would be abnormal for another. The usual means of estimating the quantity of blood lost is by. counting the napkins used. The average number is fourteen. Nothing approaching exactness is gained by this method, since napkins vary in size and capacity for absorption, and since one woman will tolerate an oversaturated napkin while another will scarcely permit the soiling.

Anatomy of Menstruation.-Although menstruation has been the subject of many strange superstitions and speculations, yet nothing is known of the utility, cause, or significance of it. Numerous conflicting opinions concerning the anatomy of menstruation have been put forth: one, that the corporeal mucosa is stripped off clear to the muscular layer at each recurring flow ; another, that only the epithelial layer is shed; another, that a newly organized tissue is developed during the intermenstrual period, and that this alone is cast off. The notion that the surface epithelium is thrown off in the process of menstruation has arisen from faulty methods of investigation. $\mathrm{Ob}-$ servations made on the uteri of women who had died from freezing or from infectious disease during menstruation, or upon uteri removed twenty-four hours or longer after death; or upon freshly removed specimens in which the surface epithelium had been injured in the handling, have supported the conclusion that the surface epithelium is shed during menstruation, when in reality the loss of epithelium was post mortem. Gebhard, of Berlin, has put forth the correct interpretation of the anatomical changes of menstruation. His observations upon fresh material carefully prepared demonstrate that in menstruation there is no shedding of the surface epithelium.

Three stages of menstruation are recognized:

1. Premenstrual congestion. Plate II., Figure 1.

2. Subepithelial hæmatoma. Plate II., Figure 2.

3. Bursting of blood through surface epithelium and post-menstrual absorption. Plate II., Figure 3.

The connective tissue of the endometrium is of the embryonal type, 


\section{PLATE II}

FIGURE

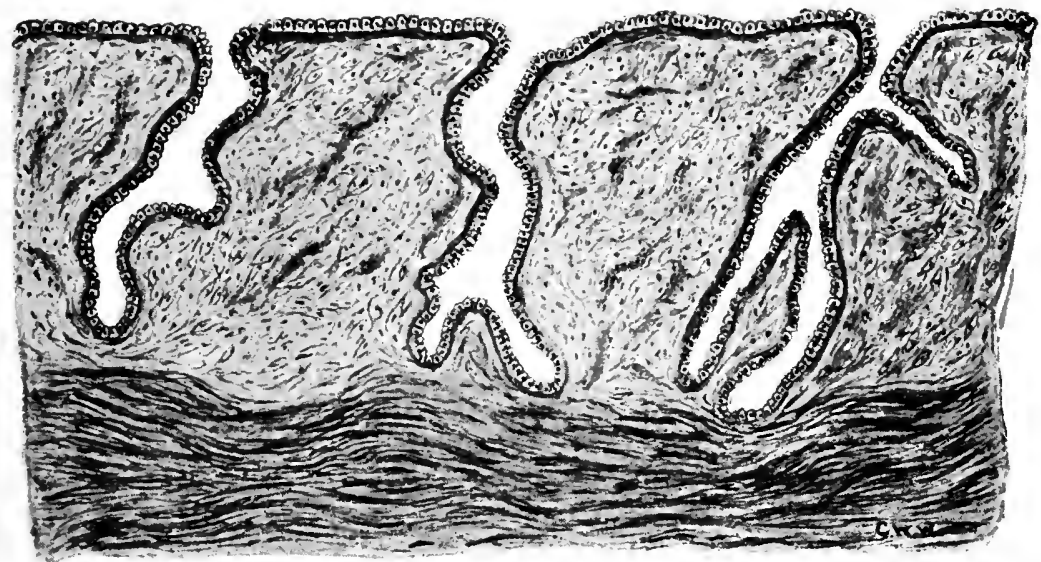

FIGURE 2

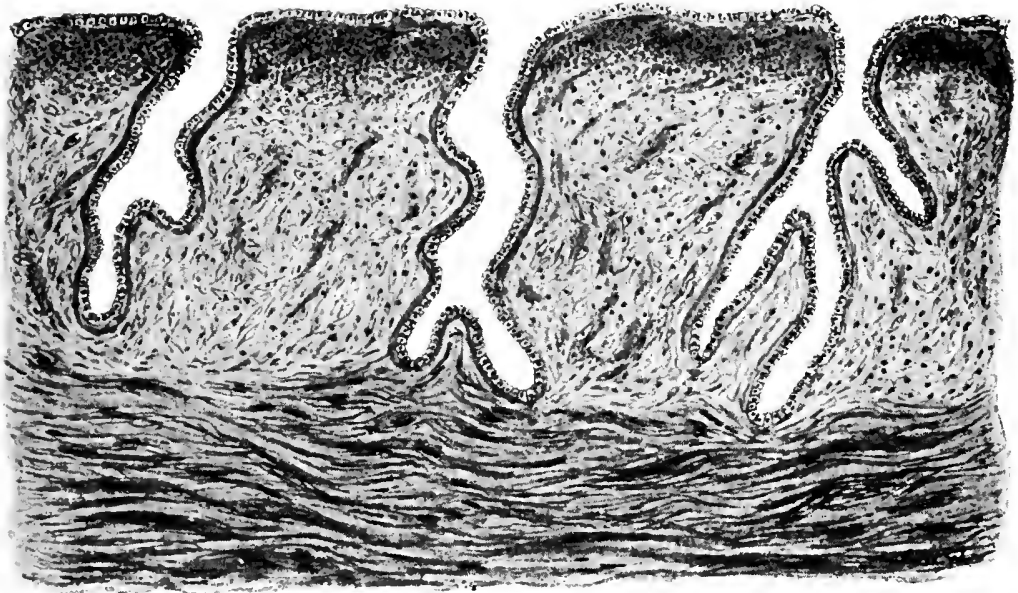

FIGURE 3

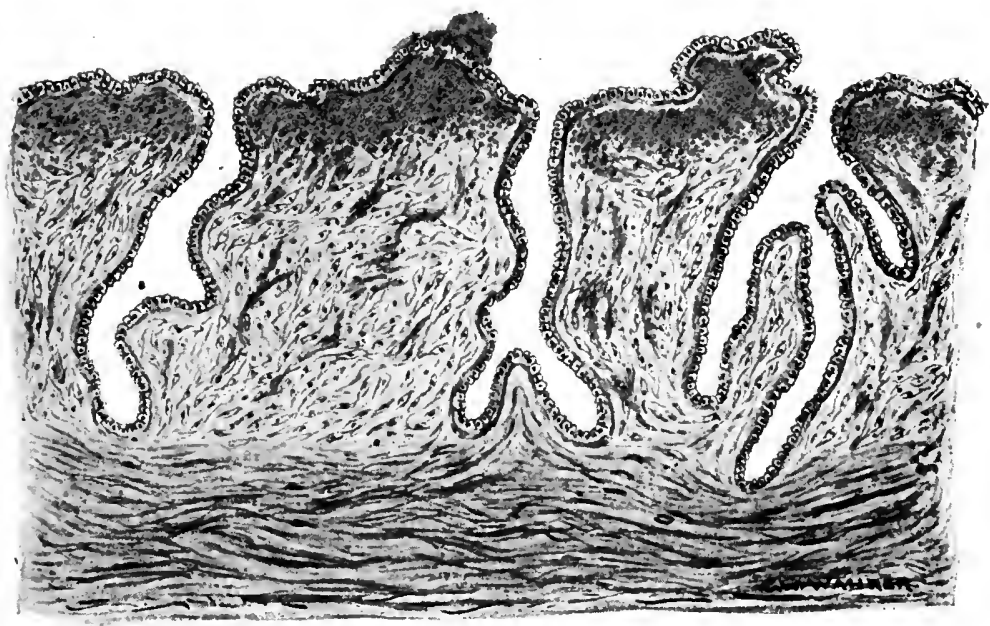

Anatomy of Menstruation (Modified from Gebhard). 

and is permeated with delicate blood-vessels. These vessels participate in the general pelvie congestion that preedes menstruation, and readily give forth an effusion of blood into the embryonal connective tissue ; the effused blood takes the direction of least resistance-that is, to the surface of the endometrium. Under the surface epithelium the blood collects in small quantities, forming what may be termed subepithelial hæmatomata. With increasing pressure the blood passes between the epithelial eells of the surface, elevating groups of cells from the basement membrane and oceasionally breaking off small fragments of epithelium. With lessening blood-pressure the hemorrhage beeomes less abundant, and finally the blood eeases to pass through the epithelial barrier; then follows absorption of the effused blood from the connective tissue and subepithelial sjaces. The epithelium that had been lifted from the basement membrane sinks back into its former relations. Any minute areas aceidentally denuded are quickly covered by new epithelium regenerated from adjoining surface epithelium and gland epithelium. Such are the anatomical events of menstruation.

\section{Ovulation.}

Ovulation involves the maturing and rupture of the Graafian follicle and the escape of the ovum. Formerly, menstruation was commonly

Frgtre 2.

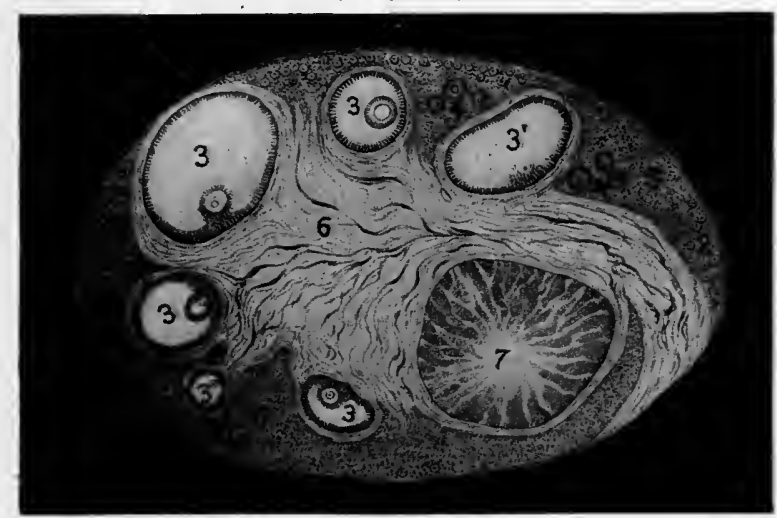

Section of ovary (magnified). 1. Outer covering. 2. Graaflan follicles in earliest stage of development. 3. Graafian follicles in more advanced stage of development; the largest follicle is almost mature. ' 3 '. Follicle from which ovum has escaped. 4. Slightly developed follicles. 5. Peripheral stroma. 6. Central stroma. 7. Corpus luteum. Modified from Schron's drawing of the ovary of a cat.

thought to be an external manifestation of ovulation and dependent upon it; but whatever may be the relation between these two funetions, that of eause and effeet, for the following reasons, is no longer tenable :

1. There is a cyclical periodicity in menstruation, and there is no such periodicity in the maturing of the Graafian follicle and discharge of the ovum ; the process of ovulation is eontinuous, and ocenrs even in the mature fotus. 
2. Menstruation sometimes continues after removal of the ovaries.

3. On opening the abdominal cavity during menstruation one frequently fails to find a fresh corpus luteum in either ovary; on the contrary, he frequently finds it during the intermenstrual period.

4. Ovulation oceurs in the absence of menstruation; this is proved by the fact that conception may take place during the period of lactation, and even after the menopause.

Although the dependence of menstruation on ovulation has not been established, there is yet reason to conclude that ovulation and menstruation are both under the control of the same nerve apparatus,

Figure 3.

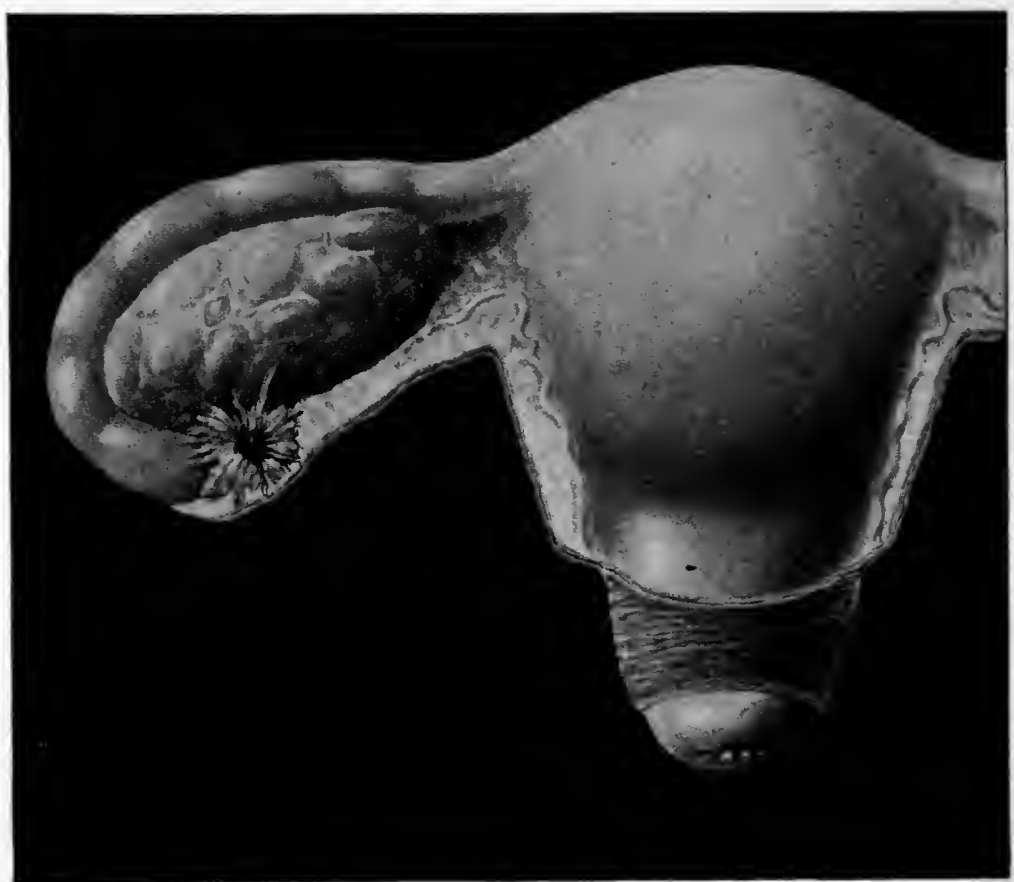

Mature ovary, Fallopian tube, and uterus, from a woman twenty-five years of age. Natural size.

and that the nerves of the uterus and ovaries have a certain co-ordination.

Care during Puberty.-Although the appearance of menstruation indicates that maternity is possible, it by no means follows that the development of the individual is complete at this time, nor that she is capable of fulfilling the requirements of maternity. Before the twentieth year the nervous system is unequal to the strain of childbearing and child-rearing; the muscles are inadequate to the carrying and expulsion of the child; and the pelvis is often too small to give it safe cxit. The period of puberty should be taken as extending not only over the few months required for the establishment of menstrua- 
tion, but as including the time necessary for full physical development. During this period the energy of the girl is taxed by the rapidity of sexual development, by the great liability to circulatory disturbances, by the physical and mental strain of education, and by the conventionalities of societr. The necessity, therefore, for great care is apparent. Nutritious and simple diet, frequent rest, moderate amusements, and adequate exercise are essential. Study, especially during menstruation, should never be pressed to the point of fatigue. Inasmuch as passional life now begins, and the whole nervous organization is therefore subject to new impulses and requirements, books and associates should be selected carefully, and whatever may unduly excite the emotions should be excluded. Errors committed now may have grave consequences, such as malnutrition, psychoses, sterility, menstrual and other funetional disorders, and may make the woman a

Figure 4.

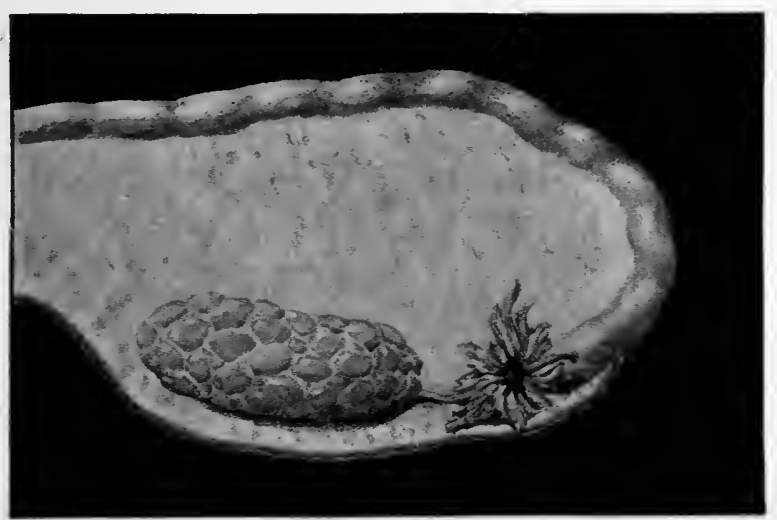

Ovary and Fallopian tube, from a woman forty-one years of age. Natural size. Atrophic processes and consequent decrease in size of the ovary and tube already begun.

hopeless invalid. For reasons already given, one of the most serious errors is premature marriage.

Education.-According to prevailing ideas, the higher education and civilization strongly tend to check and pervert the development of woman, to cause numerous weaknesses, to increase the burdens and dangers of maternity, and to lessen the rigor of the offspring. We are told that the republic is in danger from deterioration of the edueated classes. These pessimistic forebodings have arisen and gained headway rather upon assertion than upon known fact. The ability of the squaw immediately after parturition to resume the march is urged often as an argument against the higher education of woman; on the other hand, observation among Indian wonen has shown abundantly that want of care, during and after labor, is the coustant eause of complete prolapse of the uterns, vagina, and bladder, and of numerous other diseases which are relatively much more prevalent among them than among the higher classes of eivilized women. The edueated woman conld " resume the march" if it were necessary ; history 
has shown many heroie examples; but education has taught her that this is unsafe. The savage woman looks old and withered at thirty ; the civilized woman preserves something of youth until after the age of fifty. The highest eivilization if earried forward under proper conditions should more than off'set any deteriorating influence whieh may come of a departure from primitive conditions; it should give to the civilized race a vitality much greater than that of the savage, and to the civilized woman a power of resistance which, if properly trained and directed, will enable her to endure and to survive many trials to which a savage woman would succumb. To make the deterioration of woman, and through this the enfeeblement of the race, a price which must be paid for the higher education and civilization, would be seemingly to reverse the law of evolution and to put in its place a law of the survival of the unfittest.

The Goitre of Puberty.- The changes of puberty are in some eases associated with an enlargement of the thyroid gland, called goitre, a condition that often disappears with the complete establishment of menstruation. In early goitre the glands are soft and almost fluctuating. If the enlargement persists, the tumor becomes fibrous, hard, and intractable. Such enlargement may be treated in the early stage with inunctions of biniodide of mercury, 30 grains to the ounce. This should be applied daily for periods of four or five days. When the skin becomes irritated the application should be interrupted until the irritation has subsided, and then resumed. These inunetions, together with the continued use of calomel or the bichloride of mercury, in minute doses, will result sometimes in rather prompt disappearance of the swelling. The thyroid extract in doses of 2 grains three times a day will in some cases effect a rapid cure; if distinct improvement is not apparent in two or three weeks, the drug should be discontinued; in any case the use of it should be guarded, and the dose regulated if necessary to an amount that will not cause disagreeable nervous symptoms.

\section{MATURITY.}

The time of sexual maturity extends from the end of puberty to about the forty-second year, and under normal conditions is a relatively healthy period. Unlike puberty and the menopause, it is comparatively free from neuroses and psychoses, except those connected with pregnancy. The woman is subject, however, to the burdens and accidents of menstruation, ovulation, pregnancy, maternity, physical and mental overstrain, and to the dangers of puerperal and other infection, amnng which especially may be nentioned gonorrhea-a potent cause of vulvo-vaginitis, metritis, salpingitis, ovaritis, peritonitis, cystitis, pyelitis, and nephritis.

During this period the non-malignant neoplasms more frequently, and the malignant neoplasms less frequently, endanger life and bealth. 


\section{THE MENOPAUSE.}

The menopause, sometimes called the climacteric, sometimes the change of life, is the second critical period. It usually takes place between the ages of forty and fifty; the occurrence of this erisis before the fortieth or after the fifty-second year is abnormal; it continues from three to five years. Pathological causes more or less recognizable, and the influence of heredity, may shorten or lengthen it. In very cold climates both puberty and the menopause are delayed. The opposite is true in warm climates.

The Anatomical and Physiological Basis of the Menopause is atrophy and cessation of function. This critical period is charac-

Figure 5.

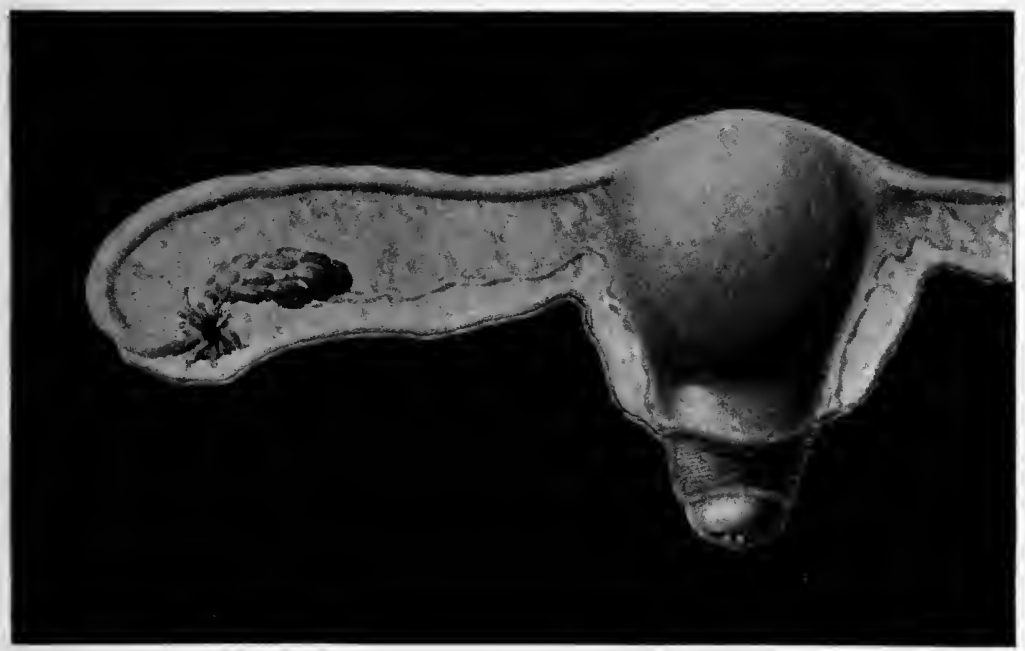

Natural size of ovary, Fallopian tube, and uterus of a woman seventy years of age. Senile atrophy of the reproductive organs complete. Rudimentary ovary and tube. Uterus atrophied to about two-thirds of the mature size.

terized by the following senile changes in all the reproductive organs, some of which are pathological.

1. Senile changes in the ovary:

a. Atrophy, induration, and shrinkage to rudimentary size.

b. Disappearance of Graafian follicles.

c. Cessation of function.

2. Senile changes in the Fallopian tubes:

a. Shortening and narrowing; often complete obliteration of lumen.

b. Destruction of epithelial elements.

3. Senile changes in the uterus :

a. Atrophy of entire organ to rudimentary size; may be reduced to a hard, wedge-shaped body, one-fourth size of mature organ.

b. Muscular and glandular elements become rudimentary. 
c. Canal may close at internal os, or external os, or become obliterated throughout.

d. Secretions may be locked up by obliteration of the cervical canal producing pyometra or hydrometra.

e. Vaginal portion may disappear, making the upper part of the vagina continuous with the uterine canal.

4. Senile changes in the vagina:

a. Shortening, narrowing, and loss of elasticity.

b. Loss of parement epithelium and substitution of a hard surface containing more or less cicatricial tissue.

c. Contraction of introitus vaginæ.

5. Senile changes in the vulva:

a. Same as in vagina-great contraction and loss of elasticity.

b. Destruction or impairment of vulvovaginal glands and vulvar follicles.

c. Cutaneous surface dry and sealy.

d. Hair on mons veneris may turn gray.

6. Senile changes in the mammce:

a. Loss of glandular elements and cessation of function.

b. Atrophy and shrinkage; sometimes the atrophic loss is made up or more than made up by the deposition of fat.

The Essential Phenomenon of the Menopause is permanent arrest of all functions peculiar to the reproductive organs. It is the inversion of the developmental process of puberty. It marks the end of active sexual life. The atrophic changes are known as senile atrophy.

The Symptoms of the Normal Menopause are referable to two stages : a stage of menstrual irregularity preceding the cessation of the menses, and a post-cessation stage of variable systemic disturbances. In normal or nearly normal cases the menstrual irregularities and the systemic disturbances are slight. The woman may at times be unusually capricious and emotional; yet she passes through this physiological crisis with only a few minor perturbations, such as the characteristic vasomotor flushes, perspiration, vertigo, somnolence, numbness, and faintness. The menstrual function ceases as it began, with marked symptoms referable to the nervous system.

Symptoms of Abnormal Menopause.-Irritability, apprehensiveness, hysteria, melancholia, and other psychic disturbances, more or less exaggerated, are common in the abnormal cases. The flow may become continuous; it may beeome so excessive as almost to amount to dangerous hemorrhage; or life may be jeopardized by a slow, continuous drain. There is an increased tendency to malignant disease of the uterus and breasts during this period, the excessive fear of which may almost amount to a symptom of melancholia.

The menopause often cures pelvic disease; this is because pathology is physiology modified by disease, and because atrophic changes when they arrest physiological processes may also at the same time put an end to pathological processes. Fspecially is this true if the pathological processes have depended upon the functional activity of 
the organs involved. It therefore follows that a woman who has suffered for years from chronic uterine or ovarian disease may now enter upon a long period of inereased vigor and robust health. It may, however, be a dangerous, even a fatal mistake to assume that the ills oceurring at this time of life properly belong to the menopause; that they need give no anxiety; that they will disappear with it; and that they therefore require no attention. Although such a notion prevails, yet some of the most grave disorders of the menopause are consequent upon pathologieal states for which atrophy of the reproductive organs gives no relief. Continuons and profuse hemorrhages and excessive nervous disturbances are matters of specially grave solieitude, and since the one may indieate malignant disease and the other may tend to mental derangement, prompt diagnosis and energetic treatinent may be imperative.

\section{SENILITY.}

The period of senility follows the menopause and continues to the end of life; it is the decline of life, and is normally a period of repose. The functions of the reproductive organs having ceased, the organs have little physiological significance. The speeial disorders and dangers of this period, sueh as malignant growths, senile vulvovaginitis, and senile endometritis, will be considered in the proper connections. 


\section{CHAPTER II.}

\section{SEPTIC INFECTION AND ASEPTIC TECHNIQUE.}

\section{General Considerations.}

Micro-organisms are clearly the cause of the septic-that is, the so-called inflammatory-diseases of women. The millions of cocci found on nicroscopical examination of a single drop of fluid taken from the abdomen of a case, for example, of scptic peritonitis show the tremendous developmental power of micro-organisms. It is criminal for a physician to go immediately from a case of virulent infection to visit other patients without any disinfection except the ordinary washing. It is usually unsafe to go after the ordinary disinfection. It is desirable, and may be necessary, that the disinfection be not only most painstaking, but even repeated on two or three consecutive days. The rcader is referred to the paragraph on the use of rubber gloves in another part of this chapter.

Septic infection formerly caused an appalling mortality in the major gynecological operations and made the minor manipulations extra perilous. The fear of infection was so great that when the malady was neither fatal nor very disabling the practitioner often used temporizing measures however unpromising, to the exclusion of surgical measures however rational. Now the application of the aseptic principle has made all gynecological procedures relatively safe.

Scpsis is the general term for all surgical infections of microbic origin. The term asepsis, and the corresponding adjective aseptic, are used to imply the absence of these infections. The phenomena of sepsis are due doubtless to the products of bacteria more than to the bacteria themselves.

The genital tract of the newborn is sterile. In mature life, under normal conditions, the cervix and corpus uteri and Fallopian tubes are sterile, but the vagina contains numerous non-pathogenic microorganisms. See Chapter XI.

\section{Forms of Infection.}

Septicæmia.-The presence of infectious microbes in the circulation, together with the chemical action of their products, gives rise to the condition called scpticæmia.

Toxæmia.-Certain microbes exist locally and may send out their products through the circulation, thereby producing septic toxæmia. 


\section{PLATE III}

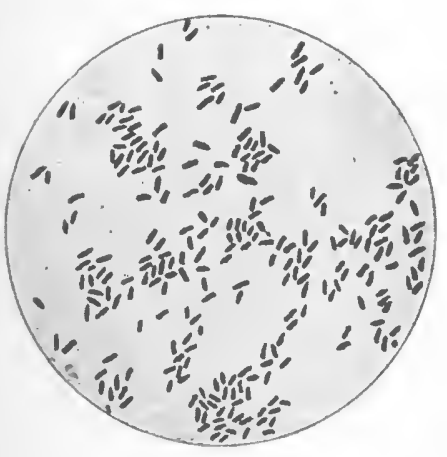

Bacillus Coli Communis.

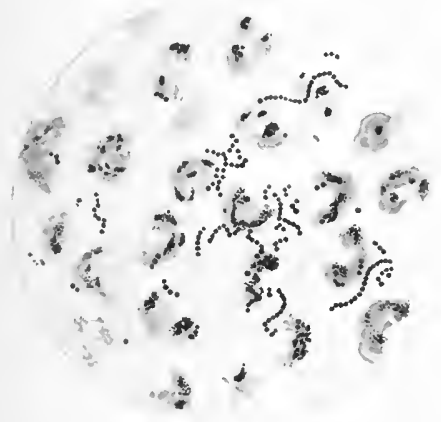

Streptococcus Pyogenes.

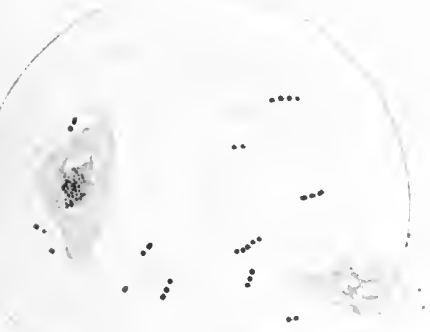

Pneumococcus.

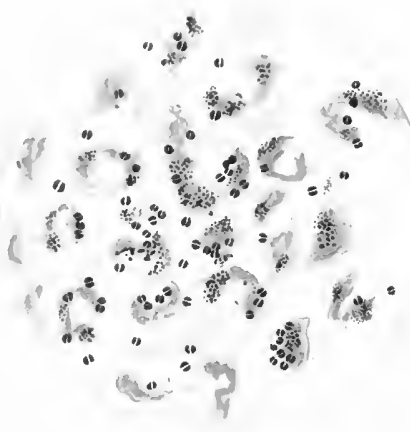

Gonococeus.

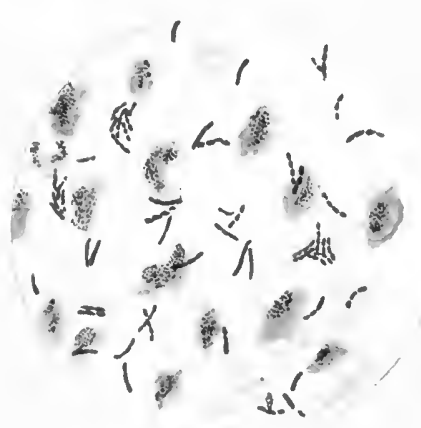

Bacillus Tuberculosis.

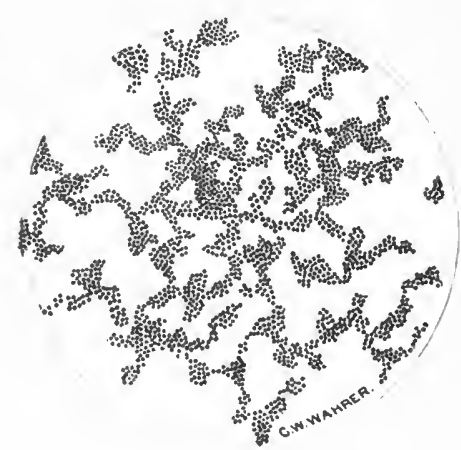

Staphylococcus. 

Sapræmia.-When the toxæmia is due to the products of putrefactive bacteria, it often is called sapræmia.

Pyæmia.-When pus emboli are carried through the circulation from a focus of suppuration to set up other foci in different portions of the body, the condition is called pyremia.

The above terms and others like them. although widely used, are not absolutely definite. An appreciation of their meaning, however, is essential to a knowledge of modern surgical literature.

Microbic invasion may be in the form of wound infection or may occur in the tinbroken cutancous and mucous structures.

The micro-organisms most common in gynecology are:
Gonococcus of Neisser,
Staphylococens pyogenes aureus, Streptococcus pyogenes,
Staphylococeus pyogenes albus, Bacillus tubereulosis.

Among the microbes less common in gynecology are:
Bacillus pyocyaneus,
Bacillus typhosus,
Bacillus diphtherise,
Pneumococcus,
Streptothrix actinomyces,
Bacillus aërogenes eapsulatus.

Gonococcus.-The gonococcus is the microbe of gonorrhoea and is invariably present in the urethral discharge in gonorrhœa, and also in other parts of the genital tract when they are the seat of gonorrhœal infection. A most striking peculiarity of the germ is the power to penetrate and intrench itself in the deeper layers beneath the mucous surfaces, especially in glandular structures. It may also migrate to distant organs, having been found in the joints in cases of gonorrhoal rheumatism, in the perspiration, and in the structures of the heart. The greatest pathogenic significance of the germ is due to the destructive action which it exerts upon infected organs. It does not set up general septicæmia, but rather acts locally; and is very persistent and destructive in the conjunctiva, in the infantile vagina, and in the Fallopian tubes of adults.

Staphylococcus Pyogenes Aureus and Albus.-Staphylococcus pyogenes aureus and albus, which resemble one another in form, are the most widely distributed and most common canses of suppuration. The former is found in almost all localized abscesses and in various other pyogenic conditions. It varies in pathogenic power from comparative mildness to great virulence. The latter is less virulent. Both varieties often are associated with other pyogenic microbes.

Streptococcus Pyogenes.-Streptococeus pyogenes is one of the most virulent, fatal, and important of the pyogenic microbes, and is especially dangerons as a cause of puerperal and traumatic septicrmia and septic peritonitis. It is the micro-organism of erysipelas. Unlike the gonococcus, which spreads preferably by way of the mucosa, this germ follows the lymph-ressels and blood-vessels.

Bacillus Coli Communis.-Bacillus coli communis is a normal inhabitant of the intestine, and is a frequent cause of peritonitis following intestinal lesions and of puerperal infection and of cystitis. 
Bacillus Tuberculosis.-Bacillus tuberculosis has been found in all of the genito-turinary organs and very frequently in tuberculous peritonitis and salpingitis. It is seldom a cause of traumatic infection, and is therefore not to be feared as a factor in surgery. On the contrary, surgical operations are said to have a decided inhibitory effect on the progress of tuberculous peritonitis, the discase having in some cases disappeared after simple exploratory incision. It is, however, doubtful whether such a result should be regarded as post hoc or propter hoc.

\section{ASEPTIC TECHNIQUE.}

\section{General Considerations.}

The causation and course of infection necessarily depend upon the source, virulence, and number of the organisms; upon the volume and nature of their products-that is, the toxins; and upon such local conditions as the presence of foreign bodies, pathological secretions, bruised, congested, and necrotic tissnes, and dead spaces. In the vast majority of cases infection is introduced by the hand of the surgeon or by instruments and other appliances, and when so introduced from without it is called hetero-infection. Auto-infection is caused by organisms existing in the patient. Some bacteria reach the wound through the air, but they are usually not virulent, and are therefore not dangerous.

Fortunately, the fluids and living tissues of the body have germicidal power, and consequently offer a degree of resistance to bacterial invasion. Many germs, therefore, which in artificial media would flourish, may become inert when exposed to the resistance of living tissue. Since this resistance is often inadequate, it becomes necessary so far as possible to exclude the organisms from the field of operation by aseptic measures, or to destroy them by antiseptic agents. It is clearly important that the power of tissues to resist organisms be not impaired by the too free use of chemical antiseptics or mechanical agents.

The mere acceptance of the aseptic idea without a thorough and systematic application of it, not only in major operations, but even in simple manipulations, is inadequate. Efficient technique is essential, and is the outgrowth of a comprehensive grasp and an intelligent appreciation of the causes, prevention, and remedies of septic infection. It requires, above all, the development of what has aptly been called the aseptic conscience.

Asepsis is the absence of infections bacteria. Strictly speaking, this may be an ideal condition, since it is not always fully realized; but it is usually possible to limit the number of bacteria to a safe minimum, or to render them harmless by means of drugs, chemicals, and other agents. Such agents are called antiseptics. When the antiseptic has the power to destroy germs, it often is called a gernicide.

Asepsis involves a great number of details variable and difficult to anticipate. A complete description is impossible and unnecessary. 
Once grasp the great principle of asepsis, and the subordinate details, otherwise complex, become simple. 'The intelligent operator, for example, who knows that septie infection is the result of contact, need not be told that during an operation he must keep his hand off from whatever is not sterile. The danger of sepsis is in a measure proportionate to the length of the operation, to the exposure of the wound or cavity, and to the extent of the traumatism. It follows, therefore, that an operation should be finished as rapidly and with as little operating as possible. Af the same time, the slow operator, if gentle in his movements, is less dangerous than one who is rapid and violent.

\section{Therapeutic Use of Antiseptics.}

The therapentic use of antiseptics is indicated when infection actually has occurred. Then the field of infection, if local, may be opened and disinfected or drained; if the infection is systemic, the internal use of antiseptic drugs may be indicated. When there is no infection, and the use of antiseptic drugs is therefore prophylactic, they should be used but sparingly, if at all, and not in contact with the wound. This is because they have injurious properties which may give rise to dangerous, even fatal, results. The use of these drugs is to secure surgical cleanliness, as soap is used to sccure æsthetic cleanliness; and, the object having been attained, they should be washed off with sterile water from the hands and instruments before these are brought in contact with the patient.

\section{Prophylactic Use of Antiseptics.}

The object of the prophylactic use of antiseptics is asepsis. Before any operation or manipulation the operator's hands, instruments and other appliances, and the field of operation or manipulation should be rendered surgically clean and so maintained thronghout-the prophylactic use of antiseptics is an antiseptic procedure to an aseptic result.

Antiseptic Agents.-Among the antiseptics in most common use are :

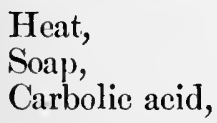

Mereuric bichloride, Formalin, Alcohol.

Heat.-The actual flame and the hot-air sterilizers have been discarded for the most part in gynecological practice. Moist heat is employed in the form of boiled water and of steam.

Sterilization by Boiling.-Absolute sterilization for bacteriological work requires boiling for thirty minutes on three consecutive days; but for surgical purposes one boiling for thirty minutes is ample. Ordinary pathogenic microbes are destroyed in a much shorter time.

Sterilization by Steam is efficient, available, and widely applicable. Everything connected with an operation that is not injured by heat may be made aseptic by this means. For this purpose, numerous 
steam sterilizers have been devised, that of Arnold being most widely used. It contains a chamber for the articles to be sterilized. The steam displaces the air from this chamber and coming in contact with the instruments, ligatures, towels, gowns, aprons, dressings, and other articles, renders them sterile, or at least practically safe for surgical purposes, in about sixty minutes. 'The Boeckmann steam sterilizer, which accomplishes so-called "over-steam sterilization," is possibly more effective than the "under-steam" sterilizers of Arnold and others. The Boeckmann sterilizer has the advantage of not wetting the dressings very much, and is provided with means of drying them before they are taken out. Steam sterilization repeated for thirty minutes on three consecutive days, insures the final destruction of any spores that might otherwise survive the first exposure and germinate the next day.

Soap, although not a strong germicide, is, perhaps, the most valuable of all antiseptics. It is used for cleansing instruments, clothing, and other things needed in connection with operations, and for washing the skin of the patient and operator, but more especially for scrubbing the hands and arms of the surgeon and his assistants. The familiar sapo viridis, usually called green soap, is the variety in general use.

Carbolic Acid is a chemical antiseptic of great power. It also has the highest germicidal and deodorant properties, and has been used more freely and generally than any other antiseptic; but it has properties that render it dangerous for the patient and inconvenient for the operator. It corrodes instruments, irritates the skin, and by its local anæsthetic properties inipairs the tactile sense. The use of this drug is limited now to the disinfection of very small areas of local infection, where the quantity used is not sufficient to cause systemic poisoning, even though the acid be used in full strength. The danger of washing out septic cavities with $1,2,3$, or 5 per cent. solutions is, generally speaking, prohibitory ; for example, profound shock repeatedly has followed the introduction of weak solutions into the rectum. It is soluble in hot water to the amount of 5 per cent., and may be rendered much more soluble by the addition of 10 per cent. of glycerin.

Mercuric Bichloride, like carbolic acid, is a germicide of considerable power, but is dangerous if brought freely into contact with the patient. It may be used for disinfecting the hands after prolonged scrubbing, for the sterilization of surgical dressings, and for solutions in which ligatures and sponges may be kept. The drug, however, should be washed out of the sponges with sterilized water before they are used. Irrigation of the bladder with a solution as weak as $1: 10,000$ has been followed by most violent exfoliative cystitis. It should never be used in the urinary system.

Sodium Carbonate.-Common washing-soda is an active germieide when used in a 1 per cent. solution with water, but it does not become active until the solution has been raised to the boiling-point; then sterilization is much more rapid than in plain boiling water. The boiling solution is said to dissolve the capsule of the germ and to 
destroy it in five minutes. This form of sterilization is suited best to instruments and other appliances that are not injured by heat.

Other antiseptics, such as formalin, alcohol, potassium permanganate, oxalic acid, sulphuric ether, essential oils, turpentine, boric acid, and nosophene, are useful in their places and will be considered later. Iodoform may be rejected bccause of its poisonous properties and offensive odor, which may produce protracted nausea and vomiting.

\section{Hospital Sterilizer.}

The High-pressure Steam Sterilizer for Hospital Use.-This sterilizer is almost indispensable in hospitals where it is necessary to sterilize rapidly large quantities of dressings and other appliances; but is too complicated and usually too expensive for general use in private practice; it consists of a large circular chamber in which the articles to be sterilized are placed and subjected in vacuo to highpressure steam. The creation of a vacuum before admitting the steam insures greater thoroughness in sterilization. This sterilizer is furnished in different sizes, the diameter varying from fourteen to eighteen inches and the length from twenty-two to thirty inches.

\section{Instruments, Pouches, Bags, and Cases.}

The conventional leather instrument-pouch is a prolific incubator of disease, and therefore must give place to the aseptic ponch of some washable fabric which may be sterilized by boiling and changed frequently. Figure 6 shows the instrument-pouch B unrolled and C rolled and tied. Figure $6 \mathrm{~A}$ shows an ordinary instrument-case.

The leather instrument-bag is certain to become unclean, and is therefore dangerous. The canvas-covered telescope valise is inexpensive, practical, and easily cleaned.

\section{Instrument-case, Low-pressure Sterilizers, Sponge-basins, and Trays combined, for Use in Private Practice.}

The apparatus shown in Figures 6,7 , and 8 is designed to lighten the burden and add to the safety of surgical work in private houses, especially in the country. From a satisfactory experience of sereral years the writer offers it in place of the septic instrument-bags, the conventional sterilizer, the cumbersome trays and sponge-basins which make up the usual impedimenta of surgical practice away from hospitals. The apparatus fulfils the requirements, first, of an aseptic instrument-case; second, of a steam sterilizer ; third, of instrument-trays and sponge-basins. It consists of two rectangular sterilizers made of copper, nickel-plated, in which may be packed all instruments and other appliances requisite for an abdominal section or for any other ordinary surgical operation. The component parts may be used separately as pans, sponge-basins, and trays. The whole outfit, enclosed in a telescope valise, is sixteen inches long, nine inches wide, twelve inches high, and when packed ready for an operation 
Figure 6.

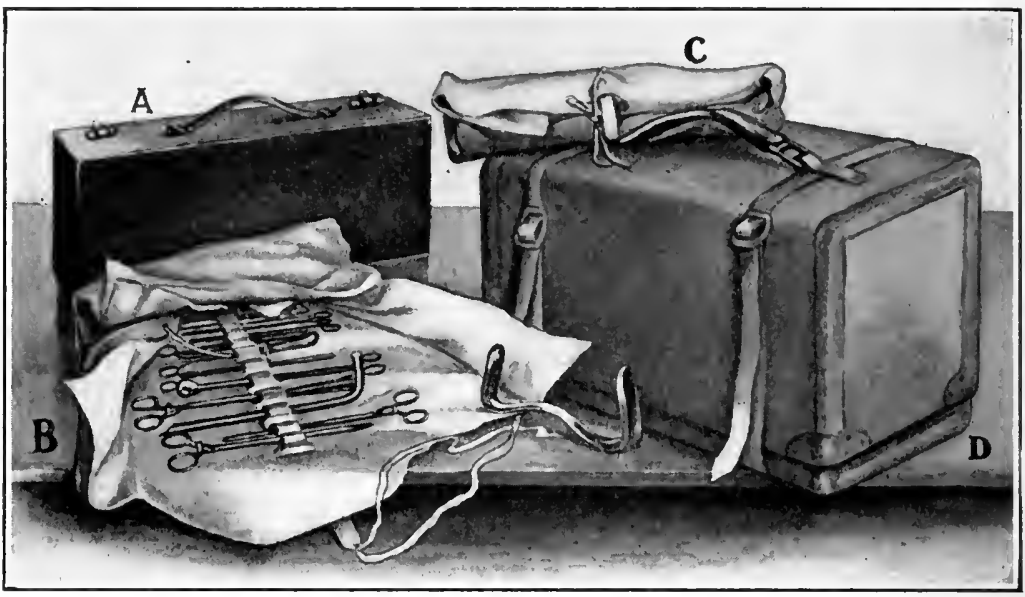

$A$, ordinary instrument-case ; B, washable instrument pouch, unrolled; $C$, poneh rolled and tied; D contains combination jistrument-case, sterilizers, sponge-basins, and trays, packed and ready to be taken to an operation.

Figure 7.

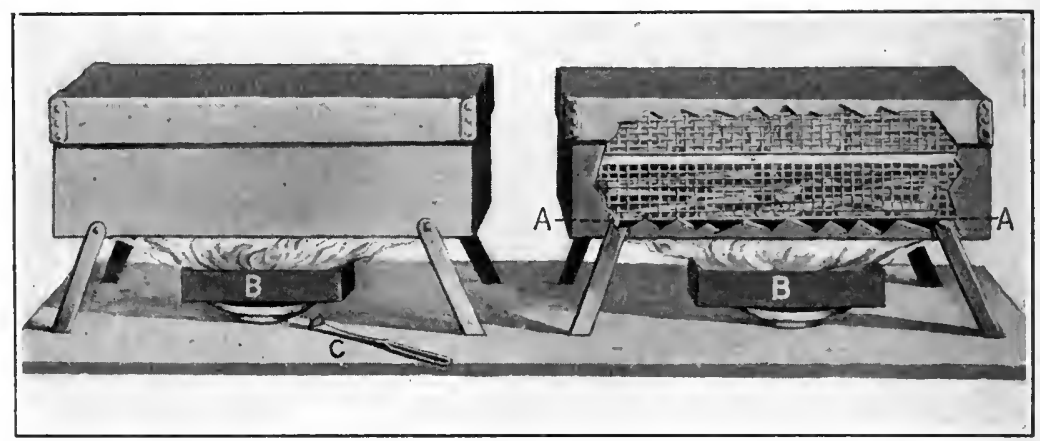

Ifetallie instrument-cases removed from telescope-ease and transformed into two sterilizers under steam.

Figure 8.

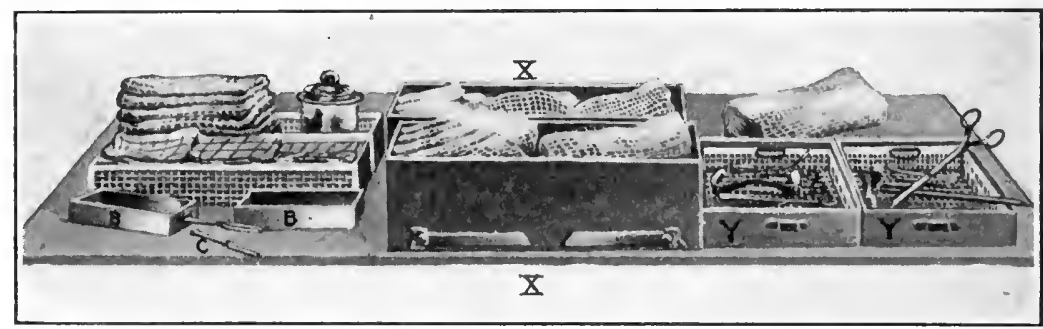

The several part of the combination instrument-cases being used as sponge-basins, pans, and trays

weighs about twenty-five pounds. Figure 6, D. 'This case contains a complete set of instruments, towels, sponges, ligatures, suitings, 
dressings, aprons, nail-brushes, sterilized green soap, ether, chloroform, alcohol, antiseptic drugs, rubber sheet, douche-bag, etc. The equipment is adapted for work anywhere. It especially solves the problem of aseptic surgery outside of hospitals, whether at the house of prince or pauper.

Figure 7 represents the two rectangular copper boxes as they appear under steam when used as sterilizers. Observe that each sterilizer is supplied with four legs, which may be folded against the sides of the box when the box is not in use as a sterilizer. Each box contains two gauze-wire trays, as shown through the broken side of the sterilizer in the right-hand cut of Figure 7. The lower tray is one inch above the bottom of the sterilizer, and contains instruments. The upper tray, resting upon the lower, contains towels, dressings, ligatures, etc. The space of one inch between the bottom of the lower tray and the bottom of the sterilizer-i.e., below the line $\mathbf{A}-\mathbf{A}$, Figure $7-$ is filled with sterilized water. The small trays, B and B, are filled with burning alcohol. These trays are set upon saucers to prevent burning the tablc-top. The burning alcohol converts the water into steam, which sterilizes the contents of the wire-gauze trays. One of the two detachable handles resting on the table between the two trays may be used to put out the flame by lifting the small alcohol-tray in contact with the bottom of the sterilizer. These detachable handles are designed also for use in separating the different parts of the sterilizers after the sterilization is complete.

Finally, the several parts of this apparatus may be utilized as sponge-basins, pans, and trays. Figure 8. The two large copper boxes become sponge-basins, $\mathrm{X}, \mathrm{X}$. The two top covers become trays, $\mathrm{Y}$, $Y$, holding sterilized water, inside of which two of the ganze-wire trays containing the instruments are placed. The gauze-trays may be lifted out by the detachable handles and placed in the covers without handling the sterilized instruments.

The other two gauze-trays to the left of the sponge-basins (Figure 8) hold the towels, gauze, sponges, dressings, and other things which have been sterilized in them. The two small, square, shallow cups which contained the alcohol in Figure 7 now become trays for needles, ligatures, and other small appliances. Figure 8, B and B. Observe that this sterilizer is quite as well adapted for sterilization by boiling water as by steam. After the apparatus has been under steam for thirty minutes, especially if this process has been repeated three times on consecutive days, not only the contents, but also the various parts which are to be used as sponge-basins and trays, are sterilized thoroughly. Each member of the apparatus is supplied with one or more slots or rings, into which fit the detachable metallic handles already mentioned. These handles are useful to separate the sterilizer into its several parts while hot, and to avoid unnecessary handling. After an operation, even upon a septic case, all the parts of the apparatus may be washed and then sterilized by boiling in a large wash-boiler. The boiling water should contain 2 per cent. of sodium carbonate. 


\section{Preparation for an Aseptic Abdominal Section.}

Asepsis necessitates a number of antiseptic procedures all looking to an aseptic result. The scrupulous preparations about to be outlined for major operations are not intended to imply that equal care is unnecessary for minor operations, because the latter are by no means free from danger of fatal sepsis, and because a performance seemingly of minor importance in the beginning may end, accidentally or purposely, in opening the abdomen or in some other capital procedure. Traumatic infection of the peritoneum involves the gravest consequences, hence the need of extreme precautions in technique; and since the greater may include the lesser, the same technique will suffice for the minor procedures.

The recklessness which results in the unnecessary removal of pelvic organs seldom escapes criticism. The recklessness which results in the unnecessary introduction of sepsis into the peritoneum is often passed by without comment. The danger to life, houcerer, is determined less by what the surgeon takes out than by what he puts in. The development of sepsis requires two conditions : first, pathogenic bacteria must be present; second, the way must be opened for them to enter. Experiment has shown that they may be transnitted even through the unbroken skin or mucous membrane, but that traumatism makes an open door. Pathogenic bacteria have their source, first, in the operator or his assistants; second, in the instruments and other appliances; third, in the patient. The antiseptic procedures to an aseptic result therefore must be these:

1. Preparation of the operator and his assistants.

2. Preparation of the instruments, sponges, dressings, and other appliances.

3. Preparation of the patient.

1. Preparation of the Operator and His Assistants.-Health of the Operator.-The operator and assistants should be in good health, and, since the breath may be a medium of infection, they should especially be free from nasal catarrh and coryza. Disorders of nutrition which involve deficient elimination through the bowels and kidneys may throw that function upon the lungs, and canse the breath to be loaded with fetid products, an undoubted source of infection. The bacteria of saliva may be most infectious ; hence unnecessary talking over the field of operation is objectionable, for small particles of saliva and its bacteria may reach the wound. Experience has shown that an orator in speaking may throw particles of saliva thirty feet into the andience. The necessity, therefore, of protecting wounds and open cavities, by means of a mask, against the germ-laden breath of the operator and assistant is apparent and urgent. A mask of ten layers of gauze tied over mouth and nose is effective; fewer layers have been found inadequate. A striking case in point is the following: A colleague, while operating for varicose vein of the leg, noticed a slight particle of saliva thrown into the wound from the mouth of his assistant, whose saliva, on examination, was proved to contain streptococei. The patient, despite most careful antiseptic care, died of streptococeus infection. 
The daily bath is an important part of the routine of aseptic surgery. Special clothing made of washable material is desirable; for women the usual costume of the trained nursc, and for mentrousers and shirts or short coats. Special clothing for operation has a threefold advautage. It protects the operator from taking cold after leaving the operating-room in his ordinary clothing, which, if worn during the operation, might be wet with perspiration. It saves the ordinary clothing from contamination when the operation is upon a septic case. It is, above all, an antiseptic measure in the interest of the patient.

Sterilization of the Hands and Arms.-Formerly the extreme mortality of abdominal sections was due in great part to direct infection from the hand of the operator. To wash the hands rapidly in soap and water and then to dip them in some autiseptic solution, a not uncommon practice even now, gives little protection against infection. Absolute sterilization of the skin without injuring it is ideal and impossible. Practical asepsis, however, is possible. To bring this about numerous antiseptics have been used; by antiseptics are meant antiseptic drugs and antiseptic measures. Of these, prolonged scrubbing with green soap sterilized by heat and with hot water is the most effective. A mixture of alcohol and sulphuric ether, which have germicidal properties, each one part, with four parts of green soap, makes a valuable liquid antiseptic soap. The green soap should be of good quality and previously sterilized by heat. Beat one pound of this soap in a receptacle with two ounces of alcohol until uniformly smooth. Transfer to a glass bottle of at least three pints capacity and add two ounces of ether. Cork well, agitate, and set aside for two hours. Then add, with thorough shaking, two ounces each of ether and alcohol previously mixed. The scrubbing of the hands and forearms, to be effective, must be in soap and water as hot as can be borne without positive discomfort. The heat is a valuable aid in the removal of dirt. The scrubbing must be thorough and vigorous, and prolonged for at least fifteen minutes. The longer the scrubbing the more difficult it is to cultivate bacteria from the scrapings of the skin.

Prolonged Scrubbing makes the hands safe, not so much by the destruction as by the mechanical removal of bacteria. They are removed together with the secretions of the skin and other foreign matter upon which bacteria flourish. To scrub the hands and forearms, always use a very large brush, preferably without handle. The large brush is indispensable; it camnot, however, be made to reach those strongholds of bacteria so often overlooked or neglected, the angles between the fingers; to scrub out these angles thoroughly, use a brush with a handle of ordinary size, but do not attempt to scrub the other parts of the hands and the arms with such a brush; it is too small. Destroy all brushes that have been used in septic cases. Brushes not in actual use should be made aseptic and kept in aseptic gauze or towels.

Alcoholic Solution of Mercuric Bichloride.-After scrubbing witl green soap or the liquid antiseptic soap just mentioned, wash off all 
trace of soap with clean water. Then wash with $1: 3000$ solution of mercuric bichloride in 70 per cent. alcohol. Frequent washing of the hands and arms in this solution as often as they become dry for a period of ten minutes is more practical and, therefore, more likely to be carried out effectively than the usual soaking for an equal length of time. Aqueous solutions sterilize less surely and less quickly and, therefore, are unnecessary for hand sterilization if this alcoliolic solution of mercuric bichloride be used.

Rubber Gloves.-Rubber-gloves serve as proteetion alike to the operator and the patient, and in all abdominal operations should be worn by all persons whose hands are brought into relation directly or indirectly with the ficld of operation. After an operation they may be sterilized by boiling, and used again. Only two plausible objections to the use of the gloves have been raised: first, that the hands can be sterilized adequately, and that the gloves are therefore unnecessary ; second, that they impede the operator to such an extent as to increase rather than diminish the danger of the operation. The reason for the first objection is not true; but if it were true that the hands can be sterilized adequately, it by no means follows that they always will be. The second objection, that gloves impede the operator to any considerable degree, ean be urged by no one unless he would thereby give such evidence against his own dexterity as will raise the question of his fitness for surgical work. Gloves may be dispensed with in exceptional cases when very rapid work is of vital importance. The use of the gloves should in no respect lead to relaxation in the sterilization of the hands, for they may be cut or punctured during an operation, in which ease, however, they should be replaced immediately by fresh ones.

Sterilization of the Nails, Hair, and Beard.-Let the nails be cut short; long nails retain quantities of dirt which any amount of scrubbing may fail to dislodge. They are also a possible cause of unnecessary irritation, not to say traumatism, and may therefore be both the carriers of poison and the instruments for its inoculation. The shorter the hair the less dirt will there be to fall from it into the wound. The hair and scalp must be kept clean by frequent washing and brushing. The long, full beard is an unnecessary source of danger ; the less beard the better. A gauze turban about the operator's head guards the wound from fine particles of dirt which otherwise might fall from the hair; if brought well down on the forehead, the turban absorbs perspiration and thereby keeps it from dropping into the wound. The operator's forehead, if wet with perspiration, may be kept dry by means of a towel in the hand of a special assistant.

2. Preparation of Instruments, Sponges, Dressings, and Other Appliances.-Sterilization of Instruments.-All instruments not injured by heat may be sterilized by boiling or by steam. Sterilization by boiling takes only five minutes if the boiling water contains 1 per cent. of sodium carbonate. This method is perfect in its results, even though the instruments have been used in a septic case. Boiling in rarbolic acid solution is no more efficient, and it injures the instruments.

Before and after an operation instruments, sponge-basins, trays, and 
other appliances may be washed thoronghly in soap and water to remove the visible dirt, and then sterilized by boiling in a large washboiler. A good way is to sterilize instruments by boiling just after using, and by steam just before using. During an operation the instruments should be arranged in trays and eovered, not with antiseptic solutions, but with sterilized water.

Sterilization of Water.-Water may be sterilized by boiling for thirty minutes. If not already clear, it should be filtered before boiling. In aseptic surgery sterilized water is indispensable for many purposes, such as to wash the hands, to cleanse the field of operation, to irrigate the wound, to wash sponges, to cover instruments in the tray, and, when indicated, to wash out the peritoneal cavity. Ten gallons should be sterilized for an abdominal section. Hospitals usually are provided with reeeptacles for sterilized water. At the patient's house water may be sterilized and kept until the time of the operation in two large wash-boilers, preferably new, in which it has been boiled. It should be kept half hot and half eold, so that by mixing, the right temperature may be secured.

Sterilization of Towels.-Towels should be of good quality and free from lint; the so-called glass-towels used for drying glassware are best. They should be laundered in the ordinary way, then boiled in a 1 per cent. solution of sodium carbonate, ironed, done up in sterilized linen, and packed in a elean tight box. Twenty towels are required for an ordinary abdominal section. Just before operation they should be re-sterilized by steam.

The Sterilization of Sea-sponges by the usual processes of washing and soaking in antiseptic drugs is tedious, difficult, and not always adequate. The uncertain results of these methods have led most surgeons to abandon sea-sponges, and to substitute for them the readily sterilized gauze.

The Sterilization of Gauze Sponges is by boiling or by steam, after the directions given above for the sterilization of towels. They should be made of four thicknesses of sterile gauze, and should be six inches wide by eighteen to twenty inches long. Smaller sponges may be overlooked in the abdominal cavity and lost, or, at the end of a long operation in which many sponges have been used, they may be difficult to find. The frayed edges of the gauze should be turned in and stitched; otherwise loose threads may stick to the wound or be left in the eavity and become irritating foreign bodies. See preeaution relative to sponges in Chapter VI.

Sterilization of Silkworm Gut and Dreasings.-Silkworm gut and gauze dressings may be sterilized by steam, or by boiling, or by both.

Sterilization of Catgut. - Frequently eatgut is sterilized by boiling in alcohol and then soaking in antiseptic solutions. Not only are the results of this method unsatisfaetory, but numerous distressing injuries have oecurred to those using it from accidental explosion or burning of the alcohol. Iodized eatgnt and eatgut sterilized by the dry heat process of Boeckmann will resist absorption only a few days. Catgut sterilized by the chromic acid process will resist absorption, according to circumstances, from ten to forty days. 
Iodization by Claudius' Solution.-For general use in abdominal surgery iodized catgut should have the preference over all others. The gut, being loosely wound on glass reels, is easily prepared by soaking in Claudius' solution of iodine for eight davs, at the end of which period the solution is poured off and the gut left in the same jar, which should be sealed. The solution is composed of :

Iodine crystals, 1 part.

Potassium iodide, 1 part.

Alcohol, 95 per cent., 50 parts.

Water, 50 parts.

The Dry Heat Process.-The individual strands, cut in lengths of two or three feet, are coiled, and each is donble wrapped in paraffin paper and placed in a small envelope and carefully sealed. 'The envelopes then are placed in a wire basket. This is exposed to dry heat, $284^{\circ} \mathrm{F}$., for a period of three hours on each of three successive days. It is necessary that the temperature on the first day be raised gradually to the required degree; this is because the gut is rendered brittle by a rapid increase of temperature before the moisture has been dried out and replacel by the absorption of paraffin from the paper. Let the temperature be raised to $212^{\circ} \mathrm{F}$. at the end of the first hour, and maintained at this point for one hour continuously; then raise it gradually so that at the end of the third hour it will be $284^{\circ} \mathrm{F}$. The temperature must now be held between $284^{\circ}$ and $300^{\circ} \mathrm{F}$. for three hours. In repeating the process on the second and third days the temperature may be raised rapidly to the required degree.

'I'he Chromic Acid Process.-Catgut may be chromicized, as follows :

1. Soak the raw catgut, sizes $0,1,2$, and 3, in Squibb's ether for fourteen days, changing the ether twice.

2. Wind on glass tubes.

3. Soak in an aqueous solution of chromic acid $(1: 3000)$ for fifteen to eighteen hours. Catgut soaked for eighteen hours will resist absorption much longer than that soaked for fifteen hours.

4. Boil in Merck's saturated solution of ammonium sulphate for twenty minutes.

5. Remove from the ammonium sulphate solution and rinse for fifteen minutes in cold sterile water.

6. Preserve in air-tight jars containing an alcoholic solution of nercuric chloride, $1: 2000$.

Aseptic and Antiseptic Dressings, such as gauze and absorbent cotton, are now articles of commerce. If obtained from the best sources, they may be reliable. Absolutely safe antiseptic and aseptic gauze may be prepared readily by the surgeon or nurse. Many kinds of antiseptic gauze are used; two varicties, however, the sublinated and the borated, fulfil all indications. Aseptic gauze is also necessary. Sublimated gauze is useful for external dressings; it is contraindicated in the dressing of exposed surfices, because dangerous, even fatal, poisoning has resulted from absorption of the bichloride of mercury. It should never be put into the abdominal cavity. Borated and aseptic gauze may be used with safety on exposed surfaces or even in the peritoneum. 
To Prepare Sublimated Gauze, boil plain commereial gauze ten minutes in a 2 per cent. solution of sodium earbonate, wash thoroughly with clean water, boil for thirty minutes in a $1: 10,000$ aqueous solution of biehloride of mercury containing 5 per cent. of glycerin, let it stand in the solution for twelve hours and then dry.

To Prepare Borated Gauze, boil plain commercial gauze ten minutes in a 2 per cent. solution of sodium carbonate, wash with elean water, boil for thirty minutes in a saturated aqueous solution of borie aeid, and dry.

To Prepare Aseptic Gauze, boil plain commercial gauze thirty minutes in a 2 per cent. solution of sodium carbonate and wash with sterilized water. Plain aseptic gauze is adequate for all surgieal purposes. For several years I have used it to the exclusion of all others.

Sterile gloves should be worn in handling instruments and dressings which are to be used in an operation.

General Precautions.-All varieties of gauze and all forms of dressings should be sterilized by steam just before they are used. See description for dressing abdominal wounds in Chapter VI. The operating-room should be clean, well ventilated, well heated, well lighted, free from carpets, stuffed furniture, infeetious drains, and everv other object liable to be a medium of infection. Dcor-knobs and other parts of the room, if liable to be in contact with the hand of the operator or his assistants, should be covered with aseptic gauze.

3. Preparation of the Patient.- The antiseptic preparation of the patient has a twofold purpose: First, to remove, destroy, and limit the power of pathogenic bacteria; this requires the local application of antiseptic measures to the abdomen, external genitals, and ragina. Second, to enable the patient to resist any bacteria that may remain or develop. This may require both regulative and medicinal treatment. A searehing general examiuation from the standpoint of internal medicine should be made in every case. This examination may show phthisis or diabetes, or some other contraindication or condition which would make the operation extra-perilous. Then the preparatory treatment should be direeted to that condition. To be forewarned is to be forearmed.

When the operation is not one of emergency the preparation may well include several days of observation and treatment. In this way often the patient's peeuliarities may be measured, and her power to resist infeetion may be increased. The abdominal and thoracic organs should be examined, especially the lungs, heart, and kidneys. A quantitative examination of urine may show a defieiency, for example, of urea; then a few days of judicions diet and diuretics may turn the result of an operation in the patient's favor. The daily general bath, with frietion, besides being an antiseptic measure, increases the action of the skin and relieves the kidneys.

The Drinking of Water in large quantities during the week before a capital operation is most advantageous and when practicable never to be neglected. Three or four pints a day should be taken.

The Bowels.-Bowel distention impedes the operator and lengthens the operation. It is a dangerous complication both during and after 
the operation, and is the cause of a great deal of mortality. So far as practicable, then, let the bowels be emptied of gases and solids and of whatever may ferment and form gas. Experiment has shown that the countless myriads of bacteria habitually present in the intestine may be redueed by catharsis and intestinal antisepties to a relatively insignificant number; henee the following measures are suggested to render the bowels, as nearly as possible, aseptic :

1. For several days before the operation exclude all food that is apt to ferment.

2. On the third night before the operation give five grains of blue mass. If the bowels do not act freely the next morning, give an ounce of castor oil. On the day before the operation give a Seidlitz powder or some other active saline purge. Two componnd cathartic pills may be substituted for the blue mass. Repeat the cathartics if neessary. A large dose-2 ounces-of eastor oil one or two days before the operation is most satisfactory and may take the place of all other cathartics.

3. Give repeated high copious enemas during the two days before the operation. The enemas may be of soapsuds, each pint containing, thoroughly mixed, a drachm of turpentine. Persevere in this until no considerable amount of gas remains. If the turpentine and soapsuds enema does not suffice, try a mixture containing two ounces each of glycerin, Epsom salt, and water. Use a flexible rectal tube with firm walls, three feet long, and give the enema as high as possible:

Cleansing the Field of Operation.-Every abdominal section may require, for drainage or for other reasons, that an opening be made from the peritoneal cavity into the vagina; hence the necessity of cleansing not only the abdominal wall, but also the vaginal surfaces and external genitals.

Clensing the Abdomen to Prevent Stitch-hole Abscesses. -Twenty-four hours, or, if possible, forty-eight hours before operation the entire abdominal, pudic, and perineal areas are shaved earefully, including not only the coarse hair, but, so far as possible, the white downy hairs, especially those about the navel. A poultice of liquid antiseptic soap, already described, is then placed over the abdomen, including the epigastrium, and allowed to remain one and one-half hours; upon the removal of this poultice the remaining soap is washed off first with sterile water, and then with commercial ether. The abdomen then is covered with thick layers of gauze wet with an aqueous mercuric bichloride solution, $1: 3000$, the whole being covered with oiled muslin or gutta pereha and an abdominal binder. This dressing is rewet every four hours and retained until three hours before the operation, when fresh sterile gauze wet with a $1: 2000$ alcoholic mercuric bichloride solution is substituted and retained until the patient is under anæsthesia. The last dressing then is removed and over the abdomen liquid antiseptic soap containing ether is poured. The umbilicus is filled completely with soap. As little water as possible is used, and the soap is worked up into a layer of paste, a towel being used for the purpose instead of a brush. The entire abdo- 
men, epigastrinm, and hips are scrubbed until a delicate erythema is produced. The umbilicus is cleansed carefully and the soap washed off thoroughly with sterile water. The abdomen then is dried with a sterile towel, and over the whole area sublimated alcohol, $1: 2000$, is poured. The umbilicus is filled with sublimated alcohol, to be nopped ont with ganze just before the initial incision is made. The reison for working the soap into a paste with as little sterile water as possible is because the excess of alkali saponifies the fat of the skin and probably destroys the capsules- $i$. e., outer coverings-of the microorganisms.

The rationale of preparing a large area when only a small portion of the abdomen is to be incised is that in some cases the incision may have to be extended greatly so that skin surfaces which one has no previous idea of handling may come in contact with the hands or gloves. A process so extensive and detailed as this may seem unnecessary, but there is proof that it almost entirely prevents wound-infection and stitch-hole abscesses.

Cleansixg the External Pudenda and Vagina.-The mons veneris and vulva having been shaved, three vaginal douches are to be given on each of the three consecutive days before the operation. Each douche should consist of, first, strong soapsuds made of green soap; second, sterilized water; third, solution of bichloride of mercury, 1:3000.

Just before beginning an operation, when the patient is under the anesthetic, the external genitals and surrounding parts should be scrubbed thoroughly with a large sterile towel, and the vagina should be scrubbed and swabbed out with a wad of gauze in the grasp of a long hæmostatic forceps. Sterilized green soap or the liquid antiseptic soap already described should be used. All soap should now be washed away by a stream of sterilized water poured from a pitcher, and the parts further sterilized by filling the vagina with a 1:3000 70 per cent. alcoholic solution of bichloride of mercury. Finally the vagina should be packed with a continuous strip of gauze saturated with the same solution; this packing should remain in the vagina during the operation unless it becomes necessary to open from the peritoneum into the vagina, in which case the gauze should be removed; it insures asepsis of the vagina and is a precaution of the utmost moment, in view of the possibility of opening from the pelvic cavity into the vagina.

Curettage.-It is wise, especially in a case of infectious endometritis, to curette and disinfect the endometrium before proceeding to open into the pelvic cavity. This precaution may prevent postoperative infection in the pelvic cavity by extension from the uterus.

In the giving of the donche the familiar Kelly pad will be found more useful and more practical than the bed-pan. The objections to this pad are, first, that it is not always obtainable; second, it is difficult to keep clean, and is therefore, for surgical purposes, apt to be septic.

The writer uses a practical substitute for the Kelly pad that 
obviates both objections. It is simply a piece of sheet rubber, three feet wide and four and one-half feet long. The rubber sheet at its upper end and sides is folded over rolls of toweling or muslin, so that, as in Kelly's pad, the water will be directed into the bucket below. See Figure 9. Rubber sheeting is available everywhere, is cleaned easily, and is so inexpensive that it may be renewed frequently. Sheeting which has the rubber finish on both sides is preferable. The ordinary oil-cloth used to cover a kitchen table is serviceable, and may be obtained in almost every house.

Figure 9.

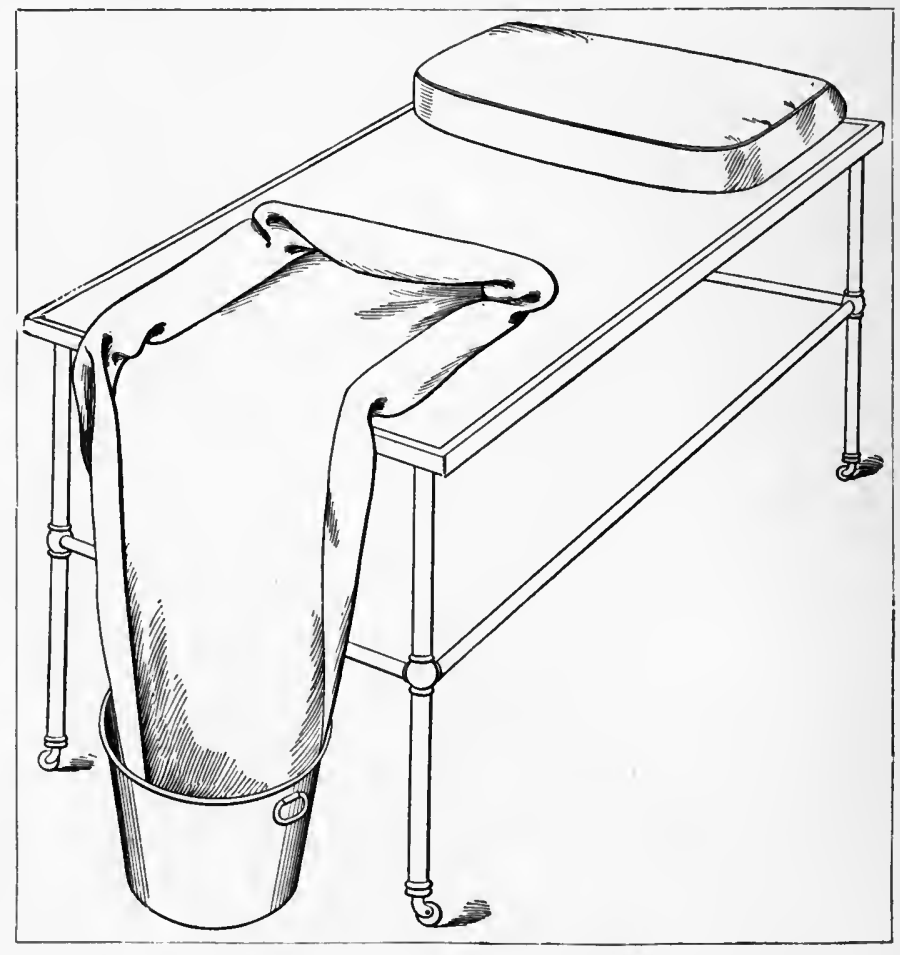

Practical substitute for the Kelly pad.

Protection for the Feet and Legs.-When the patient is placed on the table before the anæsthesia begins, it is well to cover her feet and legs with long sterilized fiannel stookings.

\section{Preparations of an Aseptic Vaginal Operation.}

The surgery of the vaginal portion of the pelvic floor is classified ustually under the head of minor operations. This designation, since it implies that the operations are trivial and safe even without full precautions, is misleading and dangerous. High vaginal amputation of the cervix uteri and the removal of an intra-uterine tumor by 
morcellement, for example, are clearly major operations. Curettement, perineorrhaphy, trachclorrhaphy, dilatation of the cervix, closure of vaginal fistulas, though relatively safe, are in an absolute sense dangerous. Failure to observe aseptic technique in vaginal operations, although less frequently fatal, is yet full of danger. The possibility of a fatal pneumonia, nephritis, phlebitis, or lymphangitis as the direct result of an unclean "minor" vaginal operation is not sufficiently appreciated. The same cleansing process as set forth above for abdominal section is imperative.

\section{The Asepsis of Minor Manipulations and Examinations.}

Since the unclean uterine probe repeatedly has cansed fatal metroperitonitis, and since "death has been carried to many a woman under the finger-nails," it follows that the same principles which apply to surgical work also hold good in the ordinary rontine examinations and local treatment of the pelvic organs.

Asepsis of the Patient.-Sterilization of the endometrium, vagina, and vulva preparatory to ordinary office manipulation is impracticable, not to say impossible. Reasonable safety is secured, however, by the hot vaginal douche which the patient usually takes before applying for treatment. As a supplement to this it is best to wipe out the vagina with dry absorbent cotton on long lock forceps, and then with absorbent cotton saturated with a 5 per cent. solution of carbolic acid in glycerin, or with a 1:2000 aqueous solution of bichloride of mereury. Disinfection of the vagina or vulva in this way is especially essential if the uterine cavity is to be instrumentally or digitally explored or treated. By this means the endometrium is protected against the entrance of septic matter, which otherwise may easily be carried in from the vulva or vagina on the instrument-a very common mode of infection.

Asepsis of the Hands ; Rubber Gloves.-The cleaning and disinfection of the hands and nails before and after the most ordinary digital examination are imperative, not only to guard against the carrying of infection from patient to patient, but to prevent self-inoculation with specific or non-specific virus through some abrasion upon the hand. The nails should be trimmed short; what has been said about rubber gloves in surgery is equally applicable here in the interest of the patient and more applicable in the interest of the examiner. By the use of gloves for examination many a physician would be saved from the calamity of specific and other infections.

Asepsis of the Instruments.-The former practice of simply washing instruments in soap and water after each treatment is unsafe. Ordinary washing does not remove micro-organisms. Surgical cleanliness may be secured by washing the instruments in hot water and green soap and boiling them for five minutes in a 3 per cent. solution of sodium carbonate. It is convenient for this purpose to have always during the office-hour a deep tray of the solution constantly boiling over the flame of a spirit-lamp or a gas-burner, or more convenient to have several set of instruments, which may be used one 
after the other, and then all disinfected together at the end of the office-hour.

The Lubricant of Vaseline or Oil, usually kept near the examination-table, is unnecessary for lubricating purposes when the natural secretions are profuse and themsclves serve that purpose. Some artificial lubricant is always uscful, however, to protect the operator's fingers against infection; but the lubricant is often a source of sepsis in itself, or it may easily become so by contact with the unclean finger or instrument. Gonorrhœal and other infection frequently is carried from patient to patient in this way. Neither fingers nor instruments, therefore, should come in contact with the lubricant unless they are free from vaginal and other secretions-unless absolutely clean. The lubricant should be aseptic and non-irritating. Olive oil and vaseline are often septic and difficult to wash off. Soap is apt to irritate the sensitive vulva. For several years the author has used glycerin. It is a most excellent lubricant and deodorant. Even after digital examination of cxtremely fetid uterovaginal cancer, the foul nauseating order, usually so lasting, may be washed off the examiner's hand by placing it under a stream of running water, if before the examination the hand was lubricated freely with glycerin. For this purpose a superior quality of glycerin is required. In cancer and other septic cases gloves should be worn.

Glycerin Emollient.-CThe adaptability of glycerin for this purpose has led to the preparation of a glyeerin ointment. It is put up in soft metallic collapsible tubes, such as are used for vaseline and paints. The ointment is forced out of the tube by compressing the botton between the thumb and finger and folding the flattened end as the tube is emptied. The use of the tube obviates the risk of contaminating the lubricant by the soiled fingers. The preparation is a sterilized combination of the following ingredients : oil of gaultheria, $2 \mathrm{gm}$; boric acid, 23 gm.; corn-starch, 88 gm.; pure glyccrin, 885 gm.; tragacanth, $17 \mathrm{gm}$. The formula is the outcome of numerous experiments by Parke, Davis \& Company, who, upon the author's suggestion, have perfected the preparation. It is furnished by them under the name "Glycerin Emollient."

A Word of Caution. - Many a distressing pelvic infection owes its: origin to meddlesome office gynecology. Instrumental invasion of the endometrium and other manipulations which require much force are procedures which, under any conditions, may be far from trifling. The physician's office does not furnish for them a uniformly safe environment. They require and should have the safeguards of the home or the hospital. 


\section{CHAPTER III.}

\section{DIAGNOSIS.}

THE subject of diagnosis is divided into two parts : first, the clinica! history; second, the physical examination.

\section{THE CLINICAL HISTORY.}

Before asking questions or recording any of the history it is well to let the patient make her own statement without suggestion; this will relieve her of nervousness and compose her mind for the systematic questioning.

Histories usually are kept in blank case-books printed and bound for the purpose. A very practical way is to keep them in individual envelopes, made of strong manila paper, each history in an envelope by itself, with the name, residence, and date of the first visit written across the end. The histories are kept in alphabctically arranged pigeon-holes, where they may be found readily. The great advantage of this plan is that the histories may be written away from the office on seraps of paper, and do not have to be copied, but may be filed away together with any subsequent correspondence, preseriptions, or additional notes.

Form for Record of Cases.-The skeleton form given on the following pages is suggested for the convenient and systematic record of cases. The printed blank is subject to snch erasures and additions as the individual case may require. In using such a blank one must keep in mind the fact that no stereotyped form can include suggestions for all the points that are liable to come up in eonnection with a case. Unless, therefore, one supplements the inquiry by such questions as each special ease may call for, he will fall into a dangerous routine.

The record form which follows will help the student and young practitioner to form the habit of aceurate and systematic diagnosis. As one gains experience and automatic grasp, and judges less from: multiform details and more from principles, he will eliminate gradually from his histories and records all that is not essential to the efficient analysis of his cases. A few general statements then may serve the purpose of a practical memorandum.

In recording a case one may use conveniently abbreviations and signs, for example, the plus sign $(+)$, the minus sign $(-)$, the plus or minus sign $( \pm)$, the zero sign $(0)$, the sign of equality $(=)$; and the letter $\mathbf{n}$ may signify $(+)$ excessive, $(-)$ less than normal, $( \pm)$ variable, $(0)$ no, none, or negative results, $(\Longrightarrow$ equals or amounts to, (n) normal; v.s. $=0$ would be, for example a short expression to indicate the absence of vesieal symptonis. 
52

GENERAL PRINCIPLES.

RECORD OF A CASE;

ABBREvitions: The sign + signifies excessive; - less than normal; v, variable; 0 , no, none or negative results:

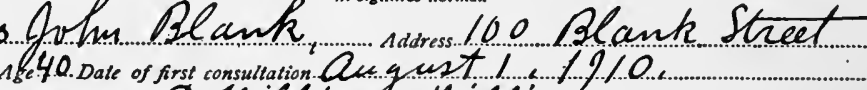
recommended by Dr.pilliag

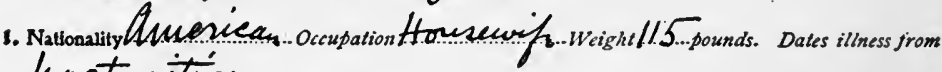
kusturitin.

2. Single, Married, Widow /.2u.years. 3. Well, til developed. Anemic,

4. Family History....... Key atisiz

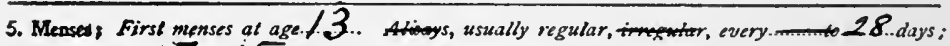

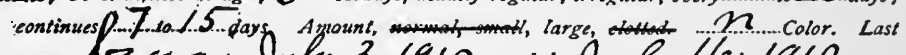

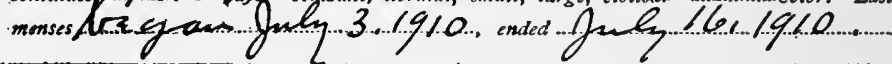

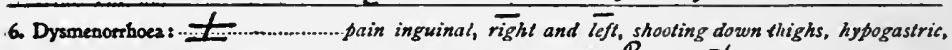

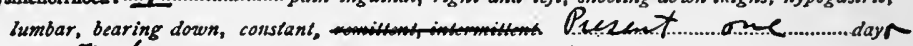

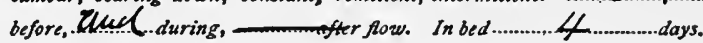

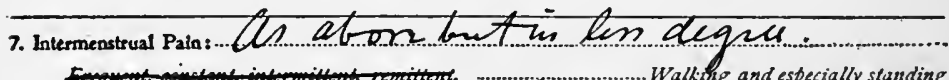

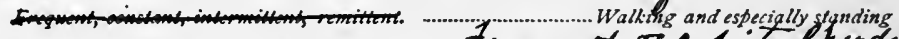

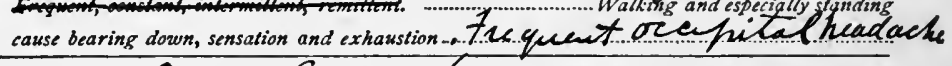

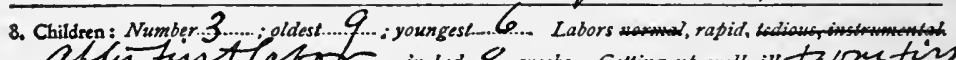

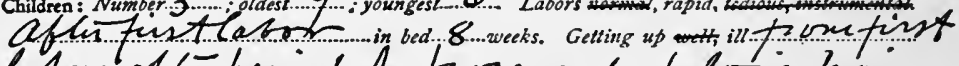

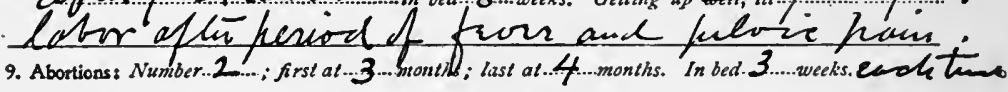

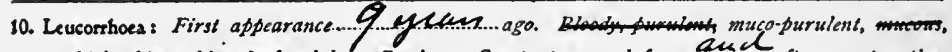

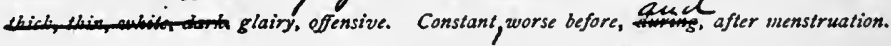

11. Bladder Symptoms: Urinates ..t times during night, t..times during day. Frequency increased. by standing or walking.

12. Digestion: Teeth...............; appetite..._...; eating causes.....

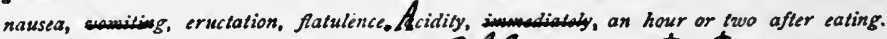

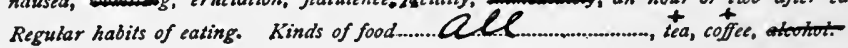

13. Bowels: Argon, constipation and diarrhea, alternating. Action fun, scanty, digit, offensive,

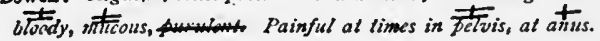

5. Menomagan sima bisthpreand child 8. Aflinfist later soidanty had julie inflammation. 
DIAGNOSIS.

53

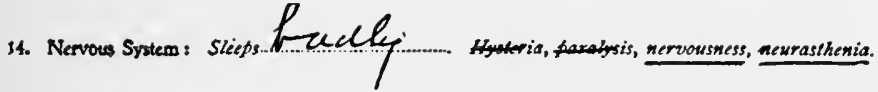

15. Extra Pelvic Organs: Paypintation of th teant intestanal indigestion and ganeral functional distus Fandes of tho digistir system

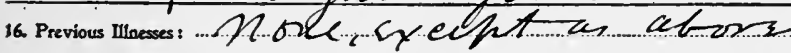

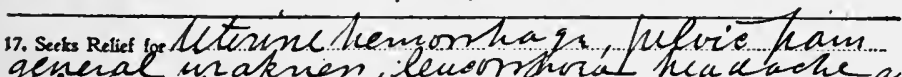

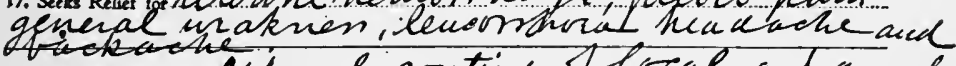
18. Previous Treatment: Minal noutine of lowal and gnuml thestiment at home and at sanitaria.

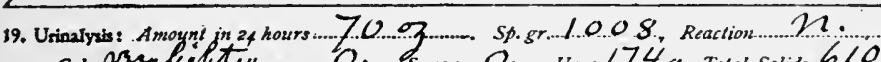

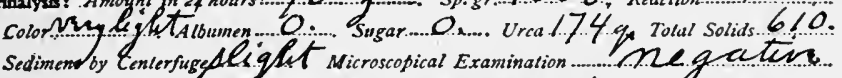

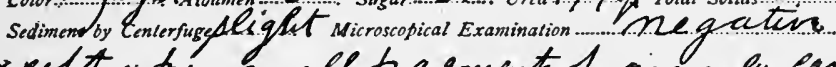

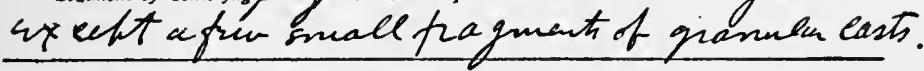

20. Physical Examination, Diagnosis and Treatment

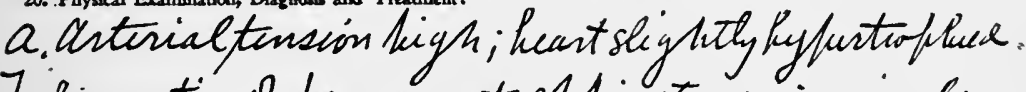
7. Liacerotion of ferencum to Sphinets ari misele c. Lucuation of Cesory uters and sudmetsity d. Retroussion of th uteris, $3^{2}$ degree.

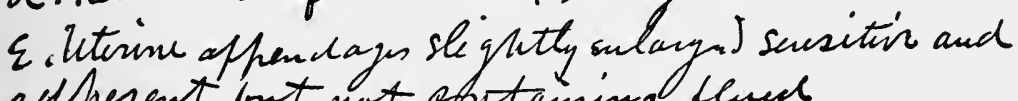
adherent but not ofrotaining flued $f$ cystrich and rectriche.

g. haenompoides, sxtreme.

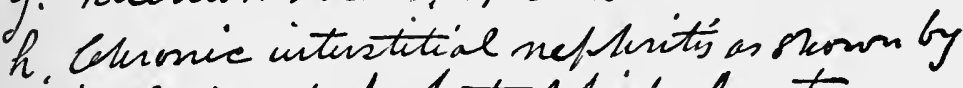
irinalysir and hyfurtiophied keact.

Juatiment acloised

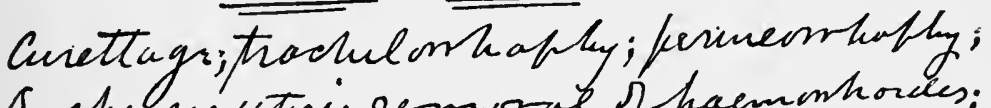

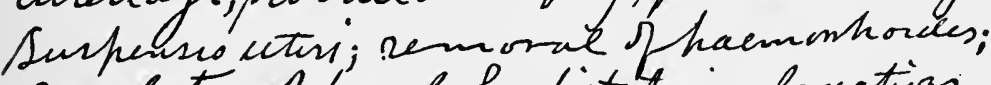
regulation of bouses by diet tonic lax ativs, and axnase; quenal mossograngegulation of mode of life, copuciall, dlet, writhefor-

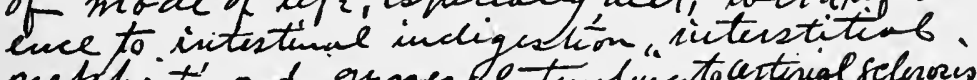
nefthisty and grmende tending to artivial seleroris. 
The hypothetical case outlined in the history blank is not out of the common. The patient had been a well-developed woman, of good family history. The menstrual and other functions had been perfectly normal until after the first child was born. Then came the abnormal developments recorded in seetions 6 to 19 . Neglected lacerations of the cervix and perineum opened the door for the entrance of infection; hence infection spread through the endometrium and possibly also through the parametric lynuphaties and veins to the tubes, ovaries, and adjacent peritoneum. Adhesions formed, binding the uterus with its appendages together in posterior displacement; this displacement was increased and perpetuated by the excessive weight of the uterus, by impairment of support from the lacerated perineum ; that is, from injury to the pelvic floor and from the now relaxed, subinvolnted state of all the pelvic organs and their supports.

Endometritis and metritis give rise to menorrhagia and leucorrhœea, which explains, partly at least, the anæmia, neurasthenia, nervous irritation, and impaired general nutrition. Diffieulty of walking and standing, both from general weakness and from displacement of the pelvic organs, interferes with necessary exercise, and still further adds to the causes of malnutrition. The increased frequency of urination when the patient is on her feet may be explained by the fact that the organs at that time descend to a lower level and drag on the bladder. Intestinal indigestion, sluggish liver, faulty metabolism, constipation, deficiency of urea and of other urinary solids, excess of uric acid, and finally chronic interstitial nephritis, are associated not uncommonly with pelvie traumatism and infection.

The difficulties of gynecological diagnosis often are increased by the fact that pelvic lesions may exist and cause no definite local symptoms. Even greater confusion may arise from the presence of pelvic symptoms which are caused not by pelvic, but by extrapelvic, disorders.

The nerve counterfeits of pelvic disease are most realistic and bewildering, and have been expressed well in the following paragraphs by the late William Goodell :

"Nerve-strain, or nerve exhaustion, comes largely from the frets, the griefs, the jealousies, the worries, the bustles, the carks and cares of life. Yet, strangely enough, the most common symptoms of this form of nerve disorder in women are the very ones which lay tradition and dogmatic empiricism attribute to ailments of the womb. They are, in the usual order of their frequency, great weariness and more or less nervousness and wakefulness, inability to walk any distance, and a bearing-down feeling; then headache, napeache, and backache. Next come scanty, or painful, or delayed, or suppressed menstruation, cold feet, and irritable bladder; general spinal and pelvic soreness and pain in one ovary, usually the left, or in both ovaries. The sense of exhaustion is a remarkable one : the woman is always tired; she spends the day tired, she gres to bed tired, and she wakes up tired-often, indeed, more tired than when she fell asleep. She sighs a great deal; she has low spirits, and she often fancies that she will lose her mind. Her arms and legs become numb so frequently that she fears palsy or paralysis. Nor does the skin escape the general sympathy. It becomes dry, harsh, and scurfy, and pig- 
mentary deposits appear under the eyes, around the nipples, and in the chin and forehead. The symptom-group of nervous exhanstion - anæmia, backache, bearing-down, difficult walking, ovarian pain, and menstrual disorders-althongh often without the least gynecological significance, is usually the signal for a gynecological diagnosis. Any pelvic organ showing the slightest irregularity is singled out as the culprit and promptly placed on trial. Endless injurious local treatment and grave surgical operations may now cause the woman to suffer miany things from many physicians."

As Goodell aptly remarks: "If no tangible disorder of the sexual organs be discoverable, the invisible endometrium or ovaries must take the blame and receive the local treatment. Whatever the inlook or the outlook, a local treatment, more or less severe, is liable to be the issue. Yet these very exacting symptoms may be due wholly to nerve-strain, or, what is synonymous, to loss of brain-control over the lower nerve-centres, and not to direct or to reflex action from some supposed uterine disorder. Neither, for that matter, may they come from some real, tangible, and visible uterine lesion which positively exists. 'Thus it happens that a harmless anteflexion, a trifling leucorrhœa, a slight displacement of the womb, a small tear in the cervix, an insignificant rent of the perineum, or, what is almost always present, an ovarian ache, each plays the part of the will-o'-the-wisp to allure the physician from the bottom factor. To these paltry lesions -because they are visible, palpable, and ponderable, and because he has by education and by tradition a uterine bias-he attributes all his patient's troubles; whereas a greater and subtler force, the invisible, impalpable, and imponderable nervous system, may be the sole delinquent. The sufferer may be a jilted maiden, a bereaved mother, a grieving widow, or a neglected wife, and all her uterine symptomsyes, every one of them-may be the outcome of her sorrows and not of her local lesions. She is suffering from a sore brain, and not from a sore womb."

We may admit the extreme wisdom of Goodcll's summing up; at the same time we must insist that an exhaustive analysis of a patient's condition often will lead to conclusions less imponderable than his ex parte statement would imply. The case above outlined on the record blank will not only show an example of possible diagnosis; but, if analyzed, will show also that the cure of aggravated local lesions may not result in the complete recovery of the patient; such cure, will, however, be an important step in the right direction. A common mistake, when there are other more general and, perhaps, more serious anomalies, is to expect prompt and complete relief upon the correction of local lesions. It would also be equally a mistake to follow the possible implication of Goodell, and, because we know that local treatment of palpable local lesions cannot completely cure the patient, fail to give that treatment, and thereby fail to cure her so far as we can.

It is, moreover, improbable that a harmless anteflexion, a trifling leucorrhoa, a slight displacement of the womb, a small tear of the cervix, an insignificant rent of the perineum, or an ovarian ache would often lead a serious practitioner away from the "bottom factor" to useless or injurious gynecological treatment. 
Not less essential than the gyneeological part of the record is that which belongs to the general condition of the patient. Age, temperament, bodily habit, heredity, color, the heart and bloodvessels, the digestive tract, the liver, spleen, and, even more important, the lidneys, all demand elose and careful attention.

\section{THE PHYSICAL EXAMINATION.}

Examination calls into use the special senses, supplemented by such conditions, instruments, and appliances as will inerease the power or widen the range of the senses.

The conditions to be fulfilled for an adequate examination are numerous and variable. Among them are: 1. Cleanliness. 2. A suitable table. 3. Proper attitude and position for the patient, and empty bladder and rectum.

Cleanliness and Asepsis have been emphasized in the last chapter; their importance cannot be exaggerated. Exception: if it is desired to study the eharacter of the uterine, vaginal, or vulvar seeretions, the preliminary douche and disinfection of the parts may be omitted.

The Rectum and Bladder should be empty for the following reasons: 1 . These viseera, when full, displace the pelvic org:ms by pressure. 2. Retained feees and urine may be mistaken for solid and cystic tumors. The full bladder pushes the uterus and its appendages upward and backward and greatly increases the difficulty of conjoined examination. Even a small quantity of urine in the bladder may cause the patient to make the abdominal museles so tense that the uterus cannot be felt between the hand over the pubes and the examining finger in the vagina. A preliminary eathartic to clear the bowels of feces and gas should therefore precede the first examination.

The Examining Table.-The digital examination may be made with the patient lying on a sofa or bed; but, as Marion Sims has tanght, "the one is too low and the other is too soft and yielding for a speculum examination." Even the digital tonch and palpation are much better made on a table. If the bed is used, the patient should lie across it, with the hips well to the edge, and not lengthwise of the bed. The table is essential for a thorough speculum examination. The eonventional office chair, although less objectionable than the sofa or bed, is, by comparison with the table, inferior. An ordinarr pine kitchen table, two feet wide, four feet long, and two-and-one-half feet high, covered with a blanket and sheet and supplied with a pillow, will answer every purpose almost as well as the more elaborate table commonly used in office and hospital work. There is some advantage in having the end of the table upon which the pelvis rests about three inches higher than the end upon which the head rests.

In making a digital or conjoined examination with the patient lying on the table, the examiner stands at the foot of the table facing the patient, and passes the examining hands between the knees.

The Position of the Patient.- Two positions are in common use, the dorsal and the left lateroprone position of Sims. The kneebreast, the standing, and the prone positions are used less frequently; 
each has advantages peculiar to itself and to the conditions under which it is employed.

Examination of Young Girls. - The first examination of a young girl should be approached with reluctance, and, if possible, avoided. The advantages of anæsthesia from the standpoint of modesty must be apparent to all. If the hymen is intact, an effort should be made to gain the necessary information by a conjoined digital exploration through the rectum, the palpating hand being over the luypogastrium.

Placing the Patient on the Table.-The clothing about the waist having been loosened, the patient steps upon a chair which has been placed at the foot of the table, and, the skirts having been raised behind, sits upon the extreme end of the table. She is then assisted to lie upon her back, the head, not the shoulders, being supported by a pillow. Before lying down she is covered with a sheet. Under the sheet, and withont exposture, the feet are lifted from the ehair to the table and placed six inches apart; the clothing in front is pushed above the knees and the knees are widely separated. The flexure of the thighs, secured by placing the feet on the table, relaxes the abdominal museles and facilitates the palpation. The edge of the sheet as it falls over the knees is parted back between the thighs so as to expose only the part to be inspected-that is, the vulva. The patient is assured that she is neither to be hurt nor unduly exposed. She is now rearly for :

1. Inspection.

2. Digital examination of the vagina and rectum.

3. Conjoined examination.

4. Pereussion, palpation, and auseultation.

5. Mensuration.

6. Instrumental examination.

\section{Inspection.}

General inspection of the whole patient is not only essential for careful general diagnosis, but it is also important to inspect the external genitals as a forewarning against possible inoculation of the examining finger with venereal or other infection. Some historic cases there are of surgeons who have gone to their death from this cause. The writer is acquainted with not less than twenty physicians who have enntracted syphilis, some of them fatal syphilis, through digital tonch. Any abrasion on the hand shonld be protected with a finger-cot or a collodion and cotton dressing : a very thin layer of eotton is placed over the abrasion before the collodion is applied. One should look for lacerations, scars, and other evidences of parturition, vulvitis, tumors, urethral caruncles, urethritis, eruptions, hemorrhoids, anal fissure, fistula in ano, pin-worms, eczema, œdema, cystocele, rectocele, ulcers, inflammation of Skene's glands, and other anomalies, and should note the calibre and elasticity of the vulvar orifice. Is the elitoris enlarged or imprisoned under an adberent prepuce? Such adhesions may give rise to pronounced reflex disorders. If the vulva is that of a virgin, it is apt to be small, with the hymen perfor. ated only by one or more small openings. 'The absence of such a hy. men, however, is neither proof nor even strong evidence of unclastity. 
The virgin labia minora are small, firm, donble folds of skin. If they are long, loose, and flabby, and especially if the vulvar orifice is patulous, the indications are that the woman has had one or more children, or has had much treatment, or has practised self-abuse, or has been the subject of some other mechanical interference.

Inspection, however, is not limited to the reproductive organs nor to external parts; it may extend through the aid of the speculum to the vagina, the interior of the bladder, the urethra, the rectum, and, by abdominal section, to the interior of the abdomen and pelvis. The surface of the abdomen is also open to visual examination, and by its enlargement and contour may disclose the presence or character of a tumor or ascitic accumulation.

\section{Digital Examination.}

Left-hand Method.--The advantage of the left index-finger for digital touch in preference to the right was demonstrated and its use popularized by Marion Sins. The great superiority of the left-hand method is acknowledged ustally by those who have accustomed themselves to both. The following are some of the reasons for this superiority : 1. The tactile sense of the left finger is educated more easily. 2. Its palmar surface more readily comes in close relations with the left side of the pelvis, and disease is more frequent on the left than on the right side. 3. The stronger right hand is reserved for external palpation. 4. The right hand is left free to pass the probe or sound or to manipulate any instrument. One finger usually will gain as much information as two. The introduction of two fingers, except in a capacious vagina, is painful. Two fingers may, however, sometimes be of use in the examination of tumors.

Lightness of Touch.-The manner of digital touch has been well described by Emmet as follows: "When the sense of touch has been cultivated, it yields more information upon which to base a diagnosis than can be gained by the eye alone, even when used under equally favorable cireumstances. Therefore the digital examination should always be thoroughly and systematically made. It is all-essential to possess a knowledge of departures from a healthy condition. The lighter the tonch the more acute it will be, and the more clearly will it appreciate slight changes. It is, indeed, remarkable how individuals vary in their method of making examinations. One will proceed with as much vigor as if he were boring a hole, and finds little more than the cervix, which feels like an obstruction in his way. $\mathrm{He}$ gains no information of importance, and inflicts unnecessary pain. Another will pass his finger lightly over every portion of the vagina, and, without having caused any pain, quickly ascertain enough to enable him fully to understand the case."

Distension of the Bladder During Examination.-In the chapter on Displacements of the Uterus stress is laid on the great importance of having the bladder and rectum empty when digital examinations are being made. The author's experience has shown that when palpation of the ovaries is desired, they sometimes are forced down within reach of digital touch with startling distinctness when the 
bladder is distended with fluid. The required distension may be secured by throwing in sterile salt water 1 per cent. through a eatheter attached to a fountain syringe.

Conduct of Digital Examination. - The hand having been washed earefully, the left index-finger is lubricated with glycerin, mild eastile soap, or glyeerin emollient (Chapter II.), and then slowly introdueed, the palmar surfaee being direeted downwarl so as to depress the perineum toward the reetum; it notes the rigidity of the perineum,

Figure 10.

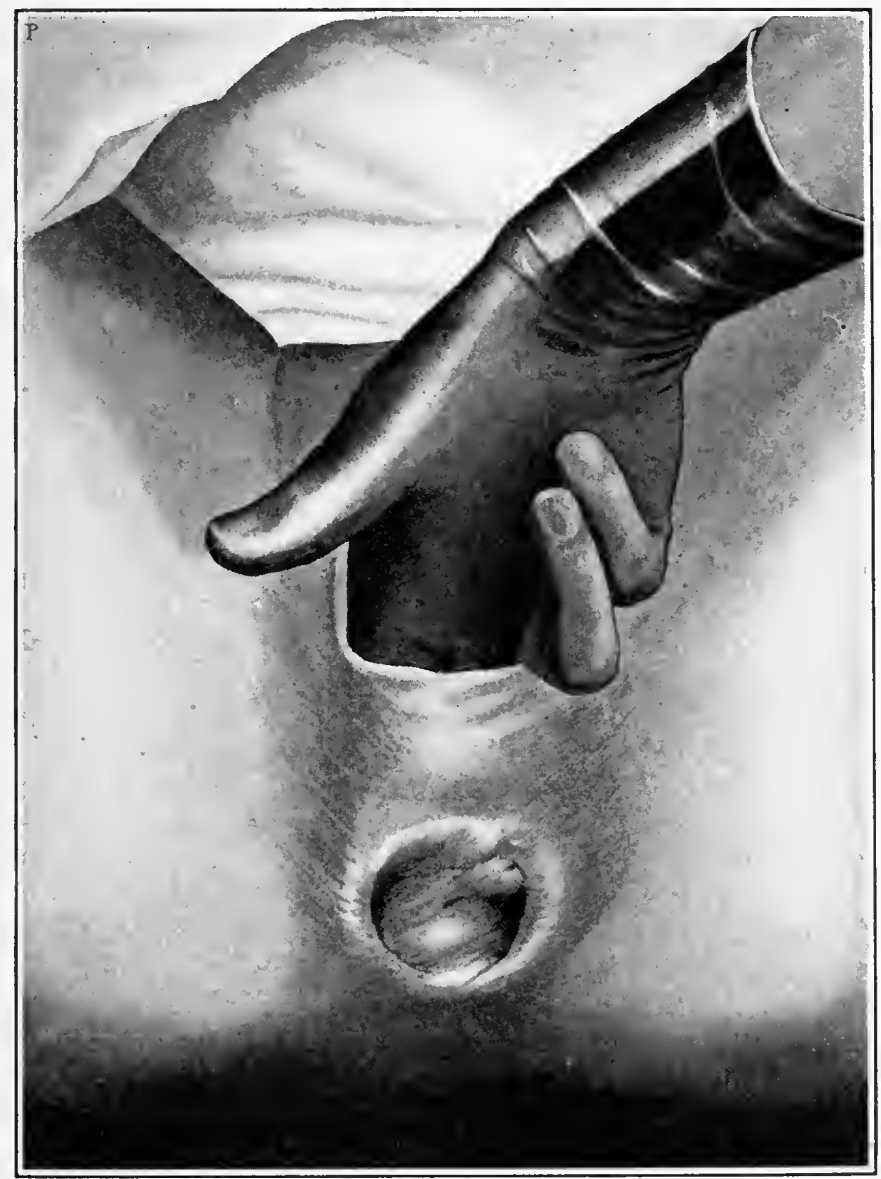

Digital eversıon of the anus.

the presence, absence, or eonsistence of the feees in the rectum, the calibre and relaxation or rigidity of the vagina, and the condition of the sacrum and coccyx. The palmar surface of the finger now is direeted alternately toward the lateral and the anterior portions of the pelvis and swept around the cervix. The direetion, size, form, and consistenee of the cervix, the ealibre and form of the os externum, and the presence or absence of laceration become apparent. The right 
hand now is placed over the abdomen behind the pubes, and the inquiry continued by conjoined examination. Irritation of the elitoris may give rise to sexual excitement; hence the examining hand should be kept well away from it.

Digital examination with the patient standing has some value as a means of diagnosis in uterine displacements. The examination may be made with the woman in the left lateroprone position, but this position is reserved rather for speculum examinations and operations.

Eversion of the Anus, as shown in Figure 10, enables the examiner to judge of the condition of the lower part of the rectum and anus, and may be done either in the dorsal or lateral position.

\section{Conjoined Examination.}

Conjoined Vaginal Examination, often called bimanual palpation, is designed to bring within the range of touch all the pelvic organs. 'These organs, one by one, in some eases are lifted forward, by the finger or fingers in the vagina, toward the anterior abdominal wall, where they can be palpated by the right hand pressed down behind the pubes. Usually, however, the right hand forces them down to a point where they may be examined readily by digital touch. The latter method is usually preferable, because the application of much force in the vaginal or rectal touch may be harmfiul to the patient or may impair the tactile sense of the finger. A combination of both methods is desirable. The necessary amount of force will vary with the tolerance of the patient and the skill of the examiner. The reach of the examining finger is increased materially by foreing the elastic perinenm backward toward the interior of the pelvis.

Bimanual examination, to be effective, requires long practice. The beginner is, first, to bring the organs properly between the two hands; second, to appreciate what may be within his reach. Should the thickness or rigidity of the abdominal walls prevent the downward pressure of the hand behind the pubes, the resistance may be overcome by continuous firm pressure, or by successive short strokes of vibratory massage, or by circular massage. The difficulty is often the result of the patient's nervousness. The examiner should, therefore, avoid sudden movements in manipulation. A deep inspiration by the patient, followed by a quick expiration, while steady pressure is being made, may momentarily relax the muscles, and thereby afford the examiner an opportunity of rapidly palpating the pelvic organs. An examiner of acute touch and quick perception will gain sometimes instantaneously the required information in this way. During the examination the patient should keep the mouth open.

If the uterus and its appendages are sensitive, or fixed by adhesions, the attempt to force them up toward the outside hand may be futile or even dangerous. Deep palpation behind the pubes is then necessary. One should remember, however, that even a little force injudiciously applied by either hand may rupture a pus-pocket or tube, and thereby lead to serious results. 
Bimanual palpation enables one to judge of the following conditions : the size, form, location, position, eonsistence, and mobility of the uterus, the presence or absenee of a pelvie tumor. If the uterus is displaced, is it replaceable, or is it bound by adhesions, and therefore irreplaceable? If there is a tumor in the pelvis, is it a neoplasm or an inflammatory swelling? If the former, it is not sensitive; if

Figure 11.

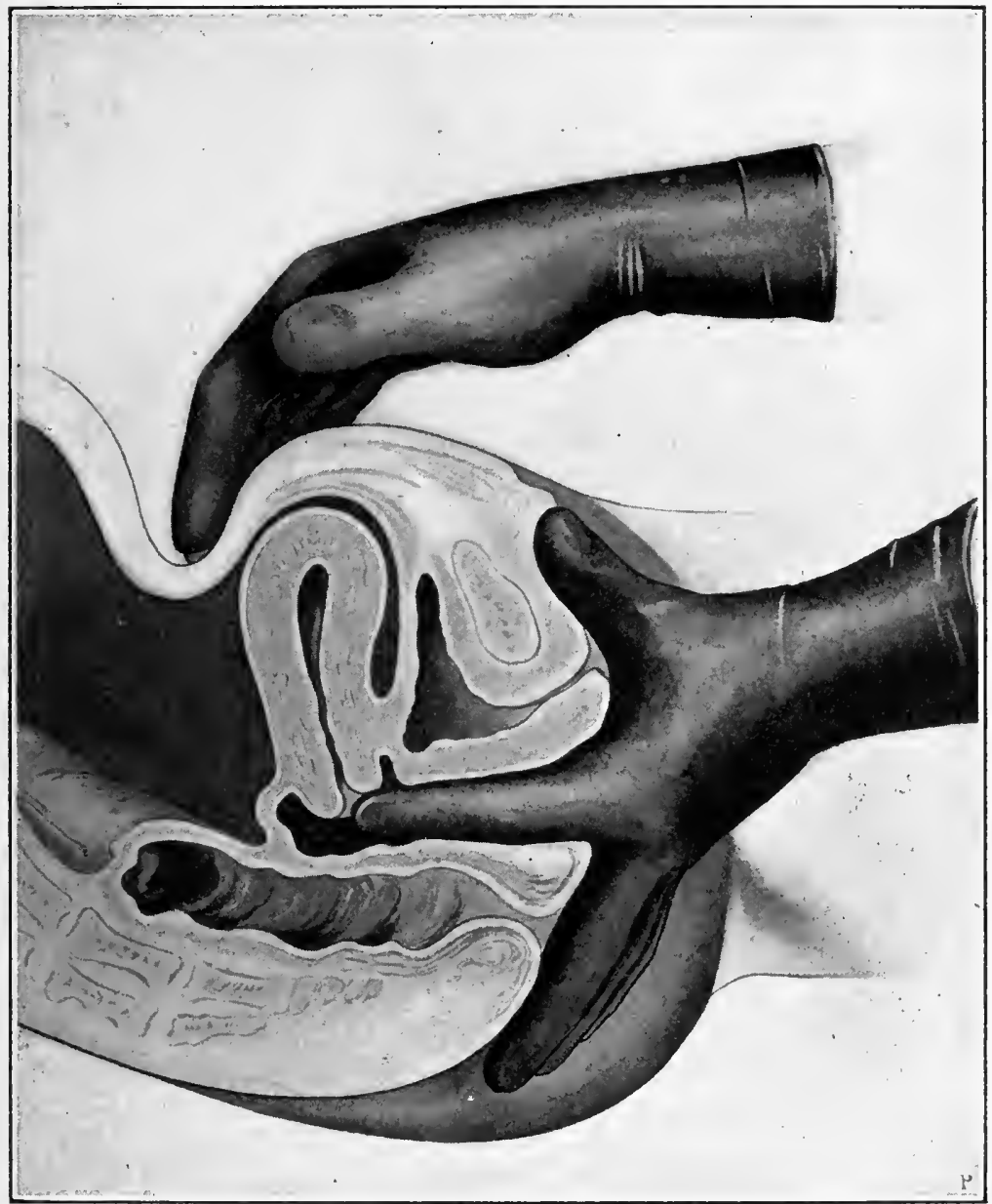

Vaginal touch, conjoined examination.

the latter, it is tender on pressure. Is it connected with the uterus, or the Fallopian tube, or the broad ligament, or the ovary? Is it cystie or solid, malignant or benign? Does it originate in the pelvis, or in the abdominal eavity, and, above all, is it possibly due to pregnaney? These questions will eome up again under the diagnosis of special disorders. 
Conjoined Rectal Examination.-Conjoined examination by rectal instead of vaginal touch may confirm, disprove, or supplement the previons observations and impressions. Figure 12. Rectal touch, whether digital or conjoined, may be impeded by coils of intestine in the pelvis interposed between the finger and the viseera to be palpated.

FIGURe 12.

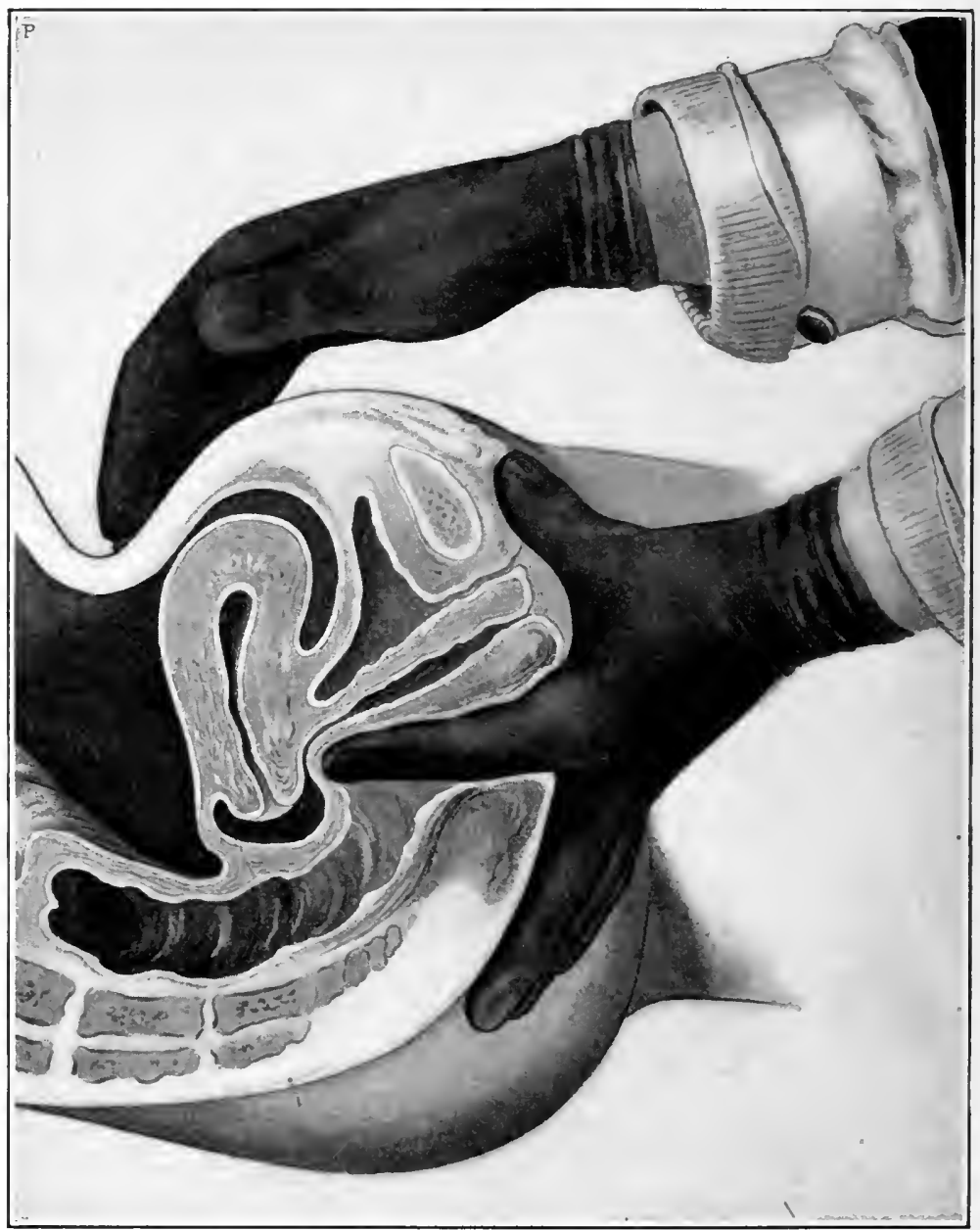

Reetal touch, eonjoined examination. In examining at the patient's house without removing the coat or loosening the starched shirt-cuft's, the examiner may push them up orer the wrists and retain them there by pulling down the sleeves of the undershirt, and turning them baek over the cufis, as shown in this illustration.

This may be avoided by a simple device of Kelly's: "The reetum and bladder are first evacuated, the patient is put in the knee-chest posture, and a speculum is introduced into the rectum. This lets in a large amount of air, and the bowel balloons out and applies itself broadly over the sacral hollow and the posterior surfaces of the uterus and 
left broad ligament, and in a minute or two the small intestine falls away into the upper abdomen. The patient must then be turned on her baek, care being taken to keep the pelvis constantly higher than the rest of the abdomen, so as not to let the intestines gravitate again into the pelvic cavity. On making the bimanual examination the pelvic viscera are felt with startling distinctness, the rectal finger enters a large air-cavity no longer impeded by the mucous folds; the

Figure 13.

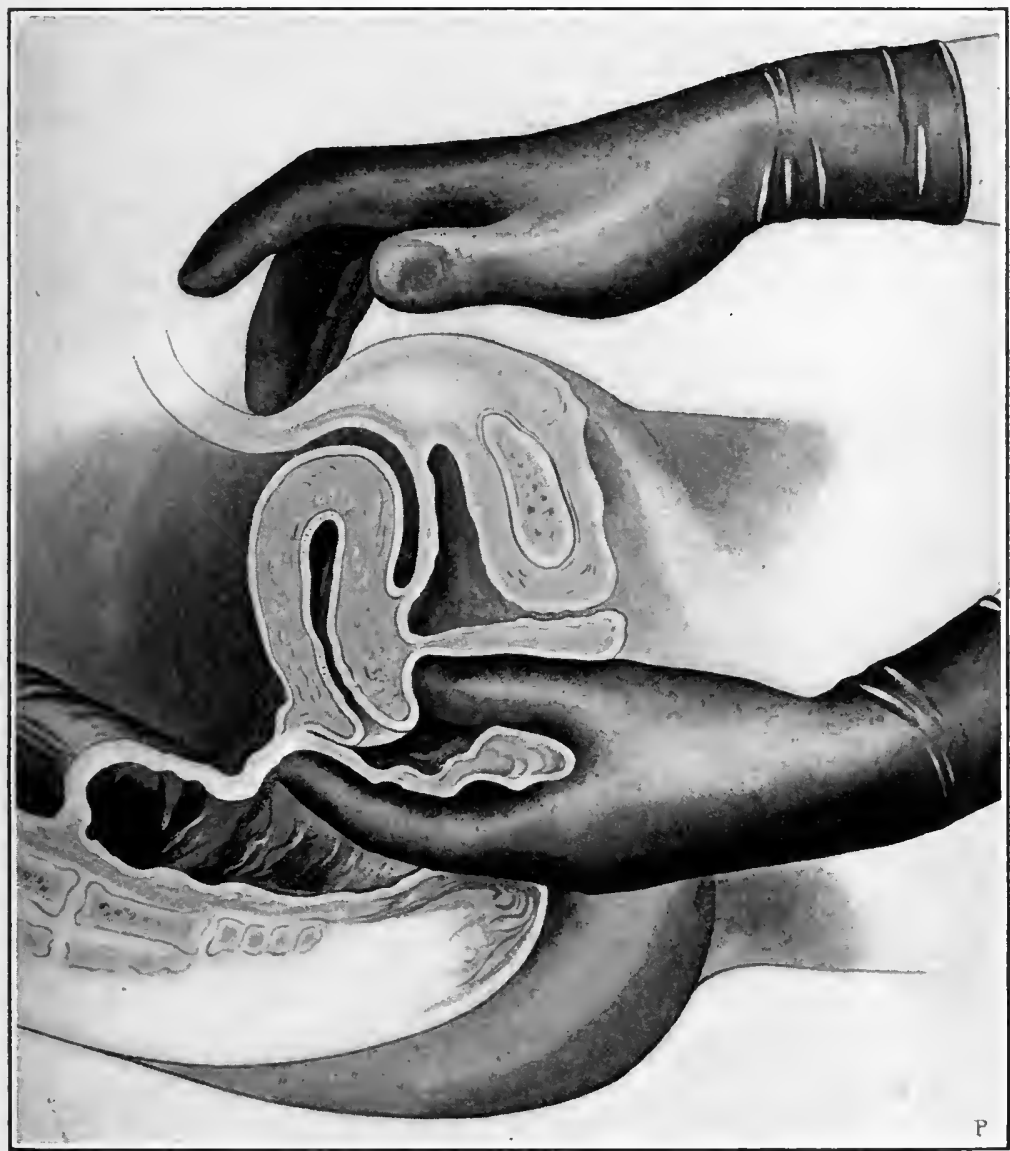

Conjoined rectovaginal palpation.

opening from the lower into the upper rectum is readily found; and the posterior surface of the uterus and the ovaries and tubes feel as if skeletonized in the pelvis. They lie so elearly exposed to touch that their minuter surface-peculiarities, fissures and elevations and variations in consistence can be detected." This peculiar ballooning of the rectum is observed often in obstruction and paresis of the bowel, and may be felt with the patient in the dorsal position without recourse to the device of Kelly. 
Conjoined Rectovaginal Examination is made with the left index-finger in the reetum, the thumb in the vagina, and the right hand behind the pubes. See Figure 13. In this way the perineum is pushed well up toward the interior of the pelvis. If the abdominal wall is thin and relaxed, the various pelvie organs, when forced down by the hand behind the pubes, may be picked up, so to speak, between the thumb and finger and definitely palpated.

Traction as an Aid to Conjoined Examination.-Palpation of the pelvie organs, especially the ovaries and Fallopian tubes, is facili-

\section{Figure 14 .}

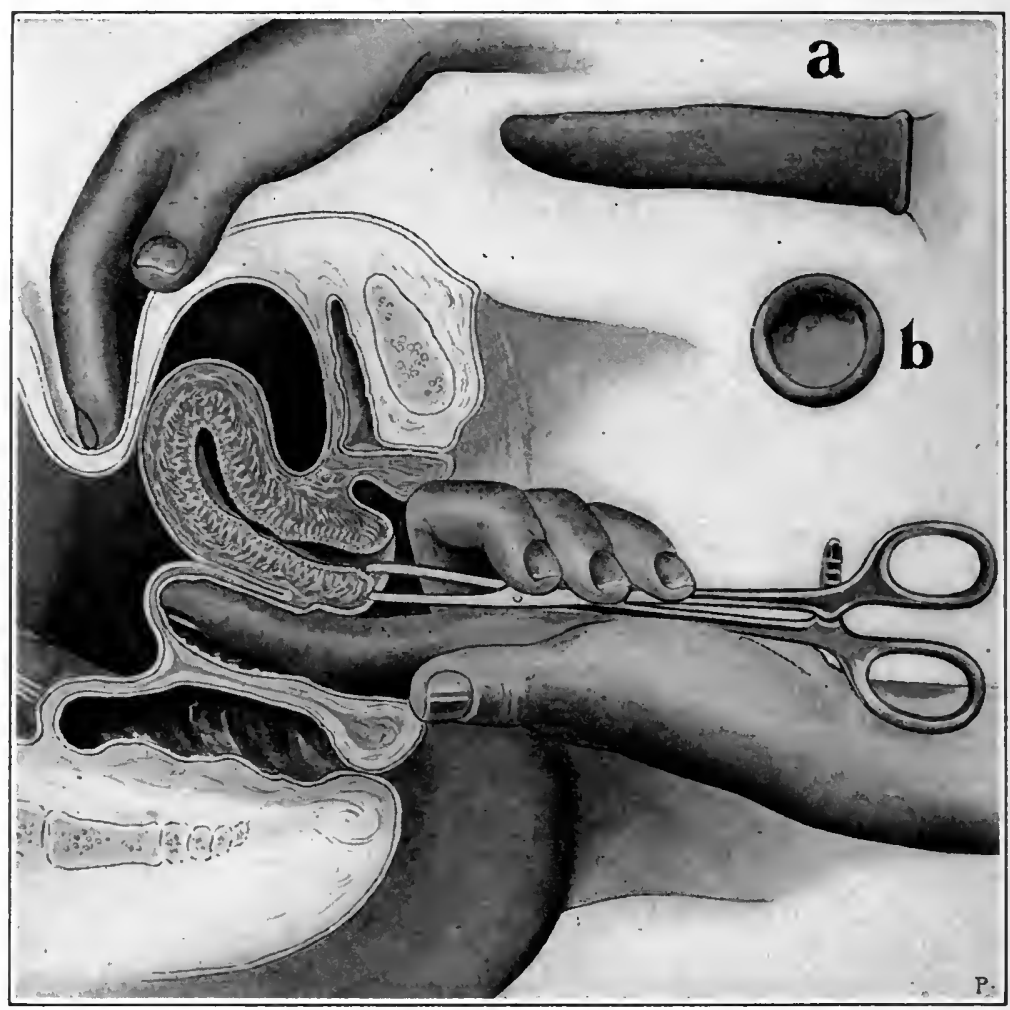

Uterus drawn down by means of tooth-forceps to facilitate manual examination or replacement: $a$, rubber tinger-cot on finger; $b$, rubber finger-cot, rolled up.

tated often by drawing the uterus toward the vulva by means of a uterine tenaculum or small tooth-forceps. Figure 14. During the palpation these instruments may, if necessary, be held by an assistant.

Anæsthesia.-Failure to engage the uterus between the hands in conjoined examination may be due to fixed retroversion or to rigidity of the abdominal museles, or to sensitiveness of the parts under examination, or to the nervousness of the patient. Intelligent treatment may be impossible under these conditions without anæsthesia. The 
accurate and adequate diagnosis thus obtained lessens the number of exploratory incisions and unnecessary operations, prevents a vast amount of indefinite injurious local treatment, and substitutes rational medicine and surgery.

The Roots of the Sciatic Nerve may be palpated through the rectum, as shown in Fignre 15; such an examination sometimes will reveal the source of obseure pelvic pain which has previously been attributed to ovarian or uterine origin. The patient must be examined

Figure 15.

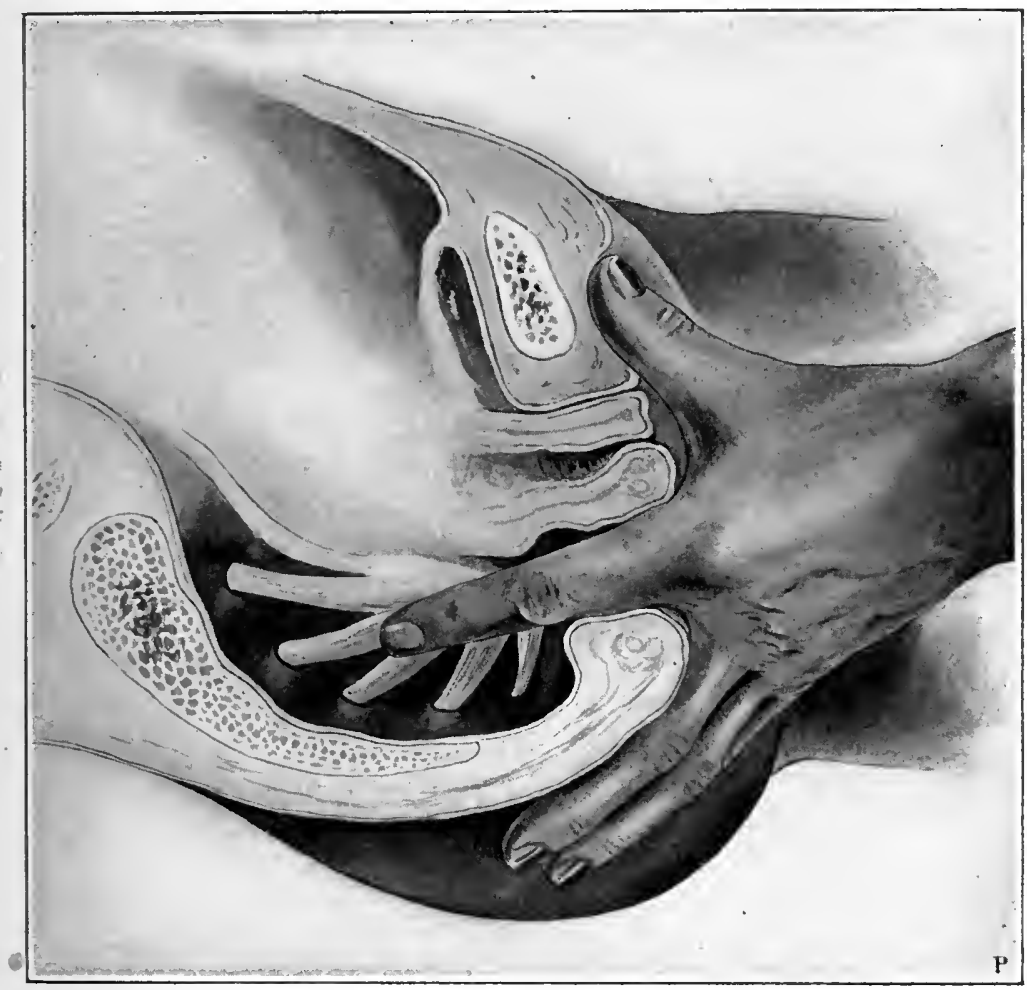

Palpation of the roots of the sciatic nerve by rectal touch.

withont anæsthesia, and as the fingers are drawn over the tender cord a cry of pain will be elicited.

Conjoined Examination with the Sound.-One may be unable by touch to decide whether a tumor is of uterine or extra-nterine origin. The uterus may then be immobilized by the sound passed into the uterine canal and held immovable by the hand of an assistant, or the uterus may be steadied by a tooth-forceps or tenaculum attached to the cervix. The examiner then may determine whether the tumor moves with the uterus or independently of it. In case of a nterine tumor with a long pedicle, or of an extra-uterine tumor adherent to the uterus, the test may fail. 
The necessity of conjoined examination is apparent in Figures 16 and 18. Vaginal touch alone in Figure 16, A, which represents a myomatous uterus, would give the same inpression as in Figure 16, B, which shows a retroflexed uterns. Conjoined examination in Figure 16, $A$ and $B$, would establish the fact of myoma in one case and the retroflexion in the other. The exact direction of the uterine canal

Figure 16.

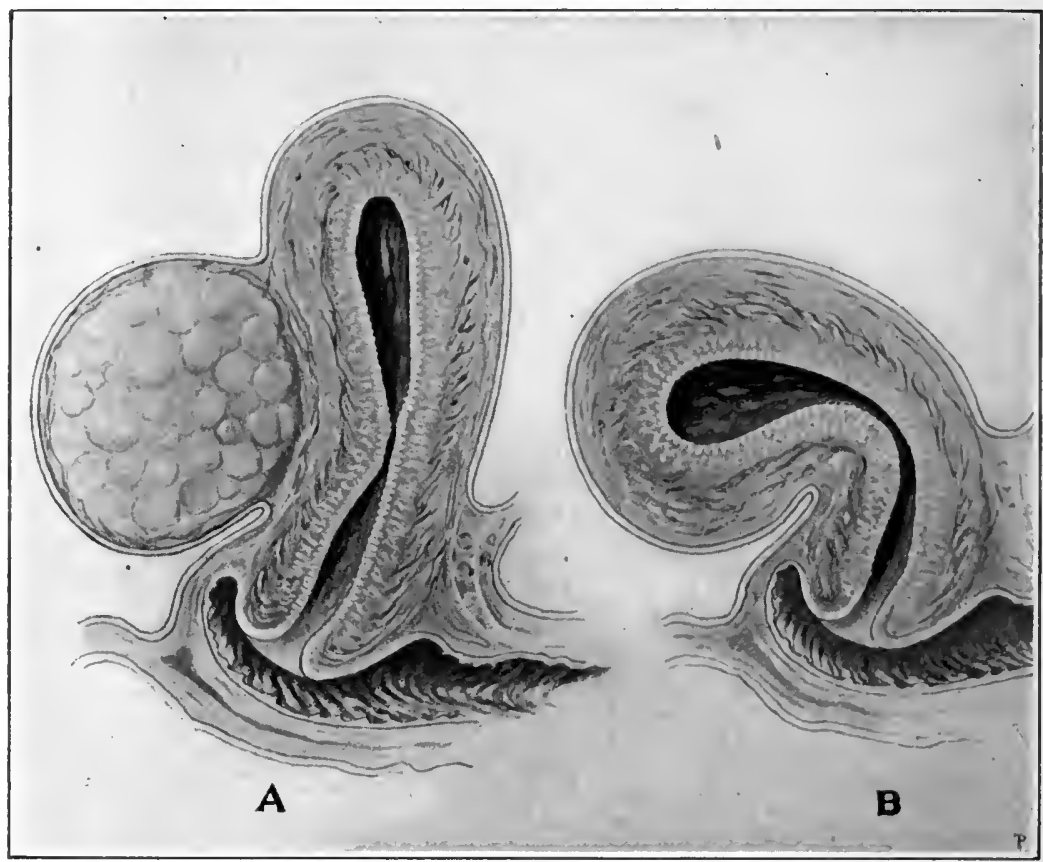

A. Myoma in the posterior wall of the uterus.

$B$. Retroflexion of the uterus.

and the relations of the uterus to the tumor might in such cases be learned by passing the probe or the sound.

\section{Percussion, Palpation, and Auscultation.}

These means of diagnosis are applicable to the differentiation of abdominal tumors and enlargements of inflammatory origin, especially pregnancy. The inquiry should include both gynecologieal disorders and others that simulate or eomplieate them. Among the latter may especially be mentioned appendicitis, a condition frequently associated with infection of the uterine appendages, especially on the right side. One who has not svstematically included the renal organs in his ex. amination will be astounded at the revelations of such investigations. Hydronephrosis, abscess and stone in the kidney, tubereular kidney, loose and floating kidney, and stricture of the ureter are among the pathological conditions commonly overlooked. The mere mention of intesti- 
nal, gastric, splenic, and hepatic disorders should be sufficient. Relaxation of the abdominal walls, with consequent falling of the intestines -enteroptosis-associated also with the falling of other abdominal organs, especially the stomach and kidney, is a frequent and unrocognized cause of abdominal and pelvic disorders. See Pendulous Abdomen and Displacement of Abdominal Viscera, at the close of Chapter XLIV.

\section{Mensuration.}

Mensuration is important in the examination of new growths and other lesions causing abdominal enlargement, and will be considered

FIGURE 17.

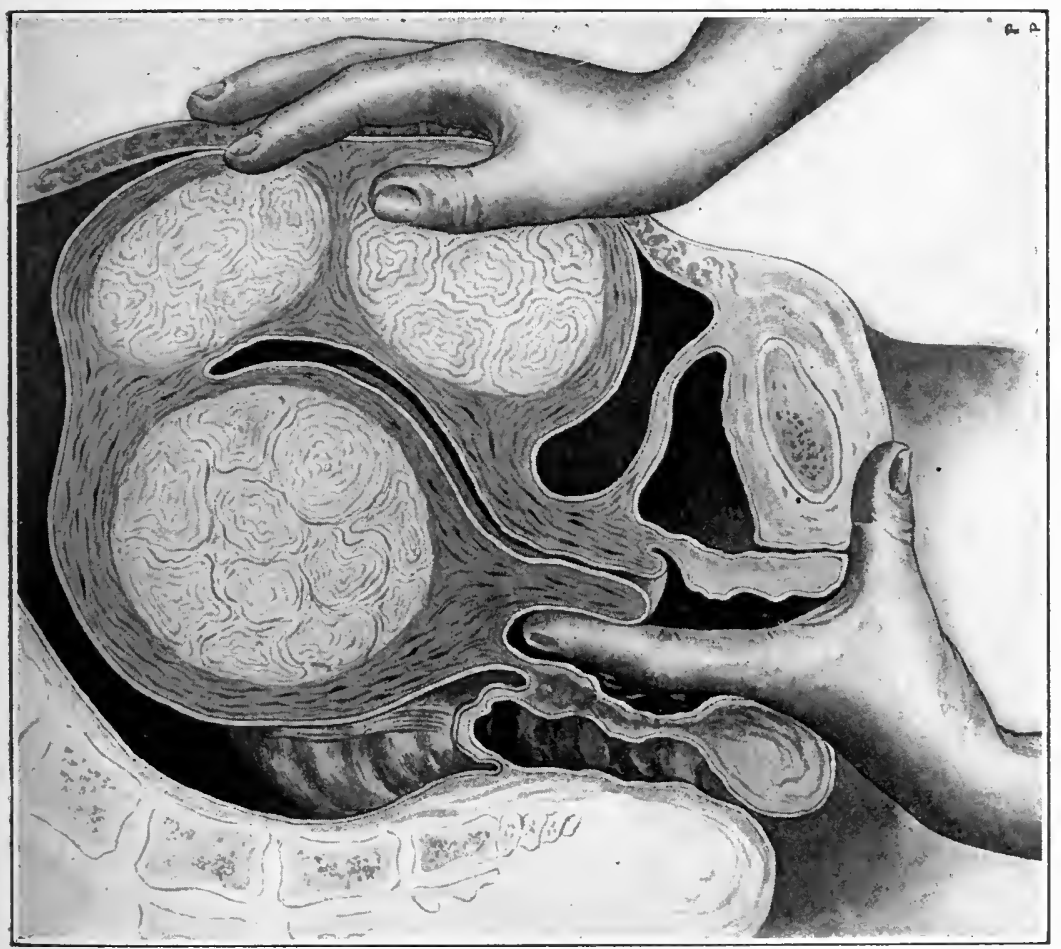

Conjoined examination of a solid tumor of the uterus. Here the tumor-mass is palpably a part of the uterus.

further in connection with the special diagnosis of these disorders. The measurements of the bony pelvis frequently have great significance, not only from the obstetrical, but also, especially in the matter of displacements and malformations, from the gynecological point of view. The reader is referred for pelvic mensuration to the literature of obstetrics. 


\section{Instrumental Examinations.}

As already stated, the development of modern gyneeology has been made possible by the use of instruments of precision designed to increase the power or widen the range of the senses. The diagnostic methods already described usually will furnish the groundwork for

Figure 18.

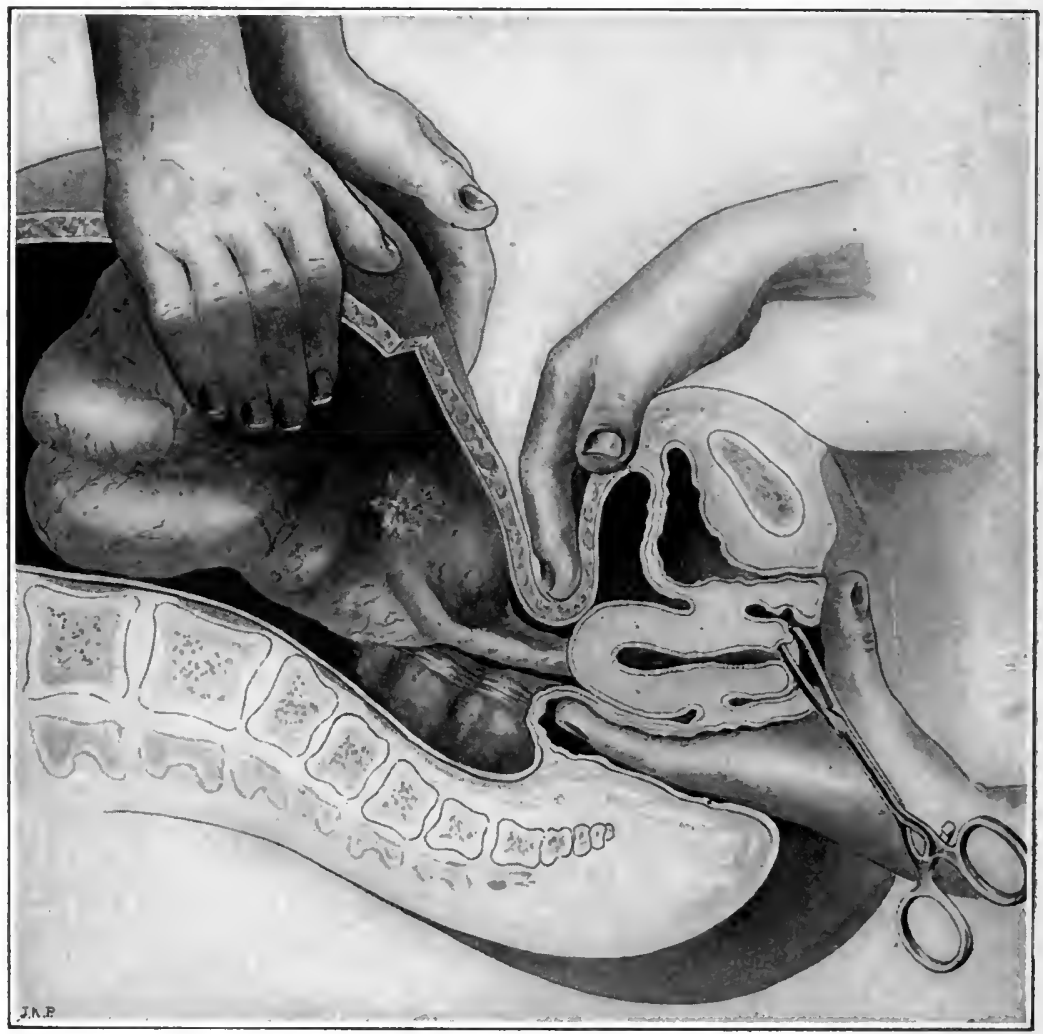

Conjoined examination of a cystic tumor of the ovary. The hands of an assistant are moving the tumor from side to side. The uterus does not move with the eyst. 'The hands of the examiner are separating the cyst from the nterus. 'The separation of the cyst from tho uterus and the independent movement of it demonstrate it to be of extra-uterine origin.

diagnosis. Instrumental examination may supplement and verify conclusions already foreshadowed. Some of the instruments used for diagnostic purposes are:

1. The speculum, 5. The exploratory needle and aspirator,

2. The sound and probe, 6 . 'The stethoscope,

3. The dilator, 7. The microscope,

4. The curette, 8 . The urethroscope and cystoscope.

The Speculum.-The choice of the speculum is simplified by the statement that of the innumerable varieties only two require serious consideration, and that these two act on the same principle--as perineal retractors. They are: 
Sims' speculum.

Simon's speculum.

Sims' speculum is an instrument of great simplicity and effectiveness. The objection, sometimes urged, that the efficient use of it requires long practice, is a mistake. Whoever once masters the simple principles of the left lateroprone position will have little or no difficulty. The failure to appreeiate the mechanical relations of this position to Sins' speeulum will explain most of the disappointnents resulting from its use. Another alleged disadvantage of Sims'speculum is

Figure 19.

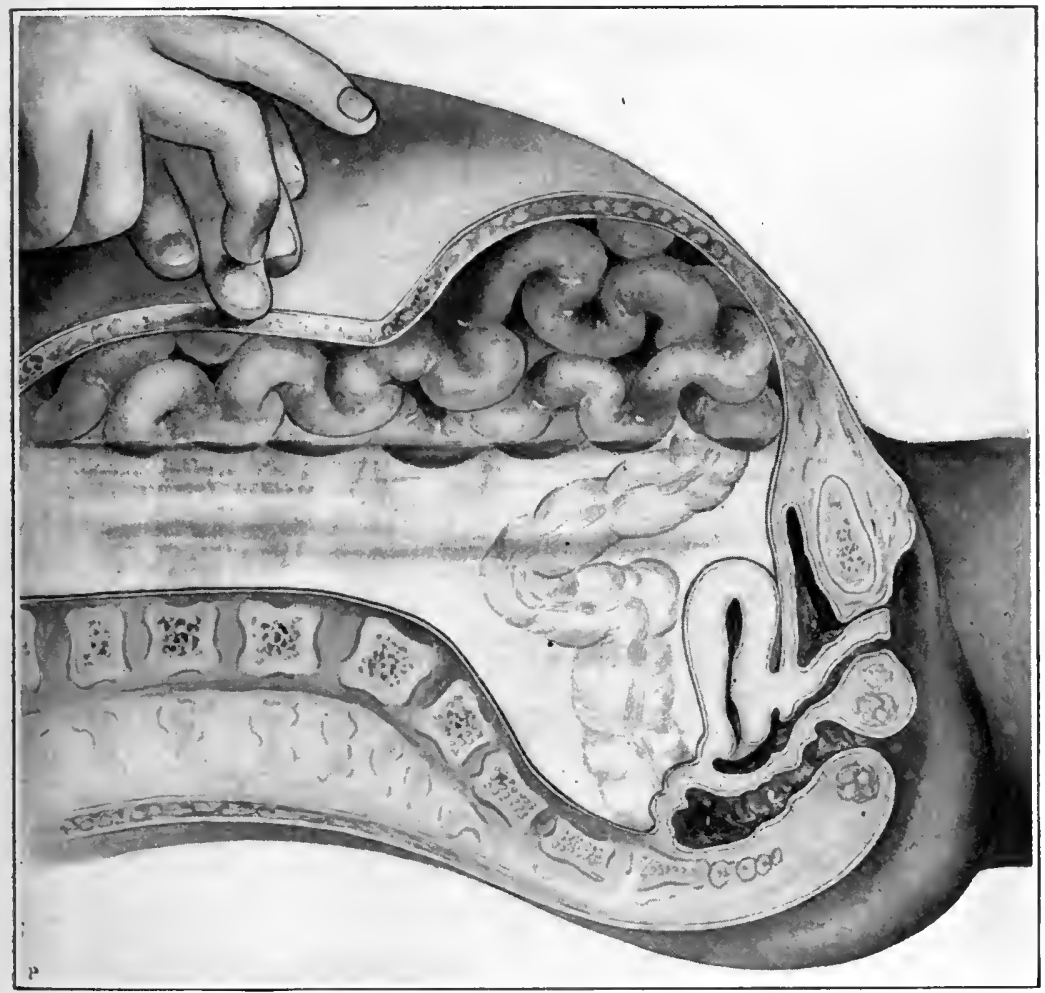

Percussion in the diagnosis of abdominal enlargement due to accumulation of ascitic fluid. Intestines float to the surfice of the fluid, Resrnance over intestines. Dulness in flanks below level of fluid. Change in position of the patient would make corresponding change in areas of resonance and dulness-that is, the higher parts would give resonance, and the lower parts dulness.

the necessity of a trained assistant to hold it. If the examiner himself knows how the instrument should be held, the assistant need not be trained. In gynecological examinations the presence of a third person is, for obvious reasons, an advantage. Examinations at the patient's house may be made usually with the assistance of some member of the family. 'The physician who has a large office practice should have the assistance of an office attendant; or if this is impracticable, a modified self-retaining Sims' speculum may be used. 
Thomas, after long experience with other instruments, makes a statement something like this: "Learn the nse of Sims' speculum, persevere in the method for three months, and you will never give it up." Emmet, whose experience with the instrument is, perhaps, greater than that of any other, says: "This instrument is so simple in design, and so perfectly does it fulfil every requirement, that it will probably never be superseded.

The Self-retaining Sims' Speculum.-Morlifications of Sims' speeulum to make it self-retaining have been devised by Emmet, Cleveland, and others. They are all inferior to the original Sims' instrument, but superior to the multiform eylindrical and bivalve instruments. Cleveland's self-retaining speculum is one of the best examples of its kind.

ThF Left Lateroprone Position.-In order to appreciate the action of Sims' speculum it beeomes necessary to study the effect of

Figure 20.

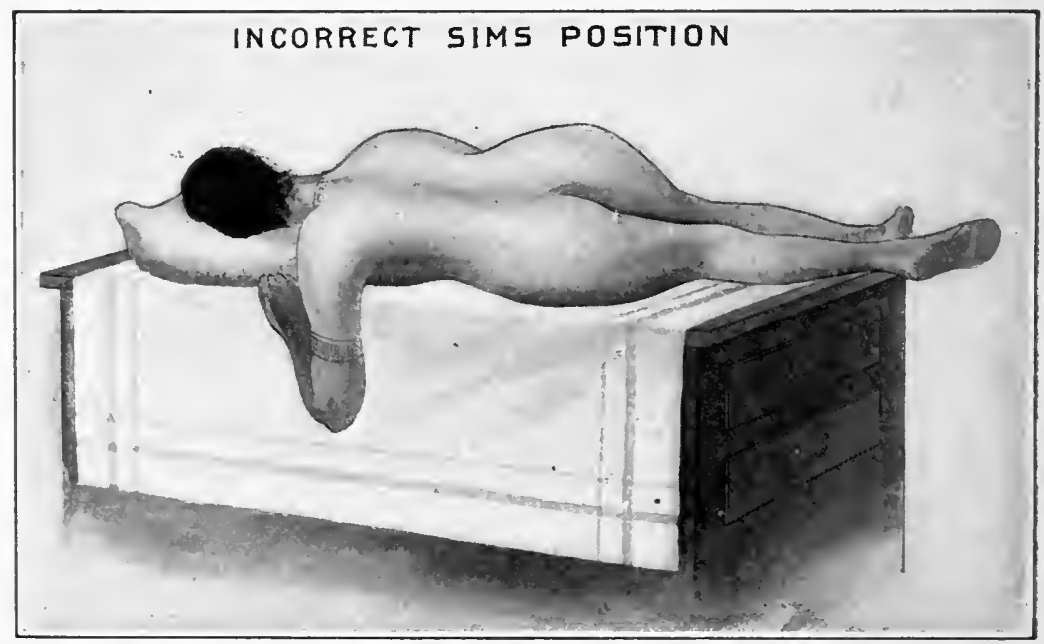

Incorrect representation of Sims' left lateroprone position, taken from a standard text-book.

Sims' lateroprone position upon the pelvic organs. Like the kneebreast position, of which it is a modification, it causes the vagina to fill with air, and the anterior and posterior vaginal walls—or, to speak more comprehensively, the pubic and sacral segments of the pelvic floor-to separate. The speculum then exaggerates the effeet of this position by hooking or drawing back the perineum, which exposes almost the entire surface of the widely opened vagina, and causes the cervix to be drawn somewhat toward the vulva.

Two requirements are essential to the snceessful use of Sims' speculum-correct position of the patient and proper holding of the instrument. The patient is to be placed on the left side, the hips being over the left-hand corner of that end of the table which is toward the operator; the knees are to be drawn up toward the abdo- 
a smaller though similar retractor which acts in the opposite direction, like the anterior blade of the bivalve speculum, and, if necessary, lateral depressors on either side. All of these instruments are more or less in the operator's way; besides, the introduction of the sound, curette, or other instruments to the interior of the uterus is more difficult in the dorsal than in the Sims position; moreover, if the organ is anteverted or anteflexed, the instrument is especially liable to be arrested at some point on the posterior wall of the cervix or at the internal os, and refuse to pass further. Simon's speculum is held less easily, and requires more assistants, more attachments and depressors, than Sims'; it gives less light and space, and for general diagnostic and surgical use, therefore, should seldom have the preference over the Sims instrument. On the other hand, the Simon

Figure 25.

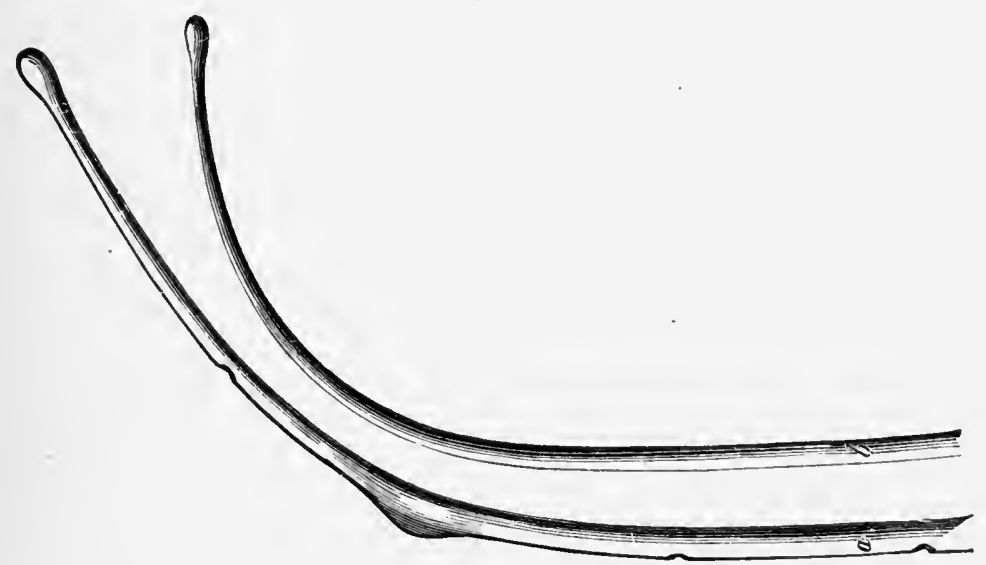

Sounds of Simpson and Sims compared: sections of full size. The upper sound is Sims' : the lower simpson's.

instrument is preferable for vaginal hysterectomy and many other operations involving vaginal section.

The Probe and Sound have been mentioned in connection with conjoined palpation as a means of diagnosis in tumors. In some cases the somd, and especially the probe, nay be difficult or impossible to pass in the dorsal position, but may readily be passed with the aid of Sims' speculum in the lateral position.

To Pass the Probe or Sound, the Patient being in the Dorsal Position, without a speculum, first introduce the left index-finger to the os externum, then, on the finger as a guide, introduce the instrument into the os and let it find its own way, judiciously aided by slight force, to the fundus.

To Pass the Sound or Probe through the Speculum, first bring the cervix into view, seize it with a uterine tenaculum or with a small vulsellum forceps, gently draw it toward the vulva, and pass the instrument, having bent it before introduction to conform, as nearly as the surgeon can judge, to the direction of the canal. 'The forward trac- 
tion of the uterus greatly facilitates the passage-in fact, is sometimes essential.

Dangers of the Sound and Probe.-Numerous eases of grave infeetion following the use of these instruments have given rise to an impression that they are dangerous. The risk, however, is practieally nothing if complete asepsis is maintained. Even a clean instrument may earry infection from the vagina or vulva; hence the necessity of thorough asepsis of these parts. The sound without asepsis is more objectionable than the probe, for it is not only equally liable to be the carrier of sepsis, but is more liable to wound the sensitive endometrium, and thereby open the door to microlic invasion. The passage of the fine probe is usually painless. The sound in a sensitive, inflamed uterus may be intolerable.

Figure 26.

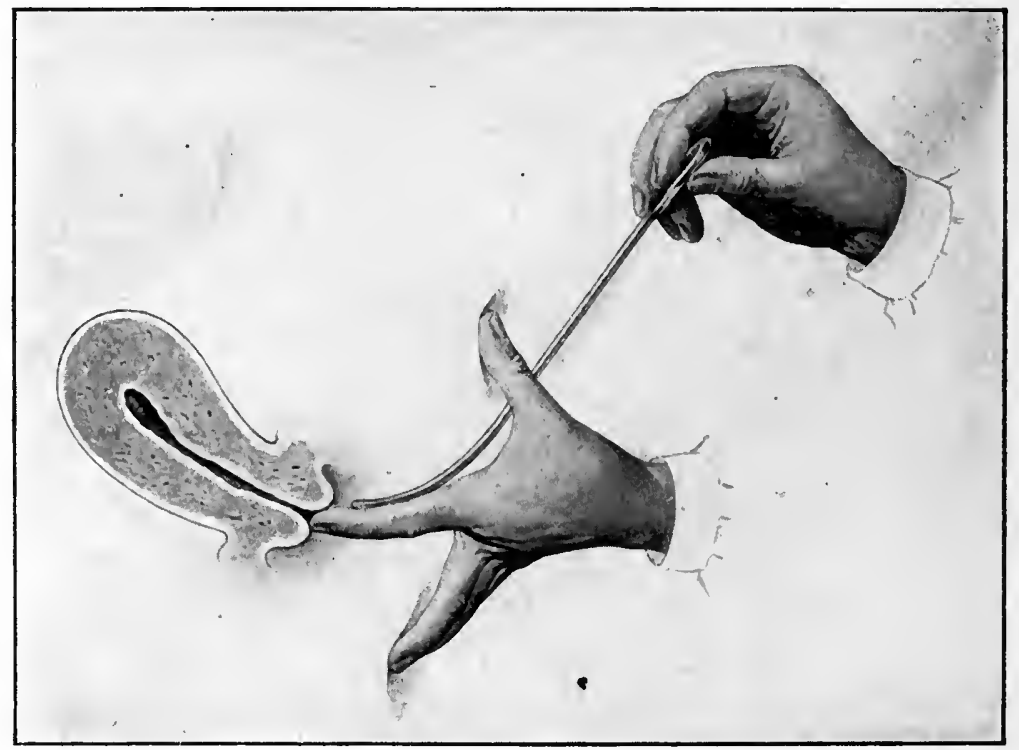

Passing sound; first step: patient in dorsal position without speculum; point of sound is guided along palmar surface of left index-tinger to os exteruum.

The diagnostic value of the sotund and probe is sometimes very great. One may, for example, be unable to locate the uterus except by the direction which the sound takes. The tortuosity of the canal, may at once show the relations of a myoma to the uterus. The sensations imparted to the hand from the point of the sound will sometimes give evidence of pathologieal eonditions inside the uterus. The length of the canal in a myomatous uterus is increased, but not materially inereased by the presence of ovarian and other extra-uterine tumors. The ease, however, is rather exceptional in which the sound or probe is a necessary means of diagnosis. The more experience one has, the more educated one's touch, the less one will need to use these instruments for diagnostic purposes. 
Uterine Dilatation may be accomplished in the following ways:

1. By graduated bougies, or sounds, after the method of dilatation of the male urethra.

2. By instruments of diverging blades constructed on the principle of the glove-streteher.

3. By water dilators.

4. By tents.

The object of diagnostic dilatation is to open the endometrium in order that by means of the curette a specimen may be removed for microscopical examination, or in order that the finger may be used for intra-uterine digital touch. Dilatation is required more frequently for therapeutic than for diagnostic purposes. The technique is the

\section{Figure 27.}

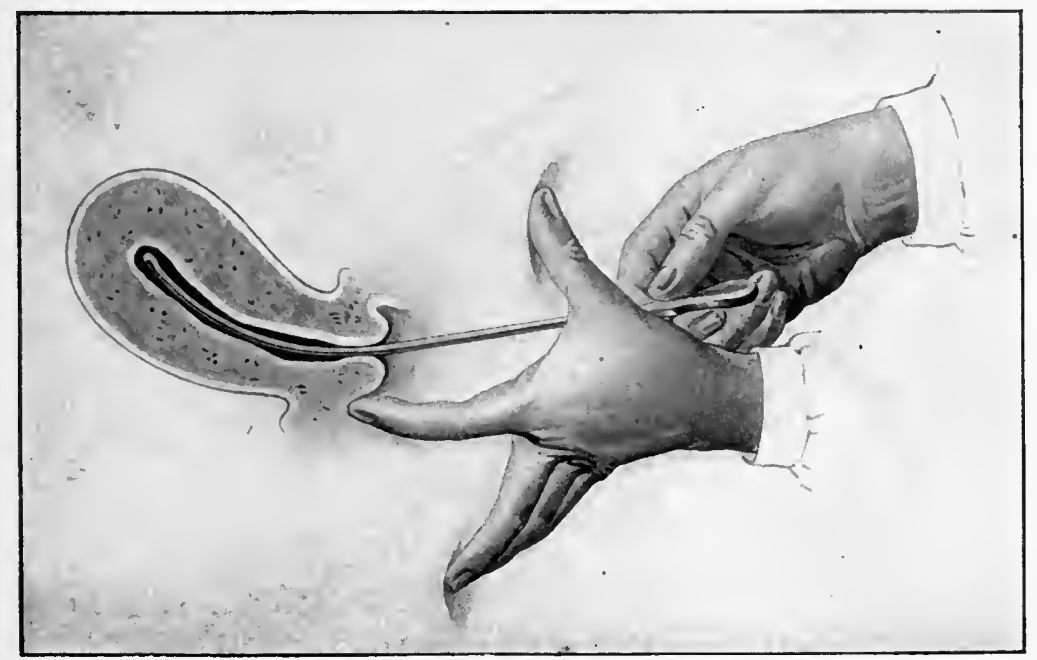

Passing sound; second step: patient in dorsal position without speculum. As sound passes from os externum to fundus index-finger is moved from os externum to posterior vaginal fornix.

same for diagnostic as for therapentic dilatation. See, therefore, a description of the latter in Chapter V., on Minor Operations.

Diagnostic Curettage. - The object of diagnostic curettage is to remove enough diseased tissue for microscopieal or other examination. If the curette is small, and the os patulous, curettage is sonetimes possible without anæsthesia or previous dilatation. Usually, however, the procedure requires both. Microseopical examination of the scrapings is frequently the only means of differentiation between hemorrhagic endometritis, the remains of abortion, post-abortum endometritis, carcinoma, and sarcoma. The technique of curettage is described in Chapter V.

The Exploratory Needle and Aspirator have the same diagnostic and therapeutic significance in gynecology as in other departments of surgery-i.e., the removal of fluid. The contents, for example, of a 
sactosalpinx, a renal cyst, a pelvic abscess, or an ovarian cyst may be removed for visual, chemical, or microscopical examination.

The uses of the stethoscope and microscope will, as the occasion requires, be mentioned in the diagnosis of special diseases.

\section{Examination of the Anus and Rectum.}

Rectal tonch and eversion of the anus by means of the finger in the vagina have been noticed in the earlier pages of this chapter. Numerous specula have been devised for inspection of the interior of the rectum. For examination of the lower part of the rectum, Sims' speculum is inmeasurably superior to all others. It is used for this purpose the same as for vaginal examination-i. e., with the patient in the left lateroprone position.

The Proctoscope and Sigmoidoscope.-The frequent association or confusion of rectal disease with the diseases of women may render necessary the inspection of the upper part of the rectum; for this purpose Kelly uses a tubular speculum, called a proctoscope, about 1 inch in diameter and 8 inches long. For still higher examinations he uses the sigmoidoscope, of the same diameter, but 14 inches long. The patient is examined in the knee-breast position, and the light is thrown in by a head-mirror. Examination through these instruments is most satisfactory.

\section{Examination of the Urinary Organs.}

The means of examination are these:

1. Urinalysis.

2. Palpation, percussion, and inspection.

3. Urethroscopy.

4. Cystoscopy and ureteral exploration and catheterization.

5. Segregation of urine.

1. Urinalysis.-The study of the urine involves, first, chemical examination; second, microscopical examination.

The chemical examination will show changes in the proportion or quality of solids, and will suggest the possible relation of these changes to pathological conditions and functional disorders. For example, decrease in urea may signify nephritis. Abundance of uric acid would indicate that nore exercise and less nitrogenous food should be taken. Excessive acidity would account for irritation of the bladder and frequent urination. Microscopical examination may locate the existence of disease in either the kidney, ureter, or bladder.

2. Palpation, Percussion, and Inspection.-Palpation and percussion over the hypogastrium may give strong evidence of distention of the bladder; further evidence would be the bulging of the anterior vaginal wall toward the vulva, and constant dribbling of urine. The evacuation of a large quantity of urine through the catheter would be proof.

Palpation with conjoined examination may show a tumor in the bladder. Vaginal and rectal touch also may give much information 
relative to the urethra, bladder, and ureter. Vaginal touch will enable one to judge of sensitiveness in the urethra and neck of the bladder. In the anterior wall of the vagina to either side of the

Figtre 28.

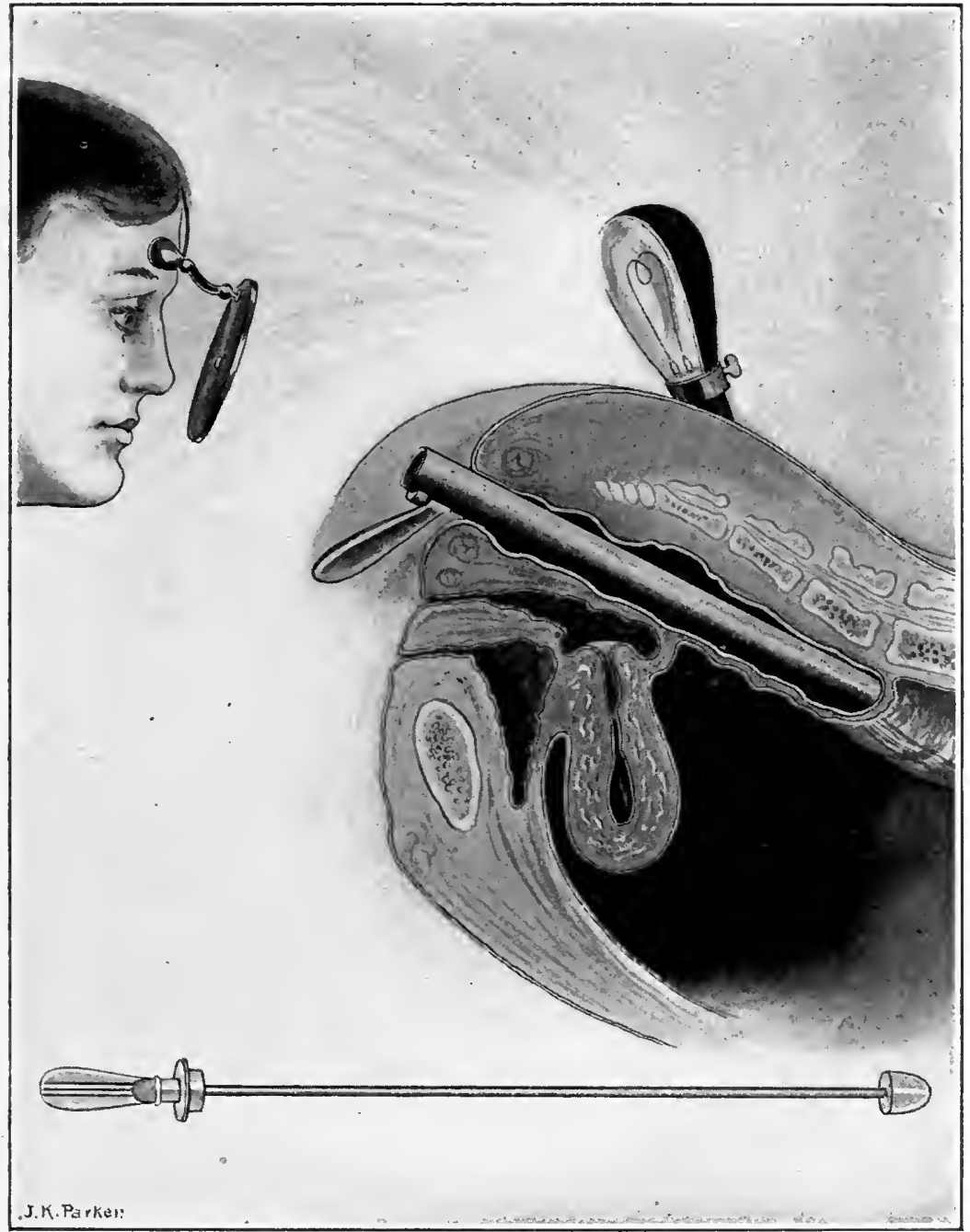

Proctoscopy: proctoscope 8 inches long and 1 inch wide. The sigmoidoscope is the samo except jn length, which is 14 inches. The instrument is provided with an obturator (shown detached in the lower part of the Figure); it is in nearly all respects except size identical with the cylindrical cystoscope.

median line the ureter may often be felt as it passes in a posterior and lateral direction on either side of the eervix toward the kidney. It is normally a flattened, eord-like, soft, yielding band. Pathological changes sometimes may make it easy to recognize as a hard, 
round, large resisting cord. A bougie introduced through the urethra into the ureter facilitates the palpation. Tenderness along the line of the ureter indicates inflammation ; this inflammation of the ureter, when unrecognized, often leads to disappointment in the treatment of cystitis.

The interior of the bladder may be palpated by the sound or by the finger. The sound enables one to judge of the presence or absence of a stone or a tumor. Vesical hemorrhage following the introduction of the sound indicates the possible presence of intlammation or of a tumor. Palpation by the finger through a dilated urethra is to be condemned, for two reasons : first, it gives no information which cannot be obtained better by means of the cystoscope ; second, permanent incurable incontinence of urine from injury to the urethra occurs in about 3 per cent. of the cases. Digital exploration, if marle at all, should be made through a vesicovaginal fistula opened for the purpose. See Cystotomy for Cystitis.

The presence or absence of cystocele, urethrocele, prolapse of the urethra, inflammation, and new growths about the meatus may be recognized by direct visual examination. See Inflammation of Skene's Glands, under Vulvovaginitis.

3. Urethroscopy.-The entire mucous membrane of the urethra may be brought into view by means of a urethroseope. There are many varieties, most of them of the cylindrical form. The urethroscope is inserted with the obturater the whole length of the urethra and the mucosa is brought into view as the tube is withdrawn. The cylindrical cystoscope of Kelly answers the purpose, the only objection to it being its excessive length.

4. Cystoscopy and Ureteral Exploration and Catheterization.-There are two classes of cystoscopes : they are-

'The cylindrical cystoscope ;

The electrical cystoscope.

The Cylindrical Cystoscope.-Numerous instruments have been devised for inspection of the interior of the bladder. It is the great merit of Howard Kelly to have popularized and perfected an effective and satisfactory means of intravesical inspection. - The following is a description of the instruments and methods used by Kelly.

The essential features of the method are:

1. Atmospheric dilatation of the bladder induced by posture.

2. Introduction of a simple straight speculum withont fenestrum.

3. Examination of the interior of the bladder and urethra by reflected light.

The instruments required are :

1. A good light and a head-mirror.

2. A urethral dilator, Figure 30 , a.

3. A vesical speculum with an obturator, Figure 29, e.

4. A suction apparatus to empty the bladder, Figure $31, \mathrm{X}$.

5. A long mouse-tooth forceps, Figure 30, d.

6. A searcher for discovering the ureteral orifice, Figure 29, b.

7. Ureteral bougies and ureteral catheters.

The speculum in most common use has a diameter of $1 \mathrm{~cm}$. If 
The Electrical Cystoscope.-This instrument was invented by Leiter, of Vienna, and later improved by Casper. Both the Leiter and Casper instruments earried the electrie light ray into the bladder by means of refracting prisms at the external end of the tube. In 1876 Nitze placed the vacuum light at the inner extremity of the tube in such a manner as to give direct illumination and to transmit to the eye through a series of lenses an exact pieture of the bladder mueosa magnified. In all these instruments the eleetric current is furnished by a battery from which insulated conductors pass through the tube to and from the lamp. This instrument is used with the bladder filled with

Figure 32.

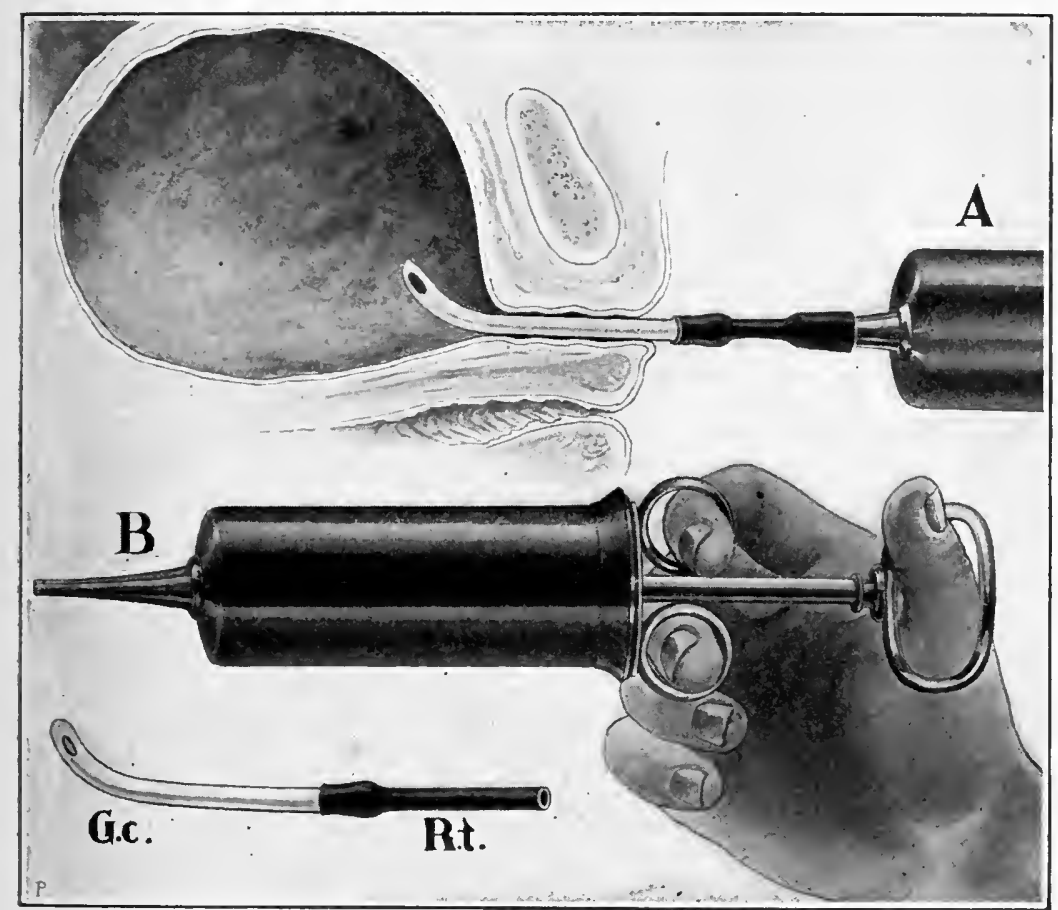

The syringe is represented here in hard rubber. An instrument with metallic barrel and metallic piston is preferable because it permits frequent disinfection by boiling, and beeause boiling injures the rubber instrument. A, filling of bladder with water, preparatory to cystoscopy, by means of syringe, to which glass eatheter is attached by rubber tube; 1 , complete syringe; G.c., glass eatheter; R.t., rubber tube.

water and with the patient in the ordinary dorsal position. Four conditions are essential to the use of the electrical cystoscope:

1. Permeability of the urethra- $5 \mathrm{~mm}$.- - sufficient to pernit the ready passage of the instrument.

2. The capacity of the bladder must be sufficient to hold not less than 100 c.e. of injected fluid. fluid.

3. The sphincter vesicæ must have the power to retain the injected

4. The injected fluid must remain transparent, and not become clouded by. admixture of blood or mucus. 
Advocates of this method claim superiority over the Kelly method for the following reasons:

1. General or local anesthesia is less often necessary.

2. The more convenient lithotomy position is used instead of the knee-breast or Trendelenburg position.

3. The bladder is distended more satisfactorily by water than by air.

4. The urethra is dilated less widely.

5. The examination requires less time and less skill, and requires no assistant.

Comparison of Cystoscopes. - It is evident that the Nitze cystoscope and the modifications of it, such as Casper's, are superior to the

Figure 33.

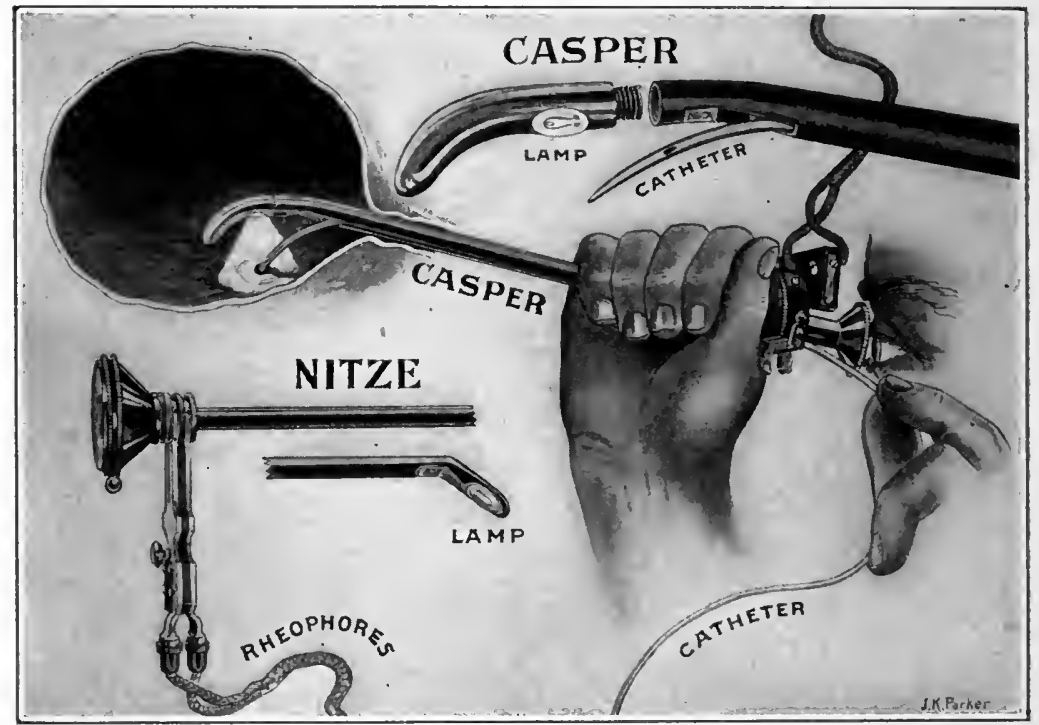

Catheterization of left ureter by Casper cystoscope. Upper right-hand figure shows terminal part of Casper eystoscope, with lamp and catheter, slightly reduced size. Lower lelt-hand figure shows Nitze cystoscope, about one-third natural size.

Kelly instrument when the sphineter vesicæ will not retain the injected fluid, when the injected fluid becomes turbid and bloody, and when the bladder is so contracted that it fails to distend sufficiently. Cystoscopy and ureteral exploration in women, owing to the shortness and dilatability of the urethra, may be accomplished satisfactorily by means of the simple Kelly cystoscope already described. In examinations of the male urethra the prismatic elfetroseope, on account of its magnifying power and the greater distance of the ficld of inspection from the eye, is indispensable.

The Kelly instrument has the advantage over the electrical cystoscope of not exposing the bladkler to burns; and of giving a more accessible field for topical applications.

Value of Cystoscopy and Ureteral Catheterization.-By means 
of the eystoseope the entire interior of the bladder may be brought into view; foreign bodies, tumors, and other pathological

Figure 34.

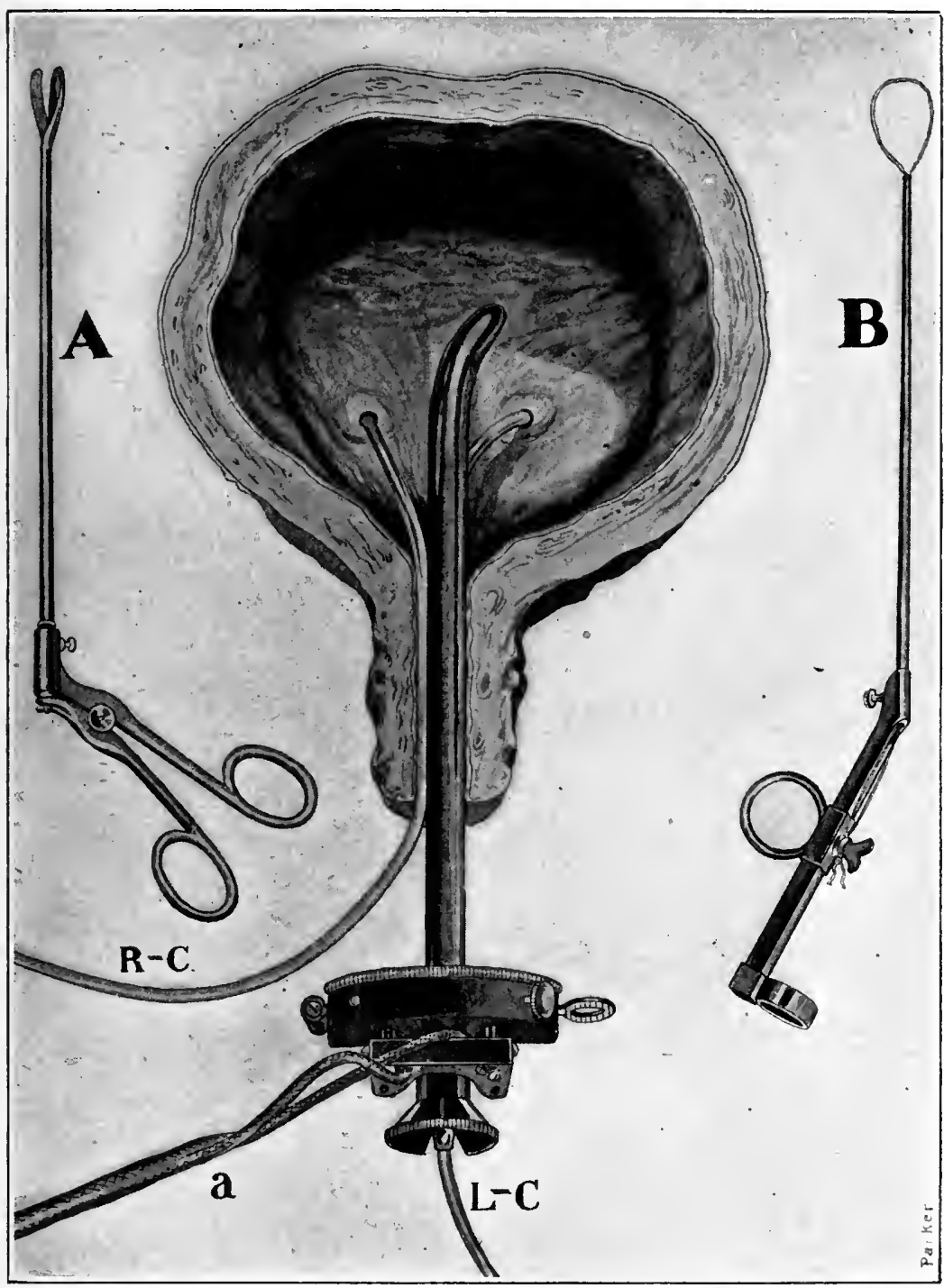

Catheterization of both ureters by Casper cystoscope. Right ureter has been eatheterized and eystoseope withdrawn, leaving eatheter in ureter. Cystoseope again introduced, and left ureter being eatheterized; R-C, eatheter in right ureter: L $-\mathrm{C}$, eatheter being passed into left ureter; A and B, foreeps and snare for intravesical operations; a, rheophores.

ehanges may be recognized. The instrument often has revealed the presenee of stones, tumors, and ulcers which had escaped deteetion by the sound. Numerous eases in which eystitis is of only 
secondary importance to other associated lesions, such, for example, as tumors, tuberculous nleers, hemorrhoids of the bladder, are now daily observed by the cystoscope. Cystoscopy is of great value in preventing blind and meddlesome treatment for a class of eases which present the subjective symptoms of cystitis, but in which inspection fails to show any lesion whatever of the bladder mucosa. The value of the instrument is incalculable when only limited areas are diseased, as, for example, in the mild inflammations of the trigone and in fissure at the neck of the bladder. Under such conditions the operator, instead of treating the entire vesical mucosa by means of injections more or less strong, may direct to the diseased part only any application which may be indicated.

Figure 35.

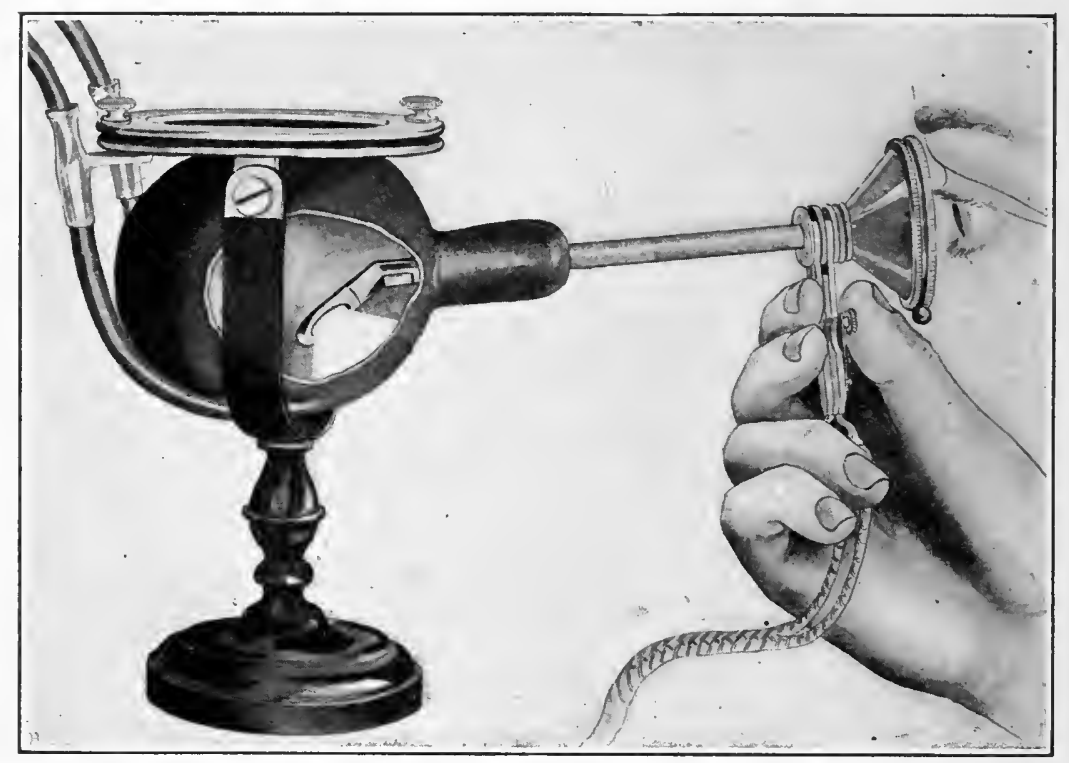

Cystoscopy of phantom bladder through the Nitze cystoscope.

By ureteral catheterization we are enabled to separate the urine of the one kidney from that of the other, and temporarily, as it were, to eliminate the bladder from the urinary tract. In case of a diseased kidney marked for removal one may demonstrate the presence or absence or ascertain the condition of the other kidney, and thereby avoid the post-mortem embarrassment of finding it either absent or useless from disease.

It is necessary to a correct diaguosis of cystitis, for example, that we know what abnormal constituents in the urine have their origin within the bladder itself. Normal urine suffers no change in a normal bladder free from microbes; hence a comparison of analyses of urine taken from the bladder, with urine taken directly from each kidney, may at once indicate the exact location of the disease. There may 
be present the subjective symptoms of cystitis-that is, pyuria, painful and frequent urination, and ammoniacal urine-and yet the bladder may be free from disease.

The points to be observed in urine thus obtained are the reaction and the presence or absence of pathological products, such as pus, blood, epithelial cells, bacteria, and crystals. The reaction of the urine should be taken at once, as secondary changes sometimes oceur quite rapidly. If urine taken directly from the kidneys possess a normal degree of acidity, while that from the bladder be alkaline, it is evident that the pathological process producing the alkalinity must be within the bladder. If urine from the kidneys be free

Figure 36.

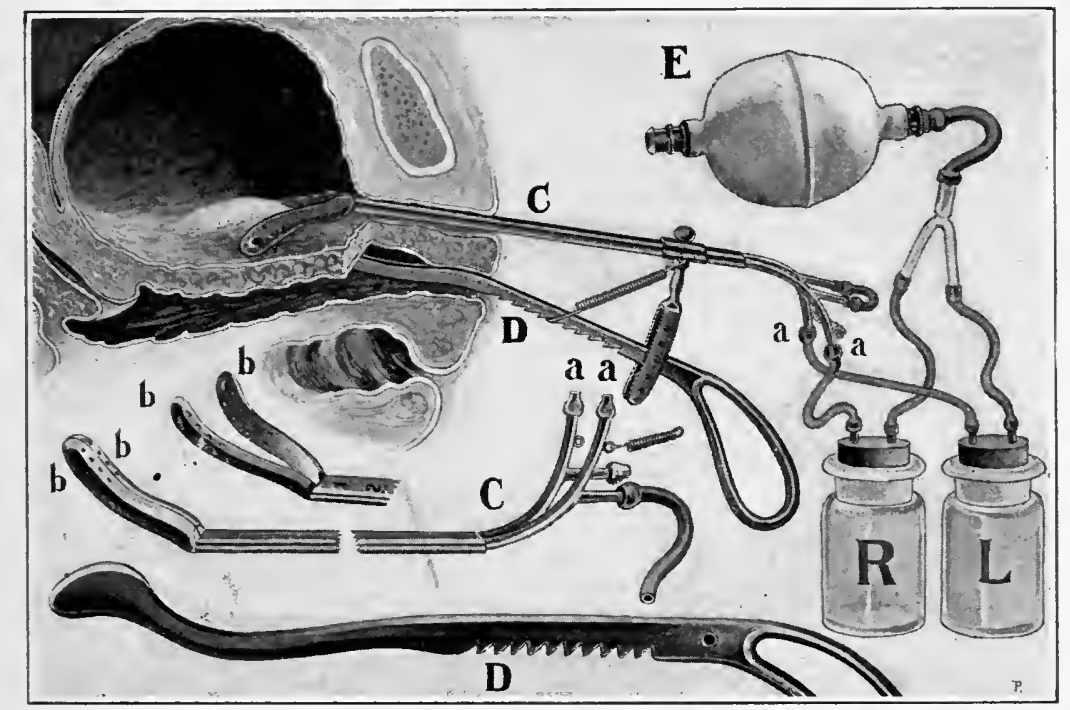

Urine being taken through each ureter separately by means of the Harris segregator: C, catheters; D, watershed lever attached to catheters, and in use; bb and bb, catheters closed and partially open; $\mathrm{D}$. watershed lever detached from catheters : $\mathrm{R}$, bottle connected by rubber tube to right catheter; L, bottle comnected by rubber tube to left catheter. These bottles receive the urine from the right and left ureters respectively : $\mathrm{E}$, rubber bulb attached by rubber tubes to bottles. This bulb is used to exhaust the air partially from the bottles, so that the urine may flow more readily : aa and aa, ends of catheters where bottles are attached.

from pathological products, while that fiom the bladder contains pus, epithelium, or bacteria, the involvement of the bladder is unquestionable.

5. Segregation.-Another instrument of importance is the segregator of Harris; it colleets the urine directly and separately as it passes from each ureter into the bladder. The instrument has two advantages over the ureteral catheter: first, unlike that instrument, it is available for the non-expert; second, it does not invade and therefore cannot infect the ureters. The instrument consists of two eatheters, their straight portions being enelosed in a flattened tube, and each being separate and movable on its longitudinal axis. Figure 36 shows the tube graduated to 19 centimetres and enclosing the two 
catheters. Their vesical ends protrude from the tube to the right and their outer ends to the left. 'The meehanism is such that, the instrument having been introduced into the bladder, the two catheters may be rotated upon their long axis so that their curved bladder-ends will lie as indicated in Figure 36, C, one on one side and the other on the other side of the trigone. A metallic lever, $\mathrm{D}$, is introduced into the vagina of the female, or the rectum in the male, and attached to the shaft of the instrument. This lever is represented detached in the lower part of the Figure. It is attachable to the eatheter tube by means of a forked metallic appliance, and held up by a spiral spring; the function of it is to elevate that portion of the bladder which lies between the two separated ends of the rotated catheters and thereby to form a watershed. The urine as it drops on either side from each ureteral orifice is now separated and flows ont through the eatheter on the corresponding side. Each catheter is continued by a rubber tube to a bottle for the reception of urine. The bottles are provided with a rubber suction bulb which may serve to create a partial vacuum and thereby to attract the urine. For obvious reasons the bladder should be washed out before the segregator is introduced. Before using the segregator one should study carefully the directions of the inventor, which accompany the instrument.

\section{Exploratory Incision.}

When other means of diagnosis have failed, and it is necessary to examine the pelvic or abdominal organs directly by toueh or by sight, the surgeon will for that purpose open the peritoneum by exploratory incision. The incision is made either throngh the vagina-vaginal section, or through the abdomen-abdominal section. The incision having been made, the finger is introduced and the diagnosis made by direct touch. The section may be enlarged, if necessary, so as to bring the pelvic and abdominal contents into view. Simple touch, however, through the incision only large enough to admit the finger, is always safer and usually gives more information than visual examination. All vaginal and abdominal incisions shonld be first exploratory.

${ }_{1}$ Harris, Transactions of the Chicago Gynecological Society, November, 1898; and Medicine, April, 1898. 


\section{CHAPTER IV.}

\section{LOCAL TREATMENT.}

THE principal procedures in local treatment are these :

1. The hot-water vaginal douche.

2. Tamponade.

3. Topical applications.

\section{THE HOT VAGINAL DOUCHE.}

The choice of the syringe, the frequency of the douche, the time and length of each application, the temperature of the water, the proper use of the bed-pan, the position of the patient, and persistence in the treatment, are all essential to good results.

Figure 37.

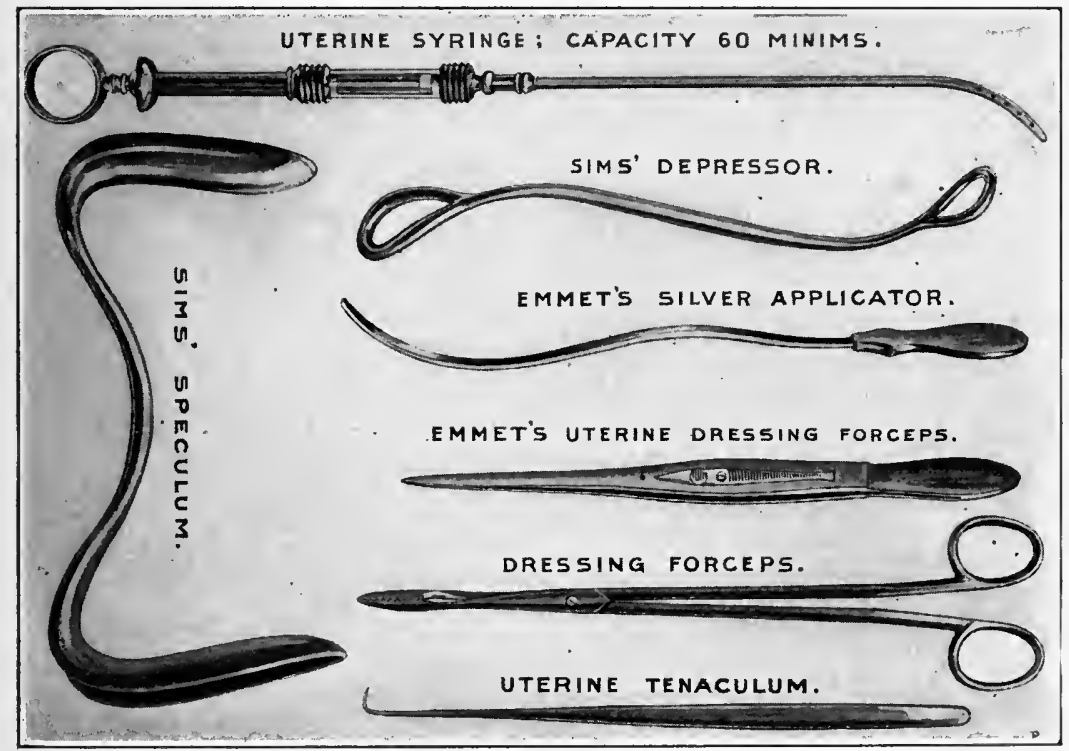

Some of the instruments commonly used in local treatment. About one-third natural size.

The small fountain syringe in general use requires refilling several times during the applieation of the donche. A large syringe-bag or receptacle of some kind to supply the water is therefore desirable. The common bed-pan is objectionable becanse it must frequently be emptied. To overcome this difficnlty, F. H. Lord attaches a rubber 
tube to the bed-pan through which the water is drained continuously to a bucket below the couch.

A satisfactory substitute for the bed-pan may be made as follows: at the side of an ordinary bed place two chairs with space enough between them to admit the lower bucket; spread a rubber sheet over the side of the bed so that one end of the sheet may fall into the bueket below in the form of a trough. The donehe may then be given with the patient lying across the bed, the hips resting over the edge of the

\section{Figure 38.}

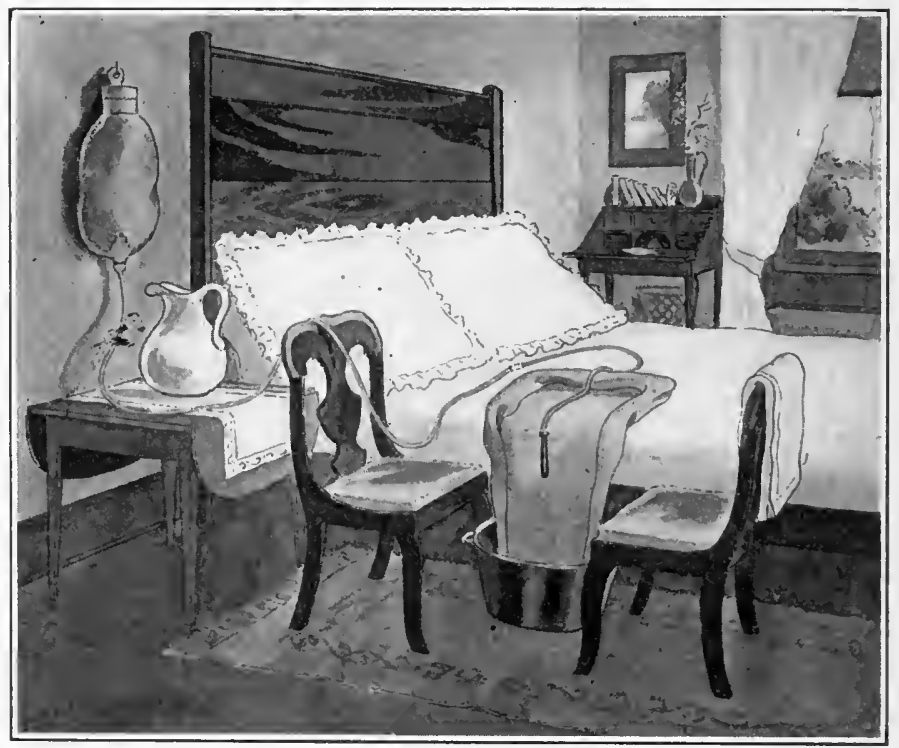

Rubber sheet substituted for the bed-pan.

bed and one foot on each chair. The water will find its way along the rubber trough into the bucket below.

The following is designed to impress the importance of strict observance of detail in the application of the douche. In no other manner will its good effects be realized :

Ordinary method of application.

I.

The douche is applied with the patient in the sitting posture, so that the injected water caunot fill the vagina and bathe the rervix uteri, but instcad returns along the tube of the syringe as it flows in.

II.

The patient is not impressed with the importance of regularity in its administration.
Proper method of application.

I.

The douche should invariably be given with the patient lying on the back, with the shoulders low, the knees drawn up, the hips elevated so that the outlet of the ragina may be above every other part of it. Then the vagina will be kept continually overflowing while the douche is being given.

\section{II.}

It should be given at least twice every day, morning and evening, and generally the length of each application should be not less than twenty minutes. 
Ordinary method of application.

III.

The temperature is not specified, or if specified, is not heeded by the patient.

IV.

The patient abandons its use after a short time.
Proper method of application.

$$
\text { III. }
$$

The temperature should be as high as the patient eau endure without distress. It may be inereased from day to day, from $100^{\circ}$ or $105^{\circ}$ to $115^{\circ}$ or $120^{\circ} \mathrm{Fahr}$.

\section{IV.}

Its use, in the majority of eases, should be continued at least for months. Perseverance is of prime importance.

The pressure of the water should be low and the douche-point short, for cases have been recorded in which the water was forced with unfortunate results through the Fallopian tubes.

Modes of Action.- The douche acts in a twofold way:

1. As a vascular stimulant.

2. As a cleansing agent.

1. Vascular Stimulant.-Enmet; the strongest advocate of the douche, attributes its good effects to the stimulating influence of the hot water on the blood-vessels. The dilated congested vessels are, he says, made to contract, and in this way congestion is lessened, circulation quickened, absorption of morbid products hastened, and local nutrition improved.

2. Cleansing Agent.-The vagina in pelvic inflammation is a passage-way, and to some extent a receptacle, for pathological secretions. These secretions flow into it from the uterus, the Fallopian tubes, pelvic abscesses, and from the vaginal mucous membranc itself. Un. less kept clean, the vagina may become an incubator and a distributing-point for bacteria. The value of the douche, therefore, as a means of asepsis is self-evident. When local disinfection is required, the hot-water douche may have in solution some antiseptic substance, such as lysol, carbolic acid, corrosive sublimate, boric acid, salicylic acid, peroxide of hydrogen, or formalin.

The Indications for the douche, as suggested in the foregoing paragraphs, are chiefly in the treatment of ehronic pelvic inflammations. The power of heat to stimulate and contract blood-ressels makes the douche also usefil in the treatment of uterine hemorrhage. The prevailing disposition to extend the use of it to the routine treatment of all pelvic disorders should be diseouraged.

Contraindications and Dangers.- The douche should not ordinarily be given during menstruation for fear of exciting pelvic congestion, nor during pregnancy for fear of causing uterine contractions. In the presence of a bleeding cancer or sarcoma of the cervix caution is required to avoid hemorrhage. In some cases of patulous Fallopian tubes the douche fluid may be forced into the uterus, through the tubes and into the peritoneal cavity, with most serious results.

There are constantly present in the normal vagina numbers of lactic-acid-producing bacteria whose function is to render the vaginal secretions acid, and therefore to make it an unfit culture-ground for about 90 per cent. of all pathogenic bacteria. The washing out of these normal germs and the acid secretion may make the vagina possibly a less difficult barrier for disease-germs to pass, and therefore 
may open the way for infection in the higher zones of the pelvis. The rontine use of the donche in the normal vagina except for ordinary purposes of bathing is for this reason of questionable propriety.

\section{TAMPONADE.}

The principal indications for tamponade are:

1. Inflammation.

2. Hemorrhage.

1. Inflammation.-Tamponade in the treatment of inflammation is designed, according to the indication and manner of application, to fulfil one or more of three purposes. It may be used: $\mathrm{A}$, as a means of pressure; $\mathrm{B}$, as a vehicle for the application of medieinal substances; $\mathrm{C}$, for drainage.

Figiure 39.

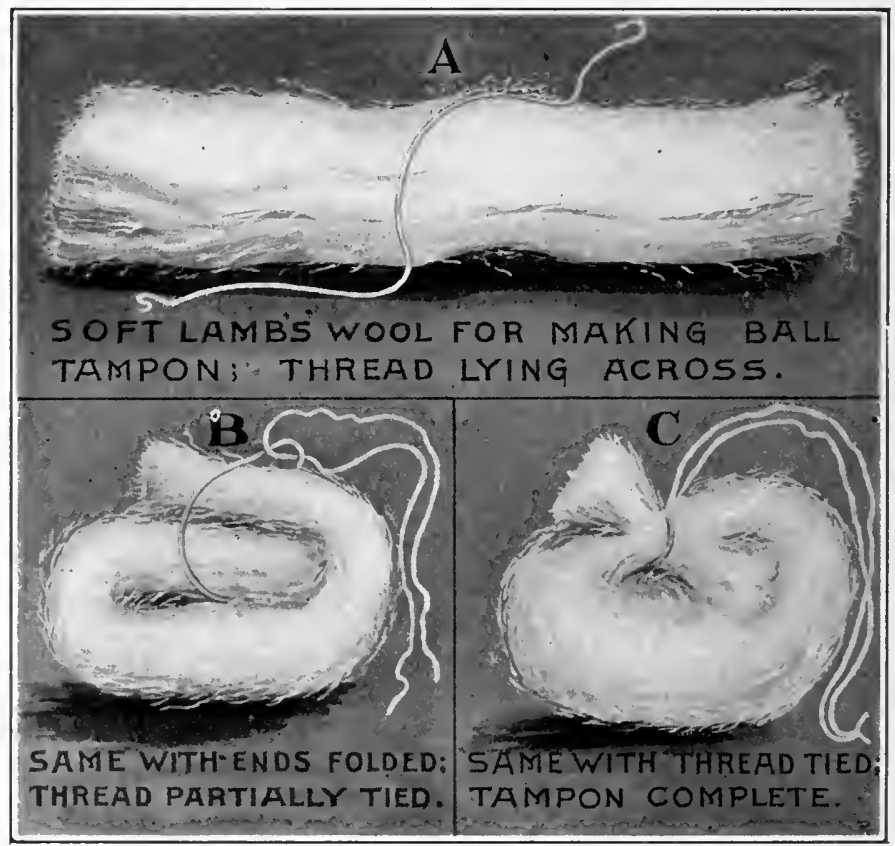

The making of a vaginal tampon of lamb's wool. One-half natural size.

A. The Pressure-effect of the tampon is chiefly useful in the treatment of displacements, especially displacements due to inflammatory causes. The subject will be discussed further under the head of Pelvic Inflammations and Displacements.

B. As a Vehicle for the Introduction of Medicaments the vaginal tampon has become a routine factor in gynecology. It is used most frequently as a carrier of glyccrin. The effect of the glycerin is to cause a watery discharge from the genital tract, and thereby to deplete the vessels and overcome congestion. Good results often have fol- 
lowed this treatment. How far they should be attributed to the tampon, and how far to the eurative forces of nature, or to associated systemie treatment, it is often difficult to say. If the tampon is left in for more than. twenty-four hours, it beeomes offensive, and may become a hotbed of infection; hence, if used at all, it shoukd be

Figure 40.

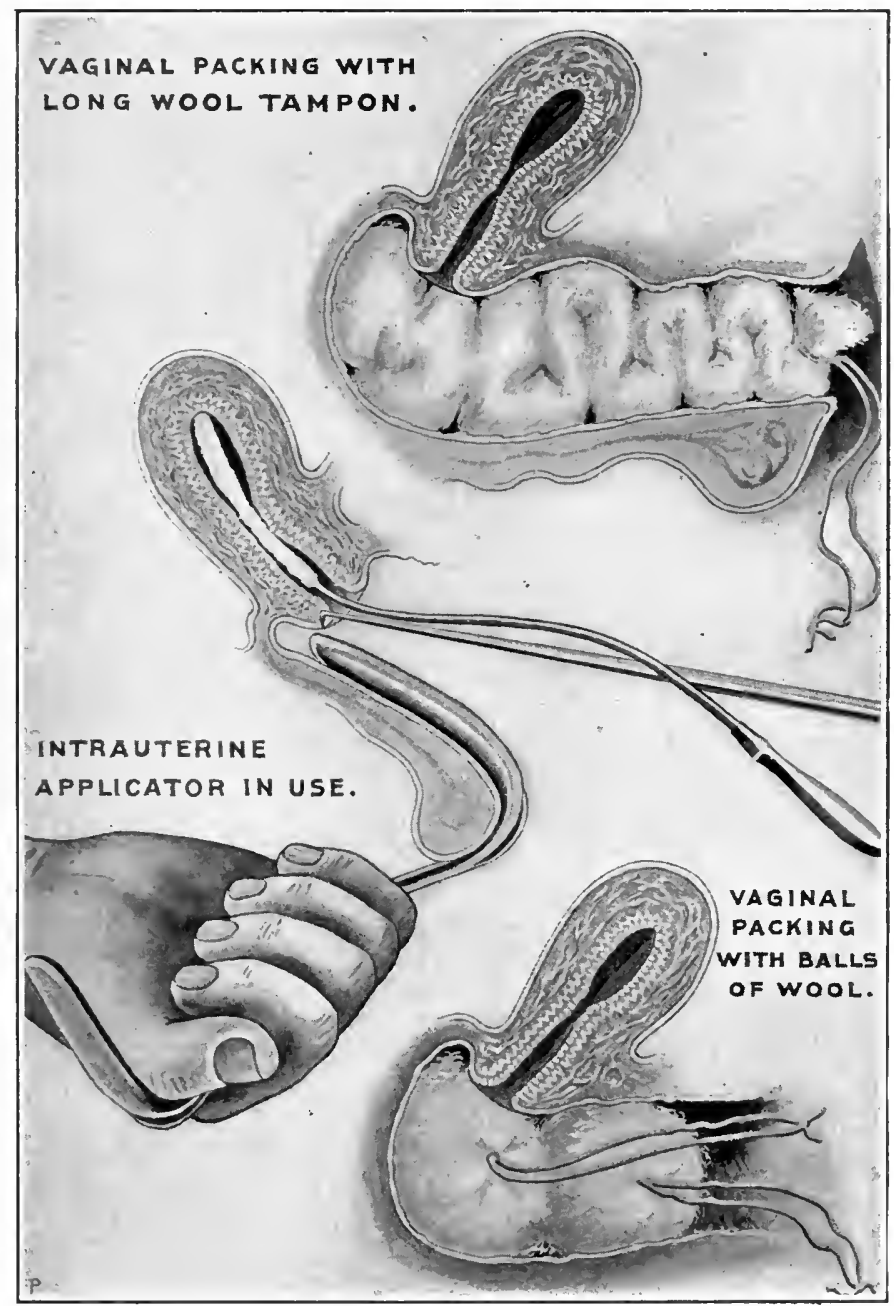

Intra-uterine application and vaginal tamponade.

removed at the end of twenty-four hours. The therapeutic value of the tampon has been much overestimated.

C. Drainage of the Endometrium for' endometritis, by means of the intra-uterine tampon of aseptic or antiseptic galuze, has been a favorite means of treatment. See chapter on Treatment of Endometritis. 
2. Hemorrhage.-Hemorhage from the vagina often may be controlled by means of a tight vaginal tampon. It is, however, better to find the bleeding-point and secure it by more definite surgical means. Uterine hemorhage, whether from endometritis, uterine tumors, or abortion, may demand immediate control. 'The vaginal tampon is used most commonly for this purpose. It is, however, a cumbersone measure in severe cases and often fails. Great distention of the ragina by a large tampon interferes with the function of the bladder and rectum, and is a mechanical cause of discomfort. The intra-uterine tamponade is the most practical and the most effective treatment for uterine hemorrhage. It shonld be in the form of a continuous strip of aseptic or antiseptic ganze two inches or more wide. The cervix having been exposed by a Sims speculum and steadied by a vulsellum forceps, the strip is introdnced by means of a slender dressing-forceps, sound, or similar instrument. The secretions absorbed by the tampon decompose rapidly, and become a prolific source of infection; hence the gauze should be renewed daily or at most every two days.

Material of the Tampon.-If elastic pressure is required, fine lamb's wool is superior to absorbent cotton. For other purposes the continuous strip of aseptic gauze is preferable to either.

\section{TOPICAL APPLICATIONS.}

Applications to the Endometrium.-Intra-uterine medication commonly results in failure and disappointment, for two principal reasons : first, it often is used in unsuitable cases; second, even though the cases be suitable, it often is used improperly.

Efficient intra-nterine medication requires that the medicinal substance be brought in contact with the nterine mueosa. Ordinarily the medicament is carried into the endometrium when that cavity is full of uterine secretions. These secretions form a thick protective coating over the mucosa. The application mixes with and may exhaust its virtue in chemical combination with the secretions, but does not reach the diseased mucons membrane. It frequently occurs that the applicator at varions points inflicts slight wounds upon the endometrium, and thereby opens the door to septic invasion. Pelvic infection may be the result. 'The treatment, therefore, unless carefully applied, may be dangerous.

The prerequisites to safe and efficient intra-uterine applications are : first, a clear indication and definite appreciation of what the application is to accomplish-that is, the case nust be selected properly ; second, preparatory disinfection of the vulvovaginal surfaces and dilatation and cleansing of the endometrium ; the disinfection is specially essential as a precaution against infection.

The Proper Selection of Cases will exclude, at least, three large classes of cases :

A. Those cases in which the increased uterine discharge is due simply to an effort on the part of the organ to relieve itself of congestion by increased seeretion-that is, cases in which the discharge is not due to infection. The congestion of the mucosa under such conditions is 
very apt to be associated with some systemic disorder, such as cholæmia, malaria, diabetes, gout, anæmia, or heart lesions. These cases should be treated by general rather than local remedies, and should be referred to a physician. The disappearance of such a discharge during local treatment should be attributed not to the meddlesome applications, but to the associated systemic treatment or to the eurative force of nature.

Figure 41.

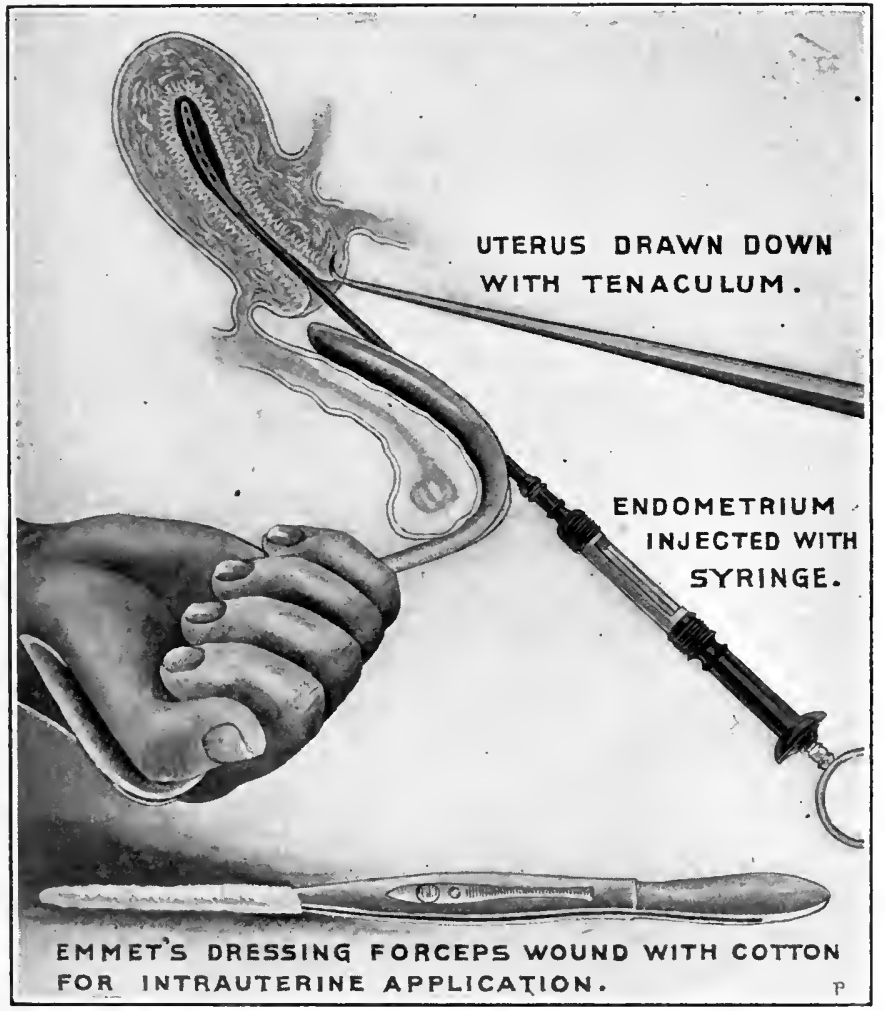

Intra-uterine injection, and Ernmet's dressing-forceps wound with cotton for intrauterine application.

B. Those cases in which the parametria, Fallopian tubes, and other eireumuterine structures are infected, or in which there is a nterine or extra-uterine tumor, or some other anomaly which would render topical applications useless or dangerons. This class of cases should be referred to the surgeon.

C. Those eases in which the uterine discharge is due to some noninfectious local irritant of non-bacterial origin, such, for example, as temporary uterine displacement from an overcrowded bowel or an overdistended bladder. When the local irritation is removed, the disorder usually disappears.

For selected cases in which the uterine mucosa is the subject of uncomplieated bacterial infection, or in which the complieations are 
not such as to contraindicate intra-uterine unedication, it may be wise to introduce medicinal substances to the endometrium; the steps of procedure will have to be as follows:

1. The preparatory dilatation (unless the uterine canal is already quite open) and cleansing by irrigation having been made, expose the cervix by means of a speculum, preferably Sims'.

2. Seize the cervix by means of a snall tenaculum, or tenaculumforceps, in the left land, and hold the cervix steady.

3. With the right hand pass the applicator, wound with cotton which has been saturated with the required medicament, into the uterine canal; or if it be desired to use intra-uterine injections, the fluid to be injected by means of a suitable intra-nterine syringe.

Iany patients will not tolerate the necessary dilatation without anresthesia; hence, intra-nterine medication as an office procedure must be restricted. Within the limitations above outlined, it becomes in many cases a surgical measure, and as such is no longer a potent cause of pelvic infection. The general subject of uterine applications is set forth more fully in the chapter on the Treatment of Endometritis.

It follows from the above that a very large proportion of the women who formerly were made the subject of extensive intra-uterine treatment should be treated rather by medical or surgical means, or by both combined. Many cases of excessive uterinc secretion which do not present well-defined indications for surgical treatment should be relegated to the field of interual medicine. The legitimate field for rontine topical applications to the uterus is limited. Some gynecologists restrict intra-uterine medication almost entirely to supplemental treatment after curettage, when a limited number of disinfecting irrigations or applications may be useful.

The use of bongies containing various nedicaments, the introduction of intra-nterine suppositories, the injection of various fluids into the uterus, the packing of the endometrium with ganze, and other similar procedures, will, according to their value, be presented, or omitted, under the treatment of special disorders.

Biers' Cupping Treatment.-Biers and others practice a method of dry cupping the cervix uteri by means of a suction pump in order to stimulate the circulation of the organ; it is called the congestive treatment and is said to modify the nutrition of the past favorably by morlifying the circulation. The idea is rational, inasmuch as it tends to correct those morbid conditions which depend upon faulty circulation. Massage of other parts is given on the same principles.

Applications to the Vulva or Vagina, including the vaginal portion of the uterus, are indicated for the cure or palliation of the various inflammatory affections of those organs. Ointments, lotions, douches, and strong caustics may be applied precisely as they would under similar conditions to other parts. Sce Treatment of Vulvovaginitis, Chapter $\mathrm{XI}$.

Direct treatment to the urethra, bladder, and ureters will be diseussed in Chapter XXIV., on Inflammation of the Urinary Organs.

Other forms of local treatment, such as scarification, leeching, and electrotherapenties, are of little value, and in the indiscriminate hand are of much harm. 


\section{CHA P'TER V.}

\section{MINOR OPERATIONS.}

Minor surgery involves a consideration of the preparatory treatment, the operating-table, anæsthesia, instruments, appliances, sutures, ligatures, dressings, the time and place of operation, assistants, operative technique, and after-treatment.

\section{PREPARATORY TREATMENT.}

The preparation for an operation, largely a matter of antiseptics and asepsis, is set forth in Chapter II.

Faulty nutrition from any cause, such as syphilis, gout, rheumatism, nephritis, diabetes, or purpura, may interfere with the suecess of an operation, and may therefore eall for systemic and hygienic treatment.

\section{OPERATING-TABLES.}

For vaginal operations the table should be approximately fortyeight inches long, twenty-four inches wide, and twenty-seven inches high. Operations in private houses are performed usually on the common kitchen table or laundry table, or upon the narrow diningtable. The length of the table should not be greater than that given above, for when the thighs are flexed and the patient drawn toward the operator the head should not be too far from the anæsthetizer, who stands at the end of the table opposite the operator. While the patient is being anæsthetized the feet and legs may rest temporarily on a chair or small stand at the foot of the table.

Clover's Crutch is one of the best of numerons devices to hold the thighs flexed and the legs in position during those vaginal operations which are done with the pationt in the dorsal position. Such an apparatus is convenient, but unnecessary, for the knees may be held by two assistants, one on cach sile.

Acute synovitis of the knee-joint followed by anchylosis has occasionally been observed to follow vaginal operations. This was mexplained until E. H. Webster, of Evanston, Illinois, suggested that an assistant, while holding the thighs in this flexed position, might carelessly throw his weight upon the leg, or lean heavily upon it, and thereby flex the joint to a dangerous degree.

All gynecological tables, whether used for examination or operation, should be made as suggested in Chapter III., with an inelination of three or four inches, the foot of the table being to that extent above the level of the head of it.

The accessories to the operating-table include knee-rests, rubber sheets, and smaller tables for instruments, dressings, and ligatures. 


\section{ANASTHESIA.}

The choice and mode of administration of anæsthetics in gynecology follow unmodified the general principles of surgery.

Ether and Chloroform.-In the absence of heart or kidney lesions the operator, aceording to his preference, is justified in the choice of ether or chloroform. Ether is preferred generally in cases of heart disease, and ehloroform in cases of kidney disease.

Local Anæsthesia by Cocaine.-A solution of cocaine injected hypodermically will produce local anæsthesia in the infiltrated tissues for a distance of about one-half inch from the needle puncture. The 1 per cent. solution commonly is used; a sufficient quantity of this solntion to produce satisfactory anæsthesia-one to two grains-has given rise repeatedly to alarming depression in the circulation and respirationso alarming as to call for the strenuous use of whiskey, strychnine, and nitroglycerin. The cocaine solutions of Schleich are designed to produce local anæsthesia with less cocaine, and thus to minimize the danger; they are nade in three strengths as follows. The basis of the solution is:

Distilled water, 1000 parts ;

Sodium ehloride, 2 parts;

Morphine hydrochlorate, $\frac{1}{4}$ part.

To the above solution is added cocaine hydrochlorate :

$\frac{1}{100}$ of 1 per cent. for the weaker solution;

$\frac{1}{10}$ of 1 per cent. for the medium solntion;

$\frac{1}{5}$ of 1 per cent. for the stronger solution.

The medium solution is for ordinary use; the weaker solution is for use where very large areas have to be anæsthetized for extensive incisions; the stronger solution is for use in very sensitive inflamed parts.

After the solntion has been injected several minutes are required for surgical anæsthesia. The experiments of Schleich show that the salt water lessens the pain of infiltration, and that even the minute dose of morphine prolongs the anæsthesia.

Large quantities of the solution may be injected and extensive operations be performed under this method of anæsthesia.

Technique of Cocainization with Schleich's solution:

1. Careful sterilization of the site for operation.

2. Numbing the surface with ether spray.

3. Hypodermic injection with the sterilized solution until a wheal about five-eighths inch in diameter is raised.

4. Repeat injections at the margins of the wheal successively until the extent of the anæsthesia equals the extent of the proposed incision. After the first puncture there is little or no pain ; the anæsthesia lasts about ten minutes.

Central Anæsthesia by Cocaine.-A 2 per cent. solution of cocaine sterilized by heating to $80^{\circ} \mathrm{C}$. three times on consecutive days, if injected into the arachnoid space of the spinal cord by means of a sterilized hypodermic syringe, will produce surgical anæsthesia (or perhaps, more properly speaking, analgesia) of all structures below 
the diaphragm. The dose of the solution varies, according to the weight of the patient, from 1.5 c.c. to 2 c.c. Under central anæsthesia by cocaine major operations, including hysterectomy, have been performed successfully. 'The methorl is said to be especially applicable to cases in which ether and chloroform are contraindicated by cardiac, pulmonary, or kidney lesions. Central cocainization obviously demands the most scrupulous asepsis to avoid the disastrous results of infection in the arachnoid space. The utility and safety of this method have yet to be demonstrated.

Adrenalin alone or in combination with cocaine gives excellent results ; it should be used in 0.1 per cent. solution $(1: 1000)$, of which 3 drops may be employed in each hypodermic injection of cocaine or it may be applied alone externally on mucous membranes.

Anæsthesia by Salt Water.-The hypodermic use of aqueous salt solution ( 2 per cent.) is said to produce surgical anæsthesia; and, therefore, to be a satisfactory substitute for the weaker solutions of Schleich. The painless removal of hemorrhoids under this method of anæsthesia has been reported.

\section{INSTRUMENTS.}

Sims' speculum and Simon's speculum have been described in the chapter on Diagnosis. For operations on the vaginal walls, such as the closure of vaginal fistulæ, repair of the lacerated cervix, division of the cervix, dilatation of the cervix, and curettage, Sims' speculum is regarded generally by all who have familiarized themselves with its use as the preferable instrument. See Chapter III.

Simon's speculum, though for plastic vaginal work often inferior to Sims', is yet for some purposes a more practical instrument. It has one advantage over Sims'-i. e., the patient being in the dorsal position, on a Kelly pad or rubber sheet, the operation may, with Simon's speculum, be done under constant vaginal irrigation.

Simon's instrument and the dorsal position are superior to Sims' and the lateroprone position for all operations in which the pelvic cavity is to be opened through the vagina, such, for example, as vaginal hysterectomy, vaginal salpingectomy, and vaginal ovariotomy.

Vulsella forceps similar in construction to those shown in Figure 45 , are useful in various operations on the uterus and about the cervix. They serve to grasp and draw down the cervix, to grasp an intrauterine tumor, and to steady the cervix during the passage of a suture or during curettage.

Scissors.-The minor gynecological operations may be performed either with the scissors or with a knife. The choice depends much upon the education and habits of the operator. The scissors eause less hemorrhage, and when one becomes accustomed to their use he can work more accurately and more rapidly. Any strong, well-made, slightly curved scissors will suffice, but those of Emmet are adapted especially to intravaginal, perineal, and vulvar operations.

Emmet's slightly and fully curved scissors are almost indispensable for denuding in plastic operations, Figure 43; the slightly 
Figure 42.

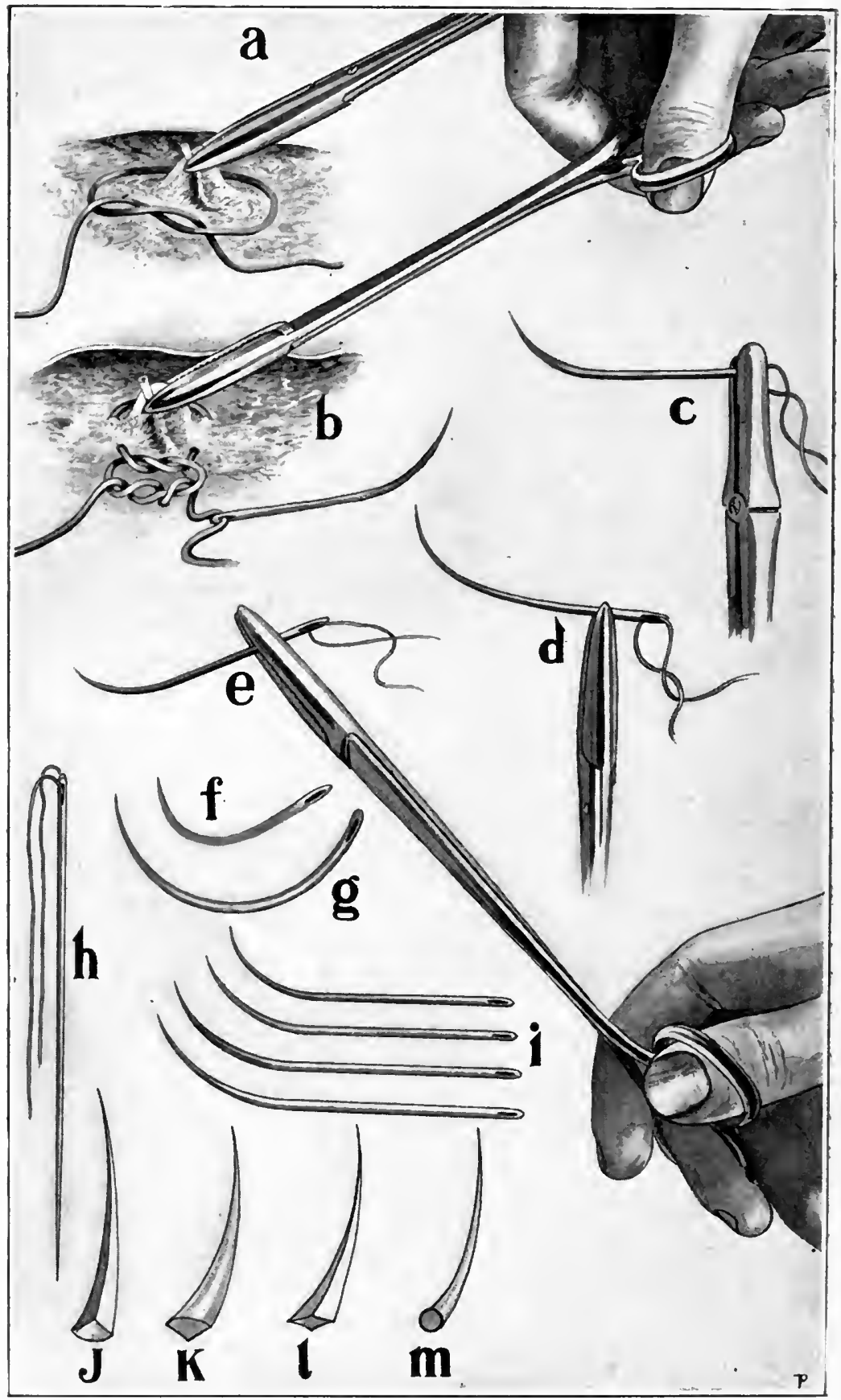


curved are used for perineal and for ordinary intravaginal denudation; the strongly curved are convenient for denuding a strip high up across the vagina or cervix uteri in fistula and cervix operations. These scissors are curved toward the left, and are intended to be used in the right hand. Emmet mentions also two others, with curves to the right; but it is scarcely possible to imagine an operation in which the latter would be necessary. In ordering these scissors one should be careful to explain that he wants those which are curved to the left for use in the right hand; otherwise the instrument-maker will send those which curve to the right for use in the left hand. In fact, a good deal of confusion has arisen in this matter, and consequently orders often are filled with scissors which are useless.

Sponge-holders.-Ordinary hæmostatic forceps with handles eight inches long serve the purpose of sponge-holders much better than instruments made expressly for the purpose.

Uterine Tenaculum.-Numerous tissue-forceps have been devised for grasping the tissues to be denuded or excised, but a properly constructed tenaculum in the educated hand is the most convenient and effeetive instrument for this purpose. With the tenaculum the operator ean pick up and hold a smaller amount of tissue, and therefore can denude nore superficially than is possible with the tissueforceps. The instrument, Figure 43 , has a perfectly straight hook a little more than a quarter of an inch long and bent at right angles to the shaft. It should be so strong and stiff that considerable force may be applied in the line of the instrument without breaking or bending the hook, or in a lateral direction without bending the shaft. The uterine tenaeulum is useful not only in denudation, but also in almost every step of a gyneeological examination or operation. In some operations as many as four of them may be required.

\section{WHEN TO OPERATE.}

Although it is a general rule not to operate during menstruation, it has by no means been proved that operations are more dangerous during this period. When menstruation is so long continued or so profuse as to endanger life or health, immediate operation may be imperative. The presence of menstrual fluid, however, is unfavorable, though usually not a bar to union by first intention in a uterine operation. An operation performed immediately upon the close of menstruation might cause it to reappear; if too near the anticipated period, it might excite a premature flow. One may operate safely

\section{Explanation of Figure 42.}

a. Ligature applied in this manner may slip. Reduced one-half.

b. Ligature applied in this manner cannot slip. Catgut tied in this manner not apt to become loose. Reduced one-half.

$c$. The forceps grasping the needle over the eye is apt to crush it.

d. The forceps grasping the needle in this way does not hold it firmly.

e. Correct grasp.

$f, g, h, i$. Different forms of needles. Ordinary size.

$j, k, l, m$. Needle-points of different varieties, magnified. 
between the third day after the close of one period and the tenth day before the anticipated appearance of the next.

Figure 43.

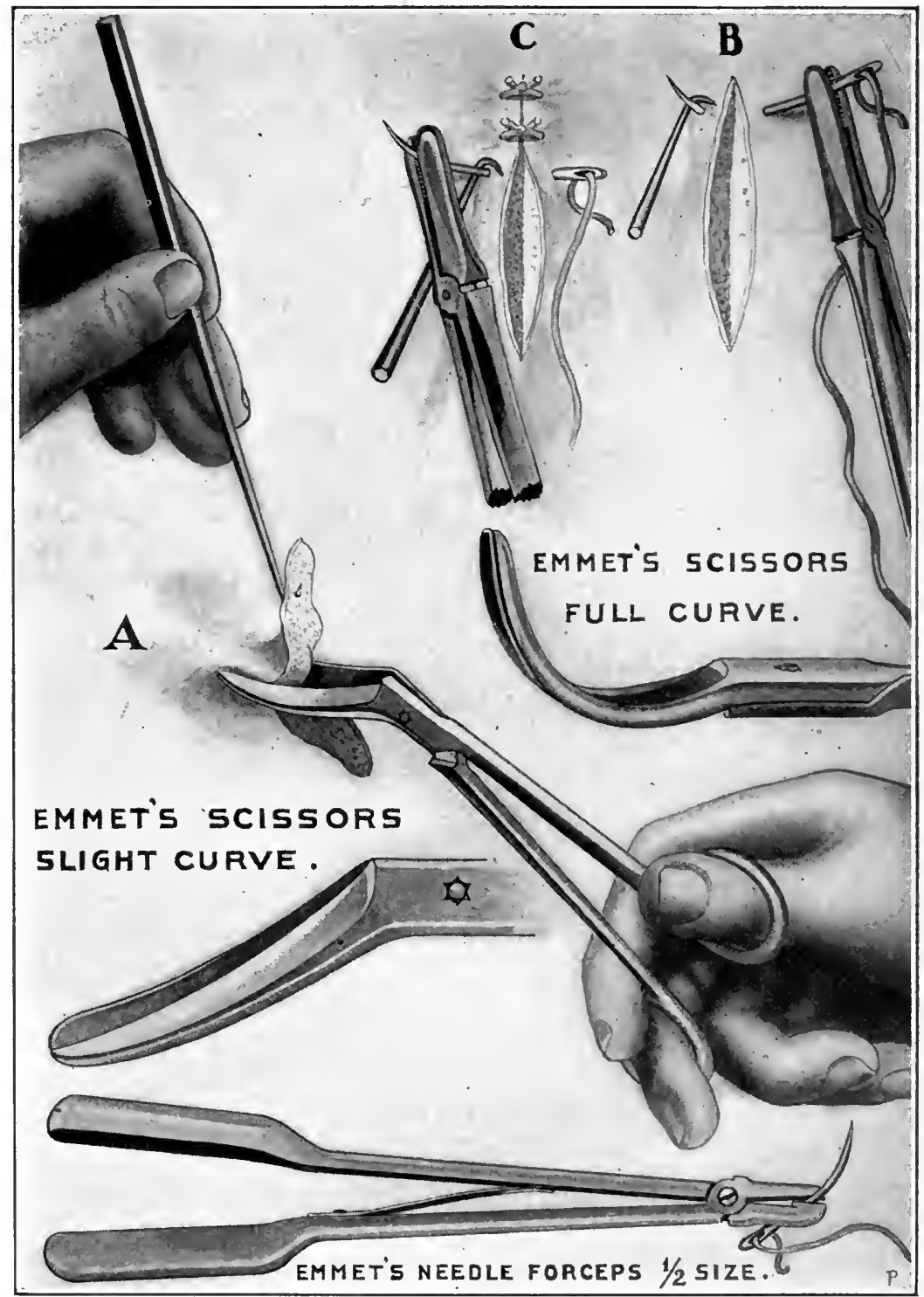

$A$, denuding a surface with Emmet's curved scissors; $B, C$, suturing a wound.

The question of primary or secondary operations after puerperal lacerations has been much diseussed. Emmet's operation for laceration of the cervix, unless there be hemorrhage from the torn sur- 
faces, is delayed ordinarily until after the puerperium. Many successful cases of immediate operation, however, have been reported. For laceration of the perineum, however extensive, the immediate operation is desirable for two reasons: The torn parts can be adjusted accurately to their former relations, which is almost impossible in the secondary operation; and the operation, if well performed, generally results in union, and thereby protects the patient against septic infection through the torn surfaces. The writer, therefore, would advise the primary operation of perineorrhaphy even as late as two days after delivery. He has operated repeatedly on the second and third days, and once on the ninth, and, with scarcely an exception, the delayed operation has resulted in satisfactory union. If, however, the primary operation has been delayed for a number of days, it.is best, before introducing the sutures, to denude with the curved scissors a narrow strip all around and over the torn surfaces, in order that fresh surfaces may be brought together. A delay of a few hours after labor insures greater freedom from capillary oozing, which sometimes occurs after closure of the wound, and which may prevent union. Moreover, if anæsthesia be required, it is better to wait for permanent retraction of the uterus; otherwise the anæsthetic may cause relaxation and consequent uterine hemorrhage.

It is the duty of the accoucheur at the close of the puerperium to examine the uterus, vagina, and perineum, and to repair any significant laceration or injury before evil results have developed from it. Operations may be necessary even during lactation. The child should be kept from the breast only until the mother has recovered fully from the anæsthetic.

Operations during Pregnancy should be restricted to cases of immediate and urgent necessity. Plastic operations, as a rule, may be deferred. Tumors connected with the reproductive organs, such as carcinoma of the cervix uteri, ovarian cyst, uterine polypi, vaginal tumors, vulvar and rectal tumors, may have to be removed. The danger of abortion following operations during pregnancy is due chiefly to possible sepsis or to some other form of toxæmia; even the toxæmia of diffusible poisons and drugs, such as iodine, carbolic acid, bichloride of mercury, quinine, and the bromides, may induce abortion; hence the use of such drugs should be limited and judicions.

Multiple Operations. - When several operations are necessary, it may be proper to perform them at one sitting. A rapid operator may perform safely dilatation of the uterine canal, curettage, trachelorrhaphy, elytrorrhaphy, perineorrhaphy, and the removal of hemorrhoids at one time. This amount of operating at one sitting would hardly be permissible for a slow operator or a beginner. The duration of an operation should usually be less than an hour and a half or two hours. Abdominal section or vaginal section is combined sometimes with plastic vaginal work. This combination, except in the hands of a rapid and expert operator, is not approved. 


\section{PLASTIC OPERATIONS.}

The subject of plastic operations comprehends all operations for the repair of lacerations of the cervix and perineum, and of vaginal fistula; it also includes certain operations on the vaginal walls known as elytrorrhaphy, and numerous operations on the urethra, vulva, and anus.

A clcar appreciation of the causes of failure will contribute to success in plastic surgery. Two principal causes of fuilure are: first, parts which never ought to be united are brought often into apposition; second, faulty technique may result in failure of union.

One of the most common bad results of the repair of the lacerated cervix uteri or perineum, for example, is the union of parts which were not together before the injury, and cannot be united without harm. Commonly a plastic operation which results in union is called successful. If, however, there has been union of wrong parts, actual harm may have been done. The flap-splitting operation of perineorrhaphy, for example, too often gives such a result.

Union by First Intention will result almost always from a correct operation. True, in certain cases of vaginal fistula in which there has becn great loss of tissue from sloughing, failures may arise from the cicatricial character of the parts or from diffieulty in holding the edges together. In very fat subjects perineorrhaphy, especially when the rupture extends through the sphincter ani muscle, may fail even after the most skilful operation. Certain systemic diseases, among them diabetes, are unfavorable for union. Generally the conditions of success are within the control of the operator. He should put the parts to be united in such a condition that non-union would be contraiy to nature. These conditions are simple, but absolute; and the operator who has neglected them can neither fairly attribute his failure to the debilitated state of the patient, nor to chance, nor to accident. Indeed, union almost invariably follows if the surfaces to be united are prepared properly and kept in contact for a week. The first condition, asepsis, has been discussed. The others will be presented in the following paragraphs.

Denudation.-The patient having been etherized, placed in position, and the field of operation exposed, the surfaces to be united should be denuded. Correct denudiation is a prerequisite to healing by first intention. Surfaces to be united should be so denuded that when brought together they will fit accurately, otherwise a part of the denuded surface, being in contact with an undenuded surface, must heal by granulation and suppuration, which may irritate the rest of the wound excessively, and always produces cicatricial tissue, which is very objectionable. The denuded surface should, moreover, be smooth and free from shreds, which might die and become sources of septic infection. Every particle of membrane or skin within the area of denudation should scrupulonsly be removed. If the surface be perfectly healthy, the more superficial the denudation the better; but diseased and cicatricial tissues do not unite readily, and should therefore, when practicable, be removed. 
Figure 43 shows the action of the tenaculum and scissors in denuding. The superiority of the tenaculum as a substitute for the tissue-foreeps must become apparent to any one who will familiarize himself with its use.

Needles.-A round needle is preferable to one with a cutting edge. The incised wound made by the latter is generally too large for the suture, bleeds freely, is prone to suppurate, and requires nore time for healing. The punctured wound made by the former readily shrinks down upon the suture, is less liable to bleed or to suppurate, and, after removal of the suture, heals more quickly. The tissue, especially in the cervix uteri, is, however, often so dense as to neeessitate the use of a needle with a cutting edge.

Many of the most dexterous operators prefer the straight needle to the curved. The straight needle has two advantages: first, however deeply it may be buried in the tissues, the position of its point can always be determined from its direction and length; second, the foree necessary to introduce it being in the direction of the needle, it is nuch less than that required to introduce a ciurved needle, for that force must be applied on the tangent to the curve; hence, the thickness of the eurved needle must be greater in order to avoid breaking. The straight needle, in a word, requires less force for its introduetion, is less liable to break, and makes a smaller wound. The plain round point, however sharp, sometimes encounters great resistance in passing through dense tissues. 'The trocar point or the saddle point represented in Figure 43 is less objectionable than the cutting edge, and may be introduced almost as easily.

Varions needles with handles attached or detached, and of different curves and shapes, have been devised, some with eyes at their points, some without eyes, and others of eylindrical form, through which the suture is passed lengthwise from one end to the other. They complicate rather than simplify an operation, and are in no respect superior to the simple needle and thread.

How to Handle a Needle-forceps. - The simple rotation of the needle-forceps on its long axis by a turn of the wrist enables the operator to sweep the straight needle around a eurve in the vertical plane, or it may be carried around a curve in the horizontal plane by loosening and tightening the foreeps grasp upon the needle at very short intervals, so that the angle between the foreeps and the needle may change almost constantly during the passage of the needle. In this way the straight needle may be made to carry a suture around a curve more accurately than the curved needle, and often more easily. Obviously, the lock forceps, which do not permit of this freedon of motion, are unsuited to such manipulations. Figure 43 represents Emmet's needle-forceps withont lock.

The Application of Sutures.-The most practical materials for sutures are silkworm-gut and catgut. The peculiar advantages of each will be presented in the description of speeial operations. Before the introduction of the sutures approximate the denuded surfaces with tenacula to determine whether they are of such size and shape that the union will produce the desired result, and whether accurate 
coaptation of the margins can be seeured without undue traetion, which might cause the suture to ent out; then hook up the margin of the wound with a tenaculum, introduce the needle, and apply counterpressure, Figure 43, C, B, until the needle-point ean be seized and drawn through with the foreeps. Some operators use the blunt hook for counterpressure; but a strong tenaculum which will neither hreak nor bend is preferable, especially in dense nterine tissue, beeanse it may also be fixed in the tissues at the very point where the operator desires to force the needle through, and it thereby insures greater precision in directing the needle to the point of exit.

Figure 44.

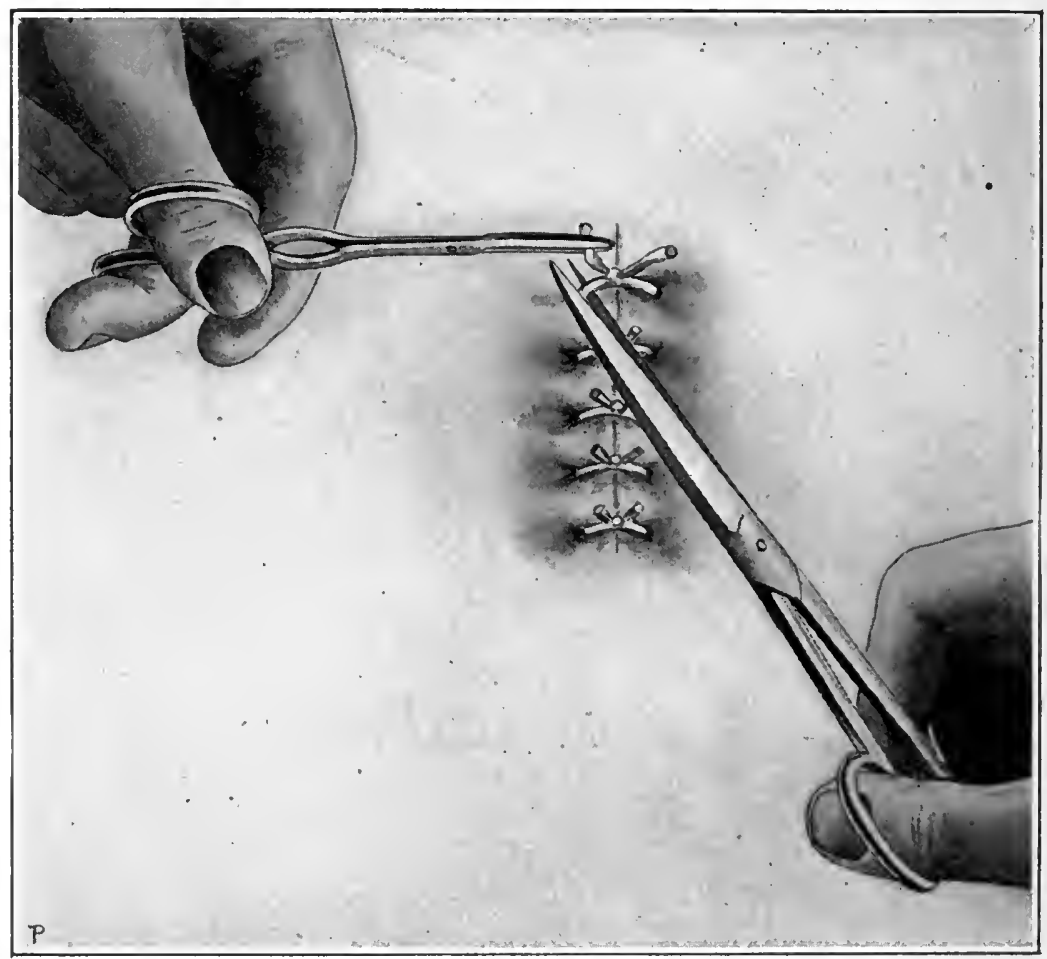

Removing a suture.

In making counterpressure the tenaculum may slip and the uterus receive a violent and sudden jerk, which is not without danger, especially when often repeated; this may be avoided and the operation facilitated by holding the flap in the vulsellum forceps while the needle is being forced through between its teeth.

Sutures slionld be about one-fourth of an inch apart, should include considerable tissue, and, if practicable, should pass entirely muder, not through, the denuded surface, so as not to be in contact with any portion of the womd. When at a listance from the denuded surface they are less liable to irritate and give rise to swelling and infeetion. 
The sutures should be tied with the greatest care, and should be drawn just tightly enough to hold the denuded surfaces in contact. If drawn too tightly, the tissue will become strangulated and swollen, the sutures will eut. out, and the operation may fail.

Before tying a suture the bleeding should be stopped; otherwise small quantities of blood may aceumulate in the track of the wound and serve as a mechanical bar to mion. A constant stream of hot, sterilized water playing on the wound during the tying of the sutures is desirable.

The After-treatment will be presented under the speeial subjects. Generally speaking, the field of operation is to be kept clean and inimobile.

Removal of Sutures.-Ordinarily the sutures shonld be removed at the end of a period varying from ten to fifteen days; if suppuration occur, earlier. Sutures about the vulva and perineum should be removed in about ten days. If left much longer, they may become loose or cause suppuration. In the vaginal walls they may be left several days longer. In the eervix, where suppuration seldom oecurs, they should be removed in about two weeks, unless perineorrhaphy has been done at the same time, in which case remoral eannot safely be undertaken in less than three or four weeks. To remove a suture, seize the free end with a foreeps, and with the seissors cut the nearest side of the loop. See Figure 44. Cutting the nearest side tends to hold the edges of the freshly united wound together during the withdrawal of the suture; if the loop were cut on the farther side, removal would tend to reopen the wound. It is well to seize with the forceps only one of the free ends, for the other will then be available in case this one is cut off accidentally. Always make sufficient traction to bring the loop in sight before eutting, otherwise both sides may be cut off below the knot and the loop left. If then the ends of the loop retract, as they usually do, the loop may remain indefinitely, keep up constant suppuration, and finally have to be removed under anæsthesia by incision.

Assistants.-Four assistants usually arc required for at gynecological operation-one to give the ether, one at the operator's left, to hold the speculum, and one at the operator's right, to sponge, and one to thread needles and render other assistance. If the operation be on the perineum or vulva, and the patient be in the dorsal decubitus, the thighs must be flexed and held in the lithotomy position by the two assistants on the right and left. The assistants in charge of the ether and sponging should be physicians. The holding of the speculum and threading of needles are better done by nurses.

\section{DILATATION OF THE UTERUS.}

The cavity of the uterus may be made surgically aceessible to the examining finger or to instrumentation by dilatation. The indications for dilatation may be diagnostic or therapeutic, or both. Among these indications are stenosis or stricture of the canal, uterine hemor- 
rhage due to endometritis, neoplasms, abortions, and pathologieal anteflexions. The means and methods are these:

1. Ineision.

2. Tents.

3. Graduated bougies or sounds.

4. Instruments with diverging blades.

1. Incision of any portion of the uterine eanal may be required in order to render the endometrim aceessible for instrumental or manual interferenee. But incision is especially applicable to the lower part of the cervical canal and to the extermal os, and is performed for congenital or acquired stenosis. Its object is to insure the free outflow, not only of menstrual fluid, but also of the uterine mueus,

Figure 45.

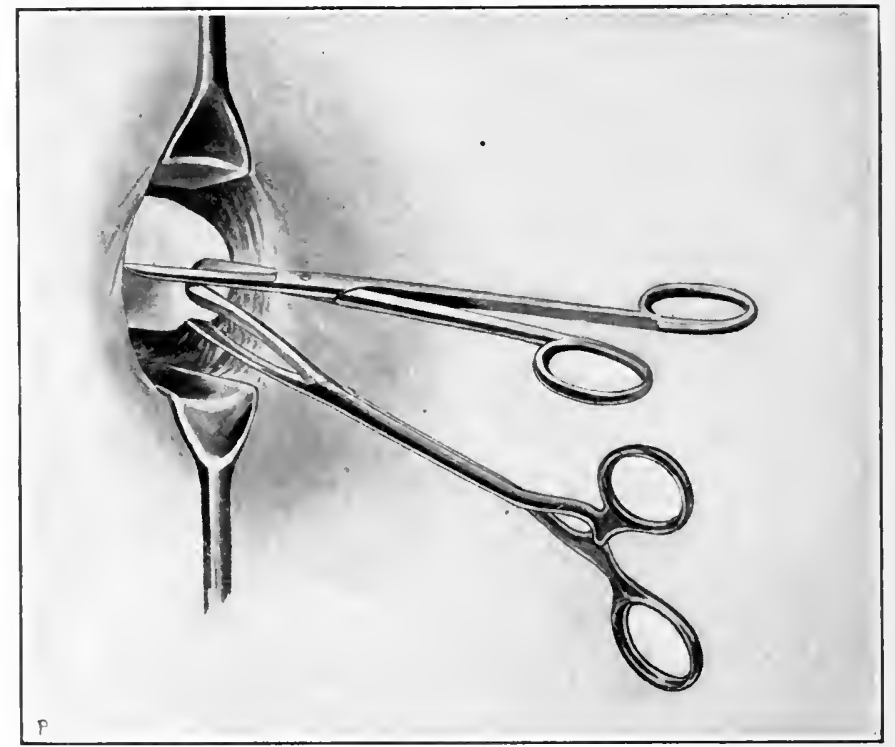

Incision of the cervix uteri with straight scissors; the cervix is steadied by a vulsella forceps.

whieh, if retained, beeomes offensive, irritates the uterine mueosa, and causes hypersecretion. Oftentimes the uterine secretions are so impeded in their passage through the strictured os internum or externum that they accnmulate, distend the uterine eavity, and are thrown off at irregular intervals with expulsive pains simulating labor-pains. This explains certain cases in which there is a recurrence in the intermenstrual period of all the painful phenomena of obstruetive dysmenorrhœa.

Schroeder's Method.-Sehroeder, in certain eases, especially of intrauterine polypi, incises the cervix bilaterally, seizes the posterior lip with a vulsellum forceps, and, with his finger as a dilator, works his way to the nterine cavity. The uterus, dilated in this way and well drawn down, is very accessible. Since the lateral incisions extend into a 
neighborhood that is very liable to infection, the safety of the operation must depend upon thorongh asepsis. In a rigid uterus, moreover, it is often impracticable to incise and dilate according to the methor of Sehroeder.

The Author's Method of rendering the entire uterine eavity and the uterine walls accessible for surgical operations, sucl as the

Figure 46.

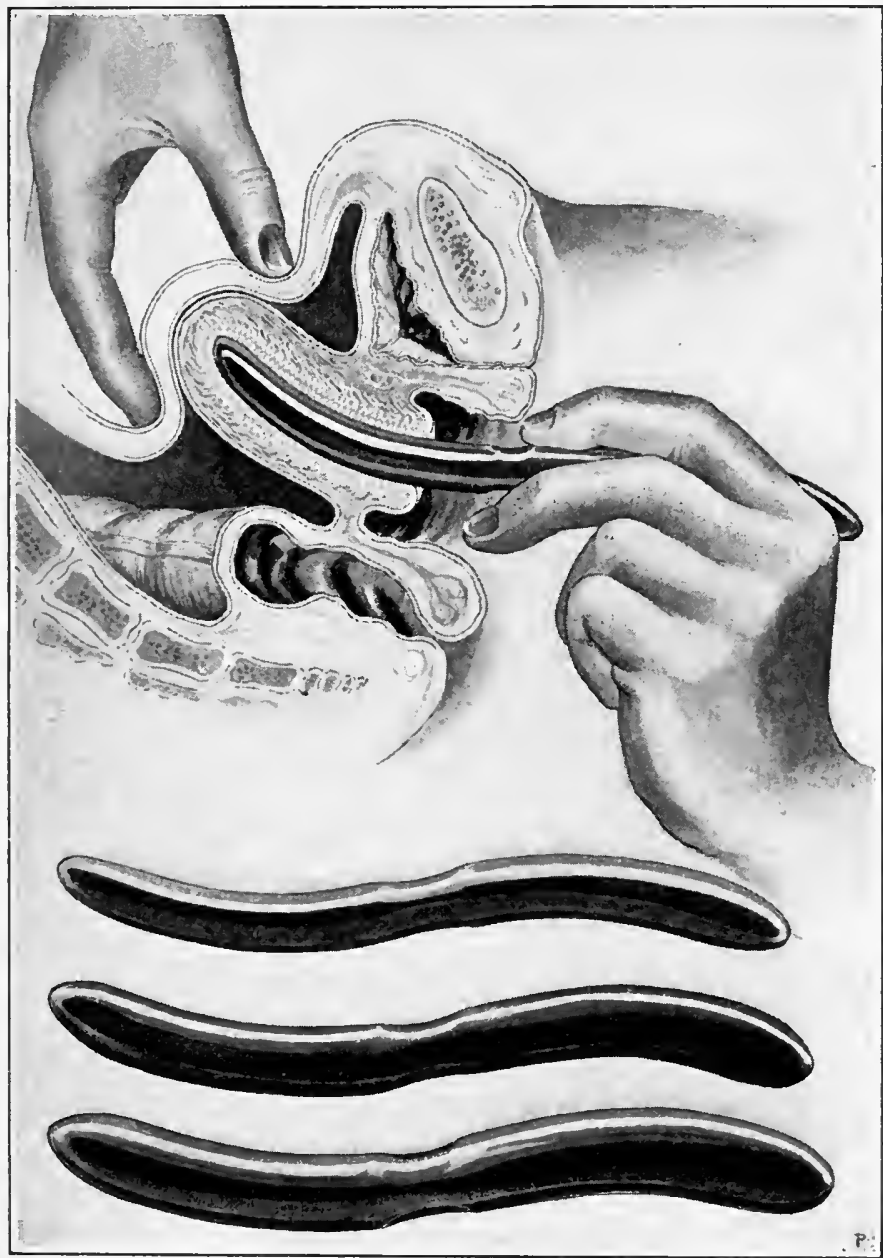

Dilatation by graduated bougies. Six gradually increasing sizes shown in the three instruments at the lower part of the illustration. One end of eaeh dilator is slightly larger than the other.

removal of myomata through the vagina by free median ineision of the anterior nterine wall, is illustrated and described under the Surgical Treatment of Myomata, Chapter XVII.

2. Tents.-Sponge, tupelo, and sea-tangle are the materials of 
which tents commonly are made; if they are introduced into the uterus in the dry, compressed state, the mueous secretion, stimulated by their presence, causes them to swell laterally to the extent of two or three diameters, and, correspondingly, to dilate the canal.

The danger of sepsis after continuous dilatation by introducing one tent after another is very great. Alarming results often have followed the use of the second or the third tent, seldom the first. A tent should not under any circumstances be allowed to remain in the uterus more than twelve hours. The tents furnished by instrument-makers are usually not aseptic. Before using them, therefore, it is always well to subject them to the dry-heat process of Boeckmann, as described in Clapter II. for the disinfection of eatgut. After the removal of a tent the endometrium should be washed out with sterile water, and disinfected with a topical application of a strong solution of iodine in 95 per cent. carbolic acid; this applieation shonld be made by means of an applicator wound with cotton. The danger of infection from tents is so great that the use of them generally is disapproved.

3. Graduated Bougies.-CThe nterus may be dilated by means of graduated bougies. Figure 46 shows this method of dilatation; it is adapted particularly to eases in which the abdominal walls are thin and lax, so that the uterus may easily be fixed by the hand over the abdomen, while one sound after another is forced into the canal until the required dilatation is aceomplished. If the abdominal walls are thick and tense, it is necessary to use Sims' or Simon's speenlum, and during dilatation to fix the cervix with the vulsella forceps, but in such eases the forceps are apt to tear out, and therefore the diverging instruments are preferable.

4. Diverging Instruments.-Innumerable instruments have been devised with blades which diverge and dilate the nterus when the handles are pressed or screwed together. See Figure 47.

Wathen's dilator, Figure $47, \mathrm{~B}$, is the most serviceable. These dilators are generally too heavy to be inserted until the way has been opened by a lighter instrument, like Palmer's, Figure 47, A, or by the smaller graduated sounds. It is important that all instruments for powerful dilatation be supplied with the thumb-screw for screwing the handles together. If the handles are compressed with the hand, rupture of the uterus is apt to oceur. The smaller dilator of Palmer does not require the screw.

Extent of Dilatation. - Goodell was foremost among the adrocates of forcible dilatation. In a large experience with extreme dilatation under ether he had no fatal result and no serious inflammatory disturbance. He carried the dilatation to three-fourths of an inch in the thin-walled, unyielding infantile uterus, and to one and one-quarter inches in ordinary eases. In ease of a rigid, mnyielding, or thin-walled uterus, which might tear from rapid expansion of the dilating blades, it is permissible with rigid aseptic eare to begin dilatation with a sponge- or tupelo-tent, the softening influence of which prepares the canal for more easy and thorongh dilatation by the foreible method.

The Dangers of Forcible Dilatation are from traumatism and sepsis. There may be extensive rupture from overdistention by rapid dilata- 
tion of a rigid uterus, and dangerous hemorrhage, peritonitis, and death may result. A uterus ruptured by dilatation should be packed and drained by aseptie ganze. An abdominal or vaginal section may be necessary to control hemorrhage.

It would be a mistake to suppose that antisepsis deprives dilatation by any method of all its perils. All manipulations of this class are

FIGURE 47.

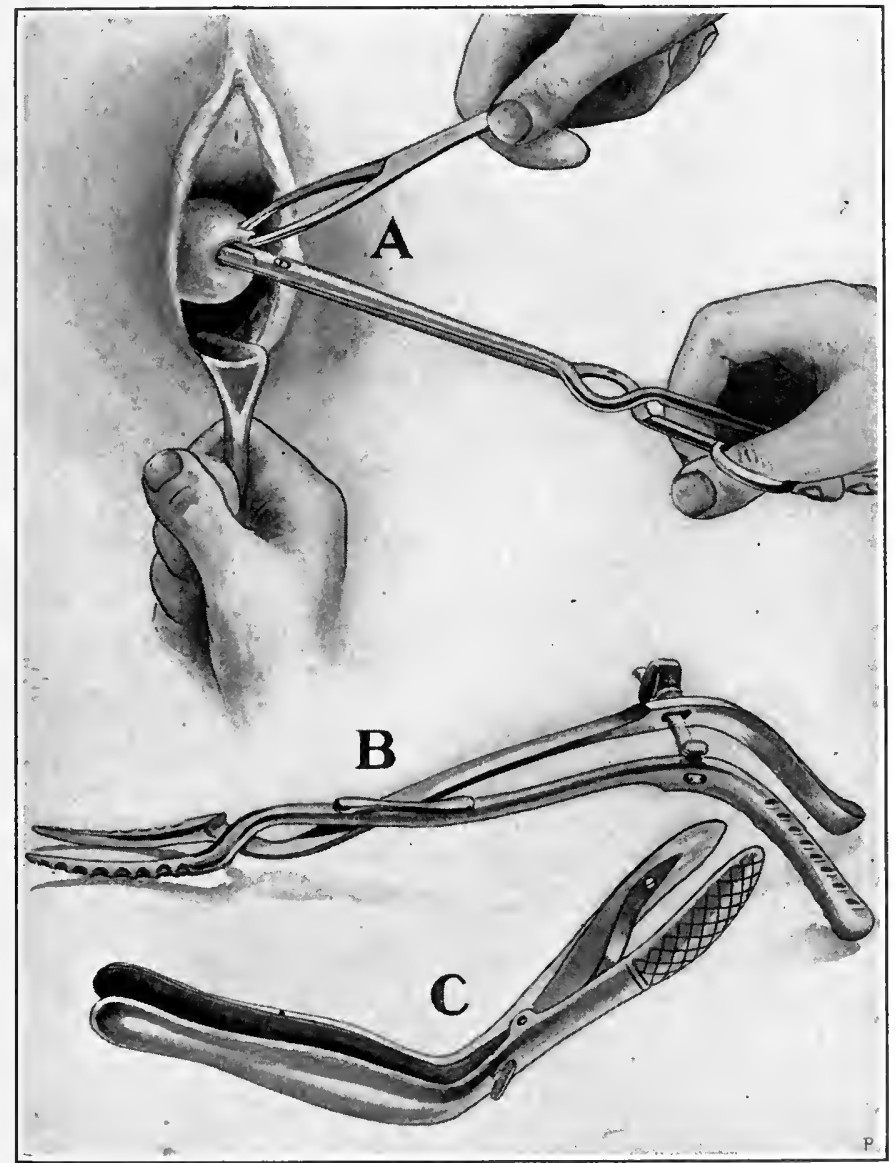

Forcible dilatation of the uterus: A, Palmer's dilator in use: B, Wathen's dilator, to be used for eontinuing the operation: C, anal dilator sometimes useful for extreme dilatation, especially in cases of abortion. About onc-third natural size.

dangerons, and not to be employed unless the indieation is quite clear. Existing pelvic inflammation, acute or chronic, is a scrious contraindication. Indeed, the history of a majority of fatal cases includes previons cellulitis, peritonitis, or metritis. Dilatation, however slight, by any method, should be regarded as a surgieal operation, should always be done at the patient's house or a hospital, never at the office, and should be followed by rest in bed for a time varying from one to 
seven days. Foreible dilatation, either by sounds or by diverging instruments, except when the dilatation is to be slight, requires an amresthetic. Tenderness and other signs of inflammation about the uterus contraindicate the operation.

If, in performing forcible dilatation of a rigid cervix, the force required suddenly becomes less, the operator should cease dilating at once, for the lessened resistance usually indicates beginning rupture.

\section{Special Advantages of Each Method of Dilatation.}

Incision.-Contraction of the os externum and lower portion of the uterine canal is treated best by Schroeder's operation of bilateral incision of the cervix. See Treatment of Cervical Endometritis.

Tents.-Sponge-tents are the most dangerous, tupelo the least. Laminaria has but one adrantage over tupelo-flexibility and adaptability to a tortuous canal. In a case of rigid hyperplastic or thin-

FIgURE 48.

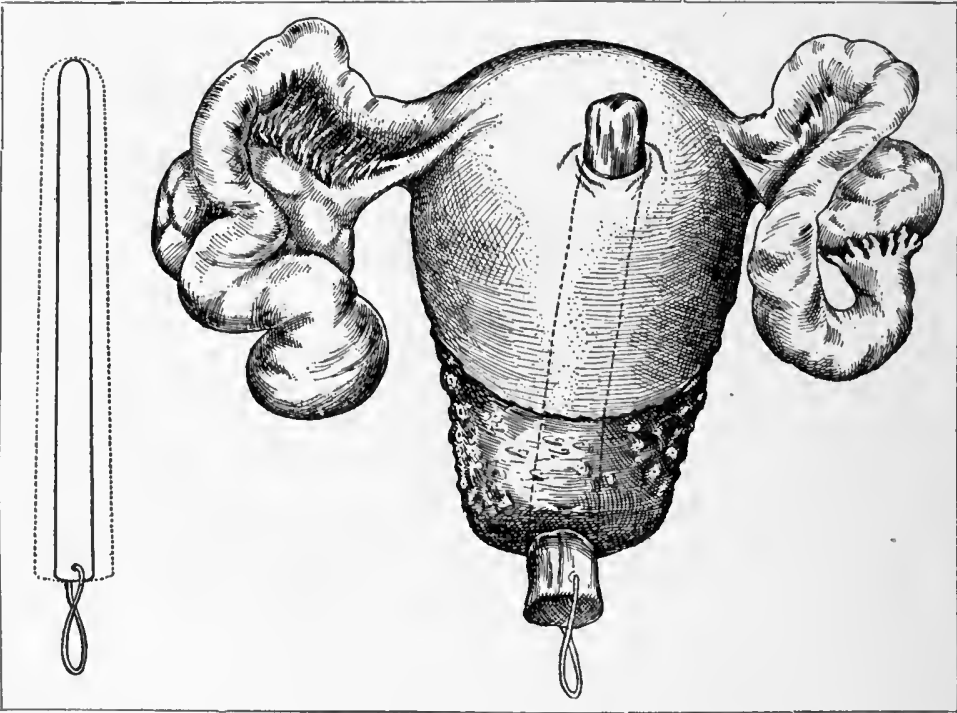

Uterus perforated by a tupelo-tent. Figure to left shows size of tent before and after expansion.

walled cervix not safely dilatable by rapid means, the tent is sometimes permissible as a means of preparation for rapid dilatation by graduated sounds or diverging instruments. The use of it, however, even in careful hands, many times has cansed futal pelvic infection.

Graduated Sounds and Diverging Dilators are generally the safest and most effective means of dilatation, and usually should have the preference.

One may combine the principle of graduated sounds in the use of diverging dilators. This requires a series of dilators of graduated sizes. The small instrument is inserted first, and the blades spread; - then the dilator next larger is used in the same manner; and so on 
through the series. Before spreading the blades each instrument acts as a graduated sound; as the blades diverge they act on the principle of the glove-stretcher. At least four dilators are required : two of the Palmer, and two of the Wathen variety.

A small light dilator as a means of complete dilatation has two disadvantages : first, the light blades may bend and fail to stretch the canal beyond a limited degree; second, if they do not spring or bend, they are apt to imbed themselves-that is, crush their way into the uterine walls. The result is not dilatation by stretching, but by tearing. The wound thus inflicted may be dangerons. This unfortunate result may be avoided by the use of a graduated series of instruments.

Technique of Forcible Dilatation.-1. Disinfect the vagina and vulva. 2. Expose the cervix by a Sims or a Simon speculum. 3.

FiriUre 49.

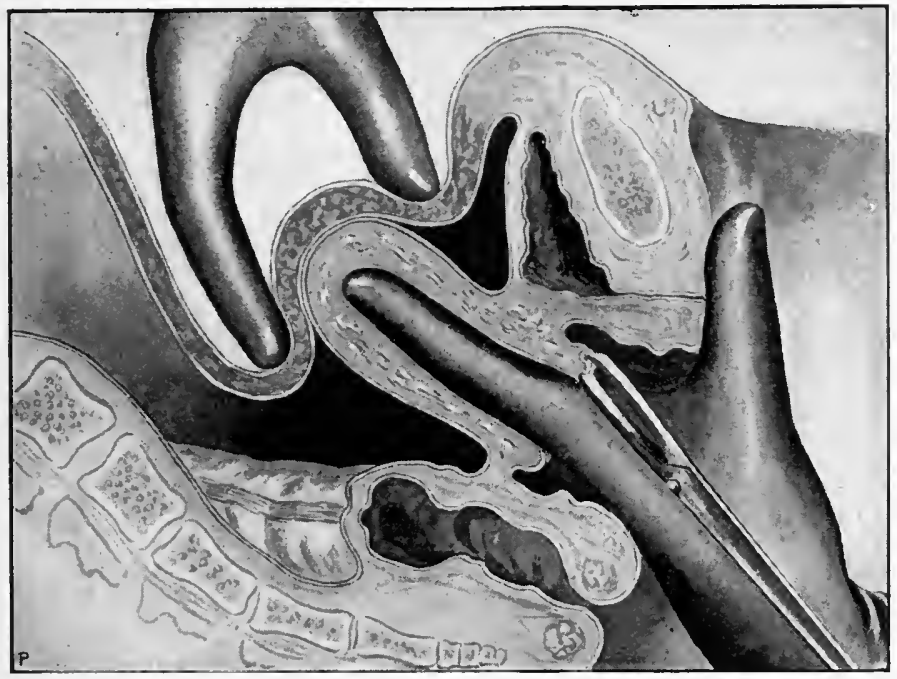

Examination of the dilated uterus by conjoined digital toueh.

Grasp the cervix firmly in the teeth of a vulsellum forceps. Figure 47. 4. Introduce the snceessive dilators and slowly screw the blades apart. 5. Wash ont the uterine cavity with sterilized water from a fountain-syringe through a rubber tube and canula. The ordinary glass female eatheter is a good canula. The dilatation should be sufficient to give a free return flow by the side of a single canula. During the washing-out it is well to remove and reintroduce the canula every two or three seconds alternately to prevent injection of a possibly patulous Fallopian tube. See Treatment of Endometritis in Chapter XVII.

\section{CURETTAGE.}

The diagnostic significance of the curette has been given in Chapter III. The therapentic purpose is the removal of diseased tissue or 
foreign bodies from the interior of the nterus. The symptomatic indications are usually hemorrhage, uterine discharges, or infection due to some intra-uterine cause. The instrument first was used in 1843 by Récamier; it has passed through numerous modifications, and on account of the disastrous results that have followed the use of

Figure 50.

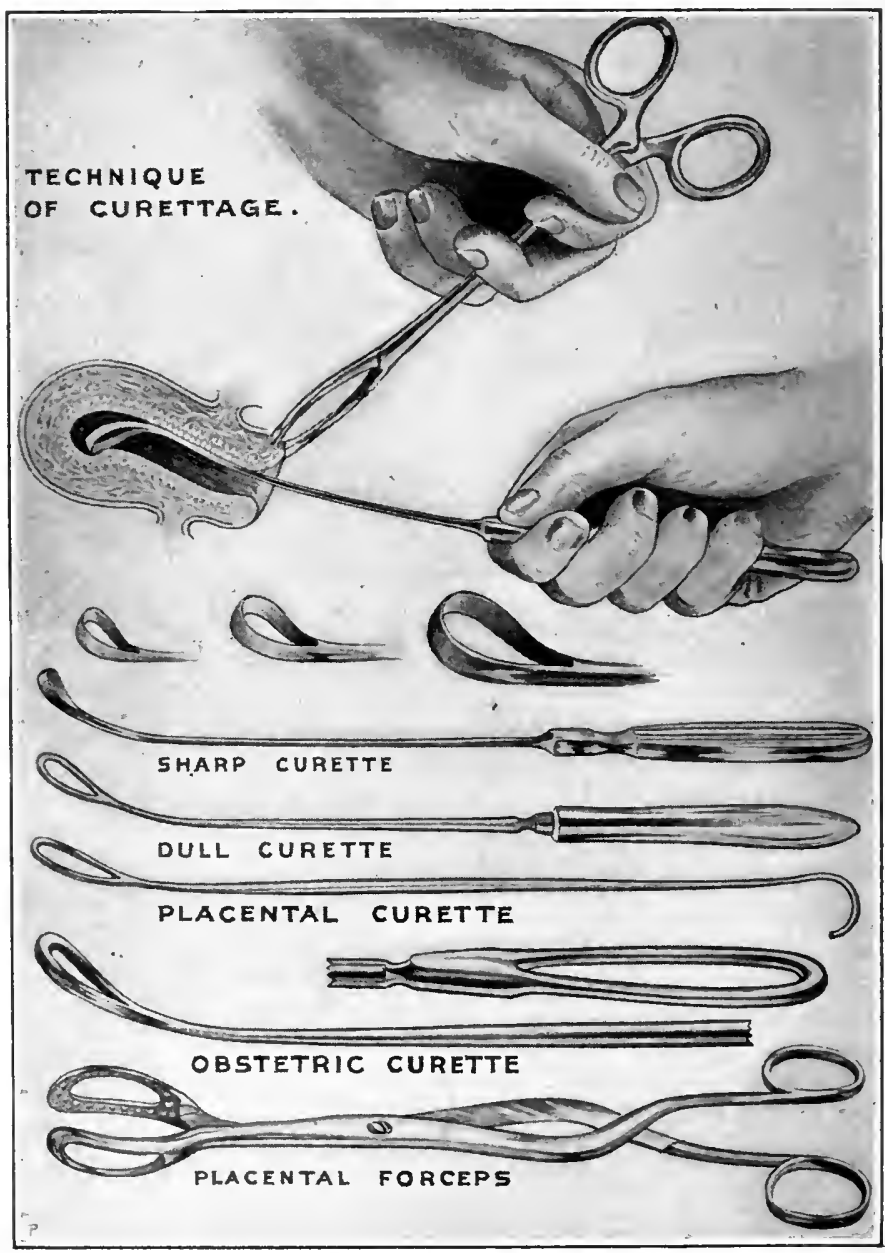

Technique of curettage; varieties of curettes; placental forceps. One-third natural size.

it-perforation of the uterus, metritis, salpingitis, cellnlitis, peritonitis-it has received at times the severest censure, not wholly undeserved.

The dull eurette, shown in Figure 50, is made of flexible copper wire. The loop has slightly flattened but not cutting edges; the malleable shank may be bent like a probe to conform to the direction of the uterine canal. Whatever the force applied, it is not likely to 
injure the sound tissue, although it will remove loose foreign bodies, such as the secundines of abortion.

The sharp curette, shown in Figure 50, is designed to remove such diseased tissues as are connected more intimately with the uterns; for example, an infected endometrium or a malignant growth. The loop is of steel, and has a sharp cutting-edge. The shank is of flexible copper, and may be bent to conform to the direction of the uterine canal.

The following is a summary of the indications for the use of the curette :

I. For diagnosis of-

a. New growths of the uterus-fibroids, carcinoma, sarcoma, deciduoma malignum.

b. Inflammatory products-endometritis.

c. Retained products of conception-placenta, fœetus, hydatid mole, fleshy mole.

II. For therapentic purposes in cases of-

a. Endometritis.

b. Mucous polypi.

c. Inoperable malignant growths.

d. Hemorrhage in inoperable fibroids.

$e$. Foreign bodies, such as secundines of abortion.

The dangers of the curette are in causing:

1. Septic infection.

2. Perforation of the uterus.

3. Hemorrhage in eases of malignancy.

4. Hemorrhage and abortion in eases of unsuspeeted pregnancy.

5. Permanent destruction of the endometrium by scraping too much.

Technique of Curettage. - The steps of curettage are these (see Chapter XVII.):

1. Dilate through a speculum sufficiently for the easy admission of the curette. Figure 47.

2. Steady the cervix with the vulsellum forceps and introduce the curette. Figure 50.

3. Should the object be to remove some foreign body, the dull curette will accomplish this readily if used like a rake. Little force is required. The sensation imparted to the fingers will show whether all the foreign substance has been removed-i. e., whether the loop glides over a smooth surface.

4. If the object be to remove diseased tissue, the sharp eurette should be nsed with a back-and-forth scraping motion round and round the endometrinm. The operator will know when the tissue has been removed sufficiently: first, by the fact that no more comes away; second, by the sensation which the curette imparts to the fingers, of a hard, resisting, more or less healthy, intra-uterine surface.

5. The diseased tissue having been scraped away, the endometrium should be washed out with sterilized water.

6. If it is desirable to apply a medicinal substance, such, for example, as a saturated solution of iodine crystals in pure carbolic 
acid, this may be done by means of an applicator or a fine dressingforceps wound with absorbent cotton. Before making the application, pack absorbent cotton under the eervix, to absorb any fluid which otherwise might run ont and irritate the vagina.

7. The after-treatment is rest in bed for a week, with vaginal donehes twice daily of some disinfectant such as 0.5 per cent. solution of lysol in sterilized water.

\section{THE STAFFORDSHIRE KNOT.}

It is sometimes necessary in minor and major operations to apply a ligature en masse. In many cases this ligature may to great advan-

Figure 51.

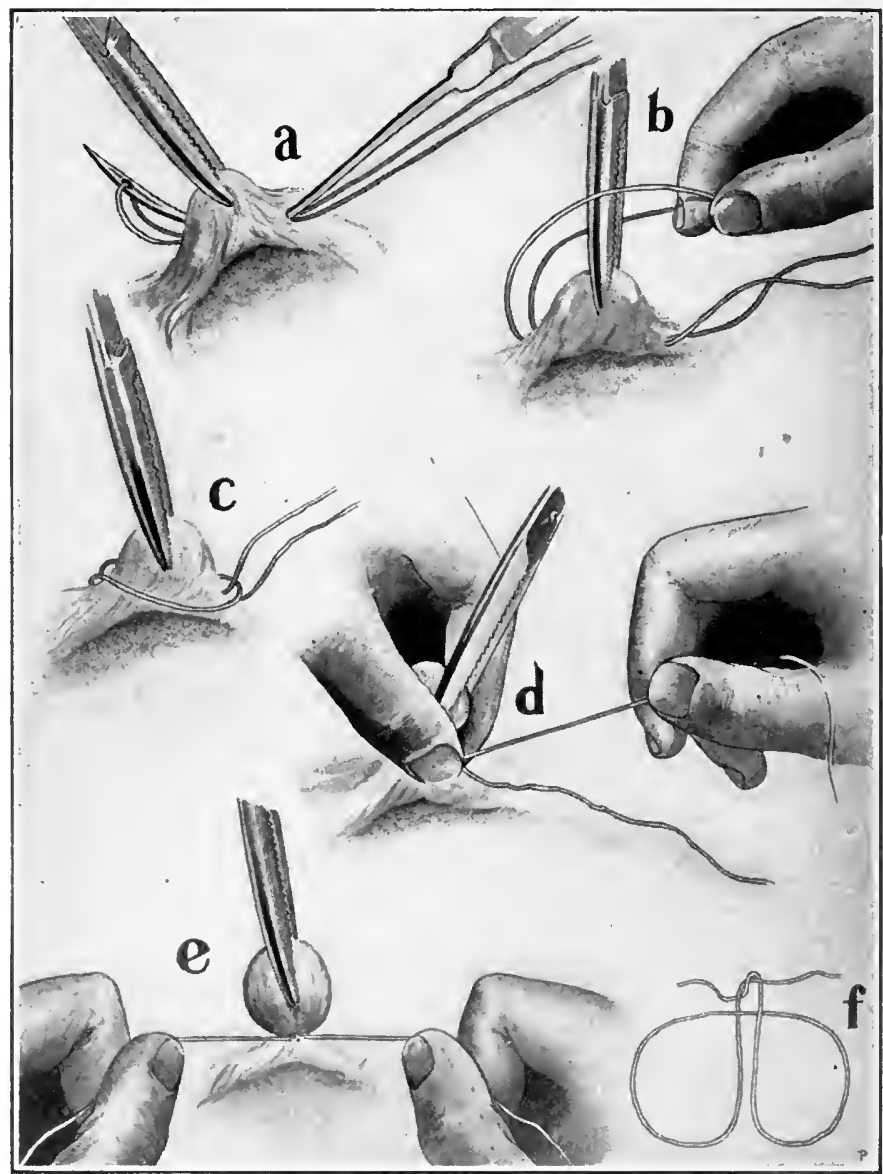

Technique of the Staffordshire knot. One-third natural size.

tage take the form of the Staffordshire knot. This knot will be found especially applicable to the ligating of hemorrhoids. The applica- 
tion of it, Figure 51, is as follows: The part to be ligated is transfixed with the needle and the needle withdrawn so as to leave the loop of the thread on the farther side of the stump $a$. The loop then is drawn over the mass to be ligated and one of the free ends drawn through, so that one free end is under and the other over the retracted loop, $b$ and $c$; both free ends being seized by the right hand are drawn tightly through the mass while the thumb and forefinger of the left hand grasp the ligature where the free ends cross the loop and make firm counterpressure against the mass until complete constriction is secured, $d$. Finally the ligature is tied securely, $e$. It then may be passed around the pedicle and tied again. The advantages of the knot are : 1 , it ties the pedicle in two halves; 2 , these halves are uniformly and strongly compressed into one mass. It is quite essential to draw the ligature very tight and to retain the constriction thereby secured until the knot is tied. 


\section{CHAP'TER VI.}

\section{MAJOR OPERATIONS.}

THIs chapter is a general consideration of those procedures which are common to the opening of the peritoneal cavity. Peritoneal section may be made through the abdominal walls or through the vagina; hence the subject is divided into

1. Abdominal section.

2. Vaginal section.

\section{ABDOMINAL SECTION.}

Operating-tables. - The table already described for examination and for vaginal operations will suffice for abdominal section, if lengthened so that the patient may lie upon it at full length. For this purpose a short table may be supplanted by a stand or by another shorter table.

Figure 52.

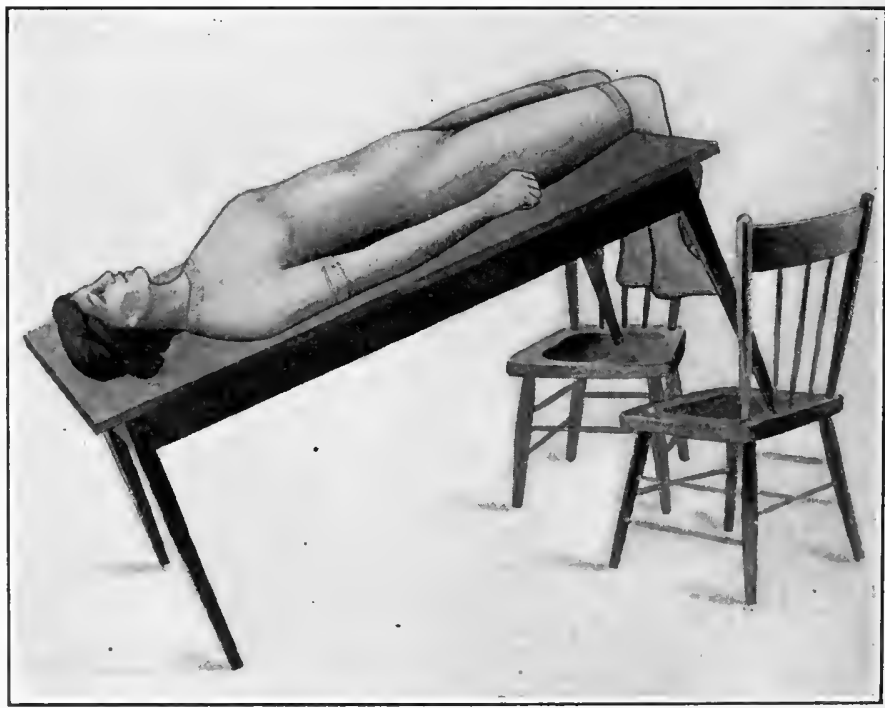

Trendelenburg position: table improvised.

The Trendelenburg Position.-A favorite table for hospital use, and especially for abdominal section, is that of 'Trendelenburg or some modification thereof. The top of the table may, at any time during an operation. be adjusted readily to any desired angle, and by this 
means the hips may be elevated so as to cause the intestine to gravitate away from the pelvis toward the diaphragm. The surgeon may then gain, in favorable cases, an almost unobstructed view of the pelvic basin and may work deep in the pelvic cavity unimpeded by the distended intestine. It is even maintained by advocates of this position that in these favorable cases the operation may be proceeded with as readily as if it were on the external surface. Extravagant claims are made that this position makes pelvic surgery easy, so that an indifferent operator may undertake it safely. The table is useful

Figure 53.

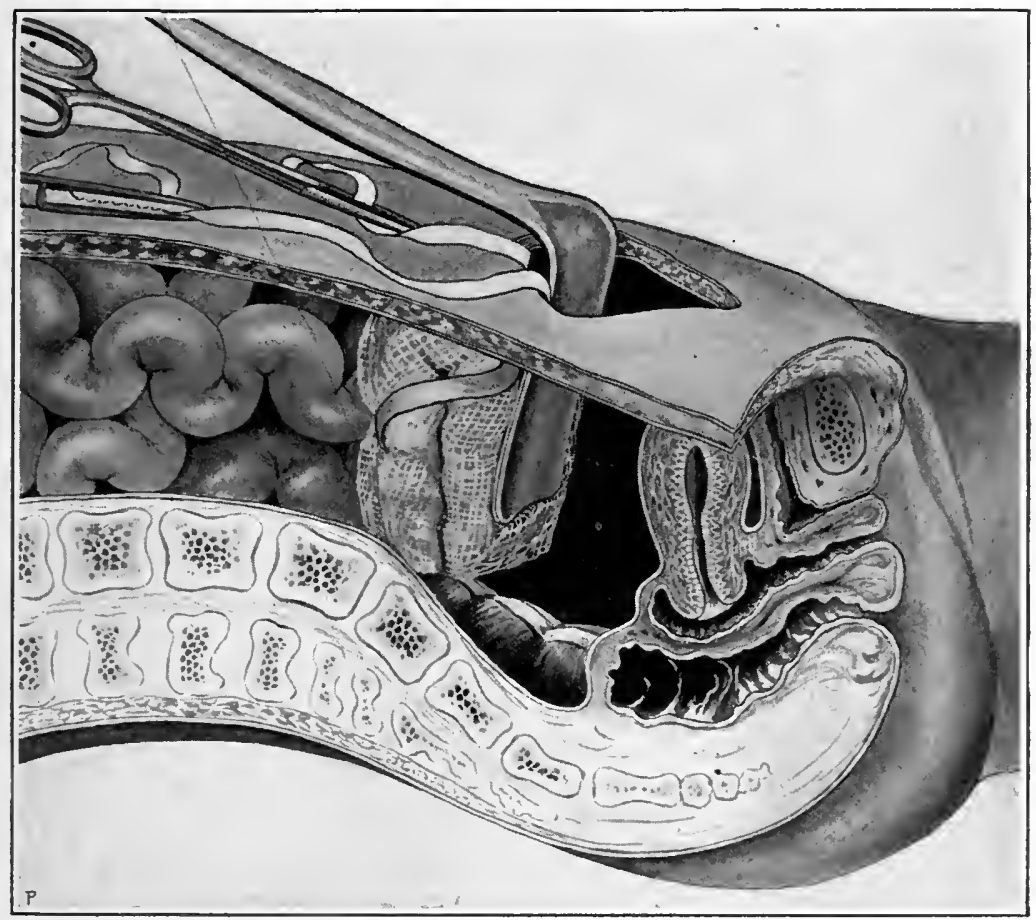

Ordinary gauze sponges held by a retractor to keep the intestines out of the operator's way.

during anresthesia when the pulse and respiration fail and it becomes desirable to elevate the lower extrenities and lower the head.

The advantages of this position, although admitted, should not be overestimated. Besides the fact that in many cases the field of operation is not rendered more accessible, the position has several disadvantages : first, infectious fluids which escape during the operation are certain to gravitate toward the diaphragm, and may infect the general peritoneum; second, the abdominal muscles often are made more rigid. The Trendelenburg position does not overeome, but rather lessens, a few-only a few-of the difficulties and dangers of abdominal surgery. With the patient on an ordinary table, large gauze pads may 
be used in such a way as to keep the intestine out of the way, and thereby to render accessible the deeper parts of the pelvis.

Improvised Substitute for the Trendelenburg Table.-The end of a common table may be raised on a block or chair so as to give it the required slant. The patient then, with the legs hanging over the foot of the table, may readily be adjusted to the desired angle without recourse to the more or less complicated Trendelenburg table.

The Preparatory Treatment.-The necessary antiseptic procedures to an aseptic result have been set forth in Chapter II. After the patient is on the table and under anæsthesia it is well to scrub the abdomen again with the sterilized soap and water, then wash with clean water, then with alcohol, and finally with a $1: 1000$ solution of bichloride of mercury. This is especially important in cases of acute pelvic suppuration, in which thorough scrubbing before anæsthesia is not tolerated. The patient's clothing should be of light flannelundervest, drawers, woollen stockings, and night-gown.

It is furthermore important, before beginning a grave operation, that the various organs of elimination be sufficiently active, so that the danger of auto-intoxication from the retention of waste-products may be reduced to the minimum. The demand made upon the patient by the operation itself reduces the eliminating capacity of these organs, sometimes to the point of danger; hence the imperative necessity of lightening the burden. Careful examination of the kidneys and heart may lead to essential preparatory treatment of these organs.

The Incision.- To open the abdomen only a few instruments are required; in fact, it may be laid down as a general proposition that the most skilful surgeons operate with the fewest instruments: A scalpel, a few strong hæmostatic forceps, long and short, and a pair of strong straight-bladed scissors are quite sufficient. Twelve short and six long hæmostatic forceps will suffice for any operation. Sir Spencer Wells and others have reported cases in which, after the operation, hæmostatic forceps were found post mortem in the peritoneal cavity. In order to avoid this, one should operate always with the same number of forceps, or at least carefully count and record the number before the operation is begun, and before closure of the wound. Unless the operator is certain of his assistant, he will do well to count them himself. The incision for gynecological exploration or operation is usually in the median line near the pubes.

Exploration.-Every abdominal section should begin as an exploratory incision, which at first should be made-long enough only to admit the index finger for examination. If it is necessary to introduce the hand, the incision may be extended in either direction. The operator now decides whether he will close the wound after the simple diagnostic exploration or proceed to a complete operation. Mr. Tait, in urging the exploratory incision as the first step of an abdominal operation, once wisely said: "It is always easy to turn an exploratory incision into an operation, but often quite impossible to turn an incomplete operation into an exploratory incision."

The Median Incision through the Linea Alba does not expose nor wound the recti muscles. If, however, the linea alba has been dis- 
placed by a tumor or by other causes and is not readily found, one may properly ignore it, cut directly through the upper fascial sheath, separate the fibres of the muscle longitudinally, and then divide the structures beneath until the cavity is reached. When cutting down upon a tumor, one often reaches the linea alba with the first stroke of the scalpel, and the subperitoneal fat with the second. The fat is then separated by the finger and handle of the scalpel, and the peritoneal membrane exposed. Bleeding points usually are secured by pressure-forceps; ligatures seldom are required. The peritoneum is then superficially caught by two small pressure-forceps. The operator's left hand retains one, and that of the assistant the other. The peritoneum is usually so translucent that the viscera just beneath can be seen as it glides over them; it is now lifted from the viscera by the pressure-forceps, and by a single stroke of the scalpel divided

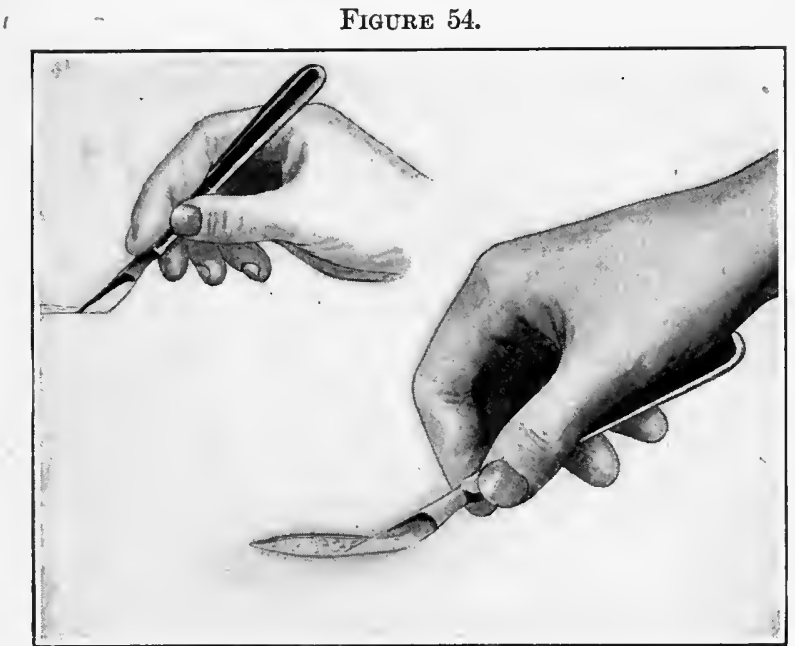

Abdominal incision: lower hand holding knife correctly; upper hand holding knife incorrectly.

between them. The grooved director formerly in use is rather a hindrance than a help. In grasping the peritoneum in the two forceps for incision, one should be careful not to include a bit of intestinal wall. The writer once in this way opened the intestine; immediate suture, however, resulted in prompt union, and no permanent harm was done. Sometimes the intestine is adherent to the parietal peritoneum and is very liable to be cut unless the incision is made slowly and with great care. Sometimes one may avoid cutting through the bladder-wall by recognizing in time its greater vascularity and the numerous little bleeding points. If the intestines or bladder are adherent and unrecognizable, this fact will be apparent by the failure of the operator to see the viscera through the translucent peritoneum, or by the fact that the peritoneum does not, as in an ordinary case, glide over them. It is then better to prolong the incision upward or downward and enter the abdomen above or below. 
The adherent viseera may then be detached and the ineision completed to its original point. Deliberation, eare, and judgment will enable the beginner usually to find his way safely to the abdominal eavity.

The cavity being open, the incision may be lengthened as desired by the scissors on the inserted index-finger as a guide. The length of the incision will vary with the requirements of the case and the dexterity of the operator; other things being equal, the shorter the incision the less the danger. Sufficient room, however, should be given for effective work. The added risk of a longer incision by comparison with the added safety of an unimpeded operation is insignificant. The pressure-foreeps may now be removed from the bleeding-points; if at any point the bleeding continues, it may be controlled by torsion or by fine catgut ligature.

Before invading the abdominal cavity for purposes of examination or operation, one shonld seize the margins of the peritoneum by two or three foreeps on either side, and draw it out through the wound toward its cutaneous edges so as to make it cover the cut surfaces. The wound thereby is protected and the peritoneum is in no danger of being stripped off from its adjacent tissues as it might otherwise be during the subsequent manipnlations.

Adhesions.-The conditions which give rise to adhesions usually also eanse more or less thickening of the peritoneum. Sometimes the parietal peritoneum is so thick as to be unrecognizable. The operator may be uneertain whether he has cut through the peritoneum, and this uncertainty may be increased by the presence of adherent intestine. Large areas of peritoneum have been detached from the adjacent abdominal wall under the impression that the peritoneum had been divided, and that intraperitoneal adhesions were being separated. Experience and sense are the only guides. There are no safe rules. Adhesions usually are separated by means of the finger, the hand, or the sponge. If great care is not used in separating intestinal adhesions, one or more coats of the bowel wall may be stripped off with the adherent tissues; this might result in sloughing and a consequent feeal fistula. Such traumatism should be repaired promptly by drawing together the peritoneal margins with fine chromic eatgut or silk sutures. The sponge, as used by the late Thomas Keith, is a most useful means of separating intestinal or omental adhesions. By firm and gentle sponge pressure against the adherent bowel at the point of attachment, one literally may sponge it away from the tumor. It is surprising to note the faeility with which rather firm adhesions may thus be broken. In breaking the adhesions in this way the surgeon avoids stripping off one or more coats of the bowel. On the contrary, the peritoncal covering of the tumor is apt to remain on the bowel. The sponge method is more gentle, more effective, and less productive of shoek than the usual method of tearing with the finger. Adhesions too strong for the sponge or finger have to be cut.

Intraperitoneal Hæmostasis.-Hemorrhage during an operation is treated on general surgical prineiples by forcipressure, ligature, torsion, sponge-pressure, or styptics. 
FIGURE 55.

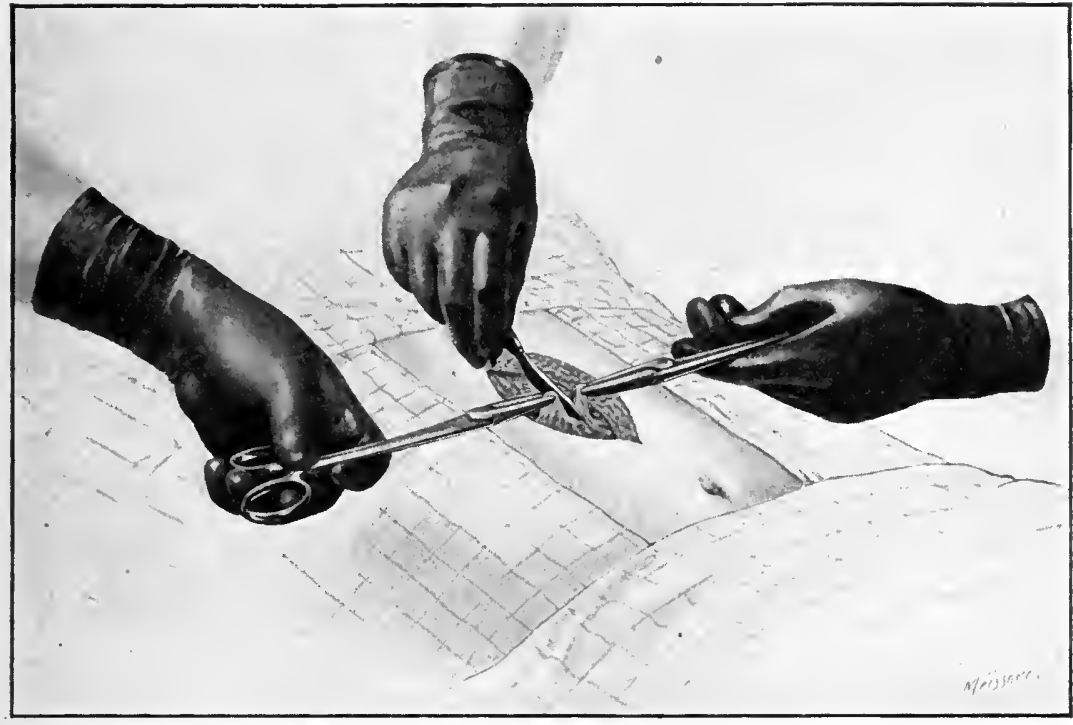

Abdominal incision, cutting through peritonetum. Peritoneum held up away from abdominal viscera by pressure-forceps. Forceps on left held by left hand of assistant; forceps on right held by left hand of operator.

Figure 56.

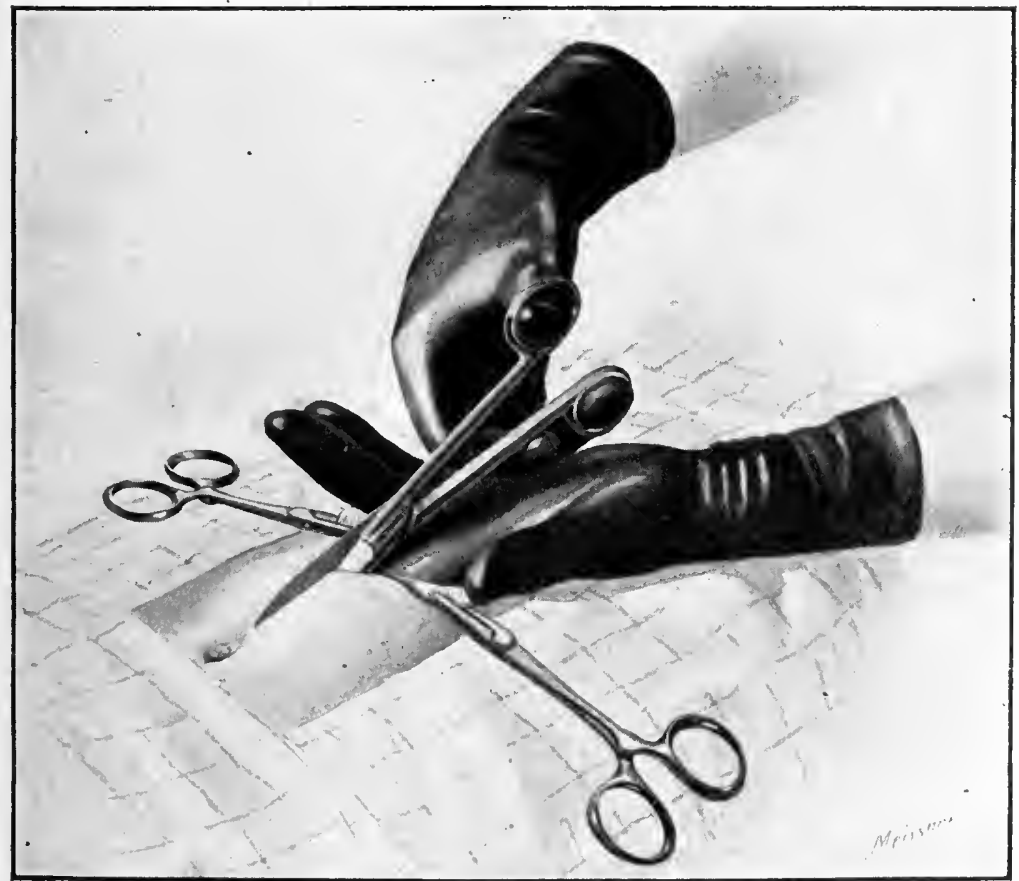

Enlarging abdominal incision. Forceps shown in Figure 55 are lying one on elther side of wound with everted margins of peritoneum in their grasp. 
Pressure-forceps placed on small bleeding points as the operation proceeds, and left there a few minutes, usually will suffice. If the hemorrhage continues, each point may be seeured by torsion or by a fine eatgut ligature; or several points, by a basting process, may be included in a ligature. Tronblesome oozing, deep in the pelyic wall, often subsides on long-continued sponge-pressure. The sponge should

Figure 57.

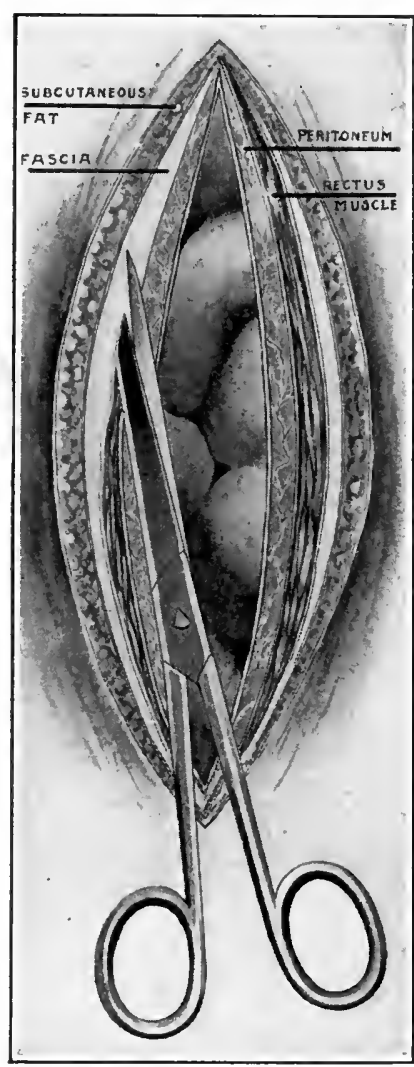

Fucial sheaths of rectus muscle on one side, being split by scissors. be wrung out in very hot water, and very firmly packed against the bleeding surface, and left there for severil minutes. Iron, tamin, and alum, since they are apt to leave masses of coagulated blood which mav decompose in the pelvis, are objectionable. A sterilized 10 per cent. solution of antipyrin applied with the sponge is a safe and often effective styptic.

Catgut versus Silk for Ligatures.Hremostasis is secured best by catgutsee Sterilization of Catgut, Chapter II. Catgut is preferable to silk because in case of localized infection around the ligature the non-absorbable silk remains as a foreign boly and is apt to perpetuate a suppurative process. 'This process, if the patient survives, may form a sinus leading from the ligature to the external surface, usually through some point in the abdominal wound. Such a sinus may continue to suppurate for weeks, months, or years, until the ligature is cast out or manually removed. Catgut sutures and ligatures disappear by absorption in a few days or weeks, and give no further trouble; if of good quality and properly disinfected, they are perfectly reliable and safe.

Closure of the Wound.-The ordinary method by through-and-through interrupted sutures, including the entire thickness of the abdominal wall, and tying upon the skin, should be abandoned, and the method of buried chromic catgut suture should be substituted.

The Buried Catgut Suture Throughout. ${ }^{1}-\mathrm{Number} 2$ catgut sufficiently chromicized to resist absorption for six weeks, should be used. The technique is as follows: In order to give broader surfaces for union, and consequently greater strength, the incision is made into the sheath of the rectus muscle on that side on which the muscle was not exposed by the abdominal incision. If, perchance, the abdominal

\footnotetext{
${ }^{1}$ Modified from George M. Edebohls. American Gynecological and Obstetrical Journal, May, 1896 , consulted.
} 
Figdre 58.

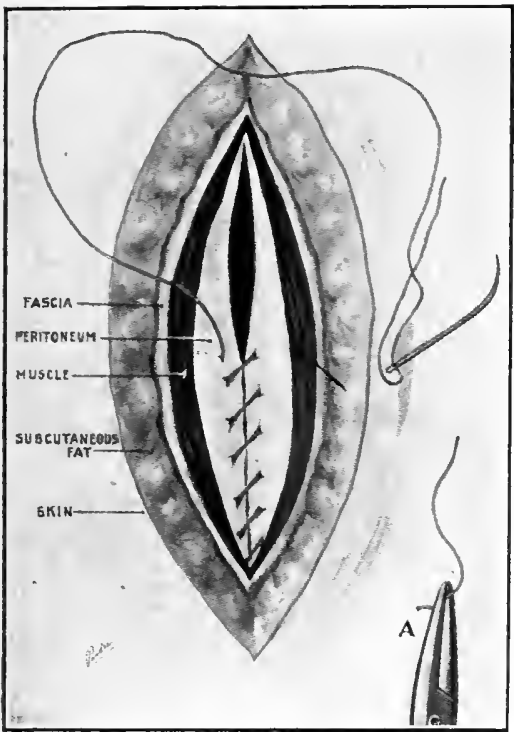

Figure 59.

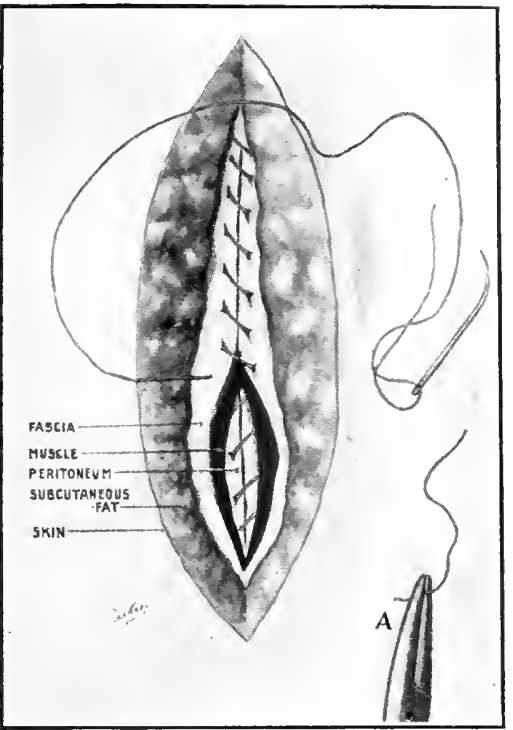

FIGURE 58.-Showing deep tier of buried running catgut suture, which unites the peritoneum. The suture may also embrace the posterior fascia and muscle. Observe that the suture was introduced at $\mathrm{A}$ and is there being held in place by neans of forceps; it first closes peritoneum from the lower to the upper end of the incision; it then returns to the lower end. closing the anterior fascia, as shown in Figures 59 and 60, and finally emerges and is tied at point of original ent rance-point A.

FIGURE 59.-Fascial margins of wound closed by second tier of buried running suture. The recti muscles are now being covered in by the united fascia.

Figure: 60 .

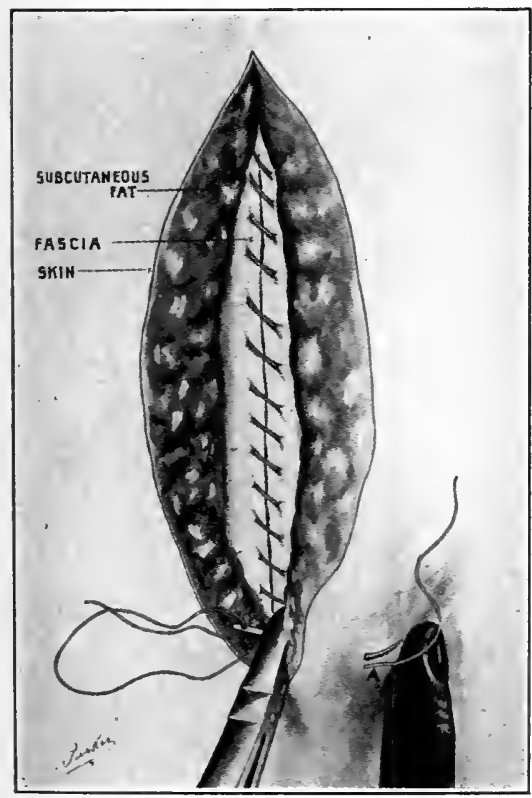

Figure: 61 .

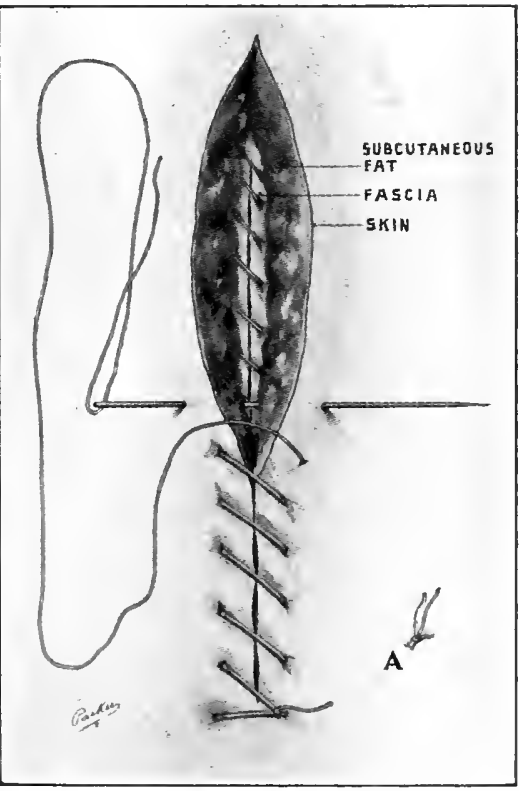

FIgURE 60 -Union of fascial margins complete; suture is being passed out by necdle to point of entrance A preparatory to tying. Suture is shown as tied at $\mathrm{A}$ in figure 61 .

FIGURE 61.-The running suture, which has closed peritoneum, recti muscles, and fascia in two tiers has been tied at point of entrance and exit A. A final suture first tied at lower end of wound is being deeply introduced to close subcutaneous fat and skin, observe that there are no buried knots to cause suppuration and that the deep, wide external suture closes the subcutaneous fat securely and prevents dead spaces. Moreover, its relations with the margins of the wound are so remote that it causes little irritation. Above all, it should not he tightly drawnjust tightly enough to hold the cut margins in apposition; this suture should be iodized gut. 
incision was made directly through the linea alba, without exposing a rectus muscle, the sheath is deliberately to be divided on either side with the seissors, as shown in Figure 57. This gives double fascial edges and broad muscular surfaces for union. The purpose of the buried suture is to approximate the muscular and fascial layers of the wound, so as to insure apposition of homologous parts, and to retain them in approximation long enough to secure firm union.

The running suture is preferable to the interrupted, first, because it brings corresponding structures more accurately and more quickly together ; second, because, in the method described, it gives no buried knots. The second advantage is considerable, for the bulky catgut knot tends to cause suppuration or failure of union.

Closure of the abdominal wound by buried catgut sutures is made as follows:

The needle is introduced at the lower extremity of the wound ou the right side (A, Figure 58), and at the first thrust is carried through skin, fat, anterior fascia, muscle, and peritoneum. The suture then is continued as a running suture the length of the wound, and unites the peritoneum. The posterior fascia and muscle may be united by this suture at the same time with the peritoneum. The suture then is carried back to the starting-point, whipping together the onter fragments of the divided anterior fascia, thus bringing the recti museles firmly together. It finally emerges at the point of entrance, $A$, where it was introduced first, and is tied. Figures 60 and 61.

In connection with the subject of suture for abdominal incision the reader is referred to the Surgical Treatment of Enteroptosis and Umbilical Hernia at the end of Chapter XLV.

The splitting of the sheaths of the recti muscles is essential in cases of relaxed abdominal walls, especially when there are enteroptosis and pendulous abdomen; this method insures a good result so far as the correction of the side-to-side relaxation is concerned, but does not always overcome the longitudinal relaxation. Figure 62 shows a method by which the upper end of the wound is closed in a line at right angles to the incision so as to hold up the relaxed pendulons abdomen. The transverse part of the closure may be at the upper or lower end or anywhere in the continuity of the wound or both at the upper and lower ends. An ordinary continuous chromic or iodized catgut suture is sufficient. For the cutaneous part of the wound Clandius' iodized gut is preferred.

Stitch-abscesses are very liable to occur unless the following precautions are observed:

1. The abdomen should be opened with a sharp scalpel, which will make a clean cut, not a ragged, uneven incision.

2. Great eare should be used during the operation not to bruise or tear the wounded surfaces.

3. All bleeding should be arrested before closure of the wound.

4. Absolute asepsis should be secured in hands, instruments, sponges, sutures, and, above all, in the field of incision. See Chapter II. 
5. The sutures should not be drawn so tightly as to strangulate the parts.

6. Buried knots should be avoided.

Should suppuration in the wound or along the sutures occur, the sutures, if of the through-and-through variety and tied on the skin, should be removed at once. The buried suture must be left in place until it is absorbed. A dressing, wet with 70 per eent. alcohol, should

Figure 62.

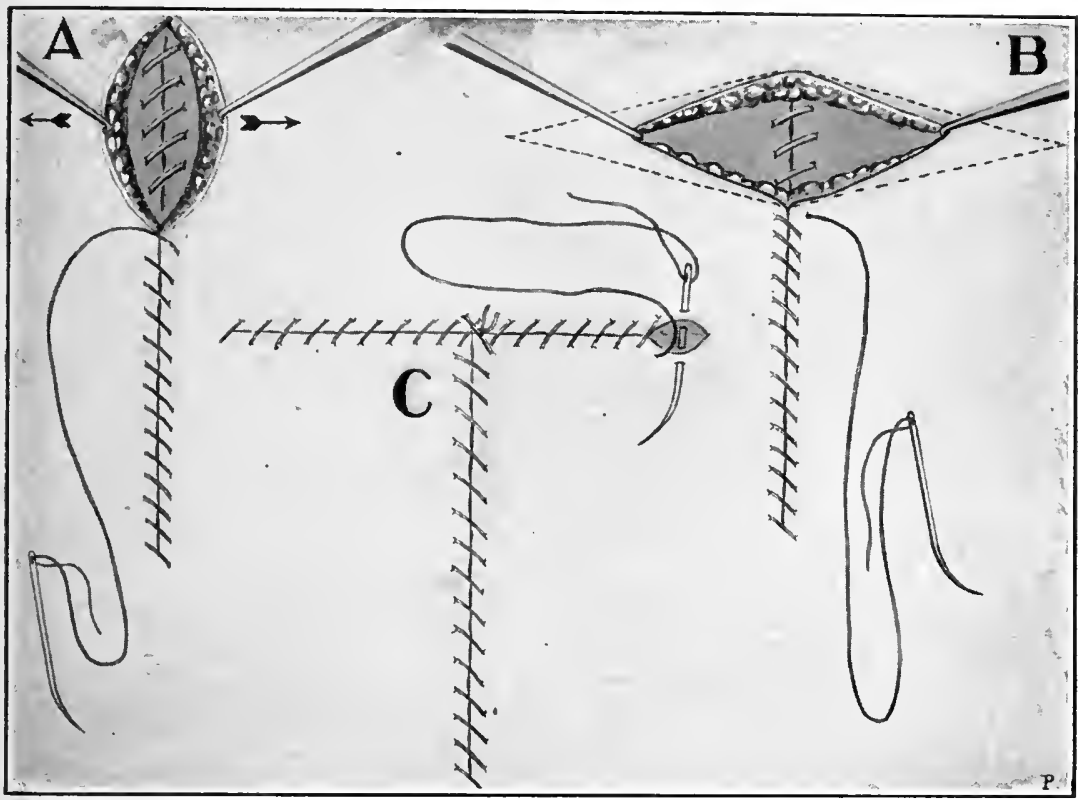

In cases of enteroptosis and pendulous abdomen it may be neeessary not only to split the sheaths of the recti muscles, but also to remove a strip of skin and subcutaneous fat on either side of the wound in order to take up the lateral slack in the abdominal wall. Not infrequently there will still remain considerable longitudinal slaek, which should be disposed of by uniting some portion of the wound in a direction at right angles to the line of ineision, as follows:

A. The peritoneal and fascial margins of the wound have been elosed eompletely. The cutaneous margins of the lower two-thirds of the wound have been elosed in the usual manner. The upper third is being held slightly apart by two tenacula.

$B$. The upper part of the wound is being drawn widely apart by two tenacula so that it may be elosed in a direction running at right angles to the line of ineision. The dotted lines indieate that part of the skin and subeutaneous fat to be removed so that the wound when closed may have a level surfaee all around it free from folds.

C. The elosure of the wound is being completed by the introduetion of the last stitch. In this Figure the suture is not subcutaneous but over-and-over, although the subcutaneons suture would be permissible. The over-and-over stiteh is shown here in order to demonstrate it.

be maintained continuously. Free drainage by incision should be established if necessary to drain out any considerable accumulations of pus. Immobilization of the abdominal walls by a firm bandage will tend to prevent separation of the suppurating wound.

Sponges.-In nearly every extensive abdominal section numerous sponges, preferably gauze sponges, are packed into the abdominal cavity, not only to absorb blood and other fluid, but to control hemor- 
rhage by pressure, and to hold the intestine and other viseera out of the way of the operator.

How to Avoid the Leaving of Sponges in the Abdomen.-It is quite impossible during the progress of an abdominal section for the operator to keep track of the exact number of sponges which may be inside of the abdomen; hence numerous humiliating, not to say fatal, results of closure of the wound and completion of the operation with one or more sponges remaining in the peritoneal eavity. The not infrequent occurrence of this deplorable aceident, even at the hands of careful men, is the writer's excuse for introducing two personal experiences; verily, how much experience one may get from a single case!

The first case was one of extensive suppuration of the uterine appendages with nearly universal old, firm adhesions throughout the pelvis, and with the uterus enlarged by chronic endometritis and metritis to about four times its natural size. All the diseased organs

Figure 63.

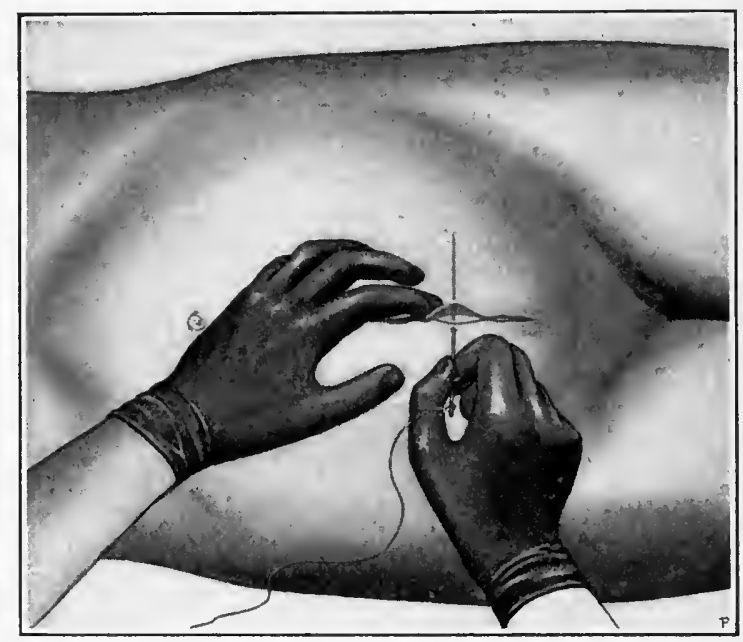

Abdominal wound has been closed by cutaneous and buried sutures. In some cases the skin-margins do not fall closely enough together; it is then necessary to unite them by means of a continuous or interrupted suture with a very fine long needle, with sharp point, threaded. with fine catgut.

were removed by abdominal and vaginal section. The operation, especially the hysterectomy, was exceptionally difficult and tedious. The broad ligaments were so short and thick as to be inaccessible for the ligature, and almost for the clamps. Each ligament was so thick that through the vagina it had to be clamped in three parts. The patient was put to bed apparently nearer dead than alive. The writer's usual preeautions had been taken to prevent closing the wound with a sponge inside. The sponges had been brought to the operation in sterilized packages each containing eight, so that the number must have been eight or some multiple of eight. Only large, flat ganze sponges were used. The operation was begun with the 
eight sponges of one package, which were counted. Two additional packages of eight each were required in the course of the operation, all of which were supposed to have been counted accurately by the nurse in charge of them. Just before the abdominal sutures were introdnced the nurse was directed to comt the sponges. She reported them "all out." After the introduction of the sutures, and before they were tied, she was told to count them again, and this comnt also made the number twenty-four and "all out." "With the evidence of a double count, that there could be no sponge in the abdomen, the wound was closed.

Three hours later the nurse reported that one of the gauze sponges used in the abdomen could not be found. After consultation with two colleagues it was decided to assume for the time that the missing sponge had been lost outside the abdomen, and that consequently the peritoneal cavity was clear.

Convalescence was minterrupted till the tenth day, when the stitches were removed. At this time there was noticed a semi-resonant mass of irregular ovoid shape, as large as a inedium-size orange, in the region of the right kidney; it gave to the palpating hand the sensation of a mass of gauze mingled with adherent intestine. Two colleagues agreed that it would be wise to wait for developments. Sixteen hours later, at 11 P. M., the mass had increased in size, become painful, the pulse had risen from 100 to 120 , and the temperature from $99^{\circ}$ to $101^{\circ} \mathrm{F}$. There was slightly increased distention, accompanied by a tendency to pronounced nausea. After a hasty consultation, the family being informed of our suspicions and fears, chloroform was given and the abdomen opened directly over the mass. The incision was made withont the usual assistants, at midnight, and revealed, not a sponge, but a much enlarged kidney surrounded and covered by firmly adherent intestine looped and matted together in an irregular mass. In working through the thickened, unrecognizable, adherent parietal peritoneum, and between the layers of visceral peritoneum and the adherent intestine, also thickened and difficult to recognize, the intestine was opened accidentally. The opening immediately was repaired with interrupted Lembert sutures, and the abdominal wound closed without drain.

Three days later the contents of the small intestine, probably the upper part of the ileum, came through the abdominal wound, and an intestinal fistula thereby was demonstrated. During the following five weeks no feces passed by the anus : all bowel evacuations came through the fistula. The opening was so high in the bowel that nutrition seriously was impaired and emaciation began. The fear of a formidable operation to restore the integrity of the bowel increased day by day. Finally, to the writer's unspeakable relief, in the sixth week fecal matter appeared at the anus. The fistula began to contract, and in a few days was closed completely. The kidney enlargement entirely subsided, and repeated urinalysis showed no evidence of functional impairment.

The prolonged anxiety and distress of such a case are beyond description. They are, both for the surgeon and for the patient, a 
life-shortening experience. The burden of this case was lightened, first, by the ultimate recovery of the patient; second, by the complete relief which she has experienced since from a distressing intestinal catarrh whieh had made her a semi-invalid for fifteen years. This relief is attributed to the continuous rest to which that portion of the bowel below the injury was subject while the fistula was open.

The second case was one of intraligamentous ovarian eyst on each side, with double sactosalpinx serosa and universal adhesions. The sponges were counted carefully before the incision was made. Before the wound was closed, the nurse again counted them and reported one missing. After a search of fifteen minutes among the abdominal viscera, the nurse in the meantime looking for the sponge outside, it conld not be found. In the hope of finding the sponge, the incision, previously short, was extended to the navel, preparatory to turning ont the intestines, when the nurse found the sponge outside; it had carelessly been nisplaced in a jar and overlooked. The patient fortunately recovered.

These two eases illustrate the degree to which a surgeon, with all the responsibility, may be powerless to protect his patient against the inefficiency or earelessness of an assistant whose shortcomings, perchance, he may be unable to discover until it is too late.

The precautions which may be taken in order, so far as possible, to guard against accidentally leaving a sponge in the abdominal cavity are as follows :

1. All sponges shonld be so large as not easily to be overlooked by the operator. If sea-sponges are used, let them all be the largest flat sponges, and of as nearly uniform size as possible. Gauze sponges are, however, preferable. They should be made of good absorbent gauze in four thicknesses, and should be of uniform size, at least six inches wide by twelve to sixteen inehes long. All sponging can be done with large as well as with small sponges. Let the smaller ones, then, be discarded. They serve no necessary purpose.

2. All sponges designed for abdominal section shonld be kept in packages of eight each. This number will suffice for the ordinary operation. If more are needed, additional packages may be opened. As soon as a package is opened, the sponges should accurately be recounted and recorded. Invariably this precaution will fix the number for any operation at eight or a multiple of eight.

3. Toward the close of the operation the sponges again should be counted. Experience has shown that under the demoralizing influence of hurry and exeitement which often attend the close of a desperate operation, the nurse in charge of the sponges is liable to blunder in the count. It is well, therefore, that the count be repeated two or three times, and, if possible, by different individuals.

Another practical means of avoiding the loss of sponges that may have been packed into the abdomen is to have them fastened in groups of two by narrow strips of tape, Figure $64, \mathrm{~B}$, the strips being about twelve inches long. One might readily overlook one sponge in the cavity, but he could hardly overlook two. Moreover, sponges fastened together in this way are eounted easily when removed. The plan of 
attaching a tape to each sponge and letting the end remain outside is objectionable, for many protruding strips of tape would be in the way of the operator, and, what is worse, one or more strips, even thongh held by forceps, might accidentally slip in and be lost. The long sponge roll of Mayo, Figure 64, $\mathrm{C}$, is most serviceable for packing intestine out of the way of the operator.

Figure 64 .

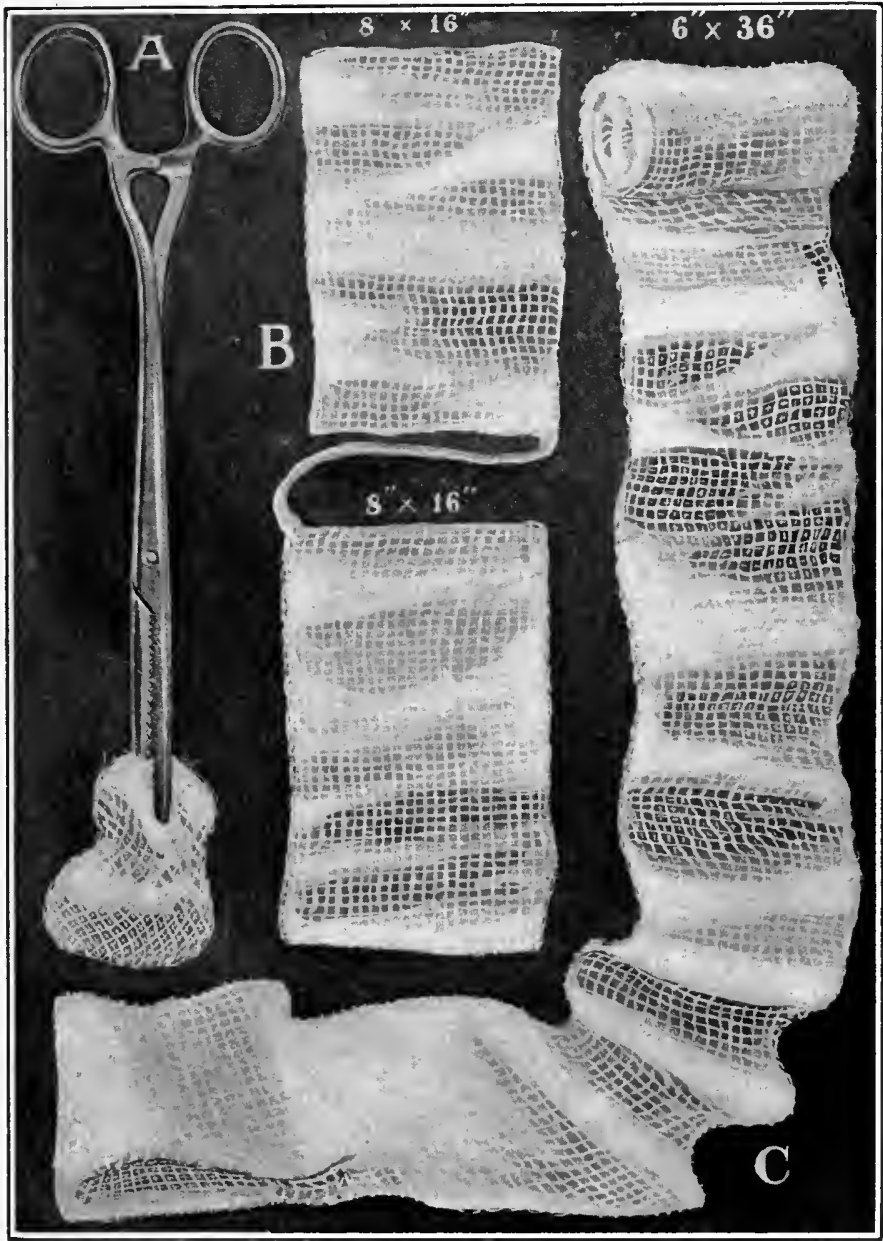

A, gauze sponge on long forceps for deep sponging in the pelvic or abdominal cavity; B, gauze sponge fastened together with tape as a precaution against leaving it in the peritoneal cavity; C. long spunge roll for packing intestiues out of operator's way.

The operator, whose every energy is employed in the effort to shorten the time of operation, camnot stop for sponge-counting; ret only a surgeon can appreciate the satisfaction which lies in the absolute knowledge that every sponge is out. The writer, therefore, now uses a simple device by which the number of sponges may at a glance 
be apparent to any one. It is this : At the time of closing the wound the sponges are arranged in uniform rows on the floor. The subjeet is so urgent that, even at the risk of seeming triviality, the accompanying illustration is introduced.

There are manifest advantages in not having the sponges washed during the operation. A sufficient number should be provided, so

Figure 65.

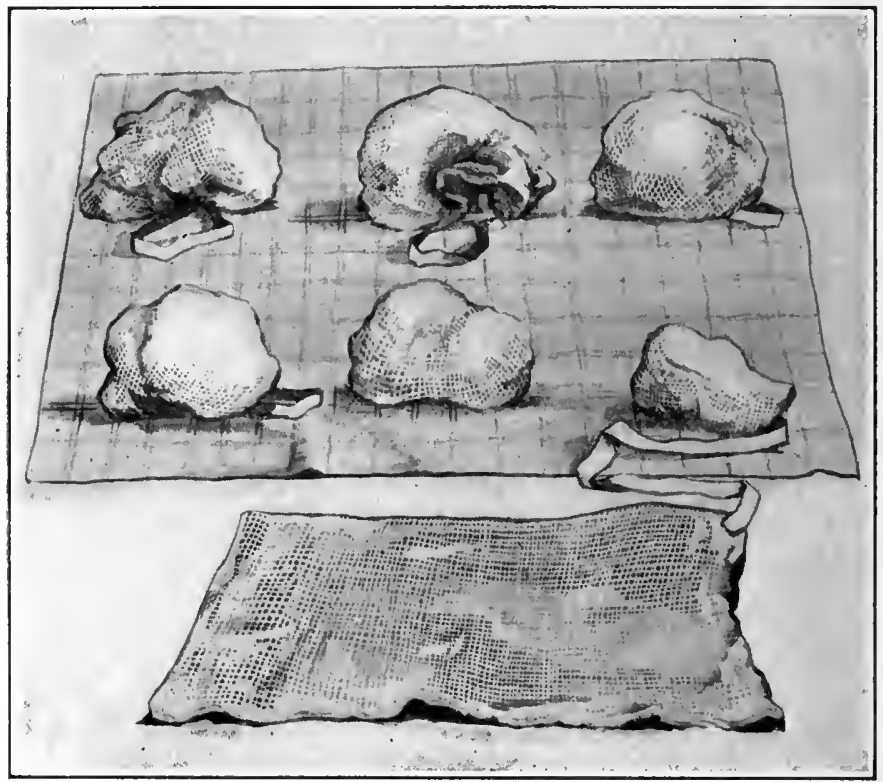

Sponges arranged in rows to facilitate ceunting at the end of an operation.

that they may always be used dry, and discarded as soon as they are soiled. In this way the operator may dispense with one assistant, the sponge-washer, and so limit the danger of infection.

Dressings and Bandages.-The usual combination aseptie dressing of gauze and wood-wool or cotton, seeured by strips of adhesive plaster and a firm abdominal bandage, will suffice. The nurse should be cautioned to use eare lest the dressing and bandage slip up and expose the lower end of the wound. If a vulvar dressing also is used, it should be kept separate from the abdominal dressing, for otherwise fluids may pass by capillary attraction from one to the other; this may explain the faet that stiteh abscesses often begin at the lower end of the wound. It is well to use two abdominal bandages, one to reach from the hips to the umbilieus, or, if neeessary; higher, and the other to lap over the lower part of this and reach to the middle of the thighs. The lower bandage keeps the dressing from slipping upward. It may be loosened for movement of bowels or urination.

Dusting Powders for the Wound.-The author has used dusting powders extensively and with good results, but recent experience has 
Figure 66.

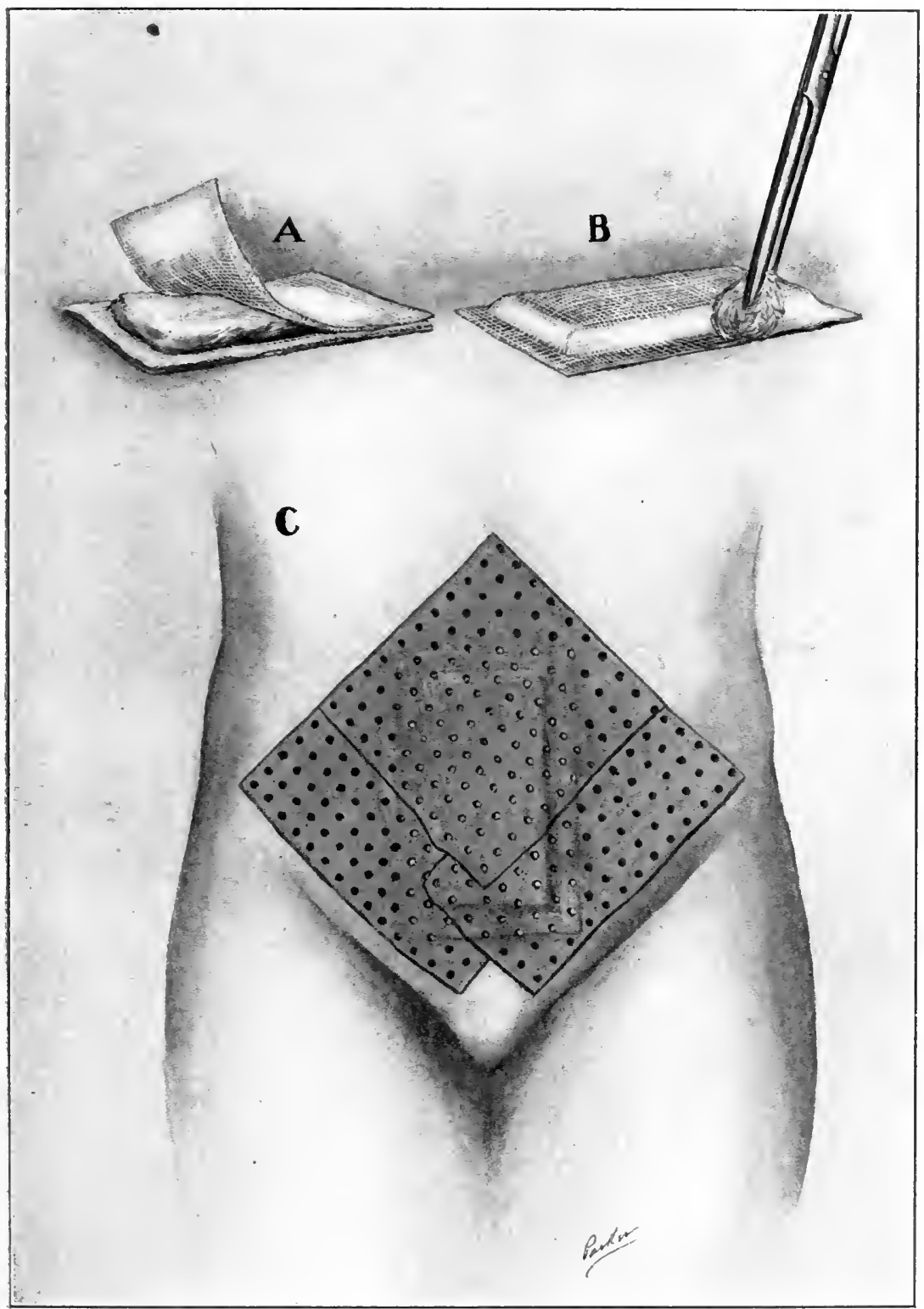

A, Two layers of gauze with eotton between lying on the elosed wound; $B$, gauze and eotton dressing being secured in plaee by means of a wad of cotton held in the grasp of a foreeps and saturated with collodion; C, gauze and eotton dressing further held in place by means of perforated adhesive plaster. Observe this plaster in three pieces, the two lower pieces being placed parallel to Poupart's ligament so that on retraction of the thighs the plaster will not be lifted off.

convineed him that in careful aseptic surgery all dusting powders may be dispensed with to advantage.

In ordinary cases in which there is little probability that the 
wound will have to be dressed or otherwise disturbed, it is better to apply the dressings and secure them by perforated adhesiye plaster, as shown in Figure 66 . The wound is covered first by a single layer of gauze. This gauze is made fast by means of collodion. In order not to confine any possible secretion which may escape from the wound, the collodion should surround, but should not eover, the line of union. On this layer of gauze is placed a layer of absorbent cotton, and over the cotton a second layer of gauze, Figure 66, A and B, which also is made fast by collodion. A few additional layers of gauze are now loosely placed over that part of the dressings already deseribed, and the whole is held in place by perforated adhesive plaster, as shown in Figure $66, \mathrm{C}$. The advantages of holding the dressings in place by means of adhesive plaster instead of the usual abdominal binder are

FIGURE 67.

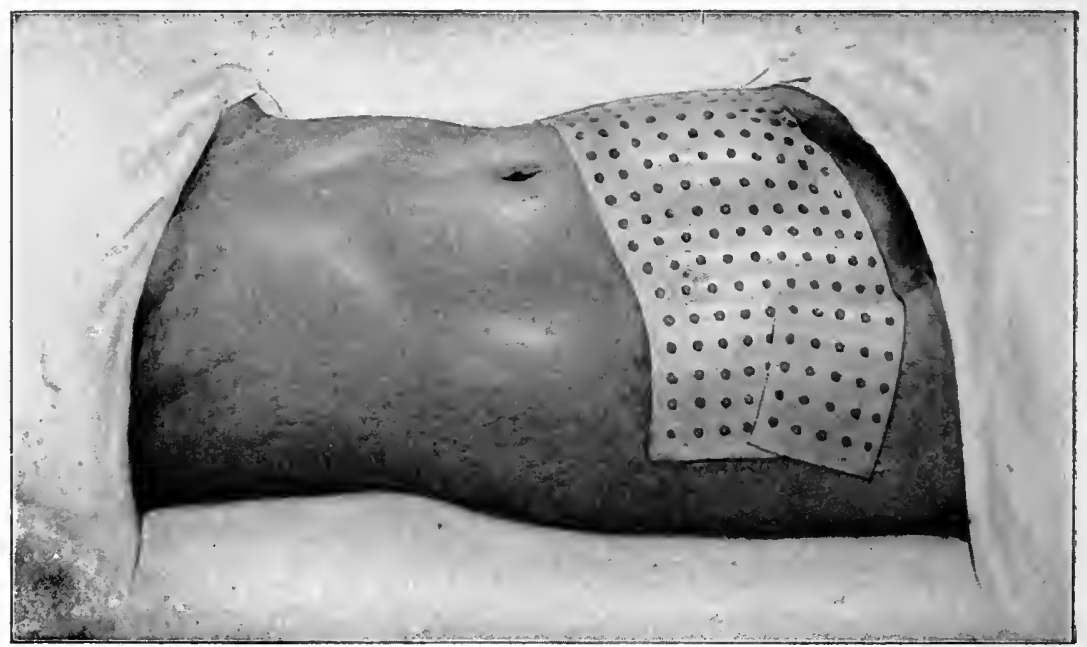

This Figure shows perforated plaster over the dressings of an abdominal wound. This is the usual method of application, but is faulty, because when the thighs a re retracted the plaster is apt to be lifted from the wound. The correct method is shown in Figure 66 .

as follows: 1, the wound is protected absolutely against exposure by the carelessness of nurses, who are prone to permit the abdoninal binder to be displaced upward, and thereby to leave the wound exposed; 2 , the dressings of the gauze and cotton, secured by collodion and covered by perforated plaster, give the patient much less discomfort than the ordinary cumbersome dressings of large quantities of gauze, cotton, and other absorbent materials, which are held in place by the conventional abdominal binder.

\section{VAGINAL SECTION.}

The vaginal route for opening into the peritoneal cavity is often preferable. The incision may be made between the uterus and rectum or between the uterus and bladder. 'The technique of the procedure 
varies within wide limits, and will be described under special operations. See Vaginal Seetion in the ehapter on the Treatment of Pelvic Inflammation.

\section{SACRAL RESECTION.}

Hysterectomy and other intrapelvic operations have been performed through an opening made by resection of the sacrum after the method of Kraske. The preeise value of the method has not been established. 


\title{
CHA P T E V I I.
}

\author{
DRAINAGE IN MAJOR OPERATIONS.
}

\section{INFECTIOUS AND NON-INFECTIOUS CASES.}

Two classes of drainage cases present themselves; first, cases which, up to the time of operation, are free from infection; second, eases in which infection has occurred previous to the operation. To the first class belong solid and cystic tumors and tubal pregnancies which have not become infected; to the second class belongs pelvic inflanmation in its various forms and stages, snch as inflammation of the Fallopian tube and ovary, including pelvic abscess, pyosalpinx, and infected tumors.

In the non-infectious cases drainage is resorted to for the removal of blood, serum, or other non-infections fluids such as otherwise might accumulate in the peritoneum, and, if left there, become infectious. Experiment and experience, however, have shown that the noninfected blood and serum which may accumulate in the peritoneum after a clean, adequate operation have little or no power for harm. Serum and liquid blood are absorbed rapidly. Coagulated blood may be absorbed, or it may become encapsulated and gradually removed by the action of lencocytes; or it may become organized and remain harmless for an indefinite period. Both blood and serum are excellent culture-nedia for microbes; hence the necessity to keep them noninfectious by aseptic surgery. The peritoneum has great power to resist infection, and is known to take up and dispose of large quantities of infectious material, even without drainage. Recent studies and experience prove that the drain is often more potent as a medium for the introduction of sepsis than for the removal of it. Drainage, on account of infection, therefore, after a clean operation in a case not hitherto infected, is contraindicated.

Bacteriological examinations of reproductive organs removed for chronic inflammatory disease frequently show that the pus is sterile, or, if organisms are present, they may be inactive at the time of the operation. Formerly the escape of the smallest quantity of pus into the peritoneum during an operation was considered an imperative indication for drainage. Now, the eseape of even large quantities if free from virulent or active microbes, does not call for drainage.

Comparison of Results.-Large numbers of drained pus-cases and equal numbers of like cases not drained uniformly show a strong preponderance of recoveries in the non-drainage series. This preponderance is proof that the drainage was useless, and that the larger mortality in the drained cases is attributable to infection introduced through the drain. 
Evil Results of Drainage.-In addition to the greatly increased danger already mentioned from the direct introduction of infection through the medium of the drain, the following evil results of drainage may be mentioned as not infrequent :

1. Obstruction of the bowel.

2. Fecal fistula.

3. Vesical complications.

4. Hernia.

5. Intoxication by iodoform gauze or other medicated gauze.

1. Obstruction may occur from adhesions set up by the irritating presence of the drain. An adherent intestine sharply kinked may become suddenly impermeable, or gradually contracting bands may shnt off its lumen slowly. Most frequently the obstruction is partial, and gives rise to constipation and griping pains for days or weeks after the operation. In such cases, when fatal, the autopsy usually has shown the intestines matted around the drainage cavity in an unrecognizable mass.

2. Fecal Fistula is the occasional result of necrosis from direct pressure of the drain or from the irritating presence of a gauze drain and consequent infection so destructive as to produce necrosis and consequent fistula. If the bowel has been opened during an operation, and has been well repaired, drainage is unfavorable to union and is contraindicated. If, however, the intestinal opening has been made deep in the pelvis, or is otherwise so inaccessible as to prevent thorough suturing; if, in a word, union is improbable, drainage is indicated as a means of exit for fecal matter.

The greatest care in covering all raw surfaces with peritoneum and stitching it down with fine continuous catgut sutures, is one of the most imperative measures for the reduction of mortality in abdominal surgery ; even the bad condition of a patient and the necessity for rapid operating will seldom be au excuse for neglect of this precaution.

3. Vesical Complications. - The territory to be drained is usually in close relation with the bladder. Infection around the drain, therefore, may give rise to adhesions between the bladder and adjacent organs, or may invade the bladder; in either case vesical disturbance more or less severe may arise.

4. Hernia in drained cases is much more common than usually is supposed. This is because the drain separates the fascial sheaths of the recti muscles and other surfaces which otherwise would unite immediately; the small breach thus made in the wall increases, and more or less hernia is the result. Hernia less often results from vaginal than from abdominal drainage.

To Prevent Infection, and thereby to avoid the necessity for drainage, is an essential purpose of every abdominal section. The subject may be summed up in the proposition that the operation should be performed in such a way as not to require drainage. This involves the following precautions:

1. Insure thorough asepsis of hands, instruments, and other appliances. See Chapter II., on Antiseptics and Asepsis.

2. Wherever the peritoneum is injured or sacrificed, let the injured 
part, if possible, be covered by adjacent peritoneum. This may require numerous sutures and careful plastie work.

3. Control hemorrhage, if practicable, even to small oozing points. This, for want of time or for other reasons, may be impracticable. It may then be safe to leave small accumulations to be taken up by the peritoneum rather than by a drain.

4. A void all unnecessary injury of the tissues. All traumatisms favor sepsis. Do the operation adequately, but with the least possible amount of operating.

5. As a most important precaution, let the bowels be evacuated thoroughly before beginning the operation. Any considerable quantity of gas in the bowel is a source of danger.

If during the operation pus ruptures into the peritoneal cavity, it should be removed as soon as possible by careful sponging. If the pus is sterile, the sponging is sufficient. If there is reason to fear that it is septic, the peritoneal cavity should be irrigated freely with a normal salt solution, 0.6 per cent. Clark advocates thorough peritoneal irrigation whenever any pus comes in contact with the peritoneum. He says: "For the last three years it has been our custom not only to irrigate the abdominal cavity thoroughly after all operations where pus or other fluids have escaped, but frequently also to leave as much as one litre of salt solution in the peritoneal cavity before closing the abdominal wound." The writer cannot too strongly urge the retention of as much salt solution in the abdomen after irrigation as the ordinary abdomen will hold; an abdomen of very large capacity with excessively lax walls would hold too much. The solution being absorbed rapidly, carries out septic matter, increases arterial pressure, and relieves the intolerable thirst of which most laparotomy patients complain. Sueh a sense of well-being follows the retention of considerable quantities of salt-water in the abdomen that the use of it as an almost routine measure even for the non-pus cases is permissible. Moreover, the filling of the abdomen with fluid displaces the air that would otherwise be retained in the peritoneal cavity after closure of the wound.

The usual method of flushing the cavity by means of salt solution poured through the wound into the cavity from a pitcher or flask is in may cases quite inadequate, because the cavity may be so filled with viscera that the solution thus introduced camnot reach the deeper portions of the abdomen or pelvis. In order to make the flushing effective, the solution may be introduced through a canula attached to a rubber tube, the tube leading from a reservoir above. The end of this canula can be passed through the wound and made to transnit the solution thoroughly to all parts of the peritoneal cavity. A very practical substitute for the canula is shown in Figure 68. The salt solution is held in a funnel-like metallic reservoir, to which is attached a rubber tube at least three-fourths of an inch in diameter. At the end of this tube is fastened a long straight pressure-forceps, as shown in Figure 68. By means of this forceps the end of the rubber tube may be carried into any part of the peritoneal cavity, and the solution rapidly and thoroughly introduced in very large quantities. During 
the process of flushing additional quantities of solution may be poured from pitchers into the metallic reservoir. By this means many gallons of solution may be brought rapidly in eontaet with every part of the peritoneal eavity. In using this apparatus it is essential that there be a free outlet through the wound, for otherwise the pressure exerted upon the diaphragm by the column of water might be dangerous.

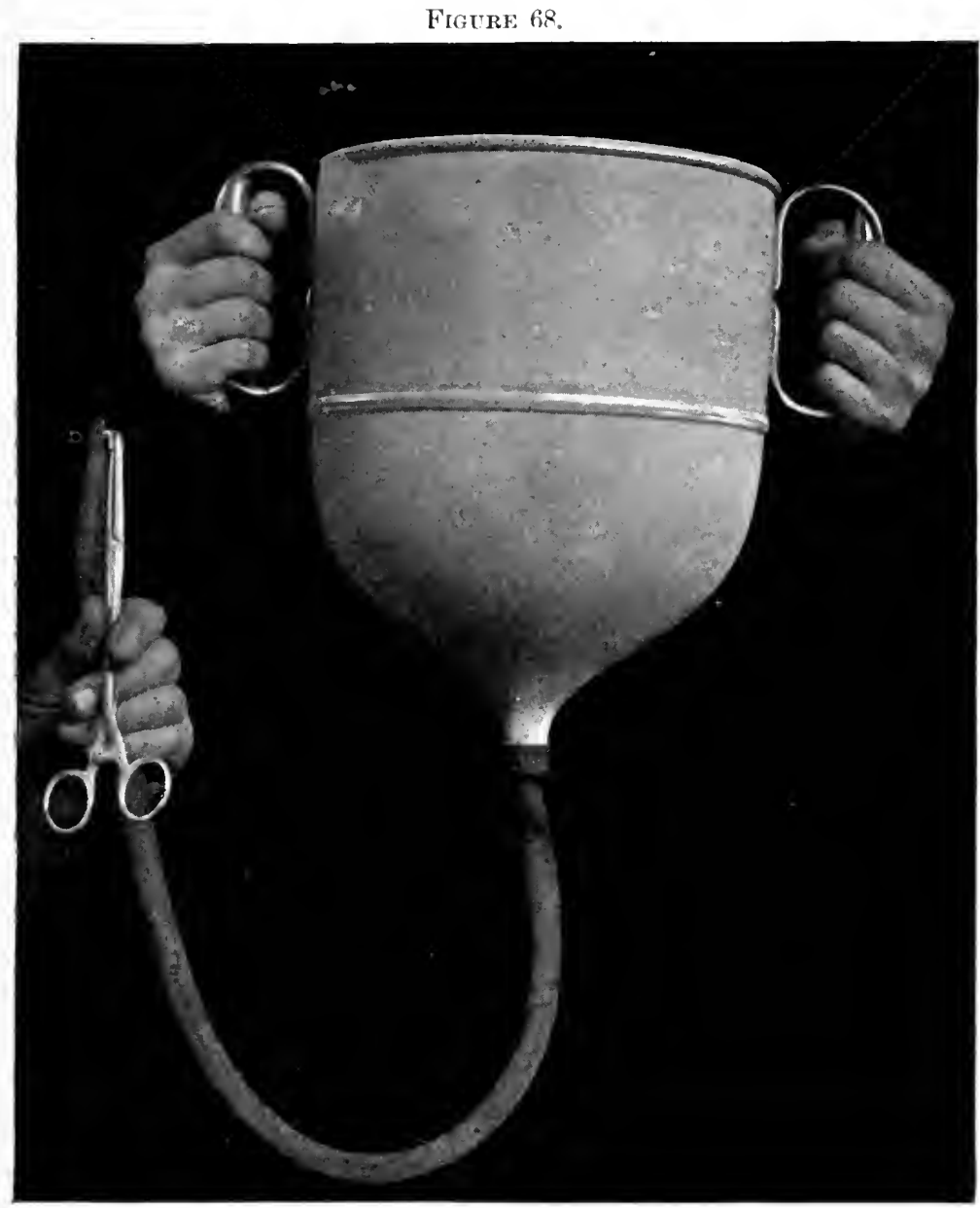

Apparatus for flushing out abdominal eavity, eonsisting of a metaltic funnel-like reservoir with two handles to be helil above the level of the patient. A rubber tube three-fourths of an ineh indiameter leads from the reservoir. At the end of the tube may be attached a canula, or as shown in the figure a foreeps, for the purpose of earrying the solution to the deeper parts of the peritoneal cavity.

It is a well-known principle in physies that a substance will undergo dissipation much more rapidly in an attenuated state than when it is massed. The same principle may be applied to the disposal of foreign matter in the peritoneal eavity; hence the advantage of attenuating any infectious matter that may be in the eavity with 
great dilutions in salt solution ; this advantage is inereased by the salt, which has antiseptic properties.

\section{CONTRAINDICATIONS FOR PERITONEAL DRAINAGE.}

Drainage is contraindicated under the following conditions :

1. Aseptic operations in which there is no pus or other source of infection.

2. Operations in which there is pus, but in which the sac containing it is removed intact so that no pus has escaped.

3. Operations in which pus has escaped, but where immediate microscopical examination of a smear shows it to be sterile.

4. Operations in which a moderate quantity of infectious pus has escaped and has been washed out thoroughly with large quantities of normal salt solution. The question of drainage in this class of cases is not fully settled, but the trend of opinion is in favor of irrigation and against drainage, for in most cases the peritoneum will take up and safely dispose of considerable infectious matter.

\section{INDICATIONS FOR PERITONEAL DRAINAGE.}

Drainage is indicated in the following class of cases :

1. General septic peritonitis.

2. The presence of a localized pus-producing surface which may continue to secrete a greater amount of septic matter than the peritoneum can take up, and which therefore may be a dangerous source of infection. Under these conditions drainage is indicated at the time of operation. The source of infection may be an open intestine; an abseess; all adherent necrotic irremovable cyst; an infected hæmatocele, or an abundance of necrotic tissue which cannot safely be removed. If after an operation infectious fluid becomes walled off by plastic effusion, thereby forming an abscess, no time should be lost in opening and draining it.

3. Hemorrhage, for the control of which the ligature, forcipressure, and hot sponge packing are inadequate.

\section{DIFFERENT FORMS OF DRAINAGE.}

Tubular Drainage is usually through soft rubber or small glass tubes. For drainage through the vagina rubber is preferable to glass. The tube is especially useful as a medium for drainage and for washing out septic cavities, such as abscesses which have been walled off by plastic lymph from the general peritoneum. 'The presence, however, of a tube in the peritoneal cavity usually causes in a few hours the surrounding organs to be fused together. The space which the tube occupied is isolated then from the remainder of the peritoneum, and is the only space which it possibly can drain. For this reason tubular drainage in the abdomen for the most part has been discarded.

Capillary Drainage.-The continuous strip of gauze has been used extensively for capillary peritoneal drainage through both the vaginal and the abdominal wound. 
There are two principal indications for the use of ganze packing in abdominal and pelvic operations: 1 . Hemorrhage which cannot be controlled practically in any other way without unduly prolonging the operation; the packing then used is immediately a compress, but if

\section{FIGURE 69.}

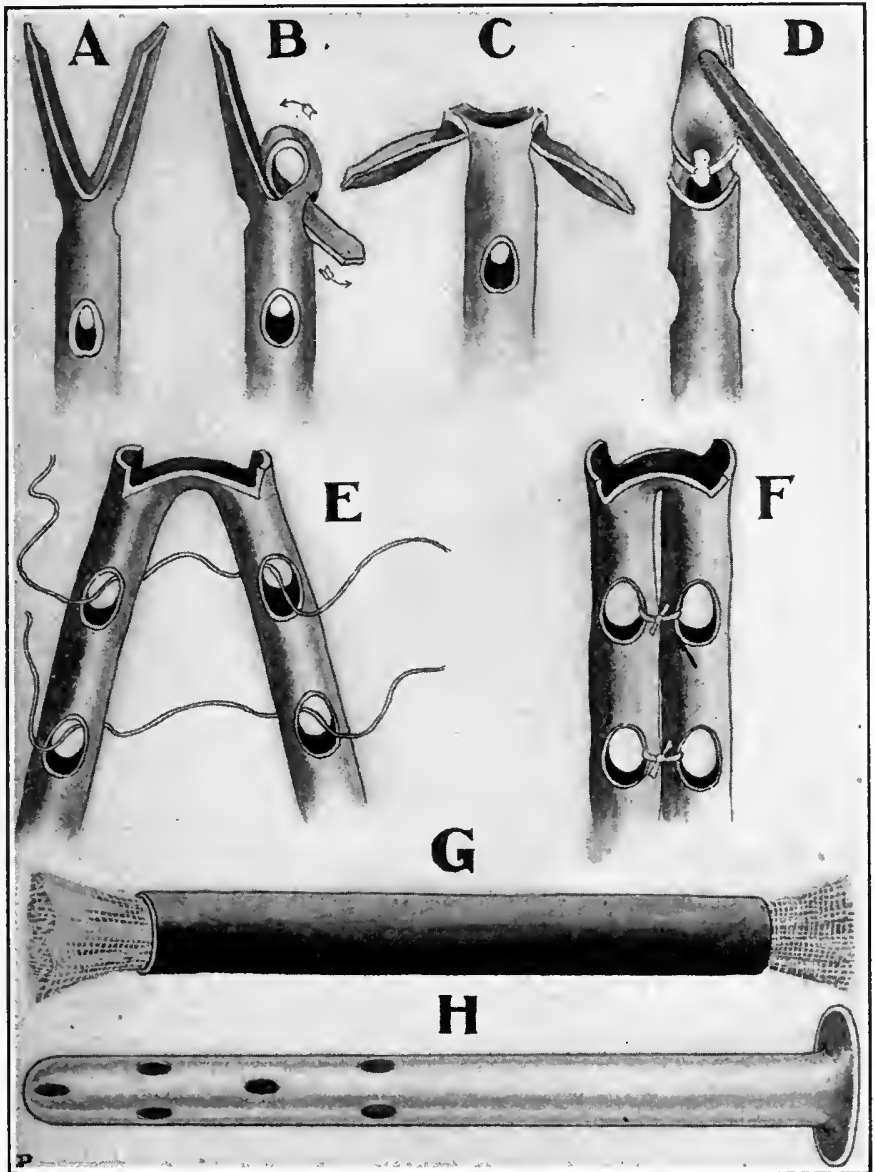

Reid's rubber drain, A, B, C, D. This drain has two wings, C, whieh make it self-retaining when placed in a pus-eavity. The wings may be held together by forceps, D, to faeilitate introduction: $\mathrm{A}, \mathrm{B}$, and $\mathrm{C}$ show the steps in the formation of the drain: $\mathbf{E}$, $\mathrm{F}$, ordiuary double rubber drains; G, a rubber drain surrounding a roll of gauze for capillary drainage; $H$, ordinary glass drainage-tube for abdominal drainage.

left longer than is necessary tor hæmostatic purposes it becomes a capillary drain. 2. The desirability of quarantining the field of operation from the general peritoneal eavity. The rapidity with which adhesions form around the packing is well known. In a few hours a septic area may be shut off from the general peritoneum by adhesions which form around the gauze packing, and in this way septic fluid is walled off and mostly confined within narrow limits. 
The use of gauze for packing should not be eonfounded with the use of it as a drain. The value of gauze for drainage as usually understood-that is, for the removal by eapillary attraction of any fluid

FIGURE 70.

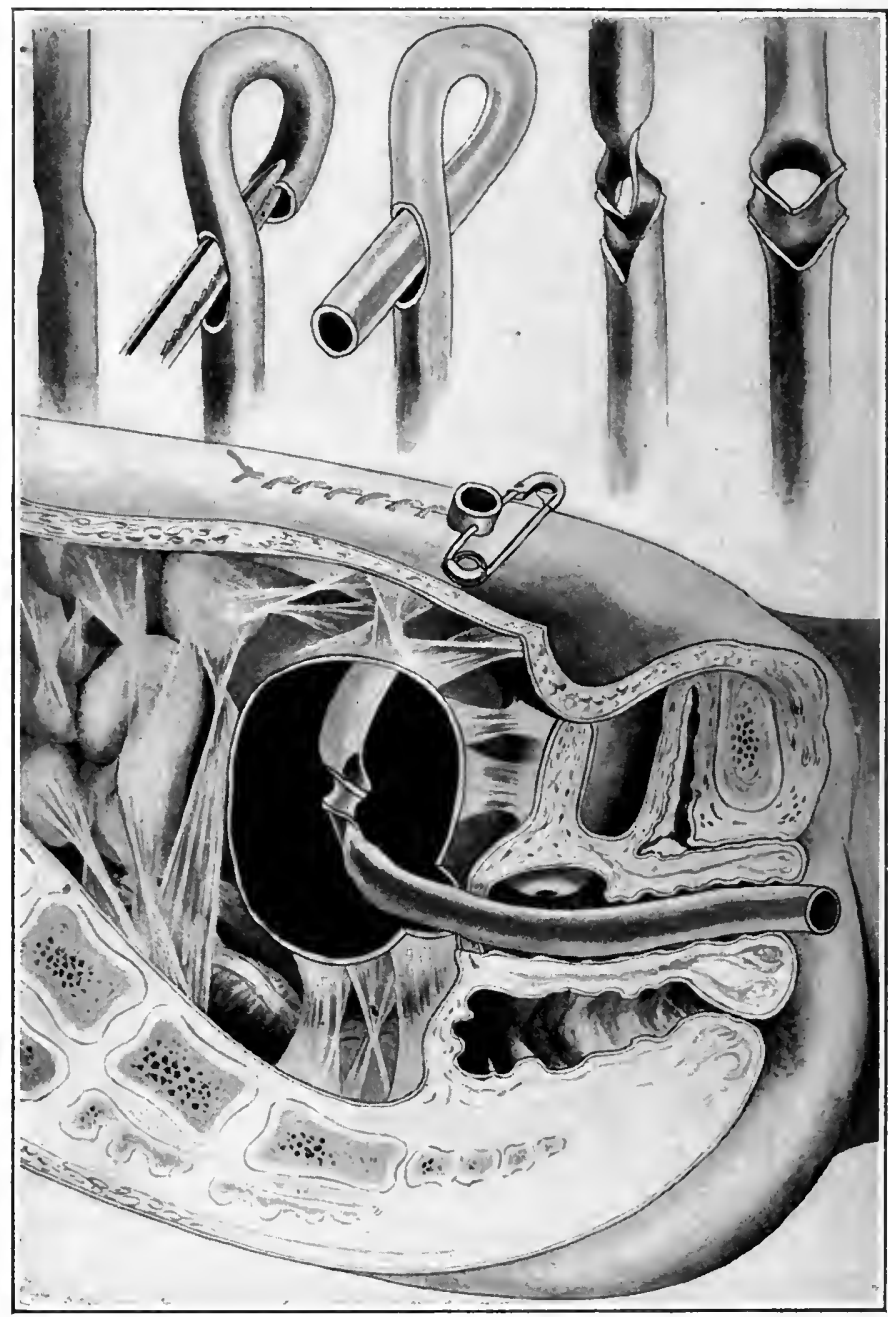

The upper five rubber tubes show the steps in the formation of Reid's rubber drain for through-and-through abdominal drainage. The Figure below shows the drain in place projeeting upward through the lower end of the abdominal wound and held there by a safety-pin. The other end of the drain is projected downward from the abscess-cavity through Douglas' pouch and the ragina to the vilva. Each end of this drain is protected by a gauze pad. These pads are heid in place by an abdominal binder and a T-bandage, and should be changed sufficiently often to keep them dry.

which mav form in the peritoneal eavity-is overestimated. The peritoneum often has demonstrated its ability to take care of large quantities of seeretion. If, as many assert, it be true that the presence 
of a drain excites the secretion of large quantities 'of fluid which would not otherwise be secreted, it follows that the drain is often not so necessary as the large quantities of fluid which it carries off would seem to indicate. Clearly it would be absurd to use a drain for the purpose of carrying off secretions which it had itself excited. Moreover, the gauze packing often acts as an obstruction to the removal of fluids, and may therefore defeat the purpose of drainage.

Abdominal Drainage, if employed, may be either by the tubular or by the capillary method. The route from the pelvis to the abdominal wound is long and in close relations with the bladder, intestines,

\section{Figure 71.}

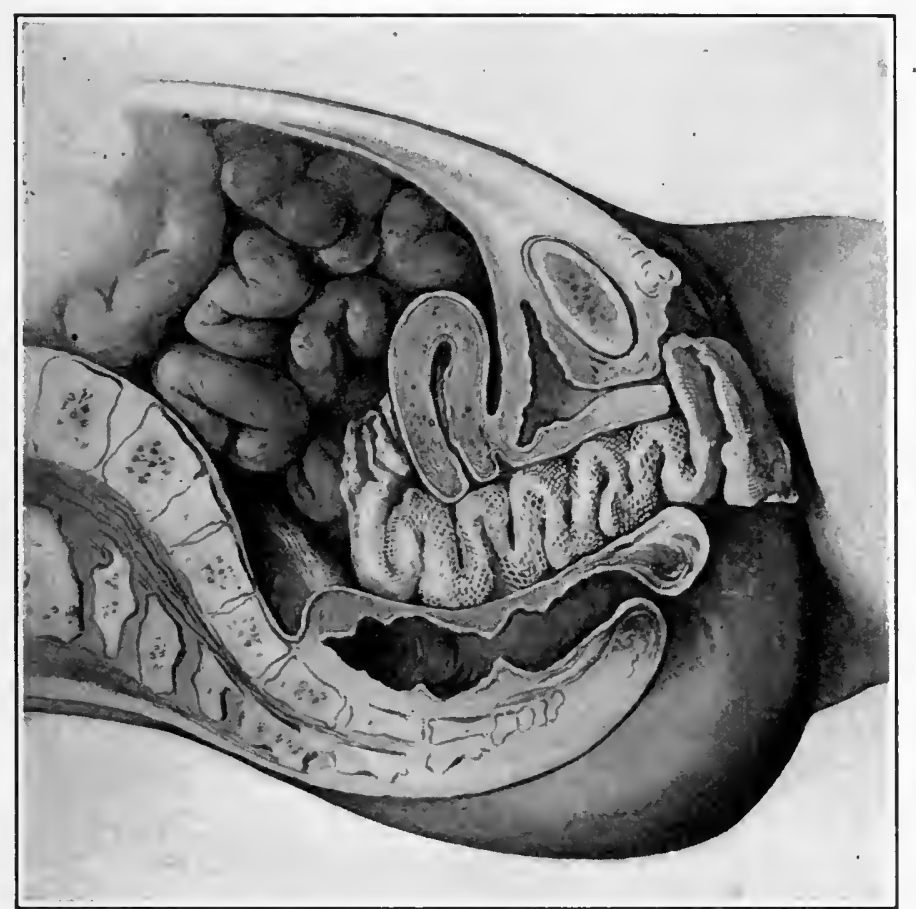

Vaginal gauze drain extending from Douglas's pouch to the vulva for capillary drainage. A large gauze pad should be kept over the vulva, and changed often enough to keep it dry. This pad is secured by a T-bandage.

and omentum, which organs should have no necessary relation with the field of operation, but which, from contact with the drain, are unfortunately liable to infection, adhesions, perforation, and hernia. Moreover, the long sinus left after removal of the drain is often slow to heal, and its outer end is prone to contract rapidly and leave in the pelvis a troublesome, undrained or imperfectly drained pocket. For these reasons the abdominal route for drainage is objectionable and should be limited to special cases.

Fowler's Position and Postural Drainage.-If abdominal drainage is required at all, it should be most thorough and is best secured 
by means of rubber tubes, an inch in diameter, split on one side the whole length, and containing a roll of ganze-cigaret drain. Three of these drains should be used: one in each flank and one in the cul-desate of Douglas. The patient then should be placed in the exaggerated Fowler position, that is, the shoulder should be at least twenty inches above the hips, so that dependent and deep depressions in the perito-

Figure 72.

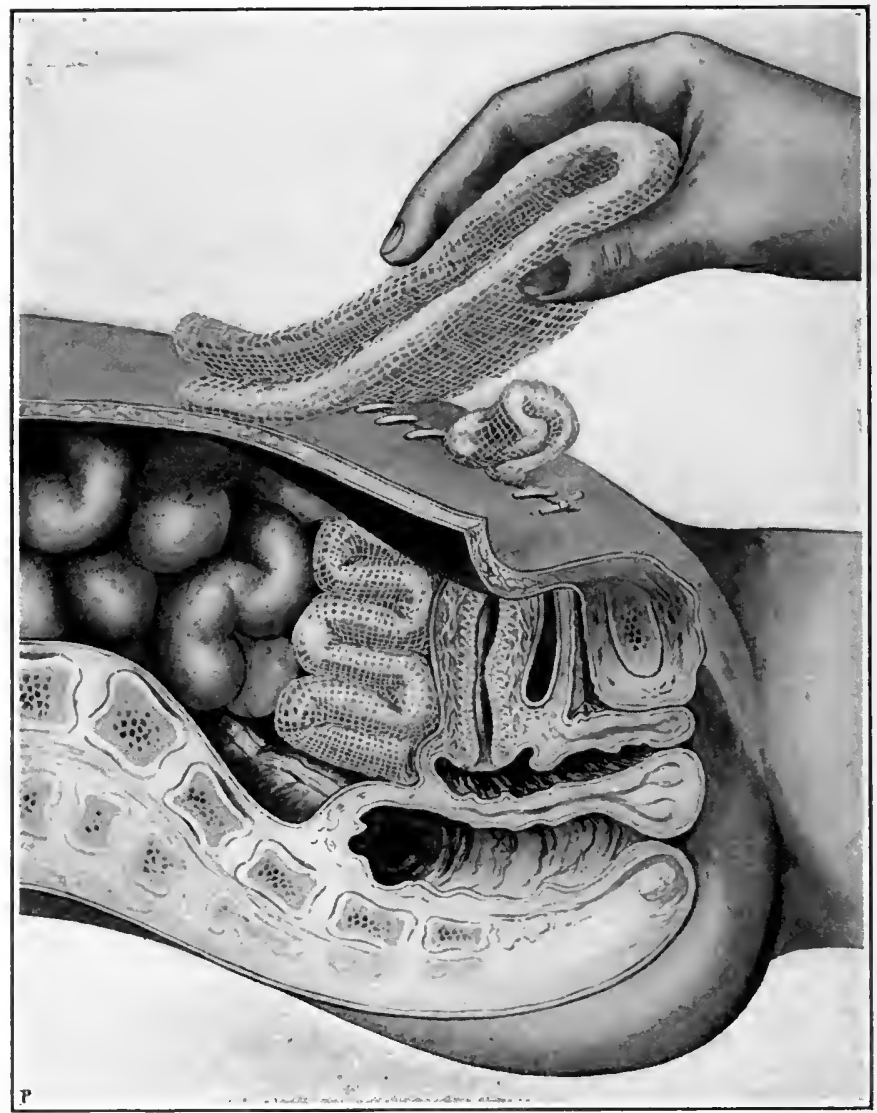

Capillary drainage through abdominal wound: the gauze pad held by the hand is to be placed over the wound in contact with the protruding portion of the drain. This pad should be secured by an abdominal binder, and changed often enough to keep it dry.

neal eavity may be drained by gravity. Such drainage is demanded only in extremc acute cases of present or threatened peritonitis and should be undertaken only by surgeons of great experience and judgment.

Vaginal Drainage.-The route from the pelvic eavity to the vagina is short and direct; hence the vaginal drain is generally preferable. If in the operation an opening between the pelvic earity and the vagina has not been male and drainage is necessary, it is often better 
to make an opening for that purpose. The great advantages of the vaginal route are : 1 , minimum risk of hernia; 2 , natural and dependent drainage ; 3 , more satisfactory convalescence. The safety of this route must depend largely upon previous thorough disinfection of the vagina.

If the vaginal gauze drain is to be passed after an abdominal operation through an opening made from the peritoneal cavity into the vagina for that purpose, one may first pack the strip of gauze into a previously disinfected vagina, crowding it well into the posterior vaginal fornix, where it easily can be felt with the finger in the pouch of Douglas. The operator then, using this packing as a guide, may eut down directly upon it. This incision thus made may he enlarged to the required degree, and the gauze already being in place, that part of the operation is complete.

The manner of introducing a gauze drain, whether abdominal or vaginal, is as follows: The end of a continuous strip of double gauze, with the edges turned in and stitched together to prevent fraying, is doubled backward and forward upon itself, like the folds of a fan, from the part to be drained to the surface. Over this an external dressing is placed and changed as often as it becomes saturated. Figures 70,71 , and 72 show vaginal and abdominal drains in place.

The time for removal of gauze varies with the purpose for which it was used; if to control hemorrhage, it should be removed in twentyfour hours; if for drainage, it may be left five days, unless, as occasionally happens, it acts as an impediment to drainage. Some surgeons consider the gauze objectionable as soon as it becomes saturated. In this connection we may again emphasize the importance of keeping the dressing over the drain absorbent and dry. The writer repeatedly has observed patients to show signs of septic absorption on the second or third day after an abdominal operation, when removal of the gauze was followed by a gush of pent-up fluid and prompt relief of all urgent symptoms. It is not usually necessary, after removal of the criginal drain, to introduce fresh gauze. If at any time the opening tends to contract too rapidly, or drainage becomes imperfect, the gauze may be renewed or a tube may be inserted.

The classical dictum has been, "When in doubt, drain." If, however, the irritating infuence of the drain is to cause the sccretion of fluid which otherwise would not be secreted; if the peritoneum, left to itself, is capable of taking up and disposing of large quantities of fluid, even, to some extent, of septic fluid; if the drain is more prone to introduce than to carry out sepsis-then the dictum may have to be reversed, when in doubt, don't drain. 


\section{CHA PTER VIII.}

\section{AFTER-TREATMENT IN MAJOR OPERATIONS.}

\section{AFTER-TREATMENT IN SIMPLE CASES.}

WATCHFUL expectancy is the rule of after-treatment in peritoneal surgery. Indeed, if properly performed, an abdominal section usually is followed by normal convalescence, and therefore requires little or no active treatment.

Importance of Rest.-Bodily and mental rest is the first consideration. The exclusion of relatives and friends from the bedside is usually imperative, and will not be difficult if properly managed. They have, perhaps, travelled long distances, and seriously believe that the comfort and consolation which they alone can give are highly essential to the patient's recovery. They must be told in kindly but positive words that the results of experience in thousands of cases demonstrate the necessity of absolute quiet; that the presenee of the husband, the mother, or other near relatives excites emotion; that emotion consumes energy, of which the patient has none to spare. Such a statement is usually sufficient; if not, the surgeon must enforce whatever regulations the welfare of the patient may demand. If she becomes restless and anxious because the relatives are kept out, it may be well to admit them. Most patients, however, during the first two or three days do not ask for them, and many prefer not to see them. Frequently sponge-bathing, eare to keep the bed-clothing under the patient smooth, and such other minor attentions as only a good nurse can suggest, all contribute to the desired end, rest.

Position.-The expression "absolute rest" is misleading if it implies the enforcement of the custom of keeping the patient "absnlutely on her back during the first two or three days." The patient not only becomes wearied of the fixed position, but is apt to attribute to it the inevitable pain and discomfort of the anæsthesia and operation. Unless there is some special objection, she should freely take whatever position is most comfortable. She will then wait more patiently for the natural subsidence of the disconfort, which if all goes well, a little time will bring. It is quite safe and may be desirable at any time, if the patient wishes, to elevate the head and shoulders on a back-rest, even to the extent of thirty degrees.

Fowler's Position, which is secured by raising the head of the bed from ten to twenty inches, often gives relief from nausea and nervous irritation. Many surgeons use this position as a routine measure immediately after operation.

Rest for the Stomach.-A variable degree of irritability of the whole digestive tract is the common result of anæsthesia, especially 
in cases of abdominal seetion. The voniting and nausea of this state are increased rather than diminished by drugs and food. The usual treatment is to withhold then until the effeets of the anæsthesia have passed off. A teacupful of hot water slowly given, even though promptly thrown up, will wash out the stomach and, perchanee, give a little relief. The knees may be drawn up into the most eomfortable position and supported on a pillow or roll. The judgment and discretion of a wise nurse will furnish a guide more useful than the most elaborate rules. The best nurse will move, when possible, along the lines of least resistance, or, when neesssary, will use a gentle firmness that inspires confidence. She will carry her patient past the critical period with the minimum of friction and diseomfort.

Thirst.—"Oh, for a good drink!" is one of the first calls. The common practice of withholding water as a routine is to be condenmed. In the absence of nausea it may be given cold or hot in satisfying quantities. Charged waters, ginger ale, champagne, and other sueh drinks, while not excluded, are not required, and may do harm. If water is not tolerated by the stomach, a pint or less of normal salt solution should be thrown into the rectum every six hours.

If in the preparatory treatment the patient has taken liberal quantities of water for several days, and especially if before closing the wound the abdomen has been filled with normal salt solution, Chapter VII., there will be little complaint of thirst; on the contrary, there will be a relative freedom from nervousness and irritation.

Hot-water Bottles and Bags placed about the patient immediately after the operation are useless exeept in eases of shock, and should be avoided as a routine. The careless use of hot-water bottles or rubber bags before recovery from ancesthesia has resulted occasionally in serious burns. In one ease the writer observed an enormous blister on the outer surface of each thigh; in another, on the sole of the foot. Both patients sustained deep sloughing of the cutaneous and subcutaneous struetures, which finally required extensive skingrafting. As the freezing of water in a pipe more readily takes place when there is no eireulation of water, so in conditions of shoek, when the circulation of blood is feeble, burns are more liable to occur.

The Bowels.-If the temperature, pulse, and respirations are normal, or nearly so, and there is no abdominal distention nor other unfavorable sign, movement of the bowels may be deferred until the seeond day; then they should be moved by an enema or a mild eathartic. Some surgeons begin immediately after the operation with half-grain doses of ealomel given every fourth hour until eight or ten doses have been given or the bowels act. If there is no aetion, the calomel is followed immediately by a saline purge, or an enema, or both.

Early eatharsis is a good precaution against sepsis and peritonitis, and may be used in all eases in whieh these complications are feared. In normal eases it is unnecessary. Early movement of the bowels, however, is desirable in all eases. After the initial movement they should be kept regular by eathartics or enemas, or both.

The Bladder.-Great caution is necessary to protect the patient against the deplorable aceident of distention and overflow of the blad- 
der. Frequent urination should always excite suspicion, and should be regarded as an urgent indication to pass the eatheter.

Pain of variable degree is usually present during the first day or two. Opium and its preparations lock up the secretions, induce nausea, arrest peristalsis, cause distention, and mask any symptoms which otherwise might give warning of approaching danger; they, moreover, counteraet the influence of cathartics, and would prove therefore a scrious obstacle if it became necessary to move the bowels. Such drugs, if given at all, should be given with great circumspection. Codeine phosphate creates less nausea, constipation, and other disturbances, than opium or morphine ; the hypodermic use of it in halfgrain doses is sometimes permissible, during the first twenty-four hours, to allay nervous irritation and pain and to insure neeled rest. There are conditions of great nervous irritation in which morphine in full doses is indicated strongly. See Hysterical Vomiting in this chapter. The operator is largely dependent upon the wisdon and discretion of nurses and internes. The best nurses and the best assistants manage their patients with the least morphine.

Food.-Except in cases of exhaustion, food is to be withheld for one or two days. It is usual to begin feeding after the bowels act or flatus passes. Eructations of gas from the stomach are an indication for withholding food. The downward passage of flatus is a good prognostic sign. "Qui crepitat vitat," said the early Roman physicians. The diet for the first few days is preferably broth, beginning in small doses and, according to the tolerance of the stomach, gradually inercasing. A teaspoonful at a time may be given at first, and repeafed in thirty minutes. If this is tolerated, the amount may after a few hours be doubled, and so on till several ounces at a time are taken. Finally, after two or three days, if all goes well, the anount may be increased largely until full quantities are taken.

Getting Up.-Ordinarily the paticnt may sit up about the end of the second week; if the incision was long and union is not quite firm, she should be kept in bed longer.

Care of the Cicatrix.-If the sutures were non-absorbable and have been removed, the wound is to be dressed as before, except with progressively lighter dressings, for a period of two weeks. The new cicatrix should be supported first by straps of adhesive plaster, and later, when the patient begins to walk, by a properly adjusted elastic bandage. A variety of suitable bandages may be found at the instrument or dry-goods shops. The bandage should be worn continuously in daytime for six months. A lighter flannel bandage may be used at night. If the wound was closed with chromic catgut, as described in Chapter VI., and is not more than two or three inches long, and strong union has taken place, no abdominal bandage is necessary.

\section{AFTER-TREATMENT IN COMPLICATED CASES.}

Shock associated with abdominal section is the same as after other operations and injuries. If it occurs during the operation, use at once the extreme Trendelenburg position, and flood the peritoneal cavity 
with a quart or more of normal salt solution, 0.6 per cent., at a temperature of $105^{\circ} \mathrm{F}$, and complete the operation as soon as possible. After the operation elevate the foot of the ber. Among other measures for the treatment of shock are the application of dry heat to the surface; the hypodermic administration of strychnine sulphate every four hours in doses of one-thirtieth of a grain each; the free hypodermic use of whiskey, at least four drachms every hour; the hypodermic injection of two grains of camphor dissolved in ten minims of sterilized olive oil, to be given every hour; and copious high rectal enemata of warm normal salt solution, to be retained if possible. Shock is most apt to occur when considerable blood has been lost during the operation; whether from this cause or not, the urgent indication is to fill the blood-vessels, and thereby increase arterial pressure. See Hypodermoclysis, below.

Secondary Hemorrhage.-It is often difficult to differentiate hemorrhage from shock. The former, if post operative, is usually slow and may not declare itself until several hours after the operation. The latter more commonly begins some time during the operation.

Diagnosis.-The symptoms of hemorrhage are well known. The patient has, perhaps, rallied well from the operation, with good pulse and temperature. Presently, or within a few hours, there are symptoms of approaching collapse-i.e., rapid, thready pulse, subnormal temperature, pallor, sighing, gaping, and cold surface. If these symptoms appear, the presence of much clotted blood in the drainage-tube, if there be one, may clear the diagnosis. The gauze drain would show a stain of deeper red than ordinary blood-serum should make. Both of these signs, however, may fail. If there is no drain, one often may work a small glass fenale catheter througl the wound between the stitches. Hemorrhage then would declare itself by the presence of clear blood in the catheter.

Treatment.-To reopen the wound, find the source of hemorrhage, apply a ligature or a pressure-forceps, sponge or wash out the cavity, and close the wound with the patient bordering on collapse, is, indeed, a serious undertaking. If, however, there is hemorrhage, any other attempt to check the bleeding is not only useless, but a dangerous waste of time as well.

Hypodermoclysis after Hemorrage. - $A$ most effective means of combating the results of hemorrhage is the hypodernoclysis injection of large quantities of normal salt solution. "The strength, according to Bacon, should be not, as generally directed, 0.6 per cent., but about 0.8 per cent. - i. e., 8 parts in 1000 . An even teaspoonful of table salt in a pint of water is a safe and reliable approximation to the required strength.

The technique of this simple and valuable procedure is as follows: The saline solution and the apparatus for its injection are sterilized by boiling. The solution having been boiled, is now cooled to the proper temperature, say $110^{\circ} \mathrm{F}$. The surface through which the needles are to be introduced is sterilized, and the needles, as shown in the diagram, are thrust deeply into the cellular tissue under the skin. 'The solution 
flows from the bottle or funnel by its own weight. An elevation of four or five feet is neeessary to make the fluid flow freely. Constant gentle massige over the injeeted area will promote the distribution and absorption of the fluid. 'Ten or fifteen minutes usually will suffice for the introduetion of a pint of solution. The apparatus consists of a glass funnel attached to a large hypodermic needle, single or double, by means of a long rubber tube. The fluid passes rapidly into the circulation, immediately increasing arterial pressure; the procedure gives rise to little or no pain. It is sometimes necessary, after an

Figure 73.

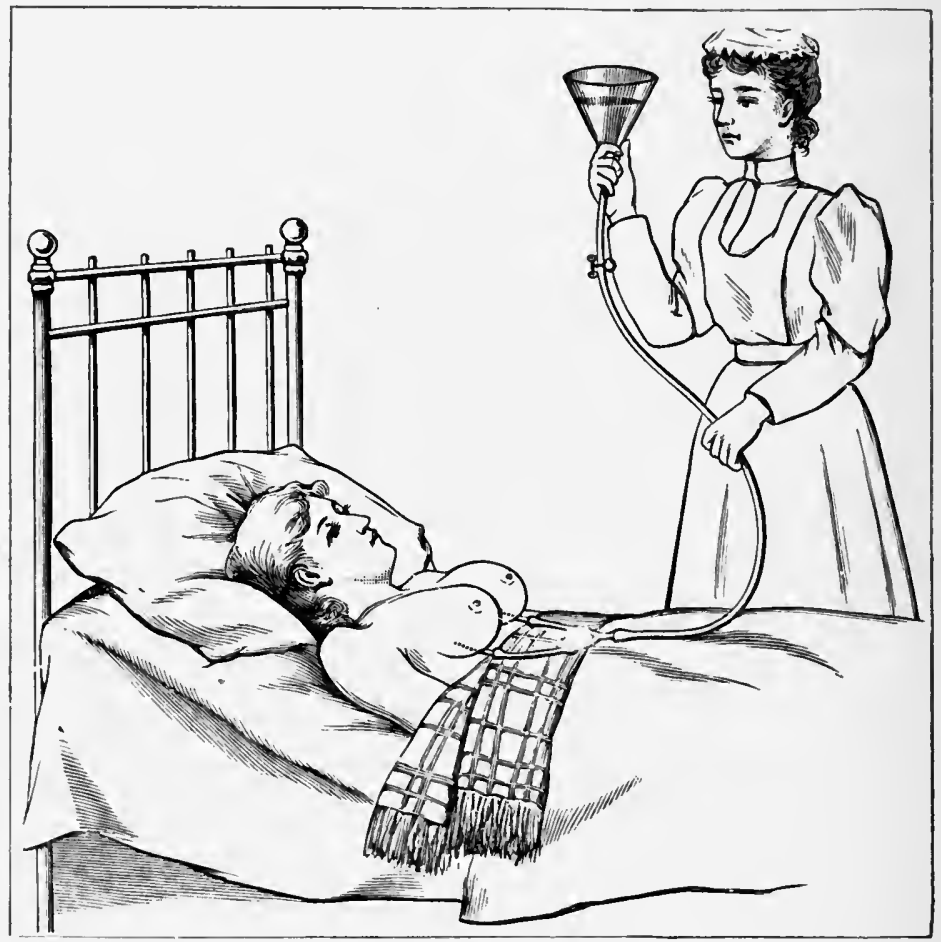

Apparatus for hypodermoclysis. The funnel contains twenty ounces. A rubber tube, with shut-off attached, connects it with a $Y$-shaped glass tube. Two small rubber tubes connect this with aspirator-needles or large hypodermic points. The injection may be thrown into the thighs, abdominal wall, or under the breasts. The submamnary region is usually selected.

exhausting hemorrhage, to inject at intervals into the breasts or abdominal wall or outer surface of the thighs, as much as three quarts in a single day.

The prime indieation to inerease arterial pressure is ordinarily more safely and quite as effeetively finfilled by this method as by the direet injection of blood or salt solution into the vessels. When the loss of blood has amounted to between one-fourth and one-half of the entire quantity in the body, sone prefer to throw the solution directly into a vein. "This demands the greatest care in asepsis and extreme preeaution against the introduction of air. 
High rectal enemata of salt solution are useful, but less rapidly effective than hypodermoclysis.

Drop Method of Salt Water Infusion.-If not very immediate action of normal salt solution is desired, one may use with great satisfaction the drop method of infusion into the rectum. For this purpose the apparatus shown in Figure 73 should be modified as follows: Substitute a small-calibre rectal tube for the needles and introduce this tube high into the rectum. Suspend the funnel on some fixture which will hold it steady about four feet above the patient. Hang a fountain syringe bag with a short rubber tube above the fumnel in such a way that water will flow from the bag into the funnel. Regulate the current to a drop-by-drop supply by means of a pressure forceps placed on the supply-tube, so that the solution passing drop by drop into the bowel will run at the rate of not over a pint in an hour. In my own practice this method largely takes the place both of hypodernoclysis and ordinary enemata of salt solution. It may be continued for many hours.

The hemorrhage having ceased, the subsequent treatment is the same as that for shock. If food is not tolerated by the stomach, rectal alimentation should be used every four hours. A good conbination for this purpose cousists of the white of an egg, three ounces of peptonized milk, and one ounce of whiskey.

Sepsis.-The.phenomena of sepsis often are considered under the name peritonitis. There are two varieties : first, plastic or adhesive; second, exudative. In the plastic variety adhesions may form around the diseased area; in this way the infection may be shut off' from the general peritoneum and confined within narrow limits. In the exudative variety the plastic or defensive action is absent or inadequate, and the infection therefore spreads throughout the peritoneum and sets up rapid and fatal blood-poisoning. It is a mistake to attribute the evils of sepsis to the associated peritonitis. The inflammatory process is an effort of nature to protect the general system against infection: if plastic and adhesive, it mav succeed; if exudative, it usually fails. It is the infection that specially endangers life, not the associated peritonitis, which may or may not save it. Sepsis, then, or to use a better term, infection, may clinically be classified as follows :

1. Localized infection.

2. General infection.

1. Localized Infection.-This usually finds expression in the form of an abscess at the seat of the operation. It may be around an infected pedicle, suture, or ligature. The nidus may be a surface laid bare in the operation and not covered by peritoneum, or it may be pathological tissue which could not be, or at least was not, removed. Localized infection is apt to declare itself a few days after operation. The prognosis is usually good.

The symptoms are those of septic absorption : rapid but usually strong pulse, varible elevation in temperature, localized pain, sweats, chilly sensations, with little or no tendency to collapse. Examination usually will show a progressively enlarged swelling situated in the pelvis and felt usually on conjoined examination. Stitch-abscess may give rise to the same symptoms, but usually in less degree. 
Treatment is simple and satisfaetory. Under anæsthesia the abseess should promptly be opened and drained. The drainage-ehannel is usually through the incision by which the peritoneum was entered in the original operation - i. e., through the abdomen or vagina. If a drainage-tube is already in the wound, there may be spontaneous rupture of the abseess into the tube. In an aggravated case it is sometimes best to make throngh-and-through drainage from the abdominal wound to the vagina. Rubber tubes, not gauze, are best for drainage.

In ease of stiteh-abscess-that is, suppuration in the line of the wound-the sutures, if of the through-and-through variety, should be removed, if of the buried catgut variety they should be left. Any aceessible aceumulation of pus should be opened with a blunt-grooved direetor and evacuated; then the wound should be treated with a 95 per eent. alcohol gauze dressing, the gauze being changed often and kept wet with the alcohol.

2. General Infection of the Peritoneum-i.e., exudative peritonitis, so-ealled-which is apt to deelare itself very soon after the operation -is fatal. Every abdominal surgeon is painfully familiar with the charaeteristie symptoms. He has deseried them from afar as one may diseern the dark eloud upon the horizon. In the balanee between hope and fear he has watehed the anxious face, the drawn expression, the progressively rising temperature, the nausea, at first attributed to anæsthesia, then as this subsides the vomiting of sepsis which takes its place, the frequent regurgitation of bile mixed with blood and mueus and growing darker and darker. He has reeognized the gradual failure of the pulse, first weak, then running, then thready to the vanishing-point, the paretie and distending bowels, which refuse to act, the rapid respirations, the cold extremities, the staring eyes, the wide nostrils, and, finally, the inevitable collapse.

Treatment is useless. The symptom-group just outlined may, however, be present in less grave eonditions, among them the local, eircumseribed infeetion above deseribed. Bowel distention, vomiting, fever, and rapid, weak pulse may also be due to eanses other than general peritoneal infection. In view of this possibility, therefore, active treatment may be indieated.

The first effort should be directed to the movement of the bowels. Try ealomel, one-half grain every half-hour until the bowels have acted. Let this be followed, if necessary, by the solution of eitrate of magnesium, a wineglassful every fifteen ininutes, or more if the stomach will tolerate it. In obstinate cases a single soft capsule containing eastor oil 2 drachms and eroton oil one-half drop will be found serviceable. Copious reetal enemata may stimulate the bowels to act, or at least to expel the flatus. The enemata may be of stiff Castile soapsuds, with a draehm of turpentine thoroughly mixed in each pint. It may be a mixture of glyeerin, Epsom salt, and water, each two ounces, or a quart of olive oil or linseed oil. A large enema should be given slowly through a long rectal tube introdnced as high as possible, with the patient on the left side. The museular walls of the bowel in this condition are generally paretic; 
hence the great difficulty in stimulating them to contract and by peristalsis to expel their contents.

Whiskey, strychnine, eamphor, ammonia, rectal alimentation, and other supporting measures, as described for the treatment of shock, may be used in moderation. Under such management patients with symptoms like those of general peritoneal infection may recover.

Credé's ointment of argentum colloidale, one drachm a day thoroughly rubbed in for two to twenty minutes, and intravenous injections of a 2 per cent. emulsion of argentum colloidale once a day in doses of 15 grains, are among the strongly recommended measures. Antistreptococcic serum, given hypodermically 10 to $40 \mathrm{cc}$. a day, may be useful for streptococcic infection.

The free use of powerful toxic drugs as a routine practice is deplorable; desperate conditions do not necessarily call for desperate measures; these drugs, unless used with the best judgment, may take away the only remaining chance for life from a patient already overburdened with the toxæmia of sepsis.

Hysterical Vomiting. - In abont 1 per cent. of abdominal sections the operation is followed by vomiting, frequent, violent, prolonged, and exhausting. The nervous depression is profound ; the pulse may rise to 170 or 180 to the minute. The condition may continue for several days, with final recovery, or may pass into collapse. The pathology of this phenomenal nerve-storm, with the stomach for the storm-centre, is unexplained. It may be due to toxæmia, or to irritation similar to that which produces the vomiting of pregnancy. The causes are widely different from those of the sepsis above described. There is little or no fever ; the temperature may be subnormal; as in shock; the bowels seldom are distended. There is simply colossal, almost incessant, vomiting sometimes even to the extent of fecal vomiting. Starvation and the violent exertion of the vomiting soon exhanst the patient.

Treatment.- The vomiting sometimes suddenly ceases without apparent cause. The removal of the sutures or of a drainage-tube has been followed by prompt relief. In one case in the writer's practice the vomiting promptly and permanently eeased upon simply re-opening the lower end of the abdominal wound; nothing abnormal was found, and the wound was closed immediately.

'The diagnosis once made to the exclusion of septic peritonitis, the treatment is simple and effective. It is the free hypodermic use of morphine in doses sufficient to allay all nervous irritation, to induce sleep, and, above all, to give the stomach and bowels rest. Under the influence of morphine food is retained, and in two or three days the patient recovers. The indication also is for hypodermic injections of strychnine, one-sixtieth of a grain every four to six hours, for rectal alimentation and for washing out the stomach through a stomach-tube.

Obstruction of the Bowels as a post-operative accident is not uncommon.

Causes.-In addition to non-surgical causes which may at any time be present, there are those causes that result directly from the 
operation. The bowel may be bent sharply upon itself-i. e., knuckled so as to make ocelusion at the point of flexure. If at the same time adhesions form at or near the point of flexure, immobilization takes place; the bowel cannot straighten itself, and obstruetion is established. Sometimes a part only of the eircumference of the bowel is constricted either in a hernial opening-Littrés hernia-or between bands of adhesion. The divertieulum looks like a nipple as it protrudes from the convex surface of the intestinal loop. On relieving the constriction the nipple disappears, leaving a deeply indented, darkblue ring. This form of hernial obstruction is partial, and therefore less severe than when the bowel is oceluded entirely. Vomiting is less free and less apt to be fecal. Flatus in small quantities may continue to pass. The downward passage of feees is not always wholly interrupted. In cases of vaginal section when the wound is left open and the gauze drain used, the space occupied by the drain may upon the removal of the drain receive a mass of intestine. The result may be adhesion and obstruetion. Occasionally a loop of bowel works its way between the margins of the wound and becomes pinehed, oeeluded, and adherent. This is not a very infrequent result of eapillary vaginal drainage. The evils of drainage have been stated more fully in Chapter Vî́.

Clearly, adhesions are more apt to oceur between surfaces not covered with peritoneum ; henee the importance of careful plastic work during the operation, to cover, so far as possible, all exposed surfaees.

Acute obstruction frequently has followed the use of solid food too soon after an abdominal operation.

Diagnosis and Prognosis.- It is important to distinguish mechanical obstruction, due to kinking, intussusception, or adhesions, from mere failure of the paretic bowel to act. The two eonditions may resemble one another so elosely as to make the distinetion impossible. Reverse peristalsis and consequent feeal vomiting, a common symptom of obstruction, seldom oceurs in paresis. The bowel may be paretic from a grave eause like septic peritonitis, or from some trivial cause.

The diagnosis is the same as for obstruction of the bowel from other causes. Nausea, vomiting, first of bile, finally of feces, abdominal distention, and rapid pulse are among the prominent symptoms. Peritonitis is first loeal and confined to the affected part; but later may become general. Death usually follows within a few days, unless the patient is relieved by surgieal means.

Treatment.-Before proceeding to the dangerous operation of reopening the wound and looking for the eause of obstruetion an attempt should be made to secure relief by means of high reetal enemas and position. In an aggravated case of apparent meelanical obstruction in which the abdomen is distended to the size of full-term pregnaney and strenuous attempts to secure action of the bowels by means of enemas and catharties have failed, the following measures may give prompt relief : 1 . Croton oil, one-half drop to one drop; 2 . A hot stupe of 25 per cent. turpentine covering the entire abdomen, the dressing over the wound having all been removed in order that the stupe nay be applied directly to the skin ; 3 . Frequent change in the 
position of the patient, especially turning on the abdomen. It is most essential to distinguish between obstruction from shutting off the lumen of the bowel and simple failure of action from other causes, for in actual obstruction eathartics might do much harm.

The diagnosis of mechanical obstruction once established, no time should be lost in the attempt to relieve the bowels. If the obstruction has been continuous for thirty hours, and upon reopening the abdomen the operator cannot immediately locate the cause of it and promptly open the way through it, the safer treatment would be to establish an artificial anus, even though a later operation may be necessary to restore the integrity of the bowel and close the sinus.

Obstruction and paresis are much less likely to occur if the bowels have been relieved thoroughly of feces and gas before the operation. See Preparatory Treatment in Chapter II.

Sinuses.-The localized infection described in a preceding paragraph commonly subsides on drainage. Sometimes the source of infection is continuous; then the drainage-track becomes a sinus, and may continue to transmit pus until the infective substance is removed. This substance is usually an infected ligature or intra-abdominal suture which refuses to be cast off. It may remain for months or years a continual nidus of infection and suppuration, or may at any time come away. Spontaneous closure of the sinus upon removal of the infective substance is the almost invariable rule. If not spontaneously thrown off, such ligatures or sutures often may be caught and fished out by means of an instrument aeting on the principle of a crochetneedle, or by means of a very small dull curette. Should these fail and the discharge continue for a number of months, the indication is to cut down and remove the offending cause. 'The operation is usually simple and relatively safe. An incision through the abdominal wall in the track of the original wound commonly enables the operator to dilate the deeper part of the sinus and seize the ligature; if not, the adherent viscera nay be separated carefully until the nidus is reached and removed.

Long-continued suppuration is a reproach to the surgeon; it is annoying, irritating, and, even though slight, tends to produce degeneration of the kidneys and other important organs; hence the necessity of efficient methods for the prevention of it or for removal of the offending source.

Prevention.-The use of absorbable catgut sutures and ligatures, which may be sterilized absolutely - see Chapter II.-is a most satisfactory preventive. Silk, silkworm-gut, metallie, and other nonabsorbable sutures and ligatures, for the reasons indicated, are not generally advised in peritoneal surgery.

Fecal Fistula. - The bowel during an operation may be opened, or so injured that an opening is liable to occur later. In either event the injury should be repaired before closing the abdomen. In a small proportion of such cases the sutures fail, or the bowel opens at some unsuspected point. The result usually is loeal infection, as already described, followed, if the patient survives, by a fecal fistula with discharge of the bowel-contents through a sinus in the wound. 
The fistula, in a majority of cases, if left a few days, weeks, or months, will close spontaneously. Closure is usually more prompt in sinuses through the vaginal than through the abdominal wound. The explanation of this may be that the sinus is shorter and the vaginal wound less accessible, and therefore less tampered with by the surgeon. If the fistula does not finally heal, an operation for the closure of it may be necessary.

Urinary fistula follows the same general laws as fecal fistula. The former seldom occurs except when the bladder or the ureter has accidentally been opened in the operation and the sutures for its closure have failed. The presence of the fistula is recognized by the appearance of urine in a sinus opening through the wound. The treatment is to introduce a self-retaining catheter and keep it in the urethra until the fistula closes. Secondary sutures seldom are required. A ureteral opening is more serious and requires special operative measures. See Chapter XLIII.

Stitch-abscess. - Suppuration in the abdominal wound usually may be avoided by serupulous asepsis. If it occurs, the sutures, unless buried, should be removed, and gauze saturated with 95 per cent. alcohol should be applied, and changed frequently. The alcohol dressing should be continued until healing is complete. In aggravated cases the abscess may have to be opened and drained.

Removal of Sutures.-One carelessly may cut the loop on both sides of the knot; in such a case the ends of the loop retract below the surface and cannot be reached. If the loop does not become encysted, there may be suppuration around it, which will persist until it works out, is fished out with a crochet-needle, or is removed through an incision. Figure 44.

Ventral Hernia.-The chief causes of ventral hernia in connection with abdominal operations are the drainage-tube-see Drainageimproper closure of the wound, and want of proper support to the abdomen by elastic bandages during the first few months after the operation. The longer the incision and the thinner the abdominal wall the greatcr the need of the bandage; incisions not more than two inches long, if properly closed, seldom require it. The treatment is to reopen the abdomen through or near the old cicatrix, split the sheaths of the reeti muscles, and reunite the wound as already directed tor ordinary elosure of an abdominal wound. 


\section{CHAPTER IX.}

\section{THE RELATIONS OF DRESS TO THE DISEASES OF WOMEN. 1}

Maxner of living,' environment, food, sleep, work, rest, recreation, exercise, and clothing necessarily must have a determinate influence on the prophylaxis and cure of disease. The gynecologist, therefore, who gives to this subject its true weight will stand upon a decided vantage-ground over that one whose resources are limited to drugs, local treatment, and operative measures. One of the most serious of all obstacles to the prevention and cure of the diseases of women is fashion in dress.

So long as sensible dress appears ecentric and excites ridicule women will adhere to the prevailing modes, and will therefore be hampered not only in the pursuit of recreation and exereise, but also in the performance of the more essential physiological functions. Under such eonditions fashion must continue to prevail against strong nerves, powerful muscles, and robust health. As soon as the girl passes from the nursery to the drawing-room, and the dress of childhood is changed for the conventional dress of fashion, some of the evils of what we call civilization become manifest. She can neither walk, run, nor even breathe without embarrassment. The fact that women has endured and survived the tyranny of dress for centuries without more serious results, says Emmet, is convincing proof of her power of endurance.

The prevention and cure of the diseases peculiar to women require the fulfilment of three principal conditions in dress :

1. Even distribution for uniform protection against cold and wet.

2. Freedom from waist eonstriction.

3. Freedom from traction.

1. Even Distribution.-Uneven distribution is conspicuous in the prevailing modes of dress. The undergarments are usually of cotton or other light material and are often sleeveless and low in the neck. Numerous skirts hang about the lower extremities and give them relatively little protection. The outer garments are usually of thin material, and, according to the caprice of fashion, may or may not cover the arms, neck, and upper part of the bust. The bonnet is useless for protection. The feet often are held in the vice-like grasp of thin, high-heeled coverings which more resemble stilts than shoes. They fail to protect the woman against cold and prevent free exercise. In contrast with such inadequate protection for the upper and lower extremities, the waist and hips are swathed and compressed

1 The writer, in this presentation of the subject, has adapted freely from the works of Robert L. Dickinson, of Brooklyn, and J. H. Kellogg, of Battle Creek. 
in a "torrid zone" of whalebone, corsets, belts, steels, skirts, and other cumbersome material.

2. Waist Constriction comes chiefly from the corset, which not only constricts the waist, but also dislocates the thoracic viscera upward and the abdominal viscera downward. It restrains the abdominal and dorsal muscles, and may eanse them to atrophy from disuse. It prevents, by its stiffness, the undulatory movements of the abdominal walls and restricts peristalsis.

Normal breathing requires the lungs to be expanded in all directions, and is therefore not costal nor abdominal, but a combination of both. Waist constriction immobilizes the abdomen, and thereby prevents abdominal breathing. This involves a loss in lung-power which cannot be supplied by any compensatory increase in costal breathing.

Figine 74.

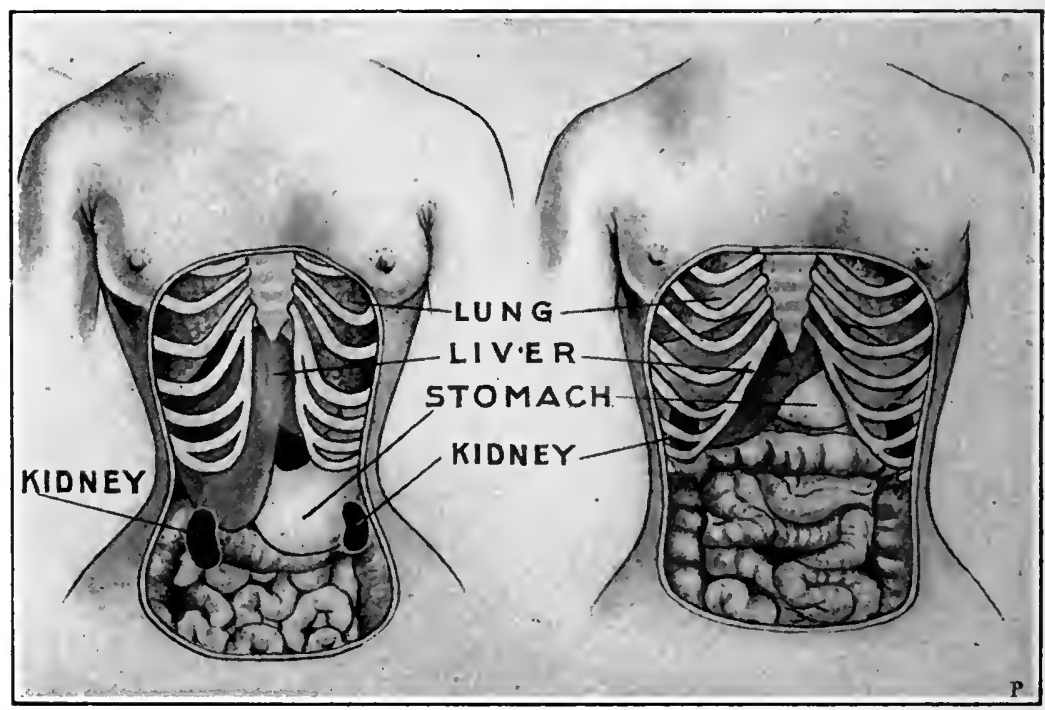

The left-hand figure is corset-deformed, with the thoracic and abdominal organs displaced The right-hand figure is normal, with the thoracic and abdominal organs in place.

Moreover, the diaphragm from upward pressure, and the pelvic floor from downward pressure, are rendered inactive and atrophic, and are thereby unable to make their upward and downward movements which normally should be transmitted to the abdoninal and pelvic viscera. The physiological importance of these respiratory movements is very great. They are a sort of natural massage. The descent of the diaphragm with each inspiration increases pressure in the abdominal cavity and lessens that in the ehest. The reverse of this occurs with expiration.

Alternating pressure and relaxation upon the blood- and lymphvessels secure free circulation. Alternating contraction and relaxation of the muscular bundles of the uterine ligaments and of the other elastic and muscular parts of the pelvic floor serve to maintain their 
normal nutrition and tone. Alternating rest and motion are essential to the health of the organs and their supports ; waist constriction immobilizes them and stops their physiological movements. 'The pelvic veins empty into the greatest area of corset pressure; the long and perpendicular column of blood of this area is by such pressure dammed back upon the pelvic organs, especially upon the ovaries. The consequence is passive congestion, an unfailing source of disease. Even the loosely worn corset excites great downward pressure whenever the woman stoops forward, as she must do in sitting and rising. Sewing-women, clerks, writers, and students, who wear corsets, are especially subject to this evil. ${ }^{1}$

The garter is injurious from its tendency to obstruct the venous circulation in the legs.

3. Freedom from Traction. - The abdominal and dorsal muscles and the hips have to carry the weight of numerous skirts and such other garments as usually oppress that area. In the effort to sustain this weight the muscles become permanently tired, lose their tonicity, and are powerless to prevent a still further increase of downward pressure upon the pelvic floor and pelvic organs.

Figure 74 is given to illustrate some of the evils of undue pressure and uneven traction.

To compare ordinary modes of dress with those which give freedom of motion, "one has only to look at a lot of girls on the way to the gymnasium," said a Vassar teacher. "They drag along; they have no spirit nor spring in them; they are in their ordinary clothes. Look at the same set coming on the gyinmasium floor in their light toggery ; they skip and dance and run in the liberty of unrestrained and untrammelled motion; they are different beings."

In laying aside waist constriction aroid half-way measures, such as loosening the corset or substituting the so-called health-waist, which too often is only an aggravated form of corset. Leaving off the corset altogether and retaining the numerous skirts with their bands and belts to drag upon the waist and hips rather increase than lessen the evil. The only judicious compromise is temporary support by means of a suitable waist having little or no stiffness, which shall cover the shoulders, and upon which skirts, drawers, and other garments may be buttoned, so that their weight may be distributed over the shoulders. This should be worn, if at all, only during the period of agrarated weakness, especially weakness of the back, which follows the withdrawal of the corset and continues until the weakened abdominal muscles have regained their tone.

The conventional dress has, until recently at least, consisted of nine garments four hanging from the shoulders and five from the waist, namely :

1. Undershirt.

2. Chemise.

3. Corset-cover.

4. Dress waist.

${ }^{1}$ Adapted from R. L. Dickinson. Harc's System of Practical Therapeutics, vol. iii pp.
733. 
5. Underdrawers.

6. White drawers.

7. Corset.

8. Flannel skirt.

9. Dress skirt.

Counting each band as two thicknesses, these make seventeen layers about the waist; and allowing twenty-five inches as waist eircumference, these seventeen layers if joined end to end would make a bandage thirty-four feet long.

Hygienic dress requires four garments, namely:

1. Union undergarment.

2. Equestrienne tights.

3. Muslin waist and skirt.

4. Dress in one piece, or so made that its principal weight may be distributed over the shoulders, bust, and hips. This makes four layers about the waist.

1. The union undergarment is a union of the modershirt and drawers in one piece; the open stride is supplied with the broad flap, as a protection to the external genitalia and to guard the other garments from their secretions. The material of the suit may be silk, wool, or cotton, or any mixture of these. In winter it should be heavy, with high neek and long sleeves, and should reach to the ankle. In summer it may be lighter, with lower neek and short sleeves, and should reach to the knee.

2. The equestrienne tights are the substitute for the heavy woollen petticoat, and are designed for out-door use in winter. 'They reach from waist to ankle, corresponding to the man's trousers.

3. The muslin skirt and waist often are made in one piece, but there are practical advantages in making them separate. The waist, if separate, should reach well down over the hips, and the skirt, made without band, should be buttoned to it. The open stride of women's garments is a great source of infection, since, in conjunction with the dust-sweeping skirts, it exposes the external genitals to the entrance of dust and other fine particles, which are always irritating and often the vehicle of infections bacteria. Closed muslin drawers are therefore desirable as a means of protection, and these also may be buttoned to the waist.

4. The dress may be in one piece, after the "princess" pattern; or, if in two pieces, the skirt, unless too heavy, may be attached to the waist with hooks, in which case its lining may be continued over the shoulders in the form of a carefully fitted skeleton waist.

The garments just described may be modified in many ways to suit individual taste and changing fashion, but the essential principle must be observed, viz. : uniform distribution, freedom from undue weight and traction, and freedon from constriction. Light whalebones may be useful in the waist-seams for very stout women with pendulous breasts. Proper dress and eonsequent freedom of motion will stimulate the woman to ont-door exereise and in-door gymnasties, which, if followed with system and perseverance, usually will give normal tone to the abdominal and thoracic muscles and normal firmness to the breasts. 
Artificial support, therefore, except in aggravated cases, is to be discouraged.

Union undergarments of all grades and deseriptions, adapted to the needs and eircumstances of all classes, now may be found in the shops. Eeonomy, health, ccmfort, and, to the properly educated sense, beauty, all combine on the side of proper dress. It is marvellous that the monstrosities of fashion have overshadowed so completely the natural beauty of form and figure. From the standpoint of beauty shall we choose the natural lines of the body or the artifieial lines of the eorset, the garment fitted to the woman or the woman fitted to the garment? Imagine the attempt to add to the dignity of the lion or to the beanty and grace of the greyhound by the use of artificial means to change the natural lines of their bodies. Throwing aside the all-controlling bias of fashion, who shall say that the woman is so inferior to the lower animals in form and figure that she nust be taken-in in some places and let-ont in others? In this connection the words of Herbert Spencer have peculiar force: "Nature is made better by no mean, but Nature makes that mean; over that art which you say adds to Nature, is an art that Nature makes. 



\title{
P A R T II.
}

\section{INFECTIONS, INFLAMMATIONS, AND ALLIED DISORDERS.}

\author{
CHA PTER X.
}

\section{GENERAL CONSIDERATIONS OF INFECTION AND INFLAM- MATION OF THE REPRODUCTIVE ORGANS.}

INFECTION of any one of the reproductive organs is liable to have the closest relations to similar infection of a part or all of the others; for this reason an intelligent consideration and satisfactory explanation of the morbid process in any one organ may necessitate a study of infection in the pelvic organs as a whole. The distinction between infection and inflammation is of the greatest practical importance.

\section{Definition of Infection.}

Infection is that condition in which foreign media of irritation have gained access to an organ, and either mechanically or by means of their products, disturbed its functions. These media are capable of being transmitted to other organs and other individuals. In most cases, at least, the invading irritant, if known, is of bacterial origin. The organisms, unless arrested, are prone to multiply rapidly, to invade new territory, to transmit themselves and their toxic products to the general circulation, and to destroy or seriously endanger the life of the patient.

\section{Definition of Inflammation.}

The local territory irritated by the organisms and their toxins becomes a centre to which lencocytes in variable numbers rapidly migrate, and in this way the process often called seroplastic infiltration is established. By this infiltration a limiting wall is formed around the infected space. This wall confines the infective process to narrow limits, and may protect the general system against the toxins. The formation of the limiting wall gives rise to heat, redness, pain, and swelling: this is inflammation. In view of these facts, inflanmation is not really the disease, but an effort to limit the disease. The almost universal use of the worl inflammation to signify the disease makes it difficult in the description of the morbid proc- 
esses to conform to the ideas above expressed. The attempt will, however, be made to use the two words infection and inflammation in their proper relations.

\section{Etiology of Infection and Inflammation.}

It is important to remember that the study of a morbid process in an organ or group of organs is simply the study of their anatomy and physiology as modified by that process. The inflammatory process has been defined as the reaction which living tissue exhibits to morbid irritation. This definition being correct, two conditions must be essential for the development of infection and inflammation : The soil must be prepared and ready to react to the morbid irritation. Clearly, tissue which has the power to resist the irritation and to hold it within physiological bounds will not inflame. There must then be :

1. Favoring conditions.

2. Exciting causes.

1. Favoring Conditions.-The various so-called diatheses, such as gout, rheumatism, anæmia, diabetes, lithæmia and cholæmia, have been set down as favoring conditions which act as predisposing causes of infection in the pelvic organs, but the exact relation which they have to the infection is somewhat speculative.

Local favoring conditions are apparent in the anatomical and physiological arrangement of the pelvic organs. The genital tract, from the vulva to the peritoneum, is an open canal, patent to the atmosphere below and terminating above in the free open ends of the Fallopian tubes. It is not only open to such microbic germs as abound in the air and penetrate everywhere, but is also a place of deposit for virulent bacteria.

Rupture of the capillary vessels of the endometrium in menstruation and of the Graatian follicles in ovulation, although physiological, results in solutions of continuity and in hemorrhage, and is therefore traumatic. These traumatisms and the menstrual engorgement of the pelvic organs under healthy conditions pass by with little or no discomfort; but if some morbid irritation upset the normal balance of nutrition, the menstrual congestion may become pathological and may be the first stage of an inflammation. Morbid congestion, though less frequently, also may be set up in the intermenstrual period independently of the menstrual congestion.

In addition to the physiological traumatisms already mentioned, the traumatisms of parturition, of abortion, of improper local treatment, and of operations still further open the way for the entrance of infection. Violent coitus, masturbation, the careless use of the unclean catheter, impure water in bathing, and soiled linen in the toilet are some of the means by which gonorrhoal, syphilitic, and other infections may develop in the genital tract.

The conditions of uterogestation, parturition, and the puerperium are specially perilous; hence infection of the puerperal woman is specially destructive. Decomposed secretions and the products of fatty degeneration from involution and from the menopause favor 
the development of pathogenic microbes. Tumors, displacements, tight lacing, and constipation are among the common local predisposing causes of morbid congestion and consequently of infection in the pelvis. Clearly the faroring conditions above outlined contribute to the preparation of the soil for infection.

2. The Exciting Causes comprise agents that have the power to produce and to mainitain morbid irritation. Greatly preponderating, at least among these, are the pathogenic microbes and their produets. The extent to which inflammation may be produced by irritants of non-bacterial origin without the presence of any bacteria whatever is largely a laboratory question, and is not fully settled. Among the pathogenic microbes not seldom found in the genitalia are the staphylococci and streptococei of suppuration, bacillus tubereulosis, bacillus coli communis, and the pneumococeus of Fränkel. Bladder parasites and the saprophytes from the rectum and colon have easy access to the genitalia. See Chapter II., on Antisepties and Asepsis. The bacillus coli communis lives in acid media, and can thus easily pass through the acid secretion of the vagina to the uterus.

The gonococcus of Neisser, one of the most frequent, destructive, and insidious factors in genito-urinary infection, is discussed partially elsewhere in connection with Vulvoraginitis, Salpingitis, and Acute Metritis; its chief power for harm lies in the lasting vitality of the germ long after apparent cure. The gonococeus may remain inactive in the mucous crypts, liable at any time, even while quiescent in the individual, to be communicated to another. Hence many an innocent and previously healthy woman, shortly after marriage to a man who supposed himself to have been cured of gonorrhoea years before, may by contact with the attenuated virus get a destructive gonorrhoal infection of the genito-urinary organs.

Some most important observations upon this subject have been made by Wertheim. A direct experiment with pure eulture from a gleety discharge of two years' standing gave the following interesting results: 1. Attempted reinfection of the original urethra with this culture was always a failure. 2. The culture when transplanted to a coceus-free urethra produced typical acute gonorrhoea. 3. Infection from this back again to the original urethra gave a fresh gonorrhoa, which after a typical acute course of five or six weels again subsided into a chronic gleet. Thus, by passing the gonococei through another individual-that is, through a new culture-ground-they became again virulent to the urethra which was invulnerable to them before.

This explains the fact that an apparently healthy subject of ehronic gonorrhoea may infect his hitherto minfected wife and become again infected from her-i.e., the gonococei by passing through the new culture of the wife again become virulent for the husband. In due time each becomes tolerant of the germ ; which, however, may develop acute infection in another person. The common notion that gonorrhoea in women may be chronic from the beginning is wakened by the experiments of Wertheim. Wc can now understand why the gonococcus, even after years of apparent cure, may regain its full virulence. 
The greatest danger of gonorrhœa, extension to the Fallopian tubes, will be considered in the chapter on Salpingitis. The microbe may be found in the uterus and tubes long after it has disappcared from the vagina. The parement epithelium of the vagina and the acid reaction of the fluid normally found there make the vagina relatively immune. The crypts of the uterine and tubal mucosa furnish a ready restingplace for the germ. Even here, in many cases, it is found only during the exacerbations. Menstruation favors, but does not insure its revival. It may for long periods remain concealed in a semiquiescent state, a destroyer of health, a menace to life. The frequency of chronic gonorrhoa-the latent gonorrhoea of Noeggerath-as set forth in valious hospital statistics ranges from 25 to 80 per cent.; at any rate the percentage is very high.

The statistics quoted above are taken from clinics largely made up of prostitutes and semiprostitutes, a fact which necessarily will modify a juclicial estimate of their value. It is, moreover, essential to appreciate two other facts : first, the evidence on this most complicated question, although sufficient to lead to the greatest apprehension, is not yet sufficient to establish definite and undeniable proof on the extreme side of the question; second, many excellent clinieal observers in private practice are disposed, on the whole, to qualify the danger and to conelude that it is vastly overestimated. If the questions involved were matters only of scientific interest, their solution conld properly wait for further and more exact observation; but the "danger and duty of the hour" are concerned with moral, not scientific, problems, and the moral obligations are serious enough to lead the writer to present the subject even from the ex parte standpoint.

Why do large numbers of apparently healthy young women date their pelvic infection from the marriage-week? Is it, as one author declares, the "fatigue and excitement of the wedding-journey"? Why do so many women with perfectly developed reproductive organs remain sterile from the time of marriage or after the birth of a single child and a dangerous "childbed fever"? The causation of too many such cases of hopelessly diseased uteri, tubes, and ovaries, not to mention proctitis, with sometimes rectal stricture, urethritis, cystitis, pyelitis, and nephritis, has been explained by the word idiopathic. The histories, if written, would tell often of an apparently cured gonorrhoa, before or after marriage, in the husband. If the most destructive infection may follow contact with a subject of gonorrhœa after the discharge has ceased, how perilous must be the slight gleety discharge so often disregarded! Young men sometimes are advised to marry in order to improve their sexual hygiene, and so to cure an intractable chronic but "innocent gleet." Such advice may result in the destruction of the reproductive organs of an innocent woman. It is doubtless possible, perhaps not unusual, for gonorrhea to be so cured that the individual cannot transmit the disease. Failure, however, to eultivate the gonococeus from the urethral secretions does not prove its absence. So long as it can be cultivated marriage should be prohibited. In every suspected case marriage should be deferred at least until repeated attempts at culture have failed. A gonorrhoal 
record does not necessarily settle, but it always complicates the question whether the individual may safely marry.

\section{Pathology and Course of Infection and Inflammation.}

Bacterial invasion and consequent infection may spread and involve any or all of the genito-urinary organs by either or both of two routes :

1. By continuity of mueosa.

2. By the lymphatics or blood-vessels.

1. Infection by Continuity of Mucosa.-The course is usually upward from the vulva or vagina, through the uterus and Fallopian tubes to the ovaries and peritoneum, or through the urethra, vagina, bladder, and ureter to the kidneys. The numerous glands of the vulva are strongholds where the infection may intrench itself and whence a eonstant supply may find its way to the organs above.

The vagina, advantageously covered with pavement epithelium, is relatively smooth, like skin, and is supplied with an acid secretion. Bacteria, accordingly, find lodgement there less easily than in the vulva. Moreover, the acid medium unfavorable to the growth of about 90 per cent. of all pathogenic microbes makes the vagina a barrier difficult to pass.

The uterus, although protected by the above anatomieal and physiological conditions of the vagina, is itself especially vulnerable on account of the loose arrangement and thinness of the epithelial covering, the villous network of the arbor vitæ of the cervix, the confluence and ramifications of the glands, and the richness of the periglandular and perivascular network. By reason of these conditions the cervix uteri is adapted to receive, retain, and distribute infection. Were it not for the nuscular constriction at the external and internal ora and the uterotubal constrictions the frequency of infection of the endometrium would be much greater.

The Fallopian tubes are embryologically and anatomically continuous with the uterus; they are, in fact, a part of it, and subject to the same causes of infection. The ovaries and pelvic peritoneum, in direct communication with the tubes, may receive infection from below. Infection by continuity of mucosa, however, although usually from below, does not always come from that direction; it may reach the ovaries and pelvic peritoneum from above, and descend through the tubes, uterus, and vagina to the vulva. Tubcrcular infection, for example, usually goes in this direction.

2. Infection by the Lymphatics and Blood-vessels is undeniable in puerperal women. The traumatism of parturition, often very extensive all the way from the uterus to the vulva, nay open wide the door for infection to be transmitted by the vessels. The destructive influence of the infection-i.e., phlebitis and lymphangitis-on the vessels themselves may impair seriously and permanently the nutrition of all the pelvic organs.

It is believed commonly that, except in puerperal cases, infection travels by eontinuity of mucosa, and not by lymph-and blood-ressels 
in the deeper structures. It is clear, however, that since infection, as is proved by the bubo, often is transmitted by way of the lymph-vessels to the inguinal glands, it may travel also by way of the lymph-vessels a much shorter distance, from the vagina or cervix to the parametria and Fallopian tubes. This reasoning by analogr has been verified by experiment. Some observers, notably Lucas-Championière, ${ }^{1}$ maintain that this is the more common mode of infection. Wertheim, from experimental investigation on white mice, rabbits, dogs, and guinea-pigs, concludes that gonococcus infection can pass through pavement epithelium and connective tissue so as to reach the deeper lymphatic and vasenlar channels, and be carried by them from the vagina or cervix to the ovaries, tubes, and peritonenm, producing thus ovaritis, salpingitis, and peritonitis. Giglio ${ }^{2}$ also experimentally demonstrated that infection may travel from the vagina, cervix, and bladder to the broad ligaments and may produce extratubal pelvic abscess. He maintains that infection by the vessels is more frequent than by continuity of surface. When the latter occurs he asserts, but withont proof, that it is more commonly in the descending order from the tubes to the uterus.

Continuous infection does not always mark the course of the microbes through the vessels. The microbes develop at the points of least resistance; hence the tubes may suppurate and the ligaments and ovaries go free. When, however, the inicrobes travel by way of the mucosa a continuous inflammation is usual, though not invariable.

Infection by the veins is especially common in puerperal cases. It often has produced general septicemia and pyæmia through very slight lesions. The arteries also may carry infection. This is proved by the fact that bacteria have been found in places where they must have been carried by the centrifugal circulation; for example, the gonococcus in the knee-joint. ${ }^{3}$ Hetero-infection of the genitaliai.e., infection from withont-is not the invariable rule. Diseased extrapelvic organs may send their germs by way of the lymphaties or blood-vessels, and produce secondary infection of the pelvic peritoneum, ovaries, tubes, and other genitalia. Tubereular infection of the tubes, secondary to that of the lungs, is a familiar example.

Experiment and clinical observation also show that both puerperal " and non-puerperal infection may travel by blood-vessels, by lymphchanuels, and by continuity of surface. The relative frequency, however, of these modes of transmission is a matter of speculation. Probably the ronte by continuity of surface is really a superficial lymph-route-that is, the infection may travel along the lymphchannels of the mucosa.

\section{Classification of Infection and Inflammation.}

Let us now raise a question relative to the looseness and confusion of the current classifications. The term simple infection as distin-

1 Paris Surgical Society Transactions, December, 1888. New York Medical Journal, March 22, 1590 .

2 Giglio. Annalio di Obstetricia e Ginecologia, May and June, 1893.

${ }^{3}$ Luther. Sammlung klinische Vorträge, 1893. 
guished from septic, for example, has no strict pathological meaning. Is the so-called simple infection aseptic or is it only slightly septic? We know that an infection seemingly very mild may take on readily a decidedly virulent character. What is there in sucl conditions to designate on the one hand as simple, on the other as septic? In the present state of our knowledge we must use for deseriptive purposes an adaptable, and therefore fiexible, nomenelature. In this nomenclature words like simple and septic can have only a loose clinical significance. They cannot be utilized as the onteome of scientific classification. We may simplify the subject by throwing out such a word as simple.

$A$ distinction between acute and chronic inflammation, since these conditions enter extensively into the pathology of the diseases of women, is most important. Many deny altogether the existence, for example, of chronic inflammation of the endometrium. Some attribute the condition which usually is classed under that name to congestion; others call it a subinflammatory state. It may be well to remark that an essential factor of inflammation-round-cell infiltration-is found in those chronic conditions, and that they may therefore be classed properly as inflammatory; the migration of white corpuscles, however, occurs more slowly, and may in some cases be very slight. In this respeet the difference between acute and chronic inflammation is one of degree. We shall avoid the question whether certain conditions should be ealled congestive, inflammatory, or subinflammatory. The discussion of this question is tiresome and unprofitable-a contest largely of words. The following ontline of some of the phenomena of inflammation will help make clear the distinction between acute inflammation and the conditions which usually are grouped under the name chronie inflammation.

The inflammatory reaction which living tissue exhibits to morloid irritation is first defensive, and then constructive or reparative. The defensive process is an effort to circumscribe the disease by throwing around it a limited wall of exudate; the morbid foree this confined and concentrated within narrow limits is within these limits nore or less intense and destructive. It may result in the sacrifice of a part for the safety of the whole. The force of the disease is spent in the destructive process, and may be active only or chiefly within the limiting wall. Finally, normal conditions of nutrition are re-established, the constructive or reparative process becomes active, and the limiting wall is absorbed. If the constructive process continues until repair is complete and then ceases, the part will resume its normal functionsthe inflammation will be at an end.

Acute Inflammation.- If the infection is of such virulence or otherwise of such character as to call forth the defensive processes; just deseribed, and to produce blood-stasis with more or less severe swelling, pain, heat, and redness, and finally to produce local destruetion, the inflammation is acute. The disease may terminate with resolution or go on to suppuration.

Chronic Inflammation.-If the irritation is of minor intensity, or in any other way of such character as to fall short of provoking 
much defensive action, there will be little or no limiting wall, and consequently no intense destructive process concentrated within a circumscribed space; heat, swelling, pain, and redness, if present, will be more diffuse and less pronounced. Chronic inflammation occurs under these conditions-a minimum of defence and an excess of construction.

Chronic inflammation may follow acute infection, or may have been subacute or chronic in the beginning. The excessive constructive action which belongs to it explains the hyperplastic and hypertrophic results of so-called chronic metritis. It also explains certain morbid nutritive changes in the blood-and lymph-vessels of the pelvis and in the cellular tissue of the pelvis. Sclerotic changes in other organs, such as arterial sclerosis and interstitial nephritis, offer a close analogy.

It is unprofitable to speculate on the question whether the conditions just described under the name chronic inflammation may better be classified as congestive or as subinflammatory states. They are recognizable under either of these names. They occur more frequently in neuropathic women, and especially in cases of the various diatheses-anæmia, lithæmia, gout, cholæmia. They are usually less dangerous to life and often more destructive to health than the acute inflammations. They constitute a large proportion of the diseases of women and include some of the most distressing ailments. They are persistent and hard, often impossible, to cure. In such cases it is frequently difficult to draw the lines between those congestions which fall short of inflammation and actual inflammation. One of the most common forms of so-called uterine catarrh is that which occurs in women of deficient eliminative power-that is, the bowels, kidneys, and other eliminative organs fail sufficiently to throw off the wasteproducts. Under these conditions the mucous glands of the uterus, for example, whose function is not excretory, may undertake vicariously to make good the deficiency. An unspeakable amount of misdirected and injurious local treatment is constantly being applied to the endometrium in such cases.

The significance of pelvic infection varies according to the resistance of the patient, to the location and nature of the structures involved, and to the virulence of the cause which produced it. Decided predisposing causes make the woman less able to resist morbid irritation; and infection once established is more likely to be severe and progressive. If infection is confined to superficial areas, its gravity is relatively much less than when deeper structures are diseased. Endometritis, for example, is less serious than an inflammation involving the uterine wall or the parametric lymphatics and veins. Moreover, the same kind of infection may be more serious in some places than in others. This may be illustrated by the ease of a man who picked his teeth with a vaccine point and experienced a most distressing result. Some bacteria are harmless and some only mildly virulent. The gonococeus is more frequent, and therefore more disabling than the staphylococcus. The streptococeus pyogenes is more dangerous than either.

From the foregoing it is easy to explain why an infection, even 
in the deeper structures, may, if not from very destructive bacteria, present in the more acute stages most of the subjective and some of the objective appearances of a fatal disease, and yet after a few days terminate in complete health. The reason is also obvious why a superficial vulvar infection, apparently innocent, may be the result of a gonococcus or of a streptococcus invasion, and may by continuity of surface, or by way of the lymphatics or veins, finally destroy life or render it miserable and useless. Some organisms may excite little or no defence-i. e., may not attract leucocytes-and may therefore sweep through the system with rapidly destructive and fatal force. The germ of tetanus, for example, gives rise to infection but does not excite defensive inflammation.

\section{Diagnosis, Prognosis, and Treatment of Infection and Inflammation.}

- The symptoms are often utterly disproportionate to the gravity of the lesions. An infection of little danger nuay cause the greatest suffering; another, which directly threatens life, may be almost painless. Objective examination, therefore, especially in acute cases, is important. The subjective symptoms may be misleading. The prognosis depends upon the region infected, the general and local resistance of the patient, and the extent and nature of the infection.

The treatment requires the individualization of each case, and the reader must therefore be referred to the special subjects. 


\section{CHAPTER XI.}

VULVITIS, VULVOVAGINITIS, VAGINITIS.

INFECTIONs of the vulva and vagina may oecur separately, but they commonly appear in combined form: to avoid repetition and to simplify the subject therefore they will so far as practicable be described together.

The importance of vulvitis and vulvovaginitis commonly is underestimated. Inflammation seemingly trivial may start in the vulva and rapidly extend to all the reproductive and urinary organs, and may, therefore, give rise to metritis, salpingitis, ovaritis, peritonitis, urethritis, eystitis, pyelitis, and nephritis.

The external genitals are the labia majora and minora; the clitoris, with its prepuee; the vestibule, including the neatus urinarius; the fossa navieularis, and the lymen. The hymen separates the external genitals from the vagina. The eovering of the external genitals is cutaneous, although it partakes somewhat of the nature of mucous membrane.

\section{Definitions.}

Vulvitis is inflammation of the external genitals.

Vaginitis is inflammation of the mucosa and submueosa of the ragina.

Vulvovaginitis is inflammation of the vulva and vagina.

\section{Classification.}

The inflammation, which may be acute or chronic, has been elassified:

1. Bacteriologically, aceording to the nature of the bacterial eause which mav have produeed it.

2. Anatomically, according to the special structures involved.

1. Etiological Classification.-The following inflammations are of bacterial origin. They may occur as vulvitis, as vaginitis, or as vulvovaginitis :

1. Gonorrlıeal, eaused by the gonocoeeus of Neisser.

2. Erysipelatous, caused by the streptococcus.

3. Diphtheritic, caused by the Kklebs-Löffler baeillus.

4. Tuberenlous, caused by the tuberenlar bacillus.

5. Mycotic, eansed by the leptothrix and Ö̈dium albicans.

6. Syphilitic, cansed by the bacillus of syphilis.

7. Chancroidal, caused by Krefting's bacillus.

Other forms of vulvovaginal infection are caused often by other micro-organisms, chief among which are staphylococeus, aureus, albus, and eitreus, and the umrecognized micro-organisms of infectious 
and contagious diseases. Psendodiphtheric vulvovaginitis is a form in which a false membrane is developed, but is not due to the bacillus of diphtheria.

Inasmuch as the specific micro-organism seldom is recognized at the bedside, the above classification is perhaps more important from a scientific than from a clinical point of view.

2. Anatomical Classification.-Vulvar and vaginal inflammation may attack special structures, such as the skin, mucons membrane, cellular tissue, glands, and follicles. This is without reference to the particular nature of the micro-organisms which may have been the irritating cause of the infection-i. e., the anatomical forms may come from bacteria of widely different natures; these forms, which may oceur singly or in combination with one another, are:
Superficial vulvovaginitis, Senile vulvovaginitis, Glandular vulvitis,

Paravaginitis, Follicular vulvitis, Furuncular vulvitis.

A peculiar vaginal disease has been described under the name emphysematous vaginitis.

The classifications above ontlined cannot from the clinical standpoint always be followed. An effort, however, to differentiate between the various forms should, for both clinical and scientific reasons, be attempted.

Eczema, kraurosis vulvæ, herpes vulvæ, and other allied disorders will be presented in the following chapters. Pruritus vulvæ and vaginismus, although often symptoms of vulvovaginal inflammation, are of neuropathic significance, and therefore sometimes are classed among the gynecological neuroses.

The general consideration of vulvar and vaginal inflammations includes certain factors in etiology, pathology, and diagnosis which are more or less common to all varieties. To avoid repetition and to give a general impression of the whole subject, these factors may be studied before taking up the special form.

\section{General Consideration of Etiology.}

The Favoring Conditions which predispose to inflammation in general have been outlined in Chapter $\mathrm{X}$. Among the conditions which specially predispose to vulvovaginal inflammation are the following:
Filth,
Obesity,
Defective nutrition,
Foreign bodies, such as pessaries, etc.,

Vaginal fistulæ,

Excessive coitus,

Masturbation.

Diabetic and other irritating urine.

Exciting Causes.-Numerous bacteria, some of which have been indicated in the etiologieal classification, are undoubtelly the essential causes of the various forms of vulvovaginal inflammation. 
Vulvovaginal inflammation is occasionally, and especially in children, a sequel of such acute infectious diseases as diphtheria, scarlatina, noma, and smallpox.

The Media of Infection may be : vagina.

Pathological discharges from the uterus, Fallopian tubes, and

Pelvic abscesses discharging into the vagina.

Urine and feces.

Carcinomatous discharges.

Pediculi pubis.

The disease may, especially in cases of severe pruritus, come by extension from the anus; very often the morbid irritation is furnished by organisms from the diseased bladder, ureters, or kidneys. Infection may originate in the vulva or from the surrounding cutaneous surface. It may result from direct infection or from an irritating discharge from some higher zone in the pelvis.

Filth outranks every other cause, with the possible exception of the gonococeus.

Epidemics and endemies of vulvitis have been recorded.

In fat women of sluggish capillary circulation the vulva is supersensitive to undue irritation. The excessive oily secretions undergo decomposition into fatty acids, which cause intense, intractable erythema of the vulva, and often of the thighs and nates, a condition aggravated by filth-i.e., by accumulated and decomposed secretions, especially in warm weather, when perspiration is free. Masturbation may be a cause or a result of the disease.

The determining factors of etiology, especially in chronic vulvovaginal infection, lie, first, in the predisposition of the patient; second, in the nature of the infection; third, in its location. Badly nourished, neuropathic, diathetic women are predisposed to chronic infection. Some bacteria, notably the gonococci, are especially apt to produce intractable infection. A principal factor in the chronicity of the disease is the intrenchment of the bacteria in the vulvar glands and follicles, from which fresh infections may travel upward to the vagina and uterus. In the same way bacteria may exist in the muciparous glands of the cervix uteri, and from this point be distributed not only to the parametria, corpus uteri, tubes, peritoneum, cellular tissue, and ovaries, but also downward to the vagina and vulva. The vulva and the cervix uteri, especially the latter, are two great distributing points of pelvic infection.

\section{General Considerations of Pathology and Pathological Anatomy.}

Catarrhal, suppurative, hemorrhagic, and ulcerative processes are rather phases than varieties of inflammation. The process is catarrhal when the product is a pathological increase of the normal secretion, suppurative when it eontains pus, hemorrhagic when it contains an appreciable amount of blood-i. e., when the destructive process has opened the walls of the vessels-and nlcerative when there is localized necrosis. The catarrhal often precedes the suppurative infection by a distinct period. 
The skin or mucous membrane in ehronic cases usually becomes thick and odematous. The pyogenic microbe does not produce suppuration until the structures are impaired in a degree. $A$ circumscribed suppurating surface may be surrounded by an area of eatarrhal inflammation. The neerotic tendency may not go beyond erosion; it merely may impair without destroying the skin or mueosa, or it may extend far below the surface and form a deep ulcer. Vulvitis and vaginitis may exist separately or together.

So-called granular vulvovaginitis is due to swelling and hypertrophy of the vulvovaginal papillæ, is found chiefly in the vagina, and,

Figure 75 .

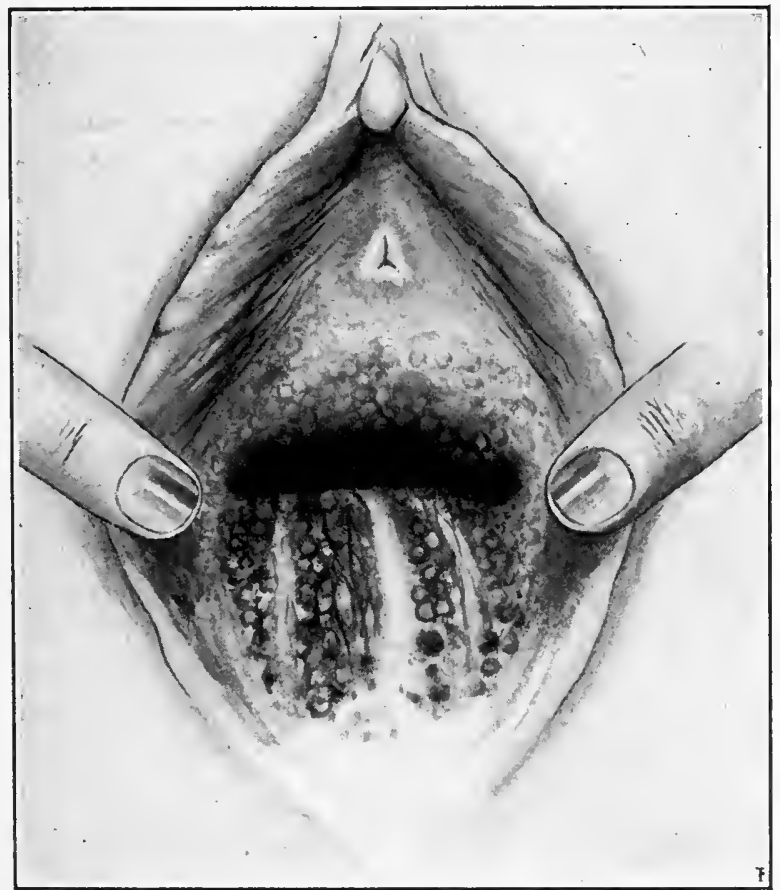

Granular vaginitis. Observe the purulent secretion on the posterior vaginal wall.

though not confined to that period, is commonest during pregnaney. It is charaeterized by small, round, protuberant granulations seattered thickly over the affeeted surface.

The inflammation may result in extensive ulceration of the volva or vagina, or of both. Sufficient plastic material may be thrown out to cause adhesions more or less firm between the nymphæ or the labia majora, or between the vaginal walls, or between the vagina and the cervix uteri. Partial or complete closure of the vulva by allhesions is not uneommon in ehildren. Such adhesions usually vield to slight force. They resemble the adhesions sometimes found between the prepuce and the glans penis of the male ehild; they also may 
occur between the clitoris and its prepuce, and may give rise to serious nervous disturbances. Strong adhesions are less likely to oceur in married women than in virgins and aged women whose organs are at rest.

Gonorrhœa excepted, suppuration is confined mostly to the vulvitis of children, especially children with defective nutriţion. The purulent secretion of vulvitis or of vaginitis is creamy, abundant, and malodorous. Numerons minute points of superficial suppuration in a limited area may run together and form an ulcer. In this way many areas of ulceration may be formed. If ulcerative changes involve the small blood-ressels, the secretions will be streaked with blood. Severe cases may present hemorrhagic areas, great swelling, and even gangrene.

Extension of vulvar inflammation to the vagina is common, though not so common as it would be were it nct for the following anatomical and physiologieal conditions of the vagina : it is smooth, and, being covered with pavernent epithelium, closely resembles skin; is almost if not quite destituce of glands, and is therefore not subject to intense eatarrhal affections.

Döderlein has distinguisher? microscopically two secretions of the vagina : one the normal secretion, a whitish, nilky, strongly acid discharge without mucous admixture ; the other a pathologieal secretion, yellowish, faintly acid, often neutral or alkaline, sometimes foamy and mixed with mucus. In the normal secretion a non-pathogenic vaginal bacillus was found constantly present. Döderlein's experiments with cultures showed that this bacillus gives to the normal secretion its acid reaction, which is due to lactic acid. These normal vaginal bacilli were found to be unfavorable to the growth of Staphylococcus pyogenes aureus. In fact, the vast majority of pathogenic bacteria do not thrive in an acid medium. In the pathological secretion Döderlein found the pathogenic bacteria to be increased and the normal vaginal microbes to be decreased. The abnormal secretion ustally originates in, the cervix uteri, is toxic to animals, and by its hostility to the normal vaginal microbes decreases or nentralizes the acidity of the vaginal secretion, thereby affording a fuvorable cultureground in the vagina for pathogenic bacteria.

When vaginitis occurs, the desquannated cells of raginal epithelium give rise to a thick, pasty accumulation of smegma not unlike vernix cascosa. When the epithelium is shed and the decper structure exposed, pus may be thrown off from the exposed surfaces.

Vulvovaginitis, if superficial, strongly tends to recovery. It becomes obstinate when the vulvar glands already described are involvel, and may be intractable when the infection reaches the muciparous glands of the uterus. Reference is made to the remarks in the preceding chapter on the relative capacities of the vulva, vagina, and cervix to receive, retain, and distribute infection.

\section{General Considerations of Symptoms and Diagnosis.}

The purpose of a diagnosis is not so much to give a name to the disease as to furnish a basis of rational treatment. A diagnosis 
should include, therefore, the source, variety, and complications of the disease. It would be absurd to confine the treatment to the area of inflammation if, for example, the disease were secondary to metritis, carcinoma, cystitis, or vaginal fistula. Attention to such complications as fissure in ano, hemorrhoids, rigid sphincter, threadworms, and endometritis often gives relief. The diagnosis should have special reference to the possible extension of the disease into the ducts of the vulvar glands and urinary organs. The discharge from a pelvic abseess has been mistaken for the seeretions of vulvovaginitis.

The symptom-group in acute vulvoraginal inflammation comprises irritation, pain, redness, swelling, heat, and inereased secretion. 'The systemic symptoms of inflammation are absent or slight, except in cases of extensive phlegmon or suppuration. The pain and swelling are often so intense that the patient must lie down with the thighs apart. The labia minora sometimes swell to twice the size of the finger, and eonsequently may close the vulva; they have a bright, glistening appearance not unlike the inflamed swollen prepuce of the male. The pain is throbbing and extreme in proportion to the swelling. The inflamed surfaces, which may include both vulva and vagina, are at first dry, but soon become moist in consequence of an effort of the glands to relieve the congestion by increased secretion. The secretion, usually profuse, is a chief evidence of the disease. In children the disease, unless due to gonorrhœal infection, is confined usually to the vulva. Carcinomatous ichor causes irritation rather than pain. Frequent difficult and painful urination are common, especially when the infection has extended to the urethra and bladiler.

Chronic Vulvitis and Vaginitis may occur separately or together. Clinically, ehronie vulvitis and vaginitis are observed more commonly than acute; they may follow the acute, or may have been ehronie or subacute in the beginning; they are reeognized by their persistence, by their tendency to recur when apparently eured-see Follieular and Glandular Vulvitis - and sometimes by the presenee of erosion of the vulvar, raginal, or vulvovaginal surfaces. They are charaeterized by a scanty, thin, yellow discharge, usually more or less purulent ; by great local irritation; by variable redness; by slight swelling, and sometimes by excessive granulation. The surfaces, especially the vulvar surfices, finally become hard, oedematous, leathery, parehment-like, and painful. $\dot{A}$ frequent symptom of chronic vulvar inflammation is an intolerable pruritus, characterized by intractable itching and burning. See Pruritus vulvæ.

\section{General Considerations of Treatment.}

A lesson to be learned from the above observations is the importance of stamping ont rulvar inflammation, and thereby preventing its invasion of the higher zones in the pelvis. The experiments of Dïlerlein would suggest vaginal donches of a 1 per cent. aqueous solution of lactic acid. This would clearly not apply to infection from bacteria which grow in acid media. 
The vulva is normally moist from its own secretions. Dust and dirt, which may contain irritants capable of exciting vulvitis, easily reach the vulva and find lodgement there. As a prophylaxis against this source of vulvitis, and as a better protection against sudden changes of temperature, the closed drawers should take the place of the commonly worn open drawers. The daily shower-bath applied to the external genitals is an excellent prophylaxis. Strong soap is irritating, and therefore injurious.

The Treatment of Acute Vulvitis is chiefly local, and includes two essentials, cleanliness and palliation. Mild alkaline solutions, such as sodium bicarbonate, when applied to the vulva, may combine with the oily secretion to form a soap which, upon being washed off with warm water, leaves the surface clean. Washing should be repeated frequently as a preparation for other applications, such as a lotion of equal parts of dilute solution of acetate of lead and fluid aqueous extract of opium, the 4 per cent. aqueous solution of antipyrine, the 4 per cent. solution of cocaine, the 4 per cent. ointment of morphine sulphate, the spray of an alkaline solution or of the 5 per cent. solution of carbolic acid. The warm sitz-bath or the ice-bag applied to the vulva is indicated in cases of extreme irritation and burning. Absolute rest in bed is important. A rectal suppository containing extract of opium, two grains, and extract of belladonna, one-fourth grain, may give relief and secure much-needed sleep. Avoid ointments containing animal fat. Vaseline, clear or stiffened with wax, is a good excipient. If the labia can be separated without too much pain, a light gauze compress saturated with a soothing antiseptic solution may be placed between then. Buboes and other abscesses if they occur should be opened.' Great care is needed to avoid carrying the infection from the vulva to the vagina or uterus by the syringe-tube or examiningfinger.

The general treatment consists of saline purges, and soporifics and anodynes as indicated by nervous irritation and pain.

The Treatment of Chronic Vulvitis includes, in addition to such of the above means as may be indicated, astringents and, in obstinate cases, causties. The surfaces should be dressed with gauze compresses saturated with a $1: 3000$ aqueous solution of the bichloride of mercury, or a 3 per cent. aqueous solution of carbolic acid. If the disease has been caused by Pediculi pubis or other parasites, mercurial ointment, in addition to the above solutions, should be used to destroy them. One of the most effective treatments is the daily packing of the vagina with gauze saturated with an aqueous solution of argyrol, 5 per cent.

The daily hot-water vaginal douche may be supplemented with a solution of copper sulphate or zinc sulphate, one drachm to the quart of water. Extensive erosion of the skin about the vulva often is cured promptly by the free use of benzoated oxide of zinc ointment. Eroded surfaces, having been dried, may be dusted daily with calomel or with the subgallate of bismuth. In neuropathic cases of severe pruritus almost miraculous relief sometimes follows the free 
withdrawal of blood from the uterus, either by scarification or by leeches.

Granular vulvitis should be treated by painting the granulated part with a $1: 20$ solution of nitrate of silver and daily packing the vagina with gauze. Obstinate cases may yield to frequent applications of 10 or 20 per cent. of ichthyolate of ammonium in glycerin. The application is made best with a compress secured by a bandage. The treatment of vulvar and vaginal infection may fail until a causal or complicating endometritis has been cured.

\section{SPECIAL FORMS OF VULVOVAGINAL INFLAMMATION.}

The special forms of vulvovaginal inflammation will be considered separately in the following paragraphs.

\section{Gonorrhœal Vulvovaginitis.}

Gonorrhœa, one of the most active and most destructive infections in the reproductive organs, is al ways the result of gonococcus infection. The disease is characterized by a strong tendency to penetrate and spread, and is prone to attack the follicles and glandular structures of the vulva, especially the vulvovaginal glands and Skene's glands. Diffuse and deep cellular inflammation and abscess of the vulva may also result from gonococcus infection. See remarks on the gonococcus and recurrent gonorrhoea in woman in Chapter X., and Urethritis in Chapter XXIV.

The infection not uncommonly extends throughout the genitourinary tract, although the constant downward current of urine may protect in a measure the more distant urinary organs. If the disease originates in the vulva, it usually extends to the vagina, and vice versa. The urethra seldom escapes. The inguinal glands may be infected through the lymphatics, and are then especially prone to suppuration.

Children are more subject to this infection than generally is supposed. It may come from infected bed-linen, from bathing with infected eloths or sponges, or from the unclean hands of infected nurses. In children the disease is less liable than in adults to extend to the vagina, because the vagina is protected in a measure by the hymen. It may, however, easily be carried upward on the douche point.

Diagnosis.-A suspicious exposure, great pain, and unusual systemic disturbance should excite suspicion. Radiation of pain to the rectum, perineum, and bladder, burning in the urethra, and involvement of the vulvar glands are strong diagnostic signs. The positive diagnosis depends upon finding the gonococcus by microscopical examination of the secretion.

Treatment.-It is highly important to stamp out the gonorrhœal infection while it is yet in the vulva or vagina, and thereby to kcep the infection from going to the higher zones of the genito-urinary system. Daily packing of the vagina loosely with tampons of sterile 
gauze or absorbent cotton saturated with an aqueous solution of formalin, $1: 1000$, will be useful during the active stage. Let the vulvovaginal surfaces be painted once or twice, as in granular vulvitis, with a solution of nitrate of silver, $1: 20$, and packed with dry sublimated or borated gauze, which should be renewed as often as it becomes moist from the secretions. At each time of changing the gauze the surfaces should be cleansed thoroughly with a warm 5 per cent. aqueous solution of carbolic acid; this is to be followed by a thorough washing with peroxide of hydrogen, which is very cleansing to the deeper glandular structures.

A solution of nitrate of silver may be used with excellent effect as follows: The patient being in the dorsal position, with the hips elevated, introduce a cylindrical speculum so as to expose the cervix uteri and vault of the vagina. Into this speculum pour a 3 per cent. aqueous solution of nitrate of silver. Allow this solution to bathe the cervix uteri and vault of the vagina for five minutes; then remove the solution by means of absorbent cotton. This treatment should be repeated two or three times a week. Argyrol may be substituted advantageously for the silver nitrate.

The diet should be non-irritating. Urethral or bladder complications call for diuretic drinks. Crayons of ichthyol in the urethra, if tolerated, may be useful.

\section{Erysipelatous Vulvovaginitis.}

Erysipelas is primarily an infiammation of the lymphatic vessels of the skin or mucous membrane. The infection is caused by a streptococcus similar to the streptococcus pyogenes-perhaps identical with it. The disease is febrile, always acute, often suppurative and superficial, and chiefly characterized by a tendency to spread. There are three varieties : the erythematous, the vesicular, and the gangrenous.

The Erythematous erysipelas of the vulva and vagina is the mildest form. It presents redness and heat of the surface. The skin or mucous membrane is but little swollen, and the tendency is strongly toward spontaneous recovery.

The Vesicular form is more severe, is characterized by intense inflammation of the skin or mucous membrane, by marked cedema, and by the appearance, under the surface, of vesicles or bulla, which, like blisters, contain serum. Finally, infection in these vesicles may cause suppuration, and the inflammation may extend to the deeper structures and become phlegmonous.

The Gangrenous is the most dangerous form of erysipelatous vulvitis. It apparently results from rapid development of streptococci and their products in the lymph-channels and connectivetissue spaces so as to shut off nutrition and cause necrosis. It destroys large areas or small patches of skin or mucous membrane and sometimes deeper structures.

Erysipelatous vulvovaginal inflammation occurs not infrequently in very young infants by extension from the navel, or it may spread from the vulva to the thighs and nates; it is observed sometimes in 
childhood, but is rare in adults, except in childbed, where it is a most dangerous affection. Bad nutrition and filth are strong predisposing causes. Generally speaking, the prognosis is farorable, doubtful, or grave according to the extent and severity of the disease. Gangrene of the vulva, especially in infants, is almost always fatal.

Treatment does not differ materially from that of the diphtheritic form ; see below. If the inflammation beeome phlegmonous and result in suppuration, the abscess should be opened. The gangrenous variety calls for strong supporting measures and rigid disinfection with pure carbolie acid.

\section{Diphtheritic Vulvovaginitis.}

This form of vulvovaginal inflammation rarely appears in the nonpuerperal adult. It is sometimes the local manifestation of a very grave form of puerperal fever which occurs in epidemies, especially in the obstetrical wards of hospitals. It sometimes attacks children during epidemics of ordinary diphtheria.

There are other forms of membranous vulvovaginitis in which the germ of diphtheria is not present-pseudodiphtheric vulvovaginitis.

Treatment.-The general treatment includes energetic supporting measures, such as quinine, the mineral acids, ferric chloride, and sometimes heart stimulants. The bowels should be regulated, if necessary, by mercurials and salines. The local treatment is the same as in the general therapeutics of vulvoraginitis: antitoxin and other measures are indicated as for diphtheria elsewhere.

\section{Tubercular Vulvovaginitis-Lupus.}

Tuberenlar inflammation has been found in every part of the genital tract, the order of frequency for the various parts being the Fallopian tubes, corpus uteri, ovaries, vagina, cervix uteri, and vulva. It gives no characteristic symptoms; the diagnosis lepends upon finding the bacillus tuberculosis. The disease may be secondary to tuberculosis in some extrapelvic organ, or may be primary in the genitals.

Tubercular vulvitis, commonly called lupus, which sometimes is described as a neoplasm, is rare and confined chiefly to the period of maturity. The characteristic lesion is the formation of tubercles and nodules, which undergo cheesy or colloid degeneration, and finally ulceration and cicatrization with much increase of connective tissue throughout the affected area. The ulcer is of red color, with a granular base, is purulent and prone to bleed. It may be superficial or so deep as to make permanent fistulæ between the bladder, vagina, and rectum. The cicatricial contraction which follows the ulceration may result even in strictures of the urethra, vagina, or rectum. Hypertrophie processes may or may not be associated with ulceration. The general thickening and induration of the affected part may be so extensive as to give the labia the appearance of elephantiasis. "The 
vulva and perineum become studded with nodules of red or violet color. Great chronicity and little pain are notable characteristics of the disease. The general health may eontinue unimpaired for many years.

The Treatment of tubercular vulvovaginitis is the same as that of tubercular disease elsewhere-i. e., systemic and local. Proper climate, outdoor life, careful attention to nutrition, and thorough cauterization. Early exeision of the diseased part together with a layer of healthy tissue around it gives good promise of radical cure. The $x$-rays have given some apparent success but the time has not arrived for a positive statement as to the value of this treatment.

\section{Mycotic Vulvovaginitis.}

Etiology.-Mycotic vulvovaginitis is most common in diabetic suljects; certain fungi-mycoses-chief among them the leptothrix, oïdium albicans, and leptomitus, often are found in the vulvar secretions, and are doubtless the exciting cause. Diabetic urine apparently favors the development of the fungi, although the disease is not always associated with sugar in the urine. Furunculosis often complicates diabetic vulvitis.

Catarrh of the genital tract and pregnancy are predisposing causes. The micro-organism may be brought in contact with the genitals by intercourse, especially with a diabetic man. The fungus may be carried on the finger of the examiner. Winckel cites two cases in which the infection apparently was traced to the touching of the genitals by the hand dusted with flour.

Symptoms.-The swelling of the vagina may extend to the vulva, and then be so great that the patient cannot stand nor walk. The epithelium may be exfoliated and the urine cause pain when in contact with the exposed surfaces. The pruritus may be extreme and paroxysmal or continuous. The depressing influence of the hypersecretion, sleeplessness, pain, and loss of appetite, are apt to hasten the fatal result of a complieating diabetes. The vulva throughout has a coppery-red color, is much swollen, is dry in some parts and may be moist in others. Scratching may cause here and there considerable bleeding. The skin is dry and brittle, wrinkled and rigid. The affection may invade the inner folds of the nymphæ, the meatus urinarius, the folds of the groin, the mons veneris, and the folds of the nates, and may surround the anus. An improvement in the general condition of the patient may lessen the local disease, which is liable to return, however, with increased severity. ${ }^{1}$

Diagnosis. - Small yellow or white spots upon the reddened mucous membrane or skin, which cannot be scraped off without at the same time removing the epithelium, are characteristic of the disease. These spots, taken together with finding the micro-organism by microscopical examination, will establish the diagnosis.

Prognosis.-The prognosis is variable. The disease may be most 
persistent or may disappear under treatment. In pregnant women it may disappear after delivery.

Treatment.-If there is an associated diabetes, a diabetic dietary, in addition to tonics and mild saline laxatives, is indicated first. The intolerable itching and burning necessitate local remedies, of which many have been used with varying and temporary success. Wash thoroughly with a tepid solution of corrosive sublimate, $1: 2000$, or with a saturated solntion of boric acid. Benzoated oxide of zine ointment, or an ointment of vaseline and salicylic acid, $1: 200$, is useful. The sitz-bath, temperature $80^{\circ} \mathrm{F}$, prolonged for an hour, often gives relief; to this bath may be added a pound of Indian meal. Astringent washes, for example, of tamnin or alum, or sulphate of zinc, may be indicated.

Since the skin in mycotic vulvitis is already dry and brittle, it is not well to dust the vulva with powder. To relieve the suffering, which is usually worse at night, place on the parts at bedtime a compress moistened with a 3 per cent. solution of carbolic acid.

Anodynes may be used locally; one part of chloroform to five parts of almond oil, ointments of belladonna and morphine, or a 6 per cent. solution or ointment of cocaine, may give temporary relief. The disease in a diabetic subject is usually intractable or incurable. See Furuncular Vulvitis and Pruritus Vulvæ. A most effective application is an ointment containing: camphor, 4 grains; chloral hydrate, 4 grains ; menthol, $\frac{1}{2}$ grain ; carbolic acid, 1 grain; lanolin, 100 grains.

Mycoses of the vulva and vagina in subjects not suffering from diabetes are usually self-limited or easily cured by the treatment above indicated. The vaginal mycoses require donches of carbolic acid, 3 per cent., or of corrosive sublimate solution, $1: 2000$.

\section{Syphilitic Vulvovaginitis and Chancroid.}

The subject includes the primary, secondary, and tertiary forms of syphilis.

Chancre develops after an incubation of from ten to twenty days, usually the latter. It is first a reddened excoriated spot or a hardened papule with or without ulceration. The characteristic feature is induration. The induration may be parchment-like and superficial, or it may be deep and reach laterally far beyond the edge of the erosion or ulceration. The indurated tissue is hard, like cartilage, but seldom so pronounced in women as in men. In the ulcerative form the ulcer is usually small and funnel-shaped, with sloping edges, superficial or deep; the edges are never undermined. The bottom of the ulcer is gray, the discharge seropurulent and never free. Rarely more than one chancre ever appears in the same person. The inguinal glands usually enlarge, but except in cases of mixed infections do not suppurate. Clancre is only the local sign of syphilis, and its pus is rarely, if ever, auto-inoculable.

The Secondary and Tertiary Lesions of Syphilis include mucous patches and gummata. These patches on the genitals have the same 
general character as when they appear elsewhere. In the seeond stage flat condylomata form. See Chapter XXV.

Chancroid, which is a purely local infection, has no period of incubation, is auto-inoculable, has a rounded or oval margin, abrupt or ragged edges, no induration, and nay develop into a large or phagedenic ulcer. The inguinal glands are prone to suppurate. Large numbers of chaneroids may occur on the same person.

Treatment.- The treatment is that of syphilis or, as the ease may be, chancroid. The local lesions may be complicated with other forms of vulvovaginitis, which should have special attention aecording to their elass.

\section{Superficial Vulvitis and Vaginitis.}

This sometimes is caller simple inflammation. When aente it often produces mild systemic fever and sometimes excessive swelling, pain, and irritation. The disorder is erythematous and resenbles urticaria. It does not give rise to much exudate, is not very virulent, and seldom or never extends to the follicular or glandular elements or to the uterus. It tends to rapid resolution on removal of the irritating cause. It often causes excessive œdema of the labia minora, which œdema may disappear in a few hours.

The causes of superficial vulvitis and vaginitis are often largely mechanical, such as masturbation, excessive coitus, rubling, scratching. Pinworms, tænia circinata, and irritating vaginal or uterine discharges are among the other causes. The inflammation may be in the form of vulvitis, vulvovaginitis, or vaginitis. It does not involve the corium in the vulva nor the submucosa in the vagina. The treatment has been described in the general therapenties of vulvovaginal inflammation.

\section{Senile Vulvovaginitis.}

Senile vulvovaginitis is usually, though not always, a somewhat deep inflammation. The retrogressive physiological processes of the menopanse which result in senile atrophy of the reproductive organs destroy in great part the epithelial portion of the nucous membrane of the uterus, vagina, and vulva, so that this membrane becomes composed largely of fibrous tissue. This fibrous tissue when inflamed is prone to granulate, to suppurate, to cicatrize, to contract, and to form adhesions of any surfaces in contact with one another. Stenosis at the internal or external os uteri may prevent free drainage of the uterine seeretions. These secretions, already pathological, when retained become excessively irritating. Similar secretions also eome from the vagina and vulva. Aged women, therefore, who have long passed the menopause, are subject to a most irritating vulvovaginitis-a most exhausting and distressing pruritus vulvæ. The adhesions often entirely envelop the vaginal portion of the cervix and may obliterate partially the vagina. The vulvar glands and mucous crypts, especially in pruritus cases, are involved extensively. Removal of them 
is the only means of relief from the intolerable itching and burning. See Treatment of Glandular Vulvitis, below. In other respects the treatment is the same as that laid down in the general therapeutics of vulvovaginal inflammation.

\section{Glandular Vulvitis.}

Inflammation of the Urethral Cysts.-Five or six small racemose glands are situated around the meatus. 'They have short ducts with wide openings; two of them are in little depressions on either side of the meatus. Inflammation in these glands or erypts, not uncommon during and after the menopause, may cause a most persistent pruritus with extreme itching and burning; this occurs most frequently in connection with senile vulvitis.

Inflammation of the Vulvovaginal Glands.-The vulvovaginal glands of Bartholin are on either side of the vaginal orifice near the posterior extremity of the bulb of the vagina. Their ducts are about one-half inch long and open into the fossa navicularis, wherc they are seen easily.

Inflammation of these glands comes by extension from the external surface. The glands, or their afferent ducts, or both, may be involved. A suppurating gland may pour out pus through the duct; or the duet may close by adhesive inflammation and form an abseess; it may become occluded and distended with the normal secretion of the gland, and thus form a retention-cyst. One or both glands nay be affected. The disease is very common. Sünger describes a red areola (nacule) about the openings of the ducts as an evidence of gonorrhœa.

Diagnosis.-Abscess is distinguished from retention-cyst of the glands by the presence of acute pain and heat in the former and the absence of them in the latter. Enlargement of the gland under either of these conditions is distinguished from phlegmonous vulvitis by the location of the former, which corresponds to that of the gland, while phlegmon may be anywhere in the vulva; and from hernia by the absence of the characteristic signs of hernia and by the location. See Figs. 76 and 79.

Glandular vulvitis, once established, becomes chronic. The glands serve as culture-ground for the infecting bacteria, hence superficial vulvovaginitis, though apparently cured, may recur again and again from the infected glands. The vulva, through its glandular structures, is a great distributing point of pelvic infection. The periodical congestion of menstruation is a recognized predisposing cause of recurring pelvic inflammation. As stated elsewhere, the capacity of glandular structures to receive, retain, and distribute infection often will explain the frequently observed attacks of recurrent gonorrhœe in women.

The explanation of so-called latent gonorrhœa in the male, discussed by Noeggerath, is the same as that given in the preceding paragraph for recurrent gonorrhœea in women.

The Treatment of Glandular Vulvitis, when acute and non-suppurative, is palliation and cleanliness, the latter to be secured chicfly by 
disinfectants. When the inflammation is chronic, the treatment raries with the different glands, as follows:

Figure 76.

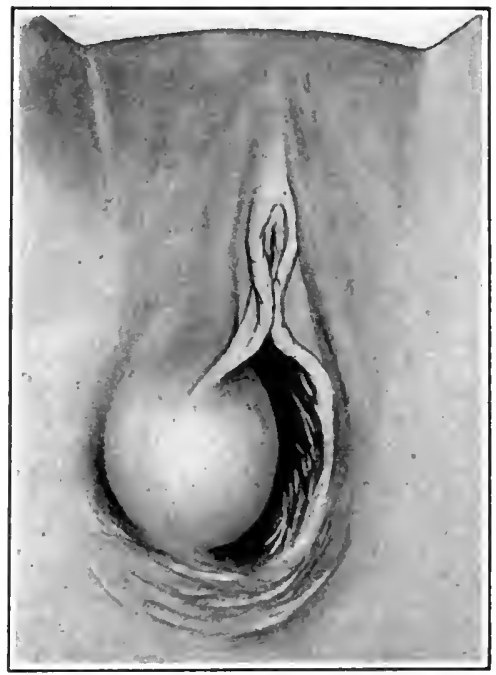

Figure 78.

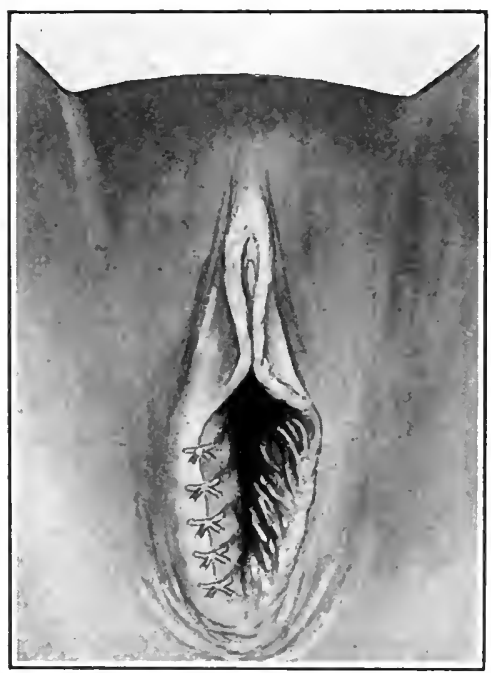

Figure $7 \%$.

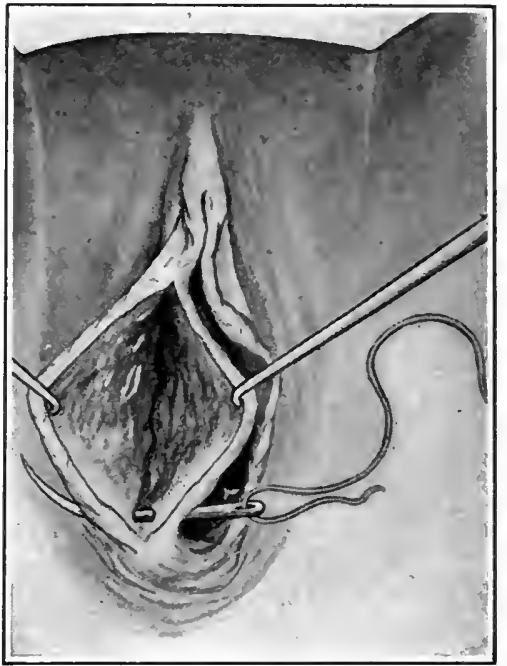

FIGURE 79.

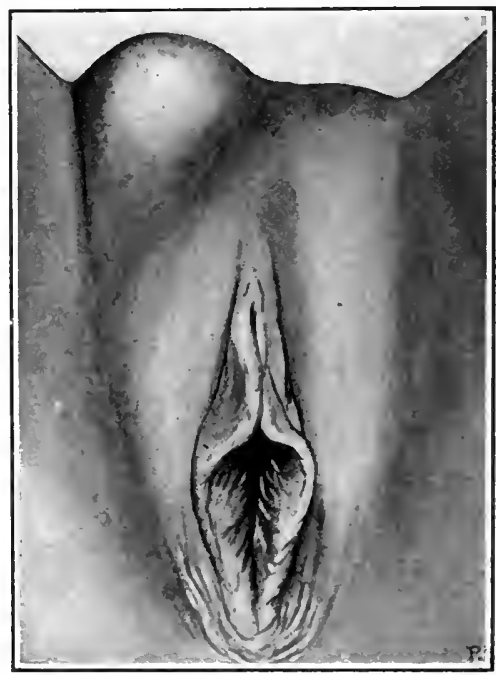

$P:$

Fifure 76.-Enlargement of the vulvovaginal gland by cyst: an abscess would have a similar appearance, but unlike the cyst would be painful on pressure.

FIGCRE 77.-Cyst wall dissected out ; wound held open by tenacula; introduction of first suture for closure of the wound; silkworm gut suture; a small tubal or gauze-wick drain is useful in these cases.

FIGURE 78.- Wound closed with fine silkworm gut sutures.

FIGURE 79.-Right inguinal hernia simulating vulvovaginal cyst or abscess.

The five or six small mucous crypts near the meatus urinarius, when infeeted, are the seat of an intolerable pruritus. 'The treatment 
is to destroy the glands by the actual cautery or to remove them by excision. 'The author's preference is to excise them, close the wounds by suture, and secure union by first intention.

The treatment of abscess of a vulvovaginal gland is the same in principle as for abseess elsewhere; it should be opened widely, the wound packed with gauze, and made to heal from the bottom by granulation. In opening the abscess find the gland, if possible, and remove it.

When a retention-cyst has formed from occlusion of the duct, the sac should be dissected out, the wound sutured, and for one or two days drained with a small rubber tube or with gauze wick. If drainage is not used, the wound is liable to suppurate.

Sometimes chronic suppuration of the gland oceurs through the open duct. Then the duct should be incised widely, the gland removed, and the wound packed with gauze.

\section{Follicular Vulvitis.}

The labia minora and majora are supplied abundantly with hairbulbs, sebaceous follicles, and sweat-follicles. Inflammation in these

Figure 80.

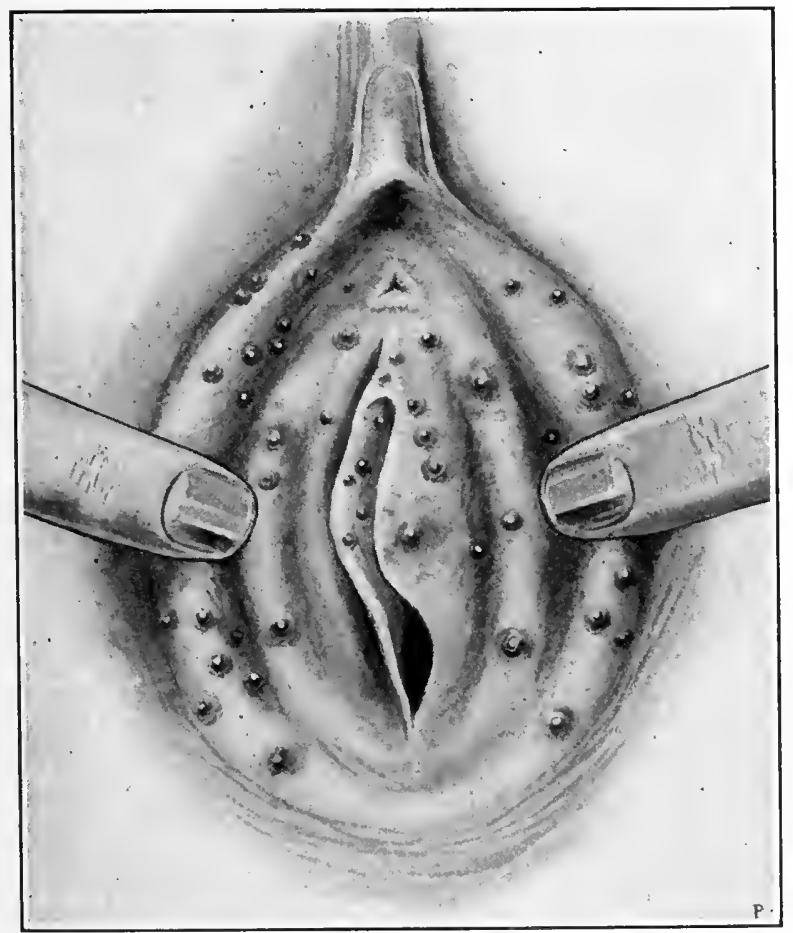

Follicular vulvitis.

structures of an acne-like character is follicular vulvitis or folliculitis. Figure 80 . The general appearance of the surface, except slight conges- 
tion, usually is unchanged. The openings of the follicles scattered over the labia ninora and majora are small, red, elevated, and swollen. Children are not subject to folliculitis. The inflammation may originate in the follicles or may extend to them from the external surface, as in glandular vulvitis. The infection often remains entrenched in the follicles after it has disappeared from the external surface, and from these lurking-places may reinfect the surface again and again.

Adhesive inflammation may close the openings of the ducts; then the secretions will be retained and form abscesses as large as a pea; otherwise the discharge is abundant, purulent, and often offensive.

Treatment. - The disease may be seated so deeply that it resists all surface applications, and vields only to direct deep eauterization strong enough to destroy the secreting structures. For this purpose, use the fine galvanocautery needle or the point of a probe made redhot in the flame of a spirit-lamp.

In follicular vulvitis with occlusion open cach follicle with a small, sharp-pointed knife, and then apply the fine-pointed conical solid stick of nitrate of silver. This may be done under eocaine without pain. A dressing of sterile gauze saturated with formalin $1: 1000$, placed as a pad over the vulva, is a most serviceable application. The $x$-rays are used with surprisingly good results.

\section{Furuncular Vulvitis.}

Furnuculosis usually starts in the hair-follicles and extends to the surrounding cellular tissue. The resultant boil may be developed at numerous points in the labia majora, where the disease usually is confined. Some women have an unexplained tendency to this form of vulvitis. Furunculosis is common in diabetes. The author has observerl that glycerin tamponade is apparently an exciting cause of boils. The incipient boil may often be aborted by pulling out the hair from the inflamed hair-bulb, thereby giving drainage.

The Treatment of furunculosis is the same in the vulva as elsewhere -i.e., open and drain the abscess. Numerous boils sometimes follow one another, or occur in successive clusters in one locality. Such recurring infection is due usually to the presence of the microbes of suppuration, which remain on the surface ready to produce reinfection at any favorable point. Daily cleansing of the surface and thorough disinfection with the ointment of biniodide of mercury $(1: 60)$ for two weeks after the last boil has disappeared are effective means of prophylaxis. The $x$-rays are most efficacious. General tonics are decidedly indicated. Fresh brewers' yeast taken in wineglassful doses four or five times a day, is a most useful remedy.

\section{Emphysematous Vaginitis.}

This rare disease occurs mostly in pregnancy. It is characterized by numerous small, soft cysts of variable size situated just under the surface and commonly on the posterior wall of the vagina. These cysts contain serous fluid and gas. The affection usually is asso- 
ciated with other forms of vaginitis. The diagnosis may be verified by pricking the cysts; then the gas escapes with a blowing sound. In pregnant women the cysts disappear without treatment at the end of pregnancy.

Treatment.-In puerperal cases the treatment is expectant. In non-puerperal cases, if the cysts do not disappear under antiseptic douches, they should be opened and the vagina packed with antiseptic gauze.

\section{Paravaginitis.}

Paravaginitis, sometimes called dissecting vaginitis, is a rare disease which involves the submucous connective tissues. Burrowing abscesses are formed with perivaginal fluctuations. The musculature of the vagina and vulva, in whole or in part, may separate and slough off in a gangrenous mass. The cicatricial contraction which follows will then cause stenosis or atresia. It is often impossible in such cases to restore the calibre of the vagina or vulva by operative measures. Secretions of blood or menstrual fluid may accumulate above the atresia in the vagina, uterus, or Fallopian tubes. This disease is due usually to a grave infection by the streptococcus or by the germ of diphtheria.

Treatment.-The pus should be evacuated freely as soon as it is discovered. If sinuses form, they should be incised and drained. Plastic operations and dilatation may be required to overcome cicatricial contraction and possible atresia. Atresia from this cause is to be distinguished from the congenital atresia described in the chapter on Malformations. 


\section{CHAPTER XII.}

\section{ECZEMA VULVA, HERPES VULVA, KRAUROSIS VULVA, PRURITUS VULVA, HYPERASTHESIA VULVA, VAG- INISMUS.}

Ayoxg the disorders allied to vulvovaginal inflammation are eczema vulvæ, herpes vulvæ, kraurosis vulvæ, pruritus vulvæ, and vaginismus.

\section{ECZEMA VULVA.}

Eczema vulvæ is an infrequent disease, may be acute or chronic, and occurs mostly during pregnancy. Rheumatism, and the uric acid diathesis are said to be predisposing eauses. The eruption consists of nodules, vesicles, pustules, and scabs, with more or less redness, swelling, and moisture of the skin. The vesicles contain serous fluid. Pus is found under the scabs in the more severe eases. The skin and sometimes the subentaneous tissues are infiltrated. Acute eczema may remain local and terminate within two weeks. Chronic eezema, often intractable, may extend to the mons veneris, thighs, and nates, with swelling and suppuration. The labia majora most commonly are involved.

\section{Treatment of Eczema Vulvæ.}

The general treatment consists of mercurials and salines, non-irritating diet, avoidance of wine and liquor, and hygienic living. The local treatment varies with the condition. Whenever the subcutaneous structures are exposed, the solid nitrate of silver point should be applied, eare being taken to touch only the exposed surfaces. Oftentimes numerous very minute abrasions may be seen with the unaided eye or through a magnifying-giass. These should be touched delicately with the finest point of nitrate of silver. The application should be repeated every five days until the abrasion disappears. The following ointment is useful :

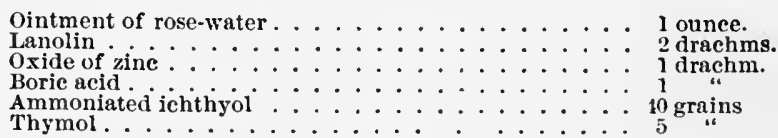

The parts should be kept elean and dry. Dusting with bismuth may give relief.

\section{HERPES VULV压.}

An herpetic eruption, not unlike herpes labialis, is observed oceasionally upon the vulvar labia. There is little redness or swelling. 
The disease usually is self-limited; like herpes in other places, it runs its course in a few days and disappears.

\section{KRAUROSIS VULVA.}

Kraurosis vulvæ is an atrophic shrinking of the vulva, and is a rare disease. ${ }^{1}$ The affection is characterized by atrophy of the cutaneous covering of the vulva, especially of the imner surface of the nymphr. The skin appears dry and sirmmen. 'The surface has the tense, glistening appearance of scar-tissue. The affection eauses distressing paroxysms of itching and burning pain in the diseased part. Sometimes the vulvar orifice is contracted extremely. The clinical features are so characteristic that once recognized they will never be mistaken for those of any other disease. The hair aromel the vulva is thin and dry, and late in the disease almost entirely absent. The vulva appears small and infantile, the labia minora are shrunken and, finally, almost absent; the skin is pale, without pigment, but studded with numerous irregularly shaped reddish-brown blood-spots, which on inspection appear slightly depressed below the surface. 'These spots are confined entirely to the restibule, but disappear in the later stages of the disease. The skin is dry, sometimes eracked, abraded, and oceasionally gives forth a slight, brown, purulent discharge. The natural elasticity of the vulva is lost entirely. The orifice is so contracted as usually to prohibit the introduction of the speculum. The sensitiveness of the parts is very great, especially while the brown spots are present. This, together with the tenseness of the vulvar orifice, causes extreme dyspareunia ; in fact, usually prohibits coition. ${ }^{2}$

\section{Pathology of Kraurosis Vulvæ.}

The pathology of this disease is not fully known. In addition to the foregoing pathological changes may be mentioned a thickening of the laver of epidermis, decrease in the number of sebaceons glands, and sclerosis of the subcutaneons connective tissue. The tightly contracted skin is so stretched over the parts that even the pressure of the examining finger may make deep rents.

Longyear has observed a deep cirrhotic-like band of fibrous tissue entirely separate from the cutaneons covering. He regards this band as the essential lesion, and gives to the superficial changes a secondary importance. This fibrous band replaces the loose cellular tissue through which the nutrient vessels pass to the skin, and by its gradual and contiunal contraction causes not only the vulvar shrinkage, but also the strangulation of the blood-ressels which pass to and from the overlying cutaneons structures. This disturbance in circulation explains the spots of ecchymosis in the earlier stages of the disease, and the atrophic changes in the later stage. The pain is explained by mechanical pressure on the nerves and by the resultant neuritis and perineuritis.

1 Rohert F. Weir: "Ichthyosis of the Tongne and Vulva," New York Medical Journal, March, 1875. Breisky: “Kraurosis Vulvæ," Centralblatt für Gynäkologie, 1885, p 358. Lawson Tait: Diseases of Women, Lea Bros \& Co., 1889, p. 53. C. A. Reed: Trans. American Association of Obste. tricians and Gynecologists, 1894. Howard Iongyear: Ibid., 1895.

2 Adapted from Longyear. American Obstetrical Journal, 1895. 
Kraurosis in a small proportion of cases is associated with epithelioma of the vulva.

\section{Treatment of Kraurosis Vulvæ.}

This new formation of fibrous tissue is of special interest from the surgical standpoint. Clearly the removal of this band, together with the contracted superficial structures, is essential to the cure of the disease. The usual operation of removing the degenerated and contracted mucocutaneous structures may relieve the acute symptoms, but can have no effeet on the stenosis. Spontaneous recovery sometimes is reported; but this is only a relief from the supersensitiveness of the vulva, never from the constriction. The fibrous band, unless removed by operation, is permanent. Longyear's operation is the removal of all the superficial discased structures, together with the fibrous band beneath, and union of the external and internal margins of the wound. An incision with scissors is made first along the lateral and posterior margins of the vulvar orifice, dividing the diseased structures from the healthy skin; then the margin of the diseased tissuc, including the fibrous band, is seized with dressing-forceps and dissected loose from the underlying tissues to the vaginal inlet. This tissue then is cut away. The anterior vulvar structures are dissected loose in the same manner, care being taken to cut carefully round the urethral orifice. After removing all the diseased structures in this way the margin of the healthy vaginal wall above is pulled down and dissected loose from the underlying parts around the whole circumference of the vagina. This loosening of the vaginal wall permits the inner margin of the wound to be brought down to the outer margin. The two margins then are united with deep silkworm gut and superficial catgut sutures, or with fine buried chromic catgut. Complete relief has followed the operation. The operation is similar to that illustrated under the surgical treatment of Pruritus Vulvæ in this chapter.

\section{PRURITUS VULV王.}

In neuropathic eases of vulvar inflammation the irritation, itching, and burning are intense, intolerable, intractable, and thereby constitute a condition called pruritus vulvæ. The nervous element often predominates, and for this reason the disease has been classed as a neurosis. It may arise from a variety of causes, may extend over the adjacent mucous surfaces, and often is aggravated by efforts to get relief by scratching; in this way the habit of masturbation sometimes is formed. The intense suffering causes loss of sleep, exhaustion, and sometimes alarming nervous depression. Sexual excitement and orgasms may occur. Pruritus may be complicated by melancholia, hysteroneuroses, and other forms of insanity. These psychoses may or may not be dependent on the disease. In many cases a paroxysmal wave recurs with great violence upon exercise or upon getting into a warm bed. The nervous element has not been explained adequately; it may be a cause, or an effect, or a coincidence. Oftentimes the inflammatory element is insignificant or apparently absent. 


\section{Pathology of Pruritus Vulvæ.}

The pathology of pruritus is considered by some authorities to be of purely nervous origin; by others, who follow the lead of modern etiological theories, to be of bacterial origin. The truth may lie between the two extremes. There may undoubtedly be an irritation of the sensory nerves of the vulvar skin of purely neuropathic origin; when this irritation occurs, it usually is aggravated by the presence of more or less vulvitis. The pathology so far as it is tangible is largely, therefore, the same as already described under Vulvitis.

\section{Etiology of Pruritus Vulvæ.}

It follows from the above that the causes of pruritus will include those of vulvitis. Numerous attempts to explain the causation of pruritus sometimes have made up in scientific elaboration what they lacked in clinical value. ${ }^{1}$

The following classes of causes are worthy of consideration :

1. Circulatory causes.

2. Secretory causes.

3. Parasitic causes.

4. Mechanical causes.

5. Thermic causes.

1. Circulatory Causes.-In certain disorders, such as icterus, diabetes, chronic nephritis, the blood contains bile, urea, or sugar, all of which by action on the nerve-endings are said to cause itching of the parts. Morphine, alcohol, and iodoform sometimes produce a similar effect.

Erythema, herpes, urticaria, and other such skin disorders which involve stasis hyperæmia, may occur in the region of the pudendal and hemorrhoidal veins; they then are characterized by the intense pruritus which they cause.

2. Secretory Causes._Abnormal secretions of the vulva, vagina, or uterus, especially if combined with the above-mentioned causes, may produce great irritation in the terminal sensory nerves of the vulva. Secretions from the diseased bowel or anus by chemical action may produce pruritus ani and, by extension, give rise to pruritus vulvæ.

3. Parasitic Causes._Animal parasites, such as pediculi and ascarides, and vegetable parasites, such as leptothrix, ö̈dium, and leptomitus, and the ordinary bacteria of inflammation, have been presented under Vulvovaginitis.

4. Mechanical Causes include inasturbation, immoderate handling, and scratching.

5. Thermic Causes.-Heat and cold are known to set up a peculiar pruritus, called in winter pruritus hiemalis and in summer pruritus æstivalis.

Above all these causes another and more essential element must be taken into the account; it is what Goodell once called the invisible,

\footnotetext{
1 A most elaborate and scientific discussion of the etiology of pruritus has been contributed by Sänger. Centralblatt fúr Gynäkologie, 1894, No. 7 .
} 
intangible, and imponderable influence on the nervous system; it is the difficulty, not to say the impossibility, of reckoning with this element that often makes the disorder persistent or intractable. The most that can be affirmed with our present limited knowledge is that there is an irritation of the sensory nerve-organ of the skin, and that many causes may contribute to its excessive development. Whatever the tangible lesion may be, nervous irritability and hypersesthesia are always essential elements.

Intolerable itching of the anus is a frequently recognized accompaniment of habitual constipation, and often is associated with pruritus vulvæ and hemorrhoids. This may be explained by the fact that the vulva is supplied by the same nerves that supply the anus. The intestinal toxins which have been recognized as a cause of pruritus ani therefore may cause pruritus vulvæ also.

\section{Symptoms and Course of Pruritus Vulvæ.}

The irritation is apt to occur in paroxysmal waves. The paroxysms may recur after vigorous exercise, especially in warm weather, before or after menstruation, or upon exposure to artifieial heat. In some cases they appear upon getting into a warm bed. The desire to rub or scratch for the relief of the irritation is almost irresistible. This instinctive effort at comterirritation greatly aggravates the pruritus. As Thomas aptly remarks, "the disease and the remedy which instinct suggests react upon one another, the first requiring the second, and the second aggravating the first, until a most rebellious and deplorable condition is developed; the patient, bereft of sleep by night and tormented constantly by day, finally gives way to despondency and depression." The loss of sleep, the use and abuse of anodynes, and the neurosis incident to the discase may contribute to the development of melancholia or some other form of insanity.

The pruritus may extend to the vagina, anus, thighs, and abdomen. In some cases the irritation begins in the anus.

\section{Diagnosis and Prognosis of Pruritus Vulvæ.}

Pruritus is not a disease, but a symptom; diagnosis must depend therefore upon the identification of the causative lesion. In so far as the disease depends upon tangible and visible conditions the diagnosis and prognosis will follow along the lines laid down in Chapter XI., on Vulvitis. In a majority of cases a clear appreciation of the special etiology of the disorder as given above will open the way to aceurate diagnosis.

Withont great care the examination may fail to disclose the point and source of irritation. An irritating discharge, for example, so sliglit as to be unknown or ignored by the patient, may be sufficient to produce the most distressing pruritus, and may therefore have the utmost significance.

In the vast majority of cases one or more of the following conditions will be found present, and will explain partially or wholly the irritation : 


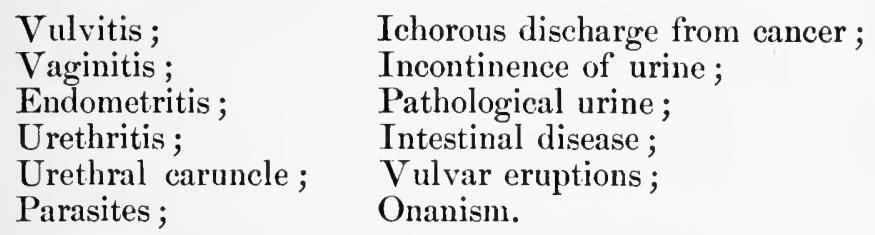

Most commonly associated with pruritus are vulvitis, vaginitis, and endometritis. The fact that these diseases do not commonly produce the disorder is explained by the absence of the essential neurosis. Senile vulvovaginitis is most prone to cause excessive irritation, and the pruritus when due to this cause is exceedingly obstinate.

The pruritus of pregnancy and the menopause is limited commonly to those states. In general the prognosis is indeterminate.

\section{Treatment of Pruritus Vulvæ.}

The treatment of vulvovaginitis already laid down is necessarily a part of the treatment of pruritus vulvæ.

A multiplicity of remedies recommended in the therapy of any disorder may be taken as evidence that our resources are limited or that the disorder may result from one or more of a wide variety of pathological conditions. Both of these propositions are true of pruritus vulvæ.

It is clear that the treatment must be directed to the cause of the irritation; to this end the reader is referred to the therapy of vulvovaginitis and of the numerous diseases and disorders already mentioned under Etiology and Diagnosis. See especially the treatment of senile and mycotic vulvovaginitis; in the treatment of the latter is the formula of an ointment containing camphor, chloral hydrate, menthol, carbolic acid, and lanolin, which may be used to advantage in various forms of pruritus.

In many cases the irritation is apparently the outcome of pent-up sexual energy. A neurotic woman who suffers intensely from pruritus has experienced entire relief upon the return of her husband from a prolonged absence.

While the radical treatment is in progress palliative measures always are demanded for the immediate relief of urgent symptoms. Fortunately, most of the palliative measures, since they allay irritation, are in a degree curative. Sitz-baths and vaginal douches of water or antiseptic solutions are useful to remove irritating discharges.

The following local applications may give relief:

The surfaces after each bath mav be dried and freely dusted with calomel, bismuth, starch, or lycopodium powder. The calomel is generally preferable.

A vaginal tampon of gauze often will protect the vulva from the discharge, and thereby give temporary relief.

Great relief sometimes is experienced from a gauze compress over the vulva, saturated with dilute solution of subacetate of lead and laudanum, equal parts. The compress should be changed frequently. 
A compress saturated with a solution of corrosive sublimate, $1: 1000$, or some form of mercurial inunetion will aet as if by magic when the cause is parasitic.

\section{Figure 81.}

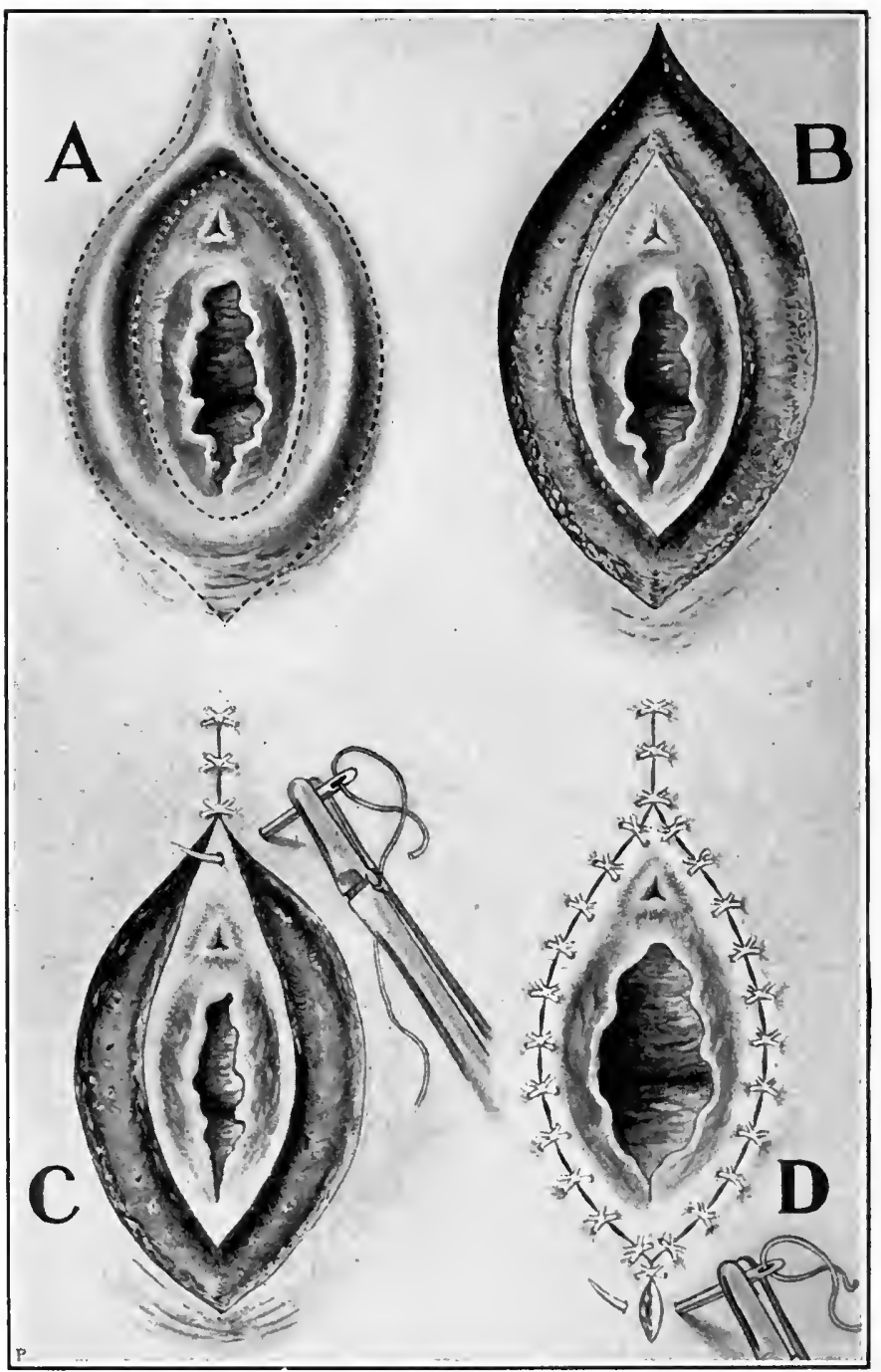

A, radical operation for krauroses or pruritus vulva. The black and white dotted lines indieate the direction of the incisions in the radieal operation for kraurosis or pruritis vulva. The inner ineision in extreme eases, involving the lower part of the vagina, espeeially kraurosis, would usually have to inelude the introitus vaginæ. B, discased structures removed. C, three sutures in place and tied, one suture being introduced. D, operation being completed by the introduetion of the last suture.

Cloths wrung out in very hot water and applied to the vulva may relieve or prevent the paroxysm which comes on after going to bed.

A strong infusion of tobaeeo, aceording to Thomas, both as a 
vaginal douche and on the vulvar compress, is most efficacious. In a case in which the neurotic element prevailed, he observed prompt and complete relief from the smoking of tobacco.

Ointments are useful from the soothing effect of their constituents and because they protect the parts from contact with irritating discharges. They are also an excellent vehicle for the application of parasiticides.

In rare cases the pruritus is due to a growth of short, stiff, inverted hair on the labia majora or pubes. This condition is called trichiasis. Prompt and permanent relief follows removal of the hairs and destruction of their bulbs by electrolysis.

The treatment of the disorder, if due to the diabetic, uric acid, or other diathesis, must include the appropriate hygienic measures, especially diet.

Painting the vulva with pure ichthyol has been known to effect a radical cure. In a case observed by the writer an accidental application of pure carbolic acid was followed by permanent cure.

Highly seasoned and highly nitrogenous food and stimulating beverages aggravate the irritation, and should be avoided. For the same reason scratching and rubbing of the part are injurious.

Finally, there is danger of forming the habit of using cocaine, morphine, or other narcotics ; for this reason their use should be guarded with judgment.

When apparent causes have received due attention, and the disease has resisted all treatment, operative interference may become necessary.

Sänger's conclusions on this point are based upon experience, and deserve attention. He says :

1. The partial or total extirpation of the vulva is a legitimate operation which should be performed in chronic, otherwise incurable pruritus vulvo. He calls the disease vulvitus pruriginosa.

2. The removal of the glans clitoridis, especially in elderly women, in whom the nerve terminations have usually lost their specific sensibility by reason of the disease, is permissible.

3. In younger persons, if the irritation is circumscribed, one may try to give relief by a partial operation without removal of the clitoris. In elderly women, and sometimes even in younger women, when the disorder is extensive, the whole vulva should be extirpated and the parts repaired by a corresponding plastic operation. See Surgical Treatment of Kraurosis Vulvæ.

\section{HYPERASTHESIA OF THE VULVA.}

Thomas has described, under this name, a rare disorder of the vulva which occurs in hysterical and despondent women at or near the menopause. It consists of an excessive sensibility of the nerves supplying the mucous membrane of some part or all of the vulva. The slightest friction excites intolerable pain and nervousness; even a current of cold air produces discomfort, and the least pressure is intolerable. Sexual intercourse is often impossible. The disease sometimes is 
associated with vulvitis or a painful urethral caruncle; in other eases no tangible or visible cause can be found. It differs from pruritus by the absence of itching, and from vaginismus in not causing spasmodic contraction of the vagina.

\section{Treatment of Hyperæsthesia of the Vulva.}

The treatment is unsatisfactory. Both the complete destruction of the mucous membrane of the sensitive area with causties, and exeision, have failed to give relief. Sexual intereourse should be prohibited and the patient placed in hygienie surroundings with cheerful company. The general treatment is by tonies, sea-bathing or warm-water bathing, and massage. Loeal lesions, if present, are treated aceording to their special indications.

\section{VAGINISMUS.}

Like pruritus vulvæ, vaginismus is not a disease, but a nervous symptom due in some eases to appreciable, in others to unknown causes. It is charaeterized by spasmodic eontractions of the muscles surrounding the vulva and lower portion of the vagina. The condition is analogous to laryngismus. The spasms oceur upon attempted coitus or upon the attempt to make a digital or speculum examination. The writer has observed one strongly neurotic case in which the wonian declared that the spasm occurred violently whenever coitus was attempted, but not the slightest objection was made to digital or speculum examination.

\section{Etiology and Clinical Course of Vaginismus.}

The condition is confined mostly to young neurotic, hysterical women. The palpable or visible lesion is usually in the form of an irritable hymen or an irritable caruncle of the meatus urinarius. If the hymen has been ruptured, the irritation will be in the remains. of it-the carunculæ myrtiformes. These caruncles and the urethral caruncle in some cases contain a superabundanee of excessively sensitive nerve-filaments. They, in fact, may resemble neuromata. In other eases the sensitive caruncles are absent, and the vaginismus is characterized only by an excessively sensitive vaginal ontlet, which may or may not be the seat of inflammation or erosion. Repeated attempts at coitus against an unyielding intaet hymen may give rise to vulvitis and extreme tenderness-a condition which should not be confounded with vaginismus.

There may be no appreciable cause of the disorder save a progressively increasing nervous apprehension on the part of the wife; each attempt gives rise to greater nervous exeitement until the pain and fear of coitus and the extreme spasnodic contraction of the levator ani and neighboring muscles which form the sphincter vaginæ preclude the possibility of a snceessful effort. Thomas has given to this distressing symptom the name "dyspareunia." "Penis captivus" has been known to result from an otherwise normal eoitus. 


\section{Treatment of Vaginismus.}

Any discoverable local cause should be removed. A vulvar tampon of gauze'saturated with a 4 per cent. solution of cocaine, kept in place ten minutes before the attempt, may lead to successful coitus, and therefore to utero-gestation and parturition, which in most eases, though not in all, effects a cure.

Vulvar inflammation and erosion require the treatment described under - Vulvovaginitis. Excision of the irritable caruncles and gradual or forcible dilatation of the vagina have in many eases given relief. The mere division of a rigid or imperforate hymen may be sufficient to remove the obstacle. Gradual dilatation is made by the introduction of graduated rectal bougies, to be worn an hour or more daily. Forcible dilatation requires ether, and should be followed by the continued wearing of a Sims vaginal plug. Meantime the patient remains in bed until the divulsed vaginal walls have healed. The plug should be removed only during urination, defecation, and the giving of the vaginal douche ; after healing, it may be introduced daily by the patient in order to retain the effects of the divulsion. In obstinate cases divulsion will be inadequate. It is sometimes necessary to incise deeply at several points or to make two quite deep lateral incisions on either side near the posterior vulvar commissure. These incisions should divide completely the underlying muscles and their fascia ; they may be closed by lines of union running at right angles to the directions in which the incisions were made, or until healing is established they may be kept open, as already described, by means of the vaginal plug. See index for Sims' vaginal plug. 


\section{CHA PTER XIII.}

\section{METRITIS-INFLAMMATION OF THE UTERUS.}

\section{General Considerations.}

THIs chapter should be read in connection with Chapter X., on the General Principles of Infection and Inflammation of the Pelvic Organs; and Chapters XIV. to XVIII., which treat of metritis as it affects the various parts of the uterus.

Inflammation, broadly defined as the reaction which living tissue exhibits to morbid irritation, may include a wide variety of lesions. These lesions, as related to the uterus, have variously and sometimes vaguely been designated as chronic metritis, subacute metritis, subinflammatory states, irritative states, and congestive states.

Anatomy and Physiology.-Before entering upon the study of metritis, which is the study of the anatomy and physiology of the uterus as modified by inflammation, the following suggestion of such parts of the anatomy and physiology as will aid in a description of these inflammatory processes will be useful.

The interior of the uterus is divided into two cavities, the cavity of the corpus and the cavity of the cervix. The former is protected somewhat from infection from above by the two muscular constrictions which divide it from the Fallopian tubes; from below by a similar arrangement at the internal os. The cavity of the cervix is protected in like manner from infection from above by the internal os; from below by the external os.

The Uterine Wall is made up of three layers: the mucous layer, called the endometrium ; the muscular layer, called the myometrium, consisting of three coats; and the serons or peritoneal layer, sometimes called the perimetrium.

The Endometrium is composed of lymphatics, blood-vessels, nerves, glands, and connective-tissue cells, and is covered by a single layer of ciliated columnar epithelium. This epithelium also lines the uterine glands and is continued through the Fallopian tubes. The same variety of epithelium, modified, also lines the cavity of the cervix uteri. Pavement epithelium, beginning at the external os, covers the external vaginal portion of the cervix.

The Glands of the Corpus Uteri are tubnlar, narrow, branching depressions. They dip down into and through the endometrium and penetrate to the muscularis. These tubular glands, penetrating everywhere throughout the endometrium, make up a very large part of its volume. They all open into the uterine cavity, sometimes two by a single orifice.

The corporeal endometrium is bound firmly to the inner coat of 202 
the muscularis by connective tissue whieh is continuous with that of the myometrium.

Lymph-and Blood-vessels and Nerves.-The lymph-spaces and lymphvessels of the uterus are abundant in the endometrium, in the muscular strata, and in the serosa. Converging, they pass by large channels outward through the broad ligaments. Figure 83, and the Frontispiece, Plate I. 'The nterus is supplied richly with nerves, both spinal and sympathetic. The arteries and veins are illustrated in the Frontispiece and in the chapter on Myomata, under myomectomy.

Figure 82.

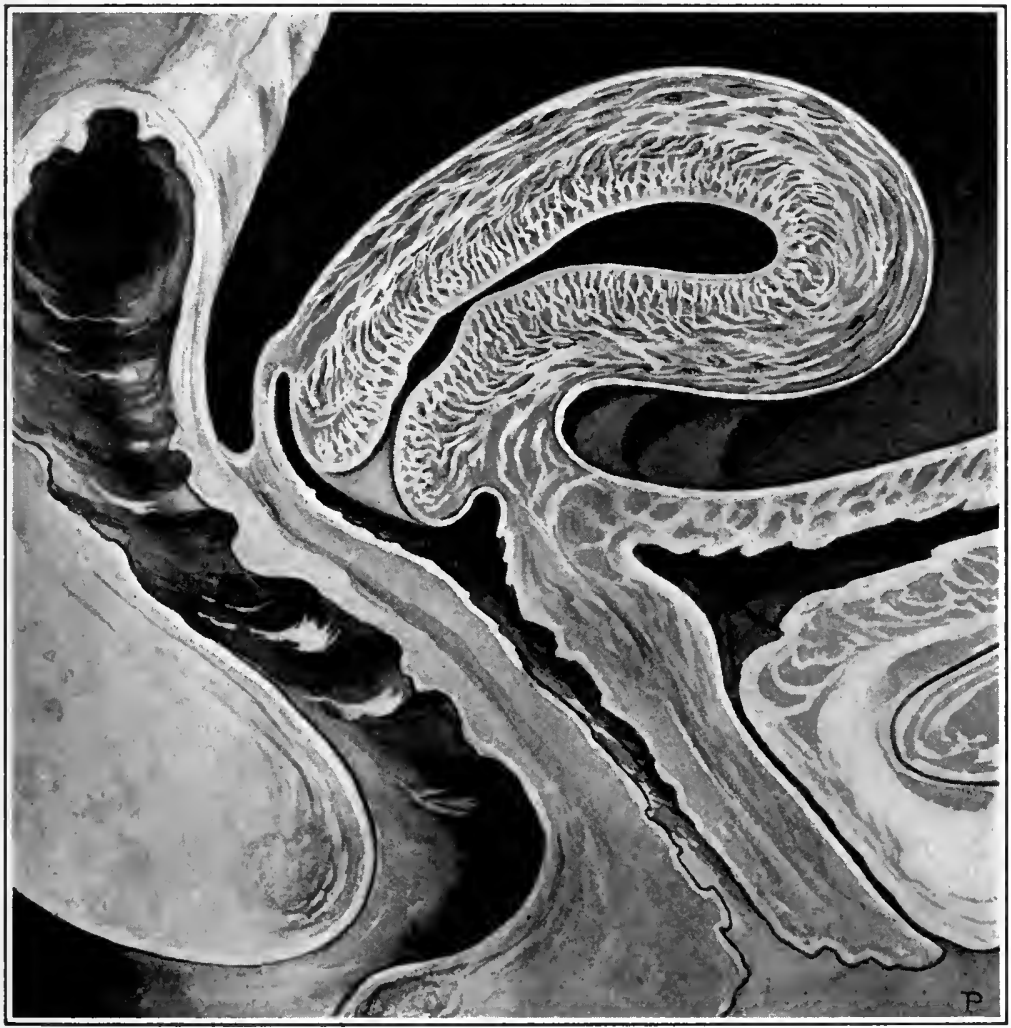

Long section of the uterus and adjacent parts-rectum, vagina, and bladder. The layers of the uterine wall are indicated ; they are the mucous layer or endometrium, the three musch. lar layers comprising the myometrium, and the serous or peritoneal layer, known as the peri. metrium.

The Minute Anatomy of the Cervix differs from that of the eorpus uteri in the following particulars. Its mucous surface has a peculiar arbor vita appearance, as shown in Figures 84 and 85 . The upper two-thirds of the intracervieal mueosa, like the corporeal, is lined with a single layer of ciliated colummar epithelial cells, which, sinnilar to those of the eorpus, and modified to the shape of a culy, pass without eilia into the cervieal glands; the epithelium in the lower 
Figure 83.

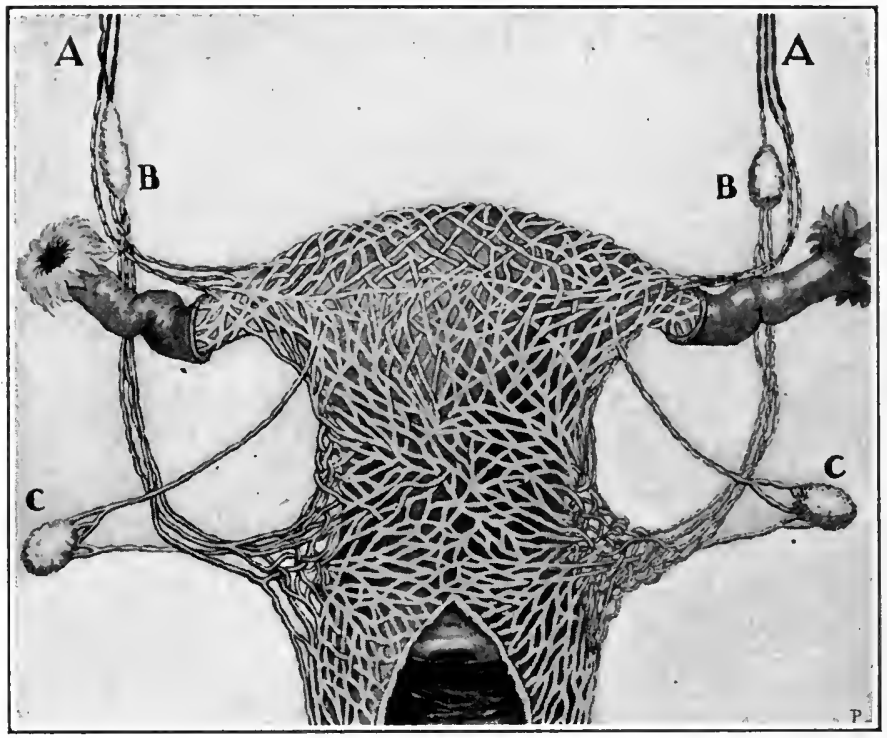

Lymphatics of the uterus.

third gradually changes to squamous epithelium, and at the os externum is continuous with the squamous epithelium of the vagina. The connective-tissue cells are closer together in the cervical than in the

Figure 84.

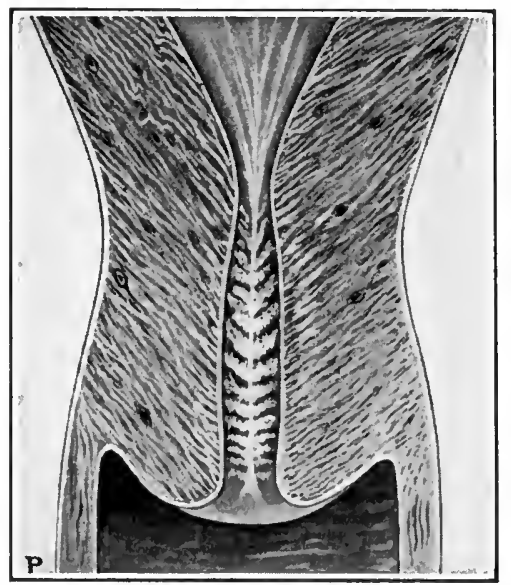

Figure 85.

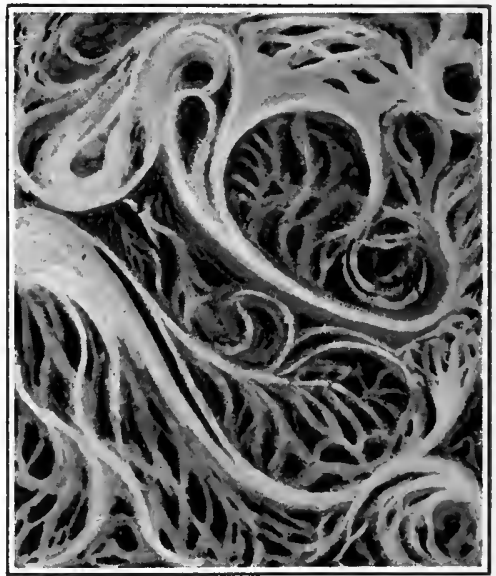

FIGUre 81.-Arbor vitea arrangement of cervical mucosa. Natural size. FigURE 85.-A Abor vita appearance of cervical mueosa. Magnified.

corporeal mucosa, but the cervical mucosa, more dense than the corporeal, is bound less firmly to the muscularis by looser connective tissue. 
An inportant function of the cervix is that of a sphincter to separate the corpus uteri from the vagina. The normal secretion of the uterine glands is alkaline, that of the corpus clcar and watery, that of the cervix clear and viscid. A yellow secretion is evidence of disease. The minute anatomy of the uterine mucosa will be described in Chapter XVI. on Chronic Endometritis.

The essential function of the corporeal mucosa is the formation of the decidua and the nourishing of the embryo. The connective-tissue cells produce the cells of the decidua of pregnancy; this, with the progress of uterogestation, matures, becomes over-ripe, degenerates, and, being of no further use, at term is cast off. The cervical mucosa has no part in menstruation.

The most significant factor in metritis is the enclometrium. It exhibits in the developmental and atrophic changes of puberty and the menopause, in the vascular changes of the menstrual ebb and flow, widely and constantly varying states. Inflammation of the uterus may occur during infancy, before the endonetrium has matured; during puberty, when it is maturing; during maturity, when it has reached its full physiological significance; during the menopause, when it is undergoing degeneration; or during senility, when in the physiological sense it has disappeared forever. The occurrence of metritis under such diverse conditions partly explains the wide and variable range of its phenomena and the difficulty of description, and partly accounts for the confusion of classification and nomenclature which runs through the literature.

\section{Classification of Metritis.}

The general subject, classification of infection and inflammation, has been discussed in Chapter $X$. The current classifications of metritis are numerous and faulty.

The Etiological Classification is based upon predisposing causes, such as parturition or traumatism, and upon the bacterial causes. The difficulty in identifying the causes and in differentiating between the many causes in a given case detracts from the value of the etiological classification as a clinical guide. A bacteriological classification may, under conditions of more exact knowledge, ultimately become a practical diagnostic, prognostic, and therapeutic guide. Already indications point strongly in this direction.

The Pathological Classification, so-called, into catarrhal, suppurative, granular, and ulccrative inflammations, is rather a designation of certain phases of the inflammatory process than a classification.

The Anatomical Classification includes endometritis (corporeal and cervical), myometritis, parametritis, and perimetritis. If these varieties usually occurred as distinct circumscribed lesions, instead of complicating one another; if each could be known by its own peculiar symptom-group; if ordinarily one could be separated clinically from the other-then the anatomical classification would not be, as it is, impractical and misleading. Endometritis, for example, cannot long continue without involvement of the myometrium, and vice versa. 
General metritis includes the peritoneal covering of the uterus. There are no sharp clinical or pathological lines of demarcation between the anatomical divisions of nterine and peri-nterine infection.

To illustrate the hopelessness of the attempt to classify metritis, observe the following from an otherwise excellent modern treatise. This work classifies metritis into (1) aeute inflammatory, (2) heinorrhagic, (3) catarrhal, (4) chronic painful. In the first division the word inflammatory is tautological. Any of the so-called varieties may be hemorrhagic, catarrhal, or painful. It is possible, therefore, to retain of this classification but two words-acute and chronic. 'The following paragraphs will show still further that any elaborate attempt at definite classification, even though diagrammatically attractive, is clinically impossible.

\section{Nomenclature of Metritis.}

The nomenclature of the foregoing paragraphs, although not the outgrowth of adequate classification, is yet useful as a means of naming certain forms and phases of metritis. Such words as gonorrhoal, parenchymatous, and catarrhal are convenient for purposes of description. The name endometritis, for example, will be used to describe not a distinct lesion independent of the rest of the uterus, but rather an essential part of uterine infection. In this way we shall not lose sight of the clinical relations between the various forms and phases of metritis. 


\section{CHAPTER XIV.}

\section{ACU'TE METRITIS.}

WHEN infection reaches the uterus, it usually attacks first the mueosa, and then may extend to the myometrium and perimetrium. Metritis is therefore a combination of endometritis, myonetritis, and perimetritis. The storm-centre of the infection is usually the endometrium, and the essential lesion endometritis. It is impossible to draw definite lines of division between these three forms of metritis. The terms endometritis, myometritis, and perimetritis will be used therefore not to describe separate and distinct lesions, but rather to identify the morbid changes that may occur in definite parts of an infected uterus.

\section{Etiology of Acute Metritis.}

The general subject of etiology has been ontlined in the ehapter on the General Principles of Pelvic Inflammations.

Predisposing Causes.-Those influences that induee pelvic congestion are favoring conditions for metritis; they are :

1. Menstrual congestion.

2. Suppression of the menses.

3. Displacements.

4. Constriction and consequent obstruction in the uterine canal.

5. The improper use of pessaries.

6. Parturition and abortion.

7. Traumatisms.

8. Excessive coitus.

Formerly these conditions and others like them were supposed to be the essential eauses. Now it is known that they contribute to the production of metritis as predisposing eauses when supplemented by some other influence. This influence is the exciting cause, is usually, at least, baeterial and produced from without, seldom from within.

Exciting Causes. - Among the exciting eauses numerous bacteria and their products predominate. These bacteria have been discussed partially in the General Principles of Inflammation, Chapter X., and in the Etiology of Vulvovaginitis. They usually invade the eavity of the ecrvix uteri from below, intrench themselves in the cervieal glands, and thence may be distributed directly by eontinuity of surface or tissue to the endometrium, the Fallopian tubes, ovaries, and pelvie peritoneum. Bacteria may also pass direetly by the lymphatic or venous cireulation from the cervix, vagina, rectum, or bladder to the ovaries and peritoneum. From these organs they may descend by continuity of the mucosa through the tubes to the endometrium. 
The cavities of the cervix and corpus uteri, especially the latter, are normally free from pathogenic bacteria; bacteria may, however, easily find access to these parts, and will then be active or inactive according to the degree of resistance which the tissues exhibit to their presence. The corporeal and cervical mucosa, penetrated throughout with a great abundance of tubular glands, are adapted especially to incubate and distribute bacteria. This accomnts for the tendency of metric and perimetric infection to become chronic.

The gonococeus of Neisser, since it has great power to penetrate the glandular elements and to intrench itself therein, is one of the most frequent and destructive causes of metritis. The staphylocoeci of suppuration are found commonly also in suppurative endometritis. The streptococcus pyogenes, very infectious and fatal, produces one of the graver forms of puerperal and traumatic pelvic infection. The great danger of this germ is in the fact that it does not strongly attract leucocytes, and therefore does not excite defensive action. The diphtheria and tubercle bacilli, Bacilli coli communis, and other bacteria may also be the exciting causes of metritis. Infection is introduced often from want of cleanliness during the puerperal state, from imperfect asepsis in parturition, in treatment, and in surgical operations. Direct infection through coition is common.

During the three or four days after parturition and just before menstruation the physiological congestion of the uterus renders it most susceptible to infection. The cervical portion at all times is apt to be the habitat of pathogenic germs; such germs often are introduced by the physician's finger, or upon septic instruments of which the unclean uterine sound is a striking example. The infectious material may be inactive unless the soil is prepared to receive and develop it; but when the traumatisms of abortion and parturition, of accident and of surgery, have opened wide the door for bacterial invasion, infection will be the natural result.

\section{Pathology of Acute Metritis.}

The lymph-channels and blood-vessels bring into direct and close communication with one another the endometrium, myometrium, parametric cellular tissue, Fallopian tubes, ovaries, and peritoneum. The uterine mucosa thus becomes both the starting-point and the distributing-point of the infection. The infected endometrium may abundantly pour its toxic products through the lymph- and blood-streams, with resultant lymphangitis, phlebitis, cellulitis, salpingitis, peritonitis, and ovaritis. The infection may spread from the endometrium not only by the vessels, but also by continuity of mucosa to the Fallopian tubes, peritoneum, and ovaries, or in like manner may descend from these organs to the endometrium.

The close physiological and anatomical relations of the lymphstream to the endometrium and uterine peritoneum partially explain the swift and terrible march of the traumatic and puerperal infections to a destructive or even fatal result. The toxins, usually streptococci or other pus cocci and their products, are taken up and widely distributed by the 
lymphaties or veins. They may be carried through the vessels without infecting them, or may infect them and produce lymphangitis, lymphadenitis, or phlebitis. The inflammation may be so intense as to destroy the vessels, or resolution may bring about complete recovery. Inflammation in the lymphaties or veins may result in lymph thrombosis or venous thrombosis. This is nature's way of limiting the spread of the infection. When recovery takes place, the lymph- or blood-stream is re-established around the thrombosed parts of these vessels by collateral circulation. Perilymphangitis and periphlebitis may oceur in the cellular tissue around the thrombosed lymphatics and veins. This process when it takes place in the parametria is pelvic cellulitis, a disease almost forgotten in these days of tubal and ovarian pathology. See Pelvic Cellulitis.

The anatomical changes may be summarized as follows:

1. Uterus enlarged, regular in ontline, and of doughy or soft consistence.

2. Congestion extreme; there may be extravasation of blood in the muscularis.

3. Endometrium and perimetrium deeply reddened in circumscribed areas throughout.

4. Small-cell infiltration of interglandular and intermuscular connective tissue.

5. Engorgement of lymph-vessels and excessive secretion from the uterine glands.

The milder cases, chiefly characterized by engorgement, increased secretion, and pain, may subside in a few days, and the uterus either may become normal or lapse into a state of chronic metritis. In the more severe forms the disease may run a destructive course to the death or permanent disability of the patient, and will in extent vary with the virulence of the exciting cause and the resistance of the inflamed structures. Abscesses rarely develop in the myometrium except in connection with myomata. Inflammation of the mucosa may be catarrhal, suppurative, ulcerative, hemorrhagic, or all combined.

A grave form of acute disease has been described under the name diphtheritic or dissecting metritis. ${ }^{1}$ The infection is usually puerperal, but is sometimes a sequel of non-puerperal diphtheria. It may be associated with gangrene of the vulva, and may occur after scarlet fever, typhoid fever, or cholera. In puerperal cases, says Garrigues, the diphtheritic infiltration may extend from the endometrium to the neighborhood of the peritonenm, cutting off a large part of the muscular layer, which after weeks or months will be expelled as a pearshaped borly. Dissecting metritis may be connected with similar disease of the vulva and vagina.

The ultimate possible changes which may follow acute metritis in the uterine glands, utcrine connective tissue and muscularis, and in the peritoneal covering of the uterus, are cliscussed elscwhere. See Chronic Endometritis, Chronic Metritis, and Peritonitis.

1 Garrigues, "Dissecting Metritis," New York Medical Journal, 1882, vol. xxxvi.p. 537; Diseases of Women. 


\section{Symptomatology of Acute Metritis.}

'The following tabular statement embraces the prineipal symptoms of acute metritis :

1. Elevation of temperature variable, sometimes preceded by a chill.

2. Pain, commonly confined to the hypogastrium, may radiate to the back or thighs.

3. Painful defecation; rectal tenesmus.

4. Frequent and painful urination.

5. Nausea ; vomiting and constipation usual.

6. Menses suppressed or decreased; occasionally increased.

The symptoms of acute metritis depend upon the extent and gravity of the disease, and therefore may vary within wide limits from those of a mild infection to those of the greatest virulence. An apparently mild metritis may, however, result in the most destructive pelvie infection with all the results of grave peritonitis. The onset usually is marked by a ehill, followed by variable high temperature and pulse; the pain is often intense. There is usually tenesmus of the rectum and bladder. Menstruation, if present, may cease suddenly, or the flow may increase. 'The menstrual fluid is mixed with the secretions of the inflamed glands. The congestion often passes off as menorrhagia comes on. 'This is nature's way of relieving the engorged ressels. The discharge, especially in the gonorrhœal form, may become rapidly purulent. When the inflamed uterus contracts to expel its abundant secretions the agony is that of exaggerated labor-pains. Bearing-down and heat in the pelvis are often excessive. When the disease has extended to the Fallopian tubes, pelvic connective tissue, ovaries, and especially when it invades the peritoneum, there may be grave, even fatal toxæmia with anxious facies, increased romiting, and tympanites.

\section{Diagnosis of Acute Metritis.}

The diagnosis is based upon the ehanges just deseribed, which, if present, will give rise to the following physical signs:

1. Tenderness on pressure over the hypogastrium and in the vaginal formix.

2. Abdominal muscles tense.

3. Vagina dry and hot to the touch.

4. Pathological seeretions from assoeiated endometritis.

5. Uterus enlarged, softened, and tender.

Examination is often so painful as to be impracticable without anresthesia; the cs uteri is usually patulons, and often surrounded by erosion. The ragina is hot, and the arteries strongly pulsating. The urgent neeessity is to watch for tubal and peritoneal extension. See Diagnosis of Salpingitis and Pelvic Peritonitis. The mere recognition of acute metritis is wholly inadequate. Unless the state of the uterine appendages and parametria is made ont accurately, therapeutic indications of the greatest urgency may be overlooked; one 
therefore may have to make an examination under anæsthesia; as the ease progresses repeated examinations may be necessary. In all acute inflammations of the genitals the use of the sound is contraindicated strictly.

\section{Prognosis of Acute Metritis.}

The prognosis of acute metritis is always disquieting, often grave. The disease may terminate in rapid resolution or in chronic metritis. Extension to the peritoneum involves immediate danger to life or remote danger to health. The relative virulence of different microbes has been discussed in the paragraphs on Etiology. Puerperal metritis is most liable to spread with the lymph-stream, and is, especially when due to streptococcus pyogenes, the gravest form ; this form even when early recognized and promptly treated by radical surgery is apt to result fatally, for the toxins are specially deficient in their power to attract leucocytes-that is, to build up a limiting wall around the infected centre and thereby to protect the general system against invasion. For this reason the streptoeoccus germ may reach the uterus in an hour ; in two or three hours more it may have passed far beyond the uterus, where surgery cannot reach, much less remove, it.

\section{Treatment of Acute Metritis.}

Future bacteriological researches may open the way for an etiological classification that will furnish a safe and definite guide to the therapentic indications. Work in this direction thus far, however, gives little promise of immediate practical results. In this connection we may add that serum therapy is undeveloped, and therefore, in a practical sense, is not very pertinent to the subject.

The treatment is not very satisfactory ; it is prophylactic, abortive, palliative, expectant, and surgical. See 'Treatment of Sepsis in Chapter VIII.

Prophylaxis includes the avoidance or removal of the predisposing and exciting causes. Reference to the etiology will suggest the appropriate indications. Susceptibility is greater during the puerperal state, parturition, abortion, and menstruation. Extra care, therefore, at such times is essential. Fspecially forbid undue exposure of all kinds. Avoid the bacterial exciting causes by asepsis. Aseptic midwifery is imperative. The minor gynecological and obstetric examinations and manipulations without asepsis are dangerous. Above all, one should use every means to prevent the spread of a vulvovaginitis, especially if it be gonorrhoal, to the uterus. See Treatment of Vulvovaginitis.

The Abortive Treatment is applicable only in the onset, and inclndes such antiphlogistic measures as may cut short the attack during the stage of congestion. The disease once established must run its course. A large blister over the hypogastrium may be useful. Leeches are of great value if early and thoroughly applied. Use five or more 
over each inguinal region and five to the perineum-two or three are useless. The ice-bag over the hypogastrium is useful. A most essential thing is early and active catharsis by a mercurial purge, two grains of calomel or five of blue mass, repeated if necessary, and followed by Rochelle salt or some other saline. The treatment is palliative and expectant in the milder cases, but may have to be energetic in the more virulent. In cases of metritis following plastic gynecological operations the sutures should be removed immediately and the denuded surfaces canterized with pure carbolic acid.

The Palliative Treatment includes rest in bed, anodynes, especially the opiates, the hot or warm water vaginal douche-the hotwater bag, and the hot hip-pack. When the acute stage is subsiding there may be use for the glycerin and wool vaginal tamponade. Chapter IV. Later, iodine counterirritation applied to the hypogastrium and vaginal fornix, though highly recommended, are of doubtful value. Severe pain may be relieved by a suppository of aqueous extract of opium, one grain, and extract of belladonna, onesixth of a grain.

Expectant Treatment.-The milder self-limited infections which have no grave systemic or local manifestations may be dismissed with palliative or expectant treatment. In grave infections it may be extremely difficult or impossible to choose wisely between the danger of the disease and the extra peril of surgical interference; hence, even in serious cases, the expectant course may be the part of wisdom.

Surgical Treatment.-When the systemic condition is grave and the nervous system indicates profound toxæmia the disease under any treatment will in a large proportion of cases terminate fatally. A number of practical and momentous questions at once arise :

Question 1. Is there simple absorption into the circulation from some focus of decomposition in the uterus? Is the toxæmia due to the products of a decomposing foreign body, such as a blood-clot, a fragment of placenta, retained membrane, or pent-up lochia? In other words, is it due to the absorbed products of putrefactive bacteria? To put the question in more concise form, Is it sapræmia? If the answer be in the affirmative, the indication is clear and imperative to remove the putrefying mass, wash out the endometrium, and establish drainage. The offending mass may be removed with the finger, the placental forceps, or, if necessary, with the dull curette, or if the uterine canal is open it may be wiped ont with a swab attached to a long forceps. Sharp curettage, powerful cauterization, and all other severe surgical measures in this connection are unnecessary.

Question 2. Is the uterine mucosa the seat of an infection, and as such is it the distributing-point of bacteria which may spread and infect the uterine appendages and peritoneum? If the bacterial invasion has extended beyond the uterus, to what extent are the uterine appendages and peritoneum invaded? Is the systemic disturbance such as to suggest that the bacteria and their products are very liable to enter the general circulation in quantities sufficient to give rise to pronounced septicæmia? 
Question 3. Have pus emboli been carried through the eireulation from one focus of suppuration to set up other foei in different parts of the body, and thereby produce metastatic abscesses? To put the question in another form, Is there pyæmia?

If the answers to the seeond and third queries are in the affirmative, it becomes essential to deeide whether the infection has spread so far beyond the uterus as to make the metritis relatively insignificant. Clearly, if there are metastatic abseesses, or if even infection has spread to the other pelvie organs, surgieal treatment of the intrauterine infeetion alone would be useless and might add to the danger. Abdominal or vaginal section and the drainage of the abseesses, or even removal of the uterus and its appendages, would then have to be considered.

The milder eases, as already stated, may safely be left to palliative and expectant treatment. The graver infeetions unfortunately have in the majority of cases passed beyond the range of intra-uterine therapeuties before the question of operative interference is forced upon the surgeon. We may, however, be eoneerned with the question, What surgieal measures, if any, are justifiable in the effort to prevent the further spread of dangerous acute uterine infection which still is confined nearly or quite to the uterus?

If dilatation, eurettage, and drainage are to be inroked, the ehoice of method at onee becomes important. In this eonsideration let us not lose sight of the purpose of these proeedures: it is to ent short and prevent the extension of uterine infeetion; or, if already in a degree extended, to limit its force by withdrawing the toxie supply. Partial, inefficient curettage, which opens up lymphaties and veins to fresh infection, but does not remove all the infected mucosa, will prepare the way for further infection, which may be more virulent and more sweeping than the first; as tersely stated by de Lee, such a procedure is like raking a pateh of lawn after scattering seed over it-a veritable insemination. It is evident, therefore, that eurettage, if indicated at all, should be thorough; should, indeed, stop at nothing short of the removal of the entire infeeted mucosa. The sharp curette, which generally has been eonsidered a more dangerous instrument than the dull one, is then less dangerous. The operations reported by Pryor, Krug, and others, indeed prove that the sharp eurette in eareful hands is much less dangerous than has been supposed. The thorongh applieation of it in seleeted eases has, aceording to reliable report, been followed by prompt deerease in the toxæmia and in the other grave symptoms. The opponents of the operation declare, not without reason, however, that most of the recoveries would have occurred withont it, and that many of the failures have oceurred in consequence of it. In the present state of our ignoranee on this perplexing subject, suspension of judgment and expectancy are perhaps more rational than dogmatic assertion or radical measures.

If the infected endometrium has become soft, spongy, friable, and macerated, and if it is decided that thorough eurettage will lessen the danger of the extension of the infection, the steps of the operation will be as follows: 
1. Anæsthesia.

2. Preparation of the vagina and external genitalia as directed for minor operations in Chapter II.

3. Dilatation of the uterus, unless it is already sufficiently open.

4. Curettage of the infected endometrium by means of the sharp curette; see Curettage, Chapter V.

5. Thorough irrigation of the endometrium with hot sterilized water.

6. Thorongh mopping out of the endometrium with cotton wound on dressing-forceps, and dipped in a saturated solution of ioline crystals with pure carbolic acid.

7. Placing of an antiseptic dressing over the vulva.

Some operators omit the iodine and carbolic acid applications and rely upon the thoroughness of the curettage to remove all infectious matter. An advantage, however, in the use of this powerful disinfectant lies in the fact that it insures thorough disinfection of any infected shreds which may have escaped the curette, and that by its cauterizing effect it so shuts the mouths of the freshly opened lymphvessels and blood-vessels that further absorption through them is less likely to occur.

It is the custom of many excellent operators after curettage to tampon the endometrium lightly with a continuous strip of antiseptic gauze, and to fill the vagina with another strip somewhat wider; after twenty-four hours they remove the gauze, repeat the intra-uterine irrigation, and introduce fresh gauze. Anæsthesia now is not usually required. Before the introduction of the ganze it is well to make a thorough intra-uterine application of creolin or of a 25 per cent. solution of ichthyolate of ammonium in glycerin. It is a mistake to saturate the gauze with such medicinal substances, because they interfere with its chief function-capillary drainage. Iodoform and sublimated ganze have caused dangerous poisoning, and, therefore, should not be used in large quantities. If the grave symptoms have subsided, the gauze may be removed at the end of twenty-four hours, and need not be renewed. In very infectious cases some operators renew the gauze and irrigate with hydrogen dioxide daily until the nterine secretions become normal. See Treatment of Chronic Endometritis, Chapter XVII., for a further discussion of intra-uterine curettage and drainage.

The operation given above is less dangerous and more rational than the meddlesome half-way measures of intra-uterine medication and irrigation of the undilated septic uterus. The judicions selection of cases is manifestly a matter of great difficulty. If proper selection can be made, the operation in careful hands may be permissible and useful.

The writer's personal conviction of the value of dilatation, curettage, and drainage of the endometrium in acute infection is that the measure should be limited in its application. Let no man be lured to the performance of this dangerous operation in an acute case because of the ease, safety, and efficacy of the similar procedure in chronic endometritis. The only cases in which it should be performed are 
those which otherwise will result in dangerous spreading of the infection.

Clearly eurettage manifestly is contraindicated in the numerous and grave cases in which the infection has passed to the parametria, not from the endometrium, but by the lymph-vessels or blood-vessels.

All admit the practical difficulty, not to say impossibility, of selection so as to limit the operation to those infections which are really dangerous and still confined to the uterus. It is, moreover, a serionis question whether the course of grave puerperal, gonorrhoal, or traumatic infection often is arrested by the procedure. At the same time few will deny that the operation repeatedly has given rise to fatal results. On the other hand, expectancy and palliation will often be rewarded by the subsidence of grave symptoms and by final recovery. There ean be, moreover, for a surgeon no greater eause of regret than the fact that he has exhausted the resisting forces of his patient by a dangerous half-way measure which itself may have contributed to the necessity for a more radical operation, and that while with the promise of such a measure he has been lulling himself into a sense of false security the infection has gained irresistible force. If urgent indications arise, the best hope of recovery may be in abdominal or vaginal section and drainage, or the removal of the uterus together with its appendages. These operations, if indicated at all, are made necessary by the rapid spread of the infective process and therefore become at once imperative. Until the necessity for such extreme measures becomes apparent, there is at least virtue in the attitude of watchful expectancy. See Vaginal Incision and Drainage, in Chapter XXIII.

Treatment of Puerperal Metritis.-Much of seeming plausibility has been written about the treatment of puerperal infection of the uterus and adjacent organs, but from the standpoint of scientific exactness it is all unsatisfactory and leaves us still on the low plane of empiricism. When called to a ease of puerperal infection in the first two or three days of the disease the author frequently has made use of the following procedures with good results :

1. Careful bimanual palpation, using rubber gloves, with or without anæsthesia, as the case may require.

2. If there are no gross lesions of the uterine appendages nor accumulations of pus in the pelvis to require a raclical operation, the patient is. placed on a table or across the bed with the buttocks well over the edge of the bed.

3. The vagina and cervix uteri are exposed with a Simon speculum and the anterior and posterior lips of the cervix are grasped with flat vulsellum forceps and the cervix drawn toward the vulva.

4. With the uterus held steady the entire endometrium is wiped out (curetted as it were) by means of a wad of gauze, attached to a long forceps, in order to remove any fragment of placenta, membranes, blood-clots, or secretions.

5. The endometrium after irrigation with a 1:5000 solution of formalin or some other disinfectant, is swabbed out (using fresh gauze on the forceps) with a 10 per cent. solution of ereolin or some 
other disinfectant, to be followed by the introduction to the endometrium of a half ounce of Crede's ointment of argentum colloidale. Thereafter the uterus is not invaded again unless some special indication arises.

6. One drachm of 'Credé's ointment may be rubbed in thoroughly for twenty minutes each day over the abdomen or back.

7. The intravenous injection of 15 grains of a 2 per cent. emulsion of argentum colloidale, once a day, is a recent and promising therapeutic resource.

8. If the infection is probably or certainly of streptococcic origin, the antistreptococcus serum may be used hypodermically 10-40 c.c. a day.

9. The bowels should be opened with mercurials and salines, or with a soft capsule containing two drachms of castor oil and one-half drop of eroton oil.

10. The author frequently has seen gratifying results from the free use of rum and milk, twelve ounces of rum and a quart of milk daily, given in divided doses at intervals of one or two hours. 


\section{CHAPTER XV.}

\section{CHRONIC ENDOCERVICITIS.}

THE synonyms of endocervicitis are cervical catarrh and cervical endometritis. Since the endometrium is situated entirely above the internal os, there is a manifest impropriety in using the word endometritis in connection with the cervix uteri.

In studying this subject the reader should have constantly in mind the physiological and pathological unity of the reproductive organs. Infection seldom is confined to a single part of the uterus; on the contrary, it extends usually to other parts and commonly spreads to adjacent organs. We are considering nothing less than the whole subject of metritis, but with special reference to the cervical mucosa.

The single layer of columnar epithelium, the underlying connective tissue, the lymph-spaces, the lymphatics, the veins, the arteries, and the nerves which make up the intracervical mucosa, are subject to certain chronic changes which are known under the name chronic endocervicitis. Similar disease of the corporeal mucosa is called chronic endometritis.

\section{Etiology of Endocervicitis.}

Endocervicitis is inflammation of the cervical mucosa. The predisposing systemic and local causes and the bacterial exciting causes have been pointed out in Chapter $\mathrm{X}$.

The disease is in some respects like, in others unlike, corporeal endometritis. It often occurs by extension from vulvovaginitis. It rarely descends from the corpus uteri. It may have been carried as a primary infection, without intermediate infection of the vulva or vagina, direct to the cervical mucosa. As in the corpus, it may involve not only the mucosa, but also the muscularis.

Although the normal endometrium is free from pathogenic bacteria, the cervical cavity is quite accessible to them. This explains the greater tendency of the cervix at all times, especially upon slight traumatisms, to become infected. The cervical glands, well adapted to receive, retain, and distribute infection, easily become a culture-ground for bacteria. Once intrenched in the gland-crypts, the germs may remain relatively quiescent for long periods, and then may develop new cultures and spread.

Among the more frequent predisposing causes of endocervicitis are the following:

Puerperal laceration of the cervix.

Excessive coitus.

Foreign bodies, tumors, polypi. 
The Exciting Causes are bacteria, especially gonorrhœal, which may reach the cervix direct or be carried by extension from the vulvovagina or endometrium.

\section{Sequence and Pathology of Endocervicitis.}

The pathological sequence of a seemingly insignificant infection of the cervix uteri, especially if acute, may be either by continnity of surface to endometritis, salpingitis, peritonitis, and ovaritis; or by the

Figure 86.

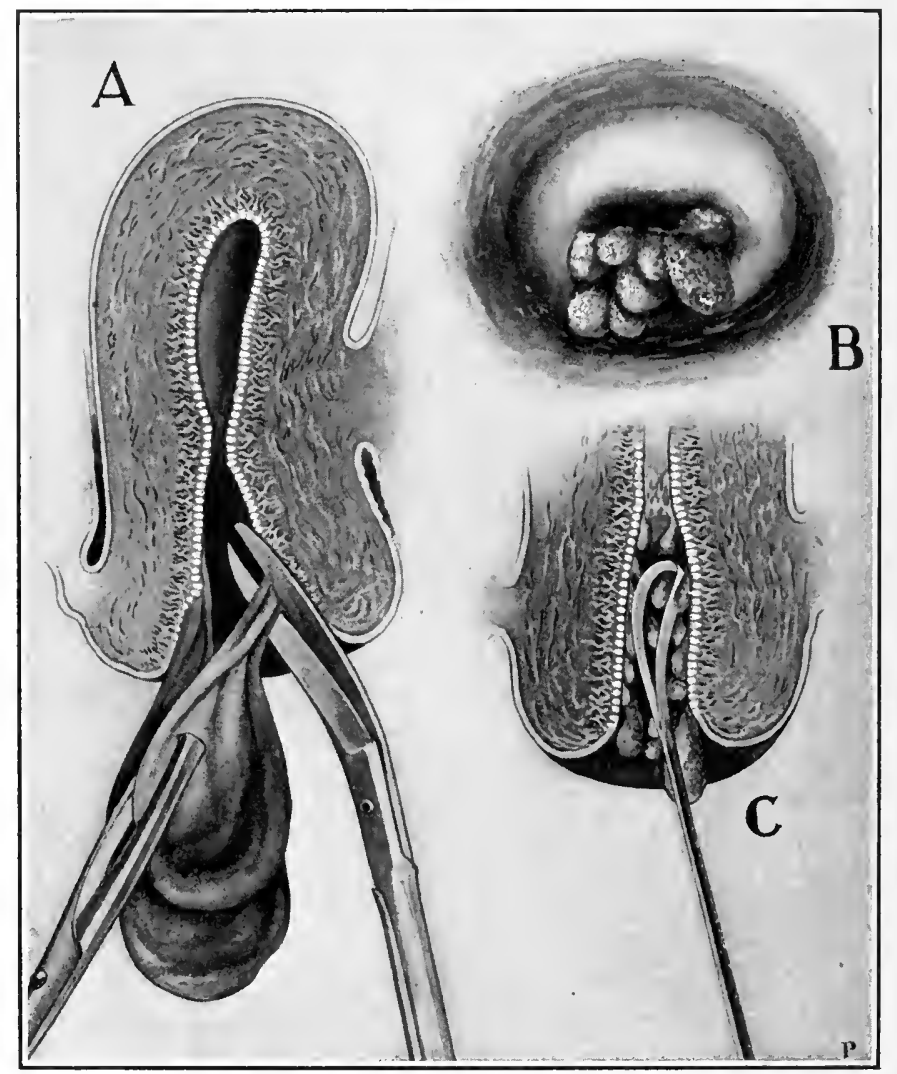

A, mucous polypi of the cervix uteri-follicular hypertrophy. One polypus has been seized with forceps and is being removed with scissors. $B$, small mucous polypi hanging out of the cervix uteri; C, mucous polypi being removed from the cervical canal with the sharp curette.

pelvic lymplaties and veins to pelvic lymphangitis, phlebitis, peritonitis, and ovaritis: thus acute infection seldom is confined to the cervix, but is apt to involve the other parts of the nterus. The corpus uteri and adjacent organs are more likely to be involved if the chronic cervicitis has followed an acnte inflammation; less likely if it was chronic from the beginning.

The swollen mucosa, especially if the eervix be lacerated, takes 
the direction of least resistance, and may protrude through the os externum. The thickened everted mucous membrane may give to the cervix the appearance of great enlargement. The condition is not unlike that of the prolapsed hemorrhoidal anus. The engorged open cervical glands in great numbers pour out their secretion upon the vulvovaginal surface. The discharge, mulike that of endometritis, is thick, ropy, viseid, abundant, and gelatinous. It may be dislodged only with difficulty from its anchorage in the cervical glands.

In nullipara the internal and external ora are sometimes so constricted as to cause retention of the cervical secretions and consequent dilatation of the cervical cavity. See Figures 93 and 94.

The chief pathological factors are erosion of epithelium and glandular enlargement. In this respect endocervicitis offers a elose analogy to corporeal endometritis.

Erosion of the Cervical Epithelium.-Erosion of the cervix is cliaracterized by a red or purple, livid color, is confined usually to the area immediately around the external os uteri, and not infrequently extends over the entire vaginal portion of the cervix; it has the appearance of ulceration, and in the older literature was so called. The affected area, as first demonstrated microscopically by Ruge and Veit, is covered by a layer of newly formed inflamed epithelium. The epithelium is not destroyed as in ulceration, but simply is eroded, and forms a mucous patch. Ulcerative processes-localized necrosis -have nothing in common with this condition; ulceration is the destruction of the superficial epithelium and inflammatory involvement of the underlying tissue, and as compared with erosion is very rare. Two varieties of erosion of the cervix have been deseribed:

1. Simple erosion.

2. Papillary erosion-coek's-comb granulations.

1. Simple Erosion, which answers to the description given in the preceding paragraph, presents a smooth, uniform, velvety surface, with little or no formation of new glands, and, althongh having the appearance of ulceration, shows, on microseopical examination, the characteristic non-specific mucous patehes covered by a single layer of newly formed epithelium.

2. Papillary Erosion.-This form of erosion, from its livid, red color and characteristic projections, has been ealled cock's-comb granulation. The irregularity of surface is due $(a)$ to newly formed glands; (b) to hyperplasia of the connective tissue beneath the surface epithelium and the glands; $(c)$ to elevations of the interglandular surfaces. The surface, as in simple erosion, is covered by a single layer of columnar epithelium. The papillary projections in gross appearance may resemble closely early cancer, but, unlike that growth, are nonfriable and have little disposition to bleed on handling. There is abundant small round-cell infiltration of the connective tissue and free secretion of mueus.

Glandular Enlargement oceurs in two forms :

1. Polypoid glandular enlargement-mucous poly pi.

2. Cystic glandular enlargement-cystic degencration-sometimes called follicular erosion, sometimes ovula Nabothi. 
1. The Polypoid Glandular Enlargement produces diminutive mucous polypi, smaller, of different origin, and softer than fibrous polypi. 'The genesis of mucous polypi is as follows: The enlarged glands protrude upon the surface; their mouths become obliterated; the glandular tissue is celematous from retained secretions; the bases constrict and the little masses become polypoid; they correspond to the so-called adenoids of nasal pathology. A similar development sometimes occurs in the endometrium as the result of endometritis. These mucous polypi, although the result of inflammation, are classified as benign adenoma by some pathologists.

2. Cystic Glandular Enlargement-Follicular Erosion.-Very often as the result of erosion the openings of the cervical glands become occluded by adhesive inflammation, so that the glands are distended by their own secretions. This process, known as cystic degeneration, results in the formation of numerous round submucous bodies, sometimes called ovula Nabothi, but more commonly known as follicular cysts or retention-cysts; they may be present in number from one to several hundred; they are hard, tense, spheroidal bodies, varying in size from that of a millet-seed to that of a pigeon's egg; and on digital touch if small feel like shot under the skin. Seen through the speculum, they appear as rounded elevations of yellow, blue, or gray color; they contain inspissated mucus, which sometimes is infected by pus micro-organisms, forming small circumscribed abscesses. These cysts when small are lined with the typical gland epithelium of the cervix, but as they become distended the epithelium flattens and finally disappears through pressure atrophy. Cystic degeneration, according to Emmet, is a cause of numerous reflex nervous disturbances; it rarely is seen on the nulliparous cervix, but is a frequent result of laceration, and as such will be described further in the chapter on that subject.

\section{Symptoms of Endocervicitis.}

Endocervicitis may cause no characteristic symptoms. The symptoms associated with it may be due to complications, and therefore have little or no diagnostic value; among then are disordered menstruation, sterility, pain in the back, a sense of weight in the pelvis, and functional disturbances of - extrapelvic organs, especially the organs of digestion.

\section{Diagnosis of Endocervicitis.}

The diagnosis is simplified by the accessibility of the diseased structures, especially when the inflamed swollen mucosa is rolled out in contact with the vagina and when the erosion extends ont over the external os; it must depend upon the physical signs, upon microscopical examination of tissues, and upon examination of the secretions.

Digital and Sight Examination.-Iacerations and cystic glandular enlargement are examined better by digital touch than by sight. Erosions and mucous polypi are soft and elusive, and therefore are seen better than felt. 
Speculum Examination.-The speculum, Sims' speculum preferred, will disclose some or all of the following conditions if they exist outside the external os:

1. Margins and scars of lacerations.

2. Retention-cysts-rounded yellowish or bluish projections.

3. Mucous polypi, protruding from the external os.

4. Erosions, as described under pathology.

5. External os filled with a plug of tenacious mucus or mueopus.

6. Ulcerations, which are seen rarely execpt those of malignant, tubercular, or cancerous origin.

Examination of Secretions.-The secretion is always abundant and viscid, and is usually clear, but may be murky from the admixtare of epithelium and leucocytes; it may also be yellow or greenish yellow from the presence of pus, or red from streaks of blood.

To obtain secretions for bacteriological examination for gonococei, tubercle bacilli, or other organisms, the method of Schultze may be employed, as follows :

1. Sterilize the vagina with douches.

2. Place a sterile cotton tampon against the cervix uteri.

3. On the day following remove the tampon, collect the secretions with a swab, and examine immediately.

The early differential diagnosis of erosions, both simple and papillary, from earcinoma is most important. Friability and bleeding upon handling with fingers or instruments are the two most reliable clinical signs of carcinoma as distinguished from erosions. The symptoms are not to be relied upon, because erosions may give all of the typical signs of carcinoma, and beginning carcinoma may give rise to few or no signs. For an absolute diagnosis a microscopical examination should be made of an excised part of the suspected growth. See Differential Diagnosis in Chapter XXVIII. on Carcinoma Uteri.

Ulceration of the Cervix may be related closely to endocervicitis. The following varieties of ulcer are recognized :

1. Decubitus ulcer, caused by ill-fitting pessaries and friction, as in procidentia uteri.

2. Carcinomatous ulcer.

3. Tubereular nlecr.

4. Chancre and ehaneroid.

As compared with erosion and glandular enlargement, ulceration is rare. The causes, pathology, and diagnosis are the same as for similar conditions in other parts of the body.

\section{Treatment of Endocervicitis.}

In the treatment of cervicitis it is well to remember the physiological fact that irritation at the opening of a duct will stimulate and increase the secretion of the gland or glands from which the duct leads; and, conversely, withdrawal of the irritation will cause a decrease in the secretion. The same is pathologically true of the uterine canal and the uterine glands. The irritation caused by endo- 
cervicitis may increase nterine secretions. Whatever will allay irritation of the cervix must tend to relieve the excessive glandular activity.

In acute or reeent endocervicitis the treatment, especially if the infection be gonorrhoal, should be strongly disinfectant. The purpose is to prevent extension to the corpus uteri and parametria. First, clean out the mucous plug, then thoroughly apply a saturated solution of iodine in 95 per cent. carbolic acid over the whole intracervical mucosa. The strong tendency of the infection to spread, and the consequent danger of the lisease being carried to the corporeal endometrium by the carcless introduction of instruments past the internal $o s$, should be kept constantly in mind.

When the chronic disease-process has penetrated to the deep mucous folds and glandular pockets, superficial treatment will fail. It then becomes neeessary to destroy the infected mucosa. Deeply acting caustics may accomplish this, but the resulting cieatricial contraction, especially when the canal is not very patulous, contraindicates their 1:se. The same objection in less degree applies to removal of the mucosa by sharp curettage. Thorough excision and covering by a plastic operation the surfaces thereby exposed are usually the best treatment. The operation of Schroeder, which fulfils this indication, is performed as follows:

Schroeder's Operation.-The patient being under anæsthesia and in Sims' lateroprone position, the cervix exposed by Sims' or Simon's

Figure 87.

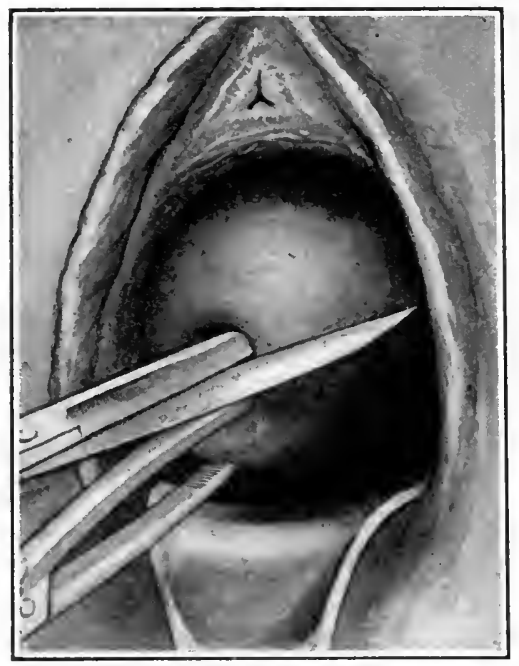

FIGURE 88.

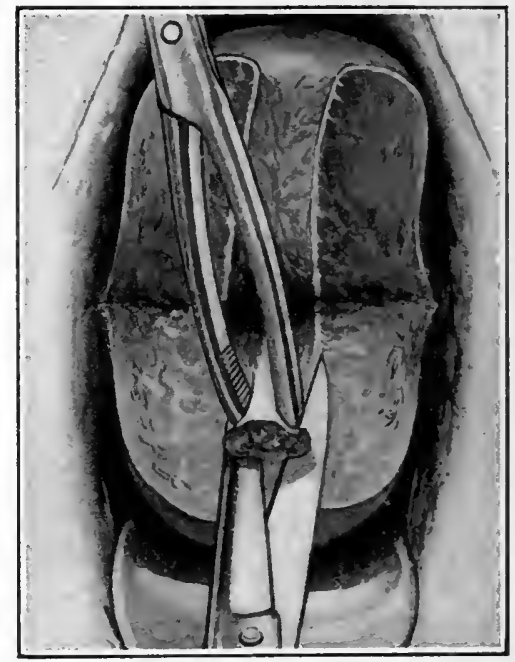

FIGURE 87.-Schroeder's operation, first step. Lateral division of the cervix uteri with straight scissors. l'osterior lip of cervix held with flat vulsellum forceps. Dorsal position. simon speculum.

FIGURE 8 s.-Schroeder's operation, second step. Removal of diseased cervical mucosa, preparatory to folding each cervical flap upon itself.

speculum is drawn toward the vulva and divided bilaterally with scissors to or beyond the uterovaginal junction. The anterior and 
Figure 89.

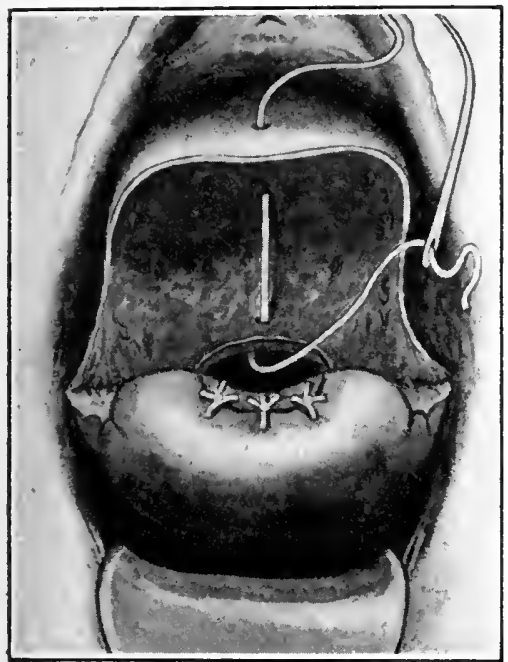

FIGURE 91.

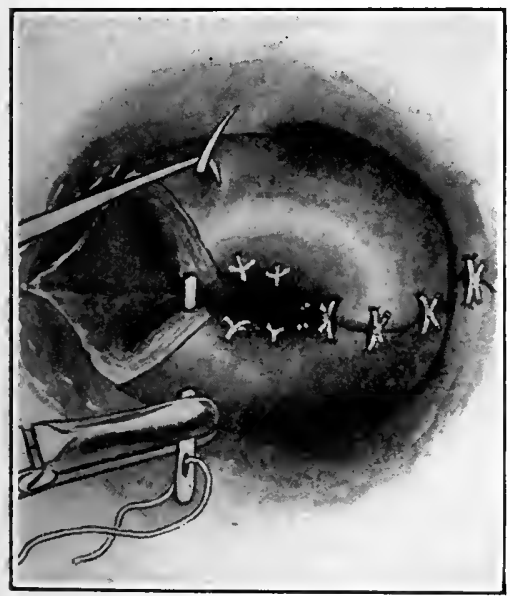

Figure 90.

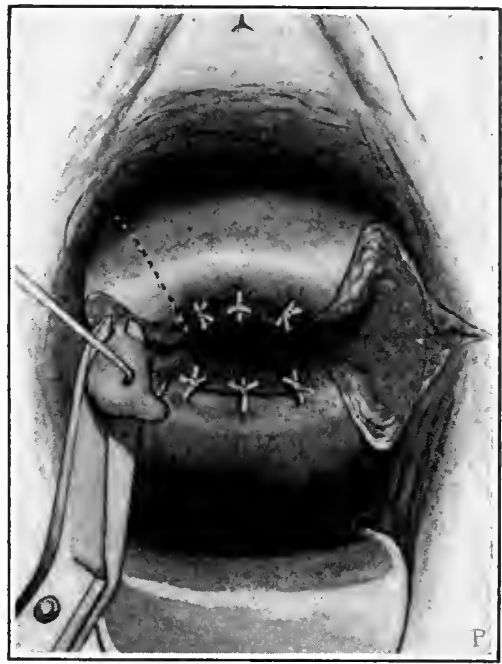

FIGURE 92.

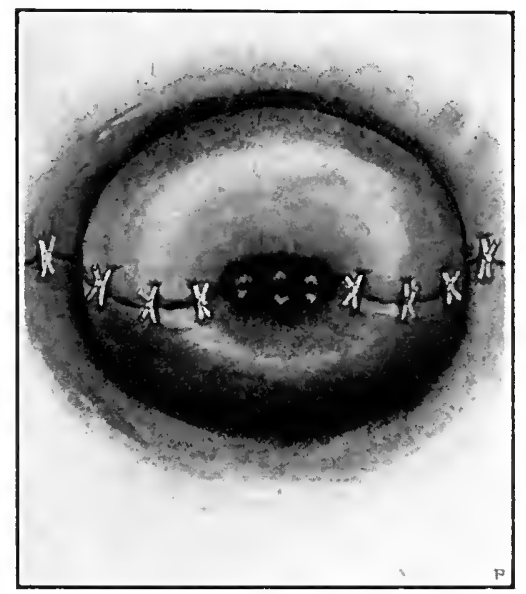

$\mathrm{p}$

FIGURE 89.-Schroeder's operation, third step. Cut margin of the vaginal portion of the Three sutures in place and tied on the anterior lip. Niddle suture passed but not tied on the posterior lip.

Figure 90.-Schroeder's operation, fourth step. Lateral denudation of the cervix uteri precisely as it would be done in trachelorrhaphy. This is preparatory to the introduction of the lateral sutures.

FIGURE 91,-Sehroeder's operation, fifth step. All the sutures introduced and tied on one side thereby rolling in the exyosed cervical mucosa, the first suture being introduced on the other side. When all the required sutures have been introduced and tied the operation will be complete.

Figtre 92.-Schroeder's operation, complete. The sutures in the cervical canal have been rolled in and are almost out of sight, only the ends showing. Each side of the cervix uteri has been brought together by means of four interrupted sutures.

posterior lips are then separated widely with tenacula. The condition is now like that of extensive bilateral laceration of the cervix. The lateral incision should be deep enough so that when the lips are forced apart all the diseased intracervical mucosa may be exposed and excised. 
The anterior and posterior flaps are now turned in each upon itself and united with sutures to the intracervieal margins of the wound. Two or three sutures are required to seeure each flap. The lateral incisions, now much shortened by the folding in of the flaps, may after suitable denudation be elosed by suture, as in Emmet's operation for laceration of the cervix. Upon completion of the operation the flap sutures will be situated deep in the cervical canal, where removal of them would be diffieult. They should therefore be of eatgut. The lateral sutures should be of silkworm gut or chromie eatgut. If silkworm gut, they should be removed in about two weeks. The operation, if well done, is followed by permanent cure and freedom from stenosis. Great eversion through the os externum, giving the outrolled mueosa an uleerated appearanee, is due usually to laceration of the cervix, and should be treated as such. See Emmet's Operation. In rare eases pronounced eversion and erosion oceur in the virgin cervix, giving rise to soft, spongy, granular masses, which should be removed with curved seissors, the cut surfaces eauterized, and the cervix dressed with strips of gauze saturated with a mixture of 10 per cent. ammoniated iehthyol in glyecrin; the dressings to be ehanged daily until the surfaces have healed. This treatment will be disappointing if there be extensive endometritis above, unless that also be included in the plan of treatment. See Treatment of Endometritis. It may, however, be wholly satisfactory if the inflammation is confined essentially to the lower cervieal mucosa. If it fails, Schroeder's operation is indieated.

FigURE 93.

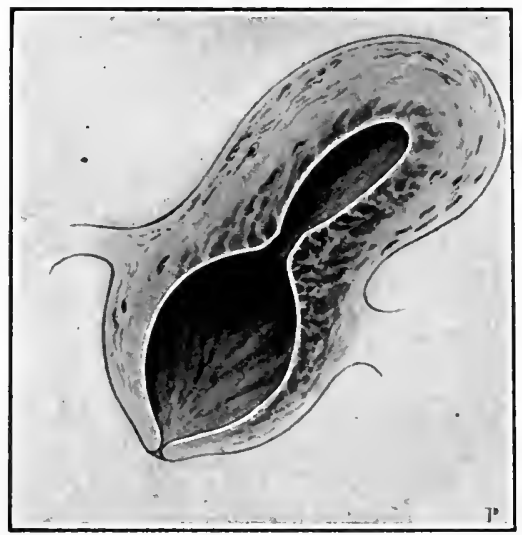

Figure 94.

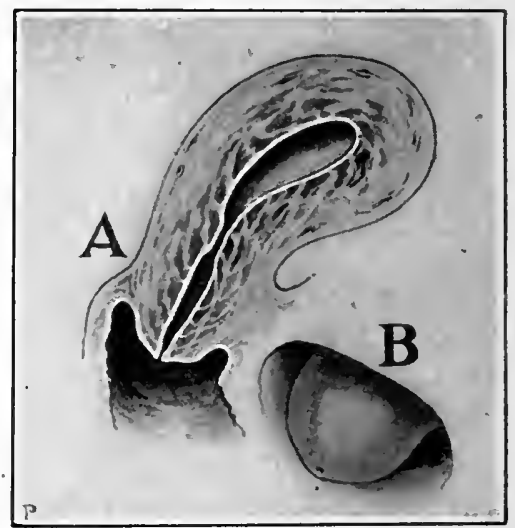

FIGURE 93.-Cervical canal distended by secretions. This is due to obstruction at the internal os uteri and partial or complete closure of the external os. Such obstruction and closure are frequently conseguent upon cicatricial contraction following eauterization.

Figure 94. - Pinhole os of congenital origin in a partially developed uterus: $A$, shows the pinhole os in section; $B$, shows the pinhole os as seen tlirongh the speculum.

Polypoid endocervicitis, so called, requires the removal of the adenoid growths by means of the sharp curette or the scissors. When glandular disease is extensive, it may be necessary to perform Schroeder's operation. 
Pinhole Os.-In nulliparæ the internal and external ora are sometimes so narrow that the cervical secretions are retained and distend the cervical cavity quite beyond its normal size. Sometimes the internal os is open, and the corpus is enlarged correspondingly from the same cause. The retained secretions give rise to great irritation and reflex disturbances. The rational treatment is to open the canal by free incision of the external os, and, if necessary, by dilatation of the internal os. Exploratory curettage will show whether the endometrium requires thorough dilatation, therapeutic curettage, and cauterization.

The pinhole os usually is congenital and eliefly confined to nulliparæ. Figure 94. It may, however, occur as the result of cauterization or of too tight closure in the operation for laceration of the cervix. The constricted external os may be opened by incision or by forcible dilatation. After the application of either of these methods the os is liable to recontract. - Schroeder's operation, which gives permanent results, is therefore preferable. 


\section{CHAPTER X VI.}

\section{CHRONIC ENDOMETRITIS.}

Is studying endometritis, one should remember that the infected endometrium is usually only a part of an infected uterus, and that this infection in many cases is not limited to the uterus, but in variable degree may involve the uterine appendages and parametria.

The layer of columnar ciliated epithelium, the connective tissue, the blood- and lymph-vessels, and the nerves which compose the endometrium, are, like the similar structures in the cervix, subject to chronic infection. Certain pathological changes result from this infection, and are the essential factors of chronic endometritis.

\section{Etiology of Chronic Endometritis.}

The predisposing and exciting causes are the same as already described for acute metritis. The most usual source of the infection is from the cervical mucosa. There is an untold amount of infection carried to the endometrium by useless and meddlesome intra-uterine treatment.

\section{Pathology of Chronic Endometritis.}

It is here important to remember that not every increased secretion is proof of endometritis. There may be an effort on the part of the mucosa to relieve, by an increased secretion, a chronic venous congestion in and about the uterus; or the mucous membrane of the uterus in common with that of other organs may be engaged in vicarious elimination of effete matter which the proper excretory organs have failed to eliminate; such conditions strongly predispose to and are present in a proportion of cases of endometritis, but are not in themselves endometritis.

In studying endometritis microscopically the beginner may be at a loss to account for occasional irregular appearances of the uterine glands, due to invagination of the glands. Figures 95 to 102, drawn after a scheme suggested by Amann, will explain these irregularities.

The general pathology has been forecast under Acute Metritis. The special pathology will be presented in the description of the different histological and clinical forms.

\section{Classification of Chronic Endometritis.}

The general divisions of chronic endometritis are :

1. Histological.

2. Clinical. 
Figure 95.

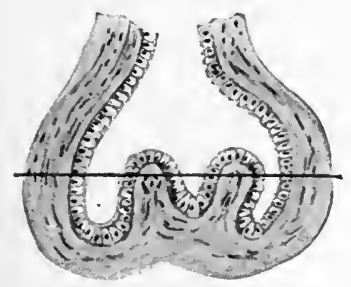

Figure 96.

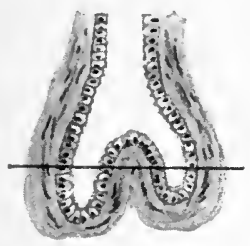

Figure 97.

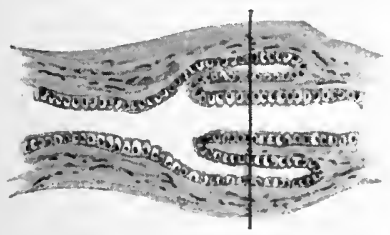

FiguRE 98.

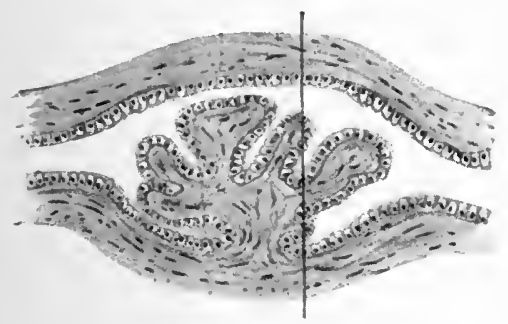

Figure 99.

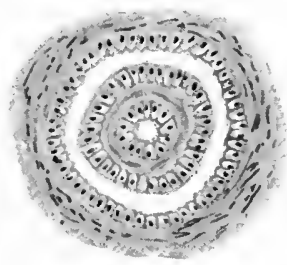

Figure 100.

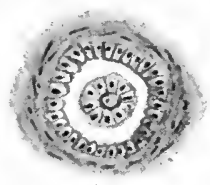

Figure 101

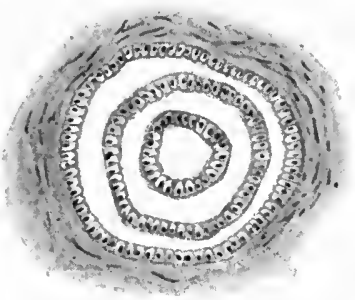

Figure 102.

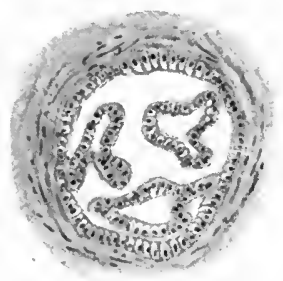

FIgures 95-102.

Explanation of scheme of gland invagination. Figures 95 to 98 show longitudinal sections of invaginated nterine glands: Figures 99 to 102 show cross-sections of the same gland. The glands shown in longitudinal section are erossed each by a line showing the plane at which the eross-sections are made. Figure 95 shows invaginated the fundus of a gland with secondary eversion. Figure 98 shows intraglandular papillary invagination of a gland epithelium from the side of the gland. Figure 96 shows simple invagination of the fundus of a gland. Figure 97 shows the inner and outer segments regular and the middle segment invaginated. 


\section{Histological Forms of Chronic Endometritis.}

The histological forms of endometritis are:

1. Glandular endometritis

(Hypertrophic glandular endometritis.

2. Interstitial endometritis.

Hyperplastie glandular endometritis.

3. Glandular and interstitial endometritis-mixed form.

1. Glandular Endometritis.-Glandular endometritis is elaracterized by increase in the size or in the size and number of the glands,

Figure 103.

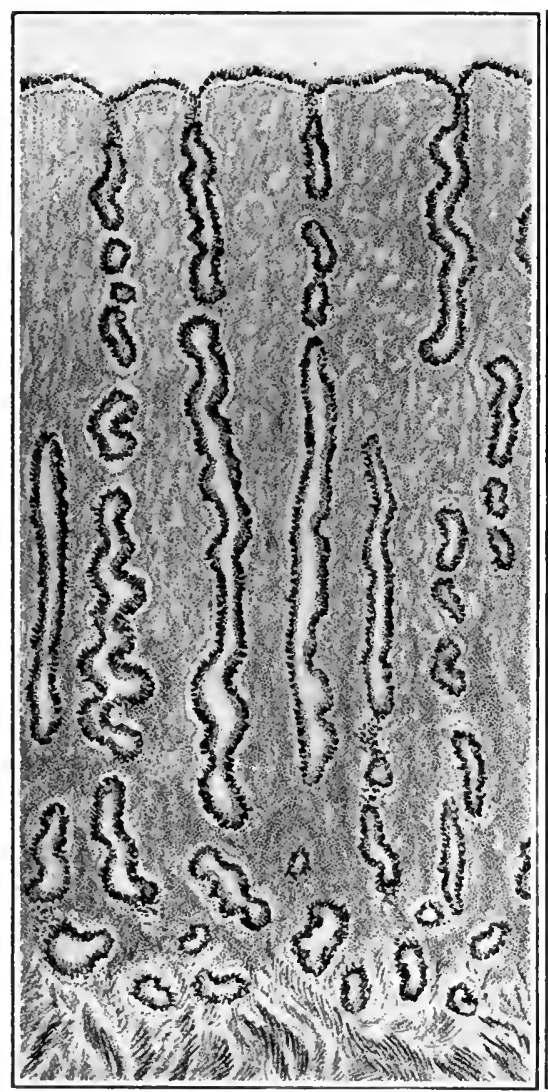

Figire 104.

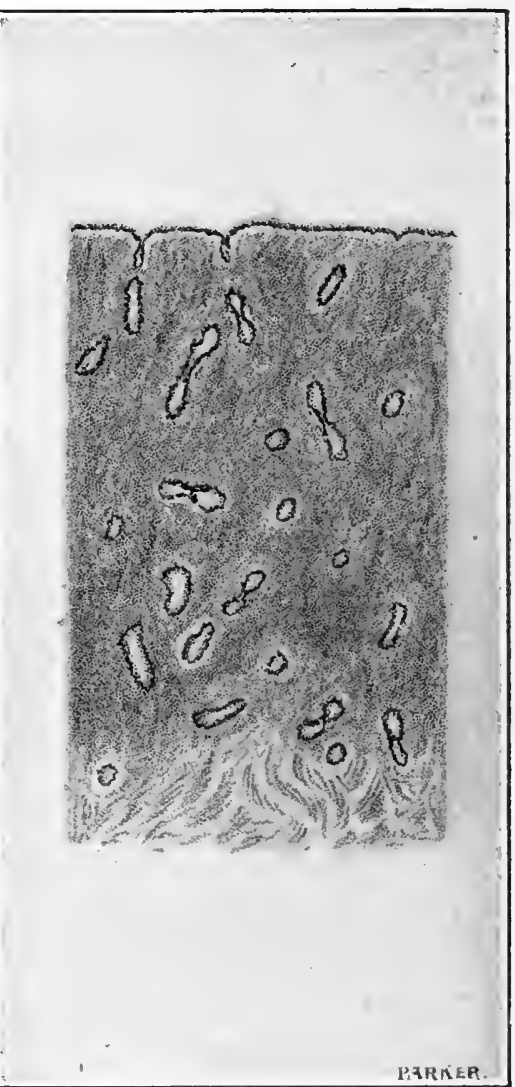

FIGRPE 103 - Vormal uterine mucosn iu a woman twenty-five years old. Four main glands are shown. The small cross-sections are branehes of the muin gland. The glands dip down to and very little into the muscularis. 40 diameters.

Figure 104.-Normal uterine mucosa after the menopanse. The size of this drawing as related to that of Figure 103 shows the shrinkage which takes place at the menopause. The nterine glands have been obliterated almost entirely by atrophy. This eondition somewhat resembles that of interstitial endometritis. 40 diameters.

and accordingly is subdivided into : a. Hypertiophic; b. Hyperplastic. a. Hypertrophic Glandular Endometritis.-The normal uterine 


\section{PLATE IV}

FIGURE 1

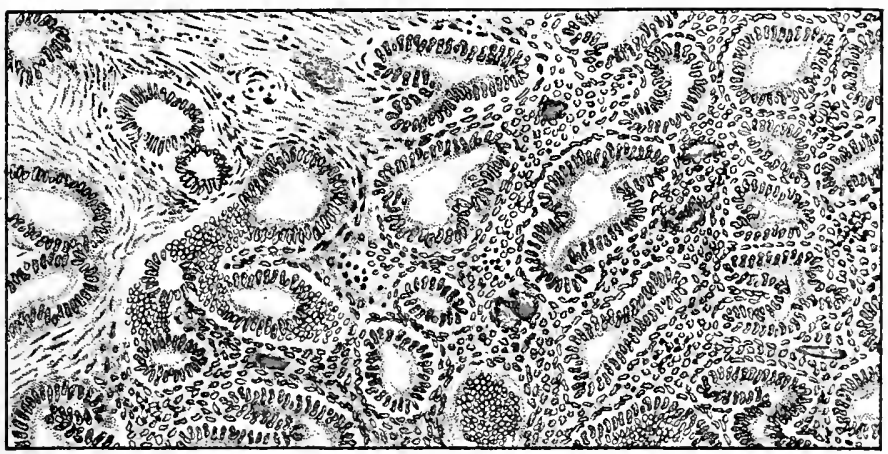

FIGURE 2

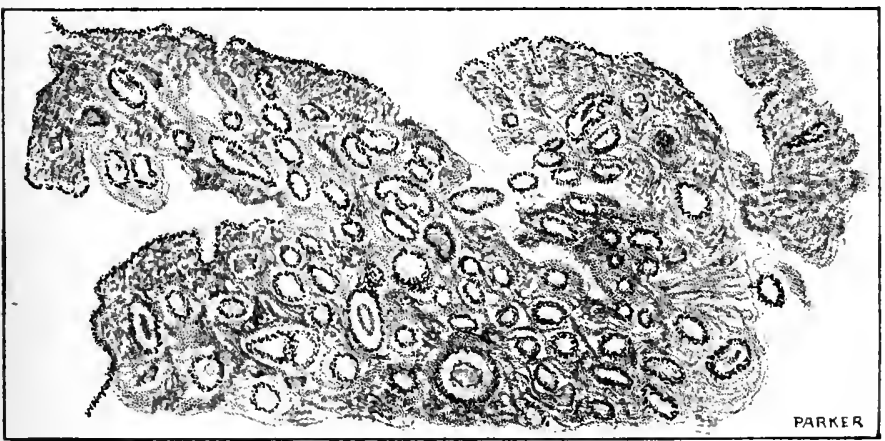

FIGURE 3

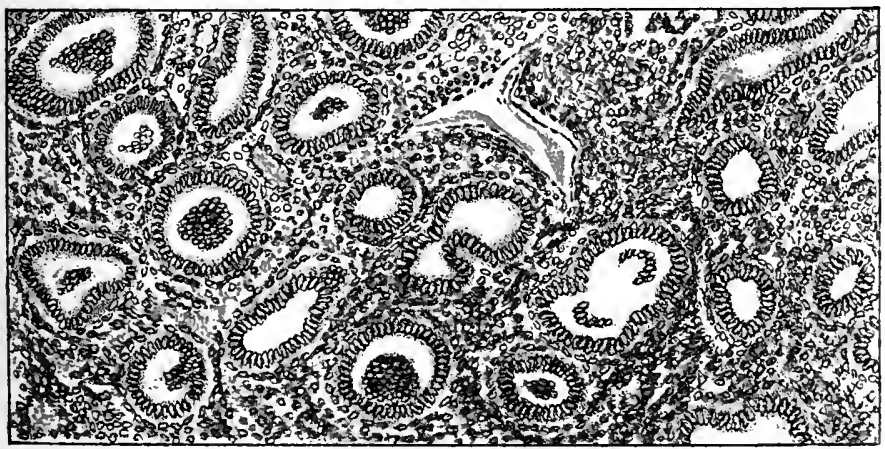



glands are tubular, approximately straiglit, branch but little, run almost perpendicular to the surface, may extend to the muscularisthat is, to the myometrium; and but rarely dip down so far as to penetrate even to the superficial layer of it. In hypertrophic endometritis the glands increase in size, become proportionately irregular in outline, pursue a direction less perpendicular to the surface, dip more deeply, develop numerous branches, become tortuous; and in consequence of dilatation in some places and constriction in others, frequently take on great irregularity of lumen. There is lining the glands a single, and only a single layer of columnar epithelium, with enlargement of the individual epithelial cells. Such is the picture of chronic hypertrophic glandular endometritis. (Plate IV., Figure 1.)

b. Hyperplastic Glandular Endometritis.-This form of endometritis not infrequently presents all the essentials of the hypertrophic variety, but, as a distinguishing characteristic, will show increase in the number of glands; this increase necessarily takes place at the expense of the interglandular connective tissue, so that the interglandular spaces no longer maintain the normal ratio of four times the diameter of the glands; but may, on the contrary, almost wholly give way to the encroachments of the newly formed glands. Increase in the number of glands results from a process of budding of the glands or of invagination from the surface epithelium. (Figures 95-102.)

The hyperplastic form of endometritis is regarded by some pathologists as a new growth, and sometimes is called benign adenoma. The consensus of opinion, however, is in favor of attributing to such growths an inflammatory origin, and of placing them in an intermediate position between inflammatory growths and new formations. In this work the term aderoma will not be used to describe hyperplastic endometritis, but will be reserved for the malignant glandular growths. (Plate IV., Figure 2.)

2. Interstitial Endometritis. - Interstitial endometritis is characterized by an increase in the connective tissue of the endometrium at the expense of the glandular elements, and is therefore the reverse

\section{Explanation of Plate IV.}

Figure 1.-Hypertrophic glandular endometritis. The glands are swollen and tortuous, but not increased in number. In the upper left-hand corner is a triangle of uterine muscular tissue containing five glands. The dipping of these glands far into the muscular tissue may sometimes raise the suspicion of carcinoma. The remaining glands are surrounded by a stroma of embryonic cells--that is, the cells which produce connective tissue. This field contains seven blood-vessels, indicated by the red color. In the middle of the left half are four glands all having tangential cuttings. This is mistaken sometimes for proliferation of epithelium. Similar cuttings of two glands zppear in the lower part of the picture on the right side. Small round cells of inflammation; as indicated by dark staining, appear here and there in groups and sometimes widlely scattered. 80 diameters.

Figure 2.-Hyperplastic glandular endometritis (liemorrhagic). Observe some of the glands invaginated and therefore characteristic of hyperplasia. The blood-ressels and the extravasated blood are seen scattered over the field. Also nuch congestion is seen near the surface epithelium at the upper margin. 15 diameters.

Figure 3.-A part of Figure 2 highly magnified. Observe the hemorrhagic and congested areas, the full blood-vessels, the swollen, invaginated, and tortuous glands, which are increased in size and number. A characteristic specimen of hemorrhagic endometritis. 100 diameters. 
of the hyperplastic glandular forms. In the normal endometrium the connective tissue is embryonal in type and composed of spindle cells loosely associated. In interstitial endometritis these cells inerease and mature into fibres which separate the glands widely. The effect of these connective-tissue changes is to shut off the nutrition of the glands, and thereby to crush out and partially or completely to destroy them. The outlets of the glands may close and give rise to retention eysts; this is ealled cystic endometritis. Finally, the endometrium may become a thin layer of contracted cicatricial tissue, not unlike that produced by the atrophic ehanges of old age. (Plate V.)

Figdre 105.

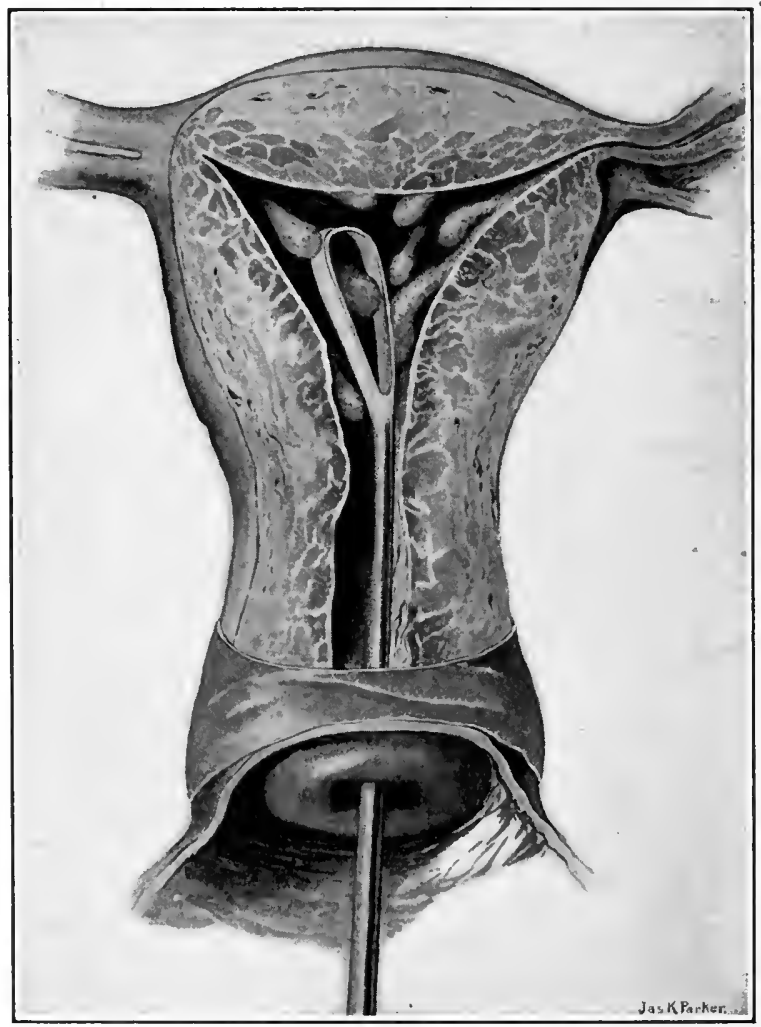

Polypoid endometritis; removal by curettage.

3. Glandular and Interstitial Endometritis.--Glandılar and interstitial endometritis often are combined in varying proportions. Part or all of the endometrium may be involved.

Mucous Polyps of the uterus, commonly ealled polypoid endometritis, are to be regarded as of inflammatory origin, are found both in interstitial and in glandular endometritis, and are apt to be developed where there is a concurrence of interstitial endometritis with great glandular enlargement; they are marked by excessive, diffuse, glan- 


\section{PLATE V}

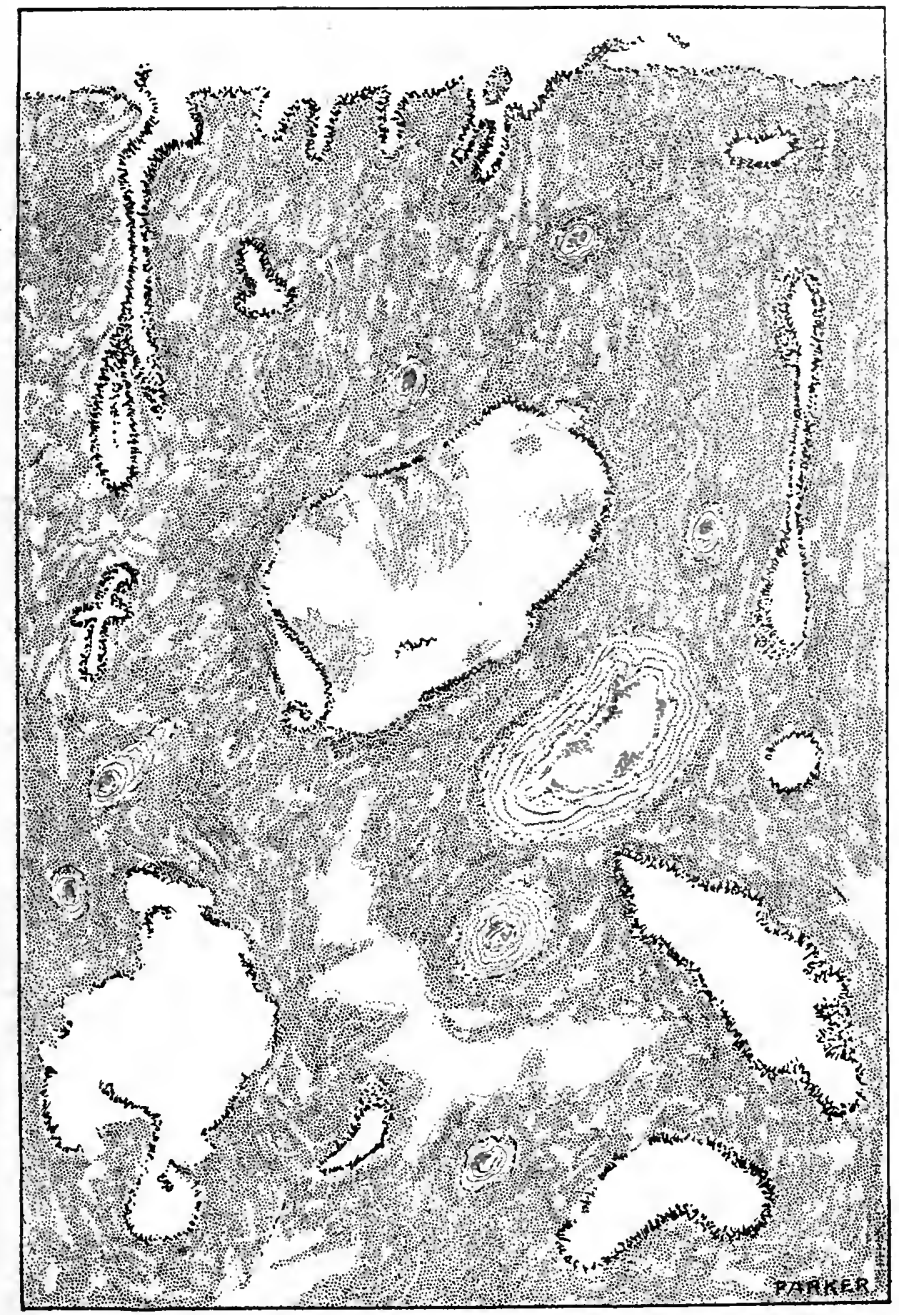

\section{Interstitial Endometritis (Cystic).}

The free mucous surface is shown in the upper margin of the picture. A gland in longitudinal section penetrates the stroma on the left. Single layers of glanduiar epithelium in some places have been detached and destroyed. In some of the glands the epithelium lining is intact, in others it partially has been shed. The large cystic gland in the centre of the figure shows much detached epithelium which at some points still preserves its form and in others has become degenerated. Near the centre of the figure is an enormously large bloodvessel, and scattered over the field are numerous small vessels, all having very thick walls. The interglandular stroma here is fibrous in character, and the quantity of it is much increased while the glands are decreased. 30 diameters. 

dular and vascular development and by eystic degeneration of the glands. Some of the cystic glands have the character described under interstitial endometritis; others become fingoid projections upon the surfitec-that is, small, soft, polypoid bodies, like nasal polypi; often pedunculated, variable in size, and œelematous from retained secretions. These changes make the endometrium excessively thick, soft, and odematous. The excessive glandular and vascular enlargement explains the chief subjective symptoms--exhaustive glandular secretions and hemorrhage. These polypoid bodies are not to be regarded as new growths, and therefore should not be classed as adenomata.

\section{Clinical Forms of Chronic Endometritis.}

The clinical varieties of endometritis usually may be referred to one or more of the histological forms. The individual peculiarities are dependent upon intercurrent conditions. Among the clinical forms are :

1. Post-abortum endometritis.

2. Exfoliative endometritis.

3. Senile endometritis.

4. Tubercular endometritis.

5. Decidual. endometritis.

In addition to tubercular endometritis may be mentioned other bacterial forms such as gonorrhœal, syphilis, and streptococcic. Here

Frgure 106.

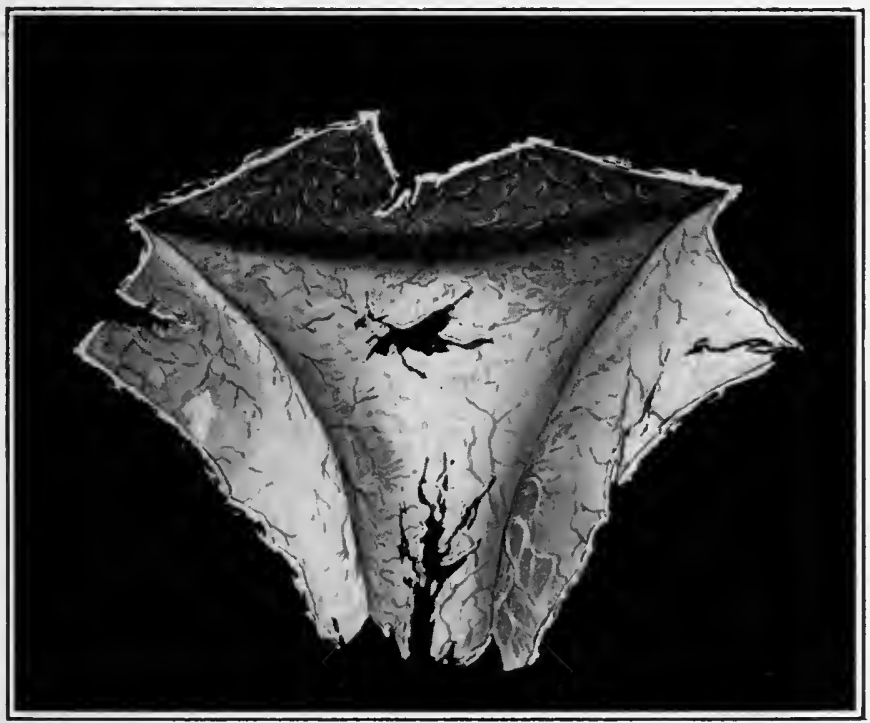

Cast from uterine cavity in exfoliative endometritis-membranous dysmenorrhoea, natural size.

belong also those forms of endometritis following the acute infections diseases-typhoid fever, diphtheria, scarlet fever, smallpox, ete. 
1. Post-abortum Endometritis.-Abortion may be a cause or an effect of endometritis. The inflammation, which is rather interstitial than glandular, eauses an arrest of involution in the mucous membrane at the site of the ovule and of the adjacent mucosa-i.e., of the decidua serotina and decidua vera. The arrest of involution in loealized areas may give rise to islands of decidual cells circumscribed within the surrounding mucous nembrane by the round cells of inflammation.

2. Exfoliative Endometritis, called membranous dysmenorrhœa, is characterized by the detachment of a membranous structure from the endometrium in fragments or as a whole, and the expulsion of it from the uterus. It may occur at puberty, with the first menstruation, and continue indefinitely, or may begin at any time during menstrual life. The character, quantity, and completeness of the thrown-off membrane vary with individuals, and from time to time in the same individual.

The membranes thrown off in the form of dysmenorrhœa elosely resemble in gross appearance those of early abortion and tubal pregnancy. The differential diagnosis between these three conditions is therefore important:

Exfoliative endometritis.
1. No history of pregnancy.
2. Dysmenorrhoic pain and
discharge of membrane at each
menstrual epoch.
3. No enlargement of uterus
or Fallopian tube.
4. Chorionic villi and am-
nion absent.
5 . No foetus.
6. Membrane may be exact
cast of endometrium or may
be in shreds.
7. Lsually incurable.

Early abortion.
1. History of pregnancy.
2. Discharge of membrane
with pain at time of abortion.
3. Enlargement of uterus, but
not of Fallopian tube.
4. Chorionic villi and am-
nion present.
5. Fotus discharged from
uterus.
6. Membranes may envelop
fatus and may be cast off
whole or may be in fragments
or shreds.
7. Self-limited, or curable by
treatment.
Tubal pregnancy.
1. Atypical history of preg- nancy.
2. Diseharge of decidual membrane usually between the fourth and nintb week of preg- nancy.
3. Enlargement of Fallopian tube on affected side.
4. Absent from uterus. Chor- innnic villi and ammion in Fallopian tube.
5. No fotus discharged from uterus.
6. Membrane, not associated with fotus, cast off entire or in irregular fibrous fragments.
7. Not incurable.

The pains of membranous dysmenorrhœa are like severe laborpains; they usually appear before, and continue with remissions thronghout, the flow. The subjective symptoms may disappear in the intermenstrual period, or they may continue as the ordinary signs of endometritis, the inflammation taking on a somewhat acute character during menstruation. The disease is intractable, and often incurable. The treatment is the same as for obstinate endometritis in generali. c., thorough sharp eurettage, with canterization of the endometrium, in the hope that the new endometrium may be healthy. Maternity sometimes effects a radical cure. See Chapter LIII. Some authors, perhaps with good reason, deny that membranous dysmenorrhoa is caused by inflammation.

1. Senile Endometritis.-After the menopause, when the uterus has undergone senile atrophy, it is subject to a most harassing form of purulent endometritis which is usually the relic of an earlier infection, and due to the action of bacteria on the atrophic, less resisting endometrium. The discharge contains numerous bacteria, is commonly offensive, purulent, often tinged with blood, and is so irritating as 


\section{PLATE VI}

FIGURE 1

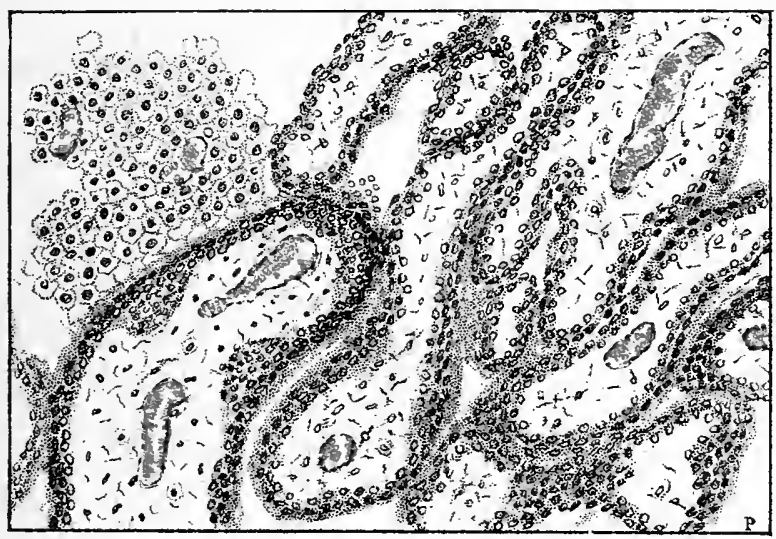

FIGURE 2

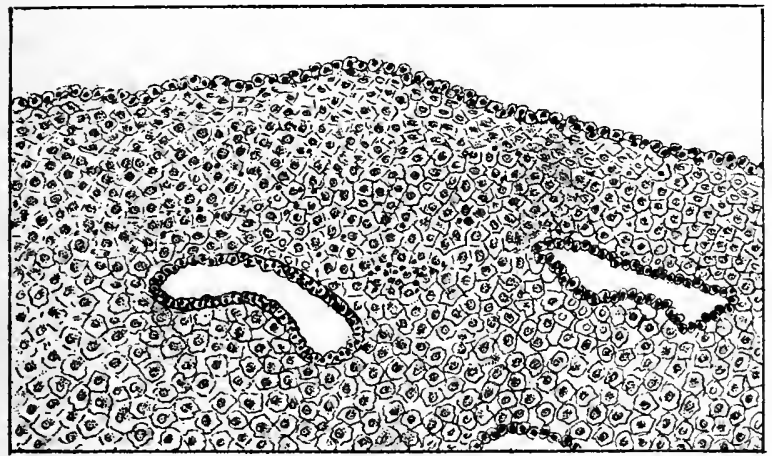

FIGURE 3

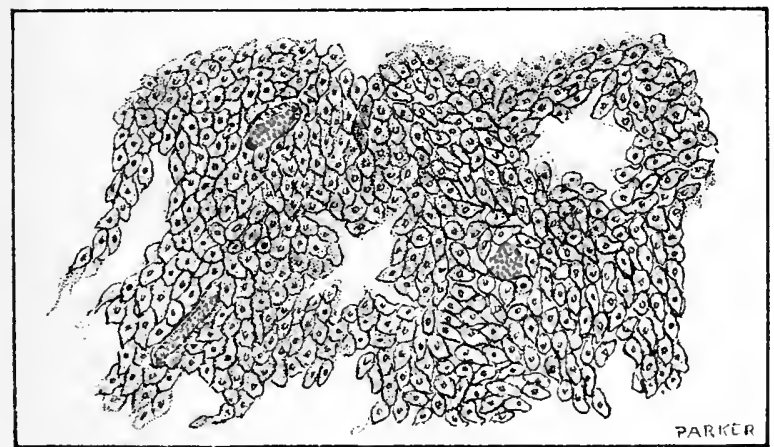



sometimes to cause a most distressing pruritus vulvæ. The infeetion may destroy the exhausted senile mucosa and penetrate into the muscularis. Cicatricial stenosis and even complete eicatricial ocelusion in the uterine eanal, usually at the internal os, may oecur causing the uterine secretions to be retained and the distended organ to become a thin-walled retention-cyst-pyometra or hydrometra, according as the retained fluid is purulent or watery; this obstruetion to the drainage of secretions aggravates the disease, and the organ may remain large from distention. The retained products of senile endometritis may give rise to reflex disturbances, innutrition, and to systemic depression, even to general chronic toxæmia. In most eases of senile endometritis the uterus is not enlarged, but rather in a state of full senile atrophy.

The microseopical ehanges are like those of atrophic interstitial endometritis, already deseribed. The glands and epithelial elements in the last stages of the disease are destroyed and the submueous structures laid bare, making eatarrhal inflammation inupossible. The exposure of fibrous tissue, on the other hand, is favorable to the development of suppuration, granulation, and uleeration-three characteristies of senile endometritis. The disease may be corporeal or eervieal, or both. The offensive discharge, the occasional uterine enlargement, and systemic depression may lead to confusion between this disease and uterine cancer. Cieatrization may bring about a spontaneous cure. Usually, however, unless eut short by treatment, the suppuration persists.

4. Tubercular Endometritis. - Tubercular endometritis may reach the uterus from withont (hetero-infection) or may be transmitted from another infeeted organ (auto-infection). See Tubereular Salpingitis. Hetero-infeetion is rare, but may be transmitted to the cervix uteri or vagina by coitus or by instrumental or digital interferenee, and may take the form of sharply eut ulcers, and when far advanced may extend to the corpus uteri ; the resemblanee to caneer is then quite marked. Auto-infection generally reaches the uterus through the Fallopian tubes. In such cases the disease finally extends from the endometrium to the myometrium, and not uncommonly to the lungs.

\section{Explanation of Plate Vi.}

Frgure 1.-Post-abortum endometritis. Section of endometrium removed by the curette after abortion. The upper left-hand corner contains decidual cells with two small blood-vessels. The remainder of the field is occupied by chorionic villi. In most of the villi blood vessels either in longitndinal or cross-section are shown. The villi are surrounded by two layers of cells, the inside layer being the ectodermal epithelial layer of Langhans, the onter layer heing the uterine epithelial layer of the chorion-that is, the syncytium. 80 diameters.

Fiaure 2.- Menstrual decidua from membranous dysmenorrhea as seen by the microscope. The upper border is composed of surface colnmnar epithelium somewhat flattened. There are three glands lined with columnar epithelium. The remainder of the field is made up of decidual cells among which are scattered, especially in the centre of the field, numerous small round cells of inflammation. 80 diameters.

FIGURe 3.-Uterine decidua commonly cast off in tubal pregnancy at the time of spurious labor. The outer margins and open spaces are torn and ragged. The drawing shows three blood-vessels full of blood. The decidual cells are large and irregular with small nuclei. The decidua of tubal pregnancy seldom shows glands and none are seen here. 80 diameters. 
There is also usually a prior involvement of the peritoneum. Often the pelvic organs-uterus, bladder, colon, rectum, ovaries, and tubes-are matted together by adhesions, with abscesses and broken-down tissues. Tubercular endometritis is relatively. rare; it is recognized by the history of the case and by microscopical examination of the scraping. The disease is incurable by any means except hysterectomy.

5. Decidual Endometritis arises during pregnancy. A positive diagnosis cannot be made until pregnancy has terminated and the decidual changes have been noted under the microscope. The symptoms suggestive of the condition are hemorrhage, leucorrhœa, and pain, all of which may continue throughout pregnancy. The pain is referable to the uterus, and may be of the cramping and bearing-down variety. Early abortion with a firmly adherent placenta is the usual result.

\section{Symptoms of Chronic Endometritis.}

The symptoms of acute endometritis-that is, liypogastric pain, pelvic weight, rectal and vesical tenesmus-may in some degree continue; but as the discase becomes chronic these symptoms cease to predominate; in their place comes a symptom-group which always contains some of the following factors:

Menstrual and intermenstrual disturbances.

Excessive mucons, purulent, or mucopurlent discharges.

Uterine hemorrhages.

Sterility or frequent abortions.

Dragging sensation in pelvis.

Pain in epigastrium.

Vesical and rectal tenesmus, frequent urination.

Systemic disturbances and reflex disorders in other organs, such as. flashes of heat and cold, insomnia, troubled dreams, and sometimes a radical change in disposition.

The above symptoms often are observed in other disorders, and are therefore not strongly diagnostic.

Pain.-Obstructive dysmenorrhœa may result from cicatricial stenosis, especially if the menstrual blood coagulates in the uterus and is forced unt by strong contractions; the pain will then be intermittent. Intermenstrual pain from the expulsion of accumulated secretion in the uterus may occur in the same way. The excessive menstrual pain, like labor-pain, in exfoliative endonetritis has already been mentioned. Congestive dysmenorrhœa often precedes the flow, but subsides as soon as the engorged ressels are relieved by the establishment of the flow. The uterine nerves, already sensitive from neuritis when crowded by the distended blood-ressels of the swollen uterus, easily become the seat of great menstrual and intermenstrual pain.

Hypersecretion is a constant and pronounced symptom. It may be catarrhal or purulent, or mixed, and often contains blood. Menorrhagia and intermenstrual hemorrhage are common.

Sterility and Abortion are associated frequently with the disease. Sterility may result from complicating ovaritis or obstruction in the 
Fallopian tubes, or from destruction of the spermatozoa by the uterine secretions, or from their mechanical exclusion from the nterus by the plug of tenacious mucus usually found in endocervicitis; or from the hostile influence of the diseased uterine mucosa on the ovulc. The failure of the ovule to implant itself upon the mucosa may give rise to no subjective symptoms, and the fact of early abortion may be unrecognized. Indeed unrecognized early abortions may occur at frequent intervals and for a long period of time.

Figure 107.

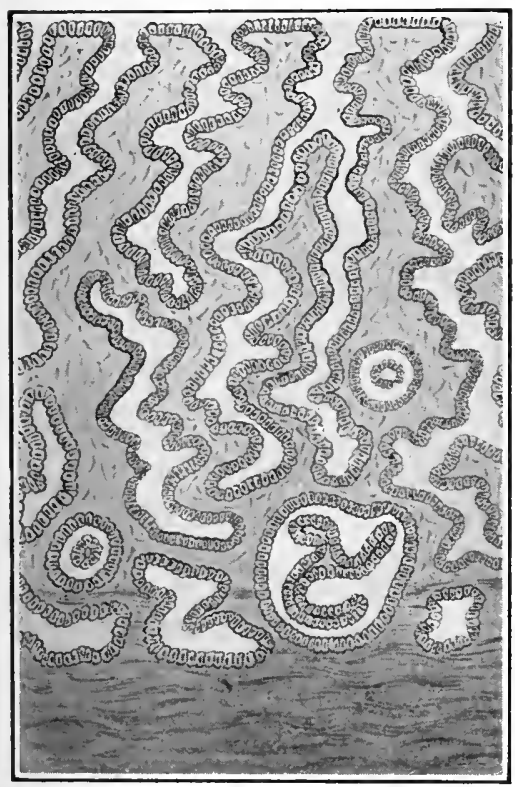

Figure 108.

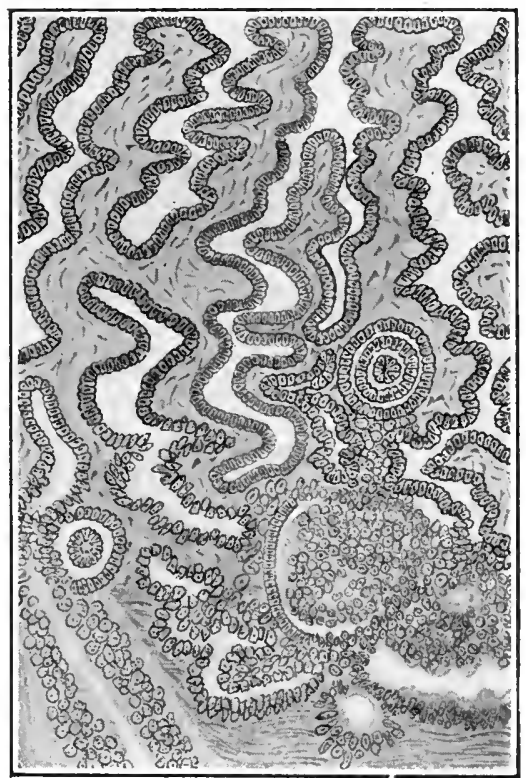

FigUre 107.-Hyperplastic glandular endometritis. The glands are increased in size and number, greatly tortuous, and dip decidedly into the muscularis. The interglandular spaces are much decreased. This eondition sometimes is called benign adenoma. Semidiagrammatic.

Figure 108. - Same as Figure 107. Nodified in the lower part by eareinoma, commonly ealled adenoearcinoma. There is great rarefaction of stroma and in the earcinomatous part the glands are so tortuous and atypical that they eannot be traced. Carcinoma is demonstrated here by the breaking through the tuniea propria into the stroma and the invasion of the stroma by proliferated gland ejithelium which also fills the glands. The lower left-hand eorner sbows a gland which shows mueh cell proliferation, and which may be regarded as the earliest stage of cancer formation, but not a condition upon which a positive diagnosis should be made. Semi-diagrammatic.

The Systemic and Reflex Disorders are chiefly referable to the nervous svstem. Among them are nenralgia, indigestion, malnutrition, nervous dyspepsia, anæmia, chlorosis, spinal irritation, and lysteria. The endometritis may be a cause or an effect of the above associated disorders, or together with them may be a concurrent resnlt of some common cause, or may have primarily no pathological connection with them. The nervous symptoms are usually most pronounced during the few days before menstruation, and may be very marked during the flow. 


\section{Diagnosis of Chronic Endometritis.}

The diagnosis of chronic endometritis, suggested by the symptoms outhined above, must depend upon curettage and a microscopical examination of the scrapings. Digital examination will in many calses show increased size and hardness of the uterus, and is especially essential as a means for the detection of complications, such as displacement and eircumuterine inflammations. A very small eurette, withont previous dilatation, will suffice often for diagnostic purposes; at least it may settle the question whether a therapeutic curettage is necessary.

A discharge from the vagina or from the Fallopian tubes may be mistaken for the product of endometritis. Inspection will show whether the discharge comes from the uterus or not. The tube may refill and empty itself through the uterus at intervals, giving rise to periodical expulsive pains in the uterus-colica serotortum.

Pus from a pelvic abscess is recognized by finding the sinus through which it discharges; such a sinus often opens into the vagina near the uterus, seldom into the uterus.

\section{Differential Diagnosis of Chronic Endometritis.}

The diagnosis between the different forms of endometritis and between endometritis and malignant disease will depend, first, upon the history of the ease, the nature of the discharge, and conjoined examination ; second, upon the findings of the curette and the microscope. The discharges from earcinoma and sarcoma, unlike those of endometritis, are more profuse, more offensive, more watery, and usually contain more blood. Cachexia and other systemic disorders are quite marked in sarcoma and carcinoma, but usually absent or slight in endometritis.

Figures 107 and 108 show in a diagrammatic way the transition from hyperplastic endometritis to andenocarcinoma.

The differential points between the two forms of chronic glandular endometritis and carcinoma are shown in the illustrations on endometritis and carcinoma and in the following parallel columns:

\begin{tabular}{|c|c|c|}
\hline $\begin{array}{c}\text { Glandular hypertrophic } \\
\text { endometritis. }\end{array}$ & $\begin{array}{c}\text { Glandular hyperplastic } \\
\text { cndomctritls. }\end{array}$ & oma \\
\hline $\begin{array}{l}\text { 1. Glands increased in size } \\
\text { ut not in number. }\end{array}$ & $\begin{array}{l}\text { 1. Glands increased in size } \\
\text { and number. }\end{array}$ & $\begin{array}{l}\text { 1. Glands rery greatly in- } \\
\text { creased in size and number. }\end{array}$ \\
\hline $\begin{array}{l}\text { 2. No proliferation of gland } \\
\text { pitlielium. }\end{array}$ & $\begin{array}{l}\text { 2. Proliferation of gland } \\
\text { cpithelium. }\end{array}$ & $\begin{array}{l}\text { 2. Very great proliferation } \\
\text { of gland epithelium. }\end{array}$ \\
\hline $\begin{array}{l}\text { 3. Gland structures nearly or } \\
\text { quite typical in outline. }\end{array}$ & $\begin{array}{l}\text { 3. Gland structures more tor- } \\
\text { tnous in outline. }\end{array}$ & $\begin{array}{l}\text { 3. Gland structures very } \\
\text { atypieal in outline. See Fig- } \\
\text { ure } 150 .\end{array}$ \\
\hline $\begin{array}{l}\text { 4. Hypertrophied cpithelinm } \\
\text { confined within the limits of } \\
\text { the tunica propria. }\end{array}$ & $\begin{array}{l}\text { 4. Proliferation con fined } \\
\text { within the limits of the tunica } \\
\text { propria. }\end{array}$ & $\begin{array}{l}\text { 4. The proliferating igland } \\
\text { epithelium hasbrokenthrough } \\
\text { the tuniea propria and is in } \\
\text { direct contact with intergland- } \\
\text { ular connective tissue, and is } \\
\text { multiplying in an atypical } \\
\text { manner. }\end{array}$ \\
\hline 5. Gland-tissue & 5. Gla & sue \\
\hline
\end{tabular}


Glandular hypertrophic endometritis.

6. Can trace tortuous glands.

7. Stroma normal in quantity.

8. Glands eveniy distributed.
Glandular hyperplastic endometritis

6. Can trace tortuous glands.

7. Stroma decreased in quantity, but clearly defined from glands. Simple filling of gland lumen with epithelium does not nccessarily denote malig naney so long as epithelium is confined within basereut membrane, $i$. $e$., within tunica propria.

8. Glands evenly distributed.
Adenocarcinoma.

6. Glandular laby rinth ; cannot trace tortuous and atypical gland.

7. Great rarefaction of stroma, so that glands touch one another. Glands have broken through basement membrane and invaded interglandular spaces and muscularis. See Chapter XXVIII.

8. Gland elements may be distributed very unevenly.

The distinction between endometritis and sarcoma is difficult, and in early sarcoma sometimes impossible. The following points are significant :

\section{Endometritis.}

1. Progress not rapid after the acute stage.

2. Cells do not vary in size or shape.

3. Walls of blood-vessels clearly separate cells from blood-supply.

4. Endometritis first involves superficial structure, and later may involve deeper structure.
Sarcoma.

1. Progress very rapid, especially in the small round-cell variety.

2. Vary most widely.

3. Intimate relation of blood-spaces to cells. Walls of vessels may be absent, leaving only blood-spaces.

4. Sarcoma often involves deeper layer first.

\section{Prognosis of Chronic Endometritis.}

Relapse is very common. The mildly infectious cases, usually called simple endometritis, are self-limited or yield readily to systemic treatment. The strongly infections cases always require surgical treatment. This in most cases will bring about at least a symptomatic cure-i.e., it will stop the discharge and may relieve other symptoms. Whether the diseased uterine mucosa can be restored to its functions will depend upon the extent to which it has been impaired by the disease or must be destroyed by treatment. The prognosis is especially discouraging in interstitial exfoliative, senile, and tubercular endometritis. 


\section{CHAPTER XVII.}

\section{CHRONIC ENDOMETRITIS (CONTINUED).}

\section{Treatment of Chronic Endometritis.}

THE treatment varies with the structures involved, the nature of the infection, the chronicity of the disease, and with the preponderance of systemic or local origin. The treatment of cervical differs from that of corporeal endometritis; that of a gonococcus infection might have to be energetic and strong, while a milder infection would require only simple or expectant treatment. Obstinate cases of long standing may yield only to the most radical surgical measures. Many authors attempt to draw a line between what they call simple endometritis and bacterial endometritis. This line can have neither a scientific nor a clinieal basis. It is better to distinguish, on the one hand, the non-purulent cases of uterine eatarrh in which general circulatory disturbances predominate; and, on the other hand, the cases in which infection predominates : in the first class of cases predisposing causes predominate; in the second elass, exciting causes. See Chapter X. The septic element is not confined to the second class, nor the systemic element to the first. An appreciation of the foregoing will suggest the following division of treatment :

1. Systemic treatment.

2. Topical treatment.

3. Surgical treatment.

1. Systemic Treatment of Chronic Endometritis.-Should Treatment be Systemic or Local ?-Systemic treatment is applicable to a very large class of cases, sometimes called subinflammatory, which arise not so mueh from local infection as from stagnation of the general circulation. The stagnation is associated not infrequently with disorders of the heart, lungs, liver, kidneys, or with such disorders as anæmia, rheumatism, and gout. The uterus may participate in the general circulatory disturbance and take on a catarrhal condition. In this class of cases the eatarrh usually involves not only the uterus, but also extrapelvie organs, especially the organs of the respiratory, digestive, and urinary systems. The mucous membranes generally become less resistant and therefore more liable to infection. Catarrh is often the vicarious act of a mueous membrane to throw off waste-products which it would not normally have to eliminate at all. When the membrane is relieved of such unnatural function the resistance to the microbe is increased, and in this way the discharge may cease.

It is elear from the foregoing that in the absence of marked local infection the treatment should be not so much local as systemic, and 
that when the uterine discharge is mainly consequent upon systemic causes, local treatment may be useless, perhaps injurious. On the other hand, the uterus may participate in the general improvement when the extrapelvic and systemic disorders mentioned in the preceding paragraph have been relieved. If the uterine secretions are purulent, or if systemic treatment proves inadequate, topical or surgical treatment may be desirable.

The Kidneys should be made to eliminate their proper amount of urea and other solids. A quantitative urinalysis should be made to estimate the total solids excreted in twenty-four hours; and if there is deficient elimination the granular effervescing sodium phosphate in copious draughts of pure soft water or mineral spring waters are indicated; the diet should include less animal and more vegetable food.

Anæmia, notably the anæmia of fat flabby women, often is associated with local engorgement, especially in the uterus. In such cases local treatment is useless. Iron, manganese, the bitter tonics, mineral waters, nutritious food, adequate exercise, and regular habits are essential. The thyroid extract has been much praised in the treatment of this class of anæmic women, and the use of it is said to be followed by reduction of fat.

Constipation is associated almost constantly with uterine catarrh. Large accumulations of old, hard fecal matter displace and keep up constant engorgement of the uterus and other pelvic organs. The successful treatment of constipation is sometimes essential in the treatment of the endometritis and should be rather regulative than medicinal. Strong laxatives tend to congest the abdominal and pelvic organs - the verv condition we want to relieve-and should therefore be avoided. Hygienic measures alone may be adequate. These include regular properly selected diet, regularity in exercise and especially in times of going to stool. Massage is a measure valuable for its direct influence on the action of the bowels and on the general circulation. Mineral waters, magnesium sulphate, magnesium citrate, lithium citrate, sodium phosphate, and Carlsbad salt are useful, and are given best in copious draughts upon rising in the morning. A large draught of cold water at the same hour will often cause free action of the bowels. The more positive cathartics like aloin or podophyllin at bedtime, which usually act strongly the next morning, are objectionable, and such drugs if given at all should be in small divided doses combined with iron and nux vomica, and given at least three times a day. The cathartic dose should be diminished each time the prescription is renewed until only the tonic remains. Polypharmacy is to be avoided.

Tablet triturates of calomel long continued in small doses-onethirtieth to one-tenth of a grain-three times a day may fulfil clear indications; it stimulates the biliary secretion, renders the glandular organs more active, dislodges morbid accumulations, and tends to secure proper elimination through the bowels and kidneys. The bichloride of mercury in minute doses-one-hundredth of a grainmay be equally useful. In the contiuned employment of mercurials 
it is well to secure normal freedom of the bowels, if neessary, by the judicions use of salines.

Colonic flushings of normal salt solution are most useful, especially in the treatment of obstinate constipation. They should be given in the left lateroprone position of Sims. To be most effective they must be copious, slowly given, and retained for at least several minutes. The disease is most obstinate in virgins. In corpulent young women cure is almost impossible unless the nutrition be improved and the weight reduced.

General Hygiene.-Dress, exercise, food, sexual relations, eare at menstruation, local and general bathing are very important. A comprehensive grasp of the subject involves the whole field of general medicine.

2. Topical Treatment of Chronic Endometritis.-Topical treatment has been as mueh overestimated as systemic treatment has been negleeted. Multitudes of women have, unfortunately, formed the habit of receiving useless rontine treatment for the relief of uterine discharges. Once eliminate the eases described in the foregoing paragraphs which require not so much local as systemic treatment, and the relatively small remainder will be made up mostly of elearly infections cases. Few seientifie gynecologists to-day place great value on topical office-treatment for clearly purulent endometritis. The number of such eases definitely cured by topical applications, when compared with the great number treated, is insignificant. In making such comparison we must exelude those which needed only systemic treatment, and have been relieved by it, notwithstanding the associated topical treatment which they did not need.

The endometrium has been the subject of a vast amount of sometimes mild, many times useless or destructive topical treatment. Other organs, the nose, stomach, intestine, bladder, and eye, are subject to the same catarrhal conditions from the same general causes. Consisteney therefore might indicate topical treatment for them also. If in a given ease, for example, the whole intestinal canal and bladder and endometrium were catarrhal, it might be quite as reasonable to apply fuming nitric aeid to all as to one. Such an experiment would not only show that the human uterus has endured an immense amount of abuse, but.would demonstrate suceessfully the absurdity of topical treatment applied to a mucous membrane when the discharge is only one of many local evidences of a general condition. Very significant is the fact that often-repeated handling of the genitals may give rise to psychic irritation or depression. A woman once habituated to local treatment may become a monomaniac on that subjeet.

Intra-nterine treatment as ordinarily practised is tedious, and whether mild or severe, if frequently repeated with indifferent aseptic eare, may set up new infection, or may earry the old infeetion to deeper structures. Many cases which do well on mild topical treatment would do better on systemic treatment alone.

Strong intra-nterine applieations which have the power to destroy the diseased structures sometimes will arrest purulent endometritis, but in doing this they may destroy the endometrium, injure the 
myometrium, and reduce the uterus to a cirrhotic-like, cicatricial condition. Numerous plastic operations have been devised with but little success to reopen the contracted uterine canals. The chloride of zinc pencil produces a slough of the endometrium and sometimes of the myometrium; the use of it having destroyed the natural secreting surface may be followed not only by a chronic purulent discharge, but also by infection of the appendages by extension from the septic sloughing endometrium. The endometrium now has lost permanently its epithelial covering, the chief protection of the uterus against microbic invasion. Contrast this condition with that in which the diseased structures have been removed by an aseptic curettage so that the healthy abraded surfaces are all ready to reproduce a new endometrium, and the objection to strong caustics in the endometrium will become apparent.

Electricity is painful, tedious, dangerous, and often unduly destructive. Great cicatricial formations and hopeless stenosis in the endometrium are among the possible results. These effects are not limited to the diseased, but may include healthy structures. The immediate dangers are greater than those of aseptic curettage. Generally speaking, the method is not to be approved.

The author has tested carefully the routine use of topical applications, the vaginal douche, the swabbing out of the uterus with cotton, the injection of astringents, the vaginal and intra-uterine application of dry powders, intra-uterine pencils of various alterative and caustic substances, wool-glycerin tamponade, electricity, and intra-uterine gauze tamponade, and the patient use of such means has been followed by a few cures, and by much disappointment, to say nothing of some positive harm. Topical treatment shonld not be continued beyond a few weeks unless good results have become apparent. It has a more legitimate place as a supplement than as a substitute for systemic and operative treatment. A reproach will be lifted from the medical profession when the indiscriminate use of topical treatment has been relegated to the dark ages of gynecology.

If topical treatment is to be used, especially if it is to be intrauterine-see Chapters II. and IV.-the cervix should be exposed with Sims' speculum, the vagina cleansed thoroughly with dry absorbent cotton on dressing-forceps and swabbed with cotton saturated with a 5 per cent. solution of carbolic acid or a 1 per cent. solution of creolin. Slight traction is now made on the cervix by tenacula or blunt-tooth foreeps, to straighten the uterine canal, and the endometrium is cleansed by means of cotton wound on an applicator. The cervical plug of mucus, if present, should be removed. The desired application may then be earried into the endometrium by means of the applicator wound with fresh absorbent cotton, or, if the canal be very open, by means of fine dressing-forceps. A pledget of cotton saturated with glycerin or a 10 per cent. mixture of ammoniated ichthyol and glycerin may be placed in the vagina as a protective and for its hygroscopic affect. Over this place a pledget of dry eotton, to keep the first in position and to absorb moisture. The vaginal tampon should be removed in twenty-four hours. Intra-uterine cleanliness is 
the first requisite. To secure this, an open canal and normal drainage are essential. During such treatment coitus is prohibited.

Intra-uterine gauze tamponade has been used extensively for dilatation and drainage in the non-operative cases. Increasing quantities of a narrow strip of antiseptic gauze are packed into the uterus in successive treatments, until the endonetrium has become gradually dilated to a diameter of one-third or one-half inch. This dilatation permits easy and thorough intra-nterime topical treatment and drainage, especially capillary drainage when the gauze is in place. This method, in

FIGURE 109.

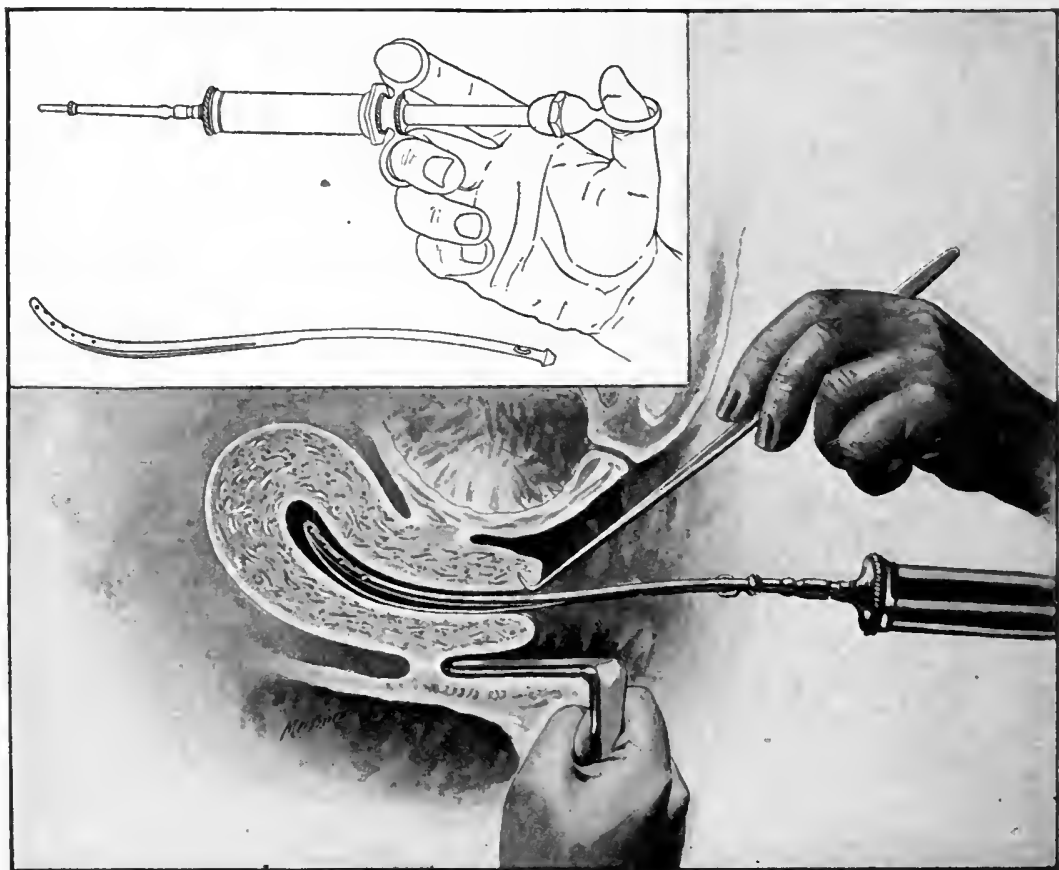

Patient in dorsal position. Cervix exposed by perineal retractor in right hand of nurse. Uterus drawn down with tenaculum in left hand of operator. Any desired fluid may be injected into endometrium by means of special canula attached to syringe. This syringe, worked by right hand of operator, is shown in the upper left-hand corner. Canula here is shown as detached from the syringe. Outflow of injected flujd is secured by a wire attachment running parallel to the canula and a little below it. Sims' speeulum and the left lateroprone position would be preferable to the simon speculum and the dorsal position.

the author's hands, has been successful occasionally, but less so than the reports of its advocates would seem to promise. Great care is necessary lest the gauze, instead of earrying out septic material, may carry it in.

It would be confusing, and it is unnecessary to name the inmumerable drugs and chemicals which are lauded for intra-uterine medication. Carbolic acid and iodine, for their disinfectant and astringent effect, meet the requirements in glandular endometritis and ichthyol in interstitial endometritis so far as topical treatment can meet them.

In cases of purulent endometritis, one may make advantageously 
a single application of 40 per cent. formalin to the endometrium, and in some very intractable cases may repeat this once or twice at intervals of a month. This application is made best by means of a uterine applicator wound with cotton, the vagina being protected by a wad of cotton placed behind the cervix uteri.

More conservative and perlaps more effective than formalin is the intra-uterine injection of tincture of iodine. Preparatory to the injection the endometrium should be cleasned thoroughly either by wiping out with cotton or by irrigation with water. One drachm shonld be injected at each treatment. In order to prevent the tincture from being forced through the Fallopian tubes, it should be thrown in gently and allowed to run out; the injection may be repeated twice a week; if in two months there is not marked improvement, the treatment should be interrupted and if necessary resumed after curettage. The technique of injecting the uterus is shown in Figure 109. In place of the instruments here presented an ordinary small glass female catheter attached to a common ear syringe by means of a short rubber tube may be used.

The Biers cupping congestive treatment, described at the end of Chapter IV., is important in connection with eleansing the endometrium, both as an independent remedial measure and as a preparation for intrauterine treatment.

\section{Surgical Treatment of Chronic Endometritis.}

Indications for Curettage.-When the endometritis is distinctly infectious and chronic, both topical and systemic treatment are usually inadequate, although either properly may supplement surgical measures. The disease of the mucosa should then be removed by the sharp curette. The operation is rendered extra-hazardous by active inflammation in the Fallopian tubes or by any other active pelvic inflammation which renders the uterus immobile or very sensitive to the touch. If for any reason it must be done under these adverse conditions, the greatest aseptic care should be taken to prevent dangerous lymphangitis, phlebitis, and peritonitis. A general description of curettage will be found in Chapter V. Formerly salpingitis, ovaritis, peritonitis, and cellulitis were considered positive contraindications for invading the uterine cavity. At present, although, these diseases, if chronic, call for especial care, they are not to be considered as necessarily prohibiting intra-uterine operations, provided these operations are of such a character as to remove the disease from the endometrium. They do, however, prohibit all intra-uterine interference which falls short of this. Ordinary intra-uterine treatment, even examinations with the sound, may be more dangerous than thorough dilatation and sharp curettage. Incomplete dull curettage is specially dangerous, for it exposes the surfaces to absorption and at the samic time may leave infections matter to be absorbed. Inflamed tubes and ovaries often become healthy, or at least symptomatically cured, after the primary source of infection has been removed from the uterus. In order to facilitate the curettage and insure drainage let the dilatation be thorongh. 
Technique of Curettage.-The aceompanying series of illustrations, Figures 110 to 114, shows the steps of the operation of sharp curettage. The dilatation should be begun with a small light dilator, Figure 110, and continued with a larger dilator of the Wathen type, Figure 111. This instrument is of heavy construction and the blades of it have great expanding power. After the uterus lias been dilated to the extent of one-half to three-quarters of an inch the endometrium is subjected to sharp curettage, as shown in Figure 112. In curettage of the uterus the perineal retractor may to advantage give place to the index and middle fingers of the left hand, while the operation is performed by means of the curette in the right hand.

Figure 110.

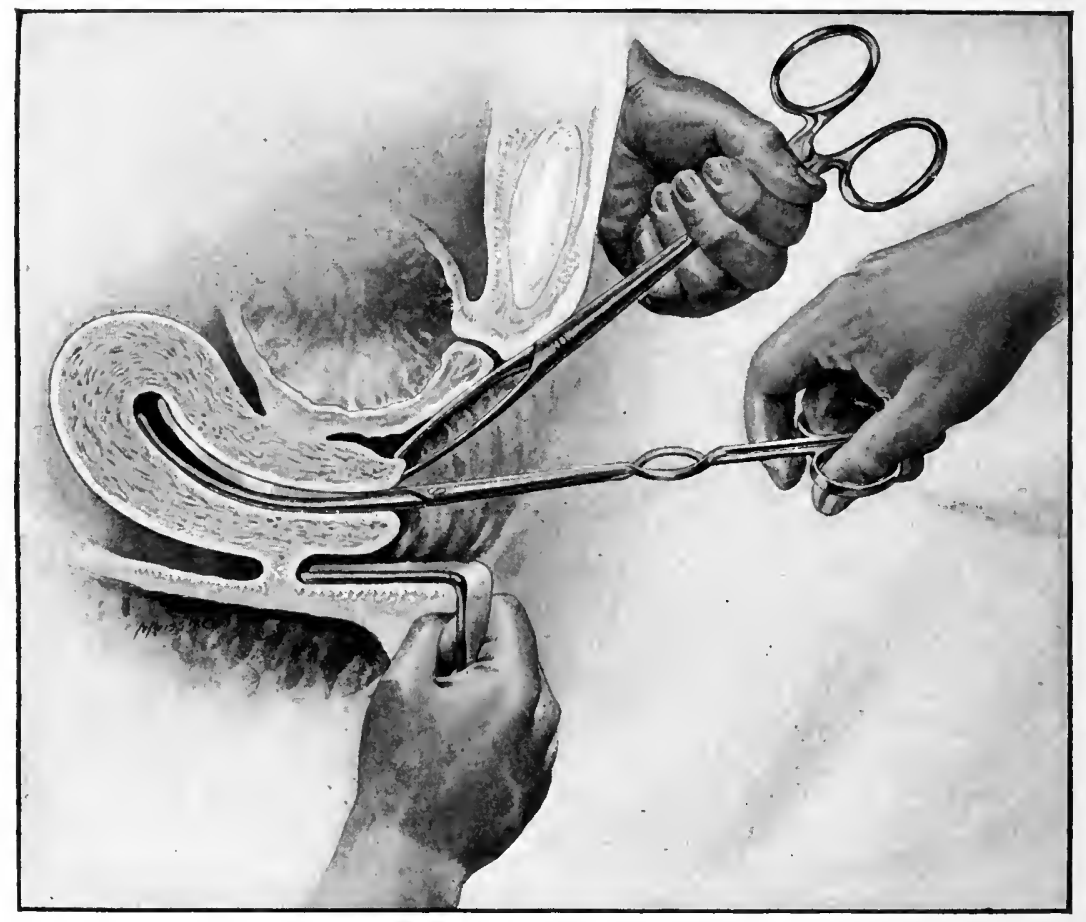

Curettage. First step: dorsal position. Cervix exposed by perineal retractor in right hand of nurse. Uterus drawn down by vulsellum forceps in left hand of assistant. Dilatation begun by small dilator in right hand of operator.

After thorough curettage the uterus is flushed out with sterile water, Figure 113. The canula used for this purpose may be a simple glass female eatheter attached to a rubber tube. This tube leads from a funnel held by a nurse above the patient. The water flows from the funnel through the tube and the glass canula into the uterus until the endometrium is irrigated thoroughly. During the irrigation the canula should continually be withdrawn and reintroduced, in order to prevent the possible forcing of the injected fluid into the Fallopian tubes. Observe the forceps fastened to the rubber tube. This is a 
Figure 111.

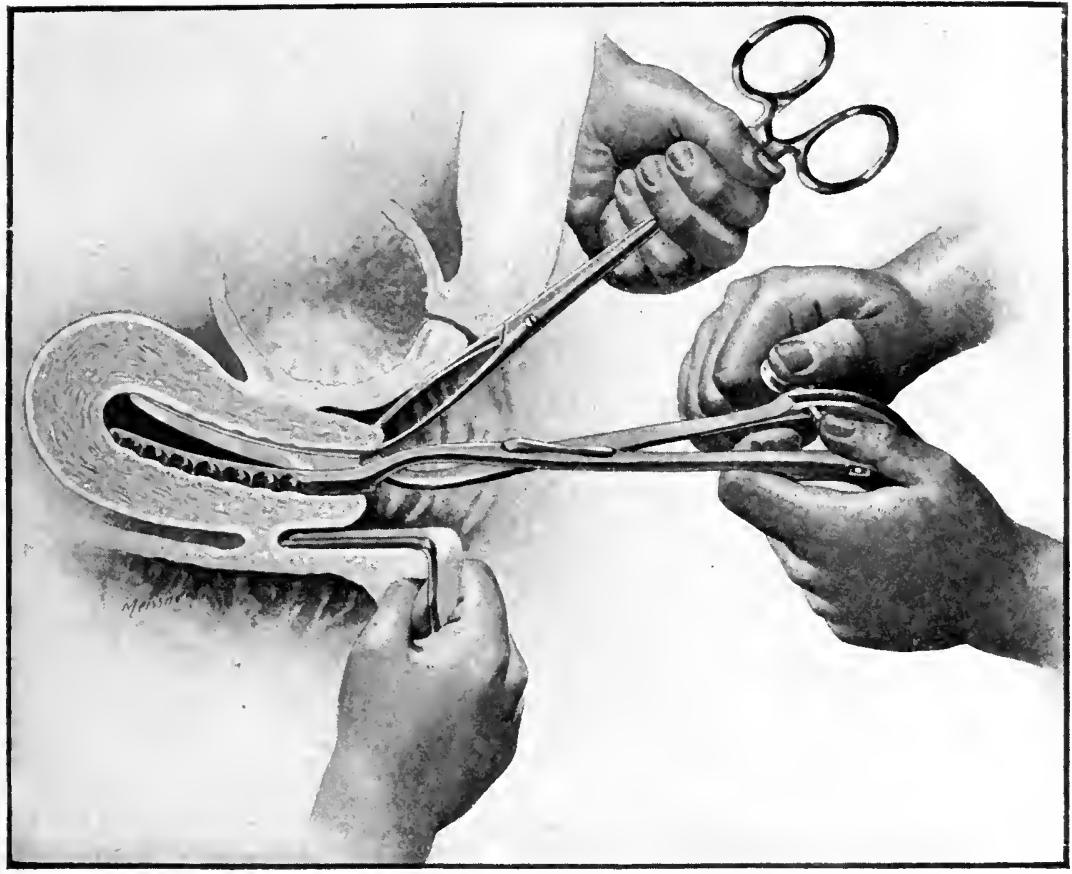

Curettage. Seeond step: dorsal position. Cervix exposed by perineal retractor in right hand of nurse. Uterus drawn down by vulsellum forceps in left hand of assistant. Dilatation completed by Wathen dilator in hands of operator.

FigURE 112.

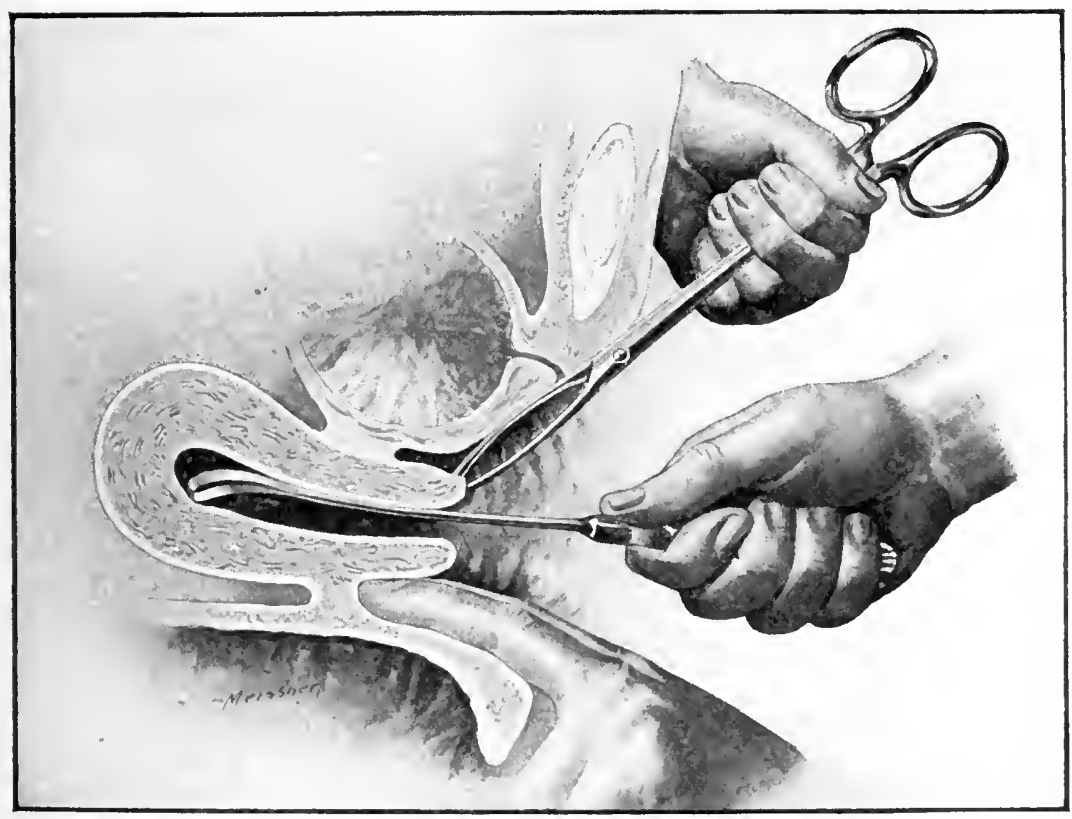

Curettage. Third step: dorsal position. Perineum retracted by two fingers of one rators left hand. Tterus drawn down by vulsellum forceps in left hand of assistant. Endometrium curetted by sharp curctte in operator's right hand. 
practical device for reducing the size of the rubber tube so that it will fit a canula or catheter of smaller size. Not infrequently this difference in calibre between the tube and cantula gives rise to eonsiderable annoyance during an operation, which may be obviated much more readily and quickly by means of the forceps thus used than by the common means of tying a strong cord tightly around the end of the tube at the point where it receives the canula.

In place of the fumnel a thoroughly sterile fountain-syringe may be used. The rubber donche-bag of the fountain-syringe is hung usually on a hook or nail at some point near to and above the patient. Sometimes, however, in private practice the operation is delayed because

Figure 113. .

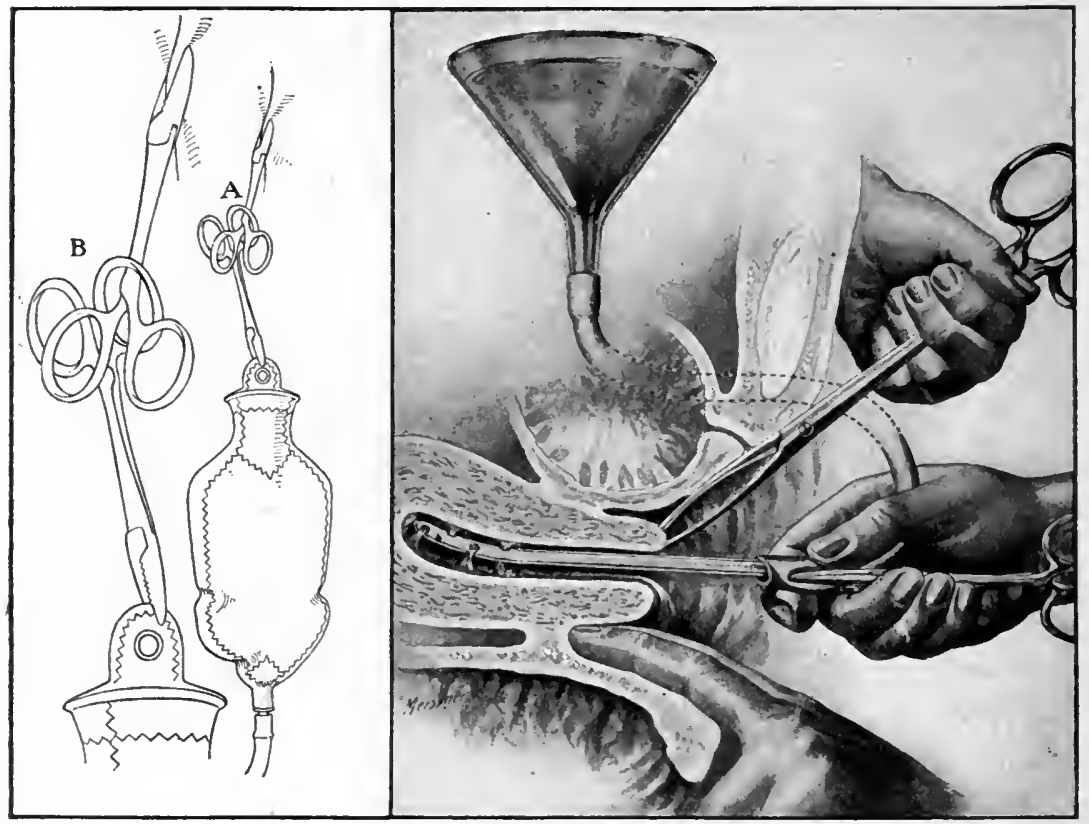

Curettage. Fourth step: dorsal position. Perineum retracted by two fingers of operator's left hand. Cterus drawn down by vulsellum forceps in left hand of assistant. Endometrium irrigated by canula inserted into rubber tube in operator's right hand. Figures A and $\mathbf{B}$ show a fountain-syringe attached to a towel by means of pressure-forceps. The towel may be fastened to a curtain or other hanging by means of safety-pins. The fountain-syringe may be used instead of the funnel.

notling is available upon which to hang the douche-bag. To overcome this diffieulty the bag may be suspended by two pairs of pressureforceps, as shown in A and B, Figure 113, the upper forceps being fistened to some fabric and the lower forceps to the donche-bag. A shows the forceps and douche-bag entire; $B$ shows the forceps and upper part of the douche-bag more in detail. The operation is performed usually in front of a window. Therefore the curtain may be used upon which to fasten a clean towel with safety-pins. The upper forceps may be attached to the towel.

The irrigation having been completed, the fingers of the operator 
are removed from the vagina, the perineum again retracted by means of the Simon speculum in the hand of the nurse or assistant, and the nterus swabbed ont by means of cotton wound on a dressing-forceps. Figure 114. The cotton may be saturated with any desired disinfectant. A saturated solution of iodine crystals in 95 per cent. of carbolic acid is used perhaps most frequently. If very thorough disinfection is required, 40 per cent. formalin nay be employed. Becanse of its destructive power formalin should be used with carc lest it cause cicatricial contraction. In order to prevent the application from

Figure 114 .

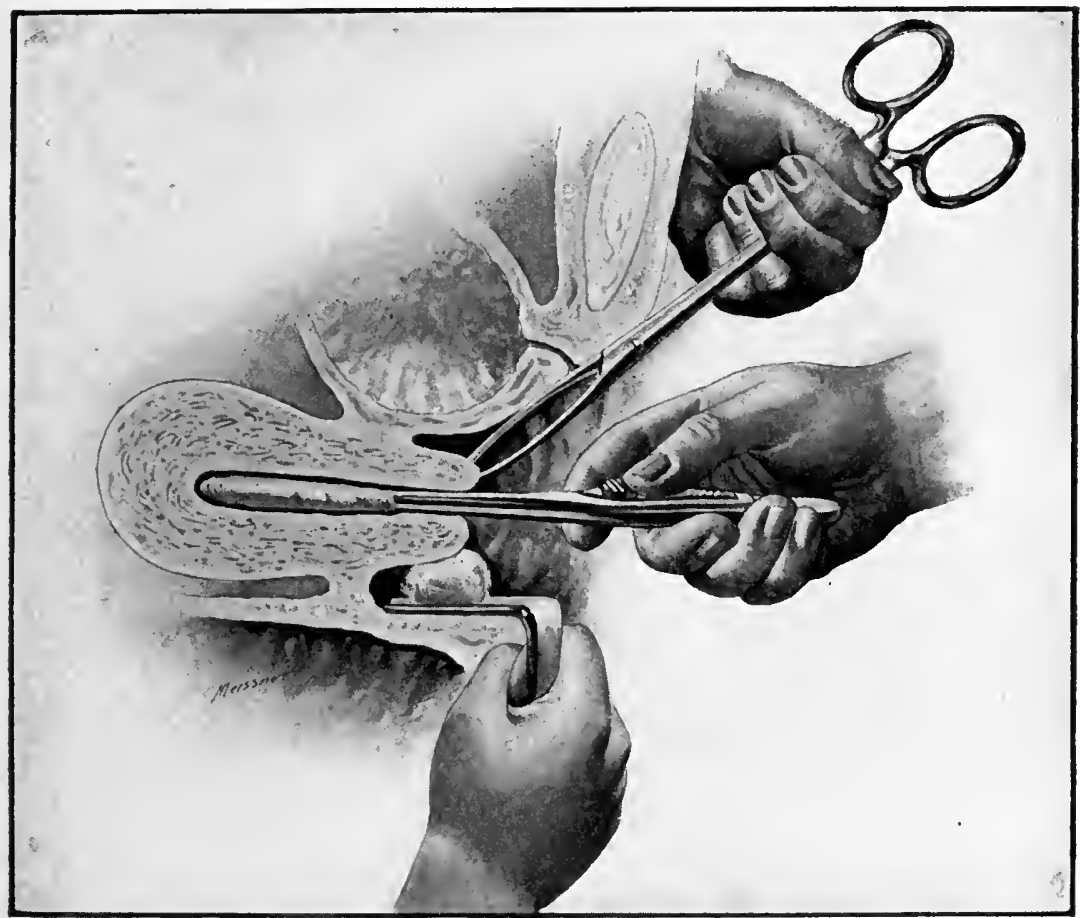

Curettage. Final step: dorsal position. Perineum retracted by Simon retractor in right hand of nurse. Uterus drawn down by vulsellum forceps in left hand of assistant. Endometrium disinfected by cotton wound on Emmet's dressing-forceps and saturated with desired disinfectant. Application made by right hand of operator.

coming in contact with the vagina, it is well, as shown in the Figure, to protect the vaginal mucosa with a pledget of cotton placed between the posterior wall of the cervix and the perineal rotractor. Before making the application two foreeps or applicators should be wound with absorbent cotton, and one should be pushed into the uterine canal with the cotton dry in order to absorb any fluid which may remain after the irrigation, and to prevent oozing, for if the application bo made in a uterus filled with blood it ma be made to the blood and not to the mucosa. The cotton on the other forceps should now be dipped in the desired disinfectant and introduced just as the first is 
withdrawn. The wad of cotton posterior to the eervix, the forceps used in making the application, and all other instruments are now removed. No dressing is required. A vaginal douche containing 0.5 per cent. lysol should be given twice daily for a period of two weeks. This is the only special after-treatment.

The treatment of endometritis, even with the curette, is not uniformly successful. Dilated and obstructed blood-ressels cannot always be restored to their proper calibre. Disorganized lymphatics, nerves, and glands do not always resume their normal functions. Regencration of lost structures is not always possible. In these respects endometritis offers a close analogy to nasal catarrh. In the glandular forms of the disease, in which the endometrium yet retains sufficient integrity to insure regeneration of the glandular and epithelial structures, the sharp curette offers both a symptomatic and a histological cure. When the disease has progressed to the atrophic stage of interstitial enclometritis and the endometrium is destroyed physiologically, anatomical cure is impossible and only a degree of symptomatic cure is possible. When endometritis is complicated with extensive chronic metritis and obstinate pelvic infection, the uterine discharge will persist regarlless of curettage or of any other intrauterine treatment. Under sueh conditions hysterectomy may be the only means of relief. Since this extreme measure might be indicated more for extra-uterine than for uterine inflammation, the consideration of it is referred to the subjeet of Inflammation of the Uterine Appendages.

Regeneration of Endometrium after Curettage.-Not only is sharp curettage efficient, but the recent investigations of Werth and others show that prompt regeneration of the uterine mucosa follows. Studies of the recently curetted endometrium show that the work often is done imperfectly, and that large portions of the diseased mueosa, particularly in the cornua and lateral walls, are apparently inaccessible to the ordinary curette. Special small curettes should be used therefore for these parts.

Werth reports histological examinations of six uteri removed at periods varying from three to sixteen days after curettage. All cases showed unequal results of the scraping on the various parts of the uterine mucosa. Some parts were untouched. In some the superficial layers had been removed and the deeper layers left, and in other parts the muscularis had been attacked. The nucosa in the fundus and in the lateral portions of the cavity most frequently was left intact. The abrisions on the anterior wall were decper than on the posterior. They were also deeper in the lower part of the corpus near the internal os. This is explained by the convergence of the downward strokes of the curette. Except in places where the muscularis had been injured by the curette, the entire lining of the uterus was covered with new mucosa, the glands opening freely on a surface of unbroken superficial epithelinm. This young mucosa was characterized by great preponderance of fibrillary conncetive tissue over the connective tissue of the stroma. The regenerating tissne was supplied with blood-ressels which grew out of the muscularis or out 
of the remaining mucosa. 'The vessels were surrounded with a broad mantle of fibrillary conneetive tissue which followed their ramifications almost to the surface of the mucosa.

The glands were regenerated from their deeper portions which the curette had spared, especially from those which were situated where the mucosa dips deep down into the muscularis; they grew out toward the surface together with the surrounding blood-vessels and fibrillary connective tissue. 'The surrounding stroma was observed frequently to grow more rapidly than the glands, and to give a somewhat irregular, jagged contour to the regenerated endometrium. The superficial epithelium was found to be regenerated principally from that of the glands. In some places the young epithelial cells were found flattened and enlarged. In the later stages of the regeneration of the mucosa the excess of fibrillary connective tissue was observed to disappear by hyaline degeneration. This process on the fifth day after eurettage was visible in the subepithelial layers; and on the tenth day only a few fibrillæ were left in the superficial stroma; in their places were large, spindle-shaped cells, with several processes of protoplasm. Only in those parts where the muscularis had been abraded did there exist a condition resembling that of granulation-tissue. 


\section{CHAPTER XVIII.}

\section{CHRONIC METRITIS.}

Chrosic metritis-usually understood to mean inflammation of the uterine muscularis, a condition more accurately described by the word myometritis-is taken here in its broader literal sense, and is used to designate chronic inflammation of the uterus as a whole, and to include therefore endocervicitis, endonetritis, myometritis, and perimetritis. The various parts of the uterus-i.e., the endometrium, inyometrium, perimetrium, corpus, and cervix-never are involved in sharply defined areas of disease, although any one of them may be the specially affected part of the diseased organ. 'The disease is commonly the consequence of endometritis and coincident with it. Infection of the uterus as observed by the clinician, except acute gonorrhoal and puerperal metritis, is generally elıronic.

The striking phenomena of acute metritis are the active infective and inflammatory processes. The term ehronic metritis stands not so much for definite processes as for certain chronic changes, more or less permanent, in the quantity and quality of the glandular elements, muscularis, blood-ressels, lymphaties, and connective tissue. These changes are usually hypertrophic, hyperplastic, or atrophic. They have been the subject of a long and unsatisfactory discussion, and have been designated variously as infective, inflammatory, irritative, subinflammatory, and congestive. Although the changes under consideration may not always conform to the strict idea of inflammation, there is yet a propriety in calling them inflammatory, because the essential element of inflammation-round-cell infiltration-is usually present. In chronic inflammations migration of these cells oceurs, if at all, more slowly than in acute inflammation.

\section{Etiology of Chronic Metritis.}

The chief predisposing eanses are these :

Acute metritis, endocervicitis, and endometritis.

Septic puerperium and abortions.

Infection following operations and examinations.

Uterine tumors.

Frequent parturition.

Uterine displacements.

Excessive venery.

The bacterial exciting causes are the same as in acute metritis, see Chapter $X$. Chronic metritis frequently is associated with obstructed cireulation in the abdominal viscera, especially the liver, and with rheumatism, gout, lithæmia, and cholæmia. 


\section{Pathology of Chronic Metritis.}

Since chronic metritis is the sum of all the inflammations of the uterine mucosa, uterine muscularis, and uterine peritoneum, it follows that the pathology of it must embrace:

I. Chronic changes in the mueosa-endometritis and endocervicitis-of which the pathology has been described in Chapter XV. and XVI.

II. Chronic ehanges in the perimetrium-peritonitis-which will be described in the ehapter on Pelvic Peritonitis.

III. Chronie changes in the muscularis which occur in two forms:

1. Hypertrophic.

2. Interstitial.

1. Pathology of Hypertrophic Metritis.-In this form of chronic metritis all the histological elements are increased. Hypertrophy of puerperal origin should be distinguished from that of nonpuerperal origin.

Puerperal Hypertrophy, commonly known as subinvolution, is produced as follows: The muscular elements, enormously increased during the evolution of pregnancy, fail to undergo the normal physiological degeneration and absorption after labor. The connective tissue also remains superabundant. The lymph-vessels and blood-vessels continue large, full, and stagnant. The uterine walls are thiekened from congestion and infiltration. The uterus is not always uniformly enlarged-that is, the hypertrophy may pertain especially to the cervix or to the corpus uteri. The uterus may be twice as large as normal, and the canal may measure three or four inches. The organ remains soft and mobile. This flexibility accounts for the fact that many uterine flexures date from the puerperium. Subinvolution may therefore be defined as the failure of physiological hypertrophy to subside after labor.

Non-puerperal Hypertrophy is pathological from the beginning, and may occur in women who have never been pregnant. It is sometimes clinically impossible to distinguish between the puerperal and nonpuerperal varieties.

Great hypertrophic elongation of the supravaginal and enlargement of the infravaginal portions of the cervix, with descent, are described in the chapters on Laceration of the Cervix and Displacements. Sometimes hypertrophic enlargement pertains ehiefly to the corpus uteri, sometimes to the eervix ; or it may involve uniformly the entire organ. Hypertrophy of the cervix often is confounded with laceration. The symptoms, like the eauses, are almost identical with those of the associated endometritis. In the absence of marked perimetritis, or parametritis, the uterus is not very sensitive to the touch. Downward displacement from increased weight is usual. Perverted menstrual and other funetions are the same as in endometritis.

The prognosis is mueh more favorable for puerperal than for nonpuerperal myometritis-i.e., subinvolution, if non-infections, is often only temporary. The disease is apt to be obstinate and destructive in proportion as the infectious element predominates. 
2. Pathology of Interstitial or Cirrhotic Metritis.-In this form of metritis, sometimes called areolar hyperplasia, there are increase of comective and loss of muscular tissne. The muscular wall is pale and indurated. The microscope will show hyperplasia of internuscular connective tissue, corresponding atroplyy of muscle-fibres, and contraction of blood-ressels as already stated. 'The chronic changes in these varions parts may be the ontcome of acute processes already described under acute metritis; or there may have been no elearly marked acnte stage-i. e., the disease, at least apparently, may have been chronic or subacute from the beginning.

Fisure 115.

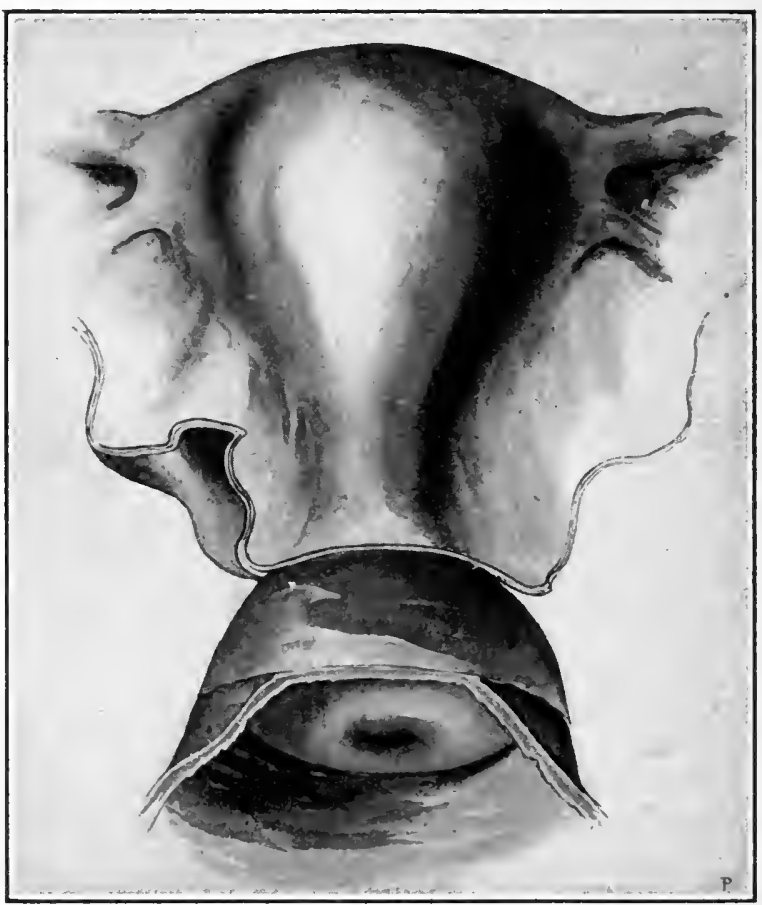

So-called hypertrophie elongation of the supravaginal portion of the cervix-rare except as a post-operative or post-mortem eondition. The cervix often becomes elongated by traction during the removal of the uterus.

Hyperplasia of connective tissue, whether puerperal or nonpuerperal, may follow hypertrophy or may develop independently of it. This form of the disease often results in a sort of pathological involution, with the following permanent changes: The lymph-vessels and blood-vessels shrink and wither, the nutrition of the muscular clements is cut off, and they disappear as if crowded out by the increasing connective tissue; the nterus now becomes lard and anæmic ; it still may remain large from the superabundant connective tissue, but finally this may contract and, cicatrix like, reduce the organ even below its normal size. The result of these changes are great uterine 
irritation and pelvic pain. The whole organ with its appendages and adjacent structures is in a state of permanent malnutrition.

In connection with the atrophic changes of interstitial and cirrhotic metritis may be mentioned two special forms of atrophy : puerperal atrophy - superinvolution-a certain form of non-puerperal atrophy and arteriosclerosis.

Puerperal Atrophy-superinvolution-is the direct opposite of subinvolution. In superinvolution the process of degeneration and absorption after labor passes beyond the physiological limits, and the uterus shrinks below the normal size and becomes soft and excessively mobile. The condition resembles senile atrophy of the menopause.

Figure 116.

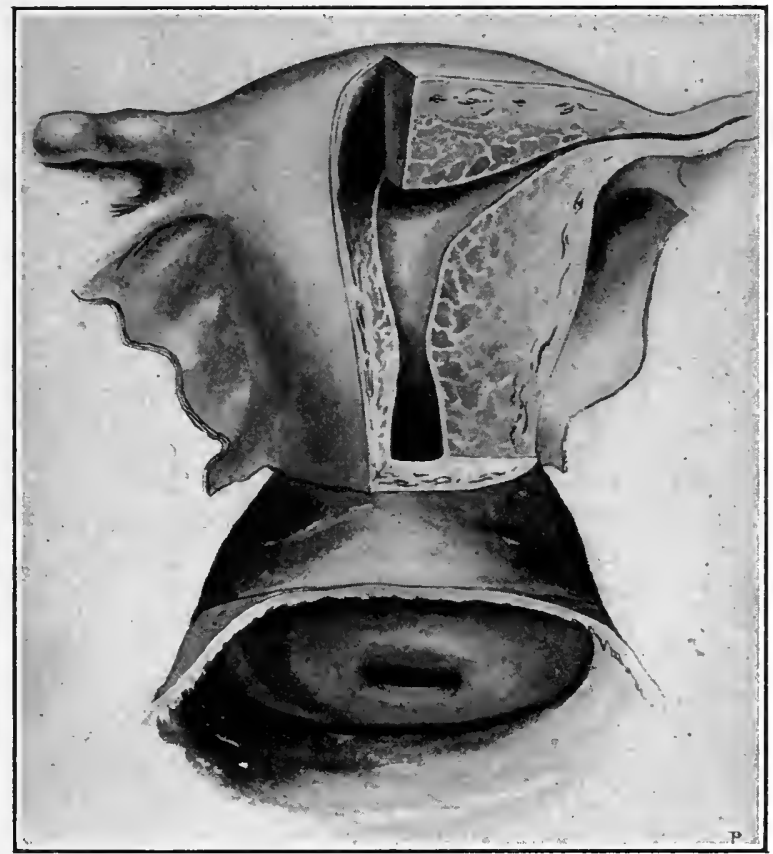

Hypertrophy of the cervix uteri. The expansion of the cervix is due partly to eversion of intra-uterine mucosa consequent on laceration of the cervix.

Apparently there are two distinct varieties of superinvolution-one temporary, the other permanent. The two forms are differentiated by the fact of a normal pnerperium, usually with prolonged lactation, in the temporary form, and by the history of a septic puerperium in the permanent variety. In the latter case one or more of the reproductive organs or parts thereof-i. e., the endometrium, myometrium, and the nterine appendages-become infected and in a physiological sense destroyed. In the temporary variety spontaneous recovery may occur and the woman again may bear children. In the destructive form there is permanent atrophy of all the structures involved. Menstruation, if it returns at all, is scanty and generally painful. 
Immediate amenorrhoa is the rule. There is sometimes a painful molimen in place of menstruation.

Non-puerperal Atrophy.-There is another class of cases in which atrophy of the reproductive organs occurs independently of parturition. This form of atrophy is generally the result of chronic wasting disease, like tuberculosis and diabetes; or of acute infectious disease, like scarlatina, rubeola, and enterie fever. There is always cessation of menstruation. This is a conservative effort of nature to save the pationt's blood and strength. Unfortunately, however, the ill-health of the patient often is attributed wrongly to the amenorrhoa, and

Figure 117.

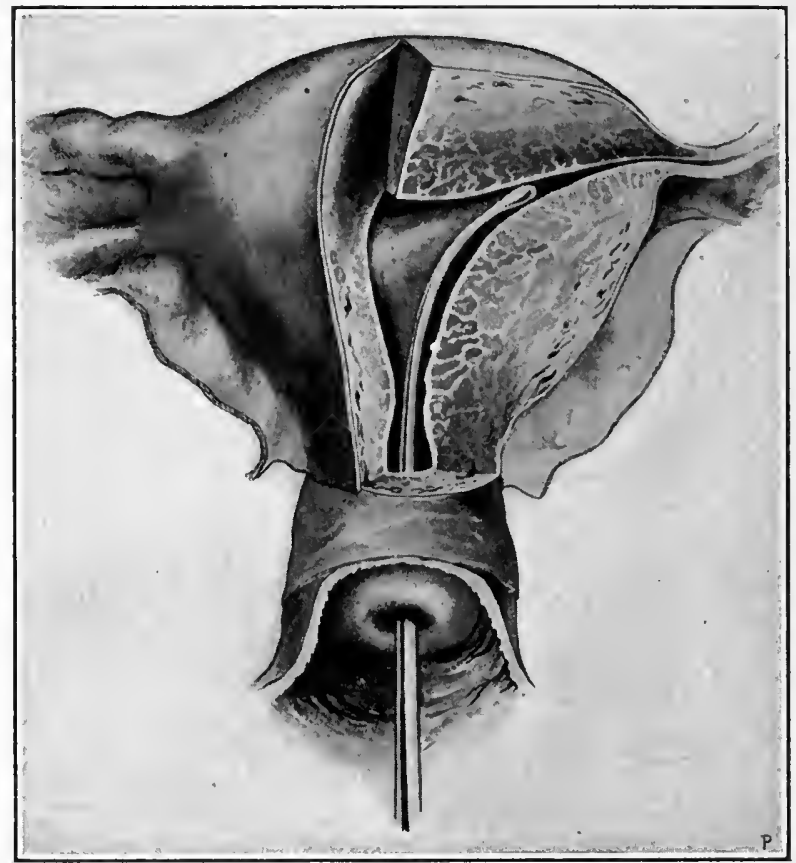

Hypertrophy of the corpus uteri : observe the great size of the corpus relative to that of the cervix.

treatment designed to stimulate and re-establish menstruation sometimes is used. By such means the woman's vitality may still further be exhausted. The above facts from the therapeutic standpoint, especially in tubercular and other wasting diseases, are very significant. Clearly the treatment should not be local, but systemie.

Superinvolution and non-puerperal atrophy are rare; the causes are obseure; the precise relation of inflammation to them is unknown. Except in the temporary non-infectious form already mentioned, recovery rarely or never takes place.

Arteriosclerosis.-Chronic metritis in advanced years is assoeiated not uncommonly with selerosis of the uterine arteries, and in some instances with ealcareous degeneration of the vessels; these 
changes may be looked upon as senile degeneration, are not therefore always, in the strict sense, pathological, but may be rather the natural ehanges of old age.

\section{The Physical Signs of Chronic Metritis.}

1. The uterus is enlarged symmetrically, and on bimanual examination, in the later stages, is harder and firmer to pressure than normal. Atrophic changes later may cause the uterus to contract to rudimentary size.

2. Tenderness on pressure is not very marked unless there is complieating inflammation of the uterine appendages.

3. The uterus may be freely movable or fixed by adhesions.

4. The uterus may be displaced; and, if so, the deviation is apt to be anteversion and descent; this gives rise to vesical and rectal irritation.

5. Enlargement of the nterine eavity may be demonstrated by passing the sound.

\section{The Symptoms and Diagnosis of Chronic Metritis.}

1. The temperature is normal or only slightly elevated.

2. Pain is not acute; there is usually a sense of aching, pressure, weight and dragging in the back, bypogastrium, and thighs.

3. Menstrual disturbances, such as menorrhagia, intermenstrual uterine henorrhages, and dysmenorrhœa, singly or combined, are commonly present.

4. Sterility, which may be due to coexisting lesions, is usual.

5. Defecation and urination in most cases are painful.

6. Reflex and sympathetic disturbances of extrapelvic organs, especially the organs of digestion, and faulty general nutrition, are generally present.

\section{Differential Diagnosis of Chronic Metritis.}

The differential signs are between metritis, small fibroid tumors, and early pregnaney.

\begin{tabular}{|c|c|c|}
\hline Chronic metritis. & Small finoid tumors. & Early pregnancy. \\
\hline $\begin{array}{l}\text { 1. Menorrhagia and intra- } \\
\text { menstrual uterine hemor- } \\
\text { rhages, not invariable. }\end{array}$ & $\begin{array}{l}\text { 1. Nenorrbagia and uterine } \\
\text { hemorrhage the rule. }\end{array}$ & 1. Amenorrhoen. \\
\hline 2. No signs of pregnancy. & 2. No signs of pregnancy. & $\begin{array}{l}\text { 2. Signs of early pregnancy: } \\
\text { a. Morning sickness. } \\
\text { b. Enlarged breasts. } \\
\text { c. Blue discoloration of } \\
\text { vaginal mucosa. } \\
\text { d. Softening of the cer- } \\
\text { vix uteri. } \\
\text { e. Hegar's symptom of } \\
\text { narrow supravaginal } \\
\text { portion of the eervix. } \\
\text { f. Rhytlmical uterine } \\
\text { contraction under } \\
\text { the palpating hand. }\end{array}$ \\
\hline $\begin{array}{l}\text { 3. Uterus hard and regular } \\
\text { in outline. }\end{array}$ & $\begin{array}{l}\text { 3. Cterus hard and irregu- } \\
\text { lar in outline. }\end{array}$ & $\begin{array}{l}\text { 3. Ltcrus soft and regular in } \\
\text { outline; may momentarily con- } \\
\text { tract and harden on handling. }\end{array}$ \\
\hline $\begin{array}{l}\text { 4. Uterus commonly in } \\
\text { pathological anteversion and } \\
\text { descent; may be in retrover- } \\
\text { sion. }\end{array}$ & $\begin{array}{l}\text { 4. Uterus, liable to be dis- } \\
\text { placed in any direction ac- } \\
\text { cording to the mechanical } \\
\text { influence of the fibroids. }\end{array}$ & $\begin{array}{l}\text { 4. Uterus commonly ante- } \\
\text { verted. }\end{array}$ \\
\hline
\end{tabular}


The least doubt as to the existence of pregnancy should lead one to await developments. Under no circumstances should the sound be passed if pregnancy is a possibility.

\section{Treatment of Chronic Metritis.}

The treatment of chronic metritis is that of the associated lesions. The reader is referred therefore to the treatment of endocervicitis, endometritis, perimetritis, parametritis, and inflammation of the uterine appendages.

Numerous operations to reduce the size and weight of the uterus have been devised, such as amputation and resection of the cervix uteri ; but as already set forth, enlargement of the cervix is generally rather apparent than real, and is due to the results of laceration. See Chapter XLII. for Emmet's operation and Schroeder's operation. As explained in the preceding ehapter, when the wall of the uterus becomes so infected that it resembles the wall of an abscess-cavity the organ should be removed. Supravaginal hysterectomy sometimes is indicated by an enormously enlarged uterus, especially when the enlargement is associated with disabling displacement. See operation for supravaginal hysteromyomectomy in Chapter XXVII. 


\section{CHAPTER XIX.}

\section{PELVIC INFLAMMATION.}

\section{ROUTES OF INFECTION-GENERAL ETIOLOGY AND SIGNIFI- CANCE OF PELVIC INFLAMMATION.}

INFECTION of the uterus, as set forth in the preceding chapters, may extend from the uterine mucosa to the surrounding lymphchannels, veins, cellular tissue, Fallopian tubes, ovaries, and peritoneum. The subject of pelvic inflammation therefore includes lymphangitis, phlebitis, eellulitis, salpingitis, ovaritis, and pelvic peritonitis.

\section{Routes of Infection.}

Inflammation around the uterus, as set forth in Chapter $\mathbf{X}$., occurs frequently by the extension of infection in the uterine mucosa to the outlying structures, less frequently from infection in the vagina, bladder, or rectum, and not seldom from extrapelvic organs. When it spreads from the uterine mucosa it passes to the outlying structures by one of two routes:

1. Continuity of uterine and tubal mucosa.

2. The lymph-vessels and blood-vessels of the uterine muscularis and of the para-uterine connective tissuc.

Route by Continuity of Mucosa.-Transmission by continuity of mucosa does not invariably involve all the epithelial surfaces over which the infection has passed. It is probably possible, altlough not usual, for infection to travel from the endometrium to the abdominal end of the tube without intervening infection of the uterine end. Even though the uterine end has been infected, it may, owing to its smoother surface and greater resistance, have recovered, lcaving the disease only at the abdominal end.

Route by Lymph-vessels and Blood-vessels.-The lymph-channel may be the mere carrier of infection, and may itself show no trace of inflammation, or it may be inflamed throughout; this is because the bacteria, by whatever route carried, may colonize only at points of least resistance; freedom from infection in the vessels therefore does not prove that infection has not passed through them.

Route from Extrapelvic Organs.-Purulent salpingitis spreading from purulent appendicitis and tubercular salpingitis extending from tubercular peritonitis have been observed very frequently ; these are examples of infection from organs outside the pelvis. 


\section{Etiology of Pelvic Inflammation.}

Since extra-uterine pelvic infection usually originates in the endometrim, the causes for the most part will correspond with those of endometritis; the infection may arise also from the intestines, bladder, peritoneum, vagina, or from the general eirculation as a sequel of the acute infectious diseases. Pelvic hæmatocele may become the seat of infection and be the predisposing cause of a pelvic abscess. See Tubal Pregnancy.

Laceration of the perineum and cervix, and other traumatisms of parturition and of surgery, may open the way for the entrance of infection through the blood- and lymph-channels. The puerperal and traumatic infections more frequently take this route. Infection of lymph-vessels and blood-vessels may be carried to the uterine appendages from external cervicitis. The micro-organisms of infectious diseases, which have been found in the genitals, are introduced most frequently by uncleanly operations, local treatnent, and examinations. Chief anong these micro-organisms are the following:

$\begin{array}{ll}\text { Gonococcus. } & \text { Diphtheria bacillus. } \\ \text { Colon bacillus. } & \text { Typhoid bacillus. } \\ \text { Tubercle bacillus. } & \text { Pneumococcus. } \\ \text { Streptococcus pyogenes. } & \text { Actinomyces. } \\ \text { Staphylococcus pyogenes albus, citreus, and aureus. }\end{array}$

\section{Significance of Inflammation.}

Extra-nterine pelvic infection, whether it passes from the uterus by the Fallopian tubes to the peritoneum by continuity of surface; or by way of the lymph-channels or veins in the pelvic cellular tissue, may be arrested, in the first route, by inflammatory occlusion of the tube; in the second route by thrombic plugging of the vessels. In the one case the infection may be confined to the tube (salpingitis), in the other to the cellular tissue around the vessels (perilymphangitis or periphlebetis-i.e., cellulitis). The infection if not so arrested may pass to the pelvic cavity and give rise to peritonitis. The inflammatory process then may localize itself by setting up peritoneal adhesions and form a protective wall against general peritonitis. If neither one of these protective processes takes place, then the infection speedily may involve the whole peritoneum and the toxins may be increased rapidly and poured in fatal quantities from the peritoneal surfaces into the general circulation.

- We are familiar with the profound depression of the nervous system, the continued nansea, the anxions facies, the paretic and distended bowels, and the tympanites, which go to make up the symptomgroup of general peritonitis. These grave symptoms of infection so often attributed wrongly to the inflammatory proeess, are rather the result of the profound toxæmia which the inflammation is striving unsuccessfully to shut off from the general circulation. If, on the 
other hand, a protective process becones effective, the result may be an almost overwhelming inflammation which may for the most part be confined so that the infection will spend its force within the narrow limits of the infected territory. The localized infective process may be so intense as to end in permanent impairment of the pelvic nutrition and in chronic invalidism; but the involved tissue has taken the brunt of the attack, sacrificed itself, and perchance saved the life of the woman. 


\section{CHAPTER XX.}

\section{PELVIC CELLULITIS.}

BEFORE reading this chapter on cellulitis the student is requested to consult the previous paragraphs on the Significance of Inflammation.

\section{Anatomy.}

An abundance of loose cellular tissue binds all the pelvic viscera together. It is continuous with the cellular tissue of the uterus and its appendages, and is found in large quantities especially in the broad ligaments; it is the medium through which the lymph- and bloodvessels, and nerves connect the uterus with its appendages, and bring them all into close anatomical, physiological, and pathological relations. The cellular tissue, and particularly that of the broad ligaments, becomes therefore a most significant factor in pelvic infection. Cellular tissue of the pelvis binds the various pelvic organs together and fills nearly all the space in the pelvis not occupied by them; it exists in great quantities aroumd the uterus, vagina, rectum, bladder, and the psoas and iliacus muscles, and furnishes, therefore, an abundance of material for the development of cellulitis.

Exception sometimes has been taken to the name cellulitis, since all tissues are cellular, and since therefore, in the wide sense, all inflammation is cellulitis. The word is used here in accordance with established usage, and is limited to inflammation of the cellular tissue around the uterus and vagina, more especially that between the folds of the broad ligaments. The term parametritis is too restricted, since the disease may occur in the lower regions of the pelvis around the vagina and bowel. Cellulitis usually is associated with some degree of peritonitis and therefore is related to it much as pneumonia is related to pleuritis.

\section{Etiology of Pelvic Cellulitis.}

Cellulitis, or, as it commonly is called, pelvic parametritis, is usually of puerperal origin; the causes therefore are largely identical with those of puerperal infection. The etiology in general is considered in Chapter $\mathrm{X}$. The most frequent bacteria in cellulitis are the common pus cocci. The gonococeus has been found in comnective tissue and in the lymph-vessels of the parametria. The source of the infection is usually the inflamed uterus; but it may arise in the perineum, vagina, bladder, or rectum. The infected rectum, urethra, and bladder are frequent sources of cellulitis in men. Unclean therapeutic appliances, septic manipulations generally, and traumatisms, 260 
especially those of parturition, open the way for the entrance of the bacteria.

\section{Pathology and Pathological Anatomy of Pelvic Cellulitis.}

Infection reaches the cellular tissue by way of the lymph-vessels and veins and may produce lymphangitis or phlebitis-that is, inflammation in the walls of the vessels. The progress of the infection may now be checked by thrombic plugging of the vessels and destruction of their walls. The infection then will spread to the surrounding structures producing perilymphangitis or periphlebitis and involving the tissue around the vessels, which is cellular or connective tissue. The disease in its full development therefore is cellulitis. Hence to define cellulitis as perilymphangitis or periphlebitis would be strictly accurate.

Cellulitis, like other inflammations, is divided into three stages : 1 , congestion ; 2 , effusion ; and 3 , suppuration.' The disease may terminate with either of these stages. If it goes to effusion, it may end in resolution and complete recovery, or continue as chronic cellulitis, or go on to suppuration and form a pelvic abscess.

The blood- and lymph-vessels here and there are plugged with firm inflammatory thromboses. If resolution does not follow, the thromboses break down and the corresponding spaces are filled with pus. The infection spreads from these small collections, which are, in fact, small abscesses, and frequently leads to the formation of single or multiple abscesses in the broad ligaments. These abscesses creep along the meshes of the loose connective tissue, avoiding firmer and stronger parts, and unless opened by incision may burst into the vagina, bladder, urethra, or intestine, or above Poupart's ligament, rarely below it, or into the labia majora, peritoneal cavity, or lumbar region, or through the obturator, sacrosciatic, or saphenous openings. Abscesses of cellulitic origin most frequently burst into the vagina; those of tubal origin, especially if surrounded by peritoneum, are more apt to break into the bowel or bladder. The bursting of an abscess through the cutaneous surface or into an organ which affords ready drainage may, if not followed by fresh infection, result in spontaneous cure. The breaking of an abscess into the peritoneum may set up fatal peritonitis.

In very severe cases, with extensive invasion of the lymphatics, the whole cellular tissue of the pelvis may be involved in paracystitis, paracolpitis, paraproctitis, parametritis, salpingitis, and ovaritis. Such infection usually results in multiple abscesses and great systemic disturbance. It is known as the erysipelas malignum internum of Virchow, or diffuse cellulitis of Pozzi. There may also be hemorrhages from destruction of the blood-vessels. The clinical picture in these cases is that of an acute general septicæmia. The infection may result in general peritonitis, and accumulations of pus may form throughout the abdominal cavity. The condition is rare and the rate of mortality high.

Formerly cellulitis was considered the central lesion in pelvic in- 
flammation. Salpingitis, ovaritis, and peritonitis scarcely were recognized as surgical diseases. A great advance was made in practical pelvic pathology when Battey, Hegar, Tait, and others showed the vastly greater relative importance, from the surgical standpoint at least, of tubal inflammation. When purulent accumulations in the pelvis. were attributed commonly to cellulitis, and therefore were left to themselves or treated by incision and drainage into the vagina, the failures were many and unexplained. As soon, however, as the majority of those abscesses were recoguized as accumulations of pus in the Fallopian tubes it was easy to understand why incision and drainage were followed so often by failure. It was because the tube is lined with mucous membrane and because chronic suppuration of mucous surfaces, even when drained, is most intractable. On the other hand, a cellulitis abscess surrounded by cellular tissue, when emptied is apt to close spontaneously. Pelvic cellulitis therefore, unless complicated by tubal communication, either terminates rapidly by resolution with complete recovery; or, if suppuration occur, it empties spontaneously or is evacuated by incision, and like a furuncle, which it resembles, promptly disappears; hence the cellulitis abscess, unless of tubal origin, seldom becomes chronic, and therefore has little or no part in the more familiar chronic pelvic suppuration for which the uterine appendages and sometimes also the uterus have to be removed.

Clinical experience shows pelvic suppuration to be prinarily almost always in the tube; rarely in the cellular tissue below; and if perchance an abscess be found there, it usually gives evidence of having burst from the tube into the broad ligament.

The above facts have led to a tendency of late years, especially among the laparotomists, to deny the existence of pelvic cellulitis, and to declare that an abscess in the broad ligament is there only when a previous infection of the Fallopian tube has forced its way throngh the mesosalpinx into the parametrium. In this connection let us remember that the disease oceurs in men, who have no Fallopian tubes, and that cellular tissue in the pelvis must be subject to the same laws of infection as in other parts of the body. The question, however, is not settled by a priori reasoning. Post-morten studies prove the frequent existence of acute cellulitis abscess not only by rupture of a sactosalpinx into the parametric cellular tissue, but also by the direct infection of the parametria by the lymphatic or venous ronte.

Pelvic cellulitis gives rise to numerous displacements and distortions of the pelvic organs, chief among which are :

$a$. Uterus drawn forward, backward, or to either side.

$b$. Ovaries and tubes displaced and fixed.

c. Bladder displaced or clistorted.

d. Rectum constricted (rarely).

Chronic Atrophic Cellulitis. - There is a form of chronic cellulitis, described by Freund, characterized by atrophic changes analogous to cirrhotic disease in other organs. 'This disease may originate in inflammation of the uterus, bladder, or rectum, and is especially apt to include chronic atrophic pericystitis and periproctitis- $i$. e., 
inflammation of the connective tissue around the rectum and bladder with resultant contraction of these viscera and shortening of the vagina.

The atrophic contracted cicatrix-like cellular tissue may cause excessive versions and flexions of the uterus. Since, however, the symptoms would be due rather to the cirrhotic disease than to the nterine deviations, mechanical support would be of little or no value. Perineuritis; neuritis; destruction of blood- and lymph-vessels; pinching of the nerves, lymphatics, blood-vessels, and ureters by the contracting eellular tissue; pain; local malnutrition; a widle variety of reflex nervous disturbances; chronic invalidism: all these are among the results of the atrophic process.

In contrast with the chronic atrophic cellulitis of Freund is the so-called cellulitis of Stapfer. It consists of odematous indurations in the abdominal walls and in the floor of the pelvis, and is characterized by pelvic discomfort and inconstant, transitory, or severe pain. The inflammation is of very mild type, with slight systemic disturbance. The transient nature of the disease suggests the analogy of urticaria and a probable angionenrotic element in its causation. Stapfer declares that the condition is common, and often mistaken for more serious affections. The author never has observed a case.

\section{Symptoms and Diagnosis of Pelvic Cellulitis.}

The symptoms are nearly identical with those of inflammation of the uterine appendages. The reader is referred therefore to that subject, especially when the inflammation is secondary to salpingitis or ovaritis, or is situated in the upper part of the broad ligament near the tubes and ovaries. When the disease is at or below the base of the broad ligament away from the nterine appendages the location of pain and swelling will correspond to that of the inflammation. In acute cellulitis there will be severe radiating pain, in many cases pain shooting down the thighs, high fever, chills, great local sensitiveness, inability to walk or stand, and painful urination and defecation. Acute symptoms may decrease, and when suppuration occurs reappear, modified by the signs of pus-formation-that is, chills and hectic fever.

The symptoms outlined in the foregoing paragraph always would suggest a tumor in the pelvis composed of products of inflammation, which, if present, may be felt as a hard or boggy mass usually in the lower portion of one of the broad ligaments, crowding the nterns to the opposite side of the pelvis and bulging into the vagina. Not infrequently the uterosacral folds, so-called ligaments, are involved. When these folds are infiltrated, the inflammatory mass will be felt posterior to the uterus crowding the organ to the anterior part of the pelvis. Later, when resolution has taken place, contraction of the ligaments may result in retroversion or anteflexion. Post-uterine cellulitis is examined best by rectal touch. If the inflammation has progressed to the third stage - that is, to the formation of an abscess - the inflammatory product, wherever situated, usually will give on digital examination the sensation of a boggy, fluctuating mass. 
The diagnosis of the effusion stage of pelvic cellulitis is determined by the presence in the pelvic connective tissue of an inflammatory infiltrate situated at some point adjacent to the uterus or to the upper part of the vagina. This infiltrate may be observed with reference to the following characteristics :

1. Location.

2. Form.

3. Relations.

4. Immobility.

5. Consistence.

6. Pain.

1. Location.-The infiltrate may be situated,

a. On one or both sides of the uterus; if milateral, it will crowd the uterus in the opposite direction and depress the lateral fornix of the vagina on the affected side.

Figure 118.

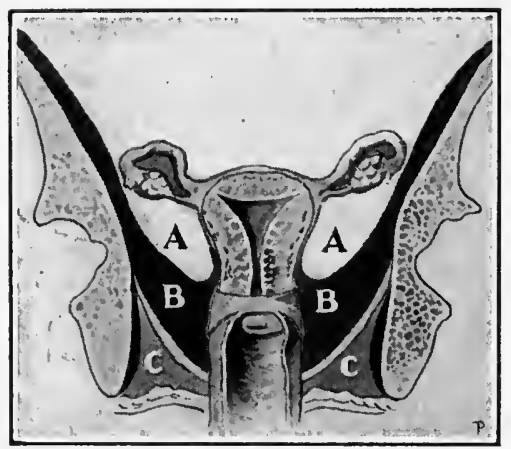

Figure 119.

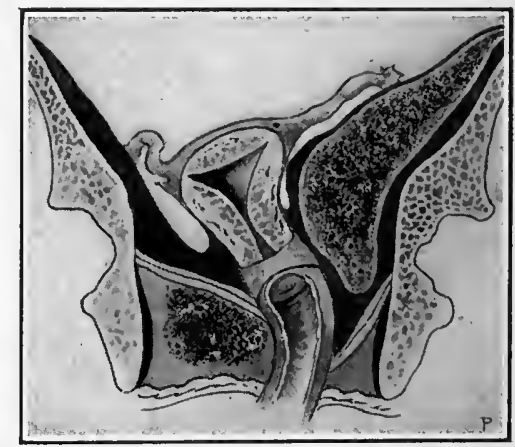

Figure 118.-Fehling's three divisions of the pelvic cavity: A, A, peritoneal division: $B, B$, subperitoneal division: $C, C$, subcntaneous division. The levator ani muscle divides the subperitoneal from the subcutaneous division.

FrgURE 119.-Parametritis. Exudate in left subperitoneal cavity, crowding corpus uteri to right. Paracolpitis in right subcutaneous cavity, erowding eervix uteri and vagina to left. This latter would produce a peri-anal abscess, and usually would be followed by fistula in ano.

b. Between the folds of the broad ligaments high in the pelvis, with a tendency to extend around the rectum.

c. In the post-cervical connective tissue, blocking up the culde-sac of Douglas and depressing the posterior fornix of

- the vagina-parametritis posterior.

d. In the comnective tissue between the cervix uteri and bladder - parametritis anterior; very rare.

$e$. In all the comnective tissue around the uterus-circumuterine cellulitis.

$f$. In the subcutaneous region, as shown in Fig. 119, crowding the cervix uteri and vagina to the opposite side and having a tendency to produce a peri-anal abscess with resultant fistula in ano.

2. Form.-The exudate, sharply circumseribed or diffuse, will take the shape of the resisting structures by which it is limited, and 
therefore will vary in form from a round to oblong, flat, or irregular mass.

3. Relations.-The exudate may surround or blend with neighboring parts, such as the rectum, cervix uteri, vaginal fornix, and bladler.

4. Immobility.-The cxudate usually is fixed, the degree of fixation increasing with the progress of the disease.

5. Consistence.-The exudate, according to the location and resistance of surrounding structures and to the composition of it, may be soft and elastic, or hard and less elastic; it may contain pus or serum, and therefore may give rise to fluctuation.

6. Pain.-In most cases tenderness and pain are present.

\section{Differential Diagnosis of Pelvic Cellulitis. ${ }^{1}$}

As set forth in the accompanying table, pelvic cellulitis has many characteristics in common with the following diseases:

Pelvic peritonitis,

Pyosalpinx,

Pelvic hæmatocele,

Perityphlitic abscess, appendicitis,

Psoas abscess,

Subserous myoma.

The frequent association of pelvic cellulitis with pelvic peritonitis and with salpingitis may render the differentiation most difficult. It is especially difficult when cellulitis and peritonitis coexist in puerperal cases. Early efficient examination in most cases of cellulitis is so painful as to be impracticable without anæsthesia.

Petvic peritonitis.

1. Uterus usually surrounded and fixed by an infiltrate.

2. Blocking up of the vaginal fornix all around uterus.

3. Mass rather high in pelvis.

4. Pain severe and paroxysmal in acute stage.

5. Tendency to suppuration not marked.

6. Cervix usually fixed in median line with corpus in anteversion or anteflexion.

7 . Frequently results in general peritonitis.

8. Anxious facial expression.

9. Both legs flexed on abdomen.

10. Nausea and vomiting frequent.

11. Exudate may extend to upper zones of pelvis.

12. Pulse rapid and weak.

13. Tongue dry.

Retro-uterine peritonitis.

1. Mass presents round, sharp outline, and may involve whole posterior surface of uterus. 2. Uterus forced forward.
.

3. Rectum pressed backward in median line.

4. Adhesions later binding posterior surface of uterus and fundus nteri to rectum.

\section{Pyosalpinx.}

1. Situated to one or both sides of corpus uteri, or bound behind uterus in cul-de sac of Douglas.

2. Sharply outlined, sausage-shaped.

3. Limited mobility of mass

4. May fluctuate or be very hard and resisting.

5. Csually bilateral.
Pelvic cellulitis.

1. Tumor usually at side of uterus.

2. Bulging of one or both lateral fornices, seldom surround uterus.

3. Mass lower in pelvis and easily palpated.

4. Pain less severe and more continuous.

5. Great tendency to suppuration.

6 . Uterus usually displaced laterally.

7. Not frequently so complicated.

8. Facial expression not characteristic.

9 . One leg flexed, seldom both.

10. Less frequent.

11. More generally confined to lower zones.

12. Not so rapid; not weak.

13. Tongue may be moist.

Retro-uterine cellutits.

1. Mass flat, diffuse, and usually limited by retro-uterine fold of peritoneum.

2. Cervix uteri forced forward.

3. Pressed to one side or backward.

4. Adhesions lower and nearer cervix uteri

\section{Lateral cellutitis.}

1. Situated low and blocking raginal fornices.

2. Usually not sharply outlined, but fiat and diffuse.

3. Isually fixation of mass.

4. Often hard and resisting before suppuration and fluctuation.

5. Usually unilateral.

- These tabular statements of differential diagnosis have been adapted from numerous works on gynecology. 
Pelvic hrematocele.

1. History of tubal pregnancy with sudden and alarming signs of iuterual hemorrhage.

2. No ehill, fever slight or absent. May be subnornal temperature.

3. Rapid development of tumor.

4. Tumor soft and doughy or fluctuating; later hard and may be elastlc.

$\overline{5}$. Usually circumscribed mass.

6. Exploratory puncture-blood.

\section{Perityphlitic absccss, appendicitis.}

1. Onset-coustipation, pain, fever, nausca, vomiting.

2. Tenderness at McBurney's point.

3. Exudate high-surrounds cæeum; not felt through vagina.

\section{Psoas abscess.}

1. U'sually history and symptoms of tuberculosis.

2. Spondylitis.

3. No history of acute inflammation.

4. Exploratory puncture-typical tubercular pus.

\section{Subserous myoma.}

1. Slow development.

2. No bistory of infection.

3. Contour of tumor-usually round, sharply circumseribed; tumor intimately connected with the uterus.
Pelvic cellulitis.

1. History of infection.

2. Chill with slight or high temperature.

3. Slower development.

4. Tumor usualy hard until suppuration.

5. Usually diffuse mass.

6. Exploratory puncture-negative, serum, or pus.

Pelvic cellulitis-right side.

1. Onset-pain, fever, little or no nausea or vomiting.

2. Not present. uterine connective tissue, easily felt through vagina.

Pelvic cellulitis.

1. Abscnt-history of non-tubular infection.

2. Absent.

3. Usually acute at first.

4. Ordinary pus or serum.

Pelvic cellulitis.

1. Development more rapid.

2. History of infection.

3. More diffuse, not so intimately connected with the uterus.

\section{Prognosis of Pelvic Cellulitis.}

The prognosis in the acute form, uncomplicated by tubal disease, is good usually. The inflammation may terminate in speedy resolution. If abseesses form, there may be rapid and complete recovery after evacuation of the pus. When pus-tubes coexist, the removal of them may be necessary. The chronic atrophic cellulitis of Freund is obstinate for symptomatic and hopeless for histological cure.

\section{Treatment of Pelvic Cellulitis.}

Treatment of Acute Cellulitis.-The prophylactic, palliative, abortive, and surgical treatment is the same as that of Acute Metritis, Chapter XIV. When the disease is secondary to salpingitis, the treatment should be directed to the uterine appendages. If the source of the acute infection has been a wound made in a surgical operation or in parturition, the exposed surfaces should be cauterized thoronghly. For this purpose, it may be necessary to remove the sutures from a recently repaired cervix or perineum. Should the source of infection be an infected endometrium, sharp curettage of the endometrium may be considered. See Chapter XIV.

If an abseess forms, it should be opened and drained; the technique of the operation is the same as that described in the following paragraph for opening and draining a pelvic abscess.

Surgical Treatment of Chronic Cellulitis.-If suppuration has taken place, the abscess should be opened promptly and drained. In most cases the opening slould be through the vagina; but if the pus points toward the abdominal wall, it nay sometimes be reached and drained by an abdominal incision direct into the abscess without 
opening the general peritoneum. Under no circumstance should the incision be made through the rectum. Persistence of suppuration after drainage indicates probable tubal disease and may require removal of the uterine appendages. In this connection the reader is referred to the treatment of pelvic suppuration by incision and drainage-Chapter XXIII.

Non-surgical Treatment of Chronic Cellulitis.-When the acute symptoms subside, absorption may be promoted by the hot water vaginal donche as described in Chapter IV.; by the internal use of calomel in doses of $\frac{1}{20}$ to $\frac{1}{10}$ grain three times a day ; by saline laxatives, sitz baths, and hot fonentations.

The treatment of chronic non-suppurative cellulitis-that is, the atrophic variety of Freund-is discouraging. The estimated value of sea-bathing, electricity, glycerin and tannin tamponade, vaginal and rectal donches, and painting with iodine, varies widely with different physicians. The author has not found such measures of great value. 


\section{CHA PTER XXI.}

\section{INFLAMMATION OF 'THE UTERINE APPENDAGES-SAL- PINGI'TIS, OVARITIS, PELVIC PERITONITIS.}

\section{SALPINGITIS.}

Salpixgitis is inflammation of the Fallopian tube.

\section{Normal Anatomy.}

The Fallopian tubes are developed by that part of Müller's ducts above the round ligaments. The part below the round ligaments, togrether with the Wolffian ducts, converges to form the uterus and vagina. The tubes therefore are directly continuous with the uterus. Chapter XXVII.

'The mucous, muscular, and peritoneal layers of the uterus are directly continuous into and form the tubes. By analogy of uterine nomenclature these three layers of the tube are named from within outward, as follows :

1. The endosalpinx.

2. The myosalpinx.

3. The perisalpinx.

The tubes extend from the horns of the uterus outward on either side and follow a bending course along the upper border of the broad ligament to a variable length of from three to five inches. They are divided into three parts :

The isthmus.

The ampulla.

The fimbriated extremity.

The Isthmus-i. e., the constricted portion of the tube-starts from the endometrium at the horn of the uterus, runs through the uterine wall, and continues outward toward the lateral wall of the pelvis about one inch. The calibre of the isthmus at the uterine junction, ostium uterinum, is so small as scarcely to admit a bristle. This constricted portion, unless dilated by disease, would prevent an intrauterine injection or secretion from entering the abdominal cavity. It also serves to protect the tube against infection from the uterus and the uterus against infection from the tube.

The Ampulla is the expanded portion of the tube, and easily admits the nterine probe. It runs from the istlimus backward and downward around the outer border of the ovary, and terminates in an expanded, trumpet-shaped part called the infundibulum.

The Fimbriated Extremity at the abdominal opening is really the termination of the ampulla. It is made up of irregularly shaped 


\section{PLATE VII}

\section{FIGURE 1}

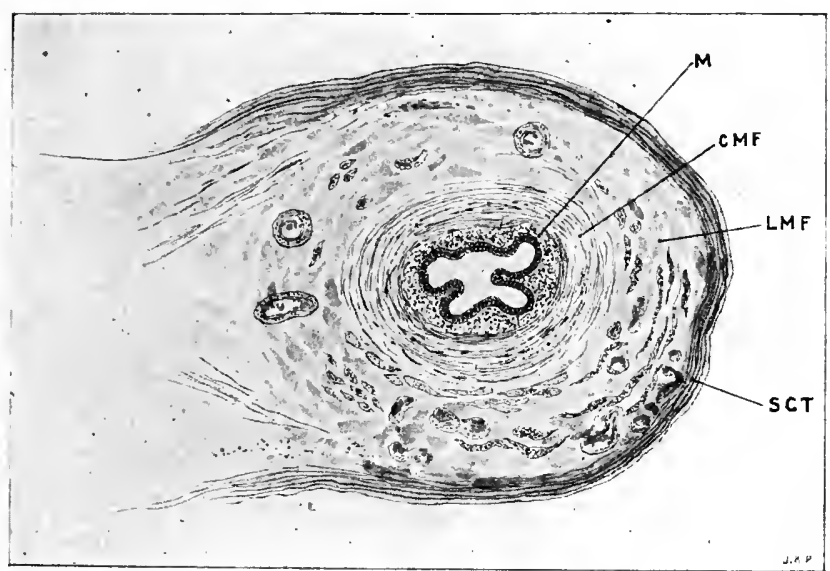

\section{Cross-section of the Normal Fallopian Tube at the Uterine}

Ostium.

II, mucosa. CMF, circular muscle fibres. L.MF, longitudinal muscle filires. SCT, subperitoneal connective tissue. Io diameters.

\section{FIGURE 2}

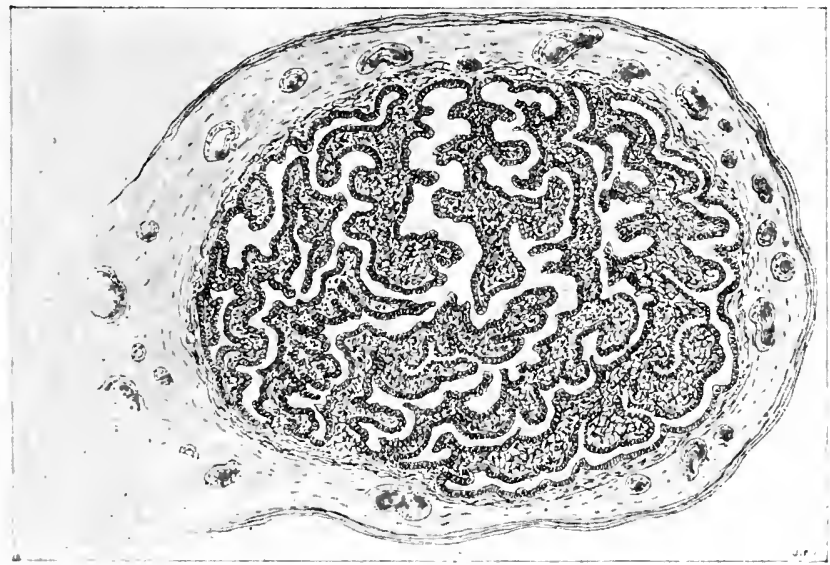

Cross-section of Fallopian Tube through Abdominal Ostium.

Observe the high mucous folds of the endosalpinx.

The walls of the tube in both figures contain numerous hoodvessels shown in red. ro diameters. 

processes, all freely movable except one, which runs along the tuboovarian ligament and joins the ovary. These fimbriæ are branches from the high mucous folds of the endosalpinx.

The abdominal openings of the tubes are sometimes multiple, with more than one fimbriated extremity for a single tube.

The Endosalpinx, or mucous lining, continuous with that of the uterus, is made of loose connective tissue covered with a single layer of ciliated columnar epithelium. The cilia always are directed toward the uterus, and probably serve to propel the ovum in that direction. The mucosa in the isthmus is relatively smooth; in the ampulla it rises in numerous high folds. This is shown abundantly in cross-section by the accompanying plate. The presence of glands in the Fallopian tube has been denied. Bland Sutton, after an exteusive comparative study of the tubes of the lower animals and of woman, declares that the plications or folds of the tubal mucous membrane "are disposed on the same principle as the glands in the uterus."

The probable function of the tubal folds is to provide an albuminous fluid for the ovum as it traverses the tube. The tube participates only in slight degree if at all in menstruation. As shown in ectopic gestation it retains somc power to develop the fertilized ovum.

The Myosalpinx is made of two muscular layers, internal circular and external longitudinal. These layers are continuous with the corresponding layers in the uterus. It is not known whether or not the tube has peristaltic power.

The Perisalpinx, or peritoneal investment of the tube, meets the mucous lining at the abdominal opening. It covers about four-fifths of the circumference of the tube, and, converging toward the broad ligament, forms a narrow mesosalpinx. Between the layers of the mesosalpinx is an abundance of loose connective tissue through which the lymph-vessels and blood-vessels and nerves directly reach the tube.

\section{Classification of Salpingitis.}

The following varicties and phases of salpingitis are distinguished :

1. Catarrhal salpingitis-salpingitis serosa.

2. Purulent salpingitis—salpingitis purulenta.

Catarrhal salpingitis may result in :

Sactosalpinx serosa-liydrosalpinx.

Purulent salpingitis may result in :

Sactosalpinx purulenta-pyosalpinx.

If sactosalpinx is complicated by hemorrhage into the tube, it is called sactosalpinx hæmorrhagica or hæmatosalpinx; this is more common in serous than in purulent infections. Tubercular salpingitis, an especially important variety of purulent infection, will be described separately.

\section{Etiology of Salpingitis.}

The causes may be classified into :

I., predisposing causes-favoring conditions.

II., exciting causes. 
I. The predisposing causes are :

1. Abortion, labor, instrumentation, and manipulations.

2. Infections in neighboring organs which may reach the tubes by extension. Tubal disease is rarely primary.

3. Menstrual congestion, injudicions exereise at the beginning of menstruation, taking cold during menstruation, excessive coitus.

4. Acute exanthemata.

5. Long tortuous tube.

6. Neoplasms.

II. The exciting causes are bacterial, and are the same as those set forth in Chapter XIX., on Pelvic Inflammation in General.

Bacterial studies of salpingitis as tabulated by Gebhard, from the elinics of eight surgeons, gave the following results:

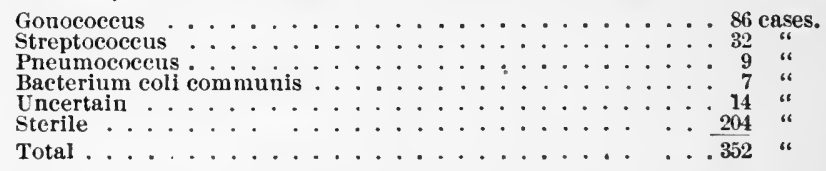

Visceral disorders as of the heart, lungs, liver, and kidneys; and systemic diseases as syphilis, rheumatism, and gout, are associated frequently with salpingitis.

\section{Pathology of Salpingitis.}

The pathology of catarrhal and purulent salpingitis presents many points in common. To some extent, therefore, one description will answer for both.

No sharp clinical lines of demarcation can be drawn between the inflammations of the different layers of the tube.

The infection usually passes to the tube from the endometrium, catarrhal endometritis giving rise to eatarrhal, salpingitis, and purulent endometritis to purulent salpingitis. Other possible routes of infection have been described in Chapters $X$. and XIX. Tubereulous salpingitis, for example, usually reaches the tube from above.

Endosalpingitis, whether catarrhal or suppurative, may extend beyond the tube in three different ways as follows:

1. If the abdominal end of the tube remains open, the secretion may flow out and infect the adjacent peritoneum and the epithelial covering of the ovary, producing periovaritis, or if there be at the time a freshly ruptured Graafian follicle, the infection may enter the ovary and produce ovaritis. Adhesions may form between the tube and whatever peritoneal surface is in entact with it. The ovary also may be in the grasp of the fimbrix, and so glued to the ampulla as completely to close the tube.

2. The infection may pass through the walls of the tube by way of the lymph-channels and produce perisalpingitis-i. e., inflammation of the peritoneal covering of the tube; thus local peritonitis may spread to the pelvic or even to the general peritoneum. All three layers of 
the tube, mucous, muscular, and serous, together with all the connective tissue of the tube, become greatly thickened, hard, and convoluted, and are now involved in a diffuse salpingitis. Adhesions usually form between the serous covering and adjacent organs. The sccretions may be clear or clouded, catarrhal or purulent, and, unless the abdominal opening has been closed by swelling or by adhesions, may be forced into the peritoneum.

3. The infection may pass through the mesosalpinx into the loose connective tissue between the folds of the broad ligament, producing perilymphangitis and periphlebitis-i. e., the cellular tissue around the lymph-channels and veins may become inflamed. This inflammation is pelvic cellulitis. Chapter XX.

The second and third modes of extension are more likely to occur if the tube has become distended by pathological secretions, a common result of plastic occlusion of the abdominal end or of mechanical closure from swelling of the uterine end. Occlusion from swelling does not continue if recovery takes place; that from adhesive inflammation is usually permanent.

The acute and chronic salpingitis shade off one to the other, so that the difference between them is one rather of degree than of kind. Chapter X.

The germs which produce the disease are demonstrable in acute salpingitis. Chronic accumulations of pus in the tubes are usually sterile-i. e., the micro-organisms have disappeared and the pus is no longer infectious. It is said that the bacteria die from the accumulation of their own products. The escape of such sterile pus into the pelvic eavity from ruptured tubes during operation, or from any other cause, is not so dangerous as it was supposed to be when pus was considered always infectious.

Sactosalpinx. - When both ends of the tube are closed either by swelling or by adhesive inflammation, and the walls become distended with the aceumulated secretions, the disease, as indicated under Classification, is called sactosalpinx. Three varieties are distinguished, as follows :

Sactosalpinx serosa-hydrosalpinx.

Sactosalpinx purulenta-pyosalpinx.

Sactosalpinx hæmorrhagica-hæmatosalpinx.

The serous accumulation of catarrhal salpingitis is known as sactosalpinx serosa, or hydrosalpinx. A purulent accumulation is sactosalpinx purulenta, or pyosalpinx. An accumulation of blood in the tube is hæmatosalpinx, or sactosalpinx hæmorrhagica.

Extensive and firm adhesions usually take place between pus-tubes and the adjacent organs, especially the ovaries. Both the tube and ovary then may be rolled together, universally adherent in the posterior fold of the broad ligament. This condition is more common in the suppurative than in the catarrhal variety. Tubo-ovarian abscess or purulent tubo-ovarian cyst may form in consequence of such adhesions.

The odor of the pus is often very offensive, and if the tube be adherent to the rectum may be fecal. 
272 INFECTIONS, INFLAMMATIONS, AND ALLIED DISORDERS.

Figure 120.

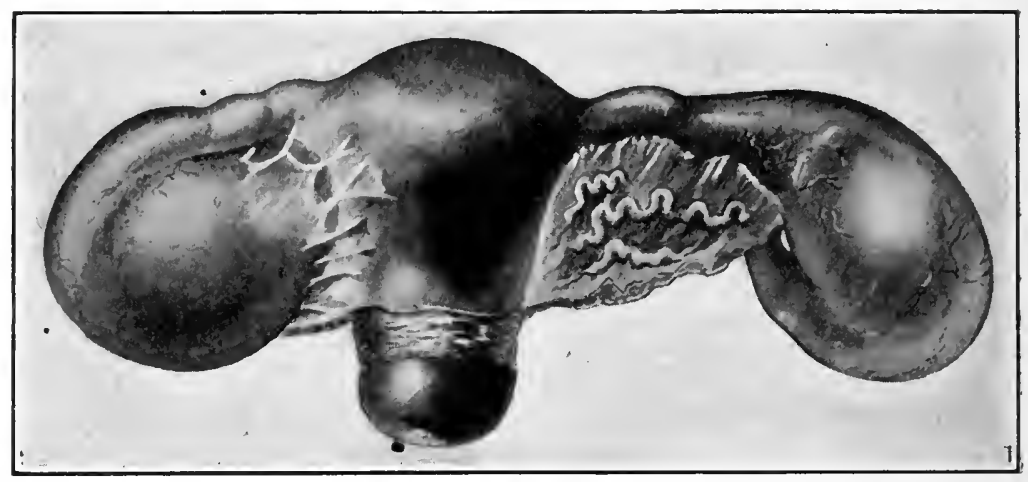

Hydrosalpinx.

Figure 121.

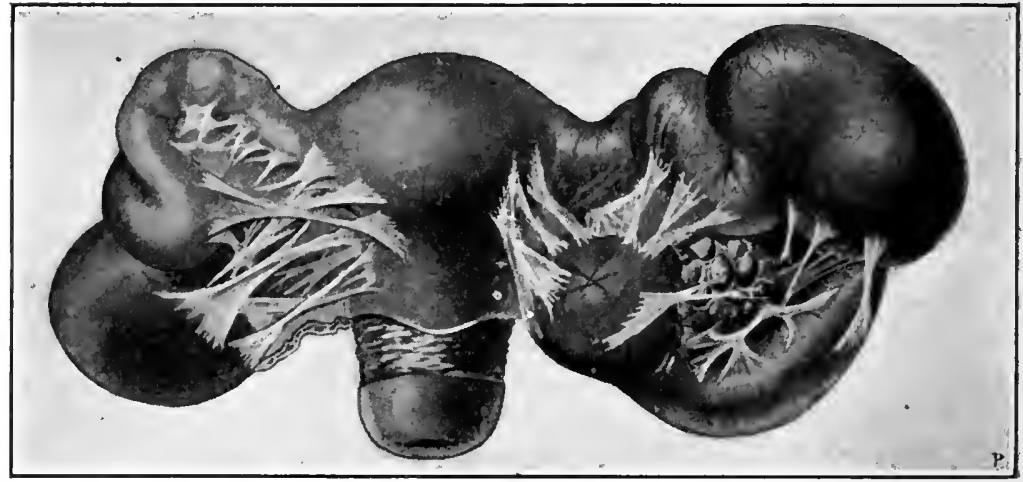

Pyosalpinx.

Figure 122.

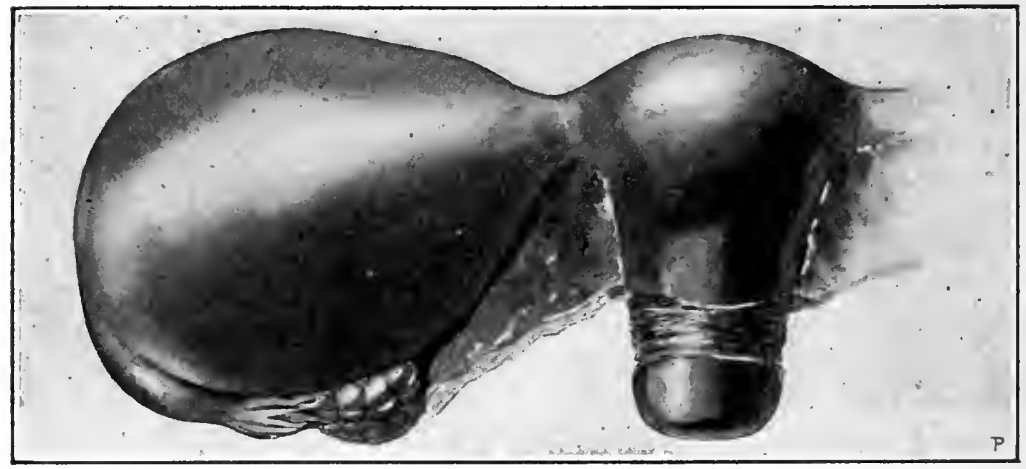

Hæmatosalpinx. 


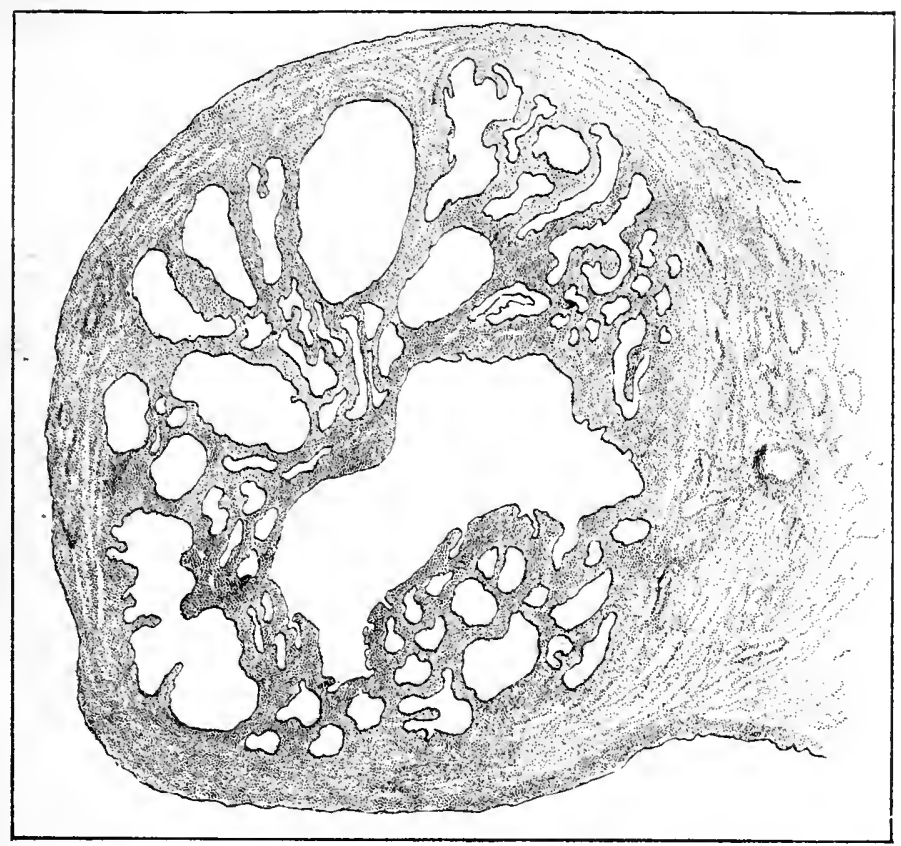

Follicular Hydrosalpinx (Catarrhal Salpingitis). Cross-section Near the Middle of the Tube.

The folds of the swollen mucosa have grown together so that the deep parts of the inflamed mucosa have been shed off partly or wholly from the lumen and have formed small crsts, a frequent result of catarrhal salpingitis. The epithelium here remains, but in the latter stage of the disease it may be destroyed. I5 diameters.

FIGURE 2

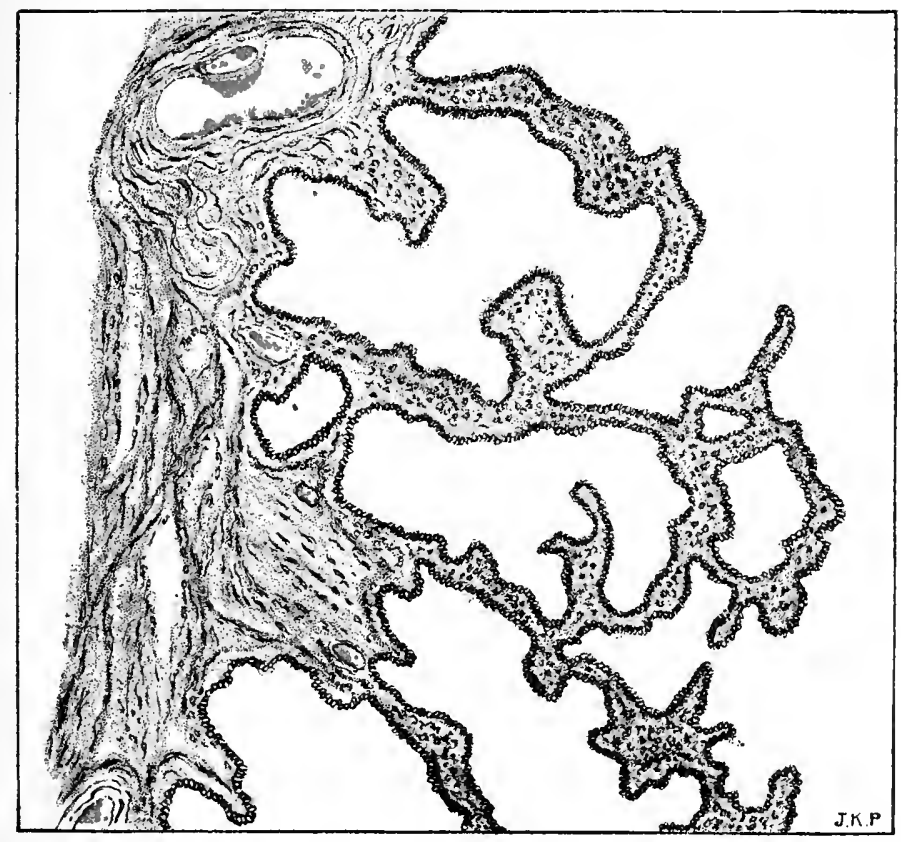

Same as Figure 1. Highly magnified. Here also are shown a number of variously shaped cavities. The high mucous folds are swollen and infiltrated with the round cells of inflammation. roo diameters. 

Pus-saes often burst into adherent organs-rectum, bladder, or intestine. Unlike eellulitis abscess, pyosalpinx does not often burst spontaneously into the vagina. The relief which comes from the rupture of a pus-tube into an adjacent organ is apt to be temporary, for the pus usually reaccumulates. The escape of fluid from a hydrosalpinx or pyosalpinx into the uterus is called salpingitis profluens.

Hydrosalpinx in one tube and pyosalpinx in the other are not uncommon. Separate compartments, formed by occlusion of a tube at different points, may result in distention of these compartments with different fluids; hence there may be in the same tube hydrosalpinx, pyosalpinx, and hæmatosalpinx.

The distended tube often has the form of a pear, the narrow part toward the isthmus corresponding to the stem and the distended ampulla to the wide part of the pear. In hydrosalpinx the fluid is clear, and in the absence of adhesions to the broad ligament the sae often is felt freely movable in the pouch of Douglas.

Hæmatosalpinx may oceur as the result of hemorrhagic salpingitis. The sac-walls, especially if the henorrhage be non-inflammatory, as in tubal pregnancy, are very thin and easily ruptured. The blood may not or may be mixed with tubal secretions, and if rupture does not occur, may be absorbed. Adnixture with blood may take place in all forms of inflammation, but an inflamed tube must contain blood in considerable quantities in order to be designated by the term hemorrhagic salpingitis or homatosalpinx. The latter, however, may occur without inflammation. Usually the blood of hæmatosalpinx due to tubal pregnancy is clotted; that due to salpingitis is often quite thick, and may resemble tar, but is not nsually clotted.

Tubo-ovarian Cyst and Tubo-ovarian Abscess may form as follows: The adhesion of a sactosalpinx to an ovary may be followed by the bursting of a small ovarian cyst or a corpus luteum into the tube and the establishment of a permanent communication between the two. During the growth of the tubal sac, which is now part of a tuboovarian cyst, the ovarian cyst is subject to the same pressure as the walls of the tube; hence the ovarian structure becomes flattened so as to form a thin wall for the ovarian portion of the composite cyst, and thus the characteristic structure of the ovary is lost. This is not to be confounded with ovarian hydrocelc. 'Tubo-ovarian eyst may occur in connection with hydrosalpinx or pyosalpinx. If the sactosalpinx communicates with an ovarian abscess, the condition is called tubo-ovarian abscess. Figure 203. gitis.

\section{Comparative Pathology of Catarrhal and Purulent Salpin-}

\section{Catarrhal salpingitis.}

1. Infection by extension from catarrhal endometritis.

2. Essentially confined to mucosa-endosalpingitis. Walls of tube in acute stage slightly thiekened; in chronic stage, more thickened. Complieating peritonitis not common.

3. After acute stage, little or no pain or tenderness.

\section{Purulent salpingitis.}

1. Infection usually by extension from purulent endometritis; may be by extension from the peritoneum, as in tubercular salpingitis.

2. Apt to extend and become diffuse, involving all layers of tube. Walls of tube very mueh thickened and infiltrated with round cells. Peritonitis common as a complication. 3. Pain and tenderness pronounced. 
Catarrhal salpingitis.

4. Tubal adhesions absent or less pronounced. Mobility and elasticity. Fluctuation through thin walls.

5. Sactosalpinx will be hydrosalpinx-i. $e$., tube distended with serum; sometimes hamatosalpinx.

6. Abdominal end of tube usually closed by adhesions of timbrix; uterine end of tube less often closed.

7. Sactosalpinx may be due to oeclusion of tube either from swelling of mucosa or adhesive inflammation: if the former, tube may periodically diseharge eontents into uterus or peritoneum, giving rise to colicky pains-hydrops tubæ profluens, salpingitis profluens.

8. Folds of mucosa pressed together by fluid contents; epithelium disappears by pressure and folds grow together; deeper parts of il)flamed mueosa may be partly or wholly shut off from lumen and form small cysts-salpingitis pseudofollicutaris.

9. Mucous folds may atrophy, become attenuated, and floating in the fluid have a wavy appearance deseribed by Sawinoff under the name satpingitis vegetans. Lumen composed of one cavity.

10. Tube thinned and translucent in proportion to distention: walls atrophy: fimbrix gradually lost. Tube moderately convoluted. Size not usually larger than the finger; shape regular. spindle, round, elongated, or convoluted.

11. Tube rarely ruptures into bowel, vagina, bladder, or peritoneum.

\section{Purulent salpingitis.}

4. Adhesions usually extensive to cul-de-sac of Douglas, walls of uterus, broad ligaments, or posterior segment of pelvic floor: tube immobile and inelastic. Fluctuation masked by thickness of tubal walls.

5. Saetosalpinx will be pyosalpin $x-i . e .$, tube distended with pus; pus niay contain blood.

6. Both ends of tube closed by adhesive inflanmution.

7. Tube usually occluded by permanent inflammatory adhesions; hence, sulpingitis prothems less frequent, althougb tube nay rupture at times and discharge contents. Purulent salpingitis proflueus is more dangerous than serous salpingitis profluens.

8. Folds of mueosa may be adherent or obliterated; lumen may be divided partially or wholly into spaces by constrictions or adhesions; these spaces may be distended by different fluids; hence the possibility of pyosallin $x$, hydrosalpin $x$, and hrematosalpin $x$ in one tube with lumen composed of several cavities. Very mueh thickened walls of tube may contain sinall abscesses usually formed between adherent folds of mucosa.

9. Salpingitis vegetans never occurs.

10. Tube more and more thickened (irregular thickening) in proportion to distention, much convoluted and nodular; size may equal that of a child's head; shape most irregular.

11. Such rupture and discharge not common.

\section{Symptoms of Salpingitis.}

There are no pathognomonie signs of salpingitis. Ovaritis and pelvic peritonitis usually are related so elosely to salpingitis that the symptoms and diagnosis of ovarian and peritoneal infections are included largely in those of salpingitis. Inflammation of the tubes and ovaries, taken as a whole, is designated sometimes as adnexal inflammation, or inflammation of the uterine appendages.

Pain.-The symptoms of inflammation of the nterine appendages vary with the extent, virulence, acuteness, complications, and mechanical eonditions of the disease. Usually the tubes are less sensitive to pain than the ovaries; the pain of salpingitis is increased therefore when the disease ineludes peritonitis' and ovaritis. The milder catarrhal inflammations, even though acute, may cause symptoms so slight as searcely to fix the patient's attention upon the diseased part; they may even run their course and disappear, leaving no trace exeept perhaps greater liability to future infection. Such unrecognized mild congestive and catarrhal attacks of salpingitis are probably more frequent than generally is supposed.

Local pain or discomfort in the affected part does not always eorrespont to the seriousness of the infeetion. There may be only a dull aching or a sensation of burning not sufficient to impress the patient seriously unless aggravated by local pressure, by vaginal examination, by exertion, or by defecation, and yet the tube may be distended ready to burst into the peritoneal cavity. 


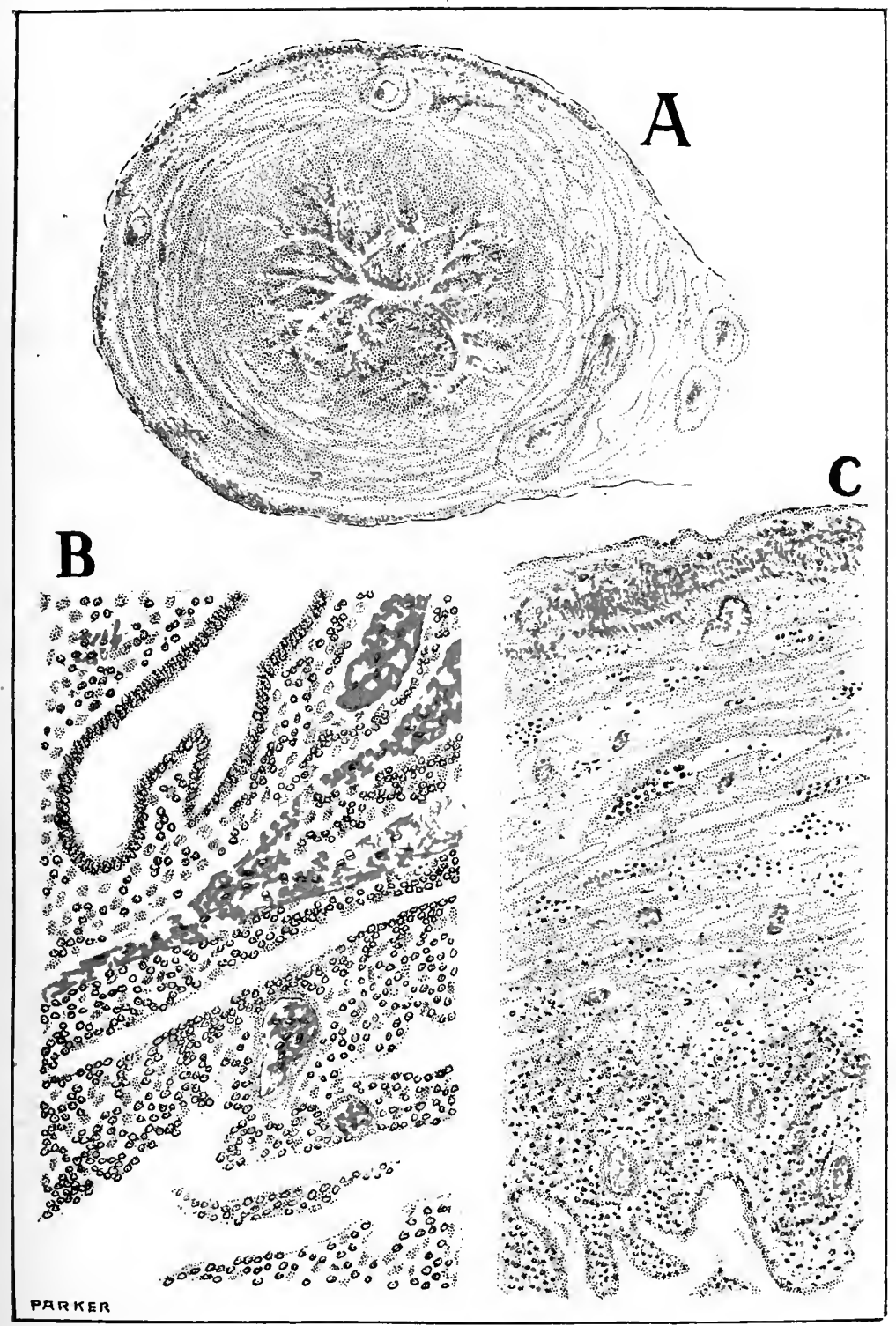

A. Diffuse Hemorrhagic Salpingitis. Section between the middle and outer third of the tube. The high mucous folds and the walls of the tube are much swollen and congested.

B. Section from the wall of the tube $A$.

C. Section from the mucous folds of the tube A. Sections A and B both show congested thickening, hemorrhagic areas and infiltration with many small round cells of inflammation.

A magnified ro diameters. B magnified soo diameters. C magnified 30 diameters. 

In a small minority of eases salpingitis is characterized from the beginning of the attack by very acute colicky pains in the region of the tubes, with intervals of comparative comfort. This synptom, from its frequency in prostitutes, has been called colica scortorum; it has been attributed to salpingitis profluens, to spasmodic contractions of the muscular wall of the tube or uterus, to peritoneal irritation, to leakage of tubal secretion, and to occasional rupture of the walls of the tube, but these explanations are unsatisfactory.

Menstrual Disturbances.-During the monthly period the pathological congestion is supplemented by that of menstruation; hence the pains are increased and dysmenorrhea is the rule. Increased menstrual flow, even to the extent of menorrhagia, is common. Amenorrhoa or scanty menstruation seldom is observed, and when present points to possible tuberculosis.

Mechanical Disturbances.-Greatly dilated and swollen tubes, especially when associated with local peritonitis, always produce meehanical disturbances. This is more marked when the swelling has been rapid. The more gradual the swelling and the more opportunity the parts have to adapt themselves to the new conditions, the less the pain. The mechanical symptoms are variable and numerous. They include painful urination and difficulty and pain on walking and standing.

\section{gitis.- \\ Comparative Symptoms of Catarrhal and Purulent Salpin-}

\section{Catarrhal salpingitis.}

1. Fever present in acute stage and usually absent in chronic stage.

2. Pain in region of tube variable in acute stage; usually absent or almost absent in chronic stage.

3. Salpingitis profuens not uncommon.

\section{Purulent salpingitis.}

1. Fever high in acute stage. Usually slight evening temperature in chronic stage. If pus becomes sterile, temperature may be normal

2. Pain and systemie disturbance (anxious facies, nausea, depression) more pronounced in acute stage. Pain and general malnutrition usually present in chronle stage. Symptoms partly due to extension of infection to neighboring organs, producing ovaritis, pelvic peritonitis, and cellulitis.

2. Salpingitis profluens uncommon.

\section{Diagnosis of Salpingitis-Sactosalpinx.}

The symptoms outlined in the foregoing paragraphs point to the probability of inflammation around the uterus. Indeed, it is usually easy to recognize the presence of acute pelvic inflammation, especially when inflamed organs are crowded with products of inflammation. Physical examination, however necessary to verify the diagnosis, will frequently not only fail to establish sharp diagnostic lines between inflamimation of the different pelvic organs, but also between pelvic inflammation and other morbid conditions, such as neoplasms, with which an inflammatory mass may be confused. The subjective symptoms in the milder cases may be overlooked. Indeed, the existence even of pyosalpinx sometimes is unrecognized until rupture of the tube and escape of pus have set up dangerous peritonitis. The presence of endometritis even should place one on guard against possible salpingitis. 
In order to avoid the accidental rupture of a pus-tube, or other abscess-wall, care in the palpation of uterine appendages is enjoined.

There is usually a recent or remote antecedent background of acute or chronic infection in some neighboring organ ; the diagnosis therefore should include both the inflamed appendages and the antecedent causative inflammation, usually endometritis, but sometimes vaginitis, vulvitis, eystitis, proctitis, or appendicitis.

Among the subjective symptoms will be dull, often burning, constant, remittent, or intermittent pain and local tenderness. The colicky pains about the tubes, already mentioned under symptoms, are strongly diagnostic. Occasional exacerbations of local peritonitis from leakage of the tube or from other sources are characteristic of adnexal inflammation. In order to establish the diagnosis the symptoms already outlined must be supplemented by physical examination.

Physical Examination is by external palpation and conjoined manipulation. The former is usually inadequate. The latter, which includes external palpation, is made with the left index-finger in the vagina and the right hand over the hypogastrium; or, as set forth in Chapter III., with the left index-finger in the rectum, the thumb in the vagina, and the right hand over the hypogastrium. Light, conjoined palpation will show an irregular elongated swelling on one or both sides of the uterus, frequently extending into the pouch of Douglas, or even sometimes in front of the nterus. It is often impossible to make out the component parts of such a mass. They will, however, usually include the inflamed tube or tubes together, in varying degree, with diseased ovaries, peritoneum, intestine, omentum, bladder, and uterus. These structures may be matted together in an irregular, indefinite tumor. The one nearly constant factor in such a mass is sactosalpinx.

The Diagnosis of Sactosalpinx depends upon the finding of a tumor connected with the uterus, sausage-shaped or retort-shaped, and tender on pressure. Gonorrhœal, puerperal, and tubercular are the three most frequent and significant forms of infection; it is therefore important to distinguish between them.

Gonorrhoal sactosalpinx will be recognized by:

a. History of suspicious exposure.

b. Presence of gonorrhœal infection in uterus, vagina, or vulva.

c. Bilateral infection; not invariable.

Tubercular Sdlpingitis.-The disease is generally secondary to tuberculosis in other organs, and is of frequent occurrence. Whitridge Williams found it in 7.7 per cent. of 91 cases of removal of the uterine appendages. It very rarely occurs from direct infection by coitus or other media. It has been observed as early as the fifth year of life, and is the most frequent form of salpingitis found in virgins. It usually attacks both tubes and extends to the surrounding parts. Tubercular pelvic disease is characterized by mild pyrexia, weakness, often splenic enlargement, and thickening of the subperitoneal tissues. The tendency of the disease is toward atresia of the tube and the formation of pyosalpinx.

Tubercular salpingitis may be acute or chronic. The abdominal 
end of the tube is in general open in the acute and closed in the - chronic cases; the contents of the closed tube are serous, purulent, or caseous. The mucosa in acute cases may contain many small tubercular nodules. In these nodules are found few giant cells and many tubercle bacilli. Chronic tubercular sactosalpinx is often a large sac containing fluid or caseous pus. The mucosa is destroyed, and the sac is lined with granular tissue which contains numcrous giant and epithelioid cells. The tubercle bacillus in this tissue is often impossible to find. The perisalpinx presents the same microscopical appearances as the mucosa-that is, there are numerous giant cells and few if any tubercle bacilli. Chronic fibroid tuberculosis of the tubes is a peculiar form described by Williams. In this variety the formation of connective tissne is the final stage of the tubercular infection. The contracting fibrous tissue around the tubercular nodules crushes out the miliary tubercles and prevents spread of the disease.

Figure 123.

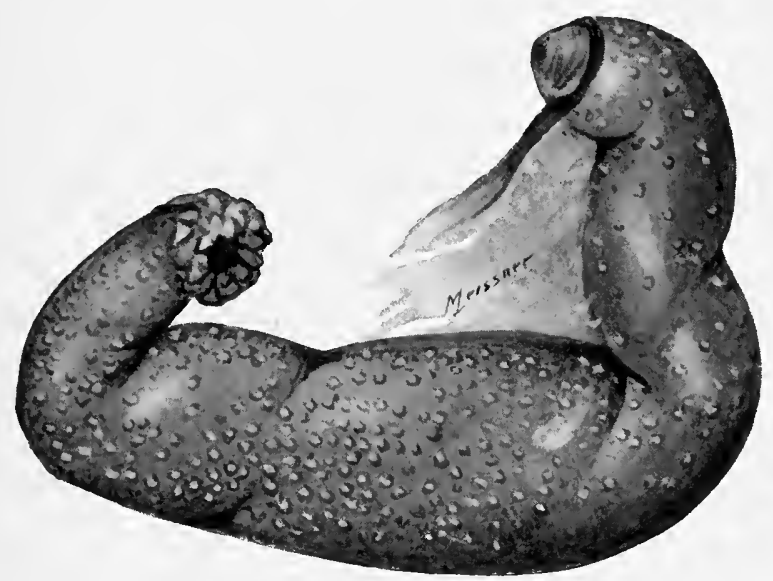

Tubercular Fallopian tube.

Diagnosis of Tubercular from other Forms of Salpingitis.-On aecount of the similarity of symptoms and physical signs the diagnosis may be very difficult. In comparison with the other forms, chronic tubercular infection is more apt to show an evening rise in temperature of about one degree, and a marked increase in the frequency of the pulse. Pain and menstrual disturbances arc not particularly diagnostic. Ascites occurs in a small minority of cases and is diagnostic. The physical signs are substantially the same as in other forms of salpingitis. The chief diagnostic points are :

a. Tuberculosis in other organs with symptoms intensified ; frequent.

b. Tuberculosis in husband.

c. Family history of tuberculosis. 
d. Salpingitis in virgins ; tubercular in 90 per cent. of all cases.

$e$. Presence of tubercle bacilli in leucorrhœea.

$f$. Scanty menstruation or amenorrhœa ; not uncommon.

g. Ascites ; not uncommon.

$h$. Palpation of tubercular nodules sometimes possible.

The Distinction of One Form of Bacterial Infection from Another must depend upon the examination of the secretions for bacteria. Such an examination is always desirable, but sometimes impracticable. The vulvovaginal and uterine secretions in the acute stage not infrequently contain the causative germs. Pus long confined in the tube is apt to become sterile. This explains the freedom from infection so often observed after a pus-tube has ruptured within the peritoneal cavity during an operation. The inflammation may continue long after the original germs have disappeared, or at least after their presence can no longer be demonstrated. The tuberculin test may serve to elear the diagnosis of early primary tubercular salpingitis.

The differentiation of the various adnexal inflammations from one another, especially in the acute stage, is often difficult. Ovaritis, usually a consequence, sometimes a cause of salpingitis, is not easily distinguished from it when the two organs are fused together by adhesions, and when the tube is distended with fluid the difficulty is increased.

Differential Diagnosis of Salpingitis-Sactosalpinx.-Sactosalpinx closely resembles many other conditions, some of them inflammatory and some non-inflammatory ; it usually may be distinguished from inflammatory growth by the location, and from non-inflammatory developments by the history of inflammation.

'The principal affections for which sactosalpinx may be mistaken are set forth in the following parallel columns:

Sactosalpinx.

1. Commonly bilateral.

2 . Tube oblong and tortuous.

3. Commonly adherent.

4. Ovary often palpated and distinguished.

5. Usually not larger than fist.

6. May be leucocytosis.

\section{Sactosalpinx.}

1. Common.

2. Usually bilateral.

3. Sensitive to pressure.

4. Usually fixed.

5 . Elastic or fluctuating.

6 . Result of infection.

\section{Sactosalpinx.}

1. Usually sharply circumscribed and of rounded contour.

2. Commonly bilateral.

3. Elastic and fluctnating. Not a reliable sign.

4. Position relative to uterus: mass usually higher in pelvis near fundus uteri ; not connected with cervix. Vaginal raul: not depressed.
Cystic ovarian tumor

1. Commonly unilateral.

2. Spheroidal or spherical.

3 . Iess commonly adherent.

4. Tumor is diseased ovary.

5 . Nay grow to enormous size.

6. No leucocytosis.

\section{Solid tumor of tube.}

1. Rare.

2. Usually unilateral.

3. Not seisitive.

4. Usually frce and mobile.

5. Firm consistence.

6. C'ause unknown.

\section{Pelvic celiulitis.}

1. Not sharply circumscribed; may be flattened.

2. Commonly unilateral.

3. Less elastic and fluctuating. Not reliable.

4. Position relative to uterus, usually lower in pelvis, often closely connected with uterus. Vaginal vault commonly de. pressed. 


\section{Sactosalpinx.}

1. Mass usually elastic; may fluctuate.

2. Adhesions common.

3 . Sensitive to pressure.

4. Uterine end of tube enlarged.

5. History of infection.

Sactosalpinx (right side).

1. Tumor felt by vaginal touch.

2. After acute stage, size of tumor may not materially diminish.

3. Recurrence less dangerous and less frequent.

4. More frequently of goworrhceal or tubercular origin.
Tubal pregnancy.

1. Consistence often quite firm.

2. Less common.

3. Not sensitive

4. Commonly normal except interstitia tubal pregnancy.

5 . History of preguancy.

a. Amenorrhoea.

$b$. Increase in size of uterus.

$c$. Enlargement of breasts.

$d$. Morning sickness.

e. Rupture of tube with great pain, collapse (pelvic hæmatocele), uterine hemorrhage, and discharge of decidual membrane.

\section{Appendicitis.}

1. Tumor not usually within reach of vaginal touch, but felt or is tender to pressure on external palpation in region of MICBurney's point.

2. After acute stage, tumor apt to disappear.

3. Recurence more dangerous and more frequent,

4. Nore frequently due to colon bacillus, less frequently of tubercular origin; never gonorrhoeal unless by extension from tube.

Among the other conditions which may in some respects resemble sactosalpinx are these :

Displaced uterus,

Tumors of the sacrum and ilium,

Fecal accumulations,

Displaced abdominal organs,

Adherent intestine,

Intestinal tumors.

Study of the diagnosis of lateral and backward versions and flexions of the uterus, in Chapter XLVI., and of Uterine Myomata, in Chapter XXVI., will enable the student to distinguish these conditions from sactosalpinx.

Tumors of the sacrum and ilium are distinguished by their location, hardness, immobility, and intimate relations with the bony pelvis.

Fecal accumulations may be recognized by palpation, and may be removed by cathartics.

Displaced kidney, spleen, and other abdominal viscera, intestinal adhesions and intestinal tumors, may usually be recognized by their remoteness from the uterus, or by the fact that they may on nanipulation be separated from that organ. The author has had a case of tumor of the sigmoid flexure adherent to the cul-de-sac of Douglas, and on conjoined examination having all the appearance of a larger sactosalpinx. Diagnosis was made only after enucleation of the mass from its surroundings.

Ancesthesia is often necessary in order to make a satisfactory diagnosis and differential diagnosis of adnexal inflammation, and should be used in cases of doubt. Relaxation of the abdominal muscles under anæsthesia permits more efficient palpation with the minimum forse, and consequently with the minimum risk. Many unnecessary laparotomies would doubtless be avoided by more careful diagnosis under anæsthesia. The more or less distended bladder or bowel often has been mistaken for a pathological collection of serum, blood, or pus ; hence evacuation of the bladder and rectum is a prerequisite to examination 
Exploratory Incision.-In serious pelvic disease the diagnosis, if not possible or satisfactory by the above means, may be made by exploratory vaginal or abdominal section. The incision may become the first step of a radical operation or, if the operation prove unnecessary, may be closed safely. It is a good rule always to begin a peritoneal operation as a diagnostic exploratory incision. As Mr. Tait wisely remarked, "It is better to turn an exploratory incision into an operation then it is to turn an operation into an exploratory incision." The late Charles T. Parkes, when questioned by a bystander at the beginning of an abdominal section, replied, "I don't know what it is, and I am tired of guessing."

\section{Prognosis of Salpingitis.}

In acute adnexal inflammation the prognosis varies with the nature of the infection and with the extent of the disease. If the tube ruptures and discharges pus into the peritoneum, a fatal peritonitis may follow. If the infection is confined to the tube, the prognosis is usually favorable, but the removal of the appendages may be necessary for permanent recovery.

Simple catarrhal salpingitis and mild ovaritis may run their courses, perchance unrecognized, to recovery. They may even leave no trace behind except an increased liability to further inflammation. The more chronic the disease the less favorable the outlook for expectant treatment. The rule that sactosalpinx, especially the purulent variety, rarely recovers without operative interference, is not without exception, for pus cavities may rupture spontaneously and discharge their contents throngh the bowel, uterus, bladder, vagina, or cutaneons surface, and recovery may follow; but such a possibility does not offer substantial hope of relief. In fact, even when such rupture and discharge are followed by relief the result is usually only temporary, and the patient may suceumb to repeated infection.

Serous sactosalpinx, although little dangerons to life, may, by permanent closure of the tubes, canse loss of function and, if the disease is bilateral, sterility. Purulent sactosalpinx is a constant danger even to life. The gonococcus is less perilons to life, though probably more dangerous to health than the streptococcus. The streptococcus is apt to destroy life, while the gonococcus in a physiological sense destroys the reproductive organs and makes a chronic invalid.

The danger of operation varies with the extent of the disease, the kind of operation, the operator, and the natnre of the causal bacteria. The mortality shown by some statisties is enormons; other reports give almost 100 per cent. of recoveries. The removal of a gonococcus sactosalpinx is less dangerons than that of a streptococcus sactosalpinx. This is especially true if the sac ruptures into the peritoneum. The more chronic the discase, the less the danger of the operation.

One hundred and forty-four cases of removal of sterile pus tubes show a mortality of 2.8 per cent. ${ }^{1}$ The avarage mortality, among the best operators, following removal of pus tubes is from 2 to 3 per cent.

1 Martin. Die Krankheiten der Eileiter. 
Operations in sixteen cases of gonococcus sactosalpinx in which the sac was removed intact show a mortality of 6.2 per cent.; in seventeen cases in which it burst during removal the mortality rises to 11.7 per cent. In another similar series the mortality was 8.35 per cent. and 11.1 per cent., respectively. ${ }^{1}$

It is evident from the above that the prognosis of the operation is affected favorably by the removal of the appendages without rupture and escape of pus into the pelvic cavity. The average mortality, however, with modern asepsis and technique should not excecd 2 per cent., except for acute cases; usually these are treated more safely by vaginal incision and drainage.

\section{Treatment of Salpingitis.}

The treatment will be found in the two following chapters.

\section{OVARITIS.}

The abdominal end of the Fallopian tube is normally close to the ovary and communicates with it by the tubo-ovarian ligament. The utero-ovarian ligament connects the ovary with the uterus. Between the insertions of these two ligaments the ovary is joined to the posterior fold of the broad ligament by a broad base, the hilum, through which pass the lymphatics, blood-vessels, and nerves. Above the hilum the ovary is covered, not by peritoneum, but by germ epithelium, so called, which forms the Graafian follicles and from which the ova originate. The anatomy of the ovary will be considered further in the chapters on Ovarian Tumors.

\section{Etiology of Ovaritis.}

Adhesions between the tube and ovary, especially when recent, contain many lymph-vessels; hence, bacteria may have a short accessible route from the tube to the ovary. Accordingly, inflammation of the ovary is usually secondary to that of the tube. It may, however, occur independently of salpingitis by extension from distant organs or directly from the peritoneum. Among the bacterial exciting causes the most frequent are these :

1. Gonococcus.

2. Colon bacillus.

3. Streptococcus and staphylococcus.

4. Tubercle bacillus.

5. Pneumococcus.

6. Typhoid bacillus.

\section{Comparative Pathology of Acute and Chronic Ovaritis.}

Acute ovaritis.

1. Usually develops by extension from some adjacent organ.

2. Ovary enlarged, tense, and elastic. Bloodvessels congested. Punctatc hemorrhages. Surface on cross-section yellowish red and Exceptions: An gonorrhoeal ovaritis adhesions form early.
Chronic ovaritis.

1. Usually develops from acute ovaritis.

2. Ovarles at first swollen and hard; later, surface uncren, nodular, and crstic. Crosssection shows numerous small cystic spaces. Tinica albuginea hard and scar like. Adheslons from periovaritis not uncommon.

1 Martin. Die Krankheiten der Eileiter. 


\section{Acute ovaritis.}

3. Small-cell infiltration of stroma-interstitial ovaritis.

4. Vessels congested and tortuous.

5. Superficial epithelium degenerated and desquamated. Small bemorrbages, cellular infiltration, and suppuration about follicles. Follicular epithelium degenerated. Liquor follieuli becomes turbid from the presence of round cells and degenerated epitbelium.

6. Infection of follicles from peritoneum or Fallopian tube.

7. Ovarian abscess may occur: $a$, in a corpus luteum; $b$, in the connective tssue; $c$, in the Graafial follicles. Non-gonorrhcal abscess usually unilateral, and not associated with pyosalpinx; infection usually travels through ymphaties in broad ligament. Gonorrhoeal abseess usually bilateral and an extension of double salpingitis.

8. MIay rupture into peritoneum or into bowel or bladder.

\section{Chronic ovaritis.}

3. Interstitial connective tissue increasedinterstitial ovaritis.

4. Same.

5. Superficial epithelium degenerated or absent. Small white bodies-corpora albicantesand hemorrhages near follicles. Ovary becomes smaller from contraction of connective tissue. Great numbers of follicles may be filled with thick, turbid, bloody fluid or may become obliterated. This follicular disease is known as microcystic degeneration, and is a common result of ovaritis; sce below. Tunica albuginea thickened and may be hyaline

6. Same.

7. Same.

See Tubo-ovarian Abscess and Tubo-ovarian Cyst under Salpingitis.

Tubo-ovarian abscess found by Martin 18 times in 110 ovarian abscesses.

8. Same.

\section{Physical Signs of Ovaritis.}

The physical signs of ovaritis are :

1. Increase in size of ovary.

2. Tenderness and sickening sensation in ovary on digital pressure.

3. Displacement of ovary common, usually backward to cul-de-sac of Donglas, and consequent upon retroposition of uterus.

4. Ovary immobile, if adherent; mobile, if not adherent.

See physical signs of salpingitis.

\section{Symptoms and Diagnosis of Ovaritis.}

Mild ovaritis commonly is associated with catarrhal endometritis and salpingitis. Severe ovaritis usually is complicated with pelvic peritonitis and suppurative salpingitis.

Orarian abscess is difficult to recognize; it is characterized by swelling and pain, and may give rise to general sepsis.

The following symptom-group will aid in the diagnosis :

1. Pain located in ovarian region, radiating to back, thighs, ischiatic nerves, navel, and breasts.

2. Fever-not a constant nor reliable symptom.

3. Nausea and vomiting-frequent.

4. Hemorrhage from uterus-not uncommon.

5. In severe septic cases of general pelvic infection local signs are masked.

6. Recurrence-usual in chronic cases.

7. Painful defecation and frequent urination.

8. Dyspareunia, hysteria.

In chronic cases the ovary is contracted and less painful on pressure. The general svmptoms-i.e., chill, fever, nausea and vomitingare absent, and the history will show long-standing irritation and discomfort in the ovarian region. Microcystic degeneration is associated often with amenorrhœa, dysmenorrhœa, and sterility. See Chapters LIII. and LIV. 


\section{Differential Diagnosis of Ovaritis.}

Ovaritis.

1. In uncomplicated ovaritis inflammatory sigus localized in ovary and sharply cireum. scribed.

2. Ovary usually mobile.

Ovaritis.

1. Ovary enlarged and tender.

2. Pain more or less constant.

3. History of pelvic infection.

Ovaritis.

1. Commonly bilateral.

2. 'Tends to localize in pelvis.

3. Digestive disturbances secondary.

4. Absence of tenderness at MIcBurney's point.

5. Inflammatory lesion deep in pelvis.

6. Inflamed organ recognized by size, formand relation to Fallopian tube.
Pelvic peritonitis and cellulitis.

1. Inflammation may involve whole pelvisll-defined.

2. Ovary usually fixed.

Ovarian neuralgia.

1. Ovary may be normal.

2. Pain inconstant.

3. No such history.

\section{Appendicitis.}

1. Confined to right side.

2. Tends to involve abdominal cavity.

3. Primary.

4. Usually present.

5. High in iliac space.

6. Ovary often recognized by bimanual examination as being separate and distinct from inflammatory area.

\section{Treatment of Ovaritis.}

The treatment will be found in the two following chapters :

\section{PELVIC PERITONITIS.}

\section{Pathology and Description of Pelvic Peritonitis.}

The two principal forms of peritonitis are :

1. Exudative peritonitis.

2. Plastic or adhesive peritonitis.

The two forms usually occur together. The plastic form, however, has been observed with little or no exudate. In addition may be mentioned tubercular peritonitis and pachyperitonitis.

Exudative Peritonitis is the result of infection which does not set up adhesions, and therefore does not strongly tend to provoke defensive action, is more liable than the plastic variety to become general, and for this reason is more dangerous.

Plastic or Adhesive Peritonitis is characterized by the formation of adhesions which tend to shut off and localize the infection and to prevent it from extending to the general peritoneum; the infection thereby is limited not only in extent and quantity, but its foree is spent within narrow limits within which the process may be very intense and the part may be sacrificed for the benefit of the whole. See remarks on Acute Inflammation and the Significance of Inflammation, in Chapters X. and XIX. The maximum of exulate with the minimum of defensive adhesion is dangerous; conversely, the minimum of exudate with the maximum of adhesion is relatively safe.

Pelvic peritonitis usually begins with perimetritis or perisalpingitis, the infection having reached the peritonenm from the nterine or tubal mucosa. Sometimes the origin is extrapelvic; in such cases the uterus and uterine appendages may not be involved. The disease, 
especially in connection with gonorrhœal salpingitis, is very common. The frequent recurrence of acute local exacerbations furnishes a familiar indication for the removal of the uterus and the uterine appendages.

The exudate consequent upon infection of the peritoneum may be serous or purulent; it may or may not be mixed with blood. In the plastic form protective adhesions often make numerous partitions through the infected parts; hence several distinct collections of fluid may be formed. These collections are sometimes serous in one part, purulent

FI(iURE 124.

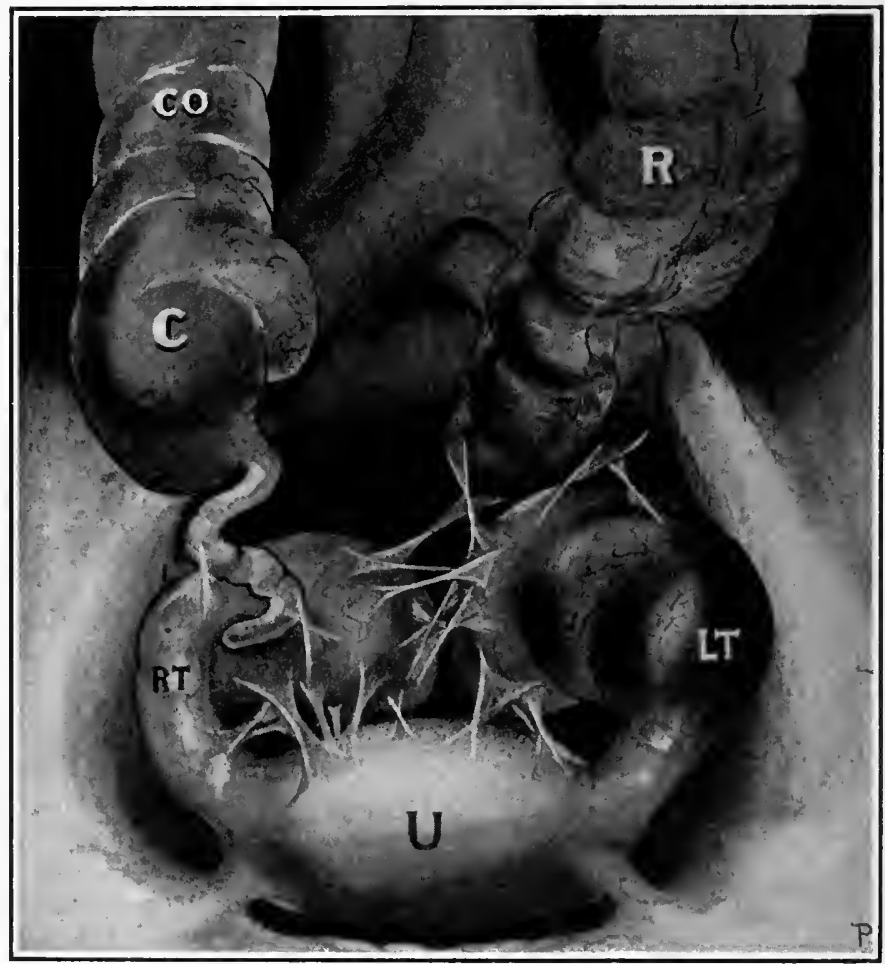

Right and left pyosalpinx; adhesions to uterus rectum, and vermiform appendix. Co, colon ; C, cæeum; $\mathrm{k}$, rectum ; $\mathrm{kt}$, right Fallopian tube. Lt, left Fallopian tube. U, uterus.

in another, and hemorrhagic in another; the whole may form a tumor filling the pelvis and the lower part of the abdomen, and having the appearance and many of the physical signs of an ovarian eyst. The adhesions may be broken up gradually by movements of the intestine and by absorption, or they may become firm and permanent; hence the organs may be matted strongly together with resultant displacement, stenosis, stricture, occlusion, kinking, embarrassed peristalsis, and defective general nutrition. The fluid may be absorbed or may break into a neighboring organ. In the latter way communica- 
tions may be formed between the pelvic cavity, and the bowel, bladder, or vagina. Sometimes the pus finds its way to the cutaneous surface. Accumulations are most frequent in the pouch of Douglas. The microseopical findings show a few round cells in the serous, numerous pus-cells with a few red-blood-corpuscles in the purulent, and numerous white and red blood-corpuscles in the hemorrlagic collections.

Tubercular Peritonitis is of frequent occurrence, and usually is characterized by small, sometimes minute, pearly tubercles or points scattered over the peritoneum.

The essential cause is the bacillus tubereulosis. The source of the infection is usually tubercular ulceration of the bowel, general tuberculosis, or localized tuberculosis of the Fallopian tubes or other pelvic viscera. The disease is rarely primary.

The pathology differs from that of ordinary peritonitis in the following particulars :

1. The peritoneum is studded with miliary tuberculosis, of which the tubercles may be large and caseous.

2. The fluid exudate (serous, hemorrhagic, or purulent) appears later, and in numerous places may be encysted.

3 . The peritoneal viseera usually are matted together by adhesions.

4. The mesenteric glands may be enlarged and palpable.

5. The bowel may become perforated or obstructed.

Pachyperitonitis.-Oftentimes the peritoneum is much thickened by the formation upon its surface of new membrane, which gives it a leathery appearance. The vessels in this new membrane early rupture with circumscribed hemorrhage. This is called pachyperitonitis.

\section{Symptoms of Pelvic Peritonitis.}

The symptoms in the acute stage vary within wide limits; may be quite disproportionate to the gravity of the infection; may be slight or absent; or may include great pain, nausea, fever, abdominal distention, retraction of the thighs, anxious facies, and profound nervous depression. The greater the tendency of the peritonitis to become general the more aggravated will be the symptoms. The chronic results of peritonitis include great discomfort, pain, and disturbance of function in the pelvis, especially about the rectum, uterus, and bladller. These symptoms may come largely from mechanical causes, such as tension on bands of adhesion and pressure and traction upon the inflamed peritoneum.

The distinctive symptoms of tubercular peritonitis are: 1, gradual onset; 2, diffuse, not severe, pain in the abdomen ; 3 , febrile condition and temperature highest at night; 4, rapid, often irregular pulse, with meteorism; 5, sweating, general malaise, relapses, and remissions.

The special local signs of tubercular peritonitis are : 1, enlargement of the abdomen ; 2 , local or general tenderness on pressure ; 3 , dulness on percussion over involved areas. 
286 INFECTIONS, INFLAMMATIONS, AND ALLIED DISORDERS.

Diagnosis and Differential Diagnosis of Pelvic Peritonitis.

The diagnosis and differential diagnosis have been given under Pelvic Cellulitis, Salpingitis, and Ovaritis.

\section{Treatment.}

The treatment of pelvie peritonitis will be found in the two following chapters. 


\section{CHAPTER XXII.}

\section{NON-SURGICAL TREATMEN'T OF PELVIC INFLAMMATION -SAlPINGITIS, OVARITIS, AND PELVIC PERITONITIS.}

THE non-surgical treatment of inflammation of the uterine appendages is :

1. General.

2. Local.

\section{General Non-surgical Treatment.}

The treatment of the milder adnexel inflammation, when early recognized, is largely the same as that of the causative endometritis. Quiet, frequent rest, judicious active and passive exercise, avoidance of sexual excitement, regulation of the bowels, nutritious and nonstimulating diet, and the prohibition of tea and coffee in neurotic cases, are among the routine measures as stated under Diagnosis. Repeated examinations and treatments, especially rough palpation of a sactosalpinx, may prove dangerous.

Medical Treatment. - The occasional practice in acute cases of locking up the bowels and preventing peristalsis by the free use of opium has largely been abandoned. On the contrary, rather active elimination through the bowels and kidney has become the more accepted practice. Pain may be relieved by opium and its derivatives; but they mask the symptoms and check the secretions, and therefore in a degree are contraindicated; hence non-constipating palliatives usually are substituted for opium. Of these, the coal-tar derivatives, chloral hydrate, hyoscyamus, and sodium bromide are among the more useful and least objectionable. The codeine phosphate repeated in half-grain doses is perhaps the least objectionable of the preparations of opium. Should the nervous symptoms predominate and demand the more dependable morphine, the constipating effect may be overcome by the addition of an equal amount of podophyllin.

Elimination often is secured well by means of rectal enemata containing magnesium sulphate, glycerin, or spirit of turpentine, or, if positive purging be required, by the use of some active cathartic. One may use to advantage repeated doses of calomel, one-half grain in each, followed by Rochelle salt, solution of magnesium citrate, or some other appropriate saline. When the stomach will not tolerate ordinary cathartics, a grain of calomel may be put upon the tongue every hour until the bowels act. A very high, retained enema of four ounces of the saturated solution of magnesium sulphate often gives prompt relief.

There is a form of chronic bilateral adnexal disturbance which scarcely goes beyond irritation and congestion. This is referred sub- 
jectively to the region of the ovaries. It is quite common among nervous, overwrought spinsters and girls, is associated usually with nervous irritability, is sometimes transient, often intractable, seldom dangerous. Overwork and overexcitement, says Lawson 'Tait, from study or social requirements, and especially the pursuit of music, by the physical strain of practice and by the power of music to excite the emotions at the developmental period of puberty, are potent and, among the higher classes, common causes of ovarian irritation. Many a hopeless neurotic invalid may in mature life date her invalidism from mental and emotional strains at the time of puberty. The treatment of this indefinite irritation should be mainly lyygienie and moral -that is, rather regulative than medicinal. Unsatisfied sexual requirements, conscious or unconscions, demand that the attention be drawn away from the reproductive organs. If the patient has reached the proper age, marriage may be desirable; at least let there be a ehange of environment and promotion of new interests. A careful, all-around examination may show some causal and removable extrapelvic fault in the patient or her environment. There will often be found disturbance of the heart, liver, or kidney or intestinal indigestion, and such disorders may explain the conditions upon which the ovarian irritation depends. There is usually an associated mild endometritis, which yields, if at all, to systemic treatment. The useless sacrifice of countless ovaries in this class of cases is a reproach to surgery. Menorrhagia, if associated with this condition, is well treated by ergot, preferably given in rectal suppositories, five to ten grains every eight hours until the flow is controlled.

Skene recommends for menorrhagic and neurotic cases the continued use of the fluid extract of hydrastis in thirty-drop doses, and, as needed for nervousness and sleeplessness, twenty to thirty grains of sodium bromide, to be given well diluted at bedtime, and repeated if necessary.

The medical treatment not only of the above form of ovarian irritation, but of chronic adnexal inflammation in general, includes the judieious use of tonies, laxatives, alteratives, and hypnotics. It must eonform to the general principles of internal medicine, and differs in no essential point from the general treatment of similar extrapelvic disorders.

\section{Local Non-surgical Treatment.}

Mechanical support for the uterus, if displaced, may open up the collapsed uterine canal, secure drainage of retained secretions, and by overcoming traction on the blood-vessels may relieve congestion.

Cold-water coils or the rubber ice-loag applied to the abdomen, or the application of a large blister to that part of the hypogastrium which lies over the seat of maximum pain, and the free use of leeches, may be serviceable, especially in the abortive treatment of acute cases. At least eight leeches should be applied : one or two are useless.

The local treatment of chronic adnexal inflammation has for its 
ehief object the quickening of the pelvic circulation and the promotion of absorption of morbid products. It includes : 1, the hot-water vaginal douche; 2 , the vaginal tamponade of lamb's wool saturated with glycerin or glycerin and ichthyol; 3 , the hot hip-pack; 4, electricity ; 5, massage.

The hot-water vaginal douche and the wool vaginal tamponade are described in Chapter IV.

Hot Hip-pack.-The application of the hot hip-pack is as follows: Let an ordinary sheet be folded lengthwise into several thicknesses, so that its width will reach from the umbilicus to the middle of the thighs. Let this be made into a roller bandage, dipped in very hot water, and wrung as nearly dry as possible, preferably by a clotheswringer. Pass this bandage several times around the pelvis, so as to envelop the zone from the umbilicus to the middle of the thighs. Cover it with a dry sheet and let the patient lie in it for thirty minutes. It is well, in order to retain the heat as long as possible, to place between the wet and dry sheet a rubber sheet, a rubber bag of hot water, or the electric heating pad. The pack repeated daily, or twice daily, according to the tolerance of the patient, is a most efficient means of stimulating the pelvic circulation, and thereby of promoting absorption of morbil products. Chronic constipation, pelvic pain, dysmenorrhœa, and other functional disturbances often give way promptly under its influenee.

Electricity.-The galvanic electrode, even with light dosage, has caused repeatedly extensive destruction and cicatricial contraction in the genital tract, especially in the upper part of the vagina. 'The intra-uterine electrode is painful, often intolerable, and the occasional cause of dangerous infection. The faradic current is used as a means of deep local massage, and the galvanic for its supposed resolvent effects. Both are said to promote absorption. The electrical treatment has proved itself neither in safety nor efficiency equal to the promise of its devotees.

Massage.-General massage is recognized as a measure of great value.

Local massage after the method of Thure Brandt is recommended for: 1 , the removal of inflammatory exudates; 2 , the breaking up and stretching of adhesions; 3 , the restoration of function to contracted or overstretched ligaments; 4 , the reposition of displaced organs. The application of local massage requires more technical skill than the physician would possess unless he had received long and special preparatory training. The objection commonly and strongly urged against massage, that it may excite sexual reflexes and produce erotic feelings, is important; this objection, however, provided that proper methods and precantions are enforced, need not necessarily, in selected cases, have prohibitory force ; that is, properly conducted massage in a suitable case should not provoke erotic feelings. The tendency to erotic excitement would usually be counteracted by the discomfort which the manipulation necessarily entails upon the patient; moreover, a subject of erotic tendencies would clearly be unfit for the treatment. Much depends upon the individ- 
uality of the operator, and upon the observance of an inviolable rule that the left hand and especially the finger in the vagina be kept perfeetly motionless, away from the clitoris and against the posterior wall of the vagina. The massage is given entirely with the right hand over the abdomen. Obviously the treatment if adopted at all should be given by a technically trained woman. The value of the method probably has been overestimated. 


\section{CHAPTER X X III.}

\section{SURGICAL TREATMENT OF SALPINGITIS, OVARITIS, AND PELVIC PERITONITIS.}

WhEN the disease has progressed to permanent obstruction of the Fallopian tube and the formation of pyosalpinx, and especially when occasional attacks of local peritonitis prove that the infection is not constantly confined to the tube, a radical operation, even to the removal of the diseased organs, may be less dangerous than the disease, and, relatively speaking, therefore, it may become a conservative measure. The inflamer tube, enlarged to the size of the finger, will seldom return to its normal state and functions, and if also there be evidence of suppuration or great local irritation, the indication for operation is clear.

For the preparatory treatment, see Chapter II.

The removal of the Fallopian tube alone is called salpingectomy; that of the ovary alone is called oophorectomy ; the removal of the tube and ovary is designated as oöphoro-salpingectomy, or salpingooophorectomy. The simple English expression, removal of the uterine appendages, would seem more appropriate. Usage reserves the word ovariotomy to signify the removal of an ovarian tumor.

\section{ROUTES OF OPERATION.}

There are two recognized routes for the removal of inflamed uterine appendages - the abdominal and the vaginal. An operation by the abdominal route necessitates abdominal section, also called celiotomy or laparotomy. An operation by the vaginal route involves vaginal section. It is sometimes necessary to combine abdominal and vaginal section in one operation.

\section{Operation by Abdominal Section.}

The reader is referred to Chapters II., VI., VII., and VIII. for preparatory treatment, for the technique of abdominal section, and for the general conduct of the operation. It is often necessary to add to this operation a vaginal section, hence the inportance of making in the vagina and about the vulva the same aseptic preparations as would be made if vaginal section were planned from the beginning.

Sometimes the inflammatory exudate has extended through the peritoneum to the subperitoneal structures, and so disorganized and disguised the parts as to render them difficult of recognition. Under these conditions careful dissection is necessary, in opening the abdomen, to avoid the unfortunate accident of opening directly through 
the thickened, leathery peritoneum into an adherent bladder or intestine.

During an operation the surrounding peritoneum should be proteeted against possible rupture of tubal or ovarian abseesses by the free use of sponges so placed as to absorb any eseaping fluid. In all operations sponges should be of gauze, should always be used dry, and as soon as they become saturated should be disearded for other dry ones. The special assistant who formerly washed sponges is no longer required, but may be utilized for connting and keeping track of sponges so that none shall be lost in the abdominal eavity.

If adherent omentum is in the way, it may be separated gently with the sponge-that is, sponged off from its attachnents. If not adherent, it may be pushed aside. Bleeding points should be secured by fine eatgut ligatures, torsion, or temporary forcipressure.

If the ease be simple, with no adhesions, or if the adhesions be few and easily broken, the operation will be relatively simple. The index-finger of the left hand finds the fundus and posterior wall of the uterus, and then maps out the diseased areas in that region. The finger, starting from the posterior wall of the nterus, sweeps alorg the posterior fold of the broad ligament on either side and examines the Fallopian tubes and ovaries. These organs, now accessible to sight and toueh, may be subjected to any necessary operation or manipulation. The incision, if too short, may be lengthened. The intestine is pushed upward and isolated by flat gauze sponges. If there are no adhesions, the appendages may be lifted gently up into the wound and examined. The surrounding exposed parts should be protected by gauze sponges. If removal of the appendages is neeessary, the operation as ordinarily performed is as follows:

It is of the utmost importanee to remove every partiele of tubal tissue, cutting it out clear into the tissue of the uterus. Failure to do this often results in the formation of stump exudates which perpetuate the evils of salpingitis in exaggerated form and may necessitate additional surgery for the removal of the offending stump.

Place a ligature on the infundibulopelvic ligament-i.e., on that portion of the broad ligament between the ovary and the wall of the pelvis. Place another ligature on the other end of the broad ligament where it joins the uterus. This ligature should not include the Fallopian tube. These two ligatures shut off the ovarian vessels and should render the remainder of the operation almost bloodless. Grasp the tube, ovary, and adjacent portion of the broad ligament in the left hand, and with the scissors remove them. As these parts are severed bleeding points may be secured by temporary forcipressure. Fine catgut ligatures are now placed upon any bleeding point between the two ligatures already tied.

Author's Reefing 0peration.-This operation is designed to shorten and strengthen the ligament and to limit the traumatism, and thereby to secure the uterus against descent and baekward displacement. If there is great tendeney to descent or retroposition, the round ligaments also may be drawn into the broal ligament wound and shortened by including them in the broad ligament sutures. The method obviates the neeessity of such supplementary operations as 
Figure 12.5.

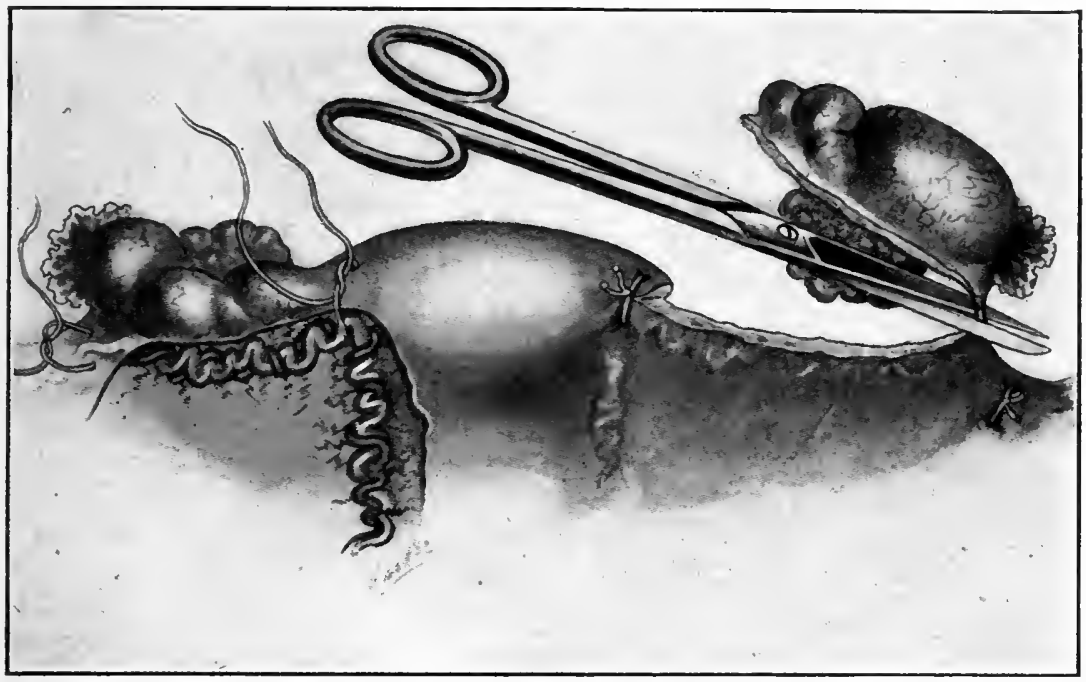

Removal of uterine appendages, first step. The tube has been freed from the adhesions and drawn up into the abdominal wound; one catgut ligature passed around the ovarian artery as it runs through the infundibulopelvic ligament and another as it enters the uterus. The right side shows the ligatures passed, but not tied; the left side shows them tied and the tube and ovary being excised by means of scissors.

FIGURE 126.

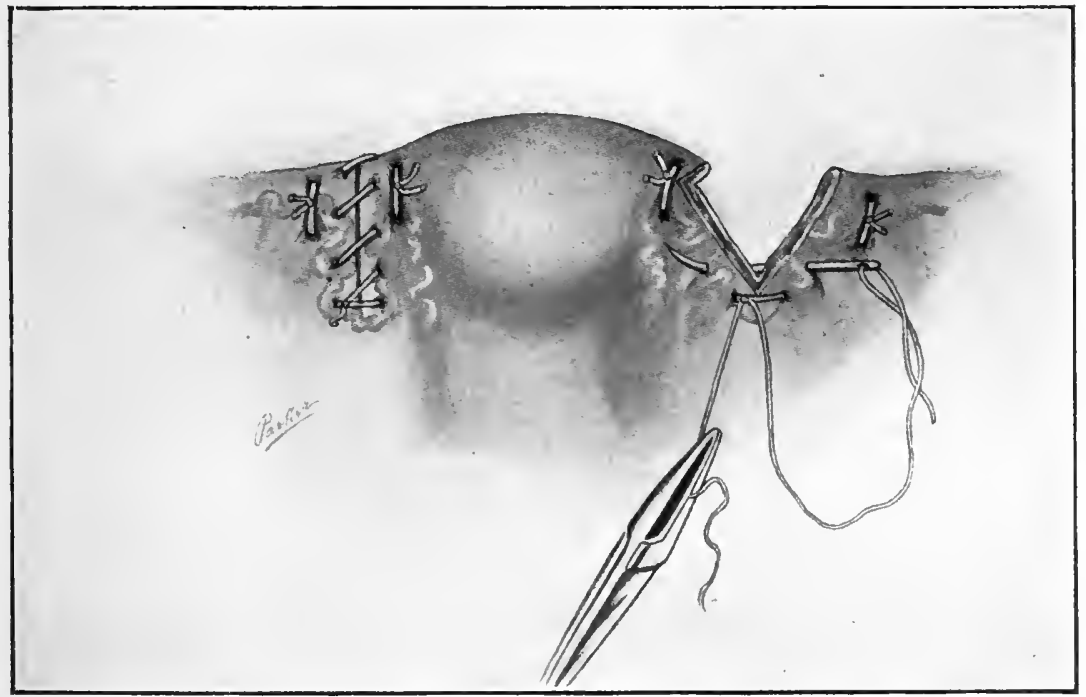

Removal of uterine appendages, second step. Ligatures on ovarian vessels drawn tight and tied, and tubes and ovaries removed. Any small bleeding points in the eut surface between the two ligatures that secure the ovarian artery should be tied by fine catgut ligatures. On left side, cut edge of broad ligament being closed by author's reefiug suture in such a way as to fold cut edge on itself and thereby strengthen and shorten the ligament. On right side closure of. ligament complete.

hysterorrhaphy and raginal hysteropexy, and is therefore specially applicable to eases in which there are great relaxation of the broad 
ligaments and consequent descent of the pelvic organs. Figures 125 and 126.

The usual method of elosing the broad ligament wound is shown in Figure 127. Observe the glover's stitch and method of introduction in the whipping togetler the cut edge of the ligaments on themselves. This method is not advised when the ligaments are relaxed.

Figure 128 illustrates removal of an ovary without sacrifice of the tube. Figure 129 shows removal of a Fallopian tube withont sacrifice of the ovary.

Complications in the Removal of the Uterine Appendages.The difficulties and dangers of the operation may be small or may be so great as to make it one of the most formidable in surgery. 'The

FigURE 127.

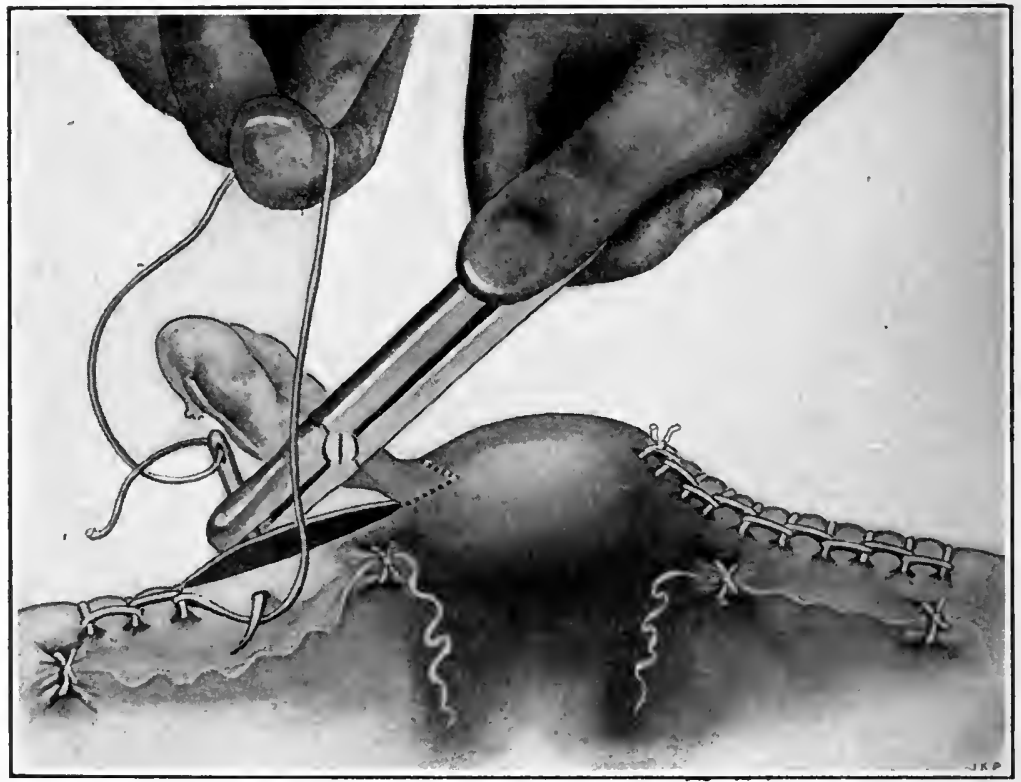

Removal of uterine appendages, final step. Showing the glover's stitch and the method of introduction.

special technique to meet the varied conditions turn upon the presence or absence of pus or of adhesions.

Technique in Pus Cases.-Although the pus in chronic pyosalpinx is usually sterile, it is not always so ; hence it is safer to proceed on the supposition that all pus or other fluid is infectious, and, if possible, therefore to enucleate the sac without breaking it. Aspiration of a part of the fluid from a very tense tube may decrease the risk of rupture. Contact of the pus of a ruptured tube with the peritoneum may have no serious results, for:

1. The fluid may be sterile, and therefore innocent.

2. Even though infectious, if thoroughly washed out with normal salt solution (Chapter VII.), the residue of septic matter, now much diluted, may be taken up by the peritoneum and thrown off by the 
Figure 128.

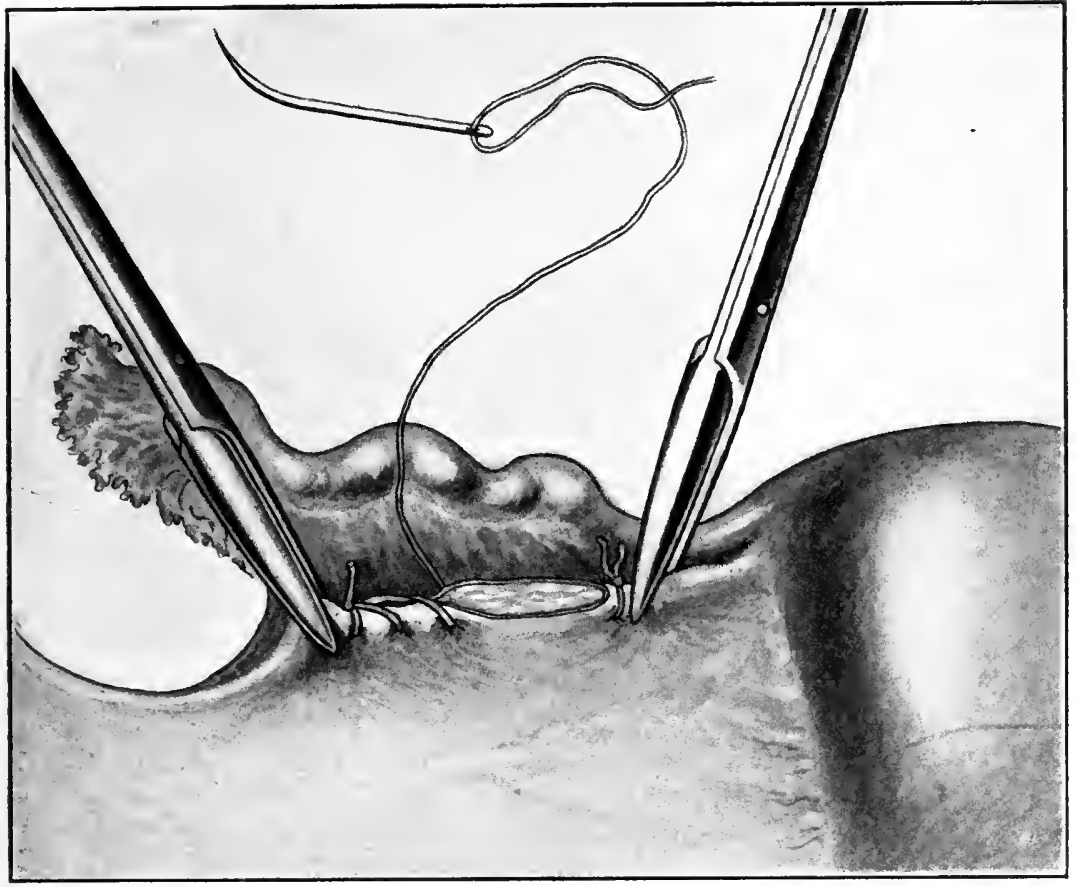

Removal of ovary; tube being normal, is not removed.

Figure 129.

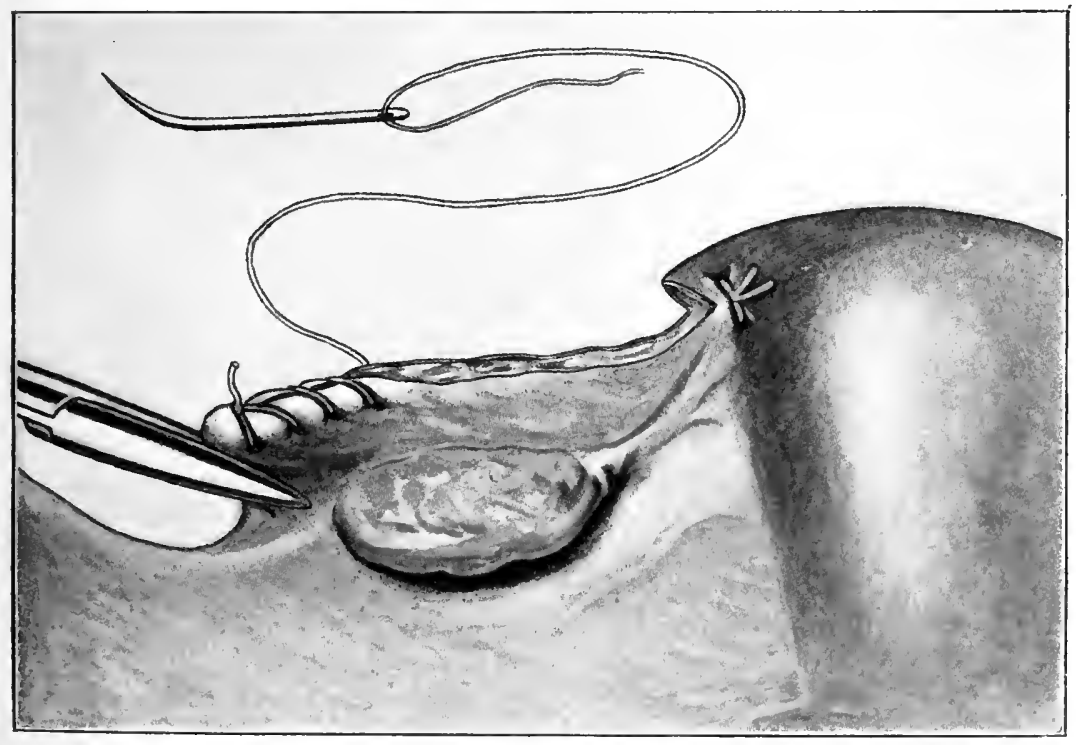

Removal of Fallopian tube : ovary being normal, is not removed. 
organs of elimination. The capacity of the peritoneum to absorb and throw off sueh matter is sometimes very great. The conditions, however, under which it does or does not do so are not fully known. See Chapter VII., on Drainage.

The coexistence of pyosalpinx and a communicating parametric abscess clearly renders the clean enucleation of the pus-sac impossible. After removal of such a tube the parametric abscess cavity will be in direct communication with the pelvic cavity, and this will necessitate a free opening from the cul-de-sac of Douglas into the vagina and effective gauze drainage by that route. Figure 71 . If possible, the general abdominal eavity should be shut off by stitching the omentum or peritoneum over the abscess-wall as shown in Figure 180. If there be a parametric abscess pure and simple, without tubal or other connections, the pus is much better evacuated and drained preferably by the vaginal rather than by the abdominal route. In some cases even with tubal involvement the vaginal route is preferable.

When the pelvic viscera are matted together with strong and extensive adhesions, including a great quantity of inflammatory infiltrate and pus, hystereetomy and vaginal drainage may be necessary in addition to the removal of the uterine appendage; such operations furnish much of the mortality in oöphoro-salpingeetomy. The danger may be so great as even to prohibit the radical operation, and to require instead the more conservative measure of simple incision and drainage by the vaginal or abdominal ronte. Sometimes the adhesions between the visceral peritoneum covering the abscess and the parietal peritoneum through which the abdominal incision is made are so extensive that the operator may find his way directly into the pus-cavity without exposure of the general peritoneum. In such a case it is wise not to attempt removal of the appendages, nor even to make a complete diagnosis, but rather to locate, incise, and drain the puscavity. A more radical operation may be made later, if necessary. Upon opening the abdomen one may locate a pus-sac adherent to some part of the abdominal wall at a distance from the incision; it might then be good surgery to close the first incision and make another through the abdomen or vagina directly into the sac. The abscess could then be evaeuated without contamination of the peritoneum.

The indications and technique of washing and sponging ont the peritoneal cavity, and the indications and modes of abdominal drainage and the toilet of the peritoneum, are set forth under the General Principles of Peritoneal Surgery, in Chapters VI. and VII.

Technique in Adhesions.-Strong and extensive adhesions are among the most common difficulties in the freeing of such hopclessly diseased organs as judicious surgery marks for removal. The first objective point, as in the simple cases, is the fundus and the posterior wall of the uterus. From this point the finger scarehes out the diseased uterine appendages on either side and recognizes their relations to adjacent structures. An ovary or tube, even though imbedded in apparently inseparable adhesions, may often be shelled out with relative ease if the weaker lines of cleavage can be found and made the starting-points of enucleation. Let the tip of the index and 
middle fingers of the left liand search for sulci between the diseased appendages and the adherent surfaces. Follow points of least resistance so long as the separation does not require undue force; then look for other such points. The finger advances with gentle firmmess, using the side-to-side and to-and-fro motion, until by pressing here and there, and by pinching the adherent structures apart, the outlines of the diseased organs are made clearer and clearer. By this means they finally are isolated and brought up into the wound. The technique of removal is then the same as for non-adherent appendages.

Technique in Hemorrhage.-During the enucleation it is not well to stop for minor bleeding points. Let the organs be isolated from the bed of adhesions as rapidly as safety will permit. Always keep sponges packed around to control hemorrhage by pressure and to absork blood, pus, or serum. When the appendages are cut off and the ordinary ligatures applied the bleeding usually will have ceased. If not, pack hot sponges firmly against the bleeding surfaces, frequently changing them to prolong the heat. A sterilized saturated solution of antipyrin, as recommended by Roswell Park, is a valuable hæmostatic. If bleeding is not controlled by prolonged hot-sponge pressure, antipyrin, and ligature of the ovarian vessels, and the bleeding points cannot be secured by isolated ligatures, it is better not to prolong the operation by temporizing, but to insure hæmostasis by immediate ligature of the uterine arteries, and if need be by removal of the nterus. The ligature is applied in the same manner as for abdominal hysterectomy.

Technique in Abdominal Hysterectomy.-If in the course of the operation the indication arises for removal of the uterus, the operator must proceed to it at once. See later paragraphs on the indications for hysterectomy. The danger now will increase rapidly with delay. The principles and technique of this operation differ in no essential respect from those of complete hysteromyomectomy described in Chapter XXVII.

The abdominal wound should be closed without drain. Drainage, if used, shonld be made through the vagina, the gauze drain being introduced through the abdominal wound and carried thence into the vagina.

Technique in Intestinal Opening.-Mention has been made of the breaking through and discharge of the contents of a pus-tube into an adherent intestine. The enucleation of such a tube necessarily would leave an opening in the intestine. Some provision then must be made to keep the contents of the bowel from eseaping through this opening into the free abdominal eavity. There are several possible plans of procedure :

1. If the opening is small and accessible, it shonld be closed with sutures and treated according to the requirements, with or without abdominal drainage.

2. If the opening is accessiblc and the loss of bowel-wall so great that repair with sutures would destroy the permeability of the bowel, the indication is for resection or for stitching the opening into the abdominal wound, and making thereby an artificial anus. Unless contraindicated by the exhausted condition of the patient, resection 
would be preferable, for if the artifieial anus did not elose spontaneously reseetion would have to be made subsequently.

3. If the opening is so deep in the pelvis as to be inaceessible or the patient is too exhausted to permit suture, the territory around the fistula may be quarantined from the general peritoneum by means of gauze packing. Adhesions will form in a few hours around the packing and thereby shut off the leaking bowel from the general peritoneum. The writer has treated successfully two cases by this method. The gauze may be brought out through the abdoninal wound; or if the fistula is deep in the pelvis, it is better to pass the gauze drain into the vagina through an opening made for the purpose and to elose the abdominal wound.

4. If the fistula is too large for suture, the parietal peritoneum may be made to take the place of the lost intestinal wall. This will require the edges of the fistula to be united to the abdominal wall by means of a plate of deealcified bone or other absorbable material. The plate should have small perforations one-sixth of an inch apart all around near its outer edge; it is placed inside the intestinal opening, and through this perforation the margin of the bowel may be stitched to the parietal peritoneun. The sutures should transfix the bowel-wall and the abdominal wall and be tied on the skin, thereby approximating serosa to serosa.

The following case is illustrative and instructive. In an operation at St. Luke's Hospital, Chicago, a large friable pus-tube was in eonmunieation with the bowel at two points. After emueleation there was a fistula too large to be elosed at each of these points. The bowelwall surrounding the-fistula was, moreover, extremely thickened and friable. The first impulse was to resect the bowel at eaeh point of injury. Instead of this most formidable operation, however, the following plan was adopted successfully: The two openings were brought together and united by three rows of fine eontinuous silk sutures, the fistula thereby being utilized as openings for an intestinal anastomosis. The abdominal wound was closed with only a slight gauze drain extending from its upper angle to the immediate neighborhood of the intestinal sutures. This drain was removed on the fourth day. The result was complete recovery. So far as the writer is informed, this principle has never been used in a ease like the above.

Technique iu Accidental Wounds of the Ureter.-In the course of a pelvic operation the ureter may be eut accidentally in the longitudinal direetion, or partially severed in the transverse direetion, or completely divided. Then one of the following operations will be indieated :

\section{For Ixcomplete Division of the Ureter.}

Ureterorrhaphy.- - If a ureter opened in the longitudinal direetion is. closed by a line of union ruming in the same direction, there will be danger of stricture at the point of elosure. To prevent this, the line of union should run at right angles to the line of incision-that is, transversely, as suggested by Fenger, who deliberately opened a strictured ureter longitudinally and then increased the calibre by elosing it transversely. 
In ease of partial transverse division, Van Hook suggests that a longitudinal incision be made directly across the middle of the transverse cut at right angles to it and twice as long as the cliameter of the tube. The sharp angles should then be rounded off with scissors and the wound sutured, as described above for longitudinal wounds.

\section{For Complete Division of the Ureter.}

Insertion into the Bladder.-If the part above the injury can be drawn down to the bladder withont mulue traction, it shonld be inserted into the bladder through an opening made for the purpose and fixed there by means of fine sutures.

Lateral Anastomosis.-If the part above the injury will not reach the bladder, it may be inserted into the lower fragment after the method of Van Hook. This operation is shown in Figure 130 .

Figure 130.
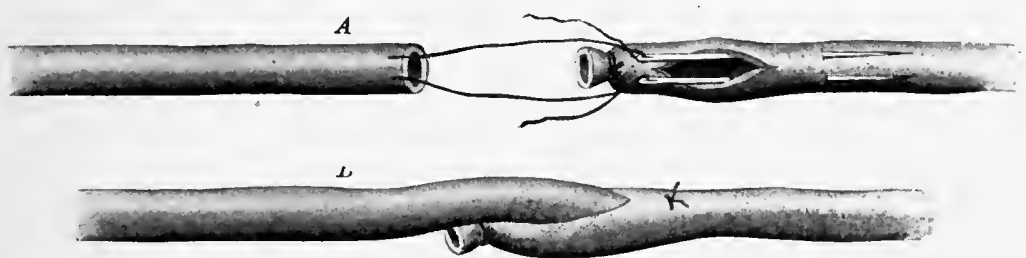

Lateral anastomosis of the ureter.

A. First step: natural size. Showing the fragment toward the bladder tied and slit longitudinally for the reception of the upper fragment. The two ends of a fine silk or chromic eatgut suture have been passed througl the ureteral wall near the end of the upper fragment froni within outward. Two tine needles on this suture are transfixing the wall of the lower fragment preparatory to drawing the end of the upper fragment $i n t o$ the longitudinal slit.

$B$. Second step: natural size. The upper fragment has been drawn into the longitudinal slit in the lower fragment and made fast by tying the suture: in order to insure security against leakage, one more similar suture should be placed and tied on the opposite side, and a few very fine interrupted sutures should be introduced quite superficially around the anastomotic union. The anastomosis thus completed should be covered by stitching omentum or some other peritoneal structure over it. If there is no apparent leakage, the abdominal wound may be closed without drail.

Ureteral Fistula to the External Surface.-If for any reason none of the above operations is practical, the ureter should be brought out through the lower end of the abdominal wound at the nearest possible point to the bladder, so that the urine may discharge temporarily to the external surface until connection with the bladder can be attempted, for example, as follows :

Ureterocystostomy by Bladder Diverticulum.--For cases in which the ureter cannot be made to reach the bladder, Van Hook proposes that the bladder be extended to the ureter by dissecting a flap fron the anterior vesical wall and reflecting it upward to meet the ureter in such a manner as to form a tubular diverticulnm from the bladkler to the ureter.

Nephrectomy.-When the ureter eannot be connected with the bladder, the only alternative may be to remove the kilney on the affected side.

\section{Operation by Vaginal Section.}

The maxim that every peritoneal section should begin as an exploration holds true as well for vaginal as for abdominal section. 
Thorough sharp curettage and cleansing of the endometrium are essential preliminaries. The object is, first, to remove the original source of infection; second, to prevent infection of the peritoneum from the uterus during the operation. Vaginal section according to the indication is made either anterior or posterior to the uterus, or both anteriorly and posteriorly.

Posterior Vaginal Section.-The posterior incision is made close to the uterus, between the cervix uteri and the rectum, from the postvaginal fornix into the pouch of Douglas. The steps of the operation are as follows :

1. The patient is to be placed in the dorsal position and the vaginal portion of the cervix exposed by Simon's retractors.

2. A semicircular incision, large enough to admit two fingers, is made directly behind the uterus in the line of the uterovaginal attachment, with blunt-pointed scissors curved on the flat, the point being directed toward the uterus and the cervix being drawn down by the vulsellum forceps.

3. The loose cellular tissue back of the cervix between the vagina and the pouch of Douglas is stripped back off from the cervix by the blunt point of the scissors, by the handle of a scalpel, or by the finger, until the peritoneum is reached.

4. The peritoneum is divided close to the uterus by a snip of the scissors. The closed scissors-points are now passed through into the pouch of Douglas and the opening is dilated by spreading the blades, and if necessary enlarged still further by careful cutting with the scissors or by tearing with the fingers.

5 . The index and middle fingers of the left hand now are introduced into the pouch of Douglas and the pelvic cavity is explored digitally. If sufficient room has not been gained, a perpendicular incision, beginning in the middle of the posterior border of the one already described and running toward the rectum, may be made. In cutting down toward the bowel the left index-finger in the rectum should be used as a guide. This finger then is withdrawn, disinfected, and with a fresh rubber glove reintroduced into the pouch of Douglas; the right hand is placed over the hypogastrium behind the pubes, and the examination is made precisely as in ordinary bimanual palpation, but with a distinct advantage-i.e., the palpating finger is in direct contact with the uterus and its appendages.

Posterior vaginal section is not well adapted to removal of the appendages; it is, however, specially applicable to the incision and drainage of pelvic pus-cavities. These cavities may be in the tubes, ovaries, or pelvic connective tissue. See Incision and Drainage of Pelvic Abscesses.

Anterior Vaginal Section.-Peritoneal section anterior to the uterus-i.e., between the uterus and bladder-renders the uterus and its appendages more accessible to conservative radical operation than posterior section, but less accessible than abdominal section. The technique is similar to that of posterior section, and is as follows:

The patient is placed in the dorsal position and the cervix exposed by Simon's retractors. The cervix is seized with vulsellum forceps 
and drawn toward the vulva. A transverse semicircular incision close to the uterus, in a line with the uterovaginal attachment, is made with scissors through the anterior vaginal fornix; or, instead of this, the incision is made in the longitudinal direction in the median line through the anterior vaginal wall from the anterior wall of the cervix toward the bladder. The latter incision is preferable, because, without great care, especially if the cervix is small, the transverse incision is liable to injure the ureters. In making the longitudinal incision, the operator should not only draw the cervix uteri well down, but also make strong downward traction on the anterior

Figure 131.

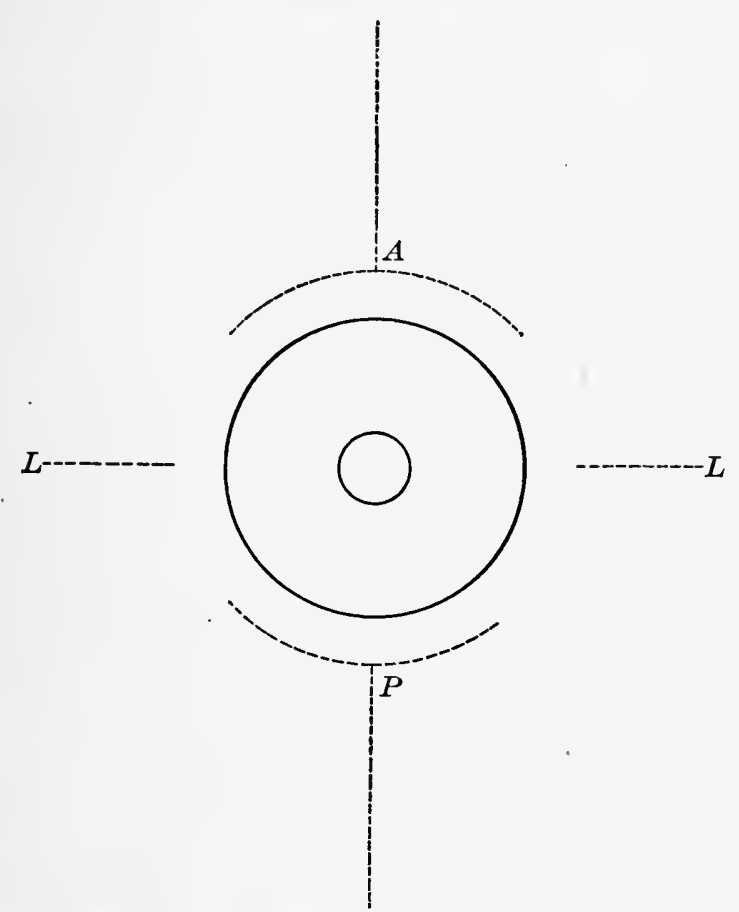

Lines of anterior, posterior, and lateral incisions in vaginal section: $A$, anterior incision : $P$, posterior incision; $L, L$, lateral incisions.

vaginal wall. This is done with a tooth-forceps attached to the wall between the cervix uteri and the urethra. If the longitudinal incision give insufficient room, it may be supplemented by the transverse. The combined longitudinal and transverse cuts have the shape of the letter T. They are shown in Figure 131, and in the surgical treatment of myomata, Chapter XXVII.

The uterus now is drawn strongly forward, and the structures adjacent to its anterior wall are stripped off, keeping close to the uterus, as described above for posterior section. As the bladder is being separated from the uterus it is held up out of the way by an anterior retractor or the finger. When the peritoneum comes into 
view, it will be recognized as a thin, translucent membrane reflected from the uterus. A sound passed into the bladder will prevent cutting that organ for the peritoneum. The peritoneum is snipped with blunt-pointed scissors. The opening thus made into the pelvic cavity is enlarged by introducing the two index-fingers and tearing laterally, and, if necessary, by careful eutting with the seissors. During the separation of the bladder from the uterus a sound in the uterine canal may be useful as a guide.

The corpus uteri may now, if adhesions do not prevent, be seized with vulsellum or bullet-foreeps and the fundus drawn into the vagina. If there be adhesions, they may be loosened with the left index-finger introduced over the fundus uteri, the corpus being at the same time drawn more and more into the vaginal opening. The Fallopian tubes and ovaries follow the corpus, and may be subjected to examination and any necessurv operation. They may be removed wholly or partially as in abdominal section. The closed fimbriated extremity of a tube may be opened or the ovary may be resected. See Conservative Operative Treatment of Adnexal Inflammation at the end of this chapter.

The removal of the appendages by anterior vaginal section does not materially differ in technique from removal by abdominal section. Hrmostasis may be secured by the usual ligature close to the uterus or by running sutures in the broad ligament. To bring the appendages into full view may require firm traction, and the uterus may have to be drawn from side to side. Ligation of the infundibulopelvic ligament, which controls the ovarian vessels, is often difficult, sometimes impossible. Sometimes the broad ligament, if short, tense, and adherent, cannot be reached through the vagina. In such case it would be safer to abandon the vaginal and resort to the abdominal route. If there is diffieulty in returning the uterus, enlarged by congestion, from torsion of the ligaments, the Simon retractor may be used in the manner of a shoe-horn, and the nterus slid in on the smooth blade.

The blood-clots having been sponged out and all bleeding points secured, the wound is closed as follows: The peritoneal margins are drawn down and approximated by means of pressure-forceps. They then are whipped together with a running fine catgut suture. The suture is continued as a buried suture to unite the vesical to the uterine surfaces of the wound, and finally, as a rumning suture to close the vaginal margins. The vagina is packed lightly with aseptic gauze.

The anterior incision, except for drainage of pus-cavities, is preferable to the posterior. It involves less danger of post-uterine adhesions, which may result in fixation of the retroverted or retroflexed uterus. Moreover, it offers by anterior vaginal fixation a cure for the retromalpositions. See Treatment of Retroversion and Retroflexion by Vaginal Fixation, Chapter XLVII. In some cases intrapelvic disease is rendered more accessible by the combined anterior and posterior incisions.

Effects of Removal of the Uterine Appendages.-Removal of the ovaries and Fallopian tubes has been usual in hydrosalpinx, and 
is the rule in pyosalpinx. The operation, if thoroughly performed, is followed generally by atrophy and consequent arrest of function in the uterus, and the precipitation of the menopause. The artificial production of this eritical period gives rise to phenomena quite similar to those which characterize the natural menopause, except in most eases menstruation is arrested permanently at once. The popular impression that the operation unsexes the woman in a mental sense or renders her masculine is a mistake. Patients frequently ask whether it will result in the growing of a beard or the development of a bass voice; but no such result has ever been observed. The operation performed on a young girl would doubtless arrest the intrapelvic and some of the extrapelvic developmental processes of puberty, but development once made is permanent.

The effect of the operation upon sexual desire is variable, but probably no more so than that of the natural menopause.

The question of insanity as a result of the operation has been raised ; it probably oceurs no more frequently than after other operations of equal gravity, probably not oftener than with the natural menopause.

The primary object of the operation is the removal of certain organs which otherwise would be dangerous to life or destructive to health. A most important secondary result is the arrest of physiological function in the remaining uterus. In this connection it is clear that, sinee pathology is pliysiology modified by discase, the atrophic changes in the uterus consequent upon the operation may, at the same time that they arrest physiologieal processes, also put an end to pathological processes. Especially is this true in the inflamed uterus, discase of which often is perpetuated by the constantly recurring menstruation. The frequent disappearance of metritis from the atrophic uterus verifies a recognized principle that physiological rest may favor the eure of disease. If the uterus is healthy or the seat of only mild eatarrhal inflammation, it usually, upon the removal of the appendages, will pass rapidly into the atrophie state, and give no more trouble than would a uterus after the usual menopause. Unfortunately, however, this very common sequence of the removal of the appendages is not coustant. The atrophic process does not always follow, or, if it follows, may fail to remove the infection. The infected uterus may be the source of pernicious menstruation, amounting at times to hemorrhage. A surviving and intractable endonetritis often gives rise to profuse uterine discharges. Exhaustive drains upon the patient's strength from such eause may destroy her resistance to disease, reinforce the uterine infection, and perpetuate a group of disabling nervous symptoms.

Should the Uterus be Removed with the Appendages ?-This question has been forced upon the surgeon by the numerous immediate and remote failures which have followed removal of the appendages alone. When the appendages on one sicle are healthy, or not suffieiently diseased to neeessitate their removal, and when enough ovarian tissue can be left to give hope that the reproductive function may be preserved, the answer is negative. The essential ques- 
tion is, What shall be done with the uterus when the appendages on both sides have to be removed completely? It may be urged with considerable force that failure to bring about atrophy of the uterus, arrest function, and to secure consequent relief from pernicious symptoms, arises in many cases from faulty technique in the operation. Arthur W. Johnstone and Lawson Tait have shown that when the tubes are taken off elose to the uterus and every particle of the appendages removed, arrest of menstruation, atrophy of the uterus, and a satisfactory menopause, even in cases of infected uteri, are apt to follow. The explanation of this is simple and as follows: The thorough removal of the tubes cuts off the ovarian artery and the supply from the uterine artery at the point of anastomosis with the ovarian. As pointed out by Johnstone, it also cuts in a similar way the nerve connections of the uterus; hence the observed atrophy and arrest of function. The claim of the enthusiastic hysterectomist, that when the appendages have been sacrificed the uterus necessarily becomes a pernicious, continuous, disabling, and dangerous source of infection, may, as a nniversal proposition, be disregarded. In order to bring about the most satisfactory results the tubes should be removed not merely close to the uterus, but the entire tubes, even as they penetrate the cornua, should be removed to the nterine mucosa, and the cornual wounds should be closed by catgut sutures; the removal of a tube by the ordinary stump and ligature method which may result in leakage of uterine seeretions into the' pelvic cavity, properly has been abandoned. In thus emphasizing the necessity of complete removal of the tubes we should have clearly in mind not only arrest of menstruation but also avoidance of stump exudation already mentioned on a previous page.

The propriety of leaving an infected uterus while the causative infection in the endometrium is still overwhelming the pelvic lymphatics with its septic supply is indeed questionable; for the uterine infection may continue to spread to the peritoneum even after removal of the appendages. If vaginal incision and drainage are deemed inadequate, the removal of the uterus, together with the appendages, may be necessary for two reasons : first, to cut off the septic supply ; second, to facilitate drainage.

If in consequence of great infection it becomes necessary to remove the uterus, the surgeon should avoid the half-way measure of removing only the corpus, for if the cervix be left, it may continue to be the source of persistent and pernicious infection in the adjacent structures, even to the extent of filling the pelvis with stump exudate. In fact, to remove the corpus and leave an infected cervix would be inexcusable, for the cervical glands, as pointed out in the Chapter on Endometritis, are specially adapted to the reception, the retention, and the distribution of infection.

\section{The Objections to Hysterectomy are as follows:}

1. The fact that the uterus is an important part of the pelvic floor and is necessary therefore to the integrity of the pelvic diaphragm.

2. The possibility that removal of the uterus, in addition to removal of the appendages, may disturb the moral and physical well-being of the woman to a greater extent than removal of the appendages alone. However this may be, since many women have the strongest 
aversion to hysterectomy, their wishes so far as may be, without harm, should be respected.

3. The fact that removal of the uterus, especially by a slow or inexpert operator, involves additional shock and danger.

4. The possibility that hysterectomy may cause secondary degenerative changes in the spinal cord or brain. This possible result has been observed as a sequel of major operations in other parts, especially those involving extensive injury to nerve structures.

5. The possibility that the uterus, like the ovary, may have an important function as an eliminative organ.

6. The absence of a clear indication.

\section{SURGICAL TREATMENT OF SALPINGITIS.}

The Indications for Hysterectomy are as follows :

1. The matting together of the reproductive organs in one infected mass, with pockets of pus. The difficulty of operation does not necessarily neutralize this indication.

2. Tuberculosis of the reproductive organs.

3. Complicating maliguant disease.

4. Complicating uterine myoma, which cannot be removed without sacrificing the uterus.

5. Involvement of the endometrium in destructive inflammation, so that the uterine wall, itself strongly infected, becomes virtually the wall of a pus-cavity; under these conditions the uterus is decidedly a source of danger.

The value of the uterus as an essential part of the pelvic floor has led some operators to remove the corpus and leave the cervix, or at least the vaginal portion of it. This is practical only when the operation is performed by the abdominal ronte, a route which contemplated originally but one wound, and that through the abdomen. To leave the cervix, therefore, after removal of the corpus, is a natural corollary of the abdominal operation. If hysterectomy, on account of the infections character of the uterus, is to be performed, the operation should include at least that part which is usually most infected and, therefore, most pernicious-the cervix. The idea of leaving it hardly would occur in connection with the vaginal operation.

\section{Vaginal Hysterectomy.}

Abdominal hysterectomy for pelvic infection, in the author's judgment, is much more frequently the operation of choice than vaginal hysterectomy. The vaginal operation, however, has recognition in the surgery of infectious and malignant disease of the uterus and of complete descent of the uterus; for this reason it is here described fully, with extended illustrations. Reference to the operation will be made in following chapters on other subjects, as the occasion may arise.

In the vaginal operation for the removal of the uterus two principal methods of hæmostasis are in use :

Hæmostasis by ligature.

Hæmostasis by forcipressure. 
Vaginal Hysterectomy with Hæmostasis by Ligature.-The technique of the operation is as follows: The patient is placed in the lithotony position; the vulvo-vaginal surfaces are thoroughly disinfected-Chapter II.; the cervical canal is dilated ; and the endometrinm euretted, washed out, and disinfeeted with formalin and iodineChapter XVII. Some operators close the os externum with temporary suture to prevent escape of uterine secretions during the operation. The eervix uteri is brought into view by means of one or two Simon retractors, seized with strong vulsellum forceps, and drawn toward the vulva. A free incision with scissors is made all around the cervix. The loose tissues around the cervix are stripped back easily by means of the finger or handle of the scalpel, keeping as close to the uterus as the disease will permit. Small bleeding points are controlled by catgut ligatures. In this way the circumuterine structures may be stripped back from the uterus until the exposed part of the cervix is measured by a zone three-quarters of an inch or more wide. This zone extends anteriorly and posteriorly to the anterior and posterior nteroperitoneal reflections, and laterally to the broad ligaments. The uterus can now be drawn down much lower, and, with the bladder thus separated from the uterus, the ureters, which lie elose to the uterus, can be avoided easily. The post-cervical struetures now are separated further by means of the finger or the handle of the scalpel, or the closed blint scissors, until the cul-de-sac of Douglas is opened. This opening is enlarged easily by introducing the two index-fingers and tearing laterally to the region of the broad ligaments. A large ganze sponge, with a string attached to facilitate removal, now is forced through into the cul-de-sac of Douglas. This will protect the pelvic viscera and absorb blood during the remainder of the operation.

A like opening anterior to the uterus between the uterus and bladder is made also into the peritoneal carity. As was done posteriorly, this opening is enlarged to the region of the broad ligaments by lateral tearing with the index-fingers, and the peritoneal edge of each opening, if the operator so elect, may be stitched to the vaginal edge. The whip-stitcli by which this is done anteriorly and posteriorly reduces the size of the wound, prevents bleeding, and thereby simplifies the operation. The anterior opening sometimes may be made more easily by passing the index-finger through the posterior opening, and, if possible, hooking it over the broad ligament, so that it may serve in some degree as a guide, and thereby prevent the operator from wounding the bladder, ureters, or anterior uterine wall. Then the index-finger of the left hand or a blunt look is hooked over the left broad ligament, the ligament is drawn down and transfixed, and tied en masse, or, if very large, in sections. The application of the ligatures may be facilitated by anteverting the uterus and drawing the eorpus through the anterior opening by means of vulsellum forceps. This twists the ligaments upon themselves, makes them smaller, and brings their upper margins within reach. Separate ligatures usually are needed for the uterine appendages. Figures 132 to 137 will 
show the technique of the operation. Each ligament usually is ligatured in two or three sections. The ligatures-preferably catgut -are passed by means of aneurism-needles, or with the ordinary threaded needle and forceps. In some cases an entire ligament may be seeured by a single ligature, but more frequently portions of it on either side are tied progressively and cut away from the uterus until the organ finally is removed. In many cases hysterectomy is facilitated by dividing the uterus into halves. Each half may then be drawn through the vagina separately and removed. The ends of the ligatures, having been left long, are used now to draw the stumps down into the vaginal wound, where they are treated as slown in Figure 137. If the stumps will not reach to the vagina, the ligatures are cut short, and the stumps returned to the pelvic cavity. Sometimes all the stumps are too short, and therefore must be treated intraperitoneally. The vaginal wound will then be closed as the conditions may require, with or without the gauze drain. See Chapter VII., on Drainage.

Vaginal Hysterectomy with Hæmostasis by Forcipressure.Hæmostasis by foreipressure is the same in general technique as that by ligature except the use of forceps in place of the ligature. After the openings anterior and posterior to the uterus have been made the ligament is drawn down and seized with hæmostatic forceps, the grasp being at a sufficient distance from the uterus to prevent the instruments from slipping off after the organ has been severed. These foreeps are constructed on the principle of Péan, but should be heavier and with jaws about two inches long. Various broad ligament clamps have been devised, but none fulfils the indication better than the straight, strong hæmostatic forceps. The forceps handles are locked securely, the ligament is severed close to the uterus, and the whole uterus pulled outside. The organ now hangs by the other broad ligament. This in turn is clamped in the same way, and the uterus is removed by a few strokes of the scissors. The ovaries and Fallopian tubes, unless already included with the broad ligaments, may be seeured by separate forceps. If, upon examination, the operator fears that the broad ligament is diseased beyond the grasp of the first forceps, he may put on other forceps back of those first applied. The first foreeps may then be removed and the diseased tissues cut away. Fatal hemorrhage has resulted from slipping of the broad ligament forceps; hence the neeessity of so making the incision through the ligament as to leave considerable tissue on the distal side of their jaws. To prevent the forceps handles from snapping apart, they should be tied together securely with strong thread.

In many cases the uterus is much enlarged, and the ligaments therefore on either side extend so high in the pelvis that they cannot be drawn down within the grasp of a single pair of forceps. Then one pair of forceps may be put on, and that part of the ligament which is in their grasp divided. The uterus can then be drawn further down, and the remaining portion of the ligament, having been clamped by one or more forceps, may be severed. If so mueh space 


\section{Explanation of Figure 132.}

A. Vaginal hysterectomy. The patient in the dorsal position.

The vagina and cervix uteri are exposed by retractors in the hands of assistants.

The os uteri externum las been closed by a continuous suture, to protect the operation wound and the peritoneum from the uterine secretions.

The cervix uteri has been seized by strong flat vulsellum forceps in the left hand of the operator and drawn strongly down toward the vulva.

The operator, with scissors in his right hand, is making a free incision through the mucosa all around the cervix uteri in the line of the utero-vaginal attachment. The black and white dotted lines indicate the direction of the incision.

The bleeding points are secured by fine catgut ligatures-not shown.

$B$. The mucosa all around the uterus has been divided by scissors. While strong traction is being made on the uterus by the forceps in the right hand of the operator, the left index finger is used to strip back the circumuterine tissue all around the cervix. The stripping process is continued until it has exposed a zone of raw tissue an inch or more wide, when the utero-peritoneal reflexion will be recognized by the loose, thin, membranous character of the tissue, and by the fact that under the finger it slips over the adjacent peritoneal covering of the uterus. 
Figtre 132

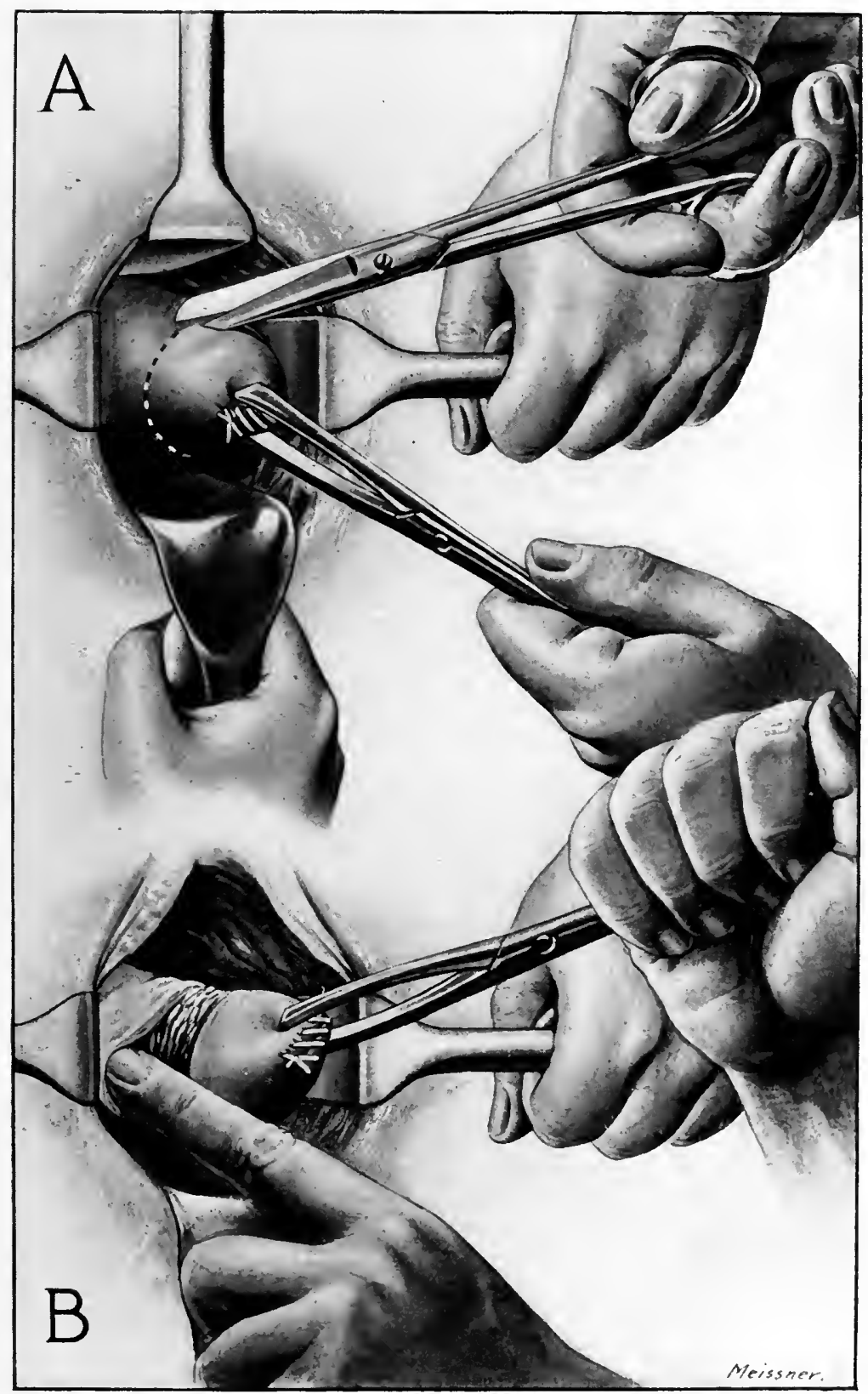


Figtre 133

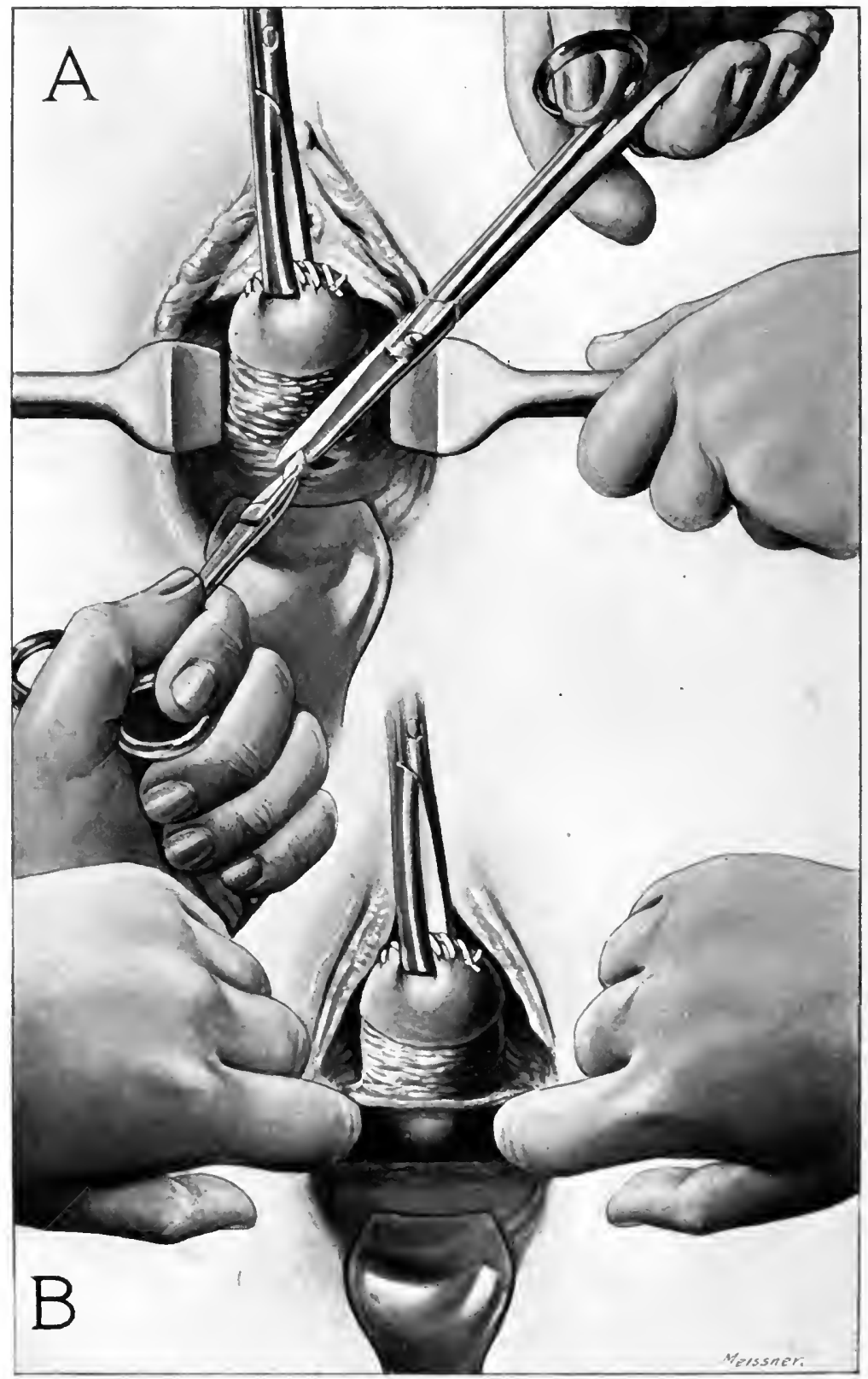




\section{Explanation of Figure 133.}

Vaginal hysterectomy. The patient is in the dorsal position.

The vagina and cervix uteri are exposed by retractors in the hands of assistants.

The os uteri externum has been closed by a continuous suture to protect the operation wound and the peritoneum from uterine seeretions.

The eervix uteri has been seized by strong flat vulsellum forceps in the left hand of the operator and drawn strongly down toward the vulvil.

$A$. The circumuterine struetures have been stripped down to the uteroperitoneal fold, as shown in Figure 132; the operator seizes this fold posterior to the uterus with a hæmostatic forceps in the left hand, and with scissors in the right hand ents through into the cul-de-sae of Douglas.

$B$. The operator, with the index fingers inserted into the eul-de-sac of Douglas through the opening shown in $A$, is tearing laterally to the region of the broad ligaments.

A similar incision is then made into the pelvic cavity anterior to the uterus, and enlarged by lateral tearing to the region of the broad ligaments in the mamner deseribed above, so that the uterus is attached to its surroundings by only the broad ligaments. 


\section{Explanation of Figure 134.}

Vaginal Hysterectomy. The patient is in the dorsal position.

The vagina and cervix uteri are exposed by retraetors in the hands of assistants.

The os uteri externum has been elosed by a continuons suture to proteet the operation wound and the peritonemm from the uterine seeretions.

The eervix uteri has been seized by strong flat rulsellum foreeps in the left hand of the operator and drawn strongly down toward the vulva.

The uterus has been freed from its surroundings anteriorly and posteriorly as deseribed in Figures 132 and 133.

$A$. While the uterus is drawn strongly downward and to one side with vulsellum foreeps in the hand of an assistant, the operator introduees the left index finger throngh the posterior vaginal opening in the eul-de-sac of Douglas and brings the finger-tip out into the vagina through the anterior opening, so as to hook it over the left broad ligament; the ligament thus held on the finger is transfixed at two points by a threaded needle passed blunt end first, so that the ligature shall inelude the entire ligament exeept the upper and lower borders. A needle passed in this mamer is a convenient substitute for the needle with the eye in the point shown in $D$.

$B$. The needle has been drawn throngh the broad ligament, learing the ligature in place ready to be tied.

C. The ligature has been tightly tied and is being seeured against possible slipping by an additional stiteh on the proximal side of it; the entire ligature when tied is shown in Figure 135. Observe that the ligature does not eompress the entire ligament, but leaves ont a margin on the upper and lower border, so that eollateral eireulation may continue to supply and keep alive the distal portion of the stump; this prevents gangrene and sloughing of the stump, and is therefore a rery essential feature of the operation.

$D$ shows the form of needle in general use for ligature of the broad ligament. The device of an ordinary needle passed by a needle-foreeps, blunt end first, is more simple, and beenuse any size, eurve, or form of needle may be used at a constantly varying angle to the forceps, is more pratical. 
Figure 134

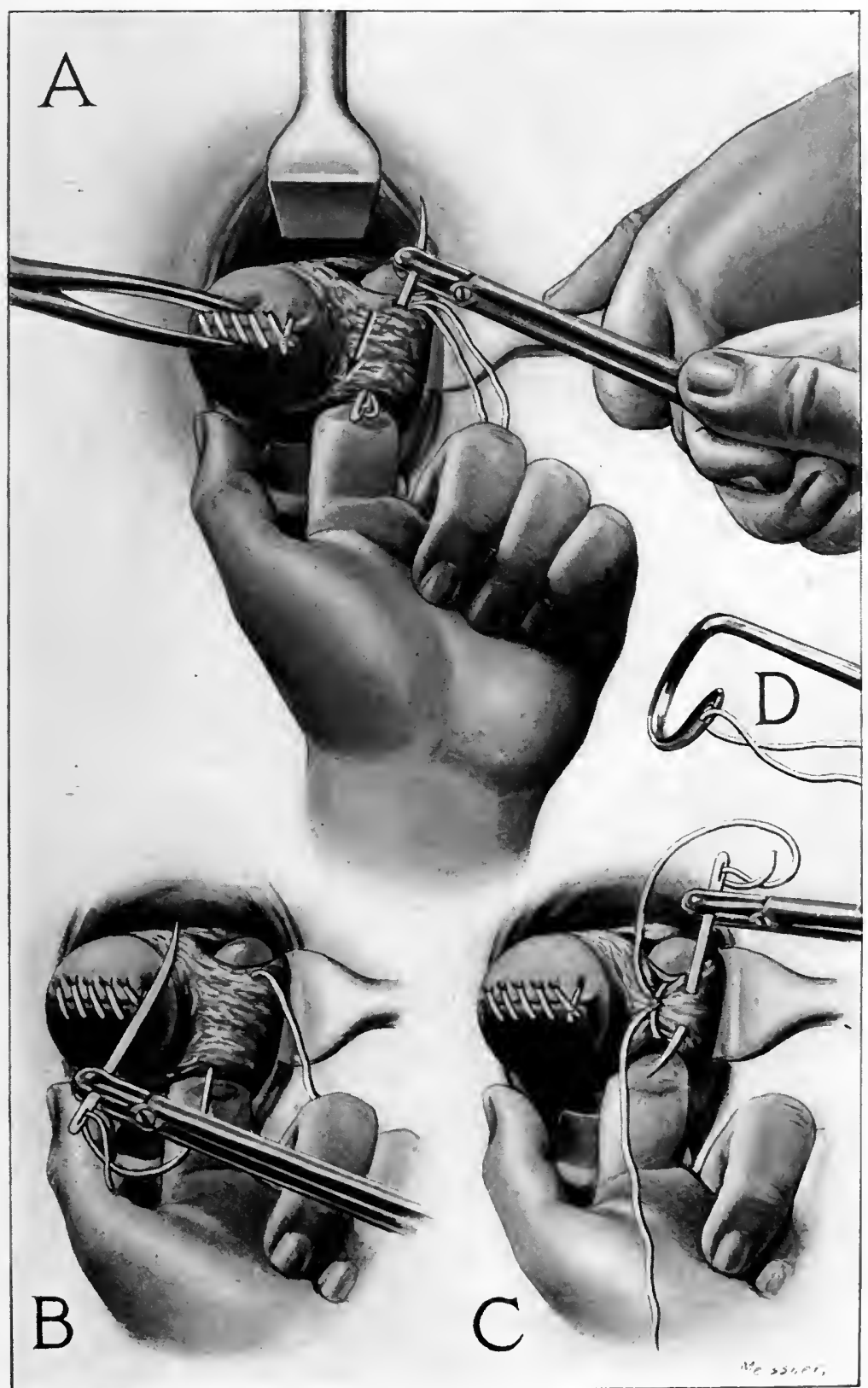




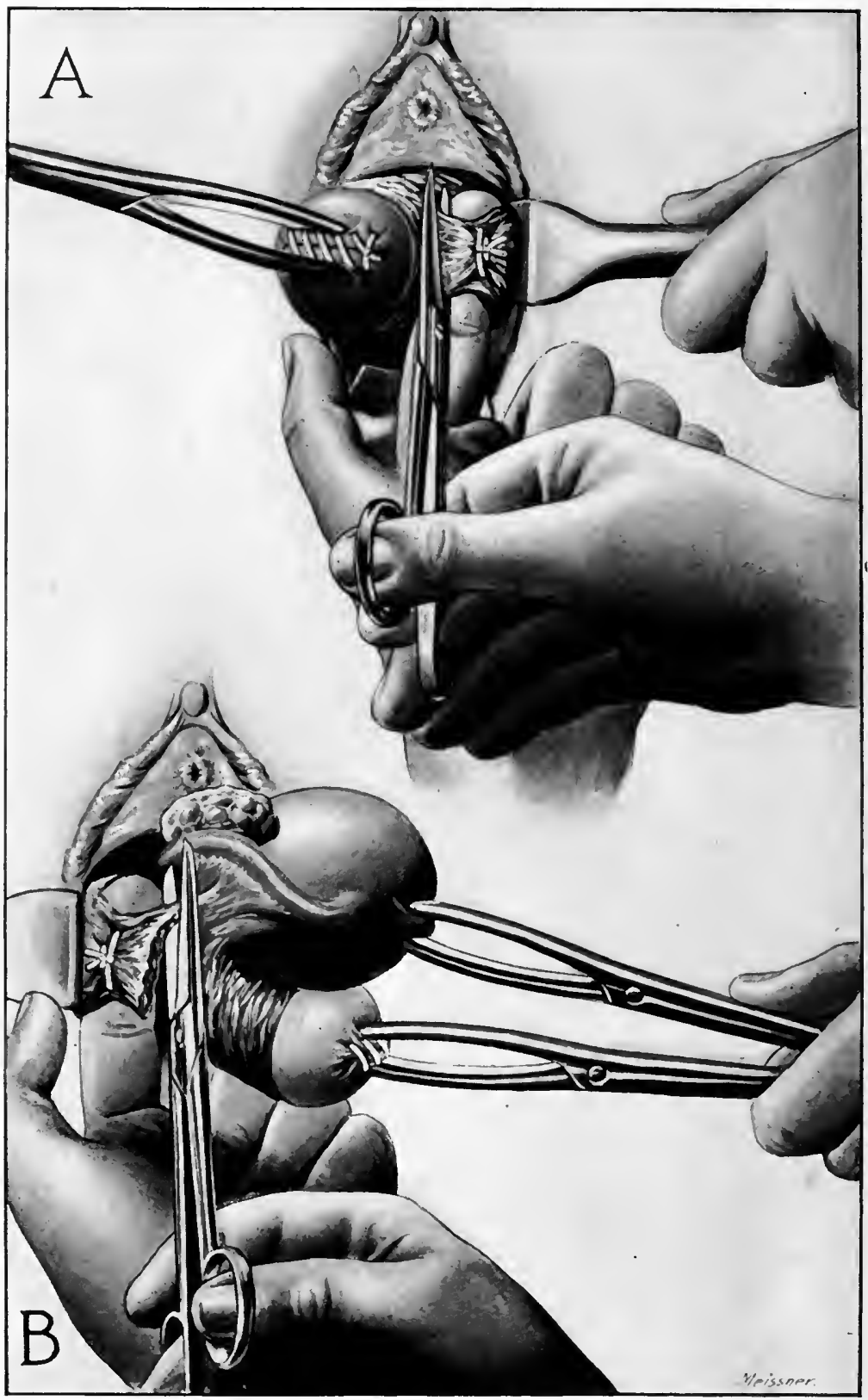




\section{Explanation of Figure 135.}

Vaginal Hysterectomy. The patient is in the doral position.

The vagina and cervix uteri are exposed by retractors in the lands of assistants.

The os uteri externum has been closed by a continuous suture to protect the operation wound and the peritoneum from the uterine secretions.

A. The cervix uteri is drawn strongly downward and to one side by vulsellum forceps in the hand of an assistant; the left broad ligament halving been ligatured en masse as shown in Figure 134, is exposed by the operator's left index-finger and is cut from the uterus about one-half inch from the ligature with scissors in the operator's right hand.

$B$. The uterus having been freed from its attachment anteriorly, posteriorly, and on the left, is drawn outside, and the corpus is seized with another pair of forceps; these forceps, together with those on the cervix, are placed in the hand of an assistant, who makes traction on them, thus pulling the doubled uterus strongly downward and to one side, while the operator ligatures en masse and severs the right broad ligament in a manner precisely similar to that already described for tying and cutting the left; the uterus having thus been removed, the vaginal wound is closed by interrupted or running catgut sutures, as shown in Figure 137. These sutures may secure both the peritoneal and vaginal margins of the wound or only the peritoneal margins; in either case they shonld so include the ligatured stumps of the broad ligaments as to fix them in the wound where they nuy give normal support to the rectum, vagina, and blarlder. If drainage is required, the wound should be left partially or wholly open for that purpose. The central third of the wonnd, if not sutured, will ustally suffice for drainage. The gatze drain is commonly preferred. 
Explanation of Figure 136.

In the majority of eases it is impracticable to include the entire broad ligament in a eingle ligature, and it is necessary therefore to tie it in parts; this is called progressive ligature of the broad ligament.

$A$ shows the broad ligament being progressively tied from the lower to the upper margin; the first ligature is being introduced on the lower nurgin. As each ligature is introduced, the ligatured portion is cut until the entire ligament is severed from the uterins.

B. In some cases the ligament is too inaccessible for progressive ligature from the lower to the upper margin; then the eorpus uteri may be delivered through the anterior vaginal wound and drawn ly strong forceps to the rnlva, so as to twist the ligament on itself and thereby reduce the size of it and render it accessible for progressive ligature from the upper to the lower border; the beginning of such a ligature is here shown.

C. In some eases the liganents are inaecessible for ligature in the manners deseribed under $A$ and $B$. The uterus may then be seized by two strong foreeps, one on either side of the cerrix, drawn strongly toward the rulva, bisected with scissors in the median line of the longitudinal axis, and each half drawn outside; the ligaments may then be ligatured and the uterns thus removed in two parts. Resection of the uterus should be avoided when possible, for it exposes the wound to infection from the endometrium. 
Figdre 136

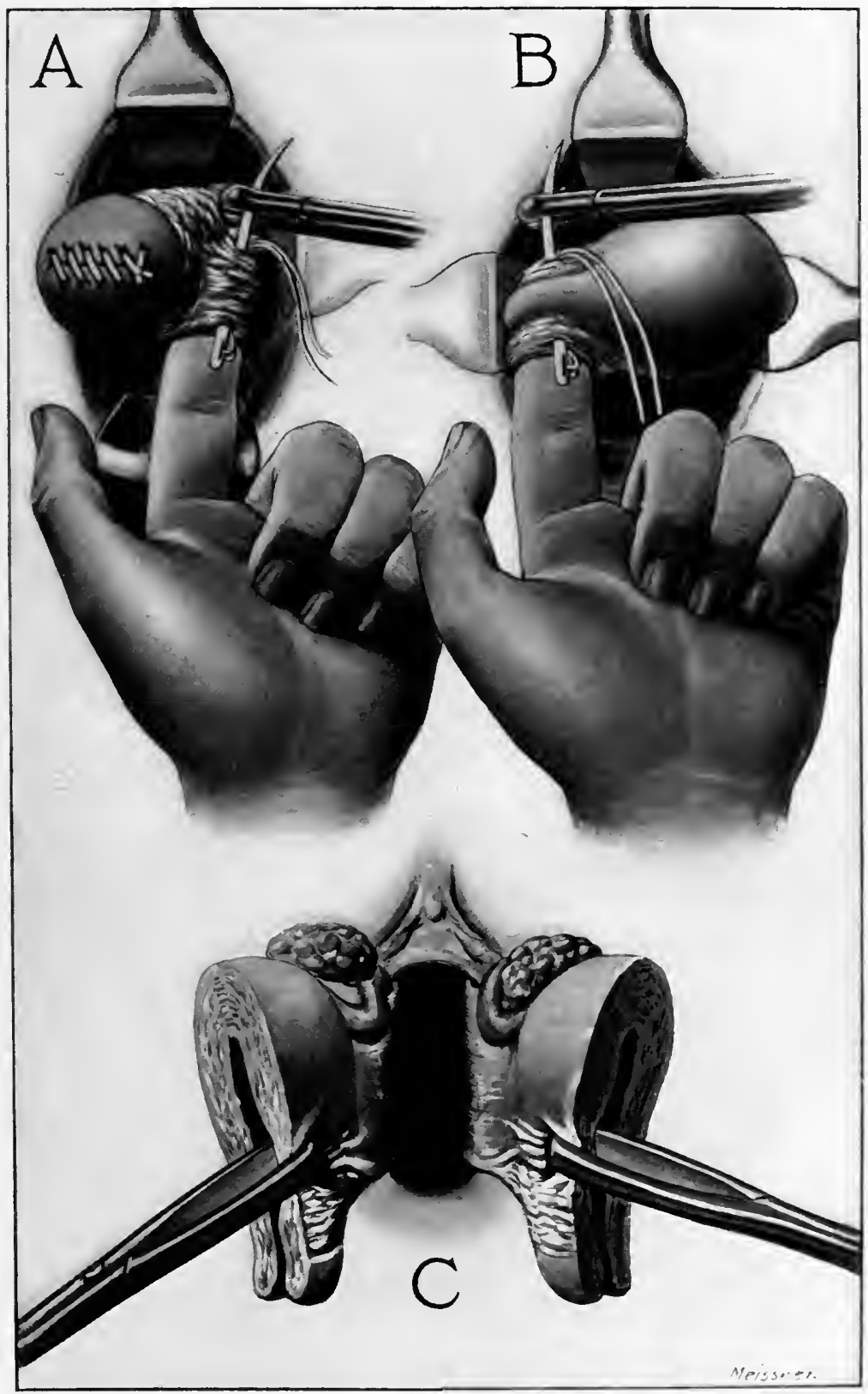




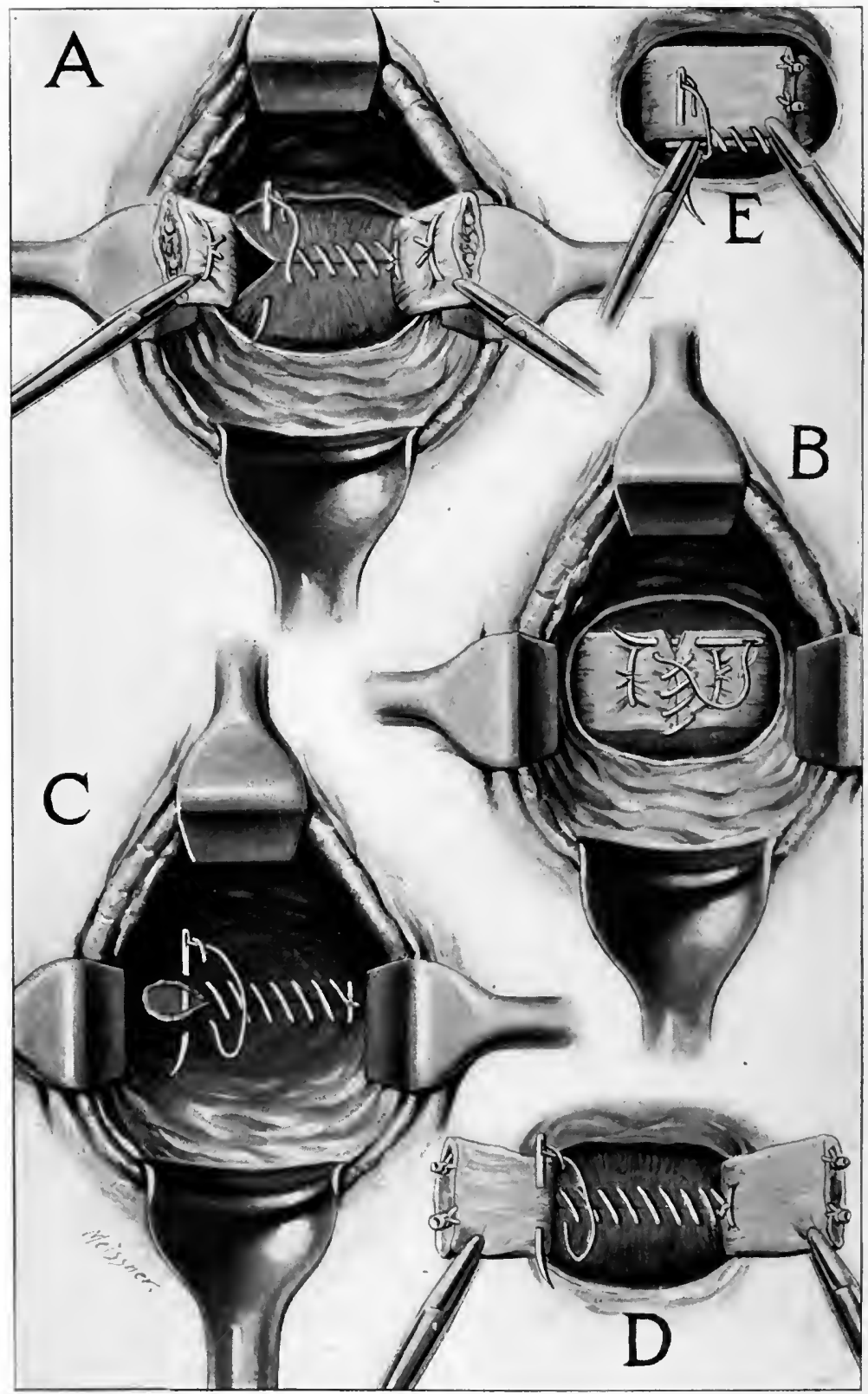




\section{Explasation of Figure 137.}

In most cases the ligatured stumps of the broad ligaments can be drawn down into the vagina. In such cases the usual method lias been to fasten them by sutures at each end of the elosed raginal wound, in such a way that the ligatured stumps shall be in the vagina below the level of the vaginal wound. The ligatures are applied by niany operators en masse around the entire ligaments in such a way that the ligatured portion will slough. It is sometimes possible to apply ligatures so that no sloughing can occur; that is, to let the ligatures include only that portion of the ligament tbrough which the arteries pass. This plate shows a very practical method of treating the ligitured ends of the broad ligaments in such a manner as to uvoid sloughing of the ligatured stumps and to fix them in the raginal wound. The method here illustrated is applicable only to those cases in which the ligaments are sufficiently long to permit either end-to-end approximation or the folding of one upon the other and the fixation of them in the raginal wound between the vaginal and peritoneal sides of it.

4 . The ligaments having been ligatured en masse in such a manner as to avoid sloughing of the ligatured stumps, are drawn down into the vagina by means of pressure-forceps. The anterior peritoneal margin of the vaginal wonnd is being united to the posterior margin by a continuous eatgut suture. At both ends of the line of union this continuous suture secures the broad ligaments, so that they cannot slip back into the pelvic carity. Only one ligature is here shown on each broatd ligament. In the majority of cases more than one ligature may be required.

$B$. The anterior and posterior peritoneal margins having been united, as shown in $A$, the broad ligaments are brought together by end-to-end approximation and united by a continuous catgut suture. The united ends of the broad ligaments are now in contact with, and in front of, the united peritoneal margins, shown in $A$.

$C$. The anterior and posterior margins of the peritoneal wound have been united, and the broad ligaments have been approximated end-to-end by continuous sutures, as shown in $A$ and $B$. The anterior and posterior margins of the vaginal mucosa are being united by a continuous catgut suture, making a line of union from side to side. This suture completes the operation.

D. In some cases the broad ligaments are so long that instead of uniting them end-to-end they may be folded one upon the other, and so fastened together. The anterior and posterior peritoneal margins have been united in precisely the same manner, as shown in $A$.

$E$. The anterior and posterior peritoneal margins of the raginal wound lave been united by a transverse line of union, as shown in $A$ and $D$. The ends of the broad ligaments have been folded upon themselves, and are being united by a continuous catgut suture along the lower borders of them. A similar suture is to be introduced along the npper borders. The ligaments having thus been united, are to be corered by union of the upper and lower margins of the vaginal mucosa, as shown in $C$.

The method of fixing the ends of the broad ligaments between the peritoneal and raginal sides of the wound will be found, when practicable, to have great value, for the ligaments so fixed can then perform the important function of holding the pelvic riscera high up in the pelvis and of preventing prolapse of the pelvic floor (rectum, vagina, and bladder), a not uncommon and most unfortumate result of vaginal hysterectomy when performed by the older methods.

The method of overlapping the ligaments will always be possible in the operation of vaginal hysterectomy when performed for complete procidentia uteri, and is strongly: urged in that class of cases; when the ligaments are not sufticiently long for end-to-end approximation, they may be fixed in the vaginal wound, as descrihed in the text, or if not sufficiently long for this, may have to be returned to the pelvic cavity.

Observe in $E$ and $D$ the isolated ligature of the arteries. This form of ligature will usually be quite practieable, except for-very short and very large ligaments, and when practicable should always be employed, because it insures normal cireulation in the stumps and is an absolnte safeguard against sloughing. It should, however, be remembered that in hysterectomy for cancer there is a decided advantage in removing as much of the ligament as possible; hence, the ligature en masse in silch cases may be preferable. 


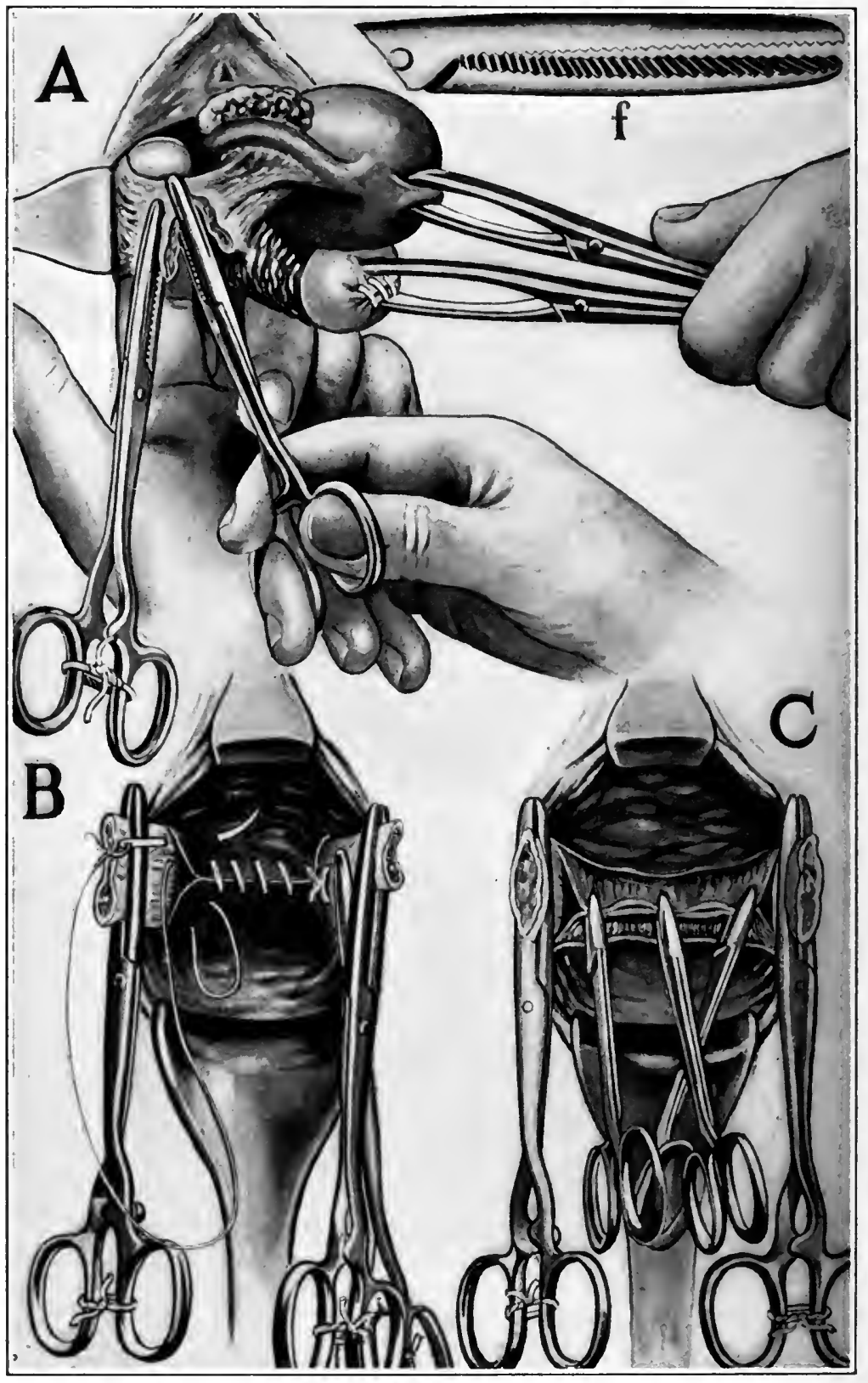




\section{Explanation of Figure 138.}

A. Same as Figure 135, B, except that forceps are used for hæmostasis instead of ligatures. One pair of forceps has been applied to the right broad ligament and the ligament has been severed partially by means of scissors. The second pair of forceps is being applied previous to cutting the ligament entirely away from the uterus. The removal of the uterus is accomplished when the same procedure has been repeated on the other broad ligament. Figure f shows full size of the jaw of the pressure-forceps here used.

B. Hæmostasis secured by one pair of pressure-forceps on the right ligament and two pairs on the left ligament. The vaginal wound is being closed by continuous catgut sutures. The peritoneal layer has been closed. The vaginal layer is being closed. Observe that the suture includes not only the margins of the wound, but also the ligament.

C. The peritoneal margins of the wound are here being held together by means of small hæmostatic forceps, which are used in place of sutures. The method of closure with forceps is indicated when, for any reason, such, for example, as drainage, the wound should not be closed entirely, or when the low condition of the patient does not permit the operation to be prolonged by suturing. The forceps here hold the peritoneal margins together and serve to keep intestines and other abdominal viscera from protruding into the vagina. When forceps are used in this way the raginal margins of the wound are left open, and the vagina is packed with gauze. All forceps and gauze should be removed within three days, and after the removal of the gauze the vagina should be gently douched twice daily with a 0.25 per cent. solution of lysol. The vulva should be dressed antiseptically. 
in the vagina is occupied by foreeps as to impede the operator, a single forceps may be applied back of two or more, and the latter then removed. Some operators leave the vaginal wound open for drainage with or without gauze packing. If no packing is used, the peritoneal margins of the wound usually fall together and promptly unite. Numerous cases, however, of annoying intestinal adhesions, protrusion of the bowel, fecal fistula, intestinal obstruction, and peritonitis prove the danger of the open treatment. The wound may be closed by the continuous or interrupted catgut suture, or, as shown in Figure 138, E, by small pressure-forceps. If drainage is required, a small rope of twisted gauze or a rubber tube, or both, may be inserted between the sutures preeisely as would be done in closing any other wound. The vagina then is packed lightly with gauze, and absorbent dressing is secured to the vulva by a T-bandage, and changed sufficiently often to keep it dry.

Whenever practicable the broad ligament stumps should be drawn down into the vagina and fixed there by eatgut sutures, so that everything included in the bite of the forceps may be in the vagina. The advantage of this is twofold: 1. All traumatisms, except the simple peritoneal wound, are excluded from the peritonemm. 2. The ligaments, when united to the upper end of the vagina, support the pelvic floor, and with it the rectum, bladder, and vagina, so that enterocele vaginalis is prevented. See Figure 138.

Accidents of Vaginal Hysterectomy.-Reetovaginal and vesicovaginal fistulæ are among the accidents of vaginal liysterectomy. Should either of these accidents occur, it is only necessary to use additional interrupted sutures at the point of the fistula in uniting the peritoneal edges to the vaginal edges of the wound anterior and posterior to the uterus by the whip-stitch already described. These sutures should not be buried, but should include the peritoneal and - vaginal margins so that peritoneum will cover the fistula. The strong tendency of peritoneal surfaces to adhere to any exposed surface renders closure of the fistula by this means almost eertain.

After-treatment of Vaginal Hysterectomy.-The general procedure in after-treatment differs in nothing from that of ordinary abdominal section. The forceps and vaginal gauze and the drain, if there be one, should be removed at the end of forty-eight hours, and a 0.5 per cent. lysol douche given. If the wound has been left open and packed with gauze, great care should be used lest in the removal of the ganze a loop of intestine be drawn into the vagina. The douche may be repeated daily, or, if the discharges are fetid, oftener. Let the douche be a weak current, lest it force its way through the fresh adhesions into the general peritoneum.

Relative Merits of the Ligature and Forceps Operation.-The advantage of pressure-forceps over the ligature are: 1. The greater facility of application very materially shortens the operation ; therefore in a difficult case, with inacessible broad ligaments, they are safer. 2. The forceps may be made to grasp a considerable portion of the broad ligament; the ligament may be drawn down and grasped 
further back by other forceps; more of the ligament may in this way be included than would be possible with the ligature. Whatever the forceps grasp will slough; by this means a very large portion of the ligament may be destroyed. This would be a more important consideration if the operation were being performed for malignant disease. Some part of the disease which the ligature might have missed therefore may be removed with the slough. 3. The forceps facilitate drainage. The secretions find their way out along the solid instrument by continuity of surface. 4. If the forceps are constructed and applied properly, the security against secondary hemorrhage is almost absolute.

The disadvantages of the forceps as compared with the ligature are: 1. They cause great suffering to the patient. 2. Their removal is painful. 3. Convalescence is apt to be more protracted and complicated.

Both the ligature and the forcipressure operations are efficient and satisfactory; therefore, whichever is most convenient or will most facilitate the operation should be used. 'The forceps will often be preferable in grave cases, especially when the ligaments are very thick and inaccessible. Both methods may be useful in the same case.

Combined Operation of Abdominal and Vaginal Section.When the vulva and vagina are small and the uterus is large, high in the pelvis, or fixed, removal of it through the vagina will be very difficult. Under these conditions, after making the vaginal incisions and separating the cervix from its surroundings, as already described, the operation may be finished better through an abdominal opening. The technique is the same as that described for hysteromyomectomy.

The difficulties of vaginal hysterectomy are much increased when the uterus and its appendages are fixed by adhesions. The uterus being drawn down and steadied by the vulsellum forceps in the right hand, the adhesions are broken up by the finger precisely as in the operation already described for removal of the appendages. The adherent appendages having been freed, the operation proceeds as if there had been no adhesions.

Drainage.-The class of cases discussed above offers a large field for drainage. Gauze is preferable to tubular drainage. The technique of vaginal drainage is described in Chapter VII. The clamps, if left on the broad ligaments for hæmostasis, also in a measure serve the purpose of drainage. They should be removed, however, at the end of forty-eight to seventy-two hours.

Hysterectomy without Removal of the Appendages.-When the appendages are firmly matted and bound together, and almost inseparable from the surrounding structures, and their removal is practically forbidden by the desperate risk of the operation, the uterus may be removed and the pus-sacs freely opened and left to drain into the vagina. Even if some pus-pockets are overlooked, they probably will break sooner or later into the wound. Such pus-sacs, whether tubal, ovarian, or parametric, when drained in this way, as a rule, undergo atrophy and become obliterated. Although this partial operation is only permissible for the reasons given above, yet it has 
been followed by entirely satisfactory results. An explanation in the following paragraph is submitted.

The removal of the appendages and consequent cutting off of the vascular and nervous connections of the uterus are followed usually by atrophy, cessation of function, and subsidence of disease in that organ; conversely, similar results in the Fallopian tubes and ovaries naturally should follow the cutting off of their vascular and nerve connections by the removal of the uterus. In one recorded case of hysterectomy the remaining tubes, however, became healthy and did not atrophy. On the contrary they were, during several years after the operation, the medium of apparent menstruation, the menstrual fluid passing from them into the vagina. ${ }^{1}$ This case speaks against the idea that the tubes do not participate in menstruation. In another case $^{2}$ pregnancy occurred in the isthmic portion of the tube; there was consequent hemorrhage into the vagina; the tubal opening was dilated and the product of conception curetted away.

\section{Aspiration of Hydrosalpinx through the Vagina.}

The contents of sactosalpinx serosa-liydrosalpinx-may be removed by aspiration, and permanent cure may result. As explained in Chapter XXI., occlusion of the ends of the distended tube may have occurred mechanically from swelling of the mucosa or organically from adhesive inflammation. Spontaneous reopening of the tube and restoration of its functions are probable under the former, improbable under the latter conditions.

Technique of Aspiration. - The diagnosis of sactosalpinx serosahydrosalpinx - having been made, the aspiration of it will include the following steps :

1. Make the vulva and vagina, so far as possible, aseptic.

2 . Locate the sac accurately by conjoined examination.

3. Keeping the right hand behind the pubes over the sac, and using the left index and middle fingers in the vagina as a guide to the point where the sac bulges most toward the vaginal fornix, push the aspirator needle into the sac with the left hand.

4. If fluid does not follow, withdraw the needle slightly and push it in again.

5. Having withdrawn the fluid, the action of the aspirator may be reversed and the sac refilled and again emptied two or three times with a 0.1 per cent. solution of formalin.

6. The aspiration and washing out of the sac having been completed, place a pledget of wool or gauze saturated with a 10 per cent. solution of ichthyol and glycerin against the cervix uteri.

The fluid having been removed, it should be subjected to a bacteriological examination, and if found sterile a permanent cure may be anticipated.

1 Weller Van Hook. Unpublished case.

2 Wendener. Centralblatt für Gynäkologie, 1896, No.4. p. 111. 


\section{Vaginal Incision and Drainage.}

Incision and drainage is a recognized procedure:

1. In the treatment of chronic sactosalpinx.

2. In the treatment of acute pelvic suppuration.

3. As a temporizing measure in grave eases.

1. Incision and Drainage for Chronic Sactosalpinx.-Incision and drainage of sactosalpinx is not expected, even when successful, to restore the function of the tube, but to produce instead complete obliteration of the lumen, thereby converting the tube into a cord. The same process sometimes occurs spontaneously as a result of recurring appendicitis or recurring salpingitis. The disease is then known as appendicitis obliterans or salpingitis obliterans, a result which incision and drainage may bring about or hasten.

There will always be great difficulty in drawing the line between those cases which may be relieved by the operation and those which demand extirpation of the diseased organs. The former treatment will be applicable to the more recent and acute cases, the latter to the older chronic suppurative cases in which permanent changes have taken place. Notwithstanding the numerous successful cases reported by Vulliet, Landau, Goulliad, Abbott, and others, the operation is not very strongly indicated in chronic pyosalpinx. Success requires the removal of old and prevention of new infection; and the fulfilment of this requirement in the many possible cavities and recesses of a pus-tube, and in the neighboring pus-pockets whose walls are infected deeply, is often beyond the power of simple drainage and disinfection.

This operation, which had been nearly obsolete for twenty years, was revived after the development of aseptic abdominal surgery. Before this time it had shown relatively few immediate cures and a discouraging number of failures to eut short tubal and ovarian suppuration. On the other hand, the more radical operation of extirpating the diseased organs has saved innumerable women from lifelong invalidism or death. The re-establishment of incision and drainage as a recognized and useful procedure has been made possible by the introduction of sharp uterine curettage and asepsis. The operration should include the thorough removal of any infection in the uterus by aseptic sharp curettage. Failure in this may lead to disastrous results.

When the distended Fallopian tube can be isolated by palpation, incision and drainage is performed as follows: First the vagina and vulva are disinfected thoroughly, the patient being in the lithotomy position; the sactosalpinx by steady pressure of the assistant's hand is now fixed downward toward the vagina, and a trocar properly curved or straight, guided by the left index-finger, is introduced into the sac. On this trocar as a guide, sharp-pointed scissors are used to enlarge the opening by working their point through the wall with altermate spreading and closing of the blades until the finger ean be introduced into this abscess cavity. The sac is washed out with a $1: 1000$ solution of formalin. 
The drainage may be capillary or tubular as described in Chapter VII. On account of a strong tendency for the vaginal opening to close, it may be necessary every few days to insert the blades of a long hæmostatic forceps and separate them by spreading the handles. After the first week or two the drains may be removed. The vagina should be kept clean by means of antiseptic douches.

The prognosis after incision and drainage is better for hydrosalpinx than for pyosalpinx and best for cellulitic abscess.

Tubercular Suppuration offers great resistance to all conservative measures, and therefore is admitted generally to be an indication for the removal of the uterus and its appendages. The great frequency of chronic tubercular infection materially cuts down the number of cases suitable for drainage. It is, moreover, usually difficult to recognize and exclude the tubercular cases until the diseased tissue has been removed and examined. The pus is often sterile. The suggestion

\section{Figure 139.}

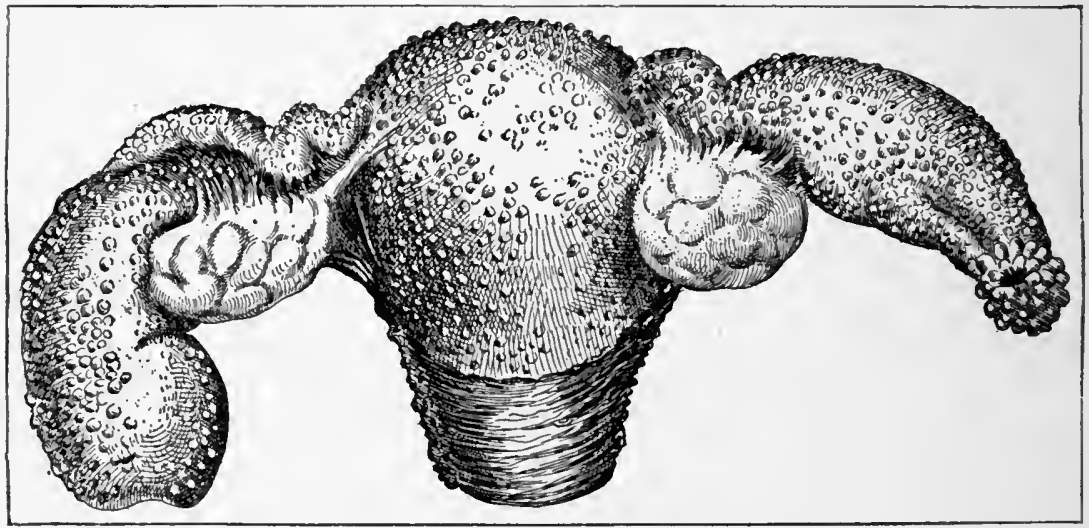

Tubercular perimetritis and sulpingitis; sactosalpinx. Uterus and appendages removed complete.

to defer the radical operation until conservative measures have been tried and failed, is weakened by the fact that after incision and arainage removal of the diseased organs is always more difficult, tedious, and dangerous.

2. Incision and Drainage for Acute Pelvic Suppuration. ${ }^{1-}$ The pelvic organs and products of inflammation may be so matted and fused together in a conglomerate mass that the individual organs are unrecognizable and the patient's general state from septic poisoning may be so grave as to prohibit a radical operation. In such conditions, whether the suppuration be tubal, ovarian, or parametric, or all combined, vaginal incision and drainage offer the following advantages : 1. Relative freedon from mortality. 2. Probable preservation and possible restoration to function of the diseased organs.

The operation begins with preliminary sharp curettage, Chapter

1 Fernand Henrotin, "The Conservative Surgical Treatment of Para- and Peri-uterine Septie Discase." Transactions of the American Gynccological Society, 1885. Adaptation. 
XXVII, and is continued as follows: The incision is made behind the uterus, as already described in this chapter, for posterior vaginal section, and the finger is introduced into Douglas's pouch, which generally is shut off from the general peritoneal cavity by the adhesions. If the post-uterine circular incision gives too little space for thorough intrapelvic exploration and manipulation, an additional perpendicular incision may be made from the centre of the first incision, Figure 131, in the median line of the posterior vaginal wall from the cervix uteri toward the rectum. During the making of the second incision the index-finger of the left hand should be in the rectum as a guide to prevent wounding the bowel. This finger, after thorough disinfection and change of rubber glove, now being returned to the pouch

Figure 140.

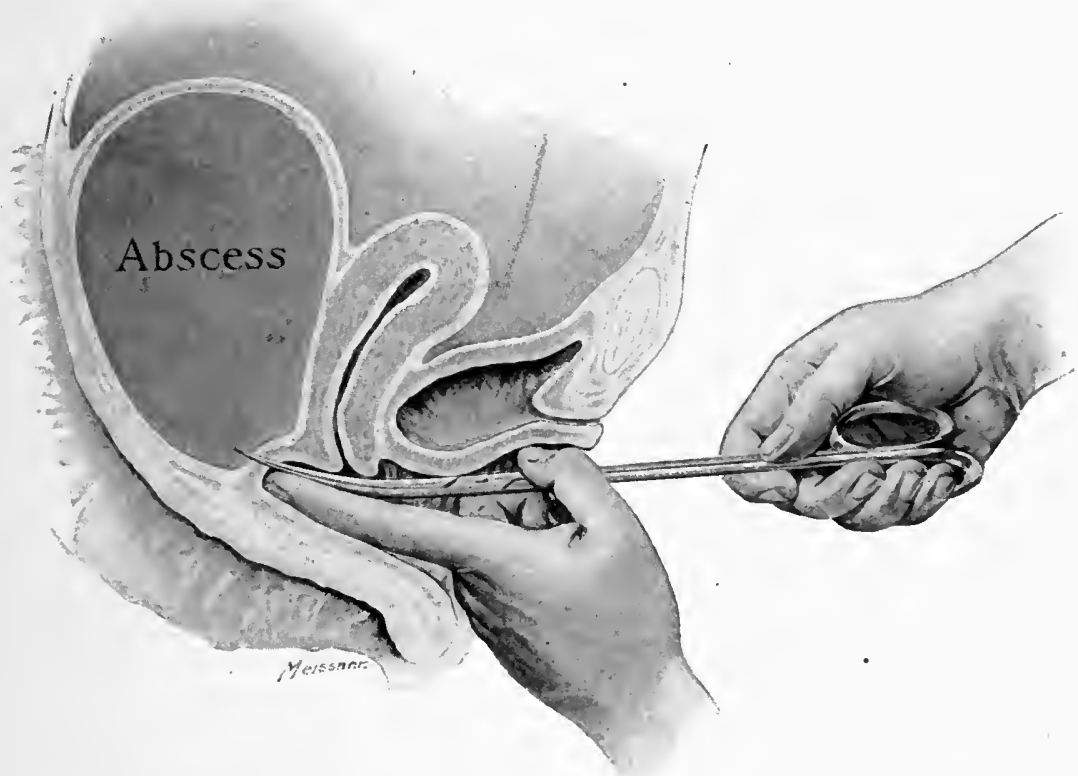

Incision through the vagina of a pelvic abscess with sharp-pointed scissors.

of Douglas, and the right hand being over the abdomen, the examination proceeds as in the ordinary bimanual palpation. The cxamining finger penetrates backward and to either side until the bimanual sensation indicates that the free peritoneum almost is reached. In shifting the finger to the right or to the left, and with it the superimposed hand, the septic mass usually will be found and penetrated without difficulty.

In most cases the infiltrated material will be evident to the touch of the examining finger and an abscess-cavity usually will be found. The accidental opening of the peritoneal cavity during these manipulations does not materially increase the danger; but if this accident 
occurs, it is well to retain the finger in the opening leading to the abscess until any escaping pus may be washed out of the vagina and the peritoneal eavity protected by gauze packing against the inflowing of pus. The finger should then be withdrawn and the puscavity evacuated. Gentle pressure upon the abdominal wall will help to empty the abscess. The protective packing in the peritoneal cavity is replaced now by fresh gauze and the finger reintroduced into the pus-cavity. This is for the purpose of finding and emptying any adjacent abscesses. Both sides of the pelvis having been thoronghly explored in this way and all hard inflammatory masses, whether pus-containing or not, having been penetrated by the finger, the cavities thus opened should be packed with a single strip of sterilized gauze about three inches wide, the outer end of which should be retained carefully in the vagina to facilitate removal. It is well also to pass into the vaginal wound by the side of the gauze a large rubber tube in order to insure efficient drainage. The operation is completed by the application of a light vaginal gauze tampon and by a pad over the vulva winich should be held in place by a T-bandage. No instrument excepting the finger should be used after the preliminary incision through the vaginal wall has been made.

The inflammatory deposit in many cases will be found in the median line posterior to the uterus; in other cases it will be found on one or both sides. In such cases the finger cannot be worked back in the median line very far without penetrating the abdominal cavity, but turning it to either side it may usually be made to separate the layers of the broad ligament, and without invading the peritoneum at all mav be pushed into the lateral masses.

3. Incision and Drainage as a Temporizing Measure.-In acute. or chronic pelvic suppuration when the condition of the patient is so grave as to prohibit a more radical operation not excluding even tubercular cases which generally require removal of the uterus and its appendages (pan-hysterectomy), incision and drainage is indicated; aid, though performed as a temporizing measure, there is usually such prompt and pronounced improvement as to pernit a radical operation later.

The mortaliy of opening into the peritoneal cavity by either route in acute suppuration before the pus has had time to become sterile is excessive. The withdrawal of such cases from laparotomy statistics and the relegation of them to the statistics of vaginal incision and drainage would reduce enormously the mortality of abdominal surgery.

\section{Relative Advantages and Disadvantages of the Abdominal and Vaginal Routes in Pelvic Surgery.}

Advantages of the Abdominal Route.-1. There is a larger field for operation.

2. The operator may see what he is doing, instead of depending largely on the sense of touch.

3. The diagnosis of unsuspected conditions and complications is much easier. 
4. It is adapted to large tumors and pus-sacs, and to conditions high in the pelvis.

5. The appendages may be removed with better chance of avoiding rupture of a pus-sac.

6. There is less danger of wounding intestine, bladder, or ureter, and greater facility in the control of hemorrhage.

7. Appendicitis and other abdominal lesions so often complicating pelvic disease are difficult to reach by the vagina.

8. It gives more light and more space for conservative work.

Advantages Claimed for the Vaginal Route.-1. It gives better drainage, and therefore is adapted specially to complicating vesical or intestinal fistula.

2. It avoids the abdominal scar and the risk of ventral hernia.

3. It is suitable for cases of small tumors without high adhesions.

4. When properly performed, it involves less danger from shock, and therefore is suited to cases of extreme pelvic infiltration and adhesions for which the abdominal route is extra-hazardous.

5. It involves less handling of the intestines, and therefore less consequent danger of shock and intestinal adhesions.

6. Recovery is less complicated and more rapid.

Unfortunately, the vaginal route is, for a large proportion of cases, impracticable. 'The long, narrow virgin vagina or the vagina which has become contracted from senile atrophy may render the field of operation almost inaccessible. A very large uterus with exceptionally short, thick, broad ligaments and greatly enlarged appendages, with adhesions extending beyond the reach of the finger, may also be difficult or impossible to manipulate through the vagina. Under such conditions the abdominal route is much safer.

In some cases it is well to begin the operation in the vagina and continue by that ronte as far as the greatest safety will permit, and then, if necessary, open the abdomen and complete the operation by the combined vaginal and abdominal method. Conversely, abdominal section may have to be supplemented by vaginal section. 'The consbined operation may be the deliberate purpose from the beginning, or the necessity for it may become apparent only in the course of the operation.

In some cases the advantages of the two routes are balanced so evenly that either is permissible; the election then must rest with the individual bias of the surgeon. The choice of procedures has in a measure been forecast in the description of speeial operations already set forth.

It will be seen from the above that each method has its special advantages and disadvantages. Some of these last arc less real than they seem; for example, an objection to vaginal hysterectomy is that it affords only a limited field of operation and small chance for visual control of the work. This objection does not necessarily appeal to the skilled operator. The danger of hemorrhage is avoidable if due precautions are used. Injuries of the bladder, ureters, and intestine may occur with either method, but in vaginal hysterectomy the perfect drainage makes them less dangerous if they do occur. 
The operator should not permit his prejudice in favor of either route to lead him to pursue it to the extreme, for that part of an operation which is easy by the vagina is often most difficult by the abdomen, and vice versa.

The vaginal ronte was for a time much in vogue, but at present, even among conservative surgeons, there is a tendency to return to the abdominal route.

\section{Conservative Operation on the Fallopian Tube. ${ }^{1}$}

Salpingo-stomatomie.-Resection of the Fallopian tubes, called salpingo-stomatomie, is designed in selected cases to sare and restore the appendages to their normal function, instead of removing them. August Martin reports 65 cases with two deaths, neither of which

Figure 141.

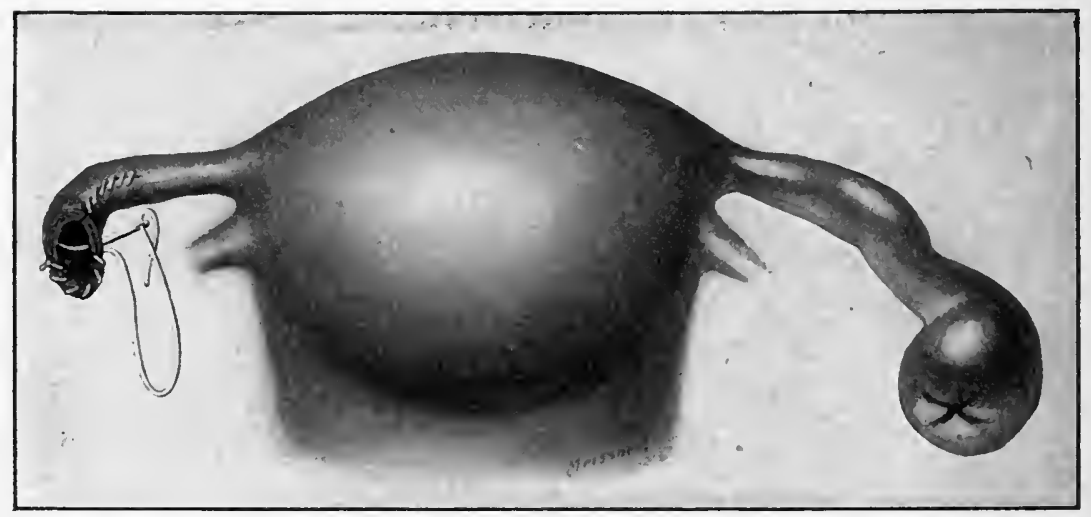

Resection of the tube. The distended ampulla to the right closed by adhesive inflammation. The ampulia of the tube to the left has been removed and the mucosa is being united to the serosa by a continuous suture; the longitudinal slit bas been closed by a continuous suture.

was attributable to the operation. In 1885 he began to open the closed abdominal ends of tubes and to study microscopically their contents and the condition of their walls. His method is as follows:

1. Bring the tube so far as possible up into the abdominal wound.

2. Protect the adjacent pelvic organs by sponge packing around the tube.

3. Open the end of the ampulla with scissors. The point of closure may be recognized by a scar in which the fimbrixe are still visible.

4. Strip the tube of fluid by pressure applied from the uterine toward the abdominal end.

5. If the contents be serous, odorless, and all fluid, and the mucosa shows only slight swelling and reddening, and the folds are flattened only by pressure, slit the tube for a distance of about one inch.

1 A. Martin. Die Krankheiten der Eileiter, p. 213. 
6. If the condition in the upper part of the tube still appears to be only catarrhal, close the longitudinal wound with three fine catgut sutures. Any large superfluous tags are to be cut off.

7. The borders of the tubal mucosa at the end of the tube and the peritoneum are to be united by fine catgut sutures so that the opening shall gape and the mucosa shall stay everted.

Henorrhage is slight and easily controlled by fine ligatures. The everting sutures at the end of the ampulla hold the new ostium close to the ovary. The now reopened tube, together with the ovary, is replaced in the abdomen. Any ovarian adhesions are to be broken up. According to Martin, this operation offers no greater danger

Figure 142.

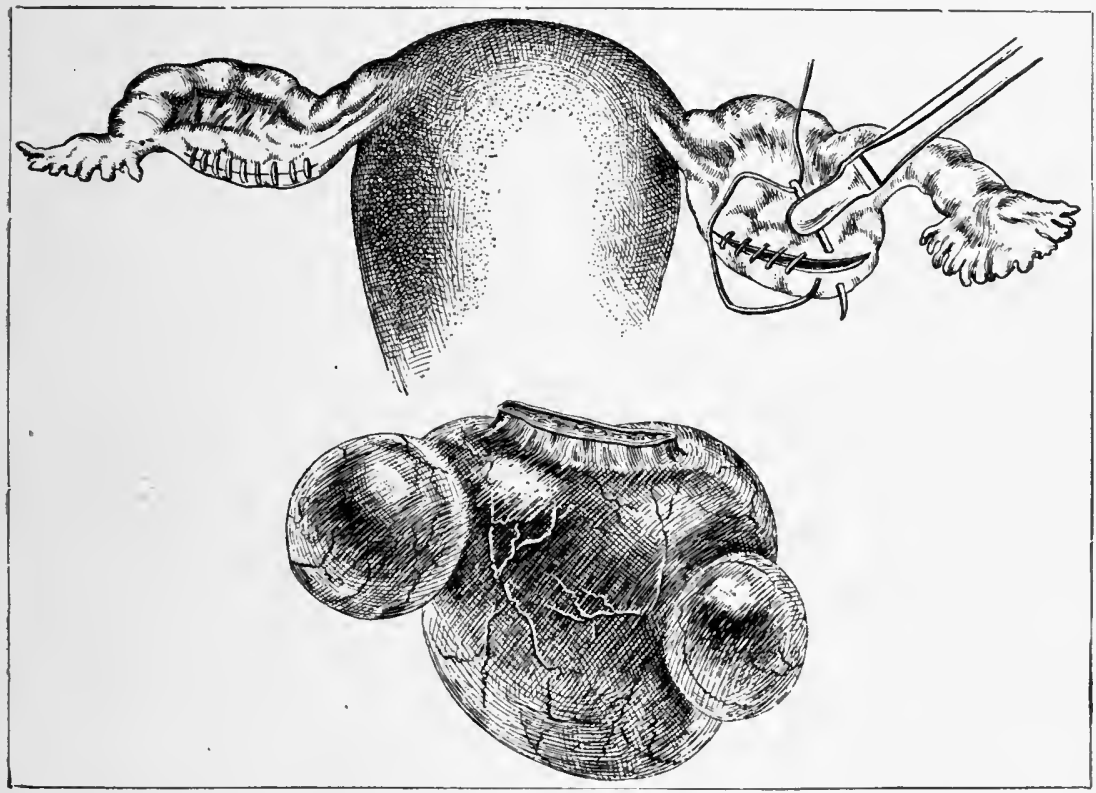

Resection of the ovary. Conservative operations on ovaries. Partial remoral of right ovary by resection. Nearly all of left ovary preserved. Small detached multiple cyst below left ovary has been removed from it. The ovaries show whip-stitches for closure of resection wounds.

than any other cœliotomy complicated by peritonitis. Pregnancy followed in two cases in which this operation had been performed on one side and the appendages had been extirpated on the other.

The general conclusion is that extirpation for atresia of tubes whose contents are not infections may be unjustifiable. 'The operation, however, can result in restoration of function only when the uterine end is open, or when, if closed, the closure is due to swelling and not to inflammatory adhesion. The surgeon should not venture to establish a communication between an occluded Fallopian tube and the abdominal cavity unless a smear taken from the secretion at the time of the operation proves the contents of the tube to be sterile. 


\section{Conservative Operation on the Ovary.}

Resection of the Ovary.-The diseased portion of an ovary may be removed by resection, and the remaining healthy part saved. The indications for resection are these :

1. The saving of a portion of the ovary in order to preserve its reproductive functions.

2. The saving of a portion of the ovary in order to preserve menstruation and other possibly important functions not definitely known, such as secretion and elimination.

Reproduction repeatedly has followed the operation when the uterus, the tube, and only a very small fragment of the ovary were left. The duty of the surgeon to leave for this purpose, when practicable, any functionating part of an ovary, is therefore clear. ${ }^{1}$ The preservation of menstruation and other possible functions is urged by many competent observers. As a rule, women are better off mentally and physically if menstruation and ovulation are maintained up to the period of nature's menopause. The possible secretory and eliminative functions of the ovary justify the operator in leaving it, or any healthy portion of it, even though the diseased tubes and uterus have to be removed. ${ }^{2}$

The Operation of Resection simply involves the excision by scalpel or scissors of the diseased portion and closure of the wound by means of fine interrupted or continuous catgut sutures.

All conservative operations for opening closed tubes and resection of ovaries should be supplemented by the release of the appendages from any adhesion which may be present.

Ovarian Extract or Desiccated Ovaries, which may be found in drug-shops prepared for internal administration, are recommended highly, and are said to give relief to the disagreeable symptoms of the menopause, whether induced by oöphorectomy or by nature.

${ }^{1}$ Polk. "Operations on the Uterine Appendages, with a View to Preserving the Functions of Ovulation and Mlenstruation." Transactions of the American Gynecological Soclety, 1893.

2 G. E. Curatulo. Secrezione Interna delle Ovaie, 1896. 


\section{CHAPTER XXIV.}

\section{URETHRITIS--URETHRITIS COMPLICATED BY PROLAPSE OF URETHRA-URETHRITIS COMPLICATED BY SUB- URETHRAL ABSCESS-CYSTITIS-PYELI'TIS.}

\section{URETHRITIS.}

\section{Etiology of Urethritis.}

The conditions which favor and excite urethritis are the same as for inflammation in general. The disease occurs by extension, most frequently from gonorrhœal, least frequently from syphilitic vulvovaginitis. It may be caused by the bacillus of diphtheria, the streptococcus, the staphylococcus, the bacillus tuberculosis, or by other bacteria which are known to infect the genitalia. Among the media of infection are: the unclean eatheter, sound, or hand; an unclean pessary, masturbation, and coitus. Among the favoring conditions are the passage of urinary calculi and irritation from new growths.

\section{Pathology and Diagnosis of Urethritis.}

The mucosa as exposed by the cystoscope-Chapter III.-is swollen and red from distention of the vessels, and, upon instrumental examination, may bleed. The inflamed urethral glands stand out prominently as oval yellow spots, and in the anterior part of the urethra sometimes give forth a secretion which looks like pus, but may be only epithelial débris. The tenderness in the milder infections is less marked than in the gonorrhœal variety.

Gonorrhoeal infection in the acute form is intense and somewhat characteristic. The swollen mucosa, at first of deep-red color and finally covered with pus, protrudes through the meatus, and has much the appearance of an inflamed, prolapsed anus. It is excessively sensitive to touch, and, especially when touched by an instrument, is apt to bleed. Burning and pain on urination may be intense. Microseopical examination of the pus will show gonococci. The eversion of the meatus usually disappears as the urethritis subsides.

Skene's Glands. - The urethral glands of Skene as described by himself are, in this connection, of great pathological significance. They consist of two glandular tubules situated one on cither side of the urethrovaginal wall. Each tubule extends, from a point just within the meatus urinarius, parallel to the urethra to a distance of about five-eighths of an inch. The tubules, lined with columnar epithelium, branch into the muscularis of the urethrovaginal wall. When the urethra is swollen and the meatus everted, the openings of the tubules 
appear just outside the urethra. The normally placed openings are seen on either side by separating the labia of the meatus urinarius.

When inflamed, these tubules give forth upon pressure a white: serous or purulent discharge. The mucous membrane around their openings, as in follieular pharyngitis, is swollen, thickened, and of a bright yellowish-gray color, or the orifices may be surrounded by a granular areola. The infection involves also the periglandular struetures. The urethrovaginal wall in the neighborhood of the tubules usually is swollen and everted. The inflammation is generally purulent, very often gonorrhœal, and may give rise to a free discharge. Occlusion of the tubules by adhesive inflammation and the consequent formation of retention-cysts is possible. There is often great tenderness on pressure. Chronic infection, as a rule, gives rise to little or no pain on urination. Inflammation in these glands, until described by Skene, had been mistaken for caruncle of the urethra. The brightred areola upon the swollen and thickened mucous membrane about the openings of the tubules closely resembles caruncle. 'The differentiation between inflammation of Skene's glands and caruncle of the urethra will become apparent on examination of the tabular statement below.

Inflammation of Skene's glands.

1. Urination not usually painful.

2. Two protuberances correspond to site of openings of tubules.
Caruncle of the Urethra.

1. Urination painful.

2. Usually only one protuberance situated anywhere in the circumference of the meatus or within the meatus, but usually on the posterior wall.

3. Removal of protuberances does not cure. 3. Removal cures.

4. Mouths of tubules inflamed.

4. Mouths of tubules normal.

The gonococens may become intrenched in these glands, as in the glands of Bartholin, and from time to time furnish infection for recurrent gonorrhœa. Even though the disease may have disappeared from the external surface, reinfection from the glands may oceur repeatedly. This source of reinfection, unless earefully sought, is liable to be overlooked. If the urethral glands are in a state of suppuration, the pus may be stripped out of them by pressure of the finger and a stroking motion against the urethrovaginal wall. Drops of pus are shown issuing from the ducts on digital pressure in Figure 149. A purulent diseharge from the urethra is strong clinical evidence of gonorrhoal infection. Tubereular infection of the glands has been observed repeatedly.

\section{Treatment of Urethritis.}

The milder non-gonorrhœal form, if not complieated by cystitis, may usually be cured promptly by a few applications made at intervals of four or five days of a 3 per cent. solution of silver nitrate. The application is made by an applicator wound with cotton, through a urethral speculum. Extreme forcible dilatation of the urethra has been much practised for the relief of this and the more intense forms of urethritis, and often has given prompt and pronounced relief. Per- 
manent injury to the urethra and eonsequent incurable incontincnee of urine have resulted, however, about three times in a hundred of such dilatations. The safe limit of dilatation is not over fire-eighths

FIGURE 143.

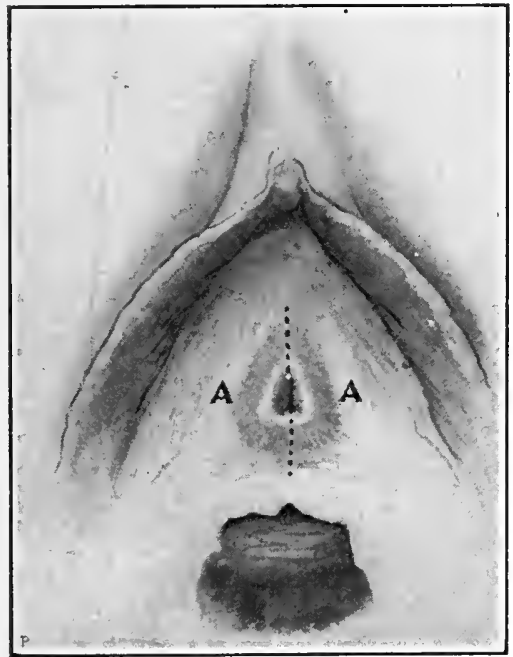

Figure 145.

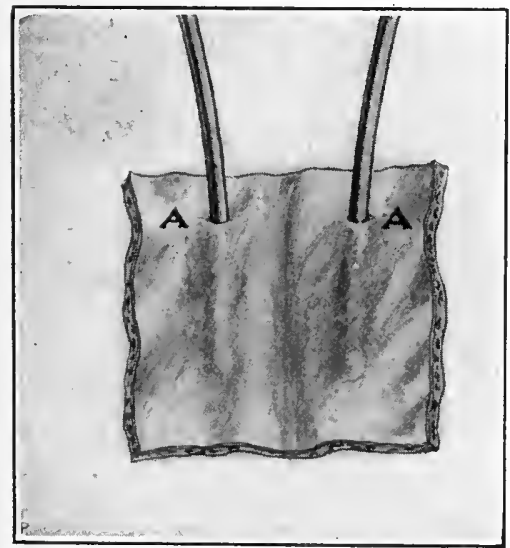

Figure 144.

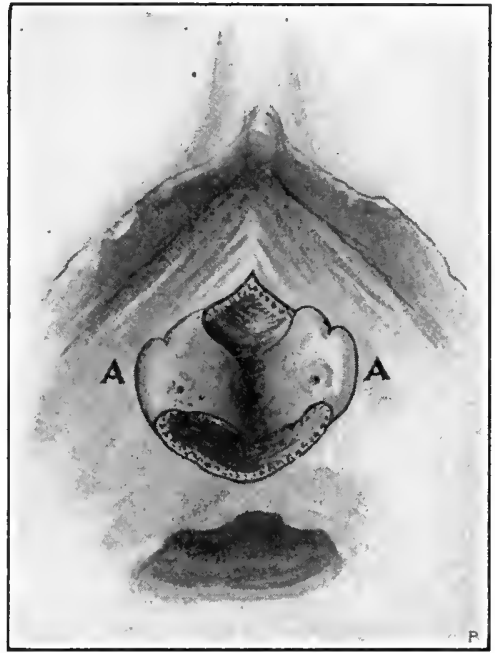

Figure 146.

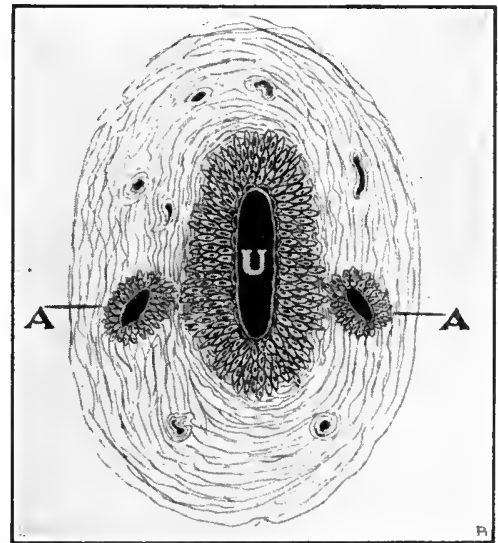

Figure 143.-Normal meatus urinarius. The openings of the duets leading from skene's glands are hidden just within the meatus in a line conneeting the letters $\mathrm{A}, \mathrm{A}$. The black and white dotted line indrcates the direction of an incision by which the meatus may be laid open, so as to expose the openings of the dnets.

Figure 144. - Meatus laid open by a vertieal ineision; the openings of the ducts luading from Skene's glands exposed. They are represented by black dots on a line drawn between the letters $\mathrm{A}, \mathrm{A}$.

Figure 145.- Urethral mucosa dissected ont and laid open by the ineision. Two fine probes are introduced into the ducts leading from Skene's glands, $A, A$.

Figure 146.-U, cross-section of urethra ; A, A, eross-scetion of skene's duets.

to three-fourths of an inch. Emmet's so-ealled button-hole operation, deseribed under stricture of the urethra, answers the therapentic indication of dilatation, and does not impair the retentive power; it 
also has the advantage of rendering the diseased mucosa accessible to direct local treatment. The opening may at any time be closed by interrupted sutures; but inasmuch as there is usually no functional impairment this repair seldom is called for.

FIGURE 147.

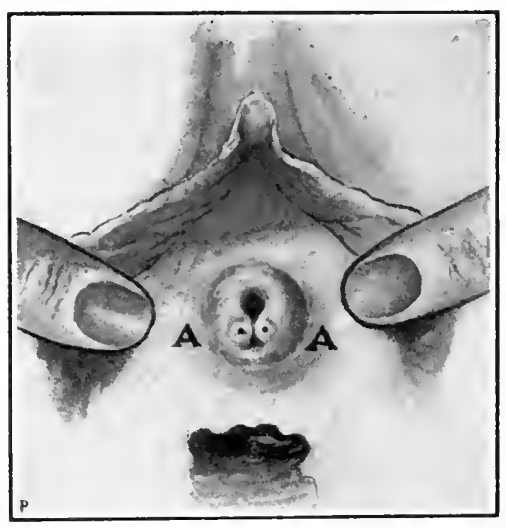

Figure 149.

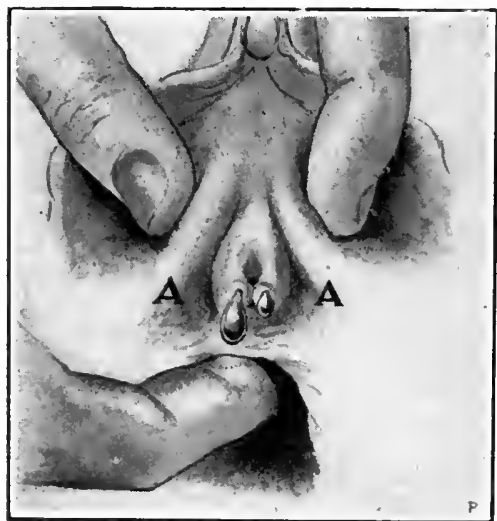

FIGURE 148.

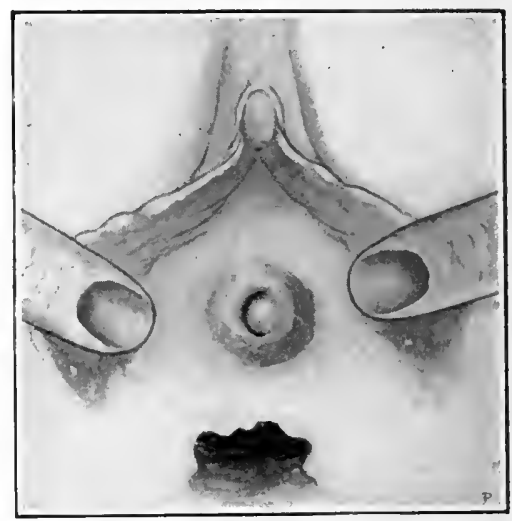

Figure 150.

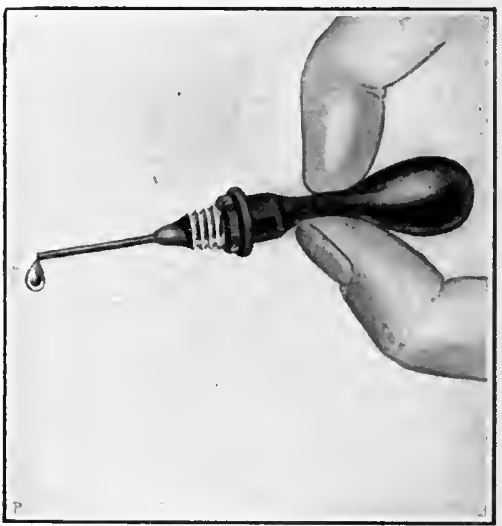

FIgUre 147.-A, A, the ducts leading from Skene's glands swollen and everted. The black dots represent the openings.

FIGURE 148. - I rethral carunele at one side of the meatus, simulating in appearance the swollen and everted sliene's duet. Ohserve the absence of the npening of a duet.

Frgtre 149.-Expression of nus from the ducts of Skene's glands.

Figure 150.-A large hypodermic syringe-needle with blunt point and a rubber bulb attaehed. This is intended as a pinette, by means of which may be injected into Skene's ducts medieinal substances for treatment of infection.

Gonorrhoal urethritis, if acute, is treated first by a single application of a 10 per cent. solution of silver nitrate, then by rest; compresses to the vulva, saturated with lead water and laudanum, or sedative suppositories in the rectum, may give relief. If the irritation is very great, the compress may be saturated with a 5 per cent. solution of cocaine muriate. 'The medicinal treatment will be the same as for gonorrhœa in the male. Urotropin is recommended highly. 
Chronic inflammation in Skene's glands, especially if gonorrhoal, usually resists all conservative measures. If it does not yield to the application of nitrate of silver fused on a fine probe, or to injections of disinfectants, the entire length of the tubules should be laid open on the vaginal side, using a probe as a guide. The glandular struetures are then to be destroyed by caustic or by excision with scissors, and the surfaces made to heal by granulation. To fuse the silver nitrate on the probe, let the salt be melted in a small receptacle over a spirit-lamp, and dip the end of the probe into it repeatedly so as to coat it over with a thin layer of the salt.

Figure 151.

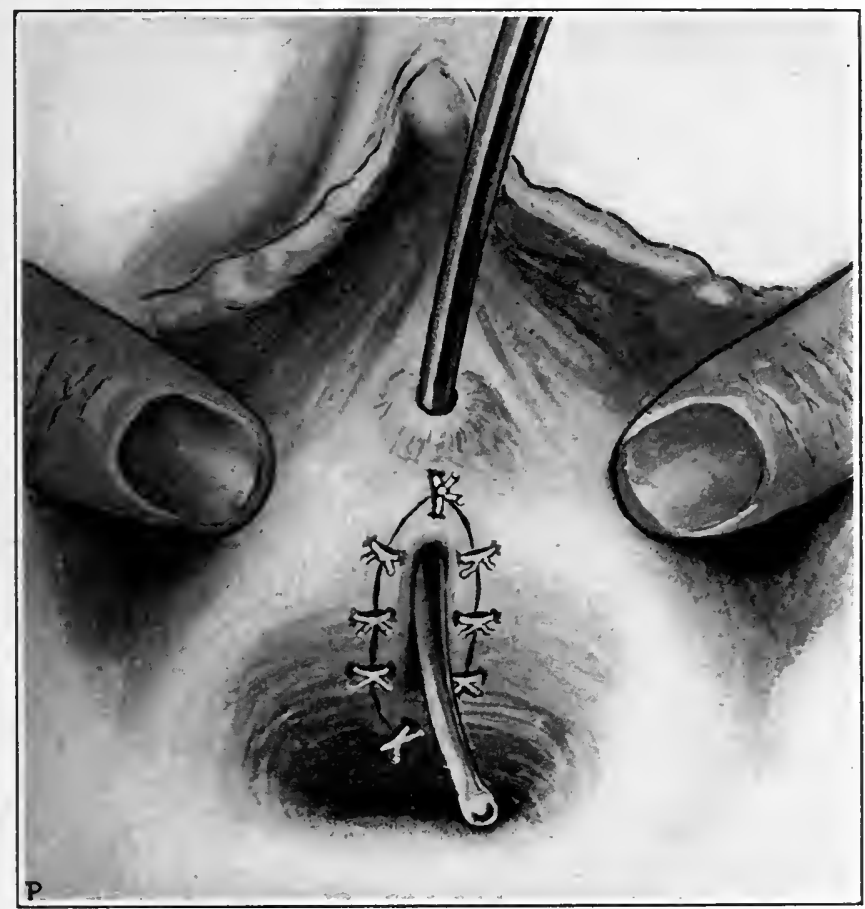

Emmet's button-hole operation.

Treatment of Urethral Stricture.-The inflammatory process may have been so intense as to produce contracting cicatricial tissue and consequent stricture. The cause of this uncommon lesion is usually gonorrhœa or trauma. The treatment is dilatation by means of graduated sounds, as in stricture of the male urethra. Should dilatation fail, a urethrovaginal fistula may be made and the raginal margins sutured to the urethral margins of the opening. When the edges have healed securely, the fistula may be closed by denudation on the vaginal surface, the interrupted silkworm gut sutures being so placed as to give ample calibre to the restored urethra. See Figure 151. 


\section{URETHRITIS COMPLICATED BY PROLAPSE OF URETHRAL MUCOSA.}

Description.-Prolapse of the urethral mucosa and submucosa, with complicating urethritis, has been described by Emmet. The prolapsed mucosa projects from the upper or lower margin of the meatus or surrounds the outlet of the urethra. The urethra is obstructed,

FIGURE 152.

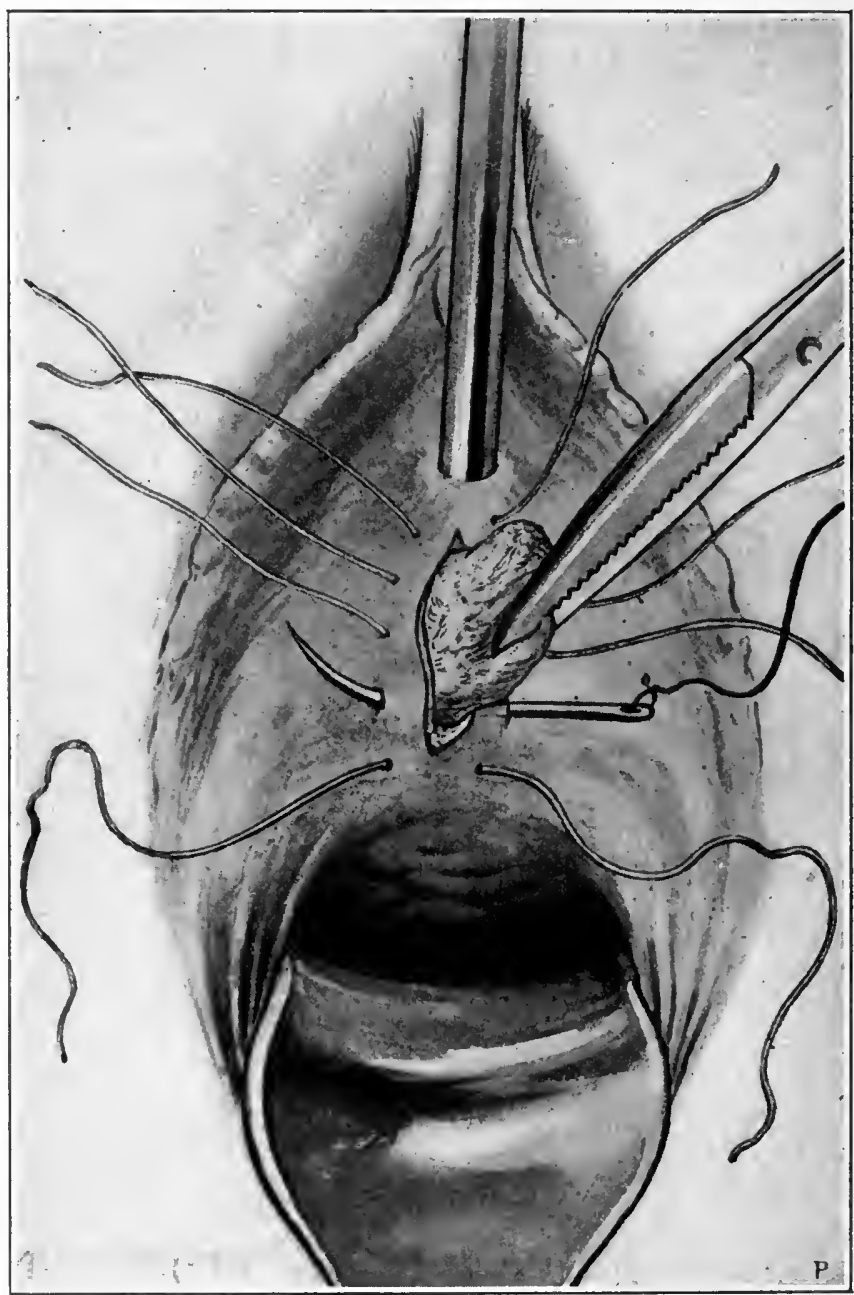

Emmet's operation for prolapse of the urethra.

and as the obstruction increases there is frequent or constant urethral tenesmus. Finally the entire urethral mucosa and submucosa may be rolled out so as to resemble a greatly prolapsed anus. The urethral canal dilates, and, as the circulation is obstructed, the rolled- 
out structures become odematous. Cystitis and infection of the kidney are possible results.

When the prolapse is confined to the upper or lower wall of the urethra and the outrolled tissues are from the outlet of the canal, they resemble hemorrhoids, and may, as in the operation for hemorrhoids, be removed by ligature.

When the prolapse is extensive and circular, removal in a mass is prohibited, first, because more prolapsed tissue usually follows and promptly takes the place of that which has been removed; second, because a distressing stricture of the urethra may result. Prolapse of the urethra may be the result of the traumatisms of labor or other causes and may have primarily at least no inflammatory history.

\section{Treatment of Prolapse of Urethral Mucosa.}

The treatment of extensive prolapse from any cause is to return the displaced mucosa, if possible; and if relief does not follow, it is well to make a small vesicovaginal fistula, and thereby give the urethra perfect rest. If this measure fails, the prolapse may be cured permanently by making what Emmet calls a button-hole slit in the urethrovaginal wall and drawing through this the excessive mucosa and cutting it away. The sutures for closure of the opening are introduced before the excision. During the placing of the sutures a sound should be in the urethra. Figure 152 .

\section{URETHRITIS COMPLICATED BY SUBURETHRAI ABSCESS.}

Suburethral Abscess occurs in the urethrovaginal wall. It has been described by Lawson Tait under the name urethral cyst. The pathology is not fully explained. In the limited number of cases described, the sac occupying the urethrovaginal wall varies in size from that of a walnut to that of a hen's egg, and communicates with the urethra by a small opening. The presence of this sac has been explained by Tait as a congenital defect, and by Kelly as a retentioncyst formed by inflammation and occlusion of Skene's ducts and the subsequent perforation of the urethral wall. Inasmuch as Skene's ducts are possibly the remnants of Gaertner's ducts-rudimentary Wolffian ducts-both the congenital and cyst theories may be true.

The tumor has the appearance of pronounced cystocele, is well defined, and very tender to the touch. Pus and ammoniacal urine often escape from it through the urethra. The tenderness is so great that anæsthesia usually is required for examination. If the communication with the urethra is exposed by a urethral speculum and pressure be made upon the sac, one may, as the sac is reduced in size, see its contents forced into the urethra.

\section{Treatment of Suburethral Abscess.}

The treatment is to dissect out the sac-wall and close the wound with interrupted silkworm gut or catgut sutures. These sutures at the same time should close the urethral opening. Complete ana- 
tomical and symptomatic cure follows this operation. If the sac has ruptured, it may be drained by gauze packing held in place by a T-bandage until it heals by granulation.

\section{CYSTITIS.}

In pathology and symptoms, inflammation of the female bladder differs in few respects from that of the male. The peculiar sources of infection, the relative shortness of the female uretlira, and the easy access to the bladder through the vesicovaginal wall, however, give to the etiology, diagnosis, and treatment a clear gynecological significance.

When the diagnosis of cystitis was based upon the presence of pus in the urine and painful and frequent urination, treatment gave less satisfaction to the physician and less relief to the patient than that of almost any other inflammatory disorder. Now these symptoms, pyuria and painful and frequent urination, are recognized as results not only of inflammation of the bladder, but also of a variety of other lesions, especially lesions of the ureter, kidney, and urethra. Moreover, the cystitis itself, which often is thought of as a distinct disease, now is relegated almost to the rank of a symptom, and is considered properly in its relations to certain deeper lesions which individually or collectively may underlie and perpetuate it or may result from it. Within a single decade the management of this symptom has risen from the plane of empiricism and has taken its place upon the scientific basis of pathology. This change has come about chiefly as the result of two causes:

Etiological investigations, especially including bacterial causes.

Improved instrumentation in diagnosis and treatment.

\section{Etiology of Cystitis. ${ }^{1}$}

In addition to most of the sources of infection common to cystitis in the male, the female bladder is more subject to concurrent infection from the same canses which give rise to infection of the reproductive organs. Susceptibility is increased during the recurring physiological congestion of menstruation, and especially during the puerperal state. Furthermore, infection may spread readily from the reproductive to the urinary organs. Vulvitis, for example, may extend to the urethra, bladder, and ureters. Such extension to the urinary organs, however, is retarded somewhat by the fact that the urinary tract is washed freely by a downward current of urine and by the further fact that the urine, being acid, is hostile to the culture of about 90 per cent. of pathogenic bacteria.

It is most important to distinguish clearly the predisposing from the exciting causes.

Predisposing Causes.-Among the predisposing causes or favoring conditions are :

1. Pathological urine.

2. Retention of urine.

1 Senn. Transactions American Surgical Association, 1898. Consulted. 
3. Tumors.

4. Foreign bodies, especially stone.

5. 'Trauma. Rupture of pelvic abscess into bladder.

6. Any local or systemic cause of congestion or blood-stasis.

7. Rheumatism and gout.

Formerly these were considered the essential causes of cystitis.

Exciting Causes.-Vastly predominating at least among the exciting causes are the pathological bacteria and their products. The bacteria most frequently found are:

1. Bacillus coli communis.

2. Gonococcus.

3. Bacillus tuberculosis.

The bacteria less frequently found are:

1. Proteus vulgaris, Hauseri.

2. Staphylococci pyogenes.

3. Streptococci pyogenes.

4. Typhoid bacillus.

It is agreed generally that the gonococcus and bacillus tuberculosis are prone to attack the healthy bladder, and that they require little if any predisposing causes. On the other hand. a normal blarder is said to offer much resistance to the other bacteria-that is, they do not become active except in conjunction with definite predisposing causes.

Ammoniacal urine is known to result from the decomposing action upon urea of certain bacteria, notably the proteus vulgaris. The frequent association of alkaline anmoniacal urine with cystitis has given rise to the nore or less common impression that the disease depends upon the irritating action of urine which has undergone ammoniacal decomposition, and that such decomposition is associated necessarily with cystitis. Johannes Miller, ${ }^{1}$ of Wurtzburg, was the first to overthrow this idea. He showed that in 73 per cent. of the cases of cystitis the urine was acid. Soon after the observations of Miller, Melchior ${ }^{2}$ reported the results of sixty-two very accurate observations. He found that ammoniacal decomposition was only a minor phenomenon, and that in many of the severest forms of cystitis acid urine was present even to the time of death. Almost all investigators now reach the uniform result that the bacillus coli communis, or a microbe very closely related, is the one most frequently found in cystitis. Ont of one hundred and twenty cases collected by Rostoski this germ was found in eighty. Whenever the bacterium coli communis was found alone the urine was acid; whenever the proteus vulgaris was found it was alkaline. Alkalinity with bacteria coli communis is said to be always due to association of other nicrobes.

\section{Instrumentation in Cystitis.}

Within a few years the cystoscope has revolutionized our knowledge of the pathology, diagnosis, and treatment, not only of cystitis, but of many other hitherto more obscure urinary disorders.

\footnotetext{
${ }^{1}$ Rostoski. Dentsche medicinische Wochenschrift, S. 235, 1898.
}

2 Monatsbericht aber den Gesamtleistungen, Heft 10. 
Formerly, when the principal factors in etiology were stricture of the urethra, foreign bodies in the bladder, and, in the male, enlargement of the prostate, and when there was no means of viewing the mucosa, the finger through the dilated urethra and the sound were almost the only means of exploring the bladder. Digital exploration with its attendant dangers was then common practice. As late as 1883 Sir Henry Thompson, ${ }^{1}$ in his work on Digital Exploration, reported as the result of two years' observation by that inethod a series of over thirty cases of tumor in the bladder upon which he had operated. Most significant in contrast is the report of Alexander Stein, ${ }^{1}$ two years earlier, in which he was able to collect from all the literature, including post-mortem observations, only about twenty cases.

By means of the cystoscope the entire interior of the bladder may be brought into view ; foreign bodies, tumors, and other pathological changes may be recognized, and the ureters and the pelvis of the kidney may be explored. The instrument has often revealed the presence of stones, tumors, and uleers which had escaped detection by the sound. Numerous cases in which eystitis is of only secondary importance to other associated lesions, such, for example, as tumors, tubereulous uleers, and piles, or hemorrhoids of the bladder, are now observed daily by the cystoseope.

Cystoscopy is also of great value in preventing blind and meddlesome treatment for a class of cases which present the subjective symptoms of cystitis, but in which inspection fails to show any lesion whatever of the bladder mucosa.

The value of the instrument is also incalculable when only limited areas are diseased ; for example, in case of mild inflammation of the trigone and in fissure at the neck of the bladder. Under such conditions the operator, instead of treating the entire vesical mucosa by means of injections more or less strong, may direct any desired application to the diseased part only.

\section{Pathology and Diagnosis of Cystitis. ${ }^{2}$}

Cystitis, in the first place, must be differentiated from simple irritability of the bladder; a condition found in neurasthenic subjects, the diagnosis and treatment of which are usually those of some underlying neurosis.

The attempt will not be made to differentiate all the phases and varieties of cystitis, but rather to outline the more pronounced types.

In the beginning of cystitis the cystoscope shows the blood-vessels to be defined less sharply than in health. Soon the normal light pink, almost whitish, color of the mucosa assumes a deeper and deeper hue until the sharp demarcation between the vessels is lost and the whole surface is finally of a uniform deep red. The epithelium may be cast off in small particles from cireumscribed areas either narrow or broad, and the surfaces thus exposed may take on a granular appearance. Finally in severe cases one may observe pus coagulation and excessive

${ }^{1}$ Belfield. Am. Gyn. and Obstet. Journal, Jan., 1899. Adaptation. 2 Ibid. 
swelling and œdema of the bladder-wall. The urine in such cases contains epithelial detritus and pus-cells in large quantities.

Mixed infection and other complications may render it impossible to distinguish between all the different bacteriological varieties ; it is, however, usually possible and is often desirable to differentiate the tubercular from other varieties, especially from the gonorrhoal.

\section{Differentiation of Tubercular form Gonorrhogal Cystitis.}

Tubercular cystitis.

1. Located chiefly about the trigone.

2. Inflammatory reaction zone absent or not well defined.

3. Tubercular cystitis not common.

4. Characterized by presence of small tubercles situated about the trigone and ureteral orifices.

5. No projecting tufts of pus.

6. No subperitoneal extravasation of blood.

7. Bacillus tuberculosis.

8. Often extension from kidney and from general tuberculosis.

9. History of tuberculosis. Great pain; hæmaturia
Gonorrhoal cystitis.

1. Not at all so confined.

2. Clear inflammatory reaction zone, later changing to dull-brown color.

3. Common.

4. Characterized early by insular areas of reactive inflammation, with healthy or nearly bealthy intermediate mucosa. Later insular areas may become confluent and extend over the whole mucose.

5. Projecting tufts of gonorrhceal pus are apt to be present. In chronic stage regions of elevation may be excavated by ulceration.

6. In very acute stage there is subperitoneal cxtravasation of blood.

7. Gonococcus.

8. Extension from vagina, vulva, or urethra.

9. History of gonorrhœa. Less pain ; seldom blood in urine.

\section{The Pathology of Tubercular Cystitis shows :}

1. At first small grayish tubercles or nodules; later larger nodules and deep ragged ulcers.

2. Trigone, base, and posterior wall of the bladder most affected; disease may extend over the entire bladder.

3. Bladder-wall thickened and contracted.

5. Disease may end fatally in few months or may continue for years.

The Diagnosis of Tubercular Cystitis is made as follows :

1. Non-gonorrhœal cystitis in a plithisical patient is usually tubercular.

2. Non-gonorrhœal cystitis in young persons is usually tubercular.

3. Chronic ulceration of the bladder is often tuberculous.

Finding of the bacillus tuberculosis in the urine or tissues is the only absolute diagnostic sign ; to obtain tissues for microscopical examination, curette the ulcerated surfaces through the endoscope. The smegma bacillus, which has the same minute appearance as the tubercle bacillus, may give rise to confusion in diagnosis. The former are decolorized by acid more than the latter.

\section{Classification of Cystitis.}

In our present state of knowledge of the subject a perfect classification is impossible. Numerous classifications have been proposed.

Anatomical Classification.-According to the special structures involved, this comprises : pericystitis, paracystitis, interstitial cystitis, 
and endocystitis. The difficulty, not to say frequent impossibility, of separating these varieties one from the other, and the fact that two or more usually coexist, render this classification, although diagrammatically attractive, clinically impossible. There are no sharp lines of demarcation between the anatomical forms.

The Pathological Classification includes numerous varieties, such as catarrhal, suppurative, ulcerative, hemorrhagic, exudative, exfoliative, and fissure cystitis. These, however, are rather phases and possible stages than distinct varieties of the inflammatory process.

FIGURE 153.

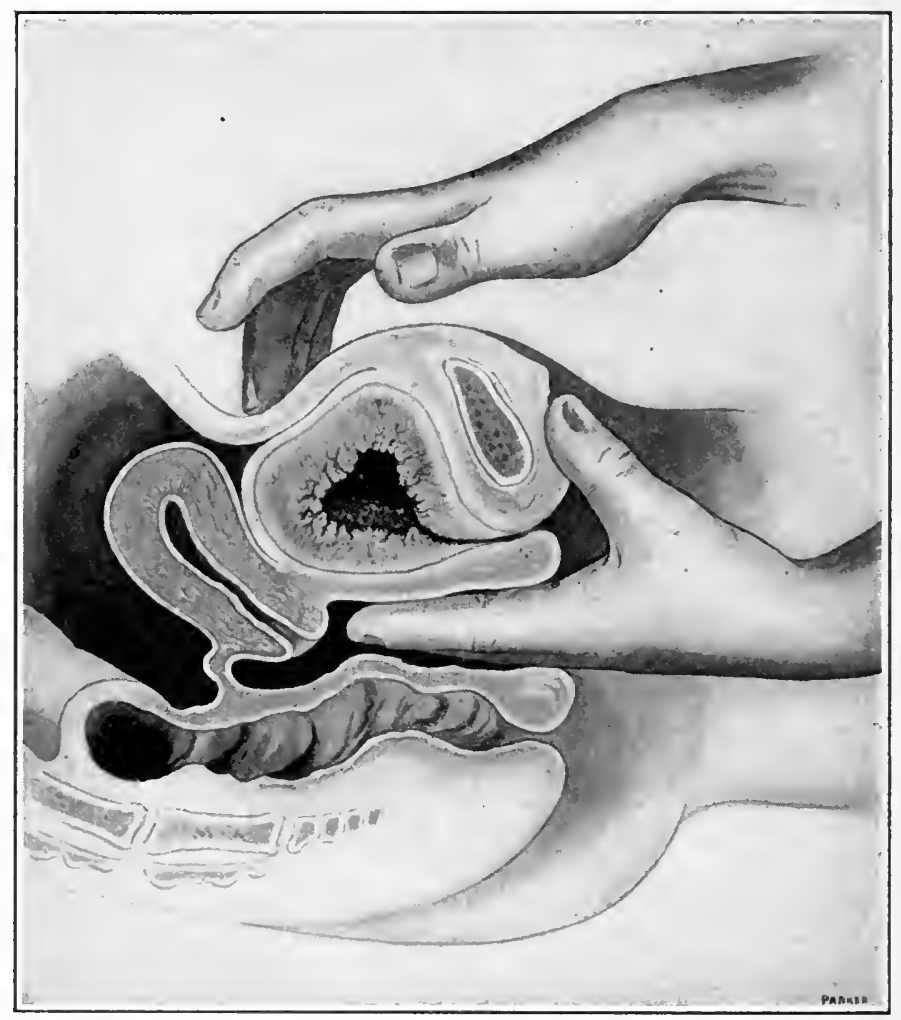

Conjoined examination of tubercular cystitis

Bacteriological Classification.-This classification might comprise as many forms as there are varieties of infective microbes. The principal bacteria have been mentioned. This classification, although quite plausible in the laboratory, is often impractical at the bedside. It is possible, however, that more exact knowledge in the future may give to it the status of a scientific working guide.

These various classifications, however, from the standpoint of nomenclature are very convenient. Such words as gonorrhœal, acute, chronic, suppurative, and interstitial are useful for purposes 



\section{PLATE X}

Figure 1. Cystitis originating in the Trigone and extending to Adjacent Surfaces. Magnified.

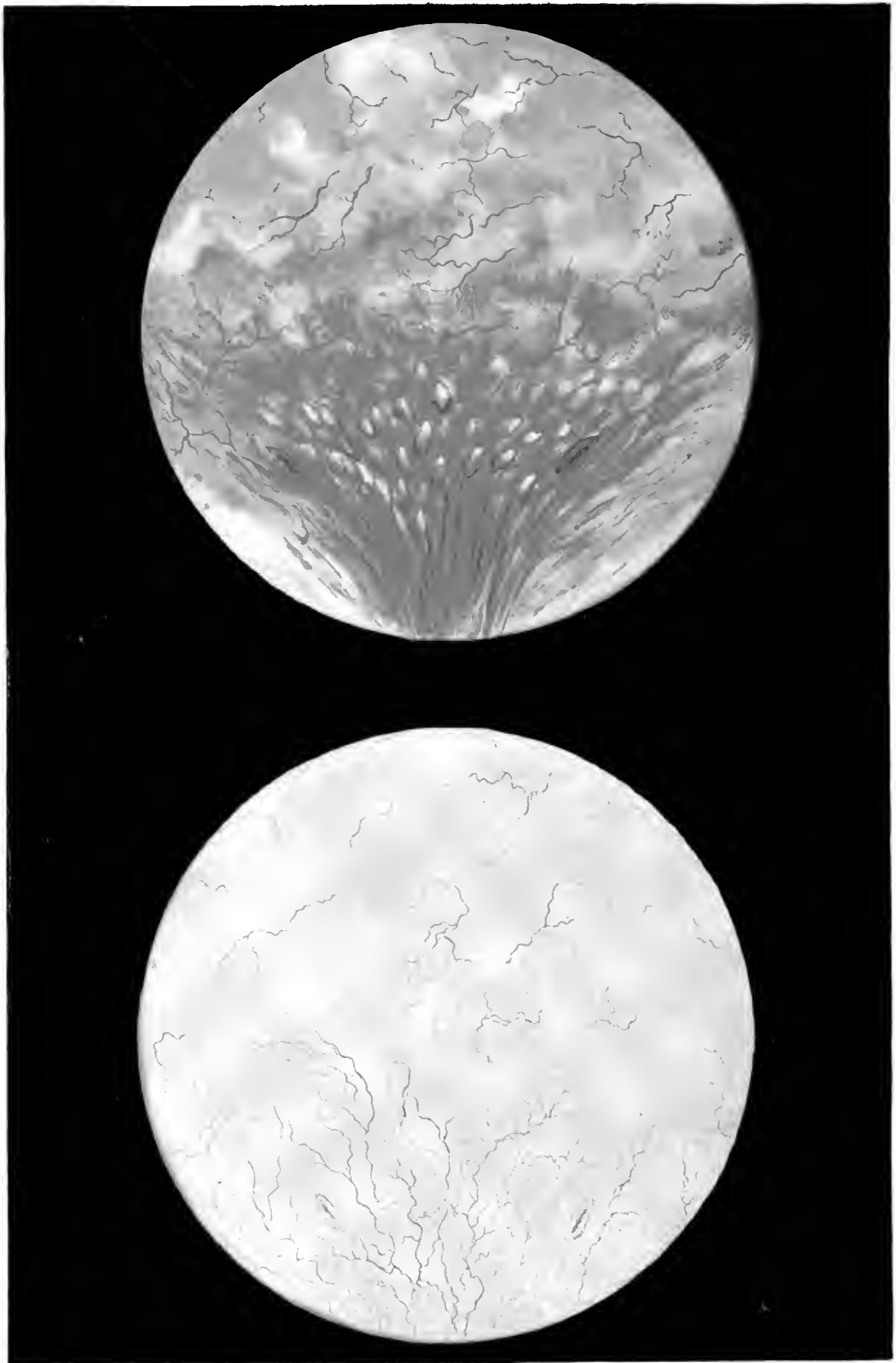

Figure 2. Normal Bladder Mucosa. Stightly magnified. 


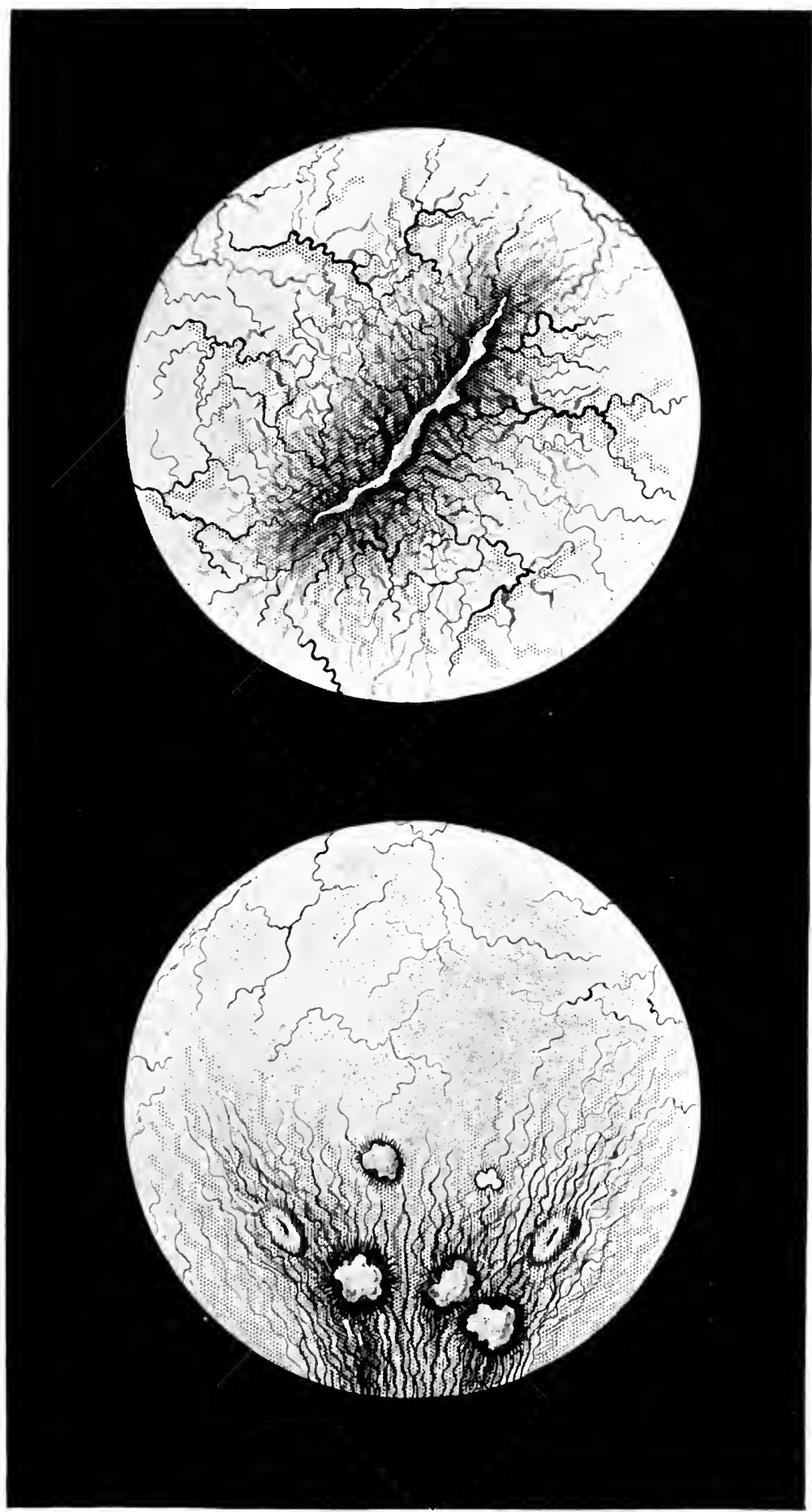

Eigure 2. Ulcerated Patches in the Trigone. Slightly magnified. 

of description and to designate the various forms and phases of infective processes. For example, we should use the word endocystitis to describe not a distinct lesion independent of the rest of the bladder, but rather an essential part of an inflammatory process.

Certain so-called clinical and pathological forms and phases of cystitis may be designated as follows, and have great diagnostic significance.

1. Superficial eystitis-catarrhal.

2. Suppurative cystitis.

3. Ulcerative cystitis.

4. Exudative cystitis.

5. Exfoliative cystitis.

6. Fissure cystitis.

7. Foreign-body cystitis.

8. Leucoplakia eystitis.

1. Superficial Cystitis.-A large proportion of the cases of chronic inflammation are of this variety. Generally it is called catarrhal cystitis; the term should be restricted to superficial infection and to cases in which the product of inflammation cones from the superficial epithelial elements of the bladder mucosa. The disease is marked by moderate swelling, redness, and exfoliation of epithelial cells; the urine contains a moderate amount of pus and is usually acid. Erosions, ulcerations, and, as a consequence, more abundant suppuration may follow - that is, cystitis, originally catarrhal, may become distinctly suppurative. Great alkalinity of the urine indicates a rather advanced stage, when the cystoscope will reveal a deposit of grayishwhite color containing mucopus.

2. Suppurative Cystitis.-In this form the inflammation may have been diffuse from the beginning and have involved both the superficial and deeper structures of the bladder-wall. As well stated by Senn, the microbic infection is of sufficient intensity to destroy the protoplasm of the pathological products of the inflammation and thus transform the leucocytes and epithelial and connective-tissue cells into pus-corpuscles. The urine contains an abundance of epithelial cells and pus. Ulcerative processes may involve the deeper structures, and in exceptional cases may lead to perforation. The urine is acid, or, if ammoniacal, may be so from decomposition due to the intercurrence of microbes other than those which produced the original infection. The proteus vulgaris will often be found in ammoniacal urine. Suppurative cystitis, both in its acute and chronic stages, is prone to invade the ureters and kidneys. In fact, chronic uncomplicated suppurative cystitis is rare. The cystoscope reveals the local conditions as already described.

3. Olcerative Cystitis. - The ulcerative phase of cystitis has been mentioned as a later stage of the catarrhal or suppurative varietics. The term is used here to designate that variety in which ulceration is the initial or, at least, a very early factor. The infection, as described by Senn, appears to be of a peculiar kind and limited in extent. 'The resulting inflammation leads quickly to circumscribed necrosis. There is at first a single circumscribed ulcer, the so-called "simple" ulcer 
of the bladder resembling gastric ulcer and the round duodenal ulcer.

The first symptom is increased desire to urinate; intermittent hæmaturia then appears. The ulcer may become incrusted with phosphates. Fragments of the deposit break off now and then, and may be passed with painful paroxysms, or may be retained to serve as nuclei for calculus formation. Finally the bladder becomes contracted and the mucous membrane extensively ulcerated. Ureteral and renal lesions may now arise. Ulcerative cystitis, like gastric and duodenal ulcer, is found quite frequently in young men, less frequently in women. There are usually no antecedent or attending predisposing local causes. In the diagnosis the cystoscope is indispensable. ${ }^{1}$

4. Exudative Cystitis.-This is characterized by the formation upon the bladder mucosa of a so-called membrane; hence it usually is designated by the rather confusing descriptive terms, "membranous," "diphtheritic," "croupous," or "fibrinous." The exudative membrane is the product of the inflammation; it is in fact apt to be the product of extensive necrotic changes, and as such indicates a grave lesion. There may be extensive destruction even in the musculature and especially in the deep blood-vessels and lymphatics. The urine usually is alkaline. The disease has been observed chiefly in puerperal women. The urine contains fibrinous shreds or cast-off patches of membrane. Cystoscopic examination reveals a yellowish-white membranous formation which often may be picked off by means of forceps passed through the cylindrical cystoscope.

5. Exfoliative Cystitis.-This variety is analogons to so-called dissecting metritis and dissecting vaginitis. The infective process and inflammatory reaction are most virulent and intense, and result in the destruction and detachment of the mucosa, and together with it sometimes of the muscular layer of the bladder; these may be expelled in fragments with the urine or may have to be removed from the bladder by a surgical procedure. It is the most grave and virulent form of cystitis, and is apt to be fatal. The conditions are like those of exudative cystitis intensified. The diagnosis between the two forms depends upon the macroscopical and microscopical character of the masses removed or thrown off from the bladder. The disease was described early and fully by Boldt. ${ }^{2}$

E'TIOLOGY.-Exfoliative cystitis is associated usually with one or more of the following mechanical conditions :

1. Retroversion of the gravid uterus in 50 per cent. of cases.

2. Protracted birth in 25 to 30 per cent. of cases.

3. Incarcerated pelvic tumors.

4. Retention of urine, especially in puerperal cases, common.

Symptoms.-

1. Membrane expelled in urine.

2. Membrane may obstruct the urethra, causing retention.

3. Membrane in the bladder canses vesical tenesmus; expulsion of it causes pain and straining.

1 Adaptation from Senn.

2 American Journal of Obstetrics, June, 1889. 
4. Death may occur from: $a$, sepsis; $b$, uræmia; $c$, pyelitis; $d$, peritonitis.

6. Fissure Cystitis.-Fissure cystitis is caused by infection through

FIGURE 154.

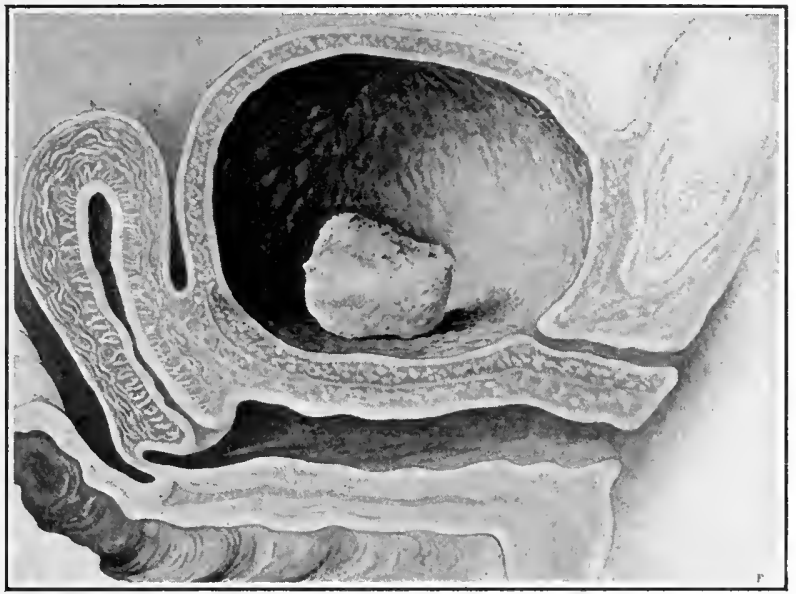

Stone in the bladder, a cause of foreign-body cystitis.

a traumatism at the neck of the bladder or in the trigone. As seen through the cystoscope, the fissure is covered usually by a brownish or yellowish exudate surrounded by an nedematous area.

\section{FIGURE 155.}

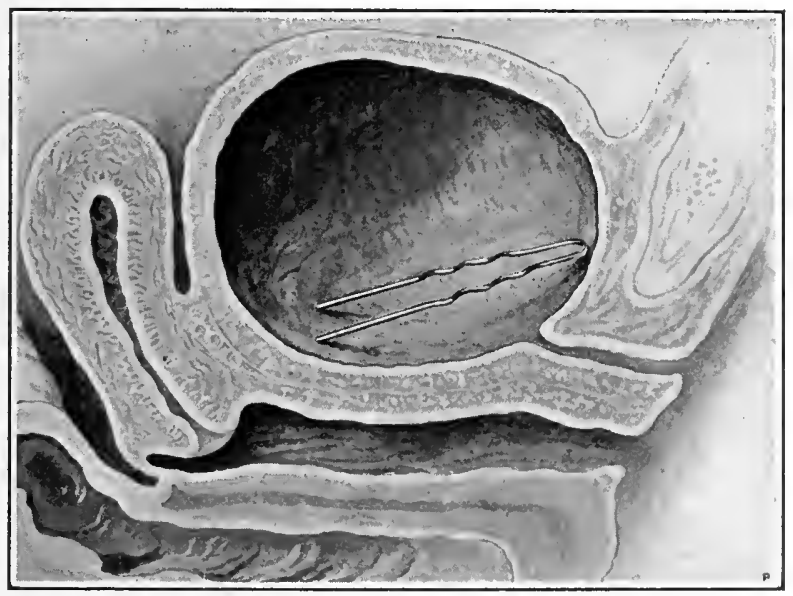

Hatrpin in the bladder, a cause of foreign-body cystitis.

7. Foreign-body Cystitis.-Cystitis caused by foreign bodies (Figures 154 and 155) varies with the character of the body and the conditions of infection. A smooth body may be tolerated without subjective 
symptoms. A rough or angular substance may produce trauma and thus open the way to any form of infection. T'he foreign body is the exciting cause and opens the way for the action of infecting bacteria.

8. Leucoplakia Cystitis. ${ }^{1}$-This affection is characterized by the appearance of grayish small circumscribed areas situated usually in the trigone. The epithelium has undergone changes which render it op:ique and which have been likened to the changes of keratitis. The cystoscope reveals a number of grayish-white reflecting spots of a diameter approximating one-fourth inch. These spots while desquamating are below the level of the surrounding nucosa; after desqnamation has ceased they become flush with the mucosa. A most pronounced subjective symptom is an intolerable and almost constant desire to urinate. Micturition may be attenıpted as often as once in fifteen minutes through the day, and almost as frequently during the night. Leucoplakia results from long-continued chronic areas of inflammation; it may be the starting-point of general cystitis.

\section{Treatment of Cystitis. ${ }^{2}$}

The treatment of cystitis falls under four heads :

1. Prophylactic.

2. Medicinal.

3. Topical.

4. Surgical.

Prophylaxis. ${ }^{3}$-Numerous autopsies upon subjects who have not suffered from cystitis have shown a hyperæmic state of the bladder so marked that it must have been of long duration, yet had not developed to the extent of infection and inflammation. The explanation must be that the infective element had not been present, or if present had not become active. On the other hand, the question has been raised, and usually answered in the negative, whether the mere presence of infective microbes alone, bacillus tuberculosis and gonococcus excepted, can easily provoke cystitis. It is agreed commonly that infection must depend usually upon: first, an abnormal condition of the soil which renders it susceptible; second, upon the presence of the bacterial exciting cause. A twofold indication is obvious : to keep the bladder in a state of resistance, and to avoid the introduction of infective material.

Susceptibility to infection may result from either systemic or local states. The systemic conditions are often the result of faulty elimination and consequent defective circulation. Hepatic and cardic disorders, kidney insufficiency, constipation, gout, lithæmia and cholæmia, anæmia, diabetes, rheumatism, at once suggest themselves as favoring conditions calling for hygienic and medical treatment, for judicious elimination and nutrition. At the risk of seeming to advocate routine measures, one may suggest the value of mercurials and salines. It is clearly essential to enforce judicious rules for food, exercise, and sleep.

The introduction of the catheter under the sheet, its passage with-

- Adaptation from Kolisher.

${ }^{3}$ Kletsch. Am. Gyn. and Obstet. Jour., 1899. Consulted. 
out preparatory disinfection of the vulva, the careless use even of the aseptic catheter and the slight traumatism which its use may cause, and the almost certain ingress of septic matter through such traumatism, are well known to every observing physician. But unfortunately many physicians, although cognizant of the facts, are not alive to the importance of them.

The possible relations of parturition to eystitis are most significant; among such relations are those which arise from certain pelvic defects. For example, contraction or excessive inclination of the pelvis may retard or obstruct labor, and thereby cause prolonged pressure of the child upon the bladder, giving rise to conditions full of danger.

Gestation in a retroflexed uterus finally enlarges the uterus until it becomes inpacted under the sacral promontory; then pressure of the cervix upon the neck of the bladder produces œedema, forces the bladder against the pubes, prevents complete evacuation, and results in the retention of residual urine. This urine may become decomposed, and is then a most favorable culture-medium for bacteria. If there be present in combination the three elements, congestion, decomposed residual urine, and bacteria, even though any one alone might be ineffective, infection is almost unaroidable. The necessity for prompt replacement of the displaced gravid uterus, therefore, is clear.

Medical Treatment.-The medical treatment already discussed as a part of the prophylaxis has, especially in connection with other forms of treatment, great value. The principles are necessarily those of general internal medicine. The particular indications have reference to the use of such drugs as may change the quality or increase the quantity of the urine. If the urine is strongly acid or concentrated, for example, it should be diluted by the free drinking of fluids or rendered less acid by the use of alkalies; if alkaline, the reaction may be modified by the use of acids. Lest there be frequent urination during the night, the drinking may be confined largely to the morning and afternoon hours. To secure a proper degree of acidity, benzoic acid, alone or combined with borax and dissolved in cinnamon-water, is a classical and useful remedy. The indication to relieve subjective symptoms is twofold : first, to allay suffering and nervous irritation; and second, to render the patient less intolerant of topical and surgical treatment.

In superficial mild cystitis, with frequent urination and painful contractions of the bladder, prompt relief sometimes follows the daily application of a rectal suppository containing two or three grains of ichthyol. In more aggravated cases opium may be substituted for ichthyol. To sccure good sleep let the ichthyol suppositories be used two or three hours before bedtime, and followed if necessary by the opium or morphine suppositories at bedtime.

The bowels should be kept normally free by mild laxatives. Drastic cathartics should be avoided. Uva ursi, triticum repens, the benzoate salts, buchu, eucalyptus, and many other time-honored and classical remedies may be useful. Urotropin appears to be the most valuable single internal remedy. It may be given in amounts varying from 15 to 30 grains daily. The writer occasionally has been gratified 
at the disappearance of irritation of the bladder after the administration three times a day for a number of weeks of ealomel in doses of onetenth to one-twentieth of a grain supplemented by the free use of natural or artificial mineral waters or of pure water. Rest, especiaily in the acute stage, is highly important.

Topical Treatment.- The washing out of the bladder as a routine procedure is not approved. Irrigation, however, is indicated positively

Figure 156.

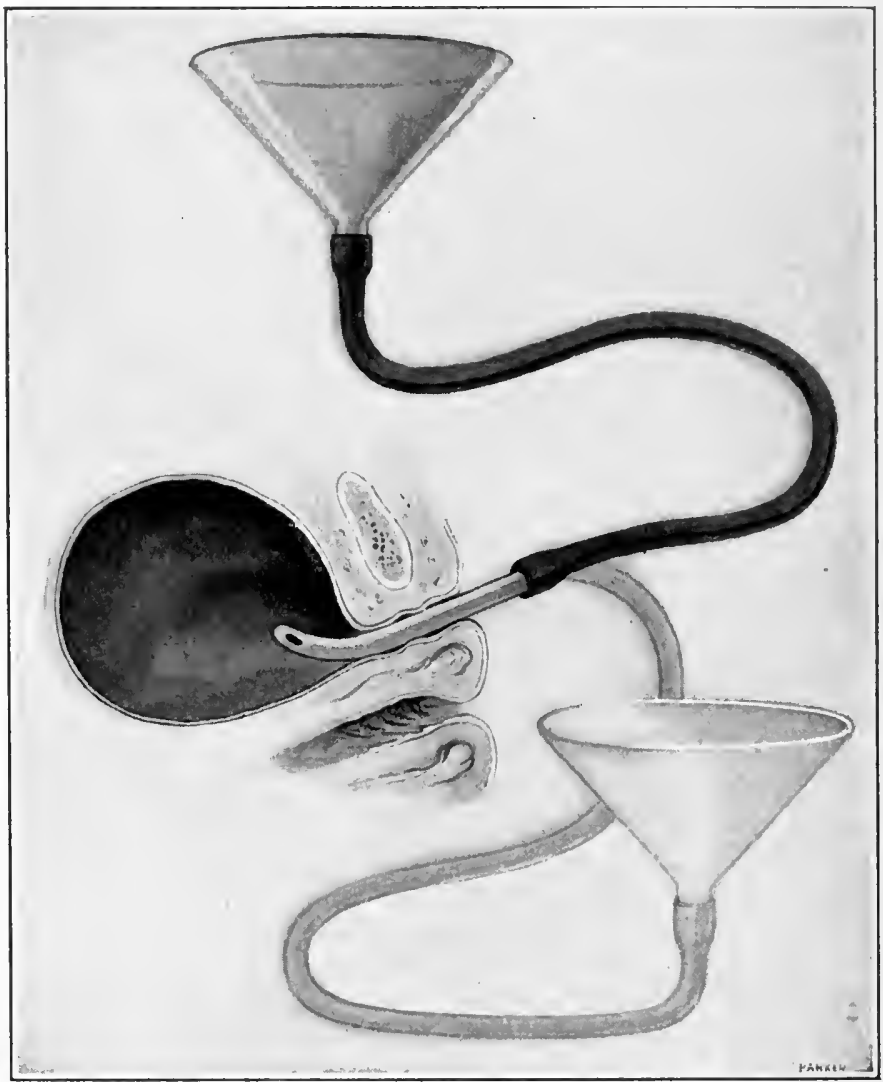

Washing ont of the bladder. The irrigation may be made repeatedly by alternately raising aud lowering the funnel; when the funnel is raised the fluid flows into the bladder, when lowered it returns to the funnel.

when necessary for the removal of loose irritating shreds or other foreign matter.

The superficial forms of eystitis respond promptly to topical treatment. If the cystitis is general and superficial, two ounces of a 10 per cent. emulsion of iodoform in oleum sesame may be thrown into the bladder with a hard-rubber syringe. If, after two or three applications of the emulsion, there is sufficient toleration, four ounces of silver nitrate solution may be injeeted into the empty bladder and 
immediately replaced by free irrigation of normal salt solution. The strength of the silver solution should vary according to the toleration of it. Begin with 1 per cent. and cautiously increase the strength if necessary even to 3 per cent. The treatment may be repeated every two or three days. Oftentimes two or three mild injections will effect a cure. Vesical injections of argyrol, 1 to 5 per cent., are non-irritating, effective, and may be substituted for the silver nitrate solution to advantage.

In many cases the infection is localized, and when localized is confined usually to the trigone or inner end of the urethra. The silver application should then be applied only to the affected part. It may be used in solution of any desired strength and applied by means of a cotton swab introduced through the cylindrical cystoscope and kept within bounds by the immediate instillation of salt solution. Mild infections in the trigone often yield completely to a single treatment. Fissure cystitis may be cured promptly and permanently by this means, but aggravated cases sometimes require the solid stick. Accompanying urethritis should be treated simultaneously with the cystitis.

When the infection has caused deep infiltration in the bladderwall, as in exudative or diphtheritic cystitis, the treatment is to be conducted in two stages-first, wash out the bladder to remove the shreds and other putrid material ; second, apply the disinfectant. In washing out the bladder use small quantities of fluid and repeat until the fluid returns clear; then apply the disinfectant, preferably the silver nitrate.

If the secretion on the bladder-wall is mucoid in character and stringy, it is better to use normal salt solution than pure water. When the bladder is so painful as to resist all efforts at treatment it may be anæsthetized with 10-20 c.c. of a 4 per cent. solution of antipyrin. This should be left in the bladder about twenty minutes. If treatment leaves the bladder very painful, cupping or hot applications over the bladder or opium and belladonna suppositories in the rectum are indicated.

Cystitis with granulations or ulcers require a long time for healing; for this purpose nothing is better than silver nitrate solution or the solid stick.

In exfoliative cystitis any systemic cause of the disturbance should, if possible, be removed. The membrane when it becomes gangrenous should be taken away by means of forceps. A permanent catheter then should be inserted both for protection to the bladder fron the results of distention and for the injection of antiseptic solutions. In these cases the systemic condition is grave and should be treated accordingly.

Surgical Treatment.-The surgical procedures in the treatment of cystitis are as follows:

1. Dilatation of the urethra.

2. Vaginal cystotomy, also called colpocystotomy.

3. Curettage of the bladder.

4. Lithotrity.

5. Extravesical operations. 
1. Dilatation of the Urethra.-The indications for this operation are as follows:

A. To cure localized cystitis in the region of the trigone, commonly ealled trigonitis, and fissure at the neck, called fissure eystitis. The mode of treatment in fissure cystitis is doubtless similar to that of anal fissure by dilatation of the sphincter ani muscle.

B. To enable the operator to see and treat surgically or topically vesical ulcers, vesical hemorrhoids, small growths, and other affections of the bladder, and to permit the crushing of stone.

The dilatation is made by means of the urethral dilator, Figure 30 . In passing the instrument one should note the extreme natural diameter of the urethra and then limit the dilatation to about twice this diameter. Further stretching is apt to rupture the urethrovaginal wall, and may cause permanent incontinence of urine. A cystoscope five-eighths of an inch in diameter, Chapter III., measures the extreme safe distention of the average urethra. Exceptional cases may arise in which this amount of dilatation could safely be increased or in which it might be dangerous. Whatever stretching can be done without tearing is usually safe. The dilatation may be started with the conical dilator, and completed with the cystoseope.

2. Vaginal Cystotomy.-This operation, Figure 150, has for its object the formation of an artificial vesicovaginal fistula. It opens the way to intravesical topical treatments and operations. Through the fistula tumors may be removed and diseased surfaces cauterized and curetted. In chronic cases of great, long-continued, and unrelieved suffering colpocystotomy has, by giving the bladder complete rest, furnished immediate and inspeakable relief. The operation may be palliative or curative. In a certain proportion of eases the disease in the bladder and upper zones of the urinary tract is so extensive that the operation can only be palliative-that is, an anatomical cure is sometimes impossible. In some of these cases the bladder is contracted permanently to the capacity of perlaps one-half ounce. No one wonld think of making a secondary operation for the closing of the fistula under such conditions. In many other cases the artificial opening may be only temporary. It gives the best opportunity for direct local treatment to diseased parts of the bladder, and for a most effective vesical douche, which can be thrown in through the urethra and allowed to flow out through the fistula and vagina. Very many cases of otherwise intractable chronic eystitis have been cured by this method with subsequent closure of the fistula, and the cures, if not anatomically complete, were at least symptomatically satisfactory. In some cases the much contracted bladder even may resume its physiological calibre. The operation of raginal cystotomy is the device of T. A. Emmet.

Operatiox.-The patient is preferably in Sims' position, with the anterior vaginal wall exposed by Sims' speculum. A large sound is introduced through the nrethra, and its point pressed against the vesical mucosa in the middle of the long axis of the vesicovaginal septum. An incision is now made upon the sound through the septum with the knife or scissors. The point of the sound then will pass 
through into the vagina. The opening thus made is enlarged so as to extend one inch in the merlian line of the long axis of the vesicovaginal septum. Its upper end will be about one-half inch from the anterior wall of the cervix uteri, and its lower end the same distance from the neck of the bladder. The margins of the vesical and raginal mucosa then are united by fine interrupted catgut sutures.

FIGURE 157.

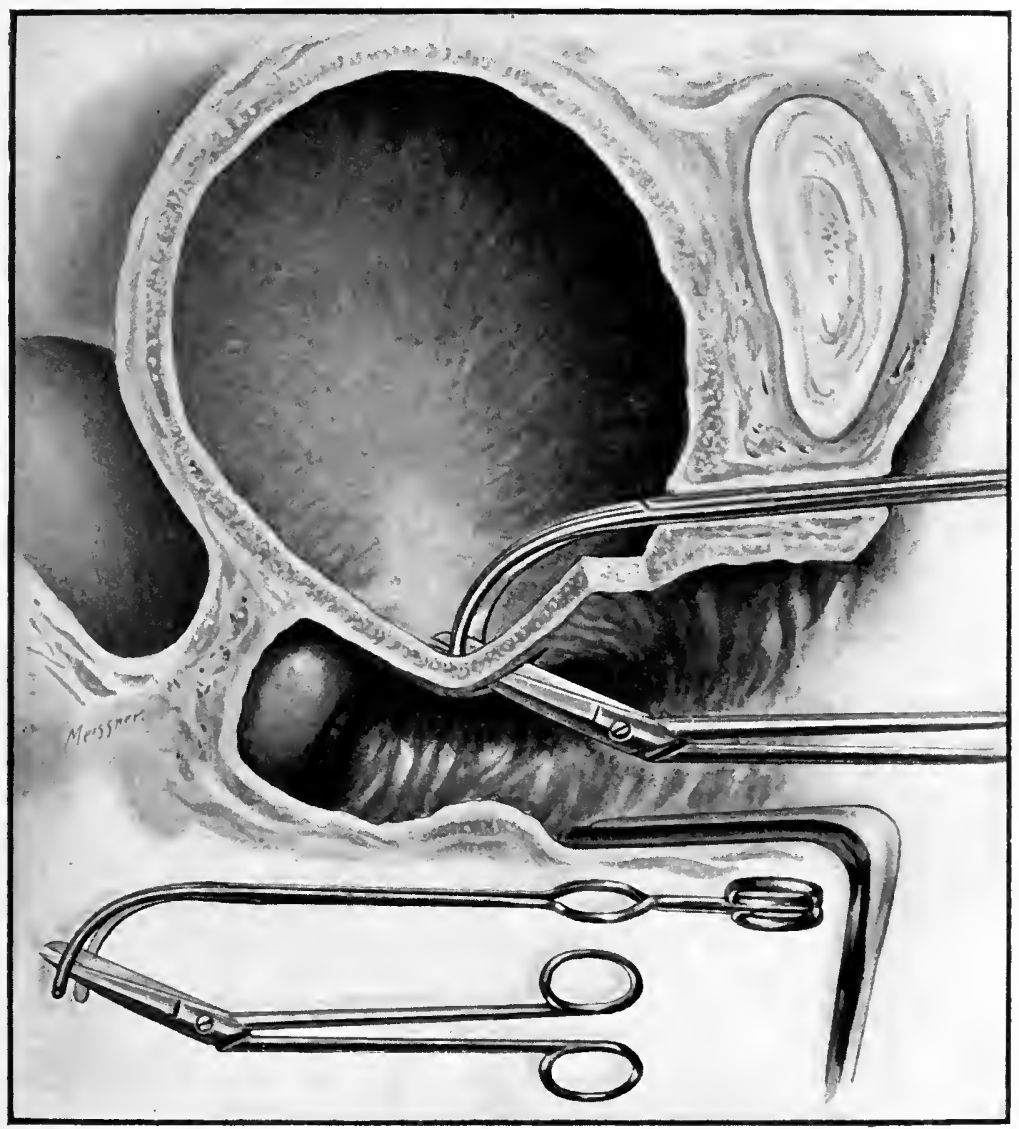

Vaginal cystotomy. A dilator is passed through the urethra into the bladder, and the blades of it are pressed against the vesieovaginal septum at a point in the median line midway between the uterus and urethra, and separated. The resicovaginal septum is incised ly means of the scalpel or pointed scissors, which are forced through the septum between the blades of the dilator. The point for incision is determined by the position of the dilator, and easily can be telt by means of the index-finger in the vagina. In the lower part of the Figure the dilator and scissors are shown complete, and in the position in wbicl they are when the incision is made.

The accompanying illnstration shows a device which renders the operation both precise and simple; it consists in the introluction of a small uterine dilator instead of the sound mentioned above, through the urethra into the bladder. The enrved blades of this dilator are turned toward the vaginal wall, the points of the blades 
are pressed against the vesicovaginal septum in the median line-that is, on a line throngh which the bladder is to be opened. The blades of the dilator are now slightly separated, and the septum, being thus fixed, is incised between the blades, as shown in the illustration. The incision may be made by the scalpel or scissors with accuracy and without danger of wounding the opposite wall of the bladder.

In some of the less severe cases sufficient improvement takes place in a few weeks to permit the closure of the fistula, with permanent relief.

In the more chronic eases in which the bladder-walls are much thickened, deeply infected, disorganized, and contracted, and particnlarly when the cystitis is complicated with pyelitis and nephritis, the fistula should remain open, for its closure will be followed inevitably by relapse.

If eystitis be complicated by stone in the bladder, the treatment may well be an artificial vesicovaginal fistula (colpocystotomy), instead of $\dot{a}$ erushing operation through the urethra. The fistula is preferred for two reasons : first, the crushing operation may involve an objectionable degree of dilatation of the urethra; second, the fistula is useful as a means of drainage for the cure of the cystitis. Colpocystotomy may be indicated further for the removal of foreign bodies; it also furnishes an opening for the canterization or curettage of ulcers.

3. Curettage of the Bladder may be done through the tubular cystoscope, but better through an artificial vesicovaginal fistula. It is indicated in indolent ulcers, especially those of tubercular origin.

4. Lithotrity and Lithotomy.-A stone in the bladder may be crushed through the urethra or removed through an artificial vesicovaginal fistula. A small stone or other foreign body may be removed entire through the dilated urethra. Prompt relief from cystitis usnally follows.

5. Extravesical Operations.-Parametritic, perimetritic, or tubal abscess may by rupture into the bladder cause eystitis. Incision, evacuation, and drainage of the pus-cavity, or removal of the pus-sae, is followed usually by prompt cure.

As a final stage in the treatment of eystitis, the bladder if contracted may often be made to return to its normal size by methodical distention with increasing quantities of salt salution; but this should be undertaken only after the cystitis has been cured and the patient is free from the dangers of recurrence.

Summary.-1. The conditions which formerly were considered the prime causes of cystitis are now recognized only as predisposing causes.

2. The recognition and appreciation of pathogenic bacteria as the exciting causes of cystitis are essential to a scientific understanding of the pathology, etiology, and treatment.

3. Alkalinity of urine depends upon the action of certain bacteria, notably the proteus vulgaris, in the decomposition of urea. The bacillns coli communis, which is one of the most frequent causes of cystitis, is one of a class which does not decompose urea and therefore does not produce ammoniacal urine. Contrary to the older opinion, 
alkalinity is not the rule in cystitis ; on the eontrary, in the majority of cases the urine remains acid. Alkalinity if present is the work of other microbes seeondarily introduced.

4. The classical symptoms of vesieal pain, frequent urination, and pus in the urine are wholly inadequate as a basis for the diagnosis of cystitis. Moreover, the condition called eystitis has eeased to rank as a distinet disease, and should be regarded only as a symptom. The mere recognition of the fact that eystitis exists is not a diagnosis; a fuct is not a diagnosis. Indeed, the recognition of cystitis may by contrast with the recognition of its eomplieations be of very minor importance.

5 . The diagnosis must comprehend not only the presence of infeetion in the bladder, but, what is more important, it must embrace the source, routes, type, complications, and variety of the associated inflammatory lesions. Uncomplieated inflammation of the bladder is rare:

-...6. The endoscope and eystoscope alone can open the way to efficient exploration and diagnosis, alone can define the indications for topical or surgical treatment; what is more essential still, they alone can prepare the way for the examiner to distinguish between cystitis and a wide variety of other affections of the bladder, urethra, ureter, and kidney. One is astounded at the revelations of the cystoseope in the recognition of most important lesions which must otherwise eseape notice.

7. The washing out of the bladder as a routine measure is not approved. The injection of disinfectants is indicated only in general or nearly general eystitis. For localized cystitis direet applications to the part affected should be made through the endoscope.

8. Dilatation of the urethra is indicated for localized eystitis at or near the neek of the bladder. The efficiency of the proeedure for such localized eystitis has given it an undeserved recognition in the treatment of general cystitis which under cystoscopy it cannot now retain.

9. The most valuable disinfecting topieal application in cystitis is nitrate of silver or argyrol.

\section{PYELITIS AND NEPHRITIS.}

This topie has a special gynecologieal signifieance in the matter of diagnosis and treatment by means of the eystoseope and the ureteral catheter, which have been deseribed in Chapter III. The ureteral catheter is introduced into the ureter through the cystoseope. $\mathrm{By}$ this means one may wash out the urinary traet up to and including the pelvis of the kidney; as a result of this treatment apparent cures in cases of hydro-ureter and pyo-ureter have been recorded.

To wash out the ureter the patient is placed in the knee-breast position; the ureteral eatheter, with a short piece of rubber tubing attached, filled with a sterilized boric-acid solution, and elamped to keep the solution from running ont, is passed through the cystoscope into the ureter and the cystoseope withdrawn. A sterilized glass 
funnel, with an attached rubber tube eighteen inches long, is filled with the irrigating solution, and the two rubber tubes are connected by a small glass tube with a point sufficiently fine to fit into the tube on the catheter. By raising the funnel above the level of the body the fluid is made to flow through the ureter into the pelvis of the kidney. When the funnel is dropped below the level of the body the fluid returns; thus, by alternately raising and lowering the funnel, the fluid is made repeatedly to flow back and forth and to wash out the ureter and pelvis of the kidney. The fluid may, if desired, be changed one or more times during the treatment. The apparatus is similar to that shown in Figure 157.

Purulent or other accumulations in the ureter should be permitted to run out through the catheter before the washing out.

The practical value of the ureteral catheter as a therapeutic agent remains to be estimated. The attempt to cure chronic infection in the uterus, nose, throat, and other mucous cavities by washing them out with various fluid has generally not been followed with great success. It is probable that the ureter and pelvis of the kidney will not be an exception to the rule. Kelly puts forth a word of wise precaution on the urgency of making all ureteral manipulations with extreme gentleness. The eatheter should never be pushed up higher than it will pass readily, for such force would injure the mucosa and might be followed by infection. 


\title{
PART III. \\ TUMORS, TUBAL PREGNANCY, MALFOR- MATIONS.
}

\author{
CHA P T E R X V. \\ TUMORS OF THE VULVA AND VAGINA.
}

\author{
Varix. \\ Hæmatoma. \\ Elephantiasis. \\ Papilloma. \\ Carcinoma. \\ Sarcoma.
}

Fibromyoma.

Lipoma.

Lupus.

Enchondroma.

Neuroma.

Cysts.

\section{VARIX.}

Varix is not a neoplasm, but an aggregation of dilated or varicose veins in the erectile tissue of the bulbi vaginæ. The varicose state is caused by obstruction to the circulation. This obstruction arises from direct pressure upon the venous trunks by the gravid uterus or may arise from pressure exerted by tumors or inflammatory exudates. Habitual constipation, portal obstruction, and visceral disease may underlie and perpetuate the disorder; it belongs rather to advanced than to early life.

The tumor is oval, globular, or serpentine, may grow to the size of a child's head, is irregular in form, of dark-blue color, and temporarily disappears on pressure. The chief subjective symptoms are pruritus and, on walking or standing, a sensation of fulness and weight. Rupture of the distended veins, spontaneous or traumatic, may occur during parturition, and if external may cause dangerous, even fatal, hemorrhage. Rupture into the tissue of the mass gives rise to an accumulation of blood called hæmatoma.

\section{Treatment of Varix.}

The treatment includes mechanical support of the uterus, if displaced, regulation of the bowels, removal of waist constriction, the application to the varix of a pad held in place by a T-bandage, the use of astringent lotions, and, especially during pregnancy, frequent rest. The radical surgical treatment is the same as would be indicated by the general principles of surgery for varix in any other part. 


\section{HAMATOMA.}

Hæmatoma, a not uncommon result of varix, is an extravasation of blood, not a new growth. The causes have been indicated in the foregoing paragraphs on varix, and in Chapter XXXIX., on Traumatisms. The tumor may develop rapidly or slowly even to the size of an orange, is commonly unilateral, globular, elastic, and of a violet color. It is distinguished from pudendal hernia by the absence of impulse on coughing and by non-reduction on taxis; it may terminate by absorption or by suppuration, or the blood-clot may become eneysted. The treatment in the early stage is to arrest bleeding by means of pressure and the ice-bag. If an abscess develops, it should be opened freely and drained. A cyst-wall, if formed, should be dissected out and the wound closed by deep sutures.

\section{ELEPHANTIASIS-PACHYDERMIA.}

This disease, primarily a ehronic recurring lymphangitis, may be assoeiated with hyperplasia of the connective tissue, skin, mucous membrane, and epidermis of the vulva. The whole process results in the formation of a tumor, sometimes of large size, and most frequent between the years of puberty and the menopause. It is rare in temperate, common in tropical elimates, and epidemic in certain lowlying countries along seacoasts and in the islands of the tropics. An organism called the filaria sanguinis hominis is found early in the disease in the lymph-vessels of the affected part, and is the exciting eause; it may not be found later, for the vessels are blocked by fibrous deposit.' The tumor may involve the whole or a part of the vulva, most frequently the two labia majora, less frequently the clitoris, and least frequently the nymphæ.

The growth, when large, is apt to be quite pendulous. The surface may be smooth, rough, fissured, warty, or uleerated. The tumor, especially if ulcerated, gives forth a sero-albuminous exudate, which may be so profuse as to demand frequent change of elothing. Ulceration is common as the result of friction. Cases of twenty years' duration have been recorded. The enlarged labia may reach the enormous weight of fifty pounds. Both labia are involved simultaneously. The inguinal glands on both sides are enlarged. Chyluria is a frequent complication. The disease does not direetly impair the general health; it is, however, disabling from its meehanical interference with urination, walking, and coitus. Other diseases of the vulva (so-ealled false elephantiasis) of entirely different character, such as papilloma, may easily be mistaken for elephantiasis.

\section{The Differential Diagnosis of Elephantiasis.}

The differential diagnosis from papilloma, carcinoma, sarcoma, fibroma, and lipoma depends upon the clinical listory as ontlined in the foregoing paragraphs and upon the mieroseopical finding. Unlike elephantiasis, all these growths are free from any induration of

1 Roterts' Gynccological Pathology, Auatomy, and Histology. 
the surrounding skin. Lupus presents more extensive uleerations, deeper induration, darker eolor, and has for its essential factor the tubercle baeillus.

\section{Treatment of Elephantiasis.}

The treatment is excision. The numerous dilated lymph-ehannels increase the danger of septie absorption, and in the operation render

FIGURE 158.

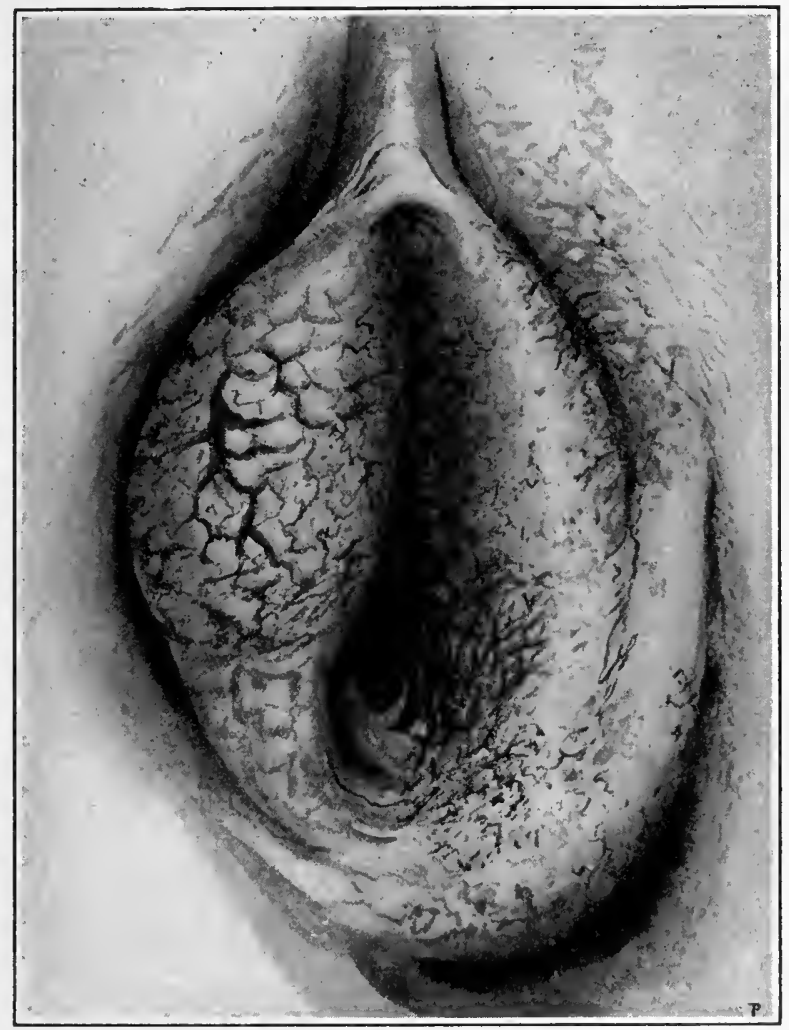

Elephantiasis of vulva.

the most extreme asepsis imperative. 'The operation is similar to that described for kraurosis and pruritus vulvæ, Figure 81.

\section{PAPILLOMATA, CONDYLOMATA, OR WARTS.}

Warty growths are epithelial in type and are characterized by hypertrophy of the papillæ of the skin or mucous membrane, increase of connective tissue, and thickening of the epithelial covering. They are divided into three elasses:

1. Non-speeific-simple papillomata-ordinary warts. 
2. Gonorrhœal-condylomata acuminata-pointed condylomata, specitic vegetations or venereal warts.

3. Syphilitie-flat condylomata.

1. Non-specific, Simple Papillomata, or ordinary warts, are found not uneommonly on the mons veneris, less frequently on the labia. They are of unknown origin, usually of dark-brown color, are not deeply divided, may have a broad base or may be peduneulated, and are not apt to coalesee into large, compact masses.

The Treatment is exeision with the sharp curette and cauterization of the base.

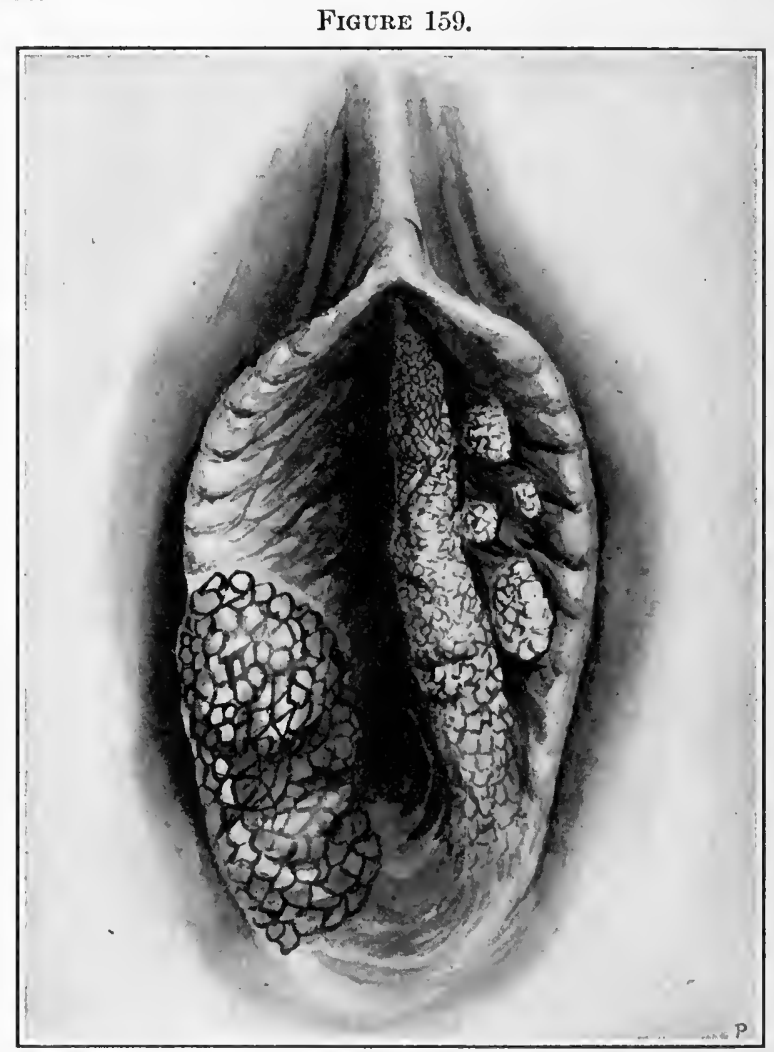

Simple warty vegetations of the vulva.

Gonorrhœal Warts-that is, warts associated with gonorrhœaare found on the vulva, vagina, eervix uteri, perineum, and about the anus. These warts oecur singly in groups, or in cauliflower-like masses. The growths may be so large as to interfere with coitus, urination, or defecation; they present a surface which is soft, moist, of bluish color, and divided into small nodules with pointed ends like a coek's comb. The growth occurs during pregnaney, is rapid, but mav disappear promptly after labor. Thiere is usually an associated fetid vaginitis. The question has been raised whether this form of condy- 


\section{PLATE XII}

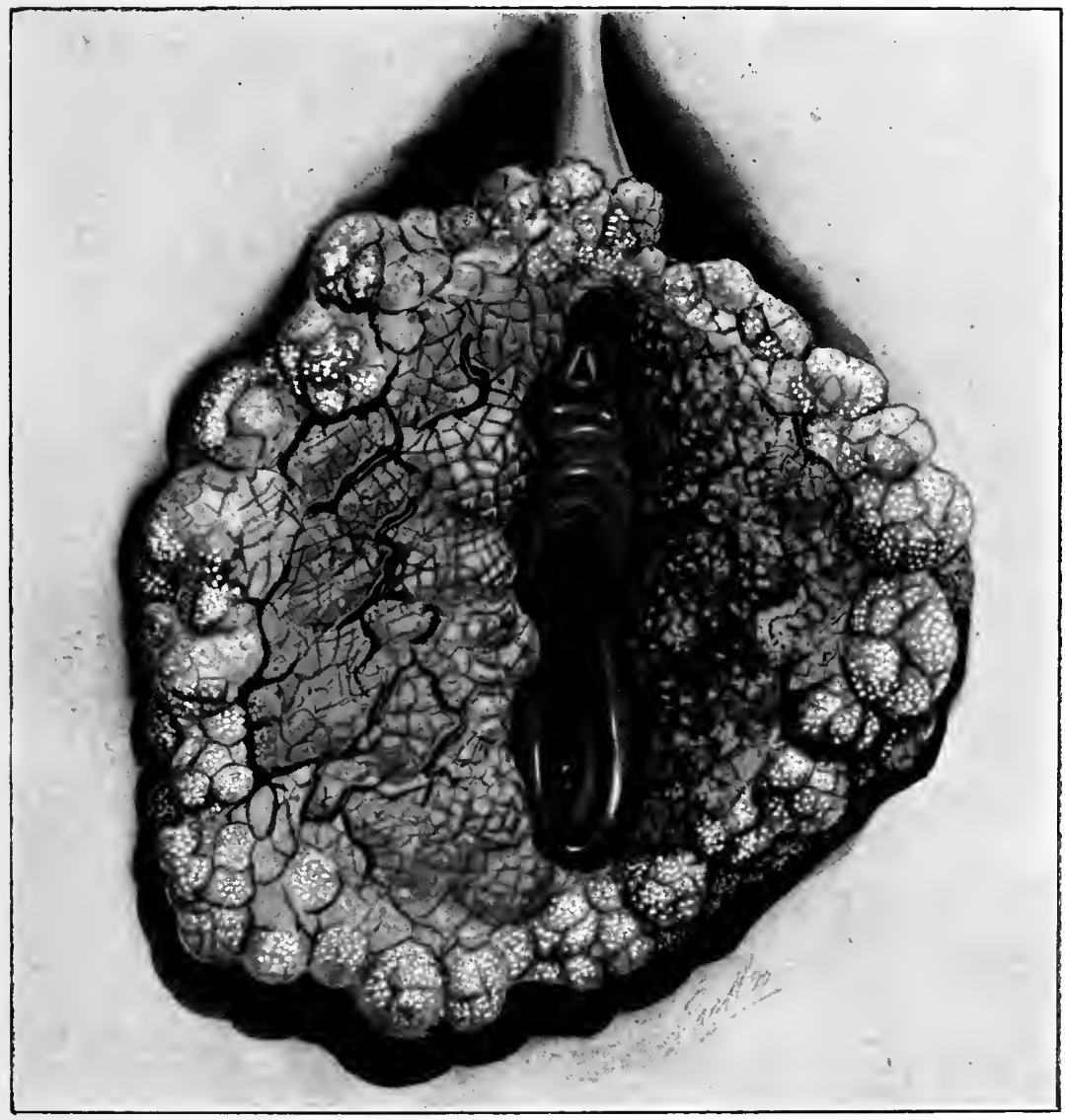

Enormous warty developments about the vulva. These growths were so extensive that after the removal of them it was impossible to draw the skin over the exposed surfaces, and recourse, therefore, was had to the device set forth in the plastic operation described in the following Plates Author's operation. (Surgery, Gynecology, and Obstetrics, June, 1906al 



\section{PLATE XIII}
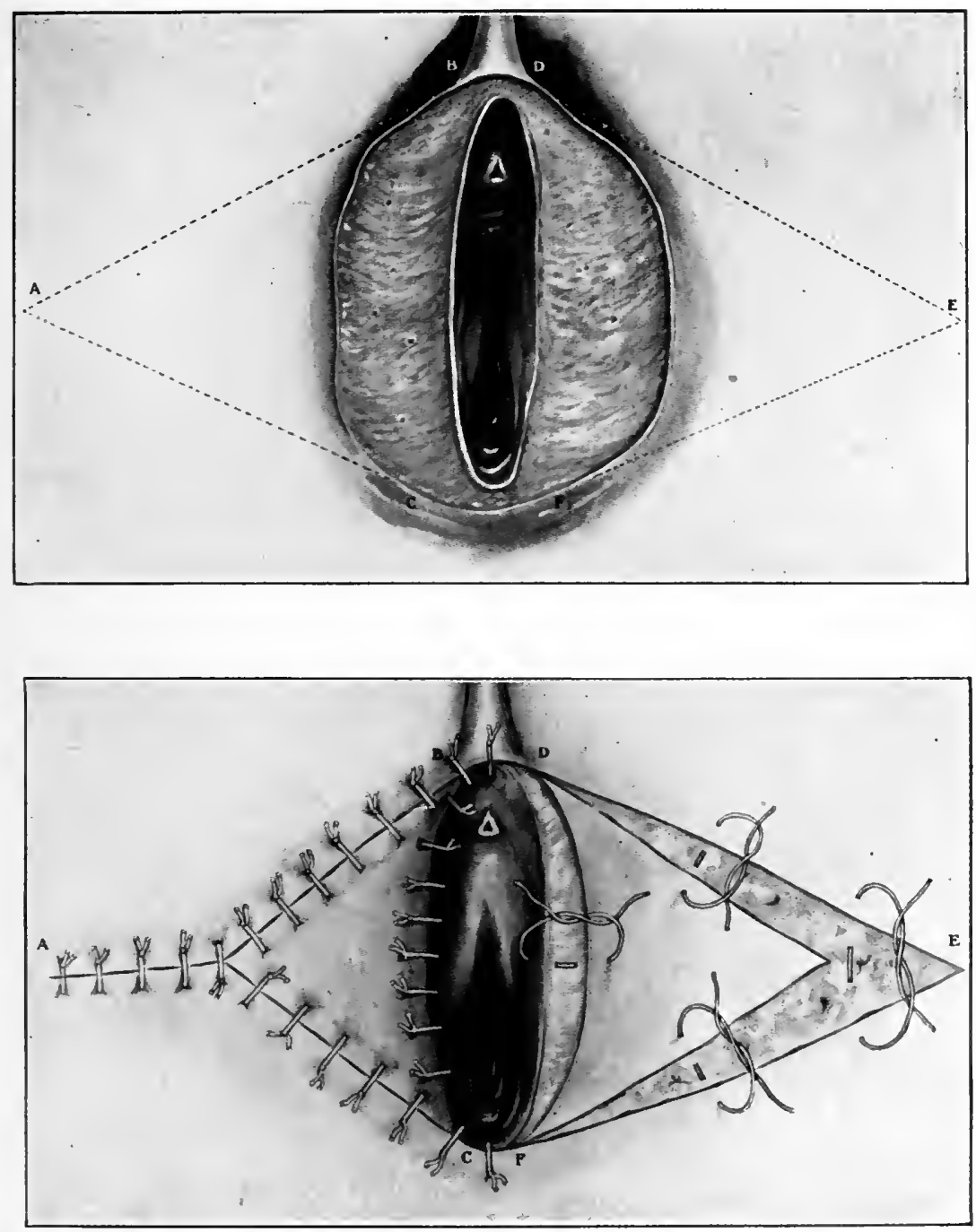

In order to cover the exposed surfaces made by the removal of the growth (Plate XII), deep lateral incisions are made, as shown in the upper figure by the dotted lines $\mathrm{A} \mathrm{B}, \mathrm{A} \mathrm{C}$, $\mathrm{D}$ E, F E. The space included in the triangles $\mathrm{A} \mathrm{B} \mathrm{C}$ and D E F are slid inward toward the vulva and the wounds are closed, as shown in the lower figure. The same device is useful also in closure of the completely lacerated perineum, when, as sometimes occurs, so much tissue has been destroyed as to prevent easy approximation. 

loma may not occur independently of the gonococcus, but the clinical evidence, including the results of bacteriological studies, strongly points to at least a coexistent gonorrhoa. The part played by the gonococcus, except as a predisposing cause, is doubtful.

The Treatment includes, first, thorough eleansing and disinfection of the diseased region; second, removal of the so-called vegetation with scissors, and cauterization of the base; third, antiseptic dressing and washes until the parts have healed. The danger of puerperal sepsis and ophthalmia of the newborn infant strongly suggests radical measures during pregnancy. The $x$-rays are among the most recent and effective means of treatment.

Flat Condylomata-modified mucous patches-are of syphilitic origin and may involve large surfaces of the vulva and vagina. They are soft, grayish, have a broad base, and should be treated by disinfectant and antisyphilitic remedies.

\section{CARCINOMA OF THE VULVA.}

Carcinoma is of epiblastic origin, and is apt to be of the pavementcell variety-epithelioma. The author observed one case of adenocarcinoma a few weeks after the removal of a cancerous uterus. In this case the disease doubtless was transplanted from an adenocarcinoma of the uterus to the vagina during the operation of hysterectomy. Prompt excision of the vulvar growth was followed by permanent cure.

Pavement-cell Carcinoma begins as a small, hard, whitish, rough and painless wart-like excrescence, situated at any point on the vulva, but more commonly on the sulcus between the labia majora and minora. There is first a gradual involvement of the superficial structures around the growth, then rapid ulceration and pain. The inguinal glands on the side corresponding to the disease are involved. The margin of the ulcer is elevated, hard, and of bluish-red color. 'The base is granular and covered by a semi-opaque, putrid secretion.

Small, pearly bodies may often be squeezed from the epithelial nests at the base; these nests are highly diagnostic. The labium becomes greatly infiltrated, very hard and thickened, and finally is destroyed by ulceration. The discharge has a most offensive odor. The disease rarely extends to the opposite labium, vagina, or abdominal wall. It may invade the perineal and peri-anal regions. The disease is analogous to epithelioma of the lip and of other parts where skin and mucous membrane meet.

The Diagnosis is chiefly from lupus and syphilis. Unlike cancer, lupus is recognized by the mildness of the pain, by the relative freedom from foul secretions, by the tendency of the ulcers to cicatrize, by the slight liability of extension to the inguinal glands, and by the slow progress of the disease. Epithelioma destroys life in about two years after the beginning of ulceration. Syphilis may be differentiated by the history of infection, by the presence of secondary and tertiary lesions elsewhere, and by the effect of spccific treatment.

Cylindrical Cell Carcinoma-Adenocarcinoma.-The swelling begins more deeply in the cellular tissue, and is characterized by irreg- 
ular-shaped cylindrical cells imbedded in the meshes of conneetivetissue fibres and by more rapid progress than that of epithelioma. The tumor more rapidly breaks down, the hemorrhage is frequent and profuse, the ichorous discharge is abundant, the inguinal glands are enlarged early, and systemic effects appear earlier and are more marked. 'The disease terminates in sepsis and marasmus. Death oceurs earlier than in epithelioma.

Figure 160.

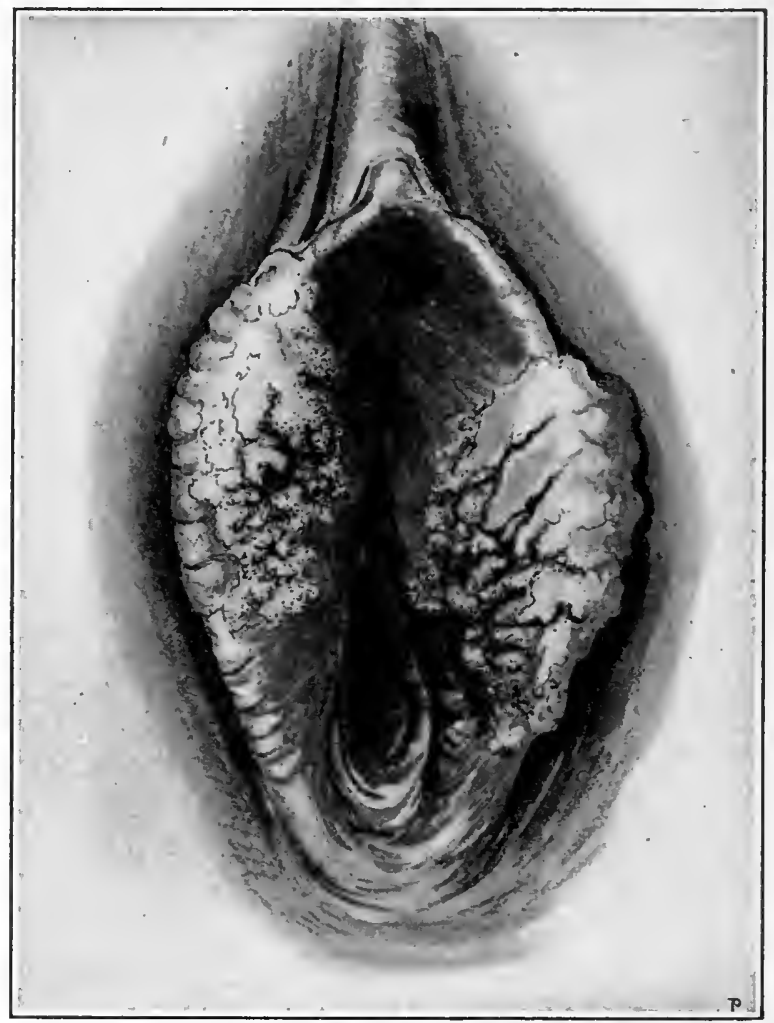

Carcinoma of the vulva.

The Treatment is radical excision if possible before glandular involvement.

\section{SARCOMA OF THE VULVA.}

Sarcoma is of mesoblastic origin and is so rare in the vulva as to preclude accurate description. The possible varieties are: first, round cell ; second, spindle cell ; third, myxosarcoma ; fourth, melanosarcoma. They preferably develop in the labia majora, but have been found in the nymphæ. The growth, according to the variety, may be slow, resembling that of lipoma $;^{1}$ or ulceration may be early, rapid, and destructive. ${ }^{2}$ The usual characteristies of sarcoma of the vulva

s Henkel.

2 Hildebrandt. 
are rapid growth, late ulceration, variable hemorrhages, and late involvement of the inguinal glands. The systemic breakdown, though more rapid and marked, resembles that of carcinoma. All recorded cases have terminated fatally. Death usually results from rapid involvement of distant organs through the venous current.

The Treatment is removal at the earliest possible date. 'The author here records a successful operation done more than fifteen years ago for the removal of a spindle-cell sarcoma of the mons veneris. There has been no recurrence.

\section{CYSTS OF THE VULVA.}

The pathology of cysts of the vulvovaginal gland has been explained in Chapter XI., under Inflammation of Bartholin's Glands. The only satisfactory treatment of such a cyst is to open the sac, dissect out the sac-wall, and close the. wound with sutures. Sebaceous cysts rarely occur.

\section{FIBROMYOMA OF THE VULVA.}

Fibromyoma belongs to the connective-tissue group of benign tumors, and is therefore of mesoblastic origin. It is composed of fibrous connective tissue and a variable amount of muscular fibres. The histological characters of this tumor will be given more fully under the subject of Fibromyoma of the Uterus. The tumor is commonly small, and when large is apt to be pedunculated; it is smooth, irregular in shape, is not adherent to the skin, and according to the amount of fluid in the interspaces may be hard or soft; it often is ulcerated from friction, but is rarely the seat of an abscess. The symptoms are mechanical, and are due to weight and pressure.

The Treatment is incision.

\section{LIPOMA-FATTY TUMOR OF THE VULVA.}

Lipoma is composed of lobuli of adipose tissue in a fibrous meshwork, and originates in the fatty tissue of the labia majora and mons veneris. It is distinguished from fibromyoma by the greater rapidity of growth, by the lobulated surface, and by a peculiar sensation to the touch. This sensation is such as would be expected from a wad of cotton under the skin. Lipoma may grow to the weight of ten pounds, may extend to the knees, and may be pedunculated; it has been mistaken for hernia.

The Treatment is excision.

\section{TUBERCULOSIS-LUPUS.}

Lupus, from the pathological point of view, should be classed as tubercular inflammation. The tumor-like mass, however, presents physical characteristics in common with certain tumors, and, therefore, is introduced here from the clinical and diagnostic points of view. See Tubercular Vulvitis, Chapter XI. 


\section{ENCHONDROMA AND NEUROMA OF THE VULVA.}

Enchondroma and neuroma are surgical curiosities. Simpson ${ }^{1}$ has reported the only authentic case of neuroma. Schneevogt and Bartholin have each recorded a case of enchondroma.

\section{CYSTS OF THE VAGINA.}

Vaginal cysts, though not common, are the most frequent of the tumors originating in the vagina; they are probably from: 1 , the

Figure 161.

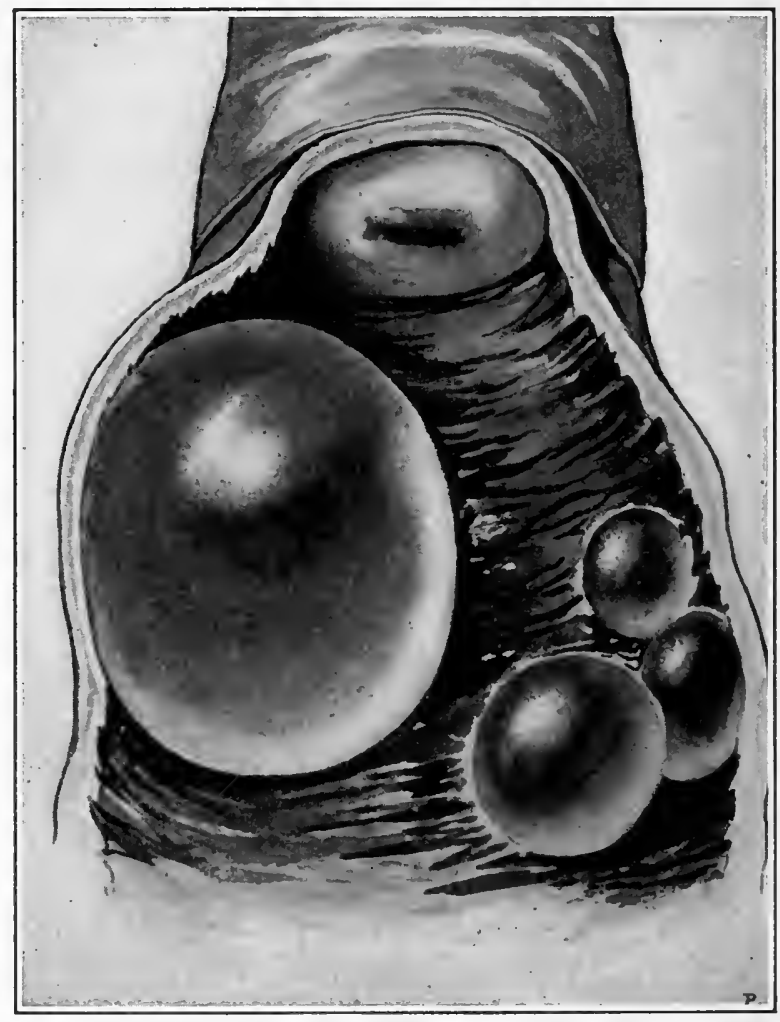

Vaginal cyst8.

embryonal remains of Gacrtner's (Wolffian) ducts ; or, 2, from diverticula of Mueller's ducts. An echinococcus cyst ${ }^{2}$ has been reported. Embryonal vaginal cysts are usually not larger than a walnut, although Veit has reported one as large as a foetal head. ${ }^{3}$ They are circumscribed, tense, elastic, rarely pedunculated, and commonly unilocular; they occur singly, or in rare instances are arranged

1 Sutton. Tumors, Innocent and Malignant.

2 Porak. Arch. de Tocologie, 1884, p. 163. Pozzi. Medical and Surgical Gynecology.

3 Pozzi. Medical and Surgical Gynecology. 
in groups of two, three, or four in a row. The cyst-wall is composed of fibrous tissue, with an inner lining of cylindrical or pavement epithelium and an outer covering of vaginal mucous membrane. The contents are commonly viscid, transparent, and of a pale-yellow color. The occasional chocolate color is explained by the presence of blood, pus, and epithelial cells.

The differential diagnosis is from cystocele, rectocele, emphysematous vaginitis, and vaginal hernia. Cystocele is demonstrated or excluded by the sound in the bladder and the finger in the vagina; rectocele by one finger in the rectum and another in the vagina. The cysts of emphysematous vaginitis contain gas, are usually multiple, and do not follow the course of Gaertner's ducts. The hernial tumor temporarily disappears on pressure and gives an impulse on coughing.

Treatment.-If the cyst is within easy reach, the treatment is excision; if it is very close to the rectum, bladder, or ureters, the vaginal side should be removed, the remainder curetted or cauterized, and the wound packed with gauze.

\section{FIBROMYOMATA OF THE VAGINA.}

Fibromyomata of the vagina differ in no essential point from similar growths of the vulva and uterus. They are of rare occurrence, and usually small, but sometimes are large enough to give the mechanical symptoms of pressure and weight.

The Treatment is enucleation.

\section{CARCINOMA AND SARCOMA OF THE VAGINA.}

Carcinoma of the vagina usually occurs by extension from primary carcinoma of the cervix, uterus, or rectum; it rarely originates in the vagina. Sarcoma of the vagina is almost unknown.

The Treatment-early excision-gives most unsatisfactory results. 


\section{CHAPTER XXVI.}

\section{TUMORS OF THE UTERUS-MIYOMA}

\section{ETIOLOGY, HISTOLOGY AND HISTOGENENIS, NOMENCLATURE, CLASSIFICATION, SYMPTOMS, DIAGNOSIS, DIFFERENTIAL DIAGNOSIS, PROGNOSIS.}

THE uterine myoma is the most common uterine tumor, and like the uterus is composed chiefly of fibrous connective tissue and nonstriated musular fibres.

\section{Etiology of Myoma.}

Myomata commonly develop during the period of sexual maturity, rarely if ever before puberty or after the menopause. The common impression that they are more common in the negro than in the white race appears to be disproved by the investigations of Howard Kelly and Daniel Williams. ${ }^{1}$ Heredity probably is an etiological factor. In the older literature they are said to result from traumatism, but the assertion is purely speculative, no evidence having been brought forward in support of it. The causes of these tumors are not known.

\section{Pathological Anatomy of Myoma.}

In most cases the tumor is circumseribed sharply, single or multiple, hard or soft, of pinkish or whitish color commonly of slow growth, and varying in size within wide limits. On cross-section the gross appearance is glistening and may be homogeneous, but more usually is striated with dense fibrous septa which divide the section into lobules. The spaces between the septa are filled with muscle-fibres. See Figures 156 and 157. In later development a loose fibrous capsule is formed which sharply defines the growtl from its surroundings; and from which the growth may be shelled ont readily; the blood-vessels of the fibrous capsule penetrate through the septa to the muscle-cells.

These growths are subject occusionally to extensive venous obstruction and dilatation which often leads to the formation of cavernous spaces ; hence the blood-supply, not only in different tumors, but at different times in the same tumor, is subject to great variation. This changeable blood-supply accounts for corresponding variation from time to time in the size of a tumor. Hard white tumors of a slow growth, containing a relatively large amount of fibrous tissue, are apt to have a limited blood-supply. On the other hand, the soft pinkish tumor of more rapid growth, with a relative preponderance of muscle-cells, is always more vascular. 


\section{PLATE XIV}

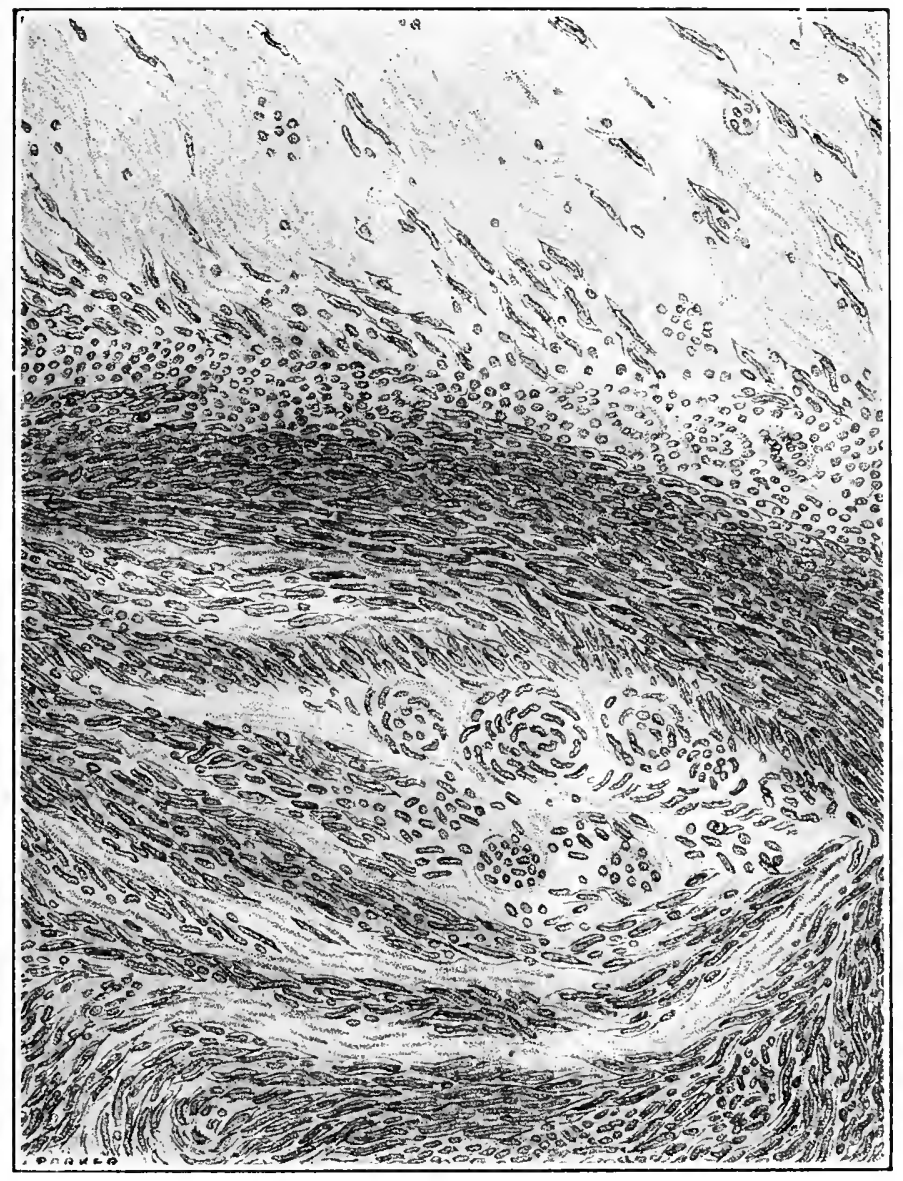

\section{Microscopic Section of a Uterine Myoma.}

The upper part of this figure contains few muscle cells and much fibrous stroma (fibromyoma). The remainder of the field shows an abundance of muscle cells and relatively speaking a small amount of fibrous stroma (myofibroma) In the lower half of the picture the spindle cells are arranged in whirls. Just above these whirls is a densely packed mass of spindle cells the nuclei of which are cut longitudinally, and just above this mass is at belt of cells the nuclei of which appear circular in shape because they are cut transversely. From a nistological point of view a uterine myoma may have all the microscopic appearance of normal uterine tissue. This drawing in different parts illustrates normal uterus tissue, fibromyoma, and myofibroma. Ioo diameters. 



\section{Histology and Histogenesis of Myoma.}

The characteristic cell elements are non-striated fusiform musclefibres with elongated nuclei which may be combined in any proportion with fibrous tissue. Nothing is known of the histogenesis of these tumors beyond the fact that they originate in the myoblast, a fact which stamps them as myomata, regardless of the relative quantity of the museular and connective tissue. The tumor does not lose its identity as a myoma even though all the muscular elements have disappeared and been replaced by fibrous tissue.

\section{Nomenclature of Myoma.}

Myofibroma, Fibromyoma, and Fibroma.-A soft vascular tumor, because it contains a large amount of muscular tissue, has been called myofibroma or leiomyoma. The hard, more fibrons myoma often is called fibromyoma or fibroma. There is no definite line between the so-called myofibroma and fibromyoma. The terms are relative and, to an extent, arbitrary, and to be used only for convenience of description.

\section{Secondary Changes of Myoma.}

The secondary changes common to uterine myoma are: Fatty degeneration. Mucoid degeneration. Cystic degeneration.

Calcification. Septic infeetion. Malignant changes.

Fatty Degeneration.-This secondary change pertains to the muscle-fibres and may destroy them completely, leaving behind a contracting formation of fibrous connective tissue which in the process of solidification is apt to crush out and destroy the blood-supply so that the tumor deprived of nutrition becomes a lard rudimentary mass; the process which may pertain to the whole tumor or to parts of it is a frequent factor in the atrophic change of the menopanse and, especially in snall tumors, explains numerous spontaneous cures occurring at this period.

Mucoid Degeneration.-This process, usually preceded by odema and rapid increase in the size of the tumor, occurs principally in large fibromyomata, and is characterized by the conversion of the fibrous tissue into mucous substance resembling the vitreous humor of the eye. The structures which form the boundary of the softened spaces may show every gradation from typical spindle cells to myxomatous cells of the spider-like shape. There may be developed numerous small cysts in a tumor or a single large spurious fibrocyst laving for its wall the fibrous capsule of the orignal tumor. This is called a fibrocystic tumor. Figure 162.

Cystic Degeneration may occur as follows: Edema may cause so much dilatation of the lymph-spaces as to give the whole tumor an appearance of marked cystic degeneration, or the dilated cavernous veins already described may be converted into blood-cysts.

Calcification occurs most frequently in atrophied subperitoneal 
tumors, and may pertain to the individual filorous septa or to the capsule or in exceptional cases the entire tumor may be displaced by lime salts and converted into a stone-so-called womb-stone. A section of such a stone made by the saw will sometimes take a high polish with the whole arrangement of the fibrous septa and capsile reproduced in the lime salts. More commonly the spaces between the septa do not calcify, but disappear by some other degenerative process giving the ealcified part a porous, worm-eaten, or coral-like appearance. When the calcification is chiefly or wholly in the

Figure 162.

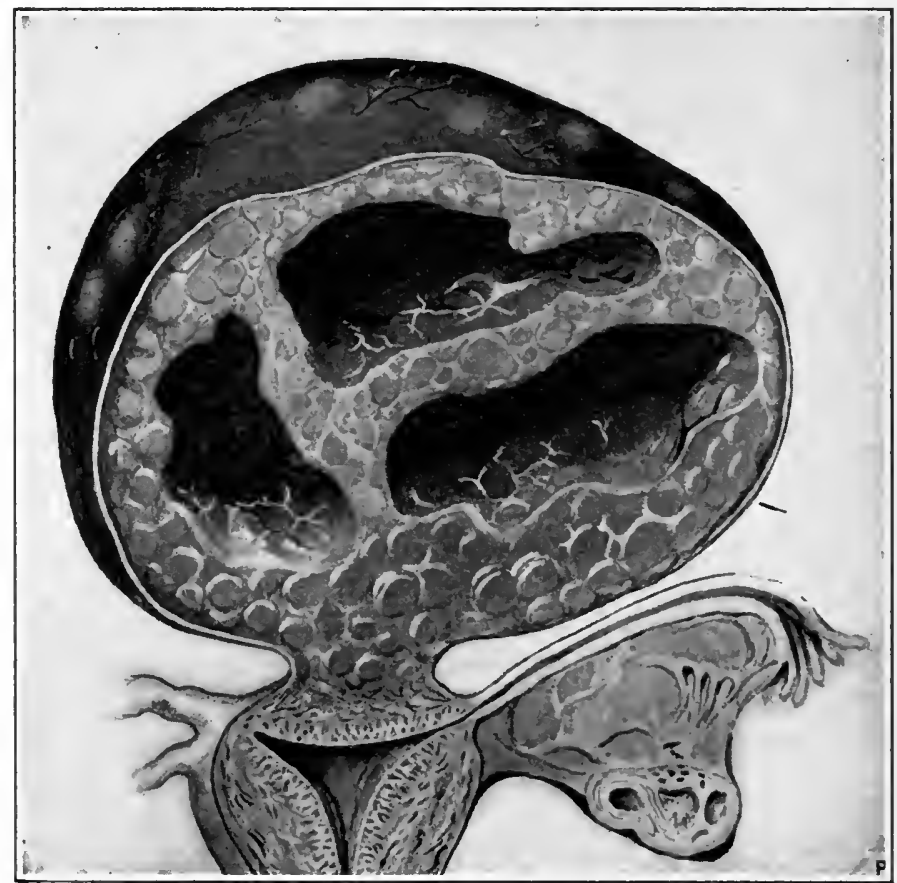

Fibrocystic myoma uteri. The interior of the tumor shows the fibrocystic changes.

fibrous capsule the tumor is covered by a thin hard erust which may resemble closely the foetal skull. In the enneleation of such a tumor from the corpus uteri the writer once found a caleified capsule which under the thin wall of the corpus uteri felt so much like a foetal head-including the sutures and fontanelles-that he was led almost to abandon the operation.

Septic Infection.-A myoma which has for years given rise to no inconvenience, may become suddenly infected, with rapid increase in size, high pulse and temperature, great pain, and evidences of septicæmia. The cause of infection, sometimes obscure, is explained usually by the presence of one or more of the known causes of pelvic inflammation, such as electrolysis, the unclean intra-uterine sound, external violence, septic operations, and extension of infection 
from an adherent intestine or bladder. A fatal result is almost inevitable unless the diagnosis is made early and the tumor renuoved.

Malignant Changes will be considered in Chapters XXVIII. and XXIX., on Carcinoma and Sarcoma.

\section{Classification of Myoma.}

Location.-The tumor may be anywhere in the nterine substance. Figure 163 ; but in the majority of eases it is in the body of the

Figure 163.

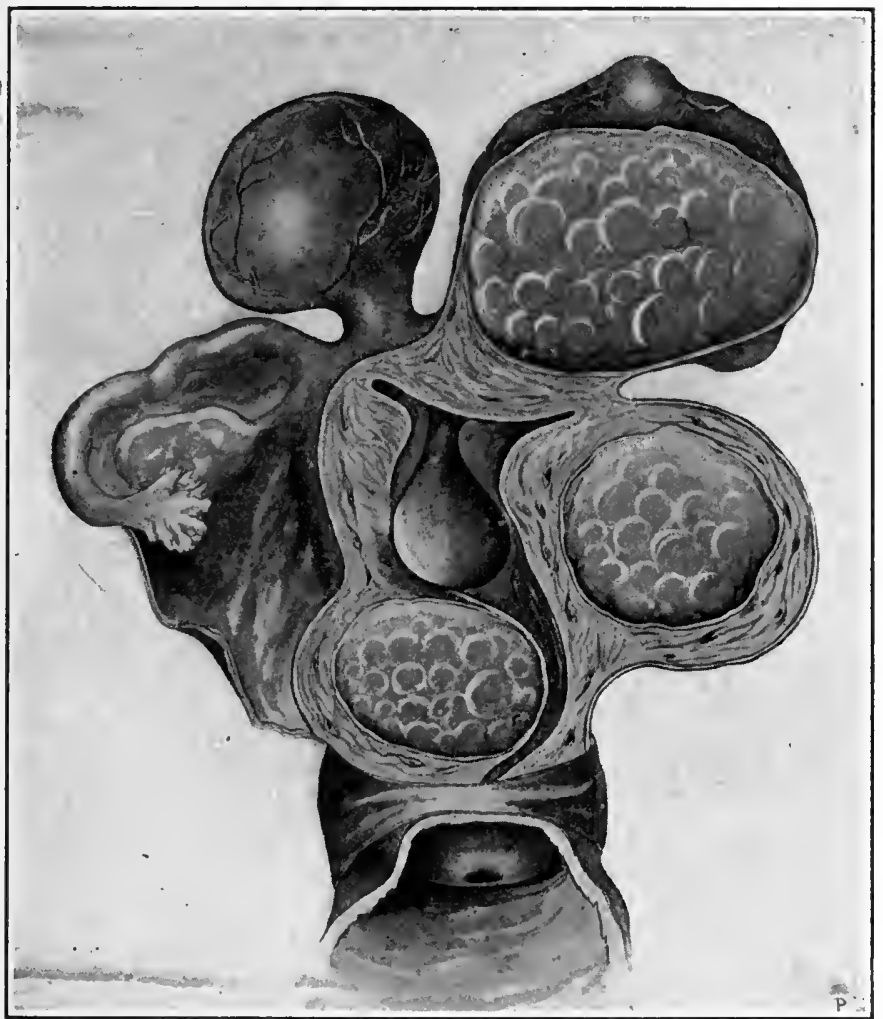

Intramural, submucous, and subperitoneal myomata. A pedunculated subperitoneal myoma sometimes is called wrongly extra-uterine myoma. A pedunculated submucous myoma is called intra-uterine polypus.

uterus. Tumors of the cervix uteri are apt to be small, those of the corpus larger.

The regional elassification, Figure 163 , is :

1. Intramural (interstitial) myomata.

2. Submueous myomata.

3. Subperitoneal myomata.

4. Cervical myomata.

1. Intramural Myomata.-All myomata are prinarily intramural- 
$i . e$, they originate in the musculature, but the term intramural is reserved here for tumors surrounded wholly by the muscular wall of the uterns. The growth in most cases is firm, sharply defined, and eneapsulated or in exceptional cases soft, ill-clefined, and without a definite capsule; it has from its situation an abundant blood-supply on all sides, and for this reason may grow rapidly to large size. It will always irritate the surrounding muscular tissues, and cause them to contract upon it so that if it is nearer to the endometrium than to the

Figure 164.

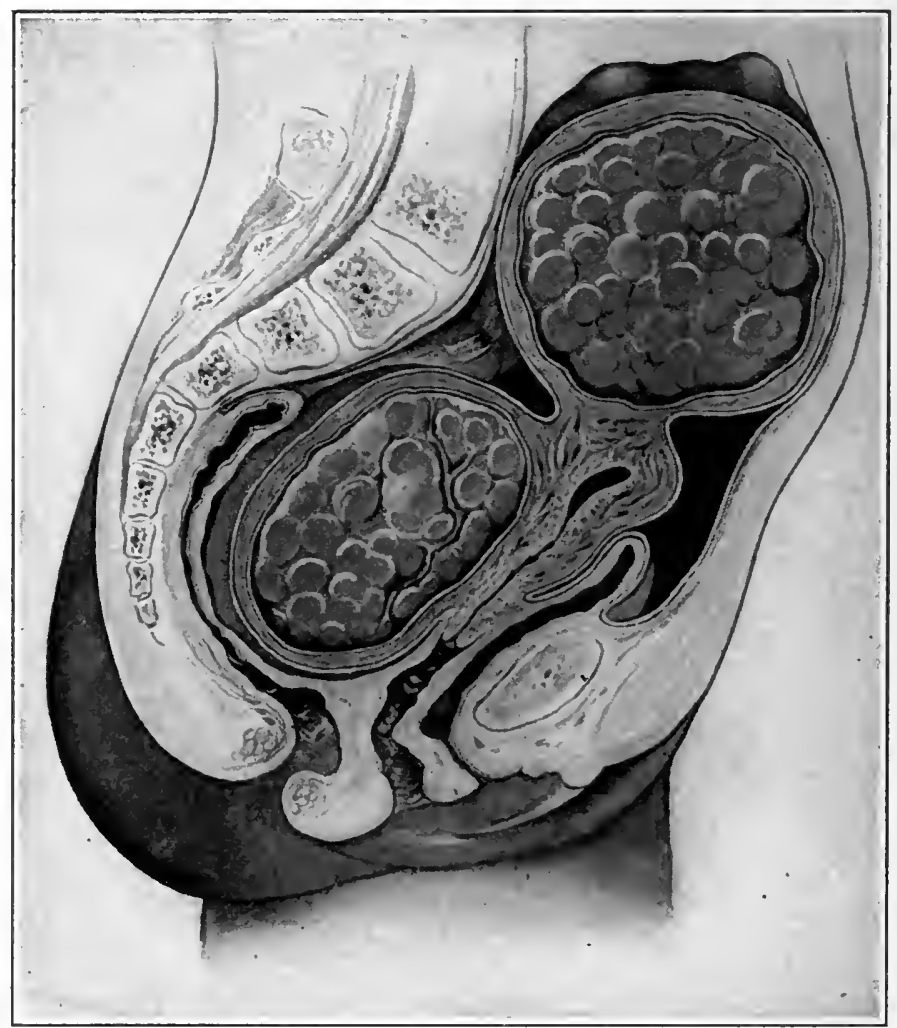

Intramural myomata. The lower tumor exerts pressure on all the pelvic organs. The upper tumor displaces the uterus upward by traction.

peritoneum, the preponderance of muscular tissue on the peritoneal side will force it slowly toward the interior of the uterus and tend to make of it a submucous tumor. If the preponderance of muscular tissue is between the tumor and the endometrium, the direction of least resistance will be toward the peritoneum, and the growth will tend to become subperitoneal. The same uterus may contain one or many intramural tumors. Figure 16j. Intramural is synonymous with interstiticl. Figure 164.

2. Submucous Myomata may originate in the muscular tissue of 
the submucosa, and be, therefore, primarily submucous; or an intramural tumor, as explained in the preceding paragraph, may become secondarily submucous. The secondarily submncous tumor is apt to remain sessile-i.e., to have little tendency to form a pedicle. The primarily submucous tumor, on the contrary, develops a pedicle. The pedunculated submucous myoma is vascular, soft, commonly single, usually corporeal, rarely cervical, in most cases small but may be large enough to distend the uterus enormously. It may be

Figure 165.

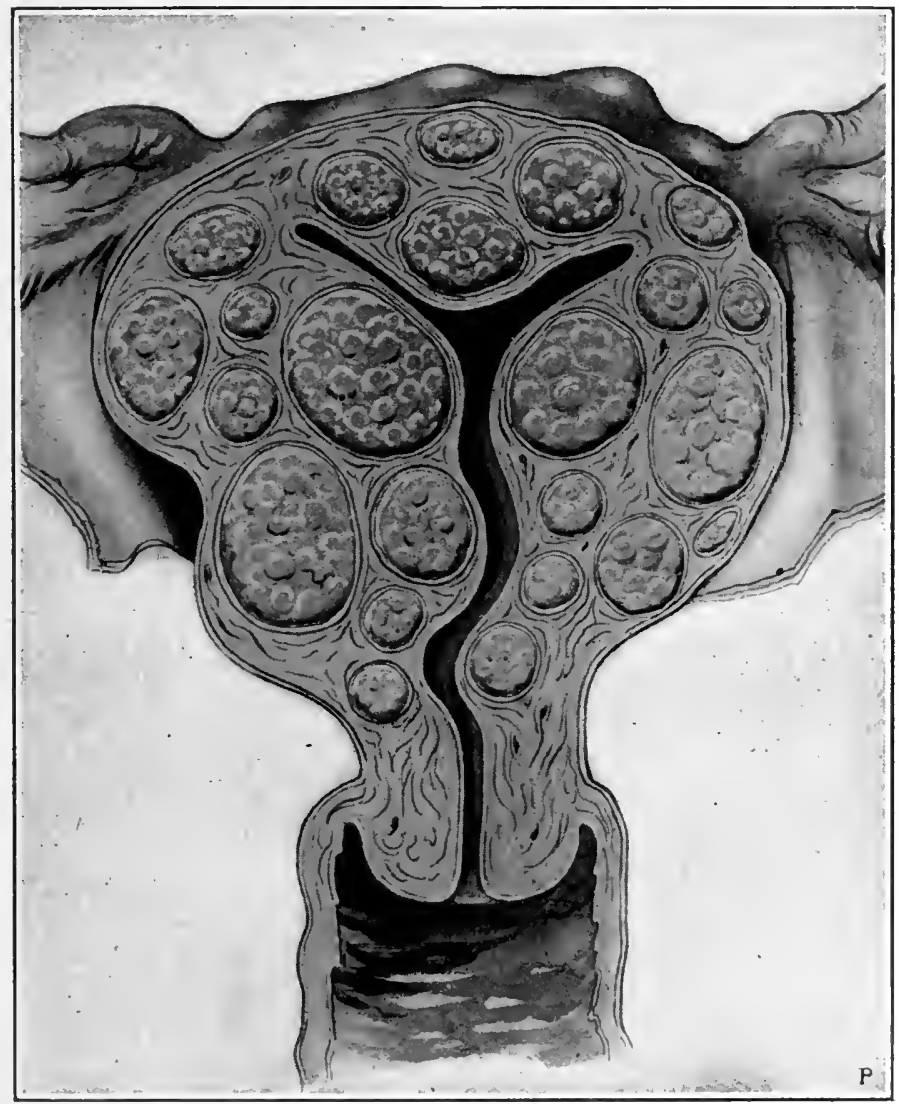

Multiple myomata, suitable for vaginal hysterectomy.

forced by uterine contractions through the cervix uteri, and the pedicle by the downward traction of the tumor may become much elongated so that the extruding mass may finally be forced through the vulva. The pedicle may be constricted by pressure of the cervical canal or may become twisted with consequent gangrene of the tumor and spontaneous detachment and cure, but not uncommonly the extruded mass remains celematous and hemorrhagic, a menace to health or a destroyer of life. 
Adhesions may form between an intra-uterine tumor and the endometrium or cervical mucosa, and by partial or complete obliteration of the uterine cavity lock the secretions within the uterus.

3. Subperitoneal Myomata-sometimes called subserous-may be either single or multiple, and occasionally may reach the enormous size of forty or fifty pounds. These tumors, primarily intramural, have been forced outward by uterine contractions until they become secondarily subserous, or such a tumor has worked its way from the point of origin into the territory between the folds of the broad ligament, and become an intraligamentous myoma. A pedunculated subserous tumor may, in rare cases, become detached from the uterus and

Figure 166.

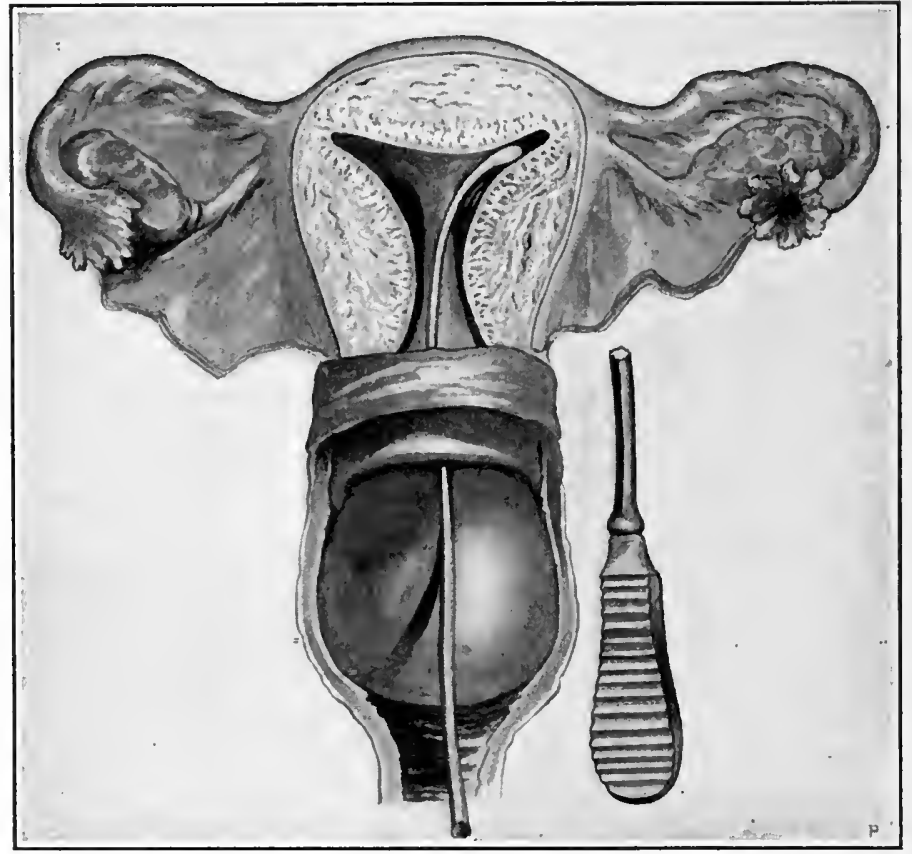

Submucous pedunculated myoma, resembling an inverted uterus.

remain as a migrating tumor, free and harmless in the abdominal cavity; or may receive its nutrition through new adhesions which have formed between it and some of the pelvic or abdominal viscera ; or may become gangrenous and give rise to serious infection ; or may atrophy and disappear.

4. Cervical Myomata.- Myomata of the cervix uteri are of rather infrequent occurrence, follow the same law as to development and location as myomata of the body of the uterus, and usually spring from the supravaginal, rarely from the infravaginal, portion of the cervix. A submucous cervical myoma is usually pedunculated, and may have the appearance of a uterus inverted into the vagina. An 
intramural cervical mycoma causes thickening of the cervical wall around it, and by pressure and stretching a corresponding thinning of the opposite wall.

\section{Symptoms of Myoma.}

The symptoms may be described under the following heads:

Hemorrhage. Congestion. Pain and discomfort.

Pressure and traction.

Miscellaneous symptoms.

Hemorrhage, the most important and the most pronounced symptom, begins not as a sudden, profuse flow, as in carcinoma, but as a gradual increase in menstruation; the bleeding occurs frequently, is prolonged, and may result from ordinary conditions, such as exercise or coitus. The irritating presence of the tumor predisposes to hemorrhagic endometritis, the hemorrhagic area being the endometrium, not usually, as sometimes supposed, the tumor itself. Fatal hemorrhage, however, has occurred from rupture of a blood-vessel in the growth.

The degree of hemorrhage depends upon the location of the tumor relative to the endometrium and the peritoneum. The closer to the uterine mucosa, the greater the hemorrhage; the nearer to the peritoneum, the less the hemorrhage; hence menorrhagia is almost invariable with the submucous variety, less severe but very common with the intramural, and usually slight or absent with the subperitoneal. The pedunculated submucous and the pedunculated subperitoneal myomata stand at the two extremes, the former producing the greatest hemorrhage, the latter little or none at all. Hemorrhage is not always proportionate to the size of the tumor; a large tumor may obstruct the flow of blood, or by pressure-atrophy of the endometrium may give rise to scanty menstruation; on the other hand, a small submucous myoma may cause alarming uterine hemorrhage.

A myoma often delays, prolongs, or prevents the menopause; it may participate in the atrophic processes of this crisis, and become smaller, or disappear ; in some cases the menopause has the opposite effect-i. $e$, great and surden increase of growth. Such increase is a strong indication for myomectomy or hysterectomy.

Pressure and Traction cause numerous mechanical and other disturbances of the rectum, bladder, ureters, urethra, and of the uterus itself, such as hemorrhoids, constipation, rectal and vesical tenesmus, mucous diarrhcea, frequent urination, dysuria, retention of urine, and uterine displacements. Pressure upon the venous trunks often causes great dilatation of the veins and passive congestion throughout the pelvis. Pressure on a ureter has given rise to obstruction and caused hydronephrosis.

A myoma in the anterior nterine wall, even though small, may, by pressure, set up extreme vesical irritation with the possible consequence of cystitis. Pressure from a myoma incarcerated under the promontory of the sacrum unless the tumor spontaneously or normally is forced up into the abdominal cavity, will cause great pain 
and interference with functions not only in the pelvis, but also in the lower extremities.

Uterine displacements may result from pressure, traction, and increased weight. A tumor situated above, below, to either side, in front, or back of the uterus may force it by pressure in the opposite direction, or may draw it by traction in the same direction, or, by increasing the weight of the uterus, may cause prolapse. A myoma, for example, which has grown too large for the pelvis to hold it, and has therefore risen above the pelvic brim into the abdomen, will cause upward displacement by traetion.

The Pain and Discomfort incident to this affection have been mentioned in the foregoing paragraphs under Pressure and Traction. Backache, bearing-down, dragging sensations in the pelvis, dysmenorrhoea, and painful uterine contractions are familiar subjective symptoms. Expulsive contractions of the uterus upon a mural or submucous myoma, especially during the period of menstrual congestion and irritation, may be transient or constant, moderate or severe.

Miscellaneous Symptoms. - Intermenstrual uterine diseharges caused by the great vaseularity of the uterine mucosa and the hypertrophic condition of the glands, usually occur in the progress of the disease ; they may be purulent or serous, or both ; are mixed commonly with blood, and are often profuse and exhansting. The watery discharge-hydrorrhœa-nsually associated with nalignant disease is very infrequent in myoma, but when present is more transient and less offensive than in eancer or sarcoma.

\section{Diagnosis of Myoma.}

Uterine myomata, unless very small and associated with metritis, usually are not difficult to recognize. The symptoms outlined in the foregoing paragraphs, although diagnostic, are far from pathognomonic. The diagnosis will depend always upon the physical signs - that is, upon inspection, palpation, conjoined examination, and exploration of the uterine cavity. See Figures 16 and 17, Chapter III.

Inspection and Palpation will show enlargement of the abdomen unless the tumor is too small to produce that result. External palpation, if the tumor is large, discloses in the pelvis and lower abdomen a solid, usually hard, though sometimes soft mass. Exeeptionally the growth has a pecuiiar elasticity which resembles fluctuation, but lacks the perenssion-wave peculiar to eystic tumors. The tumor may be single and symmetrieal, globular or oblong. The presence of multiple myomata may, with their numerons projections, give to the uterus a most irregular form. Many small tumors may be distributed so evenly throughout the uterine walls as to eause a nearly symmetrical enlargement of the uterus, but in such a case the surface usually gives to the touch a sensation of small nodular irregularities. Inspection, palpation, and perenssion will be considered further in connection with differential diagnosis.

Conjoined Examination.-The index or the index and middle fingers in the ragina, the palmar surface directed toward the uterus 
and tumor, and the palpating fingers of the right hand over the abdomen, if the abdominal museles are not too tense, will enable the examiner to outline the uterus and its myomatous projections. In the majority of cases ordinary conjoined examination will complete the diagnosis. The palpation often is facilitated by means of the thumb in the vagina and the index-finger in the rectum. This enables the operator to pick up, so to speak, the enlarged uterus between the thumb and finger. Information through the examining finger is obtained not so much by forcing it up against the tumor as by strong pressure of the tumor against it by means of the right hand over the abdomen. If the abdominal walls are rigid or thick, anæsthesia may be neeessary.

Conjoined Intra-uterine Examination with an index-finger in the uterus and a hand over the abdomen is possible only when the uterine canal is dilated, a condition which may be brought about by instrumental means or by uterine contractions upon an intra-uterine tumor. The index-finger in the dilated uterus will recognize by direct touch the presence and character of an intra-uterine growth.

Exploration by the Sound.-The sound will show:

The direction of the uterine canal.

The length of the uterine canal.

The shape of the uterine eavity.

The relations of tumor's to the uterine cavity.

The one fact constant for all uterine myomata is elongation of the uterine cavity, and the presence or absence of elongation can be ascertained by the sound or probe. The increased length is proportionate to the size of the tumor, and may reach seven or more inches. Unless care is used, a submucous tumor may obstruct the passage of the sound and lead to wrong measurement. Snbmucous and intramural tumors project into the uterine cavity, and thereby render the uterine canal tortuous, and unless the myoma is too soft and small to be recognized the sound or probe will be deflected, and as it glides over the growth the deflection will indicate the size of the growth and the degree to which it projects into the uterine cavity.

\section{Differential Diagnosis.}

The principal lesions from which myoma must be differentiated are the following:

Intra-uterine pregnaney.

Tubal pregnaney.

Carcinoma and sareoma.

Chronic metritis.

Inversion of the uterus.

Uterine displacements.

Incomplete abortion.

Ovary.

Pelvic infiltrations.

Pelvic cysts.

Sactosalpinx.

Floating kidnev.

Normal Pregnancy.-Normal utero-gestation will be excluded by the absence of the usual signs of pregnancy. The difficulties in diagnosis will arise commonly in abnormal pregnancies, especially in placenta prævia and in psendo-menstruation connected with pregnancy. If the enlargement of the uterus be symmetrical and the rate 
of growth usual for a pregnant uterus, and the os be soft and patulous, pregnancy is highly probable. If, on the other hand, the cervix be hard, the os non-patulous, and the uterus irregular in ontline from the presence of a hard, resisting mass, the diagnosis is probably myoma. Not very infrequently myoma and pregnancy coexist; then if the tumor is large and the foetus small, the difficulty of diagnosis is great. In doubtful cases the myoma, if present, will declare itself by relatively slow growth. The following tabular statement contains the chief points of difference between myoma and pregnancy.

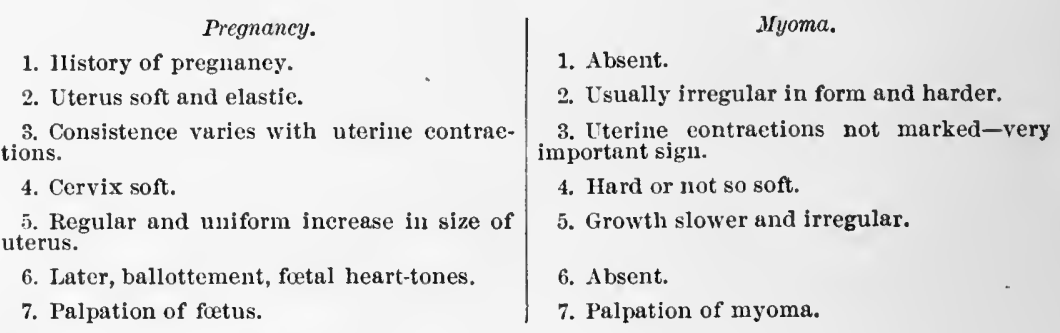

Tubal Pregnancy gives a history of gestation. The gestation-sac closely resembles sactosalpinx. Rupture of the tube produces pelvic hæematocele. A decidua may be cast out of the uterus. The reader is referred to the clinical forms of endometritis, Chapter XVII.

If the tubal pregnancy has resulted in pelvic hæmatocele by tubal rupture or tubal abortion, the differential points will be as follows:

\begin{tabular}{|c|c|}
\hline Irxmatoccle. & Myoma. \\
\hline 1. History of tubal pregnaney. & 1. Absent. \\
\hline $\begin{array}{l}\text { 2. Sudden appearanee, shoek, severe pain, } \\
\text { and evidenee of hemorrhage. }\end{array}$ & 2. Absent. \\
\hline $\begin{array}{l}\text { 3. Consistenee of mass usually soft, later } \\
\text { nay be hard. }\end{array}$ & 3. Üsually hard. \\
\hline 4. Not sharply outlined. & 4. Sharply outlined. \\
\hline $\begin{array}{l}\text { 5. Later, mass shrinks and beeomes harder } \\
\text { r may suppurate. }\end{array}$ & $\begin{array}{l}\text { 5. Commonly inereases } \\
\text { after menopause. }\end{array}$ \\
\hline
\end{tabular}

Diagnosis of Myoma complicating Pregnancy.-Myoma may be mistaken for the foetal head, elbow, or knee. Intramural myoma complicated by preguancy takes on rapid increase of growth, is softer than formerly, but fimer than the pregnant uterus; this variability in consistence is almost proof of complicating pregnancy, and calls for repeated examinations. Later, one may palpate the fotus, elicit ballottement, and hear the fotal beat.

Carcinoma and Sarcoma.-The evidences of malignant disease, including the sudden onset of hydrorrhoea, the bloody, fetid discharge, the rapid emaciation, and the microscopical finding of carcinoma or sarcoma in the scrapings, will exclude myoma definitely. A sloughing, extruding myoma may, however, both in the profuse fetid discharge and in the sensation to the examiner on touch, closely resemble carcinoma or sarcoma of the cervix. The diagnosis then will depend on the microscope. 
Metritis often complicates uterine myomata, and is difficult, often impossible, to differentiate from small, multiple, interstitial growths. The symmetrical form of the uterus is the distinguishing feature of metritis. See differential tabular statement below.

Figure 167.

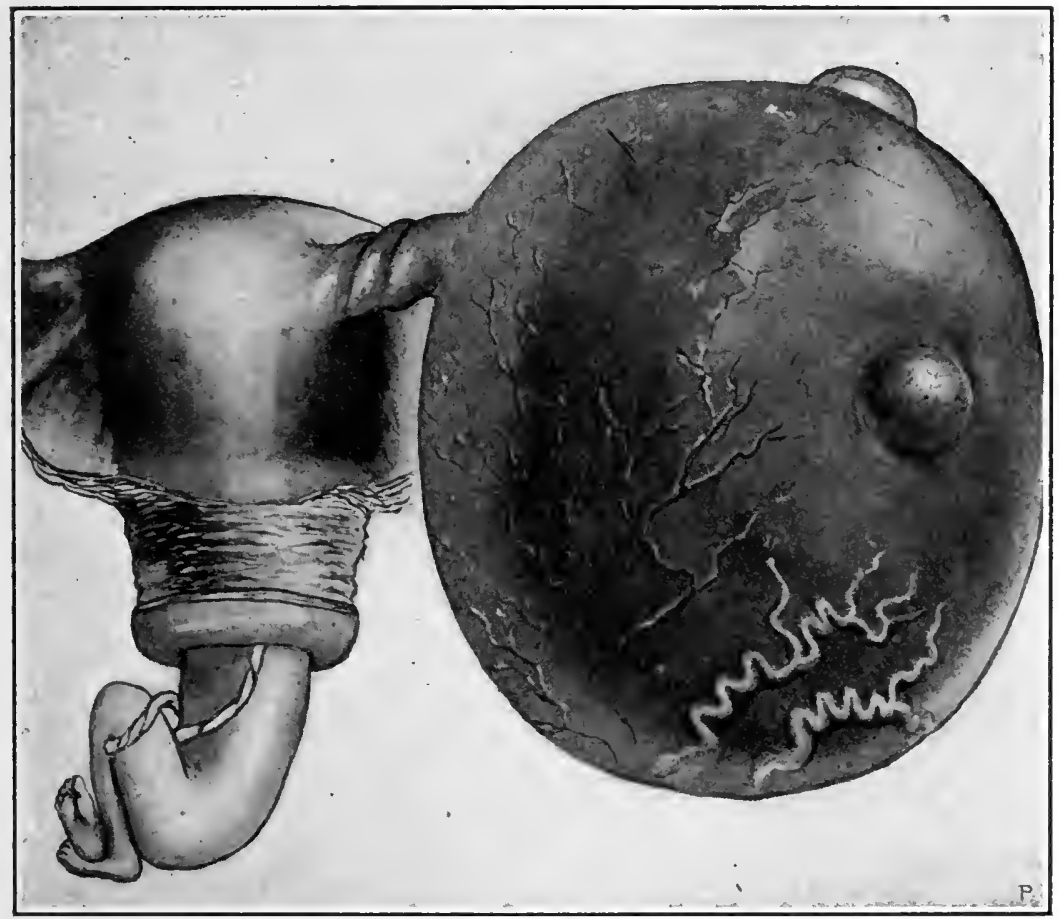

Myomatous uterus, five months pregnant. Infection of myoma, and consequent peritonitis from twisted pedicle. Hysterectomy. (Case referred by Dr. A. C. Haven.)

Chronic metritis.

1. Uniform enlargement.

2. Uniform hardness.

3. Uterus not larger than two or three times the normal size.

\section{Myoma.}

1. Enlargement usually irregular.

2. Uterus softer than tumor.

3. Size may increase to thirty or forty pounds.

Inversion of the Uterus.-A uterine myoma protruding into the vagina may have the appearance of an inverted uterus. The sound then will glide past the tumor into the uterus above. Conjoined recto-abdominal exmination will demonstrate the absence of the uterus in its normal location if it be inverted into the vagina. See Inversion of the Uterus.

Displacements of the Uterus are recognized on conjoined examination by the symmetrical contour of the uterus and by the change in the direction of the uterine canal as demonstrated by the sound.

Incomplete Abortion witl hemorrhage will be recognized by the history of interrupted pregnancy and by nicroseopical examination of the scrapings. 
The Ovary, especially if adherent to the uterus, sometimes simulates a small pedunculated subserous myoma. The myoma, however, is smoother, more firm, and less sensitive to pressure.

Pelvic Inflammatory Infiltrations, unlike myomata, always give a history of pelvic inflammation, are very tender on pressure, immobile, and prone to disappear by resolution or to undergo suppuration. See differential diagnosis of Pelvic Cellulitis, Chapter XX.

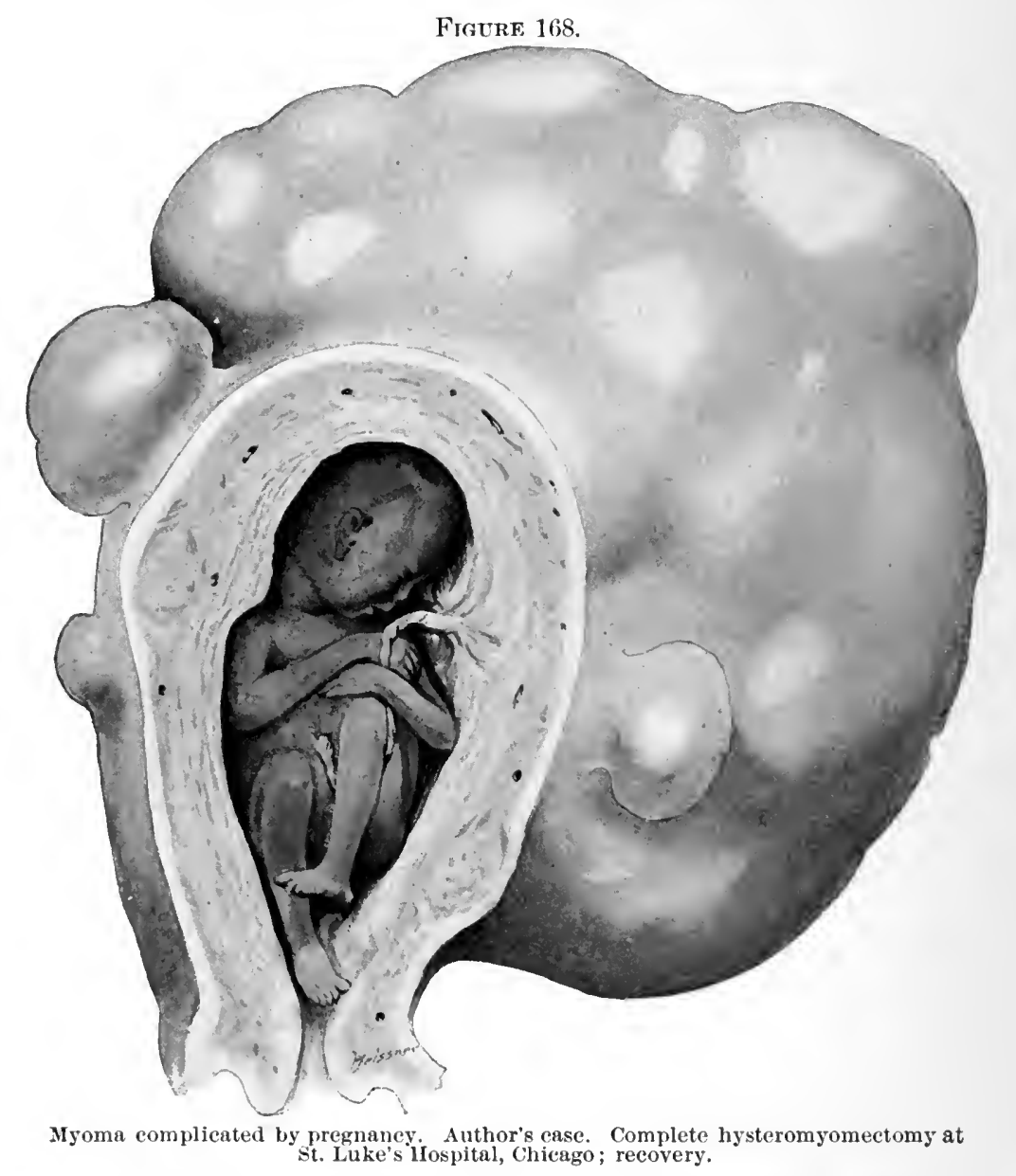

Pelvic Cysts are distinguished from myomata by fluctuation, by separability from the uterus on palpation, by more rapid growth, by the normal or nearly normal length of the uterine cavity, and by the absence of uterine hemorrhage. Figure 18.

Sactosalpinx develops more rapidly, is situated commonly at the side of the uterus, is of elongated, ovoid form, is fluctuating, is more or less tender on pressure, and does not cause material enlargement of the uterine cavity. 
Floating Kidney, unless adherent, is replaced readily, has the form of a kidney, and may be tender to pressure. See Displacement of the Kidney, Chapter XXXIII.

\section{Prognosis of Myoma.}

Non-operative Prognosis.-Myoma may be present throughout the period of sexual activity and produce no subjective symptoms, or it may give rise to the symptoms already outlined. It may participate in senile atrophy of the reproductive organs at the menopause or in involution after pregnancy, and thus become much smaller or disappear. On the other hand, at either of these times it may grow larger. It usually develops rapidly during gestation. Even small growths, if near the endometrium, may threaten life from hemorrhage. Complicating cardiac and renal diseases render the prognosis more grave. The causes of death include hemorrhage, sepsis, peritonitis, and secondary changes in the tumor itself.

Natural Cure.-There are three natural modes of cure:

1. Great shrinkage or disappearance by absorption-rare; reason unknown.

2. Detachment of intra-uterine or intramural myoma and expulsion through the cervix uteri or vagina; detachment of subserous pedunculated myoma into the peritoneum. See Subperitoneal Myomata.

3. Disintegration and gangrene.

The Operative Prognosis will be found at the end of the next chapter. 


\section{CHA PTER XXVII. \\ TUMORS OF THE UTERUS (CONTINUED).}

TREATMENT OF MYOMA.

The treatment includes medication, manipulations, intra-uterine tamponade, intra-uterine stypties, electrolysis, and surgical operations. The treatment is, therefore, non-surgical and surgical.

\section{NON-SURGICAL TREATMENT.}

1. Medication.

2. Manipulations.

3. Intra-uterine tamponade.

4. Intra-uterine styptics.

5. Electrolysis.

6. Expectant treatment.

\section{Medication.}

Ergot stands at the head of the numerous drugs that have been used in the treatment of utcrine myoma. Indeed, no other drug, except possibly hydrastis canadensis, has any special value. 'The latter is said to have some power to control hemorrhage. Ergot has some value in controlling hemorrhage, and thereby preserving the vitality of the patient until relief may come with the menopause or after surgical removal. The drug, if long continued, is not well borne by the stomach; hence it should be given either by hypodermic injection or by rectal suppositories. The dose is determined by the effect. If used at all, sufficient should be given to control the bleeding. The ice-bag over the hypogastrium to some extent controls hemorrhage.

\section{Manipulations.}

When a myoma becomes incarcerated in the small pelvis under the sacral promontory and causes pressure-symptoms, the indication is to force it up into the abdomen by manipulation. The manipulation is earried out best with the patient in the knec-breast position, the assumption of which in some cases will cause the tumor to fall out of the pelvis by foree of gravity alone. In other cases considerable pressure, with or without anæsthesia, may be required to dislodge it. The tumor, if small and very movable, may fall back readily into the pelvis minor, causing great mechanical disturbance and necessitating daily replacement. If the tumor from any cause, such, for example, as adhesions, cannot be forced up into the abdomen and pressuresymptoms are urgent, it may have to be removed. 


\section{Intra-uterine Tamponade.}

When hemorrhage is profuse and exhausting, the most effective means of temporary hæmostasis is intra-uterine tamponade made through Sims' or Simon's speculum. A continnous strip of aseptic gauze should be packed tightly into the uterus, especially into the cervical cavity, and renewed every forty-eight hours until the flow has ceased. In this way an exhausted exsanguinated patient may in a few weeks regain strength to endure the radical operation. This treatment in the hands of the author has in one case been followed not only by entire rehief of menorrhagia, but also by almost total disappearance of the tumor. The tampon was used during three consecutive menstruations, and the tumor was reduced from the size of a child's head to that of a hen's egg. The age of the patient, forty-five years, and the near approach of the menopause may account partially, at least, for this extraordinary result.

\section{Intra-uterine Styptics.}

Churchill's tincture of iodine, solution of iron persulphate, the 10 per cent. solution of antipyrin, and other styptics, may be injected into the uterus for the control of hemorrhage. These agents, especially the iron persulphate, are apt to form hard blood-clots, which may become septic and therefore dangerous. The method is altogether inferior to that of tamponade.

\section{Electrolysis.}

The observations of Vineberg upon the statistics of Keitl, Engelmann, Gautier, and other eminent electro-therapentists, show three hundred and seventy-two cases with nine reported cures and five deaths-an excessive mortality in consideration of the limited number of cures. Galvanopuncture and electrolysis in fibrocysts are condemned strongly. The earlier promises of the enthusiastic supporters of electrolysis have not been fulfilled; the immediate dangers of it also are considerable. The survival of the electrical method in gynecology depends chiefly upon the patient's ignorance of its inadequacy and dangers, upon her worship of the mysterious, upon an unreasoning dread of operative measures, and upon a desire to grasp any other promising means of relief.

\section{Expectant Treatment.}

See non-operative prognosis at the end of the last Chapter.

\section{SURGICAL TREATMENT.}

It would be unprofitable to enlarge npon a great varicty of procedures which have become or seem destined to become obsoletc. The more useful operations for the treatment of fibromyomata of the uterus will be divided as follows :

1. Palliative operations.

2. Radical vaginal operations.

3. Radical abdominal operations. 


\section{Palliative Operations.}

The only palliative operation of recognized value is curettage.

Curettage.-If the tumor by its irritating presence gives rise to hemorrhagic endometritis, curettage is indicated precisely as it would be in hemorrhagie endometritis from any other cause. Generally the operation is followed by some relief from the menorrhagia, lint is seldom permanent in its results, and usually, therefore, must be repeated again and again. It is especially useful, in connection with intra-literine gauze tamponade, to control hemorrhage until an exhausted patient can gain blood and strength for a more radical operation, or, in cases of small tumors, until the menopause has passed. The technique of eurettage is described in Chapter V.

Other palliative operations have been proposed, such, for example, as ligature of the uterine and ovarian arterics; but they are all obsolete with the possible exception of removal of the uterine appendages; this procedure, which suggests the names of Battey, Hegar, and 'Tait, when properly carried out-i.e., when the Fallopian tubes are thoroughly removed, as described in Chapter XXIII., and when the ligatures are placed close to the uterus so as to include a large part of the broad ligament-usually checks the hemorrhage and reduces the tumor, sometimes eveu causing it to disappear. It is, however, nearly if not quite as dangerous as the more radical operations, and is now obsolete except in rare eases of small tumors in which, for some special reason, hysterectomy and myomectomy are inadvisable.

\section{Radical Vaginal Operations.}

The vaginal operation is preferable when the tumor ean be reached readily by that route. All cervical fibroids, all intra-uterine peduneulated fibroids, and some of the more accessible submucous fibroids, have been removed usually by way of the vagina. In their renioval the écraseur and galvanocautery, so often used for hæmostasis, are unnecessary, because the hemorrhage is either not feared or can be controlled readily by the uterine tampon. The vaginal route usually has been reserved for tumors of a size not larger than the eapacity of the small pelvis.

The radical vaginal operations arè : A, torsion for small pedunenlated intra-uterine myomata; $\mathrm{B}$, vaginal hysterectomy; $\mathrm{C}$, vaginal enncleation and moreellation.

A. Removal of Small Pedunculated Myomata.-When the uterus is dilated, either by uterine contraction on the tumor or by instrumental means, the pedunculated tumor is seized by the vulscllum forceps or bullet forceps, drawn down, and twisted off or removed by the scissors. The uterus then is packed with aseptic gauze. In the removal of a pedunculated myoma this traction on the growth shonld not be very strong, for the uterine wall may be drawn down and accidentally cut through in the removal of the growth. Fatal peritonitis has resulted from this aceident.

B. Vaginal Hysterectomy.-When numerous small tumors are seat- 
tered throughout the uterus, Figure 165, and the number is so large that individual enucleation is impossible, and when, moreover, the mass is not too large to be delivered through the vagina, it may be

FIGURE 169.

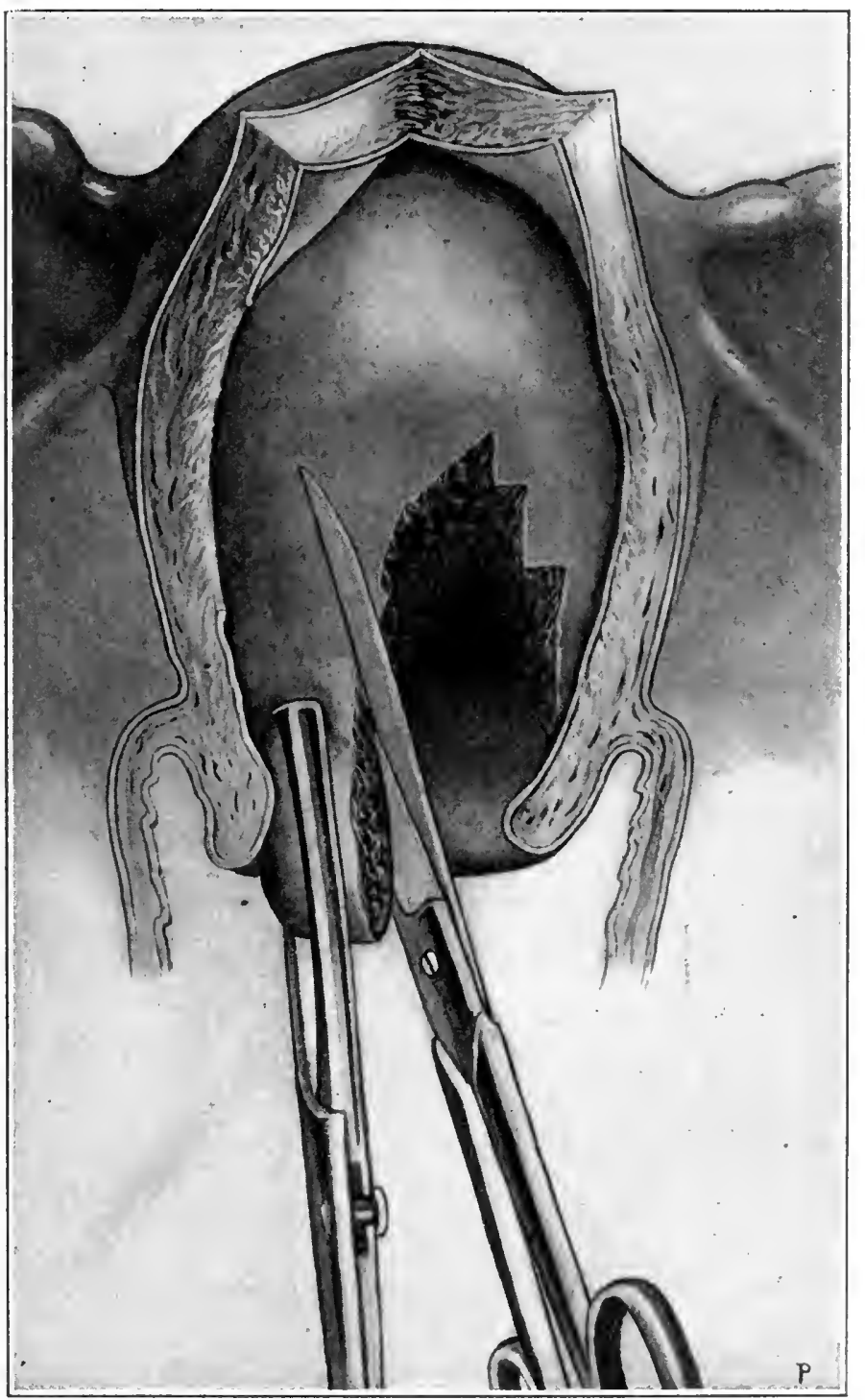

Radical raginal operation. Morcellation of an intra-uterine myoma. Emmet's method.

removed entire by vaginal hysterectomy. Delivery through the vagina sometimes presents unexpected difficulties. Preparation should therefore be made for a supplemental abdominal section. 
C. Vaginal Enucleation and Morcellation.-Intramural myona, especially if situated in the lower segments of the corpus or in the cervix uteri, and not too large, may be enucleated safely and removed through the vagina. The morcellation method has been used often and successfully by French surgeons for the removal of large intramural tumors. 'The removal is aceomplished by repeatedly seizing the presenting part of the tumor with the vulsellum forceps and cutting away as large a piece as possible with the scissors, one piece after another, until the whole tumor has been removed. This is the operation by traction and morcellement or moreellation. The method, although generally supposed to be of more recent origin, was described virtually by Thomas Addis Emmet more than thirty years ago, and has been consistently advocated and practised by him ever since. It is applicable to those cases in which the tumor is accessible through the vagina, but is too large to be enucleated and delivered entire.

The operation of traction and morcellation, when its technique is understood more generally and its advantages appreciated, will become undoubtedly more and more a procedure of election in place of hysterectomy. Many large submucous or mural tumors, for which the abdomen now is opened and the uterus sacrificed, may be removed rapidly, safely, and effectually by this method.

One clear contraindication to the vaginal route must always be, however, the possible presence of pus-tubes or ovarian abscesses, so often unrecognized or unrecognizable when they occur in connection with large, irregular fibromyomata. Many times an unsuspected pustube has been ruptured by the enueleation or morcellation of a myoma through the vagina. The vaginal route, then, should be avoided if there be reason to suspect purulent disease of the uterine appendages.

Usually the tumor is made more accessible and enueleation or morcellation of it facilitated by dilatation or by deep lateral incisions of the cervix, even to the internal os. These incisions having been made, the anterior and posterior lips of the cervix are drawn well down to the vulva and held widely apart by means of strong, double tooth-forceps in the hands of an assistant. The operator then seizes the presenting part of the tumor with heavy moreellation tooth-forceps, Figure 169, and removes it, either by enucleation or by morcellation. If the tumor be intramural, it may be necessary to divide the mucous membrane and subjacent muscular tissue before beginning the enucleation.

An improvement upon the two lateral incisions mentioned above is a simple median incision through the anterior wall of the uterus made as follows : 1

1. Make a circular incision in front of the uterus which shall separate the vaginal wall from the cervix at the uterovaginal attachment, as shown in Figures 170 and 171.

1 Published by the author in the Transactions Ninnesota State Medical Society, 1896, and republished in the Journal of the American Medical Association, August 15, 1896. 
2. Incise the anterior vaginal wall from the middle point of the first incision for a distance of about one inch, taking eare not to invade the bladder and to avoid the ureter on either side. These incisions are the same as for anterior vaginal section, described in Chapter XXIII.

FigURE 170.

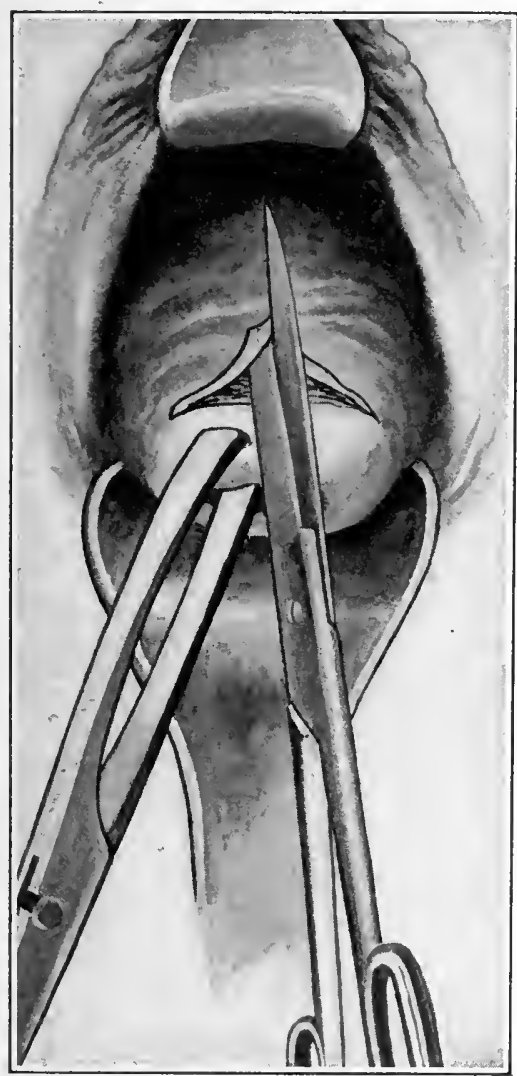

Figure 171.

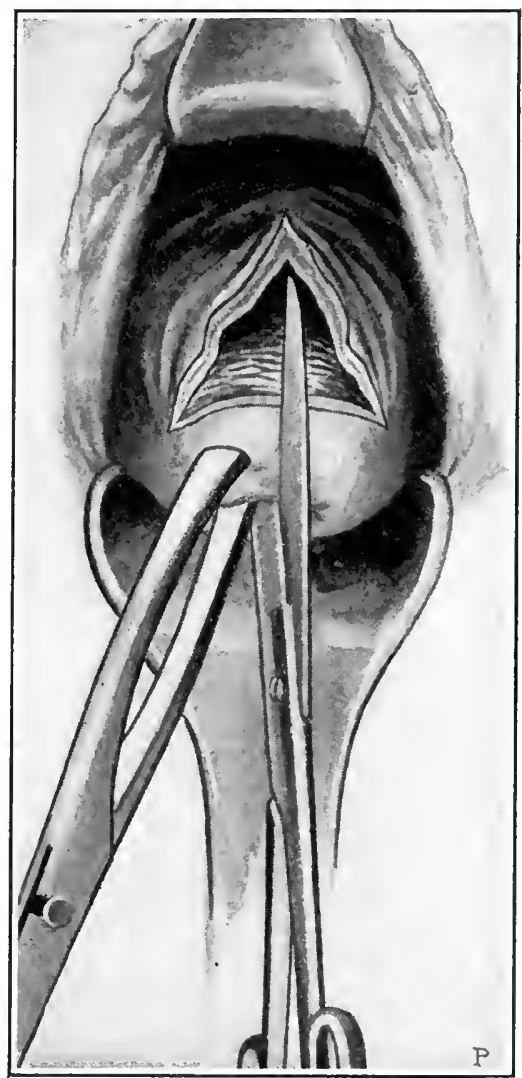

Figure 170.-Radical vaginal operation. Author's incision. Lines indicating the ragiual incisions to expose the anterior uterine wall preparatory to dividing it with scissors.

FIGURE 171,-Author's incision. IIaking longitudinal division of anterior wall of uterus, in order to expose the field of operation for the removal of a myoma.

3. Separate the bladder from the nterus by means of the fingers or some blunt instrument, keeping close to the uterus until the peritoneum is reached, but not divided. Then expose with retractor's or fingers the anterior wall of the uterus.

4. Divide the anterior wall of the nterus longitutinally in the median line by means of scissors to whatever extent may be necessary to render the tumor accessible. If neessary, the peritoneum may be opened and the incision earried up into the corpus uteri.

This simple anterior incision permits wide separation of the lateral 
fragments of the anterior nterine wall, and thereby exposes the endometrium, and may render accessible a myoma in any part of the nterus. It has the following advantages over the lateral incisions: 1. There is less traumatism-one incision instead of two. 2. 'The parametria are not opened and exposed to possible sepsis. 3.' The tumor is more accessible because the anterior uterine wall is out of the way, instead of being between the operator and the field of operation. 4. A much longer incision may be made, if necessary, because the broad ligaments are not involved. 5. There is less hemorrhage. 6. The pelvic cavity may be reaehed easily through this incision for any further operation on the uterine appendages or peritoneum. Even a small pedunculated subperitoneal tumor may be removed through this incision.

\section{Radical Abdominal Operations.}

Radical abdominal operations are adapted to large subperitoneal and intramural tumors which cannot be removed well through the vagina. 'They are:

1. Myomectomy-removal of the tumor without sacrificing any part of the uterus.

2. Supravaginal hysteromyomectomy-removal of the tumor together with the corpus uteri and the supravaginal portion of the cervix uteri.

3. Complete hysteromyomectomy-removal of the tumor and the entire uterus.

\section{Abdominal Myomectomy.}

Abdominal myomectomy-removal of the tumor without sacrificing any part of the uterus-is indicated for the tumors mentioned below under $a, b$, and $c$.

a. Pedunculated subperitoneal tumors. Figure 172.

$b$. Small intramural and subserous tumors, when the growths ean be removed with slight traumatism and the uterine wound closed with interrupted or continuous catgut sutures. Figures 173 and 174. An improvement on the interrupted and continnous sutures is the pursestring suture shown in Figures 175 to 178 , which has the following advantages: 1 , rapidity of application ; 2 , ready and reliable hæmostasis ; 3 , slight traumatism.

c. Some large intramural and subserous tumors which can readily be enueleated-shelled ont-and the uterine wounds elosed with or without drain. Figures 179 and 180.

The growths indicated under $a$ and $b$ may be treated easily and safely by enucleation and suture.

The larger growths indicated under c, even though lying deep in the uterine wall or broad ligament, in many cases may be "shelled out" with the greatest ease, and the tumor-cavities from which they have been removed may be closed successfully and obliterated by buried sutures; or, if the surfaces are too extensive to be treated safely by buried sutures, the cavities may be drained, and in this way finally obliterated. Figures 179 and 180 show the method of drain- 
age and suture. While a large growth is being enucleated and the uterine wound closed hemorrhage may be controlled by a temporary rubber ligature placed around the lower segment of the uterus. Before closing the abdominal wound this ligature is removed, and a little time is allowed to make sure that there is to be no hemorrhage from the uterine wound. Hemorrhage usually is controlled in great
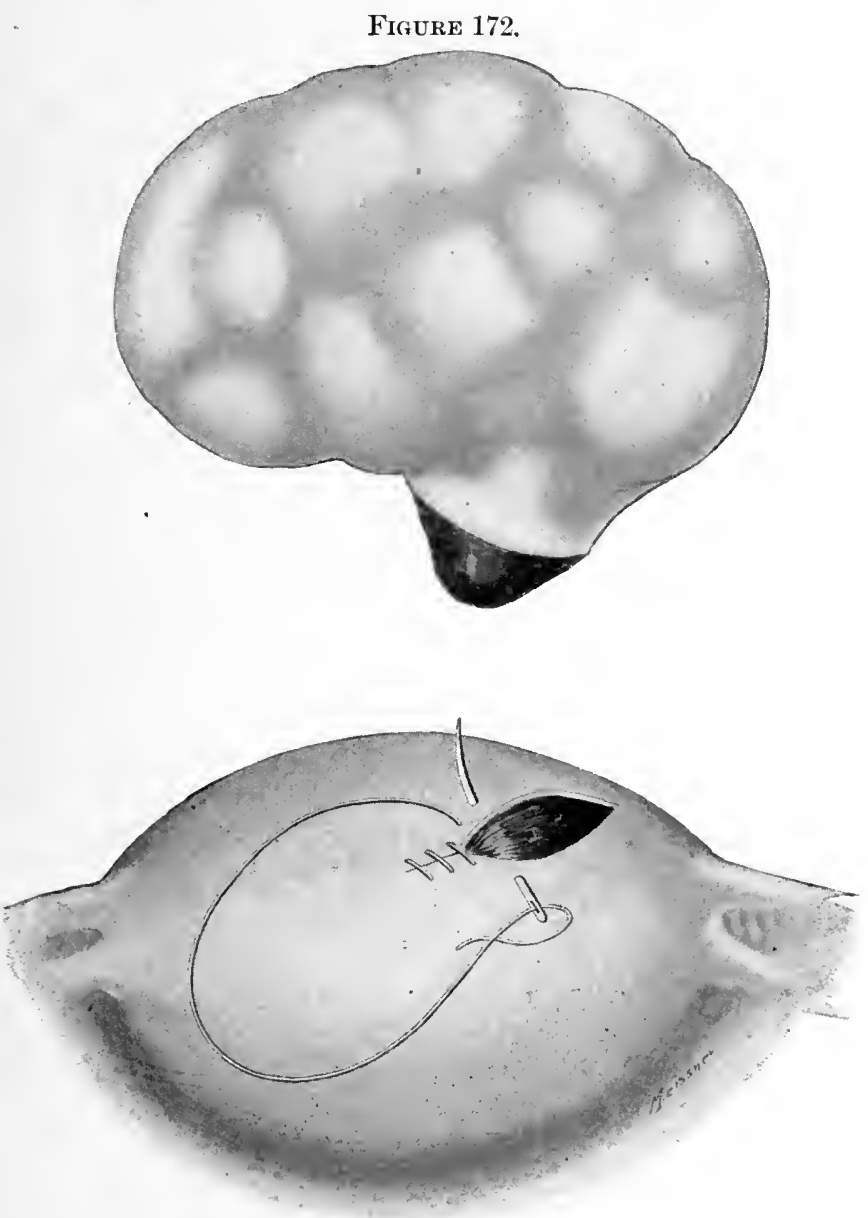

Myomectomy. Fedunculated myoma removed; wound being closed by continuous catgut suture.

measure by the uterine contraction which follows enucleation. The mortality of this method for small tumors, in which the traumatism is slight, is surprisingly small.

In case of a large intramural tumor, and extensive traumatism with enormous surfaces to be united by buried sutures, the method of abdominal myomectomy as above deseribed involves great danger of sepsis and secondary hemorrhage, and should give place to hystero- 
myomectomy or be modified by the introduction of drainage as follows :

Figure 173.

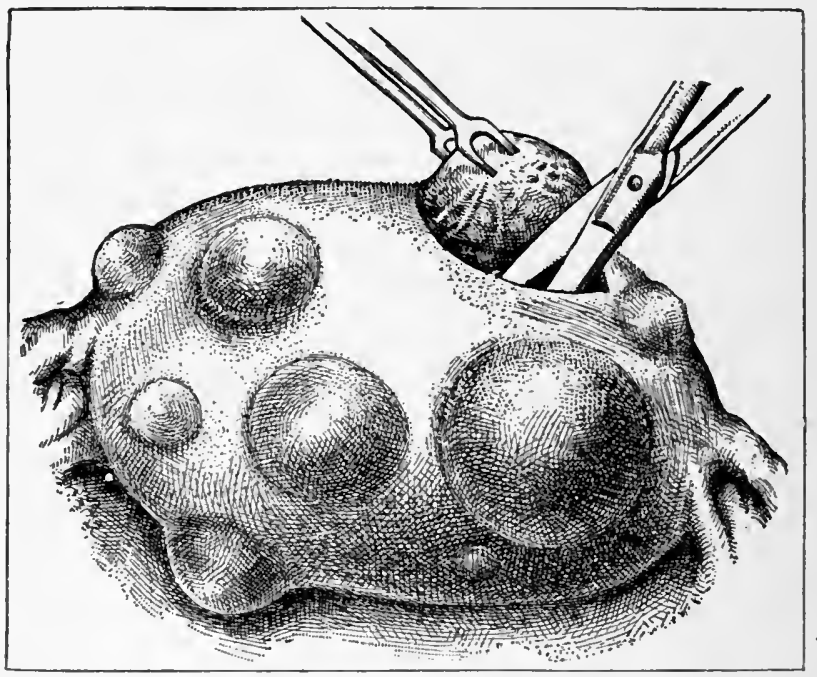

Myomectomy. Uterus with eight myomata to be removed and one being removed. The number of myomati here shoun is larger than should ordinarily be removed by this methud. Unless they are very small and subperitoneal, hysterectomy would be safer.

Drainage in Abdominal Mromectomy.-After the tumor has been enucleated an opening is made from the tumor-cavity to the

Figure 174 .

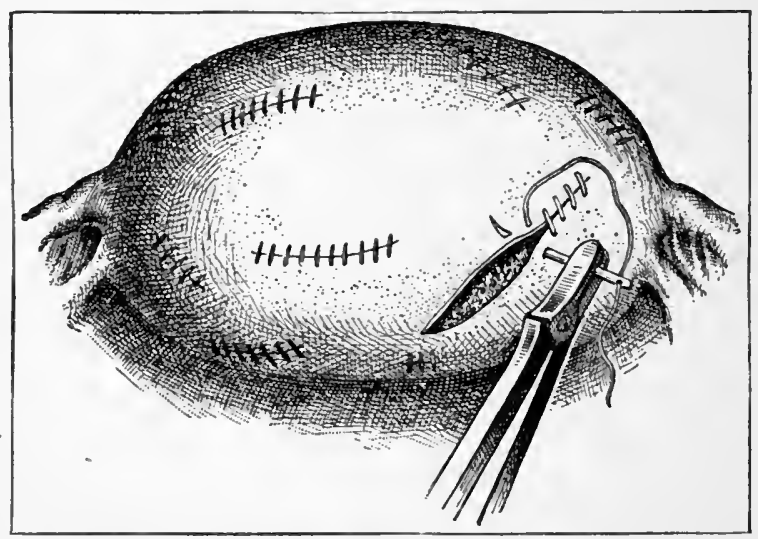

Myomectomy : usual method of suture. Same uterus as shown in Figure 166. Shows methoc of closing wounds made by removal of myomata; continuous catgut sutures.

uterine cavity. If the uterine canal is patulous, a continuous strip of gauze is carried from the tumor-cavity directly through into the vagina, the tumor-cavity being packed with the same continuous 
strip. The temporary elastic ligature around the uterus does not interfere with the introduction of the gauze. The uterine wound then is closed with deep catgut sutures, the peritoneal margins being turned in and united, as shown in Figure 179, so that the whole uterine traumatism, now isolated from the peritoneum, may be drained adequately through the vagina. If the uterine canal is not sufficiently

Figure 175.

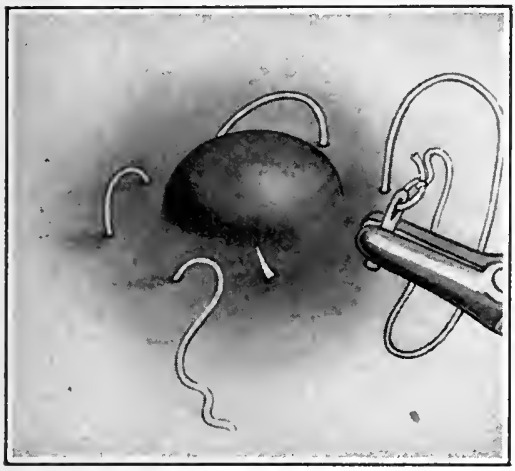

Figure 177.

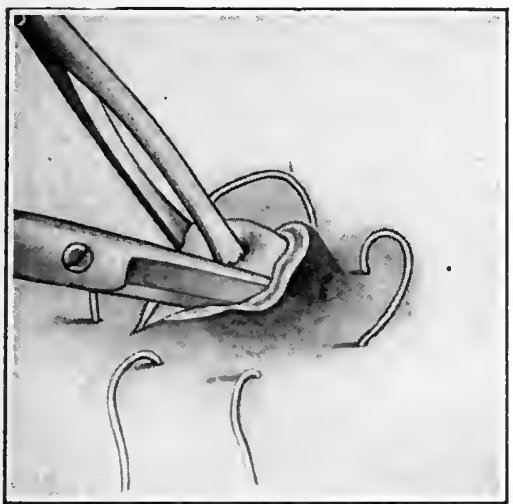

Figure 175.-Author's operation, first step. Figure 175.-Athts operar

Fra which is to be remor

Figure 176.--Seeond step. Capsule split and myoma exposed.

Figure 177.-Third step. Enueleation of myuma.

Figure 178.-Final step. Fundus uteri. One myoma has been removed, and wound elosed by tying purse-string ligature. Another myoma bas been removed, and purse-string ligature is being tited.

patulous to admit the ganze, it may be dilated or the walls of it may be incised anteriorly and posteriorly by means of a herniotony-knife, or it may be both dilated and incised. The vagina is filled loosely with gauze to meet that which protrudes from the uterus, and the vulva is covered by a gauze pad, to be changed as often as it becomes moist. The gauze should be removed in two or three days. Care is necessary in the closure of the uterine wound that the ganze be not 
caught in a suture, because then removal would have to be postponed until after absorption of the suture.

An intraligamentous myoma may be shelled out readily from its bed between the folds of the broad ligament. These same principles of drainage apply as in the case of intramural tumors, except as to the route of drainage, which should be, not through the uterine canal, but through an opening which is made readily from the tumor-cavity to a point in the vagina just back of or in front of the uterus. In exceptional cases it may be necessary for purposes of hæmostasis to ligature the ovarian or uterine artery, or both. Experience has shown

Frgure 179.

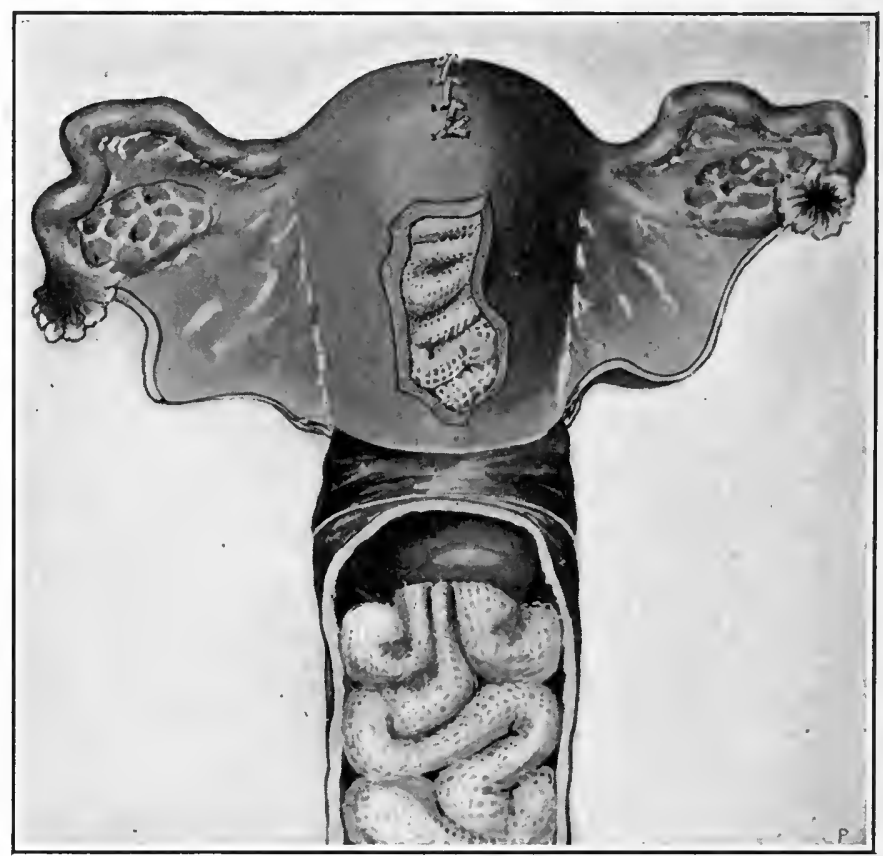

Wyomectoiny. An intramural myoma has been enucleated; an opening has been made between the tumor-eavity and the endometrium; uterovaginal gauze drain; uterine wound closed by suture. The opening in the uterine wall is intended to show the ganze.

that sloughing of the uterus from thus cutting off this blood-supply is not to be feared. See Figures 179 and 180.

Intra-abdominal closure, with vaginal drainage of the tumor-cavity, was suggested early by August Martin, but he appears not to have practised the method extensively.

The author's experience since 1889 with the above technique shows: first, almost entire freedon from mortality; second, prompt and uneventful recovery ; third, the most gratifying permanent results. The method is undonbtedly applicable to a much larger number of tumors than generally is supposed. Removal of the uterus for myoma is often necessary, but not so often as statistics would indicate. 
In the majority of cases the uterine appendages will be found normal, and in a large proportion of this majority the tumor may be enucleated and the wound successfully closed, precisely as would be done for the removal of such a tumor in any other part of the body. Cases of very large tumors, and cases in which many small tumors are seattered through the uterine wall, will require hysterectomy; but the conservative operation of simple enucleation often will be indicated when the tumor is even larger than the foetal head, and in cases of multiple myomata when there are not too many tumors. The preservation of a non-infected uterus, even when the appendages have to be removed, is desirable.

Figure 180.

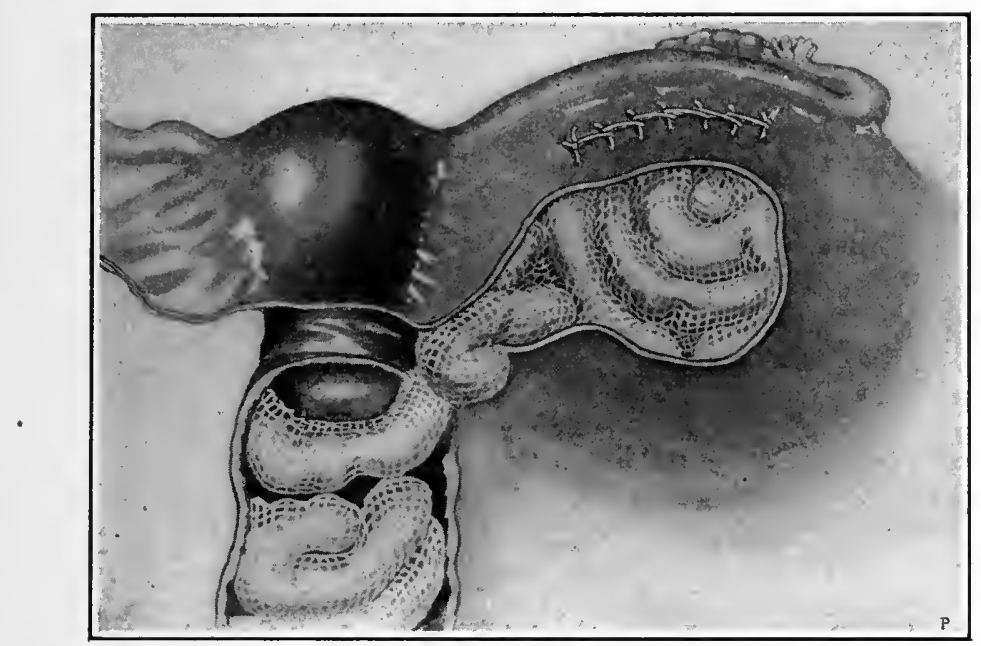

Myomectomy. Intraligamentous myoma has been removed from space between folds of broad ligament. Gauze-drain from this space through an opening, made for the purpose, direct into the vagina.

Drainage of the tumor-cavity by stitching it into the abdominal wound and packing it with gauze ${ }^{1}$ has been carried out successfully in many cases. . The vaginal route for drainage, however, offers decided advantages, and therefore usually will be preferred. ${ }^{2}$

\section{Supravaginal Hysteromyomectomy.}

Supravaginal hysteromyomectomy is removal of the tumor, the corpus uteri, and the supravaginal portion of the cervix, through an abdominal incision, leaving no part of the uterus except the vaginal portion of the cervix.

Disinfection of the Vagina.-An imperative measure, preliminary to this operation, is scrupulous disinfection of the vagina and the external genitals. After the usual cleansing of the vagina, as described in Chapter II., the vagina should be packed lightly but in all its parts with gauze saturated with a 70 per cent. alcoholic solution of mereuric

1 Polk. E. C. Dudley. Senn.

2 The author used uterovaginal drainage in myomectomy in April, 1889; his first case was reported in the American Journal of Obstetrics, September, 1889. 
bichloride. This is most essential as an aseptic measure, especially in view of the possibility that an opening for drainage may lave to be made from the pelvic eavity into the vagina.

Technique of Supravaginal Hysteromyomectomy.-The usual operation is to secure the ovarian and uterine arteries by means of strong catgut ligatures, and after removal of the tumor, corpus uteri, and supravaginal portion of the cervix, to close the uterine stump by means of a continuous suture running from side to side, and then to close the wound in the broad ligaments by means of another continuous suture also ruming in the same direction. See Figure 174: This method is open to the following objections: 1 . The severed broad ligaments retract to the sides of the pelvis, where they can no longer give adequate support to the bladder, vagina, and rectum; the frequent consequence is exaggerated descent of the pelvic floor with disabling and permanent cystocele and rectocele. 2. The rectum and bladder are brought into close relations with only a thin wall between, so that the possibility of infection from one to the other is increased. 3. In many cases the bladder is drawn over the uterine stump in order to cover it; this may give rise to mechanical irritation of the bladder.

The author has attempted to overcome the difficulties above mentioned by closing the uterine stump in the anteroposterior direction and by end-to-end approximation of the broad ligaments. A description of end-to-end approximation will be found in Figures 182 to 192. The steps of supravaginal hysteromyomectomy are as follows: $A$. Abdominal incision.

$B$. Delivery of the tumor through the abdominal wound.

$C$. Ligature of the ovarian and uterine arteries, and removal of the tumor together with the corpus uteri and supravaginal portion of the cervix uteri.

$D$. Toilet of the peritoneum.

$E$. Closure of the abdominal wound.

$A$. In case of a large tumor the abdominal incision should be made nearer the umbilicus than the pubes, to avoid the bladder, which by the growth of the tumor not infrequently is drawn up out of the pelvis. The incision, first exploratory - that is, large enough to admit one or two fingers-may be enlarged sufficiently to permit delivery of the tumor.

$B$. Delivery of the tumor through the abdominal wound is effected sometimes by pressure on the abdominal walls around the incision, so as to squeeze it out as one would squeeze pus out after opening an abscess. Usually, however, the tumor is delivered by traction with the hands or with heavy vulsellum forceps. In many cases the tumor is fixed so firmly in the pelvis that it cannot be brought through the abdominal wound until after some of the pressure-forceps or ligatures have been placed around the arteries and the mass partially severed from the broad ligaments. If the abdominal incision has been very long, and the intestines are much inclined to protrude throngh the wound, they may, as soon as the tumor has been brought through, be held back by a large flat gauze pad or by suture 
of the upper part of the wound. It is clearly important to prevent protrusion of the intestine, and thereby to lessen exposure of the peritoneum.

$C$. In a majority of all cases of hysteromyomectomy, whether complete or incomplete, the operation may be facilitated by the use of long-bladed forceps to secure temporary hæmostasis of the uterine and ovarian arteries during removal of the mass, and to be substituted-by ligatures as soon as the mass has been removed. See Figures 182 to 186 . This use of the forceps will enable the operator to get the tumor rapidly out of the way, and to complete the operation with great speed, and during the operation to avoid hemorrhage. Figures 182 to 184 show the forceps in place. The further steps of this part of the operation are as follows:

1. Clamp the arteries as shown in the diagrams; the onter forceps shut off the ovarian artery as it passes inward through the broad ligament toward the uterus; the inner forceps prevent reflex hemorrhage from the utero-ovarian anastomosis at the nterine end of the broad ligament.

2. Divide the broad ligaments by means of scissors.

3. Divide the peritoneal investment of the uterus all around the cervix just above the bladder attachment; this is done best by lightly cutting around the uterus with a scalpel or pointed scissors.

4. Strip the circumuterine peritoneum together with the attached bladder down toward the vaginal portion of the cervix to the region of the uterine arteries. During the stripping off of the bladder the relations of it may be recognized by a sound in the bladder. The stripping is accomplished best by means of gauze pressure; it is like a blunt dissection.

5. Clamp the uterine arteries by means of forceps or ligate them at once; in applying the ligatures, care is necessary to avoid the ureters, which sometimes run very close to the uterus. Some operators take the precantion to have a catheter in each ureter in order to keep track of it during the operation.

6. Remove the tumor and all the uterus except the vaginal portion by means of a wedge-shaped incision so directed that the uterine stump may be sutured in a line running from before backward, not from side to side.

7. Place permanent ligatures on the ovarian and uterine arteries and remove the pressure-forceps. It is important that the forceps be loosened by an assistant while the ligatures are being drawn tight, because if tied before the forceps are removed dangerous hemorrhage may result. The uterine arteries are located sometimes by sight, sometimes by touch, and accordingly are secured by ligature, isolated or en masse. The ovarian artery tisually is tied en masse. In tying either the ovarian or uterine arteries en masse, it is of great importance to place the ligatures so that the ligatured part will not be deprived wholly of circulation-that is, so that it will receive collateral circulation -and therefore not be subject to necrotic processes. Figures 188 to 192 show the ligatures, which have been applied in such a manner as not to produce necrosis of the stump. 
In some cases the tumor so fills the pelvis that the forceps, for lack of room, cannot be applied. Then, an elastic ligature having been thrown rapidly around the cervix for temporary hæmostasis, the tumor may be enucleated and the size of the mass so reduced that the forceps may be applied. As the incision is carried down through the broad ligament on each side additional forceps, if needed to control hemorrhage, may be used until the entire mass-tımor, corpus uteri, and supravaginal portion of the cervix-has been removed; then permanent ligatures on the ovarian and uterine arteries and on bleeding points should be substituted for the forceps.

$D$. The toilet of the peritoneum, which consists of the following steps :

1. Ligature of any bleeding points.

2. Cauterization of the remaining portion of the cervical canal with 95 per cent. carbolic acid; this may be applied on a probe or grooved director. Sponges and instruments used in connection with the cervieal canal should for reasons of asepsis not be used elsewhere.

3 . If the case is simple and requires no vaginal drain, the cervical stump should be closed by a line of sutures running in the anteroposterior direction, and the broad ligaments should be closed by endto-end approximation, as described in Figures 185, 186, and 187. If there is fear of possible sepsis in connection with the vaginal wound, or other reason to use vaginal drainage, the walls of the remaining portion of the cervix should be split, as shown in Figure 188, and a continuous strip of sterile gauze shonld be passed from the pelvic cavity between the fragments of the cervix into the vagina, as shown in Figure 189. This strip should fill the vagina loosely and present at the vulva, and should be removed about two days after the operation, and the removal of it should be followed by gentle low pressure, vaginal douches, 0.5 of 1 per cent. lysol in sterile water. The vaginal wound should be closed as indicated in Figures 189 and 190.

$E$. The abdominal wound should be closed without drain in the usual manner, as described in Chapter VI.

\section{Complete Abdominal Hysteromyomectomy.}

The removal of the entire myomatous uterus is indicated: first, when the uterus is septic or otherwise so diseased as to render the presence of any part of it unsafe ; second, when on account of extensive traumatism or suppuration vaginal drainage is required. In addition to the above indications there is a certain legitimate latitude of choice, so that the bias of the operator properly may be in the direction of complete hysterectomy. The operation demands the same antiseptic preparation as already laid down for supravaginal hysteromyomectomy.

Technique of Complete Abdominal Hysteromyomectomy. - The abdominal incision; the delivery of the tumor; the clamping and ligature of the arteries; the division of the broad ligaments; and the closure of the wounds, both pelvic and abdominal, are substantially the same as already described for supravaginal hysteromyomectomy. 


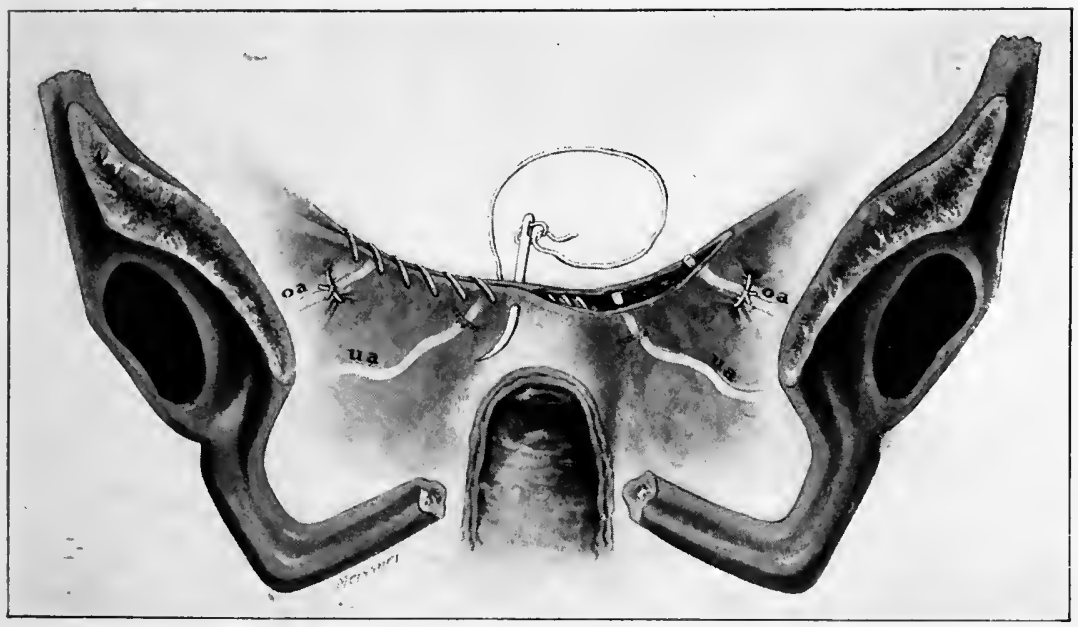

Supravaginal Hysteromyomectomy. Ordinary method of cloxing broad ligaments. The ovarian and uterine arteries have been serured by means of strong eatgut ligatures, the uterine stump has been closed by a continuous sut ure running from side to side, and the wound in the broad ligaments is being whipped together by a continuous catgut suture. The ligatures on the uterine arteries are covered in by peritoneum, those on the ovarian arteries are not so covered.

A better method of securing the broad ligaments by end-to-end approximation is described in the following figures, 182 to 192.

${ }^{1}$ This series of illustrations, Figures 181 to 192 , with text, were publisherl by the author in the Journal of the American Medical Association, December, 1906. A similar series also was publislied in the same journal, IIarch 29, 1902. 


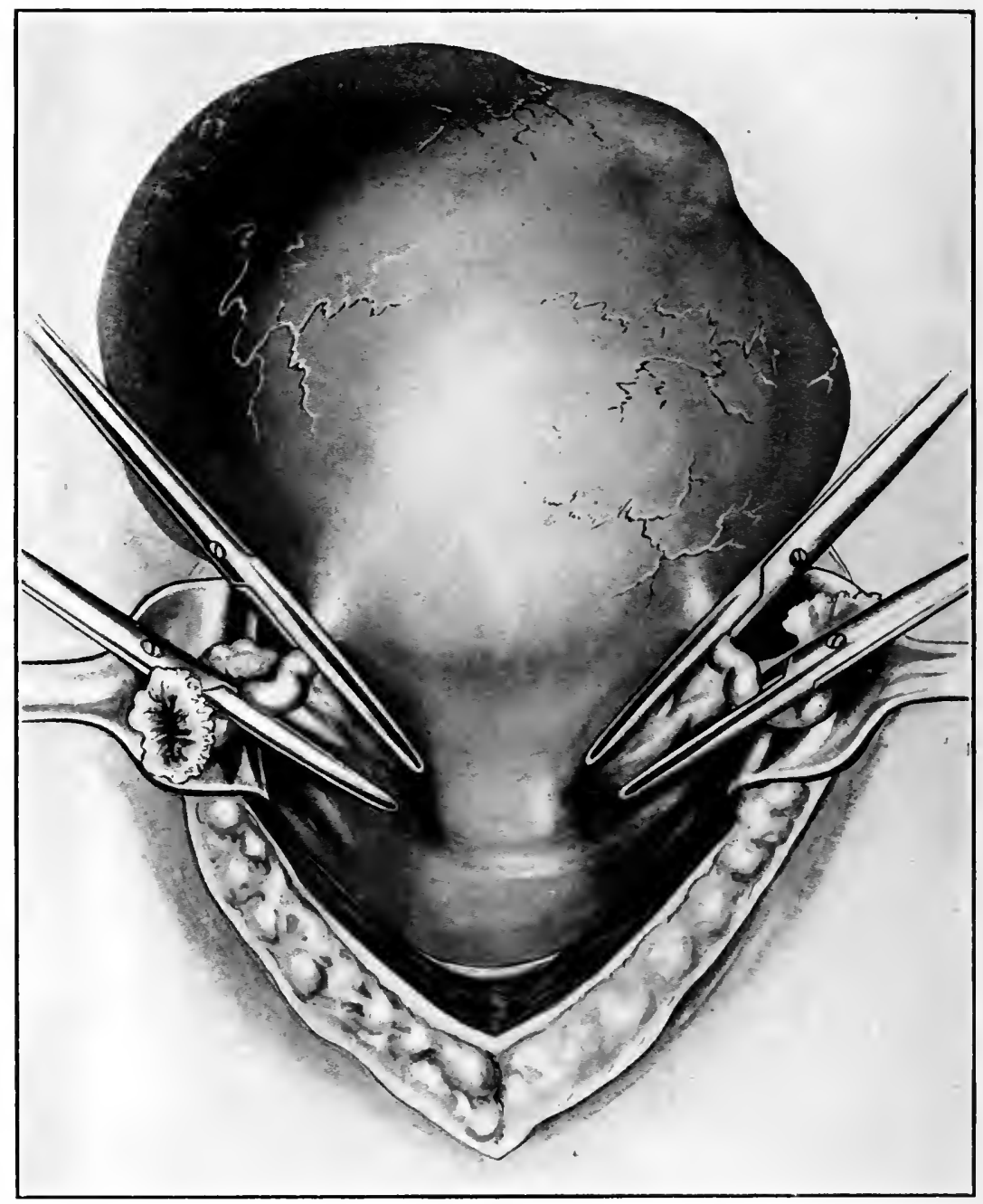

Supravaginal fiysterectomy. Median incision. Abdominal wound held apart by retractor. Myomatous uterus delivered through the wound. Broad ligament on either sicle elamped by two long forceps preparatory to supravaginal amputation of the uterus. Cervix uteri between the tips of forceps. Bladder in front. 


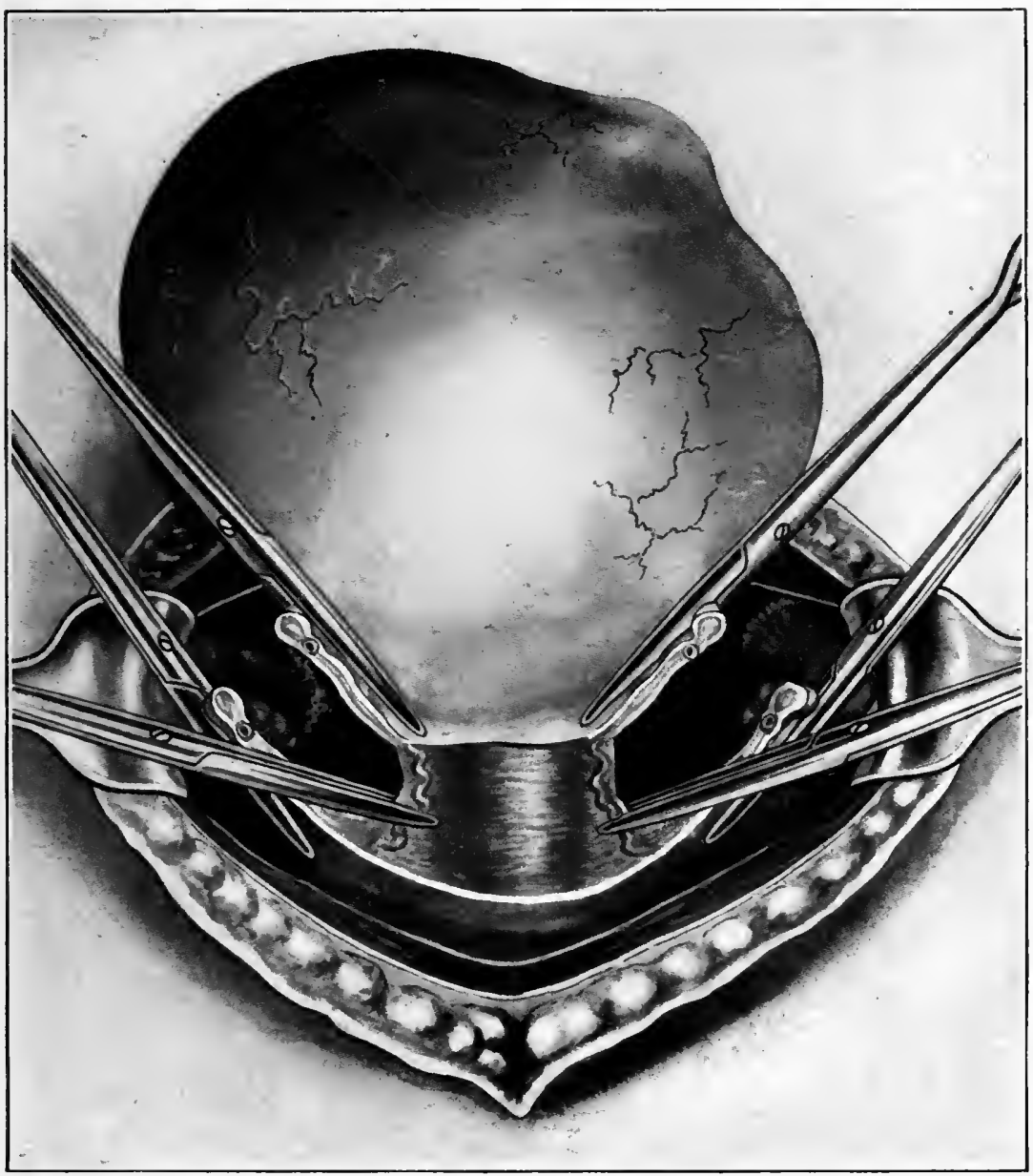

Supravaginal Hysterectomy. (Semidiagrammatic.) A transverse incision has been made in front of the cervix uteri at the vesical reflexion, each end terminating where the broad liganent joins the uterus. A similar incision has been made through the peritoneum on the posterior wall of the uterus, but on a line rather higher than the one on the front. The bladder has been stripped away from the cervix as far down as possible by the same kind of sponge pressure as shown in Figure 358. The posterior peritoneum has been stripperl down toward the vaginal portion of the cervix in the same manner. The broad ligament has been divided on either side between the forceps, and the utero-ovarian anastomosis has been clamped by an adclitional forceps. 


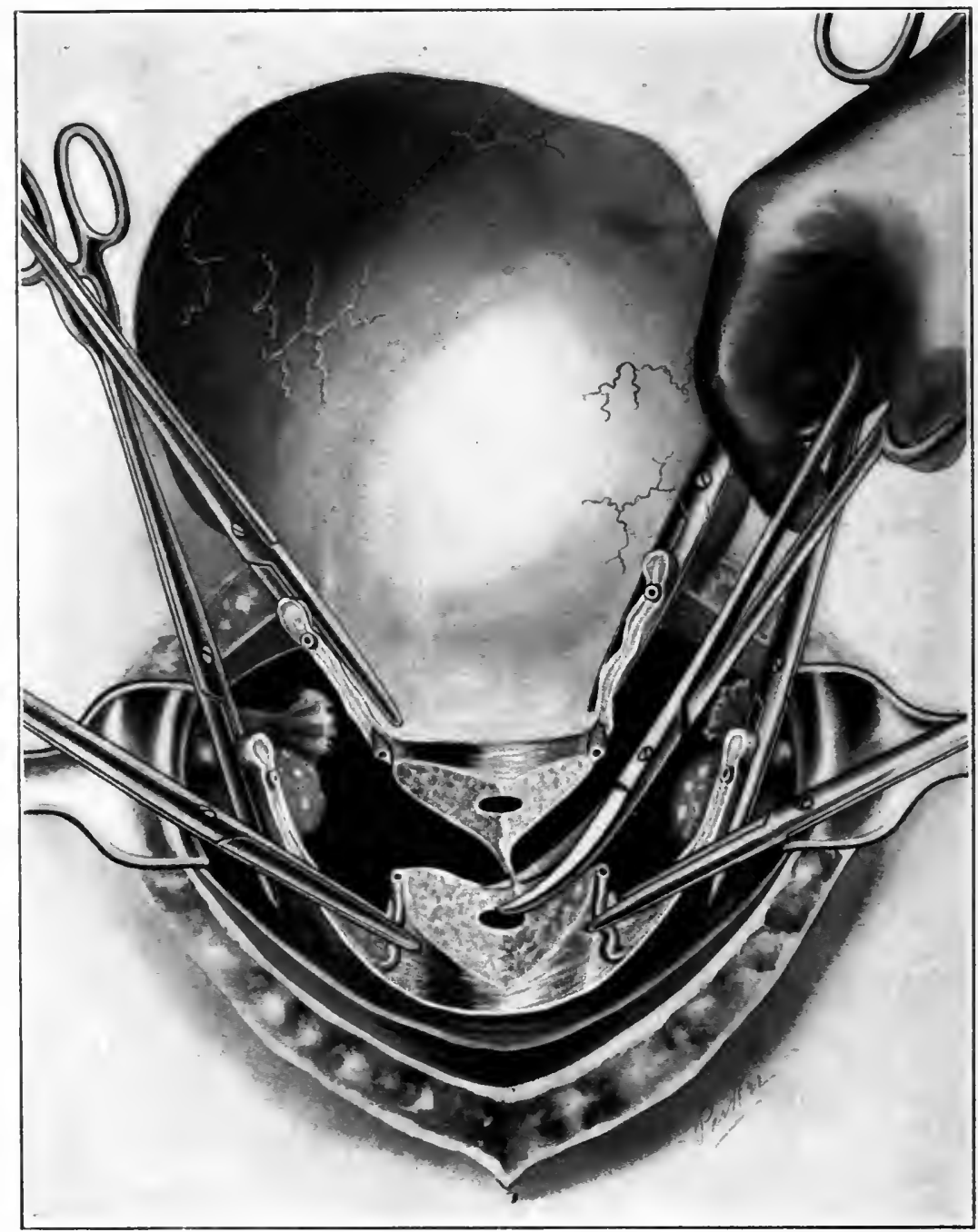

Supravaginal Ilysterectomy. (Semiliugrammatio.) The myomatous corpus uteri is being cut from the cervix by means of scisorors. Observe the triangular incision is directed not in the lateral. but in the anteroposterior clirection. The tumor having been removed, the next step will be to secure the uterine and ovarian arteries by means of eatgut ligat ure. on either side. This having been done the forcens, are $t o$ be removed. The brond ligaments and the $t w o$ sicken of the cervical st ump are then to be brought together. 


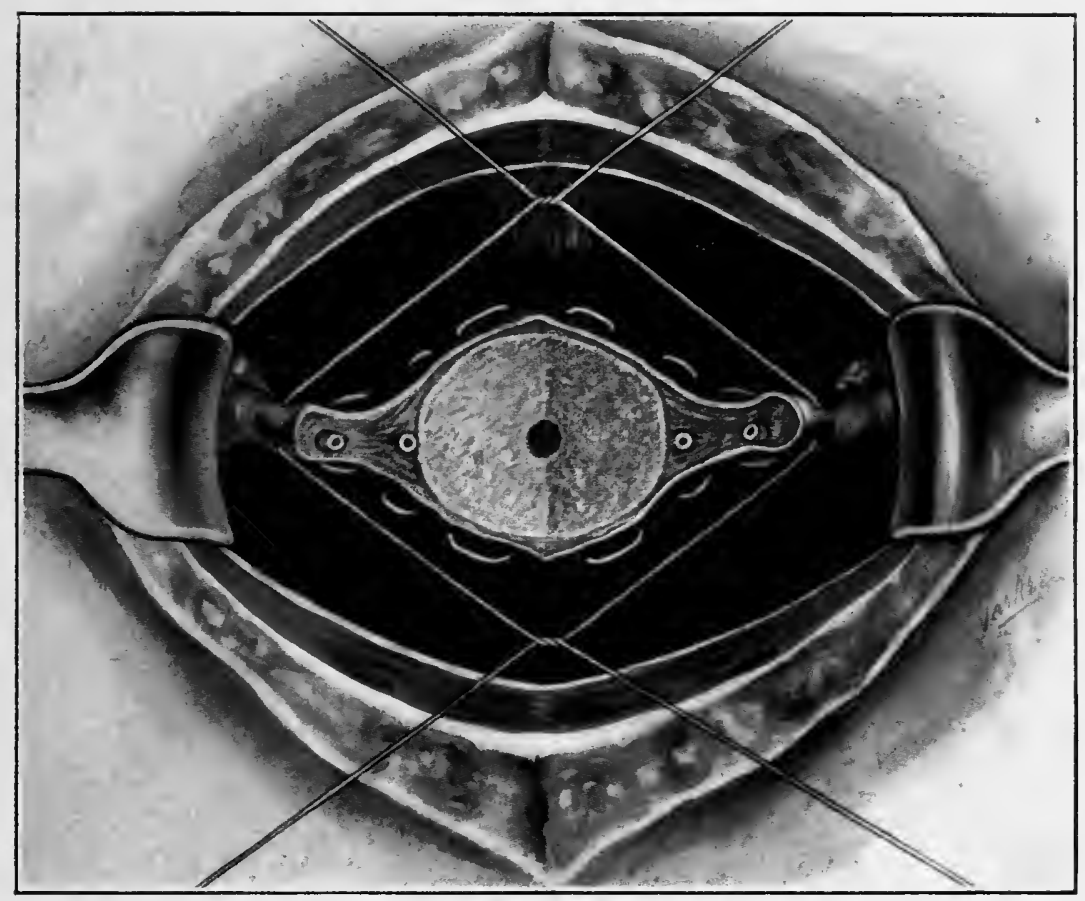

Supravaginal Hysterectomy. (Semidlagrammatic.) Looking down on the cervieal stump and severed broad ligaments instead of looking at the anterior wall of the cervix, as in Figure 184. Uterine and ovarian arteries secured by ligature. Two running catgut sutures catching up the peritoneal covering of the cervical stump and of the broad ligaments, one anterior and one posterior to the cervix and broad ligaments, have been passed in such a manner that when tied they will draw into apposition the twolateral surfaces of the cervical stump and will unite the ligaments end to end. 


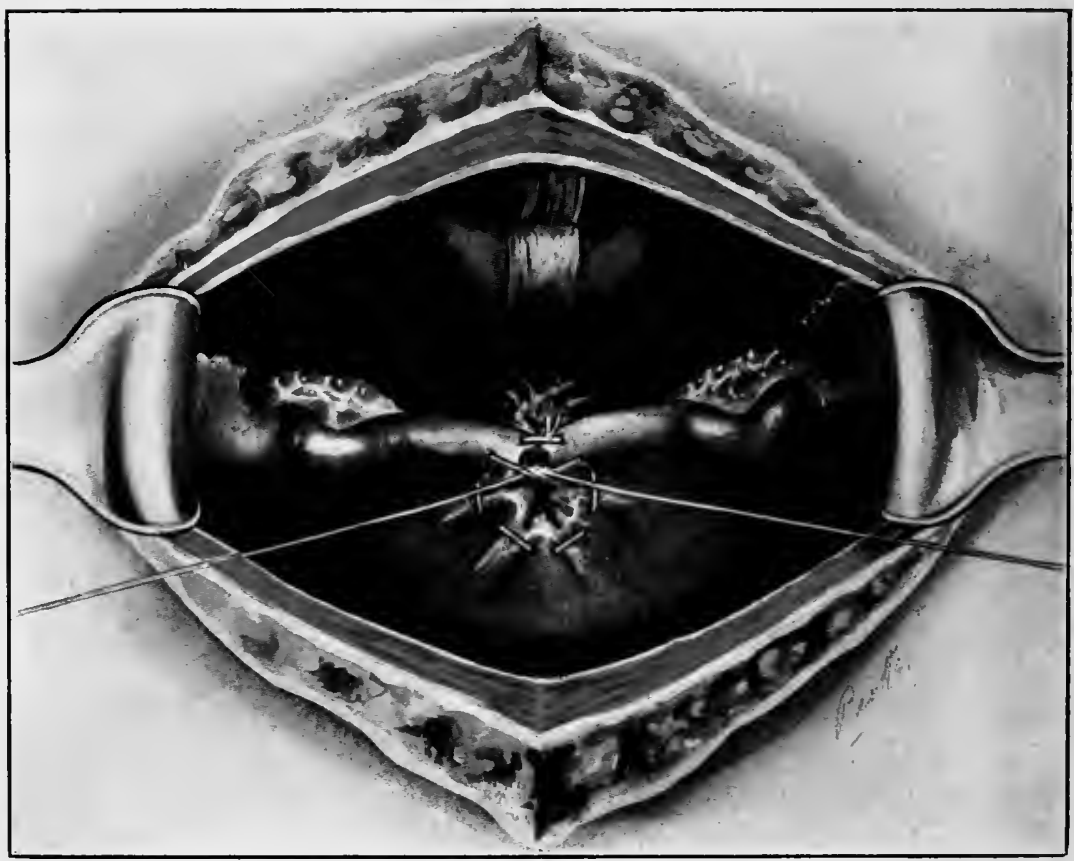

Supravaginal Hysterectomy. (Semidiagrammatic.). The posterior continuous suture shown in Figure 185 has been drawn taut and tied. The anterior suture is being drawn taut preparatory to tying. 


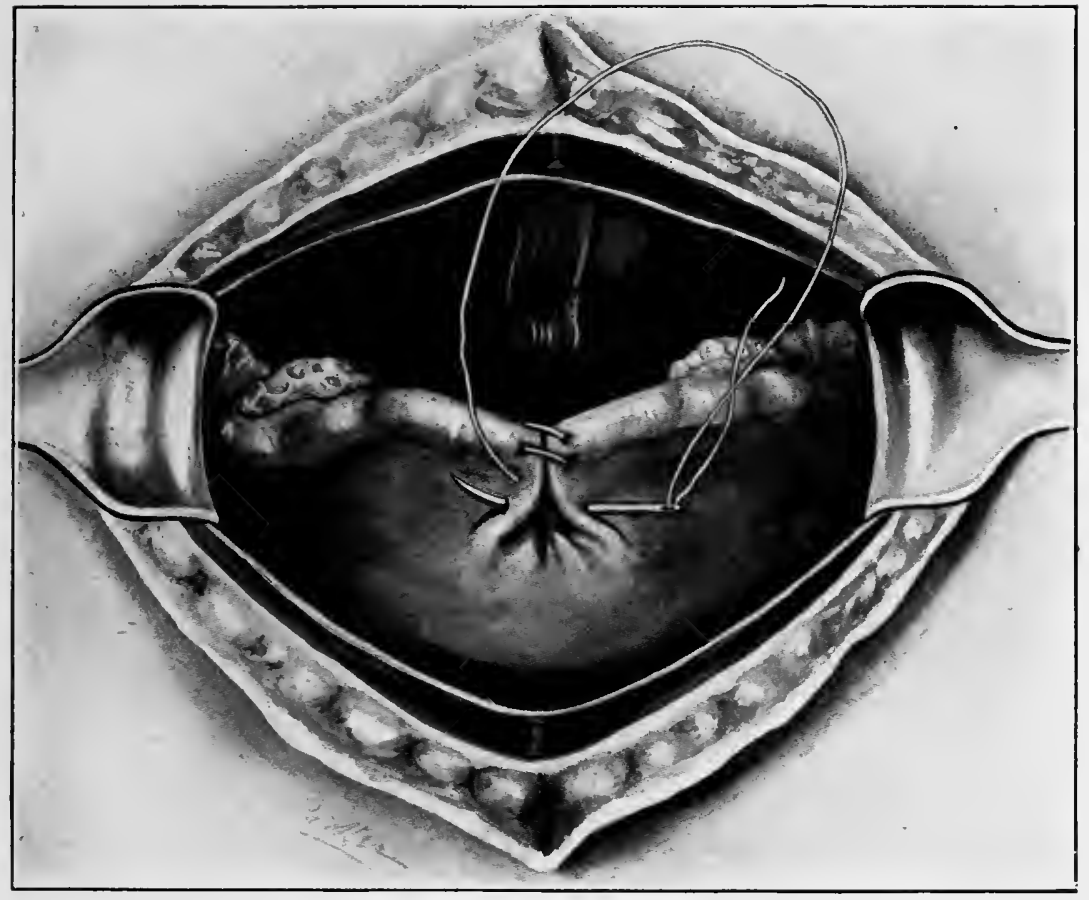

Supravaginal Hysterectomy. (Semidiagrammatic.) The broad ligaments having been drawn together as sliown in Figure 186, the approximution is completed by an additional running catgut suture beginning at the lower end of the line of union on the posterior side of the united ligaments and continuing over the edge of the ligaments down on the anterior surface to the bladder. This completes the closure of the pelvic wound. 


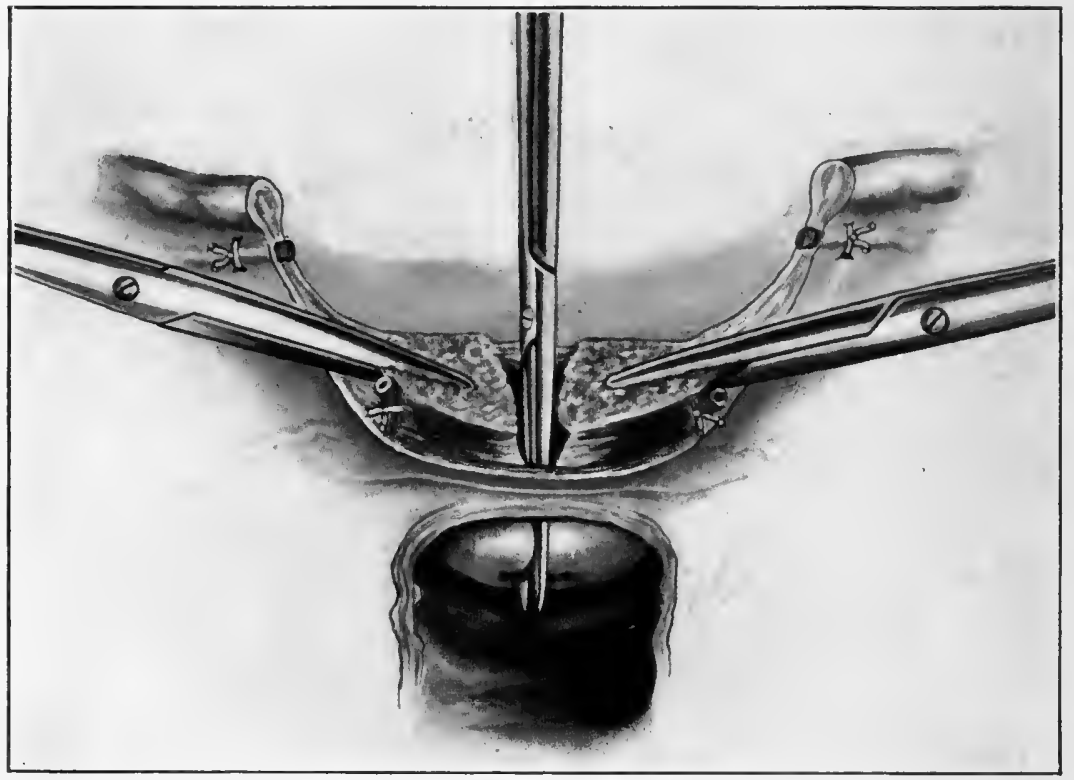

Supravaginal Hysterectomy. The corpus uteri ha" been removed, as shown in Figure 184. The uterine and ovarian arteries have been tied with catgut. The cervical stump is being held up and steadied by two forceps and divided anteroposteriorly into two equal lateral fragments clear to the vagina by means of scissors. In a very short, easily dilatable, patulous cervix the uterine dilator introduced from above downward might be used to divulse the cervical canal wiclely in place of cutting with scissors. 


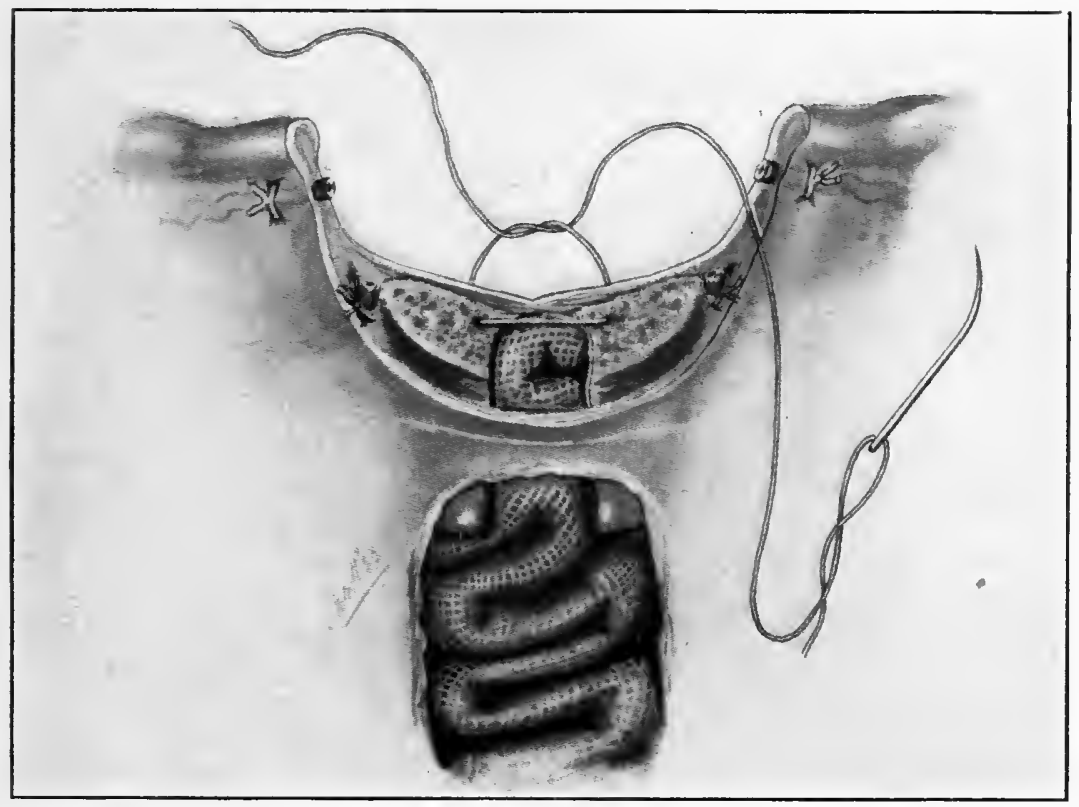

Supravaginal Hysterectomy. (Semidiagrammatic.) The cervix having been divided or dilated as explained in the legend of Figure 188, a short strip of gauze is introduced from above downward into the vagina. The two fragments of the cervix and the cut end. of the broad ligaments are then ready to be united by sutures. The first suture is introduced, but not tied. The operator may use the same form of suture as shown in Figure; 185,186 , and 187 , or may adopt any other form which the case may require. 


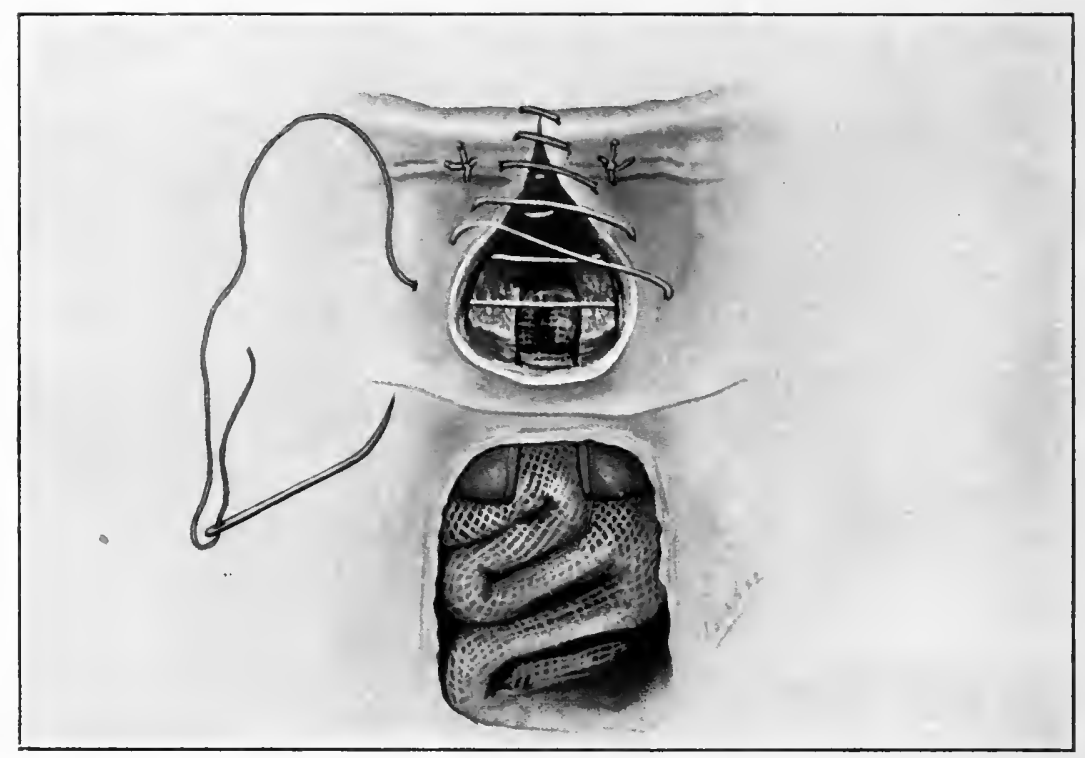

Supravaginal Hysterectomy. (Semidiagrammatic.) ('ontinuation of Figure 189. vaginal gauze drain. End-to-end approximation of the broad ligaments by continuous suture. This sut ure is here intended to suggest a method of closure. Other forms of sut ure and methods of closure are permisible. 


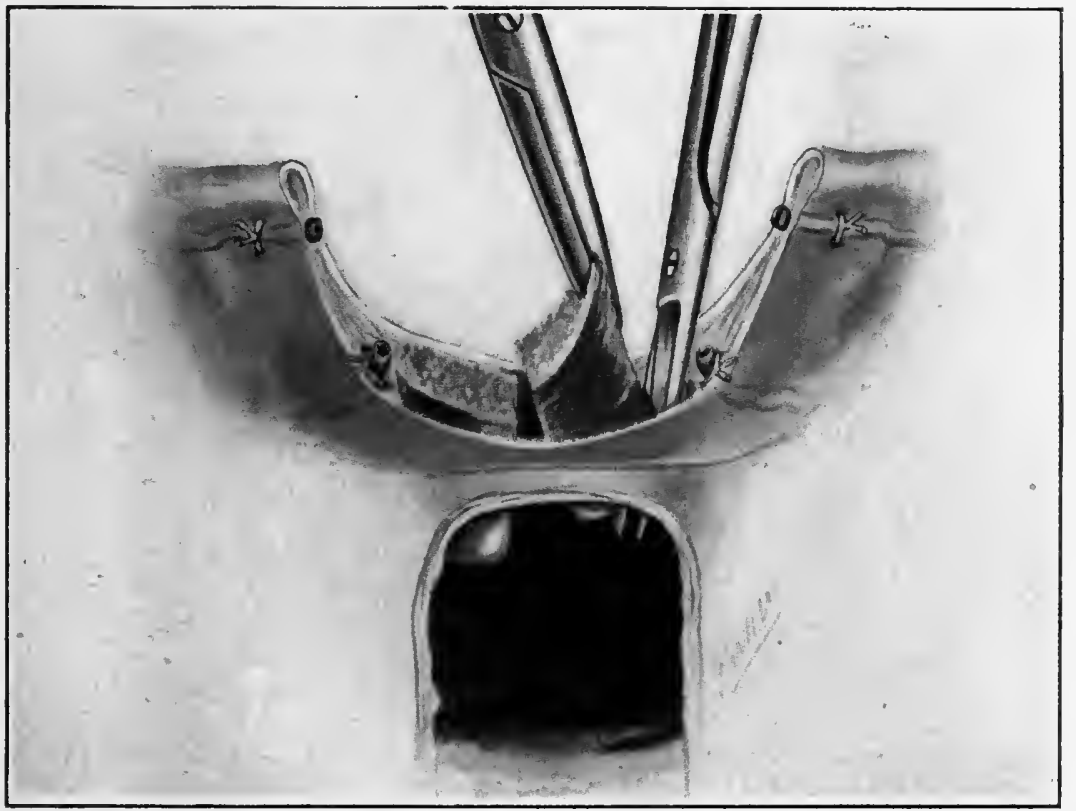

Complete Hysterectomy. (Senidiagrammatic.) The myonatous corpus having heen removed and the cervix split, the two lateral fragments are being removed by means of scissors, care being taken to keep close to the cervix in order to avoid the ureters. 


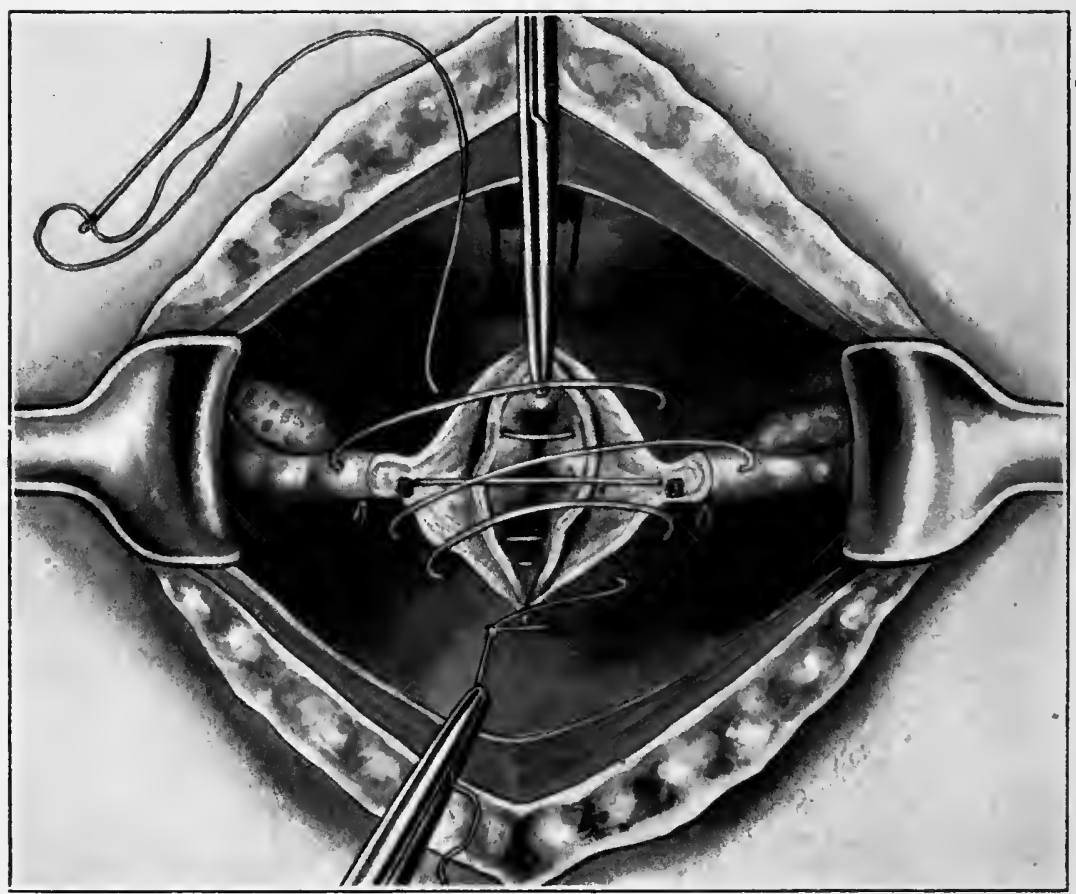

Complete Hysterectomy. (Semidiagrammatic.) Continuation of Figure 191. The cervical stump having been removed, the cut edge of the vagina is drawn up by means of forceps and included in a continuous suture, which closes the upper end of the vagina against the united broad ligaments which are brought together end to end. This form of suture is here introduced to show a method of union. Other forms of suture may be better suited to individual cases. 
Certain peculiarities in technique, however, should be observed as follows :

When the cervix is accessible through the vagina, the first incisions may be made as for vaginal hysterectomy, the bladder and the reetum being stripped away from the cervix, if practicable, as far as the peritoneal cavity. The broad ligaments may be separated through the vagina and tied off as high in some cases as the uterine arteries. The extent to which this ean be done will vary with the individual case. The vagina now is packed temporarily with a continuous strip, of gauze saturated witl a $1: 3000 \cdot 70$ per cent. aleoholic solution of mercuric bichloride. The final removal of the uterus through the abdomen is facilitated greatly by even a small amount of vaginal detachment. The abdomen then is opened and the operation continued as already described for supravaginal hystereetomy; the uterine arteries usually are clamped and tied a little further from the uterus. This necessitates the greatest care not to include the ureters, which cross the arteries very near the uterus. The broad ligaments and circumuterine structures then are divided by means of strong scissors ; in making the incisions for this purpose close to the uterus, no harm is done if, on either side, a small portion of the lateral wall of the cervix uteri be left behind. The bladder is stripped away from the cervix as far toward the vagina as practicable, and the peritoneum of the posterior wall of the uterus is stripped or dissected off in the same way.

If the vaginal incisions previously have extended into the pelvic cavity, the final removal of the uterus will be easy. If the incisions have not extended so far, the removal will not be difficult; but if no vaginal incisions have been made, the operator may in some cases find it quite tedious, if not difficult, to work his way down into the vagina. The attempt has resulted occasionally in opening the reetum, bladder, or ureter. This difficulty may be overcome largely by a simple device which has been used by the author for several years with great satisfaction ; the operation is performed as follows:

The bladder having been stripped off from the cervix as far down as possible toward the vagina, the uterus is drawn by means of vulsellum forceps well up through the abdominal wound. This traction exposes the anterior wall of the cervix, which now is divided freely with sharp scissors by a longitudinal incision and the cervical canal thereby laid open. One blade of the seissors is passed directly down through the external os to the vagina, dividing the entire anterior cervical wall. The finger now readily passes to the vagina, and serves as a guide for the rapid removal of the uterus by a circular ineision around the cervix at the uterovaginal attachment. In some cases it is convenient to reserve the ligature of the uterine arteries to this part of the operation. Small bleeding vessels are tied or twisted. Figure 191.

If drainage of the pelvic cavity is required, it should be vaginal, and the vaginal wound should be left open or partly open for this purpose. The drain is introduced as follows: the end of a long strip of gauze, double thick and two inches wide, is passed from the pelvis through the vaginal wound to the vulva; then the gauze is packed 
lightly from below upward, so as to fill the vagina and the vaginal wound, and to cover all surfaces in the pelvis left exposed by the operation. The dressing over the vulva which receives the capillary drainage from the gauze should be kept dry by frequent changing. The gauze drain, being a continuous strip, may be removed easily through the vagina in two or three days.

If drainage is not required, the wound should be closed completely both on the vaginal and the peritoneal side. This may be done by lines of union from side to side, as shown in Figure 192, or by end-to-end approximation of the broad ligaments as already described for supravaginal hysteromyomectomy. Figure 192 shows the upper cut end of the vagina closed by side-to-side union, and the peritoneal part of the wound closed by end-to-end approximation of the broad ligaments. If end-to-end approximation is employed, the same sutures that unite that part of the broad ligaments nearest to the vaginal wound should also eatch up the upper cut end of the vagina so as to draw it into the space from which the ecrvix has been excised, and mnite it to the lower portion of the broad ligament stumps at or near the point where the ligatures surround the uterine arteries; this serves to draw the vagina strongly upward and to cover the exposed surfaces between the vagina and broad ligaments.

Upon completion of the operation the vagina should be packed with ganze from the vaginal wound to the vulva, and a large ganze dressing placed over the vulva to absorb any wound secretions, and held there by a T-bandage, and changed often to keep it dry; the vaginal gauze is removed in about three days, after which low pressure vaginal douches of 0.5 per eent. lysol are given twice a day.

In hysteromyomectony, the ovaries, if normal or nearly normal, for reasons given in Chapter XXXIII., should be preserved.

In all operations for hysteromyomectomy the ligatures and sutures should be of sterilized catgut, Claudius' iodized gut preferred. See Chapter II.

\section{Advantages of End-to-end Approximation.}

1. The broad ligaments, in the anatomic sense, take the place of the excised uterus and form a pouch posteriorly like the cul-de-sac of Douglas, and anteriorly a depression that answers to the uterovesical pouch, thus conforming to the normal anatomy.

2. The broad ligaments, thus united, together with adjacent structures, hold up the rectum, bladder, vagina, and other parts of the pelvie floor, and in so doing prevent the descent of these organs, which so commonly results from hysterectomy as ordinarily performed.

3. The broad ligaments and adjacent structures, in occupying the space left by complete hysterectomy, prevent the intimate union of the rectum and bladder, a union which would leave only a thin wall between them, through which infection might pass from one to the other.

4. The operation is performed more easily and quickly by this method than by that of transverse suturing of the wounded ligaments. 
5. There is after closure much less intraperitoneal traumatism and eonsequently less danger of sepsis, adhesions, and secondary hemorrhage.

\section{Myonectomy during Pregnancy.}

The following eonditions more or less strongly contraindieate surgical treatment during pregnancy: 1 . Small size and slow growth of the tumor. 2. Location of the tumor where it will not materially interfere with uterogestation or obstruet delivery. 3. Probability that it will rise spontaneously, or that it may be forced manually out of the pelvis into the abdomen, where it will not interfere with pregnaney or parturition. 'The opposite of these conditions may eall for surgical measures. The following radical measures should be considered:

1. If the foetus is not viable, abortion, and, later, myomectomy or hystereetomy.

2. If the child is viable, Cæsarean section or the removal of the uterus entire or supravaginal hystereetomy-i.e., Porro's operation.

3. Removal of the tumor without interrupting pregnancy or saerifieing the uterus.

If surgical interference is inevitable, and gestation has not advanced beyond the end of the third month, the indieation is for abortion. Interruption of gestation at this time, and a radical operation for the removal of the tumor later, would be the safest course. After the third month the danger of induced abortion is inereased enormously. This increase comes from the difficulty of delivering the placenta, from infection, and from hemorrhage. Cæsarean scetion, to be followed immediately by eomplete hysterectomy or supravaginal hysterectomy, may now, in the interest of the child, be deferred, if possible, to the period of viability - that is, to the end of the seventh month or later. Removal of the tumor without sacrifieing the uterus or interrupting gestation may be preferred when the tumor is subperitoneal and removable with small uterine traumatism. 'This operation is indicated specially in subperitoneal pedunculated tumors.

An infected myoma, especially if eomplicated with pregnancy, demands immediate radical measures, and if the uterus also is infected may call for not only myomectomy, but for hysterectomy as well. See Plate VIII.

\section{Prognosis after Operation for Myoma Uteri.}

The danger in the removal of a uterine myoma varies with the skill of the operator, the loeation and relations of the tumor, and the eondition of the patient. The mortality of the abdominal operation in the hands of the average operator has been placed at about 15 per cent. This is too high. Under favorable conditions, including an expert surgeon, a mortality of 5 per eent. is too high. Statisties usually show a mortality of about 25 per cent. in the removal of intraligamentous tumors with broad uterine eonnections and of supravaginal tumors of the upper part of the cervix. This, again, is too 
high. The method already described for the removal of these tumors by myomectomy with gauze drainage into the vagina, when required, closure of the tumor-cavity in the abdomen, and, if necessary, ligature of the uterine vessels, las reduced the mortality to a very small percentage. Vaginal extirpation of the small myomatous uterus shows a mortality of less than 3 per cent. Removal of a tumor from the infravaginal portion of the cervix and removal of intrauterine tumors throngh the vagina are practically without danger. The long-continued menorrhagia associated so commonly with uterine myomata may so exhanst the woman as greatly to decrease her resistance, and thereby to increase the danger of an operation; hence the occasional necessity of preparatory curettage, uterine tamponade, general treatment, and delay in some cases for weeks or months, until the systemic condition is favorable. Before an abdominal operation it may be necessary to increase the hæmoglobin and the red blood-corpuscles by preparatory treatment; the hæmoglobin, if possible, should be brought up to at least 50 and the red blood count to not less than $3,000,000$.

The mortality of hysteromyomectomy with end-to-end approximation of the broad ligaments, as described above, has not risen above 1 per cent. in my awn cases during the last six years. 


\section{CHAPTER XXVIII.}

\section{TUMORS OF THE UTERUS (CoNTINUED).}

\section{CARCINOMA AND ENDOTHELIOMA.}

\section{Pathology of Carcinoma Uteri.}

Carcinora may arise from any portion of the uterine mucosai. e., from the cylindrical epithelium of the corporeal or cervical glands, from the surface cylindrical epithelium of the interior of the uterus, or from the pavement epithelium outside of the external os. The variety of the cancer usually corresponds to the type of epithelium from which it springs. Cylindrical cell carcinoma occurs on the corporeal and intracervical mucosa, and the pavement-cell variety occurs on the lower portion of the cervical canal or on the external vaginal surface of the cervix. This rule is not invariable. Eversion of the intracervical mucosa is quite common-see Laceration of the Cervix-hence the frequent formation of the cylindrical cell carcinoma outside of the apparent os externum.

From the pathological standpoint there are thus two varieties of carcinoma. One type is that in which the squamous cells of the cervix have multiplied in an atypical manner and have invaded the deeper tissues; this carcinoma is like the epithclioma which occurs at the junction of the skin and mucosa of the lip. The other type is that in which the cylindrical cell gland-acini of the interior of the cervix or corpus uteri multiply in an atypical manner, invade the interglandular stroma, and thus conform to the carcinomatous type. The two varieties of carcinoma are therefore called:

1. Cylindrical cell carcinoma, adenocarcinoma, gland carcinoma.

2. Pavement-cell carcinoma, squamous carcinoma, epithelioma.

Carcinoma of the cervix usually originates near the os externum, where the cylindrical and pavement epithelium meet, and is therefore either a cylindrical cell or a pavement-cell carcinoma, or both. Early in the disease the tendency of the former is to extend to the submucous structures, and of the latter to confine itself more to superficial areas.

When the deeper tissues are involved in carcinoma, the affected portion is enlarged, hard, marble-like, and friable. The surface is smooth, glistening, and flattened, or may be nodular. Either varicty rapidly extends and ulcerates early. The margin of the ulcer is irregular, hard, and usually raised. The base is irregular and bleeds easily. The ulcerative process may destroy the cervix slowly or rapidly.

Carcinoma may extend from the cervix uteri:

1. To the vaginal vault, anteriorly or laterally, less often poste- 
riorly. The advancing margin of the growth is raised, rounded, and hard.

2. To the broad ligaments, giving them on digital touch a hard, board-like feel.

3. To the vesicovaginal septum, less often to the rectum.

4. Rarely to the uterine appendages, urethra, or pelvic bones.

5. To the corpus uteri-frequently.

6. To the iliac glands-not usually until the disease has involved the broad ligaments; this delay is because the squamous cancer-cells are too large to pass easily through the lymph-radicles of the cervix, but not too large to traverse the lymph-vessels of the ligaments.

7. After the discase has passed beyond the cervix uteri the kidneys and ureters usually are involved in nephritis, hydronephrosis, or pyelonephrosis. Dilatation of the ureters is the common result of inflammatory constrictions near the invading growth, pressure of the growth on the ureter, or extension of the growth to the ureter.

8. Metastatic cancer extending from the cervix uteri direct to distant organs is not common.

- Carcinoma may extend from the corpus uteri:

1. To the broad ligaments and parametria, and thence to any adjacent structures, such as peritoneum, omentum, mesentery, intestine, Fallopian tubes, and ovaries; all these organs being involved, if at all, in the later stages; complicating cancer of the liver and lungs is rare.

2. To the lumbar glands, common; to the inguinal glands, not common; the iliac glands are less liable to involvement than in cancer of the cervix.

3. To the cervix uteri.

\section{Etiology of Carcinoma Uteri.}

The causes of cancer are unknown; the predisposing or favoling conditions are as follows:

1. Age-the disease occurs most frequently between forty and fifty. The extreme limitations are between eight and seventy-six.

2. Heredity - an apparent predisposing cause.

3. Social state-more frequent among the poor and ignorant.

4. Race-relatively rare among negroes.

5. Tranma of labor-laceration of the cervix a possible predisposing cause.

6. Endometritis and endocervicitis are said to be favoring conditions.

\section{Symptoms and Course of Carcinoma Uteri.}

There are no incipient symptoms, nor at any time in the course of cancer are the symptoms pathognomonic. As the disease progresses the following disorders always appear:

1. Hemorrhage.

2. Uterine discharges. 
3. Pain, especially in adenocarcinoma.

4. Visceral disorders.

5. Cachexia.

1. Hemorrhage is usually the first symptom, and is the result of accidental injury to the cancer, and to ulcerative processes. Unfortumately it is attributed often to irregularities of the menopanse or to a return of menstruation after the menopause; hence the fact that the bleeding of carcinoma often is disregarded until the disease has progressed beyond the hope of cure. The reappearance of hemorrhage one, two, three, or more years after the menopause is strong presumptive evidence of cancer, and demands immediate examination. The loss of blood, at first slight, is noticed commonly after straining at stool, or vigorous exercise, or coitus. With the progress of the disease the hemorrhage increases; it may be nearly or quite constant, may occur at irregular intervals, or in the form of menorrhagia at the catamenia. Usually the patient's strength is exlausted slowly by a persistent, slow seeping away of the watery blood. On the other hand, profuse, even dangerous, hemorrhages are possible.

The menstrual history bears no very significant relation to the development of carcinoma. The disease in many cases follows the menopause.

2. Uterine Discharge.-The character of the discharge varies with the progress of the disease :

a. Early ; discharge watery, serous, transparent, inodorous.

$b$. Later; discharge watery and fetid.

c. As ulceration increases and growth becomes friable, the discharge is more profuse, bloody, turbid, sometimes purulent, and always of a most nauseating odor. This latter symptom continues more or less constant to the end, and is characteristic of malignancy. The discharge is called "carcinomatous ichor," or "cancer juice." All excessive discharges, especially after the menopause, should be regarded with suspicion.

3. Pain is rarely present while the growth is confined to the vaginal portion of the cervix. Involvement of the corpus uteri and of the structures around the uterus may give rise to sharp, lancinating pains. These pains, although often described as pathognomonic, are by no means constant or confined to cancer. They may be sipplemented by the pains of pelvic peritonitis. The peritonitis protects the general peritoneum by adhesions which form in front of the invading carcinoma. The pains are due to pressure on the pelvic nerves or to actual involvement of those nerves in the carcinoma ; they commonly are referred to the region of the pelvis, perineum, or thighs, and usually indicate that the disease is past operative cure. The retention of secretions in the uterus from occlusion of the cervical canal by the invading carcinoma may give rise to hydrometra or pyometra, and cause spasmodic expulsive uterine pains like labor-pains.

4. Visceral Disorders may be consequent upon pressure or invasion of neighboring organs. The bladder becomes irritable. Vesical catarrh, strangury, painful urination, pyuria, and cystitis may follow. Vesicouterine or vesicovaginal fistula often results from the destruc- 
tive ulcerative processes. Uretero-uterine and ureterovaginal, rectouterine and rectovaginal fistula may occur in the same way. Nephritis, uræmia, hydronephrosis, and atrophy of the kidney are among the usual resultant complications. Constipation is explained as follows: First, the patient, through fear of pain and bleeding, voluntarily retains the feces; second, the feces become dry and hard from loss of water in the ichorous discharges; third, the bowel is incapacitated by the disease for the ready expulsion of its contents. Diarrhœa may be cansed by irritation of the bowel from the invasion of the cancer. Alternating constipation and diarrhoea are not uncommon.

5. Cachexia appears not very late in the course of the disease, and is a characteristic symptom. It is marked by emaciation, a yellowish pallor of the skin, profound anmia, and great depression of both mind and body. It is caused by toxic agents elaborated by the malignant tumors through their perverted metabolism and by malnutrition due to anorexia, vomiting, pain, and hemorrhage.

\section{Diagnosis of Carcinoma Uteri.}

The sooner the carcinomatons uterus is removed, the less the likelihood of recurrence; hence the earliest possible diagnosis is imperative. Absolute diagnosis must depend usually upon the microscopical findings. A probable diagnosis may often be made by

1. The clinical history.

2. The physical signs.

1. The Clinical History, as indicated in the foregoing paragraphs, gives strong evidence, though not proof of eancer.

2. The Physical Signs are demonstrated by conjoined examination and inspection. The extremely fetid odor, which clings to the examining finger despite much washing and the energetic use of the nail-brush, usually may be avoided by the free use of glycerin as a lubricant. Infiltrating carcinoma of the cervix is recognized as a thick, usually hard, more or less nodular, friable growth. The friability and bleeding are almost pathognomonic. The ulcers, if present, have an irregular, hard, raised margin, and uneven base, and bleed freely upon slight injury. Through the speculum the surface before ulceration appears even or nodular, marble-like, and glistening. After ulceration the surface is ragged and irregular, and may show large excavations from the sloughing of carcinomatous tissue. The entire cervix may be destroyed in this way. The papillomatous superficial variety appears as a soft, friable, bleeding, cauliflower-like mass.

\section{Explanation of Plate XV.}

A. Carcinoma of the corpus uteri complicated with small multiple myomata.

B. Carcinoma of the corpus uteri. On the right side the disease has extended nearly through the uterine wall; on the left side the disease is confined apparently to the mucosa, and in gross appearance resembles polypoid endometritis.

C. Carcinoma of the cervix uteri, the cervix nearly destroyed.

D. Carcinoma of the cervix uteri, the cervix wholly destroyed. 
PLATE XV

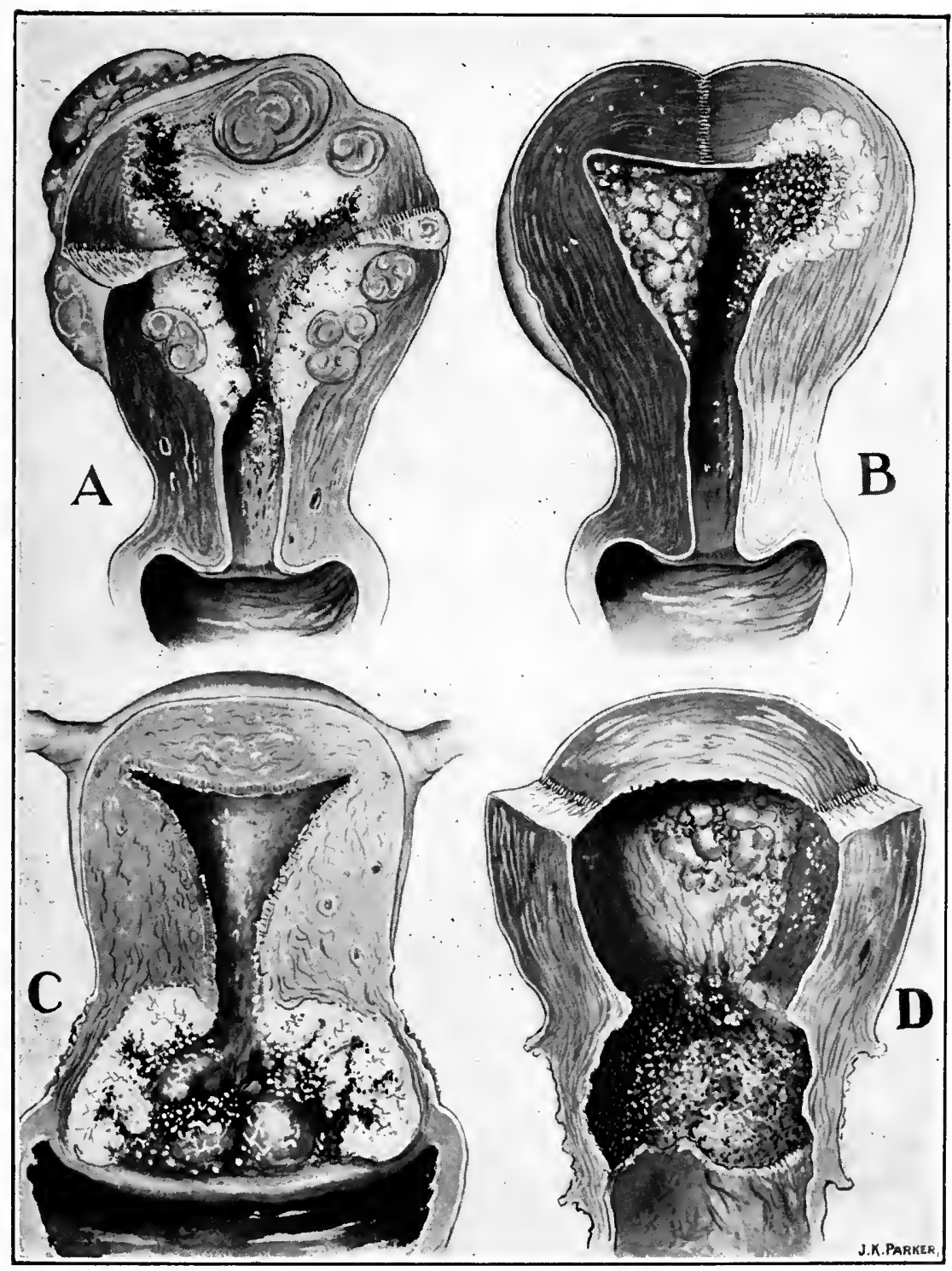




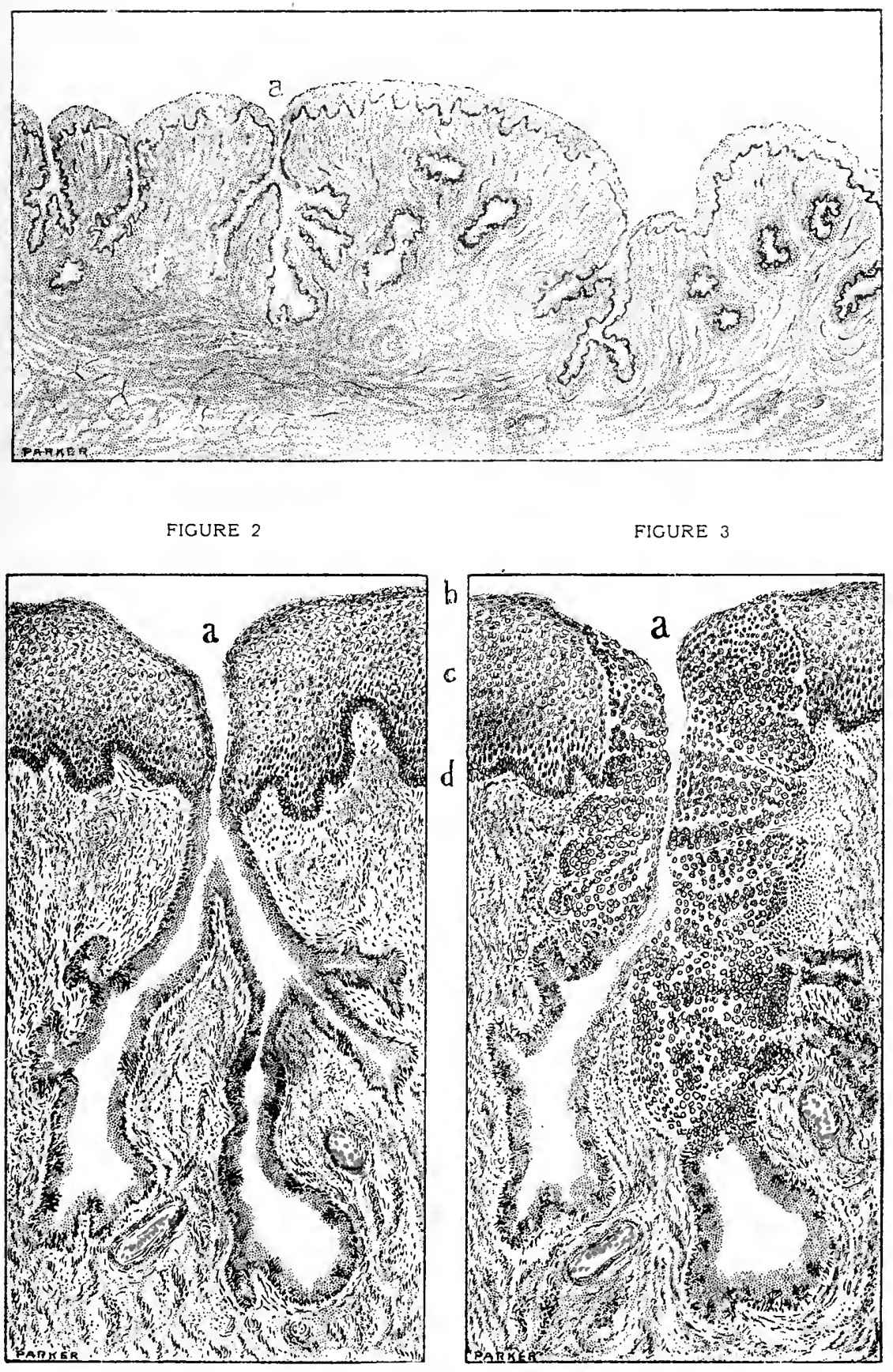



\section{Diagnosis of Carcinoma of the Cervix Uteri.}

Early carcinoma of the cervix without great care may be ocerlooked. The cervical wall around the external os may be only a little thickened on the affecter side. The indurated tissue may appear almost insignificant in amount. Extreme friability and persistent bleeding on slight abrasion will, however, be strong diagnostic factors. Subjective symptoms may even be absent. Excision of a small piece for microscopical examination is now imperative. This should be wedge-shaped, and should include a portion 'of the surrounding healthy tissue. The slight wound may be closed by one or two sutures. Cervical scrapings are usually useless for examination. Cervical carcinoma has been shown by reliable statistics to be about sixteen times more frequent than carcinoma of the corpus uteri.

\section{Diagnosis of Carcinoma of the Corpus Uteri.}

Carcinoma of the corpus uteri is in the beginning often impossible to recognize. It is apt to appear between the ages of forty and fifty. There is increased and irregular menstruation, which often is attributed wrongly to the menopause. The presence of a slight watery discharge, even though odorless, is highly diagnostic. If the discharge is very fetid, the evidence is much stronger. The general tone of the patient may be almost up to the normal standard. Conjoined examination, preferably with rubber gloves, shows nothing except perhaps slight enlargement of the uterus. Life now may depend upon speedy diagnosis. The whole question centres in the product of curettage and the microscopical findings. Should no microscopical evidence of cancer be found, the curettage should be repeated whenever the hemorrhage reappears. In cancer the discharge always recurs promptly. The scrapings are usually much more abundant than in benign growths.

Frequently recurring glandular hyperplastic endometritis with much cystic development after repeated curettage, especially if associated with free hemorrhage and a watery discharge, should give rise to grave apprehension, and would justify removal of the uterus on suspicion.

Advanced carcinoma of the body of the uterus is recognized by the symptoms already described and by conjoined examination. The

Explanation of Plate XVI.

FIGURE 1.-Mucous membrane of the cervix uteri in section taken just above the external os, showing, a, the branching racemose gland at a dips down from the free mucous surface to the muscularis. 15 diameters.

FrgURE 2.-A magnified reproduction of gland a in Figure 1. Observe the horny layer at $b$, the cuboidal epithelium at $c$, and the papillary layer at $d$. 60 diameters.

FIGURE 3.-Pavement- or squamous-cell carcinoma. A hypothetical pavenentcell carcinoma is here shown as it would appear if it attacked gland a in Figure 2. The two deep pockets of gland a are normal, but the remainder of the gland has been invaded by squamous epithelium, of which the nuclei are stained deeply and packed closely together. The surrounding stroma is infiltrated with small round cells. Invasion of the gland in this manner by squamous epithelium establishes the diagnosis of carcinoma. Two normal blood-vessels are shown in the lower part of the Figure. Diagrammatic. 60 diameters. 
uterus is enlarged-often two or more times its normal size. It is hard, nodular, and, in the later stages, more or less fixed. Early fixation also occurs in cervical cancer. The causes of fixation are similar to those which produce the same condition in pelvic inflammation-i.e., extension of the disease to the parametria. The lower extremities become oelematous from hydræmia, from pressure, and from thrombosis of the pelvic veins. The absolute diagnosis may depend upon the microscope. The recognition, however, of advanced carcinoma, whether of the cervix or corpus, even without the microscope, is usually not difficult. "He who runs may read."

The following diagnostic signs will distinguish pavement-cell carcinoma from cylindrical cell carcinoma :

\section{Pavement-cell carcinoma- epithelioma.}

1. Originates in pavement-cell epithelium and follows that type.

2. Forms solid finger-like or branching ingrowths.

3. Epithelial pearls may be formed.

4. Solid alveoli formed.

5. Breaks down rapidly and bleeds freely.

\section{Cylindrical-cell carcinoma- adenocarcinoma.}

1. Originates in cylindrical cell epithclium and follows that type.

2. Forms gland prolongations.

3. Absent.

4. Glandular structures always recognizable.

5. Breaks down more slowly.

\section{Differential Diagnosis of Carcinoma Uteri.}

The conditions most liable to be mistaken for carcinoma are :

1. Myoma.

2. Sarcoma.

3. Retained placental tissue.

4. Incomplete abortion.

5. Hypertrophy of the cervix.

6. Endocervicitis. Nabothian follicles.
7. Endometritis.

8. Syphilis.

9. Chronic metritis.

10. Ichthyosis.

11. Tuberculosis.

12. Laceration of the cervix uteri.

The differential diagnosis will be found in the following parallel columns and paragraphs:

Advanced carcinoma of the corpus uteri.

1. Cachexia, hemorrhage, and very foul discharges.

2. Sloughing tissues very friable.

3 . Cervix may be involved by extension.

4. Charaeteristic epithelial proliferations seen by microscope.

\section{Carcinoma uteri.}

1. Frequent

2. Diffuse.

3. Surface not smooth.

4. Trabeculæ seen on cross-section.

5. Gland formation, abundant stroma, bloodvessels with walls in stroma.

Carcinoma of the corpus uteri.

1. History of pregnancy commonly absent.

2. Hemorrhage from uterus.

3. Quantity of tissue removed by curette may be large.

4. Scrapings composed of short, friable threads having a shaggy appearance.

5. Alicroscopical examination shows carcinoma.
Sloughing submucous myoma.

1. Anæmia from hemorrhage; discharges less foul.

2. Tough, not friable

3. Cervix not so involved.

4. Absent.

\section{Sarcoma uteri.}

1. Very rare.

2. Rather sharply defined.

3. Previous to necrosis surface usually smooth.

4. Cross-section shows smooth, homogeneous surface.

5 . Cells round or spindle-shaped, little or no stroma; in plaee of blood-vessels with walls there are blood-spaces in direct relation with surrounding cells.

\section{Retained placental tissue.}

1. History of recent pregnancy.

2. Same.

3. Quantity always large.

4. Scrapings composed of myriads of long, slender threads having shaggy appearance.

5. Iicroscopical examination shows products of conception. 


\section{PLATE XVII}

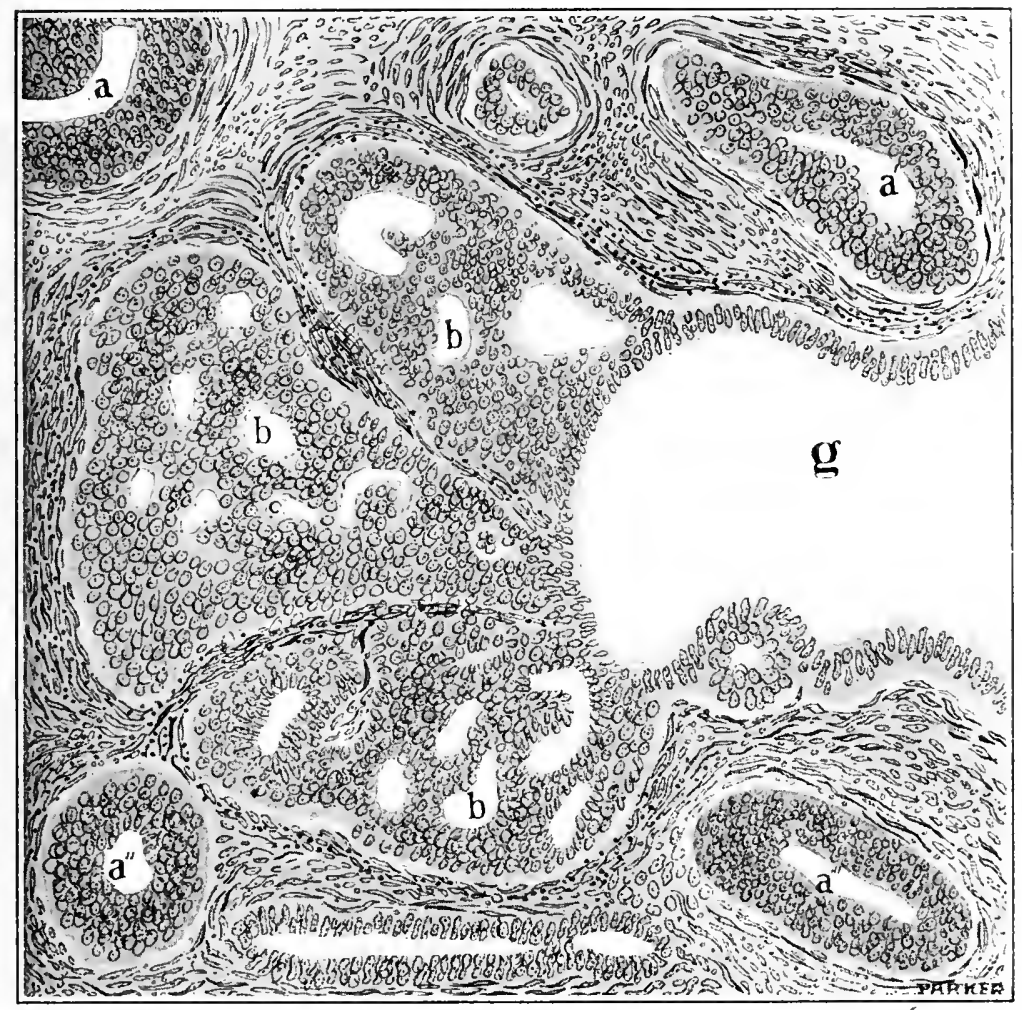

\section{Adenocarcinoma of the Corpus Uteri.}

In the lower middie part of the drawing is a longitudinal section of a normal gland. In the four corners are glands marked $a^{\prime} a^{\prime \prime} a^{\prime \prime \prime}$ which have many layers of epithelium still confined within the glands. Occupying the centre of the drawing is an enormously large gland the right half of which $(g)$ is nearly normal, but the left half shows three branches $(b b b)$ in which there is very great proliferation of epithelium filling that part of the gland cavity and invading the stromat between the branches. The great proliferation of cells at $b b b$ and the invasion gland structure into the stroma establish the diagnosis of carcinoma. The characteristic small round-cell infiltration around the carcinomatous area is present. ro diameters. 

Carcinoma of the corpus uteri.

1. No history of pregnancy.

2. Scrapings on microscopical examination show carcinoma.

3. Cachexia.

Carcinoma of the cervix uteri.

1. Cervix enlarged, friable, breaks down rapidly and bleeds freely to touch.

2. Characteristic offensive watery discharge - "cancer juice."

3. Microscopical examination of excised portion shows carcinoma.

\section{Incomplete abortion.}

1. History of pregnancy and abortion.

2. Scrapings on microscopical examination show products of conception.

3. Anæmia.

\section{Hypertrophy of the cervix uteri.}

1. Cervix enlarged, tough, and does not bleed freely to touch.

2. Absent.

3. Shows cervical structures modified only by hypertrophy.

Endometritis.-The microscopical differential diagnosis has been set forth in the chapter on Endometritis. See clinical diagnosis of the two diseases, as presented in the same chapter. Polypoid endometritis is distinguished from carcinoma of the corpus uteri by the following characteristics :

1. Polypoid eminences not sharply defined.

2. Friability not marked.

3. Underlying muscularis not invaded.

Endocervicitis.-Cystic and polypoid glandular enlargements due to endocervicitis have certain characteristics which might lead one to mistake them for cancer.

Cystic glandular enlargement (cystic degeneration) of the Nabothian follicles may be distinguished from cancer by the following characteristics :

1. The large, hard nodular cervix is smooth, non-friable, and has no tendency to bleed.

2. Puncture of cysts reveals mucous contents.

3. Progress of disease very slow.

4. Microscopical section shows eysts lined with a single layer of epithelium, with tunica propria unbroken and surrounded by normal stronia.

5. Condition generally due to laceration of cervix and eversion of the cervical mucosa.

Polypoid glandular enlargement (mucous polypi) differs from carcinoma in the following particulars:

1. Springs from area within cervical canal.

2. Lips of cervix intact.

3. Polypi rather firm, not friable, and bleed but little.

4. Microscopical section shows single layer of epithelium and normal or hypertrophied cervical glands.

Syphilis will be known by the clinical history. In doubtful cases specific treatment shonld establish the diagnosis.

Chronic Metritis.-Chronic metritis shows a history of inflammation, is associated usually with endometritis, does not cause the carcinomatous cachexia nor the offensive watery discharge. On conjoined examination the uterus is symmetrical, while a carcinomatous uterus is often nodular.

Ichthyosis Uteri.-This condition, first described by Zcller, ${ }^{1} 1884$,

1 Zeller. Plattencpithel im Uterus (ichthyosis uterina). Zeitschrift für Geburtshülfc und Gynäknlogie, Band xi. Ries. "Fine neue Operationsmethode des Utertiscarcinoma," Zeitschrift für Geburtshülfe und Gynäkologie, Band xxiv. "Ichthyosis," American Gynecological and Obstetrical Journal, February, 1886. 
is marked by the presence of two or more layers of stratified epithelium; in the cavity of the uterus it has been observed in connection with inversion of the utcrus, with cervical polypi, and, according to Zeller, with chronic endometritis. 'Transition of columnar to pavement-cell epithelium occurs in hydrometra and hæmatometra, and in extra-uterine pregnancy; the transformation may occur where, from any cause, the mucosa is stretched and flattened, so as to stratify the epithelium. The condition gives rise to no unusual symptoms except such as ordinarily would be observed in endometritis or in the beginning of carcinoma of the corpus uteri. The scrapings of stratified epithelium under the microscope may have a similar appearance in ichthyosis uteri and carcinoma uteri. If the microscopical findings show that the stratified epithelium is limited to the superficial structures, the case is one of ichthyosis uteri. If the epithelium penetrates the underlying connective tissue, or muscular layer, and if that epithelium is surrounded by round-cell infiltration, the disease is probably carcinoma. Just as glandular hypertrophy may be the startingpoint of carcinoma, so may ichthyosis.

Tuberculosis of the Uterus.-Tubercular disease in the uterus varies according to the location.

Tuberculosis of the endometrium is distinguished from carcinoma by the following characteristics :

1. Mucosa at first smooth, yellowish white, and glistening; later, yellowish-white nodules appear on surface and below surface of endometrium.

2. Finally, nodules undergo caseous degeneration and ulceration.

3. At times small yellowish tubercles surround ulcers.

4. Disease may involve entire endometrium and may extend through the muscularis to the perimetrium.

5. Hemorrhage not characteristic.

Tuberculosis of the cervix presents the characteristic ulccrative processes of lupus.

1. Margins of ulcers are well defined, or may be undermined, and are surrounded by tubercles.

2. Base of ulcers is studded with tubercles and covered with pus, necrotic tissue, or caseous matter.

3. Microscopical section shows giant cells and tubercular bacilli.

Tuberculosis differs from carcinoma in all the above particulars.

Laceration of the Cervix Uteri.-This condition is characterized by inflammatory and mechanical results which may resemble closely carcinoma of the cervix. They are :

1. Eversion of the intracervical mucosa and cystic degeneration of the Nabothian follicles (see Endocervicitis, in one of the foregoing paragraphs.

2. The everted eroded surfaces present an irregular and sharply defined line of demarcation.

3. Approximation of the lacerated margins by means of tenacula causes the everted mucosa to be rolled in and to disappear.

4. The cervix, if indurated, presents the peculiar hardness of hypertrophy; not the friability of carcinomatous infiltration. 
5. In all the above particulars this condition differs from cancer. In doubtful cases microscopical examination is essential.

The differential diagnosis of carcinoma of the body of the uterus may be rendered difficult by such associated lesions as myoma, sarcoma, endometritis, salpingitis, and ovaritis.

\section{Causes of Death from Carcinoma Uteri.}

The causes of death are :

1. Exhaustion.

2. Sepsis.

3. Hemorrhage.

4. Uræmia.

5. Intercurrent diseases, such as peritonitis and pneumonia.

Hemorrhage, although it may exhaust the vitality slowly, is rarely a direct cause of death. Fatal peritonitis seldom occurs from extension of the disease. In the vast majority of cases death is from marasmus or uræmia, or both. Fatal septic pneumonia and pulmonary œdema may be caused by embolism.

\section{Prognosis of Carcinoma Uteri.}

The sole hope of radical cure is in surgical removal of the carcinoma. Drugs are useless. If the growth has progressed beyond the limits of a radical operation, "All hope abandon ye who enter here." 'The disease sometimes will destroy life in a few months or weeks; it may for a time become apparently inactive, or develop very slowly, and then go on to a rapid termination. The prognosis as to limit of life should be guarded. A gencral statement that death is more liable to occur within one year than after two years usually will be safe.

\section{Diagnosis of Recurrence of Cancer after Removal.}

After hysterectomy, recurrence may be suspected under the following conditions :

a. Pain radiating to hip and thigh.

b. Edema in lower extremities.

c. Cachexia and failing health.

\section{Diagnosis of Extension of Carcinoma Uteri.}

Carcinoma of the uterus may extend to adjacent and other organs, as follows :

1. Extension to the Vagina, recognized by :

$a$. Cartilage-like hardness and nodules in the vaginal vault, due to carcinomatous infiltration.

b. Ulcers in vaginal vault having hard border and bleeding base.

2. Extension to the Rectum, recognized by :

a. Bloody, offensive discharge from rectum.

$b$. Rectal touch shows irregular hard post-uterine mass extending into rectal wall. 
c. Rectal mucosa fixed to mass and ulcerated; borders of uleerated surfaces hard and raised.

3. Extension to Bladder, recognized by :

a. Cystoscopy, conjoined examination, digital touch through artificial vesicovaginal fistula.

b. Digital palpation of bladder through urethra; not advised.

c. Hæmaturia.

4. Extension to Parametria, recognized by the following conditions:

a. Cancerous infiltrate is harder than inflammatory infiltrate.

The inflammatory infiltrate, unlike that of carcineoma, first forces the uterus to the opposite side, and later, as the mass disappears, contracting structures draw it toward the affected side. Fixation of the uterus is not a reliable sign, because the uterus may also be fixed by inflammatory infiltrate.

b. Carcinoma of the supravaginal portion of the cervix gives rise to early infiltration of the parametria; carcinoma of the infravaginal portion gives rise later, and carcinoma of the corpus still later.

c. Hard mass extends first from posterior wall of the eervix to the back of the pelvis, later to the sides; best palpated through the rectum.

5. Extension to the Glands:

a. The iliac glands are the first to be involved in cancer of the cervix, and are palpated best under narcosis per rectum, in front of the sacro-iliac joint; infiltration of the parametrium precedes infiltration of the glands.

b. The lumbar glands involved in cancer of the corpus uteri are palpated only under anæsthesia through the abdominal walls when the walls are thin and relaxed.

6. Extension by Metastasis to other organs is a late manifestation, of which an early positive diagnosis is impossible.

\section{Treatment of Carcinoma Uteri.}

The treatment is radical when the cancer has not extended beyond the limits of entire removal; palliative, when it eannot be removed entirely. The radical treatment should always be complete hysterectomy. The old practice of high amputation of the cervix for cervical cancer should never be resorted to, for one can never be certain that the disease is not also present and unrecognized in the corpus uteri.

\section{Indications for Hysterectomy.}

Hysterectomy always is indicated if the carcinoma is limited to the uterus; such limitation may be possible if:

1. The uterus is normally mobile and symmetrical.

2. The uterus is not excessively enlarged.

3. The iliac and lumbar lympluatic glands are not enlarged; this is difficult to determine.

4. The vaginal wall is not involved in careinoma.

5. The parametria are not infiltrated, as shown by vaginal and rectal examination. 
The reverse of the above conditions weakens the indication for hysterectomy; the stronger the reverse the weaker the indication.

Enlargement of the glands, although evidence that carcinoma has extended beyond the uterus, does not necessarily contraindicate hysterectomy, although it renders the prognosis less favorable. Such enlargement may be due to inflammatory infection, and not to extension of carcinoma. Further discussion of this part of the subject follows under Radical Abdominal Hysterectomy in this chapter.

Extension of cancer to the vaginal walls, if slight, does not definitely contraindicate hysterectomy, provided the diseased portion of the vagina can be removed together with the nterus. Extensive involvement of the vagina and fixation of the uterus in surrounding cancer contraindicate the operation.

When the disease has passed beyond the hope of radical cure, but not beyond the limits of palliative operation, hysterectomy is performed sometimes for the temporary relief of symptoms; the benefits, however, usually are not sufficient to overbalance the dangers. The removal of a carcinomatous cervix alone by galvanocautery as a radical operation is usually inadequate; as a palliative measure in advanced carcinoma it may furnish temporary relief.

\section{HYSTERECTOMY.}

Hysterectomy for carcinoma nteri, whether performed by vaginal or abdominal section or by combined vaginal and abdominal section, should be complete; that is, it should completely remove at least the entire uterus. Up to the present time the most common operation has been ordinary vaginal hysterectomy-substantially the operation described in Chapter XXIII. on Pelvic Infection. 'This operation, although having almost no mortality, per se, has fallen under deserved criticism because of the discouraging percentage of recurrences at the site of the operation. Consequently, radical efforts have been made to modify it in such a way as to increase the percentage of permanent cures. Taking the operation as described in Chapter XXIII. as a point of departure, one must consider how much further it is practical to go in the removal of broad ligaments and pelvic and lumbar glands, how far traumatism and difficulty of technique may be increased, to what extent the duration of the operation may be prolonged without adding enongh immediate mortality to offset any possible advantage accruing from increased freedom from recurrence.

In estimating the merits of different operations, one must have in mind constantly the rule which applies to the removal of cancer in other regions; that is, complete removal of all apparently diseased tissue and of as wide a margin of adjacent tissue as prudence will permit; this rule is based upon the invariable tendency of cancer to follow lymph-channels into surrounding structures and to involve neighboring lymphatic glands. The above considerations lead to a discussion of the following subjects :

1. Paravaginal hysterectomy.

2. Radical abdominal hysterectony.

3. Ignihysterectomy. 


\section{Paravaginal Hysterectomy.}

Paravaginal hystercetomy, known as Schuchardt's ${ }^{1}$ operation, is especially adapted to cervical carcinoma, is the most radical of all vaginal operations, and is performed as follows : ${ }^{2}$

With the forefinger and the thumb of the left hand the operator seizes the posterior portion of the left labium, while an assistant seizes the parts in the middle line and puts them on the stretch. The operator then makes an incision between the two sets of fingers and, so far as possible, divides at one stroke in a forward direction the vaginal wall up to the left side of its insertion into the cervix uteri and downward and backward to the middle of the coccygeal region. By this incision he splits the whole vaginal tubc, the left labium, the paravaginal and pararectal tissues, the levator ani muscle, the coccygeal muscles, the cellular tissue of the ischiorectal fossa, as well as the skin of the perineum and of the lateral anal region down to the sacrum. The wound in the pararectal tissue is carried to the left only far enough to avoid the rectum and sphincter ani muscle-that is, about a finger's breadth from the middle line. The incision on the left side is more convenient for the right-handed operator, but when a portion of cancerous rectum must be removed with the cancerous vaginal wall, bilateral incisions become necessary.

After controlling the copious venous hemorrhage the surgeon catches the vagina as far as possible away from the cancer with vulsella, and below these vulsella makes a circular incision through the vagina, connecting at right angles with the paravaginal cut; he now separates the ragina by blunt dissection in an upward direction and sews the cuff thus formed over the portio vaginalis in order to protect the field of operation from uterine secretions, the sutures being left long to serve as guides in the following steps of the operation.

The effect of this paravaginal incision is surprising. In place of a vaginal tube we have before us a shallow excavation about an inch deep, at the bottom of which the parametria are seen in full extent and within easy reach.

The operator now separates the bladder in front, partly by scissors, partly by blunt dissection, clear to the pelvic cavity, and in the same manner opens into Douglas' space behind; then passing the left forefinger successively behind each parametrium, he draws it within easy reach and ligates it, together witl its broad ligament, as far from the uterus as possible, with strong catgut, and excises the uterus, together with the adjacent parametrium on each side of it.

In the removal of considerable portions of the parametria the regions of the ureters are invaded and the ureters themselves may have to be laid bare. Dissections in these parts involve great technical difficulties, and should be undertaken only by specially expert surgeons. In this connection the reader is referred to the original

1 The reader is referred to a masterly papcr read before the Amcrican Gynecological soclety ai Niagara Falls, 1905 , in which George Gellhorn, of St. Louis, discussed fully the various radical operations with special reference to paravaginal hysterectomy.

2 Adaptation from Gellhoru, Trans. Am. Gyn. Soc., 1905. 
literature, especially papers by Schuchardt, ${ }^{1}$ Schauta, ${ }^{2}$ Kroemer, ${ }^{3}$ Kundrat, ${ }^{4}$ Bovée, ${ }^{5}$ and Mackenrodt. ${ }^{6}$

The suggestion of Clark to make the operation with catheters previously passed into the ureters is nost practical. The vaginal portion of the wound is closed loosely by catgut, in such a way as to unite the anterior to the posterior peritoneal edges and to draw into it the eut ends of the broad ligaments. The cutaneous portion is closed more firmly, as in perineorrhaphy. A gauze drain, aceording to the special indication, may or may not be indicated. Union by first intention is usual.

The advantages claimed for this most radical of all vaginal operations are less immediate danger to life, in comparison with radical abdominal operations, and greater freedom from recurrence, in comparison with the ordinary vaginal operations. The relative value of Sehuchardt's operation will be considered later.

\section{Radical Abdominal Hysterectomy.}

The development of this operation in its various phases is inseparably connected with the labors of $H$. W. Freund, Rumpf, von Rosthorn, Ries, Schauta, Clark, Cullen, Werder, Russell, and others.

After preliminary curettage and cauterization of exposed surfaces in the vagina and after thorough disinfection of the vaginal and abdominal fields of incision, the ureters, according to Clark, should be catheterized, and the eatheters left in them as guides by which the ureters may be avoided during the operation.

The patient being in the Trendelenburg position, the abdomen is opened between the symphysis pubis and umbilieus and the humbar iliac and sacral glands, the pelvie ligaments, and the parametria are examined for more aceurate diagnosis of the disease. Wertheim, Ries, and others advocate the removal of glands with varying degrees of thoroughness in connection with the radical operation. Others, notably Clark and Schauta, take the reasonable ground that in very many cases the glands are not involved, and that in all eases the removal of them so fur inereases the primary danger of operation as to outbalance any possible freedom from recurrenee. The removal of glands may be disregarded, and for purposes of this deseription it is so decided.

"Special stress," as Clark ${ }^{7}$ wisely says, "should be laid upon the neeessity of this preliminary survey, for, as the question must now be viewed, it is useless to perform more than a simple abdominal operation if the higher groups of glands are involved, for in such eases we can only hope for a palliative effect from any operation. If, on the

\footnotetext{
K. Schuchardt. Centralblatt f. Chirurgie, 1893, No. 51.

K. Schuchardt. Ueber die paravaginale Methode der Extirpatio uteri und ihre Heilerfolge beim Uteruskrebs. Monatsschrift f. Geb. u. Gyn., 1901, xiii.

K. Schuchardt. Transaction of the German Gynecological Congress, 1901.

2 F. Schauta. Dic Operation des Gebaermutterkrebses mittelst des Schuehardt'schen Paravaginalschnittes. Ionatsschrift f. Geb. u. Gyn., 1902, xv.

F. Schanta. Die Berechtigung der vaginalen Totalexstirpation bei Gebaermutterkrebs. Monatsschrift f. Geb. u. Gyn., 1904, xix.

3 P. Kroemer. Die Lymphorgane der weib. Genitalien, etc. Archiv f. Gyn., 1904, Ixxiii.

4 . Kundrat. Ueber die Ausbreitung des Carcinoms in parametranen Gewebe bei Krebs des Collum Uteri. Archiv f. Gyn., Ixix, Heft 2.

5 . W. Bovée. The Treatment of Cancer of the Cervix Uteri in Advanced Stages. American Jiedicine, January $7,190 \overline{5}$.

6 A. Mackenrodt, Zentralblatt f. Gyn., 1905, No. 11.

7 Kelly-Nobie, volume i, p. 737 .
} 
other hand, preliminary examination of the pelvic structures has been negative so far as metastasis or wide intraligamentary extension is concerned, the scope of the local operation becomes radical."

The operation now will be continued as follows: ${ }^{1}$ 'The intestines are packed well back into the abdominal cavity with gauze bolsters, wrung out of hot salt solution. Painstaking care in this step renders the operation very much easier and guards against post-operative complications. The fundus is grasped by a heavy tenaculum forceps, and forcibly drawn upward to the opposite side from that upon which the operation is to begin. The peritoneum is snipped open, as suggested by Wertheim, begimning over the bifurcation of the common iliac artery and continuing down into the pelvis to the point where the ureters enter the bladder. The infundibulopelvic ligaments at the pelvic brim are next ligated doubly and cut between the ligatures.

The round ligament is separately ligated one inch from the cornu uteri and divided. The peritoneal incision is now carried around in front of the uterus, through the uterovesical reflection to the opposite side. The bladder is detached from the uterus with sponge pressure and the broad ligaments opened by the same means. With the uterus sharply drawn upward into the abdomen, detachment of the bladder from the cervix is completed, and the dissection carried well down into the paravaginal tissues. In this way the vagina is detached from its fixed points and appears as an isolated sheath. If the bougies are in place, or if the anatomic guides are well in mind, the ureters can most easily be located at the brim of the pelvis where they cross over the external iliac vessels. From this point they are traced downward to the points where they swing inward to enter the bladder. With the ureters isolated and pushed out of the way, the ligation of the uterine vessels becomes a relatively safe procedure. The vessels lifted up on the tip of the index-finger are doubly ligated well ont against the pelvic wall. The ligature is best applied with a curved aneurysmal ligature carrier. This step having been duplicated on the opposite side, the chief danger of hemorrhage and of injury to the ureters is largely overcome.

The broad ligaments are now cut away from their pelvic attachments, leaving the uterus, its appendages, and the upper portion of the vagina held in the pelvis by the uterosacral ligaments. The uterus is now pulled upward and forward against the symphysis pubis to facilitate the division of the recto-nterine reflection of peritoneum and the uterosacral ligaments. The peritoneum should be opened well back of the cervix. The uterosacral ligaments are ligated or clamped and divided as close to their sacral attachments as possible. Small bleeding vessels may be clamped and snbsequently ligated.

$\mathrm{U}_{\mathrm{p}}$ to this point the operation has followed the general plan of all radical operations, but here the best suggestion which Wertheim has offered becomes imperative. Instead of using imbricated ligatures around the circumference of the vagina, Wertheim has employed rightangled clamps with which to compress the walls of the vagina from above while it is excised. With traction forceps the uterus is pulled

1 Adaptation from Clark. Kelly-Noble, volume i, pp. 738-744. 
as high as possible into the abdomen, thus bringing a considerable portion of the vagina into view. Two clamps are applied to the isolated vaginal tube, one on one side and the other on the opposite side and overlapping the first. These clamps are quickly placed and permit the immediate excision of the uterus with the upper portion of the vagina. In the separation of the pelvic attachments of the uterus, and in eutting through the vagina, the cautery should invariably be employed, and after the excision of these structures the surrounding tissues, with due protection to the ureter, bladder, and rectum, should be thoroughly burned. This igniextirpation is the strongest possible guard against recurrence. While he believes the ligature should never be discarded, he recognizes without reservation the splendid value of the cautery in excising cancerous tissue. By the cautery method adjacent tissue which might escape the knife is removed. Extirpation being completed, the vagina is closed below the clamps, with either a running or an interrupted catgut suture, and the clamps are removed. If a gauze drain is employed, a small opening is left, through which the gauze projects into the vagina, from which it can be removed two days later. The vesical and rectal reflections of the peritoneum are brought together with a running catgut suture. The abdomen is closed without drain in the usual manner.

\section{Ignihysterectomy.}

It is highly probable that the great difficulty, tediousness, and excessive mortality of the operation just described will so far outbalance any possible increase of permanent cures due to it that the next generally recognized procedure in the radical surgery of uterine cancer will to a greater and greater extent limit the use of hemorrhagic incisions and ligatures, and in place of them substitute the bloodless and safer method of igniextirpation. The foregoing description of technique of radical abdominal hysterectomy includes somewhat extensive use of the thermocautery and thereby emphasizes the growing tendency of the radical operator to recognize the paramount value of this agent.

Igniextirpation of the entire uterus, with adjacent tissues, has been perfected by X. O. Werder, ${ }^{1}$ of Pittsburg. The technique of his operation, substantially as set forth by himself, is as follows:

1. Thorough curettage of the cancerous surfaces in the vagina and control of resulting hemorrhage by actual cauterization.

2. An incision entirely around the cervix as far as practicable from the affected area by means of the cautery knife at a dull heat, which prevents oozing and renders the wound dry. This incision is made while the uterus is drawn well down toward the vulva, and the vaginal walls protected from the hot knife by means of retractors. Dissection by the cautery knife is continued anteriorly between the bladder and uterus, the bladder being drawn firmly away from the uterus by means of a retractor until the peritoneum is reached but not opened. The cul-de-sac of Douglas is then entered by similar careful dissection, guided by the index-finger. The lateral vaginal attach1 Werder, X. o. Surgery, Gynecology, and Obstetrics, January, 1907. 
ments are then burned through. The vaginal wound is earefully inspected now and all surfuces not thoroughly blackened and charred ale gone over again by the dome-shaped cautery until thoroughly charred. The vagina is now packed lightly with gauze.

3. The abdomen having been prepared for laparotomy, a median incision is made between the symphysis and umbilicus, and the uterus seized and drawn into the opening by means of vulsellum forceps and earefully packed off from the surrounding peritoneal cavity by means of sponge pads.

4. The bladder peritoneum at the vesical reflection is now dissected off from side to side by means of the hot knife, and the anterior vaginal pouch is opened so as to expose the vaginal gauze packing. A Downs' electro-thermic cautery clamp is then applied to the right infundibulopelvic ligament and round ligament, the surrounding parts being carefiully protected by the shield and by additional pads. The tissues included in the clamp are then thoroughly treated by the galvanic heat until they appear as a thin white, horny ribbon. The clamp is then removed and this ribbon is cut through at its inner margin, and if free from bleeding is dropped. The other side is treated in the same way.

5 . The parametrium is seized on the least affected side with the eautery clamp and burned through, the bladder and ureter being carefully aroided. The horny ribbon thus formed is cut through and the remaining portion of the broad ligament and uterosacral ligament are treated in the same manner and dropped. One side of the uterus now being freed from all its attachments, the same treatment is repeated on the other side. If the technique has been good, the cauterized surfaces will be dry, no blood except from the preliminary cauterization having been lost.

6. The stump of the vagina now exposed is closed by means of a running eatgut suture, the bladder is brought over the sutured vaginal stump, and its peritoneum sutured to the rectum. Adjacent peritoneum is now stitched over each broad ligament stump.

7. The entire field of operation now being covered and protected against infection by peritoneum, and having no exposed surfaces, the abdomen is closed without drain in the usual manner.

History and Rationale of Igniextirpation.-Werder has based his operation upon the principles of the well-known Byrne operation of amputation of the cervix by the galvanocautery, extending it to complete hysterectomy. The statisties of Byrne ${ }^{1}$ are a matter of history. They show 369 cases of cervical cancer removed by galvanocautery, with no primary mortality, and 19 per cent. of permanent cures-a very remarkable result, even allowing for some possible errors of diagnosis which may have led to the inclusion of a few cases in which the presence of undeniable cancer remains umproved.

"Byme in all his writings on this subject emphasizes the importance of thorough and repeated cauterizations of the wound surfaces and edges from which cancerous material has been removed, regarding it as the best safeguard against the recurrence of the disease." $\mathrm{He}$

1 Transactions of the American Gynecological Society, volume xiv, 18s9. Same, 188. 
thinks that "there is hardly any doubt that the developmental activity of the cancer cells and germs, in certain stages and under certain conditions, may be arrested or permanently destroyed by a degree of heat nuch below that which would be detrimental or destructive to normal tissues"; he is certain that "the thermal agent exerts some strong modifying influence on pathologic processes much deeper than the surface actually cauterized, hence the importance of repeated applications, so that every spot suspected of contamination may be thoroughly charred."

Byrne's results, especially the freedom from local recurrences, would seem to justify the above conclusions. The most remarkable feature of this operation, when compared to other operative measures, is unquestionably the absence of vaginal recurrences. Byrue says: "I have never known an instance of relapse in which the disease has returned to the part from which it had originally been excised. I have repeatedly observed the reappearance in the fundus, ovaries, and some of the adjacent tissues, but I have never known a single instance in which the disease has reappeared on or very close to the cauterized surface from which the cervix had been removed by galvanocautery." This same immunity from local recurrence is claimed by other operators using the galvanocautery, such as Pawlick, Madden, and others, so that Byrne's experience seems by no means singular. The facts apparently bear out Byrne's assertion that the influence of the cautery extends beyond the actual field of operation, carrying destruction to cancerous elements deep into the tissues, and, therefore, doing much more radical work than can be accomplished by the use of the knife or scissors under similar conditions.

Byrne's experience covers 367 cases, extending over a period of twenty years, without a single operative death; 140 of these were carcinoma of the cervix; in 219 cases both cervix and body were involved, and in only 8 cases was the disease confined to the corpus uteri; 151 cases were lost sight of during the first year; there remained, therefore, 216 cases of which the subsequent history is known. Of these, 19 remained free from the disease from ten to eighteen years, 22 for five years or more, and 93 for two years or more. 'Thus 19 per cent. were free from recurrence for five years or more, and over 43 per cent. for two years and over. ${ }^{1}$

Advantages of Ignihysterectomy. - The advantages of this operation are numerous and convincing; they are:

a. Comparative simplicity of technique.

b. Comparative freedom from post-operative infection.

c. Comparative freedom from hemorrhage; most important in view of the fact that cancerous patients are anemic.

d. Surprisingly low percentage of immediate mortality, considering the radical nature of the operation.

e. A most encouraging percentage of permanent cures, especially when due allowance has been made for the much greater immediate mortality of paravaginal hysterectomy and radical abdominal hysterectomy by the methods of Schuchardt, Wertheim, and others.

1 Quoted from Werder, Kelly-Noble, volume i. 
Mortality of Hysterectomy for Carcinoma.-The mortality depends upon the character of the operation. Schuchardt's operation will show a mortality of at least 10 per eent. The radieal vaginal operation in the hands of expert operators will show an immediate mortality of from 10 to 20 per cent. The mortality with improved technique probably cannot be reduced below 10 per cent. The ordinary vaginal hysterectomy, as deseribed in Chapter XXIII., for pelvic inflammation, in properly seleeted eases shows in expert hands not over 1 per cent. of immediate mortality. Ignihystereetomy as performed by Werder is almost as safe.

Recurrence of Carcinoma after Hysterectomy.-The recurrence of cancer after hysterectomy is less frequent than after the removal of cancer from other parts of the body. Even in eancer of the breast, where by reason of the exposed position of the growth the diagnosis usually is made earlier than in the uterus, recurrence is mueh more frequent. This is true notwithstanding the common practice of thorough attempt at removal of the subclavian and axillary glands in connection with breast operations, and notwithstanding the fact that in the usual hystereetomy the parametric glands seldom are removed. The statistics of the best operators show freedom from the disease two or more years after vaginal hysterectomy by the ordinary method, as deseribed in Chapter XIII., in an appreciable number of eases.

The percentage, however, of permanent cures with any operation is enormously greater for eorporeal than for cervical cancer-placed by some observers as high as 75 per cent. for the former and as low as 5 per cent. for the latter.

Schuchardt's operation, which if thoroughly performed will, perhaps, show almost as high an immediate mortality as the radical raginal operation without removal of the glands, will be followed, doubtless, by a somewhat larger pereentage of reeurrences. It is more than possible that ignihysterectomy, which of all radical operations has inmeasurably the least primary mortality, will give almost if not quite as much freedoin from recurrence as can be accomplished by either of the other radical operations.

The Operation of Election.-In our present unsatisfactory state of knowledge we may summarize as follows:

1. The removal of glands is of questionable value as a protection against recurrence, is extra hazardous, and involves an immediate mortality which more than offsets any probable advantage. The difficulty and danger of removal of all the lymphatic glands in comnection with hysterectomy will be apparent from examination of the frontispiece; moreover, removal of the lymph-vessels, throngh which careinoma must travel to reach the glands, is impossible; therefore, if cancer has passed far beyond the uterus, removal of glands is of donbtful value.

2. If the parametria, and especially the glands, are demonstrably affected, a palliative operation only should be elected. See Palliative Treatment.

3. If the parametria and glands are not demonstrably affected, and, therefore, a radical operation gives hope of permanent cure, the clinice will be between radical abdominal operation without removal of glands, 
on the one hand, and ignilhysterectomy on the other. The latter procedure, in consideration of its lesser immediate mortality, and its encouraging freedom from recurrence, would appear, for the present at least, to offer the maximum advantages, both from the conservative and from the radical points of view. Radical abdominal hysterectomy could not remove the parametria and broad ligaments much more than half an inch nearer to the pelvic walls than ignihysterectomy. This increased removal would hardly offset the additional mortality of the former operation. Besides, the effect of the cautery may be destructive to cancer far beyond the charred section.

Early diagnosis must be urged as a necessary factor in the successful surgical treatment of cancer of the uterus.

\section{PALLIATIVE TREATMENT.}

Unfortunately, the onset of eancer of the uterus is so insidious that early symptoms-pain, hemorrhage, watery discharge-are overlooked or attributed to other causes; hence, the diagnosis usually is not made until too late for permanent cure by radical hysterectomy. In later stages, when the cancer has extended to the bladder or rectum, or has involved demonstrably the vagina, parametria, or glands, and especially when the uterus is fixed in carcinomatous infiltrate, with thickening of the broad ligaments, hysterectomy is permissible only as a palliative measure and is of questionable value; under these conditions the operation, if done at all, should be simply local hysterectomy, as set forth in Chapter XIII. Other palliative treatment which does not in volve the removal of the uterus is both local and systemic.

The purpose of local treatment is to check the exhaustive hemorrhages and discharge. This may be accomplished by sharp curettage of the more superficial, soft, ulcerating portion of the cancerous growth. Remember that the disease in advanced cases may have extended through vesical, rectal, or uterine walls, and that without care the bladder, bowel, or peritoneum may be opened. The redundant cancerous mass having been removed by the curette, the bleeding surfaces, that is, the exposed surfaces, should be charred over with the Paquelin or galvanic cantery.

The cancerous growth may be kept down and the fetid discharges at the same time deodorized by the application, every three or four days, of a saturated solution of iodine crystals in pure carbolic acid. This application is made best on small tampons. The healthy parts of the vagina may be protected by covering the mucosa with gauze pads during the application of the solution.

Deodorizing douches are useful to destroy the nauseating fetor of the discharges. Among the best of these are peroxide of hydrogen, a 2 per cent. solution of potassium permanganate, a weak solution of formalin, or liquor sodx chlorinata (one part to ten parts of water).

Hemorrhage is controlled best by the curette and cauterization, already dcseribed, but a sudden profuse hemorrhage may be checked by a douche of hot water, hot vinegar, or hot alum solution. Should 
the vaginal tampon be used, it will become intolerably offensive, and therefore should be removed every twenty-four hours. The erosions and excoriations of the external genitals and nates, which are cansed by the ichorous diseharges from above, may be relieved by frequent bathing and by the application of benzoated zinc oxide ointment.

The general treatment ineludes regulation of the bowels and kidneys, tonics, nutritions food, and mild exercise. Pain is a clear indieation for morphine or opium in quantity sufficient to give relief. Life will be limited to a few months; hence the danger of the opium habit is not significant. Numerous drugs, both for local and systemic use, have been lauded as cancer cures; they are, so far as their merits have been investigated, useless.

\section{ENDOTHELIOMA OF THE UTERUS.}

\section{Pathology, Diagnosis, and Treatment.}

Endothelioma is a very rare malignant new formation arising from the endothelium of blood-vessels, or of lymph-vessels, or of serous surfaces; it closely resembles carcinoma in gross appearance and clinical manifestations. The entire lumen of the vessel is distended with proliferating endothelium, which assumes a variety of shapes. The cells ustially form nests and strands similar to those of earcinoma. The diagnostie point is the origin, as stated above. The diagnosis can be made only by microseopical examination. The growth is found in the cervix and corpus uteri, and very rarely also in the ovary, Fallopian tube, and vagina. The treatment is the same as that of other malignant disease. 


\section{PLATE XVIII}

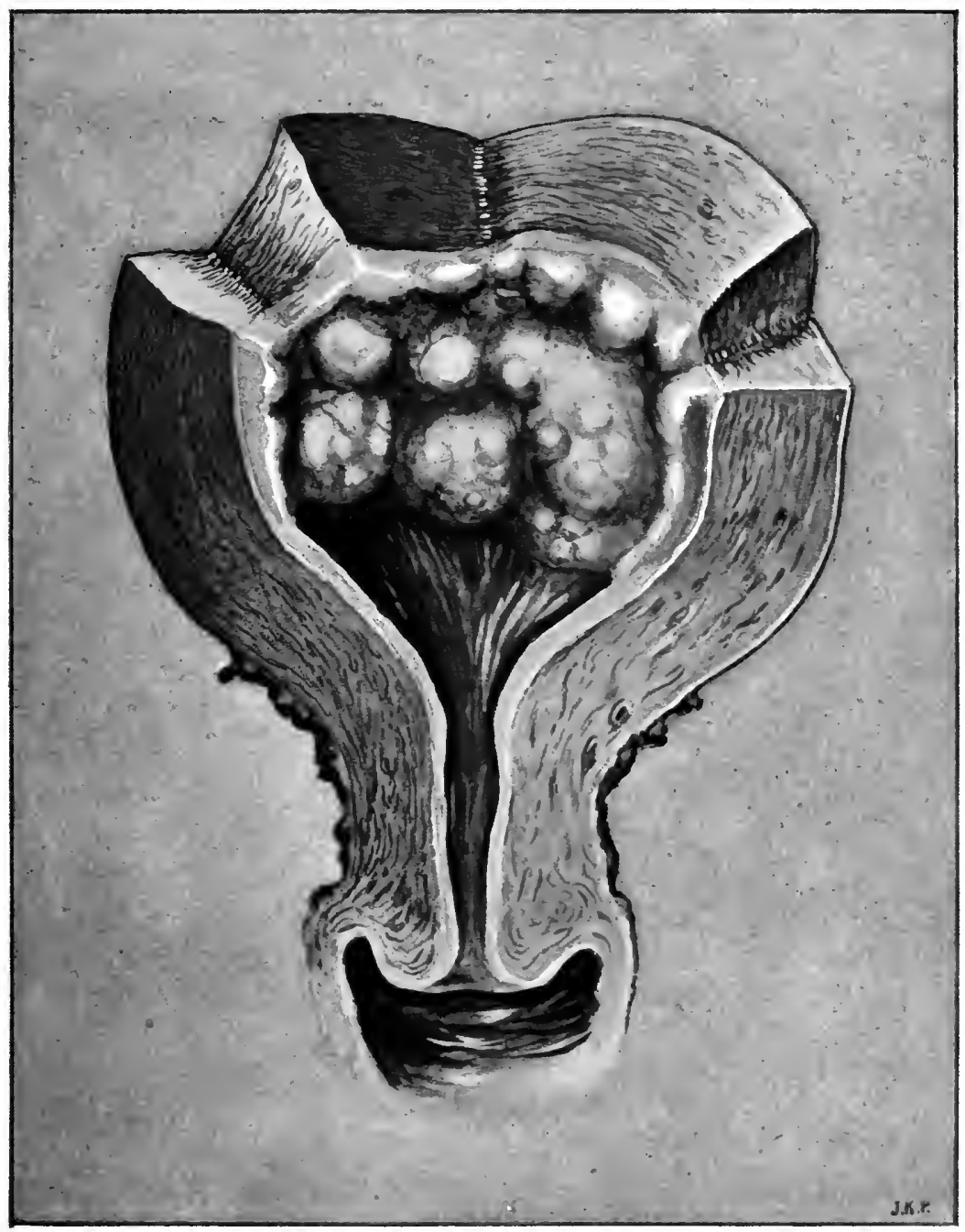

Sarcoma of the Body of the Uterus. 



\section{CHAPTER XXIX.}

\section{TUMORS OF THE UTERUS (CONTINUED).}

\section{SARCOMA OF THE UTERUS.}

SARCOMA is a malignant tumor belonging to the connective-tissue group, and, as compared with carcinoma, is of rare occurrence; it is more apt to occur during the period of sexual maturity, but has been observed as late as the age of seventy. Like carcinoma, it is more frequent between forty and sixty.

\section{Histogenesis and Etiology of Sarcoma.}

Sarcoma may develop from any of the following sources:

1. The interglandular connective tissue of the endometrinm.

2. The intermuscnlar connective tissue of the myometrium.

3. The walls of the blood-vessels.

4. The perivascular connective tissue.

5. The muscle-cells. ${ }^{1}$

6. Any of the structures of a uterine myoma.

The causes of sarcoma are unknown.

\section{Classification and Pathological Anatomy of Sarcoma.}

Sarcoma may be divided histologically into :

1. Small round-cell sarcoma.

2. Large round-cell sarcoma.

3. Spindle-cell sarcoma.

Sarcoma may be divided regionally into :

1. Sarcoma of the uterine mucosa.

2. Sarcoma of the entire uterus--diffuse sarcoma.

Sarcoma, especially when it has developed from myoma, may have many of the gross claracteristics of myoma-that is, it may be submucous, subserous, intramural, round, oblong, irregular, multinodular, soft, hard, circumscribed, or diffuse. The older pathologists designated these growths as "recurring fibroids"; they sometimes are called fibrosarcomata or interstitial sarcomata. They rarely are encapsulated, though usually well defined. The cells are round or spindle, and the spindle cells predominating are often so elongated as to appear like fibrous tissue, hence the name fibrosarcoma.

Diffuse sarcoma usually develops from the interglandular connective tissue of the endometrium. In this form the small round cell usually predominates over the spindle cell. The growth may be confined to separate areas, or may infiltrate the whole endometrium and rapidly involve the entire uterus and adjacent organs. It develops both in the endometrium and in the muscnlaris. Intra-uterine sar1 Whitridge Williams. American Journal of Obstetrics, 1894, vol. xxix. 
eoma may take the form of numerous soft medullary polypi. When removed by the curette, they have the gross appearance of carcinoma, and microseopically the small round cells of sarcoma may be difficult to distinguish fron the round cells of inflammation in endometritis.

There is a form of sarcoma which in gross appearance resembles grape-like bodies, and sometimes is called butyroides; it is extremely rare, usually originates in the cervix uteri, and has the form of cystlike masses resembling hydatid moles. The growth is composed mostly of round and spindle cells; it has been observed in the uteri of adult women and children, and in the vagina of children. ${ }^{1}$ The development is most rapid and malignant.

All sarcomata, especially the diffuse, are extremely vascular. The blood-vessels sometimes are dilated so enormonsly as to form cavernous spaces. The lymph-spaces may dilate into eystic eavities.

\section{Symptoms, Course, Diagnosis, and Prognosis of Sarcoma of the Uterus.}

The symptoms and course vary with different forms of sarcoma.

The interstitial spindle-eell sarcoma, formerly ealled recurring fibroid, is sometimes of slow growth. In exeeptional cases it may not destroy life for several years.

The diffuse, small round-cell sareoma, on the contrary, is ordinarily much more malignant than carcinoma; it often goes on to a fatal result in a few months. The small round-cell sarcoma is most malignant, the large round-cell less malignant, and the spindle-cell least malignant.

The tendency of sareoma is to seatter its nodules through the uterine walls, to penetrate the blood-vessels, to extend to the peritoneum, and to involve adjacent organs. The thickened, enlarged uterus, the bladder, and the neighboring intestines then are matted together in the sarcomatous disease and materially increase the size of the tumor. The disease is prone to send its emboli by the veins to the lungs, liver, kidney, spleen, and brain. These and other organs now may become rapidly involved in metastatic sarcoma. It is a peeuliarity of the disease that emboli pass through the veins to distant organs. In this respect it differs from carcinoma, which is apt to travel by the lymphatics and to be arrested by thrombic plugging at points much nearer to the original seat of the disease. The symptoms and clinical course of interstitial sareoma in the beginning may resemble those of myoma so closely as to make the elinical diagnosis wholly unreliable. The clinical course and physical signs of this variety in the later stages are almost identical with those of eancer.

Sarcomatous degeneration of myoma (spindle-eell sareoma) may be suspected :

1. When hemorrhages formerly attributed to the myoma suddenly inerease or, having ceased for a considerable time, begin again.

2. When after the onset of the menopause the tumor rapidly increases in size and becomes softer.

Peck. From Play fair's System of Gynecology. 


\section{PLATE XIX}

FIGURE

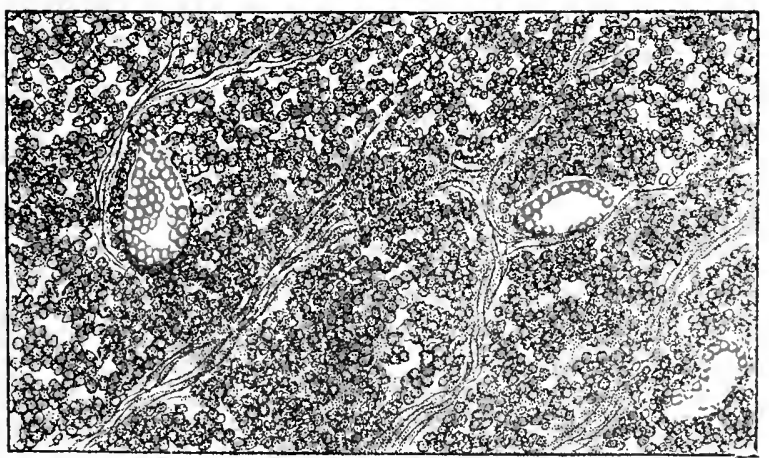

FIGURE 2

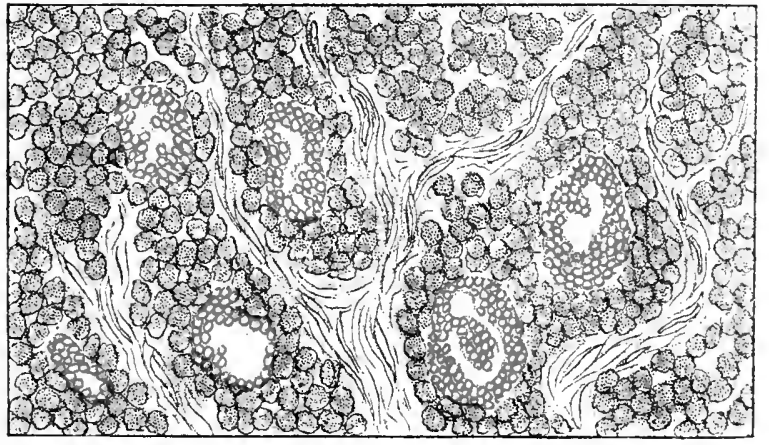

FIGURE 3

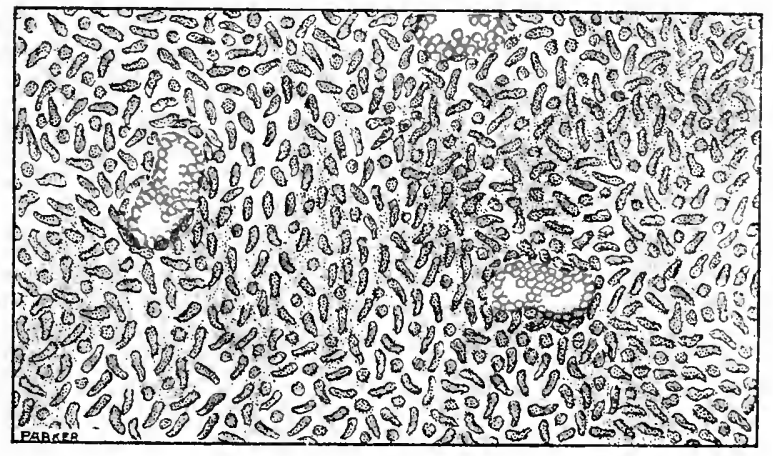


- 
3. When the growth returns after removal.

4. When ascites suddenly occurs.

5. When cachexia suddenly appears.

When sarcoma takes the place of myoma, the growth rapidly increases. Hitherto painless, it now causes intense suffering. Hemorrhages are increased and supplemented by watery, sanious discharges, which, after the onset of ulceration and gangrene, have an offensive odor. There is a facial expression of distress. The general depression is out of proportion to the anæmia and inanition. Pressure-symptoms, cachexia, and emaciation are pronounced, and of rapid development. If the sarcoma becomes polypoid, the pain from uterine contractions is spasmodic, and hemorrhages are frequent and prolonged.

The clinical symptoms and course are substantially the same as those of carcinoma, but with the following characteristics, especially in the round-cell varieties:

1. Ascites occurs earlier than in carcinoma.

2. Rectum and bladder disturbance not pronounced; these viscera not usually involved, as they are in carcinoma.

3. Cachexia earlier than in carcinoma.

4. Growth more rapid than in carcinoma.

5. Symptoms referable to metastasis more common because metastatic extension occurs much more frequently and extensively than in carcinoma.

Positive diagnosis is only possible by the microscope.

\section{Treatment of Sarcoma of the Uterus.}

The treatment may be either radical or palliative, and is the same as for carcinoma, viz., early hysterectomy, if possible. Unless all the growth can be removed, operation hastens death, for it opens the venous channels, and thereby favors metastasis. Palliative hysterectomy-a questionable remedy in carcinoma-is therefore useless in sarcoma. The operation is performed the same as for cancer. See Hysterectomy for Cancer.

\section{Explanation of Plate XIX.}

FIGURE 1.-Small round-cell sarcoma. Observe that the vessels are mere bloodspaces for the most part destitute of walls, that the cells are composed almost entirely of nuclei, and that they are of the same size as the red blood-corpuscles. Muscular elements are invaded and mostly destroyed by sarcoma. 250 diameters.

FIGURE 2.-Large round-cell sarcoma. The nuclei occupy almost the entire cells. The blood-spaces have no walls. The cells are five or six times as large as the red blood-corpuscles. The sarcoma has invaded and nearly destroyed the muscular tissine, the remainder of which is shown in the form of waving fibrous-like tissue running irregularly across the field. 250 diameters.

FIGURE 3.-Small spindle-cell sarcoma. Cells mostly composed of nuclei about twice as large as the red blood-corpuscles. Blood-spaces have no walls. 250 diameters. 


\section{CHAPTER XXX.}

\section{TUMORS OF THE UTERUS (CONTINUED).}

\section{DECIDUOMA MALIGNUM-CHORIO-EPITHELIOMA.}

\section{Etiology of Deciduoma Malignum.}

Deciduoma malignum is confined to the physiological period of maturity, occurs usually between the ages of twenty and thirty-five, and usually, perhaps always, is preceded by gestation or hydatidiform mole.

\section{Pathology of Deciduoma Malignum.}

Deciduoma malignum, sometimes designated as chorio-epithelioma, first described in 1889, ${ }^{1}$ differs radically from all other neoplasms; the essential element is a giant-cell imbedded in a sarcoma-like tissue.

The growth is of foetal origin, " ${ }^{2}$ the tissue entering into the formation of it being: 1. Syncytium-i.e., the uterine epithelial layer of the chorion. 2. The so-called cellular layer-layer of Langhiansi. e., the ectodermal epithelial layer of the chorion." The disease has been designated variously, according to the origin of the growth.

If from the decidua as:

Deciduoma adenomatosum.

Deciduoma carcinomatosum.

Deciduoma sarcomatosum.

If from the chorion as

Chorioma syncytiale.

Chorioma sarcomatosum.

Deciduoma malignum is circumscribed, reddish brown, and friable ; it presents secondary nodules, and commonly extends by early metastasis to the vagina, ovaries, broad ligaments, spleen, kidney, lungs, and brain.

The growth is rich in blood-supply, ${ }^{3}$ and the blood is confined within irregular spaces; the vessels have no adequate walls; hence the frequent hemorrhages. Necrotic changes take place early. Under the necrosed tissue is solid tumor, and under this is normal uterine tissue. In the development of the growth the normal constituents of the uterine wall are replaced rapidly by invasion of giant-cells and small round cells.

\section{Symptoms and Diagnosis of Deciduoma Malignum.}

Profuse hemorrhage occurring during the puerperium is the most characteristic symptom; it is intermittent and commonly so profuse

1 Sänger. A System of Gynecology, by Playfair.

2 Marchand. From Playfair's System of Gynecology.

$s$ H. M. Jones. "A Clinical and Pathological Study of Deciduoma Malignum." Johns Hopkins Hospital Reports, vol. vi. 
as to cause profound anæmia. Curettage gives but transient relief. The discharge is profuse, watery, and often foul-smelling. Hydatidlike moles may be discharged with added hemorrhage. The uterus rapidly enlarges. Metastasis takes place by the venous route, most commonly to the lungs, and gives rise to symptoms referable to the newly infected part. Anæmia, emaciation, and cachexia follow in rapid succession. Even though the disease be removed by early hysterectomy, death is usual in a few months.

Physical examination shows an enlarged uterus. Smooth, secondary nodules may be felt on the tubes. The uterine cavity may be open sufficiently to admit the finger. Digital exploration then will detect masses of soft tissue and coagula of blood usually localized in the uterine wall. The above history and symptoms are highly diagnostic. Microscopical examination of the scrapings will establish the diagnosis.

\section{Prognosis of Deciduoma Malignum.}

Seventy-eight per cent. of all cases terminate fatally within six months. Deciduoma malignum is the most malignant of all tumors.

\section{Treatment of Deciduoma Malignum.}

Prophylaxis requires thorough removal of all retained products of conception and prompt attention to post-abortum and puerperal hemorrhages. The surgical treatment is the same as that already laid down for carcinoma, viz., early hysterectomy. Some radical cures have been reported. 


\section{CHAPTER XXXI.}

\section{SOLID TUMORS OF THE OVARY.}

\section{Fibroma. Myoma. Sarcoma. Carcinoma. Benign Papilloma.}

SoLID tumors of the ovary, like some ovarian eysts (intraligamentous), may develop between the folds of the broad ligament. More commonly, however, solid ovarian tumors lie outside of the broad ligament. A pedicle connects the tumor with the uterus, and, as in ovarian cyst, is made up of the broad ligament, oviduct, ovarian ligament, and ovarian vessels. About 5 per cent. of all ovarian tumors are solid. Solid tumors, even though not malignant, are associated more commonly with ascites than are cystic tumors.

Fibromata are histologically identical with similar tumors in other organs. Peterson ${ }^{1}$ has collected from the literature 84 cases; he finds that fibromata are not so rare as formerly supposed, and that, contrary to the usual observation, they grow sometimes to large size; his paper is one of the most complete studies of the subject yet published.

Myomata are of rare occurrence. They are composed of the usual unstriped muscle-fibre and fibrous tissue-fibromyoma. The muscle-fibre is traceable from the ovary to the ovarian ligament. Distinction between the myoma and the spindle-cell sarcoma, even with the microscope, is not always easy. These tumors sometimes grow to large size.

Sarcomata are not of frequent occurrence. They sometimes occur, especially in childhood, in connection with dermoid cysts, or follow the removal of dermoids. The spindle-cell is more frequent than the round-cell variety. As in sarcoma elsewhere, rapid growth, speedy degeneration, and metastatic invasion of other organs characterize the disease. Both ovaries are apt to be involved primarily at the same time.

Carcinoma.-Little is known of primary carcinoma of the ovary. It arises in both ovaries at the same time. Secondary carcinoma may occur by extension from neighboring organs or by metastasis.

Benign Papillomata (solid warty growth) arise from the outer surface of the ovary; they may spread to the peritoneum and broad ligaments, and may undergo malignant changes. See Papillomatous Cysts in the following chapter.

Diagnosis of Solid Ovarian Tumors.-Solid ovarian tumors may be recognized one from the other, from cystic tumors, and from other pelvic conditions by the clinical history, conjoined manipulation, exploratory incision, and microscopical examination. The clinical history 


\section{PLATE XX}

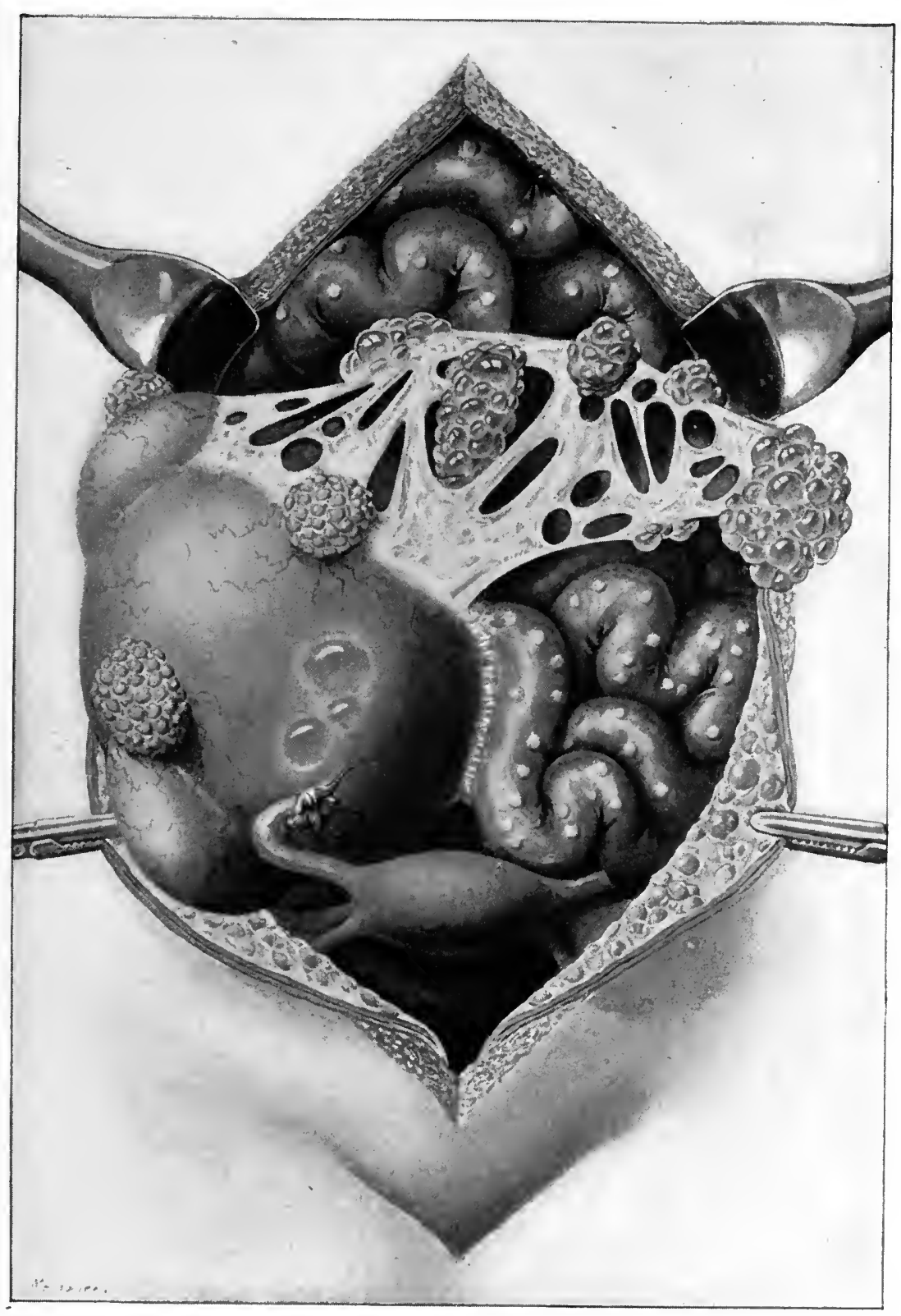

Solid Carcinoma of the Ovary, with Extension of the Disease to the Intestinal and Parietal Peritoneum and to the Omentum. 

often will suffice to separate the malignant from the benign growths. Conjoined examination will outline a tumor in the ovarian region, will show that it is not connected with the uterus, and will determine its size, form, mobility, and consistence. Exploratory incision will define further its physical characteristics and its exact relation to adjacent organs. The diagnosis is concluded by the microscope.

Figure 193.

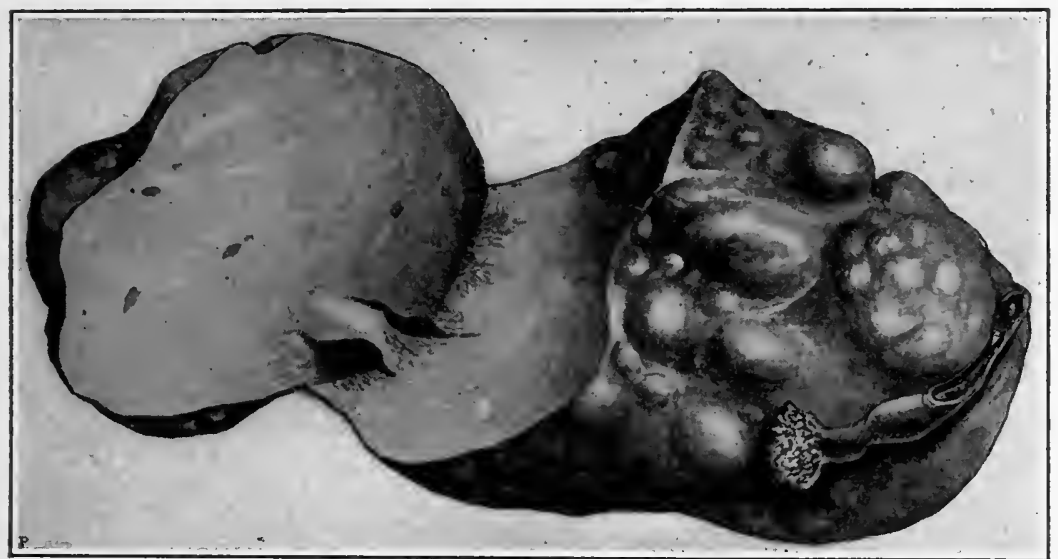

Solid sarcoma of the ovary, weight four pounds. Observe the smooth cut surface, chrracteristic of sarcoma, as compared with the uneven surface of a uterine myoma iu Chapter XXVI.
Author's case; ovariotomy; recovery.

Treatment of Solid Ovarian Tumors.-The treatment of very small benign growths without pressure-symptoms or functional disturbanec is expectant. That of large benign tumors or of malignant tumor's is early removal. The operation is the same as for cystic ovarian tumors. See Ovariotomy. 


\section{CHA P TER X X XII.}

\section{CLASSIFICATION, MODE OF DEVELOPMENT, AND PATH- OLOGY OF OVARIAN AND PAROVARIAN CYSTS, AND OVARIAN HYDROCELE.}

THE ovary consists of two parts :

1. The outer cortical or egg-bearing part, called the Cortex and containing the Graafian follicles.

2. The inner zone, which never contains follicles or ova; this part is in relation with the hilum of the ovary, is composed of fibrous tissue and traversed by numerous blood-vessels, and is called the vascular or medullary portion.

In relation with the ovary and situated in the broad ligament is a remuant of the Wolffian body, which has no physiological significance, called the Parovarium.

Cystic tumors may arise :

1. From any portion of the ovary-Ovarian Cysts.

2. From the parovarium-Parovarian Cysts.

In connnection with Figure 194, the student is referred specially to Embryology in Chapter XXXVII.

\section{OVARIAN CYSTS.}

\section{Mode of Development of Ovarian Cysts.}

Ovarian cysts may be :

1. Unilocular-monocysts.

2. Multilocular-polycysts.

1. Unilocular Ovarian Cysts may spring from the Graafian follicles, the corpus luteum, or may be simple cyst-adenomata. They are not very common. Some ovarian tumors classed as monocysts may have apparently a single cavity, but close examination usually will show numerous small loculi in their walls. Sometimes, as stated by Sutton, imperfect septa or bands running from one part of the cyst-wall to another are remnants of these walls and show that the cyst originally was multilocular. Parovarian cysts, which are usually unilocular, have often been mistaken for unilocular ovarian cysts; hence the impression that the latter are quite common. Unilocular cysts, although usually small, may have a capacity of several gallons.

2. Multilocular Ovarian Cysts are common, and will be described under multilocular cyst-adenomata.

Ovarian cysts are divided according to their origin or character as follows : 
1. Follicular eysts (degenerated Graafian follicles).

2. Corpus luteum cysts (unilocular).

3. Cyst-adenomata, subdivided as follows :

a. Simple.

b. Papillary.

4. Dermoid cysts.

Follicular cysts, corpus luteum cysts, and cyst-adenomata are epithelium neoplasms.

Figure 194.

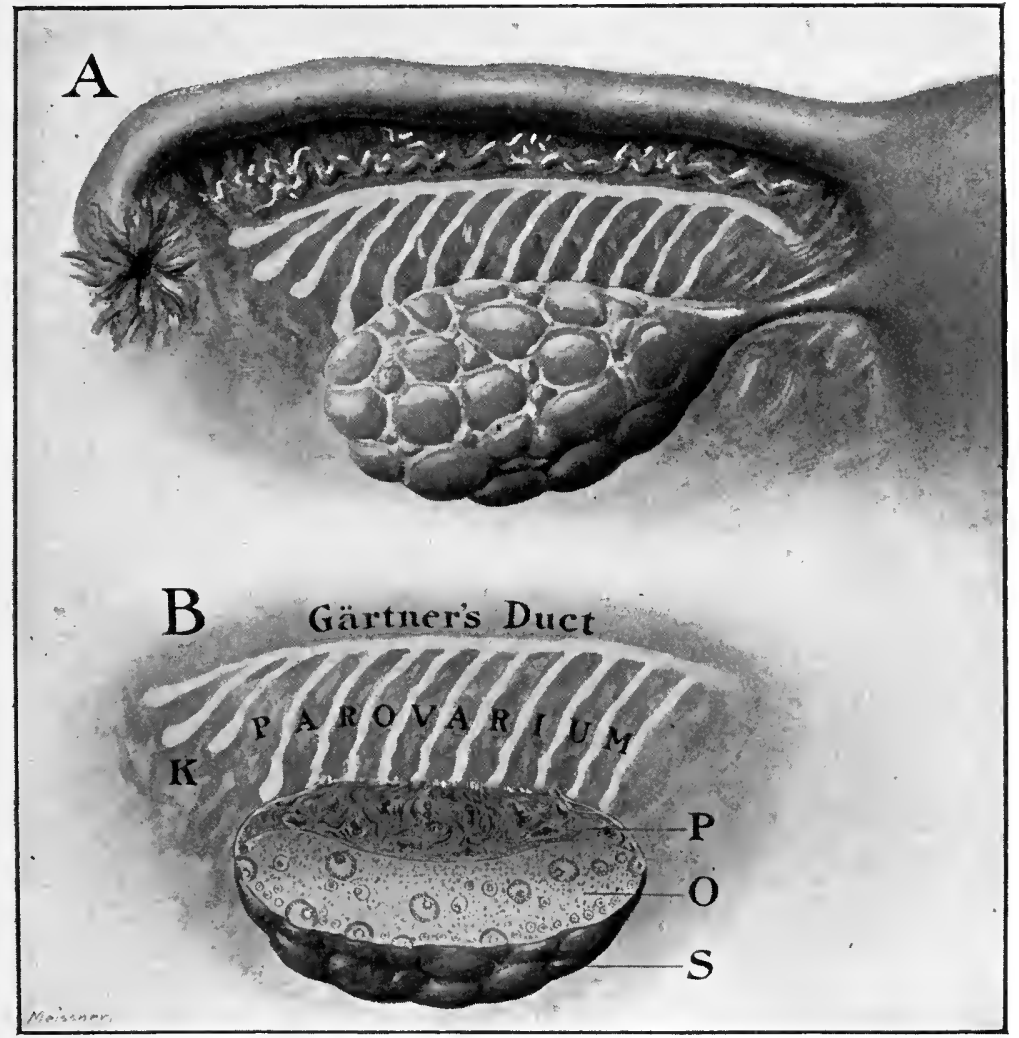

Cyst-producing region of the ovary and its surroundings :

A. Uterus, Fallopian tube, parovarium, anđ ovary.

B. Gaertner's duct (remnant of Wolffian duct), parovarium (remnant of Wolffian body or mesonephros), and ovary shown in section: $P$, vascular or medullary zone; o, oöphoron-this is the egg-bearing portion, sometimes called parenchymatous zone, sometimes the cortical portion; s, trce external surface of ovary; $\kappa$, Kobelt's tubes. Semi-diagrammatic.

\section{Follicular Ovarian Cysts.}

Follicular cysts due to inflammatory changes and shown in Figure 195 as microcystic degeneration of the ovary have been described in the chapter on Ovaritis. Putting aside the possible relations of these retention-cysts to ovarian tumors, we may describe a genuine follicular cystic neoplasm, which may cause considerable enlargement 
of the ovary. A number of follicles take on cystic degeneration, giving rise to many small adjacent cysts which may by the destruction of the partitions between them run together and form one or more larger eysts.

The wall of a follicular cyst is composed of three layers : an outer layer of endothelial cells or in small growths of cuboidal epithelium. A middle layer of white fibrous tissue containing blood-vessels and lymphatics, an inner layer of membrana granulosa like that of the Graafian follicle. This membrana granulosa is maintained until the cyst reaches the approximate size of an egg. In tumors the size of an orange the lining layer changes finally to a flat epithelium. In

Figure 195.

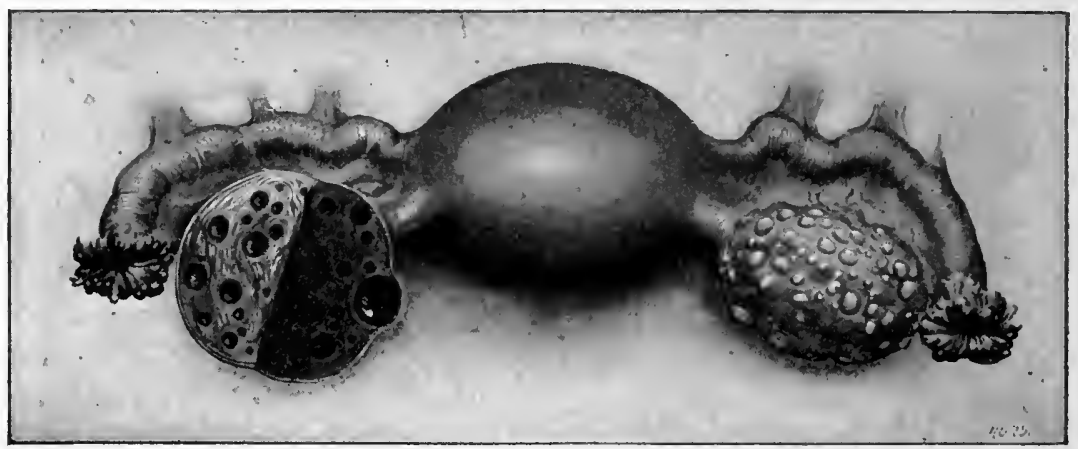

Microcystic degeneration of the ovary; the ovary to the right shows numerous small cysts scattered over the surface; these are Graafian follicles which have undergone cystic degeneration, and which it is sajd may take on excessive growth and develop into large tumors, or may remain as here represented; on the other side is shown a similar comdition of the ovary in section.

large cysts containing much more fluid the epithelium disappears by atrophy and gives way to fibrous tissue. The atrophic process is due to pressure of the fluid contents. The fluid is apt to be identical with mucus, a fact doubtless owing to the lining of epithelium.

\section{Corpus Luteum Cysts.}

The wall of a corpus luteum cyst sonetimes may be recognized by the wavy aspect and yellow color of its inner surface. The external layer is composed of connective-tissue stroma containing blood-and lymph-vessels around which at many points are gronps of round cells; these vessels pass perpendicularly to the middle layer which contains as a characteristic of this form of cyst; lutein cells. The inner layer shows cellular elements which are undergoing retrogressive changes and is not distinctly separahle from the cyst-contents. These cysts are usually small, rarely growing to the size of an orange. They commonly have a thick wall and contain a watery, yellow fluid which is said to get its color from the pigment of the lutein cells. Corpus luteum cysts are monocystic. 


\section{Cyst-adenomata.}

Cyst-adenomata, often called proliferating cysts, the nost common form of ovarian tumors, unlike follicular cysts, frequently grow to enormons size. They are characterized by excessive rapid growth and by having the capacity to secrete great quantities of fluid, and are divided into :

a. Simple cyst-adenomata, which have smooth inner walls.

$b$. Papillary cyst-adenomata, the inner walls of which produce warty growths.

a. Simple Cyst-adenomata may be unilocular or multilocular. As in follicular cysts the compartments between the cavities of a

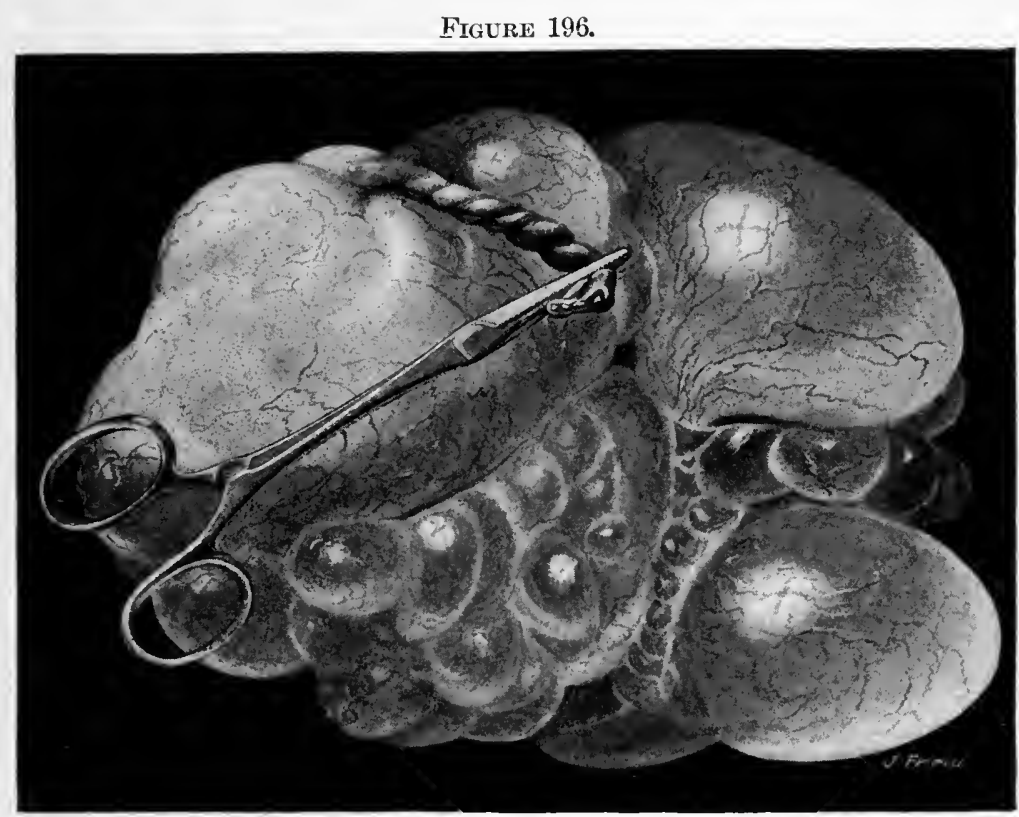

Cyst-adenoma. Multilocular ovarian cyst, sometimes called ovarian adenoma.

multilocular eyst may disappear and form a single cystic cavity, so that a multilocular cyst may become unilocular. The multilocular cyst usually has one large primary eavity and a number of smaller adjacent cavities, which have formed in the walls of the original cyst. The simple cystic development may increase until the tumor becomes cnormous, a burden to the patient and a destroyer of life.

In these simple adenomatous tumors the walls are traversed by large blood-vessels and are lined with glandular cylindrical epithelimin sometimes ciliated, which continuously produces large quantities of fluid and preserves its integrity in spite of the pressure cxerted upon it by the cyst-contents. Adenomatous ersts of the ovary have some tendency to become malignant; hence the importance of early removal. The walls are more or less thick according to the degree of 27 
distention, and are composed largely of connective tissue. Cyst-adenomata usually contain a thin clear sticky fluid which from admixture of blood may become red or chocolate colored, and in which chemical tests show the presence of pseudomucin. Figures 196 and 197 show two views of a cyst-adenoma, one of which is in section.

b. Papillary Cyst-adenomata.-Papillomatous cysts more commonly develop in the hilum or in the medullary portion of the ovary. They usually contain a clear, thin, yellowish fluid, which may be reddish from admixture of blood. In chemical properties and microseopical appearance this fluid resembles that of ordinary cysts. The

Frgure 197。

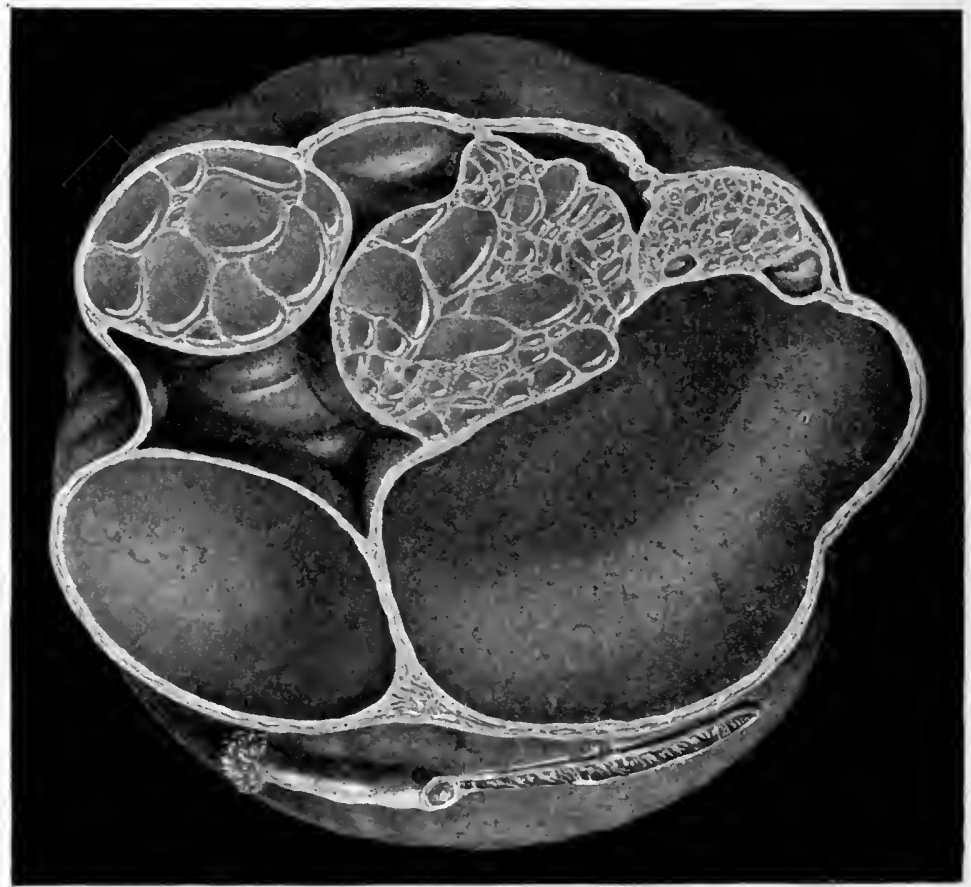

Cyst-adenoma. Multiloeular orarian cyst, sometimes called ovarian adenoma, in section; the larger cavity is primary; the smaller carities, secondary.

fluic may be mixed with a tenacious colloid formation. These cysts have the following characteristies :

They are rare hefore the twenty-fifth year, more common between the ages of thirty and fifty; seldon attain the size of ordinary ovarian eysts; are usnally nuilocular and frequently develop between the lavers of the mesosilpinx. With increased growth they may separate the layers of the broad ligament and foree their way between them to the lateral walls of the uterus, and will then feel on digital touch like an outgrowth from the uterus. They present, especially on the inside, in variable quantity, warty or papillomatous growths, which histolonically are the same as warty growth in other parts of the 
body, and which may penetrate the cyst-wall to the peritoneum and to adjacent organs.

The cyst-wall is composed of the usual fibrous tissue, which has an inner lining of eylindrieal epithelinm. The souree of this epithelium is not definitely known. It is thought by some to be from remnants of epithelium from the Wolffian body. At any rate it has the power of producing a most abundant warty growth.

The characteristic of papillomatous eystic tumors is the warty growth, which proliferates rapidly, bleeds freely on manipulation, is usually soft and friable, varies in quantity from that of the smallest wart to that of an orange, may be either sessile or pednnenlated, and according to the variable blood-supply, pale or pink. These papillomatous elements may so increase in quantity as to foree their way by rupture or perforation through the eyst-wall, spread over the outside,

Figure 198.

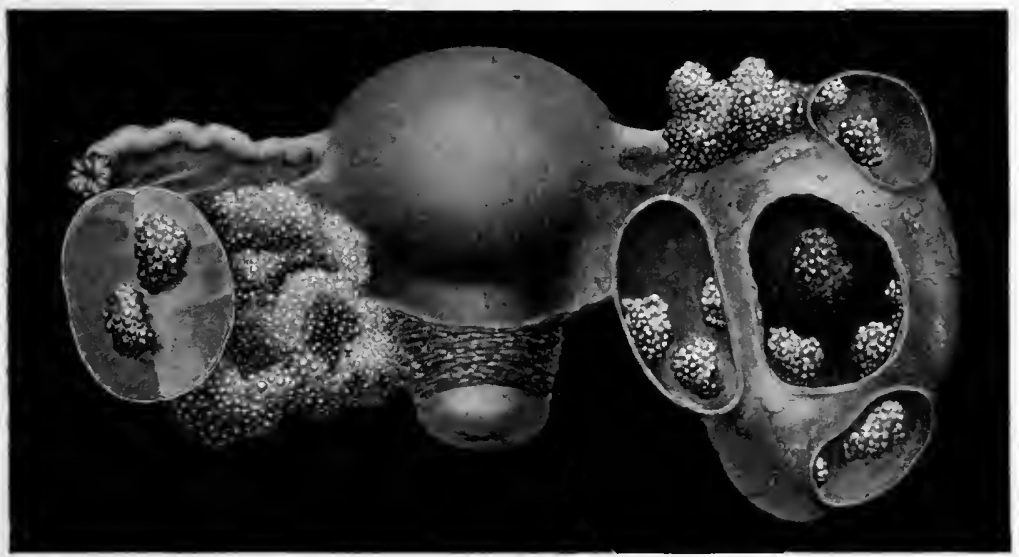

Papillomatous ovarian disease. On the right side is a cyst from the vascular zone of the ovary in the wall of this cyst have developed three secondary cysts, which are shown in section and which contain warty growths; observe also the warty growths both on the outside and inside of the cyst; to the left is a sliperficial papilloma of the ovary, which lies between the ovary and the uterus. Papillomatoug disease on the inside of this ovary is also shown in section.

and affect the adjacent peritonenm. Extension of the disease is by continuity of tissue or surface, not by way of the vessels. Warty ovarian cysts may be associated with dermoids, and oceasionally with sarcoma of the ovary. 'Tapping of the cyst is contraindicated, for if fluid eseapes into the abdominal cavity, the peritoneum may be infected; hence in removal care should be used to prevent the escape of fluid.

It should be noted in this connection that other papillomatous tumors found in the ovary and broad ligaments have not developed from the medullary portion or hilum of the orary; these eysts, according to Sutton, differ as follows from the warty cysts just teseribed :

1. They may be in any part of the ovary.

2. They are usually multiple. 
3. The warts are of almost eartilaginous hardness.

4. They are associated frequently with uterine myomata.

Clinically speaking, papilloma of the uterine appendages, whether solid or cystic, is on the border line between benign and malignant growths. Frequent tappings extending over a period of several years have been recorded in connection with inoperable cases. The stronger the malignant tendency, the nore pronounced-the ascites. Papillomata may give rise to great embarrassment in the removal of the tumor, for if any of the warty growths are left behind they are apt to spread rapidly and render the operation useless. On the contrary, if they are all removed the ascites may be expected to disappear and the patient to become permanently well. Even in the cases of extensive warty development, if there is hope of complete removal, the radical operation, even to the extent of combined ovariotomy and hysterectomy, should be attempted.

\section{Dermoid Ovarian Cysts.}

Dermoid ovarian eysts are distinguished from follieular cysts and cyst-adenomata by the inner lining of the cyst-wall, which is made up of squamous epithelium and papillæ like external skin. The cystwall contains an outer layer of connective tissue. Dermoid cysts are found not only in the ovary but also in various other parts of the body. The quantity of dermoid elements is variable. Cutaneous structures may line an entire cyst or may be confined to small isolated areas. Sometimes dermoid elements are contained in a single small compartment of a large multilocular cyst-adenoma and the dermoid character of the growth may then be overlooked. Two classes are recognized :

1. Simple dermoid cysts.

2. Conplicated dermoid cysts-teratomata.

Simple Dermoid Cysts.- The simple dermoid eyst has a very distinct inner lining of integument, consisting of flat epithelium, papillæ, and sabaceous glands. The cyst-wall and the cyst-cavity contains dermoid elements, such as hair, skin, nails, sebaceous matter, and a yellowish fat. As a rule, these tumors do not grow to the great size of cyst-adenomata. The hair is sometimes present in great abundance, and may be matted together in the form of a round ball the size of an orange. According to Sutton, the color is variable, does not necessarily correspond with that of the head of the patient, and in aged people may be grav or may have been shed, leaving the wall of the eyst bald. Extensive involvement of both ovaries in dermoid cystic disease, even though little normal ovarian tissue remains, does not positively render the woman sterile. In one case, reported by Cullingworth, the patient, at the age of thirty-nine, had had twelve children, the last being three months old at the time of the removal of the two dermoid ovaries.

Dermoid tumors occur at all ages, from infancy to extreme senility. They occasionally are found in children, and are not uncommon in young women. Unlike other forms of ovarian cysts which destroy 
life in three or four years, simple dermoids may exist for a lifetime and give little or no inconvenience. They have been found postmortem in aged women, who may have had them from the period of sexual maturity and never been aware of their presence. Like other eysts, however, they may at any time undergo suppurative malignant and other degenerative changes and therefore become dangerous.

The fluid content of a pronounced dermoid cyst is an oily fat, which is fluid at the temperature of the body, but at a lower temperature, semisolid. The fatty contents are very irritating to the peritoneum, and when a eyst breaks into the peritoneal eavity the epithelial

\section{Figure 199.}

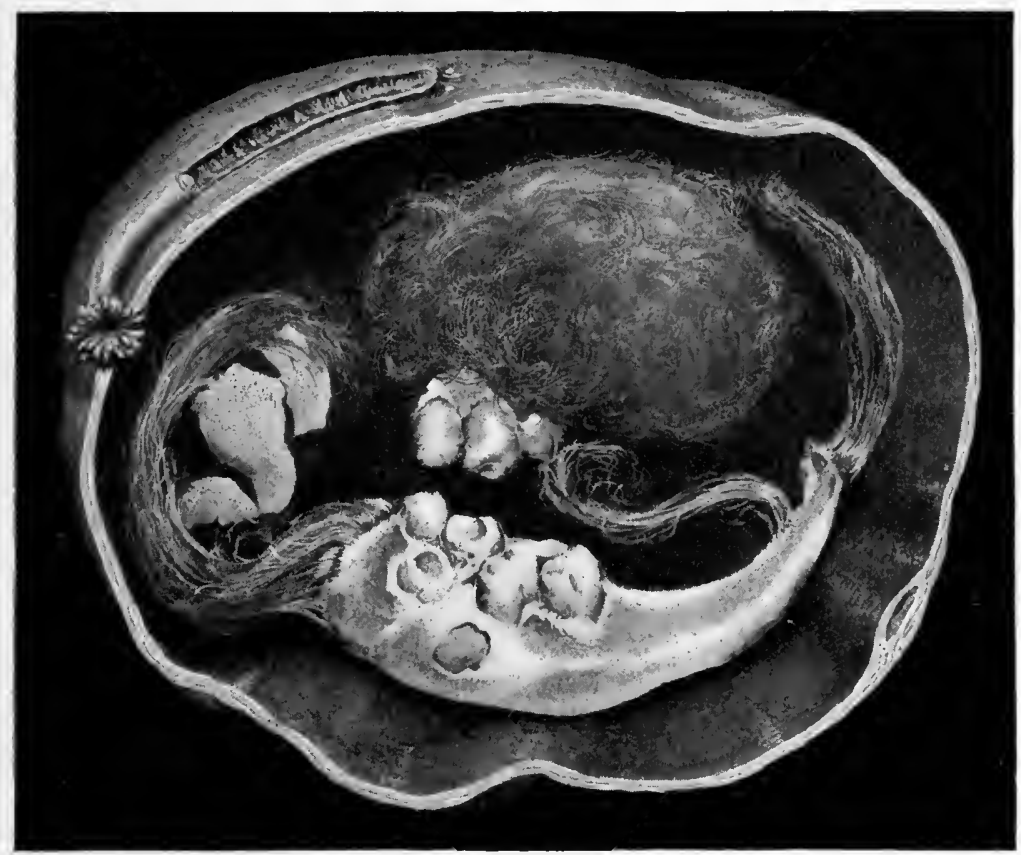

Dermoid ovarian cyst in section, showing inside of cyst-cavity, which contains a Inwer jaw and a fragment of another jaw, with teeth, small fragments of bone, and considerable hair; the upper mass of hair is in the shape of a ball, and is held together by the fatty contents of the cyst, which, at the temperature of the body, is liguid, but bccames solid upon exposure to the ordinary temperature of the air, that is, about $70^{\circ} \mathrm{F}$.

elements of it may engraft themselves upon the peritoneum and give rise to secondary growths.

2. Complicated Dermoid Cysts (Teratomata).-In addition to the dermoid elements mentioned above, dermoid eysts may contain other struetures of the body, such as bones, teeth, fragments of the brain, musele, and eartilage, even an entire finger has been observed. Such tumors are apt to be of the solid rather than of the evstic variety, and are called teratomata. The presence of bone and teeth does not characterize them as especially inclined to malignant degeneration, but when numerous other embryonal structures of the differ- 
ent layers of the blastoderm, brain, muscle, nerve, cartilage, fat, and bone are thrown together throughout the tumor in a confised mass the growth should be regarded as having a decided malignant tendeney. Teratomata have been classified as simple growths, which contain dermoid elements, teeth and bone, and as complicated growths which contain the other structures above mentioned.

\section{Formation of Pedicle of an Ovarian Cyst.}

Most ovarian cysts are pedunculated, the pedicle being made up of structures which connect the cyst with the uterus, broad ligament, round ligament, Fallopian tube, ovarian artery, and ovarian vein.

\section{PAROVARIAN CYSTS.}

The parovarium from which parovarian cysts spring is the remnant of the Wolffian body (primitive kidney) and has no knowu physiological significance. The epirlidymis and vasa efferentia in the male also spring

Figure 200.

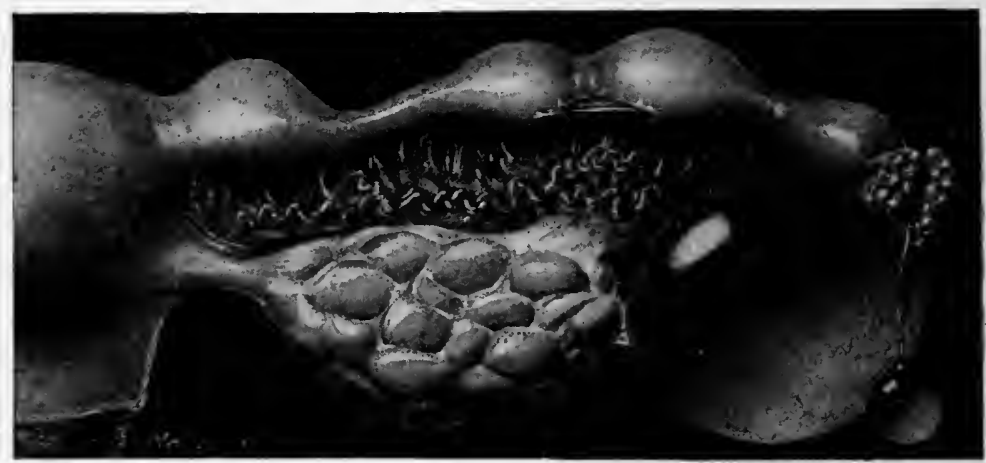

Small parovarian cyst. This cyst has sprung from the parovarium, and is therefore entirely distinct from the ovary; to the right is the hydatid of Morgagni suspended from a long, slender pedicle, which is attached to one of the fimbriated extremities of the Fallopian tube. The hydatid of Morgangi has been known to grow to the size of a small orange, and it then has the same general appearance as the parovarian cyst, but is distinguished from it by the fact that it springs from the extremity of the Fallopian tube. The Fallopian tube shows numerous points of expansion and constriction, one of them being at the isthmus; this is known as salpingitis isthmica nodosa, common in gonorrhœal salpingitis. Myoma and adenomyoma of the tube present a gross appearance similar to that of salpingitis isthmica nodosa. This condition of the tube is rarely found in connection with cysts of the parovarium.

from the Wolffian body and are the homologue of the parovarium. If the broad ligament is stretched and held up to the light, a series of small tubules will be seen radiating from the ovary and joining at right angles a longitudinal tube. The tubules are the parovarium. See Figure 194. They are of two kinds: 1. The vertical tubules. 2. The outer tubules, free at one end-Kobelt's tubes. All these tubules join a longitudinal tube-Gaertuer's duct. This duct is the homologue of the vas deferens in the male; occasionally it may be traced downward to the vagina. The parovarium lies between the folds of the mesosalpinx. 
The little tubules of Kobelt very often are distended by their fluid contents into cysts, usually not larger than a pea. These ersts, which have little or no significance, frequently are confounded witl the hydatid of Morgagni. Figure 200. A distended vertical tubule may become separated and form a peduneulated eyst. This may rupture, discharge its contents into the abdominal eavity, and become obliterated. The remnant of the eyst-wall then presents a fringe-like appearance. See Chapter XXXVII.

Figure 201.

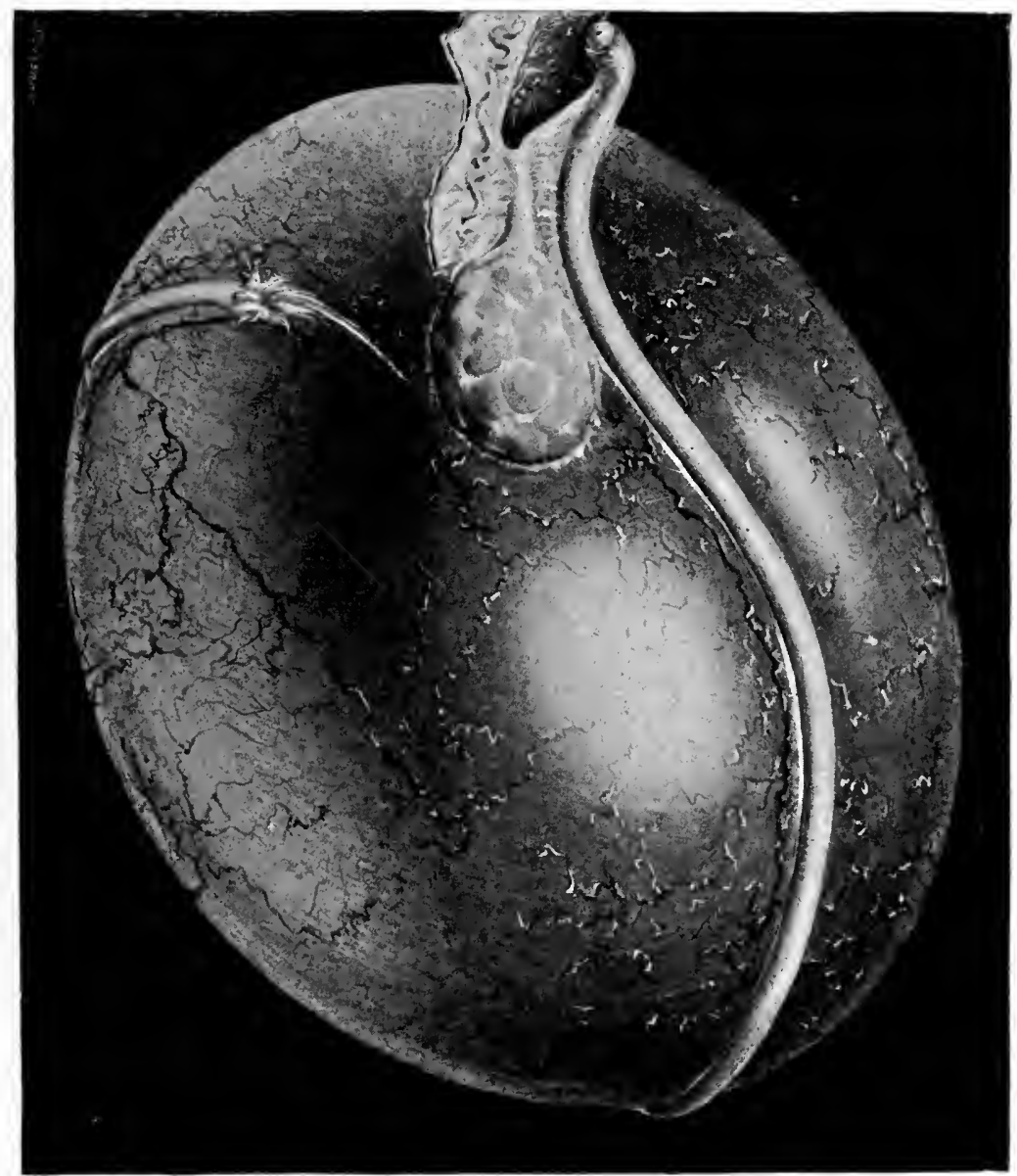

Parovarian cyst. Observe the ovary separate from the cyst and the long, stretened out Fallopian tube which surrounds the cyst-wall.

The usual parovarian eyst is unilocular, that is, it springs from a single vertical tubule, and most commonly develops without a pediele, and remains between the layers of the mesosalpinx. As it grows larger it may foree its way between the layers of the broad ligament and lies in close relation with the uterus. The Fallopian tube, with its 
fimbriated extremity attached to the ovary and its uterine end to the uterus, is stretched over the enlarging eyst-wall, and the tube in this way is often enormously clongatea.

The walls of small cysts are usually quite thin and transparent; when larger they become thick, opaque, pearly-like, and of conjunctival blue color. The lining of the small cysts preserves the columnar epithelium of the tubule; in larger cysts the epithelium becomes flattened; in the largest eysts the atrophic influence of pressure is so great as entirely to destroy the epithelium.

Figure 202.

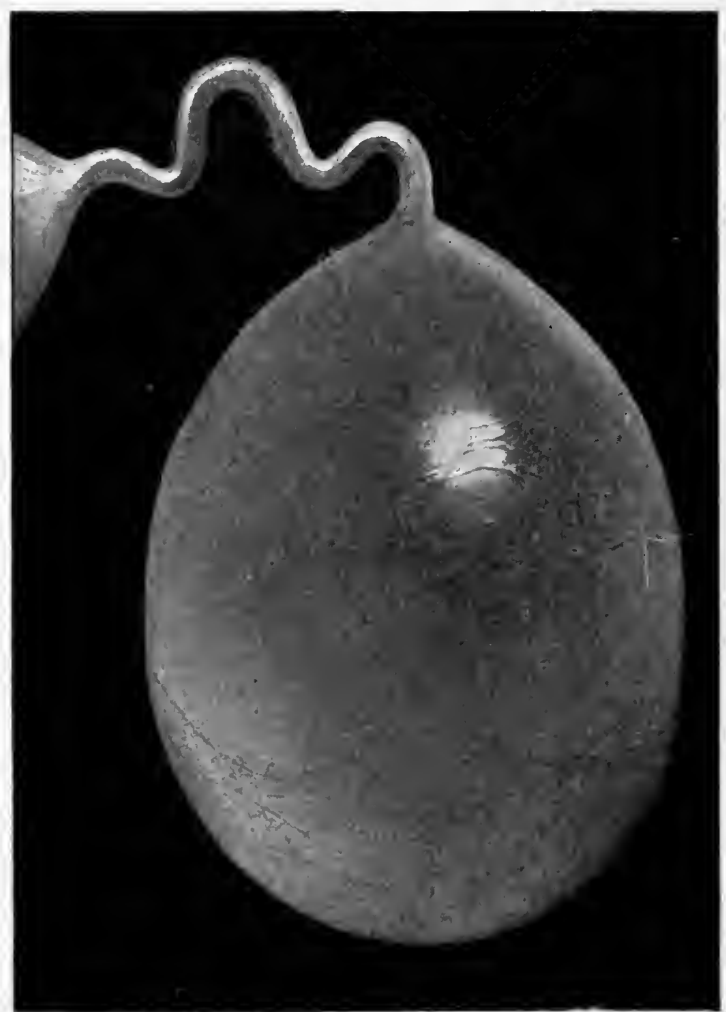
Ovarian hydrocele, natural size. The tortuous, retort-shaped Fallopian tube connects the
tumor with the uterus.

Unlike the ovarian cyst, which is a diseased ovary, the parovarian cyst usually has a normal ovary attached to the side of it. The fluid is almost always clear and colorless, like spring-water; the nitricacid and heat tests may show a trace of albumin. The specific gravity is usually nuch less than 1010. The reaction is faintly acid. See Tabular Diagnosis between Parovarian and Ovarian Cysts, in the following chapter. Adleesions rarely form about these cysts. The peritoneal covering is stripped off easily, and the cyst, therefore, easily enucleated. 
The parovarium does not often take on demonstrable eystic disease before the age of puberty, the more common age for development being from eighteen to thirty-five. These eysts do not tend to rapid degeneration, and therefore may be carried for years with little or no danger. Rupture or tapping sometimes is followed by obliteration and cure.

Cyst of the Broad Ligament is a name reserved by many to designate parovarian cysts. Various other cysts, however, also develop in the broad ligament. The name, therefore, has no definite significance beyond the fact that it designates a eyst situated between the layers of the ligament. Such a eyst may originate in the ovary and gradually

FIGURE 203.

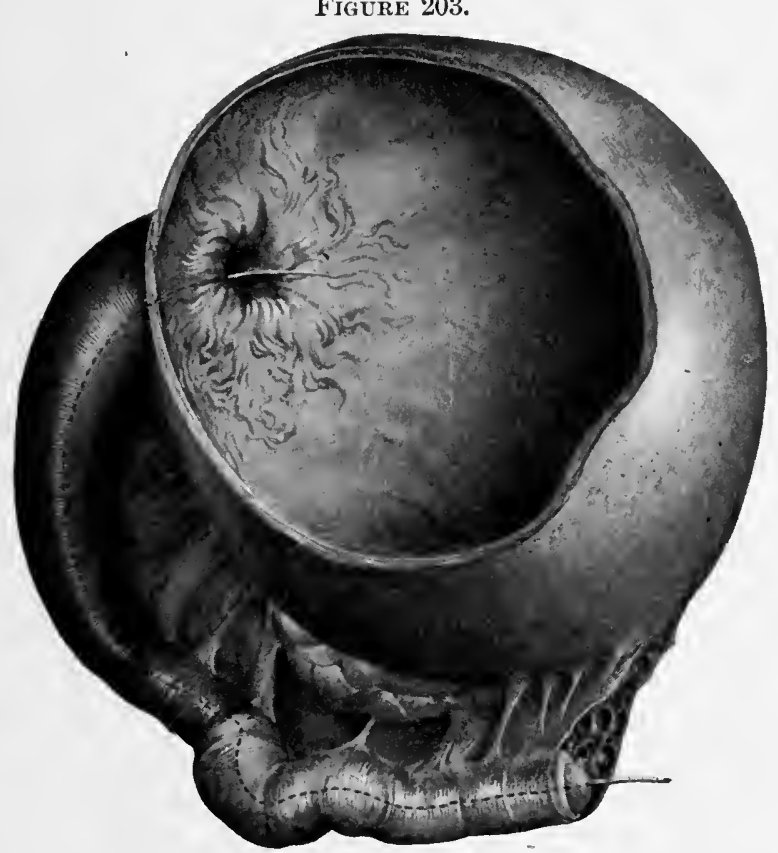

Tubo-ovarian cyst. The genesis of this cyst is set forth in Chapter XXl. Sometimes the sac of a tubo-ovarian cyst suppurates; it is then known as a tubo-ovarian abscess.

foree its way between the folds of the broad ligament. It not uncommonly originates near the hilum of the ovary, and the great majority of broad ligament eysts are either of this sort or parovarian. When they are of ovarian origin they are called intraligamentous ovarian cysts.

The Hydatid of Morgagni may grow to such considerable size as to be a mechanical irritant and require removal. 


\section{OVARIAN HYDROCELE.}

Ovarian hydrocele is a rare and curious disease, in which the dilated Fallopian tube communicates by its abclominal opening with the cavity of a cyst. The opening is usually large and circular. According to Bland Sutton, ${ }^{1}$ the formation of the cyst is analogous to that of hydrocele in the male. He gives evidence to show that it arises in a tunic of peritoneum, which sometimes invests the ovary as the tunica vaginalis covers the testis.

Ovarian hydrocele may suppurate, and may then easily be confounded with a tubo-ovarian abscess. The treatment is ovariotomy.

Ovarian hydrocele has been confounded hitherto with tubo-ovarian cyst. The distinctions between these two eysts, made by Bland Sutton, are shown in the following tabular statement:

\section{Ovarian hydrocele.}

1. Salpingitis has nothing to do with the cause, although it may be present as a complication.

2. The opening between the tube and sac is large and round or oval, and is the dilated $\mathrm{ab}$ dominal opening of the tube.

3. The tube, not large, is usually tortuous, like the worm of a retort.

4. There is apt to be an intermitting discharge of fluid from the tube through the uterus.-hydrops tubæ profluens.

\section{Tubo-ovarian cyst.}

1. Salpingitis is a cause of the communication between the tube and ovarian cyst. See Chapter XXI.

2. The opening is variable in size, and usually does not correspond to the abdominal ostium; if the cyst is purulent-i. $\epsilon_{\text {., if it is a }}$ tubo-ovarian abscess-the opening is usually sinall.

3. The tube is usually larger and not tortuous.

4. The intermitting discharge-8alpingitis profuens-not common. 


\section{CHA P'TER XXXIII.}

\section{SECONDARY CHANGES - SYMIPTOMATOLOGY-DIAGNOSIS, PROGNOSIS, AND DIFFERENTIAL DIAGNOSIS OF OVARIAN AND PAROVARIAN CYSTS.}

\section{SECONDARY CHANGES.}

THE principal secondary changes in ovarian and parovarian cysts are :

1. Infection.

2. Twisting of the pedicle.

3. Rupture of the cyst.

In addition to the above secondary changes may be mentioned the following degenerative changes in the cyst-wall :

1. Fatty degeneration.

2. Calcareous degeneration.

3. Myxomatous degeneration.

4. Malignant degeneration (sarcoma and carcinoma).

Changes in the fluid contents of a cyst may occur as follows : The fluid naturally contained in an ovarian cyst is usually transparent, clear, of a light-straw color, and of a specific gravity varying from 1010 to 1050 . In the progress of the disease secondary changes occur which make the widest variation in the physical properties of the fluid. This variation is caused by the admixture of blood, pus, fat, epithelial cells, cholesterin, and chemical changes. The fluid, therefore, may be thick, thin, dark, light, clear, muddy, or chocolate-colored. Different fluids may be present in the different compartments of the same cyst.

\section{Infection.}

The sources of infection from which bacteria may reach the cyst are the adjacent organs-viz., an adherent Fallopian tube, urinary bladder, intestine, the blood. Formerly, when tapping and aspiration were frequent and asepsis was disregarded, infection was introduced frequently by the puncture. Small cysts that remain fixed in the pelvis in close relations with the pelvic viscera are more subject to infection than the large growths that fill the abdomen. When adhesions occur in large cysts they are usually stronger and more extensive in the pelvis than in the abdomen.

The Fallopian tube is doubtless the greatest carrier of infection. This may be inferred fiom the fact that when an infected cyst becomes adherent to adjacent organs the strongest adhesions are usually where infection, if it came through the tube, would first reach the cyst-viz., about the abdominal end of the tube. 'The inference is 
strengthened by the almost constant presence of salpingitis in connection with infection of the eyst-wall. Inflammation and consequent adhesions from this source are not, however, confined to the neighborhood of the tube; they may extend indefinitely over the tumor, ghuing it to any adjacent peritoneal surface, viseeral or parietal, and the infection may even penetrate the cyst-wall.

The intestine and bladder, if inflamed, are prolific sources of infection. The inflamed gut readily adheres to the cyst-wall, and becomes softened by the inflammatory process or thin from the atrophic results of pressure. Germ-bearing gas, according to Sutton, may pass into the cyst and set up suppurative inflammation of the sac, or the adherent gut and sac-walls may become perforated; the sac-contents will then escape by way of the bowel. Extensive infection of a cyst may be traceable in some cases to an inflamed adherent vermiform appendix.

Suppuration.-Inflammation of the cyst may go on to suppuration and to the formation of extensive adhesions. In acute suppuration the symptoms rapidly become grave; they are:

1. Sudden enlargement of the tumor.

2. Severe pain and tenderness.

3. Rapid and weak pulse.

4. High temperature and exhaustion.

Aeute nephritis, with albuminuria, is a frequent complication. In some cases putrefaction leads to the formation of gases in the cyst, when a tympanitic note will be elicited by percussion over the tumor. In rare cases rupture of the sac and the discharge of its purulent contents through the intestine or some other viscus may avert the otherwise fatal result. Unsually the only hope lies in prompt ovariotomy.

Adhesions are among the most constant results of inflammation. Formerly they were the bete noir of the surgeon. Now, with improved technique, tumors that formerly would have been abandoned after an exploratory incision are removed almost always. Adhesions may be abdominal or pelvie, visceral or parietal. Visceral adhesions are those which unite the tumor to the uterns, bladder, liver, and other abdominal or pelvic viscera. Adhesions to the omentum are common and often extensive. Intestinal adhesions sometimes gives rise to dangerous, even fatal, obstruction of the bowel. Pelvic adhesions are more inaccessible, and therefore more dreaded than parietal. Two large ovarian eysts, one from the right and the other from the left ovary, may come in contact with each other and become strongly and broadly united. The difficulties of diagnosis and operative removal are then much increased.

\section{Twisting of the Pedicle.}

Rotation of the cyst, with consequent twisting of the pedicle, is an occasional and serious accident.

Acute Torsion is a sudden rotation of the cyst with sufficient twisting of the pedicle to cut off circulation and set up grave symptoms.

Chronic Torsion is a slow rotation of the cyst, with gradual twisting of the pedicle. This gives the tumor an opportunity to readjust 
itself to the changed conditions. The symptoms are less severe and the course more prolonged than in acute torsion. The impaircl circulation may be restored partially through adhesions. The pedicle in rare cases is twisted off completely. The detached tumor then must receive its blood-supply, if at all, by way of vessels which reach it only through adhesions.

Etiology.-Among the probable causes are :

1. Alternate distention and evacuation of the bladder or bowel.

2. A fall or other violence.

3. Violent exertion ; tight lacing.

4. Growing pregnant uterus.

5. Long, slender pedicle, especially if associated with ascites.

Pathology.-The pathological results of torsion are:

1. Edema from obstruction to the circulation in the sac-wall due to compression of bloodvessels as they pass through the twisted pedicle.

2. Engorgement, which may cause only occasional extravasations of blood from small vessels; or may be so intense as to cause rupture of larger vessels, and consequent profuse hemorrhage into the sac and great distention and rupture of the cyst-wall, and discharge of the cyst contents into the abdomen.

3. Strangulation of the cyst, which may result in :

1. Gangrene of the cyst.

2. Atrophy of the cyst, rare and only in small tumors.

3. Separation of the tumor from the pedicle.

4. Peritonitis with possible obstruction of the bowel from adhesions.

Diagnosis.-The diagnostic signs are not positive; they are :

1. Sudden abdominal pain and increase in the size of the tumor, cyst growing more and more tense and the pain more acute.

2. Rupture of the sac, recognized by sudden disappearance of the enlarging tense cyst.

3. Symptoms of peritonitis following the above conditions.

Prognosis.-Rotation, if sufficiently acute to cut off the circulation, would result in gangrene of the cyst, hemorrhage, peritonitis, sepsis, and suppuration, and, unless, relief come from ovariotomy, death. In the less acute cases the circulation is not entirely cut off and an operation if delayed will be more difficnlt on account of adhesions. Unless ovariotomy is performed, death ordinarily follows.

Treatment.-The only treatment of both acute and chronic torsions is immediate ovariotomy.

\section{Rupture of the Cyst.}

The cyst may rupture into the abdominal cavity, or into some one of the abdominal viscera. One locument of a multilocular cyst may rupture into another. Thin-walled secondary compartments very commonly rupture into the abdominal cavity, leaving the remaining compartments of the cyst intact. The opening made by rupture may reunite and the cyst refill. 


\section{Causes:}

1. Softening or thinning of the cyst-wall in one or more places ; this may occur as the result of inflammation or distention.

2. Hemorrhage into the cyst-wall or into the sac.

3. Fatty degeneration, necrosis, or gangrene of the cyst-wall.

4. Suppuration in the cyst.

5. Papillomatous growths penetrating the cyst-wall.

6. Direct injury from blows, falls, careless palpation in examination, and contraction of the abdominal wall in labor.

7. Torsion; see twisting of the pedicle.

Results.-A ruptured parovarian cyst may in exceptional cases become obliterated and thereby spontaneonsly cured. Cases of supposed ovarian cysts have been reported as cured by rupture or tapping, lout it is known that an ovarian cyst cannot be cured in this way; the tumors in question therefore must have been parovarian. The former practice of tapping ovarian cysts as a means of radical cure has been abandoned. Even parovarian cysts are treated better by removal.

Rupture may occur into :
1. Peritoneal cavity, most frequent.
2. Bladder.
3. Vagina.
4. Rectum.
5. Small intestine, rare.
6. Stomach, rare.
7. Fallopian tube, rare.
8. Abdominal wall, rare.

Fate of escaped contents and sequels of rupture:

I. If a parovarian or an ovarian cyst containing innocent aseptic fluid ruptures into the abdomen, the fluid may be absorbed through the peritoneum and eliminated by the kidneys without harm.

2. If rupture of a papillomatous cyst takes place into the abdomen, the cyst contents are liable to form secondary papillomata on the peritoneum.

3. The contents of a dermoid cyst may, if septic, infect the peritoneum, or dermoid elements may be engrafted on the peritoneum.

4. The colloid contents of a cyst may cause peritonitis.

5. The contents from a malignant cyst may engraft malignant disease on the peritoneum.

6. If fluid passes by rupture into a hollow viscus, the opening may close and the sac refill, or the opening may remain and transmit the contents of the viscus to the sac; thus feces and gas may escape from the bowel to the tumor and replace the tumor dulness with resonance on percussion ; or urine from the bladder may fill the sac.

The Symptoms, Diagnosis, and Prognosis of rupture will vary according to the condition outlined in the preceding paragraphs. The gravity of the case will depend upon the nature of the escaped fluid. The accident even in the non-fatal cases is apt to be marked by sudden severe pain, and by more or less severe peritonitis with adhesions. A monocyst upon discharge of its contents will collapse. A polycyst upon rupture of one or more of its locuments only changes 
its form. Rupture of an infected cyst into the peritoneum is usually fatal.

Treatment.-The greater the gravity of the case the more urgent the indication-immedicte ovariotomy.

\section{SYMPTOMATOLOGY.}

The symptoms of ovarian and parovarian tumors, of which none is pathognomonic, are :

1. Nutritive disorders; intestinal indigestion, and, in many cases, constipation.

2. Menstrual disturbances; not very significant :

a. Uterine hemorrhage or amenorrhœa.

b. Dysmenorrhœa:.

3. Sterility; not invariable, cren in bilateral cases.

4. Pregnancy may be simulated.

5. Pains from peritonitis not uncommon.

6. Pressure-symptoms :

a. Vesical and rectal tenesmus.

b. Edema of vagina, vulva, and lower extremities from pressure on iliac veins.

c. Abdominal pains.

d. Uterine displacements.

e. Hemorrhoids.

$f$. Urinary functions disturbed; albuminuria associated with pressure on renal artery; suppression of urine and hydronephrosis from pressure on ureters. Vesical irritation and cystitis from pressure on bladder.

g. Ascites from pressure on vena cava, or from malignancy.

$h$. Pressure on thoracic viscera may cause most distressing symptoms, such as weakness of the heart, rapid pulse, and dyspnœa so extreme that the patient must maintain continuously the sitting posture, night and day; pressure on the stomach and bowels may cause nausea, vomiting, and other alimentary disturbances ; pressure on the liver and bile-ducts may cause catarrhal jaundice.

7. Umbilical hernia-occasional.

8. Atrophic lines on abdominal skin when cyst is large.

9. The Facies Ovariana.-This is a peculiar facial expression that is somewhat diagnostic of the disease in the later stages. It is difficult to describe, but once seen is remembered easily. The natural facial expression is modified as follows:

a. The face is shrivelled, elongated, and has an anxious and careworn expression.

$b$. The nostrils are wide, the angles of the nose and mouth are drawn down, and the lips are thin.

c. The cheeks are furrowed and the face is marked by deep wrinkles. 
$d$. The space between the eyelids, and the bony margin of the orbits is sunken and hollow.

$e$. The whole areolar tissue of the face is atrophied.

$f$. The face is pale, but not with that peculiar leaden, sallow, or parchment-like color scen in malignant diseases. ${ }^{1}$

The facies ovariana is quite in contrast with an indescribable and less marked facial expression known as the facies uterina. This is often present in pregnaney and sometimes in cases of uterine tumors. The face is full and flushed.

\section{DIAGNOSIS.}

The recognition of a large, uncomplieated ovarian eyst is usually not difficult. The means of diagnosis are ${ }^{1}$

1. Clinical history.

2. Inspection.

3. Palpation.

4. Percussion.

5. Conjoined examination.

6. Measurement.

7. Aspiration or tapping.

8. Exploratory ineision.

The physical examination by inspection, palpation, percussion, or conjoined manipulation requires that the abdomen be exposed and that the patient lie on a harl couch or table, preferably the latter.

1. The Clinical History should include a consideration of the secondary ehanges as outlined in this chapter. It also includes the symptoms noted in the foregoing paragraphs, the age, social condition, pregnancies (if any), family history, and menstrual history of the patient.

2. Inspection.--If the tumor is small, the enlargement will be most apparent on the affected side; as it grows larger and rises out of the pelvis the swelling will be greater in the lower part of the abdomen between the pubes and the umbilicus, and will be nearer the median line. Abdominal enlargement from a unilocular cyst is obviously more symmetrieal than from a multilocular cyst. With declining strength the faeies ovariana becomes more pronounced.

3. Palpation will show usually a fluctuating tumor: if small, in the pelvis; if large, extending into the abdomen. The mass will be much more distinet on the affected side. The degree and character of elasticity will vary with the tenseness of the cyst and the consistence of the contents. A greatly distended tense sac, especially if the contents are semisolid, may feel like a solid tumor.

It is rare to find solid matter predominating in an ovarian

Adaptation from Peaslee's Ovarian Tumors.

2 In the diagnosis and differential diagnosis I have mane numerous adaptations from the classical work on ovarian tumors by my honored friend and teacher, the late Edmund Randolph Peaslee. 
cyst. Large masses of semisolid matter, and small nodules of very hard or bone-like, substance, often are detected by palpation. The more solid parts are found rather in the pelvis than in the abdomen. The different locuments of a multilocular cyst in some cases are outlined easily by palpation. The cyst sometimes may be moved from side to side, and up and down; the degree of mobility will depend upon its size, the length of the pedicle, and the extent of the adhesions. In cases of rery thick or rigid abdominal walls, and especially of small tumors, anrsthesia facilitates the examination.

4. Percussion.-The tumor-sac, with its contents, occupies the anterior part of the abdomen; the intestines are in the posterior, lateral, and upper parts; hence the maximum dulness on percussion will be over the anterior and lower portions of the abrlomen. Since the cyst extends from the pelvis, the dulness will be continuous from the abdomen into the pelvis; it, however, will cease abruptly or shade off into resonance and tympanites at the limits of the tumor, toward the sides of the abdomen and toward the diaphragm. This is because the spaces above and to the sides of the tumor are filled with intestine. For the relative areas of dulness and resonance, see Differential Diagnosis of Ovarian Cyst and Ascites. The location of the cyst does not change with change in the position of the patient; the areas of dulness correspond to the location of the tumors, and are constant.

The Percussion Wave usually present is elicited by placing the finger-tips of the left hand to one side of the tumor, and with the finger-tips of the right hand sharply tapping or thumping, or with the thumb and finger snapping the other side. In very tense cysts and in cysts with semisolid contents, like dermoids, the wave may be slight or absent.

5. Conjoined Examination, which includes vaginal and rectal touch, will show usually the relations of the uterus to the cyst. The importance of this means of diagnosis is great, for any cyst of pelvic origin not connected with the uterus is almost certainly ovarian or parovarian. If therefore, upon vaginal or rectal touch, the uterus proves to be healthy and normally mobile, with little or no increase in length, the presumption is in favor of an ovarian tumor; if, upon conjoined examination with one or two fingers of the left hand in the vagina or rectum, and the right hand over the abdomen, the uterus can be made out distinct and separate from the cyst-tumor, the evidence of ovarian tumor is very strong.

In very exceptional cases of ovarian cyst, however, the uterus may be enlarged, drawn up out of the true pelvis, immobile, and otherwise abnormal. The eyst may be so monlded to the pelvis as to press the uterus forward and upward and flatten it against the pubes. The tumor and the uterus may, through adhesions or location, be nearly or quite inseparable from each other; such conditions are very indicative of uterine tumors, but are found occasionally with ovarian cysts. See Differential Diagnosis of Ovarian Cysts and Uterine Tumors. 
6. Measurements.-The circular measurement of the abdomen is increased. 'The distance from the anterior superior process of the ilium to the umbilicus is greater on the affected side. The distance from the pubes to the umbilieus is inereased relatively more than that from the umbilicus to the ensiform cartilage.

7. Aspiration, or Tapping, once a common means of diagnosis, is now almost abandoned. This is because there is always some danger from the possible escape of fluid into the abdominal carity. The diagnosis usually can be made without tapping, and, moreover, an exploratory incision is safer and more effective.

8. Exploratory Incision is the final resort in diagnosis and differential diagnosis. When this is done the patient should be prepared for ovariotomy, and the tumor, if operable, should be removed.

\section{Diagnosis of Adhesions.}

Adhesions may be reeognized, though not with eertainty, by the following signs:

1. Immobility of the tumor.

2. Sensitiveness on pressure (peritonitis), not reliable.

3. Bands of adhesions sometimes may be felt through the vagina or at the sides of the tumor.

\section{Diagnosis of Malignancy.}

The physical sigus of malignaney, not conclusive, are as follows :

1. Nodular, hard surface.

2. Ascites, always present in malignaney, seldom mueh in benign tumors.

3. Cachexia, and œdema of the lower extremities.

4. Metastasis.

5. Rapid increase of growth and involvement of surrounding struetures.

\section{PROGNOSIS.}

The prognosis, without operation, of ovarian and parovarian tumors has been indicated partially under Secondary Changes in the first part of this ehapter. Multilocular proliferating cysts and papillomatons cysts, if not removed, usually cause death in about three years. The causes of death are:

1. Exhaustion due to interference with sleep, nutrition, and respiration.

2. Nephritis, hydronephrosis, uræmia, eystitis, pyelitis.

3. Peritonitis and intestinal obstruction.

4. Suppuration and gangrene of cyst.

5. Rupture of cyst; hemorrhages from any cause.

6. Impediment to labor.

The prognosis with ovariotomy should show a mortality of less than $\check{b}$ per eent. 


\section{DIFFERENTIAL DIAGNOSIS.}

This subject involves : first, the differential diagnosis of ovarian and parovarian cysts from one another ; second, the differentiation of these cysts from other conditions with which they have been confounded.

\section{Distinction between Ovarian and Parovarian Cysts.}

This distinction has been given under pathological anatomy and secondary changes. The following tabular statement, however, will emphasize the differential points:

Ovarian cysts.
1. Develop from the ovary.
2. Usually multilocular.
3. Apt to contract adhesions.
4. May be small or attain enormous size;
growth more rapid.
5. Never cured by tapping.
6. Fluid may be thick, thin, muddy, light,
dark, straw- or coffee-colored, albuminous.
7. Usually pedunculated unless situated be-
tween folds of broad ligament.
8. Sometimes contains warts-i. e., papillo-
mata.
9. Sac-wall does not usually have peritoneal
covering.
10. Apt to be adenomatous and may be papil-
lomatous.
11. Is fatal in three or four years. Facies ova-
riana marked.

Parovarian cysts.

1. Develop from the parovarium.

2. Almost always unilocular.

3. Do not usually contract adhesions.

4. Iay become quite large, but not so large as ovarian eysts : growth slower.

5 . Sometimes cured by tapping.

6. Fluid usually light, like spring water. Specific gravity rarely as high as 1010 . Niay be

only slightly albuminous or non-albuminous.

7. Usually not pedunculated. Development apt to be in broad ligament.

8. Seldom papillomatous.

9. Sac has peritoneal covering, from which it may be readily enucleated.

10. Is neither an adenoma nor a papilloma.

11. May not impair health for many years. Facies ovariana absent or not marked.

Dermoid Tumors develop from the cortex of the ovary, and are a special variety of ovarian cysts. They have been described specially in a preceding chapter, excepting teratomata (complicated dermoids). The diagnostic points by which dermoids (simple) may be distinguished from the two kinds of cysts just tabulated are these:

1. Facies ovariana comes very late, if at all.

3. The tumor may exist for many years without impairment of the general health.

3. Abdominal enlargement usually to one side; is otherwise symmetrical.

4. The tumor does not grow to very large size.

5. Contents are too thick to permit tapping even with a large trocar.

6. Inflammation of cysts and adhesions not very common.

7. Spontaneous rupture not common.

8. CEdena of lower extremities rare.

9. Fluctuation and percussion wave obscure or absent.

10. Sac contains dermoid element.

\section{Explanation of Figure 204.}

Ovarian cysts and other conditions which simulate them.

A, ovarian cyst with ascites; the ascites causes the umbilicus to bulge.

B, ordinary ovarian cyst.

$\mathrm{C}$, characteristic shape of abdomen in nearly symmetrical myoma.

$\mathrm{D}$, shape of abdomen in multiple myoma or carcinoma.

$\mathrm{E}$, cylindrical shape of abdomen in ascites.

$\mathrm{F}$, ascites with relaxed abdominal wall.

$G$, pendulous abdomen in fat woman.

$\mathrm{H}$, pendulous abdomen from advanced ovarian cyst ; woman much emaciated. 
460 TUMORS, TUBAL PREGNANCY, MALFORMATIONS.

Figure 204.

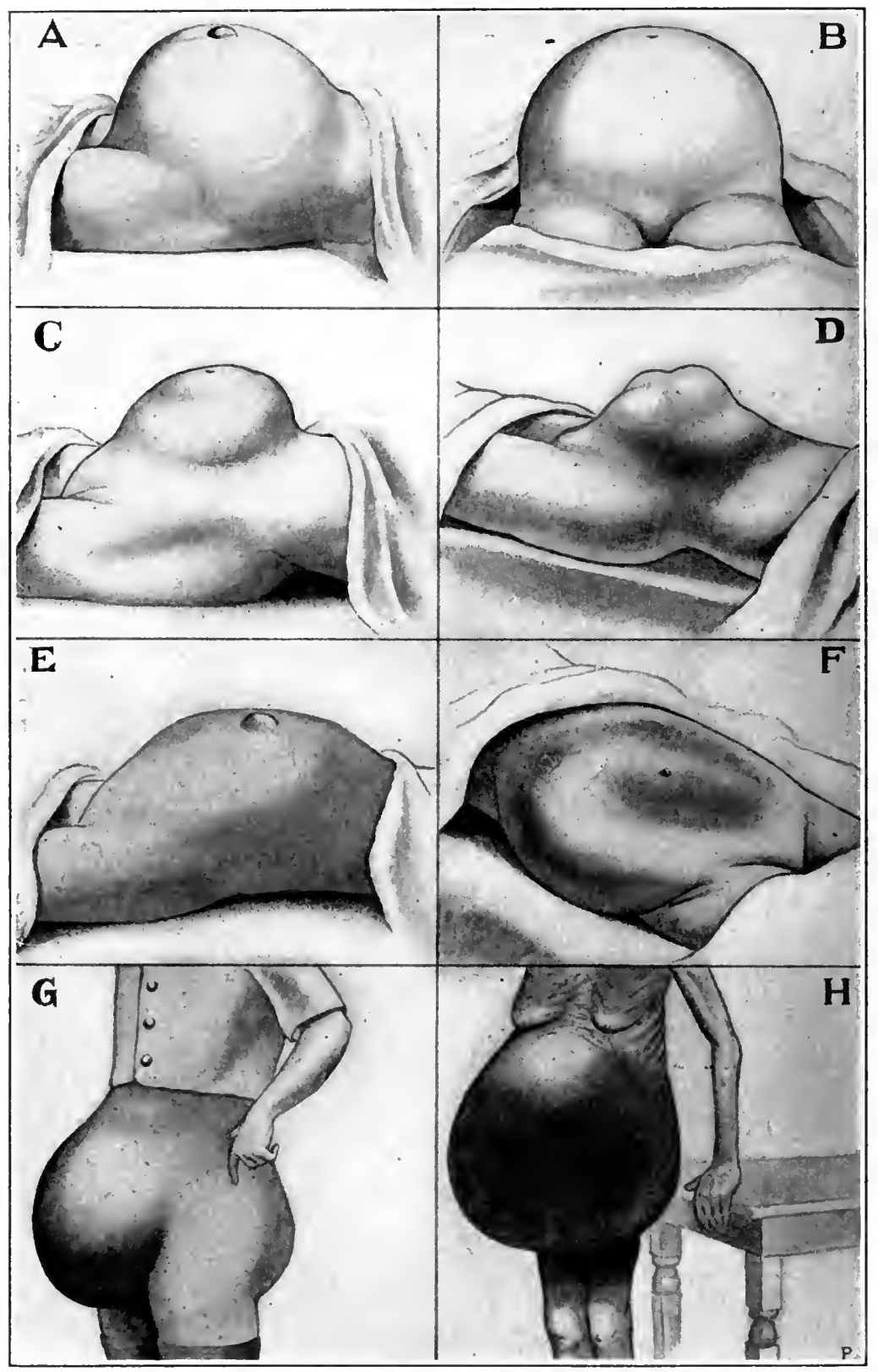


11. Location of small dermoid cysts more apt to be anterior than posterior to nterus.

12. May occur early in childhood.

Complicated teratomata, as explained in the preceding chapter, have a strong malignant tendency, may be of rapid growth, and may destroy life in a short time.

\section{The Differentiation of Ovarian Cysts from Other Conditions that may be Mistaken for Them.}

The pathological conditions that have been mistaken for ovarian cysts may, for convenience of description, be divided into those which originate in the pelvis and those which originate in the abdomen. Among the nore important of these conditions may be mentioned the following :

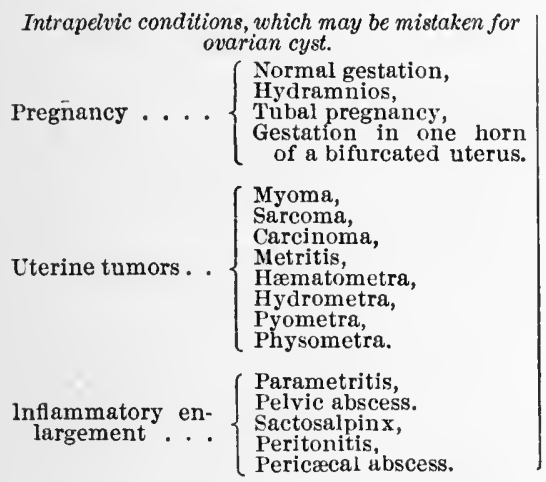

Abdominal conditions, which may be mistaken for Ascites.

Encysted ascites.

Hydatid eysts.

Renal tumors.

Floating kidney.

Pancreatic cyst.

Enlarged liver.

Mesenteric cyst.

Cysts of the urachus.

Enlarged gall-bladder.

Intestinal tumors.

Fatty tumors.

One or more of the above conditions may coexist with ovarian cystoma. The diagnosis then is complicated, difficult, and without exploratory incision may be impossible. Before taking up the subjects outlined in the foregoing table it is important to consider the following question :

QUESTION I.-Is there any tumor at all within the peritoncal eavity?

The abdomen has been opened repeatedly for the removal of a supposed ovarian tumor when no tumor of any kind existed; even more frequently, tapping and aspiration have been done when no fluid was present. One anthor, in his statistical tables, mentions no less than twenty-one cases of the kind. ${ }^{1}$ The following conditions may give the appearance of an intra-abdominal growth when no such growth exists :

1. Fat in the abdominal walls.

2. Phantom tumor.

3. Tympanites.

4. Fecal accumulations.

5. Distended bladder.

6. Dilated stomach.

1 John Clary, in Ovarian Tumors, 1872, Peasleee. 
1. Fat in the Abdominal Wall.-An eminent British surgeon once laid open the abdomen from the pubcs to the ensiform cartilage, only to find, instead of an ovarian eyst, a mass of subcutaneous fat. Similar blunders have occurred repeatedly. Such an error at the present day, however, should be almost impossible. No proper signs of ovarian eyst would be present in such a case. The mass of fat in the abdominal wall may be grasped between the hands and isolated from the abdomen. Vaginal touch would also yield negative evidence of a tumor. Great thickening of the abdominal wall from edema is differentiated by pitting on pressure.

2. Phantom Tumor.-Some hysterical women have the power so to contract the abdominal muscles as to force up the tympanitic intestines into a bunch, and in this way to make an apparent abdominal enlargement in form like that of a tumor. Prolonged firm pressure with the palms of the lands usually overcomes the muscular contraction. The percussion note is decidedly tympanitic. Anæsthesia completely exposes the deception.

3. Tympanites.-The extraordinary blunder occasionally has been made of mistaking tympanites for an abdominal tumor. This has occurred usually when the evidences of percussion and palpation were obscured by large amounts of abdominal fat. Tympanites will be known by resonance on percussion, absence of the percussion wave, and by the negative results of vaginal touch.

4. Fecal Accumulations in the bowel have led occasionally to the suspicion of an ovarian cyst. The history of constipation, supplemented by palpation, will settle the diagnosis ; if not, active catharsis will remove all doubt. Deep pressure through a thin abdominal wall or through the vagina causes pitting of the distended bowel.

5. Distended Bladder.- Retained urine may accumulate in large quantity until the bladder appears between the pubes and umbilicus as a distinct fluctuating tumor. The external appearance, on inspection, palpation, and percussion, is very like ovarian cystoma. The anterior vaginal wall, however, bulges into the vulvar orifice. There is an almost or quite continuous overflow of urine through the urethra. Hypogastric pain and distress are urgent. The use of the catheter will settle all possible doubt.

6. Dilated Stomach.-The anthor personally knows of one case in which a deservedly eminent surgeon opened the abdomen for a supposed ovarian cyst, and found instead a dilated stomach. The condition ordinarily would be distinguished from cyst by the maximum enlargement above instead of below the umbilicus, and by resonance on percussion all over the tumor. A positive test is to let the patient swallow water while the stethoscope is placed over the tumor. As the water reaches the stomach a gurgling sound will be heard clearly all over the enlargement. Inflation of the stomach through a tube would cause a decided tympanitic note over the abdomen.

Given sufficient evidence that there is a tumor, the next inquiry is-

QuESTION II.: Is the enlargement of pelvic or of abdominal origin? 
If not of pelvic origin, it cannot be ovarian, and therefore does not come within the scope of this inquiry. If the hand cannot be inserted by deep firm pressure between the tumor and the symphysis pubis, it is inferred that the tumor rises from the pelvis; if vaginal and rectal touch confirm this inference, it is so decided. ${ }^{1}$ The pelvic origin of the tumor being established, the next inquiry is-

Question III.: Is the tumor possibly due to pregnancy?

The humiliation of attempting to remove from a pregnant woman an ovarian tumor which does not exist may be avoided by assuming, until the contrary is proved, that every abdominal enlargement in a woman is due to pregnancy.

\section{Differential Diagnosis of Normal Gestation and Ovarian Cyst. ${ }^{2}$}

Normal gestation.

1. Enlargement sudden, rapid, and usually symmetrical.

2. Facies natural and healthy.

3. Superficial veins of abdomen not enlarged. Edema of ankles not uncommon after seven months.

4. Fluctuation not distinct unless liquor amnii is cxcessive.

5. Menstruation arrested.

6. Vaginal touch detects softening and apparent shortening of the cervix and enlargement of the uterus. No extra-uterine tumor.

7. Ballottement gives impulse of foetus.

8. Fotal heart-sounds after twentieth week.

9. Fotal movements about sixteenth week.

10. Enlarged sebaceous glands; areola about nipples darkened.

11. Tumor has developed in six to nine mouths.

\section{Ovarian cyst.}

1. Enlargement gradual and, until tumor becomes large, asymmetrical.

2. Fucies ovariana in later stages.

3. Veins enlarged. CEdema exceptional and only after one or two years.

4. Usually very distinct, especially in monocysts.

5. Not usually arrested unless late in the disease.

6. Uterus unchanged except by displacement, usually in front of or behind the cyst. Tumor extra-uterine.

7. Ballottement gives negative results.

8. None.

9. None.

10. Rarely imitated.

11. Development continues two to four years.

If the foetus is dead, the heart-sounds and fotal movements will, of course, not be present.

Ovarian cyst and pregnancy not infrequently coexist. The diagnosis then is made by the clinical history of both conditions, by palpation, and by conjoined examination.

Hydramnios is an excess of amniotic fluid. There are normally from six to thirty ounces; this amount may be increased enormously, giving the uterus the appearance of an immense cyst. The attempt has been made occasionally to tap or remove such a tumor by mistake for an ovarian cyst. follows :

The differential diagnosis of hydramnios and ovarian cyst is as

$$
\begin{aligned}
& \text { Hydramnios. } \\
& \text { 1. Evidence of pregnancy. } \\
& \text { 2. Rapid development. } \\
& \text { 3. Ballottement. } \\
& \text { 4. Distention symmetrical. }
\end{aligned}
$$

Ovarian cyst.

1. Not usual.
2. Less rapid.
3. Absent.

4. Distention more on one side.

Tubal Pregnancy.-The diagnosis of this condition will be found in Chapter XXXVI. Unlike ovarian cyst, it gives an early, though irregular, history, as of pregnancy. Conjoined examination before rupture shows a boggy, fluctuating, pulsating tumor at the side and back of the uterus. After rupture the tumor is less distinct, non- 
pulsating, and non-fluctuating. At or near the time of rupture the endometrium throws off a modified decidua of pregnaney. The symptoms of rupture are urgent ; they are those of pelvic hæmatocele, and are not likely to be mistaken for any synptoms of ovarian cyst unless it be those of rupture of the sac or twisting of the pedicle.

Gestation in One Horn of a Bifurcated Uterus.-The unilateral location may give bicornate pregnancy the appearance of an ovarian cyst or of a myoma.

Question IV.: Is there a uterine enlargement due to other causes than pregnancy?

The pathological conditions suggested by the question are these :
Uterine myoma.
Uterine sareoma.
Hæmatometra.
Uterine carcinoma.
Hydrometra.
Metritis.
Pyometra.
Physometra.

\section{Differentiation of Uterine Myoma from Ovarian Cystoma.'}

1. Slow growth.

\section{Uterine myoma.}

2. Facialexpression unchanged. Face may be full and flushed; later pale from hemorrhage.

3. General health not necessarily impaired except from loss of blood if submucous or mural; may be painful.

4. Abdomen often asymmetrical from irregular shape of tumor.

5. Abdominal veins not usually enlarged.

6. Action of kidneys normal.

7. Usual menorrhagia.

8. Elasticity, not fluctuation. No percussion-wave.

9. Surface firm and usually lobulated.

10. Vaginal touch and conjoined examination show tumor dense and firm, and, unless pedunculated, continuous with uterus. Uterus large and heavy.

11. Uterine cavity much elongated.

12. Uterus moves with tumor.

13. Negative results from aspiration; aspiration not advised.

Exceptions.-A subperitoneal myoma with a long pedicle moves independently of the utcrus, and the uterine cavity is not necessarily lengthened. If the myoma has degenerated to a fibroeyst, there will be more or less finctuation, and aspiration may yield positive results.
Ovarian cystoma.

1. Usually more rapid growth.

2. Facies ovariana in later stages.

3. General health early impaired from emaciation. Not painful.

4. Abdomen more symmetrical, especially when tumor is large.

5. Usually enlarged, especially in large polycysts.

6. Kidneys less active.

7. Mlenstruation unehanged or diminished.

8. Fluctuation marked. Percussion-wave marked.

9. Surface yielding; in monocysts, regular; in polycysts, irregular.

10. Uterus normal, except displacement from pressure. Tumor compressible, fluctuating, separate from uterus.

11. Not materially elongated. (This is a most important diagnostic point.)

12. Does not move with tumor.

13. Positive results from aspiration (aspiration not advised).

Exception.-A cyst with semisolid contents yiclds negative results on aspiration. Fluctuation, if present, is indistinct, and percussionwave is absent or indefinite.

Edematous myoma may give rise to an apparent fluctuationpseudofluctuation.

\section{Differentiation of Uterine Sarcoma and Carcinoma from Ovarian Cyst.}

The relations of these growths to the uterus are similar to those of myoma. The accompanying tabulated statement concerning myoma, therefore, in the main applies to malignant growths. Malignant uterine tumors differ from myoma in these particulars-viz., more

1 Adaptation from Peaslee's Ovarian Tumors. 
pain, great tendency to early ulceration and other degenerative changes, more profuse hemorrhages, offensive watery or bloody discharge, cachexia, and a speedily fatal result.

\section{Differentiation of Metritis from Ovarian Cyst.}

Metritis gives a history of inflammation, and is apt to be associated with parametritis, salpingitis, and ovaritis. The uterus is never enlarged to more than two or three times its normal size, and in form is always symmetrical. Conjoined examination will show that there is no extra-uterine growth. There are also tenderness on pressure and diminished mobility.

\section{Differentiation of Hæmatometra, Hydrometra, Pyometra, and Physometra from Ovarian Cyst.}

In the conditions above named the uterine enlargement is always symmetrical, and the uterus whether distended with blood, serum, pus, or gas, gives a greater or lesser sense of fluctuation, but not the clear fluctuation of a cyst. See Retained Menstruation, Chapter XXXVIII. Examination will show that the os externum or the cervical canal at some point is closed completely. Unless the Fallopian tubes also are distended the enlargement will be confined entirely. to the uterus.

Questior V.: Is the enlargement extra-nterine, and possibly due to inflanmation?

This question suggests the following conditions:
Parametritis,
Pelvic abscess,
Pyosalpinx,
Peritonitis,
Hydrosalpinx,
Pericæcal abscess.

The history of inflammation and the close relations of the enlargement to the uterus will aid greatly in the recognition of any of these diseases. In all, except possibly hydrosalpinx, there will be tenderness on pressure. Sactosalpinx, whether the tube be distended with serum, pus, or blood, will be identified usually by its location to the side and back of the uterus, but more especially by the irregular, elongated, tortuous, or ovoid form of the mass. A pus-tube is much more likely to be adherent than an ovarian cyst of small size. A parametric abscess situated in the broad ligament is always continuous with the side of the uterus. Suppuration, anterior or posterior to the uterus, is also inseparable from the uterus. Pericæcal abscess or appendicitis may be suspected from its location.

Question VI.: Is the tumor of abdominal origin, and therefore not ovarian?

A large ovarian cyst may have a pedicle so long as to permit the entire tumor to rise out of the pelvis into the abdominal cavity. It may even be possible to insert the hand deeply between the tumor and the symphysis pubis. Conjoined vaginal and rectal touch may not discover the pedicle, nor establish the pelvic origin of the cyst; it is sometimes difficult to differentiate such a cyst from other tumors of abdominal origin. 
The following extrapelvic pathological conditions have been mistaken for ovarian cyst:
Ordinary ascites.
Encysted ascites.
Enlarged liver.
Mesenteric cyst.
Hydatid eysts.
Cysts of the urachus.
Renal cysts.
Displaced or fioating kidney.
Pancreatic cyst.
Enlarged gall-bladder.
Intestinal tumors.
Fatty tumors.

\section{Differential Diagnosis of Ascites and Large Ovarian Cyst. ${ }^{1}$}

Figure 205.

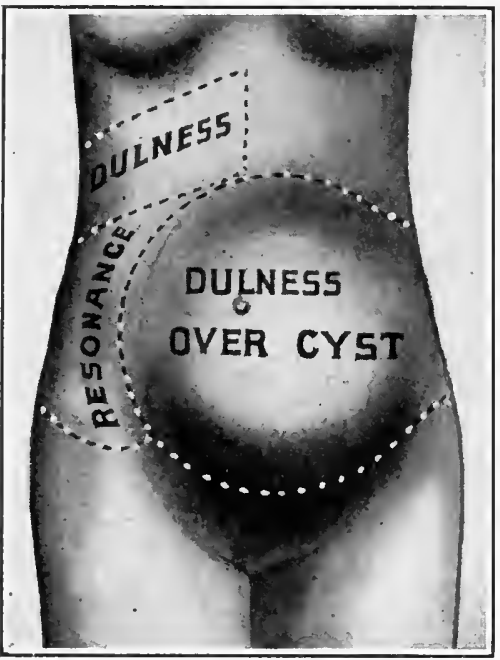

Figure 206.

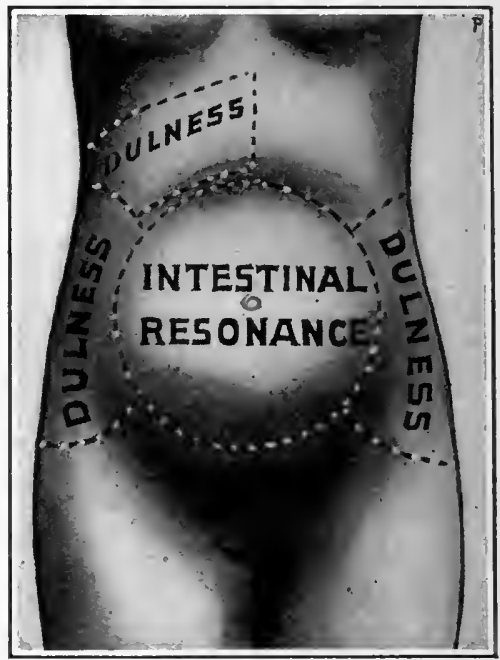

FigURE 205.-Areas of dulness and resonance in ovarian eyst; dulness over tumor; resonance over intestine in the flank opposite to the side on which the tumor has developed. There would be no change in areas of resonance and dulness with change in position of patient; dulness over liver in hepatic region.

Frgure 206-A reas of resonance and dulness in ascites; resonance over intestines; hydrostatic duluess below level of fluid in the flanks : dulness over liver in hepatic region. Constant change in areas of dulness and resonance with change in position of patient, because intestines seek high level and fluid seeks low level.

Ascites.

1. Previous history of visceral disease.

2. Enlargement eomparatively sudden.

3. Face puffy; color waxy ; carly anæmia.

4. Patient on back, enlargement symmetrical; flat in front.

5. Sitting up, abdomen bulges below.

6 . Navel prominent and thinned.

7 . Fluetuation decidedly elear, diffuse throughout abdomen, but avoids highest parts in all positions, and always has a hydrostatic level.

8 Intestines float on top of fluid: hence percussion gires elear tympanitic note over the highest parts of abdominal cavity, and dulness in lowest parts for all positions- $i$. $e_{\text {. }}$, areas of resonauce and dulncss change with position.

9. Vaginal touch detects fluctuation, bulging into vagina.

\section{Ovarian cyst.}

1. No such history.

2. Gradual.

3. Facies ovariana. Anæmia absent or less marked until later period.

4. Asymmetrical until tumor is quite large; prominent in front.

5. No appreciable change.

6 . Navel usually unchanged.

7. Less clear; limited to cyst; not modified by change of position. No hydrostatic level.

8. No change in areas of dulness and resonance with change of position. Dulness over cyst. Clear resonant note in all parts beyond cyst limits-i. $e_{\text {., }}$ in flanks and toward the diaphragm.

9. Vaginal fiuctuation less clear or absent. 
Ascites.

10. Uterus in prolapsed location. Size and mobility unchanged.

11. Hydragogues and diuretics temporarily remove the fluid.

12. Fluid light straw color and thin. Coagulates spontaneously.

Exceptions.-The intestines may be adherent to the posterior part of the abdominal cavity, and the fluid may therefore be in the anterior part, or the amount of fluid may be so great that the intestines held back by mesentery or adhesion cannot float to the surface; then the areas of resonance and dulness, except on very deep percussion, may be similar to those of a cyst.

Gas in the colon may produce clearness in the flanks.

Eucysted ascites-i. e., fluid confined to a limited part of the abdomen by adhesions-may give the same areas of dulness and resonance as a cyst.
Ovarian cyst.

10. Uterus displaced forward or backward, or laterally by pressure of eyst.

11. Mledicines have no effect.

12. Fluid light or dark and of varying consistence; albuminous, but does not coagulate spontaneously: may contain colloid matter.

Exceptions.-Flanks may be dull from feces in the colon.

Cyst may communicate with the intestines and be filled with gas. This would give a tympanitic note all over the cyst.

The cyst may be small and glued to the posterior part of the abdominal cavity by adhesions. The intestine might then be in front of it and give a tympanitic note over the most prominent part of the enlargement.

Ovarian cyst and ascites may coexist. If the cyst be small and the patient a stout woman, the diagnosis without exploratory incision may then be most difficult.

\section{Differentiation of Hydatid Cysts from Ovarian Cysts.}

Hydatid, or echinococcus, cysts are sometimes difficult to distinguish from ovarian tumors. They may originate either in the pelvis or in the abdomen. Hydatid cysts of pelvic origin may be in the broad ligament or immediately beneath the uterine or pelvic peritoneum. Hydatid abdominal cysts may originate in the omentum or liver. If of abdominal origin and of small size, their location usually will prove them to be extrapelvic, and therefore not ovarian. The qualifying word "usually" is introduced because the writer once encountered a small ovarian cyst adherent to the liver. The pedicle was in this case very slender and about seven inches long. In palpation of hydatid cysts a peculiar fremitus sometimes is imparted to the fingers.

Large abdominal hydatid cysts may extend into the pelvis, and, like those of pelvic origin, closely simulate ovarian disease. These cysts unless inflamed are rarely painful. When they distend the abdomen, they project as a mass of small, rounded, tense, elastic bodies; the indvidual projections are smaller than those of ovarian cysts. Fluctuation is distinct. Suppuration will give rise to signs of an abscess in addition to the signs of hydatids.

Definite diagnosis is impossible without exploratory incision. The fluid usually will show the characteristic hooklets. It is slightly alkaline or neutral, non-albuminous, has a specific gravity of about 1010, and contains chloride of sodium. Fragments of the characteristic laminated lining of the cyst may come away through an aspirator or trocar. ${ }^{1}$

Degenerative processes may canse rupture of the cyst and discharge of its characteristic vesicles, hooklets, or membranes through the vagina, rectum, or bladder; the diagnosis is then clear. Hydatid cysts are rare.

1 Sutton. Surgical Diseases of the Ovaries and Fallopian Tubes. 


\section{Differentiation of Renal Tumors and Ovarian Cysts.}

The distinction between renal tumors and other abdoninal and pelvic enlargements is often extremely difficult. They have been repeatedly mistaken, not only for ovirian tumors, but as well for tumors of the pancreas, liver, spleen, intestine, omentum, and uterus. Without an exploratory incision, the greatest care and the widest general knowledge may be inadequate to a diagnosis. The enlarged kidney has been found, not only so loose as to occupy almost any loeation or position in the abdomen or pelvis, but fixed by adhesions in its mal-location-for example, to the pelvic brim or to the saerum.

Figure 207.

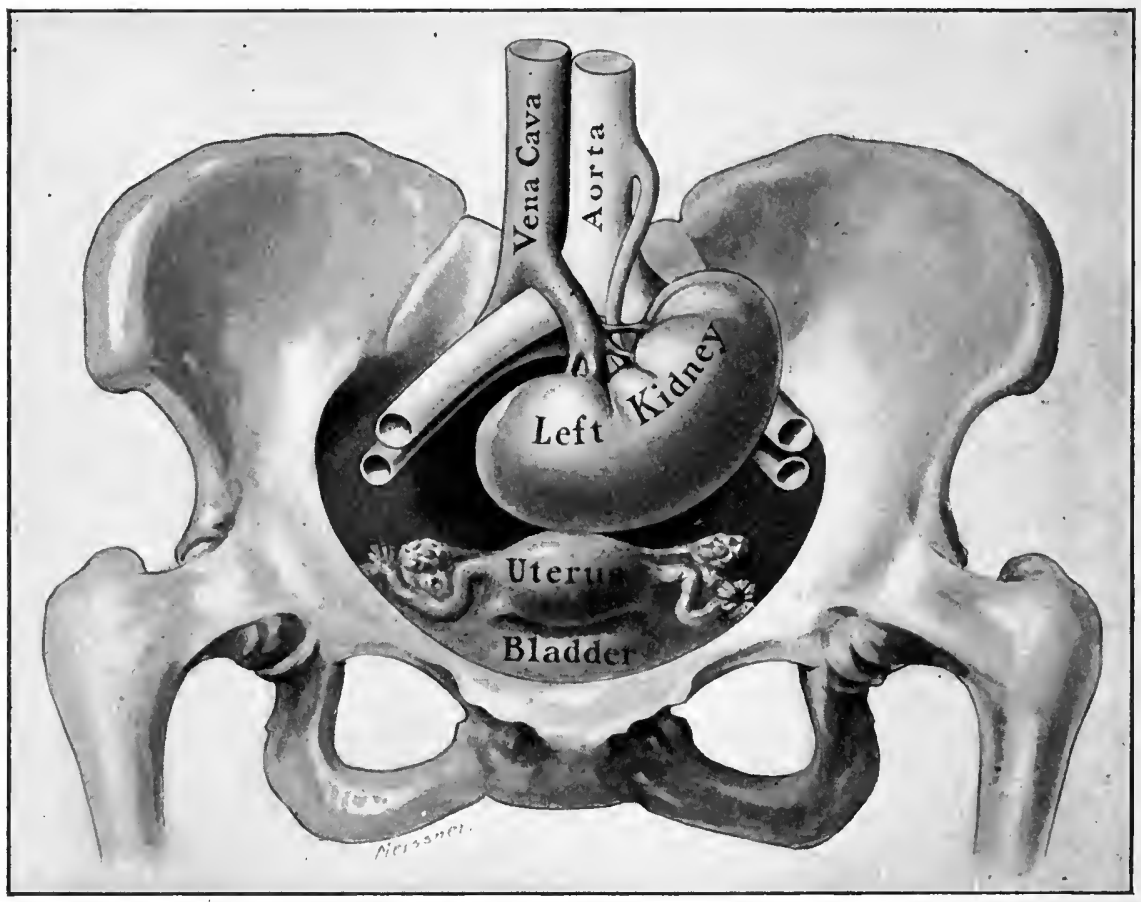

Left kidncy in the hollow of the sacrum; the renal artery and vein are dragged down wath the kidncy producing a mechanical disturbance in the urinary system and in the circulation which would necessarily have serious results

In such eases the clinical history and rational signs-including urinalysis-usually will give evidence of renal disease. A renal cyst may be hydronephrosis or pyelonephrosis. The differentiation is made as follows :

\section{IIydronephrosis.}

1. Enlargement unilateral and from above downward. Growth fixed in region of kiduey,

2. Expression unchanged.

3. Growth usually slow.

4. Intestines may be in front of tumor.
Ovarian cysts.

1. Enlargement at first unilateral : later symmetrical and from below upward, No fixation. 2. Facies ovariana.

3 . Growth relatively rapid.

4. Intestines in the flanks above and back of tumor. 


\section{Hydronephrosis.}

ह. Fluid not necessarily albuminous; may contain calculi.

6. Vaginal touch negative.

7. Urine may contaili pus, blood, or albumin. 8. Cystoscopy shows absence of urine through ureter on affected side.

Exception.-In case of a movable kidney the tumor may not be fixed.
Ovarian cysts.

5. Fluid albuminous; no calculi.

6. Tumor usually felt by vaginal touch,

7. Urine generaliy normal.

8. Urine flows through ureter on both sides.

Exception.-Iu case of adhesions the cyst may be fixed.

In pyelonephrosis the symptoms of suppuration will be present.

Pancreatic cyst, enlarged liver, mesenteric cyst, cyst of the urachus, enlarged gall-bladder, intestinal tumors, subperitoneal or omental fatty tumors, all of which may grow to large size, have been mistaken for ovarian cysts. With ordinary care and skill, however, such mistakes are not very likely to arise. All these tumors develop from above downward, and may be distinguished easily from ovarian cyst by their location and physical characteristics. Únlike ovarian cyst, they are usually beyond the reach of vaginal touch.

\section{EXPLORATORY INCISION.}

Finally, in cases of doubt, the question may be settled by exploratory incision. Indeed, every ovariotomy-yes, every abdominal section-should begin as an exploratory incision. Mr. Tait's wise caution, already quoted in connection with the diagnosis of pelvic inflammation, will bear repetition here: "One may easily turn an exploratory incision into a complete operation, but it may be a serious matter to turn an incomplete operation into an exploratory incision." 


\section{CHAPTER XXXIV.}

\section{OVARIOTOMY.}

THE general principles which apply to ovariotomy are laid down in Chapters III., VI., VII., VIII.

Eleetricity, incision, and drainage, and numerous drugs have been tried in the treatment of ovarian eysts; they are all useless. The treatment is summed up in a single word-ovariotomy. The operation was performed first in 1809 by Ephraim MeDowell, of Danville, Ky.

\section{Removal of Ovarian Cyst.}

The subdivisions of the subject are these:

Preparatory treatment.

The abdominal incision.

Emptying and delivering the cyst.

Ligature of the pedicle.

Closure of the wound.

Drainage.

After-treatment.

Aceidents and complications.

The Preparatory Treatment and arrangements for the operation, ineluding the selection of sponges, ordinary instruments, operatingtable, and assistants, have been outlined in the General Discussion of Major Operations, Chapters II. and VI. The instruments and appliances specially required are:

16 Small pressure-forceps. Figure 42, a, b, e.

6 Long pressure-foreeps. Figure 63, A.

2 Nélaton forceps. Figure 208, E.

1 Sealpel.

2 Pairs of straight scissors.

1 Ovariotomy trocar. Figure 208, A.

12 Needles, round at the point, for intraperitoneal plastic work. Figure 42.

1 Rubber sheet.

1 Bucket to catch the cyst-fluid.

1 Small, curved trocar. Figure 208, D.

4 Retractors. Figure 208, B and C.

The Abdominal Incision.-Ovariotomy, except for small, nonadherent eysts, is performed by abdominal seetion. The incision, made throngh the abdominal wall in the median line near the pubes, has been described in Chapter VI. Vaginal Section, usually not advised, but sometimes used for very small cysts, is described in Chapter XXIII. Ordinary, uneomplieated ovariotomy requires an ineision not more than two or three inches long.

Cyst fluid may be perfectly innocent, or, on the contrary, may, from suppuration or other causes, contain infectious matter. The 
Figure 208.

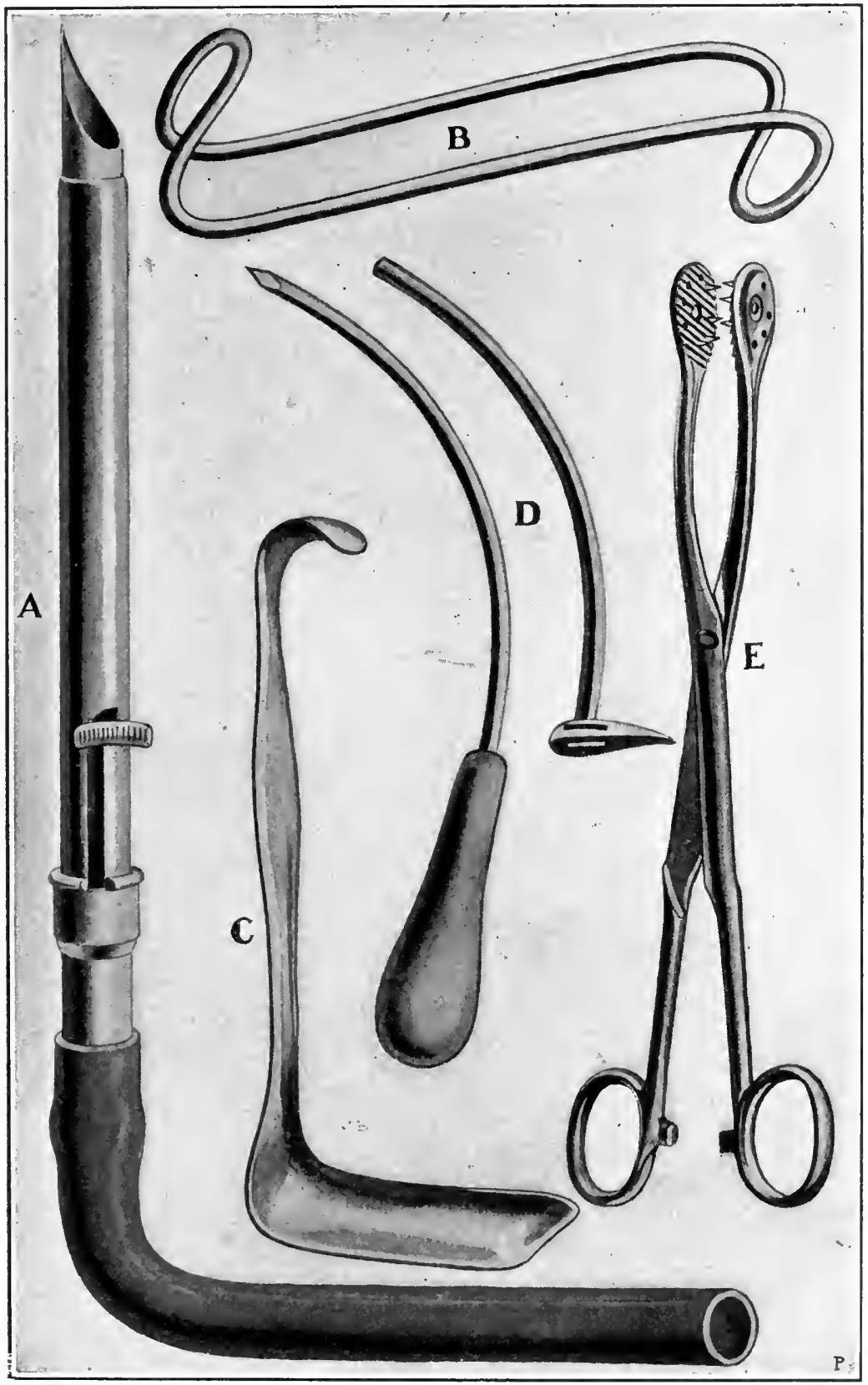

A, ovariotomy trocar; B, retractor for keeping abdominal wound open during the operation (two of them are required); C, retraetor for abdominal wound; D, small trocar for emptying small eysts; E, Nélaton's cyst-forceps for drawing out the empty and collapsed cyst. 


\section{Explanation of Figure 209.}

\section{OVARIOTOMY.}

$A$. Examining the Tumor.- The abdomen having been opened by an incision in the median line, the hand is introduced into the peritoneal cavity in order to determine the presence or absence of adhesions, and to break up any slight adhesions which may be found.

B. Tapping the Cyst. - The patient is on the right side. A folded, flat gauze sponge is partially introduced into the peritoneal cavity and held by the left hand of the operator, in order to absorb any fluid which may escape from the cyst. The operator, with his right hand, plunges the trocar into the cyst. The fluid is evacuated through the trocar and the attached rubber tube into the bucket below. 


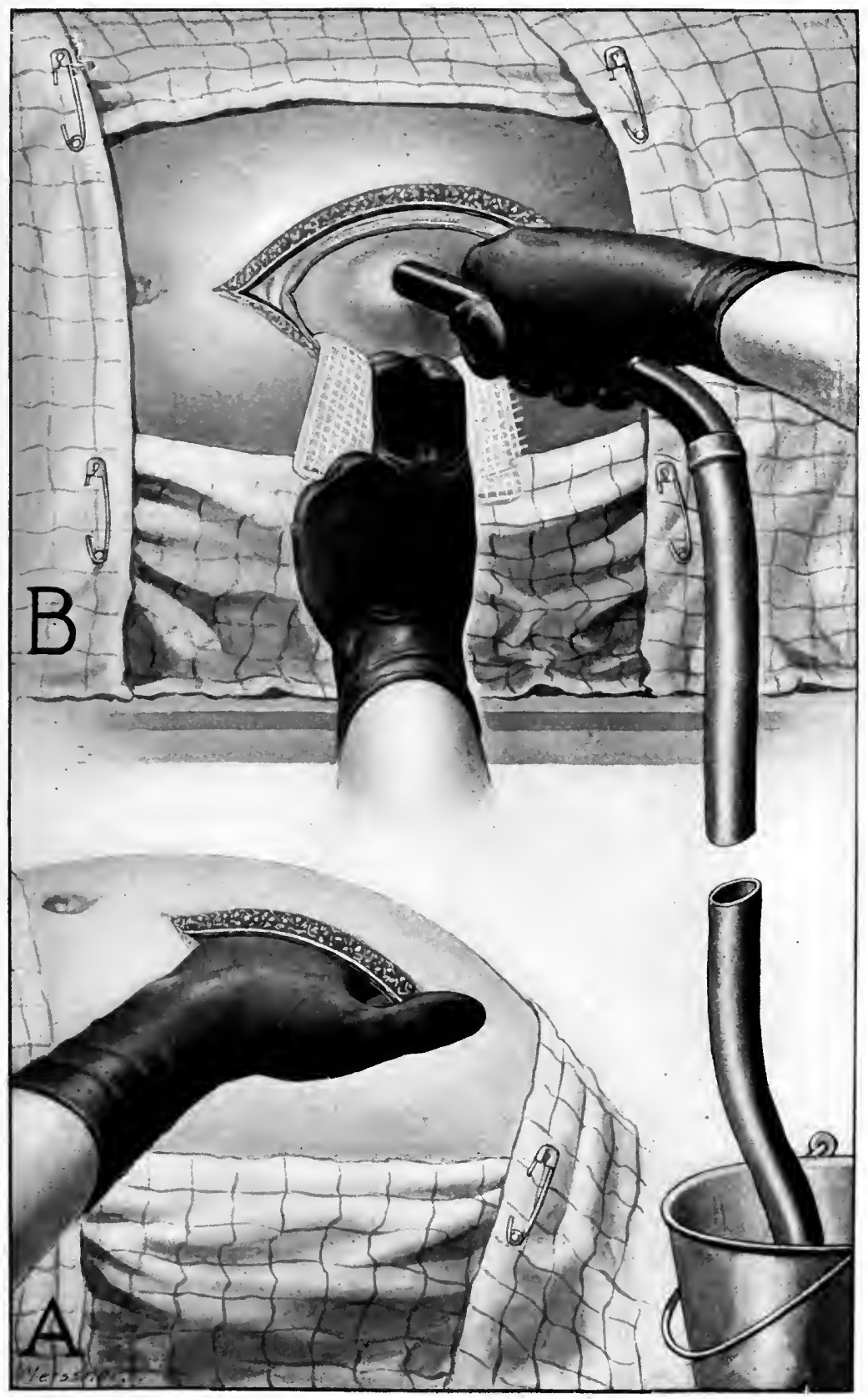




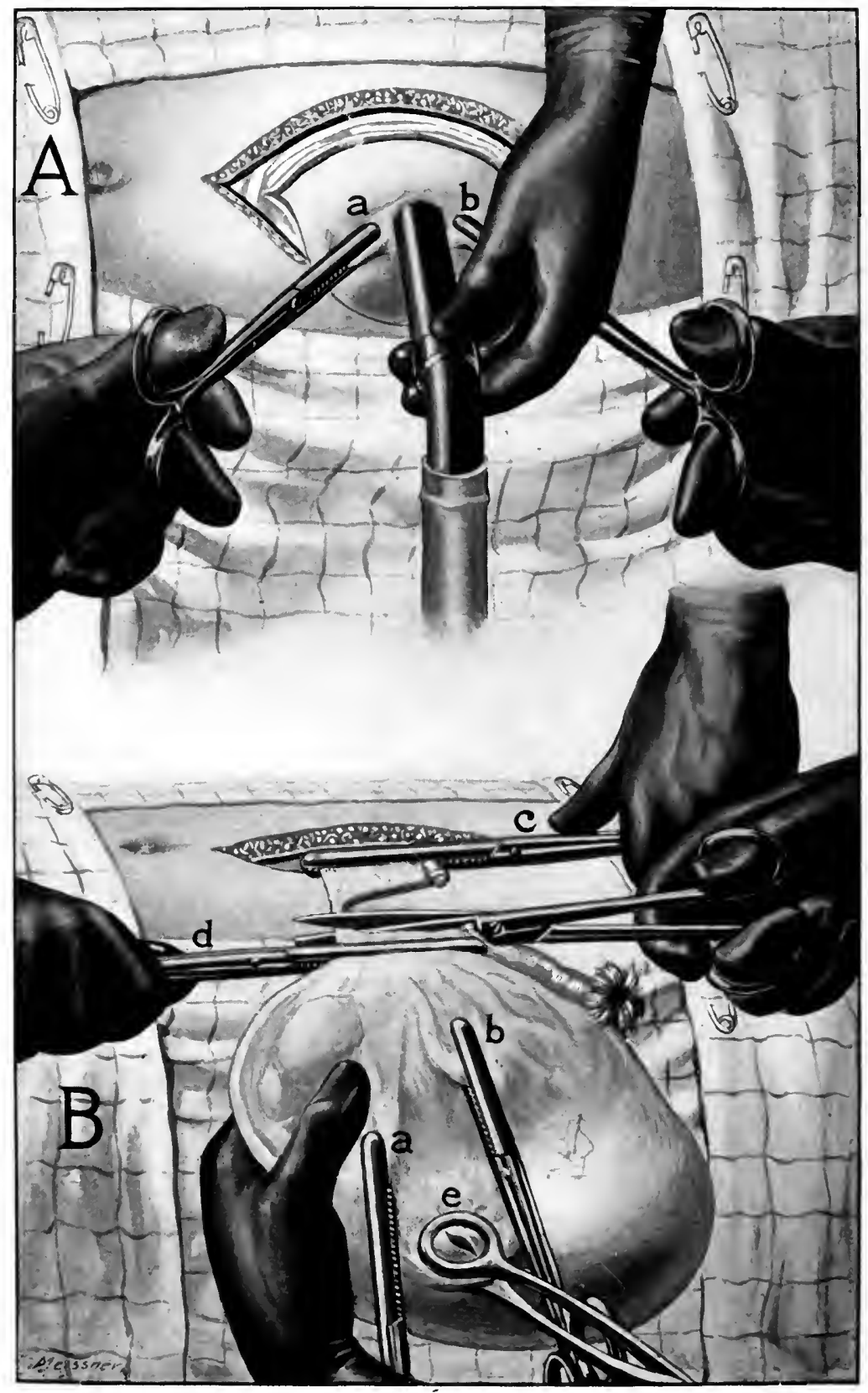




\section{Explanation of Figdre 210.}

A. As the fluid passes from the cyst throngh the trocar and the sac begins to collapse, the trocar is placed in the hands of an assistant, and the operator with a heavy long forceps in each hand seizes the sac on either side of the trocar at points $a$ and $b$, and makes steady traction, so that, as the sac is emptied and collapsed, it may be drawn out through the abdominal incision. During the emptying of the sac it is seized successively at different points by first one forceps and then the other until it is delivered. The delivery of the sac in this manner by traction usually would be rendered impracticable or impossible by adhesions; see $A$, Figure 211.

$B$. The sac has been emptied or nearly emptied. The wound in the sac-wall made by the trocar is closed temporarily by the Nélaton sac forceps $e$. Forceps $a$ and $b$, by which the sac has been drawn through the abdominal incision, are hanging upon the cyst-wall. The pedicle is clamped by two strong forceps, $c$ and $d$, which then are placed in the liands of the assistant, and the pedicle is divided between them by means of scissors in the right hand of the operator, while his left hand holds the tumor steady. 
Figure 211

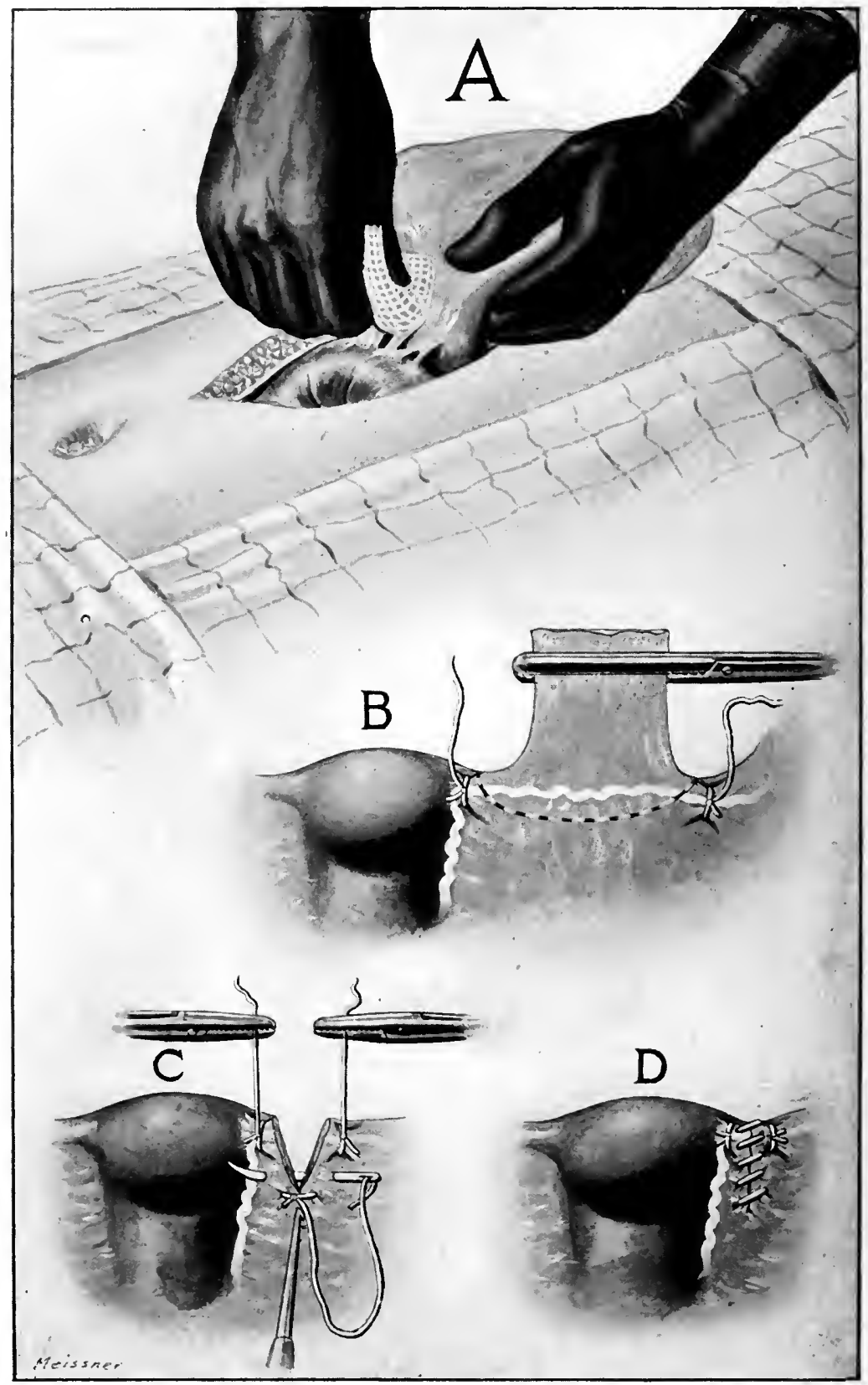




\section{Explanation of Figure 211.}

$A$. If the sac is adherent to adjacent structures, the adhesions must be broken up before it can be delivered through the abdominal wound.

Here adhesions are shown between the sac and the intestine, and are being broken up by strong pressure with the sponge in the right hand of the operator while his left hand holds the sac. Very extensive and firm adhesions may be separated-sponged off, as it were-in this way.

$B$. The sac has been delivered through the abdominal incision, the pedicle clamped, and the tumor removed. Here the pedicle is shown temporarily clamped by a strong forceps. This forceps corresponds to forceps $c$, Figure 210, $B$. Two strong ligatures en masse have been introduced and tied one on each side of the pedicle. These ligatures control the ovarian vessels. The black and white dotted line shows where the incision for the removal of the pedicle is to be made.

$C$. The pedicle has been removed by an incision along the dotted line slown in $B$. The ligatures which surround the ovarian vessels en masse are being held tant each in a pressure forceps, while a tenaculum makes downward traction on the centre of the cut edge of the broad ligament. This shows the cut edge folded and being united upon itself by a continuous suture.

$D$. The suture uniting the wound in the broad ligament is conpleted. For a full description of the technique of this procedure, see Figures 125, 126, 127, 128, and 129. 
thick, gelatinous contents of colloid eysts and the contents of dermoid cysts are usually infectious, and, if brought into contact with the peritoneum, may cause dangerous infection. In order to avoid such contamination it is often safer, when the fluid is known to be infections, to make a long incision and deliver the tumor intact without attempting to puncture the cyst and draw off the fluid.

Emptying and Delivery of the Cyst.-As soon as the peritoneal cavity is opened, the eyst, of peculiar blue or grayish-white color, is seen directly through the opening. The cyst being exposed, the assistant turns the patient partly on the side, so that the abdomen will be directed toward the operator. The trocar, with an attached rubber tube, then is thrust through the eyst-wall, and the fluid is drawn into a bucket provided for the purpose. Figure 209.

As soon as the fluid begins to flow the cyst-wall is seized close to the trocar with the Nélaton or long forceps-one or two pairs-and as the sac empties, the collapsing walls are drawn rapidly through the abdominal wound. A non-adherent monocyst with thin walls is delivered in this way with great ease.

In case of a polycyst, the point of the trocar may, withont complete withdrawal, be thrust successively into one compartment after another until all are emptied and the collapsed sac is delivered.

The obstacles to the delivery of the sac are : 1 . Secondary eysts. 2. Semisolid contents, and solid portions of the cyst. 3. Adhesions.

1. The Secondary Cysts may be too numerous to be tapped by the trocar. Delivery may then be accomplished through a larger incision, or, the trocar having been withdrawn, one or two fingers, and, finally, the left hand, introduced into the sac breaks up the partitions between the secondary cysts-as it were, eviscerates the cyst. During this manipulation the forceps in the right hand must keep the opening into the eystwall drawn well outside of the abdominal incision; this is important, in order to prevent escape of the cyst-fluid into the abdomen.

2. Semisolid Contents found in dermoid and colloid cysts will not run through the trocar. Often tumors are partly eystic and partly solid. A longer incision necessary for the delivery of such non-collapsible tumors, is made upward and to the left of the umbilicus, with scissors, the left index-finger being used as a guide. Figure 56.

3. Adhesions, which are the most common obstacle to the easy delivery of the sac, may be parietal or visceral. The general technique in adhesions is described in Chapters VI. and XXIII. The cyst usually should be tapped and the fluid drawn off before the adhesions are broken. The different parts of the sac from which alherent intestine, omentum, and other structures are to be separated may usually be brought successively into the opening, and the adhesions broken until the tumor is free. If this cannot be done, the incision is lengthened and the adhesions separated in situ. In loosening the adhesions it is well to secure bleeding points, as they occur, by forcipressure, or torsion, or fine catgut ligatures. The tumor having been freed, the operation proceeds as already described for non-adherent tumors. Figure 211, $A$, shows adhesions being separated by sponge pressure.

Ligature of the Pedicle.-The cyst having been drawn through 
the abdominal incision, the pedicle is treated as shown in Figures 128, 129 , and 211.

Closure of the Wound, Drainage, and After-treatment.-These subjects have been considered fully in Chapters VI., VII., and VIII.

The Accidents and Complications are such as may occur in abdominal sections performed for any other purpose.

Extrusion of the bowel during operation should be prevented by the assistants; if it oceur, the bowel should be returned immediately and held inside by broad gauze pads or towels.

Stripping of the parietal peritoneum from the abdominal wall, under the impression that it is an adherent cyst, has occurred even in the hands of an experienced operator. Peritoneum thus detached is apt to slough ; and therefore, if not too extensive, should be removed with the tumor; if it is not removed, there should be drainage of the space between the detached peritoneum and the subjacent structures.

Rupture of the cyst-wall and escape of its contents are harmless if the fluid is innocent; unfortunately, the thin, friable, gangrenous cysts that are apt to contain infectious fluids are the ones most liable to rupture. The clear indication after rupture is thoroughly to irrigate the cavity with normal salt solution- 0.8 per cent. If there is anticipation of rupture, one may pack sponges around and under the cyst to absorb the fluid as it escapes.

Injuries to the intestines, ureter, or bladder are sometimes unavoidable. The bowel is specially liable to be opened in breaking up adhesions. In operating deep in the pelvis the bladder or ureter may be cut even by a careful operator. Injury to the intestine or bladder should be repaired immediately by suture. If the ureter has been cut, the surgeon will have recourse to one of the following procedures: 1. The cut ends may, if practicable, be reunited by end-to-end approximation after the method of Weller Van Hook. 2. The attempt may be made to turn the ureter into the bladder. 3. The ureter may be brought out through the abdominal wound. 4. The kidney on the affected side may be removed. See Chapter XXIII.

Foreign bodies left in the abdomen, such as sponges, forceps, and other instruments, have caused numerons deaths, not only after ovariotomy, but after other abdominal operations. See Chapter VI.

Intestinal obstruction, the principles of drainage, and the after-treatment have been presented in Chapters VII. and VIII.

\section{Removal of Intraligamentous Cysts.}

Ovarian and parovarian cysts which develop between the folds of the broad ligament and are called intraligamentous, have been described fully in Chapter XXXII. The parovarian cyst is easily peeled out of the broad ligament. The papillomatous ovarian cysts may, if intraligamentous, present the greatest difficulties in removal, for such tumors often lie deep and firmly fixed in the substance of the broad ligament, and are therefore difficult to enueleate.

Before attempting the enucleation two ligatures or tenporary lock forceps should be applied, one on the infundibulopelvic ligament, the other on the uterine end of the broad ligament. 'The first cuts off 
the ovarian artery as it enters the pelvis ; the seeond, if deeply placed, cuts off the utero-ovarian anastomosis. In this way the broad ligament and included tumor are deprived of a great part of their bloodsupply, and the troublesome hemorrhage sometimes encountered in the removal of a papillomatous intraligamentous eyst therefore may be avoided measurably. In order to control hemorrhage it may be necessary to ligature also the uterine vessels, or even to remove the uterus.

The tumor may be removed, according to its depth, in one of two way : If it is not very deep, and lies rather loosely in the broad ligament, the ligament and eyst sometimes may be excised and removed together. This procedure is very much like that described in Chapter XXIII. for the removal of the uterine appendages. The other method is that of enucleation, and as indicated above may be extremely difficult and hemorrhagie. As the enucleation proceeds the bleeding points, so far as possible, are secured by fine eatgut ligatures. The sac having been removed, the raw bleeding surfaces between the folds of the broad ligament are packed temporarily with hot gauze sponges to check the oozing. The redundant portions of the ligament may be trimmed off with the scissors, the elges may be turned in and united with deep interrupted or continuous sutures. If the cavity from which the sac was enucleated is too large to be obliterated by inversion and suturing, or the oozing from its surface is uncontrollable, an opening may be made from the bottom of the eavity elose to the uterus directly into the vagina, and the end of a long strip of gauze may be carried through this opening into the vagina, the carity packed full, and the edges of the broad ligament closed over the packing. This leaves the bleeding part entirely covered by peritoneum, renders the raw surfaces extraperitoneal, eontrols hemorrhage, and provides for drainage. Care to avoid the ureters is necessary in the enueleation, in the placing of deep ligatures, and in the incision into the vagina.

The gauze drain, which is the same as that described in Chapter XXVII., may be removed through the vagina in two or three days.

\section{Ovariotomy during Pregnancy.}

An ovarian tumor complicated by pregnancy may give rise to the following accidents: 1. Twisting of the pedicle. 2. Abortion. 3. Obstruction to labor, necessitating Cæsarean section or ovariotomy during labor. From these and other possibilities the danger of labor to child and mother is extreme. Puncture of the eyst, as a temporary substitute for ovariotomy, is permissible only when ovariotomy is inpracticable. The chief danger of both puncture and ovariotomy is from possible sepsis and consequent abortion or premature labor. In the complication of pregnancy the necessity for an early, rapid, gentle, aseptic ovariotomy is apparent. The pedicle always eontains large vessels, and should therefore be tied with special eare.

\section{Vaginal 0variotomy.}

The vaginal route offers no advantage even in cases of small pedunculated non-adherent eysts, and should seldom, if ever, be preferred to the abdominal route. 


\section{CHAPTER XXXV.}

\section{TUMORS OF THE FALLOPIAN TUBES, BROAD LIGAMENTS, ROUND LIGAMENTS, AND URINARY ORGANS.}

\section{TUMORS OF THE FALLOPIAN TUBES.}

The tumors of the Fallopian tubes include myoma, adenoma, adenomyoma, cysts, carcinoma, and sareoma.

Myoma of the tube rarely occurs, seldom obstructs the oviduct, and is commonly too small to be of clinical significance. One case, however, is reported in which the tumor reached the size of a child's head. ${ }^{1}$ Salpingitis isthmica nodosa and tubercular salpingitis have been mistaken for myoma of the tube.

Adenoma, as termed by J. Bland Sutton, ${ }^{2}$ or papilloma, as first described by Doran, ${ }^{3}$ is found not uncommonly. The growth usually begins as a small papilloma or wart, and may attain the size of a large orange. It may present the appearance of a so-called hydatid mole, a multiple cyst, or a cauliflower growth. A frequent complication, according to Sutton, is hydroperitoneum. This results when the abdominal end of the tube is open and the secretion passes from the tube into the peritoneum. When the abdominal end is closed and the uterine end open, there may be a discharge through the uterus. Adenomata frequently undergo malignant degeneration; early removal of the tube therefore is indicated.

Adenomyoma is characterized by small nodular enlargements of the Fallopian tube. It has been described fully by Recklinghausen, and later by Ries, as originating in the remnants of the Wolffian body: The various nodular enlargements of the tube, including salpingitis isthmica nodosa and adenomyoma, may be cansed by a number of pathological conditions. The differential diagnosis between them must be made by the microscope. They cannot be distinguished by clinical examination.

Cysts of the tube are of frequent occurrence, but of little clinical importance. Small pedunculated cysts, known as hydatids of Morgagni, are often to be found at the fimbriated extremity. Numerous minute cysts with thin walls are seen frequently on the mucous surface of the tubes.

Carcinoma, as a primary growth, is very rare in the tube, and, when present, is usually the outgrowth of adenoma. Secondary carcinoma may be the result of extension from the ovary or the body of

1 Sir J. Y. Simpson. From System of Gynecology, Playfair and Allbutt.

2 Surgical Diseases of the Tubes and Ovaries. J. Bland Sutton.

3 Transactions of the Pathological Socicty of London, vol. xxxi., p. 174. Surglcal Diseases of the Tubes and Ovaries. J. Bland Sutton. 
the uterus. It is seldom, if cver, secondary to cancer of the cervix without first involving the body of the uterus.

Sarcoma of the tube is exceedingly rare.

\section{TUMORS OF THE BROAD LIGAMENT.}

Tumors of the broad ligament include myoma, lipoma, cystoma, carcinoma, and sarcoma.

Myoma and Lipoma are pathological curiosities and do not grow to large size. The other growths have been described.

\section{TUMORS OF THE ROUND LIGAMENT.}

Tumors of the round ligament include myoma, fibroma, cyst or hydrocele, sarcoma, and carcinoma.

Myoma and Fibroma are rare, but, according to Coe, are more common in multipara than in nullipara, and more frequent on the right than on the left side. The growth may be intraperitoncal or extraperitoneal. Myoma is commonly pedunculated, hard, of slow growth, painless, not tender to pressure, and may be either smooth or lobulated. When large, it may cause pressure-symptoms; if extraperitoneal, it may be found in the inguinal canal or in the labium majus. During pregnancy it may increase rapidly in size.

The Diagnosis is from ovarian and omental hernia, enlarged inguinal glands, and cysts of the glands of Bartholin. Ovarian hernia is differentiated from myoma of the round ligament by its ovoid form, tenderness on pressure, possibility of reduction on pressure, and by its increase in size during menstruation. Omental hernia may be as hard as myoma and impossible to recognize without an exploratory incision. Enlarged inguinal glands are distinguished by the history of infection, by the lobulated outline, and by the presence usually of more than one enlarged gland. Cysts of the glands of Bartholin are distinguished by their location. In myoma the tumor originally lics above the location of the glands of Bartholin. Exploratory puncture will serve to identify the cyst.

The Treatment is extirpation.

Cyst or Hydrocele is supposed to be developed within the canal of the embryonic round ligament, the embryonic ligament being hollow instead of solid. It may appear in the form of several cysts, or of a collection of fluid either within the inguinal canal or at the external ring. Schroeder reports a case in which there seemed to be a communication between the cyst and the peritoneal cavity; at least the fluid could be forced by pressure inside the abdomen. The writer never has observed a case of hydrocele in the round ligannent.

The Differential Diagnosis is from inyoma of the ligament and inguinal hernia. From myoma it is distinguished by the sense of fluctuation and by exploratory puncture. From hernia the growth is distinguished by not transmitting an impulse on coughing, by failure to reduce by taxis, and by fluctuation.

The Treatment is extirpation of the sac and direct suturing. 
Sarcoma and Carcinoma are so rare as to be of interest chiefly as pathological curiosities.

\section{TUMORS OF THE URETHRA.}

The principal varieties of urethral tumor are caruncle, mucous polypus, condyloma, wart, carcinoma, and sarcoma. The most frequent seat is the meatus urinarius. Carcinoma and sarcoma are apt to occur by extension from the vulva or vagina. Chapter XXV.

FIGURE 212.

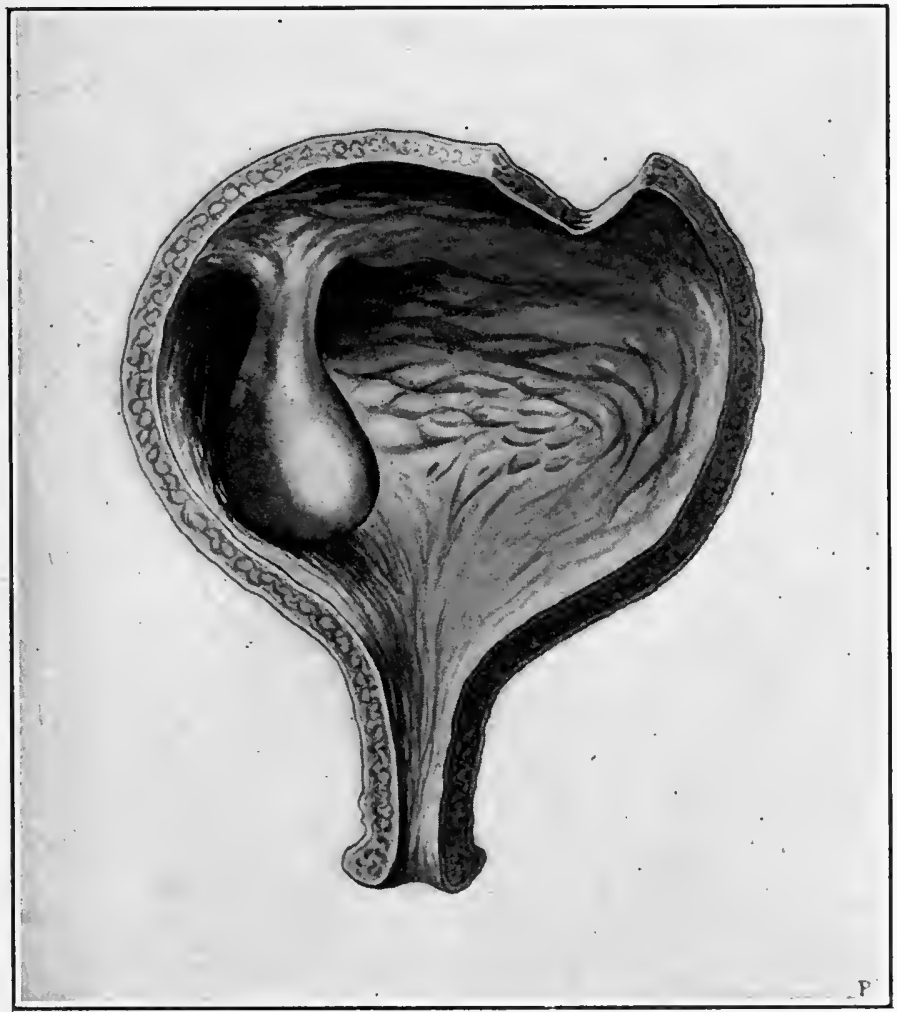

Polypus in the bladder.

Urethral Caruncle is a growth which occurs quite frequently in nervous, irritable women, and, although no age is exempt, it is most frequent near the menopause.

Pathology. ${ }^{1}$ - Urethral caruncles are usually of the granuloma, papilloma, or telangiectatic (dilated blood-vesscls) type.

Etiology.-Irritating discharges from above, especially the discharges of gonorrhoea, senile endometritis, and vulvovaginitis, are the commonly assigned causes.

\footnotetext{
1 M. Lange. Zeitschrift für Geburtshülfe und Gynäkologie.
} 
Diagnosis.-This growth is of frequent occurrence, and is a small, soft, red, friable, hemorrhagic mass situated usually at the margin and on the vaginal side of the meatus urinarius. It may, however, be anywhere in the urethra. There is usually a previous history of pelvic disease. There often is associated great sensitiveness or extreme pain on urination; but, according to Lange, this pain is due not so mueh to the growth itself as to the complieations.

The Differential Diagnosis from Skene's glands has been given in Chapter XXIV. The growths may be differentiated from other tumors in the same region by the constant finding of urethral glands in the caruncle, although the structure of the glands is modified frequently by hemorrhage, round-cell infiltration, and prolapse of the

Figure 213.

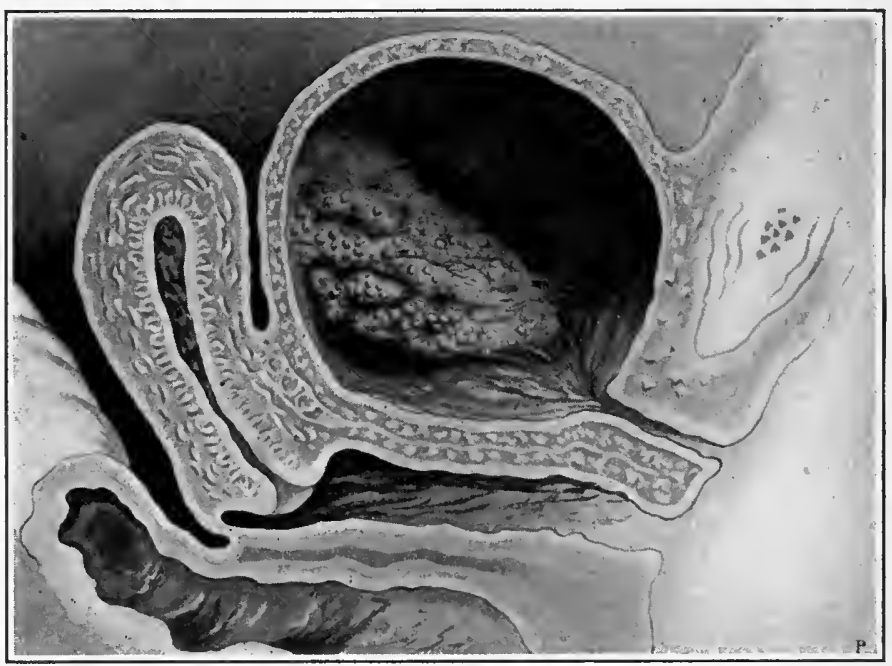

Carcinoma in the bladder.

urethra so that the free surface of the earuncle no longer bears cylindrical nor transitional epithelium.

Treatment.-The treatment is excision with the scissors under the base of the growth, and when practical union of the wound by suture. Excision may require dilatation of the urethra, as described in Chapter III., or Urethrotomy, as described in Chapter XXIV. The actual cautery, though commonly used, is objectionable on account of unreliability and because of its destructive and cicatricial effects. The frequency with which these growths return after surgical removal is due undoubtedly to the failure of operators to treat successfully the causative complications.

Warts, Mucous Polypi, Carcinoma, and Sarcoma follow the same principles of pathology, diagnosis, prognosis, and treatment as when they occur in the vulva. 


\section{TUMORS OF THE BLADDER.}

Tumors originating in the bladder are rare. They occur much less frequently in the female than in the male bladder.

Benign tumors, especially if polypoid, are removed easily through an artificial vesicovaginal fistula. Hæmostasis may, if necessary, be secured by leaving the forceps for a time on the stump. A sessile growth, on account of its inaccessibility and its hemorrhagic tendencies, is much more difficult of removal. Diagnosis is made by cystoscopy.

Ialignant disease is in a majority of cases an extension from the cervix uteri, and in such cases the treatment is wholly palliative. Snall carcinomata originating in the bladder may be removed with some hope of cure.

The differential diagnosis of vesical tumors is from calculi and other foreign bodies in the bladder., Figures 212 and 213 show polypus and carcinoma in the bladder. 


\section{CHAPTER XXXVI.}

\section{TUBAL PREGNANCY.}

TUBAL pregnancy includes all forms of gestation that originate outside the uterine cavity. The old idea, that extra-uterine pregnancy comprised three types-viz., tubal, ovarian, and abdominal-is obsolete; no authentic case of gestation originating upon the peritoneum has ever come to light. Some claims have been made of pregnancy originating in the ovary, but the evidence in support of these claims hardly amounts to proof. So far as known, all ectopic gestations with the possible exception of a very few ovarian pregnancies originate in the Fallopian tube. Pregnancy in a rudimentary horn of a bicornate uterus is virtually a tubal pregnancy.

Ectopic pregnancy formerly was considered a rare condition. Now we know it to be of relatively common occurrence. Pelvic hæmatocele, formerly attributed to other causes, is recognized now, in the vast majority of cases at least, as being due to rupture of the gestation-sac of tubal pregnancy.

\section{Etiology of Tubal Pregnancy.}

It is conceded generally that in at least a large proportion of cases normal fertilization of the ovum occurs in the Fallopian tubes. Spermatozoa have been found in the fimbriated extremity of the tube, and it is probably here that they unite normally with the ovum. The diameter of the human unimpregnated ovum is not over two-tenths of a millimetre; that of the tube, two or three millimetres; although after impregnation the ovule rapidly increases in size, yet under ordinary conditions there is ample time for it to pass into the uterus before disproportionate enlargement takes place. The question of the size of the tube, therefore, is not very pertinent to this discussion.

Tubal pregnancy is common after long periods of sterility. This is explained possibly by the fact that the sterility may have been due to chronic salpingitis, which, by thickening of the tube and destruction of the cilia, prevented the normal passage of the ovule to the uterus and at the same time favored the implanation of it in the tube.

According to Webster, ${ }^{1}$ tubal pregnancy is explained as follows : In the earlier type of mammalian development the uterus was bicornate-that is, composed of two horns, of which the Fallopian tubes in the woman are mere rudiments. In other words, the uterus consisted of two highly developed Fallopian tubes. In some women even now he believes there is a structural or functional reversion to the ancient 
type. According to this theory, the stronger the tendency to reversion the greater the liability to tubal pregnancy. This might explain the fact of repeated tubal pregnancies observed in the same individual.

Peritonitic adhesions and bands obstructing the tubes are frequent in ectopic pregnancy; but whether they cause the morbid condition or result from it, or are only incidental, is uncertain.

There is considerable authentic literature on the transmigration of the ovum from the ovary of one side to the tube of the other. Both clinical and experimental examples have been well attested in which pregnancy occurred in the tube when the ovary on that side was absent. There has been atresia of one tube and tubal pregnancy in the other, but with the corresponding corpus luteum only in the ovary of the closed side. All this proves that the ovum must have passed across the pelvic cavity to the tube in which it finally lodged. Tubal pregnancy, therefore, may occur under most unfavorable conditions.

The following is a summary of the supposed predisposing causes, none of which accounts entirely for the phenomena :

1. Inflammation of the Fallopian tubes causing:

a. Desquamation of eiliated epithelium and denuded patches which obstruct the ovum.

$b$. Loss of peristaltic action of the tube.

c. Cicatricial contraction in the tube.

2. Persistence of foetal type-tube long and tortuous, with small lumen.

3. New formations in and around the tube.

4. Torsion of the tube.

5. Diverticula in the tube.

6 . Conditions giving rise to sterility of long standing.

\section{Formation of Chorion, Amnion, Decidua, and Placenta. ${ }^{1}$}

During the first month or six weeks of tubal pregnancy that portion of the tube in which the fertilized ovum is lodged becomes thinner and very vascular and turgid. The mucous membrane becomes stretched and its folds effaced. The changes that occur in the fertilized ovum after impregnation are identical, whether it be in the tube or the uterine cavity. The membranes by which the embryo is enclosed are similar to those in intra-uterine gestation. These membranes can be studied to advantage in the so-called tubal moles, which are similar in origin to uterine moles. The chorion is shaggy with villi, and resembles in gross and microscopical appearances that found in intra-uterine gestation. The villi appear as clusters of circular bodies. The embryo lies within the amniotic cavity, and the structure of the amnion and its relations to the embryo and chorion are almost the same as in intra-uterine pregnancy.

The formation of the placenta in tubal gestation differs in several particulars from one developed in the uterus. In normal gestation

$1 \mathrm{~J}$. Bland Sutton, in Allbutt and Playfair's System of Gynecology. 
the nterine mucosa and the foetal structures both contribute to the formation of the placenta; but in tubal pregnancy the tubal mucosa plays a very insignificant part. A tubal placenta is derived almost entircly from the embryo.

Contrary to Sutton, Webster has demonstrated a decidua in the tube. It is a curious fact that in addition to this tubal decidua a decidua also forms in the uterus; it is thrown off during false labor, or, if the patient goes to term, is expelled later in small fragments and without pain. This intra-uterine decidua has all the elements of a decidua of normal intra-uterine pregnancy.

The muscular layers of the tube (myosalpinx) at first undergo hypertrophy, but soon that portion to which the placenta is attached becomes thinned, and the bundles of muscular fibres are separated; this favors early rupture.

\section{Frequency of Tubal Pregnancy.}

Tubal pregnancy is not infrequent. Indeed, pelvic hæmatocele, which is not uncommon, is almost invariably the result of ectopic gestation. In thirty-five hundred general autopsies Formad found thirty-five ectopic pregnancies, or 1 per cent. This is, perhaps, the largest percentage reported. The extirpation of diseased tubes has brought to light many cases of tubal gestation that otherwise would not have been recognized, and thereby has added to our estimate of their frequency ; this estimate is increased still further by microscopical diagnosis of the decidua cast off by the uterus in the spurious labor which always occurs at some period of tubal pregnancy.

Repetition of tubal pregnancies in the same individual has been noticed above. Both tubes may be simultaneously pregnant. Twin tubal pregnancy in the same tube and concurrent tubal and uterine gestation have been reported. There is no absolute rule as to the frequency of the condition on either side. Tubal pregnancy has been reported after extirpation of the uterus, the tube still having a connection with the vagina. ${ }^{1}$

\section{Varieties of Tubal Pregnancy.}

Tubal pregnancies occur at the uterine end, the middle region, or near the abdominal extremity of the tube, and are designated respectively :

1. Interstitial pregnancy.

2. Isthmic pregnancy.

3. Ampullar pregnaney.

The subvarieties will be noticed in describing each type. The primary classification depends upon the original site of implantation, not upon subsequent accidents of development or secondary changes. A normal pregnancy may become extra-uterine by rupture of the uterus, as in a case reported by Leopold, ${ }^{2}$ but that does not make it extra-uterine in the sense here considered.

Wendiles. Monatsehrift für Geburtshälfe und Gynäkologie, 1895. Centralblatt für Gynäkologie, No. 4, 1896.

2 Arehiv für Gy näkologie, lvii., 1896. 
1. Interstitial Tubal Pregnancy.-This is by far the least frequent form. Lodgement of the ovum takes place in that part of the tube which traverses the uterine wall, and the foetus develops in a eavity formed in the substance of the uterus. This cavity may open

FigUre 214.

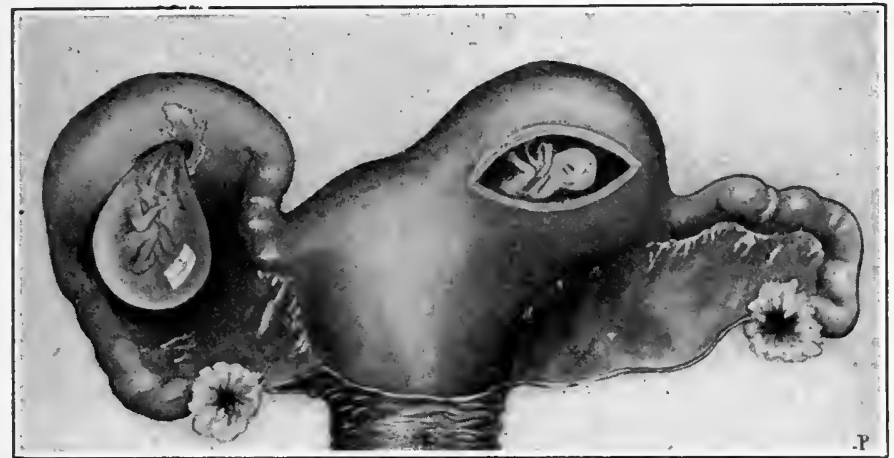

In the right tube isthmic pregnancy, about third month. Embryo with unbroken membranes protruding through the ruptured tube.

In the left tube interstitial pregnancy. The embryo lies in the uterine wall between the left horn of the uterus and the isthmus of the tube.

into that of the uterus, making a tubo-nterine pregnancy; or in rare instances it may extend ontward between the layers of the broad ligaments. Webster ${ }^{1}$ concludes that in some cases of interstitial preg-

Figure 215.

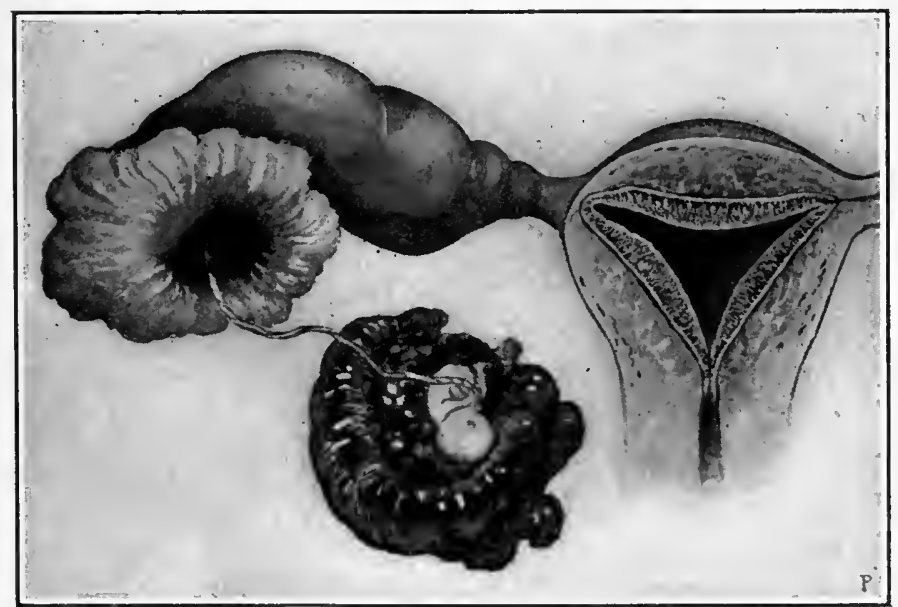

Ampullar pregnancy. Fimbriated extremity of the tube closed by adhesions, which renders tubal abortion impossibie and rupture inevitable. Observe a decidua of pregnancy which has developed in the endometrium.

nancy the ovum develops in the side wall of the uterus, in a diverticulum formed by the incomplete fusion of Mueller's ducts which sometimes occurs in this particular region. Pregnancy in a rudi- 
mentary horn of the nterus, although having a pathology of its own, is yet not nnlike tubal pregnaney. The course and ontcome of interstitial pregnancy will be noticed later in connection with that of the other forms.

2. Isthmic Pregnancy is more frequent than interstitial, less frequent than ampullar, pregnancy. The ovum is lodged in the middle region; and there is gencrally, before rupture, a spindle-shaped dilatation of the tube. So-called pedunculated tubal pregnancy is possible in this part of the tube, and in a few cases has gone to term. This occurs when the ovum is lodged in a diverticulum or angle of the tube. Under such conditions the walls of the tube may be thick or thin in parts, with consequent greater liability to rupture in the thin parts.

3. Ampullar Pregnancy.-This is the common variety. The attachment of the ovum takes place in the ampulla or outer third of the tube. Tubo-ovarian pregnancy is a subdivision of ampullar pregnancy and oceurs when there is prior adhesion of the ampulla to the ovary, so that both coutribute to form the gestation-sac.

\section{Development and Course of Tubal Gestation.}

After the ovum has attached itself to the tubal wall it continues to develop. Naturally the conditions are not so favorable as in normal gestation; the tubal walls are suited less, and contribute less fully to the nourishment and development of the embryo than does the uterus in normal pregnancy. Unless the ovum is inserted well in toward the uterus, as in interstitial pregnaney, the whole tube becomes extravascular, turgid, thinner, and, in most cases, less and less resistant. The margin of peritoneum around the ostium abdominale thickens and forms a ring about the fimbriæ. This ring by the eighth week usually eloses over and shuts the tube. 'The development of the embryo in the tube, so far as the conditions will permit, follows the same course as in the uterus.

As the foetus enlarges, the course of gestation will be modified in one of the following ways:

a. The fotus, if in or near the ampulla, may be expelled from the tube through the ostinm abdominale into the abdominal cavity. This is called tubal abortion.

$b$. The tubc may rupture and partly or wholly discharge the fotus in one of four directions :

1. Into the abdominal cavity.

2. Into the space between the broad ligaments.

3. Into a space formed by adhesions between the tube and ovary.

4. Into the uterus.

c. The foetus may remain in the tube and die before maturity or go on to term.

a. Tubal Abortion neccssarily occurs while the ostium abdominale is still open-that is, before the eighth week. The nearer the implantation of the ovule to the ostium, the greater the liability to 
abortion. In this accident the product of conception-sometimes called tubal mole-is discharged with free hemorrhage through the still open ostium into the abdominal cavity. The hemorrhage gives rise to the formation of intraperitoneal pelvic hæmatocele. The accident may be fatal from shoek and loss of blood, or the patient may recover. In some cases the mole lies quiescent in the tube ; and if only partially detached, it gives rise to repeated and dangerous hemorrhage. The false uterine decidua usually is thrown off with uterine hemorrhage when the tubal abortion takes place. The latter occurrence may be masked, as it were, by the uterine hemorrhage. Tubal abortion does not occur in interstitial and is rare in isthmic pregnancy ; after occlusion of the ostium it can hardly occur even in the ampullar variety.

b. Tubal Rupture.-Rupture of the tube may occur at any period. It is not very usual in the first month, is quite liable to occur in the second, and rapidly becomes less frequent after the beginning of the third, still less in the fourth. It may be due to direct tension on the tubal walls from the growing foetus, but is brought about commonly by hemorrhage between the ovum and the sac. Among the other causes are mechanical violence from falling, junıing, digital examination, and coitus. The rupture usually takes place where the hemorrhage begins-that is, at the placental insertion. The fotal membranes are not necessarily involved in the tear. If the ovum still retains its placental insertion, as it does in rare cases, it may continue to grow. More commonly it is extruded through the ruptured tubal wall and passes into the abdominal cavity; or it may pass downward between the folds of the broad ligament or into a cavity formed by adhesions between the tubal wall and the ovary.

Rupture in interstitial pregnancy may be either into the abdomen, where it is apt to be rapidly fatal from hemorrhage and shock, or into the uterine cavity, where the pregnancy may continue as in normal gestation. Rupture into the uterus may occur much later than the fourth month.

If the fotus in ampullar or isthmic pregnancy is not entirely cut off by rupture or abortion from its nutritive connections, or disorganized by lemorrhage, and especially if the rupture is into the space between the folds of the broad ligaments or into a tubo-ovarian cavity, gestation may go to full term. If the foetus and its investing membranes escape into the general peritoneal cavity, the placenta remaining in the tube, it is possible, though rare, for development to continue.

The notion that a free embryo can escape and ingraft itself on the peritoneum is obsolete. The experiments of Leopold on dogs demonstrate the great absorbing power of the peritoneum, and indicate that no organism thus introduced could survive.

If rupture occurs very early in pregnancy, hemorrhage may be less severe; but after the first month it is apt to be formidable and may cause death in a few hours. If the hemorrhage is slight, we have the common type of retro-uterine hrmatocele, which, if not aggravated by repeated bleedings, is generally encysted and gradually absorbed. In isthmic and ampullar gestation the rupture is often 
downward between the layers of the broad ligament. The blood is then poured out into this eonfined space. The natural tendeney of this confinement is to eheek the hemorrhage. The blood thus accumulated is called a broad-ligament hæmatocele. The more gradual the rupture and the more slight the hemorrhage, the less will be the general and local disturbance. Under such conditions the embryo and its envelopes and placenta will have a better ehance to adapt themselves to their enlarged and enlarging quarters, and may go on to term.

Figure 216.

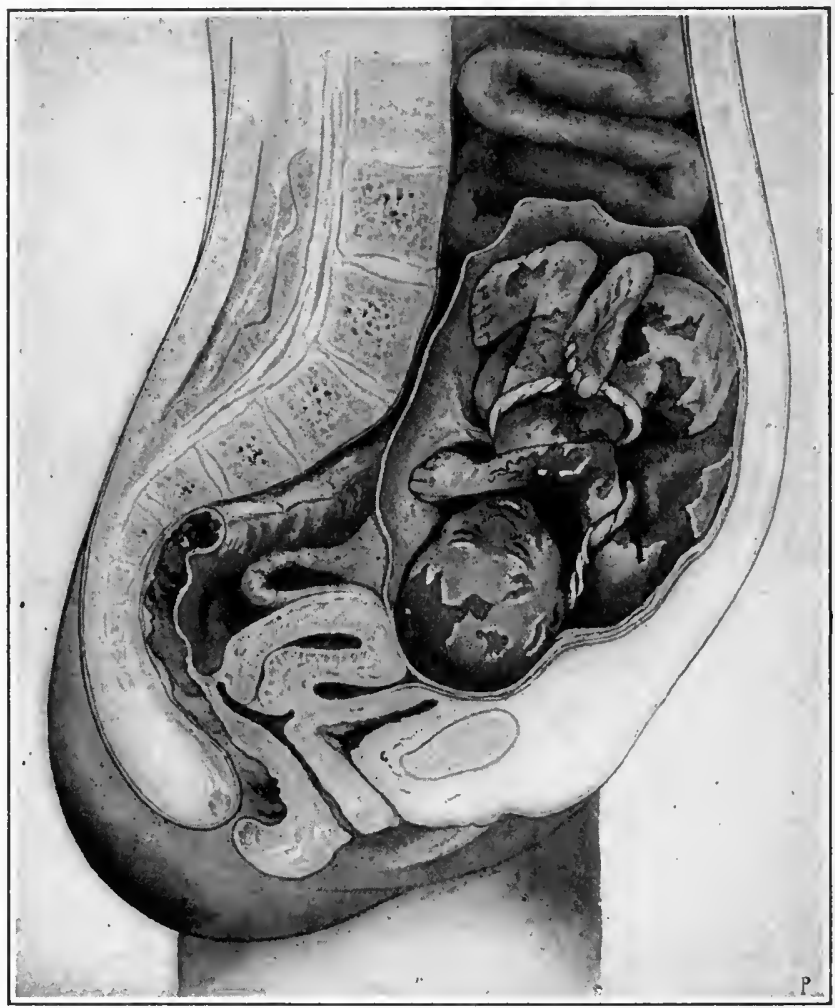

Secondary abdominal pregnancy after term; primarily tubal. The original attachment of the plaeenta may be seen in the ruptured tube. After rupture the embryo developed outward, and became adherent to the peritoneum. Operation at St. Luke's Hospital by laparotomy. Macerated child removed. The enntents of the gestation-sac were purulent and extrcmely fetid. The incision was made directly through the abdominal wall into the sac without invading the general abdominal cavity. Reeovery.

If the eseaped embryo develops in a eavity formed by the two layers of the broad ligament and the outer wall of the tube, the pregnaney is called tuboligamentous. As the foetus develops it presses aside and displaces other organs, the layers of the broad ligament beeome eompressed or thickened and form adhesions to surrounding parts, the peritoneum is pressed upward and stripped from the bladder and abdominal wall, the uterus is displaced to the opposite 
side and, according to the direction of pressure, upward or downward.

If the placenta is situated in the upper part of the tube, so that it is pressed up above the foetus toward the abdomen, forming a tubal placenta prævia, the danger from secondary rupture of the gestationsac into the abdomen is very great; such an accident is apt to be fatal. If the placenta is situated below the fotus toward the mesosalpinx, and pressed down upon the pelvic floor, this danger is less imminent ; for rupture in this situation, since it does not of necessity directly involve the placenta, is attended with less hemorrhage and less risk.

All isthmic and ampullar pregnancies, if left to nature, end with death of the foetus. The tubo-nterine variety of interstitial pregnancy may, as already explained, result in the passage of the embryo into the uterine cavity and subsequent normal gestation.

c. In rare instances the pedunculated isthmic pregnancy already mentioned may go to term in the unruptured tube.

\section{Secondary Changes in Connection with Tubal Gestation.}

If the death of the foetus oceur in the earlier weeks and the mother survive, the subsequent conditions will vary according as the embryo is retained in its envelopes or is cast out free into the abdominal cavity. In the latter case it may be absorbed quickly; in the former, absorption, although slower, is the usual ultimate result. Gestation that has advanced for several months may give rise to a variety of changes. The foetus may undergo a process of mummification and remain encapsulated in the body of the mother for years. Chiari has reported a case in which the nummified foetus was carried for fifty years. It may undergo calcareous degeneration, so-called, and becone a lithopædion, and remain in that state for years. The mummified or calcareous foetus ordinarily gives little trouble; it, however, may become the seat of suppuration, and as a consequence the patient may succumb to exhaustion from peritonitis or blood-poisoning. On the other hand, spontaneous opening of the abscess into the intestine or vagina, or through the abdominal walls, may lead to recovery. A lithopædion has been the mechanical cause of obstruction in labor. The formation of a nterine decidua and its discharge in tubal pregnancy have been mentioned. The nusculature of the uterus undergoes hypertrophy; the organ may enlarge to the size of the second or third month of pregnancy, and then to some extent diminish. If the tubal pregnancy is interrupted by abortion or rupture, the uterus generally at the same time throws off the decidua with a bloody discharge. This spurious labor may occur, however, at any time, and always does occur at some time in the course of the gestation.

\section{Symptoms of Tubal Pregnancy.}

To some extent the symptoms of tubal pregnancy have been indicated. In some cases the menstruation is uninterrupted. The usual signs of pregnancy, such as pigmentation, fulness of the breasts, 
and morning sickness, may be present or absent. During the first eight weeks the ordinary subjective signs of pregnancy usually are absent. Slight uterine hemorrhages may occur at irregular intervals from the beginning. Colicky pains, probably due to uterine contractions, appear toward the end of the second month, and are apt to continue at irregular intervals throughout the whole period of gestation. The signs of interstitial pregnancy are much like those of normal uterine gestation. This is explained by the nearness of the gestation-sac to the endometrium.

When tubal pregnancy goes on beyond the fourth month the external sign of asymmetrical enlargement in the abdomen begins to appear. The pressure-symptoms are much like those of uterine pregnancy. In tuboligamentous pregnancy there is exaggerated pressure on the pelvic organs. Finally the usual signs of fotal life are present, and in the latter months of tubal pregnancy painful foetal movements are common.

The pains of spurious labor resemble those of normal parturition, and are sometimes very deceptive. They may be slight or severe. Cases are recorded in which they continued for days and even weeks, or recurred irregularly for long periods. Cases have been reported in which the sac rupturer into the vagina at the time of spurious labor and the child was produced by the natural passage. Rupture into the intestine and expulsion of the foetus through the bowel have been. reported. This could occur only in the earlier weeks.

Pelvic Hæmatocele a Result of Tubal Pregnancy.-Pelvic hæmatocele is an accumulation of blood in the pelvis consequent upon rupture of a blood-vessel; in rare instances it may be due to traumatism or to rupture of a vessel fron disease of the vessel, but in the vast majority of cases it is the result of tubal abortion or tubal rupt-

\section{Explanation of Figures 217 to $223 .{ }^{1}$}

Figure 217.-Side view. Pregnancy complicated by hæmatocele of both broad ligaments; blood-clot posterior and to either side of the uterus, crowding the cervix forward.

Figure 218. - Retro-uterine hæmatocele extending into both broad ligaments, the mass on the one side rising much higher than on the other, so that accumulation of blood feels to the touch like two distinct masses closely set together and sharply rounded above and at the sides.

Figure 219.- Front view. Hæmatocele of left broad ligament extending anterior to the uterus; felt as a hard tumor in the left vaginal vault close to the uterus; easily felt through the vagina and in the left inguinal region.

FigURE 220.--Retro-uterine hæmatocele lifting the peritoneum high out of the culde-sac of Douglas, and extending into both broad ligaments. Easily felt on vaginal and abdominal palpation.

Figure 221. - Front view. Hæmatocele in both broad ligaments extending in front of the uterus; tumor larger on the right side than on the left, and divided on the left into two segments. The mass on the left side communicates with that on the right, high up in front of the cervix. Uterus pushed back to the posterior wall of the pelvis.

FIGURE 222.- Side view. Retro-uterine hæmatocele, not extending to the sides of the pelvis. Mass felt between the uterus and rectum, lifting the peritoneum out of the cul-de-sac of Douglas and crowding the uterus forward.

Figure 223.-Front view: Hrmatocele of the left broad ligament, lying close to the uterus; easily felt by vaginal touch and by palpation over the left iliac region. Crowds the uterus forward and to the right.

\section{Redrawn from Kuhn.}


Figure 217.

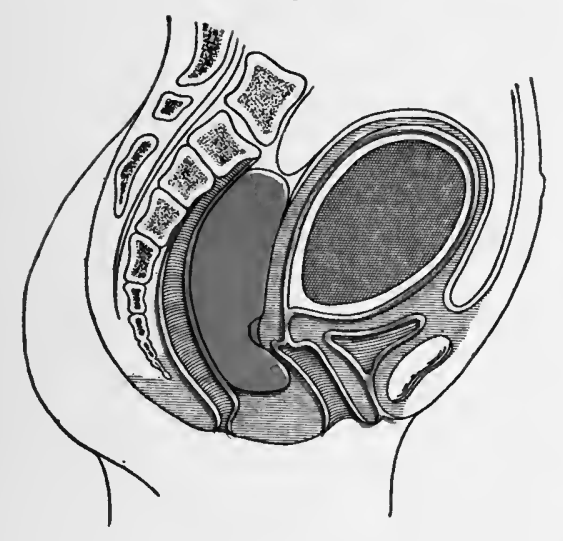

Figure 220.

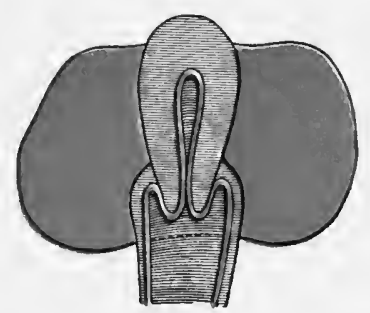

Figure 221.
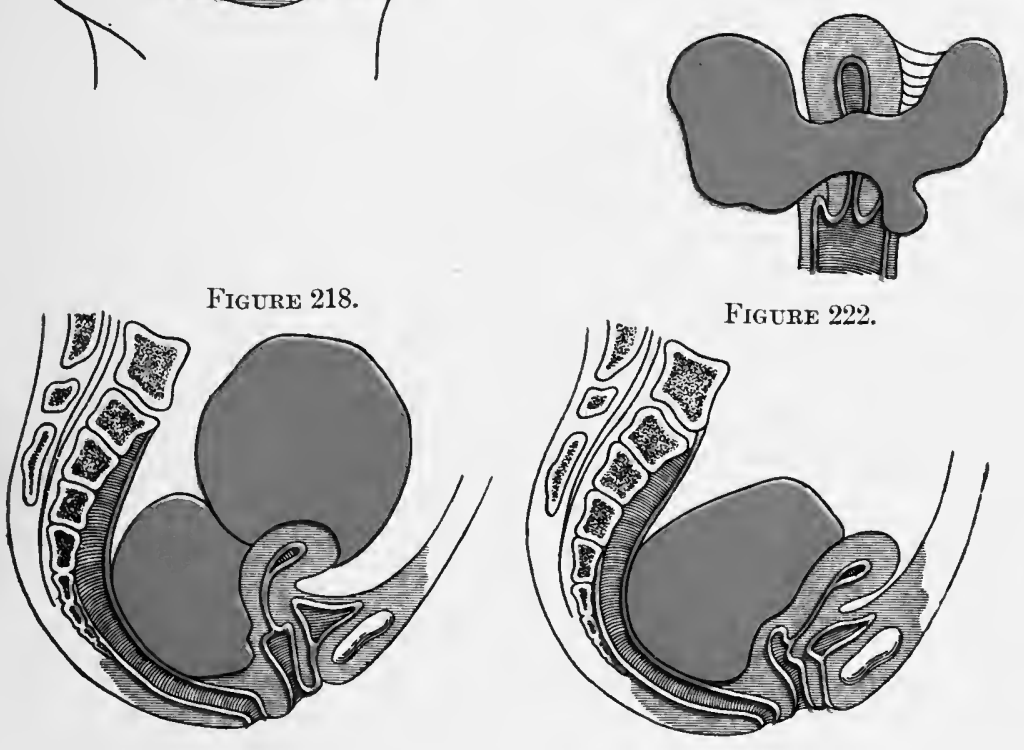

Figure 219.

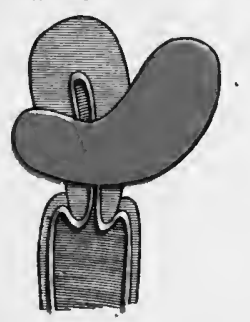

Figtre 223.

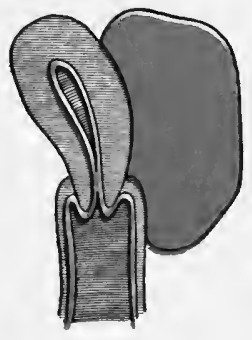


ure, and is therefore an accident of tubal pregnaney; in fact, the symptoms of tubal abortion and rupture are those of pelvic hæmatocele. There are no premonitory signs. Small hemorrhages may give rise to no marked subjective symptoms; even large accumulations of blood, if free in the peritoneal cavity, may cause little or no pain. When the blood is poured out into confined spaces, such as the space between the folds of the broad ligaments, the subjective symptoms which are due to the tearing of the parts are distressing and overwhelming to the patient.

There is sudden and excruciating pain all over the abdomen, and especially about the pelvis; then come nausea, vomiting of bile, cold extremities, bathing of the skin in cold sweat, pinching of the features, rapid and weak pulse, tenesmus, and irritability. In serions cases the shock will be as great as in Asiatic cholera; the pain outbalances every other symptom. The tissues are being literally torn asunder. Such a scene can never be forgotten. The woman tosses to and fro and stains the bedclothing with vomit. The bloodless, pinched features, the bloodshot eyes starting from their sockets, the twitehing of the facial muscles, the clinching of the fingers, the piercing shriek, the agonized bearing-down movement, as if the woman would drive the eontents of her body from her, all combine to make upon the memory an indelible impression. These symptoms may subside and convalescence may be established, with absorption of the clot; or, on the other hand, fresh hemorrhage and acute ænemia with profound collapse, or death, may occur suddenly. ${ }^{1}$

The symptoms are much more pronounced in tubal rupture than in tubal abortion. If the abortion is complete-that is, if the ovum and its envelopes are thrown out completely-the hemorrhage may be comparatively slight and may be walled in by adhesions; under such cireumstances slow recovery may take place. In this way many cases of pelvic hæmatocele reeover without operation. Such results are probably more common in the very early stages of tubal gestation than generally is supposed. In fact, many such cases are unrecognized. There is, indeed, a possibility of moderate and gradual hemorrhage without pronounced symptoms In a very large proportion of cases, however, the abortion is incomplete, and a portion of the ovum or its envelopes is left attached. Repeated hemorrhages, with severe abdominal pain, may, after days or weeks of suffering, unless relieved by operation, end in collapse. If the progress of the case is more rapid, the symptoms closely resemble those of intestinal or gastric perforation and excessive hemorrhage combined. Hæmatncele at first may be unrecognized. If the bleeding be excessive, the early sense of fulness on pereussion and palpation gives way later to the localized signs of a contracted elot.

The hemorrhage from tubal abortion is much less than from tubal rupture.

As already stated, hemorrhage into the space between the folds of the broad ligament is confined, and therefore limited. If the force is sufficiently strong to eause secondary intraperitoneal rupture-that is, 
rupture from the interior of the ligament to the peritoneum-there will be great danger of acute hemorrhage and collapse. If the blood is confined, vesical and rectal tenesmus and other symptoms due to tearing and pressure may overwhelm the patient.

\section{Diagnosis of Tubal Pregnancy.}

In the early period of tubal pregnancy there are no certain means of diagnosis. The patient may have noticed no irregularity in her physiological life, and may have been utterly unaware of her condition until the occurrence of rupture or abortion. This is especially likely to be the case when the abortion or rupture occurs very early after impreguation. Usually, however, it occurs between the fourth and ninth weeks; during this time certain anomalies already mentioned, such as irregular menstruation or pain, may have attracted attention and led to the discovery of an erlarger tube. It is a significant fact in diagnosis that tubal pregnancy often occurs after long periods of sterility. Such sterility therefore is somewhat diagnostic. The microscopical finding of the cast-off decidua together with the history of tubal pregnancy is strongly diagnostic. In the later periods of gestation many of the usual signs of pregnancy are modified and distorted by abnormal conditions.

The diagnosis may be considered with reference to three groups of cases:

I. Late cases in which neither tubal rupture nor tubal abortion has occurred and in which gestation is progressing or has progressed to term.

II. Early cases in which neither tubal rupture nor abortion has occurred.

III. Early cases in which tubal rupture or abortion has occurred.

I. Late cases in which neither tubal rupture nor abortion has occurred, and in which gestation is progressing or has progressed to term, may be recognized by the following characteristics:

1. Uterus enlarged to the size of two months pregnancy.

2. Formation of a tumor at one side of the uterus, which, like the uterus in normal gestation, gradnally increases in size, although the size of the mass does not always correspond strictly to that of normal gestation.

3. As gestation progresses the foetus may be palpated externally. Palpation may disclose occasional contractions in the gestation-sac; the foetal heart-tones become distinct at about the same time as in normal pregnancy.

4. Intermittent false labor at nine or ten months, followed by cessation of circulation in the placenta and death of the child, and finally by marked diminution in the size of the tumor.

5. General sepsis from absorption of decomposing products of gestation in most cases.

II. Early cases in which neither tubal rupture nor abortion has oceurred may be diagnosed by the following signs : 
1. Cessation of menstruation for one or two months, and other signs of pregnancy, such as nausea, mammary changes, and venosity of the vulva and vagina.

2. Ovoid mass, corresponding in position to the right or the left tube-not very sensitive to pressure.

3. Intermittent contractions of the mass-not always felt.

4. Death of the foutus in some cases without tubal rupture or abortion may be followed by absorption of the amuiotic fluid, with rapid decrease in the size of the tumor.

III. Early cases in which rupture or abortion has occurred are characterized by symptoms same as those of group II., followed by sudden onset of extreme pelvic pain, evidences of acute, alarming, and sometimes fatal hemorrhage and sudden appearance of a pelvic tumor-hæmatocele.

\section{Differential Diagnosis of Tubal Pregnancy.}

The following outlines will enable the reader to distinguish tubal pregnancy from numerous conditions, for which it sometimes is mistaken.

Ruptured tubat pregnancy. Hæunatocele.

1. No initial history of infection.

2. Great rapidity of pulse.

3. Temperature at first subnormal, later may be elevated.

4. Pain excruciating, but subsides after few hours.

5. Symptoms of hemorrhage:

a. Sudden, acute anæmia.

b. Weak, rapid heart.

c. Dyspncea.

d. Sighing respiration.

$e$. May be syncope.

Ruptured tubal pregnancy. Hrmatocele.

1. History of pregnancy.

2 . Sudden onset.

3 . Hemorrhage may cause collapse.

4. Temperature normal or submormal at first.

5. Usually mass soft : later hard.

6. Fever may finally follow appearance of hæmatocele.

7. Uterine decidua.

8. No lencocytosis at time of rupture.

Ruptured tubal pregnancy. Hæmatocele.

1. Urgent symptoms at onset.

2. Development rapid.

3. Not very sharply circumscribed.

4. Immobillty of mass.

5. Signs of pregnancy precede formation of mass.

6. Uterine decidua.

Ruptured tubal pregnancy. Hrmatocele.

1. No pre-existing tumor.

2. History of pregnancy.

3. Tumor not smooth and tense.

4. Uterus somewhat cnlarged.

5. Uterine decidua.

\section{Tubal pregnancy.}

1. Before rupture, gestation-sac harder.

2. Fluctuation and ballottement absent.

3. Uterus slightly enlarged. Tumor separate from uterus and crowds it to opposite side of pelvis.

4. Unusual history.

5. Tubal abortion, or rupture between fourth and ninth week usual.

6. Discharge of uterine decidua with false labor-pains occurring usually at time of tubal abortion.

\section{Ruptured pyosalpinx.}

1. Initial history of infection.

2. Pulse not so rapid.

3. Rise of temperature marked from onset.

4. Pain less intense but continuous.

5. Usually absent.

Pelvic peritonitis and cellulitis.

1. History of infection.

2. Onset less sudden.

3 . No hemorrhage.

4. Temperature elevated.

5. Usually mass hard; later may soften.

6. Precedes.

7. Absent.

8. Always leucocytosis in early stages.

tterine and ovarian tumors.

1. Absent.

2. Slow.

3. Mass sharply circumscribed.

4. Mobility usual.

5. Absent unless complicated by pregnancy.

6. Absent.

Hemorrhage into ovarian cyst.

1. Pre-existing tumor.

2. Absent.

3. Tumor smooth and tense.

4. Not so much enlarged.

5. Absent.

1. Uterus softer

Normal pregnancy.

2. Fluctuation and ballottement later.

3. Tumor is enlarged uterus.

4. Nothing unusual in history

5 . Does not occur.

6. Does not occur. 
Uterine displacements, pregnancy in one horn of a bicornate iterus, perfuration of the stomach or bowel, and rupture of an aneurism have been mistaken for tubal pregnaney, but none of these conditions produce the symptom group outlined in the above paragraphs on Diagnosis. In cases of hæmatocele Huppert's test for urobilin will give positive evidence of the absorption of blood and the elimination of it by the kidneys.

\section{Prognosis of Tubal Pregnancy.}

The outlook is always doubtful and serious. Spontaneous recovery is, however, not uncommon. In former times pelvic hæmatocele was not, in the majority of cases, recognized as related to tubal pregnancy, and therefore usually was treated on the expectant plan. Under such conditions spontaneous cures were frequent. Our knowledge of the true pathology and the consequent greater frequency of operative interference do not change the fact that spontaneous recovery often will occur just the same, even though the name of the condition has been changed from hæmatocele to tubal pregnancy. However, recovery occurs much more frequently with than without operation In two hundred and seventy-eight cases for which there had been no operation, collected by Schauta, Martin, and Orthmann, one hundred and eighty-seven, or a little over two-thirds, died; while five hundred and seven, or 80 per cent., of six hundred and thirtysix cases operated upon, survived. ${ }^{1}$

\section{Viability of the Child at Term.}

In all cases of ectopic pregnancy at term the viability of the child as compared to the life and welfare of the mother is a very secondary matter. Few children are produced alive, and fewer still survive many days. The few who do survive are physically and mentally inferior. Harris ${ }^{2}$ collected a number of cases of living children of extra-uterine pregnancies, and in 1895 reported to Orthmann that of fifty-seven whose histories he had been able to trace only five survived their second year.

\section{Treatment of Tubal Pregnancy.}

From the observations already made, it follows that the treatment of tubal pregnancy, as a general rule, will be operative. The safety of the patient is immeasurably greater if the diagnosis is made and the operation performed in the earlier wecks, before the time of tubal abortion or rupture. Unfortunately for the majority of cases, the first intimation of the diagnosis comes with one of these accidents.

The treatment will vary with the varying conditions. The four possibilities are :

1. That the diagnosis has been made before the time of rupture or abortion.

1 A. Martin. Die Krankheiten der Eileiter, 1895.

2 American Journal of the Medical Sciences, August-September, 1888. 
2. That rupture or abortion has just occurred.

3. That the patient has survived the immediate effects of rupture or abortion, and that gestation has ceased with death of the foetus.

4, That rupture has occurred, but the foetus is alive and gestation is still going on.

1. Treatment before Rupture or Abortion.-The tube and its contents should be removed immediately. Only by this means can the woman be protected against the extreme peril of continued tubal gestation. The danger of the operation is not greater than removal of the utcrine appendages under other cireumstances; the technique is the same. In very many eases tubal pregnaney is unrecognized until the abdomen has been opened upon the diagnosis of a supposed hydrosalpinx or pyosalpinx. 'This fact, as Penrose says, emphasizes the value of the rule to operate for all gross lesions of the tube.

2. Treatment Immediately after Rupture or Abortion.-The general rule is to operate withont delay. It may be unwise to wait for reaction from the shock and hemorrhage, for hemorrhage is the very indication for interference. Indeed, the immediate object of the operation is to stop the hemorrhage.

'The writer has recorded two cases in which the patients were in apparent collapse, and for this reason it was not deemed wise to operate unless there should be a tendency to rally. In both cases slow improvement and final recovery followed the operation. A few months later the products of conccption disappeared by absorption. These cases show that without operation the prognosis, even in the most extreme conditions, is not hopeless.

Operation.-The abdomen is opened as described in Chapter VI. The tube and, together with it, the broad ligament, are grasped and pulled into the wound; two pairs of strong hæmostatic forceps are placed on the broad ligament-one on the infundibulopelvic extension of it, near the pelvic wall, the other close to the uterus; this will control the ovarian artery at its point of entrance both to the ligament and to the uterus. Ligatures arc substituted immediatcly for the forceps, the tube removed, and hæmostasis secured as described in Chapter XXIII. If there is dead space betwcen the folds of the broad ligament, it may be obliterated by fine buried eatgut sutures; or if too large to be sutured, it may bc sutured into the abdominal wound and drained or may be drained through the vagina, as explained in Chapter XXVII. for drainage of intraligamentous. myomata.

The free infusion of normal salt solution, two or more pints, by hypodermoelysis, preferably under the breast, or the introduction of it into the abdomen before closure of the wound as described in Chapter VII., is indicated strongly. This solution, which may be used, according to the indieation, before, during, and after the operation, has turned the seale for recovery in many a desperate case. If the hemorrhage has been great, Frankenthal advises direet transfusion of blood.

3. If rupture or abortion has occurred, and the patient has reeovered from the immediate effects of it, and gestation has ceased with 
death of the foetus, there may be spontaneons cure, with absorption and disappearance of the products of conception. Under these favorable conditions, especially if there be continuous gradual improvement in the symptoms, one may adopt the plan of watchfill expectancy. Frankenthal says: "Treat conservatively only those cases seen some time after primary rupture, when you are reasonably certain of the death of the foetus, when the alarming symptoms have subsided, and when, presumably, absorption is going on." Intraligamentous rupture occurring within the first three or four weeks of gestation is rather liable to be followed by recovery and absorption. One must, however, be prepared to operate promptly upon the least cridenee of secondary rupture and hemorrhage or upon the onset of infection. Even in uncomplicated cases of this third division, however, it is permissible and possibly safer to operate, and thereby relieve the woman of the danger incident to the presence of a dead foetus in the pelvis.

Previous to the fourth or fifth month the entire gestation-sac and its contents may be removed usually withont great danger of fatal hemorrhage. At least the hemorrhage, if troublesome, may be controlled by ligature of the ovarian vessels, or, if necessary, of the ovarian and uterine vessels. After the foetus has been dead for some time there is little or no danger of hemorrhage in separating the placenta.

4. If gestation has advanced beyond the fourth or fifth month, and the child is living, the removal of the fotus, together with the placenta and gestation-sac, is practicable in only a small minority of cases, and then only in the hands of the expert operator. The conditions favorable for this radical operation are found in the rare pedunculated tubal pregnancies already mentioned, in which gestation may go to term without rupture, and in other rare cases in which the sac can be isolated, brought through the wound, and a pedicle formed, or its attachments separated without excessive hemorrhage. Ligature of the ovarian and uterine vessels, even if practicable, does not control the terrible hemorrhage which at this period and under ordinary conditions invariably follows separation of the placenta. The surgeon must assume the great responsibility of a decision, when the abdomen is open, whether he will attempt removal of the gestation-sac. The deliberate attempt to remove it has resulted many times in uncontrollable and fatal hemorrhage. In opening the sac the operator accidentally may incise or separate the placenta and find himself face to face with a most formidable, if not unmanageable, hemorrhage, Compression of the aorta and ligature of the uterine and ovarian arteries, if promptly and skilfully executed, may or may not save the patient's life. In the vast majority of eases in which gestation is in progress beyond the fourth or fifth month the operator nust be content to incise the sac, remove the foetus, stiteh the sac to the abdominal wound, and leave the placenta. J. Bland Sutton proposes to close the sac with sutures instead of stitching it to the wound. This is done in the hope that the placenta will undergo atrophy or absorption. The danger of infection in a sac thus closed would be considerable. The more usual 
and safer plan, therefore, is to leave the placenta and establish gauze or tubular drainage. After two or three weeks, when the placental circulation has ceased, the wound may, if necessary, be reopened, and the placenta taken away, but the more common and approved practice is to let the placenta disintegrate and drain away as débris.

Some operators in the fourth class of cases prefer to delay operation until after term, when the child has died and the placental circulation has ceased. The products of conception may then be removed entire, with the minimum danger of hemorrhage. This plan necessarily involves the dangers incident to the continued presence of an extra-uterine foetus, and should include as a positive requirement that the patient remain in a hospital.

The Abdominal Versus the Vaginal Route.-If the prodncts of conception are low in the pelvis, and quite accessible, if gestation has not passed beyond the eighth week, and if the tube is movable so that it can be brought ont readily through the vaginal wound, it is permissible to operate by the raginal route. If the gestation-sac is between the folds of the broad ligament and the child has been for some time dead, and the placental circulation therefore has ceased, one may remove the products of conception, unless too large, and drain through the vagina. In all other cases the difficulty of controlling hemorrhage through the vagina is too great, and the abdominal route therefore is to be preferred. The difficulty, not to say impossibility, of ligaturing the infundibulopelvic ligament through the vagina is an objection to the vaginal route. Before making the vaginal incision it is sometimes well to open the abdomen by a short incision, so that if hemorrhage occurs no time will be lost in controlling it through the abdominal incision. 


\section{CHAPTER XXXVII.}

\section{EMBRYOLOGY OF THE GENITALIA AND CONGENITAL MALFORMA'TIONS.}

\section{EMBRYOLOGY.}

Ax appreciation of the embryology of the genitalia is essential to an understanding of malformations and is important in the study of pathological growths. The various phases of embryological development cannot be madc clear by studying the human embryo alone, because in man the transitions from one morphological stage to another are less accessible for study and in many details not apparent; therefore some of the following statements are derived from the embryology of lower vertebrates. The development of the reproductive system is allied so closely to that of the excretory organs that they must be considered together. The general subject inay be skeletonized as follows :

\section{DEVELOPMENT OF THE WOLFFIAN RIDGE.}

Early in embryonic life a portion of the mesolerm (connectivetissue layer) known as the intermediate cell-mass becomes thickened and projects into the cavity of the body or colum (later peritoneal cavity). This projection forms a ridge which is concerned in the development of the genito-urinary system, and is known as the Wolfian ridge.

\section{DEVELOPMENT OF THE GENITAL RIDGE.}

On the inner surface of the Wolffian ridge a secondary projection (sexual gland) is formed which derelops later into the testis in the male or the ovary in the female, and is called the genital ridge.

\section{EMBRYOLOGY OF THE EXCRETORY ORGANS.}

In connection with the Wolffian ridge four elementary struetures appear as follows:

I. Wolffian ducts ; one on each side.

II. The pronephros or primitive kidney in connection with the Wolffian duct; one on each side.

III. The mesonephros (Wolffian body); one on each side.

IV. The metanephros; one on each side. 


\section{Development of the Wolffian (Pronephric) Ducts.}

The pronephric ducts, commonly known as the Wolfian ducts, begin as thickenings of portions of the intermediate cell-masses, one on each side. 'They undergo complete development in man and have an important part in the formation of the urogenital system. The thickenings project as solid rods of eells from before backward, beginning near the region of the heart and extending toward the cloaca-that is, the lower portion of the intestine. They lie imbedded in the substance of the Wolffian ridges. Soon after their formation each of these ccllular rods at a median point develops a cavity which extends in both directions and thus converts them into ducts, the posterior ends of which invariably open into the cloaca. As already mentioned, the thickenings from which the Wolffian ducts arise are of mesodermal origin, but in early development the posterior ends of the ducts fuse with the overlying ectoderm. This connection is temporary, and while it lasts gives the Wolffian ducts the appearance of having originated from the ectoderm; some say it does so originate. The occasional persistence of this connection may explain the presence of epithelial structures in tumors where connective-tissue structures naturally would be expected, and vice versa.

\section{Development of the Pronephros.}

The pronephros develops only to a very primitive stage in the human embryo and finally is resorbed, although traces of it have been observed in mature man. In embryos of abont $3 \mathrm{~mm}$. it forms on each side as two tubular invaginations of the epithelium lining the body-cavity and projects into the substance of the Wolffian ridge. Each of these tubules ends blindly at one extremity and at the other opens into the body-cavity. The pronephros has no physiological significance in man, but there are somc pathological growths, for example, of the kidney, which may be explained as persistence and proliferation of some of the cells of the pronephros, but which otherwise would be unexplainable.

\section{Development of the Mesonephros-Wolffian Body.}

The mesonephros is formed by a number of tubules running transversely from the Wolffian duct toward the cœlum on each side of the body and penetrating the Wolffian ridge. These tubules have a temporary excretory function in the luman embryo, and in some vertebrates they are the chief permanent renal organs throughout life.

\section{Development of the Metanephros.}

The metanephros, which in man becomes the permanent kidney, develops from an outgrowth which begins in the dorsal aspect of the 
Wolffian duct and in the tissue surrounding it. In the embryo of $5 \mathrm{~mm}$. the Wolffian duct presents a tubular eminence just above the point of its insertion into the cloaca ; this outgrowth becomes the ureter. The extremity of the ureter branches into numerius tubules, which again branch, and so on until each terminal branch ends in a distinet vesicle (primitive vesicle). The vesicles give rise to several

FIGURE 224.

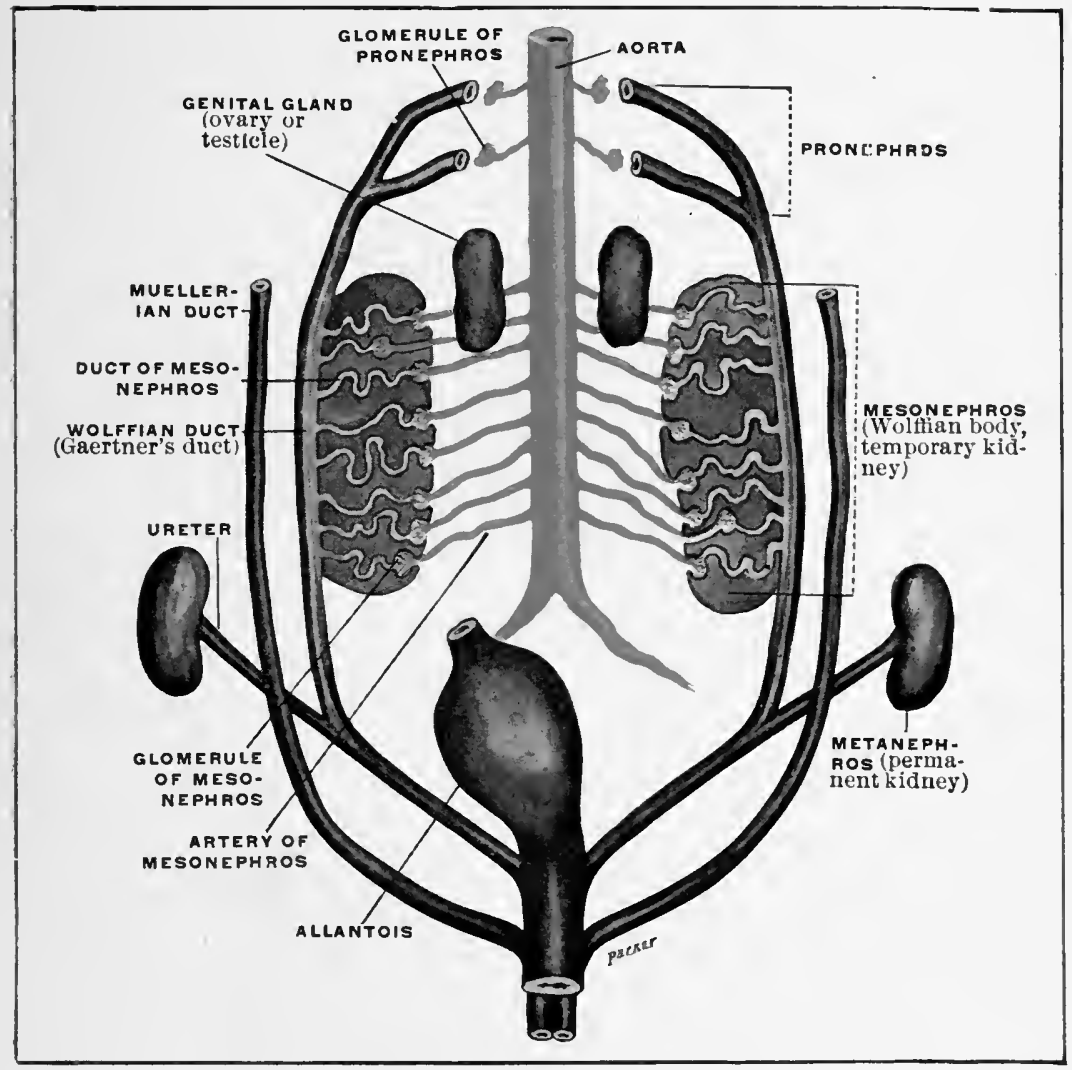

Excrementary organs of the embryo, showing genital gland, pronephros, mesonephros, metanephros, aorta, and allantois.

tubules which become the uriniferous tubules of the kidney ; they are coiled upon themselves in the form of the letter S. 'The lower portion of each tubule is surrounded by proliferating cells into which the renal artery branches so as to form the glomeruli of the kiclnev, and the walls of this lower portion of each tubule become converted into a capsule of Bowman. Additional tubules form from the primitive 
Figure 225.

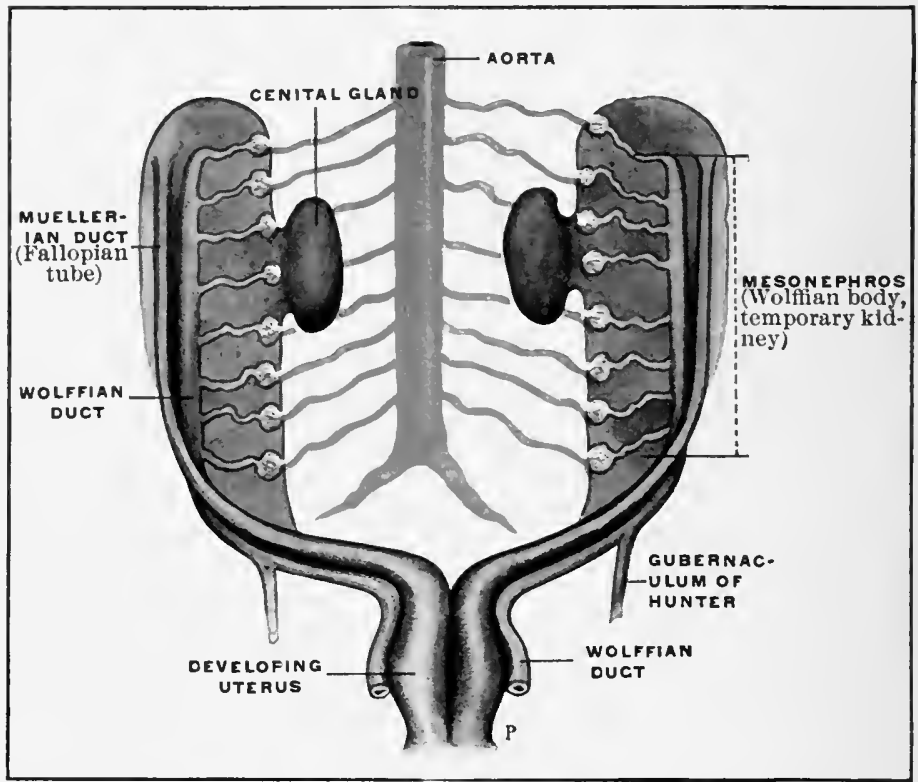

Reproductive organs of the embryo: lower part of Mueller's ducts coalescing to form the uterus and vagina. Differentiation of sex is just beginuing by the derelopment of the genital gland into an ovary.

Figure 226.

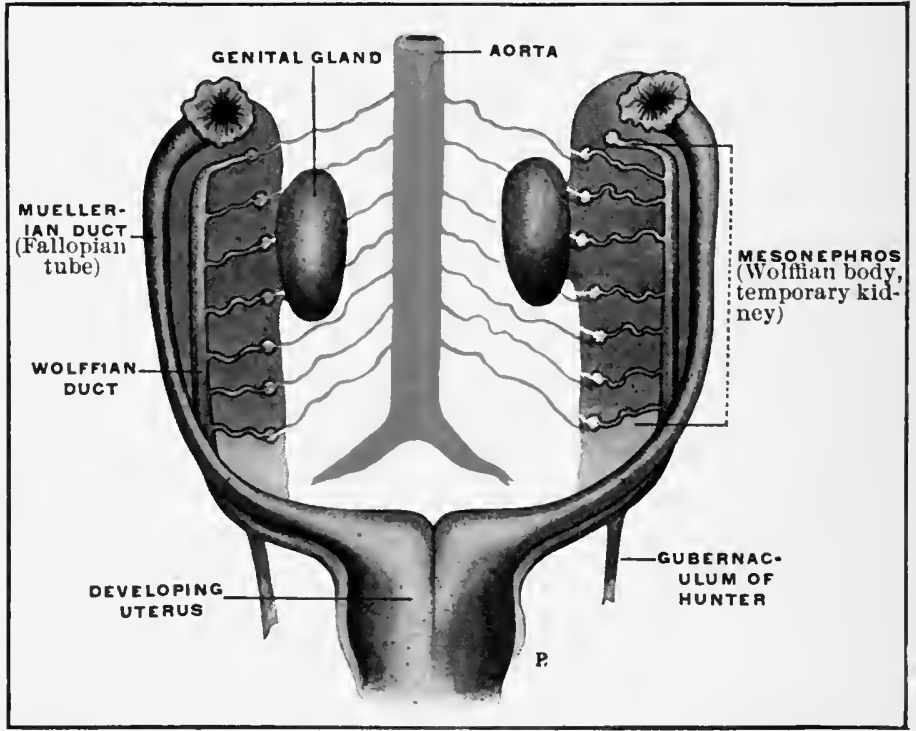

Same as Figure 225. Uterus further developed, but Mueller's ducts still separable; ovaries and Fallopian tubes more developed. Wolffian bodies and Wolffian ducts undergoing atrophy. 
Figure 227.

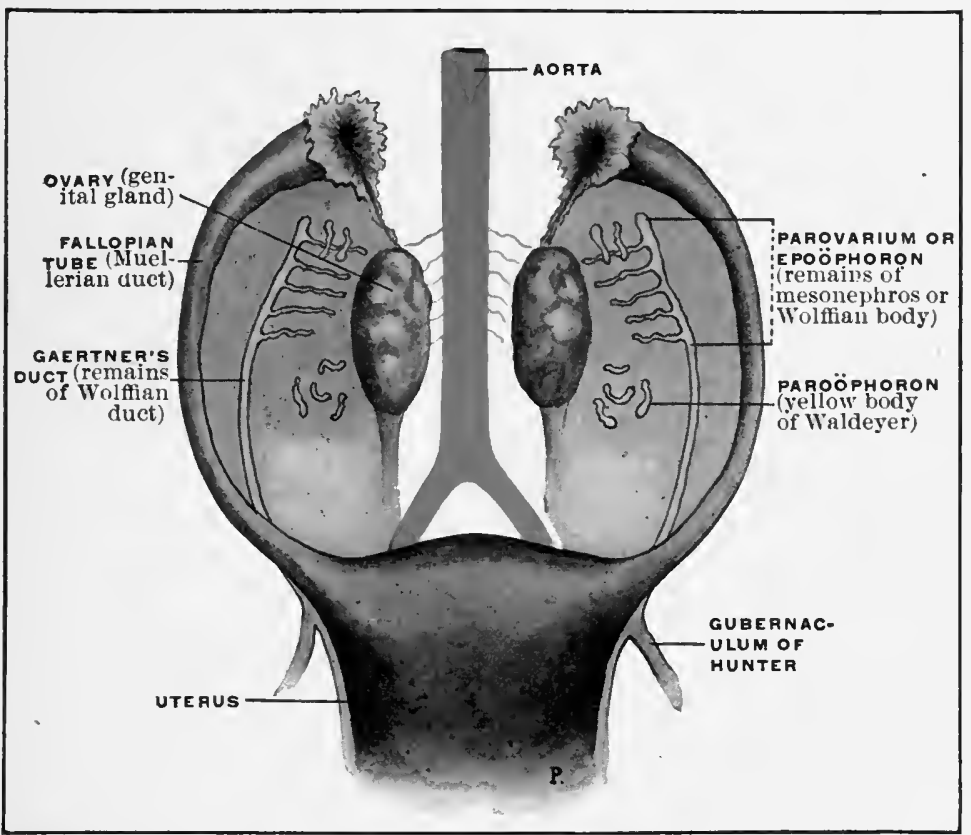

Same as Figure 226. Each rudimentary Wolffian duct and body now appear as the parovarium above, and the paroöphoron below. Uterus, Fallopian tubes, and ovarles almost fully developed. Lower part of Iueller's ducts persist as developed uterus.

vesieles, giving rise to a large number of glomeruli. The vesieles themselves elongate finally and form the collecting tubules, which open separately into the pelvis of the kidney. The number of the primitive vesicles which form in the human kidney is about eighteen, and each one corresponds to a fully developed lobe.

\section{EMBRYOLOGY OF THE GENERATIVE ORGANS.}

In elose developmental relations with the organs already deseribed, which are mainly excretory, may now be introdneed the embryonic structures which have more especially a generative significance; they are :

I. The ducts of Mueller.

II. The genital ridge.

III. The urogenital sinus.

\section{Development of the Ducts of Mueller.}

When the mesonephros (Wolffian body) has reached the height of its development, invaginations form in the peritoneum eovering the 
Wolffian ridge, and these invaginations are the beginnings of the Muellerian ducts, which are imbedded in the first part of their course in the substance of the ridge itself. The forming ducts become disconnected from the peritoneum except at one small point, and continue to grow as two solid rods of eells by proliferation of their ends. Presently they aequire a lunen which develops throughout their length.

The duets of Mueller are situated to the outer side of the Wolffian ducts, except in the lower portion of their course, where they are situated between then. In human embryos of $22 \mathrm{~mm}$. they have attained their full length, and finally they fuse together at the lower end to form the uterus and vagina, the upper portion, however, remaining separate to form the Fallopian tubes.

\section{Development of the Genital Ridge.}

As already stated, one portion of the intermediate cell-mass on each sicle of the body eavity constitutes the Wolffian ridge, and the other portion, known as the genital ridge, forms the sexual glandthat is, the ovary in the feniale and the testis in the male. The epithelial cells of that region assume a high eolumnar form and grow several layers thick, the conneetive tissue beneath proliferating at the same time so as to form a distinct elevation or ridge. This thickened epithelial layer (germinal epithelium of the ovary) is coneerned in the formation of the egg cells. Some of the cells of the germinal epithelium are seen to be larger and more spherieal than others; these are the primordial ova out of which develop the sexual glands.

\section{Development of the Urogenital Sinus.}

The terminal portion of the intestinal eanal is ealled the eloaea ; it persists as a common opening for the intestinal and the urogenital system until after the fifth week of foetal life; the allantoie duct (allantois) opens into it. The upper portion of the allantois enlarges and beeomes the bladder, while the lower portion remains narrow. Into the lower, narrow portion open the ducts of Mueller, one on each side. The junction of the Muellerian ducts with the narrow portion of the allantois divides the latter into two parts; the part above the junction represents the urethra, while the part below is the urogenital sinus. The urogenital sinus forms the vestibule in the female, while in the male it is included in the formation of the urethra.

\section{DEVELOPMENT OF EMBRYONIC STRUCTURES INTO ORGANS.}

The pronephros (primitive kidney) has no physiological significance in man. The development of the metanephros into the permanent kidney in man has been described. It remains to consider what becomes of the Wolffian ducts, Wolffian body, the ducts of Mueller, 
the genital ridge, and the urogenital tissues; the following explanations and tabular statements accordingly are set forth.

\section{What becomes of the Wolffian Body and Ducts?}

In the eighth week of the female embryo the Wolffian body begins to undergo atrophic changes and is resorbed slowly, except a small anterior portion which persists and enters into the formation of the sexual organs, the resorption being much more extensive in the female than in the male.

The Wolffian duct and the remnant of the Wolffian body give rise to the following homologous structures in the two sexes:

\begin{tabular}{|c|c|c|}
\hline In the embryo. & In the adult female. & In the adult male. \\
\hline $\begin{array}{l}\text { 1. Wolffian duct. } \\
\text { 2. Wolffian body, anterior } \\
\text { (sezual) portion. }\end{array}$ & $\begin{array}{l}\text { 1. Duct of Gaertner. } \\
\text { 2. Parovarium(epoöphoron). }\end{array}$ & $\begin{array}{l}\text { 1. Vas deferens. } \\
\text { 2. Epididymis. }\end{array}$ \\
\hline $\begin{array}{l}\text { 3. Wolffian body, inferior } \\
\text { portion. }\end{array}$ & 3. Paroöphoron. & $\begin{array}{l}\text { 3. Paradidymis (organ of } \\
\text { Giraldes). }\end{array}$ \\
\hline
\end{tabular}

At the lower middle part of the Wolffian duct is an offshoot, the gubernaculum of Hunter, which passes through the canal of Nuck, and which by contracting draws the parovarium, and with it the ovary, down to its normal location in the posterior fold of the broad ligament. The contraction of this cord therefore is concerned with the descent of the ovary. In rare cases this contraction is so excessive as to draw the ovary through the canal of Nuck, and thereby to produce a serious displacement of the ovary.

\section{The Parovarium (Epoophoron, Organ of Rosenmueller).}

The paroöphoron is a remnant of the anterior portion of the Wolffian body, and is situated between the ovary and the Fallopian tube in the folds of the broad ligament; it consists of a number of tubules which open into Gaertner's duct, this duct, as shown in thie above table, being a remnant of the Wolffian duct.

\section{Paroophoron (Yellow Body of Waldeyer).}

The paroöphoron is a remnant of the inferior portion of the Wolffian body, is situated between the folds of the broad ligament near the uterus, and is composed of a number of small blind tubules comnecting with one another.

\section{What Becomes of the Ducts of Mueller?}

The Muellerian ducts in the male become rudimentary; in the female they form the Fallopian tubes, uterus, and vagina.

The homologous structures in the two sexes developed from the ducts of Mueller are as follows : 
Duct of Mueller. In the embryo. Duet of Mueller. In the adult $\left.\right|_{\text {female. }} ^{\text {Duct of Mueller. In the adult }}$

1. Upper exîremity.

2. Middle ununited portion.

3. Lower united portion.
1. Fimbriated extremity of Fallopian tube (the hydatid of Morgagni as usually designated in the female is a vesiele attached to one frimbria).

2. Fallopian tube.

3. Uterus and vagina.
1. Hydatid of Morgagni.

2. Cornua nteri masculini (usually absent, sometimes rudimentary).

3 . Uterus masculinus (rudimentary and in elose conneetion with the prostate gland).

\section{What becones of the Genital Ridge?}

The structures originating in the genital ridge in both sexes are developed as follows:

Genital ridge in the cmbryo. Genital ridge in the adull female. Germinal epithelium of the Graaffian follieles. genital ridge.

Mesoblastic portion of the Stroma of the ovary. genital ridge

Genital ridge in the adull male. Epithelium of the seminiferous tubules.

Connective tissue of the testiele.

\section{What becoyes of the Urogenital Sinus and Neighboring STruCtURES?}

In this comnection the student is referred to Figures 231-238 and the accompanying text.

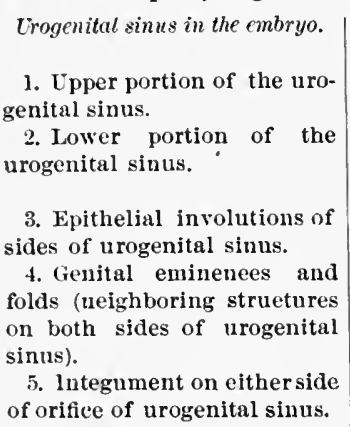

Urogenital simus in the adult
female.
1. Urethra.
2. Vestibule.

3. Glands of Bartholini.

4. Clitoris and nympliæ.

5. Labia majora.
Urogenital sinus in the adult
male.

1. Upper prostatic portion of urethra.

2. Lower prostatic portion of urethra and membranous portion of urethra.

3. Cowper's glands.

4. Penis.

5. Scrotum.

\section{DIFFERENTIATION OF SEX.}

From the foregoing paragraphs and tables it will be seen that if the genital ridge develops into an ovary the result will be a female; if into a testicle, the result will be a male.

On one hand, along with the differentiation of the genital ridge into an ovary, the Muellerian ducts develop into Fallopian tubes, uterus, and vagina, while the Wolffian ducts and body atrophy, leaving behind only rudimentary structures. On the other hand, along with the differentiation of the genital ridge into a testicle, the reverse happens - that is, the Muellerian ducts atrophy and the Wolffian ducts and Wolffian body become important male organs-epididymis and vas deferens.

The development of the external genitalia follows the same lines in both sexes, except development in the male is more exten- 
sive than in the female, being arrested in the female with the formation of the clitoris, nymphæ, and labia majora, but going on in the male to the development of the penis and scrotum. In one sense the elitoris embryologically may be regarded as an undeveloped penis and the labia as an undeveloped scrotum.

\section{CONGENITAL MALFORMATIONS.}

Malformations may be due to arrested development or to exeessive development. In the first elass of anomalies we have the malformations due to the persistence of embryonal conditions; the second elass ineludes the hypertrophies and multiplications of otherwise normal organs and tissues.

The subject should be studied in eonnection with the first part of this ehapter on embryology.

\section{MALFORMATIONS OF THE OVARIES.}

Malformations of the ovaries consist mainly in lack of development or in excessive development. The principal anomalies are:

Aceessory or constricted ovaries.

Supernumerary ovaries.

Absence of the ovaries.

Rudimentary ovaries.

Congenital hypertrophy of the ovaries.

Congenital displacement of the ovaries.

Accessory Ovaries are found in a small percentage of autopsies. They are always of small size, and generally are conneeted with normal ovaries by a peduneulated or sessile attachment. Two or three may be found in one case. They are usually parts of the original ovary separated during late foetal life by the constrietion of peritonitic bands. The ovary thus may be divided into equal halves or may be divided only partially. The presence of aecessory ovaries may account for pregnaney after both ovaries are supposed to have been removed.

Supernumerary Ovaries.-Only one authentic ease has been reported. ${ }^{1}$ This was a third ovary situated in front of the uterus in direct relation with the bladder, and connected to the uterus by a strong ovarian ligament. This ovary was twice the normal size. The two other ovaries were normal and of equal size. There was no trace of peritonitis in the neighborhood.

Absence of the Ovaries is a rare condition. It usually is associated with imperfeet development or absence of one or more of the other sexual organs. An absolute diagnosis can be made only by autopsy, for the ovary may be present in an abnormal location or in a partially developed state, and therefore may be overlooked. Absence of one ovary is apt to be associated witl absence of the corresponding half of the uterus and Fallopian tube. The writer, however, in one case has operated for the removal of the suppurating right tube and ovary, and found a perfectly developed uterus and, so far as 
could be discovered, entire absence of the left tube and ovary. There was only a slight protuberance at the left uterine cornu to mark the point where the tube should have joined the uterus. Figure 228, A. August Martin reports a similar case.

Rudimentary Ovaries are rather uncommon. They are of small size, and the Graatian follicles are absent or rudimentary. The uterus may be normal or may be also rudimentary.

Congenital Hypertrophy of the Ovaries.-Excessive growth of the ovary has been recorded, but cainnot be classed strictly as a malformation. It has been attributed to hyperæmic or inflamimatory conditions during foetal life.

Congenital Displacement of the Ovary.-The following is quoted from Ballantyne in Allbutt and Playfair's System of Gynecology: - Non-descent of an ovary is a rare but not unknown anomaly. Bland Sutton has reported a case in which the right ovary was adherent to the lower border of the kidney on the same side, and I have seen a case in the newborn infant in which it was attached by peritonitic bands to the cæcum. It has been stated that it may be found free in the peritoneal cavity. Instead of nondescent, there may be dislocation of the ovaries downward into the inguinal canal. According to Puech, congenital inguinal hernia of the ovary is much more common than acquired, and Zinnis recently has reported an instance of it; Bland Sutton states that he knows of no case in which the ovarian nature of the herniated body has been proved by microscopical examination conducted by a competent observer. Herniation of the ovary, which may be unilateral or bilateral, is associated usually with displacement of the Fallopian tube, and sometimes with malformation of the uterus and malposition of the kidney. It may be due to defective derelopment of the round ligament and a patent condition of the canal of Nuck."

\section{Clinical Significance of Ovarian Malformation.}

The absence of one ovary, if the other is developed perfectly, does not render the woman sterile. On the contrary, her reproductive functions may be in no practical respect impaired. If both ovaries are rudimentary or absent, sterility is inevitable. There is usually wanting in such cases the normal development at puberty ; there mav also be an associated faulty general nutrition, a weak nervous organization, chlorosis, and not uncommonly a growth of hair on the face, especially the upper lip. The individual may retain the general physical characteristics of infancy and childhood, or there may be an apparently full development of the extrapelvic organs.

\section{Diagnosis of Ovarian Malformation.}

The diagnosis of ovarian malformations is made by the above signs and symptoms and by the recognition on conjoined examination of undeveloped, absent, accessory, or otherwise anomalous ovaries. Early and accurate diagnosis is important, for only by this means 
will the woman be saved from a possible long-continued and useless treatment for sterility. It is often impossible to say that an apparently rudimentary ovary is engenital, for it may have been subjeet to atrophic ehanges consequent upon the acute infeetious diseases of childhood.

\section{MALFORMATIONS OF THE FALLOPIAN TUBES.}

Malformations of the tubes are analogous to those of the ovary, and are therefore as follows:

Supernumerary tubes.

Aecessory tubes and ostia.

Inereased length and exeessive eonvolution.

Rudimentary development.

Absence of the tubes.

Supernumerary Tubes may be associated with supernumerary ovaries. Only a few cases have been recorded.

Accessory Tubes and Ostia are not uneommon. As many as six accessory ostia have been observed in one tube. The anomaly has no definitely recognized signifieanee.

Increased Length and Excessive Convolution of the tubes may exist, and have been said to favor tubal pregnancy.

Rudimentry Development.-The rudimentary tube is usually imperforate, being a mere fibrons eord with, perhaps, the semblanee of an open ampulla and fimbriæ. The eorresponding ovary may or may not be also rudimentary or absent. The aceident is due to failure of development of Mueller's duet.

Absence of the Tube pertains more frequently to one than to both sides. When both tubes are absent the uterus and ovaries also are usually wanting. Cases have been reeorded in which the tube and kidney on the same side were absent. Absence of one tube is associated usually with lack of development of the corresponding side of the nterus-that is, with uterus unicornis.

The Clinical Significance of malformations of the tubes is much the same as that already outlined for malformations of the ovaries.

\section{MALFORMATIONS OF THE UTERUS.}

The developmental defeets of the uterus form a large proportion of the genital malformations. They may be ranged, for the most part, under two general heads: 1 . Those due to imperfect development of Mueller's ducts. 2. Those due to imperfect blending of Mueller's ducts.

Infantile Uterus.-If the Muellerian ducts unite but do not continue to develop, the result will be an undeveloped, infantile, or foetal uterus. If the arrest of development oceurs very early in fotal life, the uterus will be extremely rudimentary. It may consist of an infantile cervix, and in place of the eorpus only a fibrous cord extending from the site of one Fallopian opening to the other. If arrest of development does not oceur until after birth, the uterus will be 
smaller than normal, but in other respects not strikingly different from the fully developed organ.

The anomalies due to defective blending of Mueller's ducts are numerous and frequent. Nearly every degree of imperfect fusion has been observed. The following anomalies are due to this eause.

Double Uterus.-The most extreme anomaly due to defective blending is the double uterus (uterus didelphys), in which there are two complete organs lying side by side, each Muellerian duct having formed a perfect uterus with cervix and fundus, but with only one cornu, one Fallopian tube, and one round ligament. Either of these uteri may be functionally competent. Pregnancy and parturition therefore may proceed normally. On the other hand, one uterus may be rudimentary or imperforate. If the imperforate organ is functionally active, it may become distended with menstrual blood and form hæmatometra. This will require surgical interference.

Accessory Uterus.-A very curious and rare malformation is the nterus accessorius. In this condition, besides the normal uterus, there exists another uterus anteriorly, between it and the bladder. In one case a third uterine lobe was found attached to the single cervix of a bifid uterus. It is difficult to account for these anomalies. The assumption has been made that the accessory organ was developed from a diverticulum of a Muellerian duct.

Bicornate Uterus.-Next in importance to the double uterus is the much more frequent bicornate uterus, in which fusion of Mueller's ducts has occurred lower down than normal, with the result of producing a Y-shaped organ. This deformity occurs in all degrees. In one extreme, the septum extends the whole length of the cervix and gives rise to a double os externum, Figure 229 ; in the other extreme the two cornua may be separated only by a noteh at the fundus (uterus cordiformis).

A unique case occurring in the author's practice in which the uterus was bicornate is perhaps worthy of note. The patient, thirtyfive years of age, married fifteen years, the mother of two children, and a sufferer from endometritis, extreme dysmenorrhœa and neurasthenia. The faulty general nutrition was thought to depend largely upon the dysmenorrhœa. The patient gave a history of having been treated for a long time and in many ways, the treatment having included dilatation and curettage of the uterus. Examination showed that the uterus was bicornate, as represented in Figure 230, a fact which hitherto had not been recognized. With considerable hesitation and after consultation, it was decided to remove the two horns of the bicornate uterus, and the operation was performed as shown in Figures 230 to 233 . The result was complete restoration to health. Six months after the operation the general nutrition lad become normal, and there was an increase in weight of about twenty pounds. Although supravaginal hysterectomy would not ordinarily be advocated as a proper treatment for dysmenorrhœa, yet the peculiarities of this rare case were such that perhaps the operation was justifiable. At any rate it appears to have been justified by the result. The author is unable to find any record of a similar operation, or for that 
Figure 228.

A

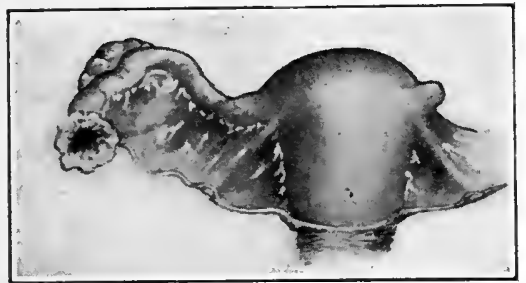

C

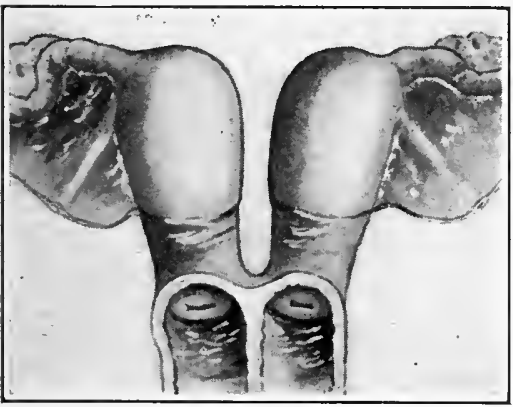

$\mathrm{E}$

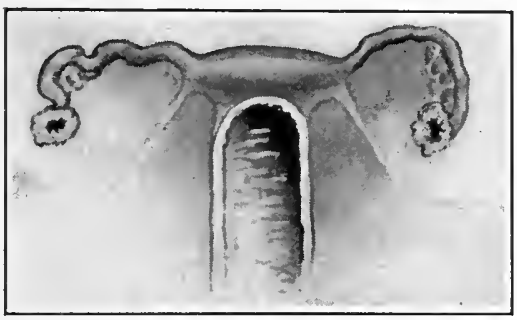

B

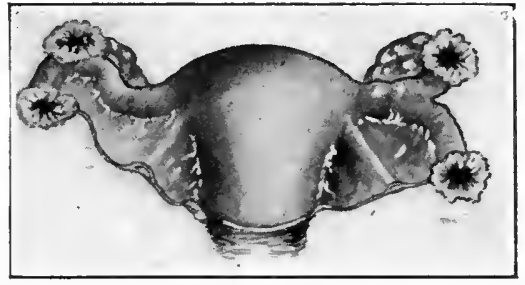

D

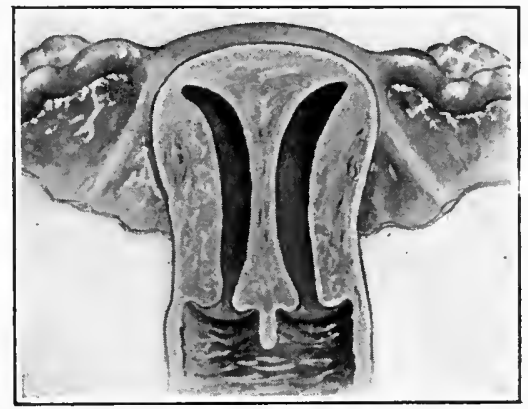

F

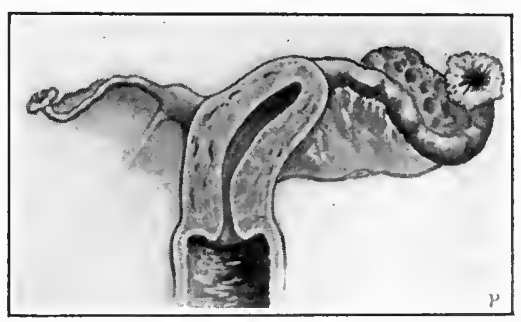

A. Absence of the left ovary and Fallopian tube, with full development of the other genital organs. Author's case. The right tube and ovary were removed for pyosalpinx and ovarian abscess ; recovery.

B. Fallopian tube with accessory ostium on right side. Fallopian tube with accessory tube on left side.

C. Double uterus $-i$. e., nterus didelphys. From each uterus there is one tube and one ovary. The vagina is also double.

D. Uterus septus duplex. Completely divided nterus and incompletely divided vagina.

E. Ribbon-shaped rudiment of uterus; rudimentary Fallopian tubes, ovaries, and round ligaments; vagina well developed.

F. Uterus with one horn (uterus unicornis). Left horn, ovary, and Fallopian tube very rudimentary. 
matter of the removal of any part of a bicornate uterus, on a similar indication.

Uterus Septus.-In this anomaly there is complete division of the uterus into two cavities by an anteroposterior vertical partition or septum. The septum may be complete or incomplete, may form only a ridge on the interior of the uterus, may extend through the cervix, or it may be confined to the cervix or to the corpus. There may be, in fact, every possible variety in the sitnation or completeness of the septum. The typical, if not the commonest, form has two lateral

Figure 229.

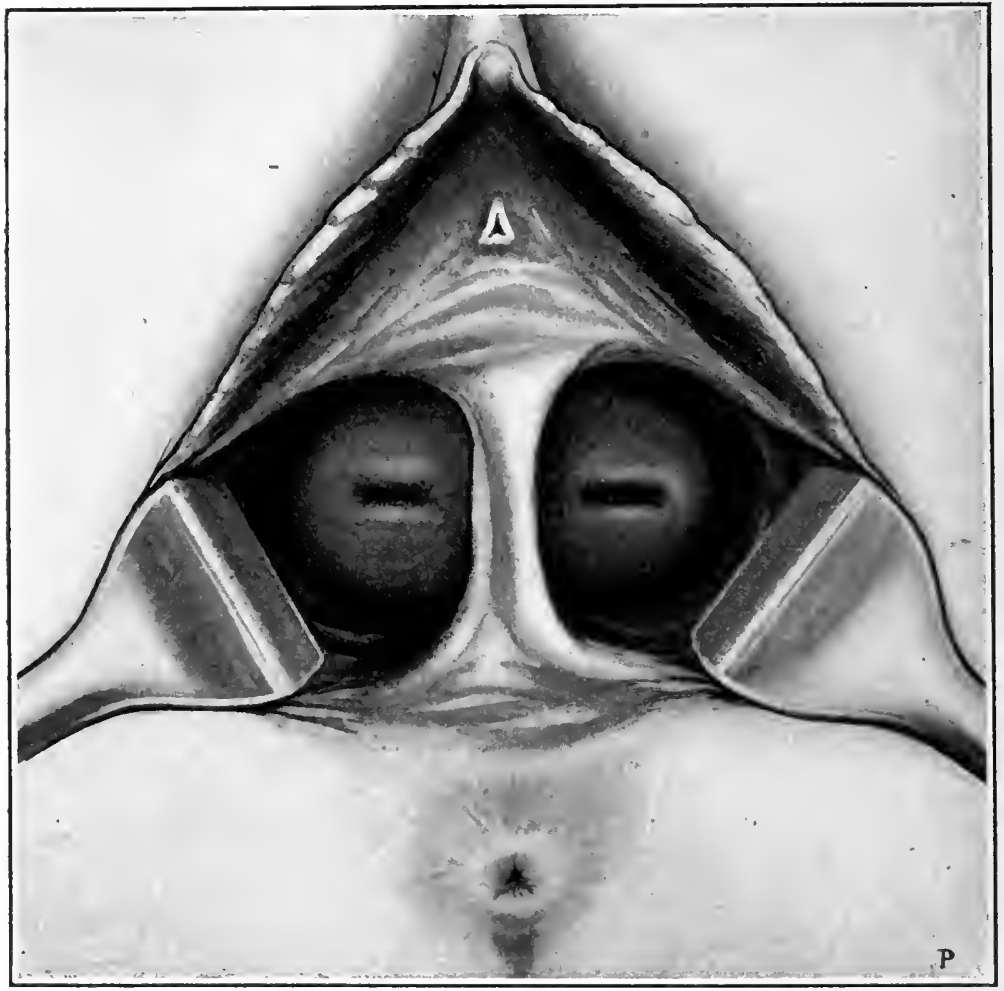

Double uterus, vagina, and vulva.

cavities for both corpus and cervix uteri. The bicornate and septate uteri have a similar clinical significance. In either there is liable to be an imperforate condition of one side of the septum or the other, with resulting hematometra. Menstruation is apt to be frequent and otherwise abnormal, anc parturition to be embarrassed. Uterus subseptus signifies an imperfect septum and eonsequent partial division. In a subseptate uterus malpresentations are prone to occur and the insertion of the placenta to be abnormal.

There is in the above varieties of malformations of the uterus a 
FIgURE 230.

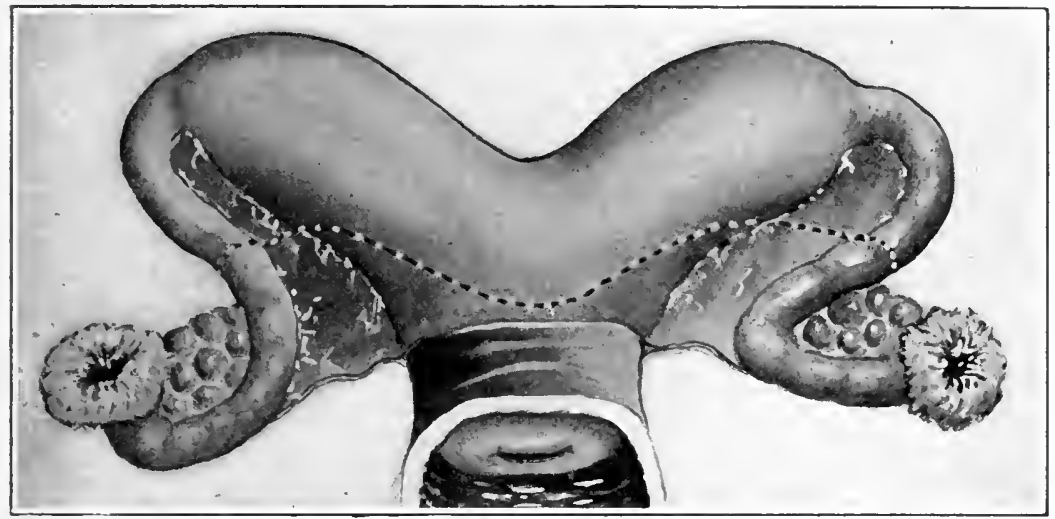

Figure 231.

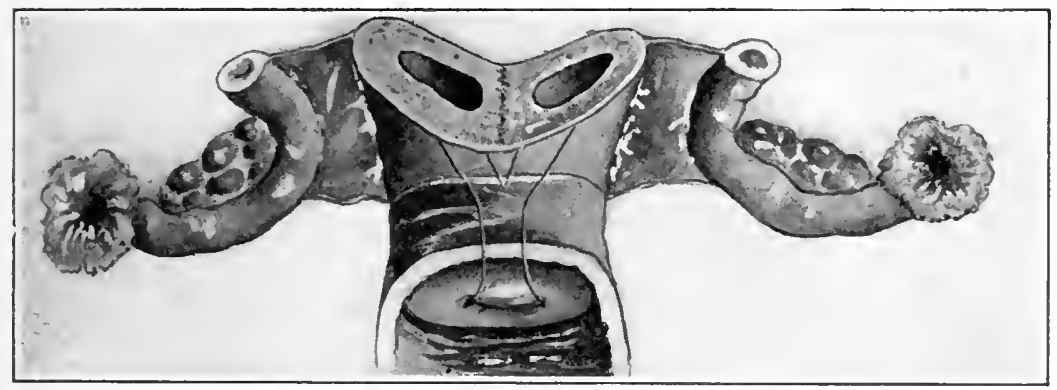

Frgure 232.

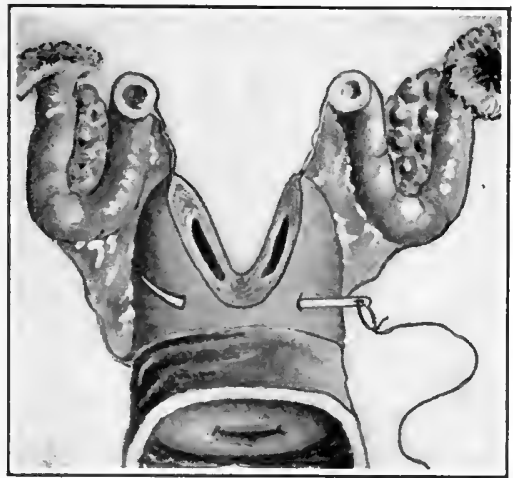

Figure 233.

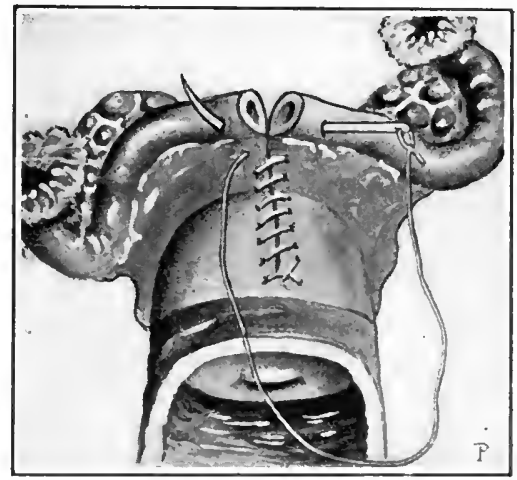

FIGURE 230.-Uterus bicornis unicollis. The horns of the bieornate uterus are continuous with one neek. The dotted line shows the line of incision for the removal of the two horns of the uterus.

Firure 231.-Same as Figure 230. The two horns of the uterus have been removed : section of Fallopian tubes; scction of uterine canal made through the two horns of the bicornate uterus Black and white lines here indicate the $\mathbf{Y}$-shape of the bicornate uterine canal.

FIGURE 232. - Same as Figure :231. First stitch in the union of the wound made by removal of the two horns of the bicornate uterus.

Figure 233. - Same as Figure 232 . Iterine part of wound brought together from side to side, and united by contınuous suture. End-to-end approximation of the broad ligaments and Fallopian tubes by same suture. 
complete gradation between double uterus on the one hand, and uteris septus on the other.

Uterus Unicornis.-When there is failure of fusion and more or less atrophy of the duct on one side, so that only one horn of the uterus is well developed, or only one exists, we have the singlehorned uterus, or uterus unicornis. The kidney, ureter, ligaments, tube, and ovary on the side of the lacking or imperfect colnu are also, as a rule, rudimentary or absent. The rudimentary horn may be hollow or solid; if the former, its cavity may or may not connect with that of the lower part of the uterus. If menstruation takes place in the closed horn, there will be hæmatometra, and the normal progress of menstruation on the other side will lead to confusion in the diagnosis. Bilateral hæmatometra, both horns being imperforate, would give rise to less difficulty in the diagnosis. Figure 2:28, F.

A mong the less important anomalies of the uterus are the following:

Defeet or absence of the vaginal portion of the cervix.

Septate os externum, with no trace of septum above.

Normal development on one side and defective development on the other; this would be an approach to a unicornate uterus.

Flat or arched fundus.

Congenital prolapse, retroversion, retroflexion, or anteflexion.

Congrenital communication between the endometrium and intestine or bladder.

In a remarkable case one side of a bipartite uterus is said to have developed on the exterior of the body.

Premature Development of the Uterus.-This usually is associated with similar precocity in the other genital organs. Young girls thus may menstruate at a very early age and show the sexual development of mature years.

\section{MALFORMATIONS OF THE VAGINA.}

The vagina, in common with the uterus, as shown above, is formed by the coalescence of Mucller's ducts, and therefore shares largely in the malformations of that organ. 'Thus the double uterus and the uterus septus may be associated with double vagina.

The congenital anomalies of the vagina are :

Double vagina (vagina septa).

Absence of vagina.

Atresia of vagina.

Vagina Septa. - A completely double vagina having two canals, each opening into an external vulva of its own, is very rare, only one ease, that of Katharin Kaufmann, having been reported. In this case $^{1}$ the pelvis was divided by a peritoneal fold into two lateral eavities ; each half contained a bladder, a unicornate uterus, an ovary, a Fallopian tube, and a rectum. The spinal cord was bifurcated at the level of the third lumbar vertebra.

The ordinary and much more common double or septate vagina is divided into two passages by a septum above the vulva. The hymen

1 Reported by Suppinger. Allbutt and Playfair, System of Gynecology. 
may have one or two openings, and the septum, as in the uterus, may be complete or partial.

Double uterus and double vagina often coexist. In some cases the vagina is double and the uterus single, with the os externum then opening into one side of the donble vagina. The other side ends in a cul-rle-sac. If, under these conditions, the blind passage alone be used for coition, sterility will result. In other cases both sides may be in communication with the uterus. The septum may be so imperfect as to constitute only a ridge along the posterior and anterior walls of the vagina.

The septum seldom divides the passage into exactly equal halves. Coitus usually is confined to one side. In case of uterus unicornis the vagina may be very small-in fact, of only half size. This is because one of Mueller's ducts has failed in development from the uterus down, and the other has developerl only on its own side, producing a unilateral vagina. In case of double uterus, or uterus septus, or uterus bicornis, one-half of the vagina may be imperforate, with resulting accumulation of menstrual blood in the uterus and vagina on that side (hæmatometra and hæmatocolpos). Chapter XXXVIII.

Aside from the possibilities of sterility and hæmatocolpos, and from the uterine conditions which may be associated, a vaginal septum is not of itself a very serious matter. It may never be suspected until parturition, and even then the septum may be destroyed or pushed to one side by the passing child.

Complete Absence of the Vagina usually is associated with absence or defect of the ovaries, tubes, and uterus, and with a generally defective sexual organization. If, however, the defect is only in that part of Mueller's ducts which forms the vagina, the uterus and tubes may be developed normally. Absence of the vagina then will lead after puberty to retention of the menstrual products and the necessity of making an artificial vaginal passage in order to give exit to retained menstrual fluid, and otherwise to establish the physiological integrity of the vagina. Impregnation and parturition have taken place through a vagina thus opened. This subject will be considered further in the next chapter.

Inflammatory Atresia of the Vagina must not be confounded with congenital absence of the organ. The former is the result of arlhesive inflammations which may be fotal and involve the whole length of the passage, or it may be dne to inflammation occurring in childhood or in adult life ; see Dissecting Vulvovaginitis. In a case of adherent vaginal walls, the walls when separated wherever the inflammation has not been destructive will retain the vaginal mucosa. In congenital absence of the vagina the mucosa never has developed. 'There is only connective tissue between the vesical and rectal walls.

Other Anomalies of the Vagina.-The remaining vaginal anomalies are rare: they include diverticula and communications between the vagina and other organs, such as the rectum and urethra. These openings are dependent not upon defects of Mueller's ducts, but rather upon fotal cloacal conditions, hereafter to be described. 


\section{MALFORMATIONS OF THE HYMEN.}

The hymen is an organ of variable strength and form. It may be annular, notched, fimbriated, fenestrated, cribriform, crescentic, thick, thin, fragile, tongh, or vascular. Some of these conditions are normal, others but slightly abnormal. Complete absence is extremely rare, if not unknown. Imperforation, so-called, is a condition usually due to closure of the end of a Muellerian duct, and is therefore in no sense an abuormal hymen.

The importance of these anomalous conditions varies. A rigid hymen makes coitus painful or impossible, a very vascular membrane may lead to a temporary profuse hemorrhage, and imperforation gives rise to hæmatocolpos, or in extreme cases also to hæmatometra, and demands operative interference ; see Congenital Atresia of the Genital Tract. A rigid hymen may, after marriage, require divulsion or incision.

\section{MALFORMATIONS OF THE VULVA AND ANUS.}

This subject becomes relatively simple when we understand the embryological development of the vulva and anus. At the end of the sixth week of fœtal life the tangible differentiation of sex begins, and the developmental changes which then normally take place are shown in Figures 234 to 238 . The student is referred to the embryology of the genitalia in the first part of this chapter.

Figure 234.

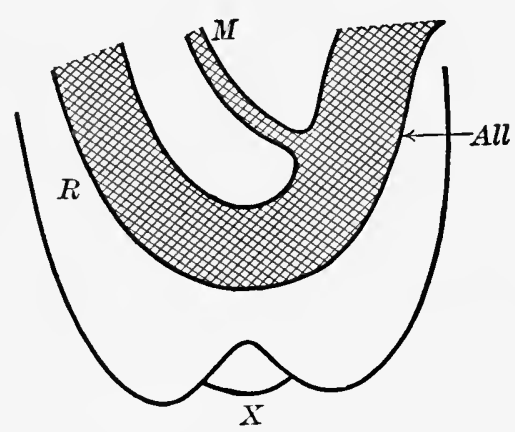

Figure 235.

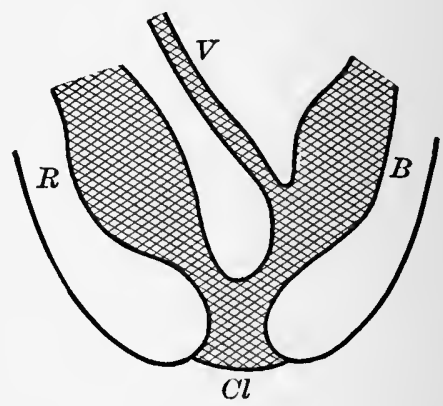

Figure 234. $-R$, rectum, continuous with, $A l l$, allantois (bladder), and, $\boldsymbol{M}$, duct of Mucller (vagina). $\bar{X}$, depression of skin below genital prominence, which grows inward and forms vulva.

Figure 235.-The depression has extended inward and, becoming continuous with the rectum and allantois, forms the cloaca. $C l$, cloaca. $B$, bladder. $V$, vagina. $R$, rectum.

At first the allantois (which forms the bladder), the rectum, and the Muellerian ducts (which form the vagina, uterus, and Fallopian tubes), all communicate with a conımon cavity, but do not at this time open on the external surface. Presently there is a depression in the skin which opens inward to this cavity, thus forming the cloaca. The cloacal opening is divided now into two parts by a septum, which later develops into the perineum. The posterior portion of the cloaca thus divided becomes the anus. The anterior part becomes the uro- 
Figure 236.

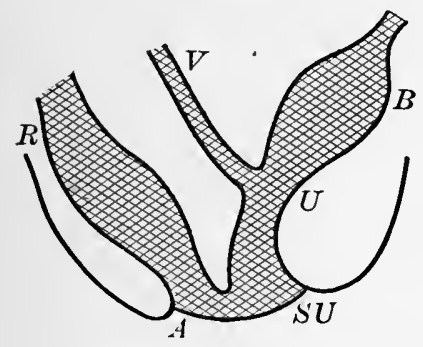

Figure 237.

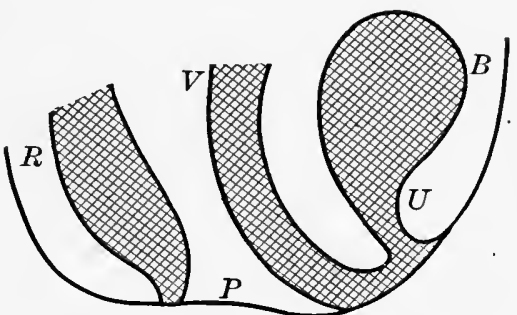

FIGURE 236.-The cloaca is becoming divided into the urogenital sinus, $S U$, and anus, $A$. by the downward growth of the perineal septum.

FIGURE 237. -The perineum is completely formed. $P$, perineum. The ducts of Mueller have united the lower portion forming the vagina.

Figure 238.

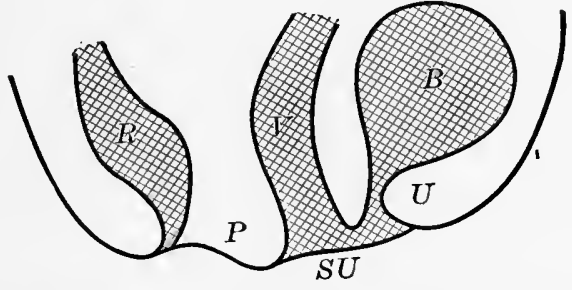

The upper part of the urogenital sinus has contracted into the urethra; the lower portion, $S U$, now becomes the vulva. $P$, perineum. $R$, rectum. $V$, vagina. $B$, bladder. $U$, urethra.

FIGURE 239.

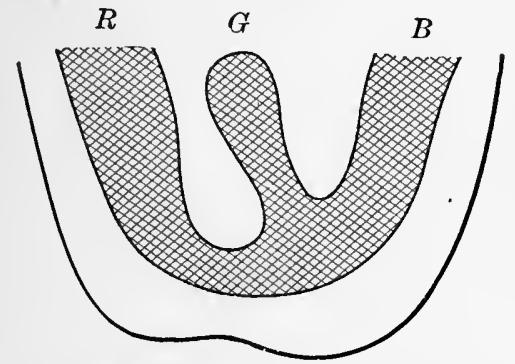

Figure 240.

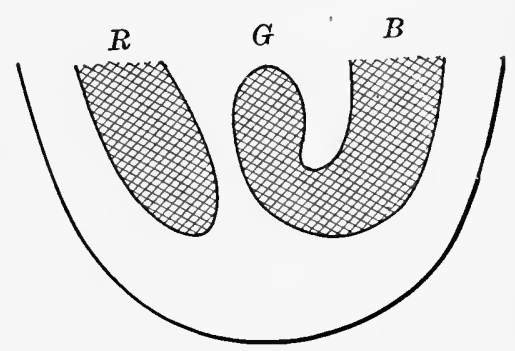

Figure 239.-Absence of cloacal division. Perineal septum wanting. $R$, rectum. $G$, genital canal. $B$, bladder.

Figure 240.-A Asenee of cloacal division. Perineal septum present.

genital sinus. This sinus in its upper part becomes the urethra, and in its lower part the vulva.

The anomalies of the vulva and anus are:

Atresia.

Persistent cloaca.

Hypospadias.

Epispadias.

Infantile vulva.

Atresia of the Urethra, Vagina, and Anus.-The cloacal division by which the urethra, vagina, and anus are opened and thereby prolonged to the external surface may fail to take place. This failure 
will result in complete atresia of the vagina, urethra, and anus. The perineal septum may be absent, as shown in Figure 239, or present, as shown in Figure 240. In the latter case the opening between the rectunn and the urogenital sinus will be closed. This condition of complete atresia has been observed only in stillborn foetal monstrosities. The bladder, urethra, and vagina-that is, the urogenital sinus - are apt to be distended with urine.

Congenital atresia is not to be eonfounded with another form of vulvar atresia in which the labia have become adherent from inflammation. This adhesion may occur before or after birth. The adhe-

Figure 241.

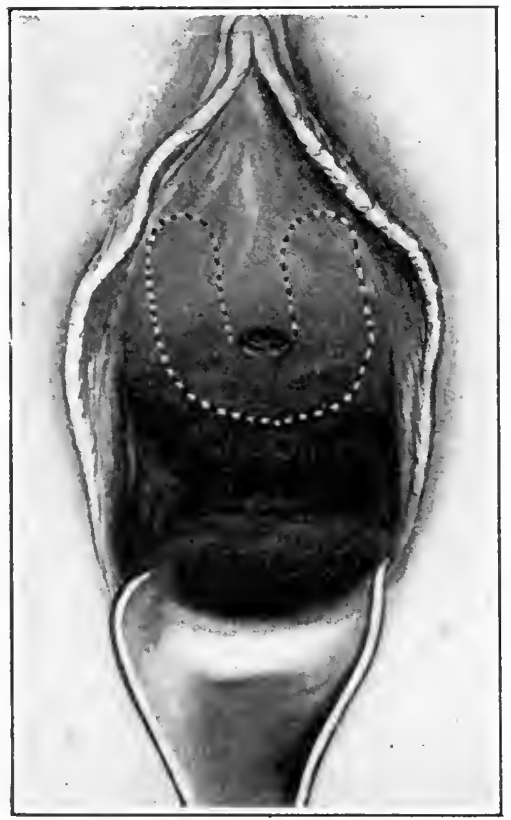

FIGURE 242.

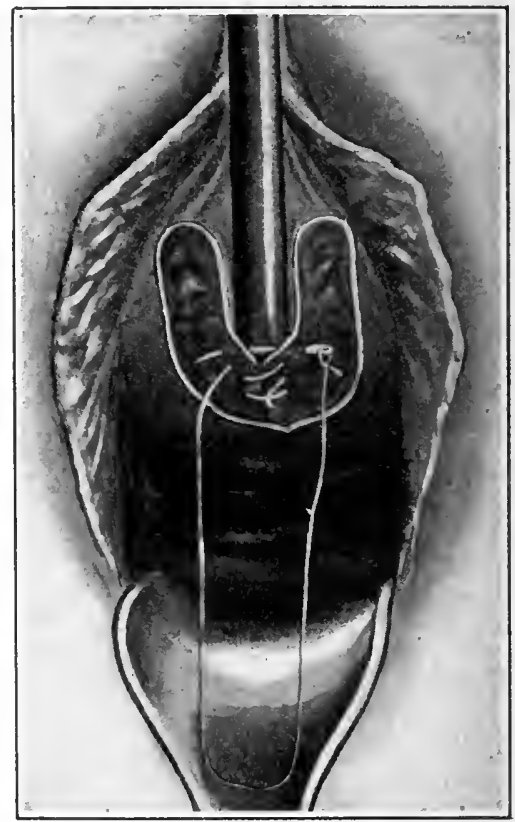

FigURe 241.-Building of a urethra. Author's operation. Congenital absence of urethra. Blaek and white dotted lines indicate area to be denuded in the construction of a new urethra.

FIGURE 242-Building of a urethra. Author's operation. Same as Figure 241. Area for construetion of a new urethra denuded. Inner margius of deunded area being brought together by continuous catgut sutures so as to undte those margins over the sound, which has been introduced into the bladder and is held by the hand of an assistant.

sion is generally incomplete, so that urine and menstrual fluid can escape. The condition has been designated superficial atresia of the vulva; it may be remedied by separating the labia, either by divulsion or by cautious dissection.

Persistent Cloaca.-In this anomaly the anus practically opens directly into the restibule; there is no perineum. The anomaly is a persistence of the condition in Figure 236. If the anal sphincter is also deficient, the condition is a pitiable one. When there is control over the feces an operation may not absolutely be needed; but when there is incontinence of feces it is always advisable, and preferably 
before maturity. The usual operation has been to pass a probe into the fistula and out at the normal position of the anus, then split up the parts, draw the rectum downward and backward to the angle of the incision, suture it into position, and close the gap in front. A modification of this operation has been suggested by Buckmaster; in this modification the new anus is made first just in front of the levator ani muscle, and at a later period the fibres of this muscle are split to make a sphincter.

Hypospadias in the female is a defect in the posterior wall of the urethra, or in extreme cases entire absence of the urethrovaginal

Figure 243.

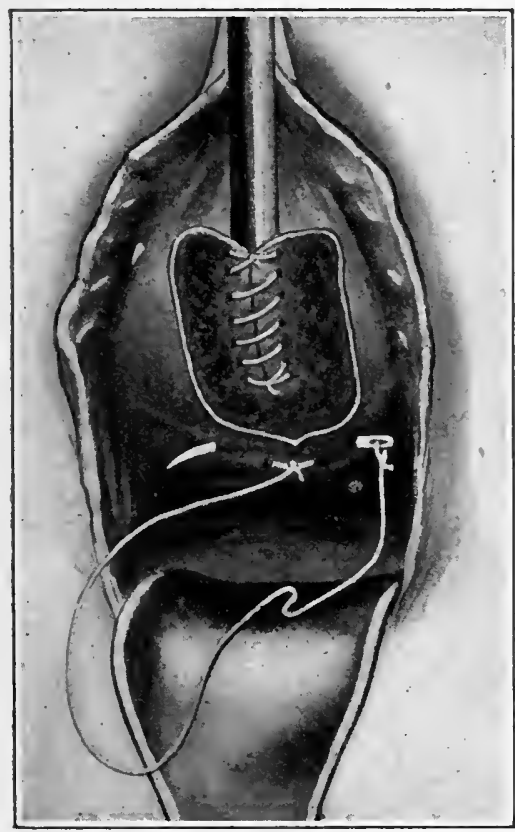

Figure 244.

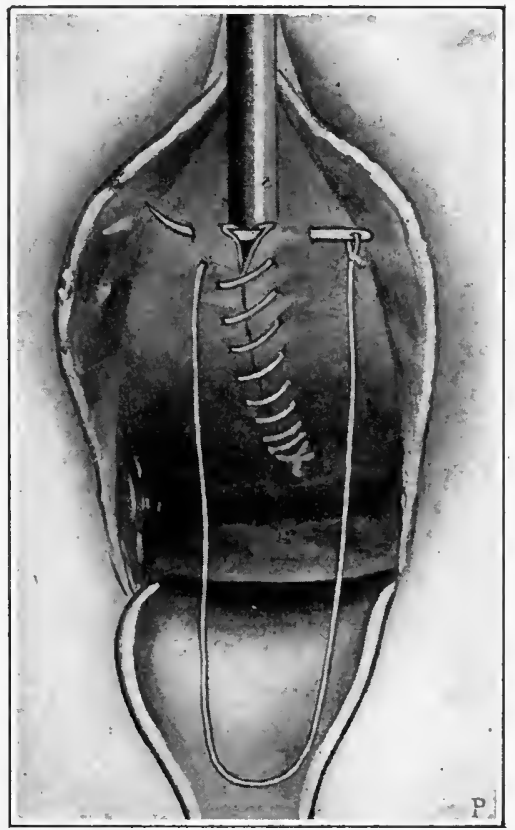

Figure 243,-Building of a urethra. Author's operation. Same as Figure '242. Margins of new urethral mucosa united over the sound by fine continuous chromic catgut suturc. The first stitch has been introduced and tied, and the needle is being introduced for the second stitch to unite the outer margins of the denuded area for the completion of the new urethra.

FIGCRE 244.-Building of a urethra. Author's operation. Same as Figure 243. Sound in position as in Flgures 242 and 243. Last stitch of a continuous suture being introduced. This stitch when tied will complete the formation of the new urethra.

wall, with resulting incontinence of urine as in a vesicovaginal fistula. The malformation is a continuation of the fotal condition, shown in Figure 236, and it is dne to persistence of the nrogenital sinus. The lower portion of the allantois has failed partially or completely at development into a urethra. In marked eases there is incontinence, but in slight cases there may be control of urine. Hypertrophy of the clitoris is apt to be an associated defect. Sometimes the clitoris may be large enough to raise the question as to sex of the individual. A condition resembling hypospadias may result from traumatism-that is, the urethrovaginal wall may be 
surgically divided, or may slough out in eonsequence of pressurenecrosis following labor. Whatever the cause of the condition may be, if there is ineontinenee of urine, a plastic operation should be made to construct an artificial urethra. Emmet was the prineipal pioneer in this operation. His method was to ntilize the labia minora as material out of which to make the new urethra. He denuded a longitudinal strip on the inner surface of each labium, and then united them by means of interrupted sutures, and in this way was successful in securing more or less retentive power. The author has for several years employed a somewhat different method with gratifying results. 'This method is illustrated in Figures 241 to 244 .

It is important in building up a new urethra to have in mind three special points : first, to introduce the sutures over a rather small sound, for the urethra will usually dilate, and, if a large sound is used, will therefore finally become too wide; second, broad areas of denudation should be made on each side so as to produce a very thick urethrovaginal wall, for this wall usually grows thinner by stretehing or absorption and therefore should be quite thick to begin. with; third, before the new urethra is attempted an artificial vesicovaginal fistula should be made midway between the inner extremity of the urethra and cervix uteri, so that during the healing process the sutures in the new urethra may not be disturbed meehanically by the outflow of urine. After union in the urethra has become solid the fistula may be closed in the usual fashion. The artificial vesicovaginal fistula should be made as described in the treatment of cystitis, Chapter XXIV.

Epispadias in woman is a defect in the upper wall of the urethra, and generally is aecompanied with fissure of the elitoris, and sometimes also with fissure of the symphysis pubes and of the whole anterior vesical wall. This gives rise to eetropion of the bladder. Incontinence of urine occurs even in the slighter forms, and an operation is required to restore the integrity of the bladder-wall. The labia are eommonly absent in extreme forms of epispadias.

Infantile Vulva.-General lack of development of the vulva and absence of the labia majora and minora are associated commonly with defeets of the internal genital organs and with a generally weak systemie development, and often coexist with chlorosis. They are not an absolute impediment to impregnation, but may be to parturition.

\section{MALFORMATIONS OF THE NYMPHA, CLITORIS, AND PREPUCE.}

Hypertrophy of the Nymphæ has been much observed in the lower races, especially among the African tribes. It is said also to be found frequently in connection with hypertrophy of the mammæ among the American Indians. See Elephantiases, Chapter XXV. The nymphæ may become so large as to require removal.

Hypertrophy of the Clitoris.-The elitoris will be reealled as 
that organ in the female which corresponds to the penis in the

Figure 245.

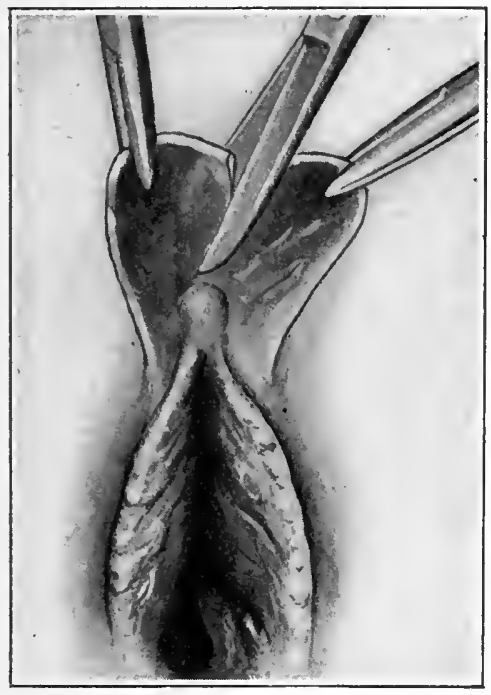

FIGURE 247.

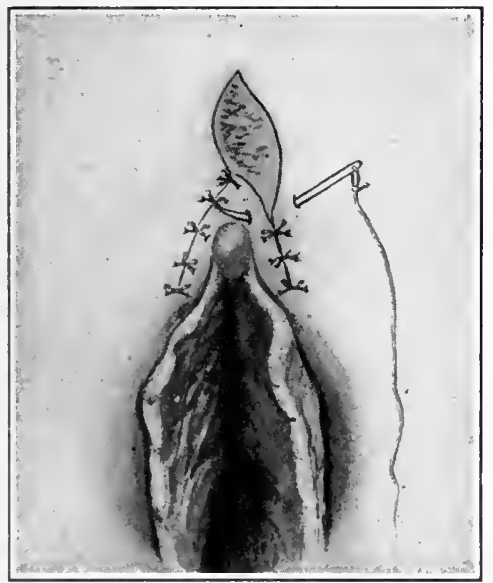

Figure 246.

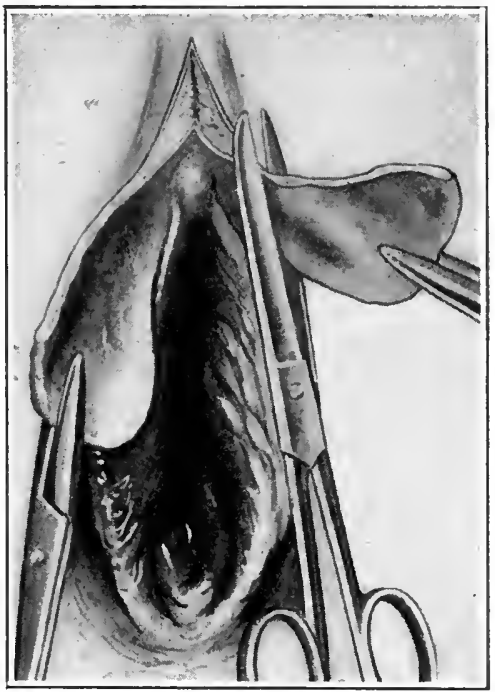

Figure 248.

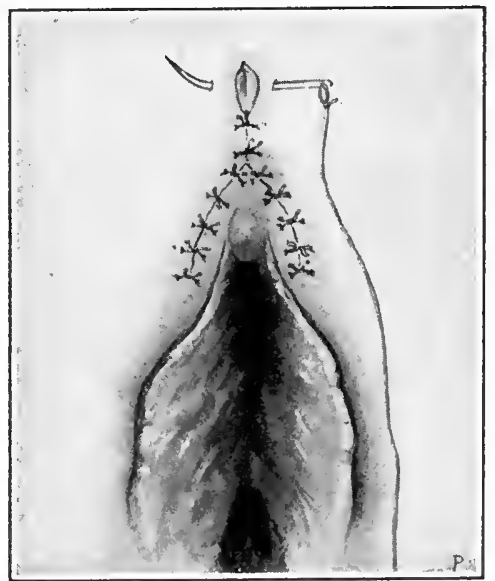

FigURE 245.-Circumeision in the female. Redundancy of the prepnee and enlargement of the clitoris in a masturbating girl of eleven. Appearanee of prepuce when pnt upon the streteh. The seissors are dividing the prepuce on the dorsum, as they would in eireumeision of the male prepuee.

FIGURE 246.-Same as Figure 245. Right half of the divided prepuce hanging loose with foreeps attaehed. Left half being removed with seissors. Right half also to be removed in same way.

FIGURE 247.-Same as Figure 246. Redundant portion of prepuee all removed. Raw sur. faces exposed. Margins of wound being brought together by means of interrupted fine eatgut sutures. tion.

FIGURE 248.-Sume as Figure 247. Last suture is being introduced completing the opera-

male. Hypertrophy of the clitoris may be congenital or aequired. Congenital hypertrophy is associated not uncommonly with other mal- 
formations, such as pseudohermaphrodism, and may be so excessive as to give the clitoris the appearance of a penis. The anomaly occurs more frequently in the tropics than in temperate zones. Congenital enlargement of the clitoris is an indication for renoval (clitorectomy). Acquired enlargement is associated usually witl masturbation, but unfortunately excision does not usually put an end to the habit. In two cases of uncontrollable erotic paroxysms occurring in the author's practice, in which the clitoris appeared to be the storm centre, but in which there had been no masturbation, excision of the clitoris was followed by relief. This operation, clitorectomy, since the career of the "brilliant and blighted Baker Brown" is almost obsolete; the indications for it being very restricted.

Acquired hypertrophy, usually the result of masturbation in childhood, does not produce great enlargement.

Hypertrophy of the Prepuce.-There is a class of cases occasionally observed among children in which the clitoris is enlarged moderately and surrounded by an abundance of loose, flabby, redundant preputial skin. In such cases the causes may be both congenital and acquired. The congenitally hypertrophied clitoris and redundant prepuce are, by reason of their size, unduly exposed to friction; this gives rise to irritation; the child instinctively rubs or scratches the part in order to obtain relief, and thus gradually forms the habit of masturbation. This frequent counterirritation and consequent congestion are then in themselves additional causes of enlargement of the clitoris, and especially of redundancy of the prepuce. The child, unless relieved of the local irritation and taught to avoid all friction of the part, soon becomes a hopeless neurotic. The treatment in such a case is circumcision.

The technique of circumcision, which closely resembles that of circumcision in the male, is set forth in Figures 245 to 248 . In the operation the same careful trimming of the loose, redundant skin is necessary as in circumcision of the male child. In suitable cases the operation, if supplemented by positive and proper moral instruction and by judicious hygiene, may be followed by a cure of the unfortunate habit and by relief from the nervous symptoms. A most important factor in the general treatment is hygiene, that is, a nonnitrogenous diet, the avoidance of sweets, spices, highly seasoned food, tea, coffee, and stimulating drinks.

Adherent Prepuce.-Adhesion of the prepuce, which may be congenital or acquired, produces the same reflex nervous symptoms in the male, and requires the same treatment-that is, separation of the prepuce from the glans by breaking up the adhesions or by incision. In some cases the indication, after loosening the adhesion is to slit up the prepuce on the dorsum. If the prepuce is not so redundant as to require removal, the wound as in the corresponding operation in the male may be reunited by a line of union at right angles to the line of incision. 


\section{HERMAPHRODISM.}

If we use the word hermaphrodism in the strict sense, to signify a combination of anatomically and functionally perfect male and female organs in one individual, a typical case has never been established satisfactorily. The condition occurs sometimes in the higher, but more frequently in the lower vertebrates, and it is perhaps possible in man, but thus far has not been demonstrated by the necessary autopsy.

The cases of so-called hermaphrodism all, or nearly all, fall under the head of pseudohermaphrodism, in which there may be an irregular development of the sexual organs, some of the female, others of the male type, but with a decided predominance of one over the other. If the individual is really a female, and resembles the male, the malformation is called Gynandry; if the male resembles the female, it is Androgyny. See Figure 249, which is presented here as a modification of a drawing by Zweifel.

Gynandry.--There are two classes of cases.

In one class the breasts approach or conform to the male type. There may also be a hairy development on the face and a masculine voice, contour, and appearance. The genitalia, although perhaps rudimentary, are yet unnistakably of the female type. There may be congenital atresia of the vagina and an infantile vulva, but the uterus and ovaries, partly or fully developed, are present.

In the other class of cases the hairy development and masculine voice, contour, and appearance are supplemented by one or more of the following anomalies:

a. Pronounced hypertrophy of the clitoris, sometimes to the size and appearance of the fully developed penis.

$b$. The labia minora and majora may be fused together so as to obliterate the vulvar entrance.

c. Ovarian hernia and a consequent pouch may be present, resembling in form and situation a scrotum with its testicles. The uterus and ovaries are developed more or less perfectly.

Androgyny.-Most of the cases of pseudohermaphrodism oceur in individuals who have testicles, and are therefore essentially male. There are several forms of this class, of which three are given below.

1. The mildest form is that in which the breasts approach or conform to the female type, and the penis and testicles are correct in form, but rudimentary.

2. An interesting subdivision of androgyny includes individuals whose generative organs are apparently female, except that they have testicles instead of ovaries, these glands being situated in the abdomen or in the inguinal canal, and the scrotum being absent. The clitoris, vulva, vagina, and uterus, more or less imperfectly developed, are present. Individuals belonging to this subdivision are usually brought up and pass as women throughout their lives.

3. The most numerous subdivision is that of hypospadic men. There is an imperfect, diminutive penis held down by a bridle, and 
having the appearance of a large clitoris. The pendulous portion of this penis is imperforate. The hypospadiac urethral opening corresponds in situation and appearance to the female urethra. The testicles are usually in the abdomen or inguinal canal, and the scrotum is wanting. There is a fissure in the median perineal raphe - a perineoscrotal fissure. The development on either side of this fissure resembles the vulvar labia. These individuals have the female mammary development, usually are brought up as girls, and in some instances have discovered the mistake only after marriage.

Figure 249.

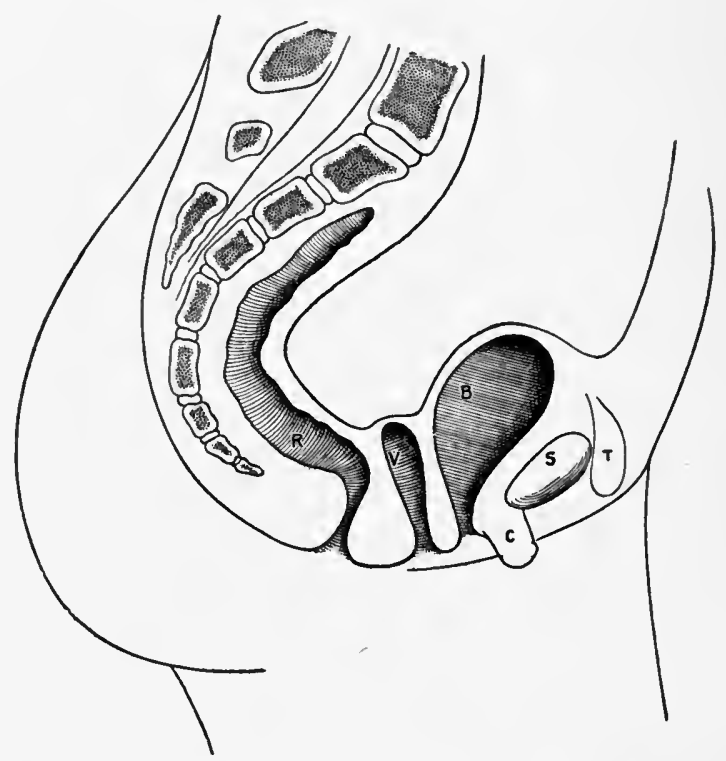

Pseudohermaphrodism by hypospadias (male). $T$, testicles, not descended. $S$, symphysis publs. $C$, undeveloped penis, resembling large clitoris. $B$, bladder. $\boldsymbol{V}$, prostatic vesicle (pseudovagina). $R$, rectum.

Intercourse may be possible either in the prostatic vesicle, see Figure 249 , or in the dilated urethra. Some of these monstrosities have been capable of coitus both as men and women.

The importance of hermaphrodism is obvious. As a clear diagnosis of sex in doubtful cases cannot always be made at birth, it is suggested that in cases of doubt the individual shonld be brought up as a boy. This course will cause less embarrassment, and in the vast majority of cases will prove to be correct.

\section{Treatment of Hermaphrodism.}

The treatment of hermaphrodism is limited to those cases in which anatomical defect can be corrected by operative measures. The labia, if fused together, may be separated by breaking the adhesions or by 
incision. The hypertrophic clitoris or labia may be removed and the wounded surfaces covered by a plastic operation.

The bridle or frænum holding down the penis in androgyny may require an operation for the liberation of the organ, and plastic work for the covering of the exposed surfaces.

Epispadias and pseudohermaphrodism may furnish indications for operative measures, and if so, must be treated in each case according to special requirements. 


\section{CHAPTER XXXVIII.}

\section{CONGENITAL GYNATRESIA WITH RETAINED MENSTRUAL FLUID.}

ATREsia in the genital tract of a menstruating woman is apt to result in retention of menstrual fluid above the point of obstruotion. The fluid thus retained, of a tar-like color and consistence, contains blood, mucus, and epithelial débris.

Figures 250 to 261 , suggested by and modified from a scheme of Sutton and Giles, show the accumulations of menstrual fluid which may take place above the various possible points of atresia in the uterus, vagina, or vulva. The accumulations come under one of the three following divisions :

1. An accumulation in the vagina will take place above the point of vaginal or vulvar atresia, and is called homatocolpos.

2. An accumulation in the uterus called homatometra is limited below according as the atresia is at the internal or external os, in the corpus, or in the cervix uteri. A distended cervix is called hæmatotrachelos.

3. The uterus having been distended, the blood may force its way into and distend the Fallopian tubes, producing homatosalpinx. The fimbriated extremity in such cases is closed by adhesive inflammation. Hæmatosalpinx due to retention of menstrual blood is associated usually with hæmatometra.

Hæmatocolpos may exist alone or may be associated with hæmatometra and hæmatosalpinx.

\section{The Pathological Results.}

The pathological results are primarily those of pressure upon the mucosa, and distention and thinning of the walls of the dilated organs; this leads to atrophy of the mucosa and muscularis. Secondarily, there may be infection and consequent admixture of pus with blood; the conditions may then be termed pyocolpos, pyometra, and pyosalpinx.

\section{Symptoms of Congenital Gynatresia.}

The symptoms are commonly absent until puberty; at this time menstruation first begins and gives rise to accumulations of menstrual blood. The young girl will then have the svmptoms of monthly recurring menstruation called the molimen, with a sense of superadded weight and heaviness due to accumulations of menstrual fluid. The sense of weight will increase with the quantity of fluid, and in cases of extreme hæmatometra will become excessive, and may resemble 
labor-pains. There may also be distressing pressure on the adjacent organs. Suppuration, if present, gives rise to the same symptoms of absorption as would result from an abscess.

\section{Diagnosis of Congenital Gynatresia.}

The physical signs will reveal a fluctuating elastic tumor corresponding to the seat and extent of the accumulations. The tumor, if in the vagina, will be felt most distinctly in that region, and may bulge between the labia; if in the uterus and tubes, it will easily come within reach of the external hand on conjoined examination, and fluctuation will be distinct on palpation between the vagina and hypogastrium. The distended tubes are marle out usually to either side of the distended nterus. Conjoined examination with the left

Figure: 250.

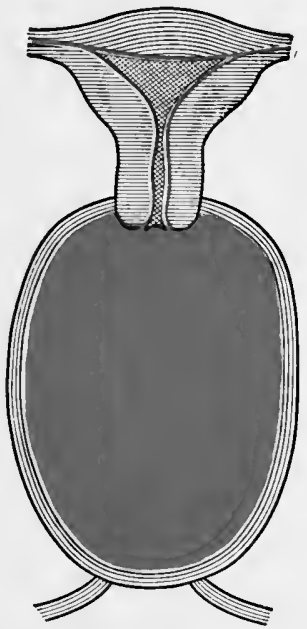

Figure 251.

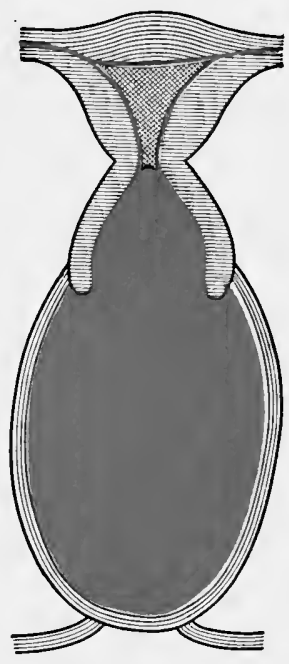

Figure 250.-Atresia at the vulva first causes distention of the vagina, producing hæmatocolpos.

FIGURE 251.-Atresia at the vulva. Hæmatotrachelos has followed hæmatocolpos.

index-finger in the rectum may give further information. The finger in the rectum and the sound in the bladder sometimes will define the upper limits of hæmatocolpos.

If the atresia is at the os internum, it gives rise to no change in the form of the cervix, but gives to the corpus uteri the appearance of pregnancy.

One side of a double vagina or uterus may be distended and the other side empty. There will then be a tumor on the affected side and lateral displacement by pressure. The symptoms, supplemented by conjoined examination and the sound, will be the means of diagnosis. The groove between the distended and empty sides may be felt on rectal touch.

Hæmatometra is distinguished from pregnancy by the absence of 
the usual signs of pregnancy, especially of discoloration, patulous os externum, and vaginal pulsation. Hæmatocolpos or. hæmatometra on

Figure 252.

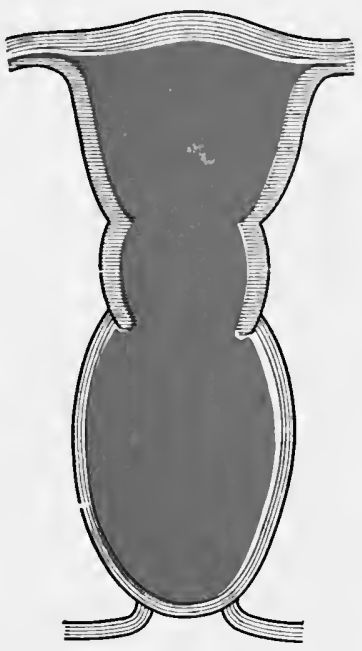

FiguRe 253.

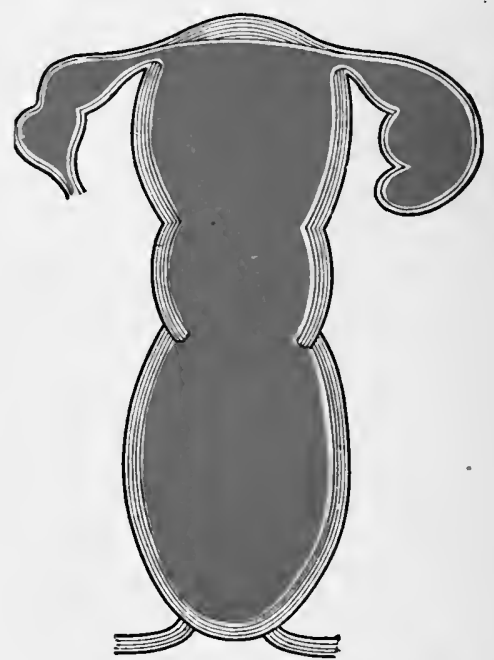

Figure 252.-Atresia at the vulva has caused hæmatocolpos, then hæmatotrachelos, and then hæmatometra.

FigURE 253.-Atresia at the vulva. In addition to the conditions in Figure 252, there is added hæmatosalpin $x$.

Figure 254.

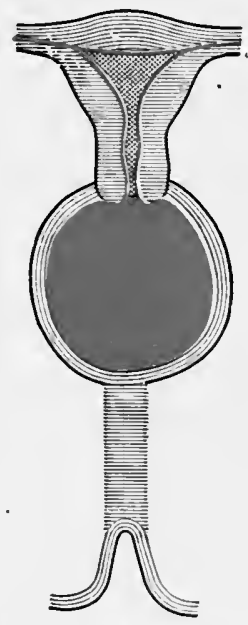

Figure: 255.

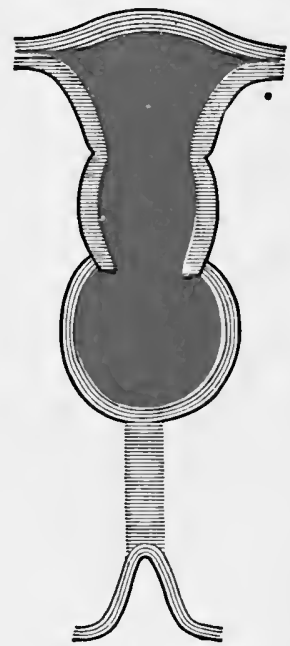

Figure 254.-Atresia in the vagina midway between the vulva and os externum, causing

hæmatocolpos in the upper half of the vagina.
FIGURE 255 . - Same as in Figure 254 , except that distention of the whole uterus has followed the partial hæmatocolpos.

one side of a double uterus or vagina may lead to great confusion. Conjoined examination and the history of the case will serve to dis- 
tinguish the former from extra-uterine pelvic tumors, and the latter from abscess or cyst of the vaginal wall.

Figure 256.

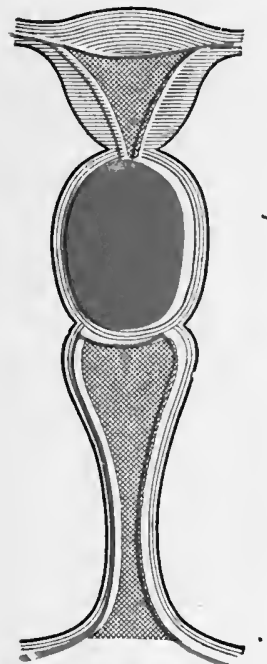

Figure 257.

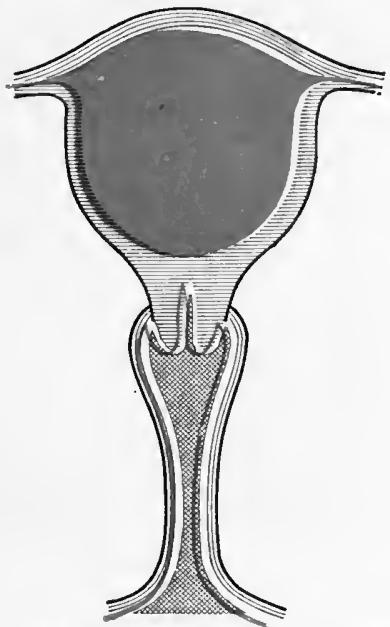

Figure 256.-Atresia at the os externum producing a hæmatotrachelos. Corpus uteri not yet distended.

FIGURE 257.-Atresia at the os internum producing hæmatometra. Fallopian tubes may become distended later.

Figure 258.

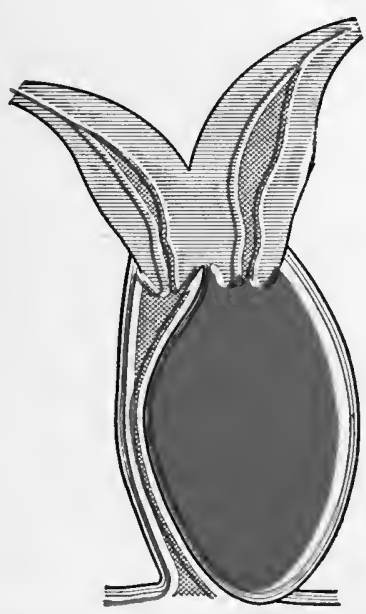

Figure 259

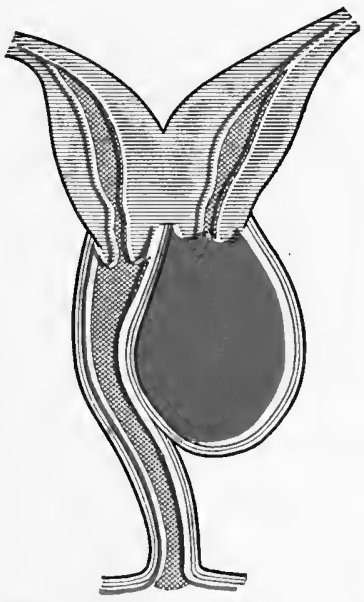

FigURE 258.-Atresia at the vulva on one side of a double uterus and vagina, causing hrematocolpos on affeeted side.

FrGURF 259. - Atresia on one side of double uterus and vagina midway between vulva and os externum. This produces partial hæmatocolpos of affected side.

The greatest care in manipulation is essential lest the sac rupture and discharge its contents into the peritoneum, or the fluid be forced through the Fallopian tubes. The fluid, however, is apt to be sterile. 


\section{Prognosis of Congenital Gynatresia.}

Unless relief comes from operation, the thin walls, especially of the Fallopian tubes, may rupture and set up peritonitis. Suppuration may give rise to the phenomena incident to pelvic abscess. If the sae ruptures into the intestine, infection and death, or a precarious recovery may follow.

\section{Treatment of Congenital Gynatresia.}

The treatment is free incision at the point of atresia, evacuation of accumulated fluids, washing out and drainage of the cavity. There is in these cases an mexplained and excessive liability to postoperative infection. Extra care therefore in the asepsis before, during, and after the operation is imperative.

Figure 260.

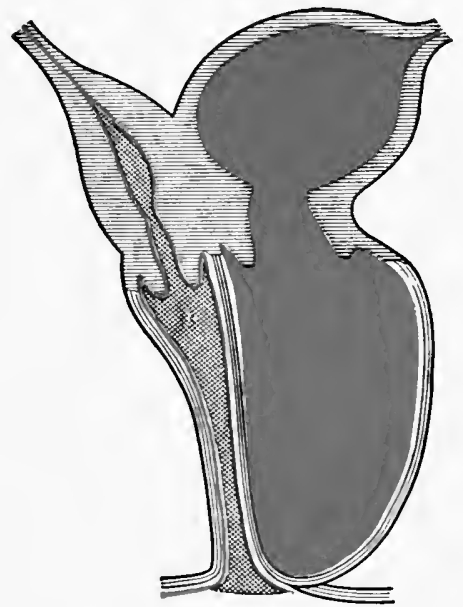

Figure 261.

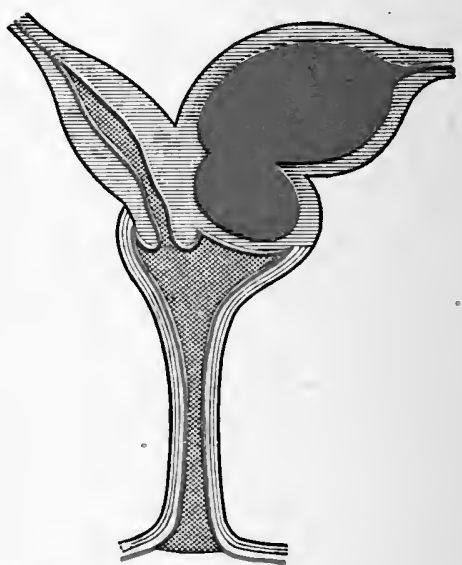

FIGURE 260.-Atresia on one side of double uterus and vagina at vulva, producing hæmatocolpos, hæmatotrachelos, and hæmatometra.

FIGURE 261.-Atresia on one side of double uterus and vagina at os externum, producing hæmatotrachelos and hæmatometra.

Operations for Hæmatometra will vary according to the location and extent of the atresia. In some cases the obstruction is slight and easily broken by a sound or by pushing a pair of blunt-pointed scissors or forceps through it, and spreading the blades to secure the necessary divulsion and dilatation. The canal once opened should be made to remain patulous, if necessarv, by immediate free incision or dilatation. The atresia may be at the internal or external os, or the whole cervical canal may be obliterated. In the latter case it is sometimes necessary to separate the bladder from the uterus as in anterior vaginal section. The separation does not necessarily extend into the peritoneum, but should be carried past the level of the internal os. The anterior wall of the uterus may then be divided longitudinally with scissors until the interior of the corpus is reached and evacuated. 
The opening thus made is rendered permanent by additional incision, gauze packing, dilatation, plastic surgery, or all these combined.

Hæmatometra may occur with complete or nearly complete absence of the vagina. See Malformations of the Vagina. Under these conditions the choice of procedure is between :

1. Artificial vagina.

2. Abdominal hysterectomy or removal of the uterine appendages.

1. Artificial Vagina consists in separating the vesical from the rectal plate of the rectovaginal septum and entering and evacuating the uterus through the canal thus made. First the incision is made freely from side to side through the vulvar skin ; the two plates of the rectovesical septum then are split realily apart by means of the two indexfingers, which easily work their way through loose cellular tissue to the uterus. The uterus, if present, will be recognized, when reached, by its relative hardness and resistance, and sometimes by the elasticity and sense of fluctuation of retained fluid, and may be opened by means of the sharp-pointed scissors, using as a guide the aspirator needle previously introduced. In working his way to the uterus, the operator may avoid entering the bladder or rectum by frequently introducing the finger into the rectum and the sound into the bladder. Emmet says that in some cases the new vagina when it heals over the glass vaginal.dilator is covered by a structure not altogether unlike mucous membrane, and that after healing has taken place the frequent use of the glass dilator will keep the vagina open. The writer's experience in three cases personally observed is that no such membrane formed; but that, on the contrary, the surfaces were entirely cicatricial or granulating in character.

It is desirable as soon as the vagina has been opened to cover it with skin flaps dissected from the external genitals. The labia minora frequently are utilized for this purpose. C. K. Fleming has published an improvement on the above method. Before splitting the rectovesical septum he dissects loose a long wide flap composed of the hymen, the posterior ends of the nymphæ and the upper part of the perineal integunient, leaving the upper part of the fiap attached, and, after splitting the septum, he ntilizes this flap for the anterior vaginal wall. He then dissects a flap from the labium majus on one side, leaving the lower part attached for the posterior vaginal wall. After splitting the septum these flaps are stitched into the new vagina, so as to make them cover the raw connective tissue surface of it. The surfaces exposed by the loosening of the flaps then are closed by means of fine chromic catgut sutures. This operation has been permanently successful.

2. Abdominal Hysterectomy or Removal of the Ovaries.-If the artificial vagina persistently contracts and cannot be kept open as an outlet for menstrual fluid, the removal of the uterus would be justifiable and preferable to removal of the ovaries.

Operations for Hæmatocolpos.-If the obstruction be only a thin membrane, it may be incised freely and the fluid let out as in

\footnotetext{
1 Denver Medical Journal.
} 
hæmatometra. In some cases there is absence of the lower and distention of the upper part of the vagina, and perhaps also of the uterus and Fallopian tubes. Then a passage must be made carefully to the point of atresia in the same manner as for opening the vagina in hæmatometra.

An operation for hæmatocolpos or hæmatometra on one side is apt to result in closure of the opening and refilling of the cavity. For this reason it is important, in the effort to secure a permanent result, to include in the operation the free division of the septum in the vagina and, so far as practicable, in the uterus.

In hæmatosalpinx the tubes usually will empty themselves through the uterus when that cavity is drained, and do not therefore have to be removed or otherwise disturbed.

Hæmatocolpos and hæmatometra may be the result of traumatic as well as of congenital atresia; the principles of treatment are then the same as for the congenital anomaly - that is, to let out the confined fluid and adopt measures to keep the passages open.

The formation of an artificial vagina in cases of vaginal atresia and rudimentary uterus has in rare instances been followed by development of the uterus and normal menstruation, and may therefore possibly result in maternity. The probability of such a result, however, is so slight as to discourage the operation.

It occasionally happens that complete atresia of the vagina and absent or extremely rudimentary uterus are not discovered until after marriage. In such a case maternity being clearly impossible, the question may arise whether or not the formation of an artificial vagina is justifiable. The operation follows the technique already laid down for the formation of artificial vagina in cases of hæmatometra. Cases have been reported in which after the operation mar. riage was happy, the woman even recovering from a tendency to melancholia, and experiencing great improvement in nervous tone and general strength. The question of the propriety of such an operation may be relegated to the department of ethics and casuistry. 


\section{PART IV.}

\section{TRAUMATISMS.}

\section{CHA PTER XXXIX.}

\section{NON-PUERPERAL INJURIES OF THE VULVA, VAGINA, AND CERVIX UTERI.}

\section{INJURIES OF THE VULVA.}

THE external genitals are protected from violence by their situation and relations to the surrounding parts, and therefore are little exposed to external traumatism.

Etiology.-The following causes of traumatism are recognized: 1. Falling upon a sharp substance. 2. Self-inflicted wounds by the insane. 3. Violent coitus. 4. A blow or fall which so bruises the soft parts against the sharp edges of the descending ramus of the pubes or the ascending ramus of the ischium as to make a deep cut. The cut may appear at the surface or may be subcutaneous.

Symptoms. - The symptoms are the same as those of similar injuries elsewhere. Hemorrhage from the abundant vessels about the vulva is usual in wounds of that region. When the wound is external the bleeding may be alarming-even fatal. Great subcutaneous extravasation of blood may occur in the bruised parts. This when clotted forms pudendal hamatoma.

Treatment.-Superficial incised wounds should be treated by suture. Bleeding points should be ligatured with fine catgut. Deep punctured wounds are treated best by compresses, which serve for dressings and to control hemorrhage. Small hæmatomata may disappear by absorption ; if too large for absorption, compresses shonld be applied for four to eight days, until all danger of hemorrhage has passed. Then a free incision should be made, the clot turned ont, the cavity packed with aseptic gauze and allowed to heal from the bottom. Suppuration is treated by incision and drainage.

\section{INJURIES OF THE VAGINA AND VAGINAL PORTION OF THE CERVIX UTERI.}

Injuries of the vagina and vaginal portion of the cervix uteri of non-puerperal origin are more rare than injuries of the vulva. They may result from violent coitus or from the violent application of any other force. The control of hemorrhage and the repair of vaginal wounds follow the general principles of surgery. 


\section{CHAPTER XL.}

\section{LACERATIONS OF THE PERINEUM AND PERINEAL REGION.}

IxJuRIEs of the vaginal outlet and pelvic floor caused by parturition, and usually designated as lacerations of the perineum, are among the most frequent of gynecological lesions, and even though the importance of these injuries sometimes may be overestimated, the fact is undeniable that they give rise to many serious disorders and inconveniences. It is not always possible to avoid the accident, but it is possible to recognize it when it occurs and, by a timely operation, prevent evil consequences. A laceration that does not extend into and destroy the functions of the sphincter ani muscle is incomplete. The laceration is complete if the sphincter is injured sufficiently to impair its functions-i.e., if the patient has lost power to retain the contents of the bowel.

\section{ANATOMY OF THE PERINEUM AND PERINEAL REGION.}

The perineum is the converging point where many of the most important parts of the pelvic floor come together; these parts are :

Figure 262.

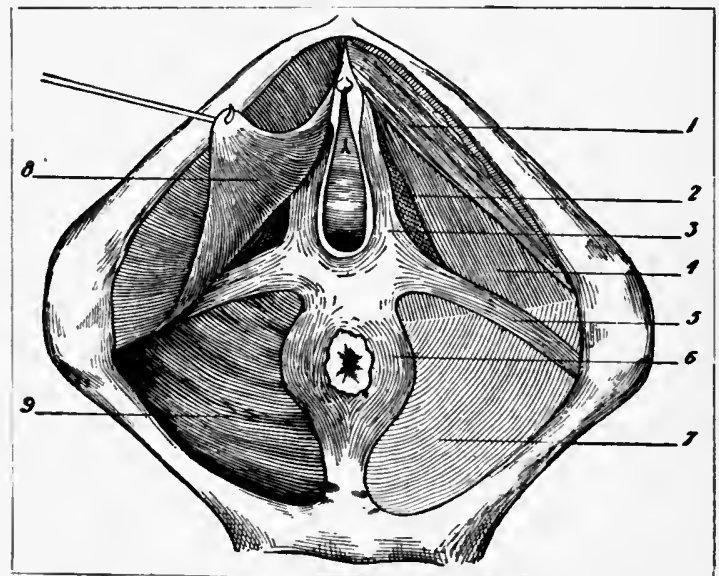

1. Erector clitoridis. 2. Bulbocavernous musele. 3. Constrictor vaginæ muscle. 4. Triangular ligament. 5. Transversus pe.dinei muscle. 6. Sphincter ani muscle. 7. Fascia of the levator ani muscle. 8 . Perineal fascia. 9. Levator ani muscle.

the bulbocavernosus muscle; the transversus perinei nuscles, superficial and deep fascia: the external sphincter ani muscle; the internal sphincter ani muscle; and the levator ani muscle. The muscles 
are surrounded and bound together by deep and superficial fascia; the fascia in some places, for example, in the triangular ligament, is quite dense, and resisting. All the perineal muscles, through the medium of tendon and faseia, are direetly or indirectly strongly connected with the pubie bones. The muscles, ligaments, and faseir

Figure 263.

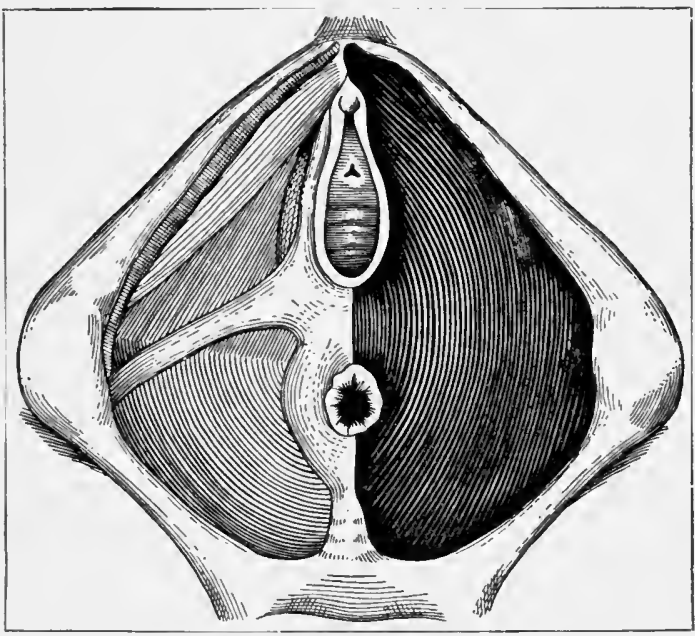

Right side, showing vesicorectal fascia.

unite in the perineum to form a diaphragm which fills the pelvic outlet. Through this diaphragm pass the lower portions of the rectum, anus, vagina, and urethra.

\section{FUNCTIONS OF THE PERINEUM AND PERINEAL REGION.}

It is clear from the foregoing paragraphs and illustrations that the museles, faseiæ, and ligaments of the perineal region constitute a most essential supporting part of the pelvic floor. They surround, bind together, hold in position, support and maintain in their mechanical relations the terminal ends of the rectum, vagina, and urethra. The reader is now prepared to take exception to the common notion that the pelvic organs derive their support from the small fleshy body called the perineum. Another false idea is that the support is in the nature of that given by a keystone to an areh. The perineal region and perineal body do not give support in the sense of being under the pelvic organs and holding them up as foundations. They are an essential and integral part of the pelvie floor, and as such contribute to the support of the abdominal organs above. The perineal muscles do not fix the parts to which they are attached. For to do this would require continnous activity and would soon exhaust their power, and would therefore be unphysiologieal. They serve to control the lower portions of the rectum, vagina, and urethra in the performance of their functions. 
The importance of the levator ani muscle as a means of support has been overestimated; on this subject a very suggestive, convincing, and clear statement comes from William W. Browning, of Brooklyn. His conclusions are as follows:

"1. That in the human subject it belongs to the class of rudimentary muscles.

" 2. That the weakness of its origin, as well as the direction and the insertion of its fibres, is inconsistent with the design of support.

"3. That it is unphysiological for a muscle to furnish a continuous support.

Figlre 264.

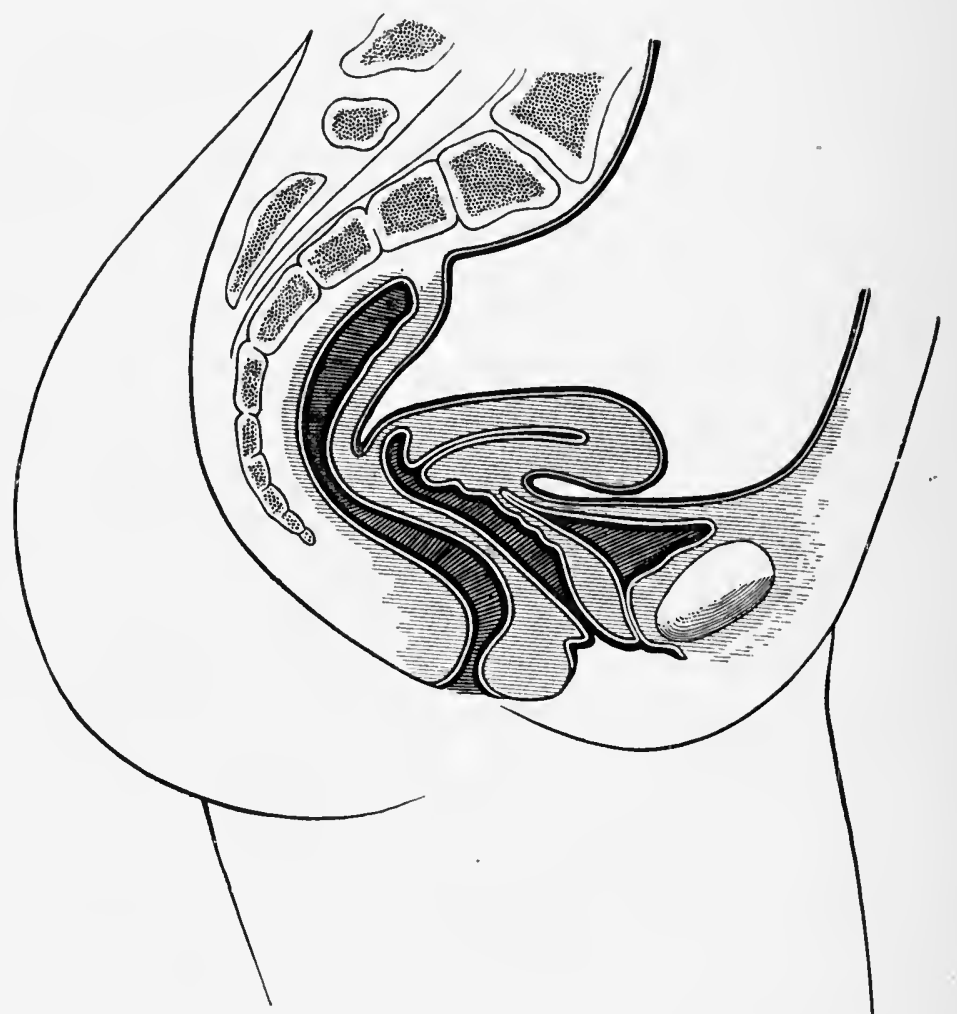

Normal relations of the pelvic organs, showing the vaginal walls resting on the perineal body.

" 4 . That the rectovesical fascia is in itself sufficient, when intact, to afford the required support.

"5. That the muscle is no better developed in the female (in whom support is more required) than in the male."

\section{LACERATIONS OF THE PERINEUM.}

A mere rupture in the perineal body where certain muscles, faseiæ, and ligaments of the pelvic floor converge is not necessarily very 
significant. If, however, the injury involves the rending asunder of these supports, especially the faseial supports; if they are so divulsed as to lose their sustaining power; and, above all, if they are torn off from their pubie attachments, the lesion becomes more serious.

\section{Causes and Prevention.}

Relative disproportion between the ehild and the perineal outlet may, unless an incision is made, render laceration inevitable. Among other unpreventable causes are rapid labor and odema of the vulva. The preventable causes and the means of protecting the perineum during labor are laid down fully in works on midwifery. If during labor rupture seems imminent, it is better to divide the vulvar ring

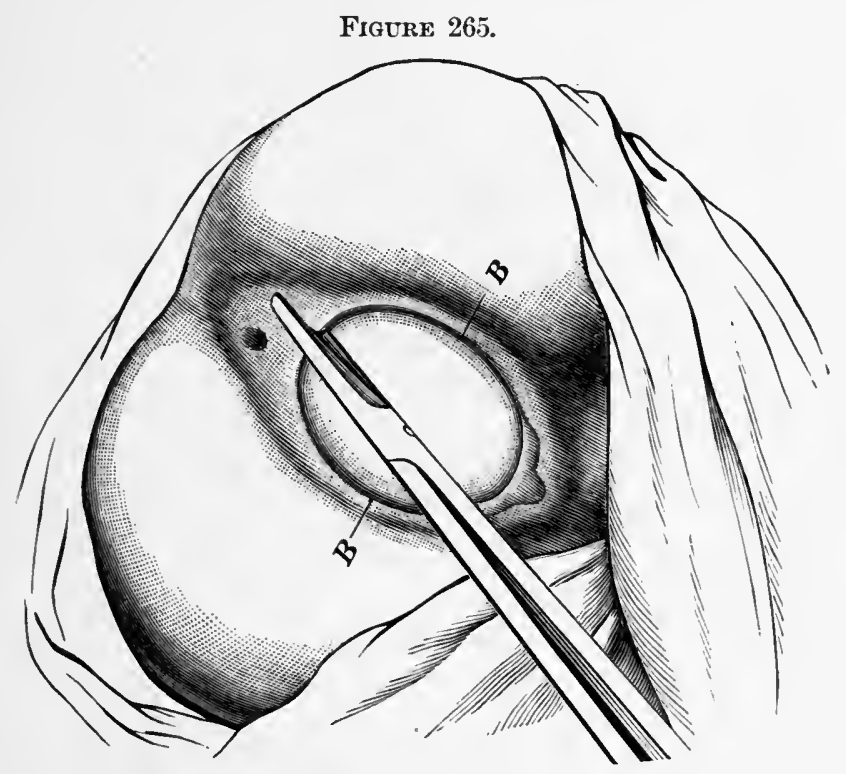

Correct incision in episiotomy. Lines $B, B$ show incorrect place for incision.

by an incision known as episiotomy, and thereby substitute a clean cut in another direction for a ragged, lacerated wound, which perhaps might involve the sphineter muscle. The usual method of episiotomy is to make a transverse ineision through the middle of the labium majus on each side. $B B$, Figure 265. The objections to these ineisions are that the rents may extend still further in the lateral direetion, as the head passes, and that an additional fresh tear may oceur at the posterior commissure of the vulva, making three wounds, all in awkward directions. A single incision in the direction shown by the seissors in Figure 265 is preferable, for any further tear will be to one side of the sphineter ani muscle instead of through it.

Complete Laceration through the sphineter ani muscle entirely 
destroys the retentive power of the bowel. The rupture in rare instanees occurs subcutaneonsly without a visible break in the eutaneons surfaces aronnd the anus. The diagnosis then depends upon the presenee of an open, relaxed anus. Relaxation of the sphineter and ineontinence of the bowel may oceur also independently of laceration.

\section{Results.}

The results of ineomplete laceration vary with the extent of injury and direction of the tear. The extent to which laceration is visible to the eye is not a safe eriterion, for, as already explained,

Figure 266.

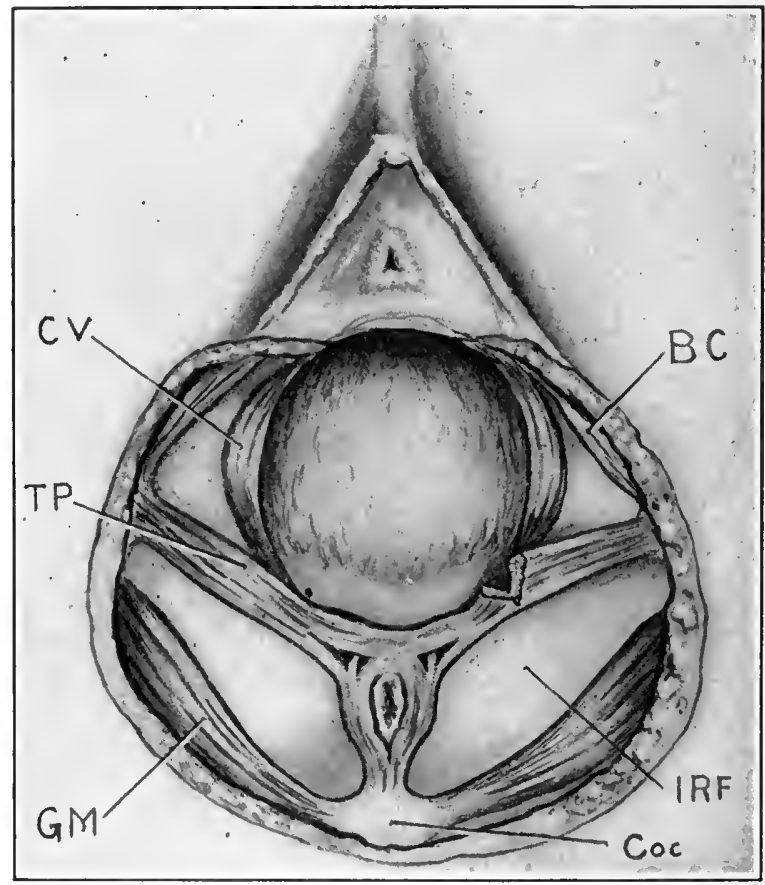

Downward pressure on the pelvic floor in labor. This figure shows how the muscles and fascia of the pelvie floor may be injured. $\mathrm{CV}$, constrictor vaginæ muscle. TP, transversus perinei muscle. Gm, gluteus maximus nuscle. BC, bulbocavernous muscle. IRF, ischiorectal fascia. Coc, coceyx. The left transversus perinei muscle is being torn.

the chief injury may be in the deeper structures, and reeognized not by sight, but by the effects. The effects may not be apparent immediately after labor; hence further examination is a necessary part of the efficient management of the puerperium. One examination should be made immediately after labor, to recognize and at onee repair such injury as may at that time be visible; another six weeks later, to reegnize and repair any deeper injuries to the perineal fascia or museles. 
Complete laceration, being usually in the median line, does not tear the supports of the pelvic floor so much as the incomplete. Moreover, if the direction of the tear, whether complete or incomplete, be in the median line, the muscles, fasciæ, and ligaments are not so seriously injured as they would be if the tear extended transversely across the perineum, especially if the structures were torn from their pubic attachments.

Figure 266 shows the child's head pressing strongly downward upon the transversus perinei and the bulbocavernous muscles and the reetovesieal fascia; such pressure gives rise to great sagging of

Figure 267.

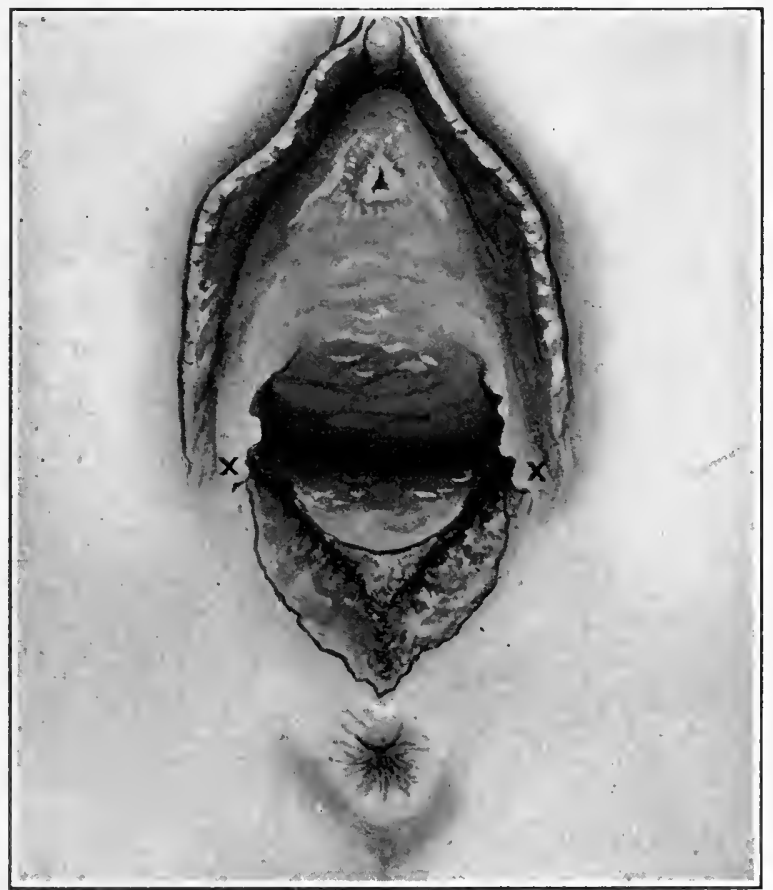

Fresh incomplete tear of the perineum. Observe the rent extending outward in the median liue toward the anus and inward in the vaginal sulci. $\mathrm{XX}$, the lowest remains of the $\mathrm{hymen}$ on each side-carunculæ myrtiformes.

the pelvic floor, and if the rectovesical and other fascia are injured extensively, the sagging, unless relieved by a suitable perineorrhaphy, is apt to be permanent. After the injury the whole pelvic floor, including the rectum, vagina, urethra, and bladder, now deprived of their support, tend to downward and backward displacement toward the tip of the encevx. The rectum, bladder, and vagina fall, as the lower jaw would fall if the masseter and temporal muscles were cut, or as a tent would fall if the guy ropes were cut. The backward displacement of the perineum is an incident and an index of the sagging. In very many of the worst cases the injury is mainly intravaginal, 
and shows little or no external evidence of laceration-that is, the cutaneous structures between the anus and the posterior commissure of the vulva may be unbroken. The palpable and visible split in the perineum may have relatively little significance; but injury to the rectoresical fascia and to the other fascire of the pelvic floor may give rise to displacement of the pelvic organs. Injury to the muscular part of the perineum accounts for frequent impairment of function

Figure 268.

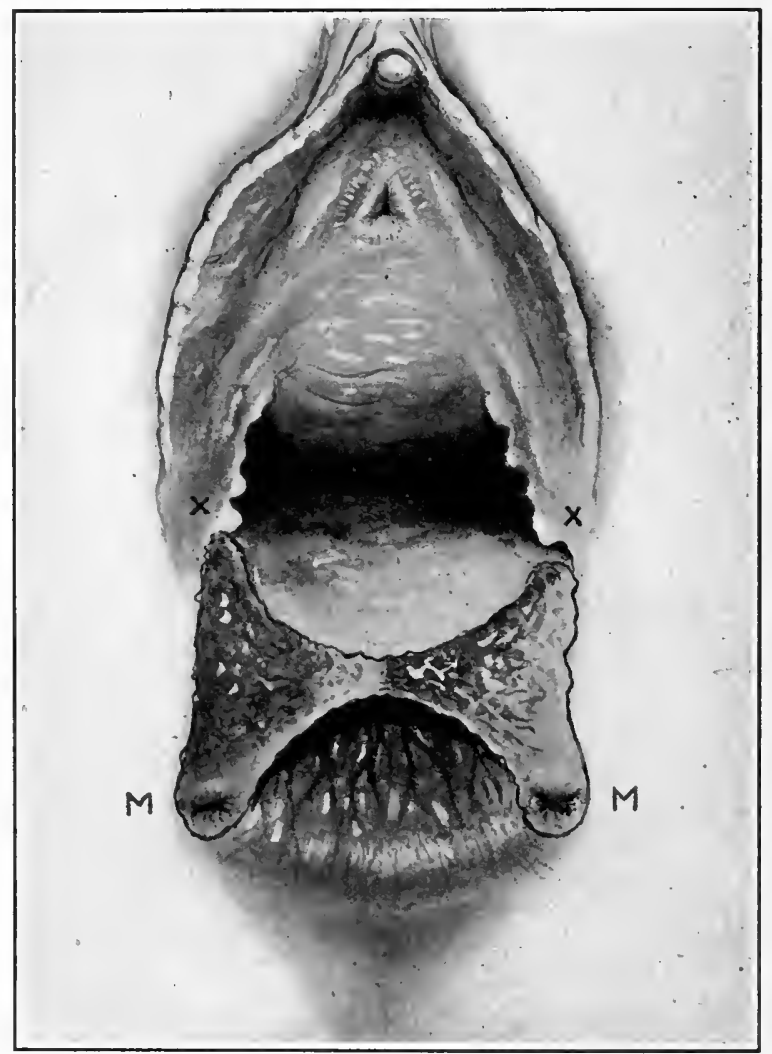

Fresh complete tear of the perineum. The direction of rent is in the median line. The rectovaginal wall is torn apart for a distance of about one inch. $\mathrm{XX}$, lowest carunculæ myrtiformes. MU, broken ends of the sphincter ani muscle.

in the organs whose outlets the muscles control-that is, in the bladder, urethra, vagina, and rectum.

Rectocele.-Figure 264 shows the direction of the anus to be at an angle to that of the rectum, so that as fecal matter comes down it must, in passing from the rectum out through the anus, turn this angle; in so doing it strikes against the rectal side of the perineum, and thence is deflected through the anus. If the injury to the perineal body has made it thin, weak, or relaxed, or otherwise impaired its 
resisting power, the downward foree of the feces, instead of being deflected backward and outward, will eause the posterior vaginal wall to pouch forward into the vagina. This pouch is a rectocele. The feeal matter thus arrested requires, with the enlarging poueh, more and more foree for its expulsion, and the pouch therefore will increase; the result will be rectal and anal tenesmus, irritation, and sometimes anal fissure or fistula, or hemorrhoids.

Figure 269.

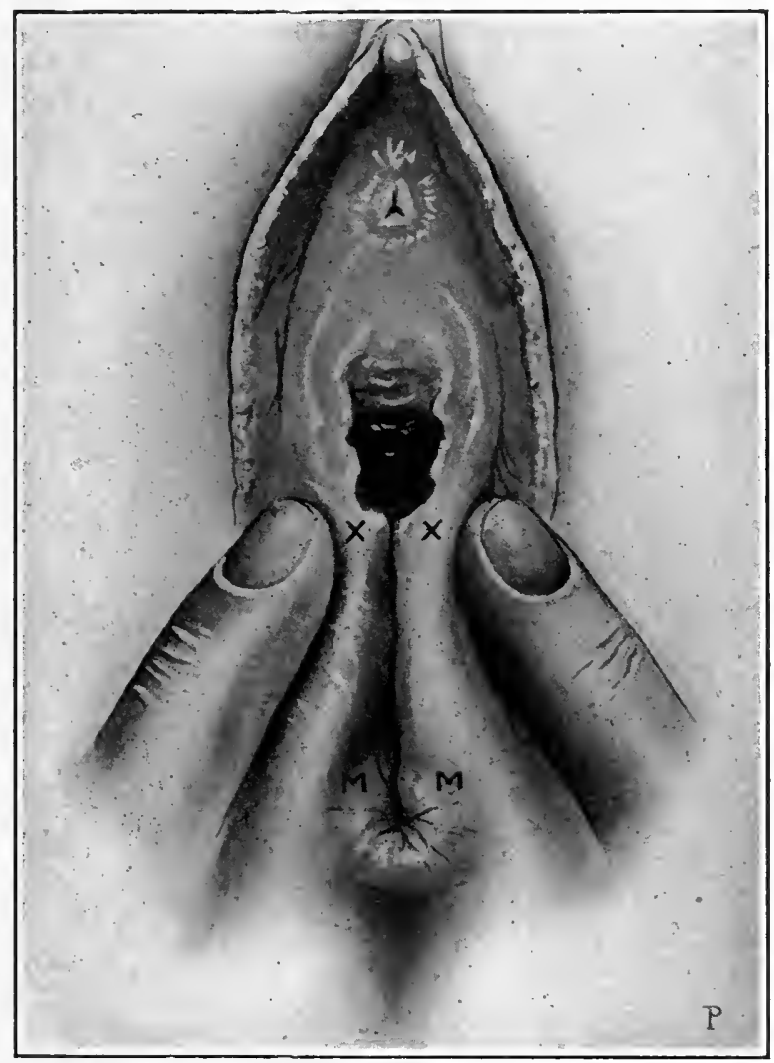

Same as Figure 268. The torn parts are being held together with the fingers, so that $\mathrm{X}$ coincides with $\mathbf{X}$ and $\mathbf{I}$ with $\mathbf{M}$. This adjustment shows what parts should be united in repair of the injury.

Cystocele.-Thə perineum having been impaired by rupture, the vesicovaginal septum, whieh normally rests upon it, tends to sag and bulge forward into the vaginal outlet in the form of a pouch. 'This pouch is ealled cystocele. The patient, exeept in the knee-chest position, may not be able to empty the bladder completely. Residual urine may aceumulate in the ponch, decompose, irritate the bladder, and may set up cystitis or nav lead to the formation of stone in the bladder. In order to expel the eontents of the bowel the woman may have to hold back the protruding organs with the hand. 
Figure 270.

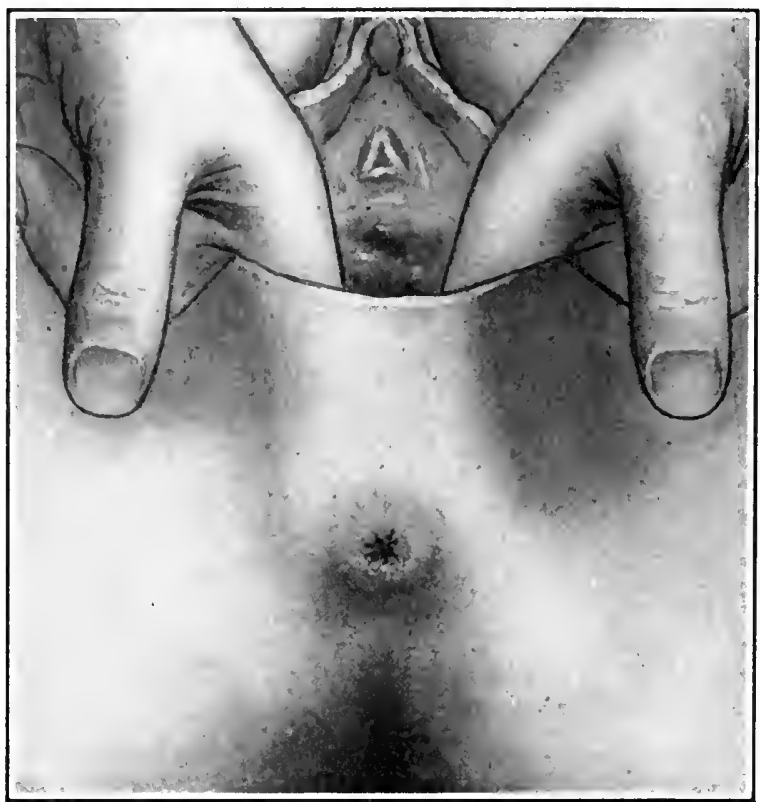

Relaxation of the vaginal outlet eonsequent upou labor. The injury is confined entirely to the interior, no cutaneous rent. 'I he perineum is extremely thin.

Figure 271.

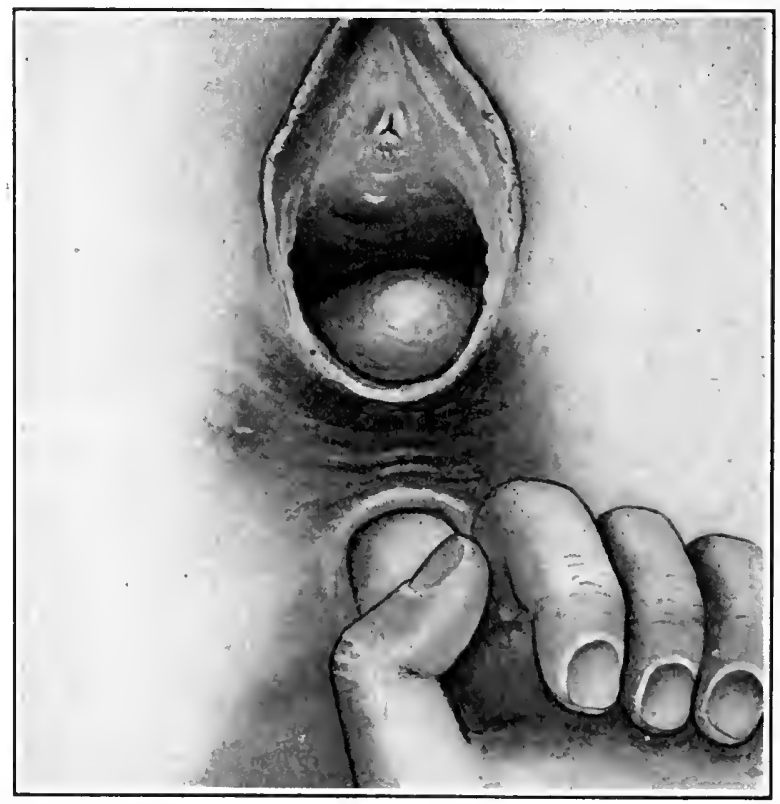

Same as Figure 270. The index-finger in the rectum forces the rectocele forward through the vuiva. 
Figure 272.

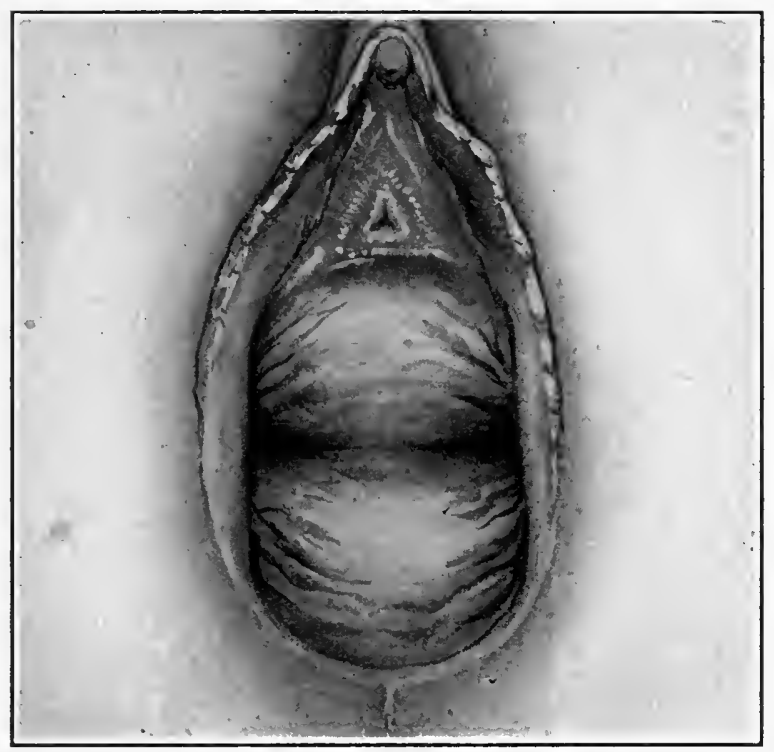

Cystocele and rectocele consequent on injury to the vaginal outlet.

The downward force of straining at stool to empty the bladder and rectum increases the prolapse of the vaginal wall, which, being

Figure 273.

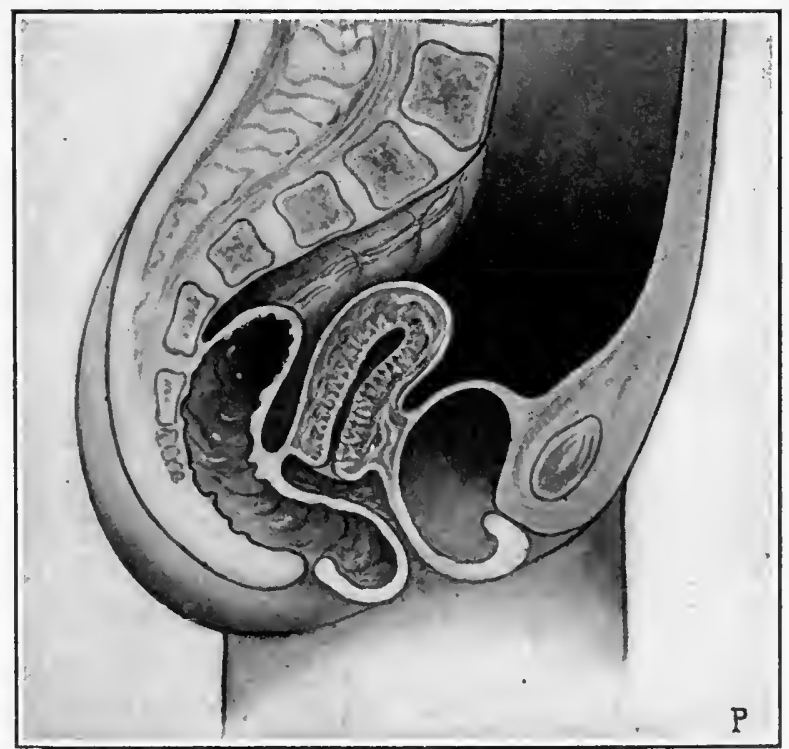

Same as Figure 272. Cystocele and rectocele shown in section. The protruding vaginal wall drags the uterus down after it. 
attached to the uterus, drags that viscus, together with its appendages and the rest of the pelvic floor, to a lower level, and thereby gives rise by traction to various displacements of the reproductive organs.

A wide range of organic and mechanical disorders naturally result from the above conditions; among them swelling of the vaginal walls, bearing-down sensations, a feeling that the "bottom has dropped out," difficulty of walking and standing, backache, constipation, and many nervous disturbances.

The relaxed or lacerated introitus vaginæ permits air to accumulate in the vagina, and at intervals, on slight change of position, to be expelled suddenly with an audible sound (garrulity of the vulva), which simulates the sound made by the passage of flatus from the bowel.

Chronic nervous invalidism is the possible indirect result of laceration of the perineum. This subject is discussed further in the chapters on displacements. 


\section{CHA PTER XLI.}

\section{PERINEORRHAPHY.}

ONE of the most important and most serious maxims in gynecology should be, "Never attempt the primary or secondary closure of a torn perineum until you have fully and clearly demonstrated and appreciated the direction or directions and extent of the injury." 1 Perineorrhaphy in a properly broad sense comprises not merely the closure of the torn perineum, but as well the repair of the injuries, both deep and superficial, of other structures in the perineal region.

\section{DIRECTION OF THE TEAR.}

A number of years ago the writer was called to make immediate repair of an incomplete though extensive rupture of the perineum. He had the belief, then commonly held, that such a rupture was usually a splitting apart of the perineal body into two lateral fragments which at once retracted to the corresponding sides. Accordingly, sutures were introduced from side to side in a way to reunite the lateral fragments by a line of union which should extend in the median line from the cutaneous to the vaginal side of the perineal body. The amazing result of this procedure was almost complete closure of the vulva so that the index-finger could only with difficulty be introduced into the vagina.

The absurdity of the situation was more apparent than the explanation; evidently the lacerated surfaces had not been brought together properly-but why? Upon removal of the sutures the torn surfaces were exposed again. A study of the injury then was made by hooking together opposite sides of the torn surfaces in different directions with tenacula. The result of the experimental approximations finally demonstrated the direction and character of the rent.

The four diagrams under Group I., Figure 274, explain the nature of this lesion and the operation of repair. Diagram 1 shows the margins of the wound before approximation. Diagran 2 shows the approximation, the correctness of which was demonstrated by the fact that all the little irregularities accurately fitted into one another, and that the integrity of the vaginal outlet was restored. Diagram 3 shows the sutures in position, but not tied. Diagram 4 shows the lines of union and the sutures tied. The line $a b c$, Diagram 4, represents the line of tear extending from side to side across the vaginal outlet inside the vulva; the point $b$ is situated in the median line; points $a$ and $c$ represent the extremities of the vaginal portion of the rupture, which extended high up across the lateral walls of the

1 E. C. Dudley. Chicago Clinical Review, April, 1894. The accompanying description of perineorrhaphy is adapted from this paper. 
Figure 274.

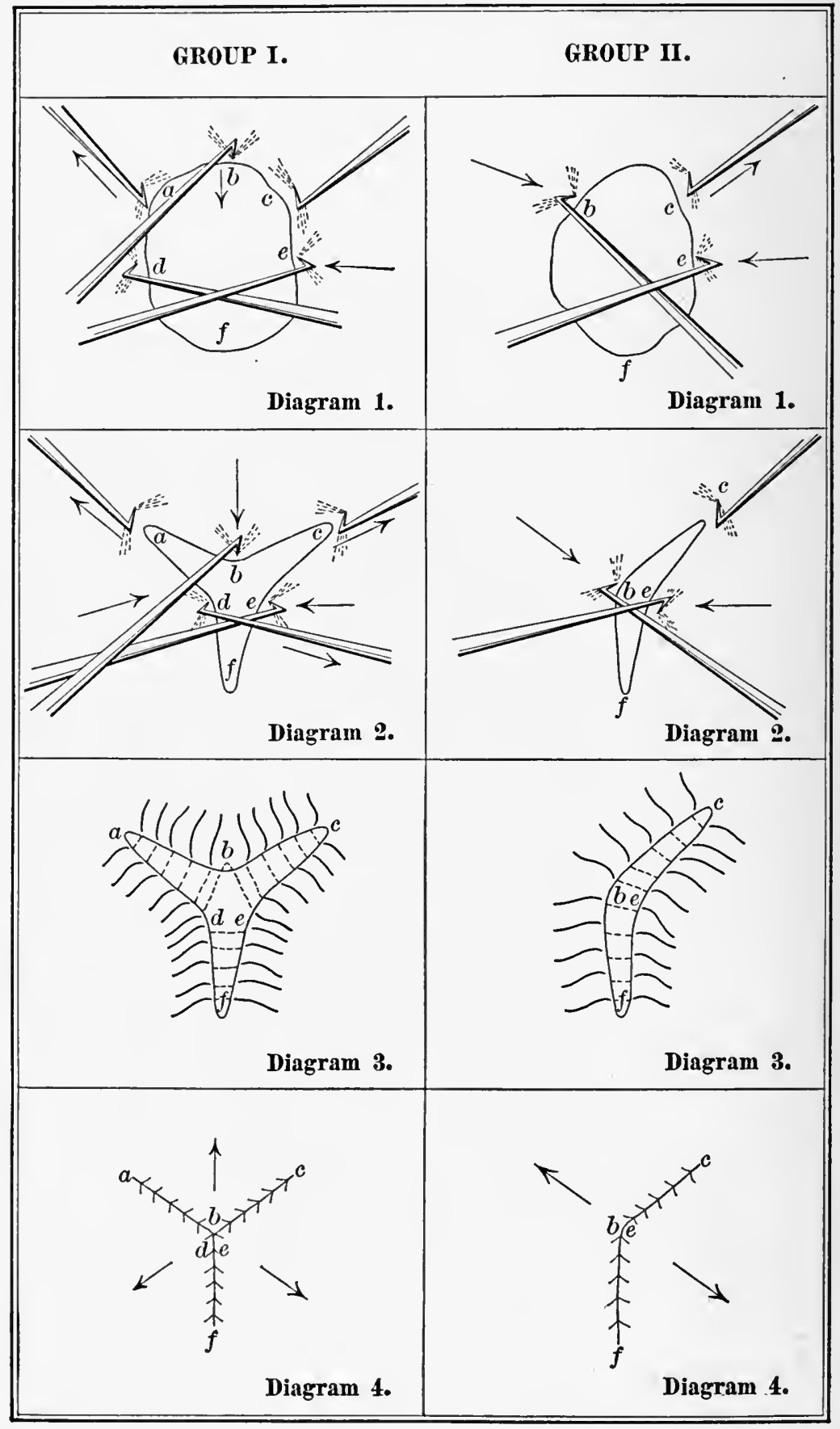


Figure 275.

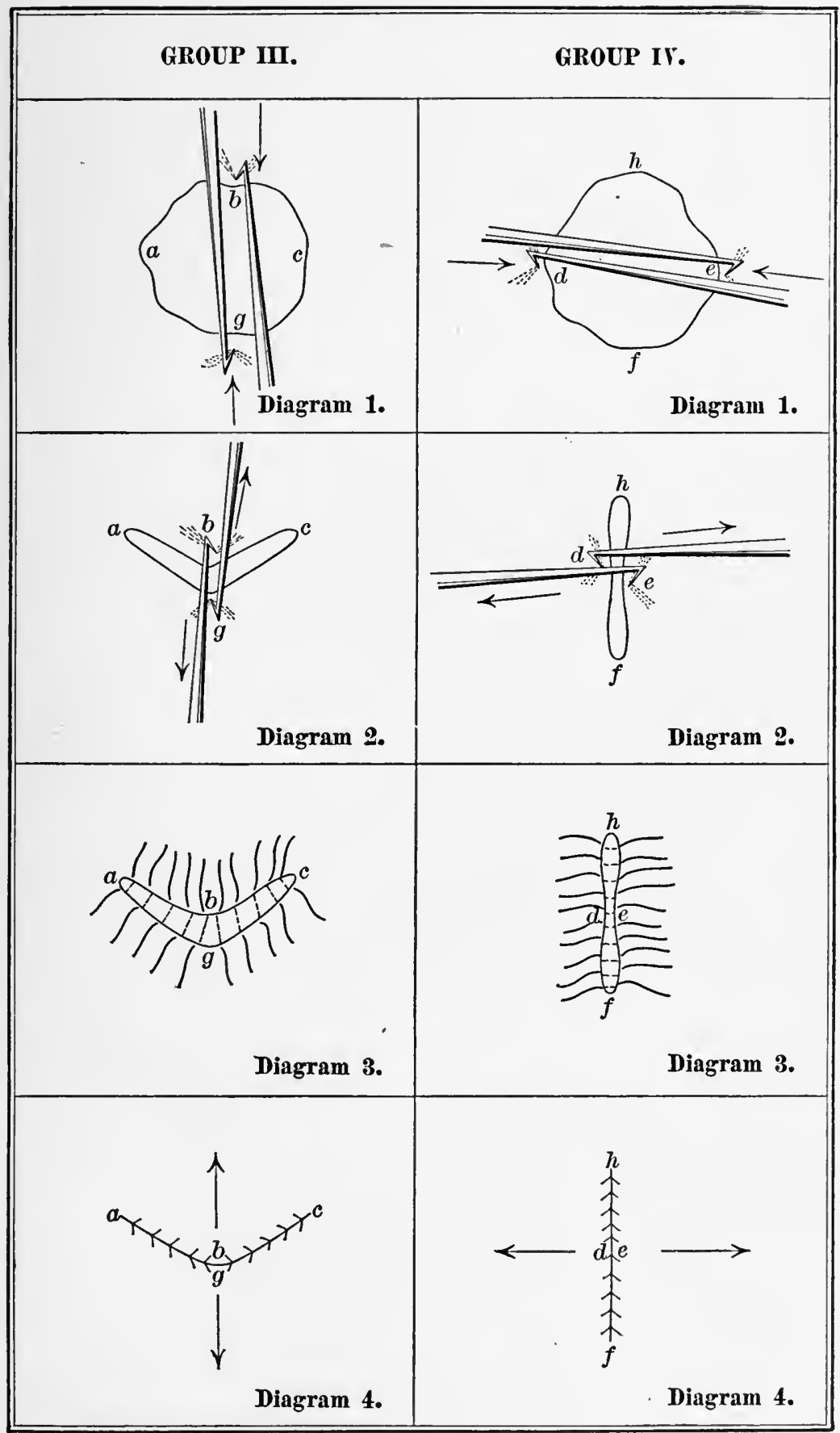


vaginal outlet in a direction parallel to the sides of the vulva. The arrow-heads show the directions in which the fragments retracted after the rupture until the exposed surfaces assumed the shape shown in Diagram 1.

The explanation of the closure of the vulva by the first procedure is now elear. It was the result of a line of union made at right angles to the actual line of tear-that is, the vaginal portion of the rupture had been from side to side, or in the transverse direction; it was closed as if it had been a longitudinal insteal of a transverse tear. This would necessarily close the vulva to a point as high as the injury extended on either side. ${ }^{1}$

In order to explain clearly the mechanism of the rupture shown in Group I., attention is called to another and very rare form of rupture, known as complete central rupture of the perineum-that is, a rupture in which the child is produced not through the vulva, but through a perforation extending from the vaginal side of the perineal body, directly through the perineal body to its cutaneous side, where the birth is completed between the vulva and the anus. This complete, central rupture of the perineum takes place in the transverse, not in the longitudinal direction. The transverse direction is determined by the general arrangement of the muscles and fascia surrounding the vulva, the fibres of which run for the most part in that direction and therefore are separated more readily in a longitudinal than in a transverse direction.

The vast majority of lacerations begin as complete central rupture, following the direction of least resistance-that is, transversely ; and continue until considerable progress has been made in the separation of the perineal structures into anterior and posterior fragments (line $a b c$, Group I., Diagram 4). Then, instead of continuing to complete central rupture and perforation of the perineal body, the expulsive forces are opposed more and more by the strength of the deeper perineal structures, the direction of least resistance changes to the longitudinal, with a corresponding change in the direction of the rupture, which now takes the longitudinal direction shown in line $b f$, Diagram 4. Notice the direction of retraction of the three torn fragments as shown by the arrow-heads in Diagram 4, a retraction which makes the irregular, torn surface of Diagram 1 . The exposed surface of Diagram 1, being partially intravaginal, often requires for its demonstration the sides of the vulva to be separated, or the perineum to be lifted forward by the index and middle fingers in the rectum.

The diagrams in Group I. represent a typical perincal laceration;

1 At the meeting of the American Medical Association in June, 1883, I described the transverse laceration of the perineum and its operative treatment. The paper was published in the Transactions of the Association of that year. This paper had reference only to the recent laceration and the immediate operation. In the first edition of Emmet's Principles and Practice of Gynæcology, which appeared about six months later, that author gave to the profession the epoeh-marking opcration on the vaginal outlet which has since been known by his name. Emmet's observations had special reference to the secondary operation, and were made without knowledge of my studies upon the recent laceration, as mine were made without knowledge of his work in the seeondary operation. It is the great credit of Fmmet to have given to the profession a secondary operation which brings the posterior vaginal wall up against the anterior
more perfectly than any other: this operation, however, though of wide, is not of universal. application. Besides, there are some matters of technique, soon to be described, which add grcatly to the result in bringing the perineum up to the pubes. 
the other three groups of Figures 274 and 275 show various modifications of this type. In each of these four groups Diagram 1 represents the exposed, torn surface of the bruised and distorted vaginal outlet, which approximates in each instance a polygon or an approximate circle bounded by a broken outline. It is an interesting fact that the description of any one of these tom surfaces will without change apply perfectly well to any other-that is, when the labia are separated, the outline of the torn surfaces does not necessarily give a definite idea of the direction of the rent. For example, the rent may be in the anteroposterior direction, and the two lateral fragments may have retracted to the corresponding sides, making the outline of the laceration as shown in Group IV., Iiagran 1; or the rent may have occurred transversely, and the torn fragments may have retracted, the one toward the raginal ontlet and the other toward the upper end of the vagina, leaving a similar torn surface, the outlines of which are shown in Group III., Diagram 1. This indefiniteness in shape of outlines in the exposed surfaces in the four groups is not only consequent upon the retraction of the torn fragments, but it is also caused by the loose, flabby, contused, rasped condition of the vaginal outlet-a condition conmon at the end of parturition.

Notice Diagram 4 in each of the four groups. In Group I. the lines of the tear correspond approximately to the shape of the letter $Y$. The upper part of the letter describes the transverse, vaginal portion of the tear; the staff describes the longitudinal, vulvovaginal portion. We really have three distinct lines of rupture : one shown by the line $a b$, another by the line $b c$, and the third by the line $b f$. Lines $b c$ and $b f$ of this figure describe the rupture of Group II. In Gromp II. the vaginal portion of the rupture runs diagonally to the patient's left. A precisely similar condition would be that in which the vaginal portion of the rupture runs diagonally to the patient's right; so that we may have, in addition to Group II., in which the laceration is left-lateral, a precisely similar injury in which it would be right-lateral. Lines $a b$ and $b c$, Group I., describe the rupture of Group III. ; line $b f$, Group I., describes the rupture of Group IV.

One may find, therefore, in the study of individual cases, by approximating the margins of the tear with tenacula, that the injury may correspond to any one or all of the lines in Group I., or to any combination of them. It may further show any variation in the length or regularity of these lines. It is a cardinal principle that, be these lines ever so variable in length and regularity, they can always be referred to the typical lines shown in Diagram 4, Group I.

The letters which have been used to designate the different points in each cut have, for purposes of convenience, been made to correspond one for all. For example, point $b$ in Diagram 3, Group III., occupies the same relative position as point $b$ in any other figure.

It is important to appreciate the mechanism of the injury as indicated by the arrow-heads in Diagram 4 of each group. They show the direction in which the torn fragments retract to make the broken outline indicated hy Diagram 1 of each group.

The upper, branching portion of the $\bar{Y}$-shaped tear indicates exten- 
sive injury to the perineal muscles and fascix, especially the transversus perinei muscle, and requires deep denudation and deep suture to catcli the impaired structures.

The further description of perineorrhaphy for incomplete rupture will be shown in Figures 276 to 287. In these Figures the letters $a$, $b, c, d, e$, and $f$ indicate the same points and landmarks which they indicate in the diagrams of Figures 274 and 275 . The chief points and landmarks have been pointed out; they are, the lowest remains of the hymen and the crest of the rectocele; if there be no rectocele, the point would be where its crest would be if there were one.

\section{PREPARATORY TREATMENT FOR PERINEORRHAPHY.}

The preparatory treatment consists of movements of the bowels and sterilization of the field of operation, as described in Chapter II.

\section{TECHNIQUE OF PERINEORRHAPHY FOR INCOMPLETE RUPTURE.}

\section{Primary Operation.}

In the primary operation-that is, the operation on the recently torn surfaces, denudation, except possibly the trimming off of any ragged edges of the wound, is unnecessary. In other respects the operation is substantially the same as the secondary operation.

\section{Secondary Operation.}

When the torn surfaces have healed over and cicatrized, after an unsuccessful primary operation or after no operation at all, correct denudation is essential, and is made possible only by studying the lines of the original tear by means of a tenaculum in each hand and having in mind the remains of the hymen and the crest of the rectocele as follows :

When labor has not resulted in laceration, the vaginal outlet will be surrounded by the remains of the hymen, which mark off the vulva from the vagina-that is, by the carunculæ myrtiformes. These consist of numerous small protuberances situated near together and surrounding the vulva, as it were, like a string of beads. They are sometimes so close together and pronounced as almost to constitute an annular hymen.

This circular line of carunculæ myrtiformes, in case of laceration, is broken at a point near the posterior commissure of the vulva, and when the break occurs the lowest caruncle on either side of the rupture is retracted to the corresponding side of the vulvar outlet. In a typical laceration, the two lowest caruncles will correspond to points $d$ and $e$ in the diagrams of Group I., Figures 274 and 275. Their location is indicated also in the corresponding points of Groups II. and IV. Figures 276 to 291 show the caruncles as they appear in the various stages of the operation. Group III., being a transverse laceration, does not involve the caruncles. The two lowest caruncles, 
on being approximated by the tenacula, show the surfaces to be united in the external parts of the rupture.

Having located the two lowest caruncles find some point near the centre of the upper fragment, point $b$, Figure $2 i 6$ (if a rectocele has formed, this will be its crest), and while the two caruncles $d$ and $e$ are being lield together, let point $b$ be drawn into coincidence with points $d$ and $e$. Then will the points $b, d$, and $e$ come together and form one and the same point. The coincidence of these three points will show what surfaces should be denuded and united upon themselves.

Remove the tenaculum at $d$, and reintroduce it at $f$. Then consider tenacula $b, e$, and $f$ as hooking up the three angles of a plane triangle. Let traction on the angles of this triangle be made by these tenacula in the hands of assistants, the direction of the traction being from the centre of the triangle toward each angle. The surfaces now put upon the stretch should be denuded. 'This completes the denudation on the left side, Figures 278 and 279 . Then remove the tenaculum at point $e$, reintroduce it at point $d$, place the included triangle $b d f$ upon the stretch, and denude as before. This completes the denudation on the right side.

Then remove the tenaculum at point $f$ and reintroduce it at point $c$, making upward traction, and at the same time approximating points $b$ and $e$ with other tenacula.

Then the surface bce, Figure 280, is to be united upon itself with a line of sutures so as to bring the line $b c$ in coincidence with the line ce. In like manner line $b a$ must be brought into coincidence with line ad. Finally, other sutures close the external rent, def, upon itself. Observe the suture of which the entrance and exit are at points $e$ and $d$, and which catches up point $b$, Figures 280 to 283. This suture, which is the "crown stitch" of Emmet, brings points $b, d$, and $e$ into coincidence. (See Diagram 4. Groups I. and IV. ; in Figures 274 and 275.)

In the introduction of sutures one of the greatest principles of plastic surgery should be observed, namely, that freedom from wounddisease and consequent union require the sutures from their points of entrance to their points of exit, so far as practicable to be buried, so that they shall not anywhere appear in the exposed surfaces.

Each suture should be tied before introducing the next, instead of first introducing all the sutures and tying them afterward, as is done nsually. One reason for this is that, under the former method, the sutures are less likely to antagonize one another.

In a typical laceration after the crown suture las been introduced (Figure 280) let the next suture be introduced and tied in the angle or sulcus on one side, point $c$. The free ends should not be cut immediately, but left long and given into the hand of an assistant, who should make firm traction upon them in the upward direction toward the pubes while the next suture is being placed and tied, when the long ends of this suture are also given, with the preceding one, into the hand of the same assistant. Then introduce the third suture, while firm traction is being made on the first two, precisely as the 
seeond was introdueed, and so on until the required number has been inserted on that side. Repeat this on the opposite side; or the sutures may be introduced as shown in Figures 280 and 281, one in one sulcus and one in the other, and so on until both sulei are closed.

Then introduce a suture under the lowest earuncle on the left side, through the erest of the rectocele, or the eentre of the upper fragment, carrying the suture around, buried all the way, out through the lowest caruncle on the opposite side. This is parallel to and supplements the crown suture already mentioned, and aids it in holding the three points, $b, d$, and $e$, together. With the tying of this and the crown suture the vaginal portion of the operation is complete. Figures 282 and 283. The buried suture mentioned above is not shown in the illustrations.

The external or vulvar portion is closed in the same way-that is, while each suture is being introduced and tied, firm traction is made upward, in the direetion of the pubes, on the preeeding sutures.

If the perineum be elosed in this way, it is surprising to see how it will be brought up so as fairly to hug the tubes. Indeed, the posterior part of the vaginal outlet will almost exert pressure upon the pubes and neek of the bladder. By this method the operator should never fail to get the perineum into its normal position and loeation.

Material for Sutures.-Silkworm gut is proferred. The free ends of the sutures are left long. Numerous devices have been used by many operators so to dispose of these ends as to prevent them from irritating the patient. Emmet ties them in a fan-shaped bundle and leaves them between the thighs; others eut them short. The irritation and suffering from this source are so extreme that some operators have used sutures of softer material, such as silk or eatgut; but sueh material is objectionable because the sutures absorb the wound-secretions, which seeretions may decompose and produce suppuration.

I have for several years used a device which entirely obviates this difficulty. All the sutures are left long enongh so that they may be laid down upon the vaginal surface and directed toward the upper end of the vagina. The sutures are well turned into the vagina and held there by an additional or binding stiteh whieh is tied over them and is situated just beyond the crown stiteh. The free ends of this binding stiteh are bent inward also in the direction of the long axis of the vagina. See Figures 284, 285, 286, and 287.

A study of Groups II., III., and IV., Figures 275 and 276 , will furnish a guide to the operation in atypical cases.

After-treatment.-The patient is not eatheterized unless unable to pass urine. Urine in eontact with the wound is harmless, the eatheter, even though used antiseptically, is apt to set up eystitis. She is permitted to lie in any position. The conventional roll under the knees and bandaging of the thighs are unnecessary except for the comfort of the patient.

The external sutures should be removed in twelve to fourteen days (Figure 286); the vaginal sutures in about twenty days. The removal of the latter is facilitated by the use of Sims' speeulum reversedthat is, hooked under the pubes, the patient being on the baek. (Figure 
Figure 276

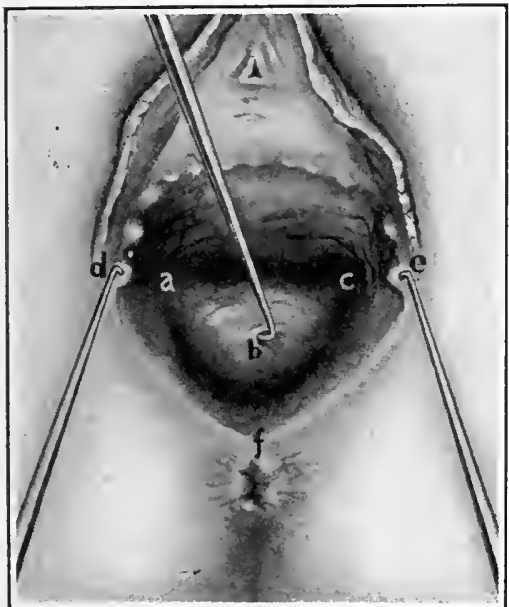

Figure 277.

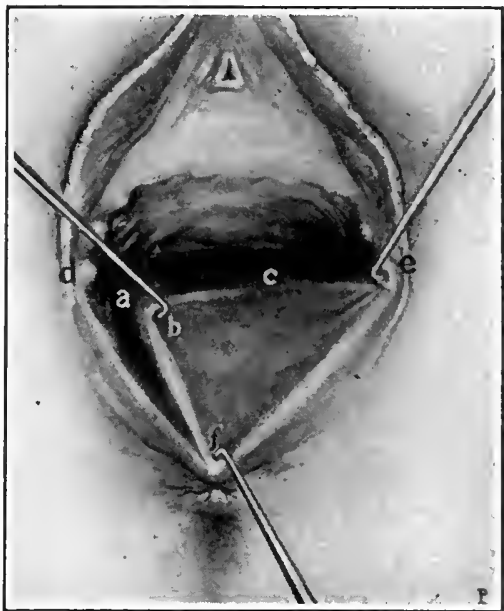

FIGURE 276.-Typical incomplete laceration of the perineum. The tenaculum hooked into the crest of the rectocele at point $b$ draws it slightly forward. The other two tenacula are hooked into the lowest remains of the hymen, points $d$ and $e$ icarunculæ myrtiformes). The three tenacula if approximated would bring into coincidence points $b, d$, and e, and would show what surfaces should be united.

FIGURE 277. - Same as 276. Tenaculum at $d$ removed and placed at $f$. Tenacula $b$, e, and $f$ make traction so as to render tense, lift up and expose for denudation the torn sulcus of the left side. The ridges formed by the structures drawn taught indicate the outline of the surface to be denuded.

Figure 278.

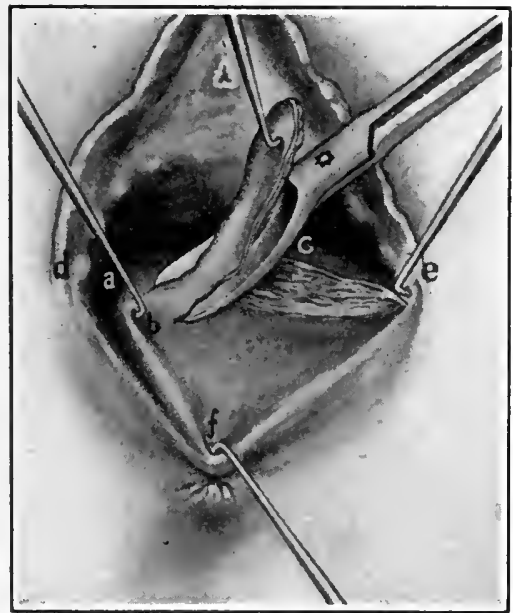

FIGURE 279

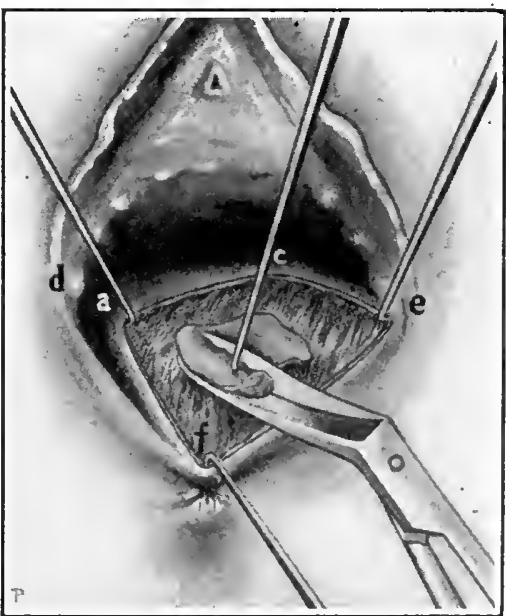

FIGURF 278.-Denudation, with Emmet's slightly curved scissors, of the surface exposed in Figure 277. A strip is denuded all around the surface, leaving an undenuded island in the centre, which retracts to small size.

FIGURE 279.-The island of undenuded surface left after denudation of the strip around the surface, exposed in Figures 277 and 288 , is being hooked up by a tenaculum and removed with scissors.

287.) During convalescence the patient may lie in any desired position. When the patient is on the back the legs and thighs may be 
Figure 280.

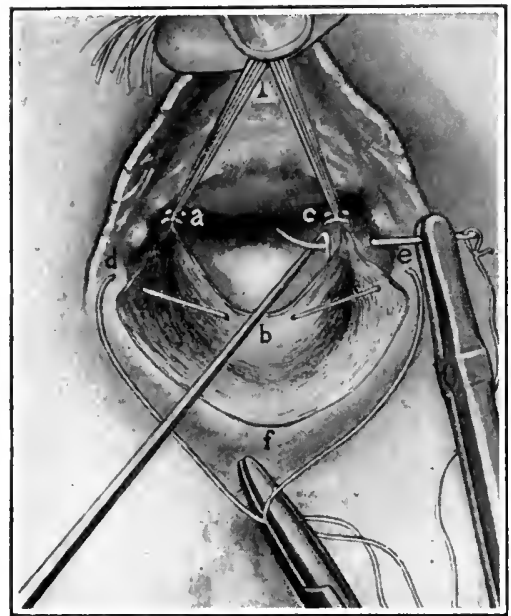

Figure 281.

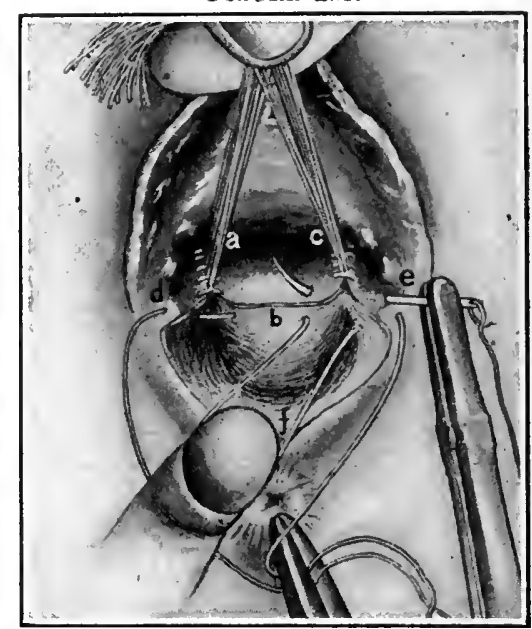

FIGURE 280.-The denudation shown on the left side of the vaginal outlet in Figures 278 and 279 has been carried out on both sides in this Figure. A suture, ealled the crown suture, has been passed under the left caruncle e, throngh the crest of the rectocele $b$, and under the right carnnele $d$. This suture, when tied later, will bring these three points together; before being tied it serves as a quide to the surfaces to be united in the two sulel. Two sutures introduced and tied in each suleus at the two lateral angles of the laceration are held taught by an assistant: a third suture is being introduced on the left side.

FigirRE $28 \mathrm{l}$. - Five sutures have been introduced and tied in the right suleus and four in the left. While the nurse is makiug strong traetion upward on these sutures, the final suture of the left suleus is being passed.

Figure: 282 .

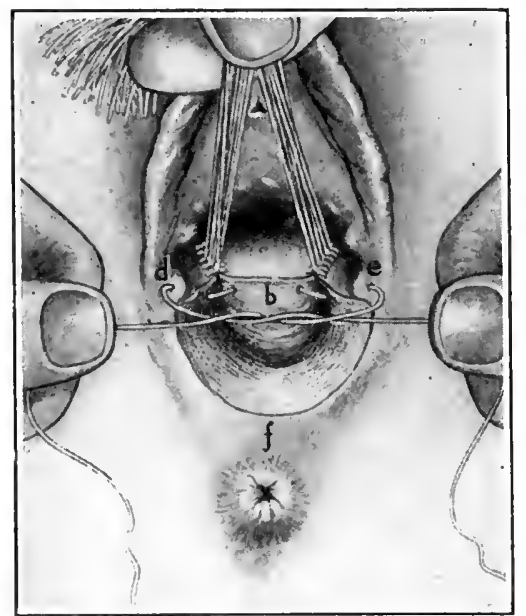

Figure 283.

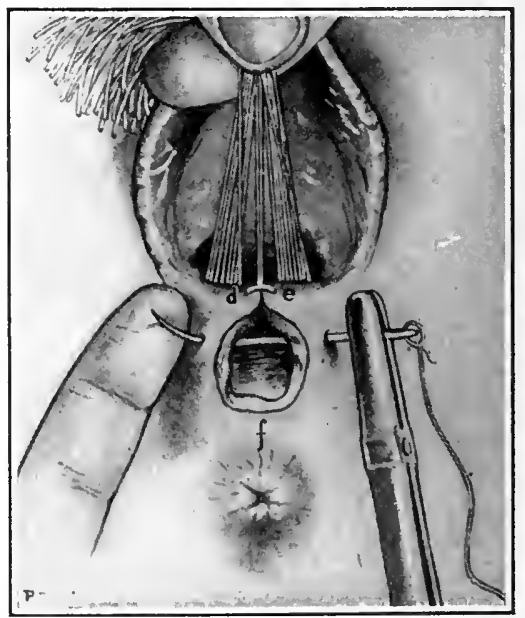

FIGURE 282.-All the sutures in the two sulei have been introdnced and tied. While the nurse is making strong truetion upward, the erown suture (shown in Figures 280 and 281), whieh brings together the two earuncles and the crest of the rectocele, is being tied. This eompletes the intravaginal part of the operation.

FIGi'RE 26:3.-The sutures which have completed the intravaginal part of the operation are being drawin strongly upward by the nurse, so as to lift the perineum toward the pubes while the first suture of the external part of the operation is bejng passed. Observe the netion of the finger in making counterpressure as the needle is passed through. Notice also that the needle is shown at the bottom of the wound. The general rule in plastic surgery to bury the suture completely under the wound although fuvorable to uniou is disregarded in the pussage of these outside sutures, for if so buried they would inchude and a raw forward into the restored perineal boly the reetocele. The rectoecle, howerer, is properly a portion of the rectovaginal wall, and in order that it may take its normal place back of the restored perineum it should not be included in these sutiires; in fact, during the passage of the external sutures it shonld be forced back to the deph of the wound by menus of the finger introduced into the wound so as to per. mit the denuded surfaces (1) be brought together in front of it. 
Figure 28.4

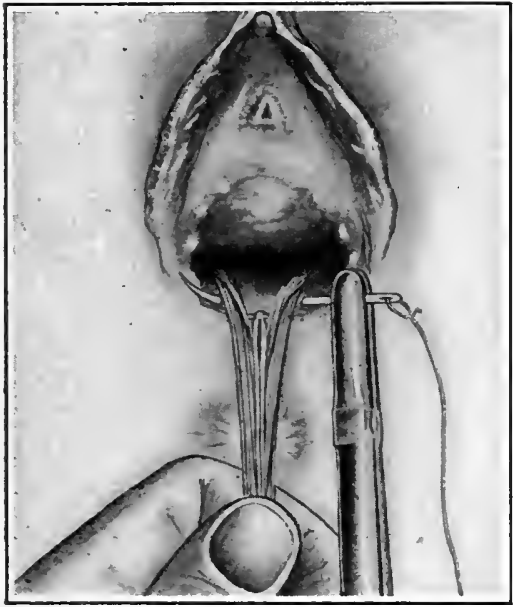

FyGUR 281 - The sutures intended for elosure of the perineum having all been tied are now temporarily held down and away from the vulva, as shown in this Figure. This is to facilitate the passage of a speeial suture just baek of the erown suture. As soon as this speeial suture has been passed, and before it is tied, the bundle of sutures is returned to its former position, as shown in the next Figure. The purpose of the suture now being passed will become apparent in the next two Figures.

FIGURE 285.-The special suture introduced in the last Figure is now being tied: its purpose is to seeure in a bundle the other sutures and hold them down against the posterior wall of the introitus vagine. The next Figure will show all the sutures turned in to the vagina. The speeial suture retains them there. The free ends of this retention-suture are earried with the others into the vagina.

Figure 286.

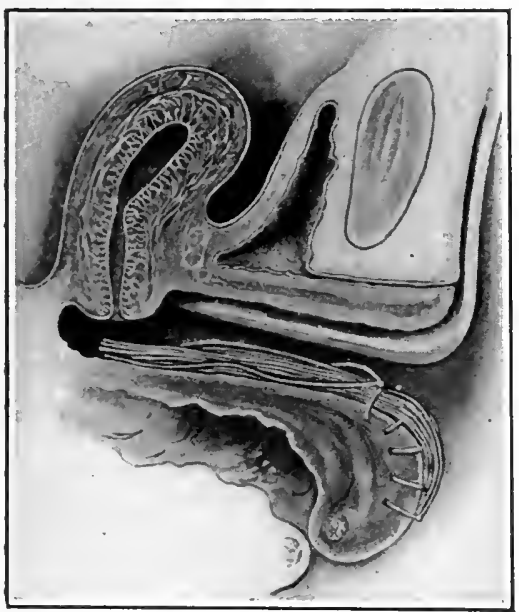

Figure 287.

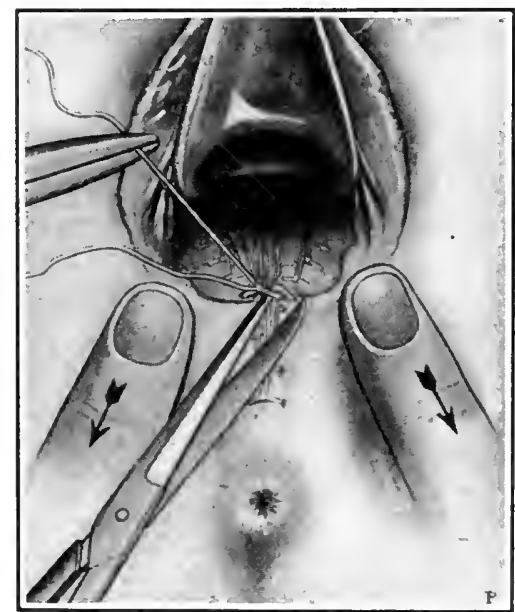

Figure 286.-This sectional view of the sutures in position and tied completes the illns trations of the seeondary operation for ineomplete laeeration of the perinenm. The entire bundle of sutures is shown turned into the vagina, where they cannot irritate the wound. This arrangement permits adequate dressings over the external part of the wound, and does away with the irritating and distressing ends of the sutures which commonly are left in eontaet with the external surfaces, and whiell always eontribute enormously to the discomfort and paiu of convaleseenee. A seetion of a sims speeulum is shown here. The instrument introdnced in this way-i.e., looked under the pubes, with the patient in the dorsal position-facilitates the turning in of the sutures at the elose of the operation, and may be used again in their remoral.

Figire 28\%.-In the removal of a suture a single free end is caught with the forceps and the loop ent with the scissors at one side of the knot. The external sutures should be removed at the end of two weeks, and the intravaginal sutures at the end of three weeks The vagina is exposed here by sinis' speeulnm hooked under the pubes and the crown suture is being removed. The remaining sutures are drawn out of the vagina and removed one by one. Observe the tengenlum point on the fine blade of the scissors, which holds up the loop and guards against the entting off of the knot which might eanse the loop to retract ont of reach. A pledget of cotton saturated with a 6 per eent. solntion of coeaine retained in the outlet of the ragina for ten minutes renders removal of the sutures painless. 
more comfortable if supported on a roll made of a blanket, comforter, or pillow. A sterilized douche should be given every twelve hours, and the external parts showered off after urination or defecation. The wound is dressed antiseptically with a pad of gauze and eotton held in place over the vulva by a T-bandage.

If secondary lemorrhage occur, the indication is for anæsthesia and prompt ligature of the bleeding point. For this purpose, the parts may be exposed by a speculum. Figure 267.

\section{Other Operations of Perineorrhaphy.}

The literature of the subject has been obscured by a countless variety of operations for the repair of the perineum. Every medical student is appalled by their number, their diversity, and their complexity. It is hardly possible, however, that perineorrhaphy should furnish an exception to the great general principle, that progress in any direction is characterized always by simplicity.

The object of perineorrhaphy is to replace rather than to enlarge the perineum. Many of the popular stereotyped operations which enlarge it really exaggerate the displacement.

The surgeon often is asked what operation he performs on the perineum. This implies that there is some fixed operation which is universally applicable. It would be no less absurd to ask what plastic operation is universally applicable to lacerated wounds of the face. It is not enough for an operator merely to get union at any cost, even though that union result in placing a solid mass of flesh where the perineum ought to be. Great harm comes if the parts brought into apposition are not parts which belong together.

Most of the stereotyped operations are prized because they make the "large, solid, perineal body" but such a perineum, composed of the union of parts which do not belong together, may be unfit for the performance of its functions, and may be very prone to subsequent rupture.

We hear much said about various stereotyped operations, the aim of which is to make a "large, solid, perineal body," a so-called "improvement on nature." This involves a radical and dangerous misconception. The large perineal body is contrary to nature, is unnecessary, is a disadvantage. The question is not of size, but of location. If the perineum, be it ever so small, is well up under the pubes, its location at that point indicates that the muscles and fascia of the pelvic floor are performing their function of supporting the pelvic organs. Let us have an end of the fallacy that the perineum supports the organs because it is large, or, for that matter, in a certain sense, that it supports them at all. By its location and integrity it only contributes to their support as an essential part of the pelvic floor. In its normal location and integrity it indicates that the pelvic floor is giving support to the pelvic organs, is doing its part in the prevention of prolapse, is fulfilling its functions. A torn perineum properly situated may be adequate. An enormous perineum, if displaced toward the coccyx, or relaxed, may require operative treatment. 
When a thoroughly scientific and satisfactory treatise is written on the subject of perineorrhaphy, it will not be an article describing the numerous and complicated operations. It will treat, in a general way, of operating in such a manner as to restore the parts to the condition in which they were before they were torn. The first step must be to find the landmarks, and Emmet has told us how to do this by bringing together the lowest caruneulæ myrtiformes on either side with tenacula; when this has been done, one may discern the direetions of the original rent and the cicatrices. On the correct observation of these landmarks will depend the method by which we must proceed to restore the perineal body so as to leave the vaginal outlet with an annular arrangement of the remains of the hymen. Failure to study these eases with the remains of the hymen as a guide accounts for the numerous and divergent methods of perineorrhaphy.

The greatest lesson in perineorrhaphy is to apply the elementary principle that, in the repair of a wound, the essential purpose is to restore the wounded part to its original state. Always individualize each case, find out the lines of tear, their direction, their length, and then put the fragments back where they were before. He who does this will do a different operation in every ease, but he will do one man's operation-the man will be himself. If one of the stereotyped operations in an individual produces a perfect. result, it will be not because it has anything like universal adaptation to the repair of an injured perineum, but because it chanced to fit that case.

The flap-splitting operation, for example, usually results in the union of parts which were not together before the rupture, and perchance cannot be united without detriment to the patient; it is often performed with little judgment, and since it is so easy that a tyro ean do it, has become popular. The prineiple of flap-splitting, however, as applied to perineorrhaphy, has great value in so far as it may enable the operator in some eases, to readjust the fragments to their original relations. If used with skill and judgment, in some eases of deep injury to the fascia it serves a most useful purpose. Its broad application beyond this has done great and irreparable harm.

The buried suture in perineorrhaphy would be beyond criticism if its use were not occasionally followed by infection. Numerous operations in the hands of careful aseptic surgeons have resulted in suppuration, burrowing of pus, formation of reetovaginal and rectoperineal fistulæ, and dangerous sepsis. The advantages of the buried over the ordinary interrupted suture which is tied on the surface do not outweigh this danger.

In cases of extreme cystocele and rectocele it is often necessary, especially in women who have passed the menopause, to combine with closure of the perineum the removal of a portion of the vaginal plate of the vesicovaginal and rectovaginal walls-an elliptical piece from the anterior vaginal wall, and a triangular pieee, with the apex toward the uterus, from the posterior vaginal wall. The margins of the vaginal wounds thus made should be united from side to side by interrupted sutures. The purse-string suture of Stoltz never should be 
used because it tends to shorten the vagina and thus to displace the uterus. See Surgical Treatment of Procidentia (Prolapsus Uteri).

\section{COMPLETE PERINEORRHAPHY.}

Perineorrhaphy involving the sphincter ani musele differs in some details from the operation just described; first, in the preparatory treatment; second, in the denudation; third, in the passage of the sutures; fourth, in the after-treatment.

Figure 288.

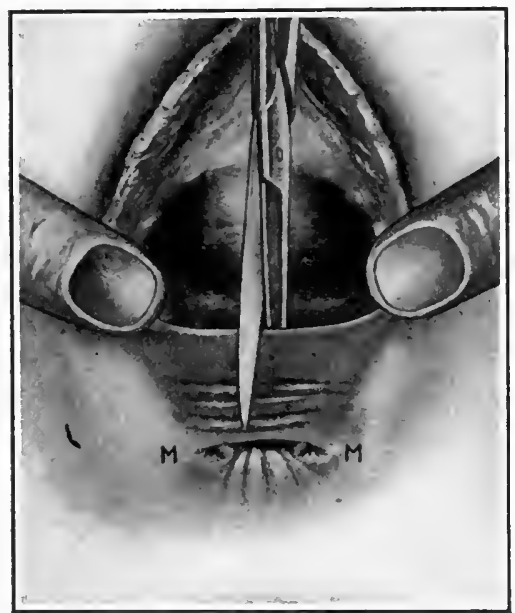

Figure 289.

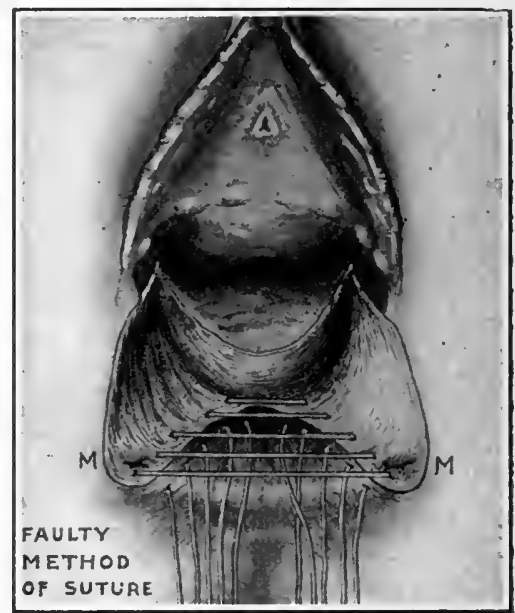

FIGURE 288.-An unsuccessful result after three operations of complete perineorrhaphy. Such failures are due commonly to the fault of the operator in not bringing together the torn ends of the sphineter ani muscle. Observe the radiating folds below the anus. After a suceessful operation these folds should radiate in all directions. After an unsuecessful operation they only radiate downward, as shown in the Figure. The retracted ends of the torn muscle are shown by the small pits marked MII, Figures 288 to 290 . The lower sutures should always be passed through the ends of the musele, as shown in Figure 290.

FIGURE 289.-Fanlty method of passing the anal sutures. Interrupted sutures placed in this manner and tied on the bowel side of the wound are open to the following objeetions: 1 . They make a long line of union whieh is exposed to the hostile environment of the bowel. 2 . The knots and free ends of the suture in the bowel may take up septic seeretions and carry them by capillary attraetion to the deeper parts of the wound, and in this way cause infection and fail. ure of union. 3. A long line of union is difficult to protect against infection.

\section{Preparatory Treatment.}

The ehances for union of the wound are increased by limiting the amount of feces which may pass over it during the first days following the operation; henee the bowels should be as nearly empty and aseptic as practicable. With this object they should be treated as in the preparation for major operations, Chapters V. and VI.

\section{Denudation.}

Figure 289 shows the rent extending up into the rectovaginal septum. At points M. and M are two pits or depressions caused by retraction of the ends of the sphineter ani musele. The prineipal object of the operation is the union of these ends and the consequent restoration of sphincteric function. The denudation, there- 
fore, must include the pits or depressions. They may be seen, though not always without eareful search, at either side of the anus. 'The denudation starts just below the pit on the patient's left, and is carried around on the margin of the torn rectovaginal septum to include the opposite pit.

A common fault in denudation is not to include these torn ends of the sphineter. Observe carefully that they are situated well down on a level with the posterior margin of the anus. Failure to carry the

Figure 290 .

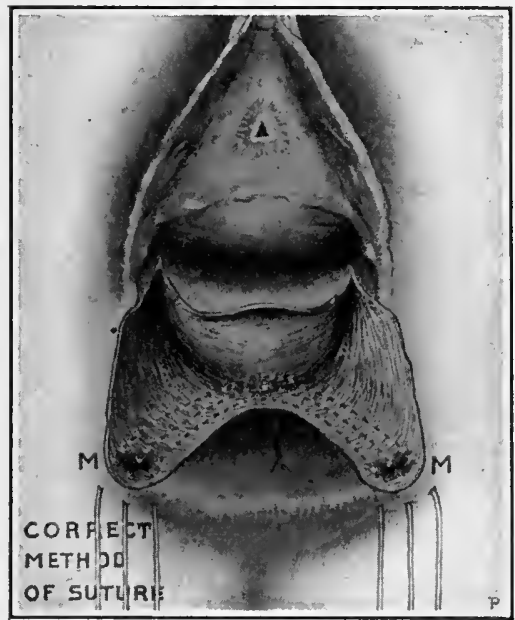

FigURE 291.

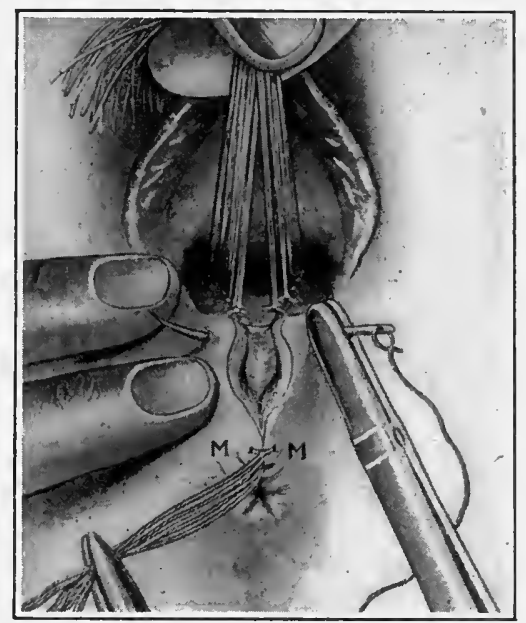

Frgure 290.-The purse-string method of suture. This draws the wound into a small compass; it leaves no part of a suture in the bowel to absorb and carry septic seeretions; the inner angle of the anal portion of the wound is drawn down to the margin of the anus, where it is less liable to infection than if the wound were longer and exposed to the interior of the bowel. Experience has shown that this method of suture is immeasurably more successful in securing primary union than that shown in Figure 289.

Figure 291.-Represents repair of a complete perineal laceration extending into the lateral sulci of the vagina. The three sutures which reunite the ends of the sphincter ani muscle are tied and held to one side by foreeps. Vaginal sulei elosed with five stitehes on each side, which are tied and held up by the assistant. The needle is being introduced for the passage of the crown stitch which unites the lowest carnneles. There is usually no reetocele in complete laceration. The remajuing sntures to be passed will close the external part of the wound. Observe that the folds about the restored anus radiate in all directions instead of downward, as in Figure 288. 'This is a reliable indication of the adequacy of the anal sutures.

denudation well below them elearly would defeat the object of suture. The remaining denudation then is done as for an incomplete rupture.

\section{Introduction of Sutures.}

The sutures should be of silkworm gut. The first two or three should be introduced to the left of the anus, should pass somewhat deeply under the left pit, as shown in Figure 290, should sweep around under the border of the torn septum, and pass under the opposite pit and emerge to the right of the anus. Figure 291 shows the ends of the sphincter united by the three lower sutures. The problem now is simplified to that of an incomplete operation, and the remaining sutures are placed as already deseribed for elostre of an incomplete laceration. The sutures, having been tied, are all 
turned into the vagina, as shown in Figure 286, and the vulva is protected by an aseptic gauze dressing.

\section{Complicated Operations.}

In some cases of complete laceration of the perineum, and more rarely in incomplete laceration, so much tissue has been lost from sloughing or from repeated denudation in former unsuccessful attempts at closure, that the denuded surfaces cannot be approximated by sutures. In such cases recourse may be had to the device shown in Plate XIII., Chapter XXV. The author published this method in Surgery, Gynecology, and Obstetrics, June, 1906. It gave a good result in one case after seven unsuccessful attempts at closure by the ordinary method.

\section{After-treatment.}

A full cathartic of castor oil or compound licorice powder should be given on the third day, and repeated, if necessary, to secure free catharsis. Excessive catharsis, producing frequently repeated liquid stools, might set up irritation of the anus sufficient to prevent healing, and therefore should be arrested by the use of a teaspoonful of paregoric every time the bowels act until stools are less frequent. After the first movement of the bowels the stools should be kept semifluid. This may require a cathartic at intervals of not more than two days. During the first week, whenever the bowels are about to move, it is well to give a rectal enema of eight ounces of olive oil. In giving the enema the syringe-tip should be passed carefully along the posterior wall of the anus away from the anal sutures. Carelessness at this point may break open the newly united surfaces and destroy the result. An inexperienced nurse should not be permitted to give the enema.

If there is no suppuration, the sutures should not be removed until about the fourteenth day. In other respects the after-treatment is the same as for incomplete laceration.

If there is retention of urine and the catheter is required, one may avoid the cystitis which sometimes follows catheterization by throwing into the bladder two drams of a 10 per cent. solution of argyrol, and leaving it there after each catheterization. 


\section{CHAPTER XLII.}

\section{PUERPERAL LACERATION OF THE CERVIX UTERI.}

THE credit of having established the pathological significance and surgical treatment of laceration of the cervix uteri belongs to Emmet. His three original communications ${ }^{1}$ not only contained the first practical information on the subject, but, what is more remarkable when we consider the great frequency and the far-reaching pathological results of the lesion, the information which they contained was at once so complete, so accurate, and so adequate that little if anything of importance has been added.

Vague allusions to the subject had appeared from time to time before the publication of Emmet's papers, but only to record the fact that such an injury could result from parturition. They contained little account of its pathological significance and none of its surgical treatment. ${ }^{2}$

\section{Causes of Laceration of the Cervix Uteri.}

The causes of laceration of the cervix uteri are:

1. Relative disproportion in size between the child and the cervix.

2. Rigidity of the cervix.

3. Rapid second stage of labor.

4. Any disease of the cervix which causes friability or impairs elasticity.

5. Instrumentation.

6. Meddlesome manipulation, such as manual dilatation of the cervix to hasten labor.

The cervix is not fully prepared for dilatation and the transmission of the child until the end of the normal period of gestation; hence the greater liability to injury in premature and immature labor. Abortion in the earlier months of pregnancy is not a frequent cause of laceration, except as it may result from forcible dilatation. A greatly prolonged labor may, by continued pressure, induce nutritive changes, and thereby decrease the elasticity and increase the liability to rupture. The condition is an approach to pressure-necrosis.

1 "Surgery of the Cervix Uteri." American Journal of Obstetrics, February, 1869. " tion of the Cervix Uteri as a Frequent and Unrecognized Cause of Disease." Ibid., November, 1874. "The Proper Treatment of Lacerations of the Cervix Uteri." American Practitioner, January, 1877.

2 The Causes and Treatment of Sterility. Gardener, 1856. Cicatricial Ectropion of the Cervix. W. Roser. Archiv für Heilkunde, ii. S. 97, 1861. 


\section{Pathological Anatomy and Results of Laceration of the Cervix Uteri.}

At the outset, let the important fact be kept clearly in mind that the injury is usually more extensive in the surrounding vaginal structures than in the cervix proper. This is, perhaps, contrary to the usual notion; but will be apparent on examination of later paragraphs in this chapter which treat of the state of the cervix before, during, and after labor.

Figure 292.

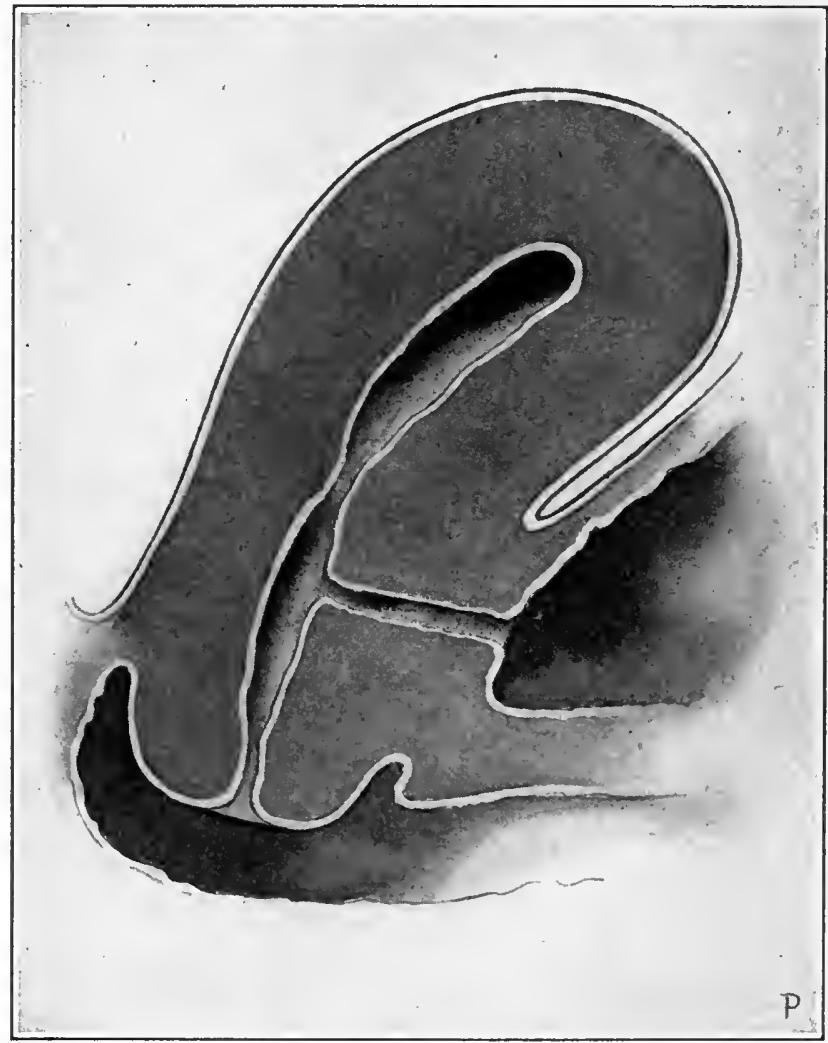

Vesico-uterine fistula at angle of laceration. The fistulous tract has been left after partial healing of an anterior laceration.

The diameter of the cervical canal in the non-pregnamt uterus is about one-fifth of an inch. This must be increased at delivery to correspond to the diameter of the child's head; it is, therefore, not surprising that some degree of laceration oceurs in the majority of labors. The lesion, however, is generally slight, and heals so readily and rapidly as to cause little or no pathological result.

The Directions and Extent of cervical laceration vary within the widest limits-i.e., the cervix may tear in any direction and to any extent. The usual directions are : anterior, posterior, and lateral. 
Anterior and posterior lacerations, especially the former, usually heal spontaneously, and, therefore, seldom are observed. This healing is explained by the anatomical arrangement of the vaginal walls, which tends to keep the torn fragments in close contact while union is taking place.

Anterior laceration in rare instances may extend so far as to invade the bladder and make a vesico-uterovaginal fistula. In such a case, if attention be paid to cleanliness, considerable spontaneous healing usually follows. There may be left, however, a small vesicovaginal fistula near the cervix uteri, or a vesico-uterine fistula at the angle of the laceration, extending from this part of the cervical canal into the bladder.

Posterior lacerations, extending into the posterior vaginal pouch, may open the way for post-nterine infection, and thus give rise to contracting cicatricial bands which may draw the uterus downward and backward, and fix it in an intractable retroversion or retroflexion. A variety of distressing and disabling functional disturbances, including menstrual disorders, sterility, and extension of infection to the parametria and the peritoneum, are among the results which may be expected from this condition.

Lateral lacerations occur most frequently to the left, less frequently to the right and left, least frequently to the right of the cervix.

\section{The False Cervix.}

Nature, instead of repairing the injury of a lateral laceration, resorts to a deception so artful that, until explained by Emmet, the lesion had been practically an unknown factor in uterine pathology. By this deception a false cervix, composed chiefly of ontrolled intra-uterine and reduplicated vaginal tissue, is substituted for the normal cervix. The evidence of laceration-that is, the irregular, fissured, uneven appearance-is so obliterated that even the practised eye may fail to recognize it. If diagnosis between the normal and the lacerated cervix were solely dependent on sight, cases commonly would arise in which increased size, congestion, and erosion would be the only diagnostic signs.

The condition of the cervix before, during, and after labor, as laid down in the following statement, ${ }^{1}$ has a determining influence upon the immediate mechanical results of laceration.

Before labor, from the moment of the pregnancy, the cervix, as well as the body of the uterus, enlarges to accommodate the growing fœetus. From the first, the entire cervix, except a small part which surrounds the external os, expands symmetrically with the body above. This expansion early in pregnancy obliterates the internal os, and converts the entire cervix into an incerted dome, which projects into the vagina, and whose walls are continnous with those of the corpus. Thus, long before term, a very large part of the fotal covering is composed of evolved and expanded cervical tissuc.

1 John Bartlett. Chicago Medical Journal, October, 1873. Wilhelm Braune. Atlas of Topographical Anatomy, Leipsic. Translation. Philadelphia, 1877. 
During labor there will be some plane in the cervix above which the muscular wall of the uterus contracts, and below which it dilates, for the expulsion of the child. Examination after delivery shows a hard, contracted, unyielding ring. This sometimes has appeared to the examiner to be the contracted external os. It is, however, above the plane of the external os, perhaps even above the uterovaginal attachment. The plates of Braune, drawn from frozen sections of the gravid uterus, show the remnants of the internal os to be on a plane far above this contracted ring. It is, therefore, neither the contracted internal os nor the external os, but is situated between the two, and is the lowest margin of the contracted part of the uterine wall. It is a temporary intracervical os, below which one must look for that part of the cervix which during labor was compelled to undergo excessive dilatation; and one must expect there to find laceration if it be present.

Without care this lowest part of the cervix, which has been so stretched that it cannot recover immediately its contractile power, will be overlooked. It can, however, always be felt projecting into the vagina as a "flabby, floating collar," not unlike a "section of large intestine," and having even less contractile power than the sphincter ani muscle after extreme forcible dilatation.

After normal labor this lowest portion of the cervix slowly recovers its contractile power, and in a few days resumes its normal shape, and the integrity of the external os thereby is restored.

In bilateral laceration, nature has especially all the conditions for the formation of the false cervix already mentioned. The anterior and posterior diverging flaps of the cervix are forced at once in the directions of least resistance : the former forward toward the vaginal outlet, the latter backward into the posterior vaginal fornix. The congested tissues about the temporary os, which in the foregoing paragraph has been called intracervical, meeting no resistance, now roll out. This eversion gives rise to obstruction in the uterine circulation. The intracervical structures, thus engorged and swollen, no longer have sufficient space for their accommodation within the uterus; hence the eversion continues until tissue enough for the formation of the false cervix has been rolled out into the vagina, and until the temporary intracervical os actually may have usurped the place of the now destroyed os externum. This everted intracervical mucosa, when rolled out into the hostile environment of the vagina becomes infected, and the infection may extend along the mucosa to the endometrium, Fallopian tubes, peritoneun, and ovaries; or by continuity of the deeper tissues to the myometrium, perimetrium, or parametrium. Laceration of the cervix, therefore, supplemented by infection, may open the way to extensive pelvic disease.

Subinvolution.-The physiological hypertrophy of pregnancy, which ought to subside after labor, under the influence of infection may fail to do so, and may become pathological. Hence the uterus remains enlarged ; this enlargement, called subinvolution, is a very common result of laceration. It usually pertains more to the cervix than to the corpus uteri. See Chapter XVIII. 
Descent and Vaginal Reduplication.-When the patient assumes the upright position the supports of the heavy congested subinvoluted

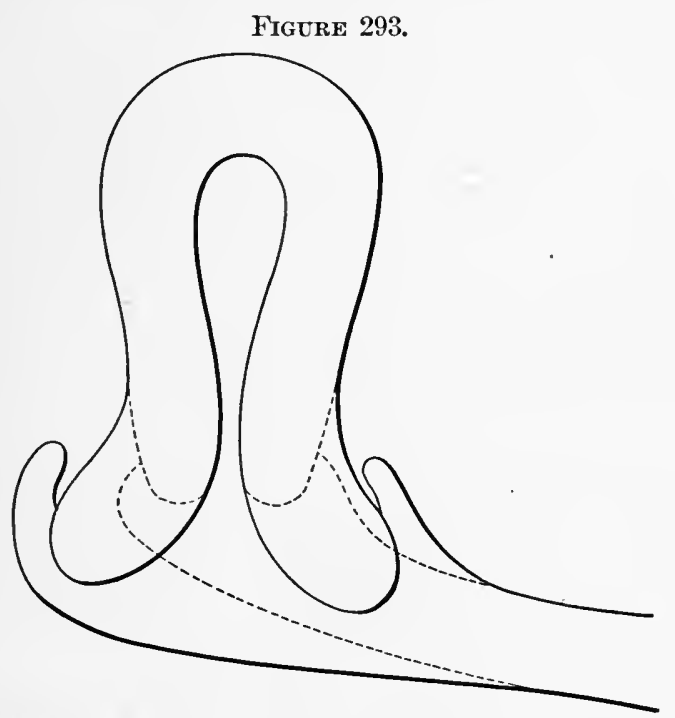

The widely separated lips of the recently lacerated cervix. The posterior lip is crowding backward into the posterior vaginal fornix, the anterior lip forward toward the vaginal outlet. The dotted lines show the contour of the uterus and the vagina before the laceration. The location of the temporary intracervical os is at the bottom or angle of the laceration.

\section{FigURE 294.}

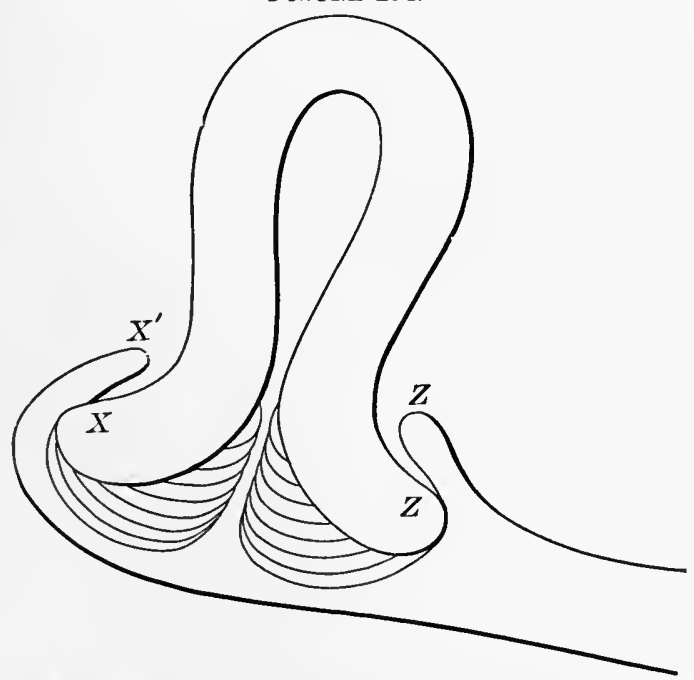

Shows the circular enlargement of the cervix due to outrolling of the intracervical tissue, and the apparent elongation due to reduplication of the vaginal walls. The actual uterovaginal attachment is at $X$ and $Z$. The reduplication makes it appear to be at $Y^{\prime}$ and ' $Z^{\prime}$. See Figure 300.

uterus are inadequate to hold it on the health level; it settles by its own weight to a lower level and carries with it a reflected fold of the 
vaginal wall. See Figures $297-299$. The vaginal portion of the cervix thus is made apparently much longer than it really is. The soft, easily moulded, outrolied intra-nterine tissue and the reflected vaginal walls may obliterate completely the fissure which is regarded commonly as the evidence of laceration; upon ordinary examination, therefore, the tear may be overlooked entirely. The deception may be exposed by placing the patient in the knee-breast position. The uterus, by its own weight, will be earried then toward the diaphragm; the vaginal wall will unfold and diselose the true uterovaginal attachment; and not uneommonly a deep laceration may be seen extending on either side far into the vaginal walls.

Figure 295

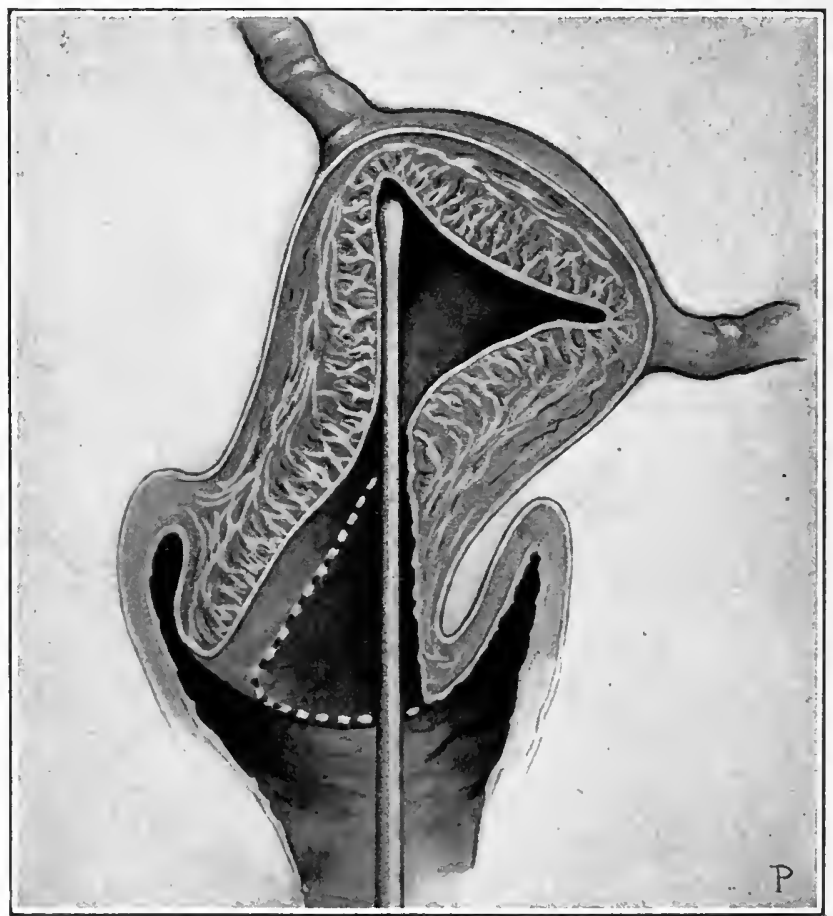

False cervix in unilateral laceration. Obliquity of the uterine axis from contraction of broad ligament.

When the laceration is confined to one side, the deception of the false cervix is quite pronouneed, for, as shown by Emmet, the fundus in sueh eases usually is drawn toward the affeeted side by inflammatory contraction of the nearest broad ligament. The effeet of this lateroversion is to raise the uninjured side of the cervix a trifle higher in the pelvis, and eorrespondingly to depress the injured side, thereby eausing a reflection of the raginal wall on the depressed side, so that, as in the bilateral injury, the apparent os externum may seem to be in the very centre of the cervix when it is really on one side. To 
add to the confusion, the sound, entering at the side, may, though passing to the horn of the opposite side of the uterus, appear to pass in the median line. Figure 295.

Cystic Degeneration.-Puerperal laceration of the eervix uteri causes marked outrolling of intra-uterine tissue and consequent permanent, passive congestion. The delicate intra-uterine membrane, instead of being in contact with the mild alkaline secretion of the uterus to which it is aceustomed, is in contaet with the irritating acid seeretion of the vagina. But the misehief does not end here. The uterine supports soon may prove unequal to the work of sustaining in position a uterus heavy from congestion, and the uterus falls to a lower plane in the pelvis. The everted membrane, in eontact with

Figure 296.

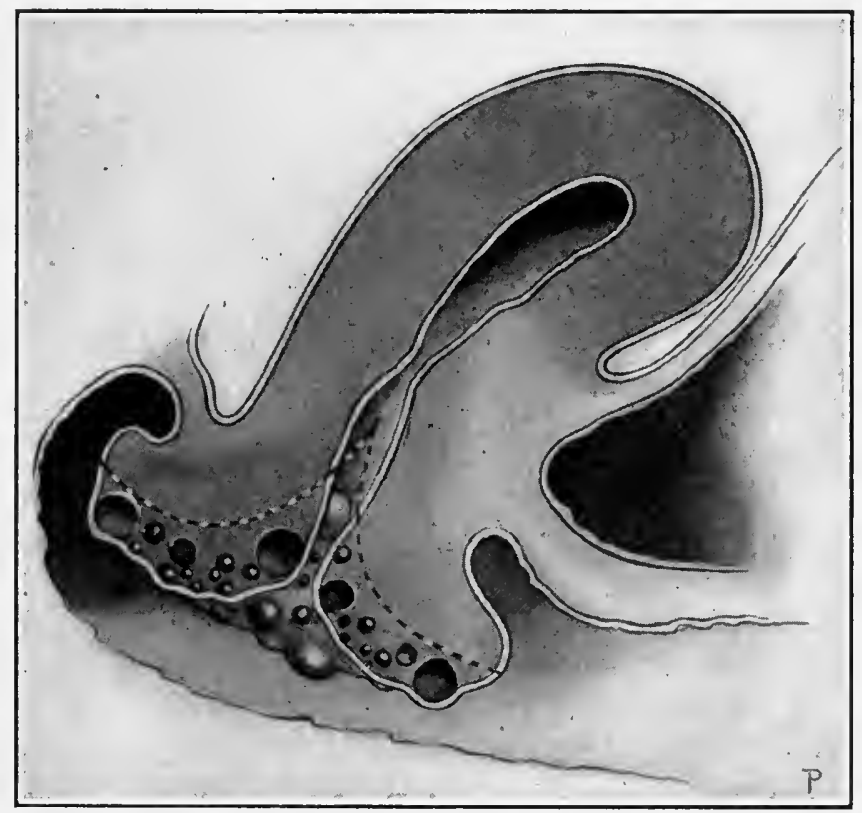

Double laecration, showing eversion of intra-uterine mucosa and enlarged mucous follicles on the false cervix.

the posterior vaginal wall, and eonstantly bathed in the vaginal secretions, is subjeet, by reason of the normal movenents of the uterus, to the additional irritation of frietion. An erosion forms, and the mucous follicles, Nabothian glands, estimated by Tyler Smith to number ten thousand in the normal virgin cervix, become discased. Some of them pour out the familiar thick, viscid, ropy, or purulent secretion. Others, in eonsequence of adhesive inflammation which has oceluded their outlets, become distended by their own secretion and undergo cystie degeneration. Figure 296. These eysts are generally present, frequently in large numbers. Subinvolution, including enlargement of the uterine blood-vessels, is a natural sequence of these clianges. 
Pathological Anatomy of the False Cervix.-As already outlined, the false cervix is composed of:

Everted intra-uterine tissue.

Reflected vaginal wall.

Cervical follicles which have undergone cystic degeneration.

Congested and inflamed mucosa and submucosa.

Enlarged uterine blood-vessels.

Apparent Hypertrophy and Elongation of the False Cervix.-Cases are frequent in which there is apparent lengthening of the cervix, so that it seems to extend from the uterovaginal attachment even to the vulva; the condition is usually described as hypertrophic elongation

Figure 297.

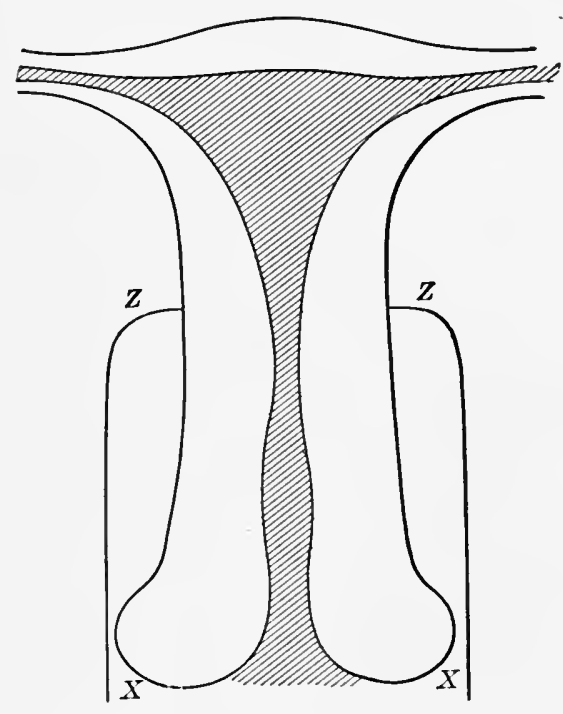

Figure 298.

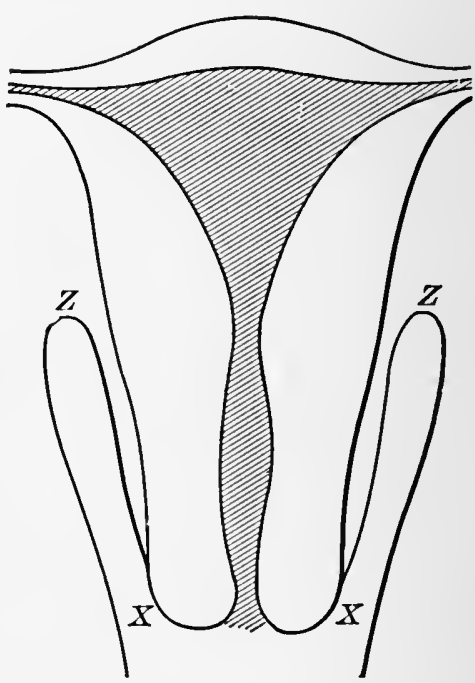

Figure 297, - This Figure is from a part of an illustration in a standard book, in which it was used to represent supposed infravaginal hypertrophy of the cervix. The raginal attachment, however, is only apparent, and is due really to reflection of the vaginal wall on a lacerated cervix. The true uterovaginal attachment is shown at $X$ and $X$ of Figure 298. Figure 298 is a correct representation of the real condition.

FIgURE 298 . - Shows the true uterovaginal attachment at $X$ and $X$, and the apparent utero. raginal attachment at $Z$ and $Z$.

of the cervix. Credit for the true explanation of this anomaly belongs to Emmet. Figure 298 shows the os externum on a very low plane. This is not because the infravaginal portion of the cervix has lengthened by hypertrophy so as to oceupy the entire length of the vagina, but because the entire uterus has prolapsed, carrying with it a reduplicated part of the vaginal wall, until the os externum has appeared at or near the vulva. If the patient be placed in the knee-breast position and the uterus be made to gravitate toward the diaphragm, the reflected vagina will be unfolded, the cervix will resume its normal distance from the vulva, and the uterovaginal attachment will appear at the proper distance from the os externum-that is, the normal relations of the vagina and uterus will be restored. 
Apparent elongation takes place occasionally in the nullipara, but it is associated more commonly with descent of the lacerated cervix. The extent of laceration will be apparent in proportion to the degree of eversion-that is, in some cases the evidence of laceration, as already explained, is obliterated by the outrolled intra-uterine tissue; in other cases of less eversion the laceration is more apparent. A striking illustration of the latter class of cases is furnished by the following case :

The patient had been sent to the hospital for amputation of a supposed "hypertrophied cervix." Superficial examination suggested

Figure 299.

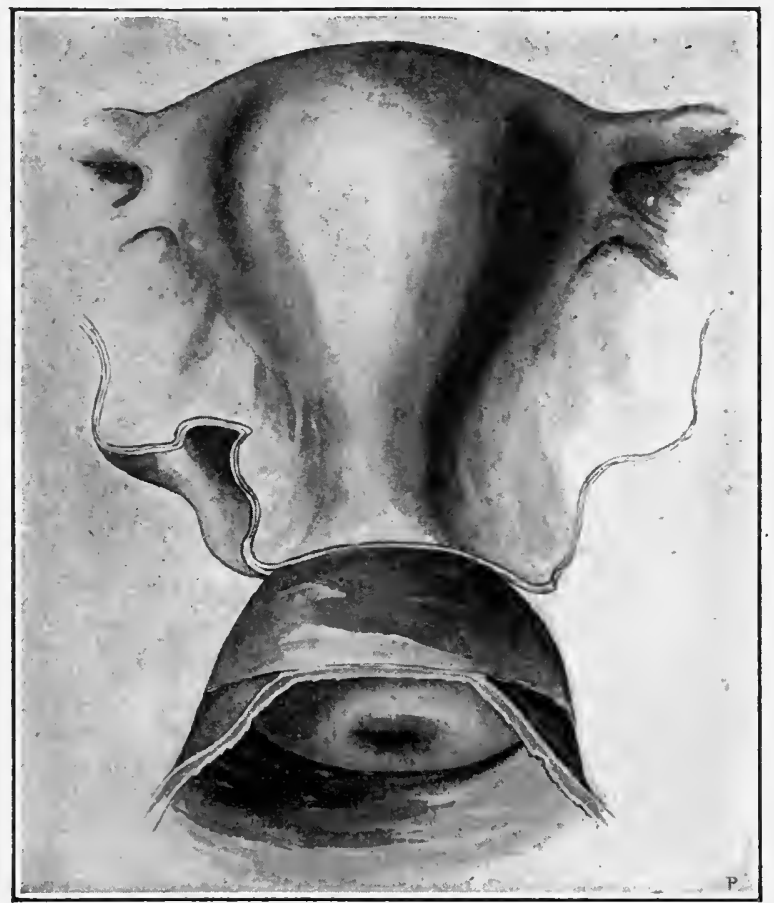

So-called hypertrophic elongation of the supravaginal portion ot the cervix-rare except as a post-operative condition. The cervix often becomes elongated by traction during the removal of it.

the presence of two large uterine polypi, one filling the anterior and the other the posterior half of the vagina, both reaching to the vulva; further examination disclosed the apparent presence of extreme hypertrophy of the anterior and posterior lips of the cervix. Between these two lips was a fissure extending into the vagina for at least two inches. When the patient was placed in the knee-breast position, however, the uterus gravitated toward the diaphragm; the uterovaginal attachment appeared in its true relation, and it was plainly to be seen that, instead of hypertrophic elongation of the infravaginal portion of the cervix, some degree of atrophy actually had taken place, for the uterovaginal attachment was nearer to the exter- 
nal os than normal. There was a fissure, however, disclosing an enormous bilateral laceration, which extended two inches into a subinvoluted uterus and far out into the vaginal walls on either side. Repair of the cervix was followed promptly by disappearance of all apparent elongation, both in the infravaginal and supravaginal portions of the cervix, and in a few weeks by complete subsidence of subinvolution. In similar cases of unilateral laceration, with extreme eversion, the apparently elongated cervix may show no fissure, but, on the contrary, the erosion may, as before stated, give to it a symmetrical form.

Amputation of the cervix and hysterectomy for so-called "hypertrophic elongation" and "hypertrophic enlargement of its circumference" are favorite operations in gynecology. "The true pathology of this condition, however, would demand not amputation nor hysterectomy, but closure of the cervix, if lacerated, and the appropriate treatment for displacement.

The existence of genuine hypertrophic enlergement and elongation of the cervix, although not absolutely denied, is of extremely rare occurrence-so rare that amputation of the cervix, except the removal of certain diseased portions, as will be explained in the operation for lacerated cervix, should become practically obsolete. In carcinoma of the cervix and in extreme intiammatory infection of the uterus, not amputation of the cervix, but hysterectomy is the operation of election.

When hypertrophic elongation of the cervix does exist, it is above the uterovaginal attachments, and is therefore supravaginal. Infravaginal elongation of the cervix-that is, elongation below the uterovaginal attachment-is often apparent, seldom or never real.

A Cause of Carcinoma.- Emmet first observed the relatively more frequent development of eancer upon the lacerated cervix and the almost entire absence of it from the nulliparous cervix. While we may not, strictly speaking, attribute cancer to laceration of the cervix, we must not ignore the fact that the irritated glands of a lacerated cervix are a fruitful soil for malignant disease.

\section{Symptoms of Laceration of the Cervix Uteri.}

Immediately after the accident occurs, arterial hemorrhage may be so profuse as to demand prompt ligature and suture. The secondary symptoms are those of the pathologieal resnlts of the lesion-that -is, the symptoms of endometritis, metritis, subinvolution, and displacements. The menorrhagia and uterine discharges so common in laceration are the symptoms of hemorrhagic and catarhal or purulent endometritis. A variety of nervous symptoms, such as may be due to faulty innervation and nutrition, have been attributed to laceration of the cervix. They include neuralgic and other pains in remote parts, dyspepsia, indigestion, constipation, menstrual disorders, backaehe, and headache. Bearing-down sensations and difficulty of walking and standing are among the results of the associated displacements of the pelvic floor. These displacements include the uterus, its appendages, the bladder, vagina, and rectum. 
Cicatricial narrowing of the uterine canal at the angle of the laceration may be so extreme, either from natural contraction or from the use of caustics, as to reduce the uterine outlet to a mere pinpoint. This reduction of calibre results in imperfect drainage of uterine secretions. Endometritis and numerous functional disturbances, including sterility, dysmenorrhœe, menorrhagia, and amenorrhœa, are common sequels.

Emmet lays great stress upon the reflex irritation prodnced by the cicatricial pling in the angle of the laceration. The cicatrix develops in an effort of nature to close the gap, or as a result of the injudicious application of caustics. He cites numerous cases in which severe neuralgia in distant organs-for example, neuralgia in the eyeball-promptly disappeared upon repair of the laceration. He attributes the reflex irritation to inclusion and pinching of nervefilaments in the cicatrix, as in the sensitive stump after amputation of the leg or arm. The cicatrix, therefore, may serve as a constant and hidden canse of nerve irritation. Microscopical stndy, however, has failed to disclose the pinched nerve-filaments. Whatever may be the explanation of the facts, the clinical observations of Emmet apparently have been verified by numerons observers, for the anæmic, nervous, neuralgic state is peculiarly liable to be associated with cicatricial cervix.

A brief report of two cases will serve to illustrate : A patient consulted one of the most distinguished ophthalmologists in America for a long-standing, severe, and obstinate neuralgia of the eyeball. As the only possible means of relief extirpation of the eye finally was advised; this operation the patient declined, and the pain continued. She subsequently was operated upon by Emmet for laceration. He removed a large, wedge-shaped piece of cicatricial tissue from the angle of laceration, which nature, in the vain attempt to bridge over the gap, had placed there. Immediate and permanent relief from the neuralgia followed.

In April, 1878, the writer performed a similar operation upon a woman who had for years suffered from almost constant pain in the top of the head. $\mathrm{U}_{\mathrm{p}}$ to the time of the operation every resource of treatment had failed. In this case the cervix was not eroded, but from the perseverance of some one in making caustic applications it had suffered considerable loss of substance. In the removal of the scar tissue, which was abundant, a large part of the cervix was sacrificed. The pain disappeared from the time of the operation and has not returned.

Clinical observation has shown sterility and repeated abortion to be associated very frequently with laceration of the cervix-an observation for which the pathological results of laceration already as detailed furnish clear explanation.

\section{Diagnosis of Laceration of the Cervix Uteri.}

Laceration of the cervix, until demonstrated by Emmet, was known only by its effects. To designate the extent and character of these effects, the following names were applied: erosion, follicular erosion, 
granular erosion, papillary erosion, granulation, excoriation, ulcer. Erosions, when exaggerated, were called coxcomb granulations; when the exaggeration was so extreme as to suggest malignant disease, it sometimes was called cauliflower excrescence, a name loosely used also to designate cancer. Inflammation of the cervical follicles, analogous to follicular pharyngitis, suggested the name follicular erosion.

The older text-books usually devoted a chapter to this subject, under the head of Ulceration of the Womb. The disease is really not ulceration, but erosion. Ulceration, except in specific and melignant disease, ravely is found on the cervix.

The presence, after parturition, of a part or all of the elements which compose the false cervix-that is, enlargement, erosion, ever-

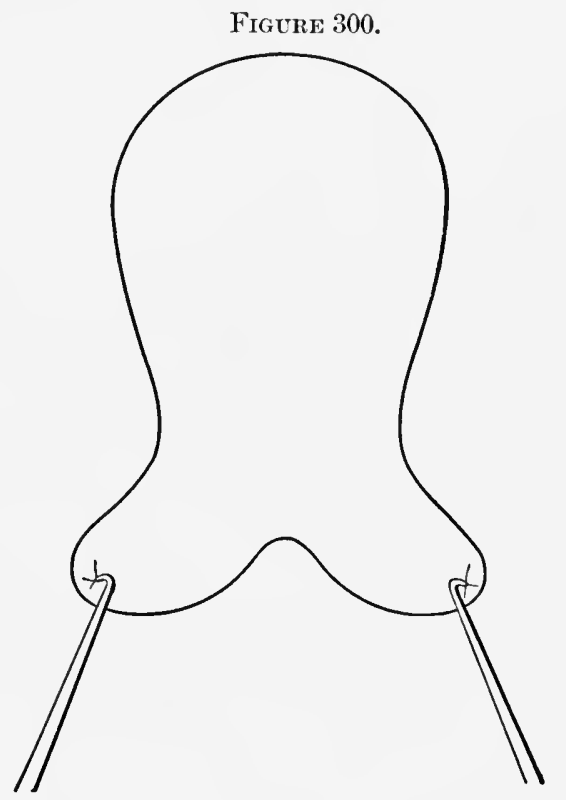

FIGURE 301.

Figure 300.-Showing everted, lacerated lips caught by tenacula and held apart.

FIGUPE 301.- Showing lacerated lips caught by tenacula and rolled in.

sion, and cystic degeneration-is strong evidence of laceration ; cystic degeneration of the mucous follicles is almost pathognomonic of laceration. The eysts, varying in size from that of a pinhead to that of a small marble, feel to the touch like shot scattered throughout the mucous tissues of the everted cervix. They rarely are found except on the lacerated cervix. As explained, they are the result of occlusion and cystic degeneration of mucous follicles, the glands of Naboth. These follicles, except in cases of abnormal distribution, are confined to the intracervical mucous membrane, and are not prone to eystic degeneration unless rolled out into the hostile environment of the vaginal secretions. This outrolling seldom occurs except as the result of laceration. Hence cystic degeneration without laceration is rare. 
Laceration, in ordinary cases, may be detected by touch and sight. Intelligent study of all cases, and accurate diagnosis in the more obscure, require the cervix to be exposed by a Sims or a Simon speculum (Sims preferred), and the everted lips to be caught and rolled in by means of two uterine tenacula, one in each hand.

For satisfactory diagnosis, in many cases the tenacula are essential. With these instruments Emmet was first to solve what was once a knotty problem, and to revolutionize the pathology and treatment of this cervical disease. Edmund Randolph Peaslee, referring to the numerous cases of so-called ulceration, said " "they were not recognized, for none of us knew anything about them till Emmet told us. It was he who, in a happy moment, brought the anterior and posterior surfaces together with tenacula, and instantly demonstrated that what we all supposed an ulceration was nothing more nor less than a laceration." Figures 300 and 301 .

I quote somewhat fully from Emmet's first systematic paper on this subject ${ }^{2}$ because it presents a graphic picture of the gynecology of the last generation, and because of the historical importance of this epoch-making contribution to surgical literature :

"November 27, 1862, I first operated for the relief of a double lateral laceration of the cervix by freshening the surfaces and bringing together the anterior and posterior flaps with interrupted silver sutures. This patient had been an invalid for several years before coming under my care, and had been treated for menorrhagia and hypertrophy of the uterus, with an extensive erosion. She was undersize, of a naturally delicate constitution, and after a severe and protracted labor, with difficulty had given birth to a large child. Her general appearance indicated incipient phthisis, but no evidence of a tuberculous deposit could be detected. The uterus was some four inches in depth, and an erosion extended about two inches in diameter over an enormons cervix. With great care this erosion had been healed several times, by maintaining the recumbent position for a sufficient length of time, but a relapse to the former condition recurred in every instance shortly after beginning to exercise by walking. I had almost despaired of being able to offer her any permanent relief, and attributed my want of success to the condition of her general health. While making a digital examination one day I was puzzled to account for the greater width of the cervix in comparison to that of the body beyond, a condition I had for the first time appreciated. I placed her on the left side and, with Sims' speculum, brought the cervix in view. I drew the posterior lip forward toward me with a tenaculum, but with no special purpose, when I was surprised to observe that it had decreased to nearly half its previous size. On lifting up the anterior lip with a tenaculum in the other hand, so as to bring the two portions into approximation, the outline of a cervix presented, of nearly normal size. The difficulty was at once apparent, for the parts had rolled back within the uterine canal, and a deep

1 Remarks after the reading of a supplementary paper on "The Proper Treatment of Laceration of the Cervix Uteri," by Emmet, before the New Fork County Medical Society, Deeember, 1876. New York Medieal Journal, January, 187\%.

2 "Laceration of the Cervix Uteri as a Frequent and Unreeognized Cause of Disease." American Journal of Obstetries, November, 1874. 
lateral fissure beeame evident, which extended on each side entirely through the cervix and beyond the vaginal junction. On separating the flaps and forcing them back to their former position, I saw the tissues gradually roll ont, and the cervix again present its previous appearance. There could then be detected no appearance of laceration, and with the reduplication of vaginal tissue over the sides of the uterus, the cervix presented a normal length above its apparent junction with the vagina. The remedy at once suggested itself; the operation was performed with the aid of ny assistant, Dr. G. S. Winston, and I believe Dr. T. G. Thomas was also present. On completing the operation the uterus was five inches in depth; it rapidly reduced in size, and in time all evidence of loeal disease subsided, but she never entirely regained her general health. Some seven years after the operation Dr. F. N. Otis, of New York, her family physician, deteeted a tuberculous deposit, and she died of phthisis within a few months, having been ten years under my observation. For two years previous to her death she had resided abroad, but, as a friend, I was kept advised of her condition, and she continued free from uterine disease. I am fully satisfied that at the time of the operation her condition was so critical that it would have been but a question of a few weeks before a tubereulons deposit would have taken place. Althongh she never recovered fully the loss of vitality to which this injury had reduced her, yet her life was beyond question prolonged many years by the operation."

After the reading of this paper before the New York County Medical Society, September, 1874, J. Marion Sims said:

"When I went abroad in 1862, among the patients I turned over to the eare of Dr. Emmet was the lady whose ease forms the basis of the paper I have just read. She belonged to the upper walks of life, and had been under my charge for twelve or eighteen months. I remember the peculiarities of her case, so well described by Emmet, as vividly as if it were but yesterday. The bilateral lacerations of the cervix, and the consequent eversion of the hypertrophied, congested eervical mucous membrane, constituted at that time a difficult problem to solve. During the whole time that I observed this ease no benefit resulted from local treatment, and I am sure that nothing short of the method so successfully adopted by Dr. Emmet could have been of the least service to her. I now only wonder that this operation had not been worked out sooner. When the perineun is lacerated, the necessity for its reconstitution is self-evident, and it is singular that the necessity for reconstituting the integrity of a lacerated cervix did not sooner foree itself upon the surgeon. The operation as devised and practised by Dr. Emmet is as simple, as safe, and as certain in its results as is the operation for a simple case of vesicovaginal fistula. The same principles underlie each. The same free denudation of tissue, the same method of suture, the same after-treatment, and the same seeurity from danger belong to both alike.

"I have performed the operation often enough to speak in positive terms of its value. The diseussion of the subjeet nust, of necessity, be one-sided. There ean be no objection, no opposition to the opera- 
tion. We must accept it as Dr. Emmet has given it to us. We callnot modify the operation; we cannot change it; we cannot improve it-for it is perfect; perfect in its method and perfect in its results.

"We owe to Dr. Emmet a debt of gratitude for this valuable contribution to uterine surgery. Like all new operations, it is likely to be abused; but the time will son arrive when it will assume its place in the foremost rank of useful improvements."

\section{Differential Diagnosis of Laceration of the Cervix Uteri.}

Laceration of the cervix uteri is to be distinguished specially from :

1. Endocervicitis.

2. Congenital eversion.

3. Cancer.

There is a form of erosion due to endometritis, associated with an irritating discharge from the endometrium or vagina, apt to occur in feeble and poorly nourished subjects, and not very uncommon in virgins; the condition is analogous to the familiar erosion and excoriation produced by prolongerl nasal discharges on the upper lips of children. Such an crosion is distinguished readily from that of laceration by absence of eversion, by the absence of marked cervical enlargement, by the presence of a normally shaped os exterum, and by physical examination soon to be described. 'The treatment is that of the causative endometritis.

Congenital eversion of the non-lacerated cervical mucosa may occur in rare cases. It has been observed even in infancy. ${ }^{1}$

The disease most liable to be mistaken for laceration is beginning cancer of the cervix. A careful reading of the description of this disease will help to show the difference between the two conditions. Cancer bleeds freely on slight abrasion, is extremely friable, does not readily permit inrolling with tenacula, and rapidly goes on to ulceration. Laceration presents none of these characteristics.

\section{Prophylaxis of Laceration of the Cervix Uteri.}

The proplyylaxis consists in the avoidance of all measures ealculated to hasten unduly the normal progress of labor-that is, the avoidance of meddlesome manipulations by digital or instrumental interference. A precipitate labor should, if practicable, be retarded. The relative disproportion between the child and the cervix may render all precautions useless and laceration inevitable.

\section{Treatment of Laceration of the Cervix Uteri.}

The Operation of Trachelorrhaphy.2-It is not necessarily the extent of laceration, but rather the degree of outrolling that indicates the necessity for repair. A relatively slight laceration may give rise to extreme eversion, and consequently to all of the pathological

\footnotetext{
1 Noble. American Gynecological and Obstetrical Journal, February, 1897.

2 E. C. Dudley, of Chicago, former interne at the Woman's Hospital in the State of New York, was the first to designate this operation trachelorrhaphy. Emmet's Principles and Practice of Gynecology.
} 
changes, above described, which belong to the false cervix. Furthermore, slight laceration without eversion may, if associated with great cicatricial formation or cystic degeneration, give rise to very distressing symptoms. On the other hand, a deep laceration may cause little or no disturbance.

Immediate Operation of Trachelorrhaphy.-Some obstetricians urge immediate closure of the torn cervix uteri. This operation, if successful, would have the same advantages as immediate perineorrhaphy - that is, less danger of infection through the exposed surfaces, relief from long-continued dread of operation, and freedom from the evil effects of any pathological changes consequent upon delay. There is, however, great difficulty in recognizing the limit of the fresh tear in the loose folds of the divulsed, soft, flabby cervix and the surrounding upper end of the vagina. The exact relations of the torn vaginal wall to the cervix are also difficult to define; for these reasons accurate adjustment of the torn surfaces may be difficult. The immediate operation, therefore, unless necessitated by profuse arterial hemorrhage, is of questionable propriety ; when it is performed, the continuous eatgut suture should be used.

Secondary Operation of Trachelorrhaphy.-In order to avoid ontrolling, subinvolution, cystic degeneration, endometritis, metritis, descent and other pathological changes, early repair of the extensively torn cervix is desirable. The operation is permissible as soon as the cervix has recovered from the immediate effects of extreme divulsion, and has regained, so far as the injury will permit, its normal formthat is, at the end of two or three months. Unfortunately, in the majority of cases, the lesion is not recognized or brought to the attention of the gynecologist until the resultant pathological changes have impaired seriously the health of the patient." It is the duty of the acconcheur, and is one of the imperative requirements of modern scientific nidwifery, to make, in the second or third week of the puerperium, an examination of the pelvic organs to determine the existence of any pathological condition which may demand attention.

Preparatory Treatment of complicating displacements and erosions, although advised by many, is not usually imperative. The treatment of a displacement may be necessary after the operation, and may be deferred properly to that time. Associated endometritis should be treated by preliminary curettage as a preparatory step in the operation for closure of the laceration. If the eroded cervix be greatly thickened or complicated by extensive cystic degeneration, the diseased tissues should be removed by Schroeder's method. Figures 309-312.

It is difficult to discriminate between certain inflammatory conditions in the pelvis which contraindicate and others which indicate trachelorrhaphy. The operation, if performed in a case of acute pelvic inflammation or of suppurative inflammation, acute or chronic, is liable to be followed by general, possibly fatal, pelvic infection, and therefore is eontraindicated. The presence, in the pelvis, of structures which are thickened, hypersensitive, or adherent-or, in other words, the non-purulent results of chronic inflammation-does not neces- 
sarily contraindicate Emmet's operation. On the contrary, the improved uterine drainage secured by the preliminary dilatation, the removal of the products of endometritis, and of the original source of the pelvic infection by thorough curettage of the inflamed endometrium, and the rolling in of the irritable everted cervical mucosa, may be the most effective treatment for such pelvic inflammation.

Puncturing of Cysts. - The follicular retention-cysts already described, if present, will, unless properly treated, render the operation for closure of the cervix not only useless, but also injurious. In fact, these diseased glands if rolled into the cervical canal by trachelorrhaphy are liable to enlarge, multiply, and remain a hidden source of irritation. Often they are so numerous and of such large size as to lead to the suspicion of cancer. If few in number and superficial, they may be punctured, or the projecting part of the cyst-wall may be caught with a tenaculum and removed by the scissors and the remaining part of the cyst-wall then destroyed by nitric acid or the galvanocautery. Several such treatments may be required before the cervix is ready for operation. Extensive cystic development, especially on the thickened cervix, extending up into the cervical canal, requires excision of the diseased tissue. Simple puncturing of the cysts by the spear-pointed lance is inadequate because, unless the secreting surface be destroyed, the cysts are prone to refill. See Schroeder's operation. Figures 309-312.

Instruments for the Operation.-The following instruments are required :

Sims' speculum and depressor, or Simon's retractor.

Two uterine tenacula.

Emmet's uterine dressing-forceps.

Emmet's slightly curved and full-curved scissors.

Emmet's needle-forceps.

Short hæmostatic forceps.

Needles.

Four sponge-holders.

Gauze or sea sponges.

Silkworm gut and chromic eatgut.

Rubber sheet or Kelly's pad.

Flat vulsellum forceps.

Instruments for dilatation and curettage.

Many operators prefer the Simon to the Sims speculum; the writer uses either indifferently. The majority of surgeons probably prefer Simon's retractor to Sims.' Education and habit will fix the choice, which should be limited to these two instruments. The needle with bayonet trocar, or glover's point, is preferable to that with the round point; the latter is difficult to introduce through the indurated tissue without breaking. The full-curved needle is unmanageable; the force required for its introduction is exerted in the line of a tangent to the curve, and is therefore more liable to break the needle than when exerted in the direct line of the straight needle. It may also be difficult to estimate the location of the point of a curved needle. There are practical advantages in a needle slightly curved at the point, but otherwise straight. 
Disinfection.--The antiseptic measures to an aseptic result have been detailed in Chapters II. and V. Under anæsthesia the patient being on her back, the raginal and external genitals are scrubbed thoroughly with water and green soap. When the soap has been washed off with hot, sterilized water, the disinfection is completed by an additional washing with a $1: 2000$ alcoholic solution of bichloride of mercury. A conjoined examination is made now in order to obtain information of any condition which before anæsthesia may have been

Figure 302.

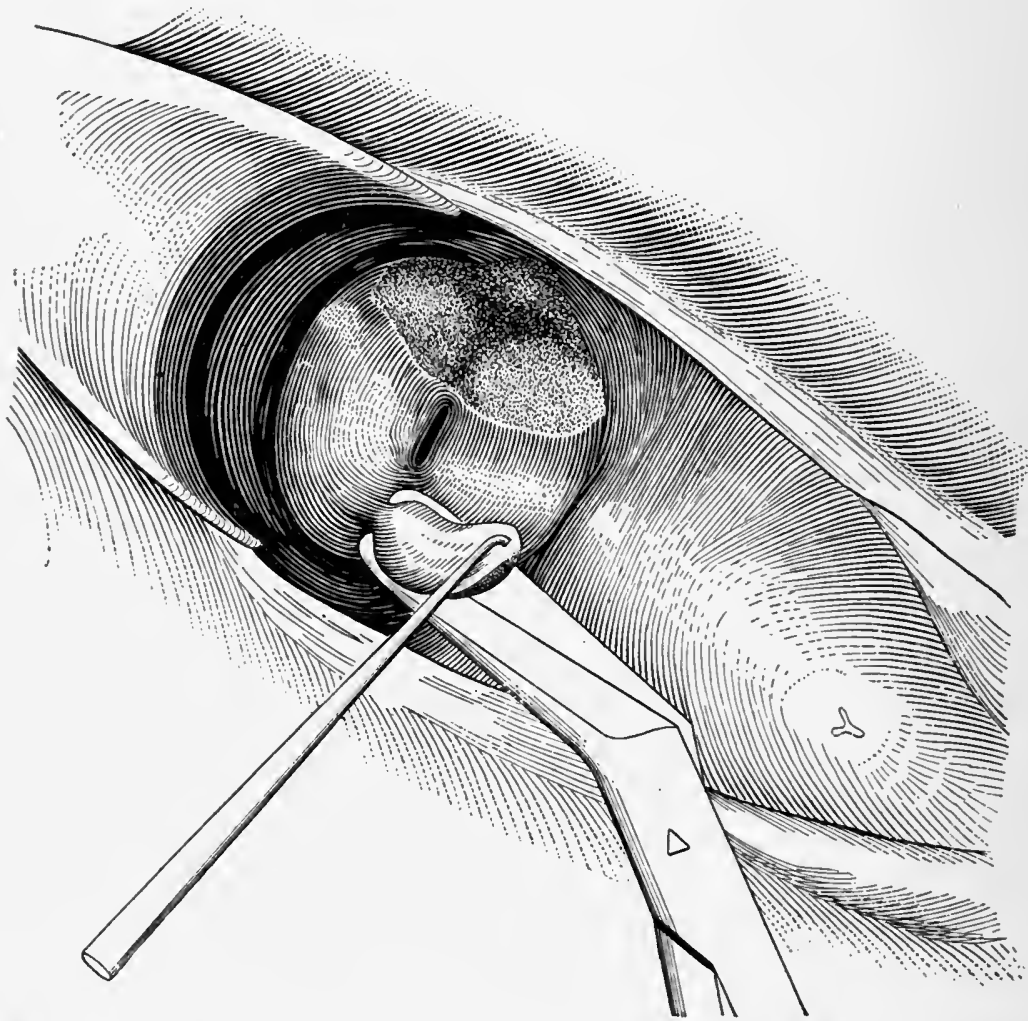

Manner of denudation with uterine tenaculum and Emmet's curved scissors. One side denuded, the other partly denuded. Left lateroprone position; exposure by Sims' speculum.

overlooked. This examination, since it occasionally reveals conditions which may modify or contraindicate the operation, is important. If no contraindication for the operation appears, the cervix is exposed by Sims' or Simon's speculnm, and the uterus dilated, curetted, washed out, aus treated with an intra-uterine auplication of a saturated solution of iodine in 95 per cent. carbolic acid, which disinfects the endometrinm and decreases the risk of infection.

Preliminary Dilatation and Curettage may be required only for the purpose of exploration, in order to deternine the presence or absence 
of complicating endometritis. The objects of dilatation and curettage are : 1 , to prevent infection of the wound and failure of union from contact of the pathological secretions of a possibly diseased endometrium ; 2 , to secure efficient drainage of the endometrium, and thereby to prevent the retention, stagnation, decomposition, and absorption of its secretions ; 3 , to avoid leaving an infected endometrium, which, after closure of the cervix, might by extension involve the parametria,

Figure 303.

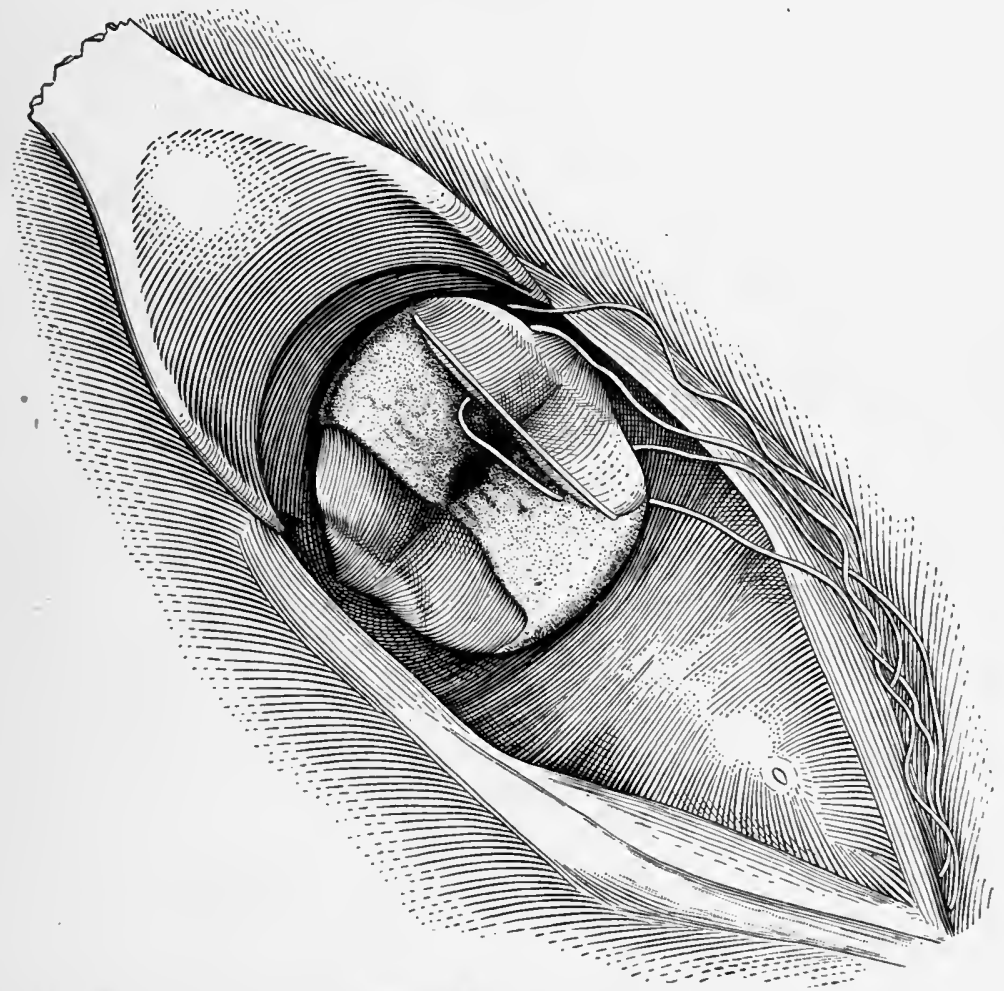

Shows the surface denuded and two sutures in place, but not tied. Left lateroprone position; exposure by Sims' speculum.

uterine appendages, and peritoneum, in disabling or dangerous infection.

Approximation.-Before proceeding to the closing of the cervix, a careful study should be made of the direction or directions and extent of the rupture, by trial approximations of the torn fragments in various ways with a tenaculum in each hand. If there be a simple bilateral laceration, the operation will be as follows:

Denudation. - With the tenaculum and curved scissors the surfaces to be united are denuded. Figure 302. Inasmuch as one of the inportant functions of the uterus is drainage, it is essential to leave a wide and free outlet at the external os. To this end, that portion of 
the undenuded mueosa which is to line the restored external os should be left wide, so that when united the normal trumpet-shape of the lower segment of the cervical canal will be preserved. Immediately after the operation the diameter of the restored external os should be even larger than normal, so that the involution that follows the operation will reduce it ultimately to the normal calibre. Figures 303 and 304 show the properly curved lines between the denuded and undenuded surfaces. Extreme stenosis at the external os, sometimes amounting to complete atresia, is a possible result of inattention to this important detail.

Among the consequences of stenosis and obstruction in the uterine canal are the following:

1. Retention and decomposition of uterine secretion and menstrual fluid.

2. Possible destruction of the Fallopian tubes.

3. Metritis, endometritis, and salpingitis.

Figure 304.

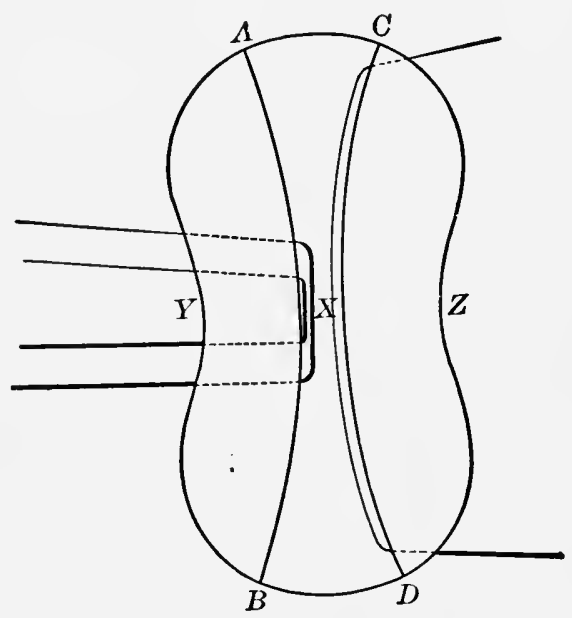

A line connecting $\boldsymbol{Y}, \boldsymbol{X}$, and $Z$ would represent angle of laceration ; $\boldsymbol{X}$, section of uterine canal at angle of laceration. Three of the sutures ill place.' Diagrammatic.

The conditions mentioned above may give rise to immediate disastrous results, or may cause persistent invalidism. The rapid and complete relief which often follows the reopening of a contracted cervical canal and os externum proves that the integrity of the uterine canal as a natural drainage-tube is essential to health.

Removal of the Cicatricial Plug.-The denudation should always include removal of the plug of cicatricial tissue which usually forms the angle of the laceration. This important step in the operation, if disregarded, may prevent easy approximation of the denuded surfaces, cause the sutures to cut out from undue tension, and result in failure of union or in imperfect union. Failure of union, however, under such conditions would be a fortunate compromise for 
the patient, since the cicatrix is much less injurious with the laceration open than closed. When, unfortunately, union has taken place, the

Figure 305.

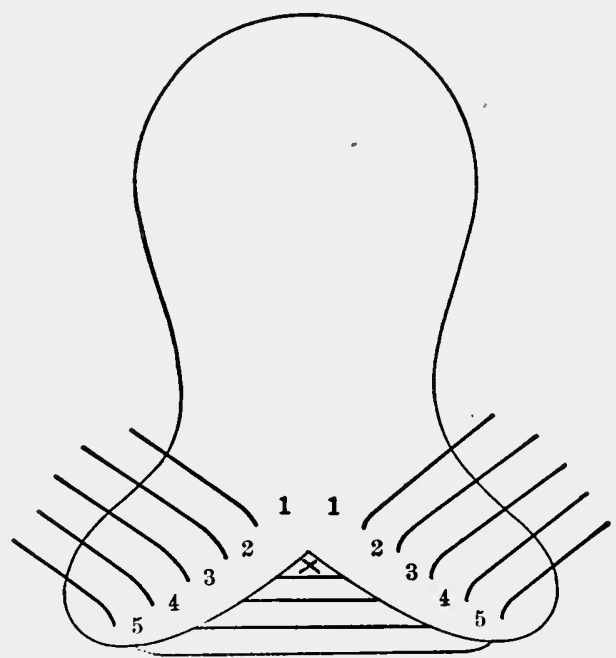

Sutures in place on one side ready to tie. Diagrammatic.

consequent train of nervous symptoms may necessitate reopening of the wound and removal of the cicatricial plug.

Hemorrhage.-The usual slight bleeding is controlled readily by sponge pressure. Arterial hemorrhage, if not controlled by foreipressure or torsion, may require a fine catgut ligature. In occasional aggravated cases the bleeding must be cheeked by the application of one or two deep sutures.

The Sutures may be of chromic catgut or silkworm gut. Silkworm gut remains aseptic longer, and is therefore superior to catgut. If the perineum is closed at the same time, the difficulty in the removal of the cervical sutures will justify the use of absorbable catgut, which does not have to be removed. Catgut may also be used in the repair of all small lacerations, especially where the surfaces readily fall together and remain in apposition without traction. The catgut should be so chromicized that it will resist absorption for twenty days. In order that the sutures may not convey possible infection to the wound, they should in all plastic surgery, so far as practicable, be passed under and not through the denuded surfaces. This principle is illustrated by the dotted lines in Figure 304; the surface between the lines $A B$ and $C D$ is left undenuded, to form that part of the cervical canal which is to be restored. The two sutures indicated on one side show the location of the sutures near the angle of laceration; and the one on the opposite side shows the location of the sutures at the os externim. When all the sutures have been tied, they will bring the surface $A Y X Z C$ in contact with 
the surfaee $B Y X Z D$ in sueh a manner that point $A$ will coincide witi point $B$, and point $C$ with point $D$. The lines $A C$ and $B D$ will then bound the restored external os.

Figure 305 shows the same laceration from another point of view, The sutures on one side are represented as all having been introduced before any are tied. This was the plan formerly pursued when the silver suture was used. It is better to tie the silkworn gut or catgut sutures as they are introduced.

Figure 306 shows the sutures tied, the everted mucosa rolled in, and the operation complete.

A stuly of Figures 305 and 306 will diselose an interesting fact in the mechanies of laceration and trachelorrhaphy. If in the subinvoluted uterus represented by Figure 305 the distance from the angle of laceration, $X$, to the fundus is, say, three inches, and the distance from

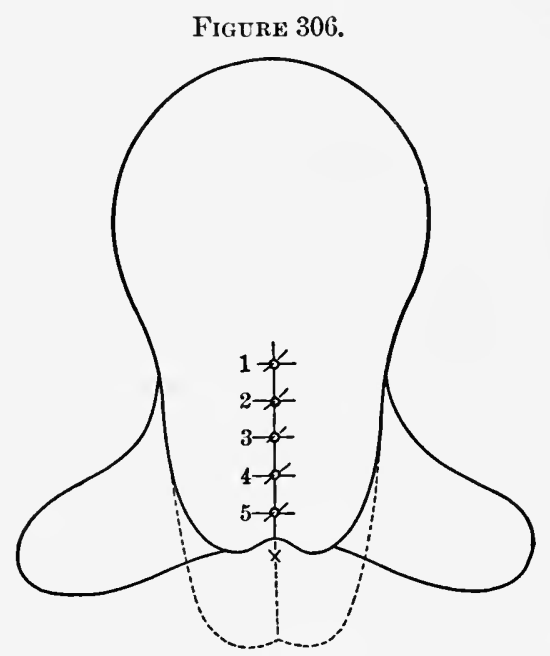

Showing the rolling-in effect of the operation. Sutures tied. Diagrammatic.

the angle of laceration to the margin of the torn lip is one inch, it would appear reasonable to assume that the uterine canal, when fully restored, would measure four inches. Aceurate measurements of the uterine eanal, however, before and after operation almost always show a decrease, not an increase, in length. In a uterus of such dimensions, the canal, after operation, usually would measure not four, but about two and three-quarters inches. The explanation of this decrease is as follows:

As shown in the pathology, the intra-uterine mucosa rolls out, and the lowest portion of the uterine canal becomes the external os of the lacerated cervix, point $\times$, Figure 306. In tying the first suture points 1 and 1 are not only brought together to form one and the same point, but all mucosa above this suture is at the same time rolled into the uterine canal, so that point $\times$ moves up and point 1 takes its place. On the successive tying of the other sutures, $2,3,4$, and 5 , 
the same mechanical result is observed, so that finally suture 5, when tied, occupies the place formerly occupied by $X$. All the mucosa between 1 and 5 on one side, and 1 and 5 on the other, is now rolled into the uterine canal above the original level of point $x$. This mechanical result alone abundantly justifies the operation; it also verifies the propositions laid down in the preceding paragraphs on the mechanical results of the lesion.

The reasons for so great a decrease in the length of the uterine canal may not be wholly apparent from the foregoing. The following reasons, in addition to the rolling-in of the cverted tissue, are therefore submitted: loss of blood and tissue in denuding; evacuation or removal of retention-cysts; contraction of muscular fibre due to the stimulus of the operation; and, above all, relief from congestion, which naturally follows restoration of everted intra-uterine structures to their normal position inside of the uterus. The ontrolled structures before the operation had been, so to speak, in a state of erection.

Operation for Atypical Lacerations.-The closure of a unilateral, anterior or posterior laceration follows the rules already down for simple bilateral injurics. Stellate lacerations in some cases may be treated by closure of each individual tear; or, if two are very near together, they may be changed into one by removal of the intervening tissue. There may be one or two major and several minor rents; in such a case the surgeon sometimes may disregard the small fissures, and by rolling in the everted cervix, as indicated by the deeper tears, find that the smaller ones disappear within the canal, and may therefore he ignored in the operation. It is impossible to anticipate every variation in the direction and effect of the injury. Each atypical case must be treated according to the special requirements.

Resection of the Cervix.-In a large proportion of cases of laceration of the cervix the lesion is unrecognized, neglected, or unskilfully treated, so that extensive pathological changes occur. These changes may prevent or contraindicate the rolling-in of the diseased tissues; or, if the cervix has been closed improperly, may require it to be reopened and closed again correctly. The changes are :

1. Great thickening and induration of thc lacerated lips, which, if possible to roll into the uterine canal at all, would cause traction upon the sutures, and result in their cutting ont; or, if union slould occur, the induration and thickening might persist and give increased trouble.

2. Extensive cystic degeneration of the Nabothian follicles. The evil results of rolling these cysts into the cervical canal have been mentioned.

3. Endocervicitis, with decp involvement of the cervical glands, and a consequent profuse discharge of a ropy, tenacious, gelatinous sccretion. The only satisfactory treatment of this condition is $\mathrm{cx}$ cision of the diseased structures. Their destruction by the cautery or sliarp curctte is apt to be followed by contraction and stenosis of the cervix and is therefore objectionable.

4. Stenosis in the lower portion of the cervical canal and os 
externum. This condition may be due to too tight closure of the cervix or to cicatricial contraction from curettage, cauterization, or other causes.

Under the conditions named above, the diseased tissue should bê removed by resection of the cervix-Sehroeder's operation. ${ }^{1}$ 'The technique of the operation is as follows: The diseased tissue is removed by incisions as indicated by the dotted lines in Figure 309, the vaginal margins of the wound then are stitched, both anteriorly and poste-

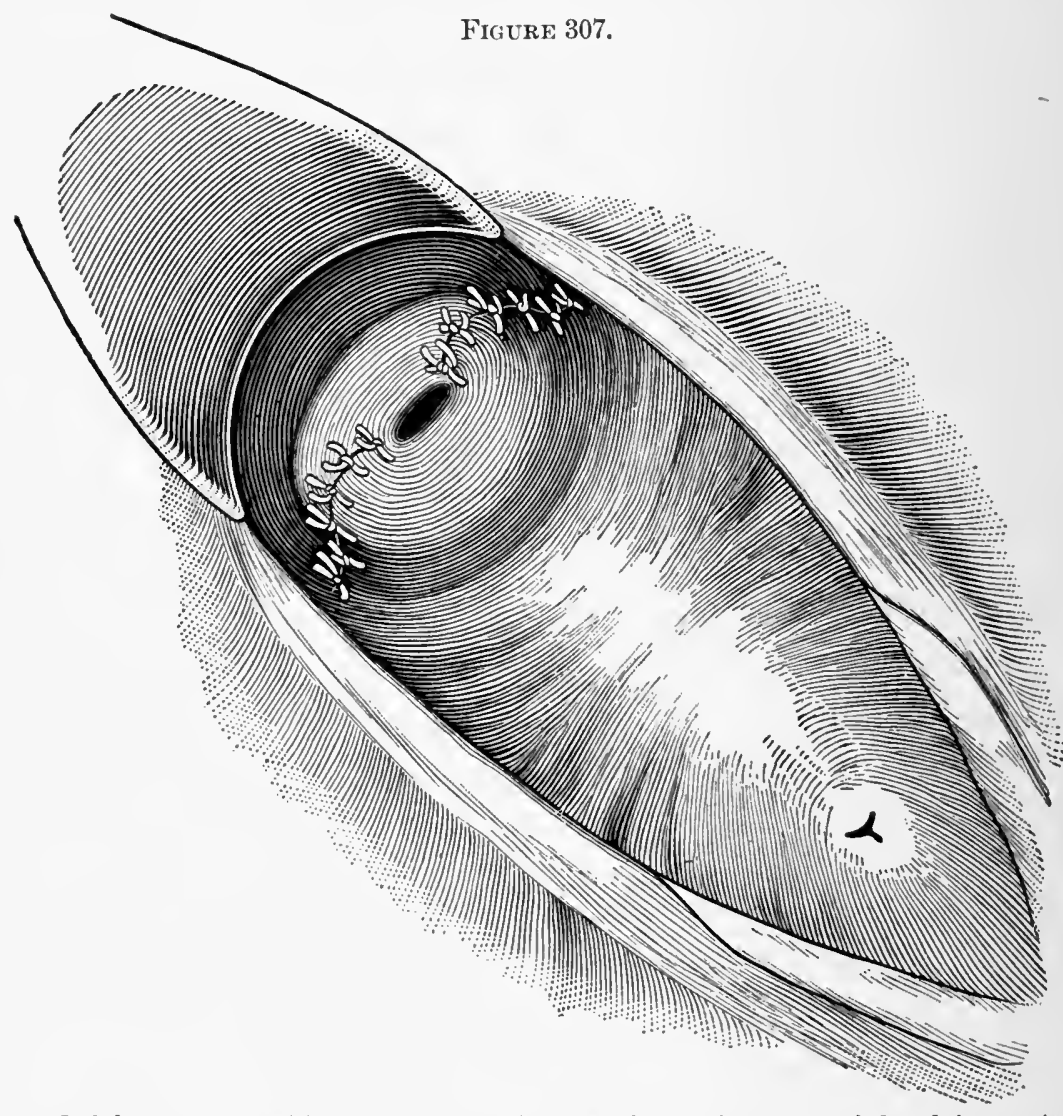

Left lateroprone position; exposure by Sims' speculum. The sutures tied and the cervix united, as seen looking through the speculum into the vagina. Notice the lines of union running from the os over the cervix across the uterovaginal at tachment into the reflected vagiual wall. In this case, as in all others, a great part of the tear is in the vaginal walls.

riorly, with fine chromicized catgut, to the margins of the intracervical mucous membrane. By this means the anterior and posterior lips of the cervix are folded upon themselves. Figure 310. The first stage of the operation is now complete, and the condition becomes that of an uncomplicated bilateral laceration. The remainder of the operation is the same as that of trachelorrhaphy, already described.

1 Emmet had for many years before the publication of Schroeder's operation performed an operation in principle like Schroeder's, but differing in technique. 
Before proceeding to excision of the diseased structures, it is often necessary to supplement Schroeder's operation by deep lateral

Figure 308.

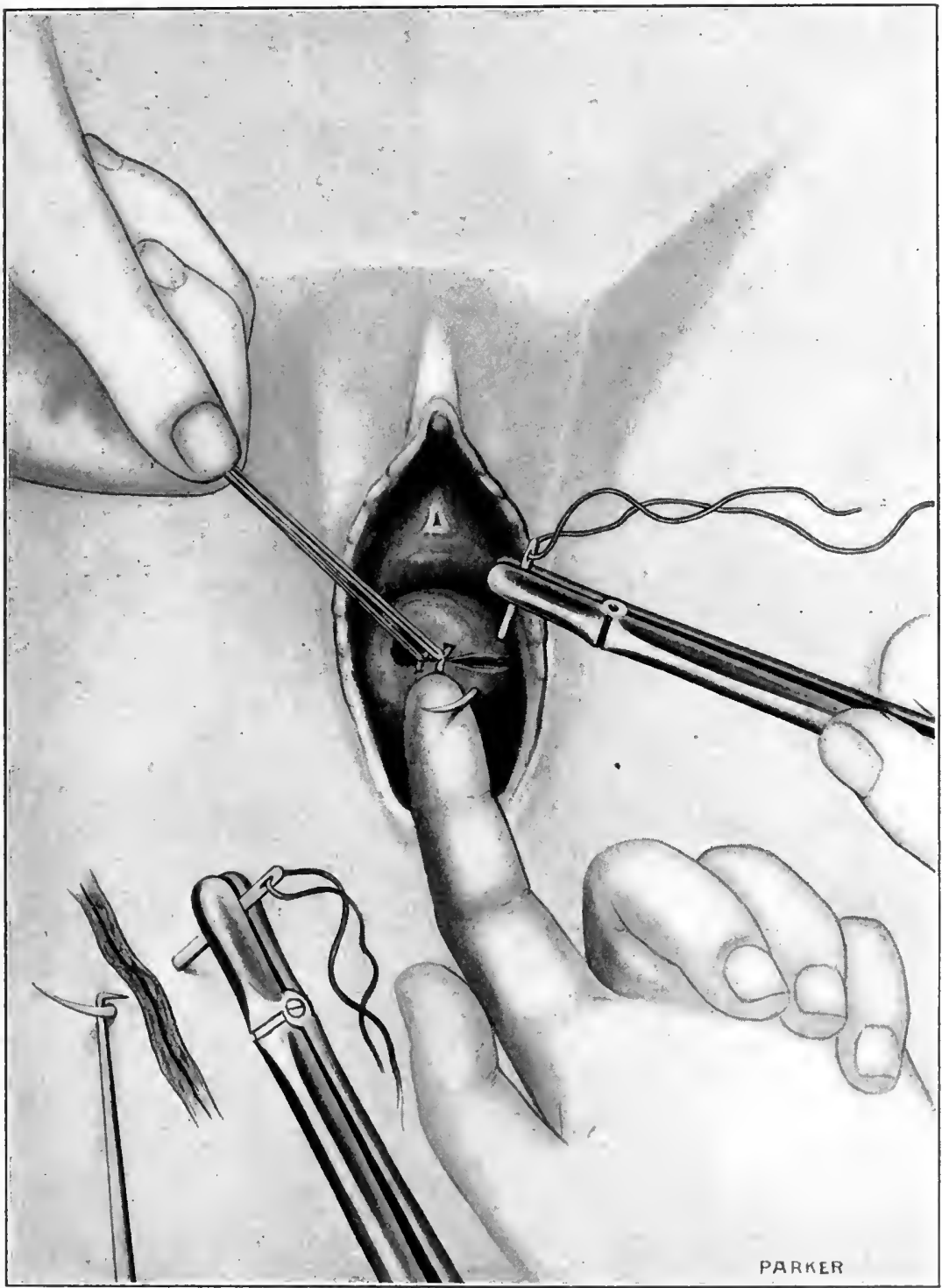

Introduction of a suture with the patient in the dorsal position and the cervix exposed by means of Simon's retractor-counter-pressure by the index finger. The illustration shows in the lower left-hand corner counter-pressure by means of the tenaculum.

incisions with the scissors. By this means the anterior and posterior lips may be separated widely far up into the uterine canal, and the 
Figure 309.

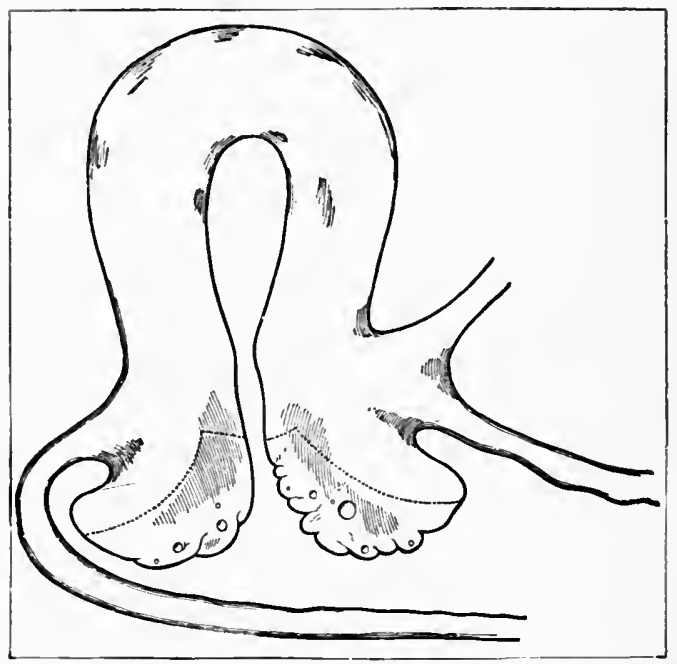

Shows a thickened diseased cervix requiring resection. The dotted lincs indicate the directions of the incisions.

diseased structures thoroughly inspected and efficiently removed. The diseased tissue is removed best by seizing it in small vulsellum

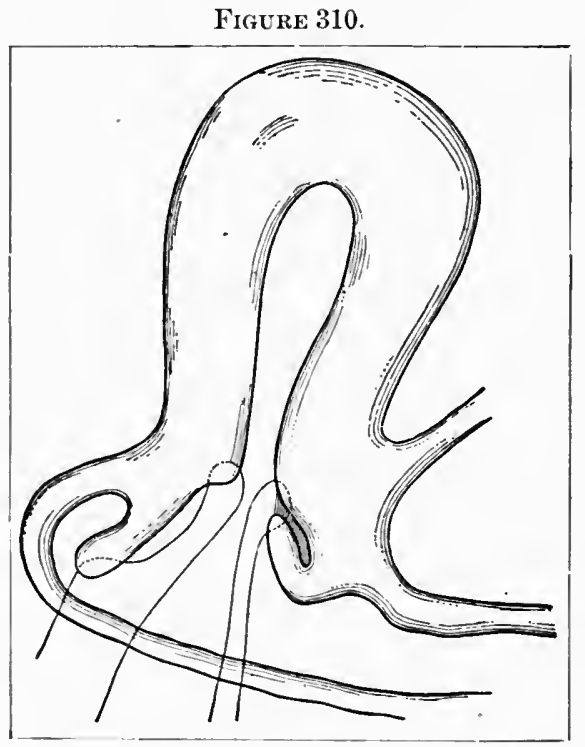

Shows the diseased tissues excised and sutures in place, but not yet tied, to unite the vaginal margin to the cervical margin of the wound.

forceps or a tenaeulum and cutting it out with two or three strokes of the scissors. The frequent closure of the lacerated cervix without 
the removal of these diseased structures accounts for numerous failures and disappointments in the operation.

The brief report of a single case ${ }^{1}$ will serve to illustrate the importance of resection. Trachelorrhaphy had been performed ten

Figure 311.

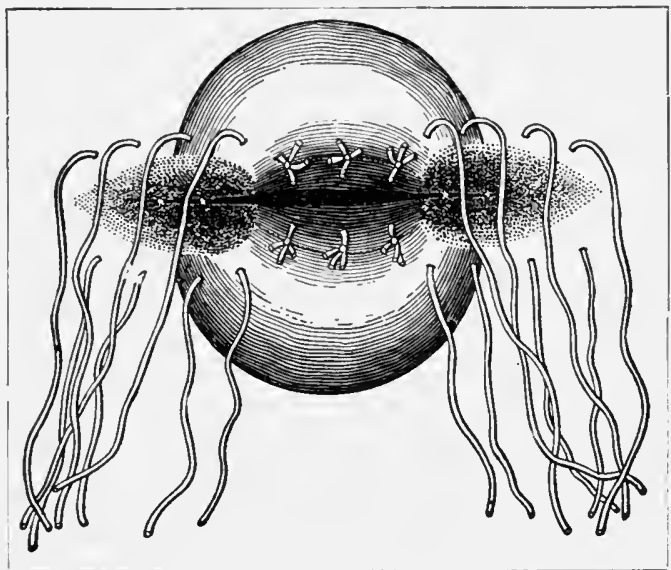

Anterior and posterior vaginal margins, each turned into the cervical canal and united by means of fine catgut sutures to the intracervical margins of the wound. Lateral surfaces denuded and sutures placed as in ordinary trachelorrhaphy, but not yet tied.

years previously. From the time of that operation the patient had suffered from pronounced catalepsy, with frequent paroxysms. Examination showed an enormously thickened cervix with a pinhole os.

\section{Figure 312.}

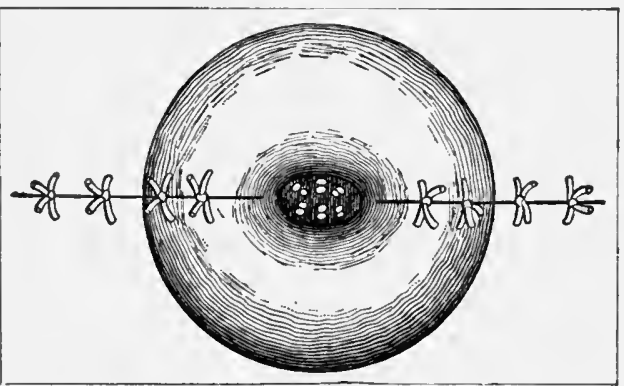

All sutures tied; operation complete. The white dots in the os represent the protruding intracervical sutures.

Both ovaries were enlarged slightly and adherent. In order to ascertain the condition of the interior of the cervix, very deep bilateral incisions wcre made: the anterior and posterior lips were separated more widely than would be nsual in an extensive laceration of the cervix. Much pent-up secretion which escaped showed that the tight closure of the os externum had converted the whole endometrium

1 Dudley. "The Abuse of Emmet's Operation for Laceration of the Cervix." Journal of the American Medical Association, September $23,1893$. 
into a retention-cyst. Numerous eysts of the Nabothian follicles, superficial and decp, large and small, appeared in the intracervical mucosa and submucosa. In excision of these cysts the cervical mucosa and submucosa were removed almost to the internal os; the vaginal and intracervical margins of the wound were united with catgut sutures, as shown in Figure 310. What remained of the lateral incisions was closed then with interrupted silkworm gut sutures. The operation, except the very deep lateral incisions, was practicaaly that of Sehroeder. Since recovery from the operation the patient, though naturally neurotic, has reported herself free from cataleptiform seizures.

The After-treatment of trachelorrhaphy, or Schroeder's operation, consists of rest in bed for about ten days, a vaginal douche of hot sterilized water twice daily, and removal of the sutures through Sims' or Simon's speculum in about two wecks. If the perineum and cervix are closed at the same time, the pressure of the speculum during the removal of the cervical sutures does not, with careful manipulation, endanger the freshly united perineum, provided the perineal sutures are still in place; if they have been removed, it is necessary to delay removal of the cervical sutures until the perineal union is solid-that is, for an additional two or three weeks. It is better, however, in the double operation, as already stated, to use in the cervix absorbable catgut sutures, which do not have to be removed at all.

Results.-Trachelorrhaphy in suitable cases, properly performed with due regard to asepsis, is one of the most satisfactory operations in gynecology. Union by first intention is the almost invariable rule. The relief from symptoms is often very great.

Disappointment in the operation, as already stated, may result :

1. From neglect to treat the complicating endometritis.

2. From the rolling in of hopelessly diseased structures, which ought to have been excised.

3. From disregarding such contraindications as pelvie suppuration.

4. From closing the os externum so tightly as to obstruct the natural outflow of uterine secretions.

5. From the unwise selection of cases.

6. Above all, from faulty technique in the operation itself. 


\section{CHA P TER XLIII。}

\section{GENITAL FISTULA.}

\section{Priority in the Operation for Genital Fistulæ.}

UP To forty years ago, when the operation for the closure of vaginal fistulæ was developed and, in practical form, given to the world by J. Marion Sims, these most distressing injuries had

Figure 313.

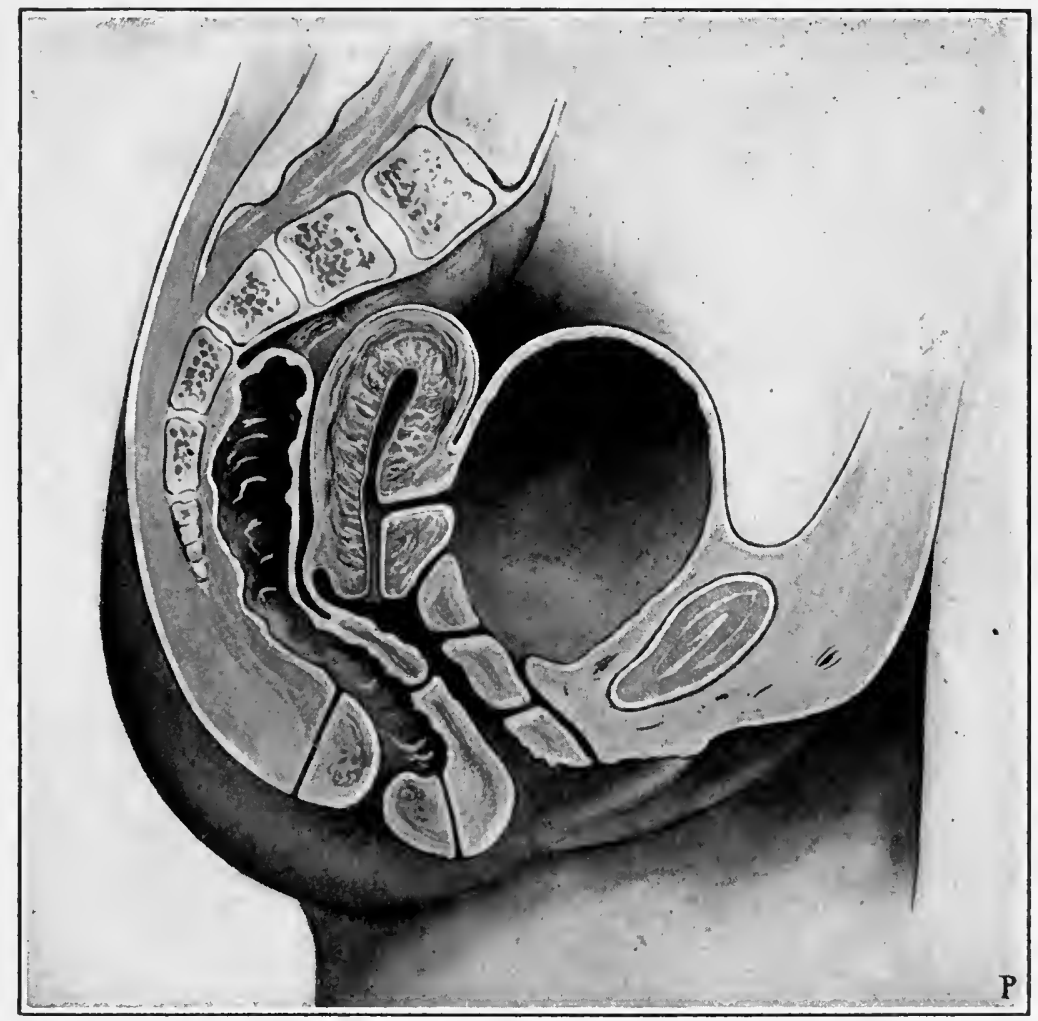

Vesico-uterine fistula. Vesico-uterovaginal fistula. Vesicovaginal fistula. Urethrovaginal
fistula. Rectovaginal fistula. Perineo-anal fistula. Anal fistula.

been ineurable. The invention of Sims' speeulum; which made the operation possible, has a signifieance, however, more far-reaching than the mere recognition of a valuable operation, for it marks an epoch in the listory of gynecology. The operation furnished the 
initiative for the period of great practical activity which followed. It will in no respect detract from the credit which justly belongs to the great pioneer if we admit the fact that the honor of perfecting and perpetuating the methods upon which his operation was based, and upon which modern gynecology has made its greatest development, must be divided between J. Marion Sims and Thomas Addis Emmet.

\section{Varieties of Genital Fistulæ.}

A fistulous opening may connect the interior of the uterus or vagina with some part of the urinary or intestinal tract. Accordingly, the varieties of genital fistulæ are urinary fistulæ and fecal fistulæ.

Figure 313 shows the more common varieties of genital fistulæ. They are :
Vesicovaginal fistula.
Vesico-uterine fistula.
Vesico-uterovaginal fistula.
Urethrovaginal fistula. Rectovaginal fistula.

The following other forms are of rare occurrence: The ureter may communicate directly with the vagina, making a ureterovaginal fistula. The ureter may open into the margin of a vesicovaginal fistula, making a uretero-vesicovaginal fistula. Various other rare forms, such as uretero-nterine fistula, should be classed as surgical curiosities.

The causes of genital fistulæ are these :

Impaction of the presenting part during labor and consequent pressure-necrosis.

Direct traumatism.

Congenital causes - that is, defective development.

Ulcerative and other destructive processes from syphilis, cancer, and inflammation.

Burrowing of pus from abscess.

\section{VESICOVAGINAL FISTULA.}

The definition of the lesion is apparent from the name-that is, an opening between the bladder and vagina.

\section{Etiology of Vesicovaginal Fistula.}

In the vast majority of cases the lesion results from impaction of the presenting part during labor and consequent pressure-necrosis in the vesicovaginal wall. Completion of the necrotic process and separation of the slough require from five to twelve days; hence, in fistula from this cause the essential symptom, escape of urine through the vagina, does not occur until several days after labor.

A fistulous opening sometimes is made purposely by a surgical operation for the treatment of eystitis or for the removal of stone in the bladder, or it may be the result of accidental traumatisn. The escape of urine will then be immediate. Congenital fistula is rare, and is characterized by the involuntary escape of urine from the time 
of birth. The ulcerative processes of syphilis, caneer, and inflammation are much more frequently the eause of fecal than of urinary fistula.

\section{Symptoms and Course of Vesicovaginal Fistula.}

The constant symptom, already mentioned, is the escape of urine from the bladder through the vagina. 'The fistula may vary from the size of a pinpoint to that of the entire vesicovaginal wall. When the opening is of appreciable size or large, the flow of urine usually is contimuous. In very small fistulæ the eseape of urine may be intermittent. The intermission is apt to occur when the woman is lying down. In rare eases of small fistula a valve-like formation may shut off the flow of urine except when the woman assumes certain positions favorable to its escape.

A Cause of Cystitis.-In the majority of cases there is more or less residual urine in the bladder. This is a good culture-medium for bacteria which now find ready access from the vagina to the bladder; hence cystitis is a usual complication. From this cause the urine becomes alkaline, ammoniacal, and excessively irritating. The vagina, external genitals, thighs, and buttocks, over which it flows, become excoriated, edematous, and ulcerated. A gritty, offensive phosphatic deposit may form and deeply incrust not only these surfaces, but also the raw margins of the fistula and the bladder mucous membrane. This deposit is specially apt to accumulate and form incrustations on ulcerated and otherwise exposed surfaces. It may fill the vagina and even extend over the ulcerated labia. The inside of the bladder, perchance deeply ulcerated, granulating, incrusted, bleeding, and excessively painful, may, in rare cases, if the fistula be large, become inverted and protrude in a semi-strangulated condition between the labia majora. The patient, within a few weeks after labor, will then, unless great care is exercised, become an object of loathing or pity.

\section{Diagnosis of Vesicovaginal Fistula.}

The opening, if sufficiently large, may be felt by the finger in the vagina. The fistula, thus having been located, the finger may be used as a guide for the passage of a sound through the urethra into the bladder, and thence through the fistulous opening into the vagina.

When the fistula is very small, it is sometimes difficult or impossible to see it even after careful search with the speculum. In such a ease, the speculum being in place, the bladder should be injected throngh the urethra with sterilized colored water. The point at which this fluid escapes into the ragina will locate the fistula.

\section{Prognosis of Vesicovaginal Fistula.}

The prognosis depends upon the extent of the injury, the amount of eicatricial tissue, and the difficulty of approximating the margins of the fistula. In exceptional cases of small opening, in which the 
margins lie in easy and close apposition, they may, if kept clean, soon unite without operative interference. The vast majority of fistulæ, however, unless united by suture, are permanent.

\section{Prophylactic Treatment of Vesicovaginal Fistulæ.}

The statistics of Emmet, covering a long series of cases of vesicovaginal fistula, show that the average duration of labor from the time of rupture of the membranes to the birth of the child was between two and three days. Statistics further prove that impaction and consequent continued pressure of the presenting part upon the vesicovaginal septum, even for a few hours, are very liable to result in cutting off the circulation and in consequent death and sloughing of. the compressed tissue. If, therefore, in any case impaction becomes apparent by the failure of the presenting part to advance during the pains and to recede in the interval between the pains, delivery should be hastened und terminated without unnecessary delay. The possible danger of a forceps operation in such a case, even by the inexperienced hand, when compared with the danger of fistula, would be insignificant.

Emmet's records show that in nearly all his cases parturition had taken place either without attendance or under the care of ignorant midwives. In some cases labor had terminated finally by the unaided efforts of nature, and in others by the use of the forceps. In the latter class of cases delivery is accomplished usually by a consultant, who is not called until after prolonged, continuous pressure has destroyed the vitality of at least a part of the vaginal wall. Sometimes the fistula is attributed wrongly to the forceps or other instruments, instead of the real cause-prolonged pressure-a cause which earlier interference would have prevented. As Thomas wisely remarks, the truth on this point should be sct forth clearly to the friends of the patient before forceps are applied, "for unless it be so, an incompetent person may shield himself from merited blame by casting censure upon a consulting physician, by whose efforts the lives of both mother and child may have been saved; and thus a skilful operator may suffer unjustly in a suit for malpractice."

Emmet's statistics show that in a large proportion of cases the bladder was not emptied during the progress of labor. This neglect would cause large accumulation of retained urine and great distention of the bladder. The result would be paralysis of the bladder and cystitis. Moreover, the impaction would be increased by the pressure urine exerted on the bladder side of the vesicovaginal septum, and this pressure would be an additional cause of necrosis. Catheterization, therefore, as a prophylactic measure is an urgent necessity.

After delivery in a case of continuous and prolonged impaction, decided antiseptic measures are indicated to prevent or limit the threatened necrosis. They are :

1. A vaginal douche of 0.5 per cent. lysol, or some other appropriate antiseptic, every eight hours.

2. Daily washing out of the bladder with a saturated solution of boric acid. 
3. Sufficiently frequent catheterization to prevent great accumulations of urine and consequent bladder distention.

\section{Surgical Treatment of Vesicovaginal Fistuia.}

The surgical treatment includes the preparatory treatment, the operation, and the after-treatment.

Preparatory Treatment.-If the parts are brought into a condition favorable for union, the operation for the cure of vesicovaginal fistula, even with ordinary skill, is one of the most satisfactory in the whole field of surgery. On the other hand, the most skilful operation, with faulty preparation, is almost certain to fail.

Phosphatic Deposits. - The margins of the fistula cannot be brought into a healthy condition and made fit for union until the phosphatic deposit already mentioned has been removed, and the further formation of it prevented. To this end, the urine should be rendered acid; otherwise the deposit will accumulate on the sutures and in the lines of union, and cause the operation to fail. It does not, however, develop in acid urine. Emmet's mixture of benzoic acid, two drachms; borax, three drachms; and cinnamon water, twelve ounces, gives uniformly good results. A tablespoonful, further diluted, should be taken four times a day until the urine becomes mildly acid. The dose then should be regulated to maintain normal acidity and to aroid deranging the digestion. The acid sodium phosphate in 30 grain dose, well diluted, is most useful to render the urine acid. In order to dilute the urine and render it less irritating, pure water should be given quite freely. If the urine is kept slightly acid and well diluted, the phosphatie deposit once removed will not return.

The removal of the deposit is accomplished best by means of a dressing-forceps, or it may be brushed off with a wad of cotton in the grasp of the forceps, after which the raw surfaces should be treated by means of a solution of silver nitrate, ten grains or more to the ounce, on an applicator wound with absorbent cotton. Sometimes the deposit adheres very firmly, as if it were interlaced with the adjacent and underlying tissue, so that immediate removal would be too difficult or painful. Emmet then applies a stronger solution or even the solid stick of silver nitrate to the deposit itself. This may be repeated every few days until the deposit is detached.

The hot vaginal douche described in Chapter IV. is of the utmost value in the preparatory treatment. It should be given freely several times a day, and large quantities of hot water 'should be used. This part of the treatment, as Emmet declares, is indispensable. The sitzbath also is most useful and gratefin to the patient. The douche may be given to advantage and with inereased comfort while the patient is in the sitz-bath. This treatment, merely insuring perfect cleanliness, has resulted in some cases, even of large fistula, in spontaneons closure. They were eases, however, in which there had not been great loss of tissue, and in which the edges of the fistula were in apposition.

The excoriated or eroded surfaces about the nates or thighs are 
treated best by frequent bathing, followed by applications of benzoated zinc oxide ointment. Napkins like menstrual napkins should be worn over the vulva to absorb the urine, and should be changed frequently; otherwise the urine which they hold will decompose and become excessively irritating to the skin. Points of ulceration may be touched with solid silver nitrate.

Cystitis, if present, is a clear contraindication to immediate closure of the fistula. The copious hot-water vesicovaginal douche, frequent and prolonged, is the best means of treating this complication. It is given as an ordinary vaginal douche, except that the hot water, instead of being thrown in by the douche point through the vulva, is introduced through the urethra. For this purpose a glass urethral catheter or canula small enough to enter the urethra is used in place of the vaginal douche point. The hot water by this means is applied first freely to the bladder, and then through the fistula to the vagina and vulva. Cystitis in these cases, as already explained, is sometimes the result of residual urine. It may therefore be necessary, especially in a very small fistula, to secure adequate drainage of the bladder by an incision in the vesicovaginal wall. If the fistula is situated in or near the median line of the vesicovaginal septum, the incision should be so made as to enlarge it, otherwise an independent opening should be made. See Treatment of Cystitis by Means of Artificial Vesicovaginal Fistula, in Chapter XXIV. Old inflammation of the kidney or ureter may be present, and if in an advanced stage might contraindicate the operation; hence the importance of the rule to examine the urine in every case. The urine may be collected for examination by keeping the woman on a bedpan until a sufficient quantity has accumulated.

Stone in the Bladder, free or encysted, may in rare cases have antedated and even been a cause of fistula-that is, the vesicoraginal septum during labor may have been compressed between the stone and the child's head. Usually, however, the calculus is deposited from the residual urine already mentioned among the frequent results of fistula. The necessity for the removal of such a stone before closing the fistula is apparent.

Direction and Manner of Closure.-The urine being normal, the vagina and bladder healthy, and the structures surrounding the fistula fit for union, the next step will be to decide upon the best direction and manner of closure. In order to preserve the length of the vagina, it is desirable, if possible, to bring the parts together from side to side, so as to make a line of union as nearly as possible in the long axis of the vagina. A line of union transversely across the vagina would shorten its anterior wall and would draw down the uterus and fix it in permanent displacement.

Unfortunately, in many cases of extensive sloughing the margins of the fistula cannot be approximated from side to side. They may even be so held apart by cicatricial bands that they cannot be approximated at all, for there may not be sufficient tissue left to fill the gap. In order to decide upon the best mode and direction of closure, the fistula should be exposed by Sims' speculum, and its margins at 
different points seized on opposite sides and drawn together with a tenaculum in each hand. In this way one may judge of the amount of force required to approximate the edges, and of the direction in which they will come together with the least traction.

It is an urgent mule never to introduce sutures unless the surfaces to be united can be held in contact without traction; even a little traction on the sutures will cause them invariably to cut out and the operation to fail.

If the restraining bands are so light and superficial that moderate traction with tenacula suffices to approximate the margins of the

\section{Figure 314.}

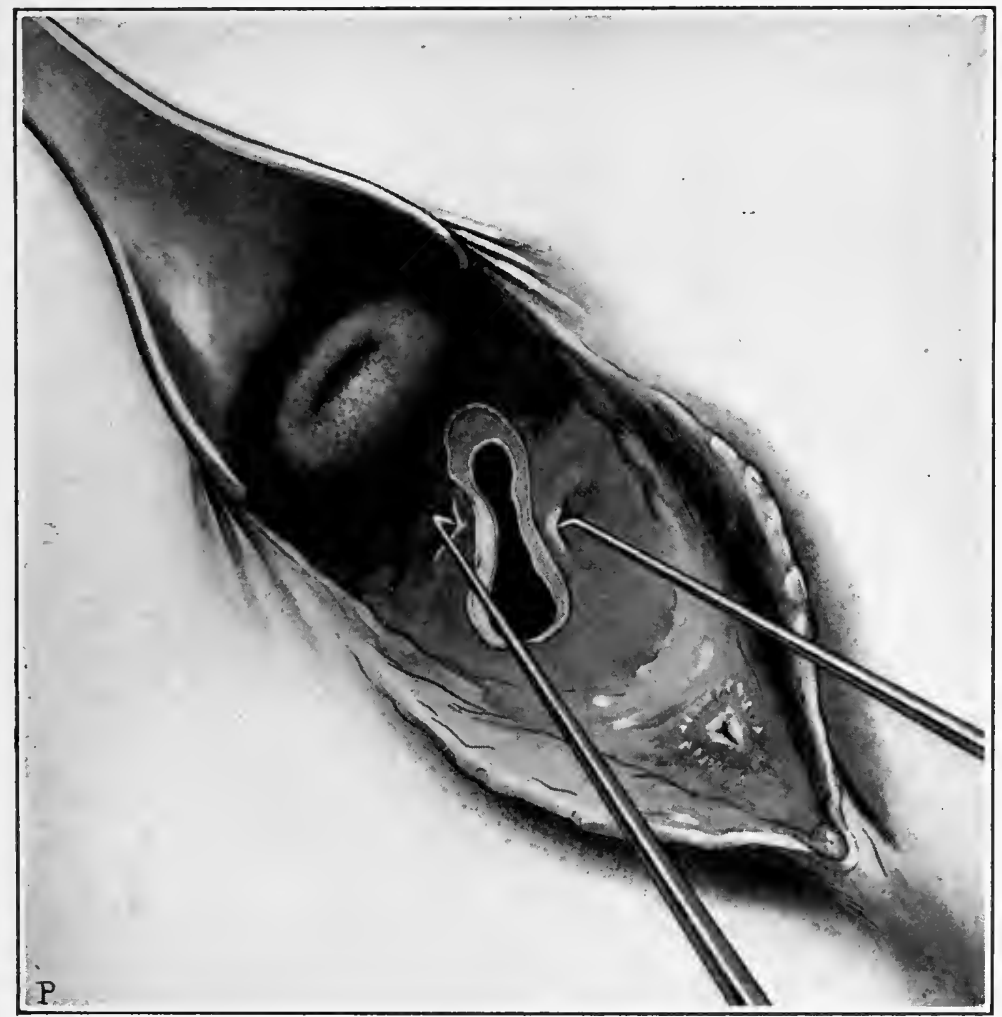

Vesicovaginal fistula exposed by Sims' speculum. Approximation of the margins attempted by means of tenacula. Left lateroprone position. ${ }^{1}$

fistula, the bands may be divided with scissors until the margins readily fall together. The surfaces then may be denuded immediately and the sutures introduced.

If the sloughing has been very extensive, one or more prcliminary operations may be necessary. Enimet places the patient on her back, introduces two fingers of the left hand into the rectum, and the thumb of the same hand into the vagina. The interior of the vagina thereby is rolled out and exposed without a speculnm. The right index-

1 Emmet. Principles and Practice of Gynecology. 
finger in the vagina now detects the points of greatest cicatricial tension. Point after point is snipped with the blunt seissors in such a way as to render the margins of the fistula more readily approximated. If the cervix uteri has sloughed, the relations of the remaining portion of the uterus to the npper part of the vagina, even by rectal touch, may be difficult to make ont. There is then great danger of wounding a misplaced ureter or of entering the peritoneum. This danger is lessened by the careful use of the sound held by the hand of an assistant in the bladder.

The restraining bands having been divided as freely as may be deemed prudent, Emmet directs that a Sims glass or hard-rubber vaginal plug be introduced and held in place by a T-bandage. It should be sufficiently long and wide to keep the vagina well stretched

Figure 315.

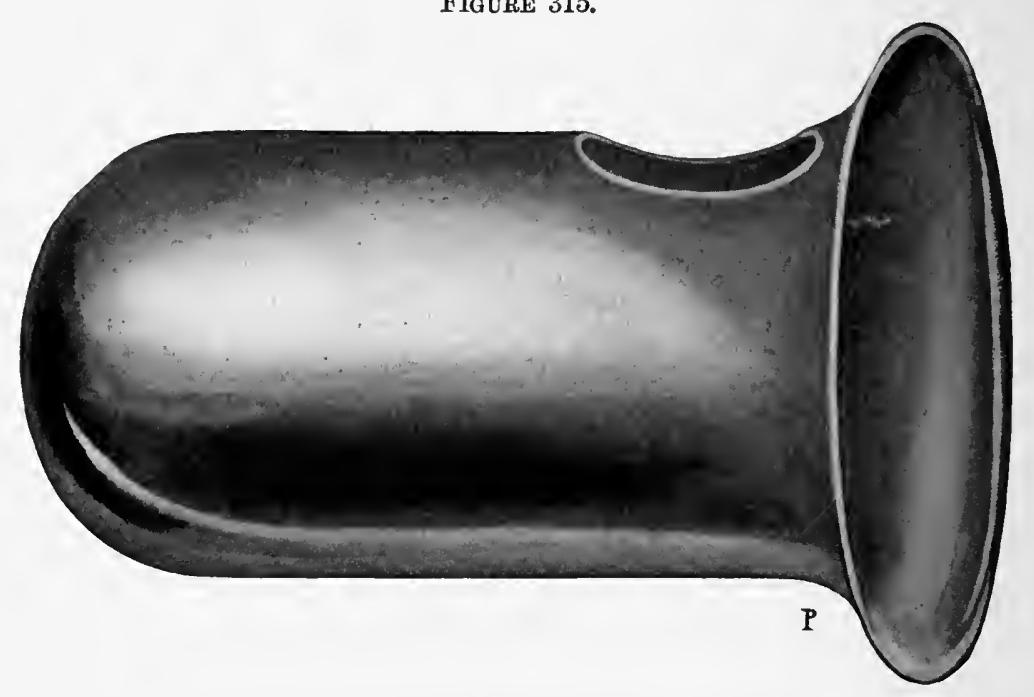

Sims' glass vaginal plug.

both longitudinally and laterally, and to eontrol hemorrhage by pressure, but not so large as to cause pressure-necrosis and sloughing. Under this pressure, absorption of cicatricial tissue is rapid. The continued stretching of the vagina also increases its calibre and renders approximation of the margins of the fistula less difficult. The dilator may have to be retained for several weeks until the incisions have healed over it; in the meantime it may be removed daily for cleansing douches. The patient shonld be kept in bed for a week or two after this preliminary operation, and the urine should, if necessary, be drawn with a catheter. After healing has taken place, the operation, if necessary, may be repeated; or, if the margins of the fistula can be brought together without tension, the sutures for closure may be introduced.

In place of the incisions and glass dilator just described, the re- 
straining cicatricial bands may be divided deeply and freely and the wounds closed at right angles to the lines of incision. The operation is illustrated in Figures 316, 317, and 318. This preliminary plastic

FigURe 316.

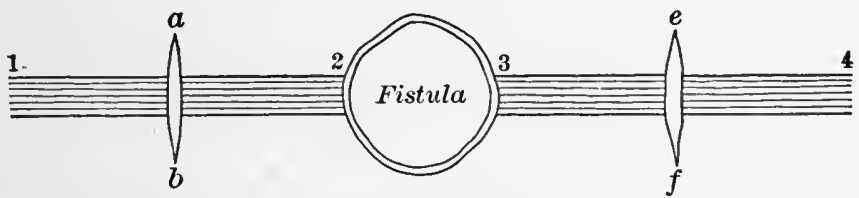

1,2 , and 3,4 , represent the restrainingeicatrical bands on each side of the fistula ; $a b$ and $e f$ show the lines of incision.

work may, according to indication, be done at the time of closing the fistula or as a separate operation.

The preparatory treatment outlined above may be difficult, long

Figure 31\%.

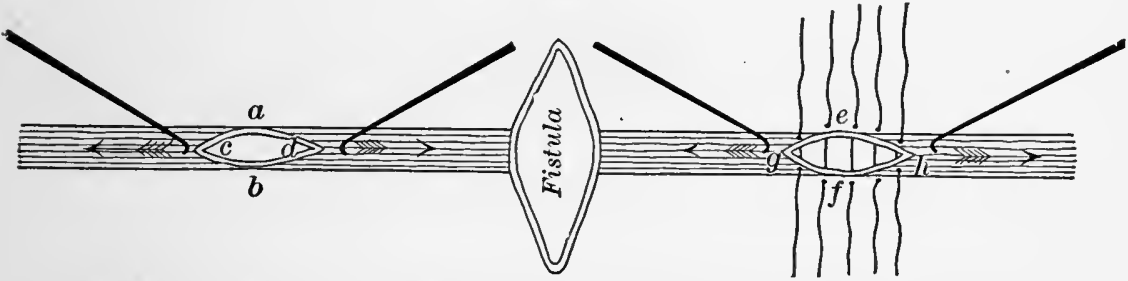

The wounds made by incisions $a b$ and of are drawn widely aport by tenacula so as to give the wound on either side the direction of $c d$ and $g h$. Sutures are in place on right side

continued, and most trying to patient and surgeon. Fortunately, there are many cases in which it is not required. When it is required, the most skilful operation will fail without it.

Figure 318.

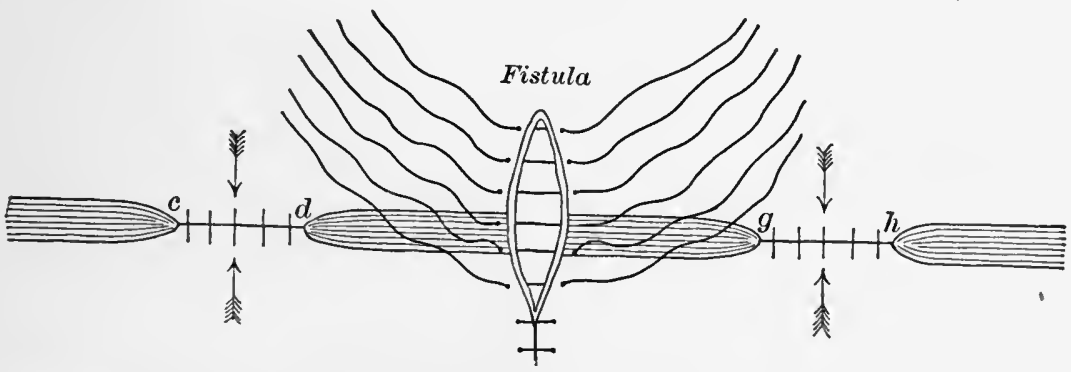

Incised wounds on both sides of the fistula closed at right angles to lines of incision. The edges of the fistula now readily fall into apposition. Sutures for closure of fistula in place, and two of them tied.

The Operation for Closing the Fistula.-This involves a consideration of the following topies:

1. General preparatory treatment.

2. Choice of speculum and method of operation. 
3. Choice of direction for closure of the fistula.

4. Denudation.

5. Introduction of sutures.

6. After-treatment.

1. The General Preparatory Treatment and arrangements for plastic operations deseribed in Chapter II. are applicable and adequate for this operation.

Figure 319.

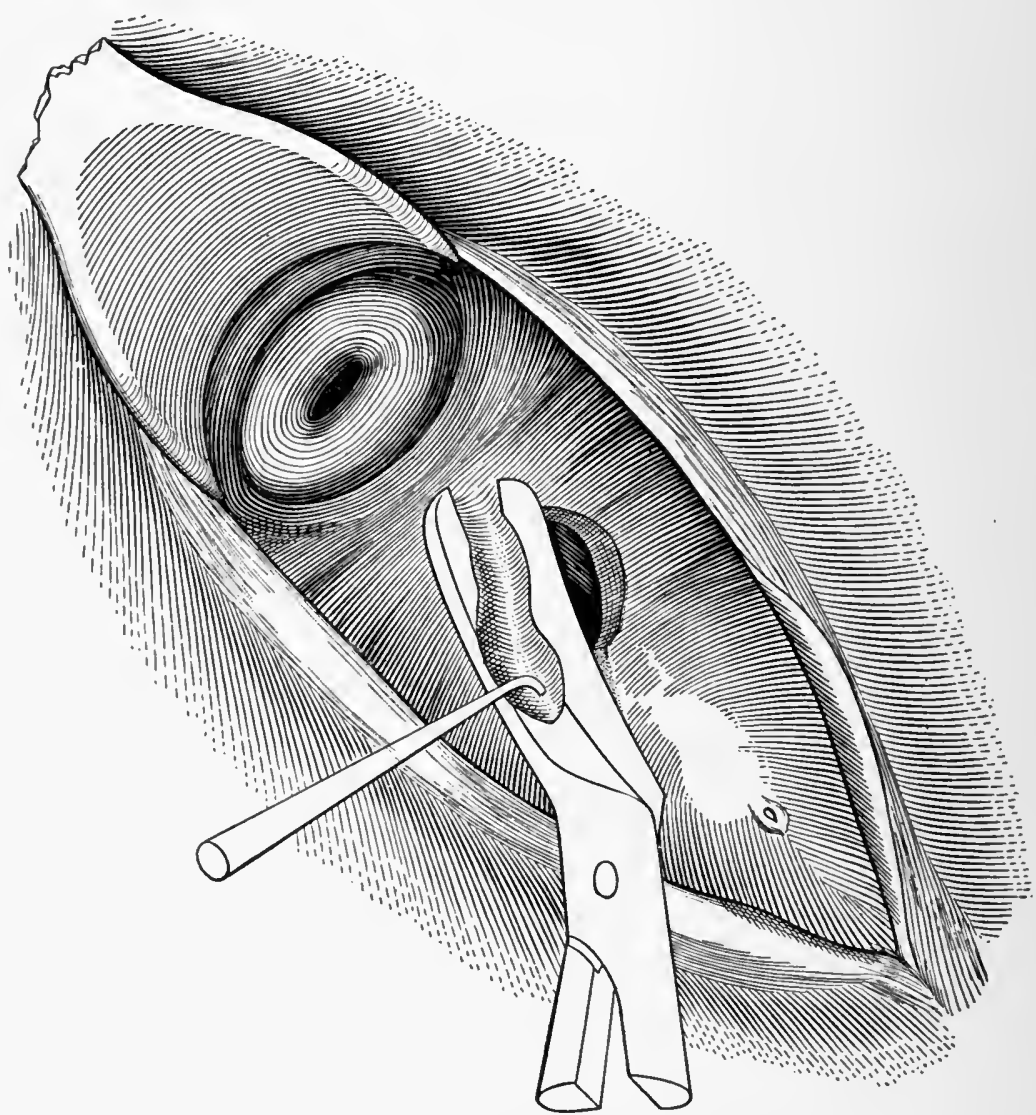

The use of the uterine tenaculum and Emmet's seissors in denudation. Left lateroprone position; exposure by sims' speeulum.

2. Choice of Speculum and Method of Operation.-The author's choice between the method of Simon, with the patient in the dorsal position, the parts being exposed by numerous vaginal retractors, and the method of Sims, with the left lateroprone position and Sims' speculum, is based upon an extensive experience with both methods. The Simon method is serviceable and adequate for the ordinary ease, but not always for difficult cases. This is especially true when the fistula is near the vaginal outlet behind the ramus of the pubes. In fat subjeets, moreover, the Sims position and speculum are almost 
indispensable. Decided preference therefore is given to the msethod of Sims as tanght and practised by Emmet. The position of the patient and the use of the speculum are described in Chapter III.

3. Direction of the Line of Union. - In order to decide upon the exact direction for closure, the edges of the fistula are approximated in different ways with tenacula, until that direction is found and adopted which permits the margins of the fistula to be approximated with the least traction. For reasons already given, it is always

Figure 320.

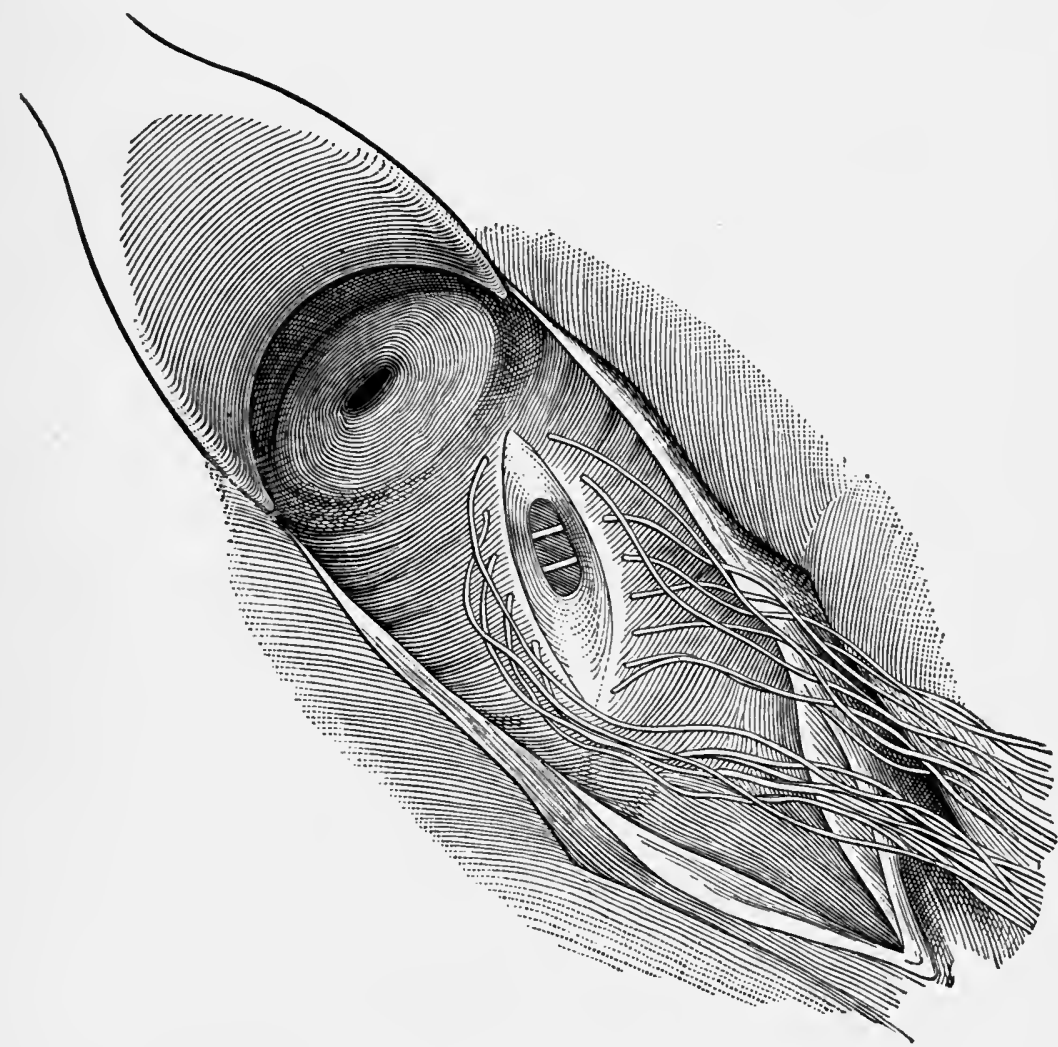

The proper area of denudation. The denuded surfaces correspond to the deck and the fistula to the manhole of a canoe. Left lateroprone position; exposure by Sims' speculum.

desirable to make the line of union, if possible, in the direction of the long axis of the vagina.

4. Denudation.- The edges of the fistula are denuded by means of the tenaculum and scissors, as shown in Figure 319.

The skilful hand will denude superficially or deeply, as may be required. The denuded surfaces should be made clean and smooth, and the bleeding should be slight. For denudation the uterine tenaculum and scissors are far superior to the tissue forceps and the scalpel. 
The margins of the fistula are seized with the tenaeulum, at the point nearest the operator, and a strip is cut away all around the

Figure 321.

\section{A FAULTY METHOD OF OPERATING.}

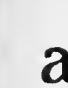

a
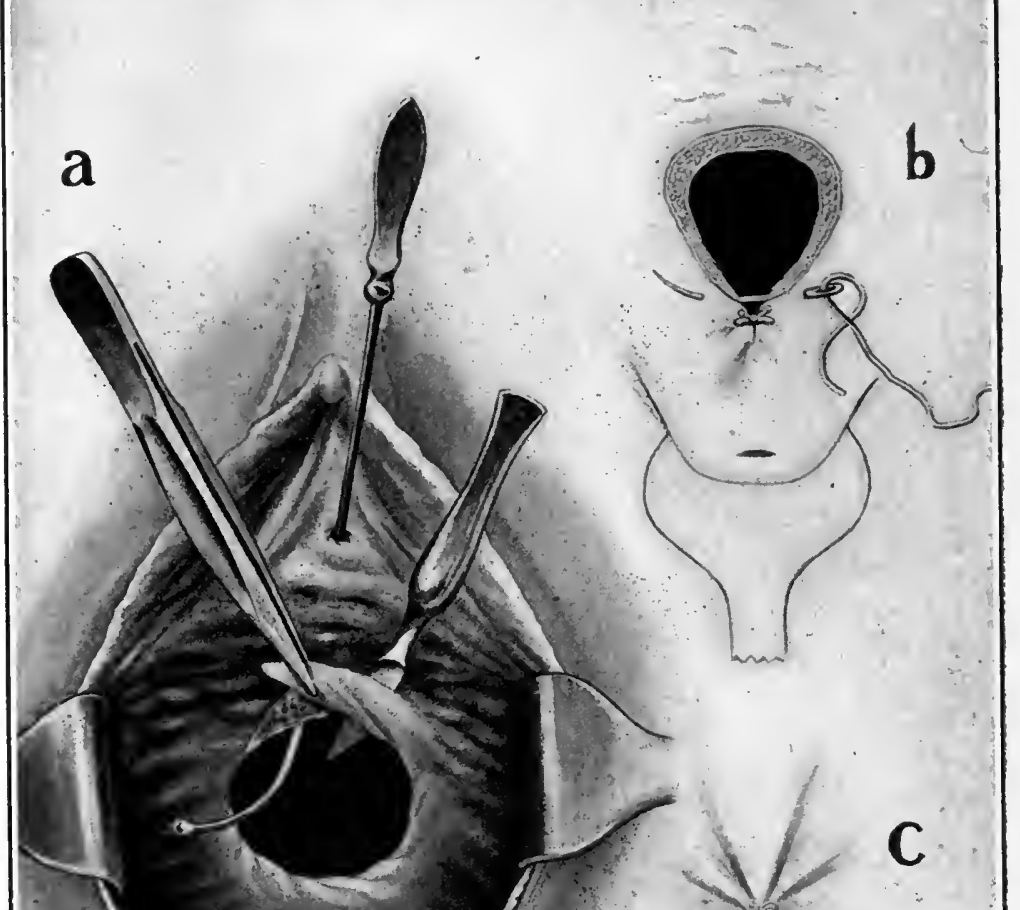
edges about the fistula quite thin, the denuded surfaces should be correspondingly broader. The two points upon which to lay the

FigURE 322.

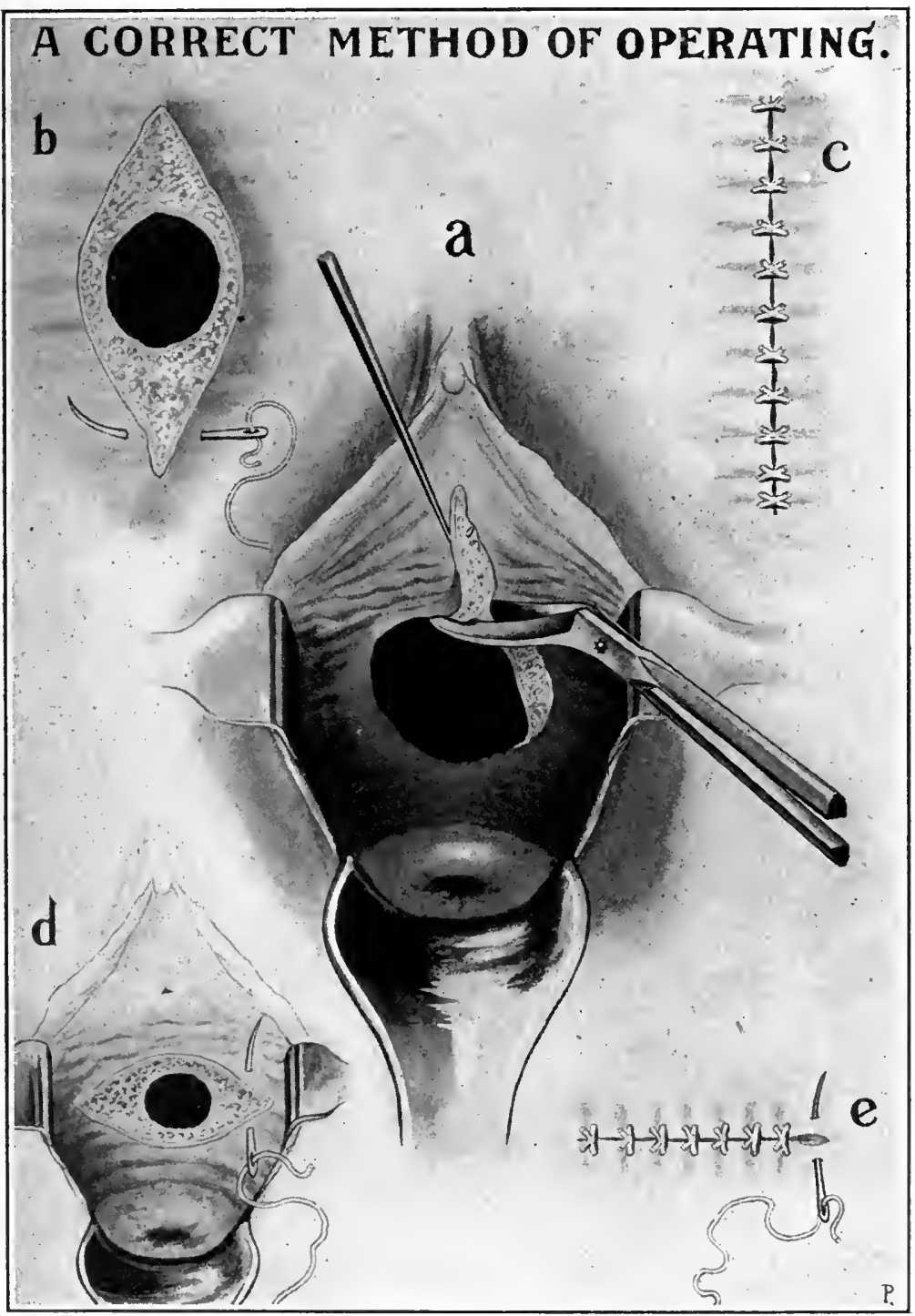

$a$, correct method of denudation with tenaculum and scissors; $b$, correct form of denuded surface. First suture being introduced for closure of fistula in direction of long axis of vagina : c, same as b, with closure of fistula completed; $d$, first suture being introduced for closure of fistula in transverse axis of vagina; $e$, same as d, with closure of fistula completed.

greatest stress are, first, adequate preparatory treatment; seeond, broad surfaces for union. The fistula may be so small as to be 
inaccessible for denudation, and therefore may have to be enlarged by incision in order that its margins may be freshened and united.

Some operators instead of denuding, split the edges of the fistula. This method, though not usual, is yet highly advantageous when the margins are thin, or when it is specially desirable to economize tissue. See Figures 323 and 324 . The bladder mucosa, if cut, is prone to bleed freely; hence denudation should ordinarily extend to, but not into it. Hemorrhage from the cut bladder mucosa, even in the careful hands of Emmet, has twice been so free as to distend the bladder with blood and endanger life. In both cases the sutures were re-

Figure 323.
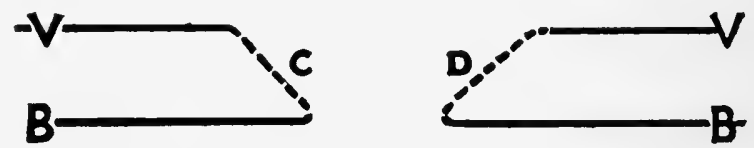

Ordinary denudatiou : V, V, vaginal surface; B, B, vesical surface; $C$ and $\mathrm{L}$, lines of denudation.

moved and the bleeding points secured. When the denuded strip includes the bladder mucous membrane, the cut margin may retract into the bladder and make the bleeding points quite inaccessible. Complete anæsthesia, a strong light, good position, and the skilful use of the speculum and uterine tenacula may then be necessary to evert and expose bleeding surfaces and control hemorrhage.

Emmet properly condemns the practice of simply denuding a strip of uniform width around on the vaginal side of the fistula. He insists that the margins be denuded to the vesical mucosa. 'The denudation at the angles of the fistula should, however, be extended some distance over the vaginal surface, as shown in Figure 320.

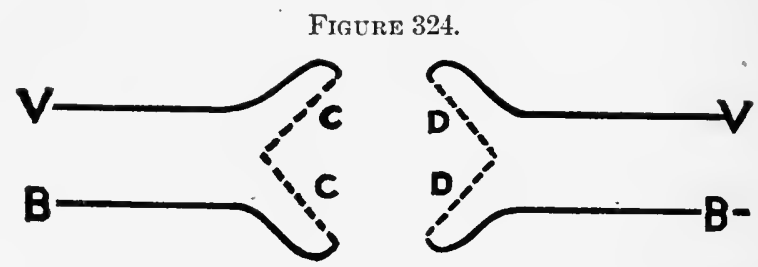

Flap-splitting on both sides seldom required: V. V, vaginal surface; $B$, B, vesical surface; C. C, and $\mathrm{D}, \mathrm{D}$, inner surfaces of split edges to be coapted.

Otherwise, there will be a double fold or pucker at each angle. One may illustrate this by picking up together two small folds of a napkin, and observing that they extend a considerable distance before they can be smoothed down to the eommon surface. The same is true of the vaginal folds at the two ends of an improperly denuded fistula, and the denudation should therefore be so extended that the folds are lost on the level of the ragina. Unless this precaution is observed, union is apt to be imperfect, or may fail altogether at the ends of the line of union.

5. Application of Sutures.-Formerly the metallic suture, usually 
silver, was almost exclusively used. Now, with aseptic methods, any suture is adequate. The writer prefers silkworm gut tied on the surface with an ordinary hard knot. The numerous devices for fastening the sutures by means of short metallic plates, quills, split shot, and other means are useless, harmful, or unnecessary.

Emmet's or Sims' needle is adapted best for ordinary use ; it is short and straight, except near the point, where it is curved slightly. Occasionally a full-curved needle may be of service. The Emmet needle is shown in Figure 325.

Figure 325.

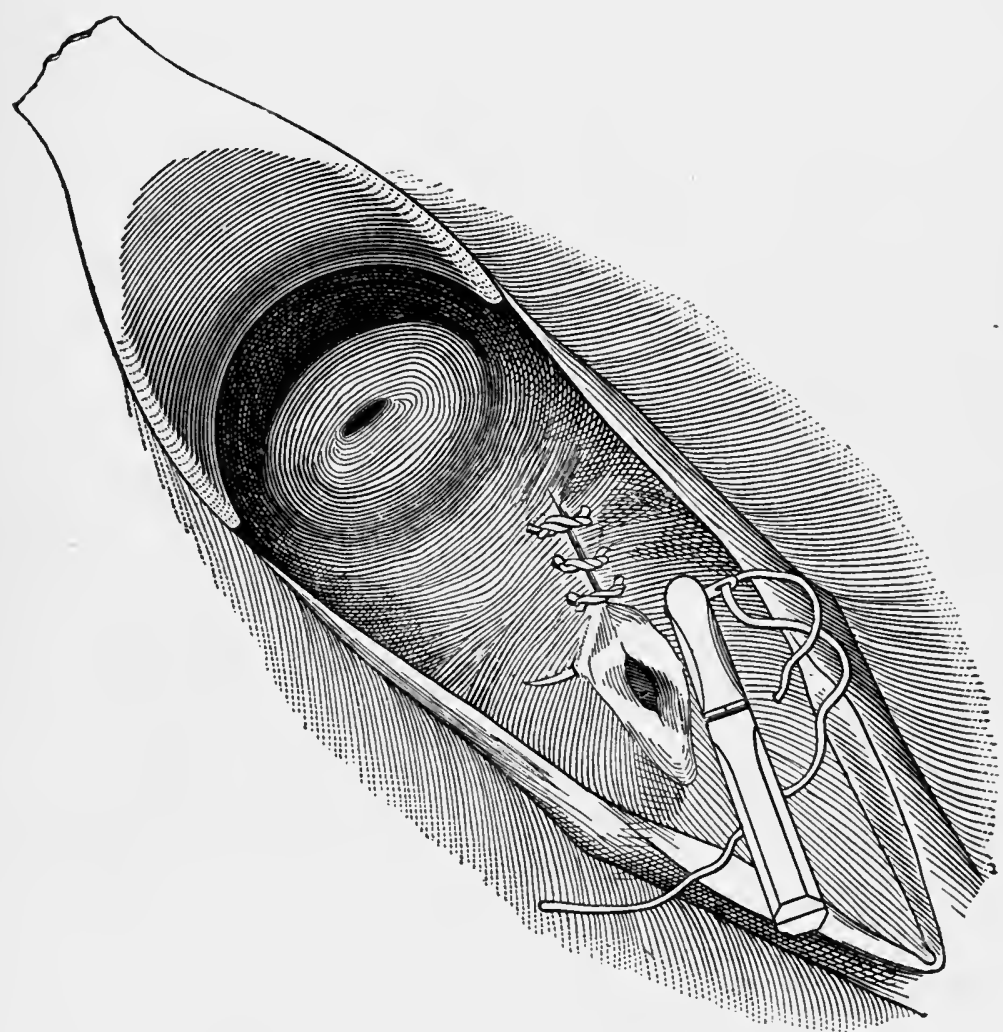

Closing of a vesicovaginal fistula: introduction of a suture. Left lateroprone position; exposure by Sims' speculum.

A needle-forceps withont locking handles is recommended because it will enable a dexterous operator constantly to vary the direction of the needle during introduction.

The suture should be attached to the noedle in the orlinary way, as a thread is attached to a common sewing-needle. The needle is grasped by the forceps and entered about one-eighth of an inch from the margin of the fistula on the vaginal sile; it transfixes the vesicovaginal wall and emerges on the bladder side, so as barely to include 
the vesieal margin ; it then is passed through the wall on the opposite side in the inverse order, and brought ont one-eighth inch from the margin of the vaginal mucosa on that side. The sutures should be placed about one-sixth of an inch apart. If silver sutures are used, they all should be passed first and then seeured by twisting. In using silkworm gut, one usually should tie each suture as it is passed. Let the sutures be tied just tightly enough to hold the parts together. If tied too tightly, they strangulate the tissnes, cut out, and fail to give union.

A elot of blood, if left in the bladder after closure, may cause great vesical tenesmus and possibly imperil the result. It is well, therefore, before tying the final sutures to throw a quantity of sterilized water through the urethra into the bladder. This water will pass through the fistula into the vagina and wash out anything remaining in the bladder.

6. After-treatment.-The patient is placed in bed on the back, with a roll under the knees for support. A self-retaining Sims' sigmoid catheter is placed in the urethra ; it should be made of block-tin or of glass, bent by the flame of a spirit lamp. The curves should be adjusted to the individual case. The urine passes through the catheter

\section{Figure 326.}

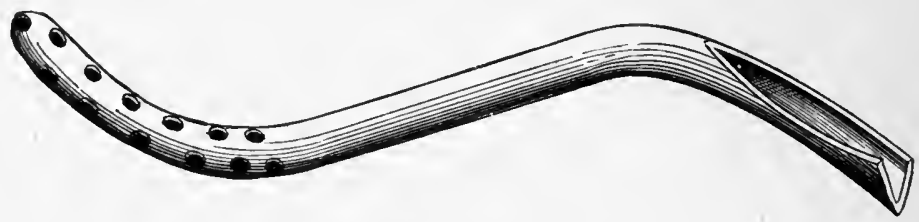

Sims' sigmoid catheter.

and is collected in a urinal placed between the thighs. The catheter is apt to become clogged with mucus or blood-clots, and, therefore, should be removed and cleaned every few hours. A second catheter is desirable, in order that one may always be introduced as soon as the other is removed. In ease of a small fistula we may dispense sometimes with the self-retaining catheter altogether and permit the patient to pass the urine in the natural way. Both patient and nurse should be cautioned to see that the flow of urine is not interrupted. The catheter should remain abont fourteen days. The sutures, unless removed earlier on account of suppuration or failure of union, may remain two or three weeks. The woman should be kept in bed a week longer, and during this time should be catheterized at frequent intervals.

During convalescence the urine should be kept normally acid, otherwise phosphatic deposits may form in the line of union and on the sutures and prevent or destroy union. The benzoic acid mixture or the acid sodium phosphate, already mentioned, should therefore be continued. The long retention of the eatheter and the continued dorsal position may give rise to great discomfort ; hence the necessity in many eases of using more or less morphine, opium, or other ano- 
dyne. A cathartic should be given on the third day, and thereafter the bowels kept regular by moderate eatharsis and enemata. After the final removal of the catheter there may be retention of urine, and it may be necessary, therefore, in order to prevent distention of the bladder, to draw the urine every few hours. In old eases the bladder, either from disuse or from cystitis, may be much eontracted, and therefore liable to distention from a small quantity of urine. The funetional powers of the bladder and urethra progressively improve as the bladder gradually becomes accustomed to the retention of con-

Figure 327.

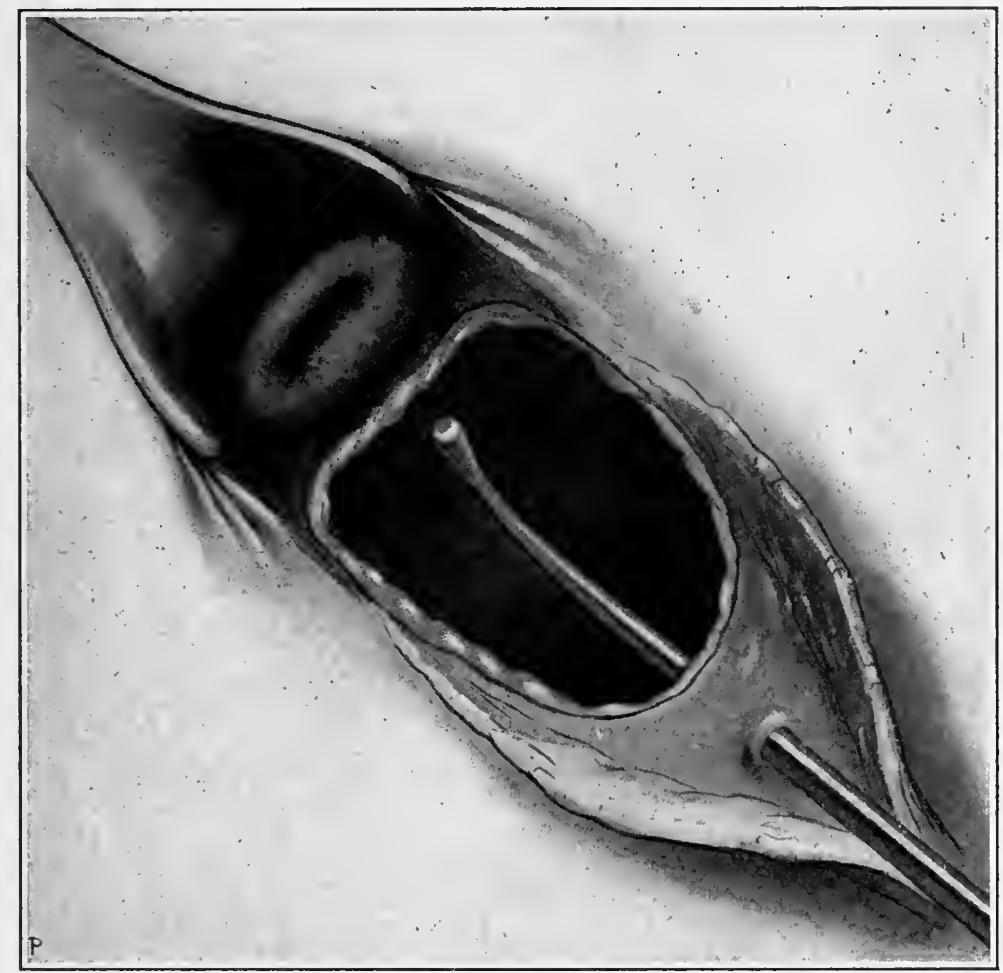

Fistula involving loss of entire vesicovaginal septum as seen through the speculum.

siderable quantities of urine, so that a bladder for many years eontracted by vesicovaginal fistula may regain its full capacity in a short time after elosure of the opening.

Atypical Operations.-The ingenuity and skill of the operator will enable him to modify the operation aceording to the requirements of an atypical ease. An operation may be only partially suecessful, and may have to be repeated again and again until the closure is complete, or it may be necessary to close the opening only in part at each one of several operations.

Loss of the Entire Vesicovaginal Septum is associated usually with 
more or less destruction of cervical tissue and cicatricial development in the posterior vaginal fornix. The usual operation in such a ease is to close by a transverse line of mion-that is, to stitch the anterior lip of the cervix uteri to the neck of the bladder. In some cases the cervix is immovable and eannot be drawn down to the neck of the bladder until the post-cervical eicatrices have been divided freely by a deep transverse incision back of the cervix. In order to gain the required reach, it may be permissible to split the cervix bilaterally. Figure 328 shows the fistula closed by union of the cervix uteri to the neek of the bladder.

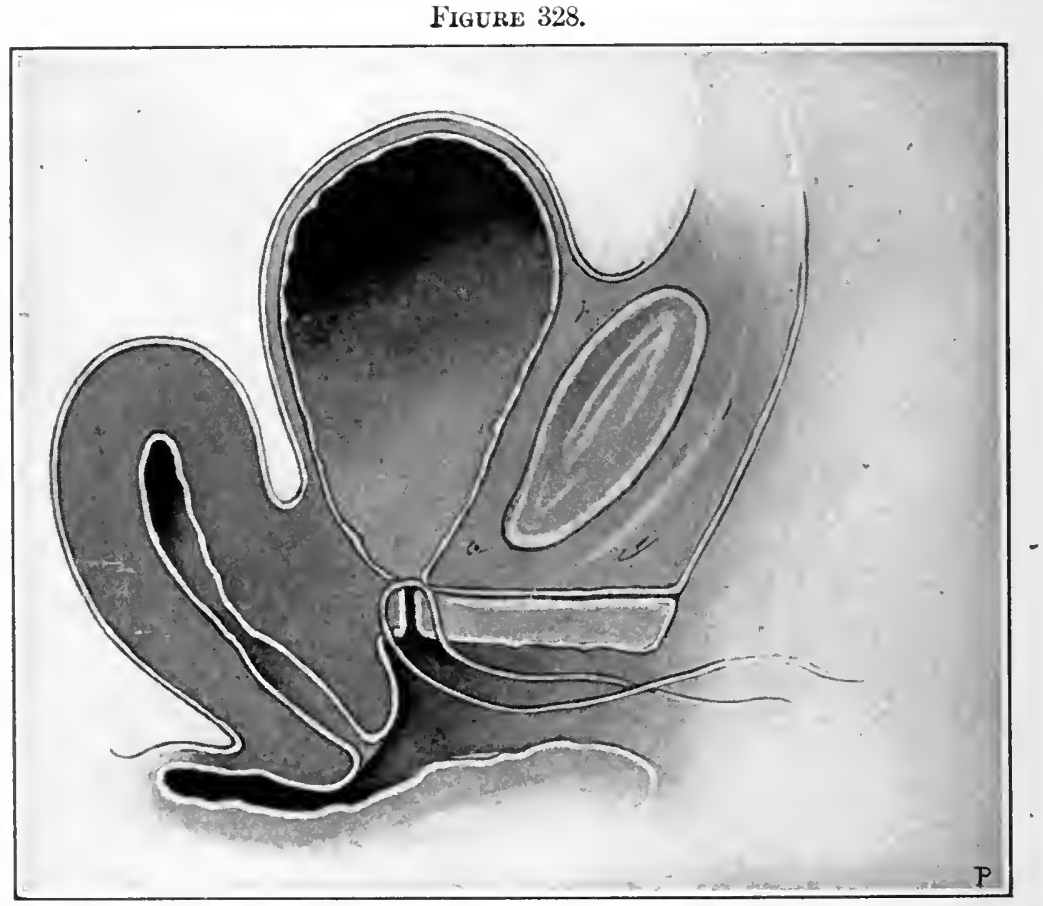

Anterior wall of cervix uteri united to the neck of the bladder. One suture in place, but not tied.

So much of the anterior lip may have sloughed a way that it cannot be drawn down to the neck of the bladder. In such a case some operators turn the cervix uteri into the bladder by union of the posterior lip of the cervix to the neek of the bladder. This would establish a communication between the interior of the iterus and the bladder. Figure 329. A great risk from this operation is in the possibility that infection may pass from the endometrium to all the urinary organs or from the bladier to the uterus, Fallopian tubes, and even to the peritoneum. The chief danger, however, is that the operation may form a pouch in which urine will stagnate, with resultant phosphatic deposit and incurable eystitis, only to be relieved by reopening the bladder and giving it drainage. See Artifieial Vesicovaginal Fistula 
for Cystitis. If the fistula be closed, so as to avoid the formation of such a ponch, cystotomy may be ummecessary; it is, unfortunately, too often impossible to avoid.

Kolpokleisis, or closure of the vagina, is an operation designed to secure retention of urine in cases of otherwise inoperable vesicovaginal fistula. It is performed by denuding a wide strip all around the vaginal ontlet just within the vulva and uniting the denuded surfaces upon themselves by means of interrupted sutures. The effect is to make one cavity of the bladder and ragina. This cavity receives the urine, menstrual blood, and uterine secretions. The operation always leads to inflammation more distressing than the condition for the relief of which it has been invoked. Emmet, in the strongest

Ftgure 329 .

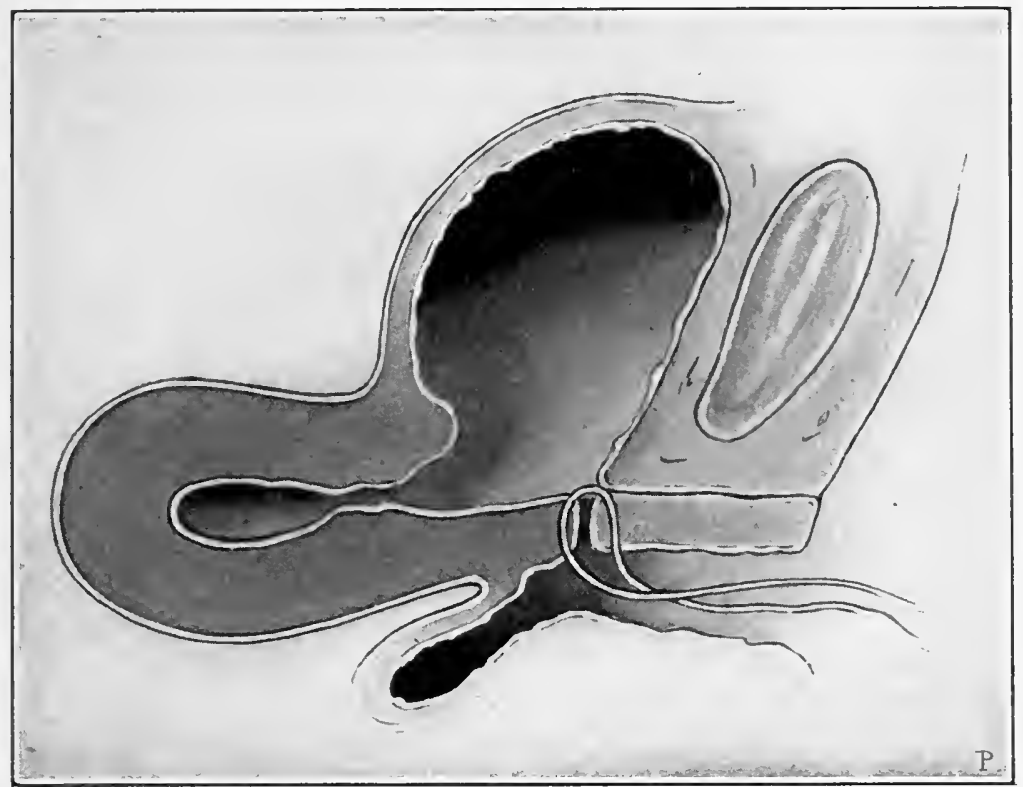

Uterus turned into bladder to secure retention of urine. Posterior lip of cervix united to neck of bladder. Anterior lip of cervix sloughed away. Lterus retroverted.

terms, condemns the operation and urges that it never be done in any case. He advises that the parts be made to heal with the opening unclosed, and that the patient be kept as comfortable without an operation as cleanliness and care can make her. 'The stagnant urine constantly present in the vaginal ponch formed by the operation always produces distressing - not to say dangerous-disease of the urinary organs. In this connection the writer offers from his practice two instructive cases: ${ }^{1}$

Case I.-The injury in this ease, Figure 330, was more extensive than would ordinarily be repaired by plastic surgery. The cervix ateri to the level of the internal os, the vesicovaginal and urethro-

IOperations in the case of Mrs. G. A. M., at St. Luke's Hospital, Chicago. 
vaginal septum, and the rectovaginal septum had sloughed away entirely; the perineum was completely lacerated through the sphineter ani muscle. The fundus of the inverted, ulcerated, and semi-strangulated bladder protruded through the pelvic outlet; this outlet was bounded by the sides of the vulva, by the posterior and lateral margins of the anus, and by the pubes. Thus all control of both urethra and anus was lost. The nterus was occluded by contracted cicatricial tissue and was full of retained menstrual fluid.

Clearly the conditions would discourage any effort to repair by ordinary methods. The problem was fourfold, and as follows:

To reopen the closed uterine canal and release the imprisoned menstrual fluid.

FIGURE 330.

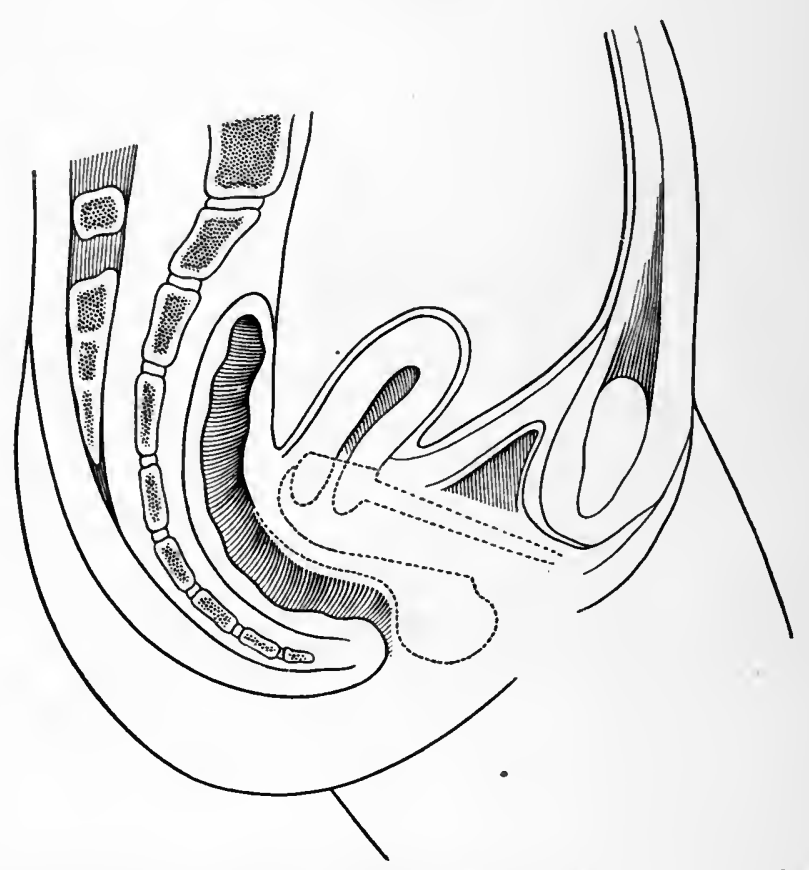

The dotted lines indicate the parts destroyed by slough. The perineum was not destroyed, but was completely torn apart.

To replace the lost vesicovaginal septum.

To replace the lost urethrovaginal septum.

To replace the lost rectovaginal septum.

To reunite the sphincter ani muscle.

A fiee incision with sharp-pointed scissors into the uterus reopened the uterine canal and re-established normal menstruation.

The labia minora were much hypertrophied, and were therefore capable of supplying abundant material for the replacement of the lost vesicovaginal wall; to this end, they, together with the adjacent tissue around and below them, were dissected off from above downward, but not detached at their lower ends. An area on each side 
just within the vulva, close to the margin of the bladder mucons membrane, was freshened by denudation and splitting, and the edge of each corresponding labium was turned in and stitched to this area with silkworm gut sutures. The flap thus formed on the right side united perfectly in its transplanted position; the flap on the left side partly sloughed away. The right transplanted labium now took its nutrition through the lower uncut end and the new tissues to which it was united. It was not possible, however, at the first transplantation to carry the labium sufficiently high to unite it with the upper margin of the fistula because it would not reach far enough to fill out the space left by the sloughed-out vesicovaginal septum; in order to make it reach, the transplanting operation had to be done three times-that is, the labium was turned end for end upon itself three times, and finally planted in place of the lost vesicovaginal wall. One face of this labium was now the bladder side, and the other was the vaginal side of the restored vesicovaginal wall. In order to maintain the nutrition of the flap during the period of its transplantation several months were allowed to intervene between the transplanting operations. Finally, after numerous attempts, in which sometimes a little was gained and sometimes nothing, the margins of the flap were united to the margins of the opening at every point and the integrity of the vesicovaginal septum was restored.

The urethra was repaired by denuding two parallel strips, threequarters of an inch apart, on either side of the urethral site, and uniting them one to the other by interrupted silkworm gut sutures. This formed a new urethrovaginal wall. The remnant of the left labium minus was utilized in this part of the work. The urethra thus formed immediately gave a measurable degree of retentive power when the woman was lying down. The blaclder, however, was much contracted from cystitis, and, having but small capacity, was at first of necessity often evacuated.

The rectovaginal septum was replaced by drawing down the loose rectal wall from above into the gap, and after denudation uniting it to the lateral walls of the vagina with fine buried eatgut sutures. At the same time the completely ruptured perineum, including the sphincter ani muscle, was remited. The bowel and sphincter nusscle at once resumed their normal functions. Nineteen operations in all were performed before this result was reached.

The patient, two years after her discharge from the hospital, reported perfect control of the bowel and practically perfect control of the urethra. In a letter written at that time she said: "I have almost perfect control of the urine at all times; I say almost because of there being a slight weakness at times; but it is not often, and even then the amount of leakage is not great. I have taken up the study of shorthand, typewriting, and telegraphy, and if I make a success of it shall feel that my life has not been a failure."

This case, a curiosity in surgery, illustrates what may sometimes be accomplished by sustained effort ; little by little, line by line, in the face of one discouragement after another, the work was done. 
The treatment continued over a period of more than two years, with an intermittent period of three years, when nothing was done. Most of the time it seemed like following the forlorn hope; now total failure, now a little success, until, finally, nineteen operations under anesthesia had been clone. Words fail to describe the bravery and patience displayed by this woman, or the diffieulties and discouragements which the surgeon must meet in such a ease.

Case $I I^{1}$ - The entire vesicovaginal septum, the vaginal portion of the cervix, and the anterior wall of the cervix to the internal os had sloughed away, leaving no bladder tissue between the inner extremity of the urethra and a point corresponding to the plane of the internal os uteri. See Figure 331. The upper and lower fragments

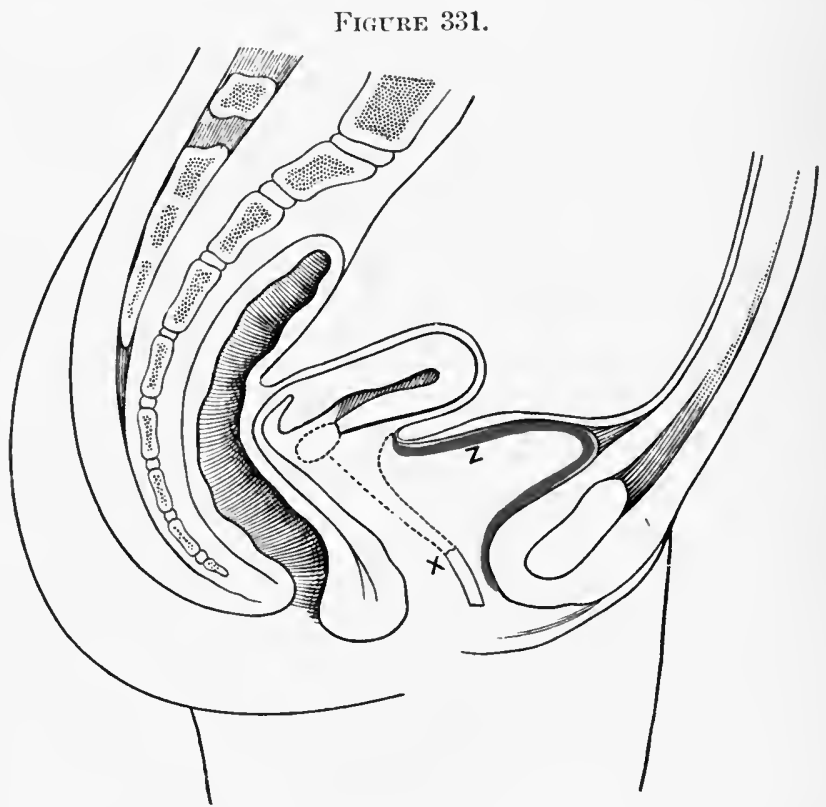

The dotted lines show the parts which had sloughed out. Red line shows remaining portion of bladder-wall.

of the opening could not be approximated-that is, the anterior wall of the uterus could not be approximated to the neek of the bladder after the method shown in Figure 329. The only operation which at first seemed possible was to unite the posterior wall of the cervix uteri to the neck of the bladder, as shown in Figure 329. This would have turned the cervix uteri into the bladder, and menstruation would have taken place through the urethra. But while this was under consideration it was found, on further examination, that the mucous membrane of the bladder, if caught with the tenaculum about an inch in front of the uterus, could be drawn to the neck of the bladder

1 E. C. Dudley. Journal American Medical Association, March 27, 1886. 
- that is, to the lower margin of the fistula-and held there without undue traction. A strip of mucous membrane across the bladder was therefore denuded from side to side an inch in front of the uterus. This denudation was continued around the lateral and lower margins of the fistula. The strip of denuded surface across the bladder was then drawn down and stitehed to the lower margin of the fistula $X Z$, Figure 332. Thus the bladker was divided into two parts, the upper elosed part communicating with the urethra and receiving the urine from the ureters; the lower open part replacing the lost anterior vaginal wall. In other words, the part of the bladder-wall, situated between the line of denudation across the fundus of the bladder and the uterus, was ntilized as a substitute for the lost vesicovaginal septum and anterior wall of the cervix. Twenty-two sutures were used. Not-

Figure 332.

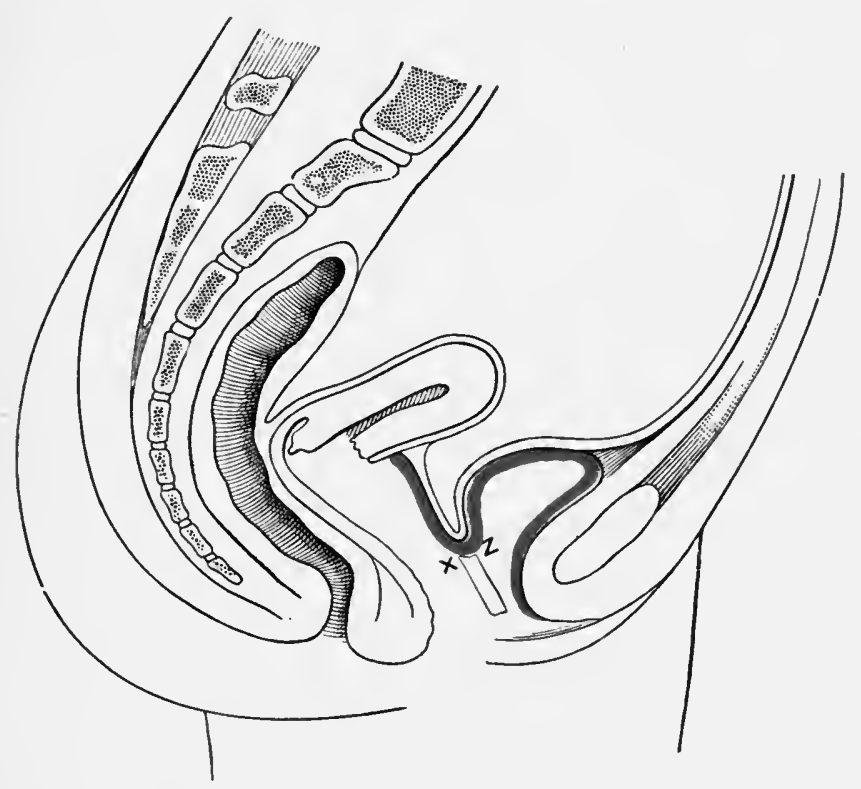

Section at $x z$ shows the fundus of the bladder stitehed to the neck of the bladder.

withstanding the failure of the nurse on the third day to keep the catheter in place, and the consequent accumulation of several ounces of urine in the bladder, union by first intention was complete. The bladder, although reduced in size by the operation, has normally performed its functions ever since. It is large enough to enable the woman to retain her urine all night. The writer is not aware that another similar operation has been reeorded.

Howard Kelly suggests a plan which might be adopted to advantage in place of the one just described. It is to dissect the bladder entirely free from the uterus, so as to make a wide opening between the vagina and the peritoneum-that is, to make an anterior vaginal section into the peritoneal eavity. The bladder-wall, anterior to the 
uterus, thus freed from its uterine attachments, may then be drawn down so as to elose the fistula by a transverse line of sutures. Sinee the bladder-wall is covered with peritonem, and since peritoneal surfaces are very prone to unite readily, such an operation would be very apt to succeed. After closure of the fistula the wound anterior to the uterus should be closed.

Figure 333.

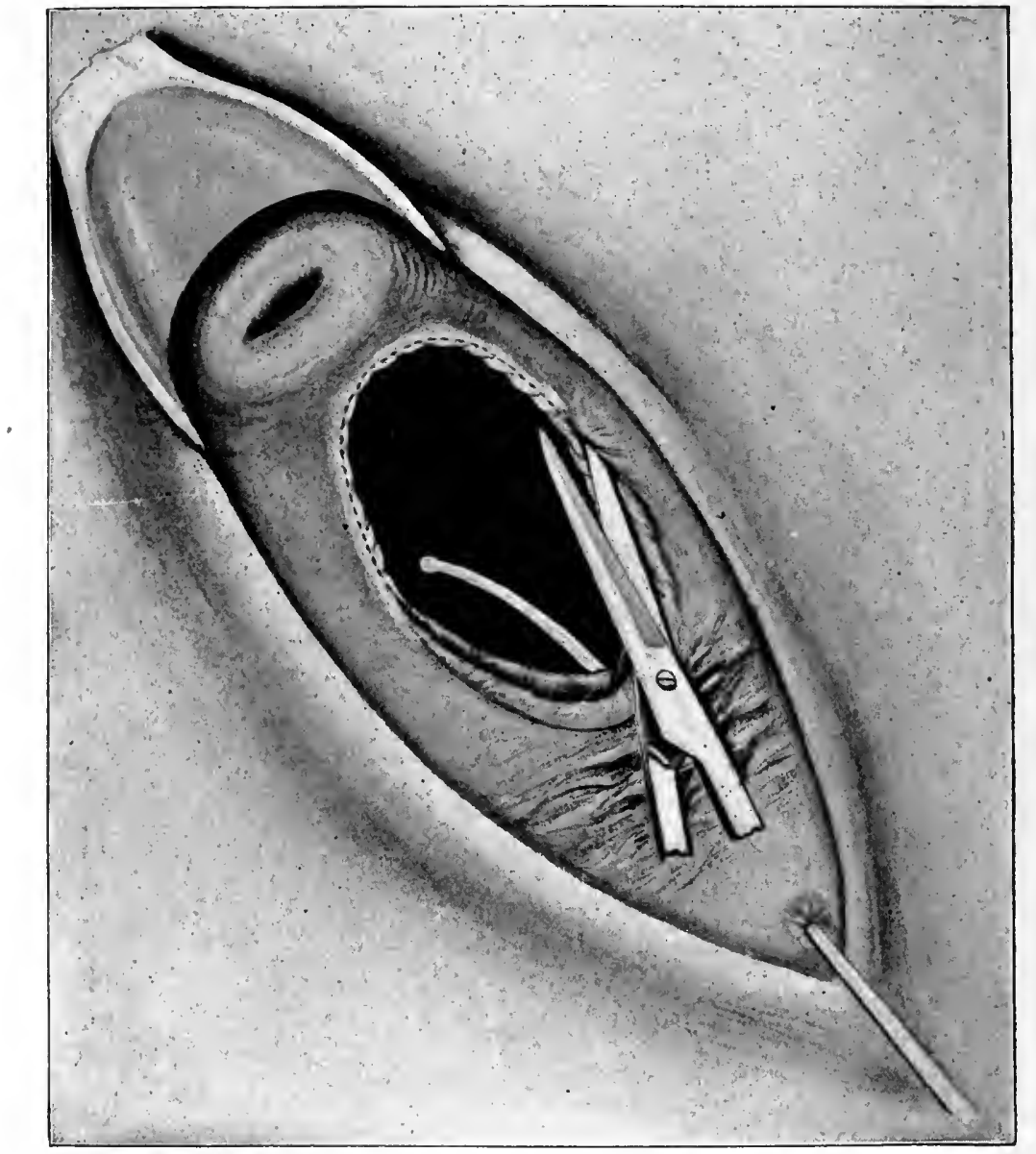

Shows act of splitting margins of fistula preparatory to approximating the fragments of the vesical plate of the vesicovaginal wall. Lateroprone position and Sims' speculum.

Another possible method, snggested by Mackinrodt, ${ }^{1}$ for such eases is to detach the vesical from the raginal plate of the vesicovaginal wall and to elose the fistula by suturing together the vesical plate independently, leaving the raginal plate open to heal by granulation. Figures 333 and 334 . 


\section{VESICO-UTERINE FISTULA.}

This form of fistula has been mentioned in the chapter on Laceration of the Cervix; it is the result of anterior laceration of the cervix extending into the bladder. Usually the effort of nature to repair produces union in the lower part of the laceration so as, to

Figure 334.

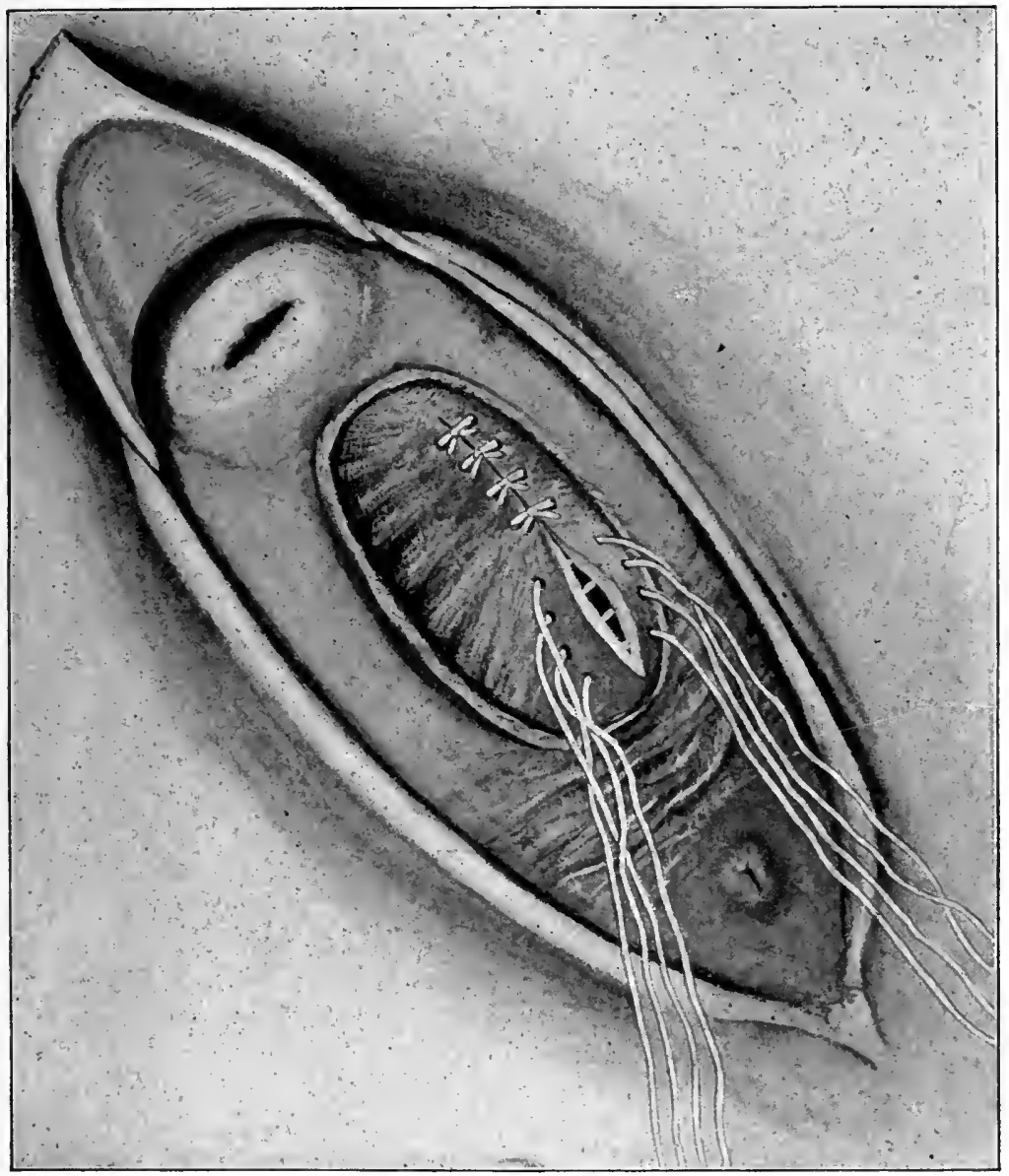

Fistula being closed by union of vesical plate of the vesicovagiual wall; this leaves the vaginai plate still open. Latero.

repair the whole vaginal part of the injury and to leave the uterine part open. Figure 292 shows the sinus extending from the bladder to the interior of the uterus.

The Diagnosis and Treatment of Vesico-uterine Fistula.

The diagnosis is based upon the history of the case and the passage of urine through the os externum. The treatment is to reproduce the 
original tear by an incision through the anterior lip of the eervix directly into the sinus. The fistula thus exposed at the angle of the incision is denuded, and the whole wound, including the fistula and the cervical laceration, is closed with silkworm gut sutures. Except that the sutures, in addition to closing the cervix, are made also to close the opening into the bladder, the operation does not differ from the ordinary operation for closure of an anterior laceration of the cervix uteri. ${ }^{1}$

\section{URETHROVAGINAL FISTULA.}

This form of fistula may be made intentionally by a surgical operation in the treatment of urethritis and other diseases of the urethra; it is occasionally the result of ulcerative processes. If the neck of the bladder is not involved, the functional power of the urethra to retain urine may be unimpaired. The operation for closure is the same as that described for vesicovaginal fistula. The after-treatment consists of the hot-water douche twice daily. The self-retaining catheter is not required. Ordinary catheterization is permissible, but if the woman can pass urine without help, it is not required.

\section{URETEROVAGINAL FISTULA.}

Ureterovaginal fistula may be :

I. Congenital ; one or both ureters may open into the vagina. II. Acquired.

\section{Causes of Ureterovaginal Fistula.}

The causes of aquired ureterovaginal fistula are :

1. Sloughing, due to pressure-necrosis during labor.

2. Necrotic processes, due to malignant or specific disease.

3. Traumatisms, usually surgical.

\section{Diagnosis of Ureterovaginal Fistula.}

The diagnosis is made by passing a ureteral catheter into the ureter at the point whence the urine escapes. In the congenital form there is no communication between the ureter and the bladder. The acquired form nay or may not be associated with a vesicovaginal fistula. If so associated, the ureteral opening is usually in the margin of the vesical opening. This combination is called uretero-vesicovaginal fistula. If there is no vesicovaginal fistula, the urine from one kidney only will escape through the vagina; that from the other will pass naturally through the urethra. If the defect is bilateral, both ureters will open into the vagina.

\section{Treatment of Ureterovaginal Fistula.}

The treatment of a uretero-vesicovaginal fistula is as follows : First, split the ureterovesical wall for a little distance back from the

เ Emmet, Principles and Practice of Gynecology, second edition. p. 635. 
margin of the vesical opening. This makes a new and larger opening for the ureter into the bladder remote from and out of the way of the vesicovaginal fistula. The latter may then be closed in the usual manner.

If the ureterovaginal fistula is not associated with a vesical opening, it should be converted into a uretero-vesicovaginal fistula by an incision at the ureterovaginal outlet directly through into the bladder. The operation then is continued as described above for a uretero-vesicovaginal fistula.

Dudley Clamp Operation.-I present herewith the report of a unique case in which stricture of the ureter was a possible result of laceration of the cervix uteri and ureterovaginal fistula a result of trachelorrhaphy.

In this case a left ureterovaginal fistula occurred three days after introducing some rather deep sutures for the control of secondary hemorrhage following trachelorrhaphy. About one-half of the urine escaped from the left ureter into the vagina; the remainder passed naturally through the urethra. The ureteral bongie passed through a Nitze cystoscope demonstrated a tight stricture-possibly obliteration of the ureter near the opening of the fistula and between this opening and the ureteral orifice in the bladder.

About four weeks after the accident, with the purpose of performing some operation to re-establish a free communication between the injured ureter and the bladder, I etherized the patient, and for more than three-quarters of an hour with uterine tenacula and a fine probe songht in vain for the point where the ureter opened into the vagina. No urine came through to mark this point, and even after some rather extensive dissection with the scissors I was unable to locate the fistula, nor was I able to make out the ureter by palpation. Finally, however, a little spurt of urine appeared just to the left of the cervix uteri, but I was unable at this point to pass even a very fine probe. Each attempt only resulted in the making of a false passagc-a thing difficult to avoid under such conditions. I then made a colpocystotomy, cutting with the scissors through the vesicovaginal wall in the median line and in the long axis of the ragina just in front of the cervix. The vesicovaginal fistula thus made was an inch long. The upper extremity of it terminated close to the anterior wall of the cervix uteri. With a pair of straight scissors I then extended the incision upward, and to the left as nearly as could be estinated, to the point whence the urine had escaped. The object was if possible to convert the ureterovaginal fistula into a uretero-vesicovaginal fistula; so that the ureter should open, not into the vagina, but into the margin of a vesicovaginal fistula. After another long search I again failed to find the fistulous opening into the ureter, until it was located by another spurt of urine, but the opening again was too small to admit a fine probe, and therefore could not be entered. I then still further enlarged the vesicovaginal fistula in a direction to the left of the uterus, and by good fortune opened into a very much dilated ureter, from which immediately there gushed two or three ounces of pent-

1 Boston Medical and Surgical Journal, volume cxlii. No. 9. 
up urine. A ureteral bongie was now passed without obstruction to the kidney.

The situation now being much simplified, the following procedures were adopted: The bladder mucosa was stitched to the vaginal mucosa all around the artificial vesicovaginal fistula. In this way the exposed surfaces were covered and hemorrhage controlled. A hæmostatic forceps, with handles about four inches long and with slender jaws about an inch long, was passed through the vesicovaginal fistula. Figure 335. The forcep jaws were then passed, one into the ureter and the other into the bladder, so that the forceps when locked included in their bite, ureteral wall, bladder-wall, and the connective tissue between. In this way the lower extremity of the cut-off ureter

Figure 335.

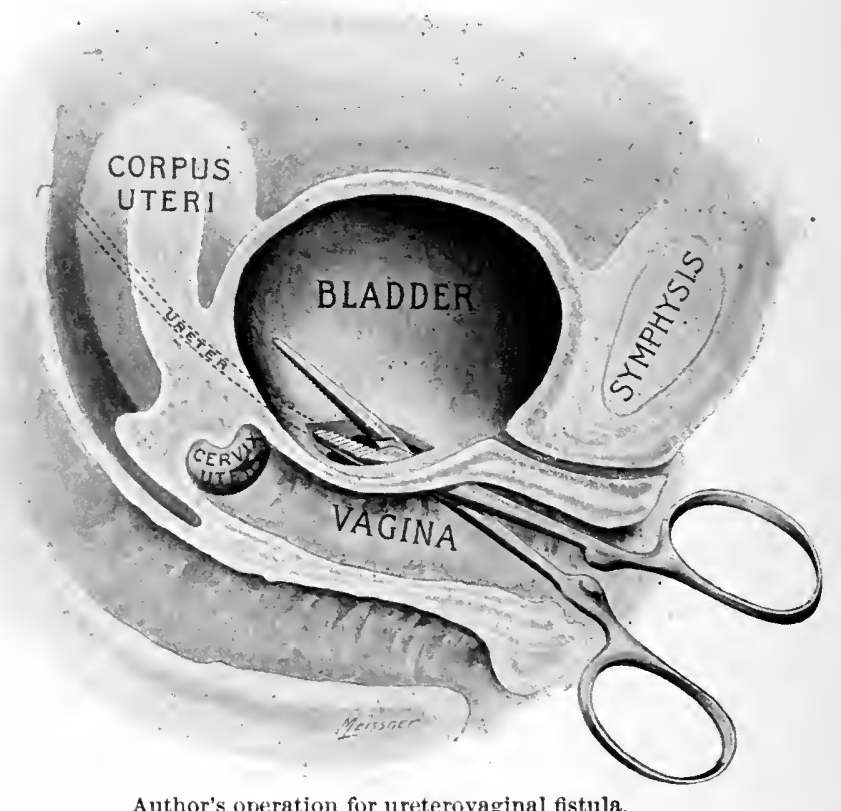

Author's operation for ureterovaginal fistula.

was clamped into close relations with the bladder. The expectation was that the structures within the bite of the forceps would be destroyed by pressure-necrosis, and that a wide, free, ureterovesical opening would be established at a point somewhat distant from the artificial opening into the bladder, and that in this way the case would become one of uncomplicated resicovaginal fistula. The forceps came off in about three days, and twelve days later the vesicovaginal fistula was closed by suture in the ordinary way. At the time of this operation the new ureteral orifice was found to be perfectly open and very patulous. The subsequent history was uncomplicated, union was complete, and in a short time the patient was discharged cured. In a letter written about six months after the final operation 
the patient reported entire freedom from pain in the left inguinal region from which she had suffered, and which had made her a semi-invalid for twenty years. I regret that the ureter was not explored before the operation on the cervix uteri, and that it has not been practicable to obtain measurements of it since.

My experience in the surgical treatment of ureterovaginal fistula is limited to two other eases, one traumatic and one congenital. In these two cases I operated at St. Luke's Hospital, Chicago, seven or eight years ago. The operative treatnent in each was like that just described, except the ureteral and vesical walls were divided by scissors instead of being clamped by pressure-forceps. In these cases, however, the ureteral openings were much nearer to the trigone, and the lower extremity of the injured ureter, therefore, was quite close to the bladder mucosa.

In the case just reported the distance and amount of tissue between the bladder and ureter was so great that it could hardly have been divided with the scissors withont danger of uncontrollable hemorrlage or of the exposure of broad surfaces to reunite, or to cicatrize and contract. These difficulties were obviated by clamping the ureter into elose contact with the bladder, so that when the forceps came off, the exposed surfaces left by the necrosis would, owing to the compression, be of small extent. The compression-forceps used in this way, therefore, nay make the operation practicable in those regions where the tissue between the ureter and the bladder is too abundant to be divided safely by seissors.

It is hoped that this operation will give great security against subsequent stricture at the new ureteral orifice-a result not obtainable by any of the usual procedures.

Traumatic ureterovaginal fistula as a result of trachelorrhaphy is rare, but as a result of vaginal hysterectomy and other vaginal sections is not of infrequent occurrence. The operation above described is applicable to the condition, whatever the cause, whether traumatic or congenital.

The alternatives to the operation are well known, and need not be describerl. To open the abdomen, sever the ureter and insert it into the bladder-wall, is an operation of great difficulty and clanger, and sometimes is of only transient value. The same may be said of dissecting or stripping the bladder from the pelvic wall, finding the ureter and inserting it into the bladder, withont invading the peritoneal cavity. The ntilization of the vaginal mucosa in a plastic operation for the purpose of diverting the urine from the vagina to the bladder usually results in failure of mnion, or, later, in cicatricial contraction and consequent stricture of the ureteral orifice. The operation of switching the ureter into the intestine or into the opposite ureter is of very questionable propricty.

As a corollary to the case just deseribed, the following observation, if well founded, nay prove to have practical significance. It is probable in this case that the laceration having extended into the parametria had torn the structures around the ureter. There may also have been injurious pressure by the presenting part of the child 
against the ureter. Such lacerated tissues would necessarily heal by cicatrization and contraction, and the eicatrix thus formed would draw the bruised ureter toward the uterus, compress it, and so give rise to obstruction both from stricture and from kinking. Contracting cicatricial tissue extending from the cervix around the ureter would necessarily draw the ureter into closer proximity to the uterus, where a deep suture applied for closure of the cervix or to control blecding would be apt to wound it, or by compression cause a narrowing of the lumen of that part lying within its grasp. In the case described the stricture extended at least a half-inch on either side of the ureteral fistula. It was evidently this constricted portion of the ureter that was caught by the needle and cut off or penetrated by the suture.

It would be quite impossible, without further observation, to estimate the proportion of cases in which laceration of the cervix uteri causes stricture or kinking of the ureter. Every gynecologist may revert to a class of cases, not small, in which there is extensive laceration of the cervix uteri on one or both sides, and in which the localized pain dating from the puerperium is not readily accounted for by palpable lesions, and is not relieved in the slightest degree by the repair of the cervix. As I look back over a long experience I recall many such cases, and among them the one just reported.

But why, one may ask, if the ureter is often drawn by cicatricial contraction close to the uterus, is it not more frequently injured by operations on the cervix? The answer is that if the sutures of trachelorrhaphy were not usually introduced close to the uterus or very superficially in the vaginal wall, more cases of ureterovaginal fistula probably would be reported. This case.was very hemorrhagic, and therefore required exceptionally deep sutures to control the bleeding. In view of the facts already set forth, I desire to submit two questions, as follows :

Question 1. In all cases of extensive laceration of the cervix uteri, in which the localized pain is not accounted for by palpable lesions, should we not pass a series of graduated ureteral bougies on the side corresponding to the laceration? This would be for the purpose of measuring the calibre of the ureter and of locating a possible stricture. The principles of examination would be similar to those of measuring the calibre of the male uretlura in the diagnosis of stricture.

Question 2. In a case of ureteral stricture due to laceration of the cervix uteri, or to any other cause, and situated within the range of a vaginal operation, would not the surgeon be warranted in opening the bladder and then proceeding, as in the case reported, to establish a new ureteral orifice? In other words, should not that condition which in this case was the result of an accident, be. reproduced deliberately in similar cases?

My answer to these questions would be in the affirmative.

Dr. Edward Reynolds ${ }^{\prime}$ has reported a successful case of a clamp

1 Boston Medical and Surgical Journal, January 24, 1901. 
operation by the method above described. Cystoscopic examination after the recovery of the patient showed a ureteral orifice which, except the high location, presented all the appearance of the natural orifice.

Accidental Wounding of the Ureter in Vaginal Hysterectomy.-On April 22, 1903, I performed vaginal hysterectomy for carcinoma of the corpus uteri upon a woman seventy years of age, a patient of Dr. Lord, of Plano, Illinois, whose pelvic organs long since liad passed into extreme senile atrophy. In making the opening into the peritoneal cavity between the bladder and the uterus the carcinomatous disease had extended so far anteriorly that the bladder was opened immediately in front of the cervix niteri. An opening into the peritoneum posterior to the uterus was made without accident, the broad ligaments then were isolated and cut close to the uterus and the uterus removed. After cutting through the right broad ligament a spurt of fluid was observed, which upon examination proved to have come from the right ureter, showing that the ureter had been divided. This accident to the ureter was consequent upon the fact that the cicatricial tissue of an old cervical laceration had caught it and drawn it into close relation with the uterus into the line of incision. Figure 336.

After a hasty consultation with Dr. Kolischer, who chanced to be present, it was decided, if possible, to establish a direct communication between the upper cut end of the ureter and the interior of the bladder. The usual method of performing this operation is to make an opening into the bladder, push the end of the ureter through, and fasten it there by means of sutures. Appreciating the wellknown tendency of the cut end of the ureter to contract when introduced into the bladder in this way, and having at hand a resicovaginal fistula which rendered the interior of the bladder quite accessible, I made use of a method which, so far as I know, had not been described. With a long slender forceps I punctured the bladder wall from within outward at the point nearest to the cut end of the ureter. Then after splitting the cut end of the ureter and denuding the bladder mucosa on either side of the punctured opening, I drew the ureter into the bladder, Figures 337 and 338, and stitched it there by means of fine chromic catgut sutures. Figure 339 . By this means the split end of the ureter was held widely apart by means of sutures, so that it could not easily contract and form a stricture. The tightly fitting ureter made the punctured bladder wound water tight.

The vesicovaginal fistula was closed immediately by drawing the anterior margin of the peritoncum down to the lower margin of the vaginal wound and fastening it there with a continuous chromic eatgut suture. In like manner the posterior margin of the peritoneum was brought into contact with the vaginal margin of the wound, after which the wound from the peritoneal cavity into the vagina was closed in the usual way, the stumps of the broad ligaments being drawn down into the vagina and fastened there by means of sutures, one at each end of the vaginal wound. During the two weeks fol- 
lowing the operation the bladder was kept empty by the continuous use of a self-retaining catheter.

Cystoscopy by Dr. Kolischer and myself four weeks later showed a perfectly patulons opening of the ureter, the divided flaps of which were united firmly to the bladder mueosa.

The special adrantages of the method, as already pointed out, are twofold: 1. A water-tight wound around the ureter where it enters

FigURe 336.

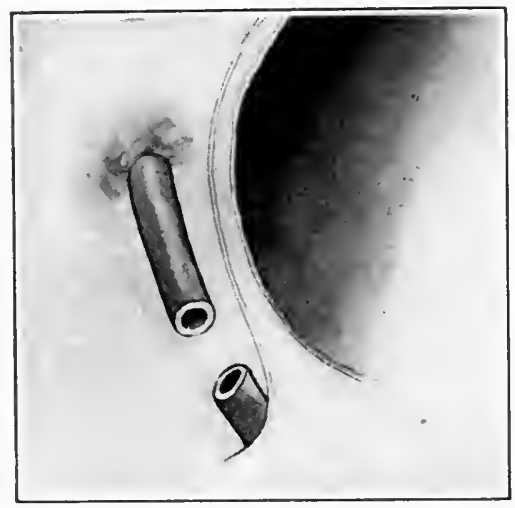

Figive 338.

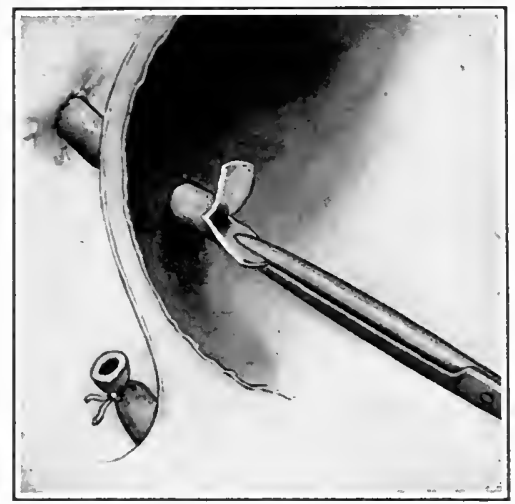

Figure 337.

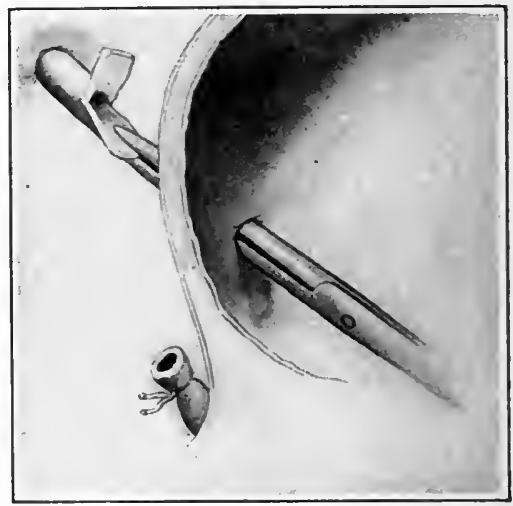

Figure 339.

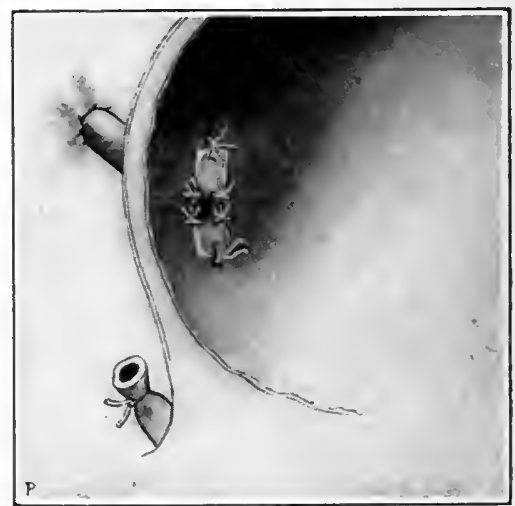

FIGURE 336.- Ureter aceidentally ent in vaginal bysterectomy. The upper cut end leads from the kidney, the lower end to the bladder. Semi-diagrammatie.

FIGTRE 337.-The upper cut end of the ureter split and in the grasp of a foreeps whieh has previously made an opening from the interior to the exterior of the bladder by puncture. Semi-diagrammatie.

FIGURE 338 . - The split end of the ureter has been drawn into the bladder by means of the foreeps. Semi-diagrammatie.

FIGURE 339.-The split end of the ureter having been drawn into the bladder and the bladder mueosa having been denuded on either side of the opening, the two flaps are fastened to the denuded mucosa by means of sutures, three on each side.

the bladder. 2. Security against contraction of the end of the ureter where it enters the bladder. These advantages in a similar case would lead me to repeat the operation if the bladder happened to be opened, and I would be inclined to make an artificial vesicovaginal fistula for this purpose if the bladder was not open. 


\section{RECTOVAGINAL FISTULA.}

\section{Causes of Rectovaginal Fistula.}

Parturition, although a frequent cause, is relatively at least a less frequent cause of rectovaginal than of vesicovaginal fistula; the lesion is observed more commonly as the result of syphilis or cancer. Occasionally a peri-anal abscess is situated in the perinenm, and in the acute stage breaks into both the vagina and the lower bowel; or, later, the perineum may be perforated from the anus to the vagina by the burrowing of pus. Such cases are apt to be syphilitic or tuberculous.

\section{Diagnosis of Rectovaginal Fistula.}

The diagnosis is made by digital or speculum examination by the probe, or by injecting milk into the rectum and observing the point at which it appears in the vagina.

\section{Prognosis of Rectovaginal Fistula.}

The lesion, when due to cancer, is incurable; when the cause is syphilis, the operation for closure, unless preceded by adequate specific treatment, usually fails. A sinus of tubercular or other inflammatory origin should be successfully closed by suture; but the prognosis is much improved by such preliminary treatment as will improve the general nutrition. In fistula due to pressure-necrosis the operation of elosure by suture, although beset by more unfavorable conditions than in urinary fistulæ, usually succeeds.

\section{Operation for Rectovaginal Fistula.}

The prineiples are the same as for urinary fistulæ. The preparation is the same as for closure of the completely lacerated perineumthat is, free eatharsis during several days before the operation, and the use of such food and intestinal antiseptics as will reduce to the minimum the amount of gas and other contents of the bowel; much depends upon making the bowel as nearly as possible aseptic.

The operation often fails from the pressure of gas and other rectal contents against the newly united womd; hence in order to give, during the healing process, a free ontlet for the rectum, the sphineter ani muscle should be stretched.

The denudation and passage of sutures should be on the raginal side of the rectoraginal wall, and should extend to but not into the rectal mucosa. The olject is to make the operation, so far as possible, in the more favorable soil of the vagina. In order to insure thorongh denudation of the whole sinus clear to the margins of intestinal mucosa, the index-finger of the left hand in the bowel is made to roll the rectal margin toward the vaginal opening, and thereby render it accessible for denudation by means of properly curved scissors. In a very small fistula the sinus may be inaccessible for 
denudation until it has been made so by free ineision on the vaginal side. Such incision should not extend into the rectum. As in urinary fistulæ, broad surfaces for union should be denuded on the vaginal wall. The method of suture is the same as for vesicovaginal fistula.

\section{ANOVAGINAL FISTULA.}

The causes, diagnosis, and prognosis are much the same as given above for reetovaginal fistula. The sinus runs through the perineum, and may therefore be inacessible for denudation, in which case it should be laid open by an ineision on the vaginal side of the perineum, but not neeessarily through the whole splincter ani musele. Most operators, however, divide the entire perineum between the sinus and the cutaneous side of the perineum. The remaining steps of the operation then are to denude freely and deeply the now exposed walls of the sinus, and then to close the wound as in the operation for complete laceration of the perineum. The advantage of complete division lies in the immobilization of the sphincter ani muscle, for unless severed, this muscle may, by continuous relaxation and contraction, imperil union. Entire division of the muscle or division of all but a few fibres of it is preferable to divulsion. The after-treatment of the operation for elosure of the fistula is the same as for complete perineorrhaphy. 


\section{PART V.}

\section{DISPLACEMENTS OF THE UTERUS AND O'THER PELVIC ORGANS.}

\section{CHAPTER XLIV.}

\section{DISPLACEMENTS OF THE UTERUS.}

\section{General Considerations.}

The title of this ehapter is not to be taken in a restricted sense, for the uterus is anatomically so connected with adjacent organs that the displacements of it eannot be considered intelligently nor presented satisfactorily without at the same time ineidentally taking into aceount the displacements-causative, resultant, and coneurrent-of the ovaries, Fallopian tubes, reetum, vagina, bladder, and perineum.

The following pathological sequence will serve as an example. The vaginal outlet may be so injured in labor as to cause displacement of the perineum backward toward the coceyx, where it ean no longer serve as a bulwark against the downward force which is exerted in the expulsion of the contents of the bladder and rectum. The foree of straining at stool and of urination is now exerted against the less resisting bladder and rectal walls; they consequently pouch into the vaginal outlet. The downward displacements thereby produced are ealled eystocele and reetocele. The vaginal walls are attached to the uterus, and, being displaced downward, must, by traction, pull that organ to a lower level. The uterus in turn is connected with the bladder, reetum, Fallopian tubes, and ovaries, and in its own descent draws these organs out of place and disturbs their relation to one another. This shows how a uterine displacement may be both causative and resultant. Coneurrent displacement of the uterus and other pelvic organs may result, for example, from the downward pressure of a tumor or from inflammatory causes.

It is convenient, because conformable to nsage, to treat the subject of displacements of the pelvie organs under the heading Uterine Displacements. At the same time it must be held elearly in mind that a uterine deviation may not be the essential factor in the morbid sequence ; on the contrary, it may, as already stated, be only an incident. The subject, therefore, properly ineludes the displacements not merely of the uterus, but of all the pelvic organs. It further em- 
braces the relations which these displacements may bear to one another, and to such associated lesions as inflammation, tumors, traumatisms, and congenital defects.

The importance of a distinction between location and position will become apparent hereafter : by the former, is meant the situation of the organ regardless of its attitude; by the latter, is meant the attitude alone. To change an object from one place to another, is to change its location; to turn it over or bend it upon itself, is to change its Dosition.

\section{Normal Position of the Uterus.}

In many works on anatomy and gynecology the uterus is represented as having a straight or nearly straight canal-as lying about midway between the symphysis pubis and the hollow of the sacrum,

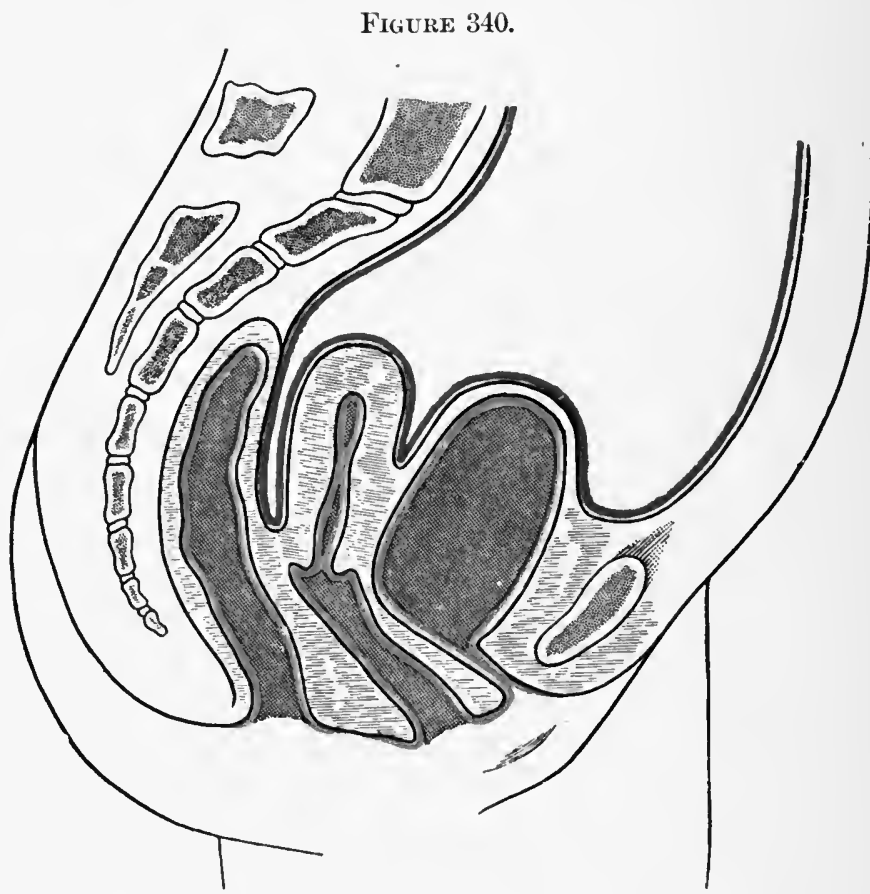

Classical representation of the pelvic organs.

its axis corresponding with that of the pelvic inlet. The position being one of slight, and only slight, anteversion and anteflexion, many authorities would pronounce the organ anteverted or anteflexed to a degree that would endanger health if by digital examination its anterior wall conld be felt through the anterior wall of the vagina. This classical idea of the normal position of the uterus wrongly presupposes a distended bladder and rectum oceupying the anterior and the posterior thirds of the pelvic cavity. Such an arrangement would 
leave for the uterus only the intermediate space, and would constitute a condition seldom or never realized in health.

Suppose a straight line coincident with the vesicovaginal wall, Figure 340 , to be continued through the cervix to the sacrum. This line represents approximately the anteroposterior diameter of the pelvis. The length of the vesicovaginal wall is two and a half inches, and, supposing the cervix to be just midway between the symphysis and the sacrum, the distanee from its posterior wall to the sacrum must also be two and a half inches. Add to the sum of these two parts of this anteroposterior diameter one inch for the diameter of the cervix, and the anteroposterior diameter of the pelvis becomes six inches, instead of the normal four and one-third, which proves that the cervix nust normally be much nearer to the hollow of the sacrum than to the symphysis. Since the length of the vesicovaginal wall plus the diameter of the cervix measures three and one-half inches,

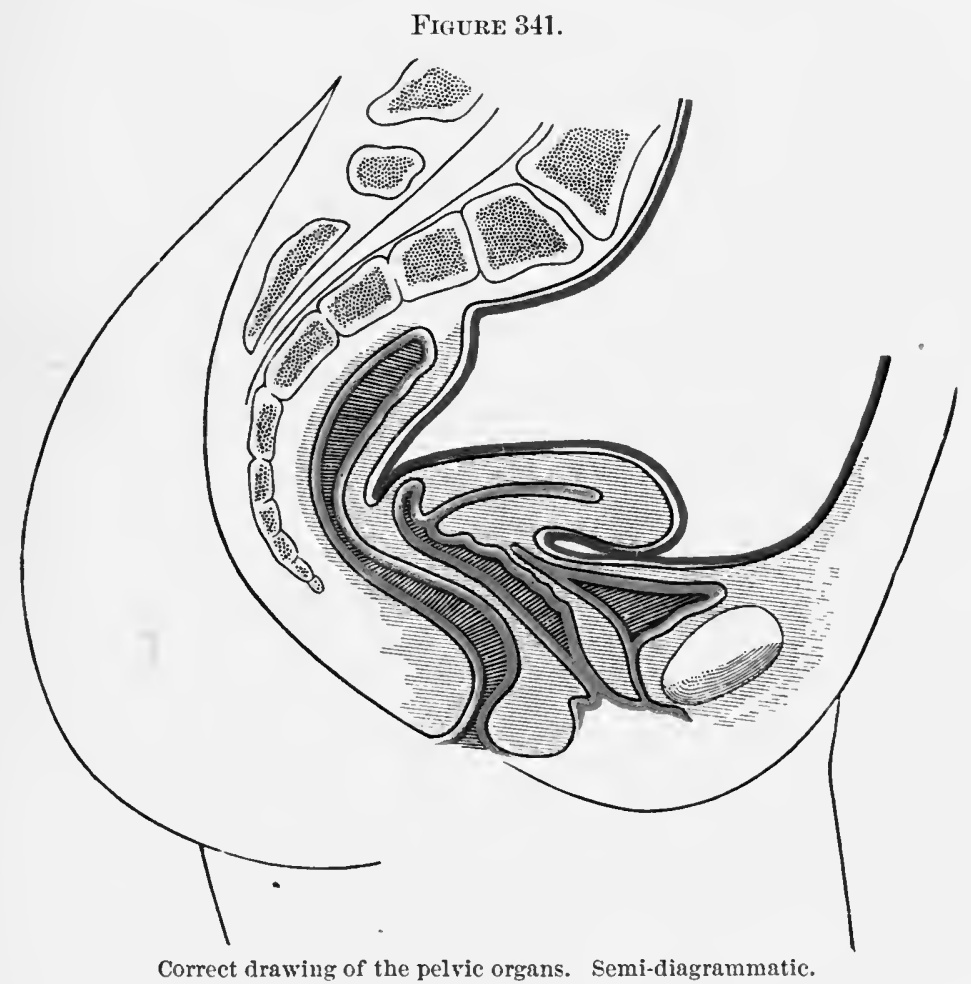

it follows that the distance from the posterior wall of the eervix to the hollow of the sacrum must be the difference between four and onethird and three and one-half inches, or five-sixths of an inch. These measurements are approximations.

Again, suppose the uterus, Figure 340 , to be earried bodily upward and backward, its axis remaining the same, until the cervix reaches its 
normal position near the hollow of the sacrum; then would the body of the uterus inpinge upon the bony sacrum. It is therefore clear that anteversion must be the normal position, because the uterus and sacrum would otherwise occupy the same space.

Figure 341 represents, according to Schultze, ${ }^{1}$ the location and position of the virgin uterus and its surroundings-the bladder, rectum, and vagina being empty and collapsed. The angle of about 90 degrees which the eervix forms with the vagina measures the forward inclination of the cervix, but is subject to variations in consequence of the physiological movements of the uterus. The body furthermore is bent forward upon the cervix, so that its anterior surface rests upon the empty bladder. The angle of normal anteflexion, according to careful measurements by Schultze, is about 48 degrees; Fritsch says that 90 degrees is the physiologieal limit. This question will be considered further under the subjeet of pathological anteflexions.

\section{Normal Movements of the Uterus.}

Strictly, the uterus can have no absolutely normal position or location, because it has a certain normal range of movements that depend to some extent upon respiration, intra-abdominal forces, and locomotion, but more especially upon the varying contents of the rectum and bladder. The normal position varies within the limits of the normal movements. If the body of the uterus rests upon the bladder, it must rise as the bladder becomes distended; and, conversely, if the urine be drawn through a catheter while the woman is lying on her back, the uterus, notwithstanding the opposing influence of its own weight, immediately follows the receding wall of the bladder and returns through an arc of 45 degrees, or possibly even 90 degrees, to its accustomed position.

The full rectum forees the uterus in the opposite direction, toward the symphysis, and thereby counteracts the influence of the full bladder. This anterior movement is, however, somewhat limited, and is confined to the cervical portion, except when the body has been forced back into close proximity with the rectum by the overdistended bladder.

\section{Normal Supports of the Uterus.}

The uterus is maintained in its normal position and location by the pelvic floor, of which the uterine ligaments are an essential part.

The uterine ligaments are physiologically in a state of relaxation; the state of tension wonld be pathological ; they do not fix the uterus; they tend only to limit its movements to their normal range. Backward displacement of the body is resisted by the round ligaments; backward displacement of the cervix, by the uterovesical ligaments and by the vesicovaginal wall. Forward and downward displacements are resisted by the uterosacral ligaments, and excessive lateral

1 Archlv für Gynäkologie, 1875, Band viii. p. 134, and Lageveränderungen der Gebärmutter, Berlin, 1881. Ely Van de Warker makes a full and critical study of the normal movements of the unimpregnated uterus, in the New York Iedical Journal, vol. xxi. p. 337; and of the normal position and movements of the unimpregnated uterus, in the American Journal of obstetrics, vol. xi. p. 314. His conclusions substantially agree with the later observations of Schultze. 
motion by the broad ligaments. This restraining power is doubtless greater in the uterosacral than in any of the other ligaments.

The Pelvic Floor, which is the chief support of the uterus, is divided into two segments, the pubic and sacral. The pubic segment includes bladder, urethra, anterior vaginal wall, and bladder peritoneum; it is attached in front to the symphysis pubis, and laterally to the anterior bony walls of the pelvis. The sacral segment includes rectum, perineum, posterior vaginal wall, and strong tendinous and muscular tissue ; it is attached to the coccyx, to the sacrum, and to the posterior wall of the bony pelvis.

Permeating the pelvie floor in all directions, entering into the composition of its single parts, binding them together, and sending its processes to the bony pelvis, is the pelvic connective tissue, upon the integrity of which largely depends the integrity of the pelvic floor as a uterine support. The idea that the uterus is supported by the vaginal walls, or by the perineum, or by the uterine ligaments is obsolete; they are important parts of the pelvic floor, and as such contribute support, but the pelvic floor as a whole supports the uterus. The various uterine supports are to a great extent the seat of motor influence. They consequently not only resist excessive movement, but also serve to return the organ from its physiological migrations.

\section{Definition and Nomenclature of Displacements of the Uterus.}

In the foregoing pages the normal location, position, movements, and supports of the uterus have been outlined. Those conditions are pathological which induce changes to positions or loeations beyond the defined limits, or which so fix the organ that its normal movements are prevented. The displacements are divided into mal-locations and malpositions.

The mal-locations, in which the uterus occupies a place outside its normal limits, are as follows:

Ascent.

Retrolocation.

Antelocation.

Lateral location.

\section{Descent.}

The malpositions are determined by excessive change in the inclination of the uterine axis. They are divided further into flexions, in which the organ is bent upon itself in an abnormal degree, manner, or direction; and versions, in which the axis of the unflexed uterus inclines in an abnormal degree or direction. The malpositions therefore are :
Retroversion.
Retroflexion.
Lateral version.
Lateral flexion.
Anteversion.
Anteflexion.

\section{Symptoms and Diagnosis of Displacements in General of the Uterus.}

Each variety of displacement may be indicated by its own group of symptoms and physical signs. These will be presented in the study of special displacements. To avoid repetition, those symptoms 
and signs which pertain to no special displacement, but which belong to all alike, will be mentioned at once. They may arise either from the displacement itself or from possible complications, of which the following are examples : metritis, ovaritis, salpingitis, atresia, stenosis, cystitis, vesical catarrh, reetitis, rectal catarrh, perimetritis, peritonitis, nterine catarrh, tumors, and cicatrices.

Uterine displacement may be a cause or an effect of associated complications ; or, together with them, it may be a concurrent result of some common cause; or it may have had primarily no pathological connection with them. The symptoms of displacement refer to the pelvic organs or to the nervous system.

A mong the symptons or associated lesions which refer to the pelvic organs are :

Difficult walking and standing.

Dysmenorrhœa.

Sterility.

Constipation.

Dysuria.

Tenesmus.

Pelvic pain.

Menorrhagia.

Frequent abortion.

Painful or difficult defecation. Polyuria.

Among the symptoms or associated lesions which refer to the nervous system are:

Neuralgia in various parts.

Hysteria.

Anæmia.

Motor disturbances.

Nervous dyspepsia.

Chlorosis.

\section{Spinal irritation.}

The final diagnosis must depend always upon direct examination of the uterus itself. The first division of the above group of symptoms is not likely to escape notice as indicative of displacement, but the nervons symptoms are disregarded constantly or treated without reference to their possible pelvic origin. The frequent dependence of these nervous phenomena upon displacement is proved by their persistence in many eases after ordinary treatment, by their prompt disappearance upon permanent replacement and retention of the uterus by mechanical means, and by their usually prompt recurrence upon removal of the support. The presence, therefore, of the seeond division of the group, or any part thereof, even though the first be absent, will justify at careful investigation into the state of the pelvic organs.

Examination that results only in giving the name to a special variety of displacement, and does not include the complicating lesions, would not furnish a sufficient guide to the therapeutic indications, and is therefore inadequate. Successful treatment, for instance, of an anteflexion dependent upon inflammation of the uterosacral ligaments must include removal of the inflammation.

An important prerequisite to examination is the absence of contents in the rectum and bladder. The full rectum distorts the vaginal walls, deprives the examiner of the space necessary for introduction of the speculum, and throws the uterus out of its accustomed position. Much more troublesome is the presence of even a small quantity of urine in the bladder, because it makes the abdominal 
muscles tense when the hand is placed over the lower portion of the abdomen for bimanual palpation, and makes it difficult to engage the uterus between the hand and the examining finger. The distended bladder, by pushing the uterus upward aud backward. makes bimanual palpation almost useless. It is not surprising that conflicting opinions are common, when one day the patient is examined with rectum and bladder full, another day with these organs empty; one day in the dorsal, another day in Sims' or the knee-breast position ; one day with the cylindrical or bivalve speculum, another day with Sins' or Simon's. The left-hand method of examination is incomparably superior to the right. The palmar surface of the left index-finger has a more acute and more easily educated tactile sense, and is directed more easily toward the left side of the pelvis, which is especially subject to disease. The stronger right hand should be free to palpate the surface of the abdomen in conjoined manipulation.

For digital examination the dorsal position is preferred : the patient should be drawn close to the edge of a bed, or preferably a table, the thighs being flexed, the feet about fifteen inches apart, and knees widely separated. The examiner should stand facing the patient or at her left side. The index-finger of the left hand, properly lubricated, then slowly advances over the perineum into the vacina, noting the condition of the perineum, the presence or absence of cicatrices, lacerations, tumors, or relaxation of the vagina or perineum, the capacity of the vagina, the condition, size, and direction of the cervix, its distance from the sacrum and vulva, its mobility or fixation. Now, the right hand is pressed well down behind the pubes, and the uterus is engaged between it and the examining finger. See Chapter III. In this way the examiner may determine quite accurately the position, location, and size of the entire organ; may detect the possible presence of complicating tumors, both inflammatory and non-inflammatory; may also note, if possible, the location and condition of the ovaries, which, especially in posterior displacements, are liable to be prolapsed and excessively sensitive, and to constitute, therefore, a most intractable complication. The index-finger sweeps around the cervix in search of tender places which may be the result of inflammation or the expression of some neurosis. Above all, the digital examination requires a light, gentle, delicate touch. The index-finger may now be removed and reintroduced into the rectum, the right hand still being behind the pubes, or the cervix may be grasped between the index-finger in the rectum and the thumb in the vagina, picked up, as it were, between the finger and the thumb thus placed, and with the aid of the right hand behind the pubes thoroughly palpated.

Adequate diagnosis of the position of the pelvic organs usually is made by tonch and conjoined palpation. It is seldom necessary or desirable to sound or probe the uterine cavity in order to learn the position of the uterus; indeed, accurate information in the majority of cases can be gained more readily and more safely by touch alone. A tumor or inflammatory mass in the pelvis may be confused with the uterus. In such a case the uterus may be located definitely- 
relative position determined-by the sound or probe. When the uterine canal thus is explored, the patient may be on her back, and the left index-finger in the vagina may be used as a guide. The exploration, however, is made most effectually and gently with Sins' speculum, the patient being in the left lateroprone position. In some cases the probe cannot be passed by any other method. The bivalve and cylindrical specula are unsatisfactory in explorations of the interior of the uterus. 


\section{CHA P'TER XLV.}

MAL-LOCATIONS OF THE UTERUS.

\section{Ascent. Retrolocation. Antelocation. Lateral Locations. Descent or Prolapse.}

\section{ASCENT OF THE UTERUS.}

Ascent of the uterus may result from traction above or pressure below. The organ may be drawn upward and backward by a contrac-

Frgure 342.

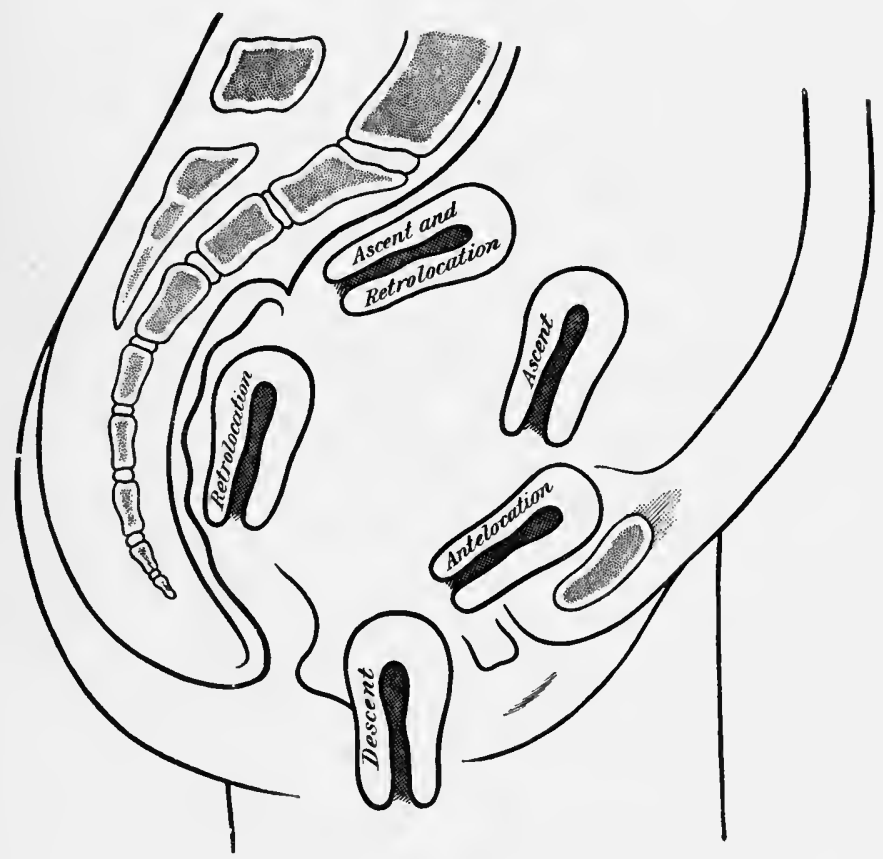

Schematic drawing of various mal-locations.

tion of the uterosacral ligaments, which results from inflammation, and which usually induces a troublesome form of anteflexion. The enlarged pregnant uterus sometimes becomes attached by adhesive inflammation to a portion of the peritoneum in one of the ligher zones of the pelvis or in the abdomen, and the organ may remain consequently fixed in its elevated position after involution. A 
tumor connected with the uterus or its appendages which has grown too large to be retained in the pelvis may, upon rising in to the abdomen, drag the uterus with it. Pressure below may come from excessive distention of the rectum or bladder, or from a large accumulation of menstrual fluid in the vagina, or from a tumor.originating in any portion of the pelvis below the level of the uterus.

\section{RETROLOCATION OF THE UTERUS.}

The uterus may be forced back into a post-normal location by the presence of a tumor in front or by the distended bladder, or it may be drawn back and fixed by peritoneal adhesions. Retrolocation is liable to induce vesical irritation by putting the vesicovaginal wall on the stretch, and thereby dragging on the neck of the bladder.

\section{ANTELOCATION OF THE UTERUS.}

The canses of this displacement are similar to those which produce retrolocation; they are: distention of the rectum, post-uterine hæmatocele, post-uterine tumors, contraction of the bladder, and peritoneal adhesions. Antelocation often causes vesical irritation, consequent upon the invasion by the uterus of space which belongs to the bladder.

\section{LATERAL LOCATION OF THE UTERUS.}

The entire uterus often is displaced to the right or to the left by a tumor or by an inflammatory mass. In either case the uterus is crowded to the opposite side of the pelvis. After resolution of an inflammatory mass the broad ligament and adjacent inflamed structures, shortened by cicatricial contraction, draw the uterus to the affected side and fix it there. Laceration of the cervix opens the way to infection, and therefore often is followed by inflammation in the parametrium on the corresponding side.

\section{Diagnosis, Symptoms, and Treatment of Ascent, Retrolocation, Antelocation, and Lateral Location of the Uterus.}

The diagnosis, symptoms, and treatment of the above mal-locations are wholly subordinate to the more significant lesions of which they are only the incidental results.

The Treatment for mal-locations due to inflammatory causes is the same as that for the inflammation. The indications for topical treatment and surgical measures, including operations on the uterus and its appendages and the removal of tumors, will vary with the causative lesions. In many eases mal-locations of the nterus give rise to no symptoms, and therefore require no treatment. Pessaries for all mal-locations except descent are useless, and may be harmful. 


\section{DESCENT OR PROLAPSE OF THE UTERUS.}

The nature of this displacement is indicated clearly by the name. It is convenient to distinguish three degrees of descent :

First Degree: The uterus is displaced downward until sufficient space has been gained between the cervix and the sacrum to permit the corpus to turn back into extreme retroversion.

Second Degree: The cervix descends to the vilva.

Third Degree : The uterus protrudes partially or wholly through the vulva. The third degree of descent sometimes is called procidentia.

\section{Etiology and Mechanism of Descent of the Uterus.}

Descent may be the result of any or all of the following causes : 1

1. Pressure from above.

2. Weakening of the uterine supports.

3 . Increased weight of the uterus.

4. Traction from below.

Any of the above conditions being the primary cause, the others singly or combined may result.

1. Pressure from Above.-Under this head may be included:

a. Pelvic or abdominal tumors.

b. Ascites.

c. Tight or heary clothing.

$d$. Straining at stool.

e. Muscular exertion.

$f$. Fecal accumulations.

g. Habitual overdistention of the bladder.

2. Weakening and Relaxation of the Uterine Supports may be consequent upon :
a. Subinvolution.
$b$. Senile atrophy of the pelvie floor.
c. Abnormally large pelvis.
$d$. Increased weight of the uterus.
e. Puerperal traumatisms.
$f$. Pressure from above.
$g$. Traction from below.

3. Increased Weight of the Uterus.-Among the pathological developments which cause increased weight are :
a. Congestion.
b. Subinvolution.
c. Metritis.
d. Pregnancy.
$e$. Fluid in the endometrium.
f. Uterine tumors.

4. Traction from Below may be due to such causes as :

a. Vaginal cicatrices.

$b$. Falling of the pelvic floor.

c. Contraction and congenital shortening of the vagina.

$d$. Tumors of the cervix or vagina. 


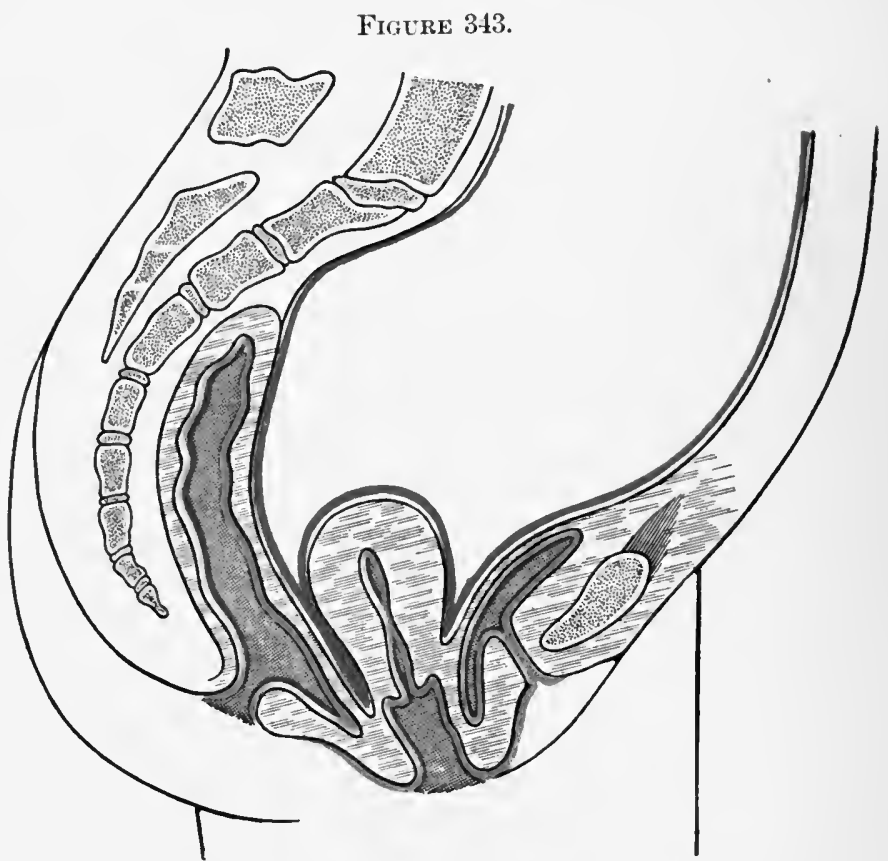

Tterus between first and second degrees of descent. Rectocele and cystocele. Semi-diagrammatic.

Figure 344.

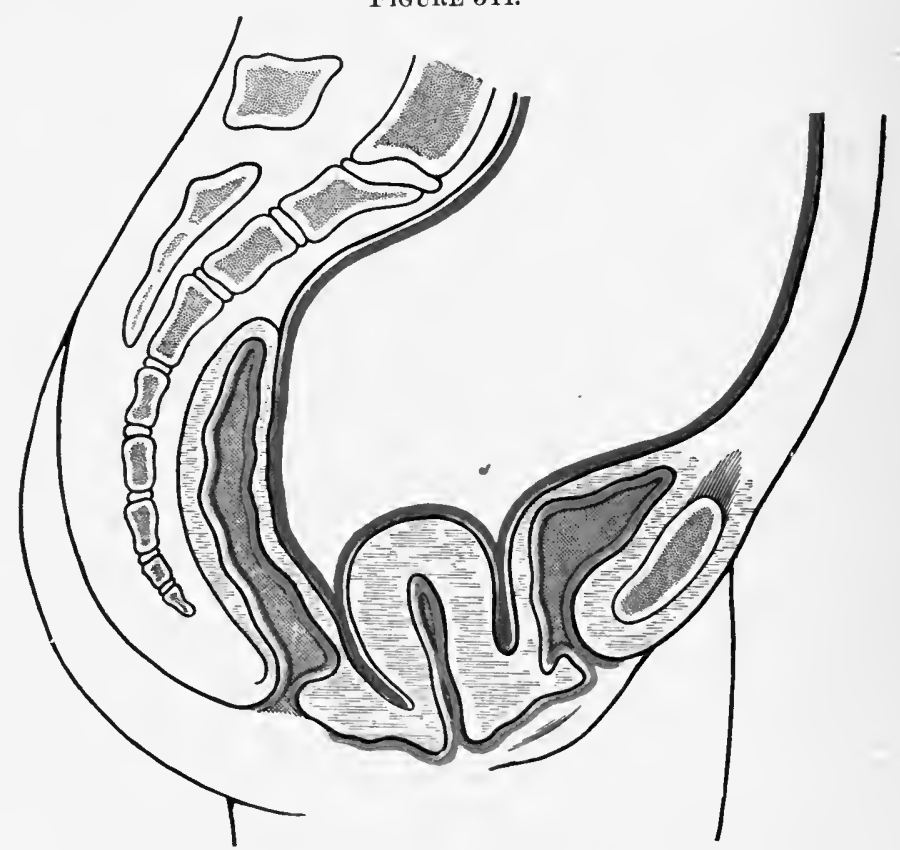

Second degree of descent. Cervix appears at the rulra. Rectocele and cystocele. Seni-diagram matic. 


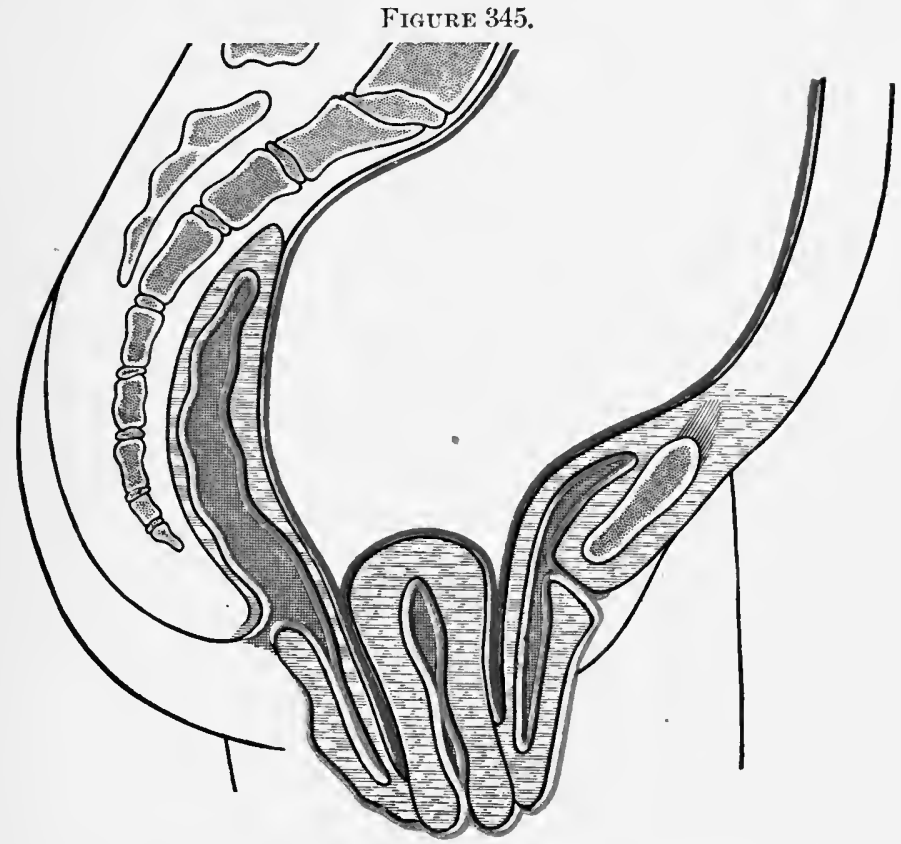

Complete or third degree of descent. Rectocele and cystocele. Rectocele forms a pouch in which scybalæ may accumulate. Semi-diagrammatic.

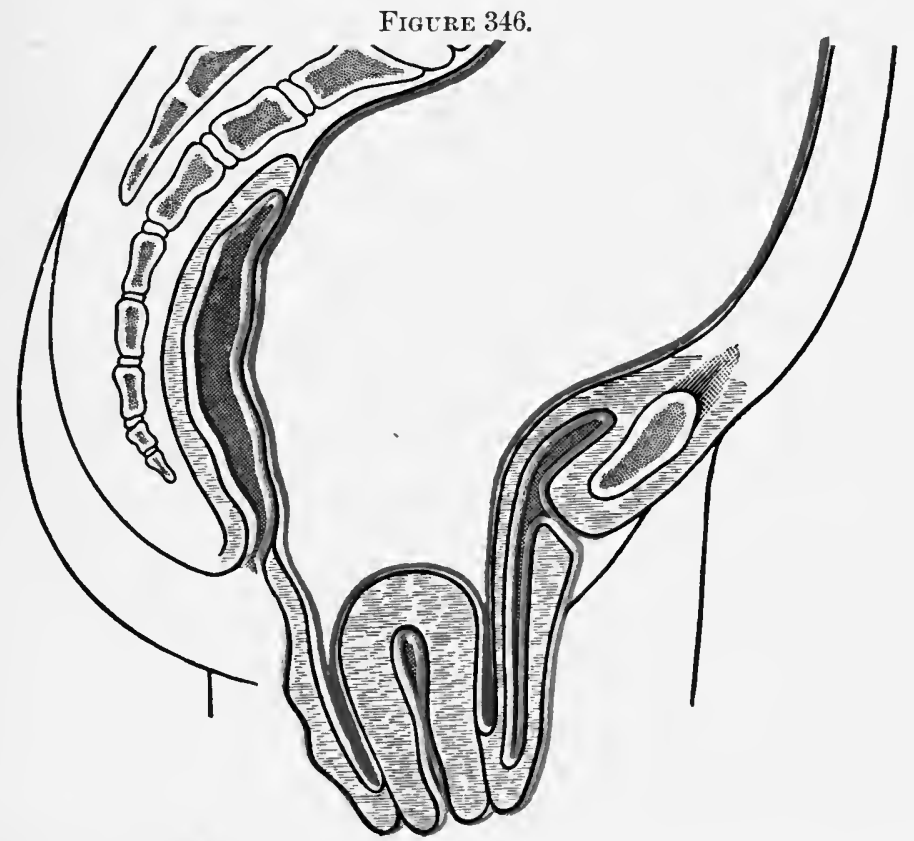

Complete or third degree of descent. Vaginal wall peeled off from the rectum leaving the rectal wall in normal position. Cystocele extreme; no rectocele. Semi-diagrammatic. Bladder displaced with the uterus. 
Uterogestation, parturition, and the puerperium may be followed by increased weight of the uterus and weakening of the supports from subinvolution. Puerperal traumatism may injure the vaginal outlet and cause the vaginal walls to fall; these in turn may drag the uterus down after them; indeed, excessive descent of the vaginal walls usually originates in parturition. Obviously, descent of the vesicovaginal and rectovaginal walls, or, more comprehensively, the sacral and pubic segments of the pelvic floor, involves also concurrent descent of the uterus and its appendages. It is clear from the above that descent of the vagina must be studied in connection with the descent of the uterus.

In labor the anterior wall of the vagina is so depressed, stretched, and shortened by the advancing head of the child that during and after the second stage the anterior lip of the cervix may be seen behind the urethra. If the puerperium progresses favorably, with prompt involution of the uterus, vagina, perineum, and peritoneum, the relaxation of the vesicovaginal wall and of the uterosacral supports disappears and the uterus resumes its normal multiparous location and position. But if the enlarged uterus remain in the long axis of the vagina, with its fundus incarcerated in the hollow of the sacrum between the uterosacral ligaments, and with its sacral supports stretched for so long a time that they cannot recover their contractile power, and with involution of all the pelvic organs arrested, the descent may not only persist, but may even progress, with constantly increasing protrusion of the vesicovaginal wall-cystocele - to the third degree of prolapse. The downward influence of the above conditions may be increased materially by rupture of the perineum and consequent prolapse of the rectovaginal wall into a pouch, called rectocele.

In the great majority of cases of complete prolapse the posterior vaginal wall in its descent is peeled off from the rectum, as shown in Figure 346 , leaving the latter in the normal position. In rare instances the lower portion of the rectum also is found to have extruded in extreme rectocele, making a pouch below and in front of the anus, in which fecal matter may accumulate and remain as hard scybalæ. See Figure 345.

Obviously, complete prolapse of the nterus is only an incident to prolapse of the pelvic floor. The whole mechanism is in all respects analogous to that of herinia. The extruded hernial mass drags after it a peritoneal sac, which, hernia-like, contains small intestine. This sac forces its way to the pelvic outlet and extrudes through the vulva, having the inverted vagina for a covering.

In the first degree of descent, Figure 354 , as we have said, the uterus is displaced downward and forward sufficiently to permit the body to turn back into retroversion; as already stated, the organ in its normal location cannot retrovert, because in so doing it would impinge upon the bony sacrum. As a consequence of the first degree of descent there are two significant possibilities: First, as the uterus falls to a lower level, where it would crowd upon and irritate the bladder, its long axis usually changes so as more and more to conform to that of the vagina, the cervix moves toward the pubes, and the 
FIGURE 347 .

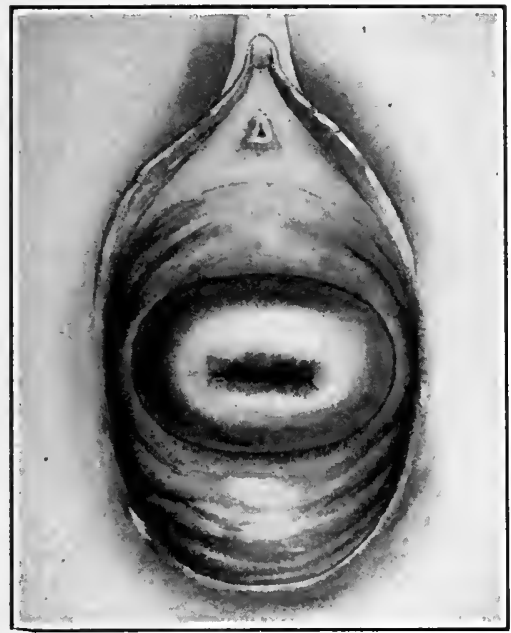

Figure 348.

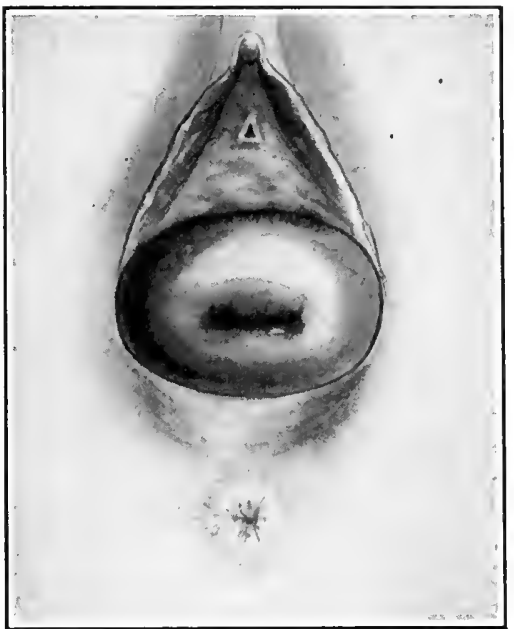

Figure 347,-Descent of the uterus to the third degree. The cervix appears at the vulva carrying with it the vesieovaginal and rectovaginal walls; that is, the reetum and bladder are in descent together with the uterus, having dragged the uterus down with them.

Figure 348. - Descent of the uterus to the third degree. The cervix appears at the vulva, not dragged down by the prolapsing rectum and vagina, but dragging them down after it, as shown in section in Figure 351.

Figure 349.

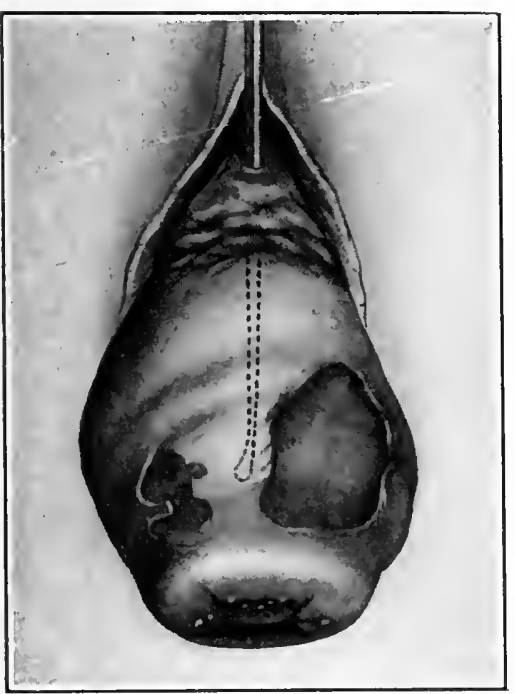

Figure 350 .

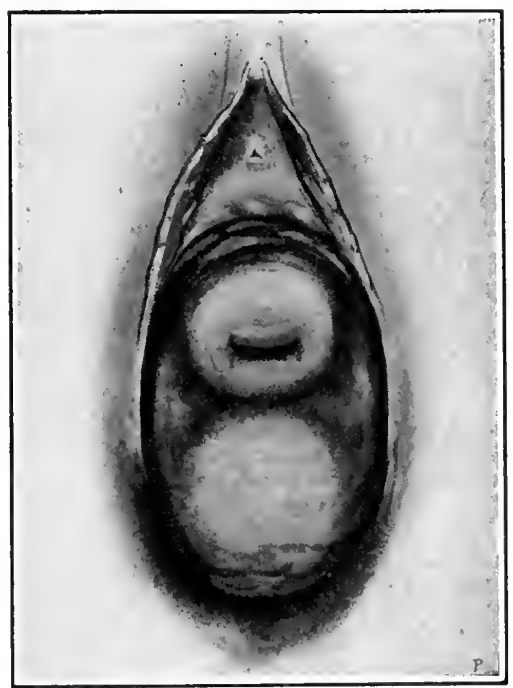

Figure 349.-Complete descent of the uterus, vagina, and bladder with ulceration of exposed surfaces. The os externum is at the bottom of the picture. That portion of the sound which is in the bladder is shown by dotted lines.

Figure 350,-Complete deseent of the retroflexed uterus: the cervix and external os uteri show in the upper part of the prolapsed structures and the corpus uteri in the lower part. 
corpus toward the sacrum-that is, it turns back away from the bladder into retroversion; this is as if the irritated bladder, in the protection of its own rights and territory, had thrown the uterus back; second, instead of turning back into retroversion, the uterus simply may change its location to a lower level, while the position remains the same-that is, the organ, still retaining its normal position of anteversion and anteflexion, only may settle to a lower plane. It must then occupy space that belongs to the bladder. The normally anteverted and anteflexed uterus in sueh descent is much more palpable

FigURE 351.

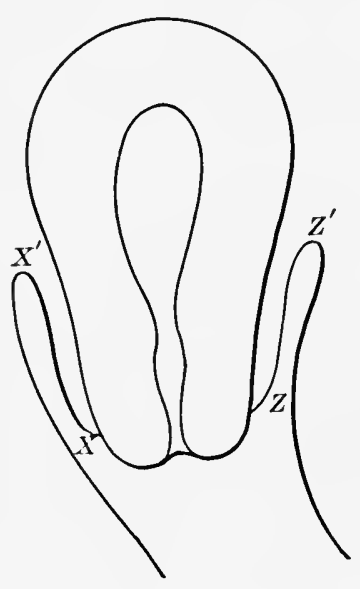

FIGURE 352.

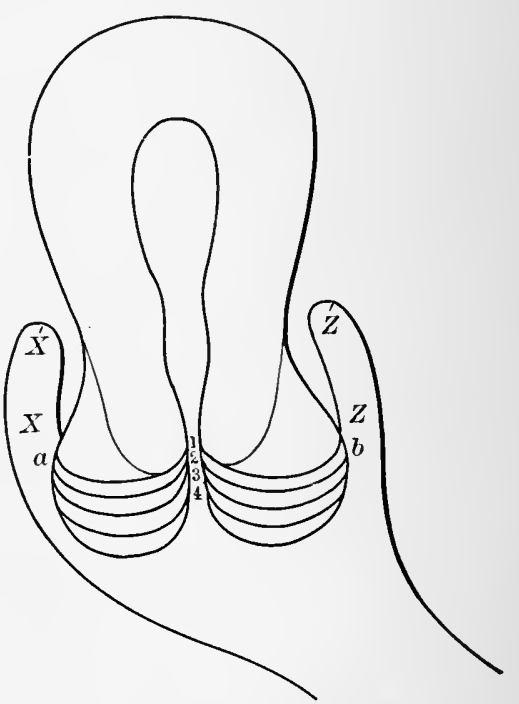

FigUre 351.-Descent of the virgin uterus into the vaginal canal, showing reduplicated vaginal walls. The uterovaginal attachment, points $X$ and $Z$, appears to be at $X^{7}$ and $Z^{\prime}$. The apparent inerease of length in the vaginal portion of the cervix, due to the reduplication, is measured by the distance from $X$ and $Z$ to $X^{7}$ and $Z^{\prime}$.

FIGURE 352,-Descent of the uterus, showing excessive circular enlargement of the lacerated cervix, conseguent upon reduplication of the vaginal walls and outrolling of intracervical tissues. The divided fragments of the os externum are at $a$ and $b$. The curved lines forming the angles 1, 2, 3, and 4 indicate the gradual process of eversion. The angle of the laceration originally at point 1 has been forced dowll by the swelling and outrolling of the mucous and submueous tissues of the cervix to point 4. The apparent os externum is at point 4 . The uterovaginal attachment $X$ and $Z$ seems to be at $X^{7}$ and $Z^{\prime}$. The vaginal portion of the cervix therefore appears much larger and longer than it actually is. This is rather descent by outrolling of the cervical mucosa rather than of the entire uterus.

to digital examination, and for this reason the vesical irritation consequent upon the descent often has been attributed wrongly to the anteversion and anteflexion. In this way has arisen much confusion in the effort to draw the line between normal and pathological anterior positions. The prompt relief which follows permanent replacement of the organ to the normal location, even thongh in so doing the anteposition be exaggerated, proves that the symptoms depend upon the mal-location, not upon the anteposition. The importance of a clear distinction therefore between location and position becomes apparent. 
Another cause of vesical irritation is the dragging of the uterus upon the neck of the bladder. This traction occurs not only in ascent, but also when the organ descends below a certain level.

In the foregoing paragraphs traction due to the falling pelvic floor has been discussed as a cause of descent. Inpairment of the uterine supports may, however, be such that, instead of falling and dragging the uterus after them, they simply permit it to descend along the vaginal canal by the force of its own weight, and to carry with it the reduplicated vaginal walls. This influence is enforced generally by increased weight of the discased organ. The vagina more readily becomes a track for the descending uterus when from any cause the normal forward direction of the vaginal canal changes toward the vertical; this change in the direction of the vagina may occur either as the result of forward displacement of its upper extremity or of retrodisplacement of its lower extremity. The former involves anteposition of the cervix ; the latter, backward displacement of the perineum. For a full discussion of backward displacement of the lower part of the vagina and vulva toward the coceyx, see Laceration of the Perineum and Injuries of the Pelvic Floor, in Chapters XL. and XLI. When the uterus descends along the track of the vagina, the long axes of the two organs will correspond ; hence, such descent must involve a degree of retroversion. See Figure 354 .

\section{Pathology of Descent of the Uterus.}

The pathology may involve all the displaced organs. The circulation throughout the pelvis is impeded by traction upon the vessels; the entire pelvic contents therefore become the subject of venous congestion, with consequences disastrous to local innervation and nutrition. The ovaries and Fallopian tubes suffer concurrent displacement. That portion of the peritoneum which enters into the formation of the uterine ligaments and of the pelvic floor is dragged along with the uterus. The vagina, also displaced, may become hypertrophied, swollen, and inflamed.

Sometimes the cul-de-sac of Douglas is distended by downward pressure of the intestine, by a small tumor, or by ascitic fluid, and a consequent hernial sac may protrude into the vagina through some portion of the posterior vaginal fornix. The anterior fornix is subject to a similar accident. These conditions are designated enterocele vaginalis anterior and posterior.

In the third degree of descent the vagina, now rolled out and exposed to external conditions, is no longer lubricated and protected hy normal secretions, and therefore becomes dry, parchment-like, œdematous, eroded, and perhaps ulcerated.

The rectum and bladder are subject to infection and chronic catarrh, and to concurrent descent. The uterus may be enlarged from any one or all of a variety of causes: congestion, subinvolution, hypertrophy, and hyperplasia. The cervix is often the seat of extreme crosion or ulceration. The endometrium, in order to relieve the organ of surplus blood, gives forth an excessive secretion of vitiated mucus from 
uterine catarrh. The enlargement of the uterus, if the cervix is lacerated, often pertains more to the cervix than to the body, especially in prolapse of the second and third degrees. An explanation of this may be found in Figures 351 and 352 .

Apparent elongation and disproportionate circular enlargement of the cervix are conditions which many standard anthors wrongly call hypertrophic elongation and circular hypertrophy. The question of infravaginal elongation is easily settled by placing the patient in the knee-breast position. Then the uterus, by its own weight, falls toward the diaphragm, the reduplicated vagina unfolds, and the apparent uterovaginal attachment, $X^{\prime} Z^{\prime}$, Figures 351 and 352 , disappears,

\section{Figure 353.}

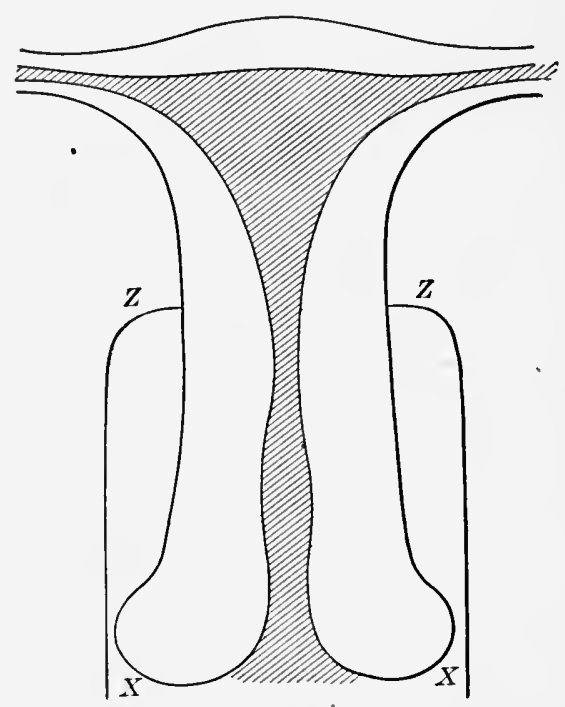

This cut is from a part of an illustration in a standard text-book. It is reproduced to illustrate the eurrent misconception of complete prolapse and apparent elongation of the cervix. The apparent elongation almost invariably disappears on replacement of the uterus. The appearance of elongation is due to congestion and vaginal reduplication. Amputation of such a cervix, so often advised, would be apt to involve the bladder in front and the cui-de-sac of Douglas behind. Actual eiongation of the cervix has seldom been demonstrated satisfactorily. Elongation, if present at all, is almost always at least in the supravaginal, not the infravaginal portion of the cervix. The nterovaginal attachment cannot be therefore as indicated at $Z Z$; it is on a plane slightly above $X \boldsymbol{X}$.

disclosing the actual attachment, $X Z$. Further, the point of the sound, passed into the bladder while the cervix is exposed by Sims' speculum, may be placed against the anterior wall of the cervix at $Z$, which would be impossible if the attachment were at $Z^{\prime}$.

The comparatively small amount of hypertrophy in disproportionate circular enlargement due to an associated laceration of the cervix uteri, is proved by the operation of traehelorrhaphy or by rolling in the ontrolled tissues with uterine tenacula, as shown in Figure 307. When the outrolled intracervical mucous tissues are rolled in, the proper diameter of the cervix is restored, and a laceration on one or both sides, extending past the vaginal attachment, becomes apparent. 
Those cases in which reduplication of the vaginal walls does not almost entirely explain the great elongation so-called, or in which great disproportionate circular enlargement has not been caused by an associated laceration of the cervix, are the rare exceptions. Formerly these mechanical conditions were attributed to hypertrophie changes, and were regarded as adequate indieations for removal of the cervix. Such elongation as is shown in Figures 300 and 353 rarely, if ever, exists. Emmet, with his enormous experience, has never seen such a case, and denies its existence. Congestion of the prolapsed uterus consequent upon obstruction in the stretehed and displaced veins is often so extreme as to induce a state analogous to ereetion. Measurements by the probe just before and a few minutes after replacement generally show a very appreciable decrease in the length of the uterine canal. If the prolapse has been of the third degree, the difference may amount to one or even two inches. It is clearly important not to confound the enlargement of congestion with increase in the solid constituents of the organ. See Laceration of the Cervix. The great merit of having secured general assent to the foregoing propositions, and of having given to the subject a new and right direction, must be accorded to Emmet. The cervix now seldom is amputated for so-called Hypertrophy.

\section{Symptoms and Course of Descent of the Uterus.}

The course of descent is ordinarily chronic, but intercurrent attacks of acute vaginitis are rather common. Peritonitis sometimes effects a spontaneous cure by peritoneal adhesions that fasten the uterus in an elevated position and hold it permanently. The symptoms of descent may be so severe as to necessitate for the patient absolute rest in bed, or they may be attended with very little discomfort; the usual symptoms are these:

1. Abdominal pains.

2. Dragging pains in the pelvis extending to the thighs.

3. Functional disturbances of the bladder and rectum-tenesmus.

4. In cases of complete prolapse; suffering from excoriation or ulceration of the exposed vagina or cervix uteri.

5. Great irritation from vaginitis and pain from possible peritonitis.

6. Uterine hemorrhages and other menstrual disorders-frequent.

7. Leucorrhoa.

8. Sterility.

\section{Diagnosis and Differential Diagnosis of Descent of the Uterus.}

The diagnosis is by inspection, palpation, and exploration. The prolapsed uterus may be distinguished from cystocele, rectocele, inverted uterus, vaginal cysts, and fibroid tumor by the presenee of the os externum. The length of the uterus may be measured by the sound; the size, shape, position, extent of descent, and difficulty of replacement may be determined by conjoined manipulation. 


\section{Diagnosis of Associated Cystocele and Rectocele.}

Cystocele may be recognized by-

a. A convex protrusion between the labia covered with rugous vaginal mucosa, easily pushed back, and diminishing when the patient lies down.

b. A sound in the bladder may be felt by the finger against the protrusion, thereby demonstrating it to be continuous with the vesicovaginal wall and formed of it.

c. After urination there will remain residual urine in the protruding sac. This often causes cystitis and stone in the bladder.

Rectocele may be recognized by-

$a$. Bulging forward of the posterior vaginal wall, the protruding mass being covered with rugous vaginal mucosa.

b. Mass increasing in size on straiming at stool and diminishing on lying down.

c. Finger in the rectum, which enters the protruding mass, and demonstrates it to be continuous with the rectovaginal septum and composed of it.

d. Lodgement of feces in the pouch and prevention of complete emptying of the bowel; it may be necessary to facilitate defecation by pushing the pouch back with the finger.

Cystocele and rectocele are apt to be associated with intermittent accumulations of air in the vagina, which may be expelled with a peculiar sound-so-called garrulity of the vulva.

\section{Prophylaxis of Descent of the Uterus.}

Prophylaxis requires such measures during labor as will prevent long and powerful pressure upon the pelvic floor. After labor any injury to the perineum shonld be repaired promptly. The vagina should be kept clean by irrigations. The urine, if retained, should be drawn regularly and the bowels moved daily without straining. If conditions be present likely to induce subinvolution, such, for example, as pelvic infection and laceration of the cervix, they should receive treatment at the proper time. Undue relaxation of the pelvic floor necessitates prolonged rest in bed, the use of astringent douches, and, when the patient resumes the upright position, the application of a pessary. If involution goes on with the uterus congested and irritated by descent, the result is apt to be perpetuation of the displacement and its attendant evils; it is therefore lighly desirable that the uterus be kept in place during the puerperium; to this end, even while the patient is in bed, a pessary may be indicated. The great prophylactic value of rest in bed, prolonged for seven or eight weeks after labor, is undeniable. The puerperium offers the best conditions for the prophylaxis and cure of descent. 


\section{Treatment of Descent of the Uterus.}

Treatment may be surgical or non-surgical.

\section{Non-surgical Treatment.}

Replacement.-The first indication is replacement, which, in the first and second degrees of descent, is not difficult unless the uterus is held down by cicatrices or by a tumor. Acute pelvic inflammation may render replacement dangerous or impossible, and may for a time contraindicate all direct treatment. Replacement of the organs from the third degree of prolapse is accomplished in the inverse order of their descent: first, the posterior vaginal wall, then the uterus, and lastly the anterior vaginal wall. Not infrequently the completely prolapsed uterus and pelvie floor, hernia-like, become strangulated. Then taxis usually will suffice; but it may have to be supplemented by hot applications, elastic pressure, anodynes, and the knee-breast position, and, should these fail, anæsthesia.

In exceptional cases of sudden descent, even to the third degree, replacement alone is followed sometimes by permanent relief; but if the descent has been gradual, it always occurs immediately after replacement. Measures are required therefore for the maintenance of the uterus in the normal location and position. This indication is fulfilled by :
Hygiene.
General and local measures.
Pessaries.
Surgieal operations.

The Hygiene prineipally relates to dress, food, exercise, and regular habit of the bowels. Undue pressure from above should, if possible, be avoided. The clothing should be loose, and the weight of the skirts supported from the shoulders either by straps or, preferably, by buttoning them upon a waist made for the purpose. The waist is a good substitute for the corset, which, under all circumstances and in all forms, is injurious. Constipation and the aceumulation of feces in the lower bowel mechanically irritate and may displace the pelvic organs; straining at stool exerts a strong downward pressure on the uterus and its appendages. Careful regulation of the bowels is therefore imperative; to this purpose food and exercise are the most essential agents.

General and Local Measures.-The value of general massage for women unable to take active exercise is very great. As a supplement to massage, or as an independent measure, one may urge strongly the knee-breast position. This position assumed several times a day causes the uterus to gravitate toward the diaphragm, and thereby gives temporary rest to the overburdened supports. While in this position the patient should separate the labia so that the air may rush in and the vagina become expanded. Mineral waters and general tonies are useful. The measures enumerated above, together with such topical treatment as local conditions may demand, are essential as adjuvants to the mechanical or surgical treatment which almost every case requires. 
Pessaries.-In the genesis of retroversion and retroflexion the first change is deseent; hence, the principles of mechanical treatment must be substantially the same for each. The reader therefore is referred to the indieations, the contraindications, modes of adjustment, and uses of pessaries in the treatment of retroversion and retroflexion.

In complete prolapsc dependent upon extensive injury of the perineum and other parts of the pelvic floor, and associated with subinvolution and relaxation of all the pelvic organs, the axis of the vagina is ehanged from its forward oblique to the vertical direction. See Figure 354. The downward traction of the prolapsing cystocele and rectocele upon the fornix of the vagina may then be so great that the pessary is inadequate to maintain in place the upper extremity of the vagina. 'The cervix uteri then moves forward, the corpus turns back, and the whole uterus easily deseends in a vertical direetion along the prolapsing walls of the vagina to the seeond or third degree of prolapse. In this condition pessaries that disappear within the vagina are liable to be forced out with the prolapsing pelvic floor, or, if retained, seldom maintain the uterus in position. In such eases the various cup pessaries, that are supplied with external attachments and abdominal belts, often are used; but they either so fix the uterus as to prevent its normal movements, or hold it in such unstable equilibrium that it may assume any one of the various malpositionsanterior, posterior, or lateral; they are open to the further serious objection of eonstantly reminding the patient of their presence, and for these reasons are not generally approved; they are, however, permissible in eases of complete prolapse when the patient refuses surgical relief. As an expedient, the uterus sometimes may be held within the pelvis by means of a large Albert Smith pessary, with extreme uterine and pubic curves; see Application of Pessaries in the Treatment of Retroversion.

\section{Explanation of Figure 354.}

$A$. Incisions have been made from the posterior vaginal fornix to the cul-de-sac of Douglas and from the anterior vaginal fornix to the utero-vesical cul-de-sac. The uterine and ovarian arteries in both ligaments have been securely ligated. The left ligament has been cut away from the uterus and is drawn to the vulva and held by forceps. The uterus has been drawn through the vulva and is hanging by a shred to the right ligament, from which it is being cut away. The wound made by the removal of the nterus with its peritoneal and vaginal margins shows between the ligaments.

$B$. The last stitch of a continnous catgut suture is being taken to unite the peritoneal margins of the wound. The line of suture thus made runs from one ligament to the other and at either end includes the ligament. Closure of the peritoneum as here shown is nearly complete.

$C$. The ends of the broad ligament are shown to be united in front of the peritoneum by means of a continuous catgut suture. Another suture is closing the vaginal margins of the wound by a line of union running across the upper end of the vagina from side to side. When this suture is complete, the united broad ligaments will lie between peritoneum and vaginal wall, and must necessarily sustain the rectum, vagina, and bladder on the health level. 
Figure 354.

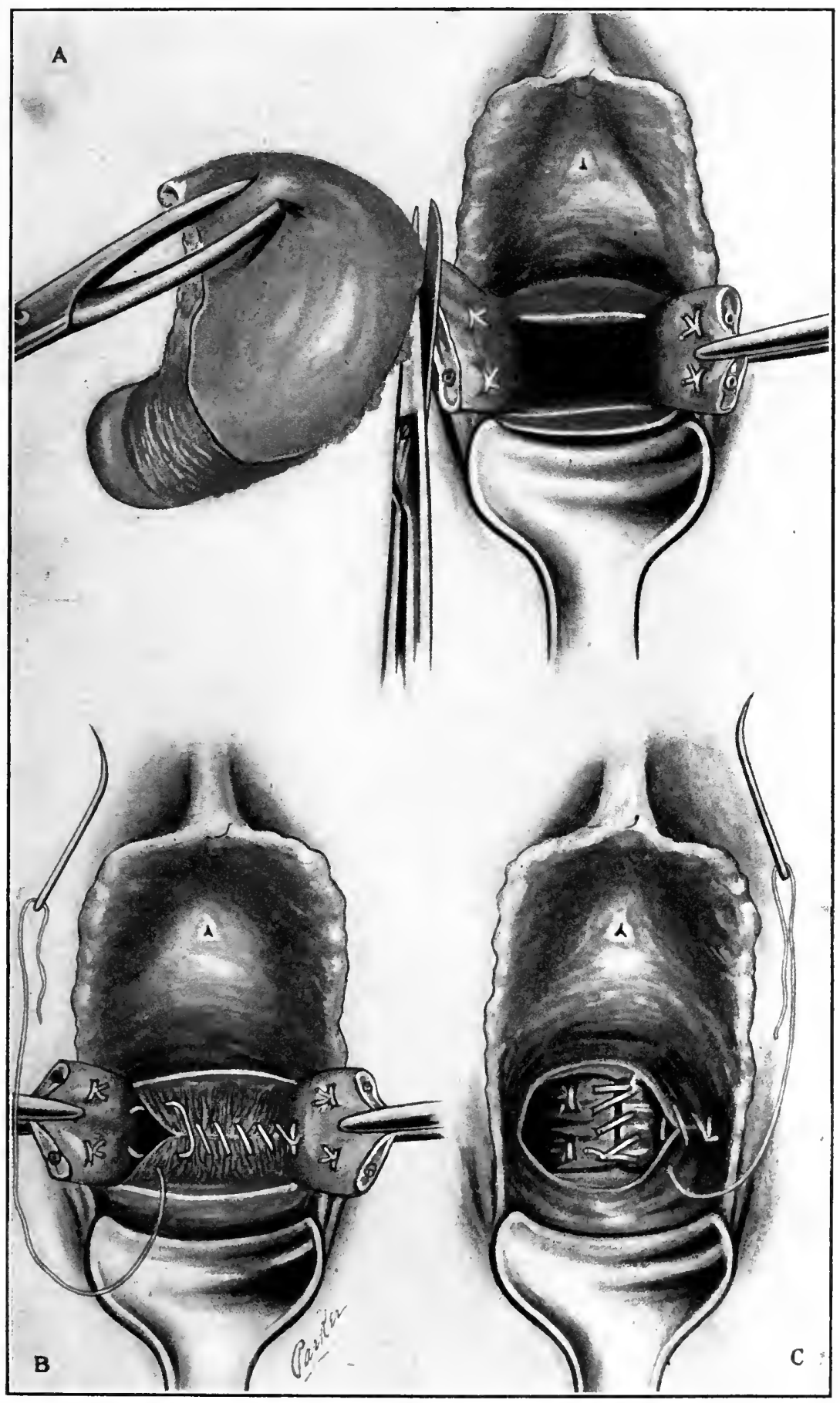




\section{Surgical Treatment.}

The surgical treatment may be removal of the uterus by hysterectomy, or the retention of it in its normal position and location by means of plastic operations.

Hysterectomy.- The failure of the older plastic operations to hold the uterus in place permanently has indueed many surgeons to adopt the more radical operation of vaginal hystereetomy - a permissible operation on women who have passed the menopause, but usually not permissible during the child-bearing period. Cases are numerous in which, after vaginal hysterectomy, the pelvic floor, and with it the rectum, vagina, and bladder, have protruded again through the vulva. For this reason the operation always should include anchorage of the upper end of the vagina to its normal location by end-to-end approximation of the severed broad ligaments in the wound made by the removal of the uterus. See Figure 354 .

Plastic Operations.-The rational treatment for complete prolapse requires: first, an operation on the anterior vaginal wall to restore the fornix of the vagina and with it the attached eervix to their normal place in the hollow of the sacrum; second, an operation at the vaginal outlet to bring the posterior vaginal wall well in contact with the anterior and thereby to restore the lower extremity of the vagina, together with the perinenm, to its normal place under the pubis. The numerous plastic operations on the anterovaginal wall for the relief of complete descent of the uterus are divisible into two classes :

1. Narrowing the Vagina.

2. Changing the Direction of the Vagina.

1. Operations designed to hold the uterus up by namowing the vagina so much that the uterus cannot pass through it, and, consequentiy, must be maintained somewhere in the pelvis above the vaginal constriction, usually consist in the removal of an elliptical piece from the anterior or posterior wall of the ragina, or from both ; or of making longitudinal denudations and bringing the edges of the exposed surfaces together from side to side. In this class of operations no effort is made to restore the normal axes of the uterus or the vagina. The whole purpose is to make the vagina so narrow that the uterus cannot pass through it. Operations of this class generally fail, because they do not restore the normal angle between the uterus and the vagina. The constricted ragina, indieated by the white lines in Figure 355, cannot resist the downward force of the uterus, which almost invariably dilates the vagina a second time, forces itself throngh, and reproduces the hernia. Moreover, the operation does permanent harm, because it shortens the vagina, therely making it draw the cervix away from the sacrum toward the pubes. This forward movement of the cervix, as already stated, is an element in the genesis of descent, and therefore should not be employed in the treatment of it.

2. Operations designed to hold the uterus in position by restoring the normal angle between the long axis of the uterus and the long axis of the vagina may narrow somewhat the vagina, but such narrowing is only 
an incident rather to be regretted than desired. It is not essential to the suecess of the operation.

There are two rational indieations : first, to fix the upper extremity of the vagina together with the cervix uteri in its normal location within an ineh of the junetion of the seeond and third sacral vertebræ, just where the uterosacral ligaments would hold it if their normal tonicity and integrity could be restored; second, to bring the lower extremity of the vagina forward, so that its posterior wall shall be elose up against the pubes. The fulfilment of these two indications will restore the normal obliquity to the vagina, and will hold the cervix so far back toward the saerum that the corpus uteri cannot

Figure 355 .

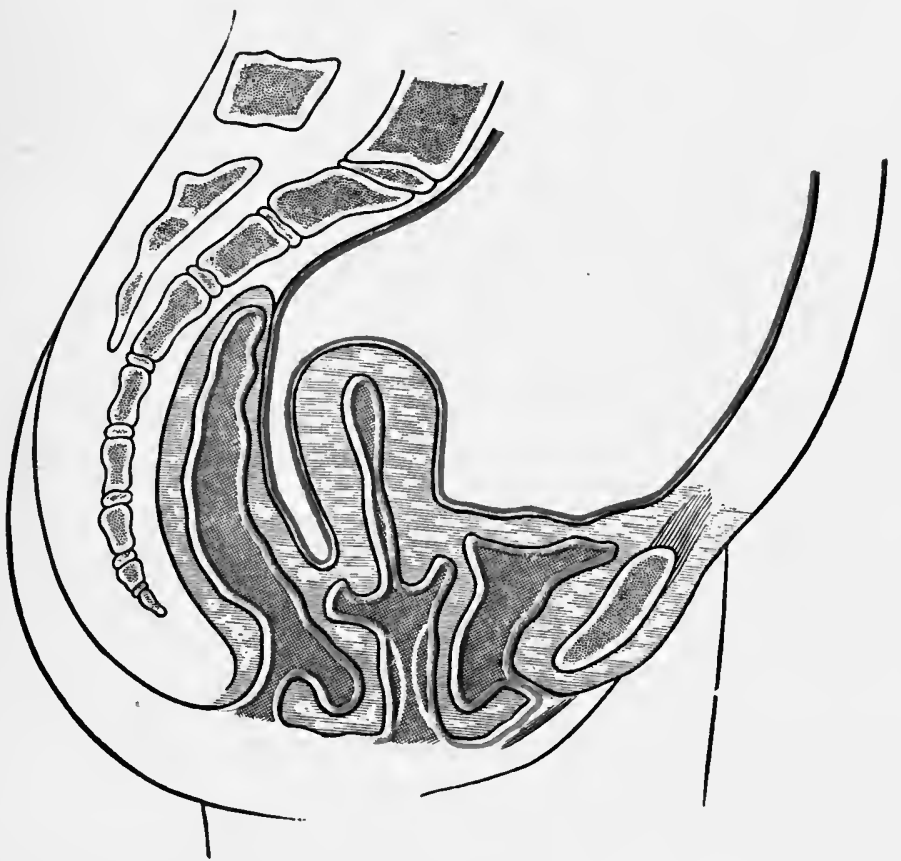

Uterus in line with the vaglna; first degree of descent. The white lines in the vagina show where it would be narrowed by the first class of operations.

retrovert or prolapse, but must be direeted forward in its normal anteverted position of mobile equilibrium. In this way the long axis of the uterus and the long axis of the vagina will form an acute angle. The indications are fulfilled best by elytrorrhaphy, including end-to-end approximation of the cut ends of the broad ligaments, and perineorrhaphy.

Reynolds wisely says: "The first point is-that to attain suceess we should ascertain and utilize the natural supports of the anterior wall instead of simply denuding and gathering together the overstretched portions. The seeond, that we should not only aroid using any part of the overstretehed portion of the wall, but should 
actually excise and do away with it; both of which objects should be attained withont the performance of an unnecessarily extensive or severe operation."

"The mechanics of pelvic support are after many years still the subject of dispute, but a few points are clear. The anterior vaginal wall has naturally two fixed points of attachment. The first is that of the lower end of the wall to the posterior surface of the pubes. This is exceedingly firm and never vields. The same cannot be said of the attachments of the upper end of the wall; they are, however, sufficient for the purpose. In prolapse complicated by cystocele, the correction of the cystocele is essential to the cure of the prolapse. Our experience of late years in total extirpation of the uterus has tanght us that the only attaehment between the genital canal and the pelvic wall, which is not readily separated with the finger, is the insertion of the hroad ligaments into the lateral edges of the uterus and the vault of the vagina. These the only firm supports of the vaginal vault furnish, then, the only upper points which rationally can be used in the restoration of the anterior wall. The utilization of the bases of the broad ligaments has, moreover, the very great incidental advantage that it not only relieves the uterus of the weight of the prolapsed anterior wall, but in itself, as will be seen, tends to restore the prolapse by throwing the cervix backward. The first point in any operation then should be the attachment to each other of these two firm portions of the wall."

The fact that vaginal hysterectomy commonly results in holding up the pelvic floor, and with it the rectum, vagina, and bladder, is because in this operation the broad ligaments usually are fixed in the vaginal wound; hence the same result may be secured by similar means, even though the uterus is not removed. Reynolds further remarks somewhat as follows:

" 'To this first principle of utilization of the broad ligaments should be added the second principle of excision of the weakened portion of the anterior vaginal wall. The wall in its natural condition is a short, firm, fascial and muscular structure, which extends from its origin at the firm bases of the broad ligaments to its still firmer pubic attachment, thus forming one of the strongest supports of the uterus and other pelvic organs. Look now at its condition in cystocele. If anyone will freely excise the anterior wall in a well-developed cystocele, he will find that the condition is that represented by Figure 356 , neglecting the diagrammatic straight lines-i. e., that the central portion of the protrusion has been covered by an overstretched, thinned, and weakened vaginal wall. 'This thin portion of the wall is overstretched because it has lost its clasticity, has lost its power of resistance to further stretching. If now we utilize for repair any part of this weakened wall, we shall have, as a result, a weak scar, which is necessarily predisposed to further stretching."

"Cystocele is in effect hernia of the bladder through the muscular and fascial structures of the anterior wall of the vagina; hence the second principle involved in dealing with it is essentially that which already is well established in the treatment of other hernias. It has 
been customary to treat cystocele by denuding the vaginal wall of its epithelinm, invaginating the protrusion, and stretching the denuded surfaces together. No one to-day would think of treating any other hernia in such a manner. On the contrary, we are accustomed to treat other hernias by reducing them and excising the sac until we lay bare strong, firmly attached fascial edges." Such should be the treatment of cystocele. The principle as set forth by Reynolds in the above quotation has led me to abandon my previous operations for elytrorrhaphy and to substitute the operation about to be deseribed.

Figure 356.

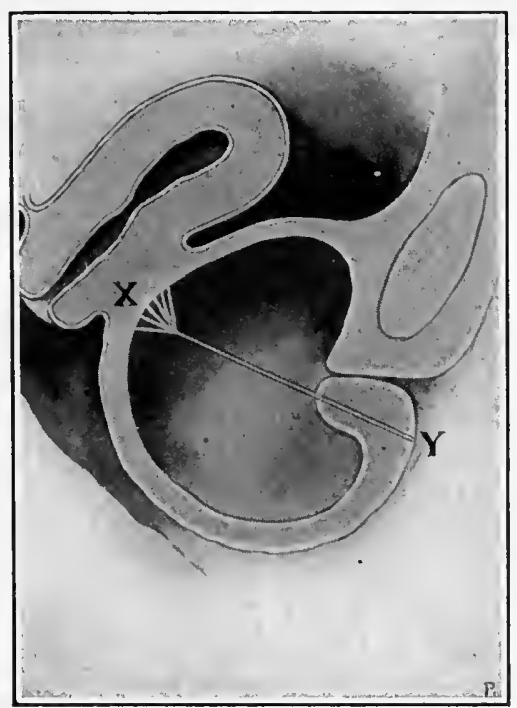

The line $\mathrm{XY}$ shows tlie direction and location of the vesicovaginal wall to be restored. The light radiating lines at $\mathrm{X}$ suggest the lines of force on the broad ligaments as caught $u p$ by sutures. The pouching vaginal wall between $X$ and $Y$ takes the form of extreme cystocele.

Before describing the proposed operation I would repeat the fact that the various plastic operations on the vaginal walls (elytrorrhaphy) have proved so unsatisfactory that many surgeons are inclined to abandon them and to adopt the radieal measure of vaginal hysterectomy in their place. This operation, however, if performed by the familiar method of ligating the broad ligaments, cutting them from the uterus, and then letting them retract to the sides of the pelvis, is inadequate, because the ligaments thus retracted camnot perform their functions of holding up the pelvic floor; consequently, many hysterectomies performed in this way have been followed by a continnance of the downward displacement of the rectum, vagina, and bladder, a condition not materially improved by the mere absence of the uterus. In order to utilize the normal supporting power of the ligaments, I devised and in 1902 published an operation by which the severed ligaments were approximated end to end between the peritoneal and vaginal sides of the wound made by the removal of the uterus. In a 
foregoing part of this chapter I have introdueed this operation unmodified, beeause I desire to make use of the principle of end-to-end approximation in the surgical treatment of complete descent of the uterus, a description of which will follow. Figure 354 will serve to illustrate the treatment of the broad ligaments by end-to-end approximation in vaginal hysterectomy.

Elytrorrhaphy, Including End-to-end Approximation of the Broad Ligaments and Excision of the Cystocele. ${ }^{1}$-The mechanism of descent would suggest at once shortening of the uterosacral ligaments to draw the cervix uteri back to its normal location near the hollow of the sacrum, and shortening the round ligaments to draw the corpus forward so as to restore the normal direction of the uterine axis. These procedures might be curative in cases of retroversion or retroflexion .without great descent, but when not only the uterus, but also the whole pelvic floor, is in extrene downward hernial displacenent the uterosacral and round ligaments, even though shortened, do not have sufficient power to give permanent support. The strain on them is so great that they are apt to stretch out and permit the descent to recur. Adequate sustaining power under the uterus is essential. To appreciate the part which the broad ligaments properly may have in a correct operation, it is only necessary to observe the fact that vaginal hysterectomy, as above described, results in holding up the pelvic floor and with it the rectum, vagina, and bladder, because in this operation the broad ligaments are fixed to the vaginal wound. Since the condition of procidentia is hernia not alone of the uterus, but of the rectum, vagina, and bladder as well, let us try to hold up the pelvic floor, including the uterus, by similar means, even though the uterus be not removed.

Following the suggestion of Emmet, Sims, Reynolds, and others who have striven to diraw structures in the neighborhood of the lower margins of the ligaments in front of the cervix for the purpose of forcing it back, I found myself stripping the structures more and more from the sides of the uterus and drawing them in front of it, but not until I actually severed a considerable portion of each ligament from the sides of the uterus did I secure the best results. In future operations I shall enphasize that part of the procedure as shown in the drawings more than I have done yet. Figures 357 to 365 , with their legends, will illustrate the proposed method of holding up not only the uterus, but also the rectum, vagina, and bladder by end-to-end approximation of the broad ligaments and adequate excision of the cystocele, without removal of the uterus.

In addition to approximation of ligaments, it must be borne in mind that other supporting structures also are brought together in front of the cervix uteri, notably the adjacent parametric structures, and if the operation is sufficiently extensive, the round ligaments. In extreme cases it wonld be well to separate the bladder entirely from the cervix uteri, as would be clone in raginal section, so as to expose the round ligaments. These ligaments then could be brought down

1 Read in the Section on Obstetrics and Diseases of Women of the American Medical Association, at Boston, June, 1906. 
in front of the eervix and included in the sutures which are used to approximate the cut ends of the broad ligaments. Such adjustment of the broad and round ligaments, together with adjacent parametria in front of the cervix, necessarily would give great strength to the pelvic floor.

The question frequently has been asked whether in cutting away the lower halves of the broad ligaments troublesome hemorrhage is not encountered. I should fear such hemorrhage if the ligaments were severed with the uterus in its normal location, but would not fear it with the uterus outside the body. Besides, since a shaving is removed from each side of the uterus, the incision usually would be safely inside the utero-ovarian anastomosis. At any rate, the uterus being outside the body, hemorrhage ean be controlled easily if it does occur.

Even at the risk of prolixity, I repeat that it is essential to remove the entire thickness of the vaginal layer of the vesicovaginal septum, as shown in Figure 357. The illustrations of this operation lere show the cervix uteri drawn well down to the vulva, but this appearance is introduced only to facilitate the illustration; the suturing part of the operation shonld be performed so far as possible with the cervix in the hollow of the sacrum where the operation is designed to fix it. The incisions may be made with the uterus drawn down, but it is well to introduce the sutures with the cervix uteri in place, and to. this end the left lateroprone position and Sims' speculum may be used advantageously. The author uses a speculum with the blade perforated at its extreme end, and before the speculum is introduced the cervix is attached to the end of the blade by means of a temporary suture which is passed through the posterior lip of the cervix and then through the perforation in the speculum, and tied. This temporary stitch, while the sutures are being applied, holds the cervix far back in the hollow of the sacrum; it should be removed at the end of the operation. When the cervix thus is held back, the space anterior to the uterus is so increased that the uterus readily falls forward into a position of decided anteversion and shows the adrantage of doing the operation with the organ in normal position.

Contraindication to Elytrorrhaphy.-Elytrorrhaphy is usually unnecessary and therefore contraindicated in descent of the first degree. The special province of the operation is in complete prolapse or procidentia when associated with cystocele. The operation further is contraindicated by tumors and adhesions which render replacement and retention impossible, and in diseases of the uterus or its appendages which demand their removal. When such eontraindications do not exist, unless the descent is extreme, elytrorrhaphy and perineorrhaphy are, usually at least, quite as effective, and are therefore to be preferred to the more dangerous and mutilating liysterectomy.

Perineorrhaphy.-As already stated, it is most important to appreciate the fact that in nearly every case of procidentia the lower extremity of the vagina is displaced backward. This is consequent upon subinvolution of the vaginal walls, and especially upon subinvolution or rupture of the perineum or of some other portion of the vaginal outlet. Unless, therefore, the posterior wall of the vagina 
Figure 357.

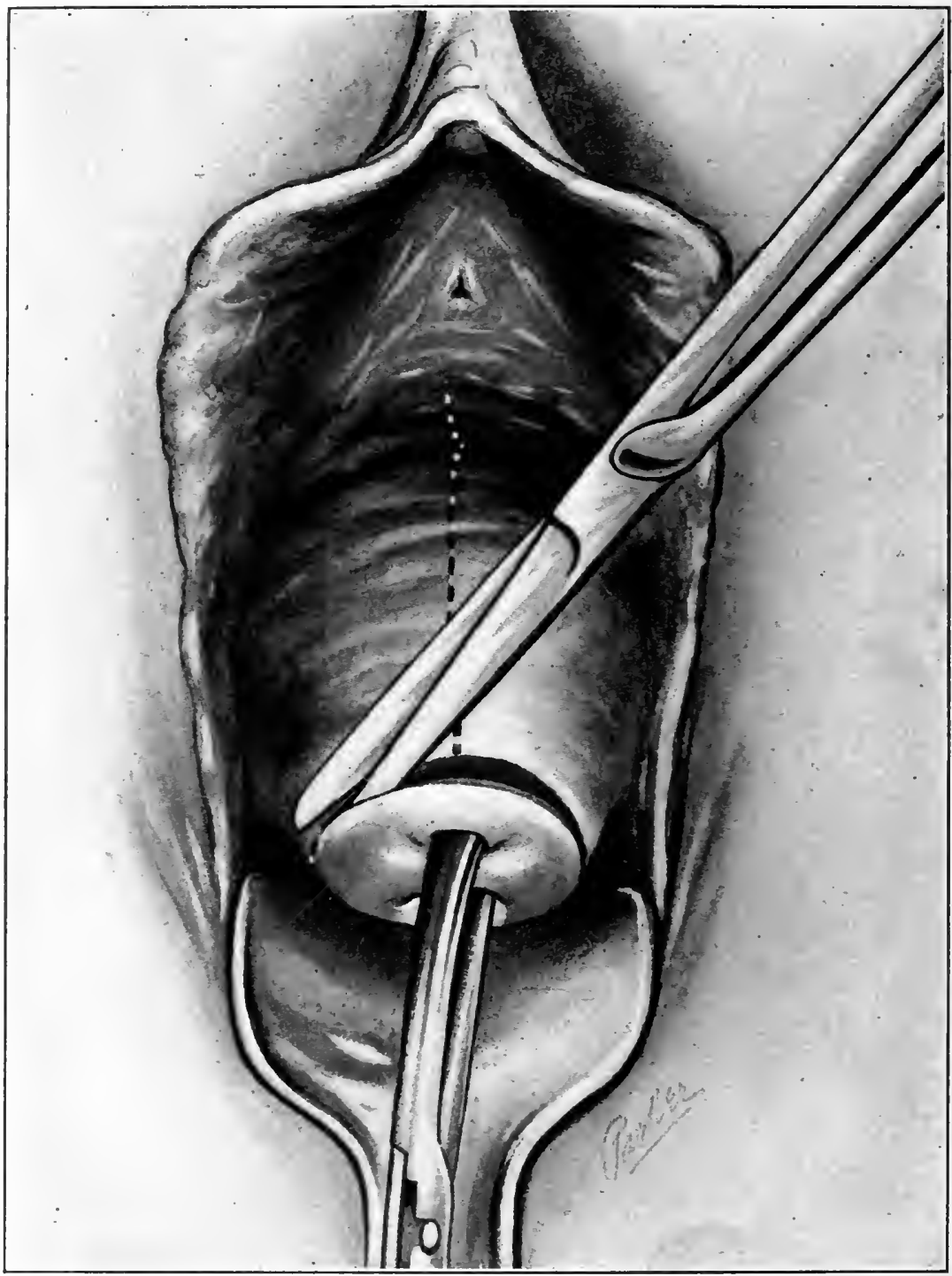

Broad ligament operation for complete descent of the uterus. Cervix held steady by flat vulsellum foreeps. The bladder lies in front of the prolapsed uterus. The dotted line extending in the median direetion on the anterior wall of the cervix from a point at the uterovaginal at taehment to the uretlrovaginal wall, marks the direction of an incision to be made by means of sharp-pointed scissors through the vaginal layer of the vesicovaginal septum. The incision extending around the anterior half of the cervix at the uterovaginal attachment is made through the vaginal wall to, but not into, uterine tissue. 


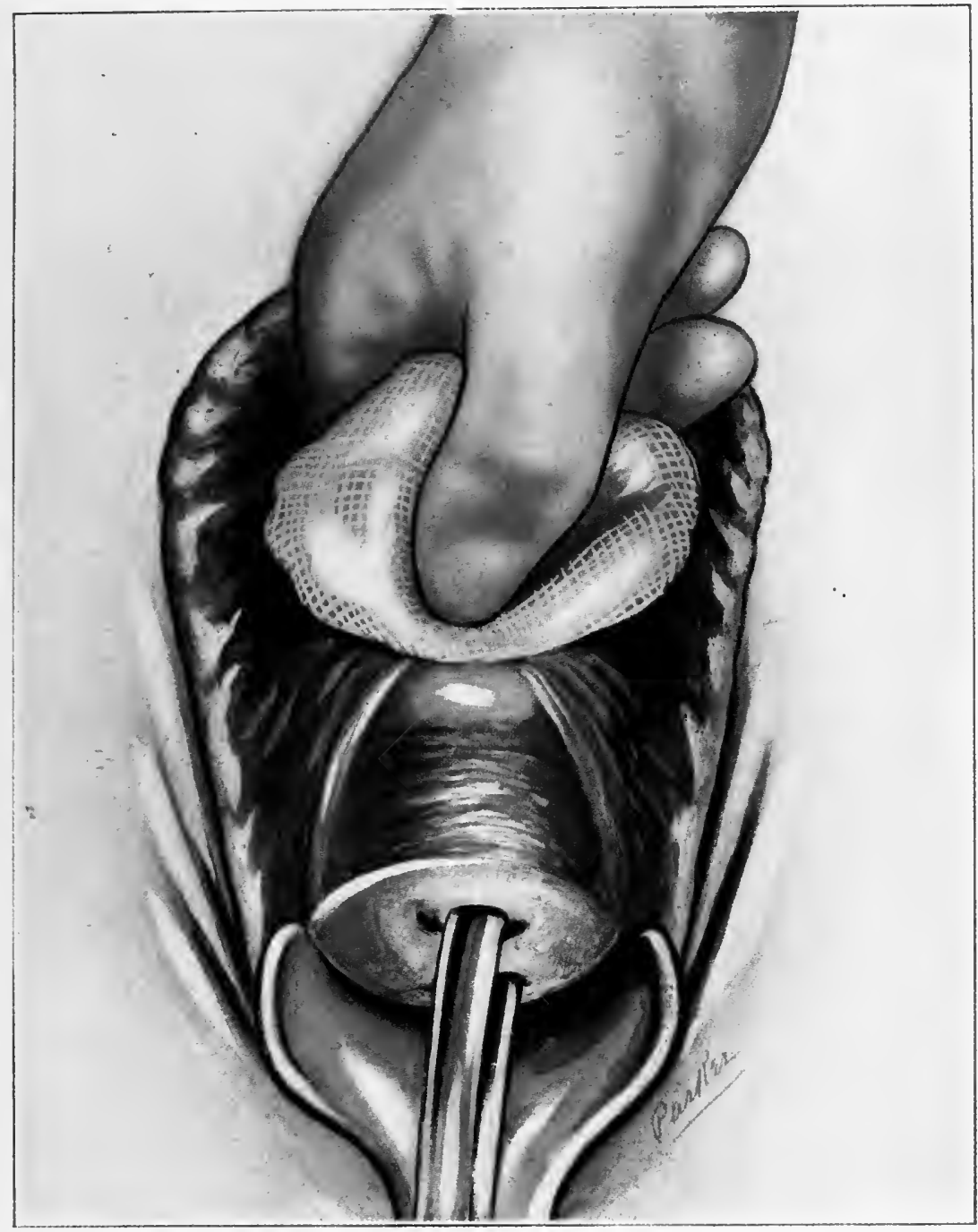

Broad Ligament Operation for Complete Deseent of the I"terus. Bladder being stripped from uterus by blunt dissection, which is accomplished readily and quiekly by sponge pressure, and is continued to the peritoneal reflexion of the vesico-uterine pouch precisely as it would be if the operator were going to open into the peritoneal eavity between the uterus and bladder. Observe here, just beneath the sponge, the translucent yeritoneum, which for the present purpose is not usually to be incised. 


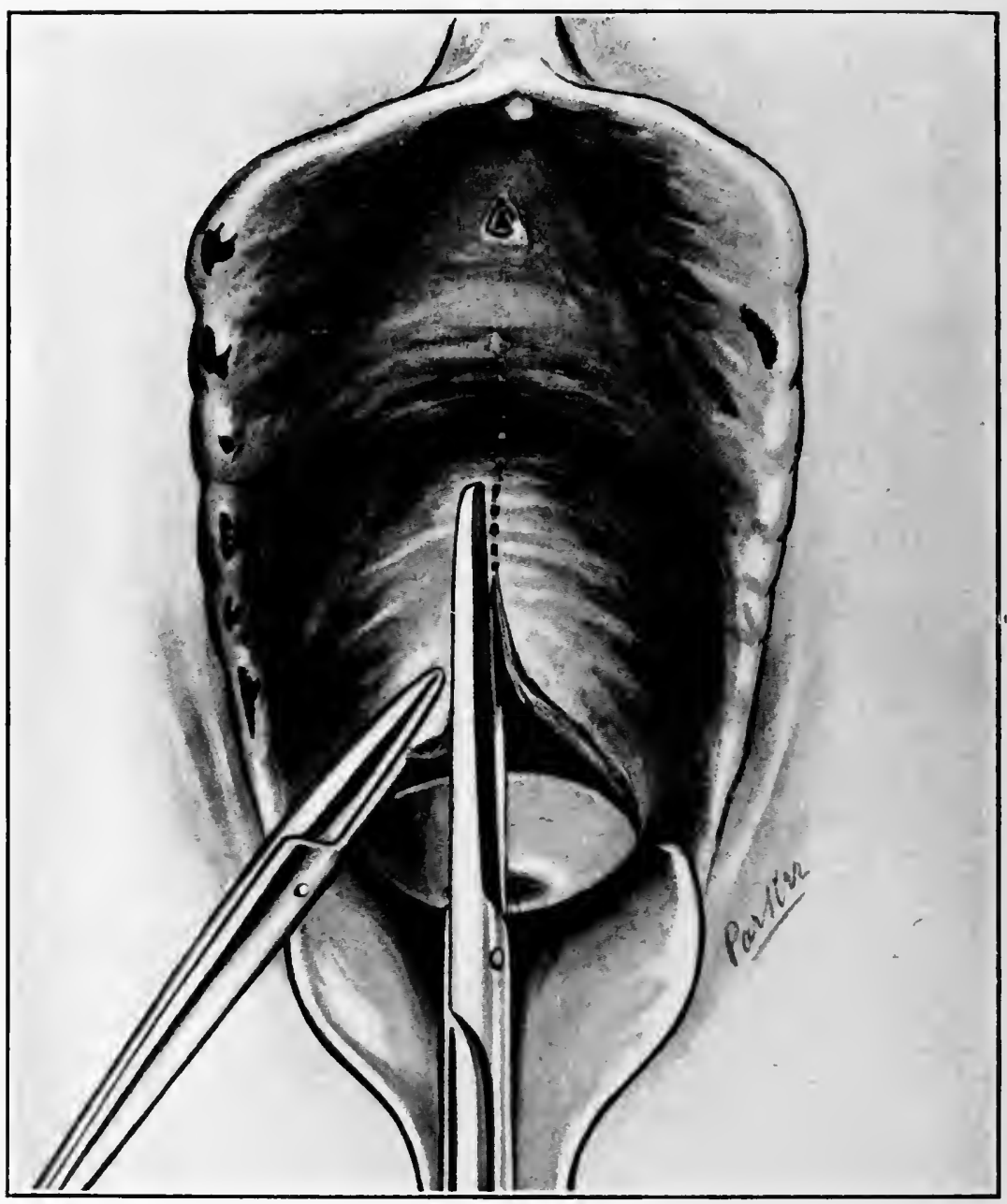

Broad Ligament Operation for Complete Descent of the Itterus. The bladder has been st ripped from the uterus, and the peritoneum exposed (Figure 358), the vesicovaginal septum is drawn down over the anterior uterine wall by a forceps, and the vaginal layer of the septum is being split on the median line with scissors. 
FIGURE 360

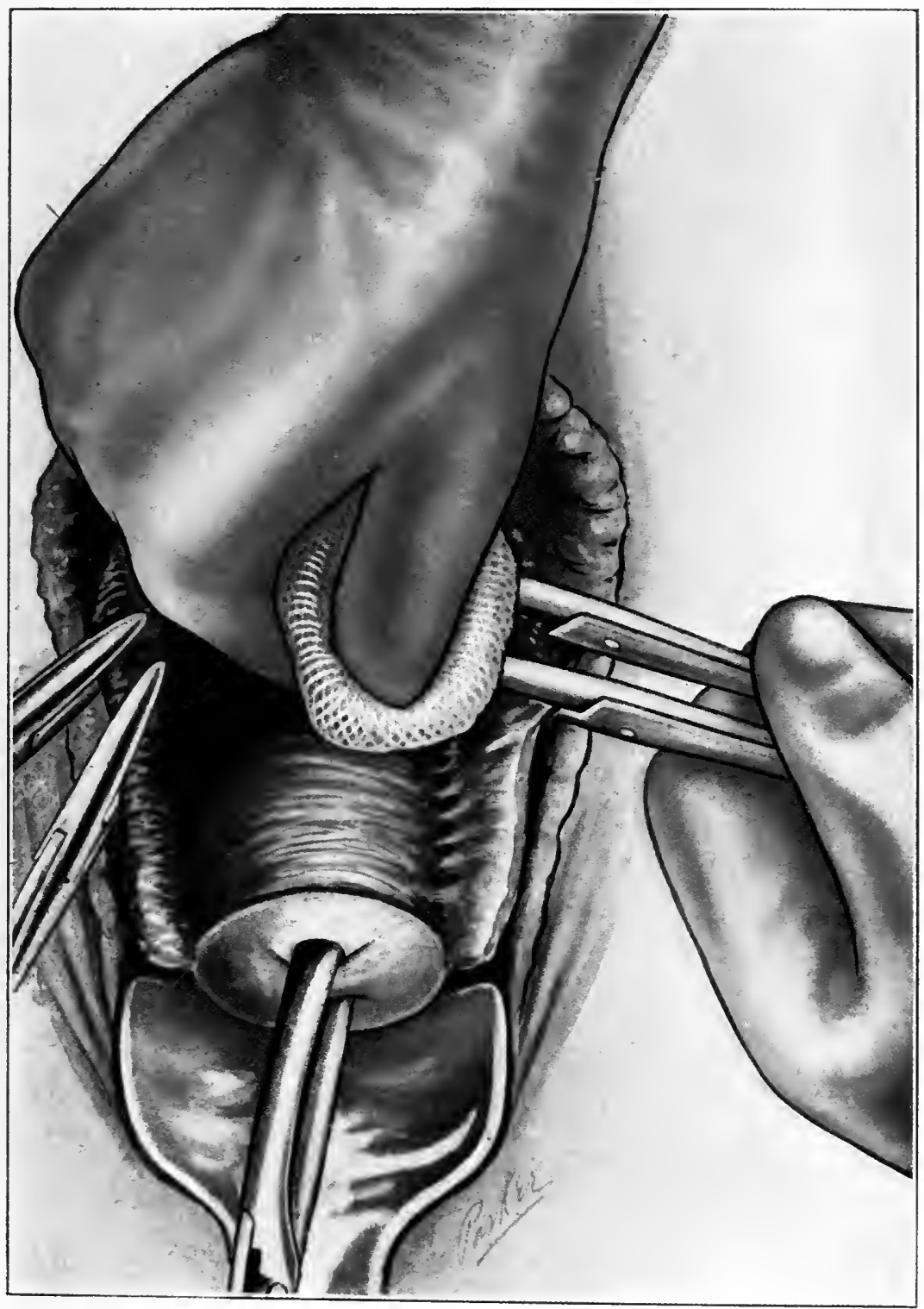

Broad Ligament Operation for Complete Descent of the L'terus. The vaginal layer of the vesicovaginal septum is being stripped away from the bladker wall by the same kind of blunt dissertion used in Figure 358 . The bladder as it is separated is mused up out of sight. 


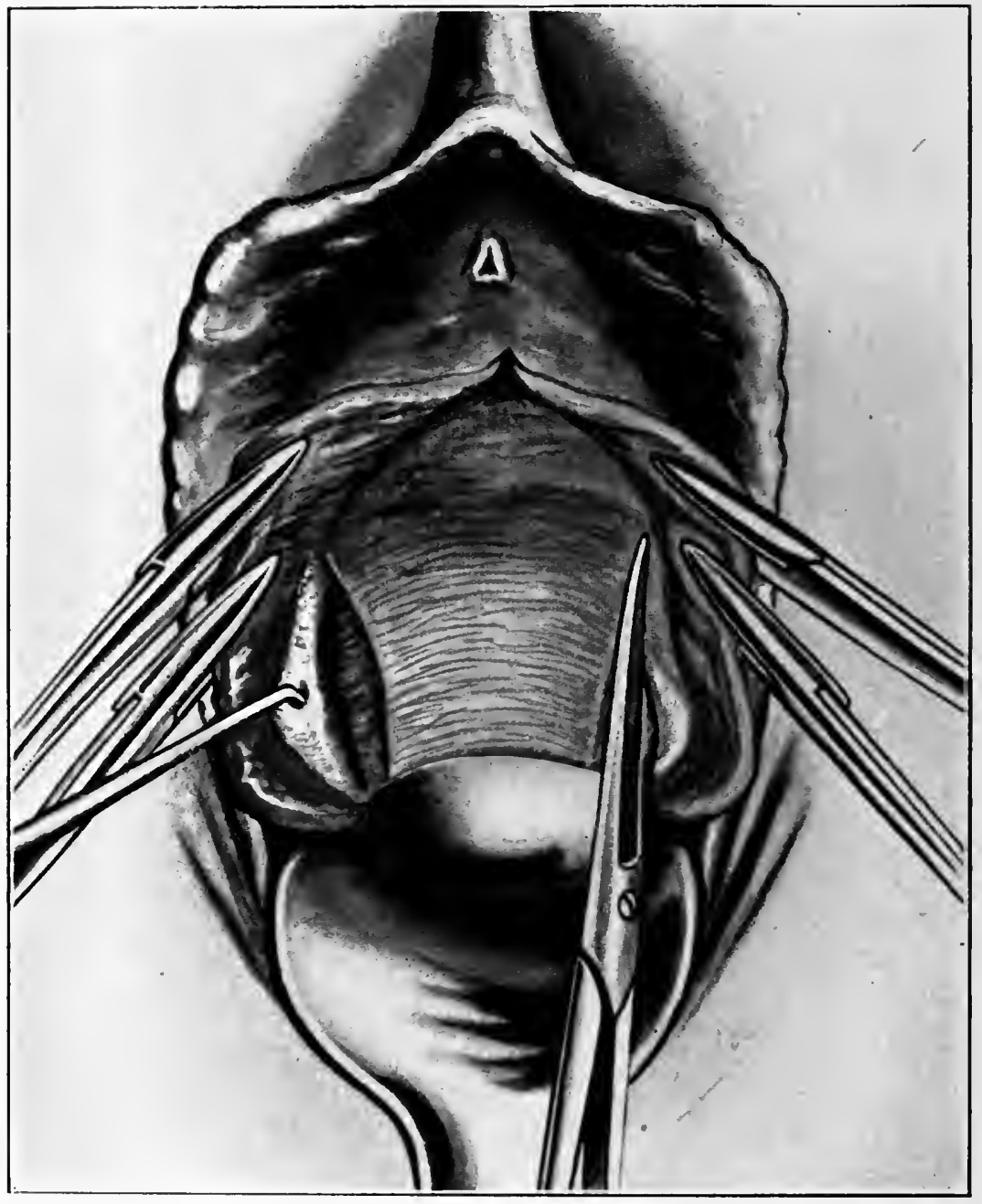

Broad Ligament Operation for Complete Descent of the C̈terus. The lower $t$ wo-thirds of each broal ligament, and, to avoid wounding the utero-ovarian anastomosis, a thin shaving also) of the cervix are being eut away with sharp-pointed scissors. The right ligament has been severed and the left is heing severed. The redundant vaginal layer (cystocele) which has been stripped off from the bladder is held by forceps out of the way to either side. 


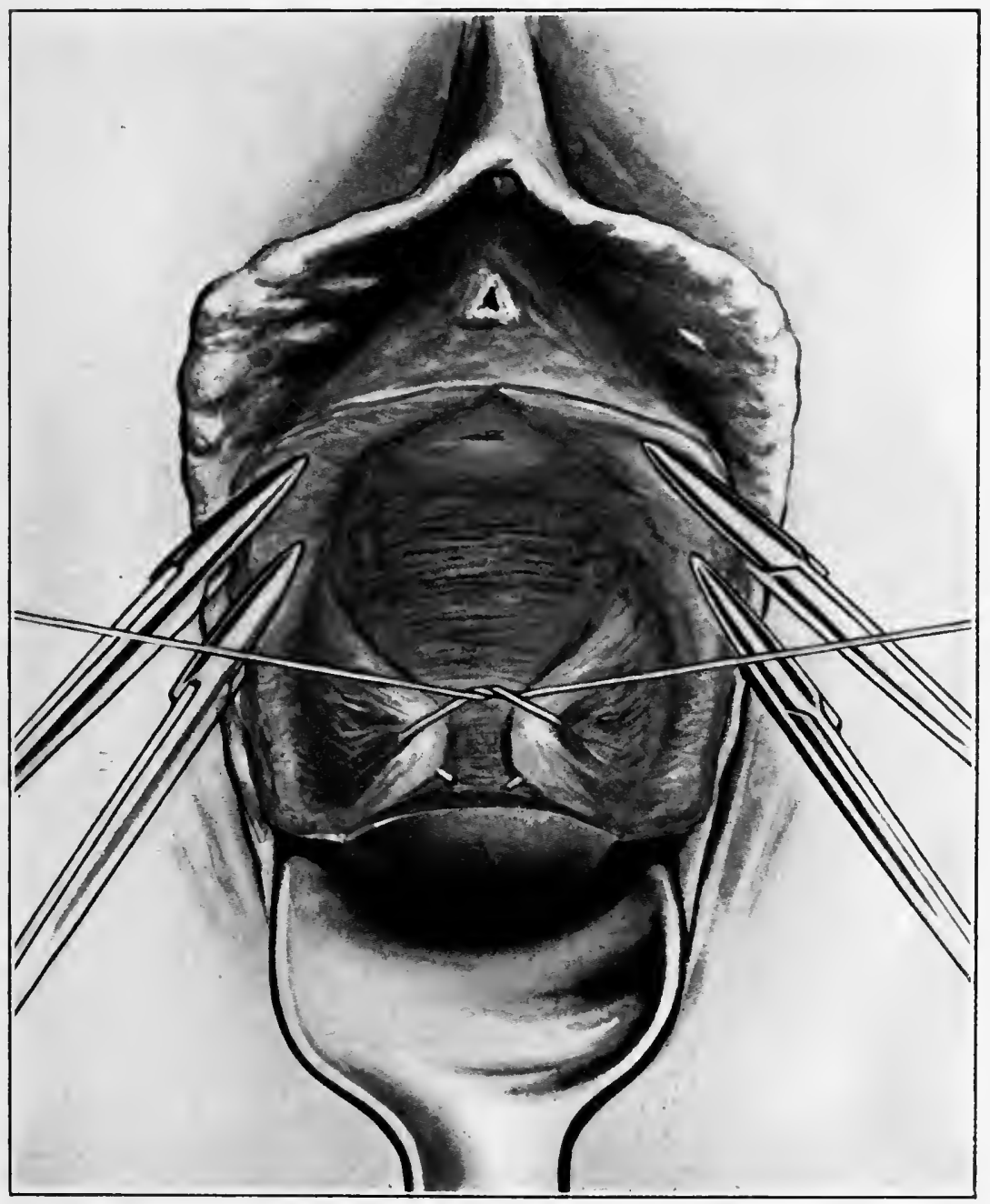

Broad Ligament Operation for Complete Descent of the Uterus. The severed lower two-thirds of the broad ligaments are being approximated end to end by catgut sutures. First suture in place and being drawn tight. These ends as they are drawn together already are forcing the cervix back. As already explained, the cut ends of these ligaments when united pull down together with them the adjacent parametric structures and in extensive operations also the round ligaments. Too tight tying is apt to strangulate the tissues. cause the sutures to cut out, and prevent union. No. 1 or No. 2 chromic catgut ordinarily is used. Silkworm gut is better, but difficult to remove. 


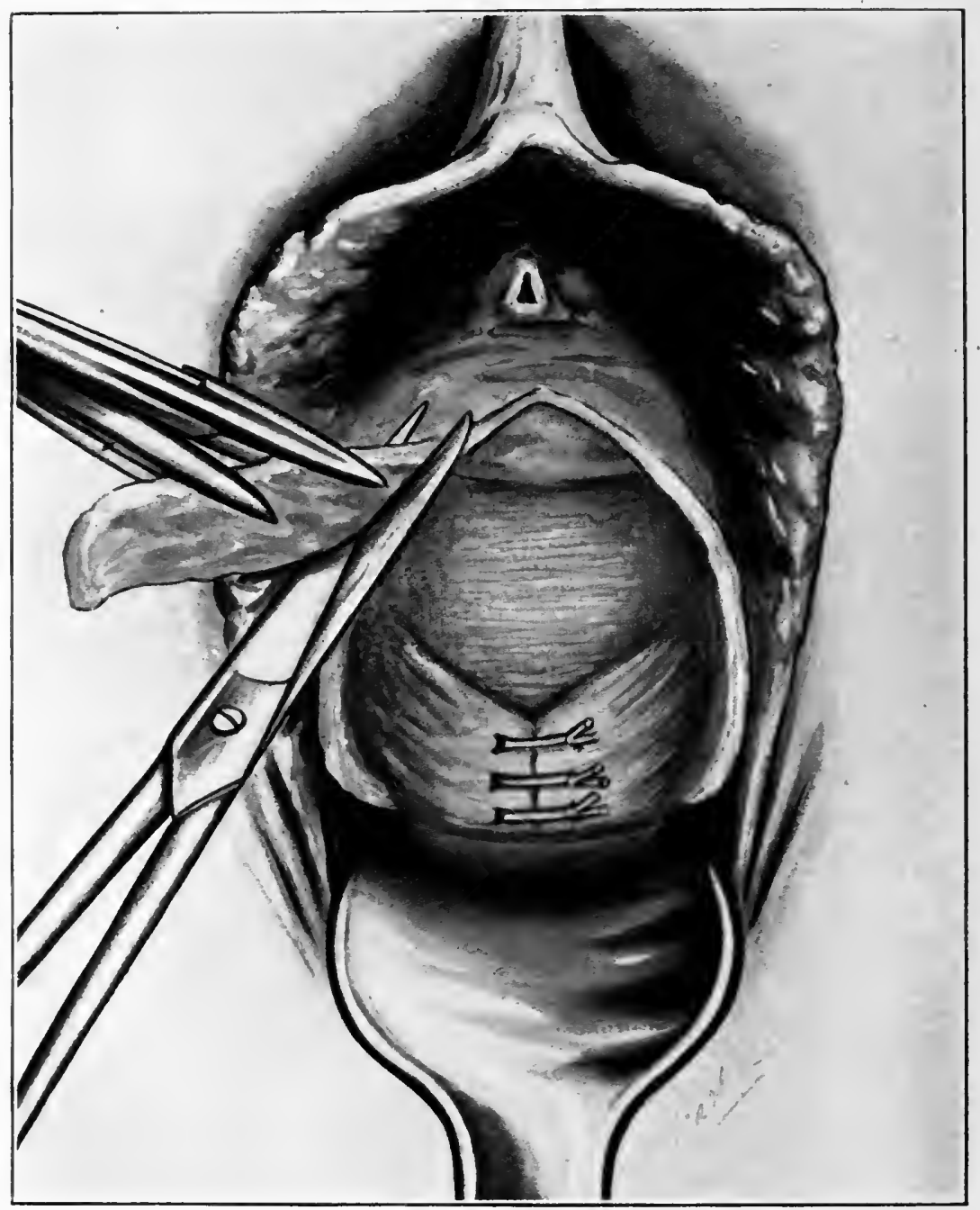

Broal Ligament Operation for complete Descent of the Cterus. The lower two-thirds of the severed broad ligaments have been united end to end by catgut sutures, thus forcing the cervix still farther back. The redundant vaginal wall (cystocele) has been cut away on the right and is being cut away on the left. The entire thickness of the vaginal wall is being cut away; this is in contrast to the older methol of superficial denudation. 


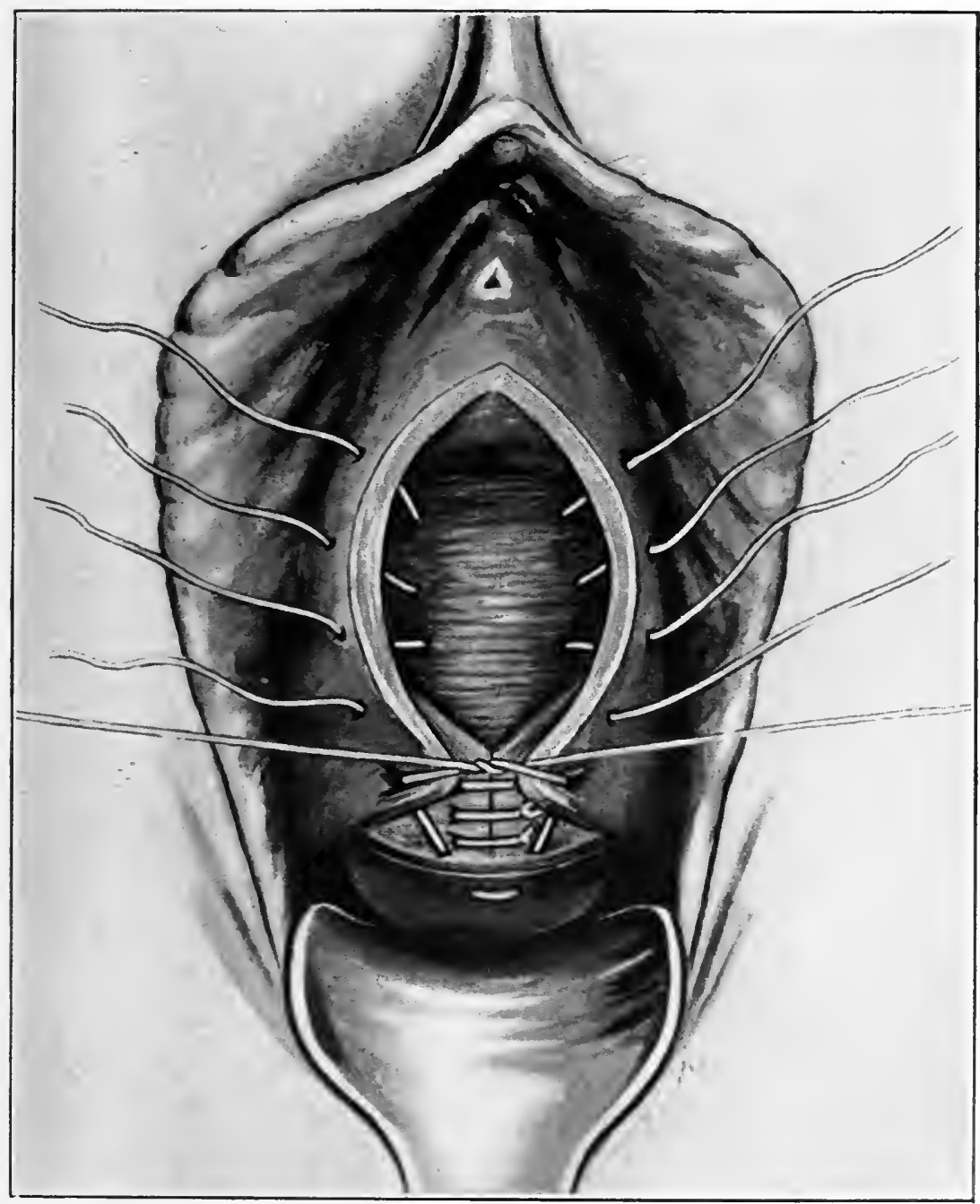

Broad Ligament Operation for Complete Descent of the Iterus. The severed ends of the broad ligamenthaving been united in front of the cervix and the cystocete excised, the cut edges of the vaginal wall are being approximated from side to side. The first suture, which is being drawn tight, shows how the margins are approximated near the cervix. The other sutures show the method of union between this first suture and the others, which are introduced but not tied. Observe that these sutures all catch up the uterine wall, thus forcing the blatder up so that it cannot come down again between the uterus and the vagina. This forcing up the bladder is a very essential factor in the success of the operation. 


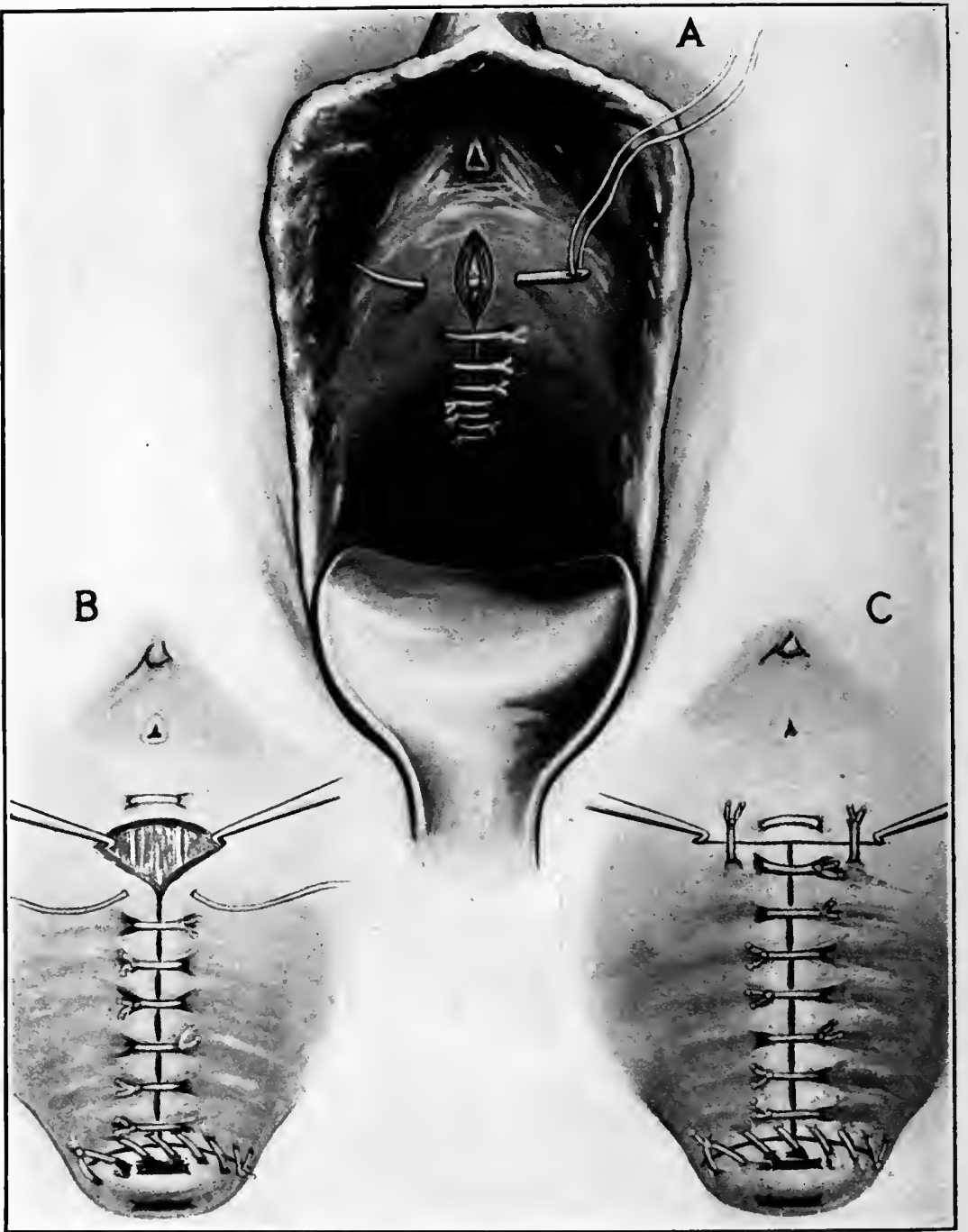

Broad Ligament Operation for Complete lescent of the ['terus. The redundant vaginal wall having been removed (Figure 363) the lateral margins of the vaginal wound are being united by interrupted sutures. As the result of this union the cervix and upper end of the vagina have been forced back to their normal location in the hollow of the sacrum. $\mathrm{B}$ and $\mathrm{C}$ show the lines of union complete. At the urethral end of the wound the redundant vaginal wall cannot be disposed of always by a median line of union. It may be necessary, as shown here, to unite that part in a direction at the right angles to the main line of union. Traction is being made by tenacula at each end of this transverse line of union. The cervical lines of union also have the $\mathrm{T}$-shape. 
and the perineum can be brought forward to their normal location under the pubes, so as to give support to the anterior vaginal wall, the latter will fall again, will drag the uterus after it, and the hernial protrusion will be reproduced. The treatment, therefore, of complete procidentia must always include an adequate operation upon the perineum, or, more comprehensively speaking, upon the posterior wall of the vaginal outlet. The operation must be performed so that it will carry the lower extremity of the vagina forward to the normal location close up under the pubes; then, if the anterior elytrorrhaphy is adequate, the whole vagina will have its normal oblique direction, and its long axis will make an acute angle to the long axis of the uterus. When this angle is maintained, the uterus cannot easily turn the sharp corner which will bring its long axis into coincidence with that of the vagina, and cannot, therefore, readily prolapse. See Chapter on Perineorrhaphy.

Comparison of Elytrorrhaphy and Hysterectomy.-As laid down in the foregoing paragraphs, the utilization of the broad ligaments is an essential factor in the treatment of complete procidentia. The operation of elvtrorrhaphy above described unfortunately either may fail to bring the lower edges of the broad ligaments sufficiently to the front of the uterus to enable them to hold up the uterus and vagina, or the ligaments having been stitched in front of the uterus the stitches may not hold. Consequently in complete procidentia elytrorrhaphy even though well performed may fail. At least this has been the author's experience in a number of cases. Therefore the completely prolapsed uterus may have to be removed in order to anchor absolutely the cut ends of the broad ligaments to the upper part of the vagina. As before stated, the operation should include the treatment of the hernial factor in the lesion-that is, removal of the redundant portion of the anterior vaginal wall. Generally speaking, the indications are somewhat as follows:

1. Extreme cystocele not associated with complete procidentia should be treated by elytrorrhaphy and perineorrhaphy.

2. Extreme cystocele associated with complete procidentia properly may be treated by hysterectomy, elytrorrhaphy, and perineorrhaphy.

3 . Conditions intermediate between the two conditions indicated above, and cases of very feeble or very aged women, will call for speeial judgment whether hysterectomy should be omitted or performed. It is, however, a fortunate fact that the completely prolapsed uterus even in aged women is removed usually with ease and with safety.

The indication for perineorrhaphy as a supplement to hysterectomy is the same as it is after elytrorrhaphy. After the senile changes of the menopause vaginal hysterectomy for obvious reasons is relatively unobjectionable, and in many cases may be preferred to elytrorrhaphy. In connection with hysterectomy, hovever, the removal of a portion of the anterior vaginal wall usually will be required.

Other Operations designed to decrease the weight of the uterus by removal of a part of it are of questionable value. The treatment of increased weight, due to subinvolution, hypertrophy, congestion, hyperplasia, and tumors, is described elsewhere under those subjects. 
Amputation of the cervix to lighten the weight of the uterus has been practised much for the spurious eircular hypertrophy and hypertrophic elongation shown in Figure 353. Since these two conditions are rare, if not indeed unknown, it follows that they could seldom furnish an indication for amputation of the cervix nteri. It is, in fact, difficult to imagine a class of cases in which this operation would be indicated. Emmet's explanation of the pathology of this class of eases has led to the substitution of trachelorrhaphy or of Schroeder's operation for amputation of the cervix.

Tumors increasing the weight of the uterus and tumors exerting pressure from above or traction from below should, if practicable, be removerl.

Alexander's operation and abdominal hysterorrhaphy are described under the surgical treatment of retroversion and retroflexion. 'The object of these operations is to suspend the uterus from above. Hysterorrhaphy, which fulfils this indication better than shortening the round ligaments, may be indicated in cases of extreme relaxation of the uterine supports and greatly increased weight of the uterus. The results of it in procidentia, however, will usually not be permanent unless it is supplemented by elytrorrhaphy and perineorrhaphy.

\section{Enteroptosis as a Complication of Descent of the Uterus.}

In many cases of descent of the uterus the displacement is aggravated by alterations in abdominal pressure associated with descent of the abdominal viscera, especially the stomach and intestine. These conditions are gastroptosis and enteroptosis. Webster has laid stress upon the fact that general weakness and laxity of the abdominal wall are not, as usually supposed, the eause of the enteroptosis, but that descent of the viscera is calsed by excessive separation of the recti muscle, due to stretching of the linea alba. All conditions that increase abdominal pressure, especially during pregnancy, such as the wearing of corsets and overwork, predispose to enteroptosis. Loss of fat is also a cause of enteroptosis.

Success in the treatment of uterine displacement will depend frequently upon associating with it some effective measure for the correction of the pendulous abdomen-that is, of the enteroptosis. 'Temporary correction may be secured by a properly fitting abdominal bandage. Permanent correction in most cases will require abdominal section and such closure of the wound as will correct the diastasis of the recti muscles. The operation is the same in principle as that described by Edebohls for closure of the abdominal wound, an operation set forth in Chapter VI. II this operation it was not proposed to open the abdomen for the purpose of treating enteroptosis, but, the abdomen having been openerl for other purposes, to close it in such a manner as to overcome any pendulous condition that might exist. The operation proposed by Webster is the deliberate opening of the abdomen for the cure of enteroptosis. It is much the same as that which for a long time has been performed for the relief of ventral hernia, and is substantially as follows: 
An incision is made in the median line divicling the skin and subcutaneous fat until the linea alba is exposed. The length of the incision should vary with the extent of the separation of the recti muscles. In aggravated cases it should extend from the symphysis pubis nearly to the ensiform cartilage. The umbilicus, if deep and difficnlt to clean thoronghly, should be removed; otherwise the mesial incision may be carried around to the left of it. 'The skin and fat should be dissected from the fascia on both sides, so as to expose the edge of each rectus muscle. The sheath of each muscle is then split longitudinally along the inner border and the incision continued to the extent of the diastasis. The inner borders of the muscles are then loosened from the sheaths and united by a series of sutures passed from side to side throngh each muscle and the corresponding anterior fascia or sheath layer. These sutures when tied will obliterate the stretched-out linea alba, approximate the muscles and cover them with fascia. Webster recommends strong linen sutures, which are left permanently buried. The anthor uses chronic catgut as a continuous suture throughout. See Chapter VI. Some bulging of the skin may follow the reduction in the size of the inner abdominal wall, but after a little time this usually disappears. In cases of excessive relaxation a strip of fat and skin may be removed before passing the superficial sutures. The use of a broad silk elastic binder, daily massage of the abdominal parietes, followed later by light gynmastic exercise and abstinence from severe exertion during a period of six months, are recommended as part of the after-treatment.

On the suggestion of Edebohls, the author has for several years, when closing the abdominal incision, felt himself justified in splitting the sheaths of the recti muscles and bringing the fascia together with continuous chromic catgut sutures as a means of protection against possible diastasis of those muscles and consequent enteroptosis. 


\section{CHAPTER XLVI.}

\section{ETIOLOGY, SYMP'TOMS, COURSE, DIAGNOSIS AND PROG- NOSIS OF RETROVERSION AND RETROFLEXION.}

\section{RETROVERSION.}

RETROVERsion is that abnormal position of the uterus in which the fundus is posterior to the axis of the pelvic inlet. If the cervix be in its normal place, near the sacrum, retroversion is scarcely possible, because prevented by the proximity of the over-arching sacrum. See Figure 341. The first degree of prolapse must, usually, precede any considerable backward turning of the uterus. When the cervix has been displaced downward and forward so far that its distanee from the sacrum is equal to or greater than the length of the uterus, retroversion to any extent becomes possible. See Figure 366 .

\section{Etiology and Description of Retroversion.}

From the above it follows that the causes of beginning retroversion must be identical with the causes of the first degree of prolapse. After the puerperium the relaxation of the supports and the weight of the displaced organ may persist, and this, together with the pressure and weight of the intestine upon the anterior surfaces of the uterus, may prevent spontaneous replacement. Every act of defecation forces the cervix forward and downward, and the uterus, being in the axis of the vagina, and having, therefore, little support below, must depend for support upon the now inadequate subinvolnted peritoneal suspensory ligaments and pelvic fascia. Abortion, with resulting increased weight and relaxation of the vaginal walls, is a common cause of descent. Metritis, parametritis, perimetritis, peritonitis, salpingitis, and ovaritis are frequent complications, and may stand in the relation of cause or effect.

Congenital retroversion is rare. Retroposition of the small senile uterus after the menopause is not abnormal. Peritoneal adhesions and cicatricial bands may fix permanently the corpus in a retroverted position. In extreme retroversion the corpus often is incarcerated between the uterosacral ligaments under the promontory of the sacrum. Chronic cystitis and consequent contraction of the bladder shorten the vesicovaginal wall, and thereby draw the cervix forward. This makes a permanent incurable displacement.

The causes of retroversion may be summarized as follows :

1. Distention of the bladder.

2. Increased weight of the uterus and relaxation of the supports - common cause in early puerperium.

3. Retro-uterine peritonitis-contracting adhesions. 668 
4. Sudden straining, violent fall, or blow-rare.

5. Chronie cystitis, which shortens the vesicovaginal septum by contraction-an intractable cause.

6. Small myoma in posterior wall of the corpus uteri.

7. The dorsal position and tight bandaging in the puerperium.

8. Congenital-rare.

\section{Symptoms and Course of Retroversion.}

The displacement of retroversion and its numerous eomplications usually cause bearing-down sensations, a feeling of heaviness in the pelvis, exhaustion upon walking and standing, especially the latter. Constipation may be a cause or an effect. After the puerperium the extreme engorgement of the pelvic organs often contributes to hemorrhagic endometritis. 'The hemorrhage then should not be confounded

Figure 366.

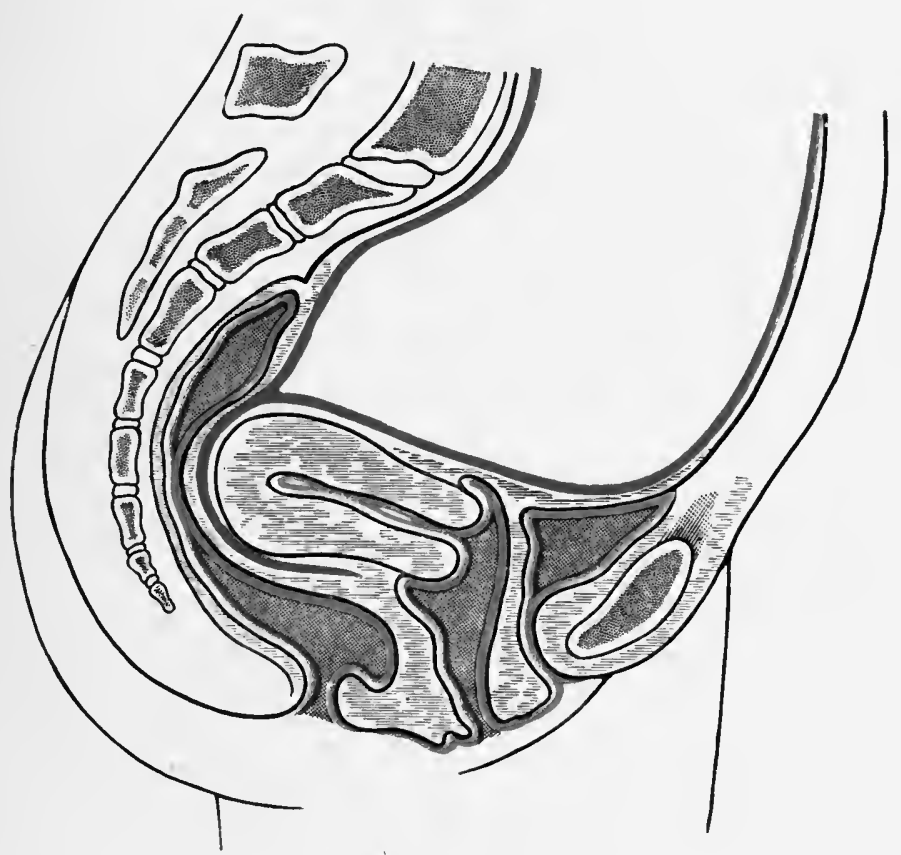

Retroversion.

with returning menstruation. The bleeding, especially after abortion, unless relieved by treatment, often persists for a long time. Gradual or sudden replacement may occur spontaneously; or, the causes continuing active, the displacement may persist and even be reinforced by cystocele and rectocele. There is usually coneurrent displacement of the ovaries and Fallopian tubes. Nutritive changes in the uterine walls may induce a superadded retroflexion. The heavy organ may descend along the relaxed, subinvoluted vaginal walls even to complete procidentia. 


\section{Diagnosis and Prognosis of Retroversion.}

The symptoms indicate the probability of displacement, but definite diagnosis depends upon direct examination. Conjoined manipulation will usually establish the diagnosis and show the organ retroverted, with the cervix displaced toward the pubes and with the corpus in the hollow of the sacrum. In certain cases of anteflexion, as represented in Chapter XLVIII., the cervix is bent forward in the vaginal

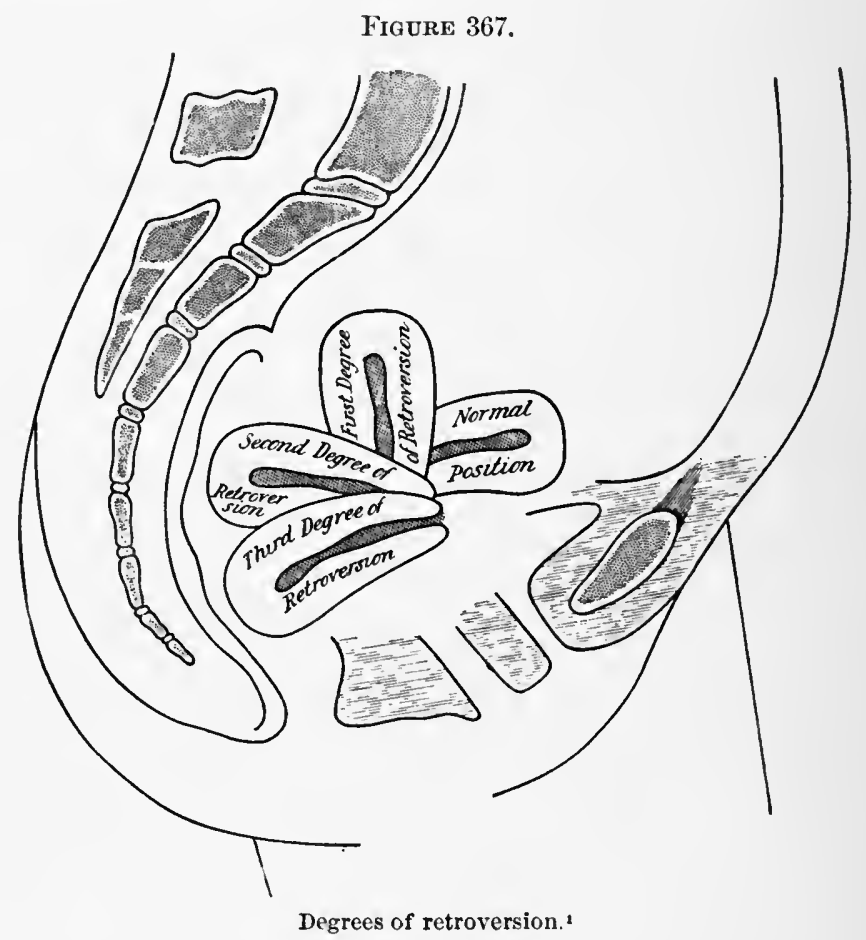

axis as in retroversion. The condition is in reality one of retroversion of the cervix, with high anteflexion of the corpus. Under treatment, the prognosis, both for speedy relief and ultimate recovery, is generally favorable.

\section{Degrees of Retroversion.}

Retroversion will be slight or extreme according to the extent to which the axis of the uterus is turned back. Three degrees of displacement usually are recognized; but the division is arbitrary, and, except for purposes of description, has no practical significance. See Figure 367. 


\section{Treatment of Retroversion.}

The treatment, as in descent, consists of the removal of the inflammatory and other complications, in the use of pessaries, and in surgical operations. Inasmuch as the treatment is similar to that of retroflexion, the treatment of retroversion and retroflexion will be presented together. See Chapter XLVII.

\section{RETROFLEXION.}

Retroflexion is that displacement in which the organ is bent back upon itself. It usually, though not always, results from, and is asso-

Figure 368.

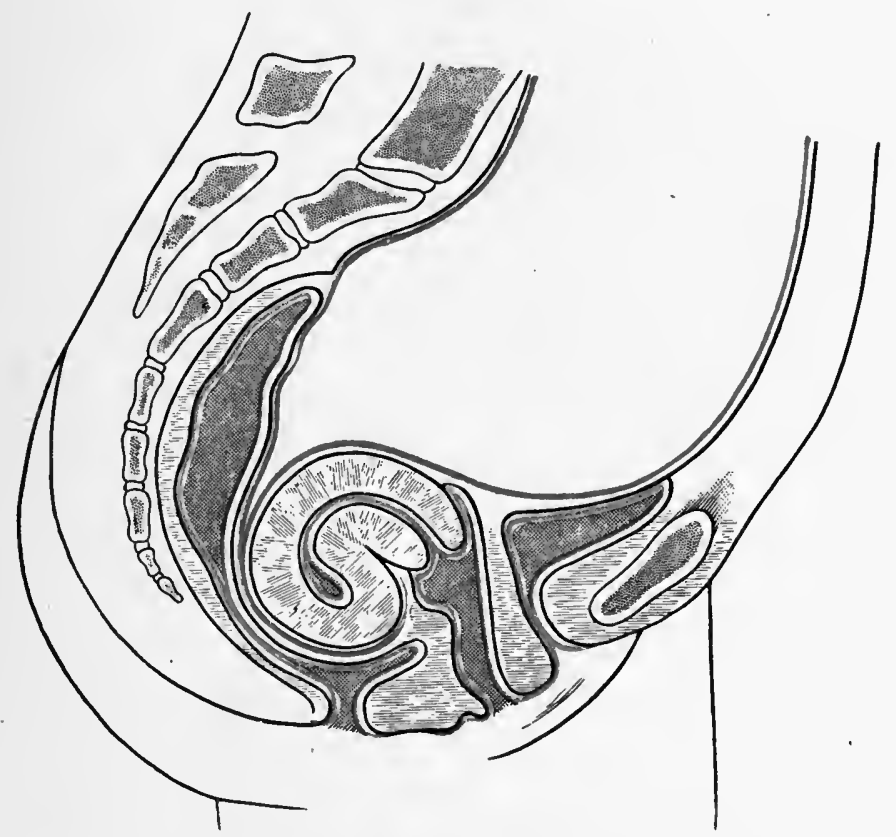

Extreme retroflexion with hypertrophy of the corpus uteri. The uterus impinges on and compresses the rectum.

ciated with, retroversion; in accordance with custom, the double displacement will be termed retroflexion.

\section{Etiology and Pathology of Retroflexion.}

The causes of retroflexion are identical with those of retroversion. which to a very great extent may be summarized as follows :

1. All causes of retroversion, see Descent and Retroversion.

2. The dorsal position and tight bandaging in the puerperium.

3. Tight lacing and tight clothing. 
4. An infectious puerperium impairing the nutrition of the uterine walls and uterine supports.

5. Pressure by tumors.

6. Congenital conditions-rare and usually associated with underdevelopment of the other reproductive organs.

7. Great weight of the corpus uteri.

8. Soft mobile condition of the uterine walls-common during the puerperium.

9. Intra-abdominal forces, such as downward pressure during defecation.

10. Metritis and perimetritis, especially when associated with post-uterine adhesions.

The ovaries and Fallopian tubes, unless fixed elsewhere by adhesions, are held down usually on either side of the corpus uteri. They are sometimes much enlarged by inflammation, often adherent, and always extremely sensitive. Infection of the uterus and its appendages from baeterial invasion is almost invariably the essential cause. The displacement often follows parturition, abortion, and injudicious treatment. Gonorrhoea and the puerperal infections are frequent causes. Peritoneal adhesions between the corpus uteri and the pouch of Douglas may render replacement impossible, except by abdominal or vaginal section.

\section{Symptoms and Course of Retroflexion.}

In some cases there are no subjective symptoms. The conditions frequently associated with retroflexion are these:

1. Sterility and frequent abortion.

2. Uterine discharges-lencorrhœa.

3. Menstrual disorders-dysmenorrhœa, amenorrhœa, uterine hemorrhages.

4. Constipation and painful defecation.

5. Bladder disturbances.

6. Weakness in the back and dragging sensation in the pelvis.

Uterine discharges, menorrhagia, and abortion usually are the result of associated endometritis, and are due to the effort of an engorged uterus to relieve itself of congestion by increased secretions or increased menstruation.

Abortion, dysmenorrhœa, and sterility mav result from a wide range of associated conditions, chief among them faulty nutrition, inflammatory complications, and mechanical obstruction in the uterine canal at the angle of flexure. The rectal symptoms are caused by the proximity of the inflamed uterus, and its appendages, to the bowel. This gives to the patient the sensation of a full bowel, and is therefore a cause of tenesmus. Passage of the bowel-contents through this sensitive zone is necessarily painful. Abdominal pains, nervous dyspepsia, nenralgia in distant parts of the body, and neurasthenia are often present ; indeed, the nervous symptoms may be of the most exaggerated character, and may comprise all that is implied by the word hysteria in its most comprehensive signification. 
Should pregnancy oeeur, the rapid growth of the uterus may induce spontaneous reposition; this is likely to take place when the fundus rises out of the pelvis at about the fourth month; but if the corpus be incarcerated under the sacral promontory from adhesions or from any other cause, the uterus, unless manually replaced, will relieve itself by a dangerous abortion.

\section{Diagnosis of Retroflexion.}

The diagnosis should include especially an inquiry into:

1. The location and position of the uterus relative to neighboring organs.

2. The mobility of the uterus.

3. The complications.

Digital touch discloses the eervix uteri low in the pelvis. The fundus uteri is felt through the posterior vaginal wall in the cul-de-sac of Douglas. Conjoined manipulation with the index-finger of the left hand, first in the vagina, then in the rectum, and the right hand over the hypogastric region, will show the size, form, consistence, and location of the uterus, the degree of flexion, and the difficulty of replacement. An inflammatory deposit or abscess posterior to the nterus, or a fibroid in the posterior uterine wall, may be mistaken for the retroflexed corpus. The probe is seldom necessary to verify the diagnosis. It should be used under strict antiseptic conditions, for otherwise additional infeetion may be introduced. In some cases of difficult diagnosis it is better at first to direct the treatment to the inflammation and defer the precise diagnosis of the displacement to a later date. Great and lasting injury may be wrought.in the attempt to complete the diagnosis at the first examination. The presence of a small myoma in the posterior uterine wall, with post-uterine inflammation, is a serious eomplication both in diagnosis and treatment. If the rectum be loaded with fecal matter, a cathartic should be given and the complete digital examination deferred. The displacement is distinguished from the presence of an ovary or small ovarian tumor in the pouch of Douglas, by careful bimanual examination and by the probe.

Diagnosis in the Puerperium.-Uterine hemorrhage which begins two or three weeks after labor, and small daily losses of blood during the puerperium, are evidences though not proof of retrodisplacement.

Diagnosis of Complications.-The following complications may have the relation either of cause or effect to the displacement :

1. Perimetritic fixation-result of perimetritis-is recognized by bands of adhesions palpated behind and to the sides of the uterus and felt about the corpus above the plane of the internal os, near the fundus. Perimetritic adhesions commonly fix the uterus to the tubes, ovaries, or broad ligaments, and such adhesions form a mass recognized by conjoined palpation.

2. Parametritic fixation-result of parametritic cellulitis-extends more usually below the plane of the os internum; it draws the 
cervix laterally, anteriorly, or posteriorly toward the pelvic wall by contraction of inflamed cellular tissue, which is thicker and more dense than in perimetritic adhesions, and lower in the pelvis.

\section{Differential Diagnosis of Retroflexion.}

The differential diagnosis should include a consideration of inflammatory retro-uterine masses, retro-uterine myomata, hæmatocele, and fecal accumulations.

Retro-uterine inflammatory products and myomata in the posterior wall of the uterus may be recognized by the location of the fundus uteri lying in front of the mass. Examination should be made by :

$a$. Conjoined palpation with or without narcosis.

$b$. The uterine sound-used with caution.

c. Conjoined rectal touch-most important.

The tumor, whether inflammatory, myomatous, or hæmic, is usually wider than the uterus, often not situated directly behind the cervix, but to one side, and may be irregular in outline. The contrary is true of the retroflexed corpus uteri.

Fecal accumulations may be excluded by cathartics. 


\section{CHAPTER X L VII.}

\section{TREATMENT OF RETROVERSION AND RETROFLEXION.}

uterus.

THE objects of treatment are replacement and retention of the

\section{Obstacles to Replacement.}

The obstacles to replacement are tumors, inflammation, and fixation of the uterus. The inflammatory complications often require weeks, and in severe cases months, of treatment preparatory to replacement; not uncommonly a tumor must be removed by a surgieal operation. Some of the general therapeutic suggestions under the subject of descent are also applicable to retropositions. Thus rest, massage, careful regulation of the bowels, forced feeding, and general tonics may be essential.

For pelvic inflammation, small blisters over the inguinal regions, frequently repeated, and the daily application of a cotton and glycerin tampon to the cervix, are common routine measures of some value. The most useful and essential topical application is the hotwater vaginal douche. The proper manner of giving the douche is described in Chapter IV. See also Chapter XXII.

As the tenderness disappears the cotton tampons may be increased in quantity, and thereby made to serve as temporary support for the uterus until the more permanent pessary can possibly be substituted. The sluggish circulation in the pelvis and torpid condition of the bowels may be much relieved by the daily application of the hot hippack ; it is applied as follows :

A small flannel sheet, folded lengthwise to the width of two feet, dipped in very hot water and dried by passing it through a wringer, is wound about the hips and covered by another dry one. At the end of half an hour, during which time the patient maintains the recumbent position, the sheets are removed. Hot-water bags between the wet and the dry sheet will serve to prolong the heat.

When the tenderness has been sufficiently reduced, gentle attempts at replacement may be made every day or two by conjoined manipulation. The patient's tolerance of manipulation may thus be observed and the way prepared for complete replacement and permanent retention after subsidence of the inflammation.

Fixation and tenderness, until overcome by appropriate treatment, are contraindications to replacement. 


\section{Methods of Replacement.}

Manipulation.-Diseased adnexæ, especially salpingitis, contraindicate forcible manipulations of the pelvic organs. The dangers incident to stretching or breaking adhesions or contractions are very great unless the manipulator possesses unusual diagnostic and manual skill. The safest and most effective method of replacement is by conjoined manipulations, as shown in the following illustrations. Efficient reposition of the nterus is very often impossible without anæsthesia. This is especially true when the corpus is wedged in and incarcerated between the uterosacral ligaments under the sacral promontory, a condition often mistaken for displacement with adhesions.

The replacement is not usually accomplished by drawing the fundus directly forward and pushing the cervix back directly in the median line, but in most eases by sweeping the fundus around the arc of a circle on the left side of the pelvis and the cervix on the right. This is owing to the greater frequency of infection on the left side, and consequent shortening of the left broad ligament. After replacement the organ is to be held in position by appropriate means.

Bimanual replacement has three great advantages over the more familiar methods of the sound or repositor: first, it is more effective and more permanent; second, the lever acticn of the sound or repositor, by which the operator may unwittingly use an undue and dangerous amount of force, is avoided in the use of the hand third, the operation is not only constantly under the operator's control, but also within his appreciation. Experience has abundantly shown that instrumental uterine reposition by means of the sound or other instruments which enter the endometrium, and act by leverage, is unnecessary, dangerous, and therefore usually disapproved.

Brandt Method.-Manipulation has some value in overcoming the obstacles to replacement and has well-defined value in the replacement of a uterus. The manipulations described below are those of Brandt. The methods of Brandt involve much complicated massage and varions gymnastic movements, and are available only to the specially trained expert ; besides, for obvious reasons, when long continued they would be regarded, at any rate in this country, as somewhat objectionable even though entrusted to a masseuse. The author has no personal experience in the administration of this form of treatment, nor has he been able usually to command the services of a competent and satisfactory masseuse. Certain manipnlations of the Brandt system, however, apart from the local massage and the associated gymnastic movements, are adapted to the detection of intrapelvic lesions, and therefore are set forth here partly for their diagnostic value, but they are presented more especially for their value in the replacement of the retroposed uterus.

Manipulation in the Treatment of Retroposition Complicated by Anterior Adhesions and Contractions.-A serious obstacle to replacement and retention of a retroposed uterus is the presence of contracted tissne between the cervix uteri and the pubes, which antelocates the cervix to snch an extent that the corpus has space to fall back under the sacral promontory. Under such conditions the corpus cannot be 
TREATMENT OF RETROVERSION AND RETROFLEXION. 677

Figure 369.

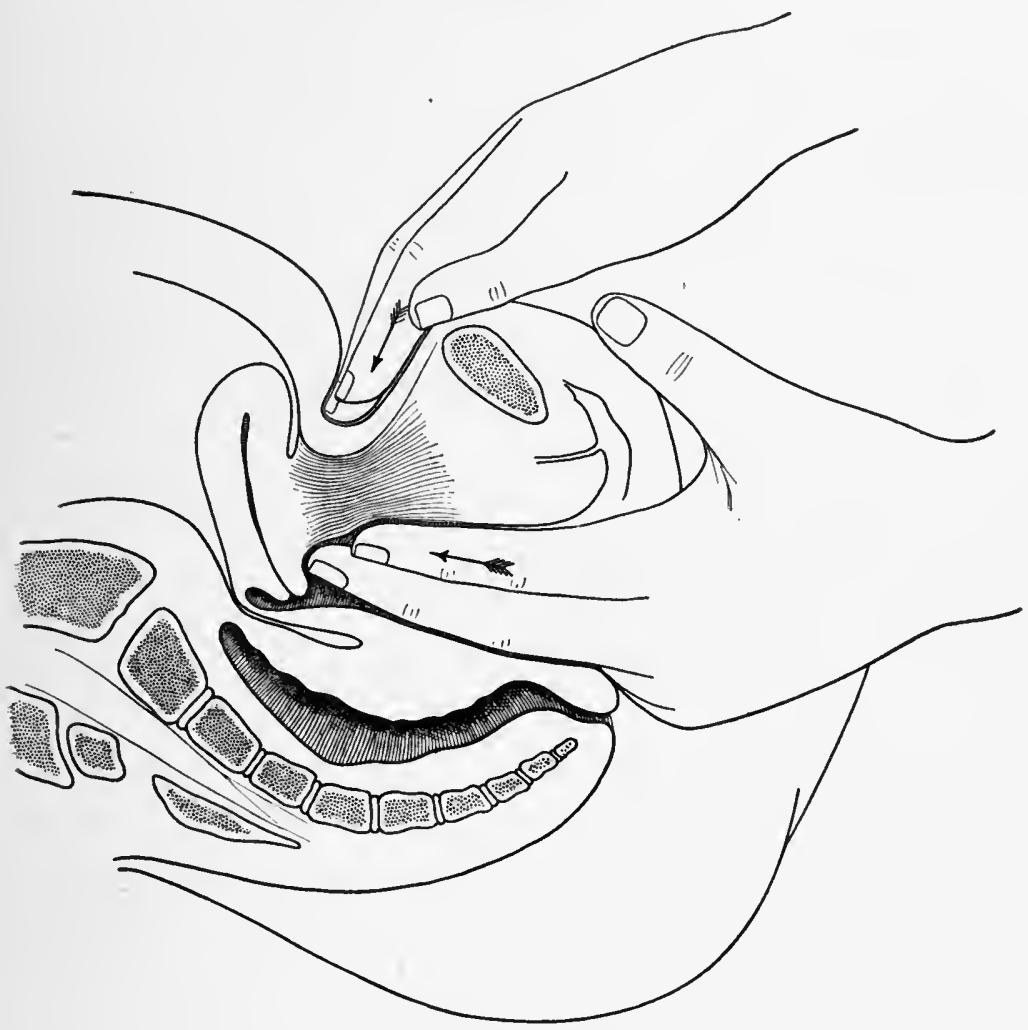

Stretching adhesions anterior to the uterus.

Figure 370.

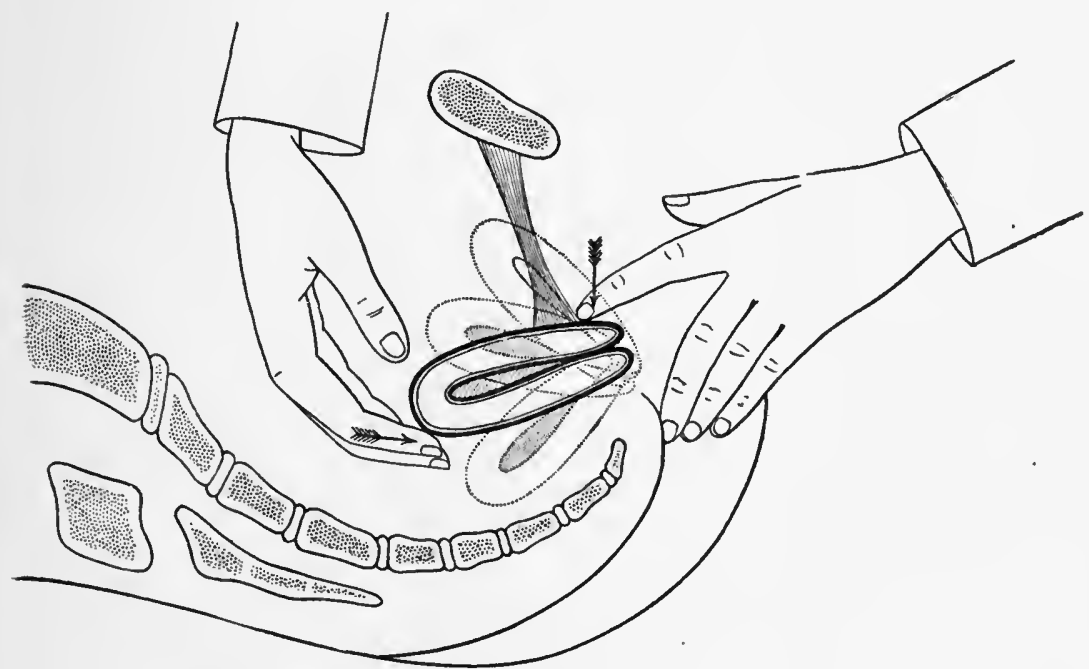

Resisting anterior attachments. Dorsal position: intravaginal finger pushes cervix downward and backward; corpus thrown forward on its transverse axis; right hand draws corpus upward and forward by exerting force through abdominal wall. 41 
brought forward and retained in normal anteversion until the contracted tissue or bands which hold the cervix forward can be so stretehed or broken as to permit the cervix to return to its normal location near the hollow of the sacrum; this may sometimes be accomplished by manipulation if the adhesions and contracting bands are not too strong. Several weeks of treatment may be required to obtain the desired result. The manipulations of anterior adhesions, shown in Figures 369 and 370 , are as follows:

The left index-finger is introduced first back of the cervix and the body of the uterus is raised as far as practical in the median line. The intravaginal finger is then transferred to the front of the cervix, while the external hand readily pushes the uterus backward, so as to move it away from the symphysis and still further stretch or break the adhesions. By pressing the fingers of the external hand down behind the symphysis they are made to meet the intravaginal fingers in front of the uterus. The fingers of the two hands thus brought together then push the uterus in the following directions: the internal fingers backward and upward, the external fingers backward and downward. This manipulation should not be carried beyond a certain limit to the ready extension of the tissues.

Manipulation in the Treatment of Retroposition Complicated by Pos. terior Adhesions and Contractions.-Posterior adhesions and contrac-

Figure 371.

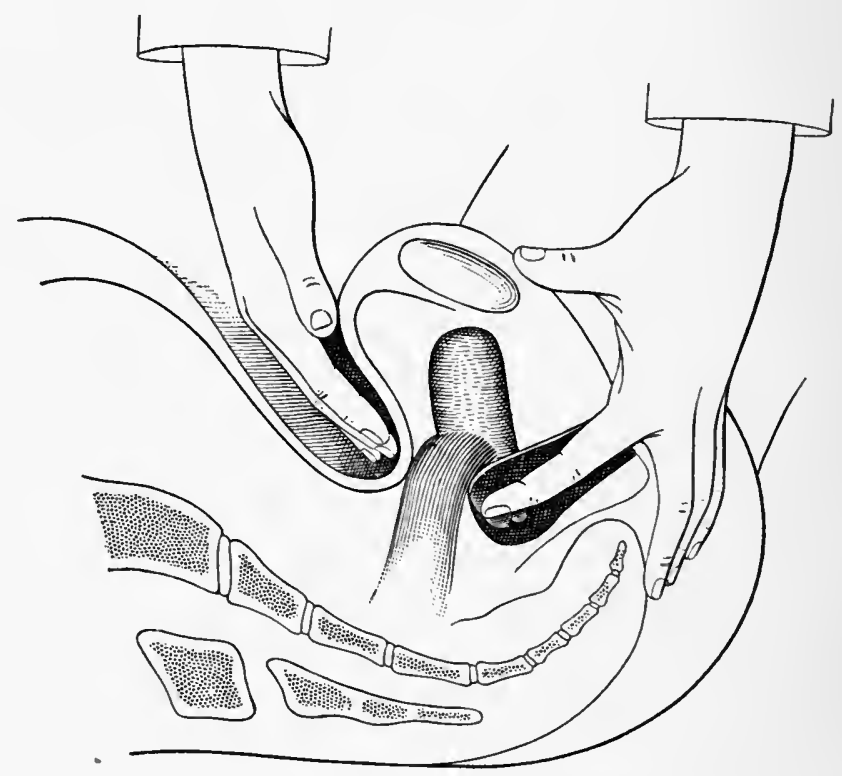

Stretching or breaking posterior adhesions.

tions may be stretched and broken on the same principles and by the same manipulations as those already set forth for anterior adhesions and contractions. Figure 371. 


\section{Replacement and Retention of the Retroposed Uterus.}

When the uterus is mobile and not too sensitive, replacement and retention in the normal position are indicated, and may be accomplished in the manner set forth in the following text and illustrations.

\section{Manual Ventrovaginal Reposition of the Retroposed Uterus.}

Figures $372-376$ taken together will explain an efficient method of replacing a retroposed uterus. The left index-finger, in the posterior vaginal fornix as high as possible, raises the uterus toward the abdominal wall-Figures 372 and 373 -while the fingers of the right hand above the symphysis press down on the cervix, the point of pressure being as nearly as possible the plane of the internal os,

Figure 372.

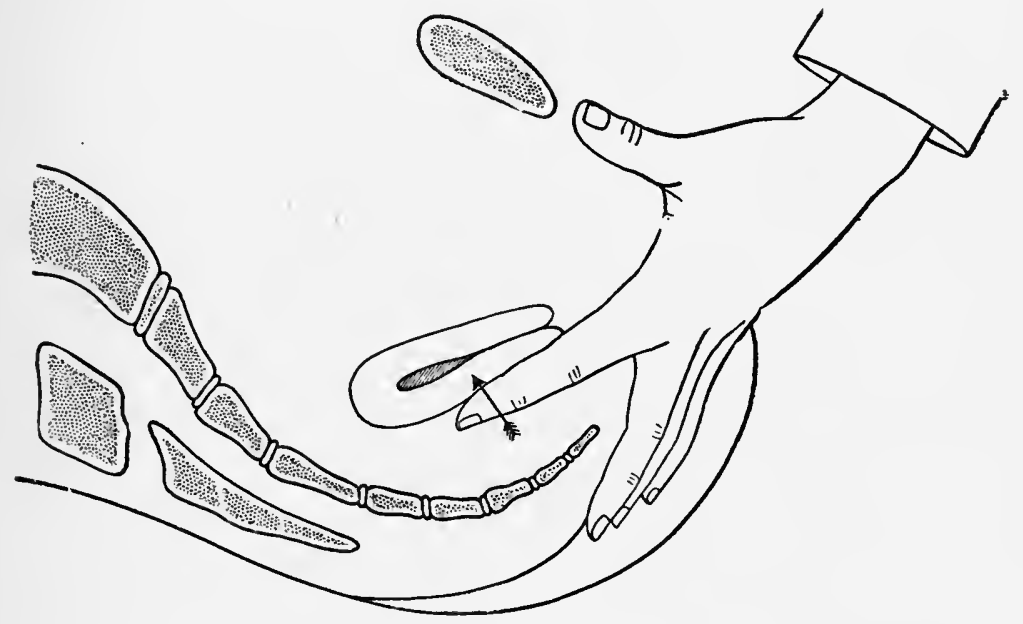

Ventrovaginal reposition; beginning of first step.

Figure 374. The left index-finger, then leaving the posterior, passes to the anterior fornix and approaches the fingers of the right hand, Figure 375. Both lands, acting together, push the cervix upward and backward, while the uterus tends to fall over slightly forward. Then, while the left index-finger is kept fixed, the fingers of the right hand are passed lightly along the right border of the uterus until they pass the fundus, which they then press forward, Figure 376. The organ then lies extended along the left index-finger. It is essential for the success of this manœuvre that the uterus be kept in the median line, or that in the replacement it be swung around slightly to the left. Reposition may be facilitated by exerting traction on the uterus by means of a vulsella foreeps during the manipulation, Figure 14 . 
Figure 373.

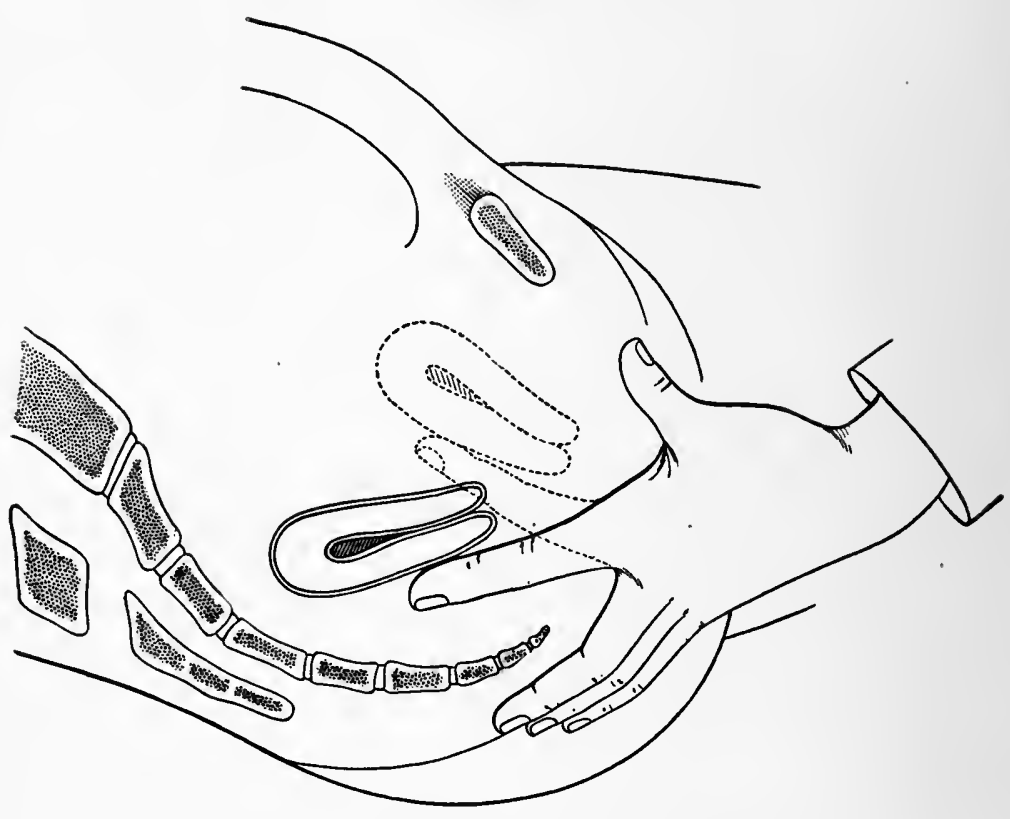

Ventrovaginal reposition; end of first step.

\section{Figure 374.}

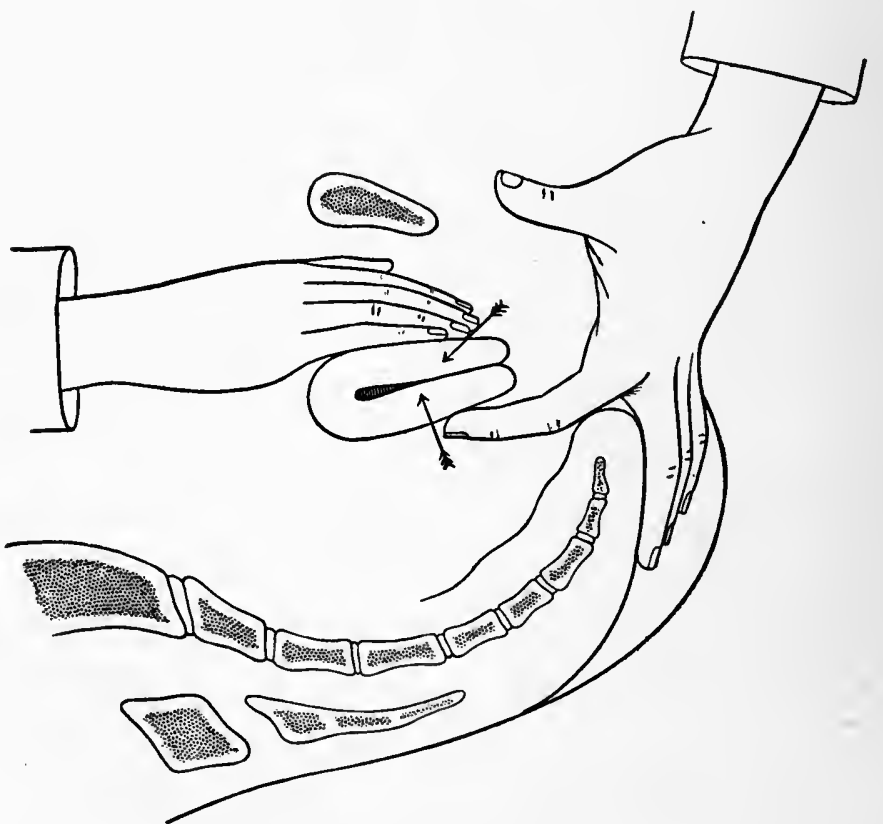

Ventrovaginal reposition; second step. 
TREATMENT OF RETROVERSION AND RETROFLEXION. 681

Figure 375.

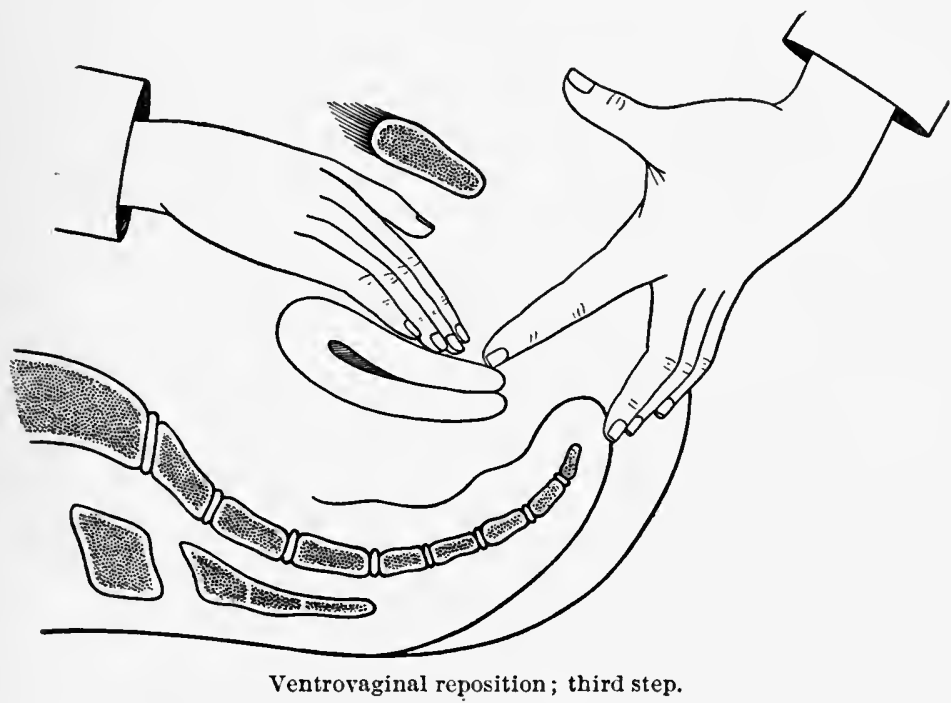

Figure 376.

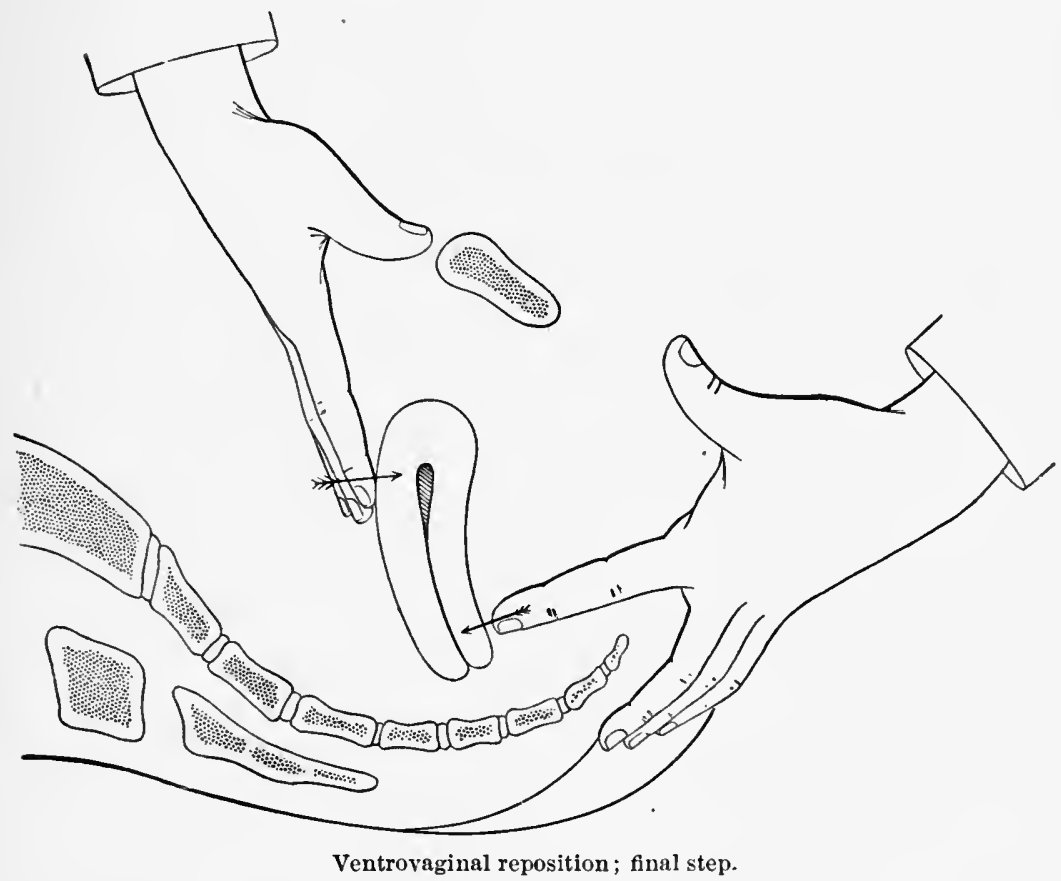


Figure 377.

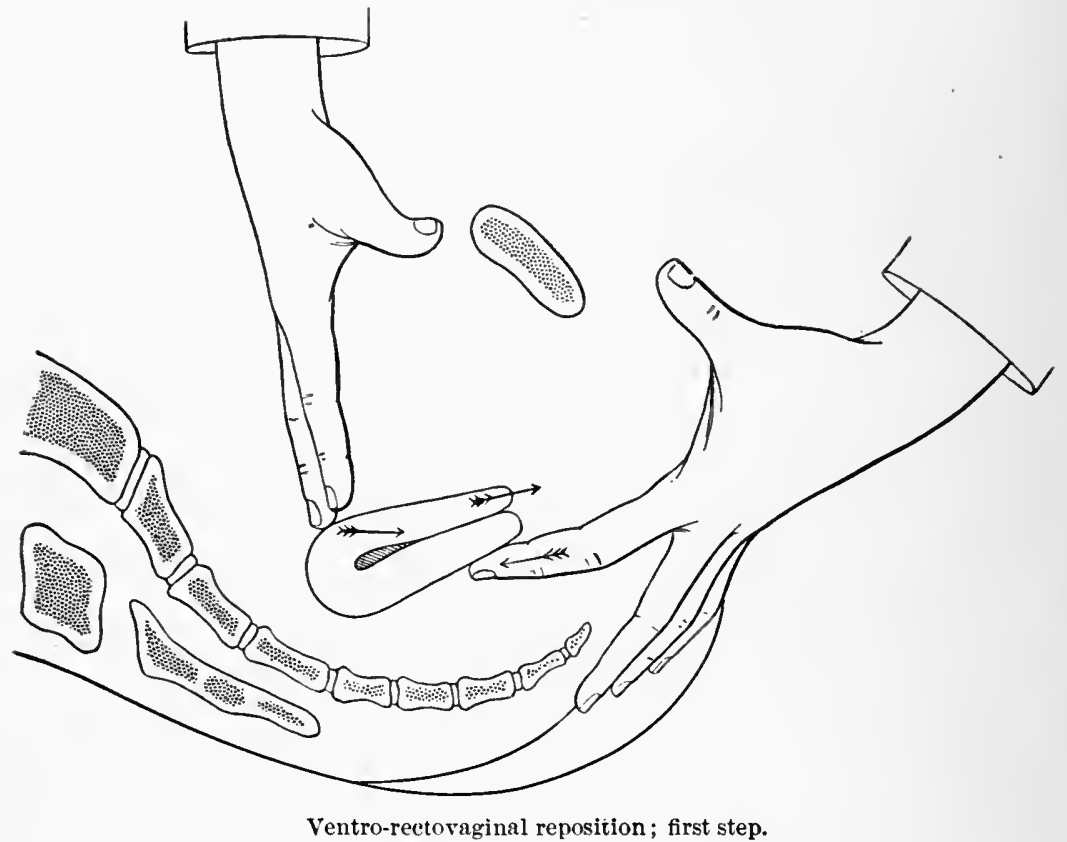

Figure 378.

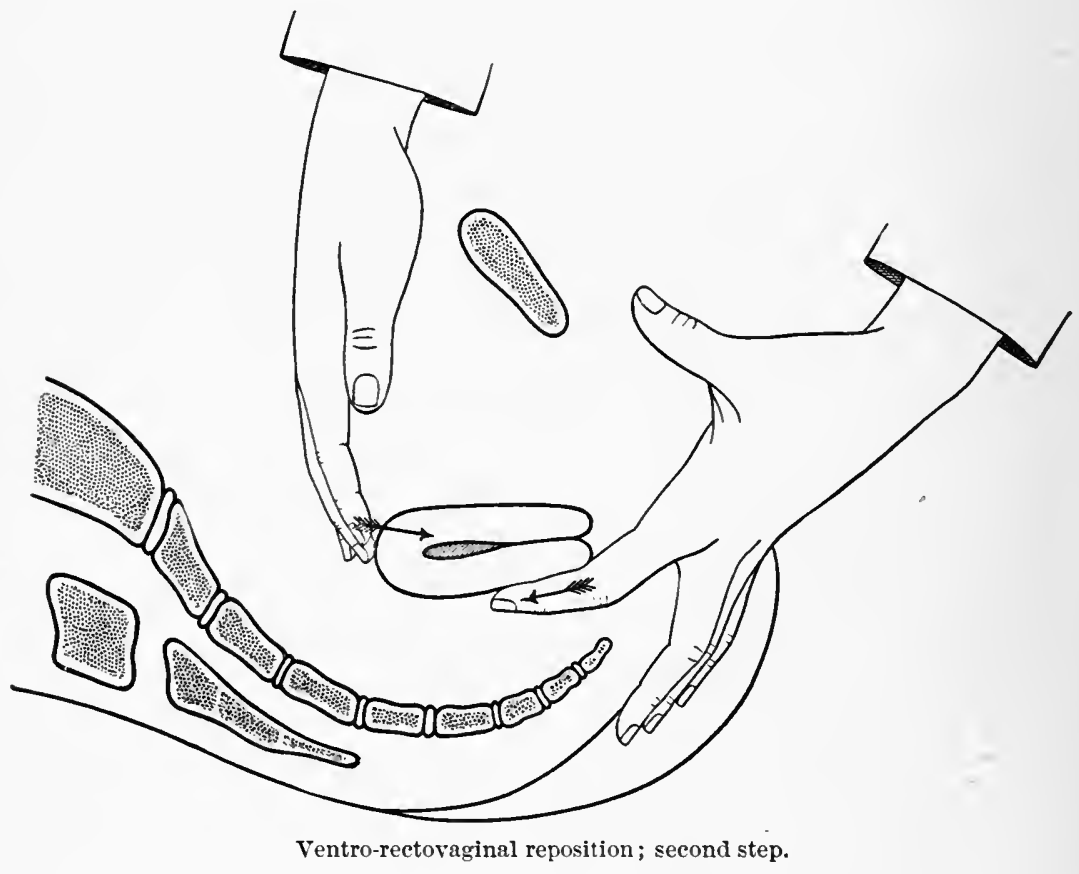




\section{Manual Ventro-rectovaginal Reposition of the Retroposed Uterus.}

This method of replacenent is illustrated by Figures 377-379. The left index-finger, high up in the rectum, pushes the fundus forward, while the right hand on the abdomen executes some circular and vibratory movements. As the muscles relax, the external fingers approach the fundus and push it downward, so that it may readily be reached by the finger in the rectum; this finger is aided by the thumb in the vagina pressing the cervix backward. The fingers of the right hand, continuing the circular movements, then insinuate themselves behind and under the fundus, and complete the replacement. Figure 379.

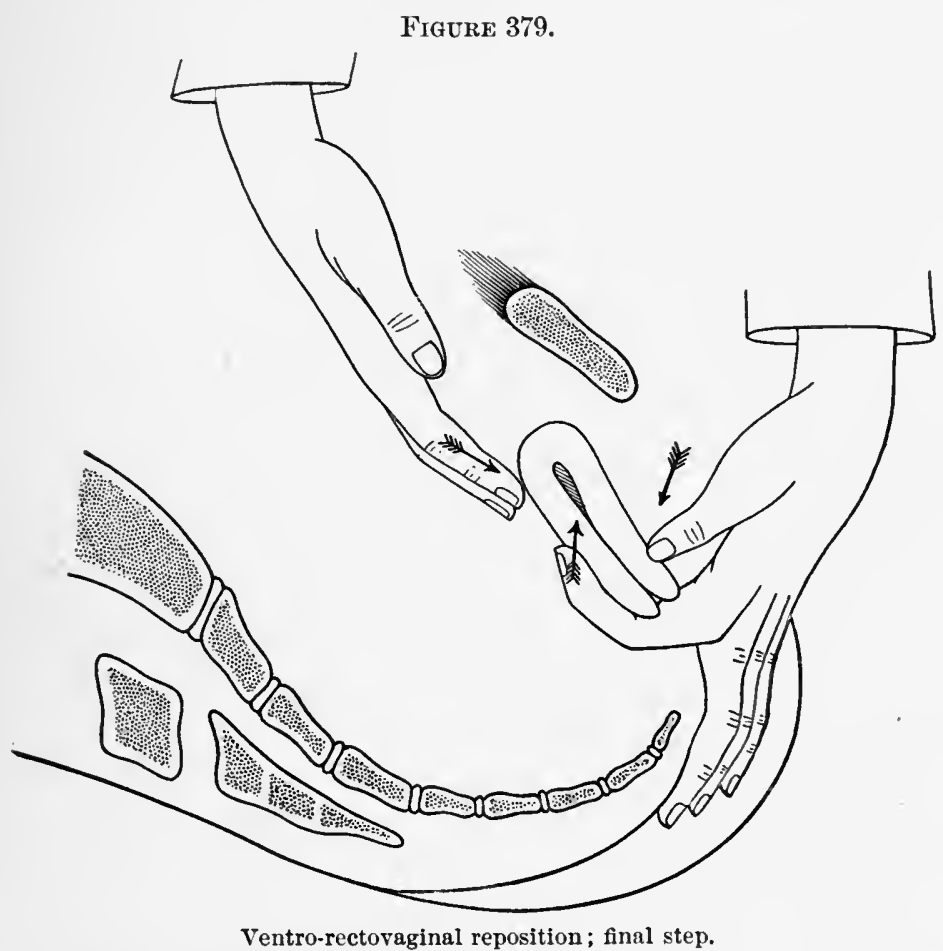

Figures 365-375 and the explanatory text are modified from Jentzer and Zeegenspeck.

The degree to which the manipulations are necessary will depend upon the breadth and strength of the adhesions and bands, and the amount of contraction in the ligaments. All manipulations should be. practised only on cases carefully selected according to the indications and contraindications set forth in the beginning of this chapter, and should be as gradual and as free from pain as practicable.

The length of time that should elapse after an acute inflammation before manipulative reposition may be undertaken with safety is not less than two months. Pyosalpinx is always a contraindication. The nearer the time to an acute infection the more virulent the pus will be; and, on the contrary, the longer the time the more likely the pus 
is to be sterile. If, therefore, on account of error in diagnosis the manipulative movements should rupture a purulent tube and force the contents of it into the abdomen, the danger of peritoneal infection would be decreased directly with the length of time that had elapsed since the acute attack.

\section{Means to Retain the Replaced Uterus.}

The uterus having been replaced will seldom retain its normal position withont artificial support. This support, according to the requirements of a given case, will be secured by means of

1. Pessaries.

2. Surgical operations.

\section{Retention by Pessaries.}

\section{Contraindications and Indications to the Pessary.}

The enthusiast in mechanical gynecology would do well to consider the four following propositions:

\section{Figure 380 .}

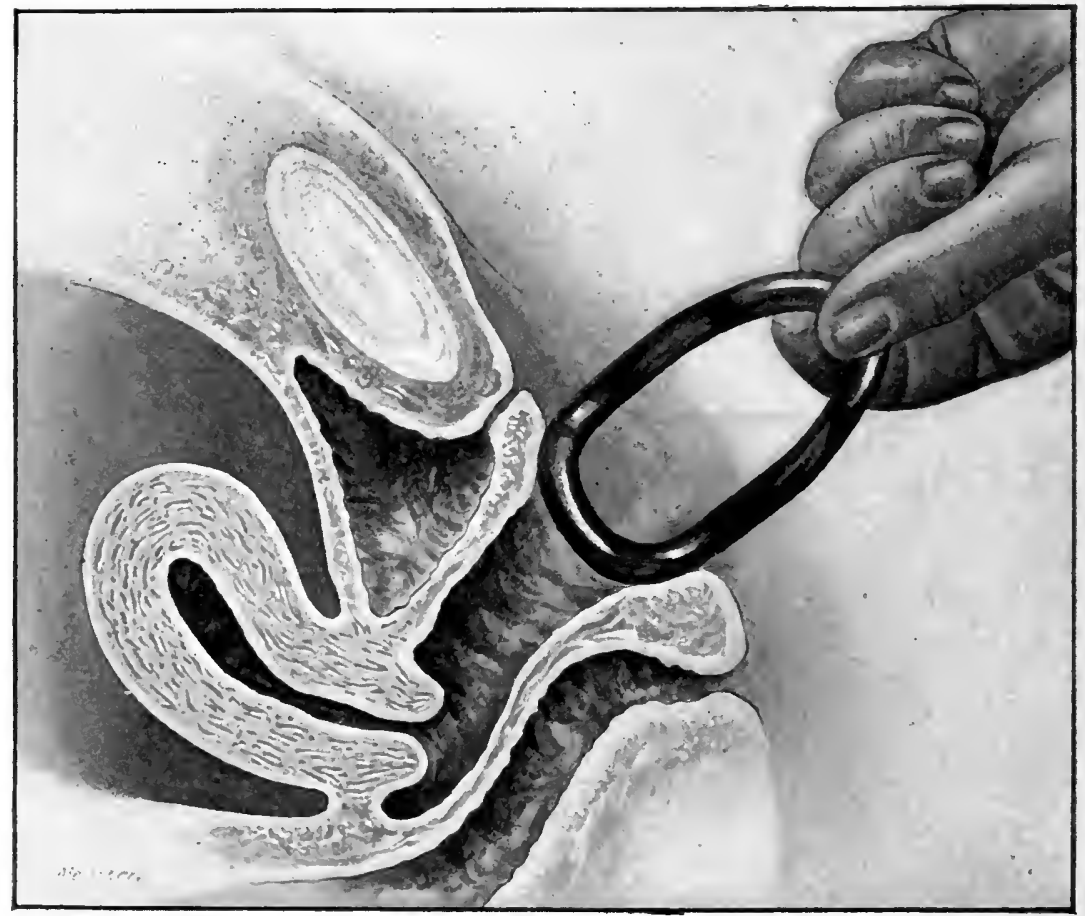

The common but faulty mode of introducing a pessary, with its breadth turned in the anteroposterior diameter of the vulva. The breadth of the instrument should be in the trans. verse direction, as shown in Figure 381.

1. In the majority of cases of retrodisplacement the essential factor is inflammation, and the resultant tenderness may render mechanical support intolerable. 
2. Adhesions and cicatricial bands may prevent or prohibit replacement, and therefore contraindicate the use of any means designed to hold the organ in place.

3. A tumor or excessive weight of the nterus may carry the corpus backward and downward with a force greater than any pessary can counteract.

4. The pelvic floor, including the fascial and ligamentous supports of the pelvic organs, may, from subinvolution or other canse, be so relaxed that no pessary can hold the organs in place.

It follows from the above that the field for the use of the pessary must be restricted to those cases in which the displaced organs are replaceable, and in which the pessary is capable of holding them in place, and can be worn without discomfort. Failnre to recognize and appreciate the contraindication accounts not only for the failures

Figure 381 .

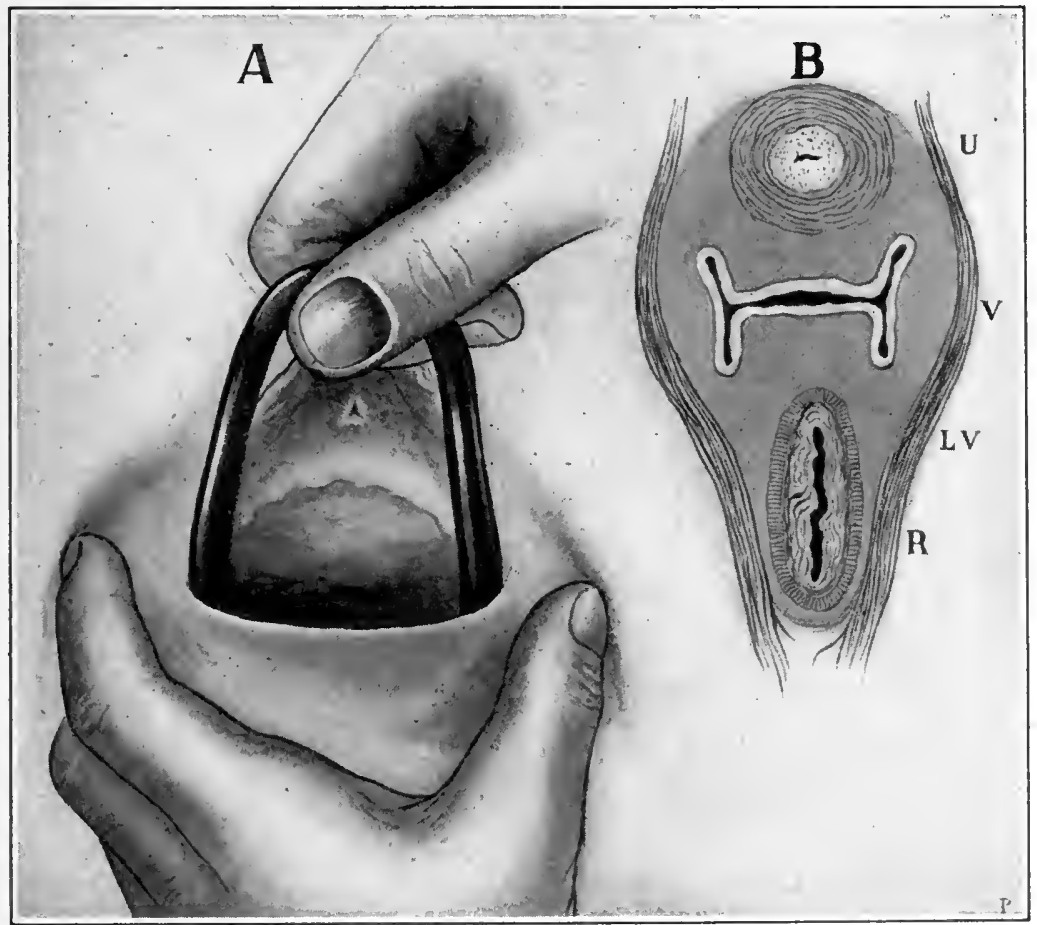

$A$, the correct mode of introducing a pessary. $B$, section through pelvis shows: $U$, urethra; $V$, vagina; $R$, rectum; $L V$, levator ani muscle.

and disappointments, but also for the many evil results which lave followed indiscriminate attempts to treat all displacements by mechanical support. The exclusion of unsuitable cases and the recognition of the necessity for accurate diagnosis are apparent. The pessary, according to the knowledge, judgment, and mechanical skill of the practitioner, will be useful, useless, or injurious. 


\section{The Function of the Pessary.}

The function of the pessary is to maintain the uterus not only on the health level in its normal location, but also, if possible, in its normal position, which requires the cervix to be about one inch from the hollow of the sacrum. The cervix in a properly selected case being thus placed, retroversion is not liable to occur, because if it does oceur the fundus uteri will be arrested in its backward course by the over-arching sacrum, and because the direction of least resistance will be forward into the normal anterior position.

Figure 382.

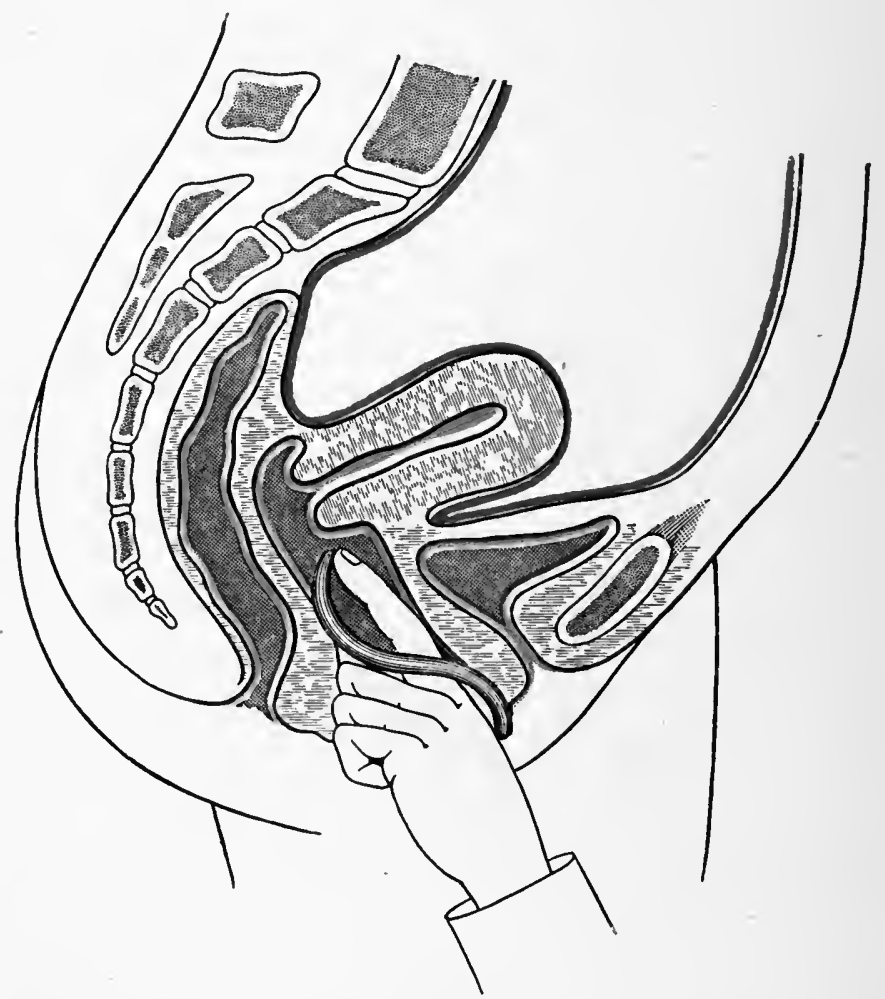

The upper end of an Albert Smith pessary being pushed into place back of the cervix uteri. The apparent lack of mobility at the normal angle of flexure in this uterus is a not uncom: mon result of the metritis which often complicates retroversion and retroflexion.

It follows that the application of the pessary is based upon the general proposition that if the cervix be normally placed the body of the uterus, in the absence of complications, will take care of itself. Since the vagina at its upper extremity is attached to the cervix, displacement of the latter is clearly impossible if the upper extremity of the-vagina be sustained in its normal location. The pessary restores and maintains the relations of the relaxed vaginal walls by crowding the posterior vaginal cul-de-sac backward into the hollow of the sacrum. It 
also holds the attached cervix at a proper distance from the sacrum, and thereby fulfils its indication by sustaining the pelvic floor. The Hodge pessary, or some modification thereof, answers this purpose in ordinary cases more satisfactorily than any other.

The same general principles-in fact, the same pessaries-which are applicable to prolapse apply also to retroversion and retroflexion, because the first step in the genesis of retro-malpositions is prolapse.

Pessaries designed to prop up the body of the uterus by pressure upon the posterior wall for the correction of posterior malpositions, and upon the anterior wall to correct anterior malpositions, are not only unnecessary, but they are also very liable to induce metritis and perimetritis, and therefore are disapproved generally. In certain cases, however, the vaginal walls, especially the posterior, may be so relaxed from subinvolution and other causes that the instrument, though very

Figure 383.

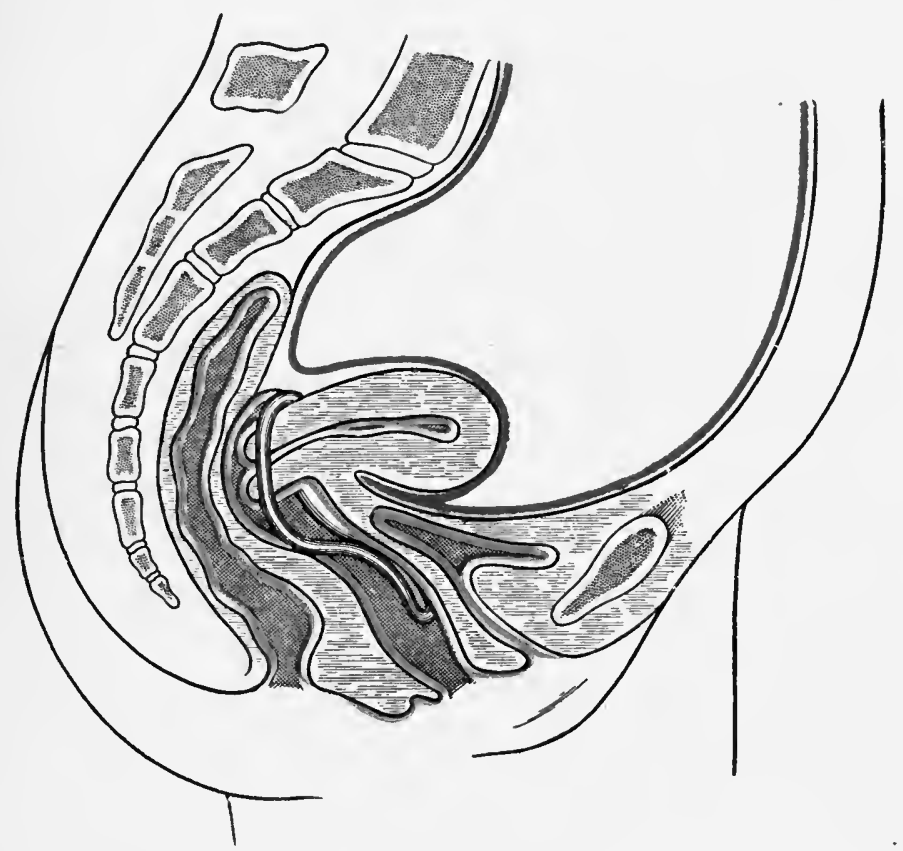

Albert Smith pessary in place and uterus maintained in normal position.

long, fails to maintain the cervix in its normal place. Under such conditions an instrument may be required to act directly upon the uterus. The Schultze sleigh pessary, although seldom indicated, represented in Fignre 386, fulfils this indication. A long Albert Smith pessary, with its uterine curve made so extreme as to bring the upper part of the instrument in front of the cervix, instead of behind, may answer the same purpose. Expedients of this kind, however, are always of doubtful value. 


\section{Adjustment of the Pessary.}

Figure 380 shows a common but faulty manner of introducing the pessary. The vagina is a collapsed tube, the anterior walls of which rest on the posterior; hence, the long diameter of a cross-section of the canal is from side to side, not anteroposterior. The pessary should, therefore, be introduced with its lateral edges to the sides of the vulva.

If introduced as shown in Figure 380, with its lateral edges in the anteroposterior direction of the vulva, the pessary is apt to press painfully against the urethra in front and the perineum behind. This pain is increased when the instrument is turned to conform to the shape of the vagina, as it must be before it can take its proper place.

In the correct mode of introduction the labia are separated by the thumb and index-finger of the left hand, and the pessary is pushed in with the right hand, its lateral edges being to the sides of the vulva; it then readily follows the eurve of the vaginal outlet. This mode of introduction requires less force and gives less discomfort. The first step toward adjustment is complete when the inner end of the pessary is in contact with the anterior wall of the cervix uteri. The second step is to pass the left index-finger, the palmar surface being in contact with the perineum, under the pessary, and push the upper end nuder the cervix and then hackward into its place in the post-vaginal fornix. See Figures 381, 382, and 383.

The curves of the pessary demand careful attention in its application. When the uterus is below the normal level, the broad ligaments necessarily are rendered more tense than natural, and the bloodsessels, more especially the veins, which are looped one upon the other, and which traverse these ligaments to and from the uterus, are made to collapse. This causes venous congestion and consequent increase in -weight of the uterus-a condition favorable to malposition, nterine catarrh, and pathological changes in structure. A pessary which will raise the uterus to the health level clearly fulfils an indication. A

Figure 384.

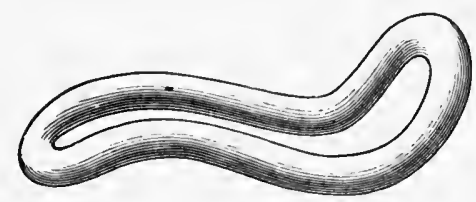

The Emmet curves.
Figure 385 .

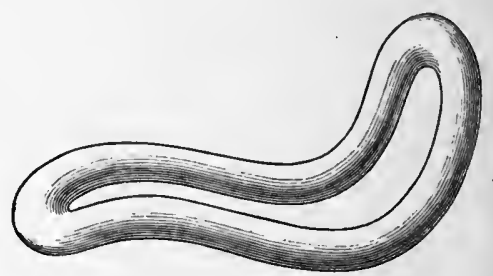

The Albert Smith curves.

pessary which raises it above the health level renders the broad ligament tense and reproduces a condition which it was designed to relieve. Maintenance of the uterus upon the health level depends largely upon the curves of the pessary. The accompanying cuts illustrate the shape and curve of the Hodge pessary as modified by Emmet and Albert Smith. Figure 384 represents the curves of Emmet, and Figure 385 
those of Albert Smith. For convenience, let us characterize that curve which rests in the posterior vaginal cul-de-sac as the uterine curve, and that which occupies the part of the vagina adjacent to the pubis the pubic curve. The acnteness and length of the nterine curve determine the height to which the pessary will lift the uterus. The longer and more acute the curve, the higher the uterus will be lifted, and vice versa. The smaller curve of the Emmet modification will answer the average indication more nearly than the sharper curve of the Albert Smith modification, which may lift the uterus too high. The pubic should generally be proportioned to the uterine curvethat is, the greater the uterine, the greater the pubic curve. A

Figure 386.

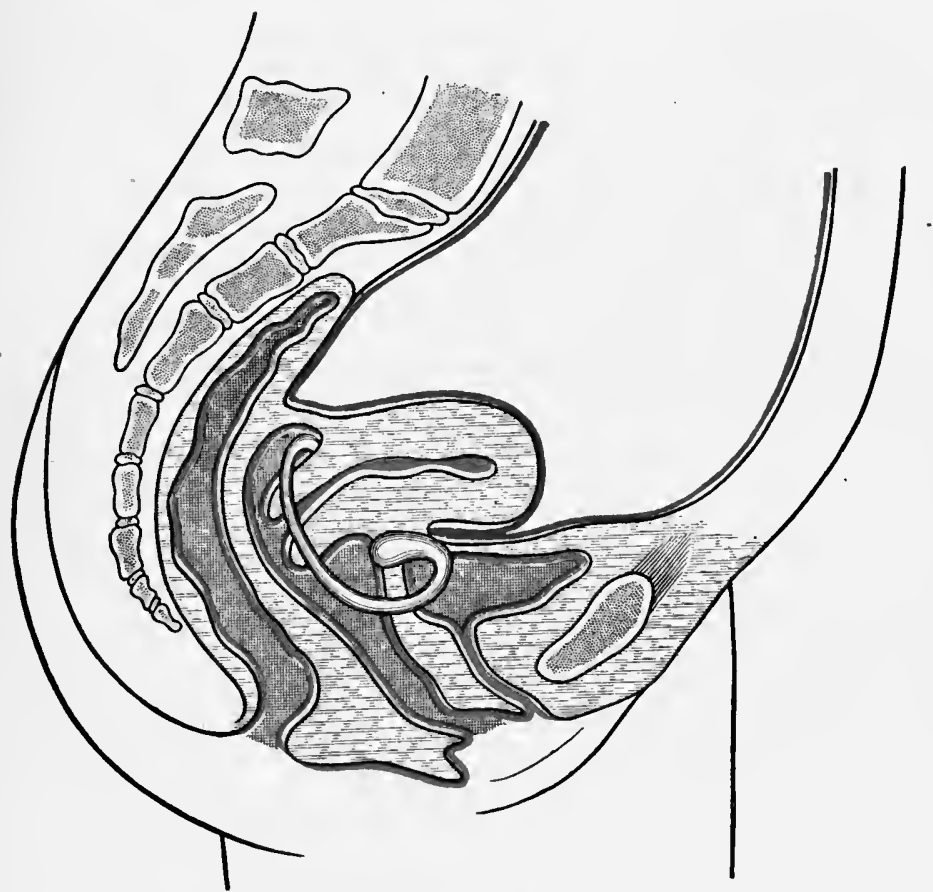

Schultze's sleigh pessary in position. This pessary is not in general use, but is serviceable to hold up the vesicovaginal wall in cases of cystocele and rectocele.

pessary properly adjusted in all other respects may, by pressure upon the urethra and neck of the bladder, create vesical tenesmus and urcthral irritation. This calls for increase in the pubic curve-that is, the pessary should be bent away from the irritated part. The pubic curve may, however, be so great that the lower part of the pessary occupies the centre of the vulva, where it is apt to create irritation. For this condition lessening of the pubic curve is the remedy. The pessary should not be so wide as to distend the vagina. The length should be measured by the distance from the lower extremity of the symphysis pubis to the posterior vaginal cul-de-sac, less the thickness 
of the finger. If properly adjusted in a suitable ease, it should ststain the pelvic floor in its normal relations and the uterus in stable equilibrium.

'Thomas' retroflexion pessary, with its bulbous upper extremity, is a long, narrow instrument of extreme uterine curve. It lifts the uterus very high, and is specially applicable in cases of great relaxation of the pelvic floor and of eomplicating prolapse of the ovaries; sometimes the bulbous portion is made of soft rubber.

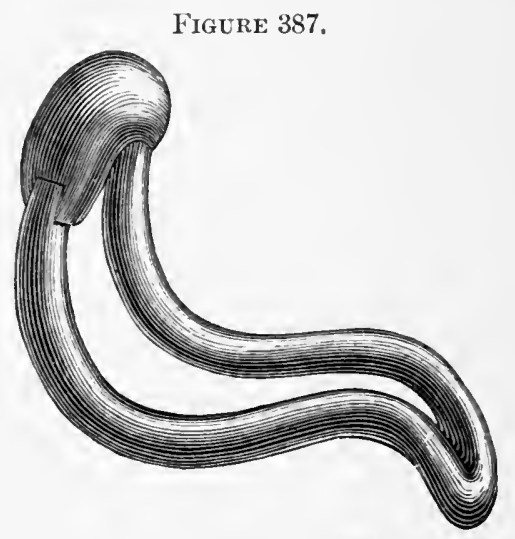

Thomas' retroflexion pessary.

In retroversion and retroflexion always replace the uterus before adjusting the pessary, otherwise the instrument will press upon the sensitive uterus, and one of three -unfortunate results may oceur: $(a)$ the pessary may not be tolerated on aceount of pain; $(b)$ it may be foreed down by pressure from above so near to the vulva that it will fail to do the least good; (c) the uterus, finding it impossible to hold its position against the pessary, instead of taking its proper position, may be bent over it in exaggerated retroflexion, with the cervix between the pessary and the pubes, or the whole organ may slip off to one side of the instrument into a malposition more serious than the one for which relief is songht.

A properly adjusted pessary gives to the patient no consciousness of its presence. If the instrument causes pain, it should be removed, and search made for the tender places; it then, if possible, should be remoulded into such shape that it will not make pressure upon them. In remoulding a hard rubber pessary, one should pass it rapidly back and forth through the flame of a spirit lamp until it is sufficiently softened to be bent to the desired form. Often a slight indentation at some point will enable the patient to wear it with comfort. If it cannot be made comfortable, it should be abandoned.

Sometimes when the corpus has been firmly bound back by peritoneal adhesions they may be broken up by very forcible conjoined manipulation under ether; but the operation is dangerous, and should therefore be undertaken only by an expert operator, if at all.

In eertain cases in which replacement is impracticable or impossi- 
ble, on account of inflammation or adhesions, a soft rubber ring may be inserted, and will sometimes give decided relief by lifting the uterus and pelvic fioor nearer to the health level. In the treatment of all displacements coition should be forbidden until the inflammatory signs have disappeared. The pessary should be kept clean by moderate daily applications of the vaginal donche. Every three or four weeks the instrument should be removed and the pelvic organs carefully examined.

No one can safely apply the pessary until he has fully appreciated its indications and contraindications. Many practitioners are deficient in the natural mechanical skill necessary to its proper adjustment-a fact of which thousands of unfortunate women bear witness. Its dangers in inefficient hands are in striking contrast with its usefulness when judiciously employed in proper cases.

\section{Retention by Surgical Operations.}

Many cases of displacement are so complicated by prolapsed and adherent ovaries, by advanced disease of the ovaries and Fallopian tubes, by tumors, by inflammatory exudates, or by peritoneal adhesions, that replacement is impossible, or, replacement being possible, the pessary is either intolerable from pain or proves inadequate to sustain the uterus. Such cases, unless relievable by non-surgical measures, furnish a definite indication for surgical treatment.

Perineorrhaphy, elytrorrhaphy, and the removal of tumors may be necessary, and have been considered under their respective heads. Whenever the perineum has been displaced downward and backward away from the pubes toward the coccyx, its restoration by perineorrhaphy or by some suitable plastic operation upon the vaginal outlet is always indicated; see Chapters XL. and XLI. Elytrorrhaphy is not usually indicated unless the retro-malposition is associated with descent to the second or third degree. If the malposition is caused by a tumor, the pathology, diagnosis, prognosis, and treatment will be those of the tumor.

The surgical treatment proper of posterior malpositions involves especially a description and comparison of the three most recognized classes of operations. Each has its special adaptation to its own class of cases. In the treatment of some cases, according to the individual preference of the surgeon, either one of the three is permissible. The operations are :

1. Alexander's operation, shortening the round ligament. ${ }^{1}$

2. Abdominal hysterorrhaphy.

3. Vaginal hysterorrhaphy.

\section{Alexander's Operation.}

The round ligaments, as already explained, restrain the uterus from excessive backward movement. They are two cords, each the size of a goose-quill, springing from the horns of the uterus, just below and in

1 A very full paper on this subject is one by Genrge M. Edebohls, in the American Gyneco. logical and Obstetrical Journal, December, 1896. This paper contains an exhaustive bibliog. raphy. 
front of the origin of the Fallopian tube. They pass forward on either side in the folds of the broad ligaments through the internal inguinal rings, through the inguinal canals and the external rings, and, spreading out in strands, are lost in the mons veneris and upper parts of the labia majora. 'These ligaments consist of unstriped muscular fibres in condensed areolar tissue. Physiologically they have some contractile power.

When the uterus is retroposed, the round ligaments are necessarily stretched to such an extent that they can no longer exert their normal restraining power upon the backward movements of the organ; hence the proposition of Alexander to shorten them extraperitoneally to such an extent that they will resume their normal functions. This is Alexander's operation.

\section{Indications and Contraindications for Alexander's Operation.}

This operation is permissible only when the displacement is not complicated by a tumor, inflammation of the uterine appendages, adhesions, or other impediments to replacement. Clearly, shortening the ligaments could not hold in place a uterus firmly bound down by adhesions or weighted down by a tumor. True, as some advise, the peritoneal cavity might be opened and the adhesions broken up or the tumor removed, as preliminary measures to the shortening of the ligaments; but under such conditions most surgeons would prefer suspension of the uterus by hysterorrhaphy as being the more rational and effective operation. If the uterus can be retained in place by a pessary, or can be treated successfully by massage, or by any of the other non-operative means already described, Alexander's operation, though not a procedure of necessity, may yet be one of expediency. The expediency will, however, depend upon the woman's ability and willingness to carry out the more conservative course. Temporizing measures may insure comfort only so long as she can be free from care, anxiety, and overwork. If she must earn her living, a radical cure by surgical measures may be necessary. After anæsthesia, befóre the operation is begun, a thorough conjoined examination should always be made, in order especially to exclude inflammation of the uterine appendages; this is because the operation, if made in the presence of unsuspected suppuration in the tube or ovary, may lead to fatal peritonitis.

\section{Preparatory Treatment for Alexander's Operation.}

The preparatory treatment is the same as that laid down in Chapter II., for abdominal and vaginal section. Endometritis is almost always present in the retroposed uterns; hence dilatation and curettage are indicated, and should be performed immediately before the shortening of the ligaments ; the reason for this is twofold: first, to cure the endometritis; second, to render the endometrium aseptic, and thereby shut off post-operative infection from that source. Necessary plastic opera- . tions on the cervix uteri, ragina, and perineum may, according to the strength of the patient and the rapidity and dexterity of the operator, 
be made at the same time. If the perineum is injured, its repair is essential to success:

\section{Steps of Alexander's Operation.}

The steps of the operation are: 1 . To find and isolate the ligaments. 2. To draw them out until their superabundant slack has been taken up. 3. To anchor or fix the drawn-out portions by means of suture, in order to keep them from slipping back into the pelvic cavity throngh the internal ring. The location and extent of the incision and the mode of isolation of the ligaments vary with different operators: For example, an incision direct to the internal ring was practised first by Alexander $;^{1}$ it is, however, often difficult to find the ligaments at this point. J. Frank and Newman ${ }^{2}$ cut directly to the internal ring. Kellogg opens the inguinal canal by a small incision near the internal ring. Edebohls opens the canal thronghout. its entire length. The method of Kellogg, with minor modifications, is the one adopted by the writer, and is substantially as follows : ${ }^{3}$

\section{Anatomical Landmarks for Alexander's Operation.}

The superficial anatomical landmarks are the anterior superior spine of the ilium, the spine of the pubes, and Poupart's ligament. The deeper landmarks on either side are the external inguinal ring, which lies just above the spine of the pubes; the internal ring, about three inches above, in the direction of the anterior superior spine of the ilium; and the inguinal canal, which lies between the internal and external rings; and the rings form the ends of the canal.

\section{The Incision for Alexander's Operation:}

The incision through the slin is made midway between the internal and external rings, one inch or more long, parallel to and just above Poupart's ligament; this is directly over the middle thirc of the roof of the canal. A clean and careful dissection is now made to the tendon of the external oblique muscle. It is important that the natural color and appearance of the cut surfaces be preserved, in order that the exact point of incision into the canal may be readily recognized; hence the necessity of clean dissection and immediate control of all bleeding points by snap-forceps.

The glistening tendon of the external oblique now exposed usually will show a dark line. This line marks a point where the fibres of the tendon begin to separate to form the columms of the external ring. The borders of the separated tendon are connected by the intercolumnar fascia, which, being thinner than the tendon, enables one to see through it to the darker tissues beneath. There may be several narrow points of separation or one broad one. In exceptional cases the separation is absent up to the very border of the external ring. When the dark line has been found, pass the finger down the line

2 Alexander. London: Churchill, 1884. Hart and Barbour.

2 American Journal of Obstetries, 1888.

The description here given is in the main an extract from the paper of Kellogg; reprint from the Proceedings of the Michigan State Nedical Society, 1898. 
toward the pubie spine, and see that it opens into the external ring. This loeates the canal. The wound is now drawn widely open by two retractors in the hands of an assistant. The opening in the tissues, overlying the tendon, thus widely separated may be slid about over a considerable area until the right point for opening the canal is found; it is abont three-quarters of an inch below the internal ring. At this point a puncture or an incision, not more than a third of an inch long, is made by a small sealpel through the tendon.

\section{Finding the Ligament in Alexander's Operation.}

To find the ligament, take two small hooks, Figure 389, one in each hand. The small opening is made to gape with the hook in the left hand, while the other is passed by the right hand into the opening and direetly backward, the flat side of the hook parallel

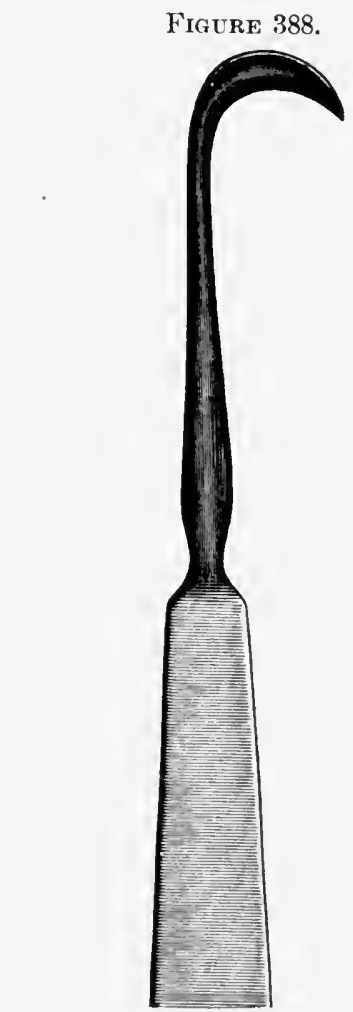

Two of these retractors are needed for opening the wound. Full length, 6 inches.

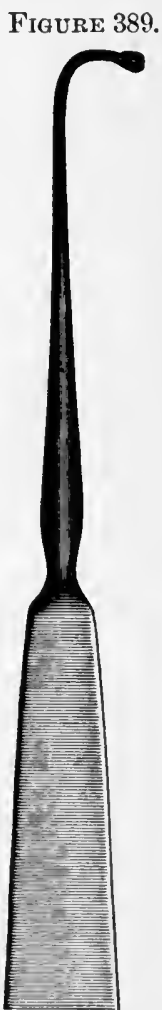

Two of these hooks arc needed for picking up the round ligament. Full length, 6 inches.

to Poupart's ligament and hugging it elosely. When the hook has penetrated to a depth of about one inch, its point is turned toward the canal, and the tissues that come in its way are hooked by a wide sweep and drawn up through the slit in the roof of the canal. The 
little mass of tissue thus brought up will often contain the ligament, but more frequently it is just below the hook and closely connected with the tissue engaged by the hook. A dip with the other hook will bring up the ligament itself. The tissue thus brought into view is a grayish-colored mass of fat, which contains anastomosing blood-vessels and the ligament, with its accompanying ilio-inguinal nerve and vessels.

The operator who attempts to find the ligament as Alexander directed, by cutting down through the mass of fat, will find himself embarrassed by the resultant bleeding and the disarrangement of the contents of the canal. He may for hours grope blindly about the region among a variety of structures which marvellously resemble, but are not, the ligament. ${ }^{1}$ The earlier operators, who cut direetly to the external ring, where the ligament spreads out just before passing, fan-shaped, to the pubes and labia, frequently were misled, and therefore often unsuccessful.

Drawing Out the Ligaments in Alexander's Operation.

The mass, having been picked up by the hook, is now spread out on the finger, and the ligament, invested in its fascial sheath, is recog-

Figure 390 .

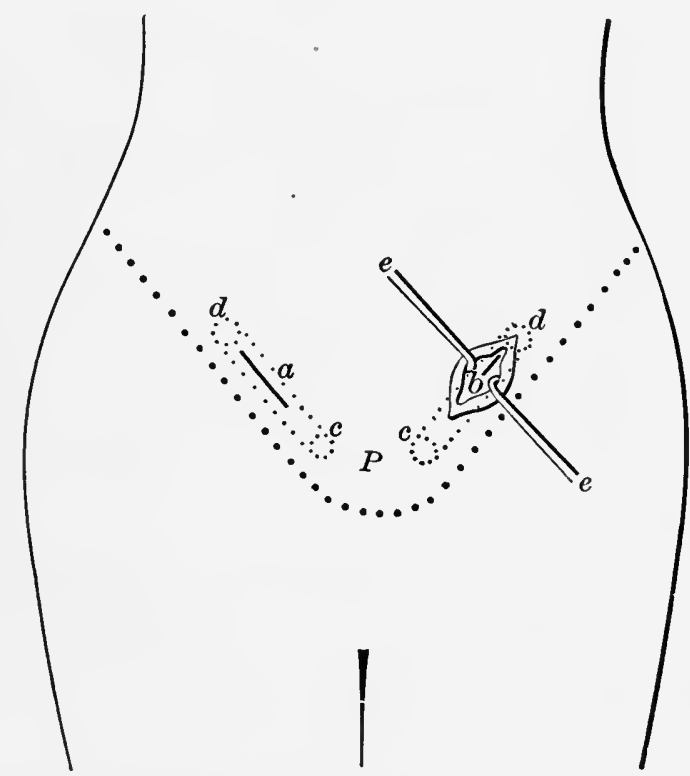

$d$, the internal ring; $c$, external ring; light dotted lines between indicate the position of the inguinal canal, the heavy dotted lines below indicate the direction of Poupart's ligament. $a$ shows position of skin incision; $\epsilon$, $e$, indieate retractors by whieh the tissues divided by the ineision are separated down to the tendon of the external oblique; $b$ shows the small ineision in the tendon of the external oblique near the internal ring; $P$, pubes. ${ }^{2}$

nized by its cord-like appearance. On making a longitudinal slit in the sheath, the smooth glistening pink surface of the round ligament

${ }_{1}$ Lapthorn Smith. The Medical News. September $29,1896$.

2 Modified from Kellogg. Proceedings Michigan State Medical Soeiety, 1889. 
appears. From this time the operation on that side is simple. As soon as the identity of the ligament is clearly established by the fact that it can be "readily pulled out from the direction of the internal ring - that is, made to run "-it is secured from slipping back into the canal by passing a ligature loosely around it and covering the wound with protective gauze, while the ligament on the opposite side is found and exposed in the same manner.

When the ligament is separated from its surroundings it will usually run freely, and this, aside from the eye, is the best test of its identity. Even the eye may be deceived, for in this region are several structures that closely resemble the ligament. If the ligament is not readily found by the hooks, as described.above, they should be introduced again, and, if necessary, yet again. Oftentimes it will be found outside of its sheath, crowded down close to Poupart's ligament at the very bottom of the eanal, or it may be at the opposite side of the canal. Give the hook a broad sweep, so as to engage the entire contents of the canal. If necessary, the incision may be prolonged to the internal ring, or another short incision may be made into the canal at the internal ring, after the method of Frank and Newman, and the ligaments songht there. A little patience and care usually will lead to success. Failure to find the ligament is to be attributed not to its absence, but to faulty technique.

The ligament, once found and isolated, will, as has been said, usually run freely. It should be drawn out by gentle, steady traction until it 'begins to increase rapidly in size and to present a sort of shoulder. This indicates that a point near the horn of the uterus has been reached. At this point the ligament is surrounded by a fold of peritoneum, the canal of Nuck; which is dragged through the internal ring into the inguinal canal. It is well to free both ligaments before pulling them out to the necessary extent. In some cases they are quite small, and therefore, if strongly pulled, are liable to break and retreat into the internal ring beyond reach. By careful and repeated trials, however, they will usually, as they are gently drawn out, become larger and appear as smooth, glistening cords.

The extent to which the ligaments should be pulled out is a matter for judgment; in each case sufficient slack should be taken up to secure the corpus uteri in its normal anterior position. The rapid enlargement of the ligament and the appearance of the canal of Nuck indicate a safe limit.

In separating the ligament and drawing it out, great care should be taken not to injure nor include the ilio-inguinal nerve. Division of this nerve has caused anæsthesia of the inguinal region repeatedly. ${ }^{1}$

\section{Anchoring the Ligaments in Alexander's Operation.}

The ligament having been drawn out to the required extent, as shown in Figure 391, the end of its loop at $C$, Figure 392, is transfixed and tied with a ligature. The ends of the ligature are left long, and together threaded into a blunt needle. The needle then is passed

1 Edebohls. American Gynecological and Obstetrical Journal, December, 1896. 
Figure 391.

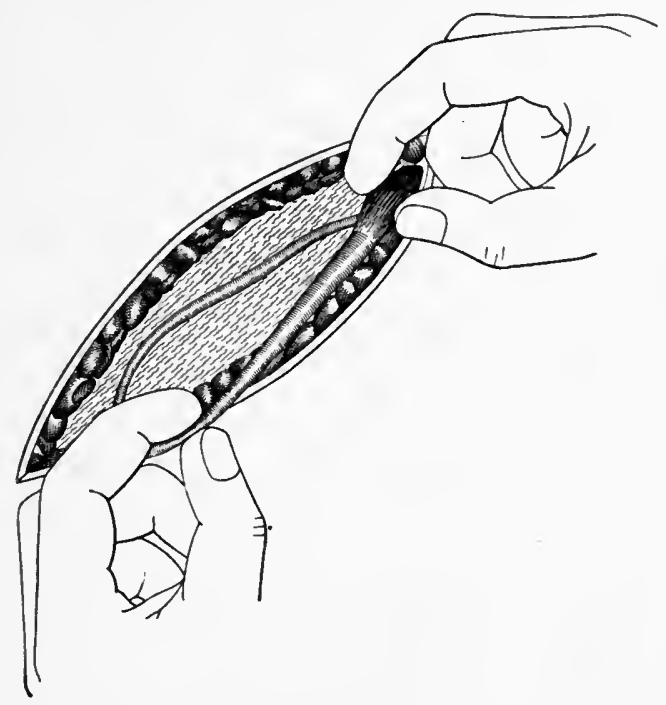

Drawing out round ligament and stripping back investing peritoneum from the broad ligament.

Figure 392.

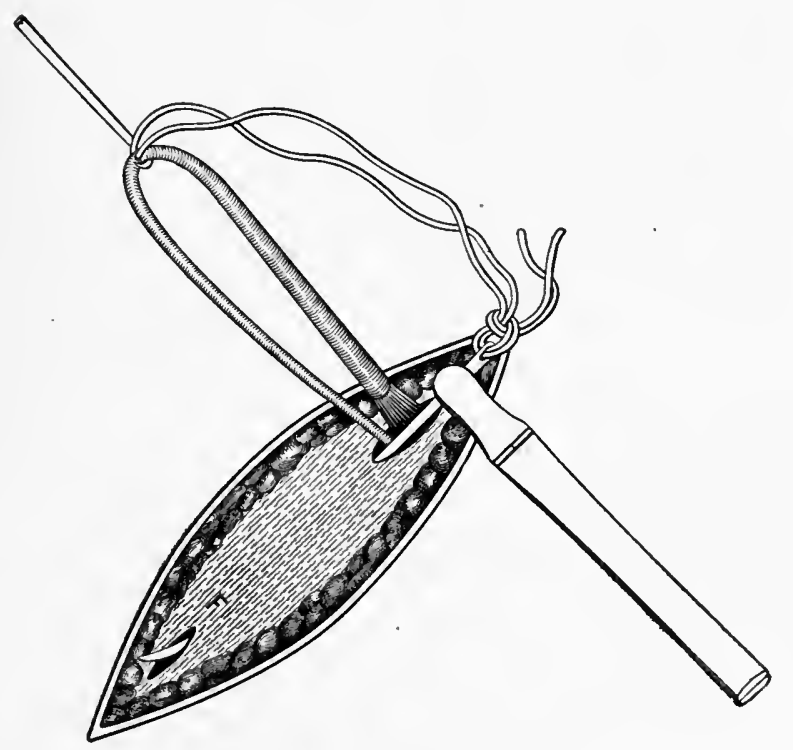

The ligament drawn out to the required extent. Ligature passed through the ligament at the loop. Loop being drawn by means of a blunt-pointed needle under the tendon of the external oblique, to emerge at the opposite end of the wound. The blunt end (eye end) of a needle wonld answer the purpose. 
under the tendon of the external oblique musele, and emerges at F, where previously there has been made into the inguinal eanal a short slit, through which the loop of round ligament is now drawn. The original slit through which the ligament was drawn first out of the inguinal canal is closed with fine catgut sutures and the remaining

Figure 393.

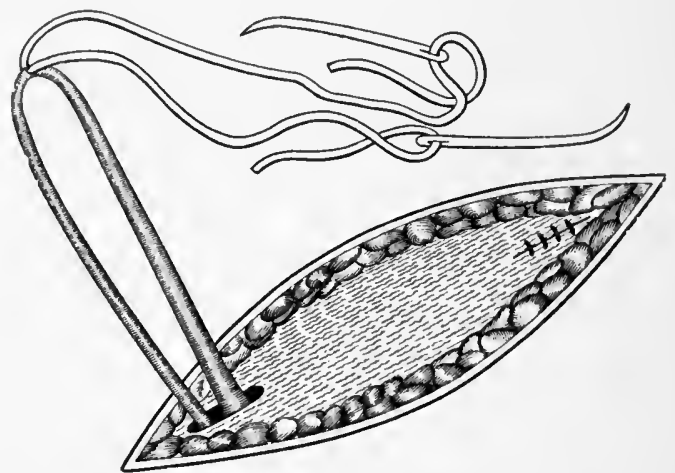

Loop drawn out at pubic end of wound. Original opening into inguinal canal closed with sutures. Two shorter needles now take the place of the single long blunt needle.

external portion of the loop is folded down on the tendon of the external oblique muscle, and stitehed there in the manner shown by Figure 394.

\section{FigURE 394.}

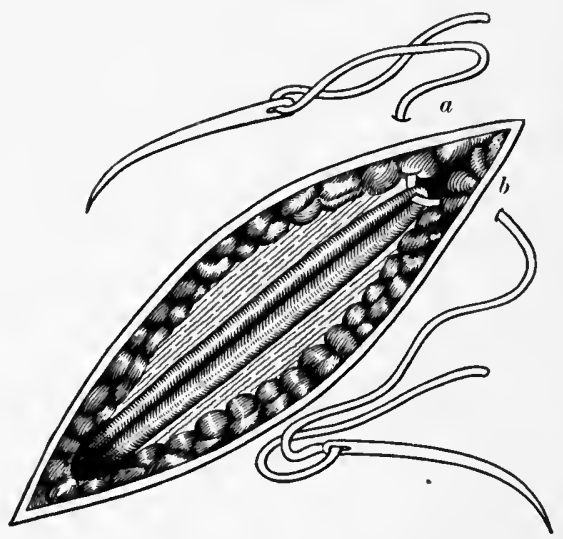

Loop of the ligament fulded down on the tendon of the external oblique muscle, and the two free ends of the ligature passed through at $a$ and $b$, to be tied for the closure of the wound.

The two ends of the ligature then are used as continuous buried sutures for the seeure anchoring of the ligament and the closure of the wound. The wound closure is similar to that laid down in Chapter VI. for closure of the abdominal wound. Chromic catgut is used for ligatures and sutures throughout the operation. 
After-treatment in Alexander's Operation.-The immediate after-treatment is the same as for laparotomy. It is a wise preeaution, though usually unneeessary, during the first month after operation-that is, until strong healing is secured-to guard against reeurrence of the displacement by the use of a pessary.

Limited Scope of Alexander's Operation.-Alexander's operation is limited to eases of slight descent with retroversion or retroflexion, in which the nterus is easily replaceable and demonstrably free from complieating adhesions, tumors, or diseased appendages. Such uncomplieated slight displacements usually may be treated satisfactorily by the mechanical support of pessaries or by other conservative measures. 'The scope, therefore, of Alexander's operation neeessarily is small.

\section{Intra-abdominal Shortening of the Round Ligaments. ${ }^{1}$}

Alexander's operation and other operations for shortening the round ligaments, whether by means of incision into each inguinal canal or through the inguinal region on either side into the pelvic cavity, have in great measure and for good reason given way in later years to more practical proeedures in which the ligaments are treated intraperitoneally through a median laparotomy incision. The raginal hysteropexies and the ordinary operations of abdominal fixation also show signs of becoming obsolete. The suspension operation of Kelly, however, by which the upper part of the posterior wall of the corpus uteri is attached to the anterior abdominal wall near the bladder, should not be set aside without some reservation. At least, after a very extensive experience with this operation, I have not observed in my own eases the injurious mechanical results which others have attributed to it. Possibly these injurious results have followed fixation, an operation always to be condenned, rather than the suspension advocated by Kelly. At any rate the two operations have been much confused with one another. Suspension for the most part is free from the objections which properly have been urged against fixation, but even better than suspension is the newer operation of shortening the round ligaments, because while being quite as effective, it is less objectionable from the mechanical, anatomical, and synptomatic points of view.

In the excellent operation of Gilliam and the improvements on it recently introduced by Barrett and others the round ligaments are drawn through an artificial canal into the laparotomy wound and fastened there either to one another or to the corresponding sides of the wound, so that this wound when elosed shall contain the loops of the ligaments thus drawn into it.

The method is satisfactory provided there is no subsequent suppuration in the abdominal wound, but it should be a fair a priori conclusion that if the wound becomes infeeted the infection would be apt to extend through the tracks of the ligaments into the peritoneal eavity, and, to say nothing of unnecessary spreading of infection, might at least defeat the purpose of the operation. The danger of such a souree of infection is obviated by the modified technique about to be described.

1 American Jourual of the Medical Sciences, June, 1906. 
This teehnique has for its objeet the malking of an artifieial inguinal canal on each side, through which a loop of the round ligament is drawn and anchored, not in the abdominal wound, but on the perito-

Figire 395.

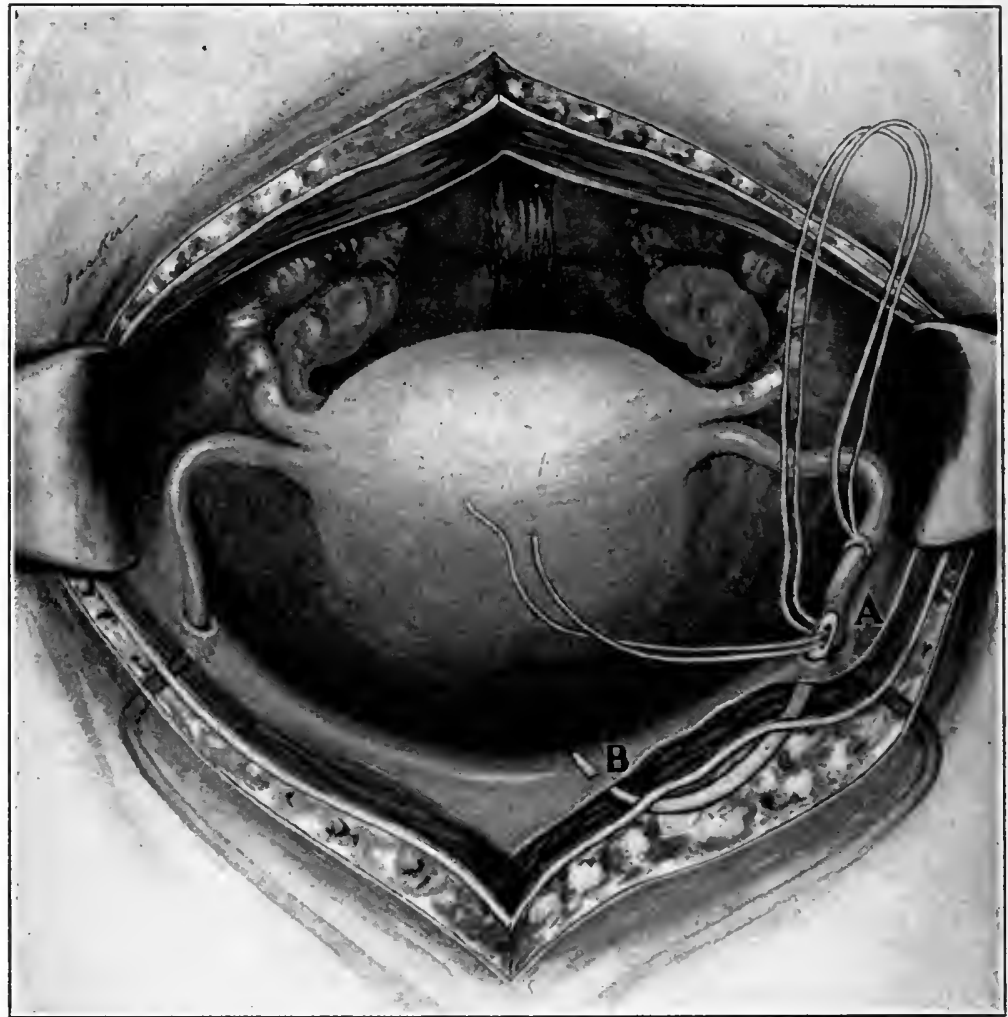

The abdomen is opened in the median line just above the pubes and the wound is held apart widely by retraetors so as to expose the pelvic contents. The middle of the relaxed round ligament is seized with foreeps and tied with a catgut suture at a point just above $\boldsymbol{A}$. The two free ends of this suture are then threaded on a curved blunt-pointed needle the size of a goose quill and this needle is then thrust through peritoneum at the internal abdominal ring. traction on the ligament having been made in order to locate the ring at point $A$. The needle is then passed on through the rectus muscle and faseia and then turned and forced through fascia. muscle, and peritoneun to its point of exit elose to the bladder refiexion, about one one-half ineh from the peritoneal margin of the abdominal ineision at point $B$. The blunt needle is then removed and the loop of ligament is drawn through the artifieial inguinal canal by means of the suture from $A$ to $B$, as shown on the right side of Figure 396 . In place of this blunt needle one may use a ligature-carrviug forceps. The artificial eanal lies between points $A$ and $B$. In order to avoid wounding the deep epigastric and other vessels the needle should be blunt-pointed and the plunge of it should not be too deep into the region of the internal ring. The needle should be about two and one-half inches long and flattened both on its convex and coneave sides in order that it may be held firmly in the needle forceps, and with a point especially blunt to make it pass by blood-ressels withont wounding them, but not so blunt as to prevent introduction.

neal surface at a point a little distant from and independent of the wound. Figures 395 and 396 will serve to illustrate the method.

It will be observed that the uterus which appears retroverted in Figure 395 takes its anteverted position in Figure 396. 
In some cases the ligament on one or botl sides will be found to be absent or so attenuated as to give little hope, even though shortened, of being adequate to sustain a heavy nterus. It may then be well to sukstitute for the above operation the procedure shown in Figure 397, whieh is to crumple the rudimentary ligament upon itself by means of a purse-string catgnt suture running from the internal inguinal ring to the uterus.

\section{Figure 396.}

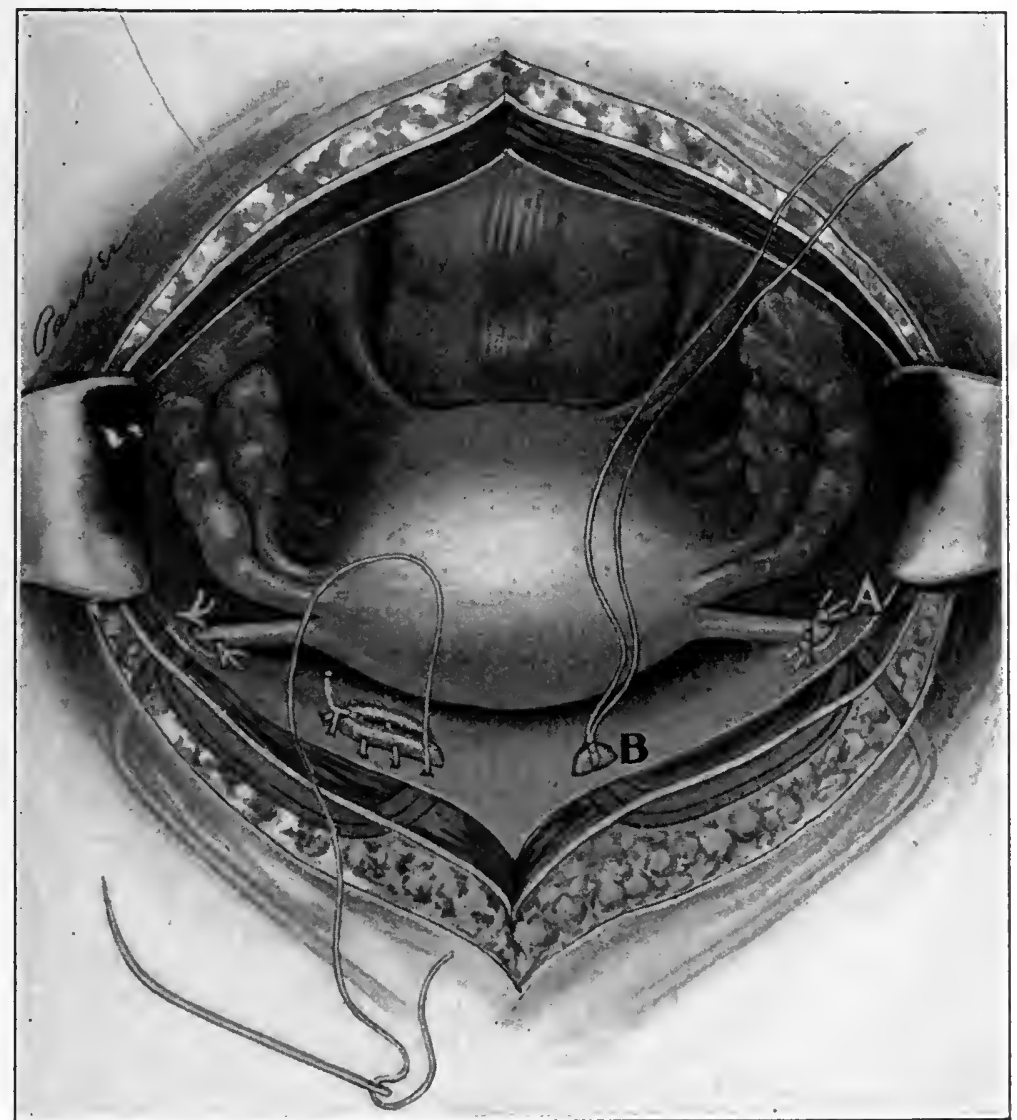

Continuation of Figure 395. The ligament having been drawn through the artificial inguinal canal is then stitched with catgut at its point of entrance at $A$, and the loop in a similar manner is stitehed down on the peritoneum at the point of exit $B$. 'The left side of the figure shows the ligament thus stitched at the point of entrance and being stitched at its point of exit. This having been done on both sides completes the shortening. The abdominal wound is then closed.

\section{Shortening the Uterosacral Ligaments.}

Any operation for shortening or strengthening the round ligaments may fail to give satisfactory results if the uterosacral ligaments are so relaxed that they cannot perform their function of holding the cervix uteri in its normal location back in the hollow of the sacrum. 
It may be necessary, therefore, that these ligaments be shortened alsc. Figures 398 and 399 illustrate the operation usually performed for this purpose, and show the change which the shortening makes in the location of the eervix uteri. Reference here is made to shortening the ligaments only through an abdominal ineision. A very exhaustive paper on the shortening, both vaginal and abdominal, of the uterosacral ligaments, with bibliography, by J. Wesley Bovée, may be found in the Transactions of the American Gynecological Society, 1902.

\section{Figure 397.}

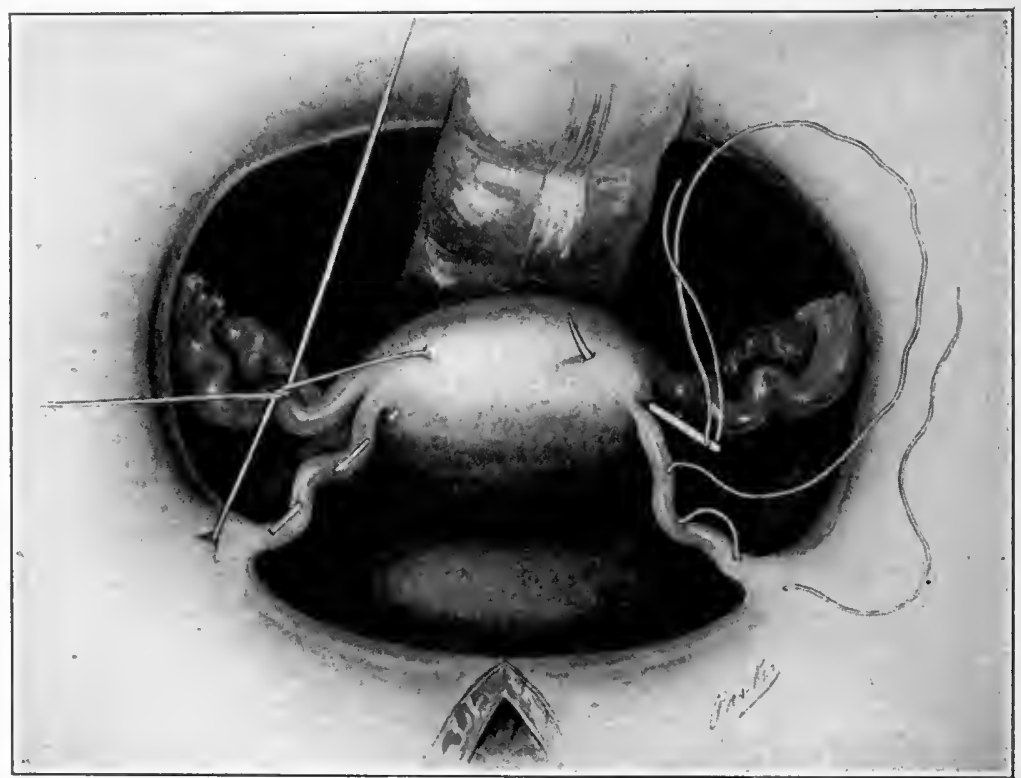

Traction being made on the ligament in order to locate its polnt of entrance at the internal inguinal ring. An ordinary needle is introduced at this point and the round ligament and the adjacent part of the broad ligament are caught up from point to point until the suture finally is brought ont in nterine tissue near the uterine end of the ronnd ligament. The tying of the suture, which should not be drawn too tightly, shortens and strengthens the ligament by crumpling it on itself. If the ligament is absent or highly attenuated, the structures of the broad ligament between the horn of the uterus and the internal ring may be brought together by a similar suture with similar result. Before tying the sutures the surfaces to be nnited shruld be scarified in order to secure strong union.

It is perhaps needless to add that the success of the operations above described may demand thorough repair of a lacerated perineum, or, more comprehensively speaking, may require adequate operative work upon a relaxed vaginal ontlet and sometimes even upon a relaxed vagina.

\section{Abdominal Hysterorrhaphy.}

Suspension of the uterus by abdominal hysterorrhaphy is known as the Howard Kelly operation. The object of the operation is to replace the uterus and secure it in its normal position by means of sntures so placed as to unite it with the anterior abdominal wall. 
FI(IURE 398

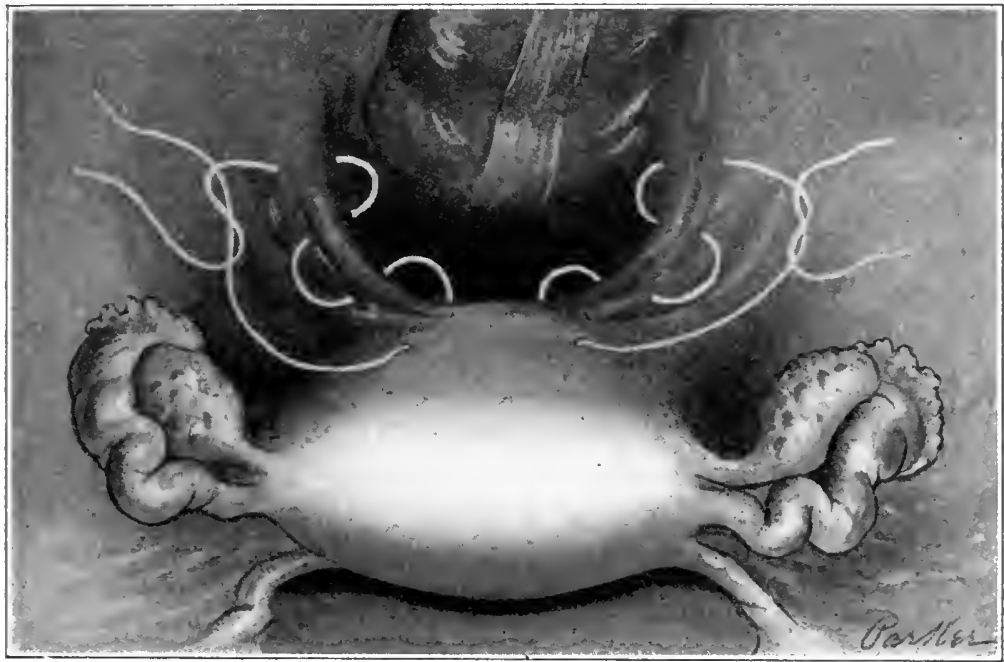

The uterosacral ligaments are exposed and a purse-string suture is introdueed for the purpnse of shortening them and partially tied, but not drawn taut. Before tightening the sutures the surfaces to be united shonld be searified in order to ensure firm union of the peritoneal surfaees. In order to avoid wounding important uerves and vessels the sutures sluould be passed supertieialiy.

Figure 399.

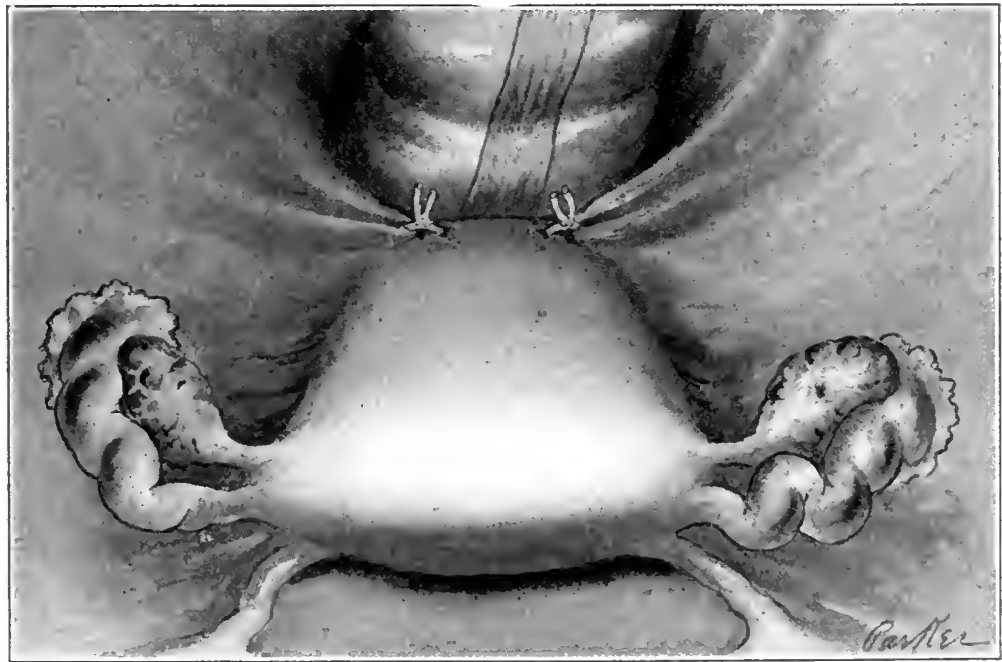

Contiuuation of Figure 39s. The catgut sutures shown in Figure 397 are now drawn tant and tied. These sutures having been tied usually sliould be fortified by an additional suture through the crumpled ligament on each side. This figure shows the cervix uteri draun well back into its normal location so near the hollow of the sacrum that the corpus cannot have room lo fall back. into its former retroverted positim.

\section{Nomenclature of Hysterorrhaphy.}

Hysterorrhaphy has been known under various names, some of them more or less deseriptive of special methods of operation. They are: rentral fixation, abdominal fixation, suspensio uteri, and hysteropexy. 


\section{Impediments to Replacement.}

The incision having been made, the left index-finger is introduced into the pelvis and a thorough study made of all the intrapelvic organs. Before the uterus can be replaced adhesions may have to be broken up, tumors of the uterus or its appendages may have to be removed, and conscrvative or radical opcrations upon the Fallopian tubes or ovaries may be necessary.

Posterior displacements of the uterus are associated often with salpingitis, ovaritis, and adhesions, which would render mechanical treatment by a pessary or shortening of the round ligaments useless or

Figure 400.

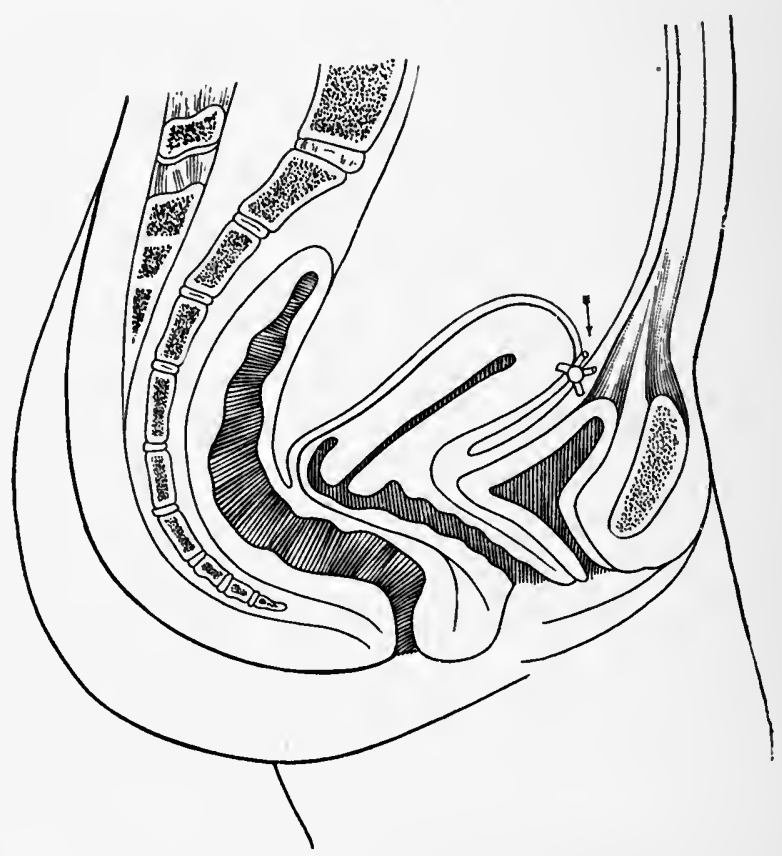

Suture wrongly placed in the anterior wall of the corpus uteri. The arrow points in the direction of the forces that fall on the anterior uterine wall and tend to force the organ back, and thereby to reproduce the displacement.

dangerous. The occasional failure to recognize these extra-nterine complications accounts for some disastrous results which have followed mechanical treatment and Alexander's operation. The great advantage of hysterorrhaphy is that the peritoneal cavity is open to direet examination and complete diagnosis. The surgeon, therefore, as he proceeds, may avoid unsuspected sources of failure or danger. The very contraindications for Alexander's operation become the indications for hysterorrhaphy. The impediments to replacement and fixation having been overcome or removed, the operation proper-that is, fixationmay be undertaken. 


\section{The Conditions of Successful Hysterorrhaphy.}

The sutures should be placed slightly posterior to a line connecting the two horns of the uterus-that is, in the posterior wall. The earlier operators stitched the anterior wall of the corpus to the abdominal wall. By this arrangement the uterus is so placed that contraction of the abdominal museles and the intra-abdominal forces must be exerted against the front of the uterus, and must therefore, by foreing the uterus back, ultimately stretch or break the adhesions and reproduce the displacement. If, on the contrary, the posterior wall of the corpus be stitched properly to the anterior abdominal wall, all the forces from above are exerted on the posterior wall of the corpus, and thereby tend to perpetuate the normal anterior position. ${ }^{1}$

Another condition of success is to limit the adhesions between the uterus and the abdominal wall. When the adhesion is to the posterior

Figure 401.

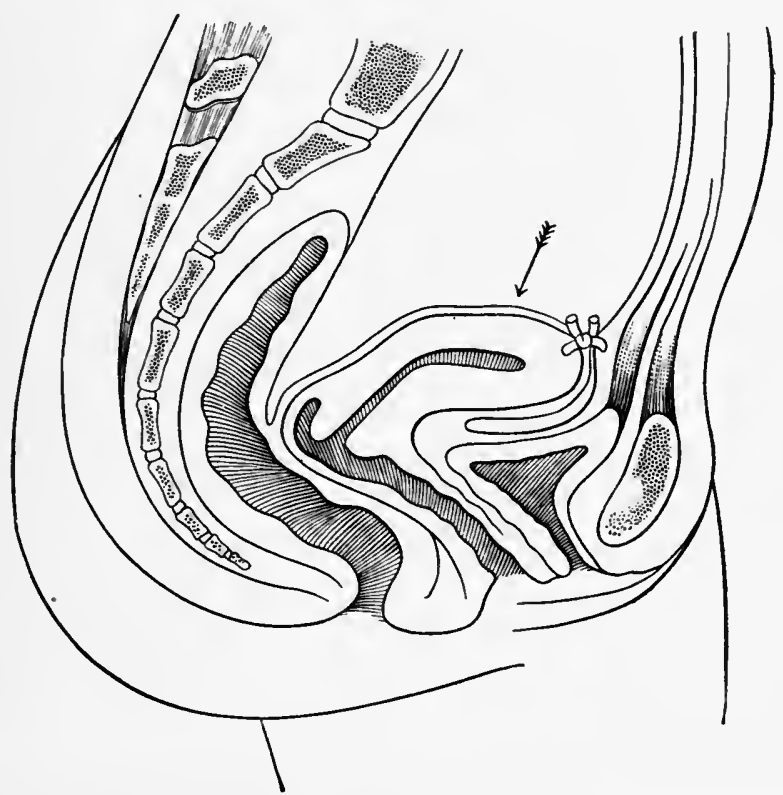

Posterior wall of the corpus properly stitched to the anterior abdominal wall. The arrow shows the direetion of forces from above so exerted as to fall on the posterior uterine wall, and thereby perpetuate the normal anterior position.

wall of the corpus uteri, it is surprising how slight it may be and yet make a permanently good result. The object of the operation is not to fix the uterus immovably to the abdominal wall by broad areas of adhesion; such a result is produced sometimes by numerons deep sutures and extensive searifications of the anterior or posterior face of the uterus. The broad unyielding adhesions thus obtained must interfere with the normal movements of the uterus, and thereby give

1 The necessity for uniting the posterior wall of the corpus uteri, instead of the anterior wall, to the parictal peritoneum of the abdominal wall, was pointed out first by Howard Kelly. 
rise to a condition more distressing than the displacement. Figure 402 shows the abdomen open and three hysterorrhaphy sutures passed, but not tied.

Occasional cases of dystocia, some of them demanding craniotomy or even Cæsarean section, bear witness to the danger of excessive uterine adhesions to the abdominal wall. After the operation the adhesions, if properly made, do not remain as such, but stretch out so far as to form a short, ribbon-like band between the uterus and the abdominal wall. This band contains connective tissue and possibly some fibres from the recti and uterine muscles, and is eovered by peritoneum; it is therefore a new suspensory ligament designed to supplement the inadequate uterine ligaments. This ligament has been demonstrated by dissection years after the operation." It is usually, when fully stretched, about two inches long. This elongation of the adherent structures into a new uterine ligament cannot oeeur if the adhesions are too extensive and too strong.

\section{Technique of Hysterorrhaphy.}

The incision, general conduct of the operation, closure of the wound, and after-treatment are the same as for any other abdominal section. See Chapters VI., VII., and VIII.

The introduction of the hysterorrhaphy sutures varies in minor details according to the individual preference of the operator. The writer uses two formaldehyde catgut sutures, one on each side of the posterior wall of the corpus uteri.

An abdominal incision from one to two inches long is made in the median line just above the pubes. The margins of the peritoneum are drawn through the wound over the cutaneous margins, and are held outside by hrmostatic forceps, as shown in Figure 393. The corpus uteri is lifted forward by the left index-finger and middle tinger introduced through the wound, and is held in place by light vulsellum forceps in the hands of an assistant.

The teeth of the forceps grasp the posterior surface of the corpus in the median line about one-hilf inch back of the summit of the fundus. The operator, standing on the patient's right, passes a short needle, slightly curved at the point and threaded with fine formaldehyde catgut, into the everted peritoneum on the left side. The needle enters just above the lower angle of the wound, about three-quarters of an inch from the peritoneal margin; it dips down about one-quarter of an inch so as to include some tibres of the rectus muscle, and emerges about one-half inch from the point of entrance. The needle then is reintroduced into the posterior wall of the corpus to one side of the median line near the hom of the uterus. Care should be taken not to puncture the Fallopian tube. The uterine part of the suture should include sufficient peritoneal and subperitoneal tissue to give it a strong hold on the uterus. The free ends of this suture now are fastened together by snap-forceps and laid to one side. Another similar suture is passed on the opposite side. It is convenient in passing this to do 
so in the reverse order-that is, to pick up the uterus first and the abrlominal peritoneum second. A third suture now is introdueed through the parietal peritonem near the first suture, then through the corpus uteri between the two first sutures and through the parietal peritoneum near the second. The three sutures being thus passed, a

Figure 402.

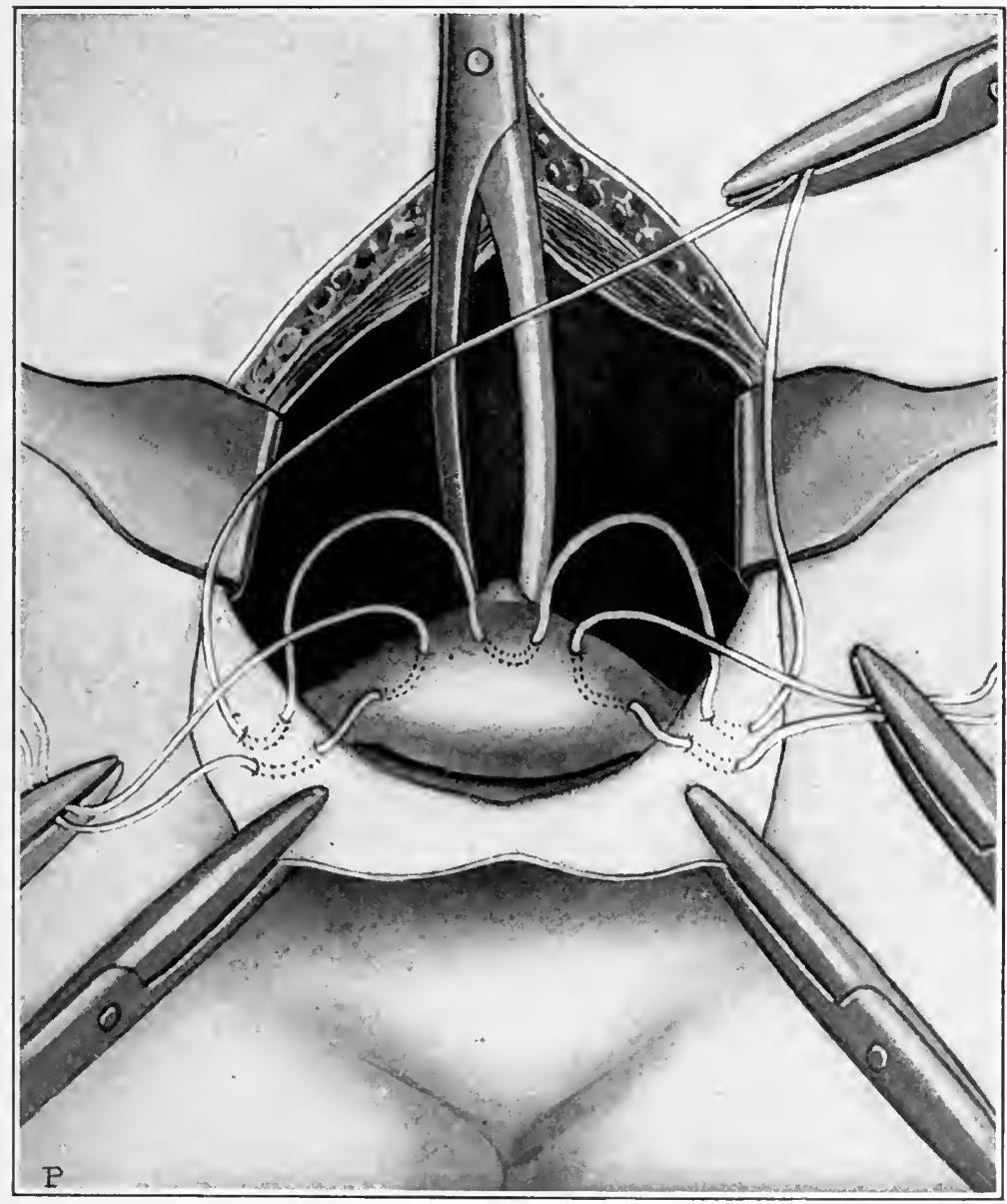

Peritoneal margins of the wound everted to the outside by snap-forceps. Corpus uteri held steady by a vulsellum forceps. Sutures on both sides and in the centre of the corpus passed but not tied.

final examination of the pelvic contents is made; and if all is well, the sutures are tied. This secures the upper posterior wall of the corpus uteri to the anterior abdominal wall. The external wound then is closed by continuous catgut suture, as directed in Chapter VI. There is no occasion for scarifying the peritoneal surfaces; indeed, this should not be done. Adequate adhesion will always form from the presence of the sutures. The pessary is not needed during convalescence. 
Immediately after the operation, while the uterus is fixed immorably, there may be some bladder irritation; but in a few weeks, when the adherent struetures have stretched and formed a new suspensory ligament and the uterus has assumed its normal state of mobile equilibrium, the vesical irritation disappears. This operation properly performed gives more security against recurrence of displacement than any of the ligament-shortering operations.

\section{Vaginal Hysterorrhaphy. ${ }^{1}$}

The purpose of this operation is to anchor the uterus in its normal anterior position by stitching the anterior wall of the uterus to the anterior wall of the vagina.

\section{Technique of Vaginal Hysterorrhaphy.}

Vaginal section is made as directed in Chapter XXIII. The patient is on her back, with the cervix uteri and anterior vaginal wall exposed by means of Simon's speculum and other retractors. The uterus is drawn downward and backward by means of vulsellum or bullet forceps. The anterior vaginal wall is put upon the stretel by means of a small vulsellum forceps fastened to the vagina in the median line midway between the meatus urinarius and the cervix uteri. A median longitudinal incision then is made in the anterior vaginal wall extending from the cervix uteri one inch or more toward the meatus. This incision, which divides the vaginal wall, but does not invade the bladder-wall, is separated by retractors, the cervix is drawn more strongly forward, and the loose cellular tissue adjacent to the anterior wall of the cervix is stripped back by means of the finger or blunt instrument until the uterovesical reflection of the peritoneum is reached. See Figures 170 and 171.

A sound in the bladder will distinguish the peritoneum from the bladder-wall. The peritoneum, then exposed, is scized with the tenaculum or snap-forceps, and divided with blunt scissors. The peritoneal opening next is enlarged by introducing the two index-fingers and tearing and stretehing it laterally, or by careful cutting with the scissors. The large opening thus made between the uterus and the bladder will permit the bladder to be pushed up out of the way and the corpus uteri to be drawn through into the vagina and down to the vulva. If there are restraining posterior or lateral adhesions, they may be broken up by the finger introduced through this opening or through a similar one made for the purpose posterior to the uterus. See Posterior Valginal Section. The uterus, being freed, is drawn into the vagina by successively grasping its anterior wall with two pairs of vulsellum forceps, one in each hand, using first one and then the other, until the fundus finally appears and with it the appendages. Any necessary operation on the uterus or its appendages may now be performed; there may be a small myoma to be enucleated, or some conservative or radical operation to be performed on the uterine

${ }^{1}$ This operation has passed throngh numerous modifications, is still sub judice, and is not strongly recommended. Among the names ehiefly associated with the evolution are those of schüeking, Sänger, Mackenrodt, Dührssen, Bỵford, and Vineberg. 
appendages. 'The uterus is now ready to be fastened to the anterior vaginal wall, as follows :

A needle, such as would be used for closing the lacerated cervix, is threaded with silkworm gut, and by means of the needle-forceps is passed through the flap of the vaginal incision near the urethra, on the patient's left, then continued transversely through the anterior wall of the uterus near the fundus, and brought out through the vaginal flap on the opposite side. Another similar suture is passed immediately below this. These uterine sutures are not tied until after closure of the vaginal incision. The vaginal incision is closed with a continuous buried catgut suture in a manner similar to that described in Chapter VI., for closure of an abdominal wound. The vagina is packed lightly with aseptic gauze and an aseptic dressing is placed over the vulva. The dressings should be changed often enough to keep them clean. After three days the vaginal gauze may be left out, and in its place may be, given a daily hot aseptic vaginal donche. The silkworm gut sutures are removed in four weeks. Chromic catgut, if used in place of silkworm, need not be removed.'

Unless the adhesions between the uterus and the vagina be very broad and very strong, they are liable in a few weeks to give way, with complete return of the displacement. If, on the other hand, the adhesions are sufficiently strong and broad to make a permanent anatomical cure, and pregnancy follows, the danger from dystocia is very great. Numerous cases have been reported, some of them fatal, in which Cæsarean section or other grave obstetric operations became necessary for delivery. The operation therefore, as described above, is not approved for cases in which pregnancy may occur.

\section{Complicatixg Versions and Flexions.}

The lateral malpositions which often complicate retroversion and retroflexion are usually the result of inflammation in a broad ligament or in the uterus, or in both. They are caused sometimes by a tumor of the uterus or its appendages. The treatment is that of the causative inflammation or tumor, and follows the general principles that have been laid down for the treatment of these conditions. . Pessaries are of little or no use.

In Congenital Retroversion and Retroflexion it is doubtful whether any treatment, surgical or non-surgical, is of the least value. This form of displacement is associated usually with faulty development, both general and local. The concurrent symptoms also are due rather to general than to local causes.

1 The method described above is similar to that advocated and successfully practised by Vineburg. New York Medical Journal, Oetober, 1894. 


\section{CHAPTER XLVIII.}

\section{AN'TEVERSION AND ANTEFLEXION OF THE UTERUS: TORSION OF 'THE U'TERUS.}

\section{PATHOLOGICAL ANTEVERSION OF THE UTERUS.}

A Cerrtaly degree and eondition of anteflexion is normal. See Normal Position and Normal Movements of the Uterus, in Chapter XLIV. 'The evils of pathological anteflexion are more a matter of the associated lesions than of the displacement per se.

Sometimes the physiologieal angle of flexure becomes obliterated in eonsequence of ehronic metritis, and results in permanent straightening of the uterus. The cervix becomes elevated and fixed above, or the corpus depressed and fixed below the normal level. This eonstitutes pathological anteversion. Figure 403.

Anterersion is associated often with pathological anteflexion. The mobility at the angle of flexure then is increased, diminished, or absent; the flexure is then the signifieant factor, and will be considered under Pathologieal Anteflexion.

\section{Etiology and Symptoms of Anteversion.}

The eauses of pathologieal anteflexion may be summarized as follows :

1. Adhesions in front of the uterus, drawing the corpus forward.

2. Tumors behind the uterus, pushing the fundus forward.

3. Metritis, inereasing the weight of the uterus.

4. Small fibroids in the anterior wall of the uterus.

5. Congenital.

The exaggerated anteversion of early pregnancy is physiological ; the exaggerated anteversion of the uterus in chronic metritis is pathological. Elevation of the cervix and depression of the eorpus uteri may be induced by peritoneal adhesions. Increased weight from a mural myoma also may depress the eorpus.

The symptoms are due to the pelvie inflammations and other eomplications already mentioned. 'The increased weight of the uterus, which usually is hypertrophied from metritis, generally eauses a dragging sensation, especially if the organ be prolapsed.' 'The enlarged corpus oecupying the territory of the bladder often induees persistent vesical irritation, or even eystitis. Menorrhagia, when present, is the result of the metritis or of a myoma, rather than of the displacement itself. The symptoms usually attributed to anteversion usually are due rather to the complications than to the malposition. 


\section{Diagnosis and Prognosis of Anteversion.}

The displacement is recognized by digital touch, which diseloses the anterior wall of the enlarged uterus parallel to the anterior wall of the vagina, with the fundus close to the symphysis and the cervix elevated. Conjoined examination will show the size, shape, hardness, and degree of fixation. Exaggerated anteversion of the healthy uterus is not necessarily pathological in its results. This is illustrated by the anteversion of early pregnancy. The prognosis is good if the complications can be removed.

Figure 403.

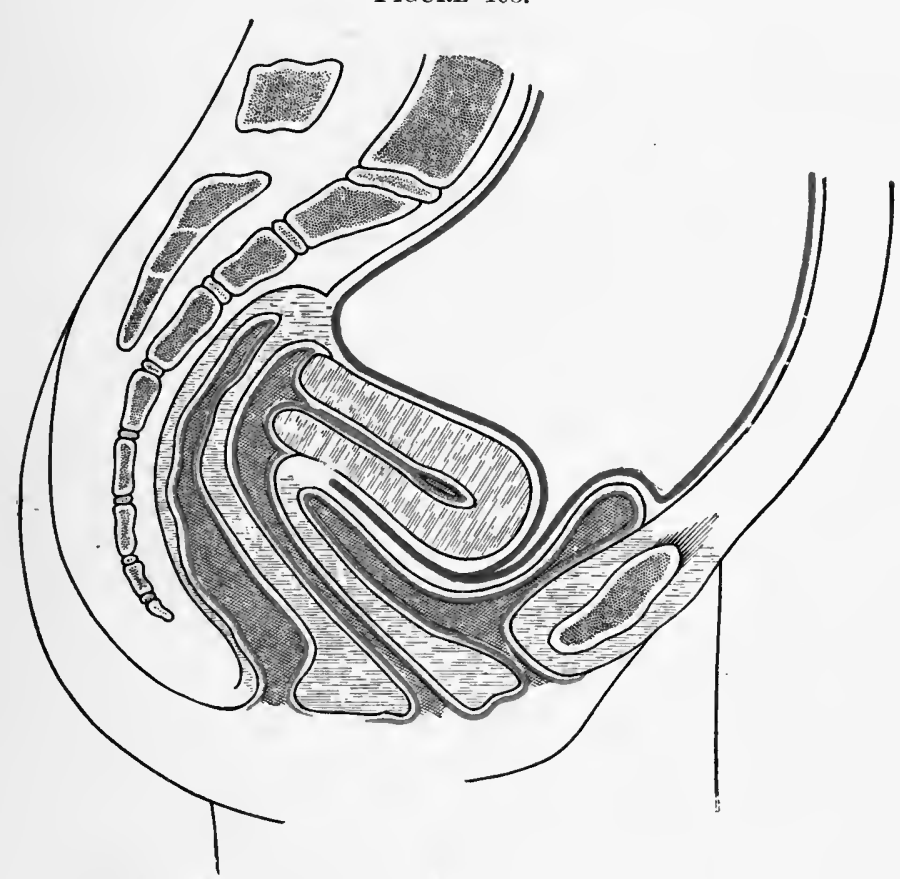

Pathologieal anteversion. Mobility at angle of flexure lost.

\section{Treatment of Anteversion.}

If exaggerated anteversion is often the position taken by the uterus in chronic metritis, it follows that the treatment often will be that of chronic metritis. For the treatment of metritis, perimetritis, myoma, menorrhagia, and other complications and lesions associated with the displacement, the reader is referred to those subjects. Irritable bladder, which is often a mechanical result of the displacement and enlargement, may be relieved sometimes by means of an Albert Smith or Hodge pessary, which lifts the organ to a higher level away from the bladder. In thus elevating the uterus the anteversion may be increased rather than diminished. This proves that the symptoms were dependent not upon the anteposition, but rather upon de- 
scent and antelocation. Should the parts be too sensitive to tolerate the hard-rubber pessary or a flexible rubber ring, the daily application of medicated pledgets of lambs' wool may give support to the uterus and decrease tenderness until the more permanent instrument can be worn. The numerous anteversion pessaries designed to elevate the corpus by direct pressure on the anterior wall of the uterus generally irritate the organ and thereby aggravate the inflammatory complications. They, therefore, are to be condemned.

Figure 404.

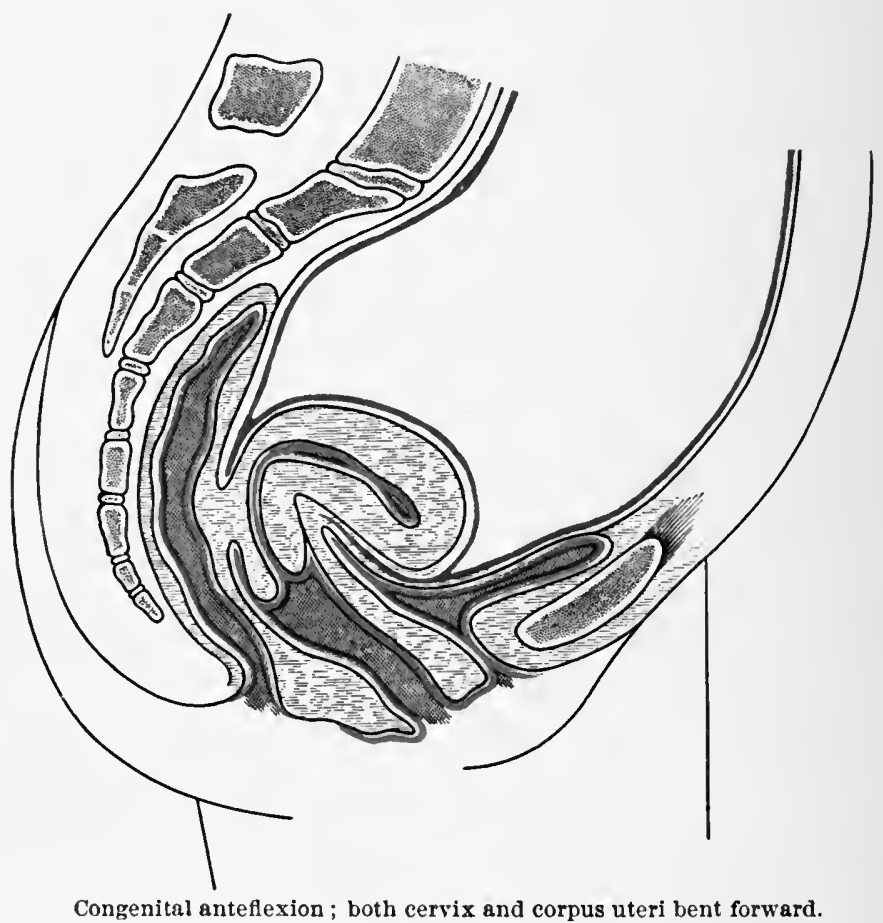

PATHOLOGICAL ANTEFLEXION OF THE UTERUS.

A comprehensive study of pathological anteflexion would have to take into account the abnormal conditions usually associated with it ; these may have the relation of cause or effect, or be a concurrent result of some common cause.

A distinetion between normal and pathological anteflexion would show that an essential factor in the former is mobility at the angle of flexure which permits the degree of flexure to vary within certain defined limits. The limit of normal anteflexion is approximately 90 degrees. The physiological variation is somewhat commensurate with the varying quantity of fluid in the bladder.

The body of the uterus rests upon the bladder, and must rise as the bladder becomes distended. Conversely, if the urine be drawn 
through a catheter, even while the woman is lying on the back, the corpus, notwithstanding the opposing influence of its own weight, immediately follows the receding wall of the bladder and returns, through an angle of 45 degrees or possibly even 90 degrees, to its aceustomed position.

The normal forward bending of the corpus upon the cervix uteri when the bladder is empty makes an angle of which the approximate physiological limits are between 45 degrees and 90 degrees; the flexure, therefore, would generally be pathological if less than 45 degrees or more than 90 degrees. Furthermore, if the flexure, whether it be normal or abnormal in extent, does not disappear upon filling the bladder, but remains constant under all conditions, the rigidity makes the flexure pathological.

Anteflexion is, therefore, pathological if the mobility at the angle of flexure is increased or diminished, or absent.

\section{Etiology and Classification of Anteflexion.}

Anteflexion may be either congenital or acquired.

Congenital and Developmental Antefléxion.-The nterus in this form of anteflexion is bent upon itself almost double, the body and the eervix both pointing in the direction of the pelvic outlet. The cervix is somewhat elongated and situated in the long axis of the vagina; see Figure 404. The cause may be defective foetal development; or failure of the immature child uterus to develop at puberty, a failure which usually pertains alike to the uterus, Fallopian tubes, ovaries, and vagina. A more proper name is infantile uterus.

Acquired Anteflexion may be simply an exaggeration of the normal flexure, due either to increased weight of the corpus from the presence of a myoma near the fundus, or to unequal growth of the uterine walls, or to unequal involution, or to an abmormally soft, mobile condition of the uterine walls. A not infrequent eause of anteflexion is thickening of the posterior wall of the uterus from the products of inflammation, and a corresponding atrophy of the anterior wall from prolonged pressure at the angle of flexure. This condition is apt to be associated with post-uterine inflammation involving the uterosacral ligaments, a frequent and discouraging eomplication. Sometimes the inflamed ligaments contract and drag the anteflexed uterus upward and backward, where it may be fixed permanently in its post-uterine location by peritoneal adhesions.

\section{Pathology of Anteflexion.}

Peri-nterine inflammations having the relation of either canse or effect to the flexure, often bind the pelvie organs together in a mass of exudate, with resulting failure of nutrition, nerve irritation, and constant pain, which sometimes render the patient's life miserable and useless. Constrietion or collapse of the uterine canal at the point of flexure may, by confining the secretions above, produce inflammation in the body of the uterus, Fallopian tubes, and ovaries. This is 
analogous to the cystitis, urethritis, pyelitis, and nephritis which follow stricture of the male urethra. As the fecal matter passes the cervix during defecation, force is applied to the posterior cervical wall in the direction of the lower arrow. Figure 405. At the same time fixation of the abdominal muscles due to straining, whether in urination or defecation, results in the application of force upon the corpus uteri in the direction of the upper arrow. Thus the flexure is increased and perpetuated with defeeation and urination.

\section{Symptoms, Course, and Complications of Anteflexion.}

The numerous symptoms due to the inflammatory and other complications should not be confounded with those that directly depend upon the displacement. The symptoms of anteflexon usually may be

\section{Figure 405.}

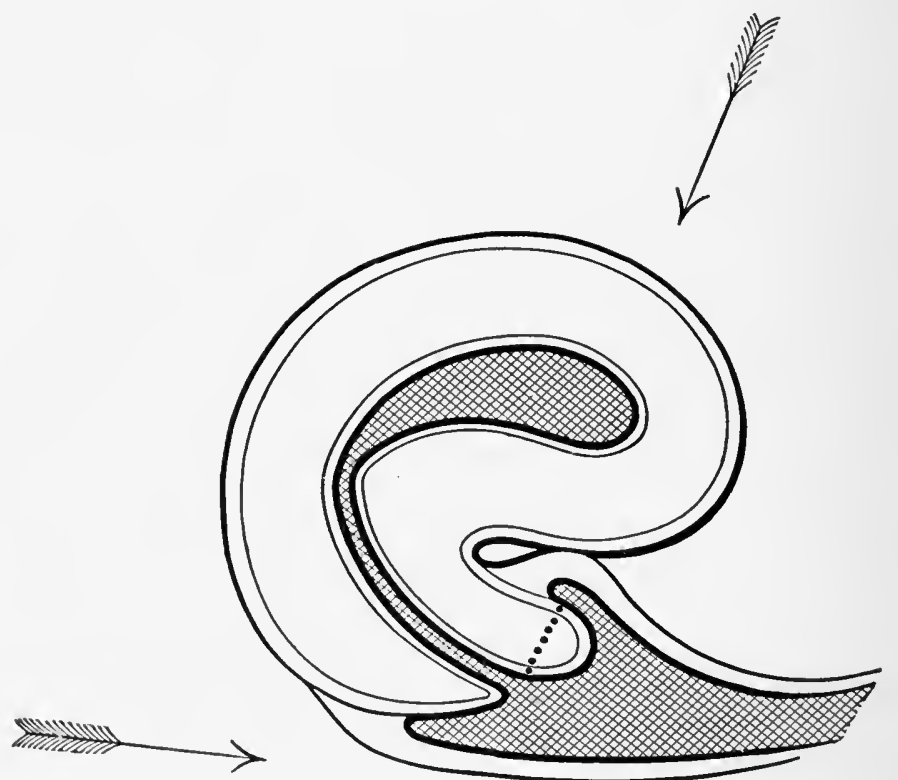

The arrows show the influence on the displacement of the forces produced by straining at stool.

referred, first, to the bladder and urethra ; and, second, to the uterus itself.

The Vesical and Urethral Symptoms are produced either by rigidity of the uterine tissue at the angle of flexure, which prevents the corpus uteri from rising out of the way of the filling bladder; or by inflammatory shortening of the uterosacral ligaments, which, by drawing the uterus upward and backward, puts the vesicovaginal wall on the stretch; this causes traction upon the neck of the bladder and consequent bladder and urethral irritation, and may be the startingpoint of cystitis and urethritis. 
Vesical irritation caused by postuterine inflammation and consequent contraction of the uterosacral ligaments often is attributed wrongly to the mechanical pressure of the anteflexed corpus uteri upon the bladder; this is manifestly impossible, for the contracted uterosacral supports hold the entire uterus far away from the bladder.

Uterine Symptoms.-When the flexure has gone beyond the normal limit and become pathological, two principal results may occur, especially if there be immobility at the angle of flexure :

1. Collapse of the blood-vessels at the angle of flexure, with consequent obstruction of the circulation, passive congestion, and hypersecretion of a vitiated mucus.

FIgURe 406.

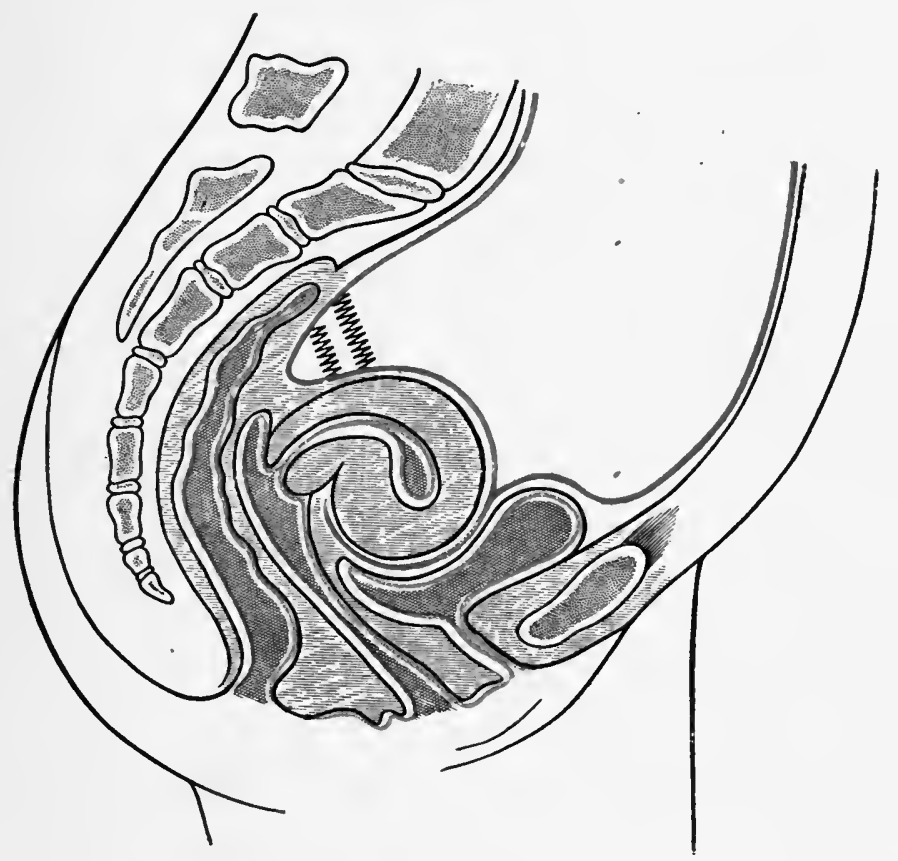

Acquired anteflexion with postuterine fixation. Want of mobility at angle of flexure.

2. Collapse and obstruction of the uterine canal at the angle of flexure, with consequent retention of the uterine secretions. The secretions may decompose and become a potent source of irritation; the uterine mucosa could then neither perform its normal part in menstruation nor furnish a safe resting-place for the impregnated ovum. The possible symptom-group dependent upon these two forms of obstruction includes endometritis, dysmenorrhoea, and sterility.

Endometritis may be caused and perpetuated by the endometrial and vascular obstruction. The causation of rhinitis from obstruction in the nasal passages and of cystitis from stricture of the urethra is analogous. 
Dysmenorrhoea may depend upon collapse and constriction of the uterine canal at the angle of flexure. This causes the blood to accumulate and to coagulate in the body of the uterus, from which it is expelled at intervals by uterine eontractions simulating labor pains. Pain, when due to this cause, is therefore always specially severe just before the passage of a clot. Dysmenorrhœa may also be caused by similar collapse and consequent obstruction in the veins at the angle of Hlexure; this causes intense venous congestion of the entire body of the uterus; pain then is due to pressure of the swollen vessels upon the nerve-filaments and to a consequent irritable condition of the muscular tissue of the uterus. Sometimes the uterine canal becomes temporarily straightened with the establishment of the flow; this removes the cause of the vascular obstruction, and the pain from congestion is relieved. It is elear that the pain would be intensified in a uterus hypersensitive from metritis, and especially from neuritis.

Sterility is consequent not so much upon failure of impregnation, as upon the fact that the ovum, if impregnated, is unable to survive in the hostile environment of an infected endometrium. It is often maintained that the constriction in the uterine canal per se prevents the entrance of spermatozoa, and therefore canses the sterility. This in a measure may be true ; but endometritis. which often results from obstruction is the more direct and frequent cause of sterility.

\section{Diagnosis of Anteflexion.}

Before the distinction was made between physiological and pathological anteflexion, it was usua! to treat all anteflexions as pathological. The reaction came, and with it a universal proposition that anteflexion had no pathological significance per se; that it was wholly a question of the assoeiated lesions. But, like other universal propositions, this one was too sweeping; it did not take into account pathological anteflexion.

The educated touch which distinguishes the normal version, flexion, and movements of the uterus will appreciate the anatomical differences between pathological and normal anteflexion. The degree of flexure, the mobility or rigidity, and the size, shape, location, and consistence of the uterus may be ascertained by conjoined manipulation. The presence of postuterine inflammation is recognized by the pain caused in drawing the uterus slightly forward, and by the increased thickness and tenderness which may be felt by vaginal or rectal tonch in the region of the uterosacral ligaments. Anteflexion is distinguished from myoma in the anterior wall of the uterus by conjoined examination and the sound. The common error of mistaking the normal version and flexion of a prolapsed uterus for pathological version and flexion should be avoided.

Congenital Anteflexion will be characterized by :

1. The small size of the uterus.

2. The small or pin-hole os uteri.

3. The relative lengths of the corpus and cervix uteri; the corpus is one-third and the cervix is two-thirds the length 
of the entire uterus. The reverse of these measurements is true of the fully developed uterus. See Chapter I.

Acquired Anteflexion will be recognized by one or more of the following conditions:

1. Resisting bands behind the uterus.

2. Downward and forward direction of the cervix uteri in the long axis of the vagina.

3. Flexure of the corpus uteri upon the cervix; the angle of flexure is easily palpated in front of the cervix.

FIGURE 407 .

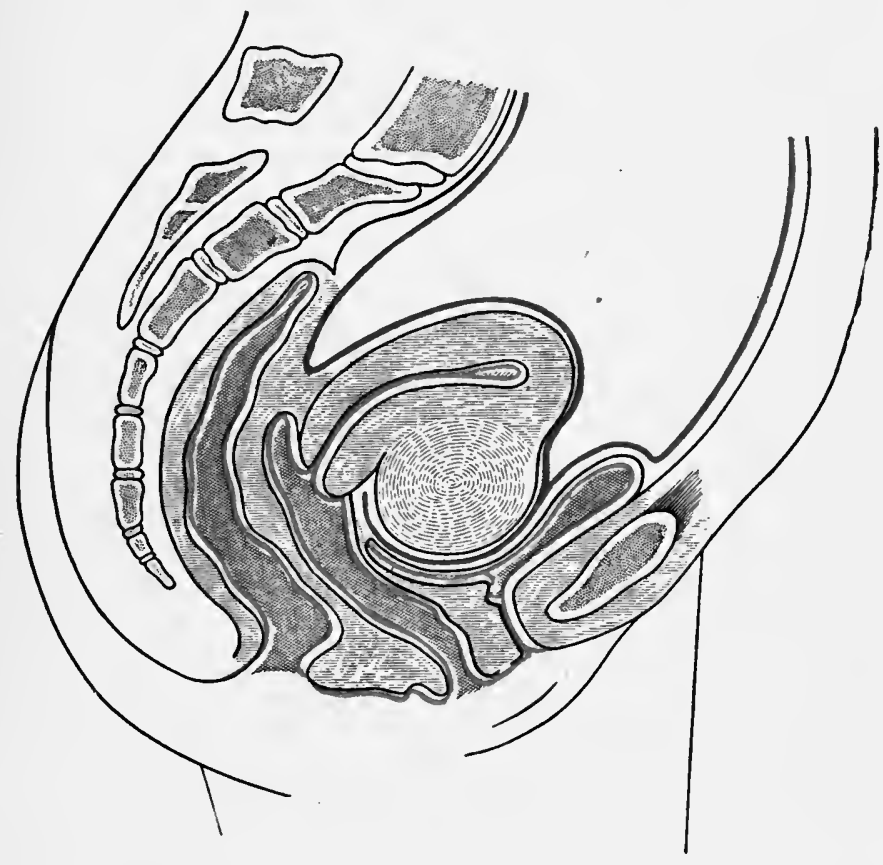

Myoma on the anterior uterine wall, simulating anteflexion.

\section{Treatment of Anteflexion.}

The treatment is directed, first, to the complications; second, to the mechanical indications for straightening the flexed uterus.

The Treatment of the Complications.-If there be inflammation of the uterus and its surroundings, in the relation of either cause or effect to the displacement, its suceessful treatment becomes the prime indication, because, unless treated, it is a contraindication to the more direct treatment of the malposition itself. It may be necessary to remove a tumor or to separate adhesions. Incurable chronic metritis may render all direet treatment useless. Improvement in the general health, treatment of other complications, and palliation then become the only resources. 
Before considering the various recognized measures for the direct treatment of the flexure itself, it is important to exclude all cases of normal anteflexion. It would be clearly absurd to treat normal anteflexion for dysmenorrhoa or sterility.

The Mechanical Indication, when the flexure is pathological, is clearly to straighten the uterus, so that :

a. The uterus may be out of the range of the forces indicated by the arrows in Figure 405.

$b$. The circulation may be relieved.

$c$. The uterine canal may perform its natural functions as a drainage-tube.

Figure 408.

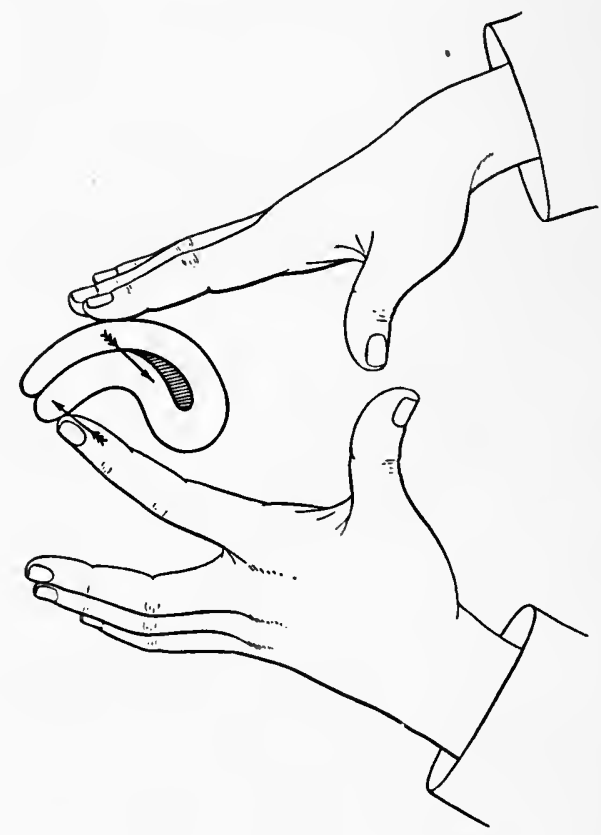

Treatment of anteflexion by massage.

The mechanical treatment includes the following measures:

1. The pessary.

2. Local massage.

3. Electricity.

4. Forcible dilatation.

5. Posterior division of the cervix.

6 . The author's operation.

1. The Pessary.-The various anteflexion and anterersion pessaries that have been devised for the purpose of propping up the corpus are almost useless. Their questionable reputation depends upon the relief they frequently give to complicating prolapse, the symptoms of which have been attributed wrongly to anteflexion and anteversion. If pessaries are indicated at all, therefore, they may be used upon 
the same prineiple as in descent. See Treatment of Deseent. Intranterine stem-pessaries designed to straighten the flexed uterus are sometimes effective-always dangerous.

2. Local Pelvic Massage applied during menstruation and in the intermenstrual period has some value for the temporary relief of dysmenorrhoa due to anteflexion. The treatment consists of pushing the cervix upward and backward with the left index-finger, while vith the right hand a forward and downward pressure is exerted on the organ ; this eonverts for the time the anteflexion into an extreme anteversion. The method is illustrated by Figure 408 .

When the displacement is associated with dysmenorrhœa the massage may be continued during menstruation, and in some eases with

Figure 409.

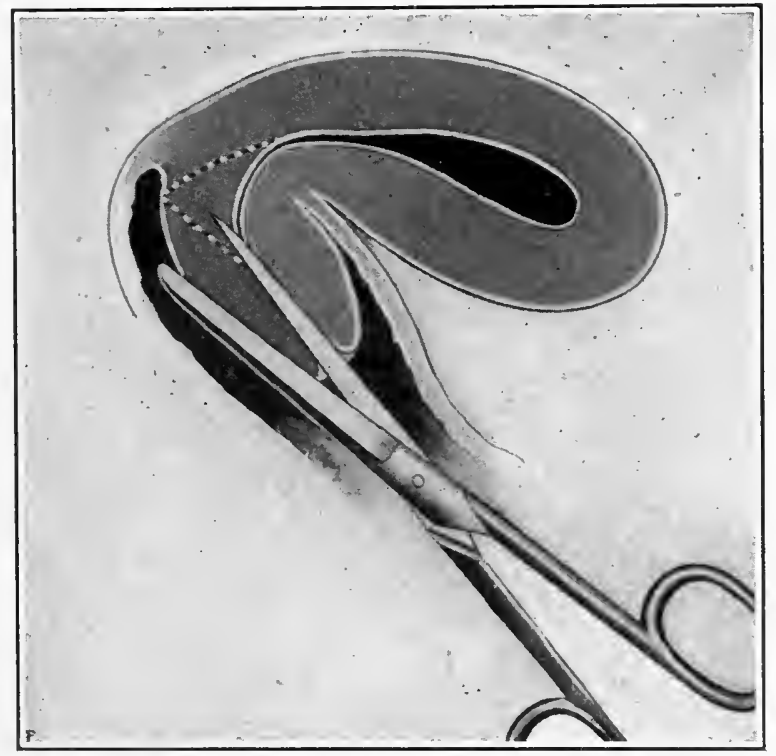

Posterior division of the cervix uteri: lines of incision in flexion of the uterus. Sims' operation.

great and immediate relief. It should be continued also for at least a number of weeks.

3. Flectricity is said by the advoeates of it to be a useful agent; it is, however, by no means effective enough to stand alone as the accepted treatment of .pathological anteflexion. After considerable personal experienee the writer has disearded it.

4. Forcible Dilatation.-This operation, usually associated with curettage, is described in Chapter V.; it is indicated in anteflexion with collapsed or stenosed uterine canal and associated endometritis, dysmenorrhœa, or sterility.

The following is an abstract, with some modifications, of a valuable contribution ${ }^{1}$ by Goodell, in which he gives positive indorsement to

1 American Journal of Obstetrics, p. 1179, 1881. 
rapid dilatation as proposed by Ellinger and others. The instruments used are two Ellinger dilators, which are recommended on account of the parallel action of their blades. The dilatation is begun with the smaller instrument and completed with the larger. The larger instrument has powerful blades that do not spring nor feather. The light instrument has only a ratchet in the handle; but the stronger one has a screw that forces the handles together and the blades apart. To prevent injury to the fundus when the instrument is open, the

FigURE 410.

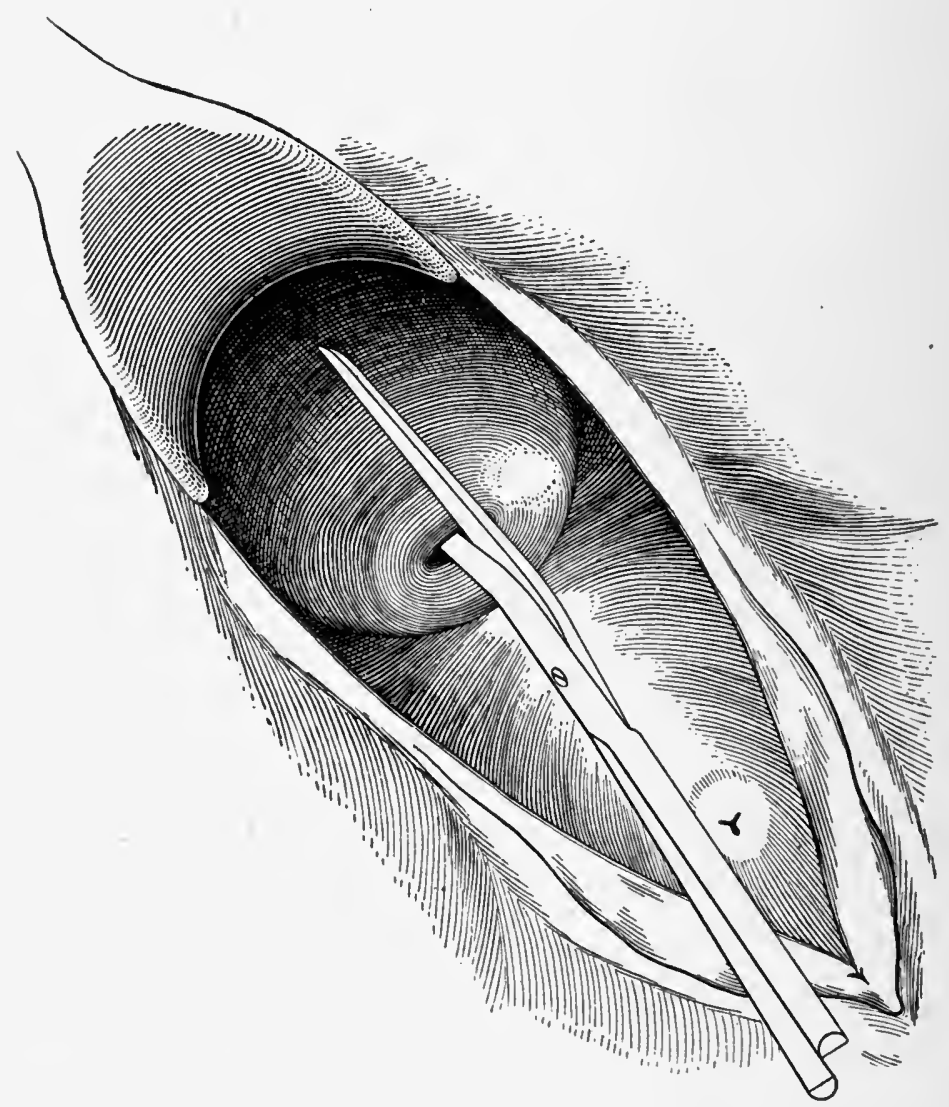

The posterior wall of the cervix being divided by scissors.

'length of the blades is limited to two inches. The larger instrument has a dilating capacity of one and a half inclies, and has a graduated are in the handles to indicate the divergence of the blades. Goodell's modification of Ellinger's dilator is provided with serrated blades, to keep them from slipping out of the canal during the process of dilatation.

For dysmenorrhœa or sterility due to flexion or stenosis the method of operation is as follows: A suppository containing a grain of the 
aqueous extract of opium is introduced into the rectum, the patient etherized, and the uterus exposed by Sims' speculum. 'The cervix is held by a tenaculum, and the smaller dilator is introduced as far as it will go. Upon gently stretching open that portion of the uterine canal which it occupies, the stricture above so yields that when the blades are closed they will pass higher. By repeating this manœurre a cervical canal is tunnelled out where before not even a fine probe could be passed. Should the os externum or cervical canal be too

Figure 411.

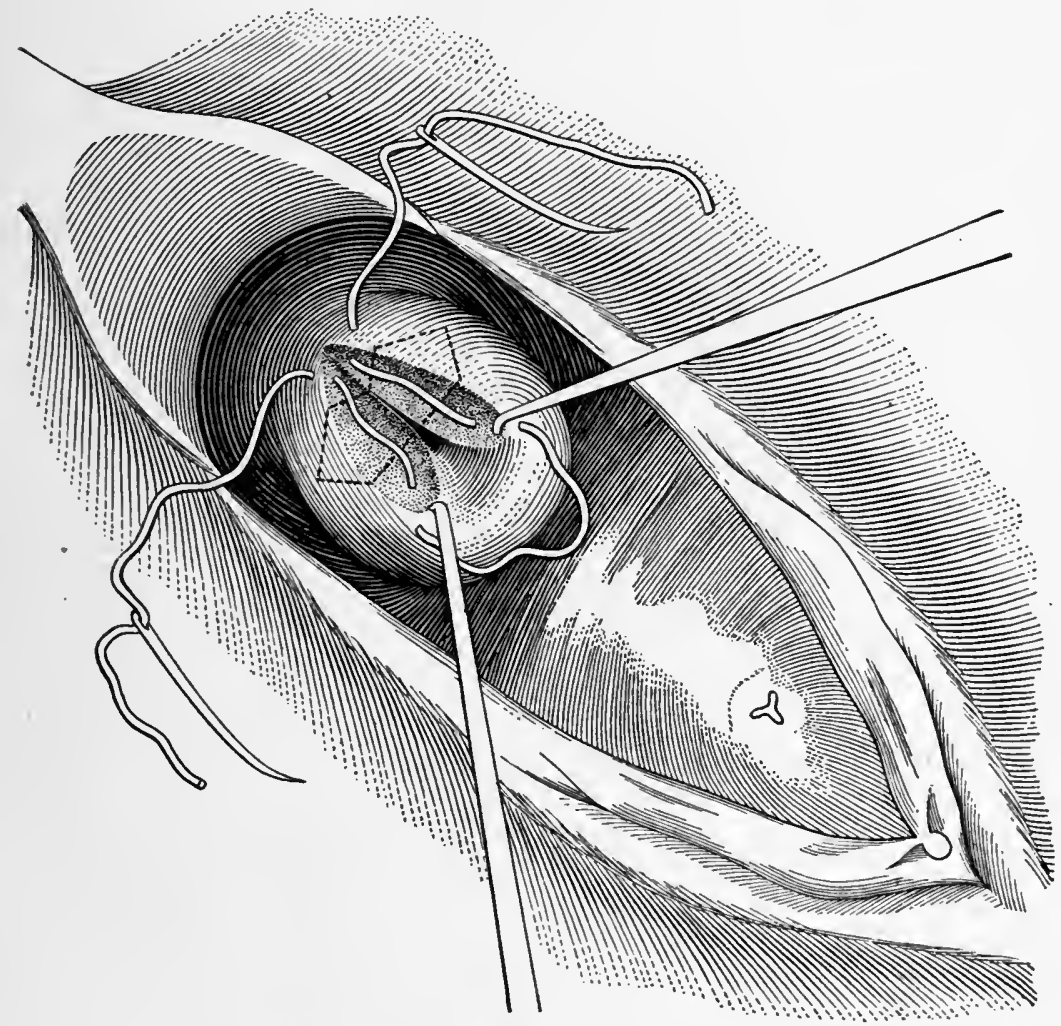

The cut surfaces held anart by tenacula. The dotted lines show wedge-shaped pieces to be removed by scissors, in order to make the cut surfaces more readily fold upon themselves. Sutures designed to fold cut surfaces on themselves in place, but not tied.

small to admit the instrument, a pair of pointed seissors may be substituted, and by the same opening and closing motions the canal may be prepared for the introduction of the smaller dilator. As soon as the cavity of the uterus has been entered the handles are brought together. This dilator is then withdrawn, the larger one introduced, and its handles slowly screwed together. If the flexure be very marked, the larger instrument, after being withdrawn, should be reintroduced with its curve in the direction opposite to that of the 
flexure, and the final dilatation made with the dilator in this position; but in reversing the curve the operator should take care not to rotate the organ upon its own axis, and not to mistake a twist thus made for a reversal of the flexure. The ether is then withheld, and the instrument allowed to remain in place until the patient begins to flinch, when it is removed. The best time for the dilatation is midway between the monthly periods. In the majority of eases the dilatation should be carried to about one and a quarter inches. The

Figure 412.

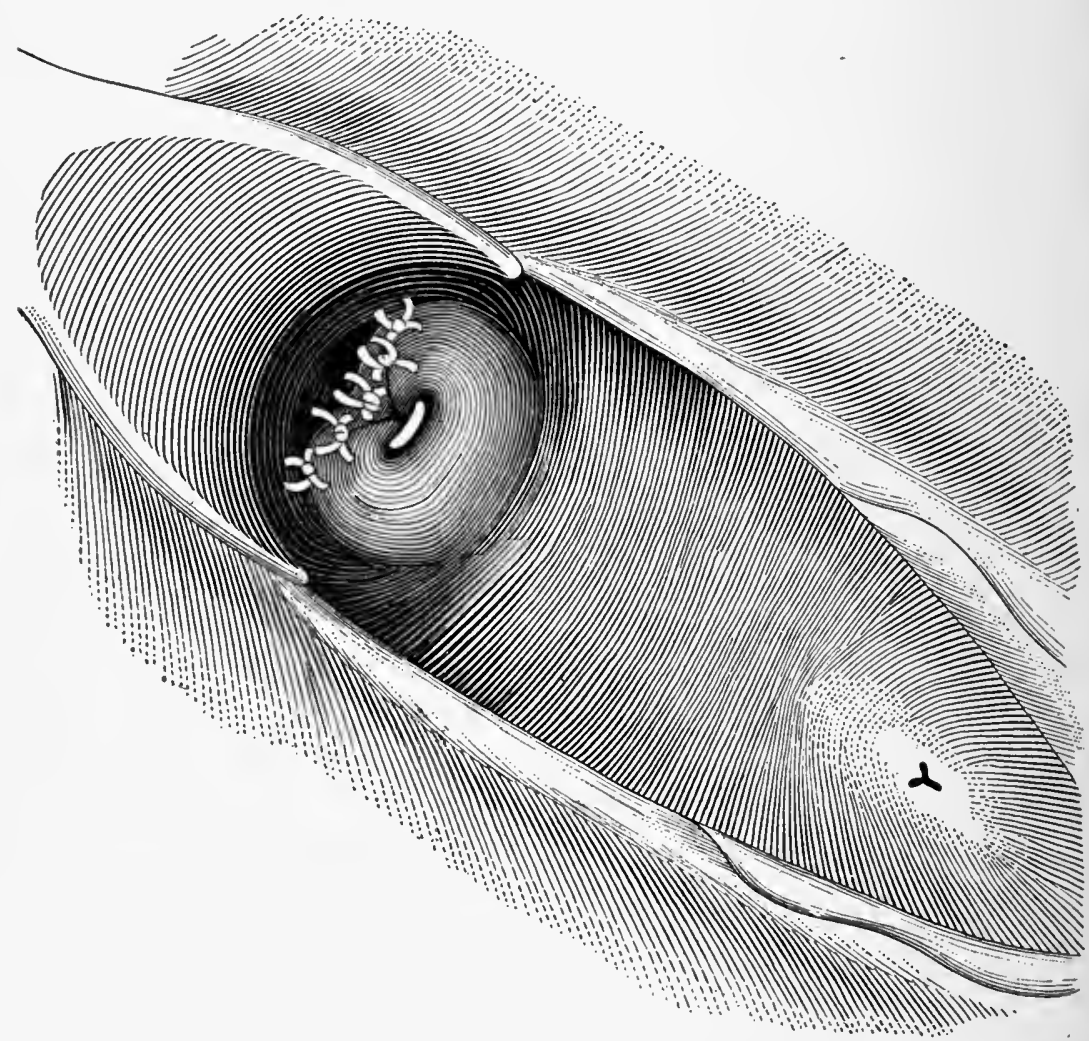

Suture shown in Figure 405 tied, and additional sutures designed to fortify this one also introduced and tied. This ordinarily completes the operation.

infantile uterus that has failed to develop at puberty has thin, unyielding walls, and should therefore not be dilated more than threefourths of an inch to an inch. In using the larger instrument it is usually necessary to have the assistant make decided countertraction with the vulsella forceps to keep the blades of the dilator from slipping out. The cervix is sometimes lacerated, but not sufficiently to produce unpleasant results. Goodell's statisties include one hundred and fifty operations of full dilatation under ether, with no fatal result and without serious inflammatory disturbance. 
After forcible dilatation under ether the cervical canal may remain relatively open and straight, and a symptomatic cure may be effected. Too often, however, the canal returns to its previously angular condition, and the dysmenorrhœa and sterility continue. The comparative safety of forcible dilatation in the hands of a skilful and experienced gynecologist may be contrasted with its great danger when undertaken by a careless septic operator unacquainted with the special requirements of uterine surgery. Peri-uterine inflammation is considered ordinarily a contraindication to the operation.

Dilatation by means of tents is transient in its results and dangerous to life. The operation has given frequent and serious warnings, in the shape of pelvic infections, which, if not destructive to life, have been overwhelmingly disastrous in their influence upon health.

5. Posterior Division of the Cervix Uteri is an operation devised and once extensively practised by Marion Sims and his followers ; it was designed to straighten the uterine canal by making a direct outlet from the point of flexure directly through the posterior wall of the cervix. The operation was not without merit, but it fell into disrepute because, first, it was done often in normal anteflexions; and second, because, while it overcame the obstruction in the uterine canal, it did not straighten the uterus and so relieve the more important obstruction in the blood-vessels. Moreover, the divided cervix was prone to reunite and leave a cicatricial contraction at the os externum. The operation was in the right direction, but was inadequate.

6. The Author's Operation, ${ }^{1}$ about to be described, has for its object the utilization of dilatation and of posterior division of the cervix in such a way as not only to enlarge the calibre of the uterine canal, but also to straighten the uterus and thereby overcome the cireulatory obstruction. The operation is performed as follows:

Everything connected with the operation has been rendered surgically clean. The patient being under ether, the uterus is exposed by Sims' speculum. The uterine canal is dilated by means of a Palmer dilator, followed by an Ellinger dilator, sufficiently to permit the introduction of a small sharp curette, but not necessarily to the extent advocated by Goodell. Curettage is performed as directed in Chapter XVII. for endometritis. The curettage may give only negative results, and may be, therefore, simply exploratory; or it may give evidence of pronounced endometritis. If the latter, it is imperative as a preliminary aseptic step, not only to the plastic part of the operation, but also as a curative measure.

The cervix is divided with scissors backward in the median line past the uterovaginal attachment nearly to the uteroperitoneal fold in the ponch of Douglas. See Figure 410 .

The cut surfaces thus incised then are held widely apart by means of two tenacula in the hands of an assistant; the incision is somewhat deepened by means of a scalpel, especially in the uterine wall next to the cervical canal, and a small angle is cut out on either side, as shown by the dotted lines in Figure 411. The cut surface on each

1 E. C. Dudley. "A Plastic Operation Designed to Straighten the Anteflexed Uterus." American Journal of Obstetrics, vol. xxiv. No. 2, 1891. 
side is now folded on itself by a single silkworm gut suture, as shown in Figure 411. This suture is tied and fortified by interrupted sntures on either side. The lines of union thus made are shown in Figure 412. By means of these sutures the os externum is earried directly back to the angle of the incision. The cervix now points backward in its normal direction toward the hollow of the sacrum, instead of forward toward the vaginal outlet. See Figure 403.

In some cases of extreme anteflexion there is a disproportionately long anterior lip, which is the result of a relatively greater. pressure

Figure 413.

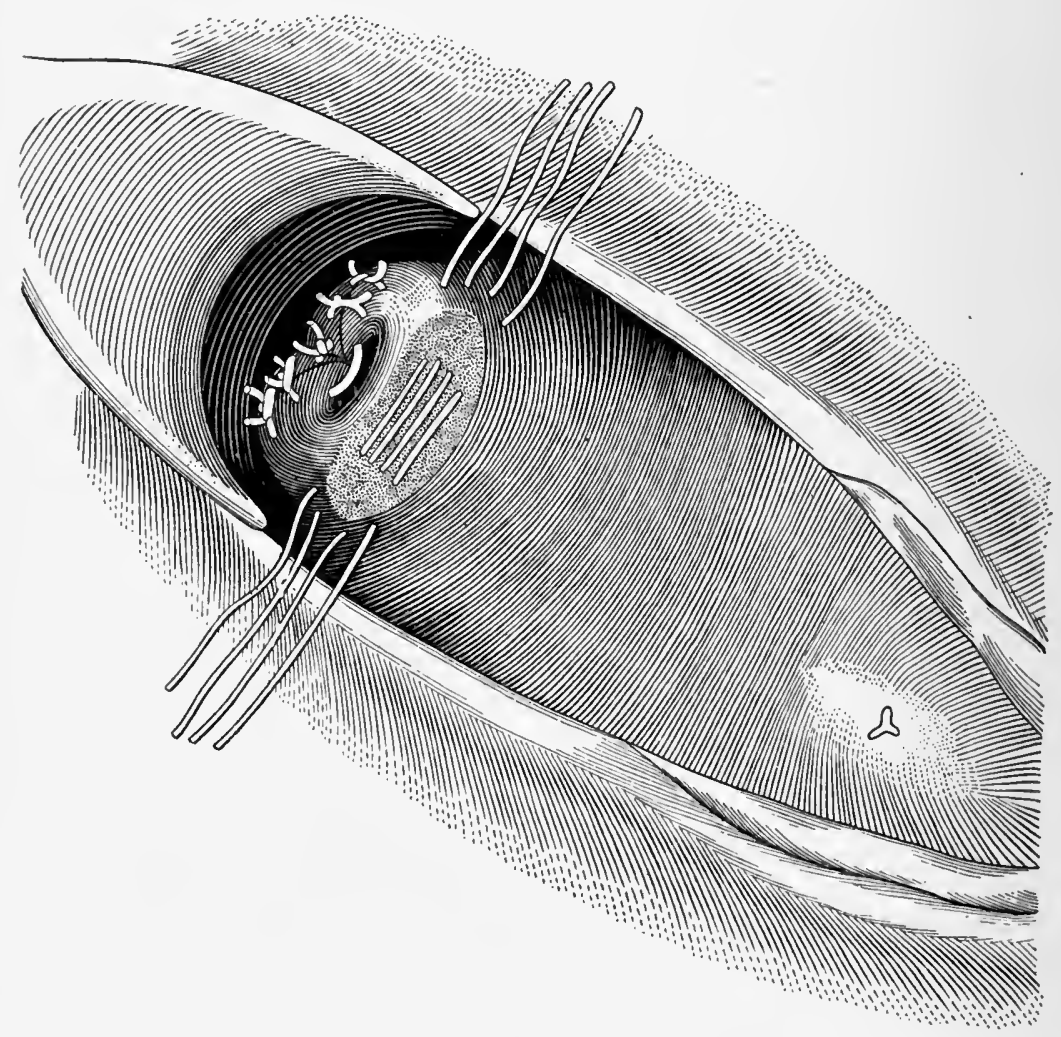

Anterior lip exciscd and sutures in place ready to tie.

on the posterior lip by the posterior vaginal wall ; this elongated lip should be eanght with the tenaculum and partially removed by the scissors. The incised surface is then elosed upon itself with sutures as shown in Figures 413 and 414. The dotted line in Figure 405 shows in section the line of incision through the protruding lip; the incision should extend to, but not into, the os externum. This part of the operation is not required muless the anterior lip decidedly protrudes, and therefore usually is omitted. The removal of a portion of the lip 
in a suitable case is not only not a mutilation, but it even contributes to the straightening of the uterus.

Conjoined examination upon completion of the operation in each of the author's cases has shown invariably the uterus either to have been straightened or the anteflexion to have been reduced to a degree quite within physiological limits. The results have been substantially the same whether the point of flexure was at the os internum or below it.

FIGURE 414.

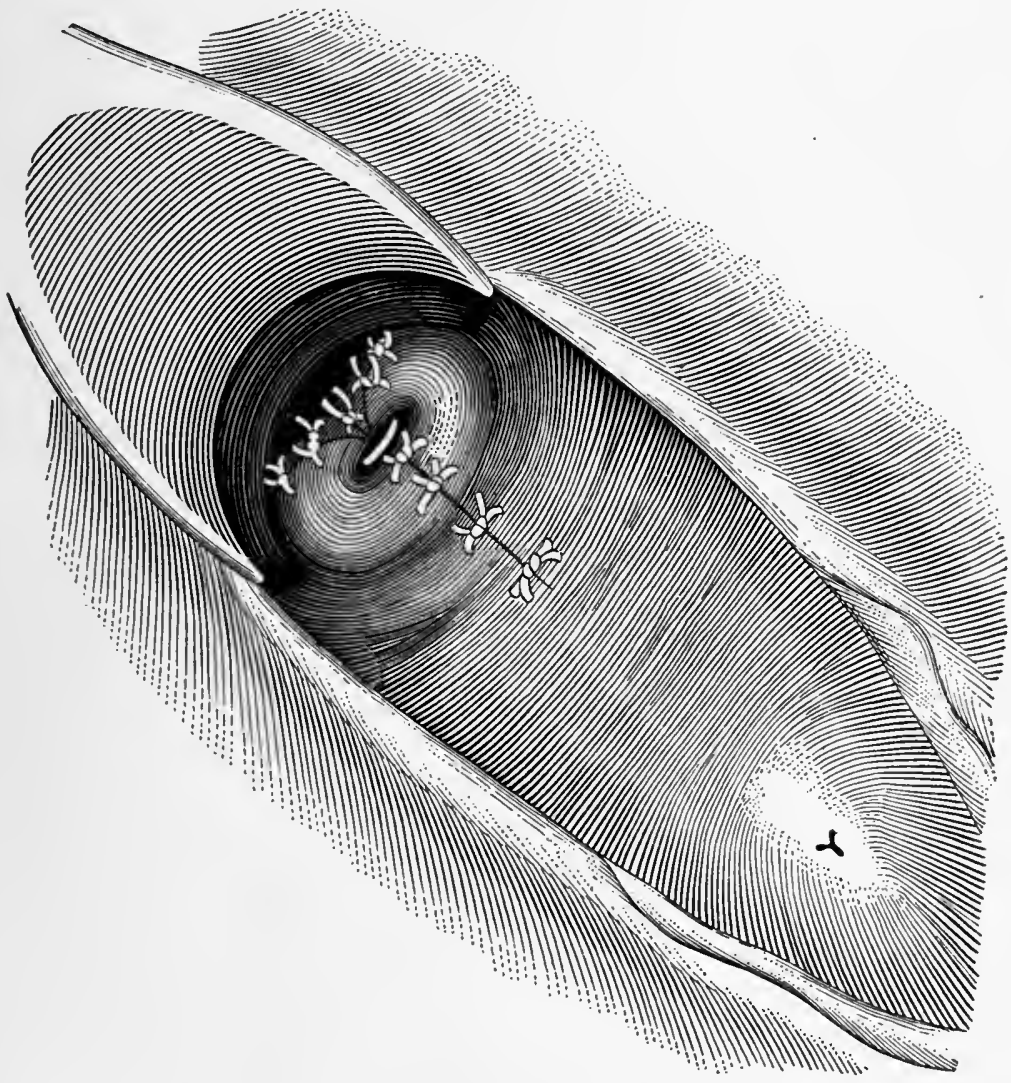

Sutures tied and operation complete, both on posterior and anterior lips.

The two posterior lines of sutures have the effect of transplanting the os externum to the very angle of the posterior incision. The anterior sutures, if used, have the effect of carrying the cervix back by a distance equal to one-half the length of the anterior cut surface, which is doubled upon itself. By these means a permanent change, quite equal to overcoming the flexure, is effected in the direction of the cervix. As the result of the anterior portion of the operation, the uterus in a suitable case is lifted also in a higher plane in the 
pelvis, where it ceases to be a mechanical irritant to the bladder. This portion of the operation may therefore be indicated for descent when complicated with anteflexion.

The writer has not undertaken this operation on the small, undeveloped infantile uterus. The so-called congenital anteflexion is only one factor in a general failure of development, a failure that pertains not to the uterus and other reproductive organs alone, but to the general system. The amenorrhœa or very scanty menstruation and the sterility usually associated with this condition, being only the local expressions of faulty general development, are not reached by any uterine treatment, surgical or non-surgical.

This operation was published first in November, $1890 .^{1}$ At that time the writer reported eighteen cases. The results were classified under two heads, one for the mechanical and one for the symptomatic results. The mechanical result was invariably a satisfactory straightening of the uterus. The symptoms were relieved satisfactorily in about three-fourths of the cases. The author's personal experience with this operation now numbers about one hundred cases. With this larger experience and larger observation of the symptomatic results he is able to verify the conclusions formed when the operation was given to the profession. In no case has the operation failed to give an anatomical cure, and the symptomatic results have been satisfactory in about 75 per cent. of all cases.

The symptomatic indication in the great majority of cases was dysmenorrhœa. This symptom, when the flexure was uncomplicated by peri-uterine inflammation - that is, when the conditions were mechanical-has been quite generally relieved. In seven cases the indication was prolonged sterility. In three of these cases normal parturition has taken place.

R. G. Wadsworth, ${ }^{2}$ of Boston, reports 31 cases for which the operation was performed by himself and other operators in the Free Hospital for Women, in Boston, and 10 cases occurring in the private practice of Dr. Reynolds, and 37 cases from the literature. Generally speaking, the results of the operation for all these cases, both anatomically and symptomatically, were substantially the same as for the series of cases originally reported by the author.

The operation is not presented as a panacea for all the maladies of pelvic origin in which there happens to be a pathological anteflexion. Cases are numerous in which anteflexion is rather an incidental than an essential factor. The hope is that the operation may prove of value when the indication to be fulfilled is wholly or in part mechanical.

There is danger that this operation will be performed indiscriminately in cases not of pathological, but of physiological anteflexion. On the other hand, those who do not consider anteflexion as having any pathological significance per se, and do not always make the distinction between the physiological and pathological position, will fail to appreciate the mechanical indication, and will therefore discard the operation altogether. Clearly a woman might have anteflexion and,

1 Paper by E. C. Dudley, read before the New York Obstetrical Society, November 18, 1890.

2 The Bulletin of the Free Hospital for Women. 
if it were normal, might have a large variety of lesions wholly independent of it. To say this; after all, might be saying only that a woman may have a variety of pathological developments in the pelvis, and at the same time have the uterus in its normal position. Normal anteflexion could, of course, have no pathological significance.

Since the investigations of Schultze and others, we may distinguish downright pathological anteflexion, in which the uterus is bent to the point of producing two kinds of obstruction at the angle of flexure: 1. Obstruction of the canal from collapse of the canal. 2. Obstruction of the blood-vessels from collapse of the blood-vessels. Under such conditions uterine congestion and catarrh, as pointed out in the foregoing paragraphs, are apt to follow; normal physiological changes cannot take place either in the decidua of menstruation or in the decidua of pregnancy; hence, menstrual disorders and sterility.

The mechanical indication is clear: straighten the uterus and thereby relieve the obstruction in both the uterine canal and the vessels.

The operation is not a substitute for dilatation and curettage, but rather supplementary to these two procedures.

The writer has practised extreme divulsion with curettement in many cases of anteflexion; but the results were not very satisfactory. They are, however, more gratifying when the plastic opcration already described is added to the dilatation and curettage.

If it is wrong to treat anteflexion mechanically, because it is a result of certain associated lesions, it follows that retroflexion and all other displacements should not be treated mechanically, because thcy also are equally the result of associated lesions. This almost amounts to the reductio ad absurdum

One hears much of inflammation of the uterosacral ligaments as the great associated lesion in pathological anteflexion, and yet is disappointed often in his search for evidence of such inflammation. Inflammation often exists there, but in a large proportion of such cases it has passed away, leaving pathological anteflexion as a permanent result.

In a few cases of anteflexion low down in the cervix the author with good anatomical and symptomatic results has substituted Schroeder's operation for his own.

\section{TORSION OF THE UTERUS.}

Any part or the whole of the uterus may turn on its own axisthat is, be twisted upon itself.

\section{Causes of Torsion of the Uterus.}

The causes of this displacement are :

1. Anteflexion associated with contraction of one uterosacral ligament.

2. Retroflexion.

3. Uterine tumors.

4. Tumors of the uterine appendages.

5. Double uterus; one horn may lie anterior to the other. 


\section{Diagnosis and Treatment of Torsion of the Uterus.}

Torsion of the uterus may be recognized by conjoined examination which will demonstrate the anterior surface of the uterus lying, not directly in front, but to one side. The long axis of the uterus generally is found, not in the median line, but having a diagonal direction across the pelvis. The treatment is that of the causative complications. 


\section{CHAPTER XLIX.}

\section{INVERSION OF THE UTERUS. HERNIA OF THE UTERUS AND OVARY.}

\section{INVERSION OF THE UTERUS.}

Inversion of the uterus is the partial or complete turning of the organ inside ont. The difference between partial and complete inversion is simply one of degree. In partial inversion some part of the wall of the corpus uteri, usually the fundus, is depressed into the uterine cavity, but the organ does not protrude through the external os into the vagina. In complete inversion the uterus has turned completely inside out. The inverted uterus is then inside the vagina, or, if the vagina is also inverted, both organs 'will be, to quote Hippocrates, between the thighs, "velut scrotum." Figures 415-424. represent the incomplete and complete forms of inversion.

\section{Etiology of Inversion of the Uterus.}

More than 10 per cent. of all cases are puerperal. The causes usually assigned are traction on the cord in the delivery of the placenta, traction in the rapid delivery of the child, traction by gravity of intrauterine tumors, or traction exerted in their delivery. These causes, however, are all inadequate to produce the accident unless the muscular walls of the uterus are predisposed by relaxation. Paralysis or great relaxation of the uterine wall is the essential cause of the accident. Undue importance has been given to the varions forms of traction; even coughing or sneezing may invert a very relaxed uterus. ${ }^{2}$

Inversion in the majority of cases occurs spontaneously as a direct result of paralysis of uterine muscles. This paralysis may pertain to any part or all of the nterine wall, but is usually most pronounced at the placental site. The paralyzed portion is depressed first into the uterine cavity, so as to give the corpus uteri the appearance of the bottom of a junk bottle. The uninverted portion of the muscular wall, not being paralyzed, may contract and seize the partially inverted paralyzed portion, and push it down further and further until inversion is complete.

The conditions that most favor paralysis and relaxation of the muscular layers are not wholly known. The accident in about 88 per cent. of all cases is associated with childbirth $;^{3}$ hence the inference that the most active causes are connected with utero-gestation and parturition: In a small proportion of cases inversion has followed distention of the

1 Hippocrates. From Thomas and Munde.

2 Adaptation from Thomas and Mundé. Diseases of Women.

3 Crosse. Loc. cit. 
730

DISPLACEMENTS.

endometrium by retained fluids or tumors. The hemorrhage, often associated with muscular relaxation of the uterus, is not a cause, but a

Figure 415 .

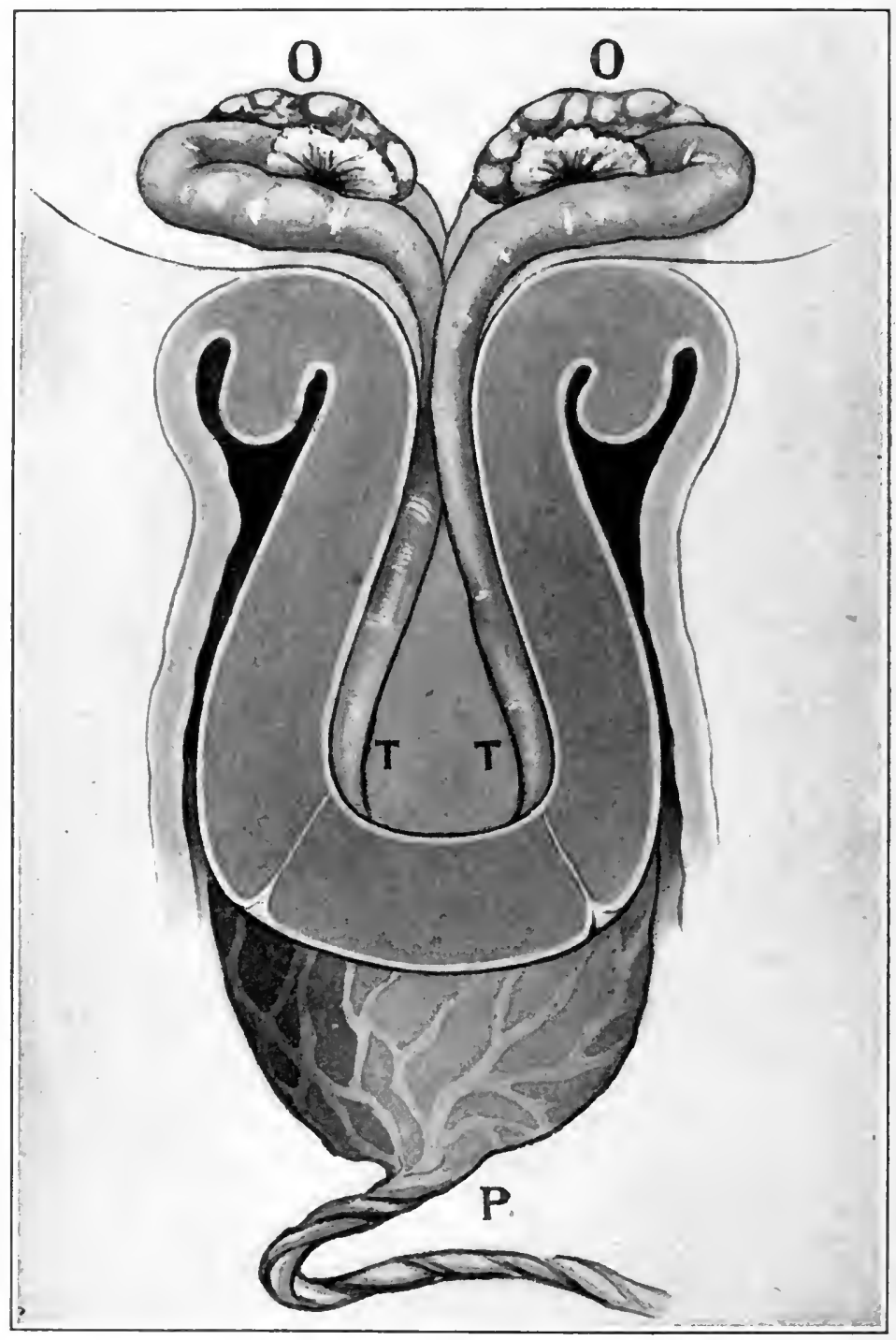

Acute case, uterus inverted completely into vagina: $O O$, ovaries; $T T$, Fallopian tubes: $P$, placenta still attached to uterus. Openings of Fallopian tubes through inverted fundus are shown in section.

result of the relaxation. Finally, we may say that the condition in a large proportion of cases arises without definite assignable cause. The 
Figure 416.

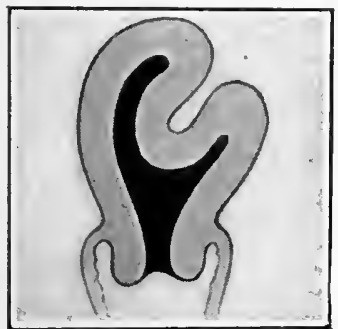

Figure 417.

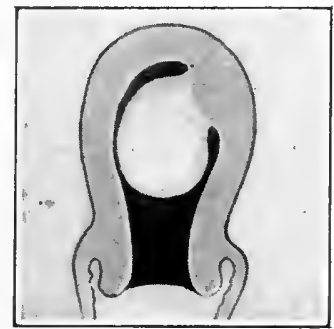

Figure: 418.

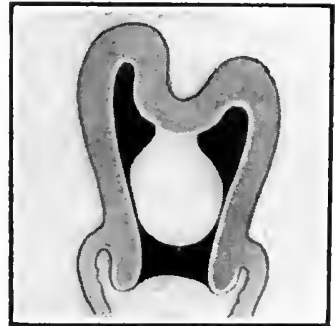

FigURE 416.-Partial inversion of the left horn of the uterus.

FigURE 417.-M yoma simulating partial inversion at the left horn of the uterus.

FigUrE 418.-Partial inversion of the uterus complicated by and eaused by a myorna.

Figure 419.

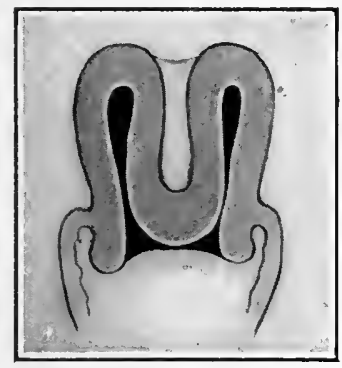

Frgure 420.

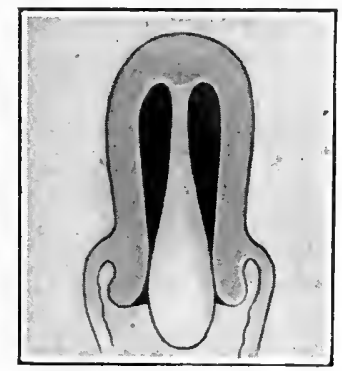

Figure 421.

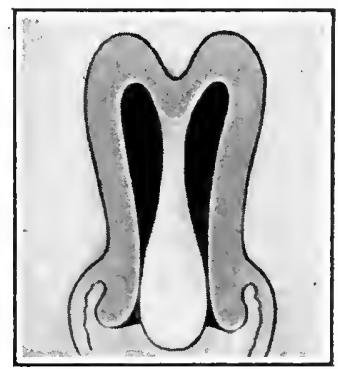

Figure 419.-Partial inversion of the uterus : the fundus is at the os externum.

Figure 420.-Peduneulated myoma protruding from the os externum, resembling an inverted fundus uteri.

FIGURE 421.- Slight inversion of the fundus uteri with pedunculated uterine myoma protruding through the os externum and resembling inversion.

Figure 422.

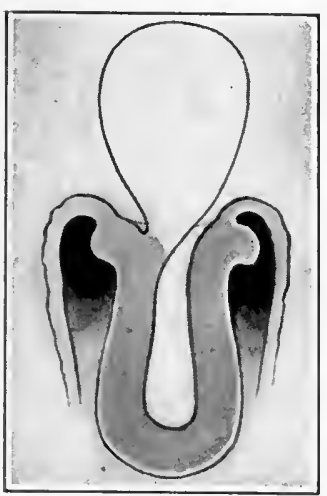

Figure 423.

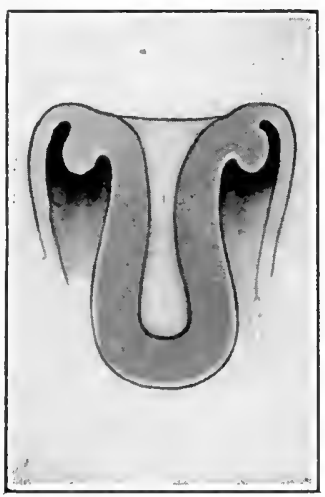

Figure 424.

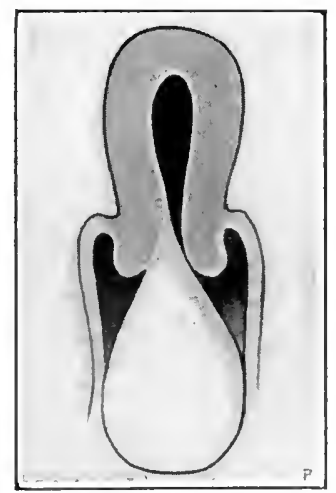

FigURE 422-Complete inrersion of the uterus complicated by a myoma in the peritoneal cavity, which has all the physical appearanee of a uterus. There is difficulty in such a case in determining which is the uterus and which is the myoma.

FIGURE 423,-The uterus is inverted completely into the vagina.

Figure 424.-The reverse of Figure 422. 
accident occurred at the Rotunda Hospital but once in 190,800, and at the Vienna Lying-in Hospital but once in 250,000 deliveries. ${ }^{1}$

A most instruetive case has been recorded by Willard Parker, as follows:

"A young woman, who had borne one ehild seven or eight years previously, and had never had any recognized uterine lisease, while making a violent effort in rolling tenpins suddenly felt something give way within her, after which she suffered the most intense pain and became completely disabled. Dr. Parker, being called to see her, after a hasty examination eoincided with the opinion of the attending physician, that a polypus had been suddenly expelled and was hanging in the vagina. Impressed with this belief, he removed the whole mass, when, to his surprise, he found in his hands the inverted uterus with its tubes and ligaments. 'The patient recovered without any bad symptoms, and subsequently menstruated regularly."2

'The occasional occurrence of spontaneous replacement of a uterus that had been inverted has been observed repeatedly, and is a fact no less remarkable than spontaneous inversion. In one ease replacement occurred while the patient was straining at stool. ${ }^{3}$

\section{Mechanism of Inversion of the Uterus.}

If the entire uterine walls are paralyzed, the organ may invert as the result of traction or coughing or sneezing, or of its own weight. Intra-abdominal force from above may push the paralyzed uterine wall through the os externum into the vagina. If the paralysis pertains to only a part of the nterine wall, the inversion, as already explained, may oceur by contraction of the non-paralyzed portion. Clearly, inversion cannot take place when the entire nterine wall is active. It nuy, however, do so when the paralysis is partial and the activity is partial. Regional paralysis, as already stated, is more apt to occur at the placental site, where the wall is thimmer and softer. It more frequently oceurs at the fundus or at one of the horns.

In some eases the inversion takes place from below upward-that is, the relaxed cervical portion comes down as in prolapse of the anus. This process begins as eversion, and continues until the whole organ is inverted.

\section{Anatomy and Pathology of Inversion of the Uterus.}

The inversion, if not eomplete, may have been arrested at any point. Thus the inverted portion may be above the internal or external os; or it may consist of the entire nterus rolled ont into the vagina; or, together with the inverted vagina, the inverted uterus may be outside of the vulva. The exposed uterine mucosa is then dark red or purple from congestion, and there may be regional ecehymosis, erosion, and nleeration. Adhesions have been known to form between

\footnotetext{
${ }^{1}$ Playfair and Allbutt. System of Gynecology. $\quad{ }^{2}$ Thomas and Munde. Diseases of Women.
} ${ }^{8}$ Thomas. Diseases of Women. 
the wholly extruded uterus and the vagina. The writer has observed one ease in which' such adhesions had formed between the partially inverted corpus and the cervical mucosa.

There is hemorrhage from the extruded and inflamed uterine mucosa. In the combined inversion of the uterus and vagina the mucosa, after long exposure to external influences, may become dry, wrinkled, and parchment-like, as does the vagina in complete procidentia of the uninverted uterus; the two conditions have been mistaken for one another.

The vessels are strangulated, circulation is impeded, the nutrition of the organ suffers, and some degree of infection is almost inevitable. In rare instances gangrene and sloughing of the inverted portion have taken place.

The uterine ligaments, Fallopian tubes, ovaries, and even intestine may at first be drawn into the peritoneal cup of the inverted organ. Rarely these organs become adherent within the cup; usually with returning utcrine aetivity and contraction they are expelled and remain outside.

\section{Symptoms of Inversion of the Uterus.}

The symptoms of acute complete inversion of sudden oceurrence are as follows:

Fixed intense pain.

Profuse hemorrhage.

Shock and collapse.

Partial inversion may occur with no characteristic symptoms, and, without physical examination, may escape notice.

Chronic inversion may have developed slowly, or may follow acute inversion ; it causes :

Mechanical disorders of the urinary organs and rectum.

Hemorrhage, more or less profuse, and consequent anæmia.

Bloody, purulent, or serous discharges.

Difficulty in walking and standing.

Pelvic pain.

Nerve exhaustion and impaired health necessarily follow. Life may be destroyed slowly by the exhaustive drain, or at any time rapidly by acute peritonitis. In rare instances, especially after the menopause, there may be only slight inconvenience or none at all.

\section{Diagnosis of Inversion of the Uterus.}

If the abdominal walls are relaxed and thin, and permit adequate palpation of all the intrapelvic organs, conjoined examination will show : first, the absence of a part or a whole of the uterus in the place where it normally belongs; and, second, its presence inverted partially or wholly in the vagina or in the uterine canal. The concavity or peritoneal depression caused by the inversion sometimes may be felt through the abdominal wall. Rectal touch or combined rectal 
and vaginal touch, with the hand over the abdomen or the sound in the bladder, may facilitate the diagnosis. The finger in the rectum may be made to meet the hand over the abdomen or the sound in the bladder, and thereby demonstrate the absence of a uterus above the vagina. The orifices of the Fallopian tubes, now rolled out and exposed, also may be demonstrable. In ease of rigid thick abdominal walls the diagnosis will be more difficult.

The differential diagnosis, in a given ease, may raise two questions: First, Is the protruding mass a uterine myoma or polypus, or a vaginal tumor? Second, Is it a prolapsed uterus?

\section{Is it complete inversion?}

I. No pedunculated attachment to uterus.

2. Uterine eavity being obliterated, sound can be passed but short distance in incomplete and not at all in complete inversion.

3. Vaginal or rectal conjoined examination shows a ring or depression where the uterus should be, and fails to show the uterus above the vagina.

4. The inverted uterus is a symmetrical pyriform body.

5. Orifices of the Fallopian tubes usually demonstrable.

6. Muciparous glands of the utcrus present and microscopically demonstrable.

Is it incomplete inversion of the uterus?

1. The uterine cavity as measured by the sound will be diminished.

2. Development usually sudden.

3. Bimanual examination shows ring-like depression in wall of uterus.

4. Usually dates from parturition.

\section{Is it myoma or polypus ?}

1. Attached to the uterine wall by broad surface or by narrow pedicle.

2. Sound passes by the side of the mass through external os far into the uterine cavity.

3. Uterus felt above vagina.

4. Not usually symmetrical and may be very asymmetrical.

5. Not present.

6. Not present, or if present less perfectly developed.

Is it an intra-uterine myoma?

1. Cavity enlarged.

2. Development gradual.

3. Uterus symmetrical or asymmetrical, but no ring-like depression.

4. No parturition.

The great difficulty in some eases of making the differential diagnosis between a polypus or myoma and an inverted uterus is emphasized by the fact that deservedly eminent surgeons have extirpated the partially or wholly inverted uterus repeatedly under the mistaken diagnosis of a myoma. Conversely, the effort has been made to reduce a supposedly inverted uterus when the extruding mass was a myoma. The author personally recalls a ease at the Woman's Hospital in the city of New York, upon which such an attempt was made persistently by T. G. Thomas.

In rare eases the diagnosis has been obseured by the presence of an inverted nterus in the vagina, and by the coexistence of a subperitoneal myoma of the size, shape, consistence, and position of a normal uterus. The distinction between the two bodies might then depend solely upon the presence or absence of the orifices of the Fallopian tubes in the vaginal mass. Ordinary eare and intelligence, however, usually will enable the surgeon to avoid serious mistakes. Velpeau, quoted by Simpson, once sagely remarked, however, that in some cases doubt is the only rational opinion.

The differential diagnosis between inversion and procidentia uteri usually will be easy. 


\section{Is it inversio uteri?}

1. The protruding mass is wider below than above.

2. External os uteri absent and tubal orifices present at lower end of mass.

3 . Sound in urethra goes upward into bladder away from mass.

Exception. - When the vagina is concurrently inverted the sound may pass downward.

4 . Obliteration of vaginal fornices.
Is it complete procidentia uteri?

1. Mass wider above.

2. External os present and tubal openings absent.

3. Sound goes downward into bladder, but also iuto anterior portion of mass.

4. Obliteration of vaginal fornices.

In the diagnosis and differential diagnosis, inspection and conjoined exanination and the sound furnish the most reliable information.

\section{Prognosis of Inversion of the Uterus.}

If replacement can be effected promptly in the acute stage just after the occurrence of the accident, the prognosis is immediately gond. If replacement be delayed until rigid contraction renders it more difficult, the prognosis will be correspondingly more serious. The possible dangers arising from acute inversion are from hemorrhage, shock, collapse, and acute infection.

Chronic inversion, unless relieved by replacement, is likely to destroy health-if not, indeed, life--by slow, exhaustive hemorrhages, uterine discharges, and consequent anæmia. Nerrous exhaustion from surgical efforts to replace the organ, and the possibility of its removal by mistake for a myoma, are positive sources of danger. Acute infection and peritonitis are among the always dreaded possibilities. Few authentic cases of spontaneous reposition have been recorded.

In rare instances the inverted uterus gives little or no trouble, even when associated with complete vaginal inversion; the uterine and vaginal mucosa possibly may undergo changes to make them resemble skin, the surfaces becoming hard, tough, parchment-like, and wrinkled. Finally, hemorrhages may cease, and the woman may live to old age in comparative comfort.

\section{Treatment of Acute Inversion of the Uterus.}

Puerperal inversion usually takes place in the presence of the attendant between the birth of the child and the delivery of the placenta, and may, therefore, in the acute stage be recognized while uterine walls still are relaxed sufficiently to permit immediate replacement.

If the placenta still is attached, it should be removed rapidly. Under anæsthesia the hand then is introduced into the vagina and the fundus pushed up through the cervical canal into place. Strong contractions, with alternating relaxations, are usual in this stage. Reduction by taxis is almost impossible during the contractions. Instead, therefore, of handling or kneading the organ to reduce its size by contraction, the attendant waits patiently for relaxation, and then makes a steady, firm, and prompt effort at replacement. The whole corpus may be carried up at once or it may be necessary with the finger-tips to indent the fundus at some point, preferably one of the cornua, and let this be the starting-point of the replacement. 
Hot water and a fountain syringe, or, better, a Davidson syringe of interrupted current, should be ready, in order that while the hand is still in the uterine cavity a hot douche may be thrown rapidly into the uterus. The hot water, by its stimulating effect, sets up strong uniform uterine contraction; this controls hemorrhage and prevents recurrence of inversion. The hot water uterine douche in the control of post-partum hemorrhage acts in the same way. Within a few hours after the accident firm uterine contraction or retraction takes place in the muscular walls of the inverted uterus. When such retraction once is established, replacement usually will be quite as difficult as if the condition had existed for months-that is, the change from acute to chronic inversion is rapid.

\section{Treatment of Chronic Inversion of the Uterus.}

Until a comparatively recent time the inverted uterus, once contracted, was regarded incurable except by hysterectony. On the possibility of replacing an inverted uterus after the organ had contracted, the late Charles D. Meigs, of Philadelphia, in his letters to the students of his class, in 1846, wrote: "You might as well attempt to invert one of the non-gravid uteri on my lecture-table as to reposit this one. The time for replacement has gone by."

The Obstacles to Reposition are these :

1. Great rigidity in the contracted cervical ring.

2 . Increase in the volume of the corpus uteri from congestion. This occurs soon after inversion.

3. Later increased firmness and hardness in the uterine structures from involution.

4. The mobility of the organ and the difficulty of opposing above sufficient counterpressure to the force applied below in the effort to replace. cup.

5. Adhesions, rare but possible, between the sides of the peritoneal

Methods of Reposition.-The difficulty of overcoming the obstacles outlined above is apparent from the manifold methods that have been practised by different surgeons. The lesson to be learned from the combined experience of these methods is that success is attained best by firm, steady, continuous, elastic pressure, and that it may depend finally upon very prolonged and patient effort.

The object is to overcome the rigidity in the cervical ring. The pressure to accomplish this may be unyielding or elastic. The treatment includes the following possible procedures:

1. Replacement by the maided hands.

2. Replacement by the hands aided by incisions or instruments.

3. Continued elastic pressure.

4. If reduction prove impossible, the final resort is hysterectomy:

If one method fails, a combination of two or more methods may succeed.

1 Emmet. Principles and Practice of Gynecology. 
Preparatory Treatment.-It is always possible in the course of an attempt at reposition that emergencies may arise that will neeessitate abdominal or vaginal section; hence, the necessity of making

Figtre 425.

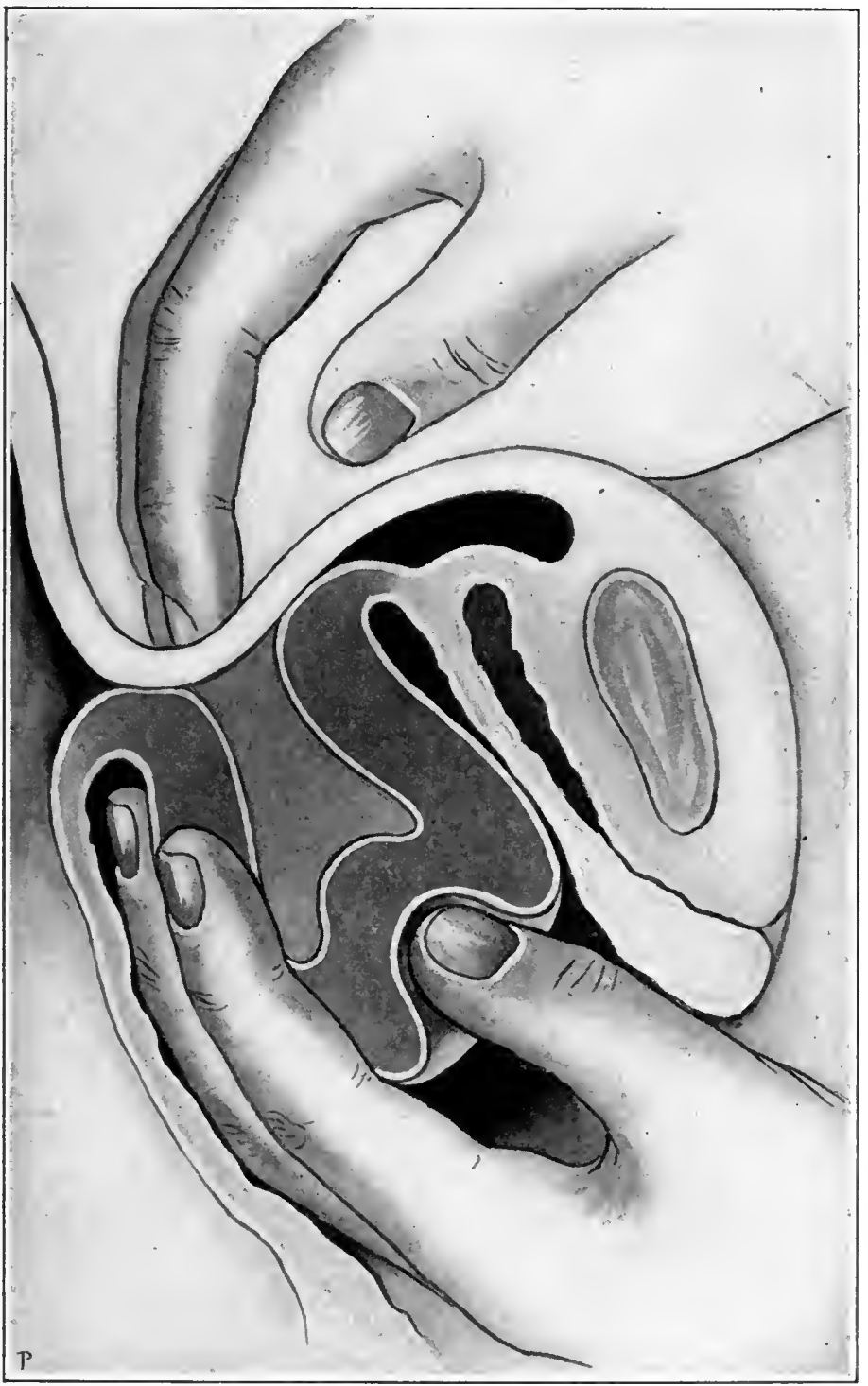

Manual reposition of an inverted uterus.

preparation for those operations. See Chapter II. In addition to the above, iron may be required for anæmia, and hot water or aseptic gauze tamponade in the vagina may be needed for hemorrhage. 
In a very anæmic case several weeks or even months of recuperative treatment may be essential.

Reposition with the Hands, Emmet's Method. ${ }^{-}$-The patient, anrsthetized, is in the lithotomy position. The left hand is passed into the vagina, the fingers and thumb are forced as far as possible into the angle of reflexion, so as to encircle the part of the corpus uteri that is close to the constricted cervical ring. The fundus is in contact with the palm of the hand, and is pressed firmly upward by it, while the fingers are separated to their utmost to open the cervix. At the same time the right hand behind the pubes slides the abdominal wall back and forth over the peritoneal depression. This effort, the object of which is to open out the contracted ring, is put forth con-

FIGURE 426.

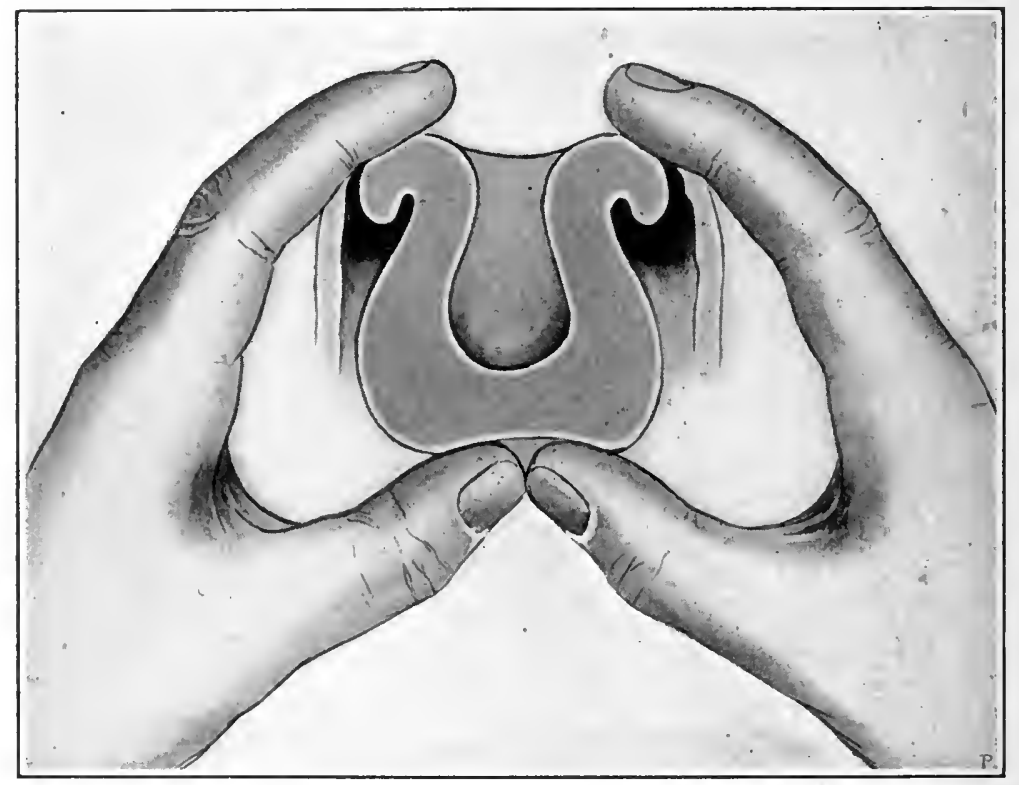

Reposition of the inverted uterus by the method of J. H. Tate. The left index-finger is in the bladder having been passed from the vagina to the bladder through a vesicovaginal fistula made for the purpose. The right index-finger is in the rectum.

tinuously. Finally, the rigid cervix uteri may begin to dilate, the corpus may grow shorter, and the extent of inversion may lessen proportionately. After the corpus has been forced partially within the cervix by steady upward pressure, the tips of the fingers are brought together as a wedge, passed through the os, and made to complete the reposition.

Emmet's method is much facilitated by keeping, for a few days previons to replacement, a widely distended Barnes elastic bag in the vagina. The bag is secured firmly by a T-bandage. This dilates the vagina, makes room for the hand, and, by the elastic upward pressure 
which it exerts, may dilate the constricted ring or even by itself effect replacement. In one case complete reposition was made by Emmet's method in three hours and fifty-five minutes.

Emmet further suggests in case of partial reposition, when the corpus uteri has been passed inside the external os, that the progress thus made be secured by temporary closure of the external os with sutures.

Tate's Method of Taxis. ${ }^{1}$ - The index- and middle fingers of the right hand are passed into the rectum, and the index-finger of the left hand into the bladder through the dilated urethra. The balls of the thumbs make constant firm pressure over the fundus, while the fingers in the rectum and bladder make counterpressure against the cervix. In this way great force is applied more directly to the constricted ring than by any other method. In a case of forty years' standing reported by Tate, the thumbs soon indented the fundus, the cervix began to dilate, the corpus was pushed through the cervix, and reduction was accomplished in a few minutes. Were it not for the danger of rupture of the urethra and consequent permanent incontinence of urine, this method would, perhaps, have the preference over all others. Every scrious objection could, however, be overcome by opening the vesicovaginal septum and passing the finger through the artificial vesicovaginal fistula thus made, instead of the urethra. The fistula, after reposition, could be closed with little difficulty or loss of time, and with practically no additional danger. $^{2}$

There are numerous other methods of reduction by taxis, but they involve no valuable principle not included in those already mentioned.

Elastic Pressure by the Water-bag or Colpeurynter has been mentioned in the preparation for Emmet's method. It is called colpeurysis. Figure 427. Reposition may be begun by depressing with the fingers one horn of the uterus, and the depressed portion, if forced onward, serves as a wedge to dilate the contracted cervix. The hand after a time becomes fatigued and useless. Colpeurysis then may be substituted and long-sustained elastic pressure, interrupted occasionally by attempts at manual replacement, may be effective. In many cases elastic pressure alone will suffice. There is, however, no short limit to the time during which it may be necessary to continue it. In some cases reposition finally has bcen made only after two or three weeks of constant effort, the rubber bag filled with water being. held constantly in place against the inverted fundus uteri by means of a tight bandage, so that it will exert constant pressure.

An effective morle of using elastic pressure is that described by Thomas and Munde. ${ }^{3}$ Through a Sims speculum place a tampon of aseptic gauze soaked with glycerin firmly around the cervix. This keeps the uterus from slipping out of the line of pressure. Shave the pubes. Introduce a large rubber bag into the vagina and fill it with water. Cut a strip of adhesive plaster two and a half inches

1.J. H. Tate. Cincinnati Lancet and Observer, March, 1878. Emmet.

2 Snggested br Fmmet. Principles and Practice of Gynecology.

3 Discases of Women, p. 454. 
wide, long enough to reaeh from the lumbar region, between the thighs, over the pubes up to the navel. There are two openings in the plaster, one for transmission of the tube of the rubber bag and one for the urethra. The plaster is cut in two just over the vulva, and that portion from the vulva to the anus lined with a layer of gauze.

FIGURE 427.

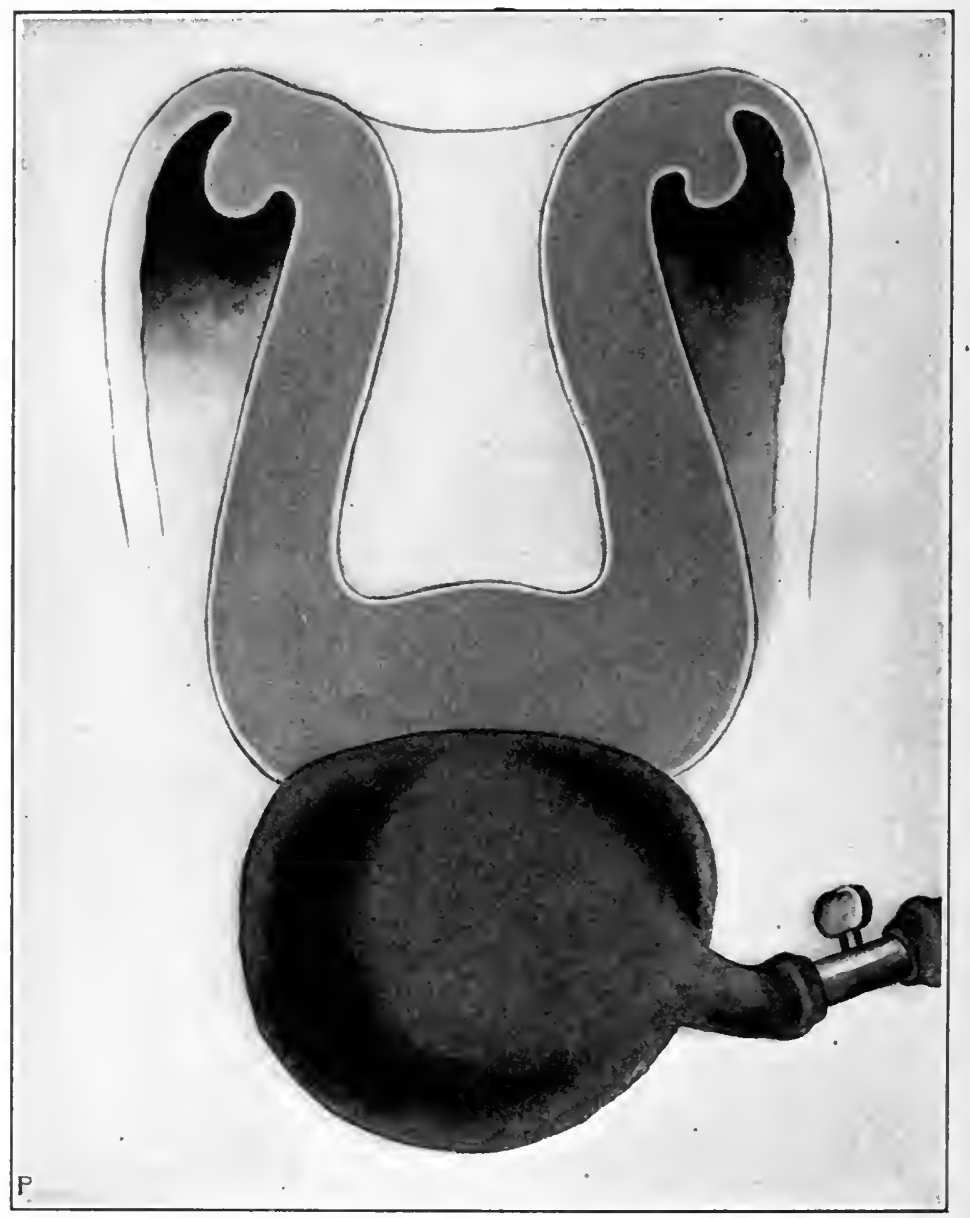

The colpeurynter.

The two parts of the plaster are held together by three safety-pins, and may be opened during defecation or urination; the urine if neeessary is drawn by a eatheter. The pressure may be inereased by tightening the plaster or pumping in more water; it may be decreased by loosening the plaster or drawing out water through the stop-cock on the tube. The patient is kept in bed, and pain is eontrolled by opium or morphine. 
Continued elastic pressure by colpeurysis is sometimes not tolerated, or it may be contraindicated by the presence of inflammation. Then anæsthesia and more energetic measures may be indicated.

The Spiral Spring (White's) Method.-A rapid and for many cases effective method of elastic pressure is that of the spiral spring attached to a rubber cup. Enmet's method may be reinforcerl effectively by the use of this instrument. Figure 428 . 'The patient is anrsthetized. 'The left hand in the vagina grasps the inverted corpus uteri, and at the same time holds the fundus in the rubber cup. Projecting outward from this cup is a slightly curved rod, having a strong spiral spring attached to its end. 'The operator's body rests against the spiral, and through it exerts pressure upon the uterus. The disengaged hand is used for counterpressure behind the pubes, as already described in Emmet's method. Figure 428 shows the instrument. 'Two or more hours of continuous effort may be required

FIGURE 428.

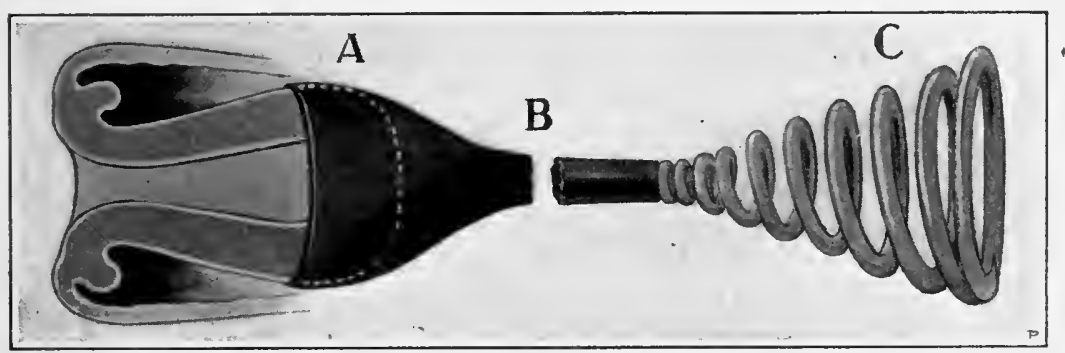

White spiral spring repositor: A, rubber cup; B, rod eonnecting cup with spring; C, spiral spring.

to reach the result. Numerous other similar instruments have been devised, but none more effective than this.

Incision.-When the various forms of taxis, supplemented by gradual or rapid elastic pressure, fail, the rigidity of the cervix may be overcome by incision. This plan was suggested by the fact that, after forcible dilatition, the cervix was usually more or less torn, and that an incision would be preferable to a tear. Various forms of incision have been advocated or practised by James Y. Simpson, J. Marion Sims, Barnes, Matthews Duncan, and others. One method is to draw down the corpus and cut nearly or quite through the constricted cervical wall at one or more points, and then reduce the inversion by taxis or rapid elastic pressure. The favorite incisions are one in the anterior and one in the posterior wall of the cervix.

J. Bernard Browne, of Baltimore, ${ }^{1}$ makes an incision through the fundus. A strong dilator then is passed through the opening into the constricted ring, and the cervix is dilated until the corpus can be forced through into place. Just before reposition the wound in the corpus is closed with eatgut sutures. If asepsis be thorough, 
intra-uterine gauze drainage may be used in place of closing the opening.

Thesc and similar methods of incision before the days of aseptic surgery were regarded as extra hazardons, and therefore were disapproved generally. Under aseptic conditions, however, the danger would doubtless be less than formerly was supposed. With an aseptie field of operation and thorough gauze drainage in the uterus, the risk should not be greater than that of other operations involving peritoncal incisions of equal extent.

The method of 'Thomas to open the abdomen, and especially that of Kïstner to open from the vagina into Douglas's pouch, in order to reach and direetly dilate the cervix on the peritoneal side, have fallen into nnmerited discredit.

Hysterectomy.-If all efforts of taxis, elastic pressure, and incision have failed, removal of the uterus may become a final resource. The operation ordinarily would be vaginal hysterectomy, and would be performed substantially as already described for cancer. The writer suggests that after the posterior and anterior incisions have been made from the vagina into the peritoneal cavity, another attempt at reposition be made. Through these openings great force could be applied by the mechanieal principle employed in the method of Tate. The fingers should be introduced not into the rectum and vagina, but through the vaginal incisions. Connterpressure then could be made most powerfully direct against the cervical ring.

\section{HERNIA OF THE UTERUS AND OVARY.}

A common form of hernia of the uterus has been described in the chapter on Descent of the Uterus.

Hysterocele, or hernia of the uterus through the inguinal canal or inguinal rings, is a rare and remarkable displacement. Cases have been reported by Olshansen, Leopold, Rectorzik, Winckel, and Scanzoni, of diplacement of the nterus into the sac of a crural or inguinal hernia. ${ }^{1}$ In two cases the displacement was complicated by pregnancy, which continued to the fourth month.

The Diagnosis is based upon the absence of the uterus from its normal place and the presence in the hernial sac of a body answering its description. If pregnant, the uterus will increase progressively in size until relieved by abortion.

The Treatment is the same as for any other form of hernia. If reduction by taxis is unsuccessful, herniotomy becomes necessary, and may involve removal of the uterus.

Hernia of the ovary may oceur in the same way as hernia of the nterus, and is subject to the same principles of diagnosis and treatment. The author personally has seen but one case; that was treated snceessfully by herniotomy and removal of the ovary.

\footnotetext{
1 Thomas and Mundé. Diseases of Women.
} 


\title{
PART VI. \\ DISORDERS OF MENSTRUATION AND ST'ERILITY ${ }^{1}$ AND INCONTINENCE OF URINE.
}

\author{
CH A PTER L. \\ PREMATURE MENSTRUATION AND PROTRACTED \\ MENSTRUATION.
}

\section{PREMATURE MENSTRUATION.}

IT is elear that precocious menstruation could not occur unless the genital organs had developed prematurely ; and that in the absence of such development there would be no evidence except flow of blood on which the fact of menstruation could be established. One should be cautions in drawing conclusions from the mere prescnce of a red stain on the napkin or elothing for such a stain is not proof of menstruation; it may be blood from vulvovaginitis, urethritis, or traumatism, or, what is more frequent, from deposit of red urates.

Development of the sexual organs has, in rarely exceptional eases, taken place long before the age of puberty, and in some instances has been observed at infancy. Perfectly developed mammæ, a full growth of hair on the mons veneris, much development of the external generative organs, and great precoeity of the internal organs have been observed in infancy and in early childhood. In many instanees of precocions development premature menstruation did not oceur; in others it made its appearance several years before the normal age of puberty; and in one or two attested eases apparently physiologieal menstruation oceurred soon after birth.

As above stated, the appearance of a bloody discharge, even thongh it be periodical, is not necessarily to be attributed to menstruation. Such a discharge may oceur as the result of tumors, erosions, ulcerations, and other pathological canses, at any period of life, from infancy to old age.

The evidence of many notable examples of early pregnancy is unquestioned; Croom tabulated from the literature a series of twelve

1 The disorders of menstruation and sterility are merely functional, and therefore must be considered, not as diseases, but as symptoms. In studring these symptoms it is escential to consider them from the standpoint of the multiform lesions that cause them. There is seareely a gynecological disease that may not have relation, direct or remote, with functional disorders.
Part VI, in a certain sense therefore, is an index to tle whole subject of gynecology. In connection with the study of these disorders, it will be profitable to read the first chapter of the book, which contains a condensed statement of the phenomena of menstruation. 
cases in which pregnancy, occurring in girls of ages varying from eight to thirteen years, resulted, with one exception, in the delivery of mature infants.

\section{Causes of Premature Menstruation.}

The causes of precocious sexual development and prentature menstruation, especially in infantile eases, are not satisfactorily explained. Among the alleged causes are: 1 , heredity; 2 , immoral associations, which vicionsly direct the attention of the child to the generative organs ; 3 , masturbation ; 4, ascarides in the rectum and other parasites about the external genitals ; 5, uncleanliness, especially the deposition of caseous secretions about the clitoris; 6 , neoplasm of the generative organs; 7 , undue nervous and mental excitement.

\section{Treatment of Premature Menstruation.}

The treatment is implied in the preceding paragraph; it consists in the removal of the cause. The necessity for cleanliness and chastity is self-evident. A young girl prone to precocity should be guarded against all influences that tend to stimulate the emotions or to provoke sexual excitation. The treatment is rather regulative than medicinal, is chiefly hygienic, and, as such, includes careful attention to diet, exercise, recreation, sleep, and study.

\section{PROTRACTED MENSTRUATION.}

The normal menopause usually takes place between the ages of forty-five and fifty years; it may occur earlier, or as late as the fiftythird year; but cessation of menstruation before the fortieth year or the continuance of it later than the fifty-second year would be presumptive evidence of a pathological cause. Apparently normal menstruation, however, has been known to continue until after the age of fifty-seven. The eoexistence of ovulation with late menstruation in a given case can be established only by the occurrence of pregnaney; of this oceurrence little satisfactory proof has been recorded after the fifty-second or at most the fifty-fourth year. The evidence is conclusive, therefore, that these ages are the practical limit of possible fertility. 'The author observed a ease of tubal pregnancy in a woman, fifty-four years old.

\footnotetext{
1 Allbutt and Playfair. System of Gynecology.
} 


\section{CHAPTER LI.}

\section{AMENORRHGA AND SCANTY MENSTRUATION.}

\section{AMENORRHEA.}

Amenorrhos is the absence of menstruation. The subject is restricted here to amenorrhœa dependent upon pathological or surgical causes, and excludes the physiological absence of menstruation before puberty, during gestation and the puerperim, and after the menopause ; it also excludes all cases in which menstrual fluid, having been retained by atresia in the genital tract, fails to make its appearance. See Chapter XXXVIII.

\section{Classification.}

Two general divisions of amenorrhcea have been recognized: 1 . The disorder may be due to absence of the reproductive organs or to failure of those organs to develop from the immaturity of infancy to the maturity of puberty. 2. Menstruation may have been established at puberty, and from pathological causes may have ceased. These two divisions are designated as primary and secondary amenorrhœa.

\section{Etiology of Amenorrhœa.}

The causes may be divided into:

I. Local causes.

II. General causes.

I. Local Causes.-Chief among the local causes are absence, imperfect development, and atrophy of the uterus and its appendages. The relative extent of these defects may be greater in the uterus and less in the appendages; or greater in the appendages and less in the nterus. Absence or imperfect development of the genitals may coexist with perfeet development of the body in general. There are two forms of uterine atroply: concentric, in which the uterus is much contracted and its canal correspondingly shortened; and excentric, in which the atrophic process has resulted in a thinning of the walls withont decrease in the length of the uterine canal.

Endometritis and metritis, especially when associated with an infectious puerperium, may give rise to atrophy of the uterus and consequent amenorrlœea. See Chapter XVIII. Oraritis may, as a cause of atrophy of the ovary, produce the same result. Mierocystic degen-. eration of the ovaries and ordinary bilateral ovarian eysts are associated frequently with atrophy of the ovary and amenorrhoea. 
Atrophy of the uterus and ovaries, especially of the former, has been observed oceasionally to follow sharp curettage for endometritis.

II. General Causes seldom produce amenorrhœa by acting directly per se; they usually do so indirectly by causing atrophy of the uterus or ovaries, or of both. 'They may be classified as follows :

1. Acute infections diseases.

2. Chronic disorders.

3. Nervous disorders.

- 1. Acute Infectious Diseases include scarlatina, diphtheria, typhoid fever, and arthritic rheumatism. 'The same infection that produces these diseases may produce also atrophy of the uterus or ovaries.

2. Chronic Disorders. - Among the chronic disorders that eause amenorrhœa are tubereulosis, diabetes, syphilis, anæmia, nephritis, chlorosis, myxœedema, malaria, and exophthalmic goitre. Amenorrhœa associated with these disorlers may not be consequent upon atrophy; it may be simply an effort of nature to conserve the blood and strength of a woman whose general nutrition would suffer even from slight menstruation. In some cases anæmia is associated with uterine hemorrhage. See Chapter LII.

3. Nervous and Mental Disorders.-The psychoses, especially those causing great mental depression, are likely, with or without atrophy of the uterus, to be associated with amenorrhoa. The influence of the nervous system is manifest in the amenorrhoea of prisoners and inmates of asylums. Sudden enotion, chill, and fright have been known, temporarily or permanently, to arrest menstruation.

In addition to the above causes may be mentioned the following : excessive hemorrhage, especially from the genitals; superinvolution of the uterus from prolonged lactation; tissue-change of unknown origin; the morphine-habit; and faulty hygiene, especially such hygiene as comes from insufficient food, overwork, and vitiated air.

Adiposity in anæmic women is often the cause of amenorhœa; the menstrual disorder, however, sometimes disappears with removal of the cause. The physiological amenorrhea of the menopause is followed not uncommonly by deposition of fat.

The amenorrhœa of delayed puberty may occur in girls whose generative organs are apparently well developed. Failure to menstruate in such cases may be due to one or more of the general causes already outlined. In some cases of apparently robust health the patient, for reasons unknown, fails to menstruate. The girl may at the period of puberty menstruate normally, and after a year or two may menstruate less and less, and perhaps finally cease altogether. In too many such cases the amenorrhœea is due cloubtless to diversion of energy, which should be used in the building up of the reproductive system, to social and mental requirements. If this energy is diverted to the brain or to the ball-room, the reproductive system may suffer.

Removal of the ovaries in the great majority of cases will arrest menstruation inmediately. The causes of continued menstruation after double ovariotomy are: 1, the presence of a supernumerary ovary not observed; 2 , failure to remove all of the ovarian tissue; 
3 , failure to remove the tubes close into the horns of the uterus; 4 , diseases of the uterns, i. e., endometritis, carcinona, and fibroids; 5 , persistence of habit.

\section{Symptoms of Amenorrhœa.}

Absence of menstruation is the prime symptom; assoeiated with this may be numerous disturbances referable to the nervous srstem; among them, defective vision, ringing in the ears, hysteria, paresis, sweating, and such skin-eruptions as acne, urticaria, eczema, and herpes. Amenorrhoa often is associated with all the symptoms of menstruation except the flow-these symptoms taken together are termed the molimen.

Vicarious menstruation is an infrequent oceurrenee. Numcrous. remarkable cases, however, have been reported in which a periodic flow of blood from sume organ other than the uterus apparently took the place of normal menstruation. Such a flow may come from the nose, ear, bowel, or bronchi, or from any exposed surface, such as an ulcer; it may be accompanied by a discharge of milk from the breasts or with diarrhoea. The cause of vicarions menstruation is unknown.

\section{Diagnosis of Amenorrhœa.}

Inasmuch as amenorrhoa is a symptom, and not a disease, the object of diagnosis must be to recognize the lesion or lesions that underlie and perpetuate the symptom. Amenorrhoea, especially that caused by atrophy of the ovaries, is characterized sometimes by the presence of a peculiar menstrual molimen above mentioned, unaceompanied by a flow of blood, but attended with great ovarian hypogastric and lumbar pain.

\section{Prognosis of -Amenorrhœa.}

The prognosis is that of the lesion that produces the amenorrhœa. Primary amenorrhœa, due to absence of the uterus or ovaries, is permanent. Full development of the rudimentary organs and consequent menstruation have been reported in rare instances; but these reports are not sufficiently definite to have practical significance.

Excentric atrophy of the nterus, that does not decrease the length of the organ, but only thins its walls, may, on removal of the cause and the establishment of correct hygiene, terminate in anatomical and physiological recovery; concentric atrophy, in which the uterus is contracted uniformly, is permanent. As a rule atrophy due to infection, especially to an infectious puerperium, is permanent.

Amenorrhnea due to prolonged lactation, or to nervous canses, such as sudden fright or violent emotion or chill, offers a favorable prognosis.

In general, the prospeet of recovery is good for all eases in which atrophy of the uterus or ovaries is only a participation in a general systemic failure of nutrition. In cases of defective local development 
and of atrophy due to loeal causes, especially to infection, the amenorrhcea is usually permanent. In the class of cases mentioned under etiology, in which girls in apparently robust health fail to menstruate at puberty, the prognosis is not necessarily bad, for in many such eases normal menstruation and maturity occur after marriage, and in some such cases pregnancy has been observed even though the amenorrhoea persisted.

\section{Treatment of Amenorrhœa.}

The treatment must vary with the cause. Certain forms of amenorrhea, mentioned under Prognosis, are permanent, and therefore incurable.

The curable eases are usually those in which the uterus or ovaries have, because of some wasting disease, such, for example, as tubereulosis, failed to perform their functions. Amenorrhoea then may, as already stated, be only an effort of nature to conserve the woman's blood and strength. 'The ill-health should not be attributed to want of menstruation, but to general want of nutrition; under such conditions treatment designed to re-establish menstruation by bocal stimulation is elearly contraindicated. Such treatnent would defeat the efforts of nature and still further exhaust the woman's vitality and strength. The treatment should be not local, but systemic.

Systemic Treatment.-It follows, from the foregoing, that systemic treatment must be that of the numerous causal diseases already mentioned, such as anæmia, chlorosis, myxœelema, exophthalmic goitre, malaria, nephritis, and tubereulosis. Among the most reliable medicinal remedies are iron, arsenic, manganese, the bitter tonics, salines, and mineral waters. Hygienic remedies, such as nutritious food, exercise, rest, bathing, and suitable climate, must have adequate attention.

In amenorrhoea due to obesity and associated anæmia the indication is to improve nutrition by cure of the anæmia and reduction of the fat,

The distressing nervous symptoms mentioned in Chapter I., that sometimes follow the menopause, should be treated on general principles. The treatment indicated for these symptoms is the same whether the menopause occurs from natural causes or is produced artificially by removal of the uterine appendages. Ovarian extract in three grain doses, taken three times a day, apparently has given much relief.

Local Treatment is generally useless. In some cases it appears to have been effective; but the improvement probably resulted from general nutritional changes. Pelvic congestion sometimes associated with suppression of the menses may be relieved by rest in bed, regulation of the bowels, hot water raginal douche (see Chapter IV.), glycerin tamponade in the vagina, scarification and leeching of the cervix. Pelvic anæmia associated with amenorrhea is an indication for pelvic massage and gymnastics. 
Electricity applied to the pelvic organs, whether from the ordinary battery or from the zine and copper pessary, is of questionable value.

\section{SCANTY MENSTRUATION.}

Scanty menstruation, like menorrhagia, is a relative condition. ${ }^{1}$ The normal flow may vary for different women between two and six days-that is, a flow of two days' duration, for example, may be normal for a woman who cannot afford to lose much blood, while for a very plethorie woman a nuch longer period of flow might be normal. A material decrease or increase, however, in the number of days or in the amount normal for a given individual should give rise to suspicion of a pathological cause. The woman's menstrual habit therefore must be considered in the diagnosis.

The canses of scanty menstruation are identical with those already laid down as the causes of amenorrhœa; the same etiology being established, it follows that the pathology, diagnosis, and treatment must also be along the same lines.

${ }^{1}$ Croom. System of Gynecology, Allbutt and Playfair. 


\section{CHAPTER LII.}

\section{UTERINE HEMORRHAGE-MENORRAGIA AND METROR- RHAGIA.}

Mexorrhagia is excessive loss of blood from abnormally profuse or abnormally prolonged menstruation. Loss of blood from the uterus in the interval between the end of one menstruation and the beginning of the next, whether scanty or profuse, is known as metrorrhagia. Hemorrhage from such causes as placenta previa, degeneration of the chorion, and inversion of the uterus, is deseribed in works on obstetrics.

The terms menorrhagia and metrorrhagia, although in general use, cannot be applied always with aceuracy. The menstrual flow may be prolonged throughout the greater part of the month, or may cease altogether for intervals of liours or days. It is therefore evident that menstrual hemorrhage-menorrhagia-and intermenstrual hemorrhage-metrorrhagia-may be indistingnishable. It is quite as well to use the more simple term, uterine hemorrhage.

\section{Etiology of Uterine Hemorrhage.}

Certain disorders which, if present, are apt to cause uterine hemorrhage will be found described more fully in other parts of the book that specially treat of them. Among the more common of these conditions are :

1. Inflammations.

2. Tumors.

3. Displacements.

4. Foreign bodies.

5. Systemic disorders.

6. Viseeral diseases.

7. Uterine moles.

1. Inflammations.-The inflammatory causes of uterine hemorrhage may be uterine or extra-uterine.

Uterine Inflammations.-Chronie glandular endometritis, as deseribed in Chapter XVI., is characterized by enlargement of the glandular and vaseular struetures of the endometrium, and by consequent excessive glandular secretion or hemorrhage, or both combined. From the pathology, therefore, it is easy to understand that an excessive flow due to endometritis is mixed ordinarily with glandular secretions; that these seeretions may form a very considerable part of the abnormal menstrual discharge; and that in some cases in which the disease is more glandular than vascular the discharge may be almost entirely a profuse uterine leneorrhea composed of vitiated muens or mncopus, and only slightly admixed with blood. Such a discharge, if profuse, even though it contains no blood, mav be quite as exlausting as if it were of a pronounced hemorrhagie character.

Arteriosclerosis of the uterine vessels alone has been assigned as 750 
the cause of uncontrollable uterine hemorrhage by Herman, August Martin, Reinscke, and Küstner. After a careful review of the few recorded cases, Findley concludes that arteriosclerosis, per se, is not an adequate cause of uterine hemorrhage. In all the reported cases there were other conditions which had resulted in obstruction to the general circulation, such as Bright's disease, heart lesions, pulmonary infection, and thrombosis of the uterine ressels. In the ease recorded by Findley arteriosclerosis and calcification of the uterine ressels undoubtedly existed long before the beginning of the hemorrhage. In this case embolic infarction of the uterus from a thrombus in the heart was the immediate cause of hemorrhage. ${ }^{1}$ See Chronic Metritis.

Extra-uterine Inflammations, such as ovaritis, salpingitis, parametritis, and perimetritis, give rise to pelvic congestion and to a consequent effort of nature to obtain relief by an increased flow. Ovaritis, according to its nature, may increase or diminish nenstruation. Parenchymatous inflammation in the cortical substance of the ovary may increase the flow. On the contrary, the atrophic process of interstitial ovaritis or of microcystic degeneration of the ovary, tends to induce amenorrlıœa.

2. Tumors.-Uterine tumors cause excessive menstruation in greater or less degree according to their situation. A growth beginning in close relation with the endometrium and developing within the uterine cavity may set up a dangerous periodical or constant bleeding; if situated in the uterine wall, between the endometrium and the perimetrium, it may excite little more than the normal flow; located near the peritoneal covering, it may, espeeially if pedunculated, give rise to no menstrual excess whatever : uterine myoma, for example, may, according as it is submucous, intramural, or subperitoneal, cause much, little, or no menorrhagia. See Chapter XXVI.

A tumor may set up exeessive flow in one or both of two ways: 1 . The irritation of its presence may give rise to a hemorrhagic endonetritis. 2. Ulcerative processes or friability of the tumor itself may cause rupture of blood-vessels and hemorrhage. In one way, the blood comes from the endometrium; in the other, from the tumor. Myomata, being slow to ulcerate and break down, are little liable to bleed per se; but if submucous, they irritate the endometrium and set up hemorrhagic endometritis. Cancer and sarcoma not only cause hemorrhagic endometritis, but themselves rapidly undergo necrotic changes, and thus become the source of hemorrhage. Extra-uterine growths may induee menorrhagia by the pelvic irritation and consequent congestion to which they give rise.

3. Displacements.-Deviations of the uterus and its appendages, through traction on the pelvic blood-ressels, may so obstruct the circulation as to cause venous congestion and a consequent excessive menstrual effort to lessen the quantity of blood in the pelvis. The complete relief from menorrhagia that sometimes follows the correction of a prolapsed or flexed uterus by means of artificial support, and the prompt return of excessive menstruation upon withdrawal of 1,1901 .

1"Arteriosclerosis of the Uterus." Findley. American Journal of Obstetries, vol. xliii., No. 
the support, are satisfactory proof that displacement may cause uterine hemorrhage. Further information on this subject may be found in Part V. on Displacements.

4. Foreign Bodies. - Tents and other foreign bodies which find their way into the uterus cither from therapeutic or criminal motives may, by their irritating presence, cause excessive flow. An embryo detached in the course of abortion is a familiar example of foreign borly in the uterus.

5. Systemic Disorders. ${ }^{1}$ - Any general disorder that will embarrass the return flow of blood from the pelvic viscera will cause an increased vascular pressure that may result in hemorrhage from the uterus. Among the systemic conditions that may underlie excessive menstruation are: hemorrhagic diathesis, scorbutus, purpura, malaria, lead-poisoning, and acute infectious diseases such as searlet fever, diphtheria, and typhoid fever. Menorrhagia associated with sirch causes is often difficult to treat, because, as Croom says, these causes interact in such a way as to form a vicious circle-the drain on the system from the hemorrhage tending to aggravate the systemic condition, which in its turn leads to the menorrhagia. Chronic mental depression, hysteria, sedentary habits, and residence in high altitudes or the tropics, all, in greater or less degree, dispose to menorrhagia. In some cases anæmia, although usually a cause of amenorrhœa or scanty menstruation, may cause uterine hemorrhage. This is explained possibly by the low specific gravity, the diminished coagulability of the blood, or faulty nutrition of the vessels.

6. Visceral Diseases.-Heart diseases, such as hypertrophy, dilatation, mitral insufficiency, or stenosis, as well as cirrhotic changes in the liver or kidney, are associated usually with such organic change or functional disturbance in the blood-vessels as to cause embarrassment of circulation and hemorrhage in various organs. Under one or more of the disorders just named, the uterus, being the seat of a normal periodic hemorrhage, and being therefore predisposed to hemorrhage, may, especially if there be disease of the endometrium, readily become the medium of exaggerated menstruation.

7. Uterine Moles.-Among the occasional causes of uterine hemorrhage may be mentioned uterine moles. There are two varieties: 1, fleshy mole; 2, hydatiform mole, sometimes called cystic mole, and sometimes wrongly called uterine hydatids.

Fleshy Mole.-The so-called fleshy mole is associated sometimes with hemorrhage from the uterus, and when so associated gives rise to abnormal signs of pregnancy. It may be recognized upon spontaneous expulsion or removal.

Hydatiform mole is the result of cystic degeneration of the chorionic villi. It gives rise to pronounced and constant or almost constant hemorrhage, and is associated with rapid increase in the size of the uterus, together with the usual signs of pregnancy. The diagnosis will depend upon the expulsion of a part or all of the vesicles. Although hydatiform mole and hydatids (echinococcus) resemble one another in gross appearance, they have no other characteristic in 
common. Uterine hydatids (echinococeus) have been observed, but they are of very rare occurrence.

When the causes of menorrhagia are so obscure that they cannot be detected, the hemorrhage has been termed idiopathic. The use of such a term explains nothing. It is better to say outright that the symptom is of unknown origin.

\section{Diagnosis of Uterine Hemorrhage.}

Before deciding what constitutes an excessive loss of blood, it is necessary to adopt at least an approximate standard of the normal amount. As stated in the chapter on Scanty Menstruation, menorrhagia is a relative condition. An anæmic woman whose blood is searcely sufficient to meet the fixed requirements of nutrition can ill afford to spare what, in a robust state, would be even less than normal; and for such a woman amenorrhœa may be a means of saving much needed blood, and in a relative sense, therefore, may be considered normal. On the other hand, in an exceptional case of plethora, eight or nine days of free menstruation may cause no illeffect-may, indeed, be beneficial, and therefore normal. We may, lowever, for practical purposes arbitrarily limit the normal duration of menstruation to six days, and the number of napkins soiled in that time to about fifteen.

In the diagnosis of menorrhagia, one must exclude all bleeding from extra-uterine sources, such as the bleeding fronı hemorrhagie vulvovaginitis, from traumatism of the vulva or vagina, and from varicose veins of the vulva.

The mere discovery of menorrhagia is only the recognition of a symptom. The practical diagnosis includes as well the discovery of the cause or causes of that symptom. Only in this way is a rational prognosis or effective plan of treatment made possible. The causes that produce menorrhagia, and that have already in a general way been outlined in preceding paragraphs of this chapter, will be found discussed more fully in the other chapters that specially treat of them.

The diagnosis of menorrhagia will vary according to the age of the patient somewhat as follows:

Uterine Hemorrhage of Girls.-Inasmuch as the menorrhagia of girls and of very young unmarried women is in a very large proportion of cases due to general systemic conditions, it is obvious that uterine examinations in such cases should at first be avoided. If the general examination has failed to disclose the causative lesion, or general treatment has failed to give relief, a pelvic examination may be the only means of diagnosis, and therefore may be imperative. It does not, however, by any means follow that a minor local disturbance, even though coexistent with menorrhagia, should be made the occasion of local treatment. $A$ case in point may be cited from the writer's experience in which there were protracted menorrhagia and increased uterine secretion, both promptly disappearing upon correction of a lateral curvature in the lumbar region of the spine by means of 
a plaster jacket. The symptoms complained of may perhaps have been due to fanlty nutrition, and this in turn may have been perpetuated by the curvature; at any rate, whatever the explanation, the relicf was complete. The author has observed one ease of profuse menstruation and intermenstrual hemorrhage in a young girl which apparently was due to chronic appendicitis.

Uterine Hemorrhage during the Period of Maturity is, in a majority of cases, dependent solely or partly upon local pelvic disease, such as infection, displacements, tumors, and products of conception. Very commonly the local disease eoexists with general disorders. Such cases frequently demand local, general, or operative treatment.

Uterine Hemorrhage during the Menopause.-To avoid repetition, the reader is referred to a partial discnssion of the subject in Chapter I. If the menopanse is characterized by a long-continued series of hemorrhages or by constant hemorrhage, the probability is that endometritis, inflammation of the uterine appendages, or a tumor exists, and retards the atrophic process and consequent normal amenorrhoea which at this time should take place. It may be laid down as a general proposition, moreover, that an abnormal menopause, even though not hemorrhagic, is a positive indication for thorough examination, both local and generil, with a view to accurate and adequate diagnosis. This indication is strengthened by the frequent development, during this critical period, of malignant disease and mental disorder.

\section{Treatment of Uterine Hemorrhage.}

The foregoing paragraphs on etiology and diagnosis, when considered in their relations to treatment, should lead to the following general statement, to which, however, numerous exceptions will arise:

The treatment of uterine hemorrhage in girls and young women is often that of a systemic eause; the treatment in married women of the childbearing age is usually that of endometritis, benign tumors, or displacements; the treatment of the menorrhagia of spinsters is commonly that of benign tumors; and of women between the ages of forty and fifty years, often that of malignant growths or myomata; the treatment during senility is often that of malignant disease.

The therapentic measures most frequently employed comprise :

1. Systemic treatment.

2. Loeal treatment.

3. Eleetrotherapeuties.

4. Surgical operations.

1. Systemic Treatment.-Rest in bed during the most excessive part of the flow, freedom from mental disturbance, bathing, passive exercise, the use of nutritious, non-irritating food, the avoidance of stimnlants, and residence in a temperate or cold climate at or near the sea-level, are the prineipal hygienic measures. Drugs, such as stypticin, salines, ergot, digitalis, cinnamon, nnelein, and hydrastis canadensis, may be selected according to special indications. When 
there is systemic hemophilia an abundance of gelatine taken with the food is known to increase the coagulability of the blood.

2. Local Treatment. - The hot-water vaginal douche and vaginal and uterine tamponade, as described in Chapters IV. and XXVII., are the two most satisfactory means of local treatment. The latter, especially as a temporary measure, if properly applied, is most effective. Intra-uterine applications of adrenalin have been recommended highly. Intra-uterine injections of strong astringents, such as concentrated solutions of iodine and tincture of iron, are used frequently, and may in some cases be promptly effective; but the liability of setting up painful uterine contractions and the danger of invasion of the Fallopian tubes and of possible pelvic infection, are serious objections to their general use.

3. Electrotherapeutics.-The subject of electrotherapeutics lias been mentioned in the treatment of endometritis and myoma, Chapters XVII. and XXVI. The value of it has been much overestimated.

4. Surgical Operations. - The operative treatment is usually not directly that of the mere symptom, hemorrhage; but rather of such diseases as endometritis, metritis, myoma, cancer, and sareoma. The surgery of these diseases may be found discussed in other parts of the book. 


\title{
CHA P'TER LIII.
}

\author{
DYSMENORRHCA AND PERIODIC INTERMENSTRUAL \\ PAIN.
}

\section{DYSMENORRHCEA.}

\section{Definition and Classification.}

Drsmexorrhera is painful menstruation. This definition does not include the slight heaviness in the loins, the general malaise, the vague sense of diseomfort, and the irritability that go to make up "the unwell feeling" of healthy women during the menstrual week. The slight "unwell symptoms" so called, although sometimes absent in normal eases, are not evidences of disease, and may therefore be disregarded.

The numerous attempts to elassify dysmenorrhoa have led to the use of a eomplieated and abundant nomenelature, of which each term is taken to designate a partieular variety of painful menstruation. Dysmenorrhœa aceordingly has been characterized varionsly as tubal, ovarian, membranous, inflammatory, congestive, neuralgic, spasmodie, intermenstrual, mechanical, or eonstitutional. Such designations, although useful for purposes of description, are, when considered from the standpoint of elassifieation, wholly misleading. The morbid conditions associated with painful menstruation are to a considerable extent eommon to most of the so-called varieties. For example, dysmenorrhoea of inflammatory eausation might originate in the ovary, tube, or uterus. It necessarily would be associated with congestion, it might take place in the intermenstrual period, or might be aggravated by eauses of a mechanieal or constitutional nature. Almost any one of the above names, therefore, might with equal propriety be applied to designate this so-ealled variety. The other terms proposed to designate special varieties are subjeet to similar eritieism. Furthermore, the diffienlty-not to say impossibility - of making in the present state of our knowledge a seientific or praetical classification of dysmenorrhœa will beeome even more apparent from what follows.

\section{Clinical History and Diagnosis of Dysmenorrhœa.}

Degrees of Pain.-Pain associated with menstruation varies in the widest limits from the general malaise of the "unwell week" to the most intense agony. In many eases the pain is assoeiated with definite lesions, and disappears upon the cure of those lesions. In other eases the suffering is wholly out of proportion to the assoeiated disease-that is, a woman presenting the most exaggerated evidence 
of pain may upon careful examination disclose little or no disease to account for it; on the other hand, extensive disease may exist and yet give rise to little or no dysmenorrhoa.

Character of Pain. - It is usual in the taking of histories of gynecological practice to note the character of the pains, and to designate them by such words as distinct, sharp, dull, heavy, radiating, dragging, bearing-down, and labor-like. The maximum pain may be in the back, loins, or pelvis; it may be constant, intermittent, or remittent ; it may come on or be most intense before or during menstruation; it may cease or may inerease with the establishment of the flow ; it may continue only during the first day or two, or with varying degrees of intensity may outlast the period.

Unfortunately, the conditions that determine the variation in the degree and character of the pain are, for the most part, not very definitely known. So far, however, as the conditions are known and have a practical clinical value they will be considered later.

The following four-fold statement relative to the phenomena of normal menstruation, if read in connection with Chapter I., will perhaps help the student to understand the variations of dysmenorrhœa :

1. There is, in normal menstruation, a general premenstrual and menstrual congestion of the pelvic organs.

2. Ovulation is associated usually with menstruation, but is not an essential factor of it.

3. There are slight premenstrual enlargement and softening of the uterus associated with congestion and swelling of the mucosa, reaching their maximum, according to Herman, on the fourth day of the flow, and eontinuing for a short time after the bleeding stops.

4. The menstrual fluid is composed chiefly of blood, but with admixture of cast-off epithelial cells and lymph-corpuscles.

Painful menstruation, in exceptional instances, nay exist unassociated with any demonstrable lesion. In the vast majority of cases, however, careful examination and close analysis will disclose either a local or a systemic cause, or both combined.

Associated Lesions.-In the absence of a scientific and adequate classification one may consider dysmenorrhœa in its relations with certain associated lesions; these lesions are designated under two heads, as follows :

A. Local diseases, usually situated in the pelvis.

B. General diseases, usually faults of nutrition.

\section{A. Dysmenorrhea associated with Local Diseases.}

The local diseases commonly associated with dysmenorrhoea are :

1. Inflammation.

2. Tumors.

3. Obstruetion.

4. Malformations.

1. Inflammation.-Chronic Endometritis.-The most pronomeed type of dysmenorrhœa dependent upon inflammation is deseribed in 
Chapter XVI., and is known as membranous dysmenorrhoea; it is due to an exfoliative endometritis, which results in the easting out, either complete or in shreds, of an entire membranons layer from the uterine mucosa. This exfoliation is consequent upon a process the nature of which is unknown, which so modifies the superficial layer of the endometrium that the blood, not being able to pass through it, aceumulates at some point behind and forcibly strips up a portion of it. There is then an effort of the uterus to expel the partially detached portion; and as this is forced toward the external os it strips off and drags after it the remaining undetached portion. The stripping off and expulsion through the narrow cervical canal of the entire laver are associated with very strong intermittent uterine contractions and consequent spasmodic pain of the most intense character. If the membrane is detached and cast off in shreds, the pain will be less severe; if it is cast off in minute particles, the pain may be very slight. Membranous dysmenorrhœa, therefore, for different individuals and at different times for the same individual may, according to the size of the exfoliated masses, be very slight or most intense.

Projections into the endometrium of granulation-tissue, the product of chronic endometritis, may stimulate the swollen irritable uterus to spasmodic contractions similar to those of membranous dysmenorrhœa, though usually less severe. See Chapter XVI.

Chronic Metritis.-Dysmenorrhœa when associated with chronic metritis may be attributed to the presence of an abnormal amount of fibrous tissue; this tissue is so dense and unyielding that it prevents the normal premenstrual softening of the uterus and the widening of the uterine canal, and in this way, especially when the organ is congested, may exert painful pressure on the uterine nerves. Metritis does not necessarily eause dysmenorrhœa. The symptom may result from the complications of metritis, such as displacement, fixation, or stenosis of the uterus, inflammation of the uterine appendages, chronic cellnlitis, chronic peritonitis, or some neurosis.

Chronic Salpingitis. - No satisfactory explanation has been given of the severe dysmenorrhoa that sometimes accompanies salpingitis. Cases in which the tube is distended moderately with pus are usually more painful than those in which there is great distention. Moreover, severe salpingitis may exist, and yet give rise to no dysmenorrhœa. For further information on the subject the reader is referred to Chapters XXI. and XXII.

Chronic Ovaritis is almost inseparable from inflammation of other pelvic organs. Its influcnee therefore as a cause of dysmenorrhoa cannot be estimated accurately. Paiuful menstruation associated with ovaritis is characterized often by a period of premenstrual suffering variable in duration, by rather pronounced nervous symptoms, and by mammary tenderness. Ovarian pain is especially apt to radiate to the thighs and nates. Pain referred to the ovaries is common, and often exists in the absence of a demonstrable lesion in the ovaries. Removal of the ovaries under such conditions seldom gives permanent relief.

2. Tumors.-The tumors most frequently associated with dys- 
menorrhœa are uterine myomata of the intramural, submucous, or intra-uterine variety. These tumors may, especially during the period of menstrual congestion, cause pain in the following ways :

1. An intra-uterine or intramural tumor may stimulate the uterus to attempt its expulsion by painful uterine contraction.

2. A tumor may by its weight produce displacement of the uterus and consequent painful menstruation.

3. A tumor, if it fills the pelvis, may produce pressure-symptoms; and these symptoms may, owing to the menstrual congestion, be aggravated during the eatamenia.

Displacements of the uterus as associated factors in dysmenorrhœa are considered further in Part $V$. In this connection the reader's attention is directed specially to the subject of anteflexion as laid down in Chapter XIVIII.

3. Obstruction.-Stenosis within the cervical canal, and consequent obstruction to the outflow of blood, have held a large place in the controversial literature of dysmenorrhoa. The claimants for the mechanical theory, on the one hand, sometimes have attributed all, or nearly all, painful menstruation to narrowing of the uterine canal; while their opponents not infrequently have denied altogether to this cause any considerable place in the causation of dysmenorrhoea. It may be sufficient, without going over the arguments for and against the mechanical theory of dysmenorrhoa, to say that contraction of the cervical canal has been properly almost excluded as a direct mechanical eause of dysmenorrhoea. The following paragraph will show, however, that this exclusion does not by any means dispose of mechanical obstruction as a frequent indireet eause of painful menstruation.

Two forms of obstruction may be due to anteflexion. One is obstruction in the uterine canal, due to collapse of the uterine canal at the point of bending; the other is obstruction in the blood-vessels, due to collapse of the walls of the vessels also at the point of bending. The obstruction which causes the dysmenorrhœa is commonly in the blood-vessels, not so commonly in the uterine canal.

Some authorities deny that dysmenorrhoea when associated with flexion is in any sense due to obstruction either in the uterine canal or in the vessels, and attribute the pain wholly to the associated uterine or extra-uterine inflammation. It is true that inflammation in a sense causes the pain ; but it is also true that it causes the flexure, and that the flexure, once formed, tends to keep up the inflammation. The two together constitute what has been called a vicions circle; the former producing the latter, and the latter reacting to aggravate and perpetuate the former. A more full statement of the two forms of obstruction may be found in Chapter XLVIII.

4. Malformations.-Malformations of the pelvic organs may be associated with dysmenorrhoea in the following way: there may be atresia somewhere in the genital tract, with consequent retention of menstrual fluid, so that during successive periods the blood accumulates with steadily increasing and painful distention. 


\section{B. Dysmexorrheea associated with General Diseases.}

The strong surgical bias in gynecological practice, emphasized by the remarkable results that have been obtained by operative and mechanical measures, and by the relative safety of such measures when aseptically employed, has led, during the last generation, to an undue estimate of the value of surgical procedures, and to a corresponding neglect of general therapeutic requirements. Accordingly, there has been during the past thirty years a strong movenent along surgical and mechanical lines, and a corresponding disposition to disregard the claims of internal medicine. On the other hand, to ignore extrapelvic eanses of pelvie pain or to disregard pelvic canses of systemic disturbances would be manifestly absurd. In either case most embarrassing blunders in diagnosis and treatment necessarily would follow.

Many constitutional and systemic conditions predispose to painful menstruation; they may be associated with dysmenorrhoea with or without demonstrable local lesions. In some cases in which local disease is not present, or, if present, is not sufficient to account for the menstrual pain, the dysmenorrhoa must be attributed chiefly or wholly to general causes. Among the general disorders often associated with dysmenorrhœa are rheumatism, gout, anæmia, chlorosis, malaria, neurasthenia, and hysteria. What Goodell aptly called the intangible, imponderable, invisible pelvic pains of neurotic women are especially liable to inerease during menstruation.

In one pronounced elass of cases the reproductive organs may, from lack of proper innervation and nutrition, have failed to mature at the age of puberty, and therefore nay perform the menstrual function imperfectly; the defect declares itself not infrequently in the form of an intensely painful effort to menstruate-that is, a painful molimen with little or no flow. The lack of innervation and nutrition in such cases is not often confined to the pelvic organs; it is usually systemic. Dysmenorrhoea of this kind, therefore, has both a systemic and a local source.

Among the neuroses most frequently associated with dysmenorrhœe are neurasthenia and hrsteria ; the one, characterized by excessive hyperesthesia and involving motor weakness; the other, eharacterized by loss of power and coördination over antomatic movements and by an excessive responsiveness to -suggestion. Either of these neuroses may coexist with dysmenorrhoe in cases that present no local disease, or local disease so slight that in an otherwise normal woman it would have little or no recognition.

\section{Treatment of Dysmenorrhœa.}

There are two possible errors that may be made in connection with treatment. One is that a distinct nervous or other general disorder elosely related to the causation of the pain may be overlooked or neglected ; the other is that exaggerated importance may be given 
to some insignificant local lesion, that this exaggeration may result in an error of judgment, and that much local treatment of what is known as the tinkering kind may be adopted or that umnecessary operations may be performed. Of course, the opposite mistake may be madethat is, unnecessary general treatment may be employed to the exclusion of necessary local or surgical treatment. ${ }^{1}$ Norman Kerr, of Chicago, reports cases of dysmenorrhœa associated with ovaritis in which relief was obtained by resection of the uterine vessels and nerves as they pass through the infundibulopelvic ligament. ${ }^{2}$

The treatment of dysmenorrhoa, like the cause, is either local or general, or both.

Local Treatment.-The term local treatment here used is extended beyond the usual meaning, and includes both non-surgical and surgical measures. These measures are described in various chapters of this book.

General Treatment.-The general treatment is that of the constitutional and systemic conditions already outlined among the possible causes of painful menstruation. The subject is almost coextensive with the whole field of gencral internal medicine, and cannot therefore adequately be discussed within the limits of a gynecological treatise.

\section{PERIODIC INTERMENSTRUAL PAIN.}

\section{(German, Mittelschmerz.)}

Periodic pain, resembling the pain of obstructive dysmenorrhœa, recurring regularly with each intermenstrual period, and continuing for a definite time, is a condition that often defies analysis and treatment. All explanations of this phenomenon are more or less speculative. The three most rational theories are that the pain is caused by:

1. Sclerosis and contraction of the ovary.

2. Salpingitis profluens.

3. Obstruction in the uterine canal.

1. Sclerosis and Contraction of the Ovary.-Hyperplastic thickening and toughening of the superficial structure covering the Graafian follicles may offer such resistance to the bursting of mature follicles as to canse pain. It is urged that there is no periodicity in the maturing and bursting of the follicles, and that the sclerosis therefore, while it might account for the pain, could not account for the periodicity of it; a speculative reply to this objection would be that ovulation from some unexplained cause, perhaps reversion to a former type, may in rare instances preserve a regular periodicity, and that in such exceptional cases sclerotic toughening would account for the periodic intermenstrual pain.

The writer once removed a sclerotic right ovary and tube, with entire relief to the patient, from a most excruciating Mittelschmer $z$ that had for a long time resisted all other treatment. The relief, however, continued only through the two periods immediately following the operation. The pain at the time of the third and fourth periods 
recurred, and was nearly as severe as before; but since the fourth period there has been partial relief.

2. Salpingitis Profluens.-In numerous instances intermenstrual pain of long standing has disappeared permanently upon the removal of leaky Fallopian tubes-hydrosalpinx or pyosalpinx. The periodicity of the pain in cases of this class is explained by the assertion that a certain definite number of days after each menstruation would be required for the tube to fill with secretions, and that being filled it discharges its contents with regularly recurring pains. There is no evidence in these cases to show absence of cirrhosis of the ovary, hence the cause of the pain may have been ovarian and not tubal.

3. There may be obstruction in the uterine canal from stenosis or flexure, and consequent periodic accumulations of uterine secretions, which are regularly expẹlled by labor pains. 


\section{CHAPTER LIV.}

\section{STERILITY.}

\section{Definition of Sterility.}

ExcLuding the physiological sterility of infancy and senility, one may define sterility as the inability of the individual to produce offspring. In a broad sense, a woman is sterile who cannot become pregnant, or, if pregnant, cannot produce a viable child. . A man is sterile who cannot produce semen that will fertilize an ovum. In a narrow sense, a woman who can conceive and imbed the ovum in the endometrium should not, if the ovum then dies and is cast off, be classed as sterile. The condition, strictly speaking, would be not one of sterility, but of abortion. From the clinical standpoint, however, such early abortion is not easily separable from sterility, for habitual abortion may occur very early in pregnancy-so early that the pregnancy could not have been recognized. Sterility in the male, except as it may have a gynecological significance in diagnosis or prognosis, will not be discussed.

\section{Statistics of Sterility.}

Pathological sterility, as distinguished from the physiological sterility of infancy and senility, is confined properly to the years between the end of puberty and the beginning of the menopausethat is, to the period of maturity; in the majority of cases the limits are even more confined, for the capacity to bear children is seldom fully developed until three or four years after puberty, and it generally ceases some years before the menopause. Failure to bear children therefore in the early years of maturity or in the late years of the menopause, even though it might indicate lateness in the development of sexual vigor or premature decadence, shonld not be considered pathological, and for this reason it should not enter into the statistics.

It is said that 10 or 12 per cent. of all marriages are sterile; this, however, is not an index to the frequency of female sterility. One must also reckon with the fact of male sterility. The proportion of cases in which the fault is in the male has never been the subject of thorough investigation, but the estimates range from 7 to 40 per cent. The percentage is undoubtedly very large, and has added greatly to the popular estimate of female sterility.

\section{Classification of Sterility.}

The varieties of sterility, according to the causes or associated conditions, have been designated as follows:

1. Complete or absolute sterility.

2. Incomplete or contingent sterility. 
Some authors have made a further classification of congenital and acquired sterility; these varieties, however, are only subdivisions of complete and contingent sterility, and should therefore be considered with them.

1. Absolute or Complete Sterility.-Sterility is absolute when due to congenital defects, or disease, or surgical operations that render the generative organs permanently incapable of performing their reproductive functions. The organs may be congenitally defective or absent, or may be impotent from disease, or may have been removed by surgery.

2. Reiative or Partial Sterility.-Sterility is relative or partial when, on account of some defect in development or nutrition, the functions of the reproductive organs are performed inadequately. The condition may be only temporary, and may disappear upon improvement of the general health or upon removal of some obstruction or disease in the genital tract. To this class belong cases of pregnancy nccurring after years of sterile marriage. Some observers designate as relative only that variety in which, on account of sexual or other defects, such as marital or mental incompatibility, a man and woman are unable to act together in reproduction. In such cases each may become fruitful immediately after a second marriage. The question then would be, not whether the individuals are positivcly sterile, but rather, what is the explanation of the sterile union between them. Investigation would show usually that the failure was due rather to anatomical or physiological defects than to mere incompatibility. Moreover, the ovum of a sexually defective woman, although not impregnable by a defective man, might, when brought in contact with the semen of a normal man, readily become fertilized; and vice versa, the semen of a defective man might fertilize the ovum of a normal woman.

\section{Etiology of Sterility.}

A knowledge of the mechanism of conception is essential to an appreciation of the causes of sterility. A large portion of the cortical substance of the ovary is occupied by Graafian follicles in all periods of growth; each follicle contains a fluid, called liquor folliculi, and the ovum. As the follicle and its contained ovum mature the former gradually becomes distended with the fluid, appears beneath the surface, ruptures, and discharges its contents. The ovum is now washed out into the pelvic cavity, and under normal conditions reaches the Fallopian tube and passes along the tube toward the uterus. If at this time, under normal conditions, coitus takes place, and seminal fluid containing virile spermatozoa is deposited in the upper part of the vagina, the spermatozoa, of their own power of movement, enter the endometrium and work their way upward along the genital tract toward the descending ovum. The exact meeting-place of the two organisms is not known. There is strong reason to infer that it is in the Fallopian tube, perhaps at the abdominal end, and that the persistent and virile spermatozoa may consume 
several days in traversing the long distance; they have been observed at the abdominal end of the tube as late as five weeks after the last sexual intercourse.

There are two necessary conditions for normal conception : one, that the ovum as it passes down the genital tract shall meet the spermatozoa, and by them become fertilized; the other, that the fertilized ovum shall find in the endometrium a place favorable to further development, and there become imbedded. If by reason of any defect, general or local, either one of these conditions be absent, conception is impossible. The ovum, failing to reach the tube, may be destroyed in the peritoneal carity; or, having reached it, may pass down the genital tract and fail to be fertilized. The spermatozoa may not be sufficiently virile to find their way upward from the vagina; or, if they reach the ovum, they may not be able to fertilize it. The two organisms may succumb to hostile environment or to some anatomical defect. As indicated in the foregoing, a sterile marriage may result from :

1. Absence of virile spermatozoa.

2. Faulty general nutrition in the woman.

3. Defective reproductive organs in the woman.

4. Intermediate causes.

\section{The Absence of Virile Spermatozoa.}

Old age, wasting diseases, congenital defects, and venereal diseases (especially gonorrhoa) may incapacitate the man to produce virile spermatozoa or may destroy entirely the procreative power. Many a woman has suffered useless-not to say injurious-treatment for supposed sterility when the fault was entirely with the husband. An examination of the husband's reproductive organs, including microscopical examination of the semen, may disclose orchitis, stricture of the urethra, hypospadias, or some other defect that may account adequately for the sterile marriage. A careful inquiry should be made also into the man's general condition. Tuberculosis is a not infrequent cause of sterility.

\section{Faulty General Nutrition in the Womax.}

Chronic wasting diseases and such nutritional disturbances as chlorosis and anæmia, and, above all, the accumulation of fat, especially the peculiar adiposity of anæmic women, due to faulty metabolism, but giving the false appearance of plethora, may lead to sterility. Enteric fever, scarlatina, cholera, variola, diabetes, and nephritis are among the diseases frequently associated with sterility. Premature menopause-that is, permanent atrophy of the reproductive organs and consequent sterility - may result from acute infectious disease. See Non-puerperal Atrophy, Chapter XVIII. 


\section{Defective Reproductive Organs in the Woman.}

Defects in the reproductive organs, both congenital and acquired, according to their nature, may give rise to the two varieties of sterility designated as complete and incomplete.

\section{Congenital Defects in the Reproductive Organs causing Complete or Absolute Sterility.}

1. Absence of the ovaries, Fallopian tubes, or uterus.

2. Rudimentary ovaries, Fallopian tubes, or uterus.

3. Inoperable atresia in the genital tract.

\section{Congenital Defects in the Reproductive Organs causing Incomplete or Contingent Sterility.}

1. Immaturity or innutrition of the ovaries, Fallopian tubes, or uterus.

2. Stenosis or atresia in the genital tract, as in the cervix uteri, vagina, or vulva; infantile vulva; abnormal backward location of the vulva.

3. Opening of the vagina into the bladder or rectum, or double vagina, preventing coition.

4. Displacements, especially flexures.

5. Elongation, shortening, or conical shape, and other irregular developments of the cervix uteri.

6. Lengthening of one lip of the cervix uteri, so as to form a flap over the os uteri externum. septum.

7. Imperforate hymen or cribriform hymen, transverse vaginal tubes.

8. Excessive convolutions or increased length of the Fallopian

9. Doirble iterus.

10. Gynandry.

Discussion of Congenital Causes.-For a more extended description of congenital defects the student is referred to Chapters XXXVII. and XXXVIII., on Congenital Malformations and - Gynatresia.

A rudimentary condition or absence of the ovary may coexist with a well-developed uterus, and vice versa; either combination is a cause of complete sterility. Absence of one ovary, if the other is normal or approximately normal, is not necessarily a cause of sterility.

Septate or double vagina, with the septum so disposed as to divide the vagina into unequal parts, may permit impregnation if the larger part is capable of coition. The smaller part may serve only for menstruation. One side of a double uterus adequately developed may receive the impregnated ovum and carry it to maturity.

Absence or impermeability of both Fallopian tubes, unless they can be opened surgically, causes complete or absolute sterility ; this defect, 
however, is associated usually with absent or rudimentary uterus. Unicorn uterus permits normal pregnancy, uterogestation, and parturition. Immaturity and innutrition of the ovaries, rendering them incapable of producing mature ova, are associated generally with some defect in the general systemic development. Rare instances have been reported in which the reproductive organs, seemingly undeveloped at the age of puberty, have later developed and become fruitful.

\section{Acquired Defects in the Reproductive Organs causing Complete or Absolute Sterility.}

1. Surgical removal of the uterus, Fallopian tubes, or ovaries.

2. Permanent atrophy of the uterus or ovaries.

3. Permanent and incurable occlusion of the Fallopian tubes or uterus.

4. Complete destruction from disease of the functionating part (cortical substance) of the ovary and microcystic degeneration of the ovary.

\section{Acquired Defects in the Reproductive Organs causing Incomplete or Contingent Sterility.}

The acquired defects that may produce incomplete sterility are so numerous and varied, so interactive and complicated, that they sometimes defy analysis; they may be designated generally as follows :

1. Inflammation in the genital tract and its results, such as displacements, adhesions, pathological secretions, stenosis, atresia, kinking of the Fallopian tubes, atresia of the cervix uteri or vagina, tuberculosis and syphilis of the genital tract, hypertrophy and hyperplasia, atrophic changes, microcystic degeneration of the ovaries, and mechanical conditions.

2. Tumors of the generative organs, and the organic and meclianical changes that the tumors produce.

3. Faulty innervation and innutrition of the organs of reproduction. Vaginismus and pruritus vulvæ are possible examples.

Discussion of Acquired Sterility.-Surgical Operations.-The surgical removal of one ovary or Fallopian tube does not cause sterility; if both are removed, sterility, except possibly in rare cases of a third ovary, is absolute; if a small part of one ovary remains, pregnancy may occur. The removal of both tubes would not necessarily cause sterility. Pregnancy has followed the removal of both tubes by ligature placed close to the uterus ; in these cases the ligatured stump having sloughed off, the extreme uterine end of the tulbe remained sufficiently open to transmit the ovum. Excision of the uterine ends of the tubes and union of the peritoneal surfaces over the wounds in both uterine cornua would canse absolute sterility. Pregnancy may ocenr in a uterus of which a part has been removed, especially the cervical part. Even considerable portions of the wall of the corpus, as in myomectomy, may be sacrificed without causing 
absolute sterility. There is a recognized persistence in the ovaries, tubes, and uterus that sometimes enables them, even though mutilated and mostly destroyed, to perform the reproductive functions. Numerous eonservative operations, therefore, designed to preserve in whole or in part the uterus and its appendages, have been substituted for the radieal operation of removal. See Myomectomy, Salpingostomatomie, Resection of the Ovary, and Vaginal Incision and Drainage.

Inflammatory Disorders of the uterus, Fallopian tubes, or ovaries are associated with a great majority of cases of acquired sterility. Ovaritis may result in atrophy or other organic changes that will render the ovary sterile or ineapable of producing mature ova. Atrophy of the ovaries, general or acquired, may or may not be associated with atrophy of the uterus.

Salpingitis may set up thickening, adhesions, kinking, stenosis, or atresia of the tube, and in this way may impede the ovum in its passage to the uterus or pathologieal seeretions may destroy it. Inflammatory thickening of the muscular layer may embarrass the tube in passing the ovum toward the uterus. Endosalpingitis may destroy the cilia of the tubal epithelium, so that they cannot aid in the transmission of the ovum. Catarrhal salpingitis may cause temporary obstruction of the tube from swelling of the mucosa.

Endometritis may produce a pathologieal secretion so abundant and so hostile to the impregnated ovum as to prevent implantation - may even destroy it and sweep it out of the uterus; or, if imbedding take place, the ovum may be unable to survive the hostile environment.

Sterility eaused by gonorrhœal or syphilitie salpingitis and endometritis may be relative or absolute; more frequently the latter. This is, perhaps, a wise provision of nature to limit reproduction by persons having venereal disease.

Endocervieitis produces a cervical plug of gelatinons mueus that mechanically prevents the ingress of spermatozoa to the endometrium.

General metritis even more than endometritis renders the uterus unfit for gestation.

Exfoliative endometritis (membranous dysmenorrhœa) generally canses complete sterility.

Pelvie peritonitis may eause obstruetion of the tube by closure of the fimbriated extremity ; or may so draw it aside by adhesions that for meehanical reasons it may fail to transmit the ovum.

Parametritis, although apt to be associated with inflammations elsewhere, is not in itself a very significant canse of sterility.

Vaginitis may produce a hyperaeid secretion that is hostile to the spermatozoa, and may induce incomplete sterility by eicatricial stenosis, or by adhesions of the vaginal portion of the cervix to the vaginal fornix, or by eicatricial shortening of the vagina. Vaginitis and vulvovaginitis prevent coition or render it painful and imperfeet, and are therefore not infrequent eanses of incomplete sterility.

Painful caruncle of the urethra and kraurosis vulvæ are other causes of dyspareunia and consequent incomplete sterility. The 
presence of urine in the vagina from a vesicovaginal fistula would destroy spermatozoa, both by its own toxins and by the vaginitis that it would cause.

Atrophy of the uterus may be either concentric or excentric; both cause sterility, the former more positively than the latter. See Atrophy of the Uterus in chapter on Amenorrhœa. Curettage has been followed occasionally by atrophy of the uterus.

Tumors of the genital organs, by their mechanical effects or by their depressing systemic influence, may induce sterility ; but they more commonly do so by the inflammatory or other organic changes which they set up. The irritating presence of a uterine myoma causing endometritis, and complete destruction of ovarian tissue by the presence of an ovarian tumor, are familiar examples. A case has been reported of a woman who had given birth to twelve children, of whom the last was only three months old at the time of the removal of two dermoid ovaries. This case illustrates the fact that an ovarian cyst, although it may cause partial sterility, yet, unless it has destroyed the entire cortical substance of the ovary, does not cause complete sterility.

Displacements and stenosis of the uterus, especially flexions, are associated very commonly with incomplete sterility. It is highly probable that these lesions cause sterility rather from associated endometritis, salpingitis and perimetritis, than from any direct influence they exert per se.

The above statement is especially true of pathological anteflexion and stenosis of the os uteri externum. Spermatozoa will pass throngh very minute openings, but catarrhal conditions above may destroy them. In sounding the uterus for the diagnosis of atresia or stenosis, care should be taken lest the sound, by catching in a fold of cervical mucosa, make the canal appear imperforate. It is also important to observe whether the sound passes on by pushing aside an unrecognized valve-like flap of mucosa, which, if left undisturbed, might give rise to obstruction.

\section{Indeterminate Causes.}

In some cases the reproductive function has always been absent, or is suspended or lost without discoverable cause. These cases occupy an indefinite ground between absolute and contingent sterility. To say that there is imperfect co-ordination between important organs involved, or that, owing to incompatibility, the husband and wife do not act together efficiently, in nowise accounts for the failure. Absence of orgasm on the part of the woman has been offered in some cases as an explanation; but there is abundant proof that, although it may have some influence, orgasm is not essential to impregnation. 


\section{Diagnosis, Prognosis, and Treatment of Sterility.}

The diagnosis, prognosis, and treatment of the symptoms of sterility necessarily conform to the various diseases that give rise to it; and are set forth at length in the preceding chapters that treat of those diseases. To those chapters the student is referred.

The Diagnosis includes, first, a careful examination of the luusband. If the fault lies with him, as it frequently does, the case is not grnecological, and should not be made the occasion for examination or treatment of the wife.

In examining the woman for sterility, it is usually necessary to make not only a careful conjoined examination of all the reproductive organs, but as well of the systemic condition. The local examination will include generally accurate measurements with the sound of the length and diameter of the uterine canal.

Prognosis.-Disease associated with sterility may offer a favorable prognosis for anatomical cure, and even for the relief of pain and other annoying symptoms; such a result, however, not always is associated with successful pregnancy. The prognosis of sterility, therefore, does not necessarily conform to that of the associated lesions.

Treatment. - The treatment is that of the associated lesions, and is in 110 respect modified by the fact that the object of treatment is impregnation. The indication will always be to secure an open genital trict, a normal state of the reproductive organs, and a good systemic condition. The treatment of sterility by mechanical aids to pregnancy. such as the transfer of seminal fluid from the vagina to the endometrium by injection inmediately after intercourse, has been tried; the method, however, is revolting and probably useless. 


\section{CHAPTER LV.}

\section{INCONTINENCE OF URINE IN WOMEN. ${ }^{1}$}

THIs discussion is confined to the in voluntary escape of urine through the urethra. Incontinence due to acquired urinary fistula or to any congenital defect of the urinary tract will not be considered here. Broadly speaking, from the point of view of the causative pathology, incontinence has been classified under two general forms, and these forms may occur singly or combined; they are:

1. Active incontinence.

2. Passive incontinence.

1. Active incontinence is due either to a hypertonic, constricted, or distended state of the bladder, which causes the urine to be involumtarily forced out through a urethra which under ordinary conditions would have adequate sphincteric power. The bladder acts to a variable extent as a reservoir, but the sphincter is inadequate to prevent involuntary escape of urine. Among the causes of this form of incontinence are abnormal urine, foreign bodies, tuberculosis, cicatrices, tumors of the bladder, cystitis, and other conditions which may give rise to such irritation and consequent contraction of the walls of the bladder as to overcome the sphincteric power of the urethra and to force out urine. Overflow of an enormously distended bladder or of a bladder very much contracted from cystitis and having thick, unyielding walls, would be an example of active incontinence. Further discussion of active incontinence does not fall within the scope of this chapter.

2. Passive incontinence is due to some sphincteric defect, such, for example, as traumatism or paralysis, which deprives the neck of the bladder and urethra of the power of retention. Complete paralysis of the sphincter which causes urine to escape involuntarily as fast as it enters the bladder, which is apt to be of spinal origin and consequently associated with paralysis of other parts, such as the bowel, bladder, and lower extremities. This form of incontinence also will be excluded from further discussion.

The present purpose is to consider the surgical treatment of another and frequent variety of passive incontinence common is! multiparous women, and usually due to the traumatisms or other results of parturition. This special form of incontinence is always characterized by a very appreciable sagging away of the urethra and neck of the bladder from the pubes, and by a dilated sacculated appearance of the urethra. This separation of the urethra from the pubes is often

\footnotetext{
1 President's address, American Gynecological Society, 1905. Journal American Medical
} Association, June 3, 1905 . 
caused by a crushing or tearing of it from the symphysis during parturition. The condition ordinarily is one of urethrocele and commonly is associated with nore or less cystocele, laceration, and relaxation of the perineum, and subinvolution of the whole vaginal outlet. The loss of urine is not constant, but is occasional or frequent, and occurs as a result of coughing, sneezing, sudden change of position, fright, or from some unassignable, perhaps neurotic, condition. The bladder is neither distended, as in cases of dribbling from overflow of urine, nor constantly empty, as in passive incontinence from paralysis of the sphincter, but normally holds more or less urine which the patient can pass or retain at will. The condition will be recognized as one of frequent occurrence, great embarrassment to the patient, and difficulty of cure.

\section{Methods of Treatment.}

In the literature up to the present time, numerous operations and procedures have been put forward for the relief of this form of incontinence; excluding those not specially pertinent to the subject, they are:

1. Injection of paraffin into the region of the urethra.

2. Massage and electricity.

3. Torsion of the urethra, after Gersuny.

4. Advancing of the urethra, after the method of Pawlik, Hummelfarb, Albarran, and others. ${ }^{1}$

1. Injection of paraffin, even if it gave good results, which it does not, would be prohibited because of danger to life from pulmonary embolism.

2. Massage and electricity, although sometimes temporarily effective, seldom give permanent results.

3. Torsion of the urethra, after the method of Gersuny, which requires the urethra to be dissected loose from its surroundings throughout its length, then twisted on itself from $180^{\circ}$ to $450^{\circ}$ and sutured in place, is apt to overcome incontinence, but is prohibited on account of danger from sloughing of the urethra.

4. The advancing of the urethra, after Pawlik's method, consists of two lateral denudations, one on either side of the urethra, and suture of the denuded surfaces in such a manner as to cause the urethra to be stretched laterally and to be drawn up toward the mons veneris. The operation is performed, according to Pawlik, in two parts, the wonnd on one side bcing allowed to unite firmly before that on the other side is made. This operation is sound in principle-that is, advancement of the urethra-but the results too often have not been permanent. Pawlik has proposed another operation in some respects similar to the one which I shall describe later, but so far as I can learn he confines the application of it to cases of congenital epispadias and congenital shortening of the urethra. Hummelfarb lengthens the urethra by union of the labia between the meatis and the clitoris in order that retention may be secured by raising the meatus above the

\footnotetext{
1 The significant literature on the subject dates back but a few years and is rather meagre. $I$ am indebted to Dr. Heliodor Schiller for numerous references to articles, mostly German, which during the past thirty years have appeared in the periodical literature, many of which i have consulted.
} 
Figure 429.

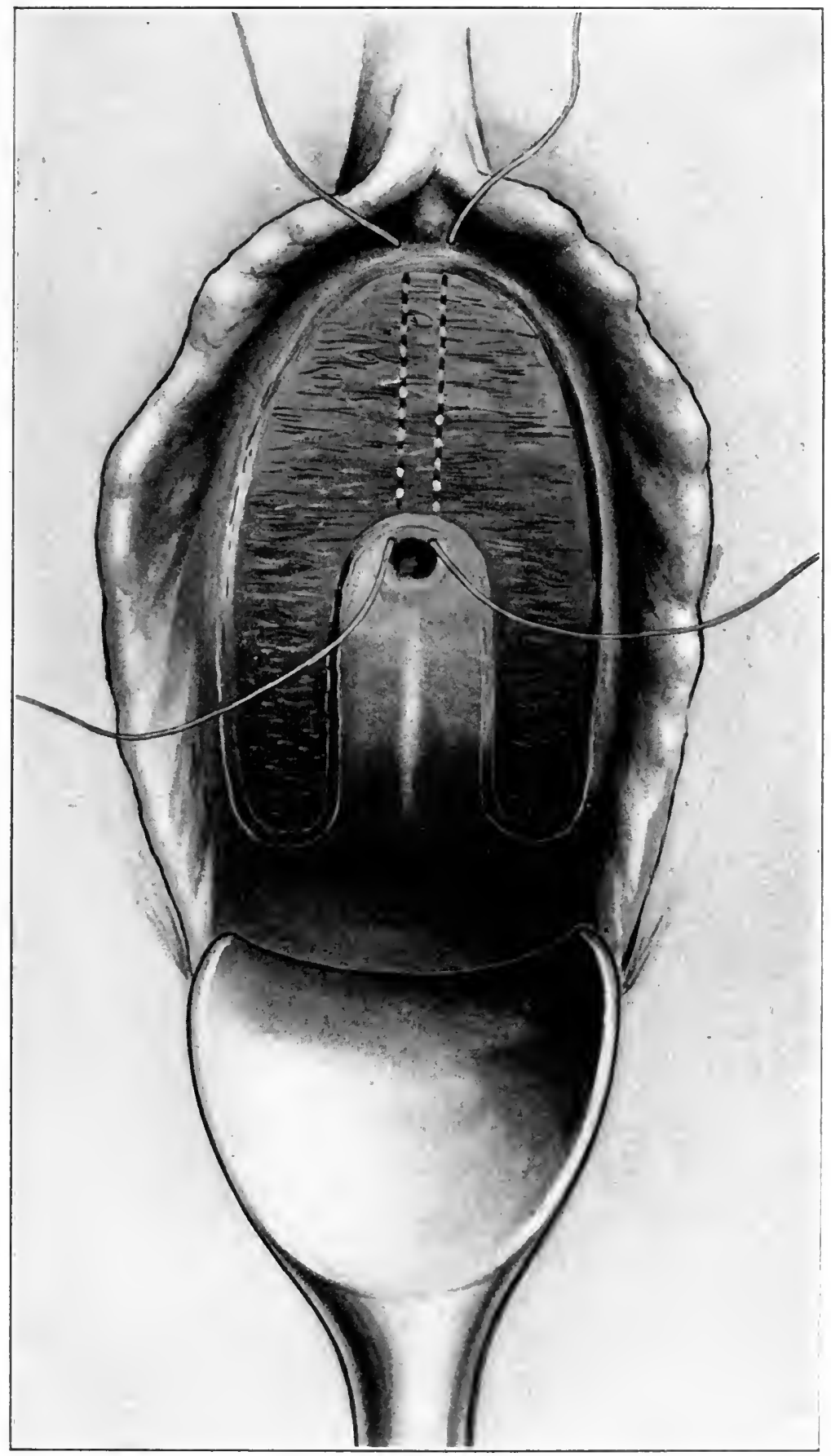

Operation for incontinence of urine; showing denuded surfaces and the first two sutures in place, but not tied. 


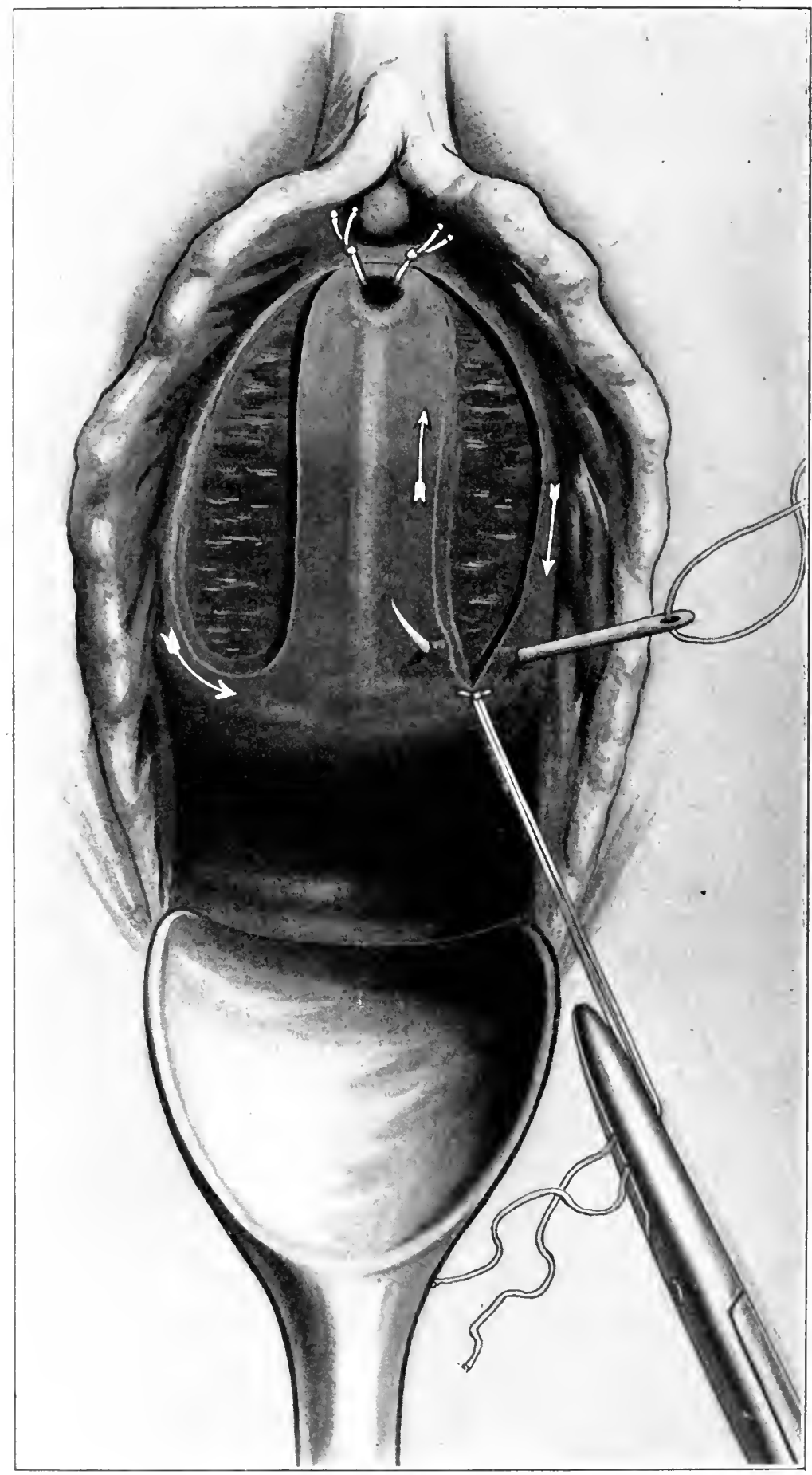

First two sutures tied and meatus urinarius drawn up to the elitoris and fastened there. The arrows leere show the margins of the lateral purts of the wonnd so rotated as to make the sutures loold the urethra firmly in its newly sequired elevated position. 


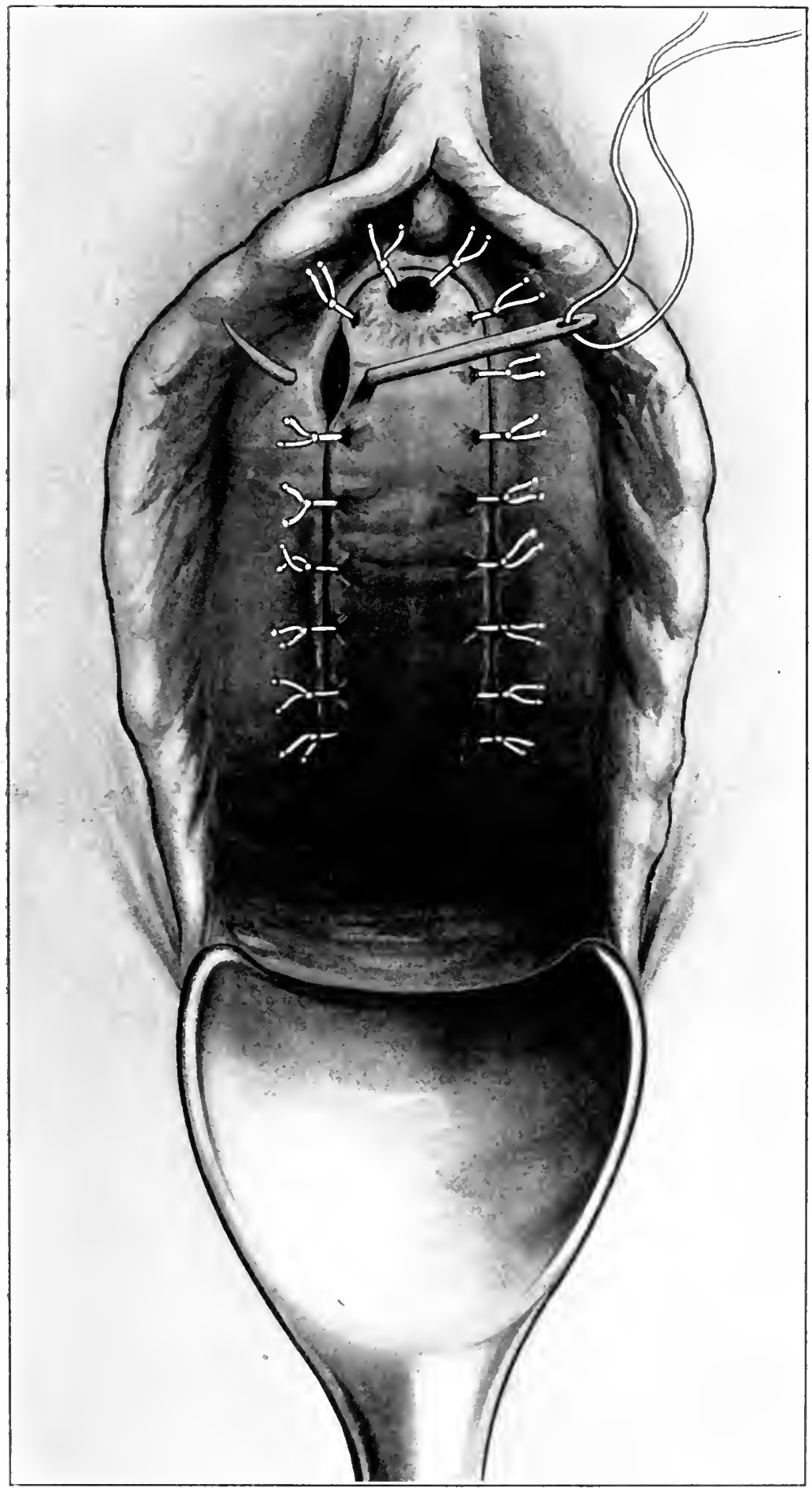

Last suture leing introduced to complete the operation. The previonsly saguing nrethra

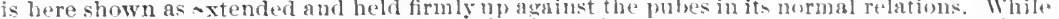
the lateral sutnres are being passef it is well to have the meatus stromgly pullect up toward the mons veneris ly means of a tenaenlum hooked into it and held in the hand of an assistant. 
level of the urine in the bladder. The virethra is then expected to act on the principle of the spout of a tea-kettle. This method, earlier described by Emmet, in a majority of cases fails on account of the activity of the contracting bladder foreing out urine (active incontinence) regardless of the level of the outlet. Albarran's operation consists of dissceting free the urethra, in making an incision from the meatus to the clitoris, in forming two flaps, in raising the urethra to the clitoris, and in suturing the flaps over it. This operation is effective unless, as sometimes oecurs, freeing of the urethra from its surroundings gives rise to slonghing.

\section{Proposed Operation.}

The operation here described is based on the same principle as that proposed by Albarran-that is, advancement of the meatus urinarius to the elitoris, but this is done without dissecting the urethra free and, therefore, it obviates the danger of sloughing of the urethra; it is performed as follows in two steps:

First Step. - A horseshoe-shaped surface is rather deeply denuded between the meatus urinarius and the elitoris, and to either side of the urethra throughout the entire length of it, as shown in Figure 429.

Second Step. - The meatus is drawn up to a point near the clitoris and is secured there by means of two sutures (Figures 429 and 430). The lateral portions of the denuded surface are now closed, as shown in Figure 431. The effect of the operation is to replace and to retain in its functional relations the sagging displaced urethra. It will be observed that the two first sutures necessarily stretch the urethra upward to the region of the clitoris and that the lateral sutures must tend to hold it in its new position. By this means it is proposed to straighten ont the urethrocele, by longitudinal traction, and by lateral traction to collapse and to hold together the dilated walls of the urethra, and thus to overeome the saeculation at the neck of the bladder where residual urine is apt to accumulate and give rise to trigonitis, eystitis, and possible incontinenee.

In many cases it will be neeessary to combine with the operation some appropriate surgical treatment for an associated cystocele, and in nearly all eases to perform perineorrhaphy to relieve, also, relaxation of the posterior vaginal outlet. In one case I saw fit to narrow the ponching urethra in order to overcome the urethral dilatation.

I have performed this operation many times and with almost uniformly gratifying results. In one case there was so much relaxation of the skin and other soft parts in the region of the elitoris that $I$ feared the sutured urethra might pull these structures down to the old malposition instead of being held up by them, but so far it remains well in place. In snch a case again, or in the event of recurrence from such a cause, however, I should be disposed to make a deep oval denudation over the pubes on the mons veneris just above the clitoris, the longer axis of the oval being directed transversely, and to unite the margins of this wound by a transverse line of union so as to make the clitoris a fixed point on a sufficiently high plane to hold the urethra 
taut. For obvious reasons, this procedure would be preferable to the removal of the elitoris and the union of the meatus to the parts thereby exposed.

The operation is so simple that I have been rather surprised not to find myself precisely antieipated in every partienlar, but so far as I have been able to learn, the operation, in some essential respects at least, is novel. If it should be found, however, that I am duplicating the work of another, I desire to make due acknowledgment in advanee. 



\section{INDEX.}

\section{A.}

Abdominal drainage, 138

comparison of results of, 138

evil results of, 139

fecal fistula, 139

hernia, 139

iodoform poisoning, 139

obstruction of bowel, 139

vesical complications, 139

forms of capillary, 142

postular, 145

tubular, 142

vaginal, 145

hysterectomy for hæmatometra, 535 radical, in carcinoma of uterus, 423

hysteromyomectomy, complete, for myoma of uterus, 394

hysterorrhaphy, 702

condition of successful, 705

impediments to, 703

nomenclature of, 703

technique of, 706

incision in ovariotomy, 470

myomectomy for myoma of uterus,

pregnancy, 486

$$
386
$$

drainage in, 388

section, 40,120

adhesions in, 124

closure of wound in, 126

curettage in, 47

buried sutures in, 126

dressings and bandages in, 134

incision in, 122

exploratory, 122

median, through linea alba, 122

intraperitoneal hæmostasis in, 124 sutures in, 126

Kelly's pad in, 48 substitute for, 48

for pelvic inflammation, 291 advantages of, 328 complications of, 294 Dudley's for, 292

preparation for, 40 operation of assistants, 40
Abdominal section, preparation for, of bowels, 45

cleansing of external pudenda, 47

of vagina, 47

by drinking of water, 45

of hands, 40

of operator, 40

preparatory treatment of, 122 sponges in, 129

stitch-hole abscesses in, prevention of, 46

Trendelenburg's position in, 120

substitute for, 122

wound of, suppuration of, 46

wall, fat in, differentiated from ovarian cyst, 462

Abortion, incomplete, differentiated from carcinoma of uterus, 417

from myoma of uterus, 375

in tubal pregnancy, 490

Abscesses, stitch-hole, prevention of, in abdominal section, 46,158

suburethral, complicating urethritis, 339

vulvovaginal, 187 treatment of, 339

Accessory tubes and ostia, 513

uterus, 514

Acid, carbolic, as an antiseptic agent, 36

oxalic, as an antiseptic agent, 35, 41

Adenocarcinoma of vulva, 361

diagnosis of, 361

treatment of, 362

Adenomatous cyst of ovary, 441

Adenomyoma of Fallopian tubes, 481

Adhesions, management of, 296

Adhesive peritonitis, 283

Alcohol as an antiscptic agent, 35

Alexander's operation, 691

after-treatment of, 699

anchoring of ligaments in, 696

contraindications for, 692

drawing out the ligament in, 695

finding the ligament in, 694

incision for, 693

indications for, 692

landmarks for, 693

limited scope of, 699

preparatory treatment for, 692

for prolapse of uterus, 666

steps in, 693 
Allantois, development of, 505

Amann on invagination of uterine glands, 226

Amenorrhœa, 745

causes of, general, 746 local, 745

classification of, 745

diagnosis of, 746

etiology of, 745

pathological, 23

physiological, 23

prognosis of, 746

symptoms of, 746

treatment of, 747

local, 747

systemic, 747

Amnion, formation of, in tubal pregnancy, 487

Ampullar pregnancy, 490

Anæsthesia in examination, 64

Androgyny, 527

Anovaginal fistula, 626

Anteflexion of uterus, 712

acquired, 713

classification of, 713

complications of, 714

congenital, 713

course of, 714

developmental, 713

diagnosis of, 716

etiology of, 713

normal, 712

pathological, 712

pathology of, 713

symptoms of, 714

urethral, 714

uterine, 715

dysmenorrhœa, 716

endometritis, 716

vesical, 714 sterility, 716

treatment of, 717

of complications, 717

by division of cervix uteri, 723

by Dudley's operation, 723 by electricity, 719

by forcible dilatation, 719

by local massage, 719

mechanical indications for, 718

by pessaries, 718

by supposit ories of opium, 720

Antelocation of uterus, 636

diagnosis of, 636

symptoms of, 636

treatment of, 636

Anteversion of uterus, 710

acquired, 713

congenital, 710

diagnosis of, 711

etiology of, 710

prognosis of, 711

symptoms of, 710

treatment of, 711
Antiseptic agents, 35

alcohol, 35

carbolic acid, 36

formalin, 35

heat, 35

mercuric bichloride, 36

oxalic acid, 41

potassium permanganate, 35

soap, 36

sodium carbonate, 36

sulphuric ether, 35

Antiseptics, prophylactic use of, 35

therapeutic use of, 35

Anus, atresia of, 521

eversion of, in digital examination, 60

examination of, 78

proctoscope in, 78

sigmoidoscope in 78

malformation of, 520

Approximation in trachelorrhaphy, 583

Arteriosclerosis of chronic metritis, 254

Ascent of uterus, 635

diagnosis of, 636

symptoms of, 636

treatment of, 636

Ascites, differentiated from ovarian cysts, 466

Asepsis in minor manipulations, hands, 49

instruments, 49
patient, 49

Aseptic technique, 34

vaginal operations, preparation for, 48

Aspiration, 77

Atresia of anus, 521

of urethra, 521

of vagina, 521

inflammatory, 519

Atrophic cellulitis, chronic, 262

metritis, 252

non-puerperal, 254

puerperal, 253

Auto-infection, 170

B.

BacılLus aërogenes capsulatus, 33

coli communis, 33

diphtheriæ, 33

in acute metritis, 208

pyocyaneus, 33

tuberculosis, 33

typhosus, 33

Bartholin's glands, development of, 510

Bed-pan, substitute for, 92

Bicornate uterus, 514

Bladder, care of, after abdominal operations, 149

curettage of, in treatment of cystitis, 354

distended, differentiated from ovarian cysts, 462

phantom, 88

stone in, in vesicovaginal fistula, 598 
Bladder, tumors of, 485

Bloodvessels, infection by, 169

Bougies, 75

graduated, in dilatation of uterus, 112

Bovée on paravaginal hysterectomy, 423

Bowels, care of, after abdominal operations, 149

obstruction of, from abdominal drainage, 139

after abdominal operations, 155 causes of, 155

diagnosis of, 156

prognosis of, 156

treatment of, 156

preparation of, for abdominal section, 45

Bowman, capsule of, development of, 505

Brandt's method in retroversion of uterus, 676

Broad ligament, cysts of, 449

end-to-end approximation of, 392,408

tumors of, 482

Brown's method of treating inversion of uterus, 741

Buboes in vulvitis, 180

Bulbocavernous muscle, 543

Byrne on ignilhysterectomy, 426

\section{C.}

Cachexia in carcinoma of uterus, 413

Canal of Nuck, development of, 509

Capillary drainage, 142

Capsule of Bowman, development of, 505

Carbolic acid as an antiseptic agent, 36

Carcinoma of cervix uteri, diagnosis of, 415

of corpus uteri, diagnosis of, 415

of endometrium, 235

of Fallopian tubes, 481

of ovary, 436

of round ligament, 483

of urethra, 484

of uterus, 411

adenocarcinoma, 411

cause of death from, 419

course of, 412

cylindrical cell, 411

diagnosis of, 414

differential, 416

from chronic metritis, 417

from endocervicitis, 417

from endometritis, 417

from hypertroplyy of cervix, 417

from ichthyosis uteri, 417

from incomplete abortion, 417

from laceration of cervix, 417

from myoma, 416
Carcinoma of uterus, diagnosis of, differential, from ovarian cysts, 464

from retained placenta, 416

from sarcoma, 416

from syphilis, 417

from tuberculosis of uterus, 418

extension of, 419

recurrence after removal, 419

etiology of, 412

extension of, 411

gland, 411

pavement cell, 411

prognosis of, 419

symptoms of, 412

cachexia, 414

hemorrhage, 413

pain, 413

utcrine discharge, 413

visceral disorders, 413

treatment of, 420

hysterectomy in, 421

ignihysterectomy, 425 mortality of, 428 operation of elcction, 428

paravaginal, 422

radical abdominal, 423

recurrence of carcinoma after, 428

of vagina, 365 palliative, 429

treatment of, 365

of vulva, 361

cylindrical-cell, 361

treatment of, 362

pavement-cell, 361

diagnosis of, 361

Caruncle of urethra, 334

Case records, form of, 52

Casper's cystoscope, 86

Catarrhal salpingitis, 273

Catgut, sterilization of, 43

by chromic acid process, 44

by dry heat process, 44

Cellular tissue, anatomy of, 260

Cellulitis, chronic atrophic, 262

diffuse, of Pozzi, 261

pelvic, 260

acute metritis and, 209

anatomy of, 260

diagnosis of, 263

differential, 265

etiology of, 260

pathological anatomy of, 261

pathology of, 261

prognosis of, 266

symptoms of, 263

consistence of, 265

form of, 264

immobility of, 265

location of, 264 
Cellulitis, pelvic, symptoms of, pain in, Cervix uteri, laceration of, treatment of, 265

relations of, 265

treatment of, 266

of Stopfer, 263

Cervical epithelium, mucous patch of, 219

myoma of uterus, 372

Eervix uteri, carcinoma of, diagnosis of, 415

division of, for anteflexion of uterus, 723

erosions of, 219

papillary, 219

simple, 219

glands of, 203

glandular enlargements of, 219 cystic, 220

polypoid, 220

hypertrophy of, differentiated from carcinoma of uterus, 417

laceration of, 565

causes of, 565

diagnosis of, 576

differential, 579

from carcinoma, 418

false cervix in, 567

apparent hypertrophy and elongation of, 572

cystic degeneration in, 571

descent and vaginal reduplication in, 569

pathological anatomy of, 572

subinvolution in, 568

pathological anatomy of, 566

prophylaxis of, 579

results of, 566

symptoms of, 574

treatment of, $\mathbf{5 7 9}$

atypical lacerations, 587

resection of cervix, 587

trachelorrhaphy in, 579

after-treatment of, 592

approximation in, 583

curettage in, 582

denudation in, 583

disinfection in, 582

immediate operation, 580

instruments for, 581 trachelorrhaphy in, hemorrhage in, $58 \overline{5}$

preliminary dilatation, 582

preparatory treatment of, 580

removal of cicatricial plug in, 584

results of, 592

secondary operation in, 580

sutures in, 585

mucous polypi of, 218

ulceration of, chronic endocervicitis and, 221

vaginal portion of, injuries of, 537

Chancroidal vulvovaginitis, 186

treatment of, 186

Chloroform in minor operations, 100

Chorio-epithelioma of uterus, 434

diagnosis of, 434

etiology of, 434

pathology of, 434

prognosis of, 435

symptoms of, 434

treatment of, 435

Chorion, formation of, in tubal pregnancy, 487

Cirrhotic metritis, 252

Clarke, J. S., on flushing of abdominal cavity, 140

on radical abdominal hysterectomy, 423

Clitoris, development of, 510

malformations of, 524

Cloaca, persistent, 522

Cocaine in minor operations, 100

Colpeurynter in treatment of inversion of uterus, 739

Condyloma of vulva. See Papilloma of vulva.

Congenital anteflexion of uterus, 713 anteversion of uterus, 710

displacement of ovary, 512

gynatresia, 530

diagnosis of, 531

pathological results of, 530

prognosis of, 534

symptoms of, 530

treatment of, 534

hypertrophy of ovaries, 512

retroflexion of uterus, 709

retroversion of uterus, 709

Conjoined examination, 60

rectal, 62

rectovaginal, 64

traction as an aid to, 64

with sound, 65

Constrictor vaginæ muscle, 543

Corpus luteum cysts, $\mathbf{4 4 0}$

uteri, carcinoma of, diagnosis of, 415 
Corpus uteri, glands of, 202

Credé's ointment, 155

Cullen on radical abdominal hysterectomy, 423

Curettage of bladder in treatment of cystitis, 354

in chronic endocervicitis, 222 endometritis, 243

diagnostic, 77

in minor operations, 115 technique of, 117

in myoma of uterus, 382

in trachelorrhaphy, 582

Curettes, dull, 118

obstetric, 118

placental, 118

Recamier's, 118

sharp, 118

Thomas', 118

Cystic glandular enlargements of cervix uteri, 219

Cystitis, 340

causes of, 340

exciting, 341

predisposing, 340

classification of 343

anatomical, 343

bacteriological, 344

clinical, 345

pathological, 344

diagnosis of, 342

differential, 343

etiology of, 340

exfoliative, 346

etiology of, 346

symptoms of, 346

exudative, 346

fissure, 347

foreign-body, 347

gonorrhœeal, 343

instrumentation in, 341

leucoplakia, 348

pathology of, 342

superficial, 345

suppurative, 345

treatment of, 348

medical, 349

prophylactic, 348

summary of, 354

surgical, 351

curettage of bladder, 354

dilatation of urethra, 352

extravesical operations in, 354

lithotomy, 354

lithotrity, 354

vaginal cystotomy, 352 topical, 350

tubercular, 343

ulcerative, 345

vesicovaginal fistula a cause of, 595

Cystocele, 545

in lacerations of perineum, 545

Cystoscope, cylindrical, 80

electrical, 85

Kelly's, 80
Cystoscope, use of, 85

Cystotomy, vaginal, in cystitis, 352

Cysts, adenomatous, of ovary, 441

of broad ligament, 449

examination of, 68

of Fallopian tubes, 467

hydatid, differentiated from ovarian cysts, 467

intraligamentous, of ovary, 479

ovarian, 438

corpus luteum cysts, 440

cystadenomata, 441

papillary, 442

simple, 441

dermoid, 444

complicated, $\$ 45$

simple, 444

diagnosis of, 456

adhesions, 458

differential, 459

from ascites, 466

from dilated stomach, 462

from distended bladder, 462

from fat in abdominal wall, 462

from fecal accumulation, 462

from gestation in one horn of bifurcated uterus, 464

from hæmatometra. 465

from hydatid cysts, 467

from hydrometra, 467

from metritis, 467

from normal gestation, 463

from phantom tumors, 462

from physometra, 465

from pyometra, 465

from renal tumors, 468

from tubal pregnancy, 463

from tympanites, 462

from uterine carcinoma, 464

sarcoma, 464

of malignancy, 458

distinction between parovarian cyst and, 459

exploratory incision in, 459

follicular, 439

formation of pedicle of, 446 mode of development of, 438 multilocular, 438

prognosis of, 458

removal of, 460

abdominal incision in, 460

accidents in, 479

after-treatment of, 479

closure of wound after, 479

complications of, 479 
Cysts, ovarian, removal of, drainage in, 479

emptying and delivery of cyst in, 472

ligature of pedicle in, 479 secondary changes in, 451 infection, 451 adhesion, 452 suppuration, 452 rupture of, 453 causes of, 454 diagnosis of, 454 prognosis of, 454 results of, 454 symptoms of, 454 treatment of, 455 twisting of pedicle, 452 diagnosis of, 453 etiology of, 453 pathology of, 453 prognosis of, 453

symptomatology of, 455 treatment of, 454

parovarian, 428, 436. See also Cysts, ovarian.

contents of, 448

distinction between ovarian cysts and, 449

pelvic, differentiated from myoma of uterus, 378

of round ligament, 482 diagnosis of, 482

tubo-ovarian, 450 treatment of, 482

urethral, 187

of vagina, 364 treatment of, 365

of vulva, 363

\section{D.}

Decidua, formation of, in tubal pregnancy, 487

Decidual endometritis, 234

Deciduoma adenomatoma, 434 carcinomatosum, 434

malignum, 434

diagnosis of, 434

etiology of, 434

prognosis of, 435

symptoms of, 434

treatment of, 435

sarcomatosum, 434

De Lee on treatment of endometritis, 213

Denudation in plastic operations, 106

in trachelorrhaphy, 583

in vesicovaginal fistula, 603

Dermoid cysts of ovary, 444

complicated, 445

simple, 444

Descent of uterus, 637

course of, 645

diagnosis of, 645

differential, 645

etiology and mechanism of, 637
Descent of uterus, pathology of, 643

prophylaxis of, 646

symptoms of, 645

treatment of, 647

change in direction of vagina in, 650

by elytrorrhaphy, 651

general, 647

hygiene, 647

hysterectomy in, 650

lıysterorrhaphy in, 666

local, 647

narrowing of vagina in, 650

perineorrhaphy in, 656

by pessaries, 648

by plastic operations, 650

Diagnostic curettage, 77

Digital examination, 58

conduct of, 59

eversion of anus in, 60

left-handed method, 58

lightness of, 58

Dilatation of uterus, 77, 109

diverging instruments in, 112

forcible, technique of, 115

graduated bougies in, 77,112

incision in, 110

Dudley's, 111

Schroeder's method, 110

special advantages of each method, 114

by steel instruments, 77

by tents, 77,111

by water dilators, 77

Dilators, uterine, 77

Diphtheria bacillus in acute metritis, 208

Diphtheritic metritis, 209

vulvovaginitis, 183

treatment of, 183

Displacement, congenital, of ovary, 512 of uterus, 627

definition of, 631

diagnosis of, 631

general consideration of, 627

nomenclature of, 631

symptoms of, 631

Döderlein on lactic acid bacteria in vaginitis, 178

Douclie, action of, 93

as cleansing agent, 93

as vascular stimulant, 93

application of, 92

contraindications for, 93

indication for, 93

Drainage, abdominal, 138

comparison of results of, 138

.evil results of, 139

fecal fistula, 139

hernia, 139

iodoform poisoning, 139

obstruction of bowel, 139

vesical complications, 139 
Drainage; forms of, 142 abdominal, 145 capillary, 142 pustular, 145 tubular, 142 vaginal, 145

peritoneal, contraindications for, 142 indications for, 142

to prevent infection, 139

tubes, glass, 144

Reid's, 143

rubber, 144

Dress, relation of, to diseases of women, 159

Dressings, antiseptic, 44

aseptic, 44

sterilization of, 43

Ducts of Mueller, development of, 507

Dudley's clamp operation for ureterovaginal fistula, 109

incision in dilatation of uterus, 111

on end-to-end approximation of broad ligaments, 392

instrument case, 38

operation for anteflexion of uterus, 723

for double uterus, 514

for oöphorectomy, 291

sterilizer, 38

Dysmenorrhcea, 756

associated lesions of, 757

classification of, 756

clinical history of, 756

due to anteflexion of uterus, 716

treatment of, 760

\section{E.}

Ectodermal layer, 434

Eczema of vulva, 192 treatment of, 192

Edebohl's enteroptosis complicating descent of uterus, 666

Eleplantiasis of vulva, 358 diagnosis of, 358 treatment of, 359

Elytrorrhaphy in treatment of descent of uterus, 651

contraindications, 655

Einbryonic structures, development of, into organs, 508

Emmet's button-hole operation, 335

method of treatment in inversion of uterus, 738

operation for prolapse of urethra, 338

pessary, 688

scissors, 101

Emphysematous vaginitis, 190 treatment of, 191

Enchondroma of vulva, 364

Endocervicitis, chronic, 217 diagnosis of, 220

digital examination in, 220
Endocervicitis, chronic, diagnosis of, examinations of secretions in, 221

sight examination in, 220

speculum examination in, 221

etiology of, 217

pathology of, 218

sequence of, 218

symptoms of, 220

treatment of, 221

by curettage, 222

Schroeder's operation in, 222

differentiated from carcinoma of uterus, 417

polypoid, 224

Endometritis, chronic, 226

classification of, 226

clinical, 231

diagnosis of, 236

differential, 236

dysmenorrhœe and, 757

etiology of, 226

histological forms of, 228

pathology of, 226

prognosis of, 237

symptoms of, 234

abortion, 234

hypersecretion, 234

pain, 234

reflex disorders, 235

sterility, 234

systemic disorders, 235

treatment of, 238

surgical, 243

curettage in, 243

indications for, 243

regeneration of endometri u m after, 248

technique of, 244

systemic, 238

anæmia in, 239

constipation in, 239

general hygiene in, 240

kidneys in, 239

topical, 240

intra-uterine tampon in, 242

decidual, 234

differentiated from carcinoma of uterus, 417

due to anteflexion of uterus, 715

exfoliative, 232

glandular, 228

hyperplastic, 229

hypertrophic, 228

interstitial and, 230

hemorrhagic, 229

interstitial, 229

post-abortum, 232

senile, 232

treatment of, 214

tubercular, 233 
Endometrium, 202

applications to, 247 of carbolic acid, 247 of formalin, 247 of iodine, 247

carcinoma of, 235

mucous polypi of, 230

regeneration of, after curettage, 248 sarcoma of, 237

Endosalpinx, 269

Endothelioma of uterus, 430 diagnosis of, 430 pathology of, 430 treatment of, 430

End-to-end approximation of broad ligaments, 39:2, 408

Enteroptosis complicating descent of uterus, 666

Enucleation of myoma of uterus, 384

Epididymis, development of, 509

Episiotomy, 541

Epispadias, 524

Epoöphoron, development of, 509

Erosions of cervix uteri, 219

$$
\text { papillary, } 219
$$$$
\text { simple, } 219
$$

Erysipelas malignum internum of Virchow, 261

Erysipelatous vulvovaginitis, 182 erythematous, 182 gangrenous, 182 treatment of, 183 vesicular, 182

Ether in minor operations, 100

Eversion of anus in digital examination, 60

Examination, anæsthesia in, 64 of anus, 78

proctoscope in, 78 sigmoidoscope in, 78

of ascites, 69

by auscultation, 66

conjoined, 60

rectal, 62

rectovaginal, 64

traction as an aid to, 64

vaginal, 60

with sound, 65

of cysts, 68

by digital touch, 58

conduct of, 59

eversion of anus in, 60

left-liand method, 58

lightness of, 58

exploratory incision in, 90

by inspection, 57

instrumental, 68

diagnostic curettage, 77

exploratory needle and aspiracor, 77

probe in, 75

sound in, 75

dangers of, 76

speculum in, 68

Simon's, 73

sims', 69
Examination, instrumental, speculum in, Sims' self-retaining, 70 uterine dilatation, 77

by palpation, 66

by percussion, 66

physical, 56

bladder in, 56

cleanliness in, 56

position of patient in, 57

rectum in, 56

table for, 56

of young girls, 57

of rectum, 78

proctoscope in, 78

sigmoidoscope in, 78

of urinary organs, 78

catheterization in, 80

cystoscopy in, 80 comparison of, 86

cylindrical, 80

electrical, 85

value of, 86

dorsal position in, 81

inspection in, 78

knee-breast position in, 83

palpation in, 78

percussion in, 78

segregation in, 89

urethral exploration in, 80

ur ethroscopy in, 80

urinalysis in, 78

of uterine tumors, 67

Excretory organs, embryology of, 503

Exfoliative cystitis, 346

etiology of, 346

symptoms of, 346

endometritis, 232

Extra-uterine pregnancy. See Tubal pregnancy.

Exudative cystitis, 346

peritonitis, 283

\section{F.}

FACIEs ovariana, 455

Fallopian tubes, conservative operation on, 330

development of, 506

inflammation of, 268

malformation of, 513

senile changes in, 29

tumors of, 481

Fecal accumulation differentiated from ovarian cyst, 462

fistula from abdominal drainage, 139,157

Fibroma of ovary, 436

of round ligament, 482

diagnosis of, 482

treatment of , 482

Fibromyoma of vagina, 365

of vulva, 363

treatment of, 363

Fissure cystitis, 347

Fistula, anovaginal, 626 
Fistula, fecal, after abdominal operations, 157 genital, priority in operation for, 593 varieties of, 594

rectovaginal, 625

causes of, 625

diagnosis of, 625

prognosis of, 625

treatment of, 625

ureterovaginal, 618

causes of, 618

diagnosis of, 618

treatment of, 618

Dudley clamp operation in, 619

urethrovaginal, 618

vesico-uterine, 617

diagnosis of, 617

treatment of, 617

vesico-uterovaginal, 593, 594

vesicovaginal, 594

cause of cystitis, $\mathbf{5 9 5}$

course of, 595

diagnosis of, 595

etiology of, 594

prognosis of, 595

symptoms of, 595

treatment of, prophylactic, 596 surgical, 597

atypical operations, 609

kolpokleisis, 611

loss of entire vesico-

vaginal septum, 609

operations for closing, 601

after-treatment of, 608

application of sutures in, 606

choice of speculum, 602

direction of line of union in, 603

denudation in, 603

method of operation, 602

preparatory treatment, 602

preparatory, 597

cystitis in, 598

direction closure, 598

phosphatic posits in, 597

stone in bladder in, 598

Flemming's operation for artificial vagina, 535

Flexion of uterus, 709

Fotus, calcareous degeneration of, 493 mummified, 493

Follicular ovarian cysts, 439 vulvovaginitis, 189
Foreign bodies in uterus, a cause of hemorrhage, 752

Foreign-body cystitis, 347

Formalin as an antiseptic agent, 35

Fowler's position in abdominal drainage, 145

Freund on radical abdominal hysterectomy, 423

Furuncular vulvitis, 190 treatment of, 190

Furunculosis, 190

treatment of, by yeast, 190

\section{G.}

Gaertner's duct, 446 development of, 505

Garrigues on diphtheritic metritis, 209 on dissecting metritis, 209

Gauze, sterilization of, $\mathbf{4 5}$

Gellhorn on paravaginal hysterectomy, 422

Generative organs, embryology of, 507

Genital fistulæ, priority in operation for, 593

varieties of, 594

glands, development of, 505

ridge, development of, 503, 508

Gestation, normal, differentiated from ovarian cysts, 463

in one horn of bifurcated uterus, differentiated from ovarian cysts, 464

Giraldès, organ of, development of, 509

Glands of cervix uteri, 203

of corpus uteri, 202

Nabothian, 219

Skene's, inflammation of, 334

uterine, invagination of, 226

Glandular endometritis, 228

hyperplastic, 229

hypertrophic, 228

interstitial and, 230

enlargements of cervix uteri, 219 cystic, 220 polypoid, 220

vulvovaginitis, 177,187 diagnosis of, 187 treatment of, 187

Gleet, 168

Goitre of puberty, 28

Gonococcus, 33

of Neisser, 167

Gonorrhœa, latent, 187

transmission of, 168

Gonorrhœal cystitis, 343

papilloma of vulva, 360

urethritis, 333

vaginitis in children, 181

vulvovaginitis, 181

diagnosis of, 181

treatment of, 181

warts of vulva, 360

Graafian follicles, anatomy of, 25

Gubernaculum of Hunter, development of, 507 
Gynandry, 527

Gynatresia, congenital, 530 diagnosis of, 531 pathological results of, 530 prognosis of, 534 symptoms of, 530 treatment of, 534

H.

Hæmatocele, pelvic, resulting from tubal pregnancy, 494

Нæmatocolpos, 530

operations for, 535

Hæmatoma of vulva, 358

Hæmatometra, differentiated from ovarian cysts, 465

operations for, 534

Hæmatosalpinx, 271, 530

Hair, sterilization of, 42

Harris, segregator of, 89

Heat as an antiseptic agent, 35

Hemorrhage in carcinoma of uterus, 413 in major operations, 151

diagnosis of, 151

treatment of, 151

in myoma of uterus, 373

in trachelorrhaphy, $\mathbf{5 8 5}$

uterine, 750

diagnosis of, 753

during maturity, 754 menopause, 754

etiology of, 750

of girls, 753

treatment of, 754 electrotherapeutic, 755

local, 755

surgical, 755

systemic, 754

Hemorrhagic endometritis, 229

Hermaphrodism, 527

treatment of, 528

Hernia from abdominal drainage, 139

inguinal, 188

of ovary, 742

diagnosis of, 742

treatment of, 742

of uterus, 640,742

diagnosis of, 742

treatment of, 742

ventral, after abdominal operations, 158

Herpes of vulva, 192

Hunter, gubernaculum of, development of, 507

Hydatid cysts, differentiated from ovarian cysts, 467

of Morgagni, development of, 510

Hydrocele, ovarian, 450

of round ligament, 482

differential diagnosis of, 482

treatment of, 482

Hydrometra, differentiated from ovarian cysts, 465
Hydrosalpinx, 271

aspiration of, through vagina, 324

Hydro-ureter, 355 technique of, 324

Hymen, malformation of, 520

Hyperæsthesia of vulva, 199 treatment of, 200

Hyperplastic glandular endometritis, 229

Hypertrophic glandular endometritis, 228

metritis, 251

non-puerperal, 251

puerperal, 251

Hypertrophy of cervix, differentiated

from carcinoma of uterus, 417

of clitoris, 524

congenital, of ovaries, 512

of prepuce, 526

Hypodermoclysis in major operations, 151

Hypospadias, 523

operative treatment of, 523 .

Hysterectomy, abdominal, for hæmatometra, 535

for procidentia, 650

in treatment of carcinoma of uterus, 421

ignihysterectomy, 425

paravaginal, 422

radical abdominal, 423

of inversion of uterus, 742

of pelvic inflammation, indications for, 305

objections to, 304

vaginal, of myoma of uterus, 383

Hysterical vomiting after abdominal operation, 155

treatment of, 155

Hysteromyomectomy, complete abdomi-

nal, for myoma of uterus, 394

I supravaginal, for myoma of uterus, 391

disinfection of vagina in, 391

Hysterorrhaphy, abdominal, 702

conditions of successful, 705

impediments to, 704

nomenclature of, 703

technique of, 706

for prolapse of uterus, 666

vaginal, 708

technique of, 708

\section{I.}

ICHTHYosis of uterus, differentiated from carcinoma, 417

Ignihysterectomy in carcinoma of uterus, 425

advantages of, 427

history and rationale of, 426

Incontinence of urine, 771

treatment of, 772

surgical, 776

Infantile uterus, 22,513

vulva, 524 
Infection, 32

by blood-vessels, 169

by continuity of surface, 169

forms of, 32

bacillus coli communis, 33

tuberculosis, 34

gonococcus, 33

pyæmia, 33

sapræmia, 33

septicæmia, 32

staphylococcus, 33

Streptococcus pyogenes, 33

toxæmia, 32

by lymph-vessels, 169

Inflammations, 165

classification of, 170

acute, 171

chronic, 171

course of, 169

definition of, 165

diagnosis of, 173

etiology of, 166

exciting causes, 167

favoring conditions, 166

of Fallopian tubes, 268

pathology of, 169

pelvic, 257

etiology of, 258

routes of infection, 257

by blood-vessels, 257

by continuity of mucosa, 257

from extrapelvic organs, 257

by lymph-vessels, 257

significance of, 258

prognosis of, 173

of Skene's glands, 333

treatment of, 173

of uterine appendages, 268

of uterus. See Metritis.

of vulvovaginal glands, 187

Inflammatory atresia of vagina, 519

Inguinal hernia, 188

Instrument cases, 37

combination, 38

pouches, 37

Dudley's, 38

trays, 37

Instrumental examination, 68

diagnostic curettage in, 77

exploratory needle and aspira-

tion in, 77

probes in, 75

dangers of, 76

sounds in, 75

dangers of, 76

speculum in, 68

Simon's, 73

Sims', 69

self-retaining, 70

uterine dilatation in, 77

pain, 761

Instruments, diverging, in dilatation of uterus, 112

sterilization of, 42

48
Interstitial endometritis, 229

metritis, 252

tubal pregnancy, 489

Intra-abdominal shortening of round ligament in retroflexion and retroversion of uterus, 700

Intraligamentous cysts of ovary, 479

Intramural myoma of uterus, 369

Inversion of uterus, 729

anatomy of, 732

diagnosis of, 733

etiology of, 729

meclianism of, 732

pathology of, 732

prognosis of, 735

symptoms of, 733

treatment of acute, 735

of chronic, 736

by colpeurynter, 739

by elastic pressure, 739

by hysterectomy, 742

by incision, 741

Brown's method, 741

manual, 738

Emmet's metliod, 738

Tate's method, 739

preparatory, 737

by spiral spray, $7+1$

White's method, 741

by water-bag, 739

varieties of, 735

Ischiorectal fascia, 543

Isthmic pregnancy, 490

K.

Kelly's cystoscope, 80

method of rectal examination, 52

pad in abdominal section, 48 substitute for, 48

Kidney, floating, differentiated from myoma of uterus, 379

glomeruli of, development of, 505

King on treatment of endometritis, 213

Kobelt's tubules, 446

Kolpokleisis for vesicovaginal fistula, 611

Kraurosis of vulva, 193

pathology of, 193

treatment of, 194

Longyear's operation, 194

Kroemer on paravaginal hysterectomy, 423

Kundrat on paravaginal hysterectomy, 423

\section{L.}

LABIA majora, developinent of, 510

minora, adhesions of, 177

Laceration of cervix uteri, 565

causes of, 565 
Laceration of cervix uteri, diagnosis of, | Leiter's cystoscope, 84

575

differential, 579

from carcinoma, 417

false cervix, 567

apparent hypertrophy and elongation of, 572

cystic degeneration in, 571

descent and vaginal reduplication, 569

pathological anatomy of, 572

subinvolution, 568

pathological anatomy of, 566

prophylaxis of, 579

results of, 566

symptoms of, 574

treatment of, 579

atypical lacerations, 587

resection of cervix, 587 trachelorrhaphy in, 579

after-treatment of, 592

approximation in, 583

curettage in, 582

denudation in, 583

disinfection in, 582

hemorrhage in, 585

immediate operation, 580

instruments for, 581

preliminary dilatation in, 582

preparatory, 580

removal of cicatricial plug in, 584

results of, 592

secondary operation, 580

of perineum, 540

sutures in, 585

complete, 541, 544

incomplete, 543

prevention of, 541

results of, 542

Lactic acid bacteria in vaginitis, 178

Langhans, layer of, 434

Lateral location of uterus, 636

diagnosis of, 636

symptoms of, 636

treatment of, 636
Leucoplakia cystitis, 348

Lipoma of vulva, 363 treatment of, 363

Lithopædion, 493

Lithotrity in treatment of cystitis, 354

Longyear's operation for kraurosis vulvæ, 194

Lord, F. H., douche of, 92

Lupus of vulva, 183,363

Lutein cells, 440

Lymphatics of uterus, 204

Lymph-vessels, infection by, 169

\section{M.}

MACKenRodT on paravaginal hysterectomy, 423

Major operations, 120

abdominal section, 120

adhesions in, 124

closure of wound in, 126

buried su-

tures in,

126

dressings and bandages in, 134

incision in, 122

exploratory, 122

median, through

linea alba, 122

intraperitoneal hæmostasis, 124

sutures in, 125

preparatory

ment of, 122

sponges in, 129

Trendelenburg position in, 120

substitute for, 122

after-treatment of, 148 complicated cases, 150 fecal fistula in, 157

hemorrhage in, 151

hysterical romiting, 155

obstruction of bowels, 155

removal of sutures in, 158

sepsis in, 153

general, 154

localized, 153

shock in, 150

stitch-abscess in, 158

ventral hernia in, 158

simple cases, 148

care of bladder, 149

bowels, 149 
Major operations, after-treatment of, Menstruation, precocious, 24 simple cases, premature, 743 care of cicatrix, 150

food in, 150

getting up in, 150

hot-water bags, 149

pain in, 150

rest, 148

drainage in, 138

thirst, 149

comparison of results of, 138

evil results of, 139

fecal fistula, 139

hernia, 139

obstruction of bowel, 139

vesical complica-

forms of, 142 tions, 139

abdominal, 145

capillary, 142

postural, 145

tubular, 142

vaginal, 147

peritoneal contraindications for, 142

indications for, 142 to prevent infection,

in sacral resection, 137

in vaginal section, 136

Malformations of anus, 520

of clitoris, 524

of Fallopian tubes, 513

of hymen, 520

of nymphæ, 524

of ovaries, 511

of prepuce, 524

of uterus, 513

of vagina, 518

of vulva, 520

Mal-locations of uterus, 635

Marion-Sims operation for genital fistula, 593

Maturity, period of, 28

uterine hemorrhage during, 754

Menopause, basis of, 29

phenomena of, 30

symptoms of, 30

abnormal, 30

normal, 30

uterine hemorrhage during, 754

Menorrhagia. See Uterine hemorrhage.

Nenstrual discharge, duration of, 24 quantity of, 24

Menstruation, age of first, 23

anatomy of, 24

disorders of, 743

frequency of, 30

periodicity of, 25

phenomena of, 23

general, 23

local, 23

causes of, 744

treatment of, 744

protracted, 24

scanty, 24, 749

Mercuric bichlorid as an antiseptic agent, 35

Mesonephros, development of, 504

glomeruli of, development of, 505

Metanephros, development of, 504

Metritis, 202

acute, 207

bacillus coli communis in, 208

causes of, 207

bacterial, 208

existing, 207

predisposing, 207

diagnosis of, 210

etiology of, 207

gonococcus of Neisser in, 208

pathology of, 208

perilymphangitis and, 209

periphlebitis and, 209

prognosis of, 211

pyæmia in, 212

sapræmia in, 211

septicæmia in, 211

staphylococcus in, 208

streptococcus in, 208

symptoms of, 210

treatment of, 211

abortive, 211

expectant, 212

palliative, 212

prophylactic, 211

surgical, 212

atrophic, 252

non-puerperal, 254

puerperal, 253

chronic, 250

arteriosclerosis of, 254

diagnosis of, 255

differential, 255

from carcinoma of uterus, 417

dysmenorrhœa and, 758

etiology of, 250

pathology of, 251

physical signs of, 255

symptoms of, 255

treatment of, 256

cirrhotic, 252

classification of, 205

anatomical, 205

etiological, 205

pathological, 205

differentiated from myoma of uterus, 387

from ovarian cysts, 465

diphtheritic, 209

dissecting, 209

hypertrophic, 251

non-puerperal, 251

puerperal, 251

interstitial, 252 
Metritis, nomenclature of, 206

puerperal, treatment of, 215

with alcohol, 215

with antistreptococcic serum, 215

with argentum colloidale, 215

with Credé's ointment, 215

with creolin, 215

by gauze curettage, 215

by irrigation, $21 j$

Metrorrhagia. See Uterine hemorrhage.

Microcystic degeneration of ovary, 440

Minor manipulations, asepsis of, 49

of liands, 49

of instruments, 49

of patient, 49

operations, 99

anæsthesia in, 100

chloroform, 100

cocaine, 100

ether, 100

salt water, 101

curettage in, 115

technique of, 117

dilatation of uterus, 109

- diverging instruments in, 112

forcible, technique of, 115

graduated bougies in, 112

incision in, 110

Dudley's, 111

S c hroeder's method, 110

special advantages of each method, 114

tents in, 111

during pregnancy, 105

instruments in, 101

seissors, 101

sponge-holders, 103

uterine tenaculum, 103

multiple, 105

plastic, 106

after-treatment of, 109

application of sutures in, 107

assistants in, 109

denudations in, 106

needles in, 107

removal of sutures, 109

union by first intention in, 106

preparatory treatment for, 99

Staffordshire knot, 118

tables for, 99

Mittelschmerz, 761

Moles, uterine, a cause of hemorrhage, 752

Morcellation of myoma of uterus, 384

Morgagni, hydatid of, development of, 510

Mucous polypi of cervix uteri, 218, 219 of endometrium, 230
Mucous polypi of uretlira, 484

Mueller, ducts of, development of, 507

Multilocular ovarian cysts, 438

Mycotic vulvovaginitis, 184

diagnosis of, 184

etiology of, 184

prognosis of, 184

symptoms of, 184

treatment of, 185

Myoma of Fallopian tubes, 481

of ovary, 436

of round ligament, 482

diagnosis of, 482

treatment of, 482

of uterus, 366

classification of, 369

cervical, 372

intramural, 369

submucous, 370

subperitoneal, 372 .

complete abdominal hysteromyomectomy for, 394

diagnosis of, 374

technique of, 394

differential, 375

from carcinoma, 376

from displacement of uterus, 377

from floating kidney, 379

from incomplete abortion, 377

from inversion of uterus, 377

from metritis, 377

from the ovary, 378

from pelvic cysts, 378

from pelvic inflammatory infiltrations, 378

from sactosalpinx, 378

from sarcoma, 376

from tubal pregnancy, 376

etiology of, 366

histogenesis of, 367

histology of, 367

intraligamentous, 390

nomenclature of, 367

pathology of, 366

prognosis of, $\mathbf{3 7 9}$

non-operative, 379

operative, 379

secondary changes of, 367

calcification, 367

cystic degeneration, 367

fatty degeneration, 367

malignant changes, 369

mucoid degeneration, 367

septic infection, 368 
Myoma of uterus, supravaginal hysteromyomectomy for, 391

symptoms of, 373

hemorrhage, 373

pain, 374

pressure and traction, 373

treatment of, 380

non-surgical, 380

electrolysis, 381

intra-uterine styptics, 381

tamponade, 381

manipulations, 380

surgical, 381 medication, 380

palliative, 382

radical abdominal operations, 386 vaginal operations, 382

Myomectomy, abdominal, drainage in, 388

in treatment of myoma of uterus, 386

during pregnancy, 409

Myometrium, 202

Myosalpinx, 269

N.

Nabothian glands, 219

Nails, sterilization of, 42

Neisser, gonococcus of, 33,167

Nephrectomy, 299

in acute metritis, 208

Nephritis, 355

Nerves, sciatic, palpation of, 65

Neuroma of vulva, 364

Nitze's cystoscope, 86

Noeggerath, latent gonorrhœa of, 187

Nuck, canal of, development of, 509

Nymphæ. See Labia minora.

development of, $510^{\circ}$

malformations of, 524

\section{O.}

Ö̈рноRECTOMY, effect of, 302

Oöphorosalpingectomy, 291

Dudley's operation for, 292

Operations, major, 120

abdominal section, 120

adhesions in, 124

closure of wound in, 126

dressings and bandages in, 134

incision in, 122 exploratory, 122

median through

linea alba, 122

intraperitoneal hæmo-

$\begin{array}{cc}\text { stasis in, } \\ 124 & \\ \text { utures } & \text { in, } \\ 125 & \end{array}$

Operations, major, abdominal section, preparatory treatment of, 122

sponges in, 129

Trendelenburg position in, 120

substitute for, 122

after-treatment of, 148 complicated cases, 150

fecal fistula in, 157

hemorrhage in, 151

hysterical vomiting, 155

obstruction of bowels in, 155

removal of sutures in, 158

sepsis in, 153 general, 154

localized, 153

shock in, 150

stitch-abscess in, 158

ventral hernia in, 158

simple cases, 148

care of bladder in, 149

of bowels in, 149

of cicatrix in, 150

food in, 150

getting-up in, 150

hot-water bags, 149

pain in, 150

rest, 148

thirst, 149

drainage in, 138

comparison of results of, 138

evil results of, 139

fecal fistula, 139

hernia, 139

iodoform poisoning, 139

obstruction of bowel, 139

vesical complica-

forms of, 142 tions, 139

abdominal, 145

capillary, 142

postural, 145

tubular, 142

vaginal, 146

peritoneal, contraindica-

tions for, 142

indications for, 142

- to prevent infection, 139

in sacral resection, 137

in vaginal section, 136

minor, 99 
Operations, minor, anæsthesia in, 100 chloroform, 100 cocaine, 100 ether, 100 salt water, 101 curettage in, 115, 117 dilatation of uterus, 109

diverging instruments in, 112

forcible, technique of, 115 graduated bougies in, 112 incision in, 110

Dudley's, 111

Scliroeder's method, 110

special advantages of each method, 114

tents in, 111

during pregnancy, 105

instruments in, 101

scissors, 101

sponge-holders, 103

uterine tenaculum, 103

multiple, 105

plastic, 106

after-treatment of, 109

assistants in, 109

denudation in, 106

needles in, 107

sutures in, application of, 107

removal of, 109

union by first intention in, 106

preparatory treatment for, 99

Staffordshire knot, 118

tables for, 99

- Os uteri, pinhole, 225

Ovarian cysts, 438

corpus luteum cysts, 440

cyst-adenomata, 441

papillary, 442

simple, 441

dermoid, 444

complicated, 445

simple, 444

diagnosis of, 456

adhesions, 458

differential, 459

from ascites, 466

from dilated stomach, 462

from distended bladder, 462

from fat in abdominal wall, 462

from fecal accumulations, 462

from gestation in one horn of 'bifurcated uterus, 464

from hæmatometra,
Ovarian cysts, diagnosis of, differential, from hydatid cysts, 467

from hydrometra, 465

from metritis, 465

from normal gestation, 463

from phaniom tumor, 462

from physometra, 465

from pyometra, 465

from renal tumors, 468

from tubal pregnancy, 463

from tympanites, 462

from uterine carcinoma, 464

sarcoma, 464

malignancy, 458

distinction between parovarian cyst and, 459

exploratory incision in, 469

follicular, 439

formation of pedicle of, 448

mode of development of, 438

multilocular, 438 .

prognosis of, 458

removal of, 470

abdominal incision, 470

accidents in, 479

after-treatment of, 479

closure of wound after, 479

complications of, 479

drainage in, 479

emptying and delivery of cyst, 472

ligature of pedicle, 479

preparatory treatment, 470 secondary changes in, 451

infection, 451 adhesions, 452 suppuration, 454

rupture of, 453

causes of, 454

diagnosis of, 454

prognosis of, 454

results of, 454

symptoms of, 454

treatment of, $45 j$

twisting of pedicle, 452

symptomatology of, 455

unilocular, 438

hydrocele, 450

pregnancy, 486

treatment of, 502

by abdomen, 502

by vagina, 502

Ovaries, absence of, 511

accessory, 511

anatomy of, 25, 438

conservative operation on, 331

contraction of, 761

cortex of, 438

cyst of, adenomatous, 441

intraligamentous, 479 
Ovaries, Graafian follicle of, 438 hernia of, 742

diagnosis of, 742

treatment of, 742

malformation of, 511

clinical significance of, 512

diagnosis of, 512

medullary zone of, 438

microcystic degeneration of, 440

papilloma of, 443

removal of, for hæmatometra, 535

sclerosis of, 761

senile changes in, 29

supernumerary, 511

tumors of, solid, 436

diagnosis of, 436

treatment of, 436

vascular zone of, 438

Ovariotomy, 470

abdominal incision in, 470

adhesions in, 478

appliances for, 470

closure of wound in, 479

delivery of cyst in, 478

during pregnancy, 480

emptying of cyst in, 478

instruments for, 470

ligature of pedicle in, 478

preparatory treatment for, 470 vaginal, 480

Ovaritis, 281

chronic, dysmenorrhœa and, 758

diagnosis of, 282

differential, 283

etiology of, 281

pathology of, comparative, 281

physical signs of, 282

symptoms of, 282

treatment of, 283

Ovula Nabothi, 219

Ovulation, 25

Oxalic acid as an antiseptic agent, 35, 41

\section{P.}

Pachydermia of vulva, 358

Pachyperitonitis, 285

Papillary erosions of cervix uteri, 219

Papilloma of ovary, 443

benign, 436

of vulva, 359

gonorrhœal, 360

treatment of, 361

non-specific, 360

simple, 360 reatment of, 360

syphilitic, 361

Paradidymis, development of, 509

Parametritis. See Pelvic cellulitis, 260

Paravaginal hysterectomy in carcinoma of uterus, 422

Paravaginitis, 191

treatment of, 191
Paroöphoron, development of, 508, 509

Parovarian cysts, 438, 446. See also Ovarian cysts.

contents of, 448

distinction between ovarian cysts and, 449

Parovarium, development of, 509

Pathological amenorrhœa, 23

Pedicle, ligature of, in removal of ovarian cysts, 478

Pediculi pubis in vulvitis, 180

Pelvic cellulitis, 260

acute metritis and, 209

anatomy of, 260

diagnosis of, 263

differential, 265

etiology of, 260

pathological anatomy of, 261

pathology of, 261

prognosis of, 266

symptoms of, 263

consistence of, 265

form of, 264

immobility, 265

location of, 264

pain in, 265

relations of, 265

treatment of, 266

non-surgical, 267

surgical, 266

cysts differentiated from myoma of uterus, 378

hæmatocele resulting from tubal

pregnancy, 494

inflammation, 257

ctiology of, 258

routes of infection, 257

by blood-vessels, 257

by continuity of $\mathrm{mu}$ cosa, 257

from extrapelvic organs, 257

by lymph-vessels, 257 significance of, 258

peritonitis, 283

description of, 283

diagnosis of, 286

differential, 286

pathology of, 283

symptoms of, 285

treatment of, 287

non-surgical, 287

by electricity, 289

general, 287

by hot hip-pack, 289

local, 288

by massage, 289

medical, 287

surgical, 291

by abdominal section, 291

advantages of, 328

complications in, 294 
Pclvis peritonitis, treatment of, surgical, by abdominal section, D u dle y's oreration, 299

by vaginal section, 299

advantages of, 329

anterior, 300

posterior, 300

suppuration, acute, vaginal incision and drainage for, 326

Penis, development of, 510

Perilymphangitis. See Pelvic cellulitis. acute metritis and, 209

Perimetritis, tubercular, 327

Perineal region, anatomy of, 538 functions of, 539

Perineorrhaphy, 549

complete, 560

treatment of, after-, 564

preparatory, 562

denudation, 562

direction of tear, 549

passing of sutures, 563

mechanism of, 549

objects of, 560

for prolapsus uteri, 556

technique of, for incomplete rupture, 554

primary operation, 554

secondary operation, 554

treatment of, preparatory, 554

Perineum, anatomy of, 538

functions of, 539

lacerations of, 540

eauses of, 541

complete, 541,544

incomplete, 543

prevention of, 541

results of, 542

Periphlebitis. See Pelvic cellulitis. acute metritis and, 209

Perisalpinx, 269

Peritoneal drainage, 142

Peritonitis, adhesive, 283

after abdominal operations, 154

exudative, 283

pelvic, 283

description of, 283

diagnosis of, 286

differential, 286

pathology of, 283

symptoms of, 285

treatment of, 287

non-surgical, 287

surgical, 291

by abdominal section, 291

plastic, 283

by vaginal section, 299

tubercular, 285

Pessaries, adjustment of, 688

in anteflexion of uterus, 718
Pessaries, Emmet's, 688

functions of, 686

Schultz's, 687

Smith's, 687, 689

Thomas', 690

Phantom tumors differentiated from ovarian cysts, 462

Phosphatic deposits in vesicovaginal fistula, 597

Physiological amenorrhœa, 23

periods, 21

infancy, 21

maturity, 28

menopause, 29

puberty, 22

senility, 31

Physometra differentiated from ovarian cysts, 465

Pinhole os uteri, 224

Placenta, formation of, in tubal pregnancy, 487

retained, differentiated from carcinoma of uterus, 416

Plastic operations, 106

after-treatment of, 109

assistants in, 109

denudation in, 106

needles in, 107

sutures in, application of, 107

removal of, 109

union by first intention in, 106

peritonitis, 283

Pneumococcus, 33

Polypoid endocervicitis, 224

glandular enlargements of cervix uteri, 219

Post-abortum endometritis, 232

Potassium permanganate as an antiseptic agent, 35,41

Pozzi, diffuse cellulitis of, 261

Precocious menstruation, 24

Pregnancy, abdominal, 486

minor operations during, 105

myomectomy during, 409

ovarian, 486

treatment of, 502

ovariotomy during, 480

tubal, 486

abortion in, 490

course of, 490

development of, 490

diagnosis of, 497

differential, 498

from myoma of uterus, 375

from ovarian cysts, 463

etiology of, 486

formation of amnion in, 487

of chorion in, 487

of decidua in, 487

of placenta in, 487

frequency of, 488

pelvic hæmatocele resulting

from, 494

prognosis of, 499 
Pregnancy, tubal, rupture in, 491 secondary changes in, 493 symptoms of, 493

treatment of, 499

abdominal versus vaginal route, 502

after abortion, 500 rupture, 500

before abortion, 500 rupture, 500

if abortion has occurred, 500

if gestation has advanced beyond fourth or fifth month, 501

if rupture has occurred, 500 varieties of, 488

ampullar, 490

interstitial, 489

isthmic, 490

viability of child at term, 499

Prepuce, adherent, 526

malformations of, 524

Probes, dangers of, 76 passage of, 75

Procidentia uteri. See Uterus, descent of.

Proctoscope in examination of anus, 78 of rectum, 78

Prolapse of urethral mucosa complicating urethritis, 338 treatment of, 339

Pronephric ducts, development of, 504

Pronephros, development of, 504 glomeruli of, development of, 505

Prostate gland, development of, 510

Protracted menstruation, 24

Pruritus of vulva, 194 course of, 196 diagnosis of, 196 etiology of, 195 pathology of, 195 prognosis of, 196 symptoms of, 196 treatment of, 197 surgical, 197

Pryor on treatment of endometritis, 213

Puberty, anatomical basis of, 22 care during, 26

education during, 27

goitre of, 28

physiological features of, 23

Puerperal atrophic metritis, 253 fever, 215

treatment of, 215

metritis, hypertrophic, 251

treatment of, 215

with alcohol, 215

with antistreptococcic serum, 215

with argentum colloidale, 215

with Créde's ointment, 215

with creolin, 215

by gauze curettage, 215

by irrigation, 215
Purulent salpingitis, 273

Руæmia, 33

in acute metritis, 212

Pyelitis, 355

Pyocolpos, 530

Pyometra, 530

differentiated from ovarian cysts, 465

Pyosalpinx, 271, 530

Pyo-ureter, 355

R.

$\mathrm{R}_{\text {ADICAL abdominal hysterectomy in }}$ carcinoma of uterus, 423

Rectal examination, conjoined, 62

Rectocele, 544

in lacerations of perineum, 54

Rectovaginal examination, conjoined, 64

fistula, 635

causes of, 635

diagnosis of, 635

prognosis of, 635

treatment of, 635

Rectum, examination of, 78

proctoscope in, 78

sigmoidoscope in, 78

Reid's drainage-tube, 143

Renal tumors differentiated from ovarian cysts, 468

Retroflexion of uterus, 671

congenital, 709

course of, 672

diagnosis of, 673

of complications, 673

differential, 674

in perineum, 673

etiology of, 671

pathology of, 671

symptoms of, 672

treatment of, 675

intra-abdominal shortening of round ligaments in, 700

method of replacement, 676

obstacle to replacement, 675

replacement and retention of retroposed uterus, 679 retention by pessaries, 684 surgical, 687

abdominal hysterorrhaphy in, 702

Alexander's operation, 691

shortening of uterosacral ligaments in, 702

vaginal hysterorrlıphy in, 708

Retrolocation of uterus, 636

diagnosis of, 636

symptoms of, 636

treatment of, 636 
Retroversion of uterus, 668 congenital, 709 course of, 669 degrees of, 670 description of, 668 diagnosis of, 670 etiology of, 668 prognosis of, 670 symptoms of, 669 treatment of, 671,675 method of replacement, 676

obstacles to replacement, 675

retention by pessaries, 684 surgical, 687

abdominal hysterorrhaphy in, 702

Alexander's operation, 691

in tra-abdominal shortening of round ligaments in, 700

shortening of uterosacral ligaments in, 702

vaginal hysterorrhaphy, 708

Reynolds on Dudley's operation for anteflexion of uterus, 726

Ries on radical abdominal hysterectomy, 423

Rosenmueller, organ of, development of, 509

Round ligament, intra-abdominal shortening of, in Alexander's operation, 700

tumors of, 482

Rubber gloves, 42

Rudimentary development of tubes, 513 ovaries, 512

Rumpf on radical abdominal hysterectomy, 423

Rupture in tubal pregnancy, 491

Russell on radical abdominal hysterectomy, 423

\section{S.}

SACRAL resection, 137

Sactosalpinx, 271

chronic, vaginal incision and drainage for, 325

differentiated from myoma of uterus, 578

hæmorrhagica, 271

purulenta, 271

serosa, 271

Salpingitis, 268

catarrhal, 273

chronic, dysmenorrhœa and, 758

classification of, 269

diagnosis of, 275

differential, 278

etiology of, 269

normal anatomy of, 268
Salpingitis, pathology of, 270

profluens, 761

prognosis of, 280

purulent, 273

symptoms of, 274

comparative, 275

mechanical disturbances, 275

menstrual disturbances, 275

pain, 274

treatment of, 281 surgical, 305

tubercular, 276 diagnosis of, 277

Salpingo-stomatomie, 330

Salt water infusion, 153

Sapræmia, 32

in acute metritis, 211

Sarcoma differentiated from myoma of uterus, 376

of endometrium, 237

of Fallopian tubes, 482

of ovary, 436

of round ligament, 483

of urethra, 484

of uterus, 431

classification of, 431

course of, 432

diagnosis of, 432

differentiated from carcinoma, 417

from ovarian cysts, 464

etiology of, 431

histogenesis of, 431

pathological anatomy of, 431

prognosis of, 432

spindle-cell, 432

symptoms of, 432

treatment of, 433

of vagina, 365

treatment of, 365

of vulva, 362

treatment of, 363

Sarcomatous degeneration of uterus, 432

Scanty menstruation, 24

Schauta on paravaginal hysterectomy, 423

on radical abdominal hysterectomy, 423

Schleich's solution of cocaine, 100

Schroeder's incision in dilatation of uterus, 110

operation for chronic endocervicitis, 222

Schuchardt's operation, 422

Schultz pessary, 687

Sciatic nerves, palpation of, 65

Sclerosis of ovary, 761

Scrotum, development of, 510

Senile endometritis, 232

vulvovaginitis, 186

Senility, 31

Sepsis after abdominal operations, 153

general, 154

treatment of, 154

localized, 153

Septic infection, 32 
Septicæmia, 32

in acute metritis, 211

Sex, differentiation of, 510

Sigmoidoscope in examination of anus, 78

of rectum, 78

Silkworm gut, sterilization of, 43

Simon's speculum, 73 use of, 73

Simpson's sound, 75

Sims' glass vaginal plug, 600

position, 70

correct, 70

incorrect, 71

sigmoid catheter, 608

sound, 75

speculum, 69 self-retaining, 70 use of, 71

Sinuses, prevention of, 157

Skene's glands, anatomy of, 333 inflammation of, 334

Smith's pessary, 687,689

Soap as an antiseptic agent, 35

Sodium carbonate as an antiseptic agent, 35

Sounds, dangers of, 76

passage of, 75

Simpson's, 75

Sims', 75

Speculum, Simon's, 73 use of, 73

Sims', 69

$$
\text { self-retaining, } 70
$$

use of, 71

Sphincter, rigid, in vulvovaginitis, 178

Sponges, sterilization of, 43

Staffordshire knot, 118

Stapfer, cellulitis of, 263

Staphylococcus, 33

Sterility, 763

acquired, 767

causes of, congenital, 766

indeterminate, 769

classification of, 763

absolute, 764

complete, 764

partial, 764

relative, 764

definition of, 763

diagnosis of, 770

due to anteflexion of uterus, 716

etiology of, 764

prognosis of, 770

statistics of, 763

treatment of, 770

Sterilization, 42

of beard, 42

by boiling, 35

of catgut, 43

by chromic acid process, 44

by dry heat process, 44

of dressings, 43

of field of operation, 46

of gauze, 45
Sterilization of hair, 42

of instruments, 42

of nails, 42

of patient for abdominal section, 45

of sea sponges, 43

of silkworm gut, 43

of sponges, 43

by steam, 35

of towels, 43

of water, 43

Sterilizers, 37

Dudley's, 38

hospital, 37

steam, 37

Stomach, dilated, differentiated from ovarian cysts, 462

Streptococcus in acute metritis, 208 pyogenes, 33

Streptothrix actinomyces, 33

Submucous myoma of uterus, 370

Subperitoneal myoma of uterus, 372

Suburethral abscess complicating metritis, 338

treatment of, 339

Sulphuric ether as an antiseptic agent, 35

Supernumerary tubes, 513

Supravaginal hysteromyomectomy for myoma of uterus, 391

disfection of vagina in, 391

Sutures in plastic operations, application of, 107

removal of, 109

removal of, after abdominal operations, 158

Syplinis of uterus, differentiated from carcinoma, 417

Syphilitic vulvoraginitis, 185

T.

TAMPONADE, 94

indications for, 94

hemorrhage, 96

inflammation, 94

Tate's method of treatment in inversion of uterus, 739

Tents in dilatation of uterus, 111

Teratomata of ovary, 445

Thomas' pessary, 690

Topical applications, 96

to endometrium, 96 selection of cases, 96 technique of, 98

Torsion of uterus, 727 causes of, 727

diagnosis of, 728

treatment of, 729

Torrels, sterilization of, 43

Toxæmia, 32

Trachelorrhaphy for lacerations of cervix, 579

after-treatment of, 592

approximation in, 583

curet tage in, 581

denudation in, 583 
Trachelorrhaphy for lacerations of cervix, disinfection in, 581 hemorrhage in, 585 immediate operation in, 580

instruments for, 581

preliminary dilatation in, 582

preparatory treatment of, 580

removal of cicatricial plug in, 584

results of, 592

secondary operation in, 530

sutures in, 585

Transversus perinei muscle, $\mathbf{5 4 3}$

Treatment of acute metritis, 211

of adenocarcinoma of vulva, 362

of amenorrliœa, 748

of anteflexion of uterus, 717

of antelocation of uterus, 636

of anteversion of uterus, 711

of ascent of uterus, 636

Bicr's cupping, 98

of carcinoma of uterus, 420

of vagina, 365

of vulva, 362

of chancroidal vulvovaginitis, 186

of chorio-epithelioma of uterus, 435

of chronic endocervicitis, 221

endometritis, 238

metritis, 256

of congenital gynatresia, 534

of cystitis, 348

of cysts of round ligaments, 482

of vagina, 364

of deciduoma malignum, 435

of descent of uterus, 647

of diphtheritic vulvovaginitis, 183

of dysmenorrhœa, 760

of cezema of vulva, 192

of emphysematous vaginitis, 191

of endometritis, 214

of epithelioma of uterus, 430

of erysipelatous vulvovaginitis, 183 of fibroma of round ligament, 482

of fibromyoma of vulva, 363

of furuncular vulvitis, 190

of glandular vulvovaginitis, 187

of gonorrhœal papilloma of vulva, 361

vulvovaginitis, 181

of hemorrhage in major operations,

151

of hermaphrodism, 528

of hernia of ovary, 742 of uterus, 742

of hydrocele of round ligament, 482

of hyperæsthesia of vulva, 200

of hysterical vomiting after abdomi-

nal operations, 155

of incontinence of urine, 772

of injuries of vulva, 537

of inversion of uterus, 735,736

of kraurosis of vulva, 194
Treatment of lacerations of cervix, 579

of lateral location of uterus, 636

of lipoma of vulva, 363

local, by tamponade, 94 by topical application, 96 by vaginal douche, 91

of mycotic vulvovaginitis, 185

of myoma of round ligament, 482 of uterus, 380

of non-specific papilloma of vulva, 360

of obstruction of bowels after

abdominal operations, 156

operative, of hypospadias, 523

of ovarian pregnancy, 502

of ovaritis, 283

of paravaginitis, 191

of pelvic cellulitis, 266

peritonitis, 287

of premature menstruation, 744

preparatory, of abdominal section, 122

for minor operations, 99

of prolapse of urethral mucosa, 339

- of pruritus of vulva, 197

of puerperal fever, 215

metritis, 215

of rectovaginal fistula, 625

of retroflexion of uterus, 675

of retrolocations of uterus, 636

of retroversion of uterus, 671,675

of salpingitis, 281

surgical, 305

of sarcoma of uterus, 433

of vagina, 365

of vulva, 363

of sepsis after abdominal operations, 154

of simple papilloma of vulva, 360

of solid tumors of ovary, 436

of sterility, 770

of suburethral abscess. 339

of torsion of uterus, 728 .

of tubal pregnancy, 499

of tuberculous vulvovaginitis, 184

of ureterovaginal fistula, 618

of urethral caruncle, 484

of urethritis, 334

of uterine hemorrhage, 754

of vaginismus, 201

of varix of vulva, 357

of vesico-uterine fistula, 617

of vesicovaginal fistula, 596, 597

of vulvitis, 180

of vulvovaginitis, 179

Trendelenburg's position in abdominal section, 120

Tubal pregnancy, 486

substitute for, 122

abortion in, 490

course of, 490

development of, 490

diagnosis of, 497

differential, 498

from myoma of uterus, 376 
Tubal pregnancy, diagnosis of, differential, from ovarian cysts, 463

etiology of, 486

formation of amnion in, 487

of chorion in, 487

of decidua in, 487

of placenta in, 487

frequency of, 488

pelvic hæmatocele resulting from, 494

prognosis of, 499

rupture in, 491

secondary changes in, 493

symptoms of, 493

treatment of, 499

abdominal versus vaginal route, 502

after abortion, 500 rupture, 500

before abortion, 500 rupture, 500

if abortion has occurred, 500

if gestation has advanced beyond fourth or fifth month, 501

if rupture has occurred, 500

varieties of, 488

ampullar, 490

interstitial, 489

isthmic, 490

viability of child at term, 499

Tubercular endometritis, 233

perimetritis, 327

peritonitis, 285

salpingitis, 276

diagnosis of, 277

suppuration, vaginal incision and drainage for, 326

Tuberculosis of uterus, differentiated from carcinoma, 418 of vulva, 363

Tuberculous vulvovaginitis, 183 treatment of, 184

Tubo-ovarian cysts, 450

Tubular drainage, 142

Tumors of bladder, 485

of broad ligament, 482

of Fallopian tubes, 481

phantom, differentiated from ovarian cysts, 462

renal, differentiated from ovarian cysts, 458

of round ligament, 482

solid, of ovary, 436

diagnosis of, 436

of urethra, 483

treatment of, 437

of uterus, 366

of vagina, 357

of vulva, 357

Tympanites, differentiated from ovarian cysts, 462
$\mathbf{U}$.

UlCERATION of cervix and chronic endocervicitis, 221

Ulcerative cystitis, 345

Unilocular ovarian cysts, 438

Ureter, fistula to external surface of, 299

injury of, in abdominal section, 298

insertion of, into bladder, 299

lateral anastomosis of, 299

repair of, in abdominal section, 298

suture of, in abdominal section, 299

wounds of, 298

Ureters, catheterization of, 84

Ureterorrhaphy, 298

Ureterovaginal fistula, 618

causes of, 618

diagnosis of, 618

treatment of, 618

Dudley clamp operation, 619

Urethra, atresia of, 521

caruncle of, 334

development of, 510

dilatation of, in treatment of cystitis, 352

reconstruction of, 523

tumors of, 483

Urethral caruncle, 483

diagnosis of, 484

differential, 484

etiology of, 483

pathology of, 483

treatment of, 484

cysts, 187

irritation due to anteflexion of uterus, 714

mucosa, prolapse of, complicating urethritis, 338

treatment of, 339

Urethritis, 333

complicated by prolapse of urethral mucosa, 338

treatment of, 339

by suburethral abscess, 339

treatment of, 339

diagnosis of, 333

etiology of, 333

gonorrhœal, 333

pathology of, 333

treatment of, 334

Urethrovaginal fistula, 618

Urinary organs, examination of, 78

catheterization in, 80

cystoscopy in, 80

comparison of, 86

cylindrical, 80

electrical, 85

value of, 86

dorsal position in, 81

inspection in, 78

knee-breast position in, 83

palpation in, 78

percussion in, 78

scgregation in, 89

urethral exploration in, 80 
Urinary organs, examination of, urethroscopy in, 80 urinalysis in, 78

Urine, incontinence of, 771

treatment of, 772 surgical, 776

Urogenital sinus, development of, 508

Uterine appendages, inflammation of, 268

removal of, 294

complications of, 294

effect of, 302

technique of, 294

in abdominal hysterectomy, 297

in adhesions, 296

in hemorrhage, 297

in intestinal opening, 297

in pus cases, 294

in urethral wounds, 298

dilators, 77

discharge in carcinoma, 413

glands, invagination of, 226

hemorrhage, 750

diagnosis of, 753

during maturity, 754 menopause, 754

etiology of, 750

of girls, 753

treatment of, 754

electrotherapeutic, 755

local, 755

surgical, 755

systemic, 754

inflammation a cause of hemorrhage, 750

moles a cause of hemorrhage, 752

tumors a cause of hemorrhage, 751

examination of, 67

Uterosacral ligaments, shortening of, in retroflexion of uterus, 702

in retroversion of uterus, 702

Uterus, anatomy of, 22, 202

blood-vessels of, 203

endometrium, 202

glands of, 202

lymphatics of, 204

lymph-vessels of, 203

minute, 202

nerves of, 203

anteflexion, 712

acquired, 713

classification of, 713

complications of, 714

congenital, 713

course of, 714

developmental, 713

diagnosis of, 716

etiology of, 713

normal, 712

pathological, 712

pathology of, 713
Uterus, anteflexion, symptoms of, 714

urethral, 714

uterine, 715

dysmenorrhœa, 716

endometritis, $715^{\circ}$

sterility, 716

vesical, 714

treatment of, 717

of complications, 717

by division of cervix uteri, 723

by Dudley's operation, 723

by electricity, 719

by forcible dilatation, 719

by local massage, 719

mechanical indications for, 718

by pessaries, 718

by suppositories of opium, 720

antelocation of, 636

diagnosis of, 636

symptoms of, 636

treatment of, 636

anteversion of, 710

acquired, 713

congenital, 710

diagnosis of, 711

etiology of, 710

prognosis of, 711

symptoms of, 710

treatment of, 711

ascent of, 635

diagnosis of, 636

symptoms of, 636

treatment of, 636

bifurcated, gestation in one horn of, differentiated from ovarian cysts, 464

carcinoma of, 411

adenocarcinoma, 411

cause of death from, 419

course of, 412

cylindrical cell, 411

diagnosis of, 414

differential, 416

from chronic metritis, 417

from endocervicitis, 417

from endometritis, 417

from hypertrophy of cervix, 417

from ichthyosis uteri, 417

from incomplete abortion, 417

from laceration of cervix, 418

from myoma, 416

from ovarian cysts, 464

from retained placenta, 416

from sarcoma, 417

from syphilis, 417 
Uterus, carcinoma of, diagnosis of, differential, from tuberculosis, 418

recurrence after removal, 419

etiology of, 412

extension of, 411

diagnosis of, 419

gland, 411

pathology of, 411

pavement-cell, 411

prognosis of, 419

symptoms of, 412

cachexia, 414

hemorrhage, 413

pain, 413

uterine discharge, 413

visceral disorders, 413

treatment of, 420

hysterectomy in, 421

ignihysterectomy, 425

mortality from, 428

paravaginal, 422

radical abdominal, 423

recurrence after, 428

operation of election in, 428

palliative, 429

descent of, 637

course of, 645

diagnosis of, 645

differential, 645

etiology and mechanism of, 637

pathology of, 643

prophylaxis of, 646

treatment of, 647

change in direction of vagina in, 650

elytrorrhaphy in, 651

general, 647

hygiene in, 647

hysterectomy in, 650

hysterorrhaphy in, 666

local, 647

narrowing of ragina in, 650

perineorrhaphy in, 655

by pessaries, 648

by plastic operations, 650

development of, 507

dilatation of, 77, 109

diverging instruments in, 112

forcible, technique of, 115

by graduated bougies, 77, 112

incision in, 110

Dudley's, 111

Schroeder's method, 110

special advantages of each

method, 114

by steel instruments, 77

by tents, 77,111

by water dilators, 77
Uterus, displacements of, 627

definition of, 631

diagnosis of, 631

from myoma, 377

general considerations of, 627

nomenclature of, 631

symptoms of, 631

endothelioma of, 430

diagnosis of, 430

pathology of, 430

treatment of, 430

flexion of, 709

foreign bodies in, hemorrhage from, 752

hernia of, 640,742

diagnosis of, 742

treatment of, 742

ichthyosis of, differentiated from carcinoma, 417

infantile, 22

inflammation of. See Metritis.

inversion of, 729

anatomy of, 732

diagnosis of, 733

from myoma, 377

etiology of, 729

mechanism of, 732

pathology of, 732

prognosis of, 735

symptoms of, 733

treatment of, acute, 735

chronic, 736

by colpeurynter, 739

by elastic pressure, 739

by hysterectomy, 742

by incision, 741

Brown's method, 741

manual, 738

Emmet's method, 738

Tate's method, 739

preparatory, 737

by spiral spray, 741

White's method, 741

by water bag, 739

varieties of, 735

lateral location of, 636

diagnosis of, 636

symptoms of, 636

treatment of, 636

malformations of 513

masculinus, development of, 510

myoma of, 366

classification of, 369

cervical, 372

intramural, 369

submucous, 370

subperitoneal, 372

complete abdominal hysteromyomectomy for, 394

diagnosis of, 374 differential, 375 
Uterus, myoma of, diagnosis of, differential, from carcinoma, 376

from displacement of uterus, 377

from floating kidney, 379

from incomplete abortion, 377

from inversion of uterus, 377

from metritis, 377

from the ovary, 378

from pelvic cysts, 378 inflammatory infiltrations, 378

from sactosalpinx, 378

from sarcoma, 376

from tubal pregnancy, 376

etiology of, 366

histogenesis of, 367

histology of, 367

intraligamentous, 390

nomenclature of, 367

pathology of, 366

prognosis of, 379

non-operative, 379

operative, 379

secondary changes of, 367

calcification, 367

cystic degeneration, 367

fatty degeneration, 367

malignant changes, 369

mucoid degeneration, 367

septic infection, 368

supravaginal hysteromyomec-

tomy for, 391

symptoms of, 373

hemorrhage, 373

pain, 374

pressure and traction, 373

treatment of, 380

non-surgical, 380

electrolysis, 381

intra-uterine styptics, 381

tamponade, 381

manipulations, 380

medication, 380

surgical, 381

palliative operations, 382

radical abdominal operations, 386

vaginal tions, 382

normal movements of, 629

position of, 628

supports of, 629

prolapse of. See Uterus, descent of. retroflexion of, 671
Uterus, retroflexion of, congenital, 709

course of, 672

diagnosis of, 673

of complications, 673

differential, 674

in perineum, 673

etiology of, 671

pathology of, 671

symptoms of, 672

treatment of, 675

method of replacement, 676

obstacles to replacement, 675

replacement and retention of retroposed uterus, 679 retention by pessaries, 684 surgical, 691

abdominal hysterorrhaphy in, 702 technique of, 706

Alexander's operation, 691

in tra-abdominal shortening of round ligaments in, 700

shortening of uterosacral ligaments in, 702

vaginal hysterorrhaphy in, 708

retrolocation of, 636

diagnosis of, 636

symptoms of, 636

treatment of, 636

retroversion of, 668

congenital, 709

course of, 669

degrees of, 670

description of, 668

diagnosis of, 670

etiology of, 668

prognosis of, 670

symptoms of, 669

treatment of, 671,675

method of replacement, 676

obstacles to replacement, 675

replacement and retention of retroposed uterus, 679 retention by pessaries, 684 surgical, 691

abdominal hysterorrhaphy in, 702

Alexander's operation, 691

intra-abdominal shortening of round ligaments in, 700

shortening of uterosacral ligaments in, 702

vaginal hysterorrhasarcoma of, 431 
Uterus, sarcoma of, classification of, 431 Vaginal incision and drainage as a temcourse of, 432

diagnosis of, 432

differentiated from carcinoma, 417

from ovarian cysts, 464

etiology of, 431

histogenesis of, 431

pathological anatomy of, 431

prognosis of, 432

spindle-cell, 432

symptoms of, 432

treatment of, 433

sarcomatous degeneration of, 432

senile changes in, 29

septus, 516

syphilis of, differentiated from carcinoma, 417

torsion of, 727

causes of, 727

diagnosis of, 728

treatment of, 728

tuberculosis of, differentiated from carcinoma, 418

tumors of, 366

unicornis, 518

ventrovaginal reposition of, 679

version of, 709

\section{V.}

Vaginisnuus, 200 porizing measure, 328

for tubercular suppuration, 326

morcellation in treatment of myoma of uterus, 383

operations, aseptic, preparation for, 48

ovariotomy, 480

section, 136

in treatment of pelvic inflammations, 299

course of, 200

etiology of, 200

treatment of, 201

Vaginitis. See Vulvovaginitis.

dissecting, 191

emphysematous, 190

treatment of, 191

gonorrhœal, in children, 181

lactic acid bacteria in, 78

superficial, 186

Van Hook on repair of ureter, 298

Varix of vulva, 357

treatment of, 357

Vas deferens, development of, 509

Ventral hernia after abdominal operations, 158

Ventro-rectovaginal reposition of uterus, 683

VAGINA, artificial, for hæmatometra, 535 aspiration of hydrosalpinx through, 324

atresia of, 521

carcinoma of, 365 treatment of, 365

changes in direction of, in descent of uterus, 650

complete absence of, 519

cysts of, 364

treatment of, 364

fibromyoma of, 365

inflammatory atresia of, 519

injuries of, 527

malformations of, 518

narrowing, in descent of uterus, 650 sarcoma of, 365

treatment of, 365

septa, 518

topical applications to, 98

tumors of, 357

Vaginal cystotomy in treatment of cystitis, 352

drainage, 146

enucleation in treatment of myoma of uterus, 383

hysterectomy in treatment of myoma of uterus, 383

hysterorrhaphy, 708 of pelvic inflammation, 304 technique of, 708

incision and drainage for acute pelvic suppuration, 326

for chronic sactosalpinx, 325

Ventro-vaginal reposition of uterus, 679

Version of uterus, 709

Vesical complications from abdominal drainage, 139

irritation due to anteflexion, 714

Vesico-uterine fistula, 617 diagnosis of, 617

treatment of, 617

Vesico-uterovaginal fistula, 593, 594

Vesicovaginal fistula, 594 cause of cystitis, 595

course of, 595

diagnosis of, 595

etiology of, 594

prognosis of, 595

symptoms of, 595

treatment of, prophylactic, 596 surgical, 597

atypical operations. 609

operations for closing, 601

preparatory, 597

Virchow, erysipelas malignum internum of, 261

Visceral diseases a cause of uterine hemorrhage, 752

Vomiting, hysterical, after abdominal operations, 155

von Rosthom on radical abdominal hysterectomy, 423

Vulva, adenocarcinoma of, 361

diagnosis of, 361

treatment of, 362

carcinoma of, 361 
Vulva, carcinoma of, cylindrical-cell, $361 \mid$ Vulvovaginal abscess, 187

pavement-cell, 361 diagnosis of, 361

cysts of, 363

eczema of, 192

treatment of, 192

elephantiasis of, 358 diagnosis of, differential, 358

enchondroma of, 364

fibromyonna of, 363

treatment of, 363

hematoma of, 358

herpes of, 192

hyperzesthesia of, 199

treatment of, 200

injuries of, $53 \overline{7}$

etiology of, 537

symptoms of, 537

treatment of, 537

kraurosis of, 193

pathology of, 193

treatment of, 194

lipoma of, 363

Longyear's operation, 194

treatment of, 363

lupus of , 183,363

malformations of, 520

neuroma of, 364

pachydermia of, 358

papilloma of, 359

gonorrhœeal, 360 treatment of, 361

non-specific, 360 treatment of, 360

simple, 360

$$
\text { treatment of, } 360
$$

syphilitic, 361

pruritus of, 194

course of, 196

diagnosis of, 196

etiology of, 195

pathology of, 195

prognosis of, 196

symptoms of, 196

treatment of, 197

sarcoma of, 362

treatment of, 363

topieal applications to, 98

tuberculosis of, 363

tumors of, 357

varix of, 357

treatment of, 357

vestibule of, development of, 510

Vulvitis. See also Vulvovaginitis.

buboes in, 180

follicular, 189

treatment of, 190

furuncular, 190

treatment of, 190

glandular, 187

diagnosis of, 187

treatment of, 187

pediculi pubis in, 180

superficial, 186

treatment of, 180 glands, inflammation of, 187

Vulvovaginitis, $\mathbf{1 7 4}$

chancroidal, 186

treatment of, 186

in children, 180

chronic, 179

classification of, 174

anatomical, 175

etiological, 174

definition of, 174

diagnosis of, 178

diphtheritic, 183

treatment of, 183

epidemics of, 176

erysipelatous, 182

erythematous, 182

gangrenous, 182

treatment of, 183

vesicular, 182

etiology of, 175

exciting causes, 175

favoring conditions, 175

follicular, 189 .

glandular, 187

diagnosis of, 187

treatment of, 187

gonorrlıeal, 181

diagnosis of, 181

treatment of, 181

mycotic, 184

diagnosis of, 184

etiology of, 184

prognosis of, 184

symptoms of, 184

treatment of, 185

pathology of, 176

senile, 186

symptoms of, 178

syphilitic, 185

treatment of, 179

tuberculous, 183

treatment of, 184

\section{W.}

WADSWORTH on Dudley's operation for anteflexion of uterus, 726

Waist constriction, 160

Waldeyer, yellow body of, development of, 509

Warts of urethra, 484

of vulva. See Papilloma of vulva.

Water, sterilization of, 42

Werder on ignihysterectomy, 425

on radical abdominal hysterectomy, 423

Werth on regeneration of epithelium, 248

Wertheim on radical abdominal hysterectomy, 424

White's method of treatment in inversion of uterus, 741

Wolffian body, development of, 504

ducts, development of, 504

ridge, development of, 503

Wounds of ureter, 298 



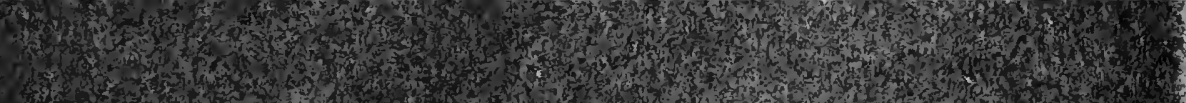

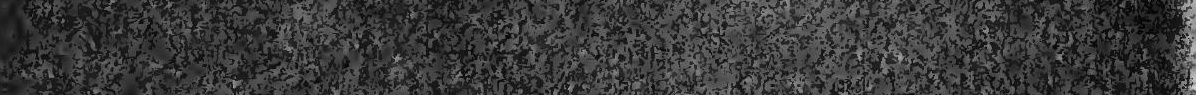

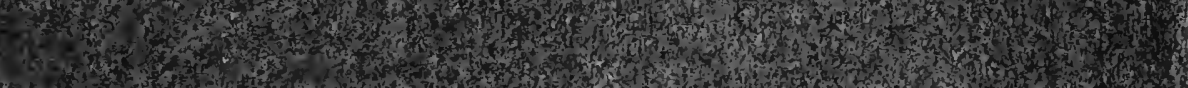

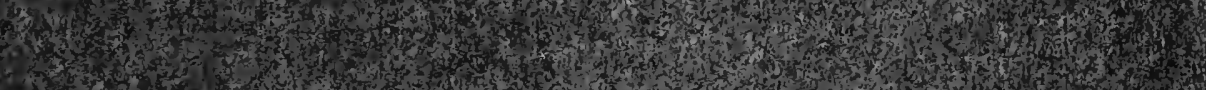

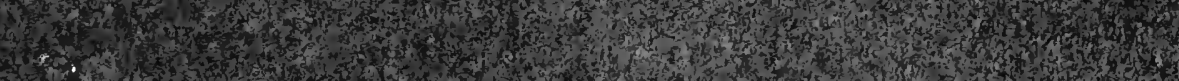

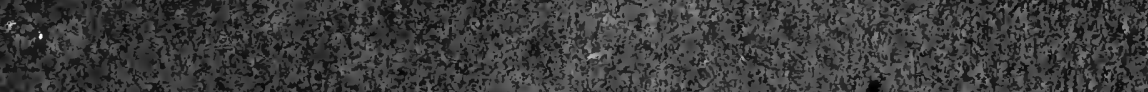

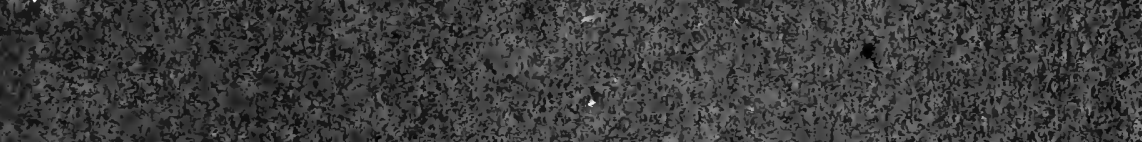
(N)

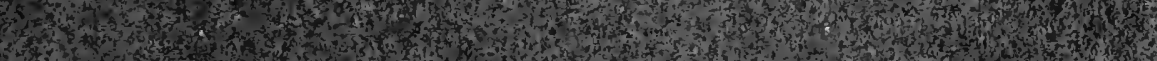

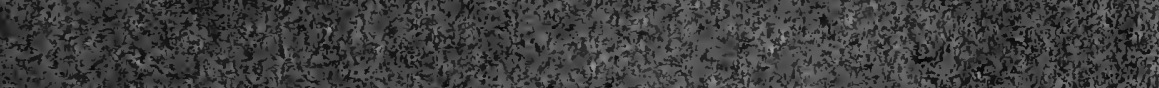
N

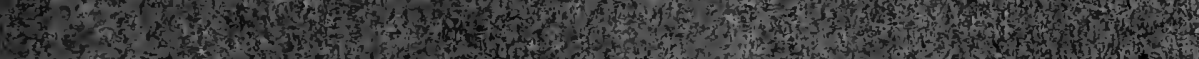

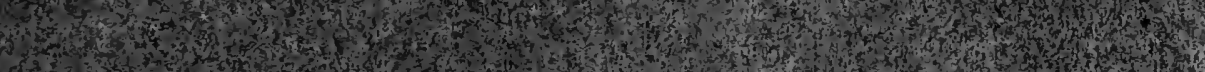

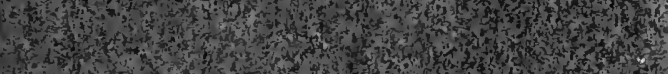

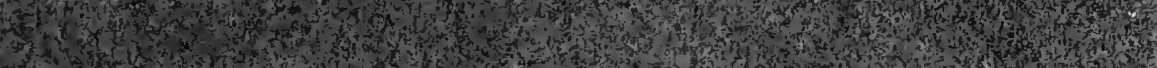
1.

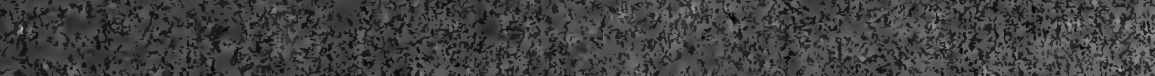

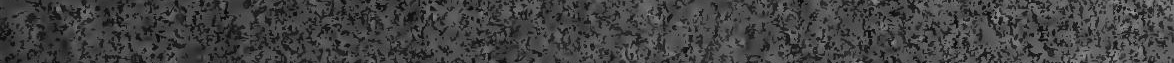
4.

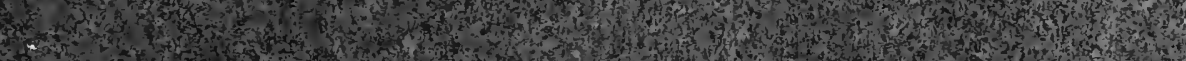

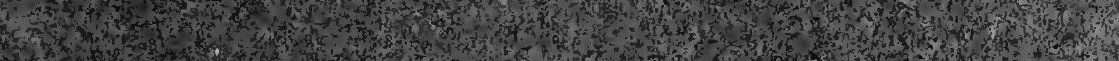

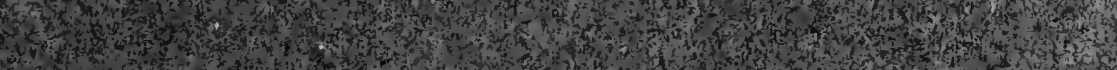

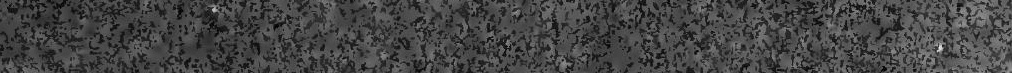

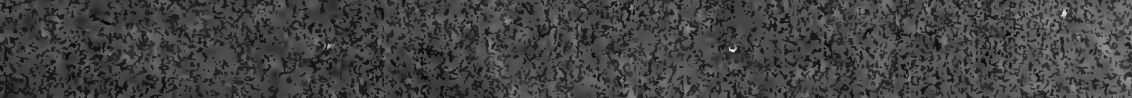

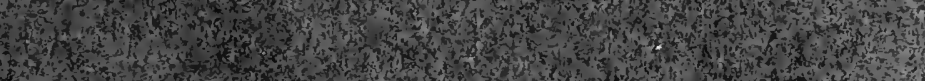
W.

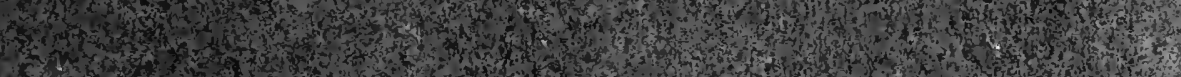
1.52

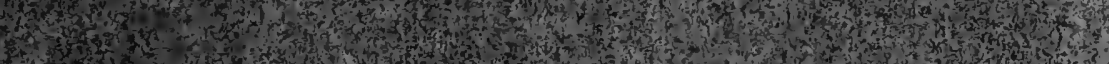
H.

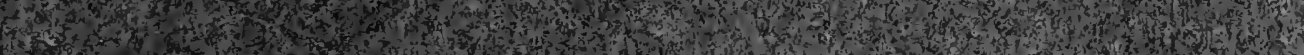

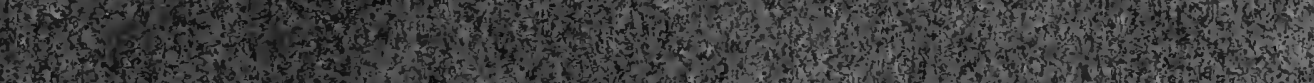

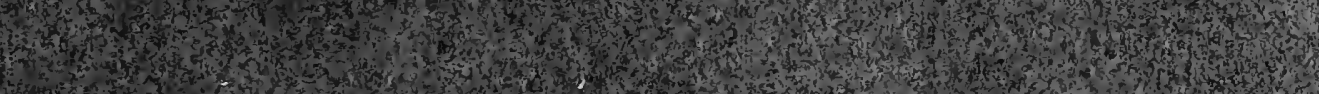

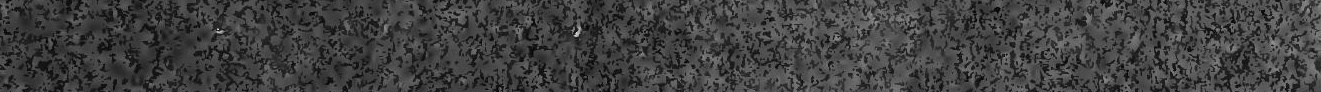

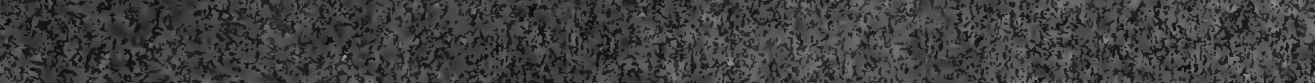

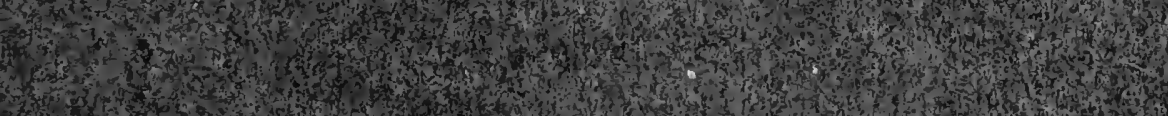
4.10 .

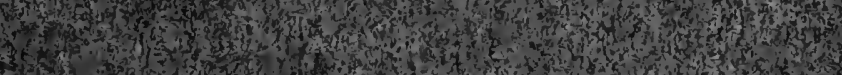

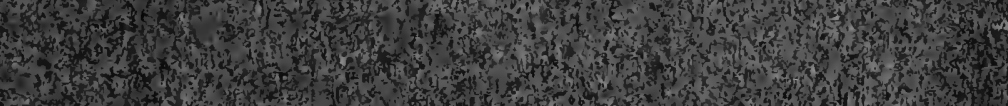

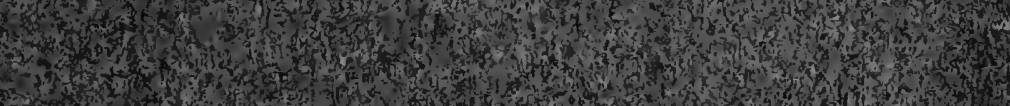
2.

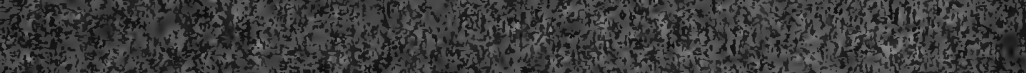

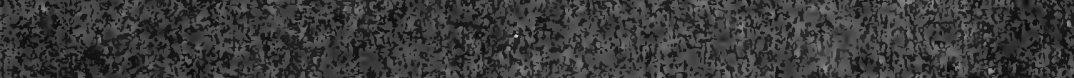
H 1.6.

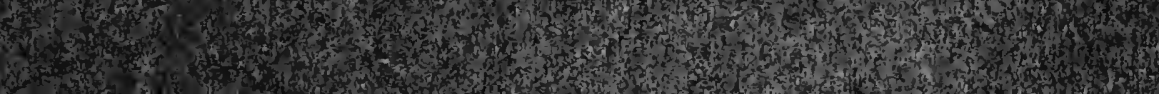




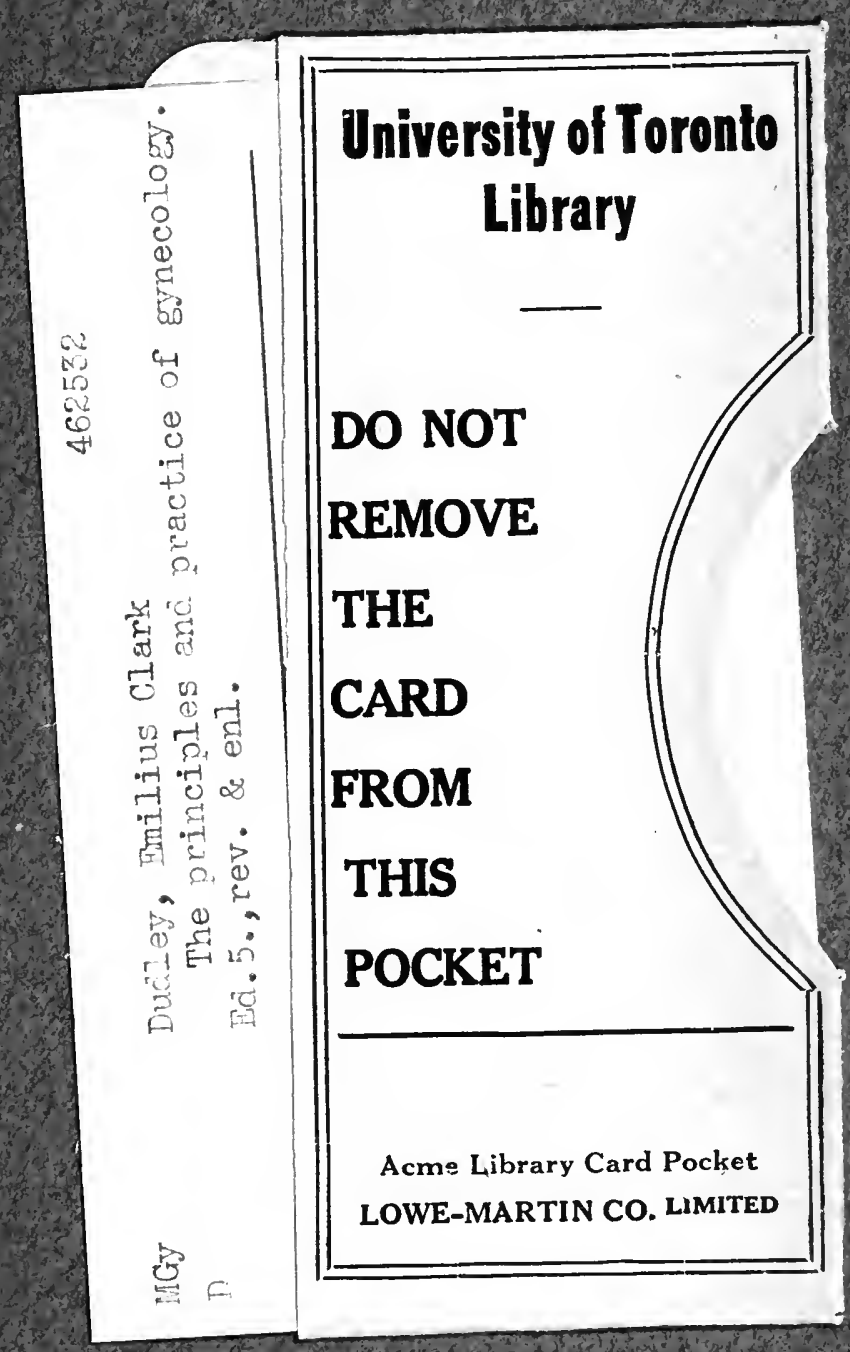


